

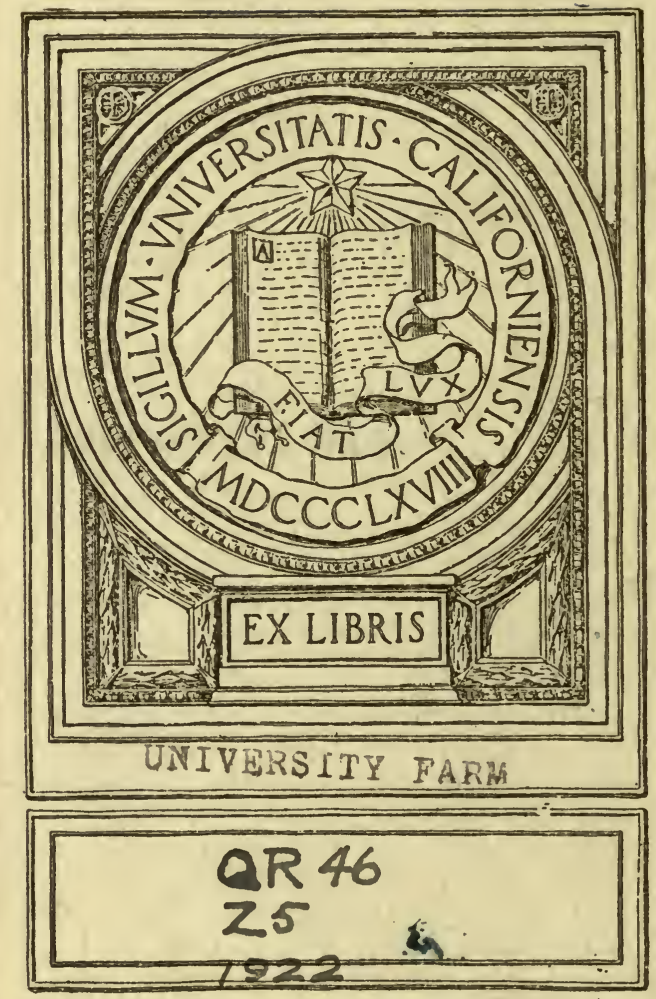


ijact Rothures

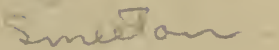

UNIVERSITY OF CALIFORNIA LIBRARY

THIS BOOK IS DUE ON THE LAST DATE

STAMPED BELOW

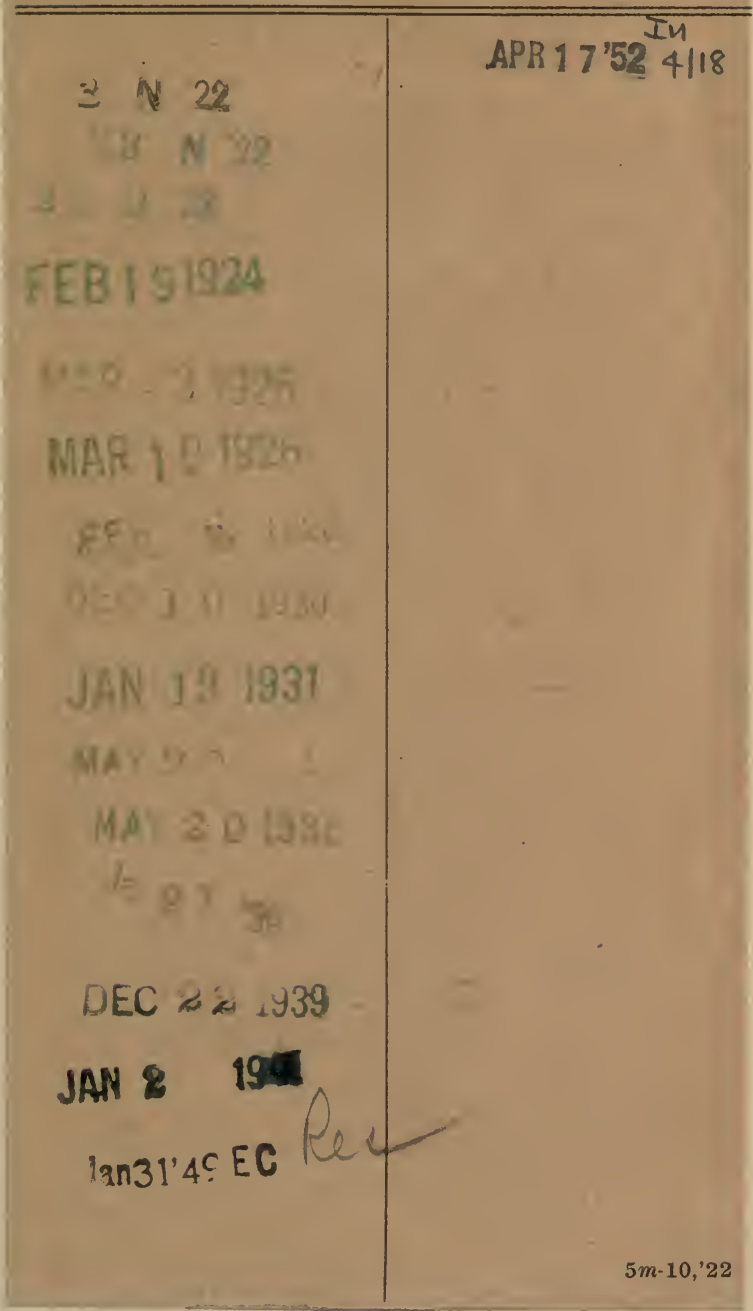


Digitized by the Internet Archive in 2007 with funding from Microsoft Corporation 
A TEX T B O K $\mathrm{OF}$ B A C T E R I OL O G Y 



\section{A TEXTBOOK OF \\ B A C T E R I O L O G Y}

A PRAC'TICAL TREATISE FOR S'TUDEN'TS

AND PRAC'TITIONERS OF MEDICINE

AND PUBLIC HEALTH

BY $\mathbf{Y}$

HANS ZINSSER, M.D.

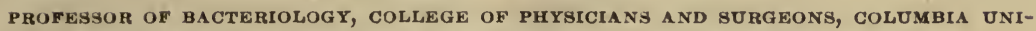

VERSTT, NEW YORK CITY; BACTERIOLOGIST TO THE PRESBYTERIAN HOSPITAL;

FORMERLY PROFESSOR OF BACTERIOLOGY AND IMMUNITY, STASTORD UNIVERSITY, CALIFORNIA; COLONEL MEDICAL OFFICERS'

RESERVE CORPS, U. S. A.

WITH A SECTION ON PATHOGENIC PROTOZOA

BY

FREDERICK F. RUSSELL, M.D.

BRIGA DIER GENERAL, MEDICAL OFFICERS' RESERVE CORPS, U. S. A., FORMERLY PROFESSOR OF BACTERIOLOGY AND PATHOLOGY, ARMY MEDICAL SCHOOL AND GEORGE WASHINGTON UNIVERSITY

(Completely revised and rewritten from the original text of $\mathrm{Hiss}$ and Zinsser)

WITH ONE HUNDRED AND SIXTY-FOUR ILLUSTRATIONS IN THE TEXT

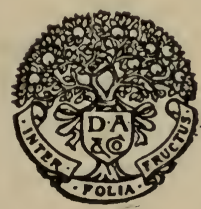

D. APPLETON AND COMPANY NEW YORK :: 1922 :: LONDON 
COPYRIGHT, 1910, 1914, 1916, 19i8, and 1922, BY

D. APPLETON AND COMPANY 


\section{PREFACE TO THE FIRST EDITION}

THE volume here presented is primarily a treatise on the fundamental laws and technique of Bacteriology, as illustrated by their application to the study of pathogenic bacteria.

So ubiquitous are the bacteria and so manifold their activities that Bacteriology, although one of the youngest of sciences, has already been divided into special fields-Medical, Sanitary, Agricultural, and Industrial-having little in common, except problems of general bacterial physiology and certain fundamental technical procedures.

From no other point of approach, however, is such a breadth of conception attainable, as through the study of bacteria in their relation to disease processes in man and animals. Through such a study one must become familiar not only with the growth characteristics and products of the bacteria apart from the animal body, thus gaining a knowledge of methods and procedures common to the study of pathogenic and non-pathogenic organisms, but also with those complicated reactions taking place between the bacteria and their products on the one hand and the cells and fluids of the animal body on the other-reactions which often manifest themselves as symptoms and lesions of disease or by visible changes in the test tube.

Through a study and comprehension of the processes underlying these reactions, our knowledge of cell physiology has been broadened, and facts of inestimable value have been discovered, which have thrown light upon some of the most obscure problems of infection and immunity and have led to hitherto unsuspected methods of treatment and diagnosis. Thus, through Medical Bacteriology-that highly specialized offshoot of General Biology and Pathology-have been given back to the parent sciences and to Medicine in general methods and knowledge of the widest application.

It has been our endeavor, therefore, to present this phase of our subject in as broad and critical a manner as possible in the sections dealing with infection and immunity and with methods of biological diagnosis and treatment of disease, so that the student and practi-

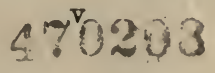


tioner of medicine, by becoming familiar with underlying laws and principles, may not only be in a position to realize the meaning and scope of some of these newer discoveries and methods, but may be in better position to decide for themselves their proper application and limitations.

We have not hesitated, whenever necessary for a proper understanding of processes of bacterial nutrition or physiology, or for breadth of view in considering problems of the relation of bacteria to our food supply and environment, to make free use of illustrations from the more special fields of agricultural and sanitary bacteriology, and some special methods of the bacteriology of sanitation are given in the last division of the book, dealing with the bacteria in relation to our food and environment.

In conclusion it may be said that the scope and arrangement of subjects treated of in this book are the direct outcome of many years of experience in the instruction of students in medical and in advanced university courses in bacteriology, and that it is our hope that this volume may not only meet the needs of such students but may prove of value to the practitioner of medicine for whom it has also been written.

It is a pleasure to acknowledge the courtesy of those who furnished us with illustrations for use in the text, and our indebtedness to Dr. Gardner Hopkins and Professor Francis Carter Wood for a number of the photomicrographs taken especially for this work.

$$
\text { P. H. H., JR., }
$$

H. Z. 


\section{PREFACE TO THE FIFTH EDITION}

The present or Fifth Edition of the Textbook of BACTERIOLOGY represents an almost complete rewriting of the book. The four preceding editions were in each case altered and brought up to date from time to time, but in all of them the original plan of presentation, conceived at the first writing, was preserved. In the present edition many and important additions of material and changes in manner of presentation have been made.

Bacteriology and the reasoning based on bacteriological and immunological discoveries have become more and more closely interwoven with the clinical and public health aspects of infectious diseases. Indeed, if this had not been sufficiently apparent before 1914, the experiences of the late war have demonstrated, conclusively, how impossible it is to organize either hospitals for infectious diseases or organizations for the control of epidemies without the intimate participation of men trained in bacteriology. It seems to us, also, to have become apparent that the bacteriologist who takes an active part in the work of a hospital or in directing sanitary undertakings, must have a very thorough understanding of the clinical and public health aspects of the problem as a whole.

The conception upon which the preparation of the new edition of the book has been based, therefore, is the belief that no thorough understanding of the clinical problems of infectious disease or of larger public health measures can be attained without thorough familiarity with the bacteriological and immunological facts upon which clinical and sanitary reasoning must be based. The book represents, therefore, in a brief way, an attempt to correlate a laboratory knowledge with the branches of medicine and prophylaxis to which it is most directly applicable.

We have felt that a Textbook of Bacteriology, primarily aimed at the needs of medical students and physicians, may be regarded as neglecting a great opportunity or, perhaps, even an obligation, if it omits emphasis upon prevention. In order to accomplish this purpose it has been necessary to add brief clinical data in the case of each 
variety of infeetion and to ineorporate in the more important chapters, brief diseussions of the principles underlying sanitary procedure.

In the sections on technique we have eliminated many methods which we have ceased to use ourselves. At the laboratory of the Bacteriological Department of the Medical School of Columbia University we have had the opportunity of having advanced students and staff try out a great many bacteriological procedures and have, in consequence, been able to eliminate a number of methods that, as matters of routine, have been practically dropped fiom our practice. We have considerably simplified the section on media. The newer methods of titration have been added.

The immunological section has been eonsiderably changed and we think simplified. It is not the purpose of a book of this kind to present a critical thesis on theoretical immunity. We have, therefore, restricted ourselves to the exposition of the more important principles and practical methods needed by routine laboratory workers. Short sections on the normal bacterial flora of the human body have been added, with partieular consideration of the important work done in recent years by Herter, Kendall, Rettger and others. Most of the sections dealing with the pathogenic microorganisms themselves have been completely rewritten and the order of presentation considerably altered in order to bring together, more logically, infections which are usually considered together from the clinical point of view. The diagnostic and therapeutic principles in which bacteriological and immunological reasoning and technique are involved have been thoroughly dealt with.

The section on Protozoa has been completely revised by one of us along the same general lines adopted for the revision of the bacteriological section.

The writers realize that the inclusion of clinical and epidemiological data in a Textbook of Bacteriology is considerably at variance with the usual treatment given to the subject in books of this kincl. But it is hoped that this manner of treatment will add considerably to the value of the book for those who are interested in microorganisms particularly in their relationship to clinical and preventive medicine.

In conclusion greatful acknowledgment is made to a number of our associates in the Department of Bacteriology at Columbia for valuable aid in the preparation of this Edition. Dr. J. G. Ilopkins, Associate in the Department who has been working with the pathogenic molds, has rewritten the section dealing with these organisms. 
Dr. J. Howard Mueller, Assistant Professor of Bacteriology, has eritically revised the sections dealing with the chemical metabolism of bacteria, and Miss Amn Kuttner, Instructor in the department, has elaborated and revised the chapter dealing with the anaerobic infections which have gained such an important place in the study of traumatic injuries. We are indebted to Dr. Osear Teague for valuable suggestions in connection with the chapters on cholera and plague.

HANS ZiNsSER

Frederick F. Russell 



\section{CONTEN'TS}

\section{SECTION I}

\section{THE GENERAL BIOLOGY OF BACTERIA AND THE TECHNIQUE OF BACTERIOLOGICAL STUDY}

OHAPTER

I. The Development and Scope of Bacteriology . . . . . . 1

II. General Morphology, Reproduction, and Chemical and Physical

Properties of the Bacteria . . . . . . . . . . . . . . . . 9

iII. The Relation of Bacteria to Environment, and Their Classification . . . . . . . . . . . . . . . 27

IV. The Biological Activities of Bacteria . . . . . . . . 45

V. 'The Destruction of Bacteria . . . . . . . . . . . 76

VI. Methods Used in the Mićoscopic Study and Staining of BacTERIA . . . . . . . . . . . . . . . . . . 111

ViI. The Preparation of Culture Media . . . . . . . . . 133

ViII. Methods Used in the Cultivation of Bacteria . . . . . . 172

IX. Methods Determining Biological Activities of Bacteria, Animal ExPERIMentation . . . . . . . . . . . . . . . . 196

X. The Bacteriological Examination of Material from Patients and Outline of Flora of the Normal Human Body . . . . . 206

\section{SECTION II}

\section{INFECTION AND IMMUNITY}

CHAPTER

XI. Fundamental Factors of Pathogenicity and Infection . . . 230

XII. Defensive Factors of the Animal Organism . . . . . . . 240

XIII. Toxins and Antitoxins . . . . . . . . . . . . . . 255

XIV. Production and Testing of Antitoxins . . . . . . . . . . 269

XV. Sensitizing Antibodies (Phenomena of Lysis, Agglutination,

Precipitation, etc.) . . . . . . . . . . . . . . . . . 277

XVi. The Technique of Serum Reactions . . . . . . . . . 301

XVII. Phagocytosis . . . . . . . . . . . . . . . . . 330 


\section{SECTION VI}

BACTERIA IN AIR, SOIL, WATER AND MILK

CHAPTER

LI. Bacteria in the Air and Soil

LII. Bacteria iN WATER

LiII. Bacteria in Milk and Milk Products. Bacteria in the

INDUSTRIES 1016

SECTION VII

\section{PATHOGENIC PROTOZOA}

CHAPTER $\quad$ PAGE

LIV. The Амсеве . . . . . . . . . . . . . . . . 1050

LV. MASTIGOPHORA . . . . . . . . . . . . . . . 1073

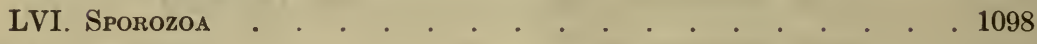

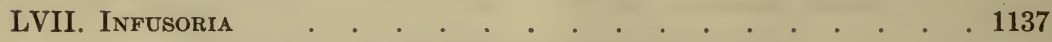

LVIII. Technique of Blood Examinations for Protozoa . . . 1140

INDEX OF AUthors . . . . . . . . . . . . . . 1145

INDEX OF SUBJECTS . . . . . . . . . . . . . . . . 1159 


\section{SECTION I}

\section{THE GENERAL BIOLOGY OF BACTERIA AND THE TECHNIQUE OF BACTERIOLOGICAL S'TUDY}

\section{CHAPTER I}

THE DEVELOPMENT AND SCOPE OF BACTERIOLOGY

As we trace back to their ultimate origins the lines of development of living beings of the animal and plant kingdoms, we find them converging toward a common type, represented by a large group of unicellular organisms, so simple in structure, so unspecialized in function, that their classification in either the realm of plants or that of animals becomes little more than an academic question. However, even such microorganisms, in which the functions of nutrition, respiration, locomotion, and reproduction are concentrated within the confines of a single cell, and in which adaptation to special conditions more readily brings about modifications leading to the production of a multitude of delicately graded transitional forms, fall into groups which, either in structure or in biological attributes show evidence of a tendency toward one or the other of the great kingdoms.

Most important of these unicellular forms, for the student of medical science, are the bacteria and the protozoa.

The former, by reason of their undifferentiated protoplasm, their occasional possession of cellulose membranes, their biological tendency to synthetize, as well as to break down organic compounds, and because of the transitional forms which seem to connect them directly with the lower plants, are generally placed in the plant kingdom. The latter, chiefly on the basis of metabolism, are classified with the animals.

Knowledge of the existence of microorganisms as minute as the ones under discussion was of necessity forced to await the perfection of instruments of magnification. It was not until the latter half of the seventeenth century, therefore, that the Jesuit, Kircher, in 
1659 , and the Dutch linen-draper, van Lecuwenhoek, in 1675, actually saw and described living beings too small to be seen with the naked eye. There can be no doubt that the small bodies seen by these men and their many immediate successors were, at least in part, bacteria. And indeed the descriptions and illustrations of several of the earliest workers correspond with many of the forms which are well known to us at the present day.

During the century following the work of these pioneers, the efforts of investigators lay chiefly in the more exact morphological description of some of the forms of unicellular life, already known. Conspicuous among the work of this period is that of Otto Friedrich Müller. In the generation following Müller's work, however, a marked advance in the study of these forms was made by Ehrenberg, ${ }^{1}$ who established a classification which, in some of its cardinal divisions, is retained until the present day.

Meanwhile the regularity with which these "animalcula" or "infusion animalcula" were demonstrable in tartar from the teeth, in intestinal contents, in well-water, etc., had begun to arouse in the minds of the more advanced physicians of the time a suspieion as to a possible relationship of these minute forms with disease. The conception of "contagion," or transmission of a disease from one human being to another, was, however, even at this time, centuries old. The fact had been recognized by Aristotle, had been reiterated by $*$ medieval philosophers, and had led, in 1546, to the division of contagious diseases by Fracastor, into those transmitted "per contactum," and those conveyed indirectly "per fomitem." It was for these mysterious facts of the transmissibility of disease, that clinicians of the eighteenth century, with remarkable insight, saw an explanation in the microorganisms discovered by Leeuwenhoek and his followers.

In fact, Plenciz of Vienna, writing in 1762, not only expressed a belief in the direct etiological connection between microorganisms and some diseases, but was the first to advance the opinion that each malady had its own specific causal agent, which multiplied enormously in the diseased body. The opinions of this author, if translated into the language of our modern knowledge of the subject, eame remarkably near to the truth, not only as regards etiology and transmission, but also in their suggestion of specific therapy.

The conception of a "contagium vivum" was thus practically 16"Die Infusionstierchen,' ete., Leipzig, 1838. 
established with the work of Plenciz and many others who followed in his train, but the astonishingly slight impression which the acute reasoning of these men left upon the medical thought of their day is illustrative of the futility of the most penetrating speculation when unsupported by experimental data.

The real advancement in the scientific development of the subject was achieved along entirely different lines. In 1837, Schwann, a botanist, showed that the yeasts, found in fermenting substances, were living beings, which bore a causal relationship to the process of fermentation. At almost the same time, similar observations were made by a French physicist, Cagniard-Latour. The opinions advanced by these men on the nature of fermentation aroused much interest and discussion, since, at that time and for a long period thereafter, fermentation was ascribed universally to protein decomposition, a process which was entirely obscure but firmly believed to be of a purely chemical nature.

Although belief in the discovery of Schwann did not master the field until after Pasteur had completed his classical studies upon the fermentations occurring in beer and wine, yet the conception of a "fermentum vivum" aroused much speculation, and the attention of physicians and scientists was attracted to the many analogies existing between phenomena of fermentation and those of disease.

The conception of such an analogy, however, was not a new thought in the philosophy of the time. Long before Schwann and Cagniard-Latour, the philosopher Robert Boyle, working in the seventeenth century, had prophesied that the mystery of infectious diseases would be solved by him who should succeed in elucidating the nature of fermentation.

Nevertheless, the diligent search for microorganisms in relation to various diseases which followed led to few results, and the successes which were attained were limited to the diseases caused by some of the larger fungi, favus (1839), thrush (1839), and pityriasis versicolor (1846). During this time of ardent but often poorly controlled etiological research, it was Henle who formulated the postulates of conservatism, almost as rigid as the later postulates of Koch, requiring that proof of the etiological relationship of a microorganism to a disease could not be brought merely by finding it in a lesion of the disease, but that constant presence in such lesions must be proven and isolation and study of the microorganism away from the diseased body must be carried out. 
It was during this period also that one of the most fundamental questions, namely, that of the origin of these minute living beings, was being discussed with much passion by the scientific world. It was held by the conservative majority that the microorganisms described by Leeuwenhoek and others after him were produced by spontaneous generation. The doctrine of spontaneous generation, in fact, was solidly established and sanctified by tradition, and had been applied in the past not alone to microorganisms. ${ }^{2}$ And it must not be forgotten that without the aid of our modern methods of study, satisfactory proof for or against such a process was not easily brought.

Needham, who published in 1749 , had spent much time in fortifying his opinions in favor of spontaneous generation by extensive experimentation. He had placed putrefying material and vegetable infusions in sealed flasks, exposing them for a short time to heat, by immersing them in a vessel of boiling water, and had later shown them to be teeming with microorganisms. He was supported in his views by no less an authority than Buffon. The work of Needham, however, showed a number of experimental inaccuracies which were thoroughly sifted by the Abbé Spallanzani. This investigator repeated the experiments of Needham, employing, however, greater care in sealing his flasks, and subjecting them to a more thorough exposure to heat. His results did not support the views of Needham, but were answered by the latter with the argument that by excessive heating he had produced chemical changes in his solutions which had made spontaneous generation impossible.

The experiments of Schulze, in 1836, who failed to find living organisms in infusions which had been boiled, and to which air had been admitted only after passage through strongly acid solutions, and similar results obtained by Schwann, who had passed the air through highly heated tubes, were open to eriticism by their opponents, who claimed that chemical alteration of the air subjected to such drastic influences had been responsible for the absence of bacteria in the infusion. Similar experiments by Schroeder and Dusch, who had stoppered their flasks with cotton plugs, were not open to

${ }^{2}$ Valleri-Radot, in his life of Pasteur, states that Van Helmont, in the sixteenth century, had given a celebrated prescription for the creation of mice from dirty linen and a few grains of wheat or pieces of cheese. During the centuries following, although, of course, such remarkable and amusing beliefs no longer held sway, nevertheless the question of spontaneous generation of minute and structureless bodies, like the bacteria, still found learned and thoughtful partisans. 
this objection, but had also failed to convince. The question was not definitely settled until the years immediately following 1860, when Pasteur conducted a series of experiments which were not only important in incontrovertibly refuting the doctrine of spontaneous generation, but in establishing the principles of scientific investigation which have influenced bacteriological research since his time. ${ }^{3}$

Pasteur attacked the problem from two points of view. In the first place he demonstrated that when air was filtered through cottonwool innumerable microorganisms were deposited upon the filter. A single shred of such a contaminated filter dropped into a flask of previously sterilized nutritive fluid sufficed to bring about a rapid and luxuriant growth of microorganisms. In the second place, he succeeded in showing that similar, sterilized "putrescible" liquids, if left in contact with air, would remain uncontaminated provided that the entrance of dust particles were prohibited. This he succeeded in doing by devising flasks, the necks of which had been drawn out into fine tubes bent in the form of a $U$. The ends of these U-tubes, being left open, permitted the sedimentation of dust from the air as far as the lowest angle of the tube, but, in the absence of an air current, no dust was carried up the second arm into the liquid. In such flasks, he showed that no contamination took place but could be immediately induced by slanting the entire apparatus until the liquid was allowed to run into the bent arm of the U-tube. Finally, by exposing a series of flasks containing sterile yeast infusion, at different atmospheric levels, in places in which the air was subject to varying degrees of dust contamination, he showed an inverse relationship between the purity of the air and the contamination of his flasks with microorganisms.

The doctrine of spontaneous generation had thus received its final refutation, except in one particular. It was not yet clear why complete sterility was not always obtained by the application of definite degrees of heat. This final link in the chain of evidence was supplied, some ten years later, by Cohn, who, in 1871, was the first to

${ }^{3}$ In a letter to his foremost opponent, at this period, Pasteur writes: "In experimental science, it is always a mistake not to doubt when facts do not compel affirmation.',

The critical spirit pervading the scientific thought of that time in France is also well expressed by Oliver Wendell Holmes, who said that he had learned three things in Paris: "Not to take authority when I can have facts, not to guess when I can know, and not to think that a man must take physic because he is sick."' 
observe and correctly interpret bacterial spores and to demonstrate their high powers of resistance against heat and other deleterious influences.

Meanwhile, Pasteur, parallel with his researches upon spontaneous generation, had been earrying on experiments upon the subject of fermentation along the lines suggested by Cagniard-Latour. As a consequence of these experiments, he not only confirmed the opinions both of this author and of Schwann concerning the fermentation of beer and wine by yeasts, but was able to show that a number of other fermentations, such as those of lactic and butyric-acid, as well as the decomposition of organic matter by putrefaction, were directly due to the action of microorganisms. It was the discovery of the living agents underlying putrefaction, especially, which exerted the most active influence upon the medical research of the day. This is illustrated by Lister's work. The suppurative processes occurring in infected wounds had long been regarded as a species of putrefaction, and Lord Lister, working directly upon the premises supplied by Pasteur, introduced into both the active and prophylactic treatment of surgical wounds the antiseptic principles which alone have made modern surgery possible.

There now followed a period in which bacteriological investigation was concentrated upon problems of etiology. Stimulated by Pasteur's successes, the long-cherished hope of finding some specific microorganism as the causal agent in each infectious disease was revived.

Pollender, in 1855, had reported the presence of rod-shaped bodies in the blood and spleen of animals dead of anthrax. Brauell, several years later, had made similar observations and had expressed definite opinions as to the causative relationship of these rods to the disease. Convincing proof, however, had not been brought by either of these observers. Finally, in 1863, Davaine, in a series of brilliant investigations, not only confirmed the observations of the two authors mentioned above, but succeeded in demonstrating that the disease could be transmitted by means of blood containing these rods and could never be transmitted by blood from which these rods were absent. Anthrax, thus, is the first disease in which definite proof of bacterial causation was brought.

Speaking before the French Academy of Medicine at this time, Davaine suggested that the manifestations of the disease might in reality represent the results of a specific fermentation produced by 
the bacilli he had found. This, in a crude way, expresses the modern conception of infectious discase.

Within a few years after this, 1868, the adherents of the parasitic theory of infectious diseases were further encouraged by the discovery, by Obermeier, of a spirillum in the blood of patients suffering from relapsing fever. It is not surprising that the successes attained in these diseases, fostering hope of analogous results in all other similar conditions, but without the aid of adequate experimental methods, should have led to many unjustified claims and to much fantastic theorizing. Thus Hallier, at about this time, advanced a theory as to the etiology of infectious diseases, in which he attributed all such conditions to the molds or hyphomycetes, regarding the smaller form or bacteria as developmental stages of these more complicated forms. Extravagant conjectures of this kind, however, did not maintain themselves for any length of time in the light of the critical attitude which was already pervading bacteriological research.

Progress was made during the years immediately following, chiefly in the elucidation of suppurative processes. Rindfleisch, von Recklinghausen, and Waldeyer, almost simultaneously, described bodies which they observed in sections of tissue containing abscesses, and which they believed to be microorganisms. Notable support was given to their opinion by similar observations made upon pus by Klebs, in 1870. In view, however, of the purcly morphological nature of their studies, the opinions of these observers did not entirely prevail. Satisfactory methods of cultivation and isolation had not yet been developed, and Billroth and his followers, with a conservatism entirely justified under existing conditions, while admitting the constant presence of bacteria in purulent lesions, denied their etiological significance. The controversy that followed was rich in suggestions which greatly facilitated the work of later investigators, but could not be definitely settled until 1880, when Koch introduced the technical methods which have made bacteriology an exact science. By the use of solid nutritive media, the isolation of bacteria and their biological study in pure culture were made possible. At about the same time the use of anilin dyes, developed by Weigert, Koch, and Ehrlich, was introduced into morphological study and facilitated the observation of the finer structural details which had been unnoticed while only the grosser methods employed for tissue staining had been available. 
With the publication of Koch's work, there began an era unusually rich in results held in leash heretofore by inadequate technical methods. The discovery of the typhoid bacillus in 1880 , of the bacillus of fowl cholera and the pneumococcus in the same year, and of the tubercle bacillus in 1882, initiated a series of etiological discoveries which, extending over not more than fifteen years, elucidated the causation of a majority of the infectious diseases.

Coincident with the elucidation of etiological facts began the inquiry into that field which is now spoken of as the science of jmmunity. The phenomena which accompany the development of insusceptibility to bacterial infections in man and in animals, first studied by Pasteur, have become the subject of innumerable researches and have led to results of the utmost practical value.

The problems which were encountered were first studied from a purely bacteriological point of vicw, but their solution has shed light upon biological principles of the broadest application. Investigations into the properties of immune sera, while making bacteriology one of the most important branches of diagnostic and therapeutic medicine, have, at the same time, inseparably linked it with physiology and experimental pathology.

By the revelations of etiological research, and by the study of the biological properties of pathogenic bacteria, contagion, an enemy hitherto unseen and mysterious, was unmasked, and rational campaigns of public sanitation and personal hygiene were made possible. Upon the same elucidations has depended the development of modern surgery-a science which without asepsis and antisepsis would have been doomed to remain in its medieval cendition.

Apart from its importance in the purely medical sciences, the study of the bacteria has shed beneficial light, moreover, upon many other fields of human activity. In their relationship to decomposition, the conditions of the soil, and to diseases of plants, the bacteria have been found to occupy a condition of great importance in agriculture. Knowledge of bacterial and yeast ferments, furthermore, has become the scientific basis of many industries, chicfly those concerned in the production of wine, beer, and dairy products.

The scope of bacteriology is thus a wide one, and none of its various fields has, as yet, been fully explored. The future of the science is rich in allurement of interest, in promise of result, and in possible benefit to mankind. 


\section{CHAPTER II}

\section{GENERAL MORPHOLOGY, REPRODUCTION, AND CHEMICAL AND PHYSICAL PROPERTIES OF THE BACTERIA}

BACTERIA are exceedingly minute unicellular organisms which may occur perfectly free and singular, or in larger or smaller aggregations, thus forming multicellular groups or colonies, the individuals of which are, however, physiologically independent.

The cells themselves have a number of basic or ground shapes which may be roughly considered in three main classes: The cocci or spheres, the bacilli or straight rods, and the spirilla or curved rod forms.

The cocei are, when fully developed and free, perfectly spherical. When two or more are in apposition, they may be slightly flattened along the tangential surface, giving an oval appearance.

The bacilli, or rod-shaped forms, consist of elongated cells whose long diameter may be from two to ten times as great as their width, with ends squarely cut off, as in the case of bacillus anthracis, or gently rounded as in the ease of the typhoid bacillus.

The spirilla may vary from small comma-shaped microorganisms, containing but a single curve, to longer or more sinuous forms which may roughly be compared to a corkscrew, being made up of five, six or more curves. The turns in the typical microorganisms of this class are always in three planes and are spiral rather than simply curved.

Among the known microorganisms, the bacilli by far outnumber other forms, and are probably the most common variety of bacteria in existence. Many variations from these fundamental types may occur even under normal conditions, but contrary to earlier opinions it is now positively known that cocei regularly reproduce cocei, bacilli hacilli, and spirilla spirilla, there being, as far as we know, no. mutation from one form into another.

The size of bacteria is subject to considerable variation. Cocci may vary from $.15 \mu$ to $2 . \mu$ in diameter. The average size of the ordinary pus coccus varies from $.8 \mu$ to $1.2 \mu$ in diameter. Fischer has given a graphic illustration of the size of a staphyloceocus by calculating that one billion micrococei could easily be contained in a drop of water hav- 
ing a volume of one cubic millimeter. Among the bacilli the range of size is subject to even greater variations. Probably the smallest of the common bacilli is the bacillus of influenza, which measures about $.5 \mu$ in length by $.2 \mu$ in thickness. The limit of the optical possibilities of the modern microscope is almost reached by some of the known mieroorganisms, and there are some diseases which are caused by organisms so small as to be invisible by any of our present methods. In fact, the virus causing the peripneumonia of cattle has been shown to pass through the pores of a Berkefeld filter, which are impenetrable to the smallest of the known bacteria. ${ }^{1}$ The coccoid or globoid bodies grown by Noguchi from poliomyelitis virus are small enough to pass through such filters, but are still visible with the highest lens magnifications. Whether or not these minute bodies should be regarded as bacteria is questionable. It seems likely that they represent an entirely different class of organisms. It is worth

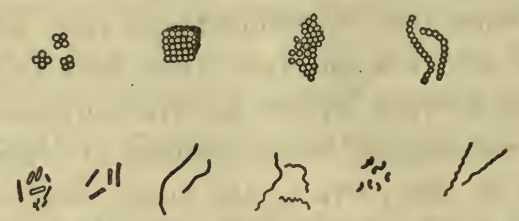

Fig. 1.-Types of Bacterial Morphology.

mentioning, also, that organisms like streptococci may show minute forms, especially when grown under anaërobic conditions, which are almost as small as the globoid bodies. It seems a general rule that anaërobically. grown cocci may assume smaller forms.

Morphology of the Bacterial Cell.-When unstained, most bacteria are transparent, colorless, and apparently homogeneous bodies with a low refractive index. The cells themselves consist of a mass of protoplasm, surrounded, in most instances, by a delicate cell membrane.

The presence of a nucleus ${ }^{2}$ in bacterial cells, though denied by the earlier writers, has been demonstrated beyond question by Zettnow, Nakanishi, ${ }^{3}$ and others. The original opinion of Zettnow was that the entire bacterial body consisted of nuclear material inti-

${ }^{1}$ Nocard and Roux, Ann. Past., 12, 1898.

${ }^{2}$ A. Fischer, Jahrbücher f. wissen. Botanik, xxvii.

${ }^{3}$ Nakanishi, Münch. med. Woch., vi, 1900. 
mately intermingled with the cytoplasm. The opinion now held by most observers who have studied this phase of the subject favors the existence of an ectoplasmic zone which includes eell membrane and flagella, but is definitely a part of the cytoplasm, and an entoplasm in which is concentrated the nuclear material. Bütschli ${ }^{4}$ claims to have demonstrated within this entoplasmic substance a reticular meshwork, between the spaces of which lie granules of chromophilie or nuclear material. Confirmation of this opinion has been brought by Zettnow ${ }^{5}$ and others. Nakanishi, working with a special staining method, asserts that some microorganisms show within the entoplasmic zone a well-defined, minute, round or oval nucleus, which possesses a definitely charaeteristic staining reaction. ${ }^{6}$

In the bodies of a large number of bacteria, notably in those of the diphtheria group, Ernst, ${ }^{7}$ Babes, ${ }^{8}$ and others have demonstrated granular, deeply staining bodies now spoken of as metachromatic granules, or Babes-Ernst granules, or, because of their frequent position at the ends of baeilli, as polar bodies. These structures are irregular in size and number, and have a strong affinity for dyes. They are stained distinctly dark in contrast to the rest of the bacterial cell with methylene blue, and may be demonstrated by the special methods of Neisser and of Roux. ${ }^{9}$ Their interpretation has been a matter of much difficulty and of varied opinion. Those who first observed them held that they were a part of the nuclear material of the cell. Others have regarded them as an early stage in spore formation, or as arthrospores. ${ }^{10}$ Again, they have been interpreted as structures comparable to the centrosomes of other unicellular

"Bütschli, “'Bau der Bakterien,' Leipzig, 1890.

${ }^{5}$ Zettnow, Zeit. f. Hyg., xxiv, 1897.

- The method of Nakanishi is carried out as follows: Thoroughly cleansed slides are covered with a saturated aqueous solution of methylene blue. This is spread over the slide in an even film and allowed to dry. After drying, the slide should be of a transparent, sky-blue color. The microorganisms to be examined are then emulsified in warm water, or are taken from the fluid media, and dropped upon a cover slip. This is placed, face downward, upon the blue ground of the slide. In this way, bacteria are stained without fixation. Nakanishi claims that by this method the entoplasm is stained blue, while the nuclear material appears of a reddish or purplish hue.

'Ernst, Zeit. f. Hyg., iv, 1888.

${ }^{8}$ See section on stains, p. - .

${ }^{9}$ Babes, Zeit. f. Hyg., v, 1889.

${ }^{10}$ See section on sporulation, p. 16. 
forms. As a matter of fact, the true nature of these bodies is by no means certain. They are present most regularly in microorganisms taken from young and vigorous cultures or in those taken directly from the lesions of disease. It is unlikely that they represent structures in any way comparable to spores, since cultures containing individuals showing metachromatic granules are not more resistant to deleterious influences than are others. Their abundant presence in young vigorous cultures may indicate a relationship between them and the growth energy of the microorganisms. There is no proof, however, that these bodies affect the virulence of the bacteria.

Cell Membrane and Capsule.-Actual proof of the existenee of a cell membrane has been brought in the cases of some of the larger

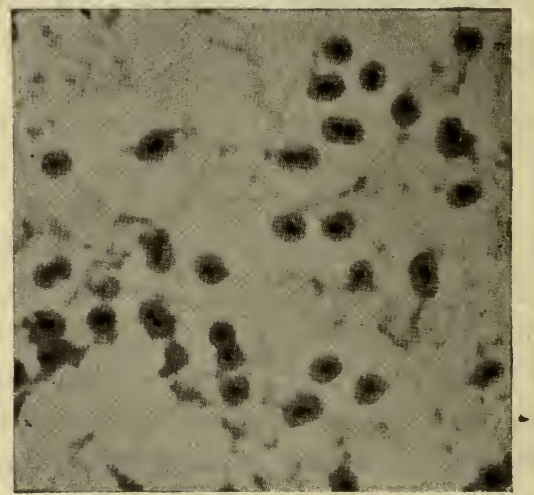

Fig. 2.-Bacterial Capsules.

forms only, ${ }^{11}$ but the presence of such envelopes may be inferred for most bacteria by their behavior during plasmolysis, where definite retraction of the protoplasm from a well-defined cell outline has been repeatedly observed. The occurrence, furthermore, of so-called "shadow forms" which appear as empty capsules, and of, occasionally, a well-outlined cell body, after the vegetative form has entirely degenerated in the course of sporulation, make the assumption of the presence of a cell membrane appear extremely well founded. Differing from the cell membranes of plant cells, cellulose has not, except in isolated instances, been demonstrable for bacteria, and the membrane is possibly to be regarded rather as a peripheral protoplasmic zone, which remains unstained by the usual manipulations. 
Zettnow, ${ }^{12}$ who has carefully studied the structure of some of the larger forms, takes the latter view, and regards the "ectoplasmic" zone as a part of the cell protoplasm devoid of nuclear material. Zettnow's opinion is borne out by the greatly increased size of the bacterial cells as seen by means of special stains.

Many bacteria have been shown to possess a mucoid or gelatinous envelope or capsule. According to Migula, ${ }^{13}$ such an envelope is present on all bacteria, though it is in only a few species that it is sufficiently well developed and stable to be easily demonstrable and of differential value. When stained, the capsule takes the ordinary anilin dyes less deeply than does the bacterial cell body, and varies greatly in thickness, ranging from a thin, just visible margin to dimensions four cr five times exceeding the actual size of the bacterial body itself. This structure is perfectly developed in a limited number of bacteria only in which it then becomes an important aid to identification. Most prominent among such bacteria are Diplococcus pneumoniæ, Micrococcus tetragenus, the bacilli of the Friedländer group, and B. aërogenes capsulatus. The development of the capsule seems to depend intimately upon the environment from which the bacteria are taken. It is most easily demonstrable in preparations of bacteria taken directly from animal tissues and fluids, or from media containing animal serum or milk. If cultivated for a prolonged period upon artificial media, many otherwise capsulated microorganisms no longer show this characteristic structure.

Capsules may be demonstrated on bacteria taken from artificial media most successfully when albuminous substances, such as ascitic fluid or blood serum, are present in the culture media, or when the bacteria are smeared upon cover slip or slide in a drop of beef or other serum. ${ }^{14}$ Most observers believe that the capsule represents a swelling of the ectoplasmic zone of bacteria. By others it is regarded as an evidence of the formation of a mucoid intercellular substanee, some of which remains adherent to the individual bacteria when removed from cultures. It is noticeable, indeed, that some of the capsulated bacteria, especially Streptococeus mucosus and B. mucosus capsulatus, develop such slimy and gelatinous colonies that, when these are touched with a platinum wire, mucoid threads and strings

${ }^{12}$ Zettnow, loc. cit.

13 Migula, "Systeme d. Bakterien," 1, p. 56.

${ }^{14}$ Hiss, Jour. Exp. Med., vi, 1905. 
adhere to the loop. Exactly what the significance of the capsules is cannot yet be decided.

There is, however, definite reason to believe that there is a direct relation between virulence and capsulation; capsulated bacteria are less easily taken up by phagocytes than are the non-capsulated members of the same species. Also, as Porges and others have shown, capsulated organisms are not easily amenable to the agglutinating action of immune sera. Many bacteria (plague, anthrax) which are habitually uncapsulated on artificial media acquire capsules within the infected animal body. Also in some species (pneumococci), the loss of capsule formation as cultivated on the simpler media is accompanied by a diminution of virulence.

Organs of Locomotion.-When suspended in a drop of fluid many bacteria are seen to be actively motile. It is important, however, in all cases to distinguish between actual motility and the so-called Brownian or molecular movement which takes place whenever small particles are held in suspension in a fluid.

Brownian or molecular movement is a phenomenon entirely explained by the physical principles of surface tension, and has absolutely no relation to independent motility. It may be seen when particles of carmine or any other insoluble substance are suspended in water, and consists in a rapid to and fro vacillation during which there is actually no permanent change in position of the moving particle except inasmuch as this is influenced by currents in the drop.

The true motility of bacteria, on the other hand, is active motion due to impulses originating in the bacteria themselves, where the actual position of the bacterium in the field is permanently changed.

The ability to move in this way is, so far as we know, limited almost entirely to the bacilli and spirilla, there being but few instances where members of the coccus group show active motility. In all cases, with the exception of some of the spirochetes, where motility may occasionally be due to an undulating membrane marginally placed along the body, bacterial motility is due to hair-like organs known as flagella. These flagella have rarely been seen during life, and their recognition and study has been made possible only by special staining methods, such as those devised by Loeffler, van Ermengem, Pitt, and others.

In such stained preparations, the bacterial cell bodies often appear thicker than when ordinary dyes are used, and the flagella apparently are seen to arise from the thickened ectoplasmic zone. 
The flagella are long filaments, averaging in thickness from onetenth to one-thirtieth that of the bacterial body, which often are delicately waved and undulating, and, judging from the positions in which they become fixed in preparations, move by a wavy or screwlike motion. 'In length they are subject to much variation, but are supposed to be generally longer in old than in young cultures. Very short flagella have been described only on nitrosomonas, one of the nitrifying bacteria discovered by Winogradsky. ${ }^{15}$

As to the finer structures of flagella, little can be made out except that they possess a higher refractive index than the cell body itself; and that they can be stained only with those dyes which bring clearly into view the supposedly true cytoplasm of the cell. Whether they penetrate this cytoplasmic membrane or whether they are a direct continuation of this peripheral zone of the bacterial body, can not be decided.

The manner in which bacteria move is naturally subject to some

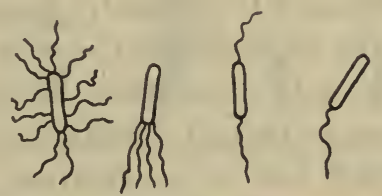

Fig. 3.-Arrangement of Bacterial FlaAgella.

variation depending upon the number and position of the flagella possessed by them. Whether bacteria exercise or not the power of motility depends to a large extent upon their present or previous environment. They are usually most motile in vigorous young cultures of from twenty-four to forty-eight hours' growth in favorable media. In old cultures motility may be diminished or even inhibited by acid formation or by other deleterious products of the bacterial metabolism.

At the optimum growth-temperature motility is most active, and a diminution or increase of the temperature to any considerable degree diminishes or inhibits it. Thus actively motile organisms, in the fluid drop, may be seen to diminish distinctly in activity when left for any prolonged time in a cold room, or when the preparation is chilled. Any influence, in other words, chemical or physical, which

${ }^{15}$ Winogradsky, Arch. des sei. biologiques, St. Petersburg, 1892, T, 1 and 2. 
tends to injure or depress physiologically the bacteria in any way, at the same time tends to inhibit their motility.

Messea ${ }^{16}$ has proposed a classification of bacteria which is based upon the arrangement of their organs of motility, as follows:

I. Gymnobacteria, possessing no flagella.

II. Trichobacteria, with flagella.

1. Monotricha, having a single flagellum at one pole.

2. Lophotricha, having a tuft of flagella at one pole.

3. Amphitricha, with flagella at both poles.

4. Peritricha, with flagella completely surrounding the bacterial body.

Bacterial Spores.-A large number of bacteria possesses the power of developing into a sort of encysted or resting stage by a process commonly spoken of as sporulation or spore formation. The formation of spores by bacteria depends largely upon environmental conditions, and the optimum environment for spore formation differs greatly for various species. It is usually necessary that a temperature of over $20^{\circ} \mathrm{C}$. exist in order that spores may be formed. Unfavorable factors, like acid formation, accumulation of bacterial products in old cultures, or lack of nutrition, frequently seem to constitute the stimuli which lead to sporulation. In the case of some species, notably the anthrax bacillus, spores are formed only in the presence of free oxygen and are therefore never formed within the tissues of infected animals. It is claimed that some of the pathogenic anaërobes, like B. tetani and the bacillus of malignant edema, may form spores anaërobically. Nevertheless it has been observed that when an absolute exclusion of oxygen is practiced in the cultivation of these bacteria, vegetative forms only are seen in the cultures. ${ }^{17}$

The process of sporulation is by no means to be regarded as a method of multiplication, since it rarely occurs that a single bacillus produces more than one spore. In some species of bacteria the formation of several spores in one individual has occasionally been observed, but there can be no question about the fact that such a condition is exceptional.

Varieties of spores are often recognized, the so-called arthrospores and the true spores or endospores. It is seriously in doult whether the structures once spoken of as arthrospores should be

${ }^{16}$ Messea, Cent. f. Bakt., I, Ref. ix, 1891.

${ }^{17}$ Zinsser, Jour. Exp. Med., viii, 1906, p. 542 
considered as in any way comparable to true spores. They are represented by the granular and globular appearances occasionally observed in old cultures of some bacteria, notably streptococeus, cholera spirillum, diphtheria baciilus, and others. It was believed that they were due to a transformation of certain individuals of the cultures into more resistant forms. It is probable, however, that such structures are merely to be regarded as evidences of involution or degeneration, since it has never been demonstrated that cultures containing them are more resistant either to disinfectants or to heat, than cultures showing no evidences of such forms. The true spores or endospores are most eommon among bacilli, and are rarely observed among the spherical bacteria. They arise within the body of the individual bacterium as a small granule which probably represents a concentration of the protoplasmic substance. Nakanishi ${ }^{18}$ elaims that there is a definite relation between these sporogenic globules and the nuclear material of the bacterial cell. At the time at which

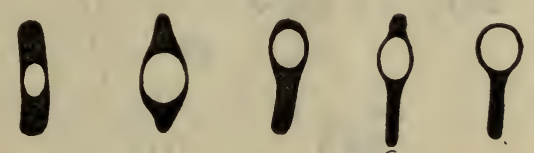

Fig. 4.-Variods Positions of Spores in Bacterial Cells.

sporulation occurs there is usually a slight and gradual thickening of the bacillary body. After the formation of this thickening, a spore membrane appears about the same thickened area. The completed spore is usually round or oval, has an extremely high refractive index, and a membrane which is very resistant. Muhlschlegel ${ }^{19}$ believes that the spore membrane is a double structure, and, as stated before, Nakanishi believes that the spore contains nuclear material.

The position of the spore in the mother cell is of some differential importance in that it is usually constant for one and the same species. Thus, the spores of the tetanus bacillus are regularly situated at the extreme ends of the bacillary bodies, while those of anthrax are situated at or near the middle.

Physiologically, sporulation is probably to be regarded as a method of encystment for the purpose of resisting unfavorable environment, and it is indeed true that species of bacteria the vegetative

${ }^{18}$ Nakanishi, Münch. med. Woch., 1900, p. 680.

${ }^{19}$ Muhlsehlegel, Cent. f. Bakt., II Abt., vi, 1900, p. 65. 
forms of which are rather easily injured by heat, light, drying, and chemicals have a comparatively enormous resistance to these agents after the formation of spores. Thus, while a 10-per-eent solution of carbolic acid will kill the vegetative forms of anthrax bacilli within twenty minutes, anthrax spores are able to resist the same disinfectant for a long period in a concentration of over 50 per eent; and while the vegetative forms of the same bacillus show little more resistance against moist heat than other vegetative forms, the spores will withstand the action of live steam for as long as ten to twelve minutes and more.

Whenever the spores of any microorganism are brought into an

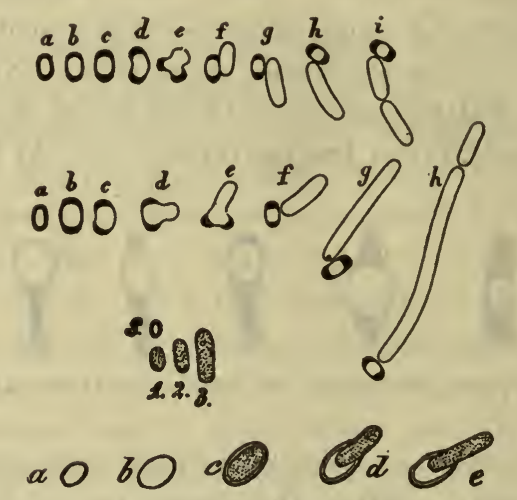

Fig. 5.-Germination of Spores.

environment suitable for bacterial growth as to temperature, mois. ture, and nutrition, the spores develop into vegetative forms. Thi process differs according to species. In general it consists of an elongation of the spore body with a loss of its highly refractile character and resistance to staining fluids. The developing vegetative cell may now rupture and slip out of the spore membrane at one of its poles, leaving the empty spore eapsule still visible and attached to the bacillary body. Again, a similar process may take place equatorially instead of at the pole. In other species again, there may be no rupture of the spore membrane at all, the vegetative form aris. ing by gradual elongation of the spore and an absorption or solution of the membrane which is indieated by change in staining reaction Division by fission in the ordinary way then ensues. 


\section{REPRODUCTION OF BACTERIA}

Bacteria multiply by cell division or fission. A young individual increases in size up to the limits of the adult form, when, by simple cleavage, at right angles to the long axis, without any discoverable process of mitosis or nuclear changes, it divides into two individuals. In spite of the claims of various bacteriologists, notably Nakanishi, ${ }^{20}$ traceable analogy to the karyokinesis of other cells has not been definitely established. In the case of the spherical bacteria a slight change to the elliptical form takes place just before cleavage and this cleavage may occur in one plane only, in two planes, or in three planes. According to the limitations of cleavage direction, the cocci assume a chained appearance (streptococci), a grape-like appearance (staphylococci), or an arrangement in packets or cubes having three dimensions (sarcinæ). In the cases of bacilli and spirilla, cleavage takes place in the direction of the short axis. The individuals, after cleavage, may separate from each other, or may remain mutually coherent. The cohesion after cleavage is pronounced in some species of bacteria and slight in others, and, together with the plane of cleavage, determines the morphology of the cell-groups. Thus among the cocci diplo- or double forms, long chains and short chains may arise and furnish a characteristic of great aid in differentiation. Similarly among the bacilli there are forms which appear characteristically as single individuals and others which form chains of varying length.

The rate of growth varies to a certain extent with the species, and also with the favorable or unfavorable character of the environment. A generation, that is, the time elapsing in the interval between one cleavage and the next, has been estimated by $A$. Fischer ${ }^{21}$ as being about twenty minutes for the cholera spirillum and 16-20 minutes for bacillus coli communis, under the most favorable conditions. The same author has calculated that under these conditions a single cholera spirillum would yield 1600 trillions in a single day. Such a multiplication rate, however, is probably not usual under natural or even artificial conditions, both on account of lack of nutritive material and because of inhibition of the growth of the bacteria by their own products.

${ }^{20}$ Nakanishi, Cent. f. Bakt., I, xxx, 1901.

${ }^{21}$ A. Fischer, "'Vorlesungen über Bakt.,", Jena, 1903. 


\section{VARIATIONS OF BACTERIAL FORMS}

Variations from the basic forms considered in the preceding section may occur, but are not common among bacteria under normal conditions. Thus the formation of club shapes by a thickening of the bacillary body at one or both ends has been frequently observed among bacteria of the diphtheria group, and in the glanders bacillus, and an irregular beading is not infrequently observed in tubercle bacilli under normal conditions. Such pictures can not, in these cases, be regarded as degeneration or involution forms, since they are

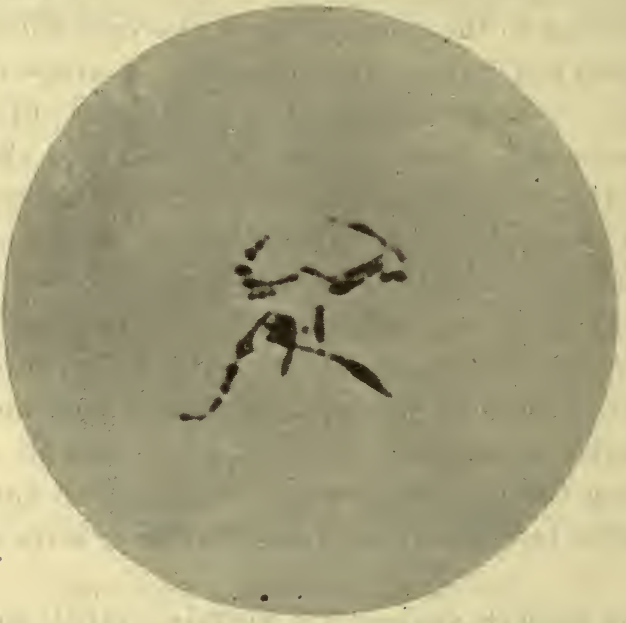

Fig. 6.-Degeneration Forms of Bacillus Diphtheriat. (After Zettnow.)

visible in young, actively growing cultures under ordinary conditions. It is a well-known fact, furthermore, that the sizes and contours of bacteria may vary to some extent according to the medium on which they are grown. This may, to a degree, be due to osmotic relations. On fluid media, for instance, many bacteria may appear larger and of a less dense consistency than do members of the same species cultivated upon solid media.

Degeneration Forms.-When bacteria are grown under conditions which are not entirely favorable for their development, or when they are grown for a prolonged period upon artificial culture media without transplantation, there may occur variations which often depart considerably from the ground type, known as degeneration or invo. lution forms. Thus, in the case of the diphtheria bacillus, old cul- 
tures may contain long, irregularly beaded forms with broad expansions at the ends. In the case of $\mathrm{B}$. pestis the fact that large numbers of oval, vacuolated bodies in old cultures are formed regularly has become of differential value. ${ }^{22}$ These degeneration forms are shown most characteristically when the bacteria are cultivated on agar containing 3 to 5 per cent $\mathrm{NaCl}$.

Among the cocci, marked evidences of involution are often seen in cultures of the meningococcus in the form of large, swollen poorlystaining spheres, and in the case of the pneumococcus in the so-called shadow forms which have the appearance of empty capsules. There

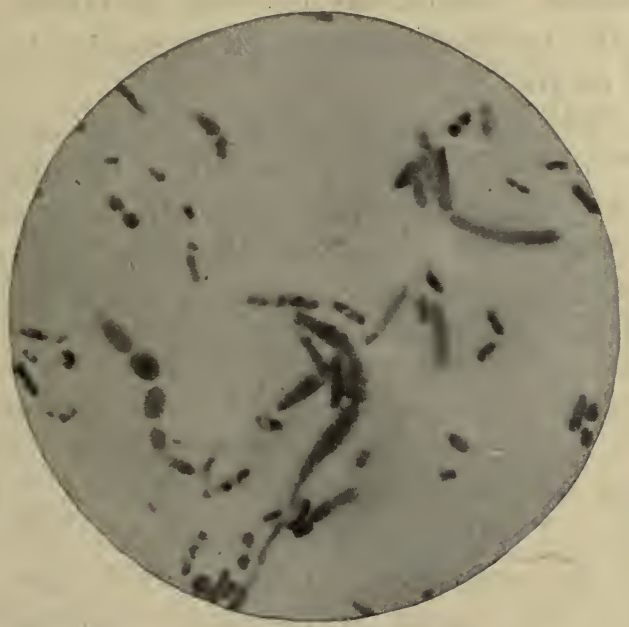

Fig. 7.-Degeneration Forms of Bacillus Pestis. (After Zettnow.)

are few microorganisms indeed, in which prolonged cultivation on artificial media or other unfavorable influences do not produce variations from the ground type which may often make the cultures morphologically unrecognizable. In the case of many of the spirilla (spirillum Milleri, spirillum Metchnikovi, etc.) the degeneration forms may appear within so short a time as two or three days after transplantation.

Chemical Constituents.-The bacterial cell has been found to contain proteins, nucleic acid, carbohydrates, fatty substances and ash. The quantitative chemical composition of dried mass cultures of bacteria, even of the same species, shows rather extreme varia-

${ }^{22}$ Hankin and Leumann, Cent. f. Bakt., I, xxii, 1897. 
tions in the pereentage of these substanees, depending upon the nature of the culture media. Thus, 80 to 90 per cent is water, and in the dry residue, the protein content, estimated from the total nitrogen, varies roughly from 50 to 80 per cent. Cramer ${ }^{23}$ has shown that the eholera vibrio, grown on bouillon, contains 69 per eent of protein and 26 per cent of ash, while on Uschinsky's medium the same organism contained only 36 per cent protein, and 14 per eent of ash. It seems probable that such variations may depend to some degree on the semi-permeable nature of the bacterial cell; and perhaps to an equal degree on the fact that analyses of the mass cultures necessarily include any insoluble metabolic products, such as pigments, the production of which is known to vary with the nature of the media.

Analyses made by Kappes ${ }^{24}$ of B. prodigiosus, and by Nencki and Scheffer ${ }^{25}$ of some of the putrefactive bacteria, may serve to illustrate the approximate proportions of the substances making up the bacterial body. The protein is calculated from the total nitrogen, and since the nucleic acid of the cell is not taken into account the results are not accurate.

\begin{tabular}{|c|c|c|c|c|c|c|}
\hline & \multicolumn{3}{|c|}{$\begin{array}{c}\text { B. } \\
\text { prodigiosus }\end{array}$} & \multicolumn{3}{|c|}{$\begin{array}{c}\text { Putrefactive } \\
\text { Bacteria }\end{array}$} \\
\hline Water. & 85.45 & per & cent. & 83.42 & per & cent. \\
\hline Proteins & 10.33 & “ & 6 & 13.96 & 6 & “6 \\
\hline Fats ... & 0.7 & 66 & 6 & 1. & ، & 66 \\
\hline Ash $\ldots \ldots \ldots \ldots \ldots \ldots \ldots$ & 1.75 & ، & “6 & 0.78 & “ & “ \\
\hline Residue $\ldots \ldots \ldots \ldots \ldots \ldots$ & 1.77 & “ & ، & 0.84 & ، & 66 \\
\hline
\end{tabular}

The bacterial protein is probably of several types. A true globulin has been described, and coagulable proteins have been demonstrated by Buchner ${ }^{26}$ in the "Pressaft" or juice obtained by subjecting bacteria to mechanical pressure. The presence of nuelear material, chromatin, in bacteria, as shown by staining reactions, together with general biological considerations, indicate that some, at least, of the protein is probably combined with nucleic acid in the form of "nucleins" or "nucleoproteins." The presence of these

${ }^{23}$ Cramer, Arch. f. Hyg., xxii, 1895, $16 \%$.

${ }^{24}$ Kappes, Analyse der Messenkulturen, etc., Diss., Leipzig, 1889.

${ }^{25}$ Nencli and Scheffer, Jour. f. prakt. Chemie, new series, xix, 1880.

${ }^{26}$ Buchner, Münch. med. Woch., xliv, 1897, 299. 
substances was established by Ruppel ${ }^{27}$ who, in an analysis of the tuberele bacillus, obtained the following values for 100 grams of the dried bacilli :

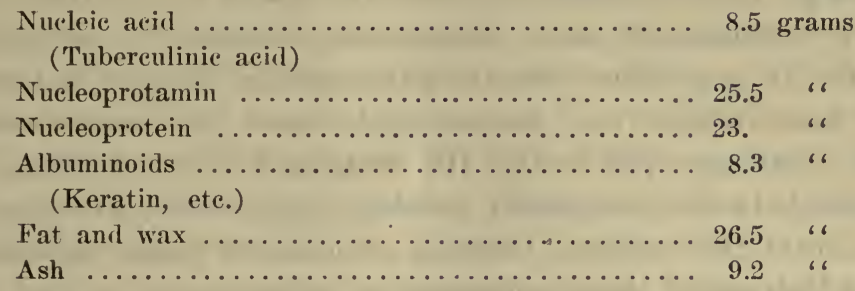

More recently, nucleic acid and the products of its hydrolysis have been shown to occur in all species of bacteria investigated. Both purine and pyrimidine bases have been isolated, and it is particularly interesting that both uracil and thymin have been found by Levene ${ }^{28}$ in nucleic acid from the tubercle bacillus. The former is found only in nucleic acid from plant cells, while the latter is obtained only from animal sources. Jones ${ }^{23}$ states, however, that uracil often results from the decomposition of cytosine of plant nucleic acids. On the other hand, pentose has been found as a decomposition product of bacterial nucleic acids, and since this substance occurs only among the cleavage products of yeast and other plant nucleic acids, it would tend to establish the relationship of bacteria to the vegetable kingdom. The evidence is, therefore, contradictory, and it may ultimately be shown that nucleic acid of bacteria is not identical with either the animal or plant type.

The basic chemical nature of the protein of bacteria does not seem to differ from protein of other kinds. Complete analysis of the amino acid components of purified preparations are wanting. However, the presence of most of the monoamino acids, as well as the hexone bases and glutamic acid have been established by the work of Tamura, ${ }^{30}$ Leach, ${ }^{31}$ Wheeler, ${ }^{32}$ and others. Tamura showed that the hexone base content of bacterial protein was the same, whether

\footnotetext{
${ }^{27}$ Ruppel, Zeit. f. physiol. Chemie, xxvi, 1898, 218.

${ }^{28}$ Levene, J. of Med. Res., vii, 1904, 251.

${ }^{29}$ Jones, " Nucleic Acids," New York, 1914.

${ }^{30}$ Tamura, Zeit. physiol. Chemie, lxxxviii, 1913, 190, and lxxxix, 1914, 289.

${ }^{31}$ Leach, J. of Biol. Chem., i, 1906, 463.

32 Wheeler, J. of Biol. Chem., vi, 1909, 509.
} 
grown on broth or on a protein-free synthetic medium, and concludes that bacteria are capable of building up their own protein, independent of the nature of their food supply.

Although there is much uncertainty about the nature of the relatively thermostable toxic substances contained in bacteria, the endotoxins, it is probable that they are also of protein nature.

The fats, which are demonstrable both by michrochemical methods, staining with Sudan III, Scharlach R., and Osmic acid, and by alcohol-ether extraction, consist of fatty acids, true fats, and in the case of the tubercle bacillus, at least, of waxy substances. ${ }^{33}$ The acid-fastness of these organisms is probably due in part to the presence of these lipoidal constituents, which are present to the extent of 20 to 40 per cent of the dry weight of the bacteria.

Cellulose has been stated to occur in certain species of bacteria, but the evidence is unsatisfactory and many investigators have failed to recognize it. A hemicellulose has also been described by Nishimura $^{34}$ and by Tamura. ${ }^{35}$ Chitin, the shell-like material making up the protective covering of lobsters, crabs, insects, etc., seems to have been definitely recognized in bacteria. by Iwanoff, ${ }^{36}$ who obtained considerable quantities of glucosamine hydrochloride from the hydrolysis of his preparation. The occurrence of chitin in bacteria would seem, again, to connect these organisms more closely to the animal than to the vegetable kingdom, thus contradicting the relation suggested by the split products of the nucleic acid.

Glycogen-like substances have been demonstrated, according to A. Fischer ${ }^{37}$ in B. subtilis and B. coli. These bacteria stained a reddish brown with iodin, and after treatment with weak acids were shown to contain dextrose. Hydrolysis of other bacteria has given reducing sugars, resulting presumably from the decomposition of mucins and perhaps of gums.

The bacterial ash consists largely of phosphates and chlorides of potassium, sodium, calcium and magnesium. The phosphate probably occurs for the most part as a constituent of the nucleic acid. Certain of the higher bacteria also contain iron oxide in granular

${ }^{33}$ De Schweintz and Dorset, Cent. f. Bakt., Erste Abt., xxii, 1897, 209.

${ }^{34}$ Nishimura, Arch. f. Hyg., xviii, 1893, 318 and xxi, 1894, 52.

${ }^{35}$ Tamura, Zeit. f. physiol. Chemie, lxxxix, 1914, 304.

${ }^{36}$ Iwanoff, Hofmeister's Beitr., 1, 1902, 524.

${ }^{37}$ Fischer, A., "Vorlesung über die Bakt.," Jena, 1903. 
form, and the thio bacteria contain insoluble sulphur in their protoplasm.

The chemical constitution of bacteria is of considerable importance in comnection with problems of immunization. It is not at all sure that the antigenic substances in bacteria consist entirely of proteins of the albumin-globulin variety. Extracts of bacterial bodies, such as those we have, on a number of occasions, produced with typhoid bacilli, tubercle bacilli, etc., contain very large amounts of material, non-coagulable by heat, which come down on the addition of weak acetic acid in the cold, and which are soluble only in dilute alkali, such as the antigenic proteins contained in many plants. It is not impossible that these may be the decisive antigenic constituents, rather than the albumin-globulin contents.

Osmotic Properties of the Bacterial Cell.-Like all other animal and vegetable cells, the bacterial cell forms in itself a small osmotic unit which reacts delicately to differences of pressure existing between its own protoplasm and the surrounding medium. The perfect and normal morphology of a microorganism, therefore, can exist only when the osmotic pressure within the protoplasm of the cell is isotonic or equal to that of its own environment. The changes produced in the morphological relations of a cell when transferred from one environment into another of varying osmotic pressure, depend intimately upon the "permeability" of the cell membrane for different substances. When such a membrane is permeable for water and not for substances in solution, it is technically spoken of as "semi-permeable." Now, as a matten of fact, the bacterial cell membrane is easily permeable for water, but its permeability differs greatly in various species of bacteria for other substances. Thus, for instance, the cholera vibrio shows great permeability for common salt and B. fluorescens liquefaciens shows a lower permeability for potassium nitrate than do many other bacteria. ${ }^{38}$

When a microorganism is suddenly removed from an environment of low osmotic pressure into one showing a high pressure, say, from a dilute to a concentrated solution of $\mathrm{NaCl}$, an abstraction of water from the cell occurs, with a consequent shrinkage of the protoplasm away from the cell membrane. This process is spoken of as "plasmolysis." Cell death does not usually occur with plasmolysis, but by slow diffusion of the salt itself into the protoplasm, the

${ }^{38}$ Gottschlich, in Flügge, "Mikroorganismen," i, p. 91. ' 
equilibrium may eventually be restored and the normal morphology of the cell resumed. In all cases the speed and completeness of the return to normal depends upon the permeability of the cell membrane for the dissolved substances. There is no evidence to support the view that the internal pressure of a cell may be in any way increased by an inherent power of the protoplasm independently of the laws of diffusion. As a general rule, old cultures are more susceptible to plasmolysis than are young and vigorous strains. Spores and, according to $\mathrm{A}$. Fischer, ${ }^{39}$ flagella are much less susceptible to osmotic changes than are the vegetative bodies.

When, on the other hand, bacteria are suddenly removed from a medium possessing a high osmotic pressure to one comparatively low, say, from a concentrated salt solution to distilled water, a bursting of the cell may occur, a process spoken of as "plasmoptysis." Plasmoptysis leads to cell death, and is probably the cause of the death of microorganisms so often observed in distilled water emulsions of bacteria.

Other Physical Properties of Bacteria.-The refractive index of the vegetative bacterial body is low, in contrast to the highly refractive character of the spores and flagella. According to Fischer, the ectoplasm or cell membrane shows a higher index than does the endoplasm.

The specific gravity of various microorganisms has been investigated by Bolton, ${ }^{40}$ Rubner, ${ }^{41}$ and others. Some of Rubner's results are the following:

Gelatin fluidifiers $\ldots \ldots \ldots \ldots \ldots \ldots \ldots \ldots \ldots \ldots$. . . . . . gr. 1.0651

Gas formers ........................6.6 66 1.0465

Cultures from potato................... 6 6 1.038

M. prodigiosus .................... "6 " 1.054

${ }^{39} \mathrm{~A}$. Fischer, quoted from Gottschlich in Flügge, "'Mikroorganismen," I, p. 91.

${ }^{40}$ Bolton, Zeit. f. Hyg., i, 1886.

${ }^{41}$ Rubner, Arch. f. Hyg., xi, 1890. 


\section{CHAPTER III}

THE RELATION OF BACTERIA TO ENVIRONMENT, AND THEIR
CLASSIFICATION

\section{NUTRITION OF BACTERIA}

LIKE all protoplasmic bodies, bacteria consist of carbon, oxygen, hydrogen, and nitrogen, to which are added inorganic salts and varying quantities of phosphorus and sulphur. In order that bacteria may develop and multiply, therefore, they must be supplied with these substances in proper quantity and in forms suitable for assimilation. To formulate definite laws based on chemical structure as to the compounds suitable, and those unsuitable for use by the bacteria, is obviously impossible owing to the great metabolic variations existing within the bacterial kingdom, and notable attempts to do so, such as those by Loew, ${ }^{1}$ have not successfully withstood critical inquiry. So unlike are the food requirements of bacteria that a basis for identification even among closely rélated species may often be found in differences in metabolism of carbohydrates, and the suggestion has frequently been made that with further knowledge of food requirements would come a more logical classification of microorganisms than any at present available. ${ }^{2}$

Carbon.-The carbon necessary for bacterial nourishment or anabolism may be obtained either directly from proteins, carbohydrates, and fats, or from the simpler derivatives of these substances. Thus, the amino-acids, ketons, and organic acids, like tartaric, citric, and acetic acids, glycerin, and even some of the alcohols, may furnish carbon in a form suitable for bacterial assimilation. A limited number of bacterial species, furthermore, notably the nitrobacteria of Winogradsky, are capable of obtaining their required carbon from atmospheric $\mathrm{CO}_{2}$, and possibly from other simple carbon compounds added to culture media. ${ }^{3}$

${ }^{1}$ Loew, Cent. f. Bakt., I, xii, 1892.

${ }^{2}$ Doryland, Jour. of Bacter., 1, 1916, 135.

${ }^{3}$ Muntz, Compt. rend. de l'acad. des sciences, t. iii. 
Oxygen.-Oxygen is obtained, by the large majority of bacteria, directly from the atmosphere in the form of free $\mathrm{O}_{2}$. For many microorganisms, moreover, the presence of free oxygen is a necessary condition for growth. These are spoken of as the "obligatory aërobes." Among the pathogenie bacteria proper, many, like the gonococcus, bacillus influenzæ, and bacillus pestis, show a marked preference for a well-oxygenated environment. Probably there is no pathogenic microorganism which, under certain conditions of nutrition, is entirely unable to exist and multiply in the complete absence of this gas. The conditions existing within the infected animal organism cause it to seem likely that all incitants of infection may, at times, thrive in the complete absence of free oxygen.

There is another class of organisms, on the other hand, for whose development the presence of free oxygen is directly injurious. These microorganisms, known as "obligatory anaërobes," obtain their supply of oxygen indirectly, by enzymatic processes of fermentative and proteolytic cleavage, from carbohydrates and proteins. or br reduction from reducible bodies. Among the pathogenic microorganisms the class of "obligatory anaërobes" is represented chiefly by Bacillus tetani, the bacillus of malignant edema, the bacillus of symptomatic anthrax, Bacillus aërogenes capsulatus, and Bacillus botulinus.

Intermediate between these two classes is a large group of bacteria which thrive well both under aërobic and anaërobic conditions. Some of these, which have a preference for free oxygen but nevertheless possess the power of thriving under anaërobic conditions, are spoken of as "facultative anaërobes." In others the reverse of this is true; these are spoken of as "facultative aërobes." These varieties of bacteria are by far the most numerous and comprise most of our parasitic and saprophytic bacteria.

The relation of microorganisms to oxygen is extremely subtle, therefore, and not to be biologically dismissed by a rigid classification into aërobes, facultative anaërobes, and obligatory anaërobes. Both Engelmann, ${ }^{4}$ by a method of observing motile bacteria in the hanging drop as to their behavior in relation to the oxygen given off by a chlorophyll-bearing alga, and Beijerinck. ${ }^{5}$ by a macroscopic method of observing similar bacteria as to their motion away from

- Engelmann, Botanische Zeitung, 1551.

s Beijerinck, Cent. f. Bakt., I, xir, 1к93. 
or toward an oxygenated area, were able to demonstrate delicately graded variations between species, favoring various degrees of oxygen pressure.

The discovery by Pasteur that certain bacteria develop only in the absence of free oxygen, produced a revolution in our conceptions of metabolic processes, since up to that time it was believed that life could be supported only when a free supply of $\mathrm{O}_{2}$ was obtainable. Pasteur's original explanation for this phenomenon was that anaërobic conditions of life were always associated with some form of carbohydrate fermentation and that oxygen was obtained by these microorganisms by a splitting of carbohydrates. As a matter of fact, for a large number of microorganisms, this is actually true, and the presence of readily fermentable carbohydrates not only increases the growth energy of a large number of anaërobic bacteria, but in many cases permits otherwise purely aërobic bacteria to thrive under anaërobic conditions. ${ }^{6}$ On the other hand, the basis of anaërobic growth can not always be found in the fermentation of carbohydrates or in the simple process of reduction. A number of strictly anaërobic bacteria may develop in the entire absence of earbohydrates or reducing substances, obtaining their oxygen supply from other suitable sources, some of which may be the complex proteins. Thus the tetanus bacillus may ${ }^{7}$ thrive when the nutritive substances in the media are entirely protein in nature.

The favorable influence of certain actively reducing bodies, like sodium formate or sodium-indigo-sulphate, upon anaërobic cultivation is probably referable to their ability to remove free oxygen from the media and thus perfect the anaërobiosis. ${ }^{8}$

Unlike plants which derive much of their energy for growth from the sun's rays, bacteria, with the exception of the pigmented sulphur bacteria, are dependent upon their food supply as a source of energy. To some extent a simple oxidation of carbohydrates and other substances takes place by means of atmospheric oxygen with the production of $\mathrm{CO}_{2}$. There is thus a type of true respiration in bacteria with the absorption of oxygen and elimination of $\mathrm{CO}_{2}$. Not all the absorbed oxygen reappears as $\mathrm{CO}_{2}$ and most of the remainder is probably used up in the formation of water or goes into the structure of the bacterial cell. The production of $\mathrm{CO}_{2}$ has also been

- Theobald Smith, Cent. f. Bakt., I, xviii, 1895.

"Chudiol:ov', Cent. f. Bakt., Ref., II, iv, 1898.

${ }^{8}$ Kitasato and Weyl, Zeiț. f. Hyg., viii, 1890. 
shown to occur in anaërobic cultures where the oxygen must have come from some constituent of the medium. It is probable that bacterial respiration is frequently of the anaërobic type in which, for example, such compounds as nitrates are reduced to nitrites and free nitrogen. Energy' may be produced, however, by the oxidation of other elements than carbon and hydrogen; thus, certain of the iron bacteria seem to obtain energy from the oxidation of ferrous to ferric compounds in their protoplasm, and the sulphur bacteria oxidize $\mathrm{H}_{2} \mathrm{~S}$ to free sulphur. The colored sulphur bacteria carry out this change under the combined influence of sunlight and their pigment in this way resembling the green plants, but the unpigmented forms do not require radiant energy.

While processes of oxidation are perhaps the most important source of energy for certain bacteria, for other types and under different conditions chemical reactions of another kind serve to produce energy. Processes of fermentation and perhaps of decay and putrefaction liberate considerable quantities of energy. For example, alcoholic fermentation in which neither oxidation nor reduction is involved produces about 33 calories for each gram molecule of sugar fermented. Since complete oxidation of the same quantity of sugar results in the liberation of about 680 calories it is apparent that relatively large amounts of glucose must be fermented to supply microorganisms with sufficient energy for their growth and, as a matter of fact, bacteria will ferment many times their weight of carbohydrates. Acid fermentation, such as the lactic fermentation of milk and probably many of the processes of protein cleavage, are exothermic and will supply bacteria with energy. Obviously these reactions are particularly adapted to anaërobic growth since for many of them oxygen is not required.

While a profuse supply of oxygen absolutely inhibits the growth of most anaërobes, a number of these may, nevertheless, develop when only small quantities of oxygen are present. Minute quantities of free oxygen in culture media have been shown by Beijerinck ${ }^{9}$ and others not to inhibit the growth of Bacillus tetani and Theobald Smith ${ }^{10}$ has recently demonstrated that when suitable nutritive material in the form of fresh liver tissue is added to bouillon, a number of anaërobic bacteria may be induced to grow in indifferently

Beijerinck, Cent. f. Bakt., II, vi, 1900.

${ }^{10}$ Th. Smith, Brown, and Walker, Jour. Med. Res., ix, 1906. 
anaërobic environment. Fêrran ${ }^{11}$ succeeded in adapting the tetanus bacillus to an aërobic environment. In this ease, however, the virulence of the bacillus was lost.

Nitrogen.-The nitrogen required by bacteria is taken, in most cases, from proteins, and many of the non-diffusible albumins may be rendered assimilable by the proteolyzing enzymes possessed by microorganisms. Among the pathogenic, more strictly parasitic bacteria, moreover, a delicate specialization may be observed as to the particular varieties of animal albumin which may be utilized by them. Thus the gonococcus grows more readily only upon uncoagulated human blood serum, and the diphtheria bacillus outgrows other bacteria upon a medium composed for the greater part of coagulated beef serum. For bacteria that do not require native animal protein for their development, the most common nitrogenous ingredient of culture media is peptone.

Many bacteria (pathogenic and saprophytic), on the other hand, may thrive on media containing absolutely no protein, in which case, of course, a synthesis of protein must be assumed. A medium used to demonstrate this, devised by Uschinski, ${ }^{12}$ contains ammonium lactate, glycerin, asparagin (the amine of anino-succinic acid), and inorganic salts.

Creatin, creatinin, urea and urates, and even ammonia compounds and nitrates, may serve as sources of nitrogen for many of the less parasitic bacteria. A limited number of species, moreover, the bacilli in the root tubercles of the leguminosae and the nitrogenfixing organisms of the soil, can obtain their nitrogen directly from the free $\mathrm{N}_{2}$ of the atmosphere.

Although the sources of carbonaceous and of nitrogenous food have been separately discussed, it should not be forgotten that, in many instances, both elements are taken up within the same compound, and that separate supplies are a necessity in isolated cases only.

Hydrogen.-Hydrogen is obtained by bacteria largely in combination as water and together with the carbon and nitrogen containing substances.

Salts.-The phosphatic constituents of the bacterial body are taken in, chiefly, as phosphates of magnesium, calcium, sodium, or

${ }^{11}$ Ferran, Cent. f. Bakt., I, xxiv, 1898.

${ }^{12}$ Uschinski, Cent. f. Bakt., I, xiv, 1893. 
potassium. The phosphates seem to be necessary constituents of culture media, while chlorides, on the other hand, according to Proskauer ${ }^{13}$ and Beck, are not absolutely essential. Sodium salts, as a rule, seem to be more advantageous for purposes of bacterial cultivation than potassium salts.

The uncombined sulphur, which is often a constituent of bacteria, is usually supplied by soluble sulphates. In the case of the thiobacteria of Winogradsky, however, free $\mathrm{H}_{2} \mathrm{~S}$ is necessary for its formation. ${ }^{14}$

The relative quantities of various nutrients in culture media are important in so far as too high concentrations may inhibit growth. In this respect, however, separate species vary. The development of bacteria is far oftener arrested by the accumulation of waste products than by an exhaustion of nutrient materials.

Substances of Unknown Composition.-Although the necessity for supplying bacteria with all the elements of protoplasm is obvious, many forms, particularly among the pathogenic bacteria, will fail to grow on media composed of amino-acids or peptones together with carbohydrates and mineral material. So far as is known, no chemical element necessary for growth is lacking in such a medium, and the failure of bacteria to multiply must indicate either that carbon and nitrogen are not present in suitable combination, or that some special accessory chemical grouping is wanting. For many practical purposes such media may be rendered suitable by the addition of meat extract or meat infusion. The components of meat infusion which the bacteria utilize in their growth have not been isolated. They seem to be somewhat labile, chemically, and the infusion loses its power to promote growth under relatively simple chemical manipulation. That there is more than one necessary component is indicated by recent work ${ }^{15}$ in which it has been shown that boiling the infusion with charcoal renders it unsuitable for the growth of certain bacteria, while the addition of an acid hydrolysate of casein and of some other proteins renders it again satisfactory, although the reactivating substance is probably not one of the known amino acids, and will not itself produce growth of the bacteria without the decolorized meat infusion.

\footnotetext{
1s Proskauer and Beck, Zeiț. f. Hyg., xviii, 1895.

${ }^{14}$ Voges, Cent. f. Bakt., I, xviii, 1893.

${ }^{15}$ Mueller, Proc. Soc. Exp. Biol. and Med., 1920-21.
} 
Meat infusion, however, will not suffice for some of the more strictly parasitic species, such as the meningococcus and gonococcus, and for satisfactory growth of these organisms a highly complex substance, such as whole blood or serum, is necessary. It is not certain at present whether the required material is the whole protein or some simpler substance occurring in the animal body. For ex-. ample, the influenza bacillus has been known for a long time to require red blood cells for its successful cultivation. It was formerly believed that the hemaglobin was the essential factor required by this organism, but recently it has been shown ${ }^{16}$ that if media are prepared by the addition of blood followed by heating to $70^{\circ}$ to $75^{\circ}$, and filtering the coagulum, much better growth is obtained than on, ordinary blood agar. Since the proteins, including the hemaglobin, have been removed by coagulation and filtration, probably some nonprotein component of the red cell is required by this organism.

Lloyd ${ }^{17}$ has suggested that these substances present in blood and animal fluids resemble the vitamines which are now recognized as playing such an important rôle in animal metabolism. She found that they were often removed from solution by filtering through paper or cotton, and on this observation are based the extremely useful hormone or vitamine media of Huntoon. ${ }^{18}$ Whether or not these substances are identical with, or merely resemble, the vitamines of animal foods remains to be established. It has been recently shown; however, that ordinary yeast ${ }^{19}$ and Sclerotinia_cinerea the organism, ${ }^{20}$ which causes brown rot disease in fruits, require water soluble B. vitamine for their growth.

\section{PARASITISM AND SAPROPHYTISM}

When we speak of bacteria as parasites or as saprophytes, we classify them, primarily, according to their relationship to the bodies of higher animals. "Parasites" are those bacteria which are capable of living and multiplying within the human or animal body, whereas the term "saprophytes" refers to the multitude of microorganisms

${ }^{16}$ Fleming, Lancet, 1919, I, 138.

${ }^{17}$ Lloyd, J. Path. and Bact., 21, 1916, 113.

${ }^{18}$ Huntoon, J. Inf. Dis., 23, 1918, 169.

${ }^{19}$ Williams, J. Biol. Chem., 38, 1919, 465.

${ }^{20}$ Willaman, J. Am. Chem. Soc., 42, 1920, 549. 
which are unable to hold their own under the environmental conditions found in the tissues of higher animals, but are found, almost ubiquitously, in air, soil, manure, and water. The separation is by no means a sharp one and carries with it other implications, which the use of these terms always conveys. While parasites are usually very fastidious as to nutritional and temperature requirements, most saprophytes are easily cultivated upon the simplest media. Thus certain parasitic bacteria, such as the bacillus of influenza, the gonococcus, and others, are dependent upon specific forms of animal proteins for their food supply, while typical saprophytes, like Bacillus proteus, may thrive and multiply upon even the simplest organic protein derivatives.

Between the strict parasites and the saprophytes, there is a large class of bacteria, to which the majority of pathogenic varicties belong, the members of which are capable of developing luxuriantly under both conditions. These bacteria are often spoken of as facultative parasites.

More recently the question of parasitism and saprophytism has become closely interwoven with our conceptions of virulence. Bail (see section on Aggressins) has classified parasites into strict parasites and half parasites. By the first term he designates bacteria like Bacillus anthracis, which actually invade all the tissues of their host, while, by the term "'half parasites," he refers to microorganisms like the spirillum of cholera which gain a foothold upon some part of the body of the host, but do not actually penetrate into the general circulation.

All pathogenic bacteria, therefore, must be grouped as parasites, strict or facultative, while the saprophytes, as a class, perform the far more important task of breaking up organic matter outside of the animal body, by putrefaction and fermentation. Absolute separation between the two classes, howéver, can not be maintained, since many ordinarily saprophytic bacteria may display parasitic qualities if administered in large numbers to animals or man in whom resistance to bacterial invasion is at a low ebb.

\section{ANTAGONISM AND SYMBIOSIS OF BACTERIA}

The ubiquity of bacteria in nature of course implies the simultaneous presence of many specics in all places where special conditions have provided a favorable environment for growth. Thus bacterio- 
logical investigation of water, milk, manure, soil, or organic infusions always reveals the presence of a large number of different varieties within the same substance. If the food supply in such a natural culture is at all limited in quantity, or the removal of waste products is prohibited, it will usually be found that gradually the numbers of varieties will diminish and a few species, or even only one, will prevail. In the case of milk, for instance, after standing for three or four days at a suitable temperature, two or three varieties will be found to have taken the place of the twenty or thirty, which may have been present originally.

This behavior is due to the influence which various microorganisms exert upon each other and is known as antagonism. Such antagonism probably depends upon the fact that the metabolic products of the predominant species (the one or ones for whom the special cultural conditions are most favorable) inhibit the growth of the less vigorous varieties. Many examples, experimentally supported, of such antagonism, can be given. Thus, the gonococcus is distinctly inhibited by the soluble products of Bacillus pyocyaneus, ${ }^{21}$ while in the preesnce of pyogenic cocei it develops luxuriously, and the bacillus of plague is completely inhibited when streptococci are present in the culture. ${ }^{22}$

Mutual inhibition may also be due to the monopolizing of the nutrition in the medium by the predominating species or to the change in reaction produced by its growth. This last consideration is probably the secret of the inhibitory effect exerted by acid-producers upon bacteria of putrefaction, and has received practical therapeutic application in Metchnikoff's lactic-acid bacillus therapy, described elsewhere.

When simultaneous presence of two species in the same environment favors the development of both, the condition is spoken of as symbiosis. Such dependence is not so frequent as antagonism, but it does occur. Examples of such a condition have been observed in cultures containing diphtheria bacilli and streptococe ${ }^{23}$ and have been frequently observed in cultures containing both aërobic and anaërobic bacteria, where the former favor the development of the latter by monopolizing the supply of free oxygen. Symbiosis may

${ }^{21}$ Schafer, Fortschr. d. Med., 5, 1896.

${ }^{22}$ Bitter, Rep. Egypt Plague Com., Cairo, 1897.

${ }^{23}$ Hilbert, Zeit. f. Hyg., xxix, 1895. 
also take place in cultures in which complex food products are split up by one species, furnishing substances for ingestion by species with a lesser digestive ability.

\section{RELATIONS OF BACTERIA TO PHYSICAL ENVIRONIENT}

Relation of Temperature.-Like all other living beings, bacteria develop and multiply by virtue of a series of chemical and physical processes, by means of which growth energy is obtained by destruction or catabolism, and the lost tissues resupplied by absorption of nutritive materials. It is natural, therefore, that the conditions of external temperature should intimately affect the metabolic processes. The range of temperature at which bacteria may grow is subject to wide variations among different species. Each species, on the other hand, may thrive within a more or less elastic range of temperature, each one having an optimum, a minimum, and a definite maximum temperature. When the optimum temperature is present in the environment, the functions of absorption and excretion keep pace with each other, and the chemical balance is well preserved. When the temperature is lower than the optimum, all metabolic processes take place more slowly, and the bacterium gradually enters into a resting or latent stage, at which actual growth may be exceedingly slow or entirely inhibited. When the temperature is higher than the optimum, the destructive processes are carried on more rapidly than the substitution of waste products by absorption, and a gradual weakening of vital energy, or even a gradual death of the bacterium, may take place. When certain bacteria form spores, they become very much more resistant against both high and low temperatures, probably because a true resting stage has been reached, during which metabolism has been reduced to a minimum, there being practically no nutritive material taken in and correspondingly little destruction taking place within the body of the microorganism.

The optimum temperature for various bacteria depends upon the habitual environment, in which the particular species is accustomed to exist. Thus, for the large majority of bacteria pathogenic for human beings, the optimum temperature is at or about $37.5^{\circ} \mathrm{C}$. There are a large number of bacteria common in water, however, which grow hardly at all at the body temperature, but thrive most luxuriantly at temperatures of about $20^{\circ} \mathrm{C}$. F. Forster, ${ }^{24}$ moreover,

${ }^{24}$ F. Forster, Cent. f. Bakt., ii, 1887. 
described certain phosphorescent bacteria, isolated from sea-water, which grow readily at $0^{\circ} \mathrm{C}$, , or a little above. On the other hand, Miquel ${ }^{25}$ has described non-motile bacilli, which he isolated from the water of the Seine, which grew" rapidly at temperatures ranging apout $70^{\circ}$ C., and the so-called "mucedinées thermophiles," described by Tsiklinski, ${ }^{26}$ develop most readily at temperatures very little above this. It is thus plain that the temperatures favored by various bacteria depend to a large extent upon an adaption of these bacteria through many generations to specific environmental conditions. A good illustration of this is furnished by the bacillus of avian tuberculosis, a microorganism differing essentially from the bacillus of human tuberculosis in that its optimum growth temperature lies at $41^{\circ}-42^{\circ} \mathrm{C}$., a temperature which exceeds the optimum temperature for the human type by as much as the normal temperature of birds exceeds that of man. The same principle is illustrated by the facts that the bacteria which have a very low optimum temperature are usually those isolated from water, and the so-called thermophile or high-temperature bacteria are obtained from hot springs and from the upper layers of the soil, where, according to Globig, ${ }^{27}$ occasionally temperatures ranging from about $55^{\circ} \mathrm{C}$. occur.

As stated before, one and the same species may develop within a wide temperature range, and it may be possible, by persistent cultivation at special temperatures, to adapt certain bacteria to grow luxuriantly at temperatures removed by several degrees from their normal optimum. In such cases it may often oceur that special characteristics of the given species may be lost. An example of this is the loss of virulence and of spore-formation which takes place when anthrax bacilli are cultivated at $42^{\circ} \mathrm{C}$., or the loss of the power to produce pigment when bacillus prodigiosus is grown at temperatures above $30^{\circ} \mathrm{C}$.

The vegetative forms of most of the pathogenic bacteria may grow at temperatures ranging between $20^{\circ}$ and $40^{\circ} \mathrm{C}$. This can, however, by no means be regarded as applicable to all of the pathogenic bacteria, as some of these, like the gonococcus, the pneumococcus, the tubercle bacillus, and others, are delicately susceptible to temperature changes and have the power of growing only within

${ }^{25}$ Miquel, Bull. de la Stat. Munic. de Paris, 1879.

${ }^{26}$ Tsiklinski, Ann. Past., 1889.

${ }^{27}$ Globig, Zeit. f. Hyg., iii. 
limits varying but a few degrees from their optimum. Others, on the other hand, like bacilli of the colon group, Bacillus anthracis, Spirillum choleræ asiaticæ, ete., may develop at temperatures as low as $10^{\circ} \mathrm{C}$. and as high as $40^{\circ} \mathrm{C}$., or over. The range of temperature at which saprophytic bacteria may develop is usually a far wider one. When temperatures exceed in any considerable degree the maximum growth temperature, the vegetative forms of bacteria perish. Thus, ten minutes' exposure to a temperature of between $55^{\circ}$ and $60^{\circ} \mathrm{C}$. causes death of the vegetative forms of most microorganisms. Death in such eases is due probably to a coagulation of the protoplasm, and since all such processes of coagulation take place best in the presence of water, the thermal death point of most bacteria is lower when heat is applied in the form of boiling water or steam, than when employed as dry heat. (See section on Sterilization.)

When spores are present in cultures, the resistance to heat is enormously increased. Exactly what the explanation of this is ean not at present be stated. It may be that the high eoneentration in which the protoplasmic mass is found in the spores renders it less easily coagulable than is the protoplasm of the vegetative body. A more detailed discussion of these relations will be found in the section on Heat sterilization.

The thermal death points of many bacteria have been earefully studied by Sternberg, ${ }^{28}$ by a technique described elsewhere.

The thermal death points ascertained by him in this way, with an exposure of ten minutes in a fluid medium, for some of the more common non-sporogenic bacteria are as follows:

Spirillum choleræ asiaticæ................... $52^{\circ} \mathrm{C}$.

Diplococcus pneumoniæ .................... $52^{\circ}$ C.

Streptococeus pyogenes ................... $54^{\circ}$ C.

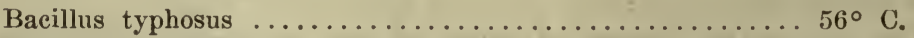

Bacillus pyocyaneus $\ldots \ldots \ldots \ldots \ldots \ldots \ldots \ldots \ldots \ldots \ldots 6^{\circ} \mathrm{C}$.

Bacillus mucosus capsulatus $\ldots \ldots \ldots \ldots \ldots \ldots \ldots \ldots \ldots 6^{\circ} \mathrm{C}$.

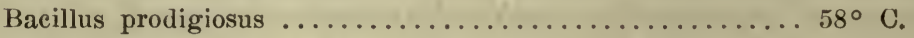

Staphylococcus pyogenes aureus .............. $58^{\circ}$ C.

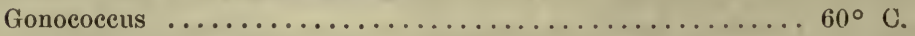

Staphylococcus pyogenes albus $\ldots \ldots \ldots \ldots \ldots \ldots \ldots \ldots 2^{\circ} \mathrm{C}$.

The bacillus tuberculosis, though not a spore bearer, seems to be slightly more resistant to heat than other purely vegetative mieroor-

${ }^{28}$ Sternberg, "Textbook of Bacteriology,' New York, 1901. 
ganisms. Thus, according to $\mathrm{Smith}^{29}$ and others, ten and twenty minutes' exposure to a temperature of $70^{\circ} \mathrm{C}$. is necessary to destroy tubercle bacilli in a fluid medium. For the effectual destruction of spores by moist heat, a temperature of $100^{\circ} \mathrm{C}$., or boiling point, is usually necessary.

Low temperatures are much less destructive than the high ones, and are even in a number of cases useful in keeping bacteria alive for long periods, inasmuch as metabolic processes are inhibited and life is maintained without actual development in a sort of resting state. Actual destruction by low temperatures rarely takes place. The exposure of diphtheria, typhoid, and other bacilli to temperatures as low as $200^{\circ} \mathrm{C}$. below zero has been carried out without destruction of the microorganisms, a fact which is of great importance in considering the possibility of infection by the vehicle of ice. Meningococci and gonococci, on the other hand, die out rapidly when exposed to $0^{\circ} \mathrm{C}$.

Relation to Pressure.-High pressure does not injure bacteria. Certes ${ }^{30}$ found that a pressure of two atmospheres had no influence upon the growth of anthrax bacilli suspended in blood.

Relation to Moisture.-For the growth and development of all bacteria, the presence of water is necessary. Nutritive materials can not be absorbed by an osmotic process unless in a state of solution. While complete dryness does not permit growth, its destructive action upon various bacteria is subject to great differences. The effect of complete drying upon bacteria will be found more fully discussed on page 76 . In the same place may be found a discussion of the effects of light, electricity, $x$-ray, and radium rays upon bacteria.

\section{THE CLASSIFICATION OF BACTERIA}

Too simple in structure, too varied in biological properties to be definitely identified with either the vegetable or animal kingdom, the bacteria are placed at the bottom of the scale of all living beings. Closely linked on the one hand to the plant kingdom by the yeasts and the molds, and on the other to the animal kingdom by the protozoa, they themselves combine, within one and the same division, attributes so widely divergent as to structure, metabolism, and biological activity that their grouping is more a matter of working con-

${ }^{29}$ Th. Smith, Jour. of Experimental Med., No. 3, 1899.

${ }^{20}$ Certes, Compt. rend. de l'acad. d. sc., 99, Paris, 1884. 
venience than of actual scientific classification. Thus, for instance, all stages of metabolic activity fill in the gap between the synthetizing sulphur and nitrifying bacteria and the purely katabolic activities of some of the aërobic and anaërobic microorganisms which cause putrefaction. Growth takes place within the limits of a wide temperature range, and the specific modes of life and cultural conditions are subject to the widest variations, from those of an indisputably useful saprophytism to those of the most exquisite parasitism. Although, therefore, strictly speaking, the bacteria can be classified as a whole neither in the animal nor in the vegetable realms, being nonchlorophyll-bearing, they are for convenience classified with the fungi or colorless plants.

The relationship of the bacteria to other simple plants may be graphically represented by the following scheme:

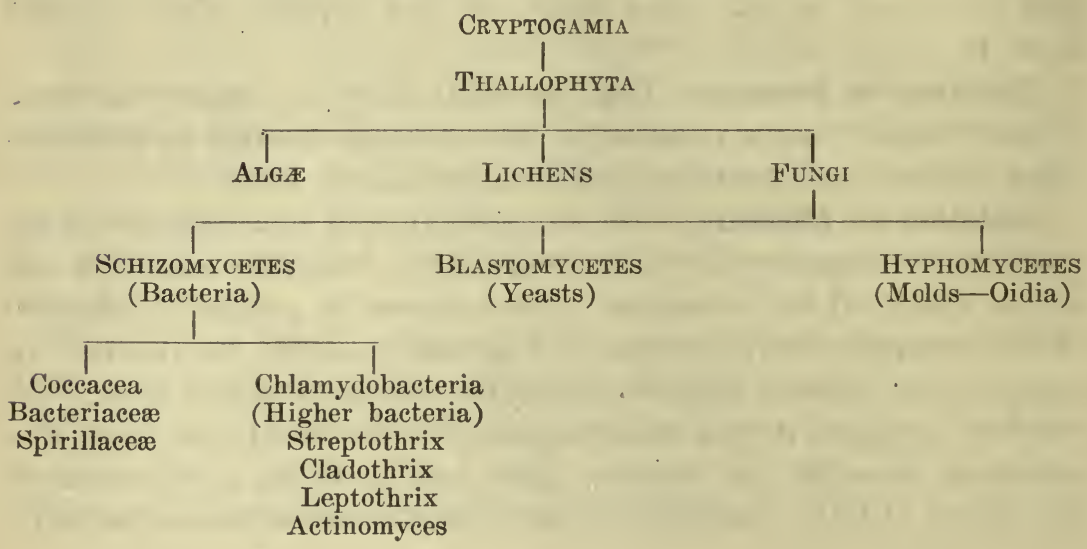

The special classification of the bacteria has offered still greater difficulties, for the lower we proceed in the phylogenetic scale of living beings, the less specialized the morphological and biological characteristics of any group become, and the more difficult it is to establish a classification which can in any way be regarded as final. It is, therefore, quite impossible to classify the bacterial varieties or species on any basis which can hope to satisfy all the demands of scientific accuracy and it is necessary to resort to the expedient of utilizing some one characteristic which remains constant for the individual genus and to base upon this an attempt at grouping. When bacteria were first discovered, and for many years following, numerous observers contended that the form of the microorganism 
observed was not a constant one for each genus, but that cocci could be converted into bacilli or spirilla according to environmental conditions. It was Cohn ${ }^{31}$ who, in 1872, first recognized the constancy of the morphology of bacteria and established, upon morphological basis, a classification which, with minor changes, has been retained until the present day. Such classifications can not, however, be regarded as anything more than a convenient make-shift pending the day when the finer structure and true biological relations of the various bacteria shall have been more accurately investigated. The scheme most commonly accepted at present is the one given below, proposed by Migula: ${ }^{32}$

Bacteria (Schizomycetes).-Fission fungi (chlorophyll free), cell division in one, two, or three directions of space. Many varieties possess power of forming endospores. Whenever motility is present, it is due to flagella, or, more rarely, to undulating membranes.

Family I. Coccacex.-Cells in free state spherical. Division in one, two, or three directions of space, by which each cell divides into two, four, or eight segments, each of which again develops into a sphere. Endospore formation rare.

Genus I. Streptococcus.-Cells divide in one direction of space only, for which reason, if they remain connected after fission, bead-like chains may be formed. No organs of locomotion.

Genus II. Micrococcus (Staphylococeus).-Cells divide in two directions of space, whereby, after fission, tetrad and grape-like clusters may be formed. No organs of locomotion.

Genus III. Sarcina.-Cells divide in three directions of space, whereby, after fission, bale-like packets are formed. No organs of locomotion.

Genus IV. Planococcus.-Cells divide in two directions of space, as in micrococcus, but possess flagella.

Genus V. Planosarcina.-Cells divide in three directions of space as in sarcina, but possess flagella.

FAmily II. BACteriace. spiral. Division in one direction of space only, after. preliminary elongation of the rods.

Genus I. Bacterium.-Cells without flagella, often with endospores.

Genus II. Bacillus.-Cells with peritrichal flagella, often with endospores.

${ }^{31}$ Cohn, "'Beiträge zur Biol. d. Pflanzen," Heft 1 u. 2, 1872.

${ }^{22}$ Migula, "System d. Bakt.,"' Jena, 1897. 
Genus III. Pseudomonas.-Cells with polar flagella. Endospores occur in a few species, but are rare.

FAMILY III. SPIRILLACEA,-Cells spirally curved or representing a part of a spiral curve. Division in one direction of space only, after preceding elongation of cell.

Genus I. Spirosoma.-Cells without organs of locomotion. Rigid.

Genus II. Microspira.-Cells rigid, with one or, more rarely, two or three polar undulated flagella.

Genus III. Spirillum.-Cells rigid, with polar tufts of five to twenty flagella usually curved in semicircular or flatly undulating eurves.

Genus IV. Spirochate.-Cells sinously flexible. Organs of locomotion unknown, perhaps a marginal undulating membrane.

Family IV. Chlamydorbacteriaced.-Forms of varying stages of evolution, all possessing a rigid sheath (Hülle), which surrounds the cells. Cells united in branched or unbranched threads.

Genus I. Streptothrix.-Cells united in simple, unbranched threads. Division in one direction of space only. Reproduction by non-motile conidia.

Genus II. Cladothrix.-Cells united or pseudodichotomously branching threads. Division in one direetion of space only. Vegetative multiplication by separation of entire branches. Reproduction by swarming forms with polar flagella.

Genus III. Crenothrix.-Cells united in unbranched threads, at first with division in one direction of space only. Later the cells divide in all three directions of space. The daughter cells become rounded and develop into reproductive cells.

Genus IV. Phragmidiothrix.-Cells at first united in unbranched threads, dividing in three directions of space, thus forming a rope of cells. Later some of the cells may penetrate through sheath, and thus give rise to branches.

Genus V. Thiothrix.-Unbranched, non-motile threads, inclosed in fine sheaths. Division of cells in one direction only. Cells contain sulphur granules.

Family V. Beggiatoacex.-Cells united in sheathless threads. Division in one direction of space only. Motility by undulating membrane as in Oscillaria.

Genus Beggiatoa.-Cells with sulphur granules.

It will be seen in reviewing the classification just given that the subdivisions are based upon questions of form, motility, and situation of flagella. While these characteristics, so far as we know, are 
constant, there are, nevertheless, many instances in which types entirely similar in these respects must be differentiated. This can be done only by careful study of staining reactions, finer structure, cultural characteristics, and biological activities.

As a matter of fact, while the botanical classification of the bacteria offers great difficulties, identification is not so complicated a task as this would indicate. Identification, once roughly made on a morphological basis, is further carried on by the aid of cultural characteristics, by biochemical reactions and by pathogenic properties. The bacteria occupy so important a place in agriculture, in medicine, and in hygiene, that it rarely becomes necessary for a worker in any particular field to survey the entire group. The habitat of a large number of species is so well known that this consideration alone often gives a clue to actual identification.

Bacterial Mutation.-The earlier views of bacteriologists concerning mutation differed greatly, Naegeli holding that extensive mutation was probably the rule; Cohn, on the other hand, holding strictly to the constancy of form and species. The accumulated experience of many bacteriologists during the years since then seems to point almost entirely in the direction indicated by Cohn, and, in fact, most of our methods of classification are based upon the assumption of such constancy.

Form alone, of course, cannot be relied upon for classification among organisms so simply constructed that the possibilities of variation in form are very limited. In classifying bacteria, therefore, we are forced to take cognizance not only of morphology, but also of staining characteristics, behavior on differential media, fermentation reactions, pathogenicity, and, as a final appeal, reactions with specific immune sera. The last especially, as utilized in agglutination and complement-fixation, seems to indicate a fundamental chemical difference in the constitution of bacteria often morphologically very much alike. It is certainly a remarkable fact that organisms such as those belonging to the colon-typhoid-dysentery group, though morphologically not differentiable, may still retain differences both in pathogenieity and in fermentation powers after being kept for ten or more years in laboratory media, and we have had the same experienee with organisms belonging to the diphtheria group. The virulence of plague and anthrax bacilli may be retained for years in storage, and such evidence shows pretty definitely that fundamental constant differences between organisms exist. 
In judging of mutation we must differentiate between temporary changes of secondary characteristics which revert to the type rapidly when brought back to the normal environment and those which constitute permanent inherited characteristics. Of recent years much work has been done on this question, which has been revived very thoroughly by Eisenberg ${ }^{33}$ and by Vaughan. ${ }^{34}$ Systematic cultivation of colon and typhoid bacilli in the hands of Twort, Penfold and others seems to have shown that agglutination as well as fermentation characteristics can be artificially changed. Furthermore, colorproducing organisms like the prodigiosus can be artificially changed to colorless strains, and it is well known that certain microorganisms rapidly lose their virulence when cultivated, and that the virulence can only be brought back by passage through animals. Rosenow ${ }^{35}$ claims recently to have converted hemolytic streptococci into typical streptococeus viridans, pneumococcus mucosus, and pneumococcuslike organisms. In just how far these observations will be shown to represent true permanent mutations we are not at present ready to determine. If it will be found that organisms typically representative of a well-known species can be changed in the animal body or in culture into forms recognizedly typical of another species, we will have to revise our classifications, and we can look upon the classes as now established merely as convenient methods of making discussion possible, but not as representing botanically constant types.

While we must therefore admit that a considerable degree of mutation is possible, we do not ourselves believe that the evidence is sufficiently strong to undermine the prevailing ideas as to the constancy of species. Most mutations so far produced have readily reverted to type when subjected to proper conditions.

\footnotetext{
${ }^{33}$ Eisenberg, Weichhardt's Ergebnesse, 1914.

* Vaughan, Jour. of Lab. \& Clin. Med., 1915, vol. 1, 145.

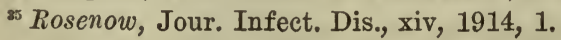




\section{CHAPTER IV}

\section{THE BIOLOGICAL ACTIVITIES OF BACTERIA}

WHILE the bacteria pathogenic to man and animals largely usurp the attention of those interested in disease processes, this group of microorganisms is after all but a small specialized off-shoot of the realm of bacteria, and, broadly speaking, actually of minor importance. Surveying the existing scheme of nature, as a whole, it is not an extravagant statement to say that without the bacterial processes which are constantly active in the reduction of complex organic substances to their simple eompounds, the chemical interchange between the animal and vegetable kingdoms would fail, and all life on earth would of necessity cease. To understand the full significance of this, it is necessary to consider for a moment the method of the interchange of matter between the animal and vegetable kingdoms.

All animals require for their sustenance organic compounds. They are unable to build up the complex protoplasmic substances which form their body cells from chemical elements or from the simple inorganic salts. They are dependent for the manufacture of their food-stuffs, therefore, directly or indirectly, upon the synthetic or anabolic activities of the green plants.

These plants, by virtue of the chlorophyll contained within the cells of their leaves and stems, and under the influence of sunlight, possess the power of utilizing the earbon of the carbonic acid gas of the atmosphere, and of combining it with water and the nitrogenous salts absorbed by their roots, building up from these simple radicles the highly complex substances required for animal sustenance.

These products of the synthetic activity of the green plants, then, are ingested by members of the animal kingdom, either directly, in the form of vegetable food, or indirectly, as animal matter. They are utilized in the complex laboratory of the animal body and are again broken down into simpler compounds, which leave the body as excreta and secreta.

The excreta and secreta of animals, however, are, in a small part 
only, made up of substances simple enough to be directly utilized by plants. The dead bodies, moreover, of both animals and plants would be of little further value as stores of matter unless new factors intervened to reduee them to that simple form in which they may again enter into the synthetie laboratory of the green plant. Agents for further eleavage of these compounds are required, and these are supplied by the varied activities of the bacteria.

On the other hand, bacteria are also important in the process of synthesis. The main supply of nitrogen available for plant life is found in the elementary state in the atmosphere-a condition in which it can not be utilized as a raw product by the plant. This gap again is bridged by the bacteria found in the root bulbs of the leguminous plants-bacteria which possess the power of assimilating or aiding in the assimilation of atmospheric nitrogen and its preparation for further use by the plant itself. Another bacterial activity which may be classified as an anabolie process is the oxidation of the ammonia, released by decomposition, into nitrites and nitrates. This is carried on by certain bacteria of the soil. These are to be treated of in greater detail in another section.

There is a constant eirculation, therefore, of nitrogen and earbon compounds, between the plant and the animal kingdoms, by virtue of an anabolic or constructive process in the one, and a katabolic or destructive process in the other, rendering them mutually interdependent and indispensable. The circuit, however, is not by any means a closed one; there are important gaps, both in the process of cleavage and in that of synthesis, which, if left unbridged by the bacteria, would effectually arrest all life-activity of plants and eventually of animals.

Far from being scourges, therefore, these minute microorganisms are paramount factors in the great eyele of living matter, supplying necessary links in the cireulation of both nitrogenous and carbon compounds.

\section{KATABOLIC ACTIVITIES OF BACTERIA}

The katabolic activities of bacteria, then, consist in the fermentation of carbohydrates and in the cleavage of proteins and fats.

Fermentation is carried out to a large extent by the yeasts, but also to no inconsiderable degree by bacteria. Protein decomposition and the cleavage of fats are carried out almost exclusively by bacteria. 
For our knowledge of the fundamental laws underlying these phenomena of fermentation and protein decomposition, we are indebted to the genius of Pasteur, ${ }^{1}$ who was the first to prove experimentally the exclusive and specific parts played by various microorganisms in these processes. While the observations and deductions made by Pasteur have not been greatly modified, a large store of information has been gained since his time, which has thrown additional light upon the chemical details and the more exact manner of action of the factors involved.

The actual work of cleavage in both fermentation and protein cleavage is carried out by substances known as enzymes or ferments, the nature of which we must further discuss before their manner of action can be fully comprehended.

Bacterial Enzymes or Ferments.-A ferment or enzyme is a substance produced by a living cell, which brings about a chemical reaction without entering into the reaction itself. The enzyme itself is not bound to any of the end products and is not appreciably diminished in quantity after the reaction is over, although its activity may be finally inhibited by one or another of the new products. The action of bacterial enzymes is thus seen to be closely similar to that of the chemical agents technically spoken of as "katalyzers," represented chiefly by dilute acids. Thus, if an aqueous solution of saccharose is brought into contact with a dilute solution of sulphuric acid, the disaccharid is hydrolyzed and is decomposed into levulose and dextrose.

Thus :

$$
\underset{\text { In contact with }}{\mathrm{C}_{12} \mathrm{H}_{22} \mathrm{O}_{11}}+\mathrm{H}_{2} \mathrm{O}=\underset{\text { Dextrose }}{\mathrm{C}_{6} \mathrm{H}_{12} \mathrm{O}_{6}}+\underset{\text { Levulose }}{\mathrm{C}_{6} \mathrm{H}_{12} \mathrm{O}_{6}}
$$$$
\text { dilute } \mathrm{H}_{2} \mathrm{~S} \mathrm{O}_{4}
$$

During this process, which is known as "inversion," the concentration of the sulphuric acid remains entirely unchanged. While theoretically the changes brought about by enzymes and katalyzers are usually such as would occur spontaneously, the time for the spontaneous occurrence would be, at ordinary temperatures, infinitely long. The definition for enzymes and katalyzers is given by Ostwald, therefore, as "substances which hasten a chemical reaction without themselves taking part in it." Exactly the same result which is obtained by the use of dilute sulphuric acid is caused by the ferment "invertase" produced, for instance, by B. megatherium. Were a solution

'Pasteur, “'Etude sur la bière,', Paris, 1876. 
of saccharose subjected to heat, without kacalyzer or ferment, a similar change would occur, but by the mediation of these substances the inversion is produced without other chemical or physical reinforcement.

This analogy between enzymes and katalyzing agents is very striking. Thus, as stated, both katalyzers and enzymes bring about ehanges without themselves being used up -in the process, both act without the aid of heat, and the reactions brought about by both have occasionally been shown to be reversible. While this last phenomenon has been variously shown for katalyzers, the process of reversibility has been demonstrated for bacterial enzyme action only in isolated cases. Thus, it has been found that by the action of the yeast enzyme maltase upon concentrated dextrose solutions, a reformation of maltose may occur. In both cases, moreover, the quantity of enzyme or katalyzer is infinitely small in proportion to the amount of material converted by their action.

There is a close similarity, furthermore, between the bacterial enzymes and the ferments produced by specialized cells of the higher animals and plants. For instance, the action of the ptyalin of the saliva or of the diastase obtained from plants is entirely analogous to the starch-splitting action of the amylase produced by many bacteria.

The action of all enzymes depends most intimately upon environmental conditions. For all of them the presence of moisture is essential. All of them depend for the development of their activity upon the existence of a specifically suitable reaction. Strong acids or alkalies always inhibit, often destroy them. Temperatures of over $70^{\circ}$ C. permanently destroy most enzymes, whereas freezing, while temporarily inhibiting their action, causes no permanent injury, so that upon thawing, their activity may be found almost unimpaired. Direct sunlight may injure, but rarely destroys, ferments. Against the weaker disinfectants in common use, enzymes often show a higher resistance than do the bacteria which give rise to them.

The optimum conditions for enzyme action, then, consist in the presence of moisture, the existence of a favorable reaction, weakly acid or alkaline, as the case may be, and a temperature ranging from $35^{\circ}-45^{\circ} \mathrm{C} .^{2}$

Proteolytic Enzymes.-In nature, the decomposition of dead animal and vegetable matter occurs only when the conditions are favor-

¿ Oppenheimer, "Die Fermente," ete. Leipzig, 1900. 
able for bacterial development. Thus, as is well known, freezing, sterilizing by heat, or the addition of disinfectants will prevent the rotting of organic material.

In the laboratory, the presence of proteolytic enzymes is determined chiefly by the power of bacteria to liquefy gelatin, fibrin, or coagulated blood serum. These ferments are not always secretions from the bacterial cell, but in some cases may be closely bound to the cell-body and separable only by extraction after death. In such cases they are spoken of as endoenzymes. Whenever they are true secretory products, however, they can be obtained separate from the microorganisms which form them by filtration through a Berkefeld candle. From such filtrates they may, in some cases, be obtained in the dry state by precipitation with alcohol. When obtained in this way the precipitated enzyme is usually much more thermostable than when in solution, for while soluble enzymes in filtrates are usually destroyed by $70^{\circ} \mathrm{C}$., and even less, the dried powder may occasionally withstand $140^{\circ} \mathrm{C}$. for as long as ten minutes. ${ }^{3}$

Apart from the general conditions of temperature and moisture, the development of these enzymes seems to depend directly upon the presence of proteins in the culture media. The number of bacterial species which produce proteolytic enzymes is legion. Among those more commonly met with are staphylococei, B. subtilis, B. proteus, B. fæcalis liquefaciens, Spirillum choleræ asiaticæ, B. anthracis, B. tetani, B. pyocyaneus, and a large number of others. The inability of any given microorganism to liquefy gelatin or fibrin by no means entirely excludes the formation by it of proteolytic enzymes, since these ferments may often be active for one particular class of protein only.

In order to study the qualitative and quantitative powers of any given bacterial proteolyzing enzyme or protease, it is, of course, necessary to study these processes in pure culture in the test tube with media of known composition. In the refuse heap, in sewage, or in rotting excreta, the process is an extremely complicated one, for besides the bacteria which attack the protein molecule itself, there are many other species supplementing these and each other, one species attacking the more or less complex end-products left by the action of the others.

Exactly what the chemical reactions are which take place in these

3Huhrmann, "Die Bakterienzyme,", p. 45. 
cleavages is not entirely clear. It is believed, however, that most of the cleavages are of an hydrolytic nature.

In general, the action of the protein-splitting ferments is comparable to that of the pancreatic ferment trypsin, and they are most often active in an alkaline enviromment. They differ, among themselves, in the extent to which they are able to reduce the protein molecule to its simple radicles, some types leading to a relatively mild cleavage, while others, of the proteus type, yield amino acids. Many species of bacteria which are unable to secrete a proteolytic enzyme, nevertheless produce erepsin-like ferments which readily attack peptones or polypeptides, with the formation of amino acids.

A distinction is oceasionally made between the terms putrefaction and decay, the former being used to refer to the decomposition taking place under anaërobic conditions, that is, in the absence of oxygen, a process resulting in the production of amino acids, $\mathrm{H}_{2} \mathrm{~S}$, indole, skatole and particularly the mercaptans, which eause the highly offensive odor characteristic of this type of decomposition. The gases generated in such decomposition are largely made up of $\mathrm{CO}_{2}$ and hydrogen. The coincident presence, furthermore, of the carbohydrate-splitting bacteria and of denitrifying microorganisms renders the actual process of putrefaction a chaos of many activities in which the end-products and by-products are qualitatively determinable only with little precision, and which completely defies any attempt at quantitative analysis. Decay is the term used to signify decomposition under aërobic conditions, leading primarily to the formation of amino acids, which are as a rule, changed further to carbon dioxide, water and ammonia, often together with $\mathrm{H}_{2} \mathrm{~S}$ and less completely decomposed substances, such as indole and various amines. Mercaptans are never formed and the foul odor of putrefaction is not present in this aërobic proteolysis. ${ }^{4}$

Ptomaines.-Very early in the study of the products of bacterial growth, a number of well defined crystalline basic substances were isolated from protein material which had undergone bacterial putrefaction. These received, as a class, the name of ptomaines (from $\pi \tau \hat{\omega} \mu a$, a dead body), and were shown to be toxic when fed to or injected into animals. It was attempted, at the time of their discovery, to explain the action of pathogenic bacteria on the basis of a production of ptomaines or related substances. This soon

'Rettger and Newell, Jour. of Biol. Chem., xii, 1912, 341. 
proved not to be the case, for not only were these bases never toxic in the minute dose sufficient for the true toxins of diphtheria and tetanus, but the anatomical lesions produced were different. Moreover, true bacterial toxins seemed to be formed to a great extent independent of the composition of the media upon which growth was obtained, while, as will be seen, ptomaine production depends directly upon the constitution of the substrate. Finally, nothing in the nature of antitoxin production could be shown, following sublethal injections of the ptomaines. While it is, therefore, not possible to account for the symptoms of bacterial action in the body on the basis of ptomaines, it is not impossible that the ptomaines themselves, if ingested in food which has undergone some putrefactive changes, may occasionally cause sickness. Food poisoning of this variety was formerly believed to be very common, and was described under various names, as kreatoxismus (meat poisoning), tyrotoxismus (cheese poisoning) and sitotoxismus (vegetable poisoning). At present there is much question as to whether food poisoning, wherever met with (excepting, of course, botulism, which is caused by the ingestion of a true bacterial toxin), is not caused by the presence of living bacteria of the Gärtner type in the food, and not to ptomaines. ${ }^{5}$ It is quite possible, on the other hand, that bacteria present in the intestine may, under certain conditions, set up putrefactive changes in the large bowel, leading to the formation of ptomaines or closely allied substances which can be absorbed directly and cause illness.

Chemically, the ptomaines are bases produced by the splitting out of $\mathrm{CO}_{2}$ from the acid group of the amino acids. Amines have been isolated resulting from this form of decomposition of practically all the known amino acids. Of historic interest are putrescine and cadaverine. The former is produced by the decarboxylation of ornithine, thus :

\section{$\mathrm{CH}_{2}\left(\mathrm{NH}_{2}\right) \mathrm{CH}_{2} \cdot \mathrm{CH}_{2} \cdot \mathrm{CH}\left(\mathrm{NH}_{2}\right) \mathrm{COOH}=$}

$\mathrm{CH}_{2}\left(\mathrm{NH}_{2}\right) \cdot \mathrm{CH}_{2} \cdot \mathrm{CH}_{2} \cdot \mathrm{CH}_{2}\left(\mathrm{NH}_{2}\right)+\mathrm{CO}_{2}$

Cadaverine is similarly formed from lysine. Both these bases possess relatively slight toxicity. Much more highly toxic is the base histamine, obtained in an entirely analogous manner from his-

\footnotetext{
'Jordan, "Food Poisoning," University of Chicago Press, 1917.
} 
tidine. Some light seems to have been shed on the mechanism of this process of decarboxylization by Koessler and Hanke, ${ }^{6}$ who found, while investigating the production of histamine by $\mathrm{B}$. coli, that it was formed only in eultures in which considerable quantities of acid were being produced at the same time, and they belicve that the production of this strongly basic substance offsets to a degree the acid production and enables the organisms to develop further before the H-ion concentration reaches the point of inhibition. Sepsine, another base of unknown composition, but probably related in structure to the other ptomaines, has been isolated by Faust from putrid yeast, and has been shown to be highly toxic.

In breaking down animal excreta, the task of the bacteria is rather a simpler one than when dealing with the cadavers themselves, for here a part of the cleavage has already been carried out either by the destructive processes accompanying metabolism, or by partial decomposition by bacteria begun within the digestive tract. This material outside of the body is further reduced by bacterial enzymes into still simpler substances, the nitrogen usually being liberated in the form of ammonia. One example of such an ammoniacal fermentation may be found in the case of the urea fermentation by Micrococcus ureæ, in which the cleavage of the urea takes place by hydrolysis according to the following formula:

$$
\left(\mathrm{NH}_{2}\right)_{2} \mathrm{CO}+2 \mathrm{H}_{2} \mathrm{O}=\mathrm{CO}_{2}+2 \mathrm{NH}_{3}+\mathrm{H}_{2} \mathrm{O}
$$

Similar ammoniacal fermentations are carried out, though perhaps according to less simple formulæ, by a large number of microorganisms. Perhaps the most common species which possesses the power is the group represented by B. proteus vulgaris (Hauser).

From what has been said it follows naturally that, so far, the decomposition of the protein molecule from its complex structure to ammonia or simple ammonia compounds is an indispensably important function, not only for agriculture, but for the maintenance of. all life processes. It is clear, on the other hand, that a further decomposition of ammonia compounds into forms too simple to be utilized by the green plants would be a decidedly harmful activity. And yet this is brought about by the so-called denitrifying bacteria which will be considered in a subsequent section.

- Koessler and Hanke, J. Biol. Chem., 1919, 39, 539. 
Lab Enzymes.-There are a number of ferments produced by baeteria which, although affecting proteins, can not properly be classified with the proteolytic enzymes. These are the so-called coagulases or lab enzymes, which have the power of producing coagulation in liquid proteins. Just what chemical process underlies this coagulation is not known. If Hammarsten's ${ }^{7}$ conclusions as to the hydrolytic nature of the changes produced by them are true, these enzymes are brought into close relationship to the proteolyzers, although a coagulation can hardly be regarded as a true katabolic process. In milk where the lab-action becomes evident by precipitation of casein, a strict differentiation must be made between this coagulation and that brought about by acids or alkalies. In the former case, casein is not only precipitated and converted into paracasein, but is actually changed so that when redissolved it is no longer precipitated by lab. ${ }^{8}$

Coagulating enzymes for milk proteins, blood, and other protein solutions are produced by a large variety of bacteria. They have been observed in cultures by the cholera vibrio, B. prodigosus, B. pyocyaneus, and several others. ${ }^{9}$

The lab enzymes are easily destroyed by temperatures of $70^{\circ} \mathrm{C}$. and over, and are very susceptible to excessive acidity or alkalinity.

Fat-Splitting Enzymes (Lipase).-The fat-splitting powers of bacteria have been less studied than some of the other bacterial functions and are correspondingly more obscure. - It is known, nevertheless, that the process is due to an enzyme and that it is probably hydrolytic in nature. The following formula represents the simplest method in which some of the molds and bacteria produce cleavage of fats into glycerin and fatty acid:

$$
\begin{aligned}
& \mathrm{C}_{3} \mathrm{H}_{5}\left(\mathrm{C}_{\mathrm{n}} \mathrm{H}_{2 \mathrm{n}-1} \mathrm{O}_{2}\right)_{3}+3 \mathrm{H}_{2} \mathrm{O}=\mathrm{C}_{3} \mathrm{H}_{5}\left(\mathrm{OH}_{3}\right)+3 \mathrm{C}_{\mathrm{n}} \mathrm{H}_{2 \mathrm{n}} \mathrm{O}_{2} \\
& \text { Glycerin Fatty acid }
\end{aligned}
$$

Some of the bacteria endowed with the power of producing lipase are the cholera spirillum, B. fluorescens liquefaciens, B. prodigiosus, B. pyocyaneus, Staphylococcus pyogenes aureus, and some members of the streptothrix family. The methods of in-

"IIammarsten," "Texthook of Physiol. Chemistry," Translation by Mandel.

"Oppenheimer, “Die Fermente u. ihre Wirkung,' I.eipzig, 1903.

${ }^{8}$ Torini, Atti dei laborat. d. sanita, Rome, 1890. 
vestigating this function of bacteria, originated by Ejkmann, ${ }^{10}$ consists in covering the bottom of a Petri dish with tallow and pouring over this a thin layer of agar. Upon this, the bacteria are planted. Any diffusion of lipase from the bacterial colonies becomes evident by a formation of white, opaque spots in the tallow. Carrière ${ }^{11}$ was able to demonstrate a fat-splitting ferment for the tubercle bacillus. Apart from the importance of these enzymes in nature for the destruction of fats, they are industrially important because of their action in rendering butter, milk, tallow, and allied products rancid, and are medically of interest for their action upon fats in the intestinal canal.

Enzymes of Fermentation (The Cleavage of Carbohydrates by Bacteria).-The power to assimilate carbon dioxide from the atmosphere is possessed only by the green plants and some of the colored alga, and the sulphur or thiobacteria. All other living beings are thus dependent for their supply of earbon upon the synthetic activities carried on by these plants to the same degree in which they are dependent upon similar processes for their nitrogen supply. The return of this carbon to the atmosphere is, of course, brought about to a large extent by the respiratory processes of the higher animals. The carbon, which; together with nitrogen, forms a part of protein combinations, is freed, as we have seen in a previous section, by the processes of protein cleavage. That, however, which is inclosed in the carbohydrate molecule, is set free by the action of yeasts, molds, or bacteria, by an enzymatic process similar in every respect to that described above for the process of protein cleavage.

Fermentation.-The power of carbohydrate cleavage is possessed by a large number of the yeasts and bacteria. The process, as has been indicated, is of great importance in the cycle of carbon compounds for the return of carbon to its simplest forms, and is, furthermore, as will be seen in a later section, of great utility in the industries. In each case the power to split a particular carbohydrate is a more or less specific characteristic of a given species of microorganism, and for this reason has been extensively used as a method for the biological differentiation of bacteria. In the eourse of much careful work upon this question it has been ascertained that the

${ }^{10}$ Ejkmann, Cent. f. Bakt., I, xxix, 1901.

${ }^{11}$ Carrière, Comptes rend. de la soc. de biol., 53, 1901. 
specific carbohydrate-splitting powers of any given species are constant and unchanged through many generations of artificial cultivation. Thus, differentiation of the Gram-negative bacteria, the diphtheria group, and to some extent of the members of the pneumococeus-streptocoreus group, ean now largely be made by a study of their sugar fermentations.

In most of these cases, as far as we know, the cleavage is produced by a process of hydrolysis. A convenient nomenclature which has been adapted for the designation of these ferments is that which employs the name of the converted carbohydrate adding the suffix "ase" to indicate the enzyme. There are thus ferments known as amylase, cellulase, lactase, etc.

Amylase (Diastase or Amylolytic Ferment).-Amylases or starchsplitting enzymes are formed by many plants (malt) and by animal organs (pancreas, saliva, liver). Among microorganisms amylase is produced by many of the streptothrix group, by the spirilla of Asiatic cholera and of Finkler-Prior, by B. anthracis, and many other bacteria. A large number of the bacteria found in the soil, furthermore, have been shown to produce amylases. By cultivating bacteria upon starch-agar plates, amylase ean be readily demonstrated by a clearing of the medium immediately surrounding the colonies. ${ }^{12}$

Since, of course, there are several varieties of starches, it follows that the exact chemical action of amylase differs in individual cases. The determination of the structural disintegration of starch by these ferments is fraught with much difficulty, owing to the polymeric constitution of the starches. Primarily, however, a cleavage takes place into a disaceharid such as maltose (hexobiose), and the nonreducing sugars and dextrin. Beyond this point, however, the further cleavages are subject to much variation and are not entirely clear. The dextrins upon further reduction yield eventually maltose, and this in turn, dextrose.

Another most interesting example of amylolytic activity is the fermentation of starch by such organisms as the B. Granulobacter Pectinovorum with the production of acetone and butyl alcohol. This reaction is now used industrially for the preparation of acetone, using a corn meal mash as the substrate. Dextrose is first formed, and from this, acetone, butyl alcohol, acetic and butyric acid, to-

${ }^{12}$ Ejkmann, Cent. f. Bakt., xxix, 1901, and $\mathrm{xxxv,} 1904$. 
gether with hydrogen and $\mathrm{CO}_{2}$ are produced. The chemical mechanism of the fermentation is thus seen to be highly complex. ${ }^{13}$

Cellulase.-Cellulose is fermented by a limited number of bacteria, most of them anaërobes. The chemical process by which this takes place is but poorly understood. ${ }^{14}$

Gelase.-An agar-splitting ferment has been found by Gran. ${ }^{15}$

Invertase.-The enzymes which hydrolytically cause cleavage of saccharose into dextrose and levulose are numerous. The chemical process takes place according to the following formula:

$$
\underset{\text { Saccharose }}{\mathrm{C}_{12} \mathrm{H}_{22} \mathrm{O}_{11}}+\mathrm{H}_{2} \mathrm{O}=\underset{\text { Dextrose }}{\mathrm{C}_{6} \mathrm{H}_{12} \mathrm{O}_{6}}+\underset{\text { Levulose }}{\mathrm{C}_{6} \mathrm{H}_{12} \mathrm{O}_{6}}
$$

Invertase is produced by many of the yeasts. It is one of the most common of the enzymes produced by bacteria, and has been found in cultures of B. megatherium, B. subtilis, pneumococcus, some streptococci, B. coli, and many others. Invertase is usually very susceptible to heat, being destroyed by temperatures of $70^{\circ} \mathrm{C}$. and over. A slightly acid reaction of media abets the inverting action of these enzymes. Strong acids and alkalies inhibit them. Inverting enzymes may be precipitated out of solution by alcohol. Antiseptics even in weak concentrations will inhibit their action.

Lactase.-Lactose-splitting ferments are extremely common both among bacteria and among the yeasts. The process is here again a hydrolytic cleavage resulting in the formation of the monosaccharich, dextrose and galactose.

Maltase.-A maltose-splitting ferment has also been found in the cultures of many bacteria, leading to the formation of dextrose.

Lactic Acid Fermentation.-Lactic acid (oxyproprionic acid, $\begin{array}{lll}\mathrm{C}_{3} & \mathrm{H}_{6} & \mathrm{O}_{3}\end{array}$ ) is one of the most common substances to appear among the products of bacterial activity, both in media containing carbohydrates and in those consisting entirely of albuminous substances. In most of these cases, the lactic acid is formed merely as a byproduct accompanying many other more complicated chemical cleavages. In some instances, however, lactic acid is produced from carbohydrates, both disaceharids and monosaceharids, as an almost

\footnotetext{
${ }^{13}$ Speakman, Jour. of Biol. Chem., xli, 1920, 319; xliii, 1920, 401.

${ }^{14}$ Omelianski, Lafar's "Handb. d. techn. Mykologie," Bd. iii, Chap. 9.

${ }^{15}$ Gran, Bergens Museum Aarbog, 1902, Hft. I.
} 
pure product due to a specific bio-chemical process. The reactions taking place in this phenomenon may be briefly expressed according to the following formulæ:

$$
\begin{aligned}
& \mathrm{C}_{12} \mathrm{H}_{22} \mathrm{O}_{11} \\
& \text { Lactose }
\end{aligned}+\mathrm{H}_{2} \mathrm{O}=\underset{\text { Lactic acid }}{4 \mathrm{C}_{3} \mathrm{H}_{6} \mathrm{O}_{3}}
$$

or

$$
\begin{aligned}
& \mathrm{C}_{6} \mathrm{H}_{12} \mathrm{O}_{6}=2 \mathrm{C}_{3} \mathrm{H}_{6} \mathrm{O}_{3} \\
& \text { Dextrose Lactic acid }
\end{aligned}
$$

In the same way lactic acid may be produced by bacteria from levulose.

Examples of lactic acid formation are furnished by the streptococcus lacticus, and B. lactis aërogenes. In the case of the former, the fermentation may indeed proceed ${ }^{\circ}$ by the simple chemical process indicated in the formulæ, since the action of the bacillus is entirely unaccompanied by the evolution of gas.

Numerous other bacteria produce large amounts of lactic acid from lactose, possibly by chemical processes less simply formulated. Among these are bacilli of the colon group, B. prodigiosus, B. proteus vulgaris, and many others. Although lactic acid is usually the chief product in the bacterial fermentation of the simpler carbohydrates, acetic, formic, and butyric acids may often be found as by-products in variable arnounts. ${ }^{16}$

Oxydases (Oxydizing Enzymes).-The most common example of oxidation by means of bacterial ferments is the production of acetic acid from weak solutions of ethyl alcohol. This process, which is the basis of vinegar production, is universally carried out by bacteriai ferments. While possessed to some extent by a considerable number of microorganisms, acetic acid formation is a function preeminently of the bacterial groups described by Hansen, including "Bacterium aceti" and "Bacterium pasteurianum." To these two original groups, a number of others have since been added.

The organisms are short, plump bacilli, with a tendency to chainformation, and occasionally showing characteristically swollen centers and many irregular involution forms. In the production of vinegar, as generally practiced by the farmer with cider or wine, these bacteria accumulate on the surface of the fluid as a pellicle

${ }^{16}$ Buchner und Meisenheimer, Ber. d. Deut. chem. Gesellsch., xxxvi, 1903. 
or scum which is popularly known as the "mother of vinegar." Destruction of these bacteria by disinfectants or by sterilization with heat promptly arrests the process of vinegar formation. Chemically, the conversion of the alcohol consists in a double oxidation through ethyl aldehyde into acetic acid as shown in the following formulæ:

$$
\begin{aligned}
& \text { 1. } \mathrm{C}_{2} \mathrm{H}_{5}(\mathrm{OH})+\mathrm{O}=\underset{\text { Alcohol }}{\mathrm{CH}_{3}(\mathrm{COH})} \\
& \text { Ethyl aldehyde }
\end{aligned}
$$

2. $\mathrm{CH}_{3}(\mathrm{COH})+\mathrm{O}=\mathrm{CH}_{3}(\mathrm{COOH})$

Acetic acid

Alcoholic Fermentation (Zymase).-The formation of alcohol as an end product of fermentation is of great importance in a number of industries, primarily in the production of wine and beer. While accomplished by a number of bacteria, this form of fermentation is carried out chiefly by the yeasts.

Expressed in formulæ the simplest varieties of alcoholic fermentation, from mono- and disaccharids, may be represented as follows:

$$
\begin{aligned}
& \mathrm{C}_{6} \mathrm{H}_{12} \mathrm{O}_{6}=2 \mathrm{C}_{2} \mathrm{H}_{5}(\mathrm{OH})+2 \mathrm{CO}_{2} \\
& \text { Dextrose Ethyl alcohol }
\end{aligned}
$$

or

$$
\underset{\text { Saccharose }}{\mathrm{C}_{12} \mathrm{H}_{22} \mathrm{O}_{11}}+\mathrm{H}_{2} \mathrm{O}=\underset{2}{4 \mathrm{C}_{2} \mathrm{H}_{5}(\mathrm{OH})}+4 \mathrm{CO}_{2}
$$

In all cases the process may not be so simple as indicated by the equations, since by-products, such as higher alcohols, glycerin, succinic and acetic acids, may often be found in small traces among the end-products of such fermentations. The conditions which favor alcoholic fermentation by the yeasts are extremely important, since, upon observance of these, depends much of the uniformity of result which is so desirable in the industries mentioned above. The optimum concentration of sugar for the production of the highest quantity of alcohol is at or about 25 per cent. The temperature favoring the process ranges about $30^{\circ} \mathrm{C}$. Under such conditions fermentation may continue until the alcohol forms almost a 20-per-eent solution. Most of the fermentations important in the wine, beer, and spirit industries, take place under anaërobic conditions, since the carbon dioxide which is formed soon shuts out any excess of air. 
In the industrial employment of yeasts for fermentative purposes, it is necessary to work with specific strains, and in scientifically conducted vineyards, breweries, and distilleries the study and pure cultivation of the yeasts form no unimportant part of the work. Certain races of yeasts are more uniform in their fermentative powers than others, and the by-products formed by some races differ sufficiently from those of other races to cause material differences in the resulting substances. In the wine industries, the yeasts differ much from one another according to climatic and other environmental conditions. In vineyards, natural inoculation of the grapes occurs by transportation of the yeast from the soil to the surface of the grapes by wasps, bees, or other insects, through whose alimentary canals the microorganisms pass uninjured. In the autumn the yeast is returned to the soil by falling berries and remains alive in the upper layers of the ground throughout the winter months. In actual practice this natural yeast inoculation is not depended upon, but pure cultures of artificially cultivated yeasts are employed for inoculation. In some of the wine-growing countries these are supplied by special government experiment stations.

Denitrifying Bacteria.-Nitrogen is most readily absorbed by plants in the form of nitrates. These are furnished to the soil chiefly by the protein decomposition induced by the proteolytic bacterial enzymes. It is self-evident, therefore, that any cleavage which reduces nitrogenous matter beyond the stage of nitrates, to nitrites and ammonia, detracts from the value of the nitrogen as a food stuff for plants, and the eventual setting free of nitrogen in the elementary state renders it entirely valueless for any but the leguminous plants.

Nevertheless, this process of nitrogen waste or denitrification is constantly going on in nature. In the course of ordinary decomposition, there is a constant reduction of nitrogenous matter to nitrites and salts of ammonia, actively taken part in by a host of bacteria, as many as 85 out of 109 investigated by Maassen ${ }^{17}$ being found to possess this power. This, however, is not nearly so harmful a source of nitrogen waste as the process technically spoken of as true denitrification, in which nitrates are reduced, through nitric and nitrous oxides, to elementary nitrogen.

This phenomenon, more widely spread among bacteria than at

${ }^{17}$ Maassen, Arb. a. d. kais. Gesundheitsamt, 1, xxviii, 1901. 
first believed, depends essentially upon simple oxygen extraction from the nitrates by the bacteria, and for this reason goes on most actively when the supply of atmospheric oxygen is low. The first bacteria described as possessing this power of denitrification were the so-called B. denitrificans I and II, the first an obligatory anaërobe, the other a facultative aërobe. Since then numerous other bacteria, among them B. coli and B. pyocyaneus, have been shown to exhibit similar activities. It is important agriculturally, therefore, to know that many species which are able to utilize atmospheric oxygen when supplied with it, will get their oxygen by the reduetion of nitrates and nitrites when free oxygen is withheld. It is thus clear that a loss of nitrogen is much more apt to proceed rapidly in manure heaps which are piled high and poorly aërated. There are other factors, however, in regard to the physiology of these microorganisms, which must be considered for practical purposes.

In order that these bacteria may develop their denitrifying powers to the best advantage, it is necessary to supply them with some carbon compound which is easily absorbed by them. This, in decomposing material, is furnished by the products of the carbohydrate cleavage going on side by side with the proteolytic processes. It is still more or less an open question whether the facilitation of denitrification brought about in manure heaps by the presence of hay and straw is due to the carbon furnished by these materials, or whether it is due to the fact that bacilli of this group are apt to adhere to the straw which acts in that case as a means of inoculation.

The actual danger of nitrogen depletion of the soil by denitrifying processes is probably much less threatening than was formerly supposed f for, in the first place, the conditions for complete denitrification are much more perfect in the experiment than they ever can be in nature, and the nitrifying processes going on side by side with denitrification make up for much of the loss sustained.

\section{ANABOLIC OR SYNTHETIC ACTIVITIES OF BACTERIA}

Nitrogen Fixation by Bacteria.-The constant withdrawal of nitrogenous substances from the soil by innumerable plants would soon lead to total depletion were it not for certain forces continually at work replenishing the supply out of the large store of free nitrogen in the atmosphere. This important function of returning 
nitregen to the soil in suitable form for consumption by the plants is performed largely by bacteria.

It is well known that specimens of agricultural soil when allowed to stand for any length of time without further interference will increase in nitrogenous content, but that similar specimens, if sterilized, will show no such increase. ${ }^{18}$ The obvious conclusion to be drawn from this phenomenon is that some living factor in the unsterilized soil has aided in increasing the nitrogen supply. Light was thrown upon this problem when Winogradsky, ${ }^{19}$ in 1893, discovered a microorganism in soil which possessed the power of assimilating large quantities of nitrogen from the air. This bacterium, which he named "Clostridium Pasteurianum," is an obligatory anaërobe which in nature always occurs in symbiosis with two other facultatively anaërobic microorganisms. In symbiosis with these, it ean be cultivated under aërobic conditions and thus grows readily in the upper well-aërated layers of the soil.

Although, until now, no other bacteria with equally well-developed nitrogen-fixing powers have been discovered, yet it is more than likely that Clostridium Pasteurianum is not the only microorganism endowed with this function. In fact, Penicillium glaucum and Aspergillus niger, two molds, and two other bacteria described by Winogradsky, have been shown to possess this power slightly, but in an incomparably less marked degree than Clostridium Pasteurianum. ${ }^{20}$ According to the calculations of Sachse, ${ }^{21}$ unsterilized soil may, under experimental conditions, gain as much as 25 milligrams of nitrogen in a season, a statement which permits the calculation of a gain of twelve kilograms of nitrogen per acre annually. ${ }^{22}$ It is very unlikely, however, that such gains actually occur in nature, where nitrogen-fixation and nitrogen-loss usually occur side by side.

Agriculturally of even greater importance than the free nitrogenfixing bacteria of the soil are the bacteria found in the root tubercles of a class of plants known as "leguminosæ." It has long been known that this class of plants, including clover, peas, beans, vetch, etc, not only does not withdraw nitrogen from the soil, but rather tends to enrich it. Upon this knowledge has depended the well-

${ }^{18}$ Berthelot, Compt. rend. de la soe. de biol., exvi, 1893.

${ }^{19}$ Winogradsky, Compt. rend. de la soe. de biol., exvi, 1893, ibid., t. cxviii, 1894.

${ }^{20}$ Tacke, Landwirtsch. Jahresber., xviii, 1889.

${ }^{21}$ Sachse, "Agr. Chem.,", 1883.

${ }_{22}$ Pfeffer, Pflügers Physiologic, p. 395. 
known method of alternation of erops employed by farmers the world over. The actual reason for the beneficial influence of the leguminosæ, however, was not known until 1887, when Hellriegel and Wilfarth ${ }^{23}$ succeeded in demonstrating that the nitrogen-accumulation was directly related to the root tubercles of the plants and to the bacteria contained within them.

These tubercles, which are extremely numerous-as many as a thousand sometimes occurring upon one and the same plant-are formed by the infection of the roots with bacteria which probably enter through the delicate root-hairs. They vary in size, are usually situated near the main root-stem, and, in appearance, are not unlike fungus growths. Their development is in many respects comparable to the development of inflammatory granulations in animals after infection, inasmuch as the formation of the tubercle is largely due to a reactionary hyperplasia of the plant tissues themselves. They appear upon the seedlings within the first few weeks of their growth as small pink nodules, and enlarge rapidly as the plant grows. At the same time, later in the season, when the plants bear fruit, the root tubercles begin to shrink and erack. When the crops are harvested, the tubercles with the root remain, rot in the ground, and re-infect the soil.

Histologically the tubercles are seen to consist of large root cells which are densely crowded with microorganisms.

The microorganism itself, "Bacillus radicicola," was first observed within the tubercles by Woronin ${ }^{24}$ in 1866 . The bacilli are large, slender, and actively motile during the early development of the tubercles, but in the later stages assume a number of characteristic involution forms, commonly spoken of as "bacteroids." They become swollen, $\mathrm{T}$ and $\mathrm{Y}$ shaped, or branching and threadlike. Their isolation from the root tubercles usually presents little difficulty, since they grow readily upon gelatin and agar under strictly aërobic conditions. On the artificial media the bacillary form is usually well retained, involution forms appearing only upon old cultures.

The classical experiments of Hellriegel and Wilfarth conclusively demonstrated the important relation of these tubercle-bacteria to nitrogen assimilation by the leguminosæ.

These observers cultivated various members of this group of

${ }^{23}$ Hellriegel und Wilfarth, Cent. f. Bakt., 1887.

${ }^{24}$ Woronin, Bot. Zeit., xxiv, 1866. 
plants upon nitrogen-free soil-sand-and prevented the formation of root tubercles in some, by sterilization of the sand, while in others they encouraged tubercle formation by inoculation. An example of their results may be given as follows $:^{25}$

Lupinus luteus was eultivated upon sterilized sand. Some of the pots were inoculated with 13. radicicola, others were kept sterile. Comparative analyses were made of the plants grown in the different pots with the following striking result:

\section{Harvested} dry weight

Root tubereles present...

No root tubercles....... (a) 38.919

(b) 33.755

(c) 0.989

(d) 0.828
$N$ added in secd, soil, and soilextract .022 .023 .020 .022
Gain or loss of $N$ $+.975$ $+.958$ $-.004$ $-.009$

The great importance of this process in agriculture is demonstrated, furthermore, by a comparison made by the same observers between a legume, the pea, and one of the common nitrogen-consuming crops, oats. ${ }^{26}$. Exactly what the process is by which the bacteria supply nitrogen to the plant is as yet uncertain. Although the degenerating bacteroids in old nodules are bodily absorbed by the plant, this can not be conceived as the only method of supply, since the total nitrogen gain many times exceeds the total weight of bacteria in the nodules. It is probable that the microorganisms during life take up atmospheric nitrogen and secrete a nitrogenous substance which is absorbed by the plant cells.

Although formerly the relationship between plant and bacterium was regarded as one of symbiosis and of mutual benefit, the opinions as to this subject show wide divergence. While, according to some authors, the entrance of the bacteria into the plants is regarded as a true infection against which the plant offers at first a determined opposition as evidenced by tissue reactions, other observers, notably

${ }^{25}$ Pfeffer, “Planzenphysiologie," Leipzig, 1897.

${ }^{26}$ Hellriegel und Wilfarth, Zeit. d. Ver. f. d. Rübenzucker Industrie, 1888. Quoted from Fischer, "Vorles. über die Bakt.," Jena, 1903.

Nitrogen contents of seed and soil.

Oats 0.027 gram

Peas 0.038

\section{Nitrogen contents}

of crop.

0.007 gram

0.459
Gain or loss.

$-.020$

$+.421$ 
A. Fischer, regard the plant as a parasite upon the bacteria, in that it derives the sole benefit from the relationship and eventually bodily eonsumes its host.

Nitrifying Bacteria.-A process diametrically opposed in its chemistry to denitrification and reduetion is that which brings about an oxidation of ammonia to nitrites and nitrates. The actual increase of nitrates in soil allowed to stand for any length of time and examined from time to time has been a well-established fact for many years; but it was believed until a comparatively short time ago that this increase was due to a simple chemical oxidation of ammonia by atmospheric oxygen. The dependence of nitrification upon the presence of living organisms was finally proved by Muntz and Schlossing ${ }^{27}$ in 1887, who demonstrated that nitrification was abruptly stopped when the soil was sterilized by heat or antisepties. It remained, however, to isolate and identify the organisms which brought about this ammonia oxidation. This last step in our knowledge of nitrifieation was taken in 1890, by Winogradsky. Winogradsky ${ }^{28}$ found that the failures experienced by others who had attempted to isolate nitrifying bacteria, were due to the fact that they had used the common culture media largely made up of organic substances. By using culture media containing no organic matter, Winogradsky succeeded in isolating free from the soil, bacteria which have since that time been confirmed as being the causative factors in nitrification. During his first experiments this author observed that in some of his cultures the oxidation of ammonia went only as far as the stage of nitrite formation, while in others complete oxidation to nitrates took place. Following the clews indicated by this discrepaney, he finally succeeded in demonstrating that nitrifieation is a double process in which two entirely different varieties of microorganisms take part, the one eapable of oxidizing ammonia to nitrites, the other continuing the process and converting the nitrites to nitrates. The nitrite-forming bacteria discovered by Winogradsky, and named Nitromonas or Nitrosomonas, are easily cultivated upon aqueous solutions containing ammonia, potassium sulphate, and magnesium carbonate. According to their discoverer they develop in this medium within a week as a gelatinous sediment. After further growth this sediment seems to break up and the

${ }^{27}$ Muntz und Schlossing, Compt. rend. de l'acad. des sciences, 1887.

${ }_{28}$ Winogradsky, Ann. Past. Inst., iv and v, 1890, 1891. 
bacteria appear as oval bodies, which swim actively about and develop flagella at one end. Upon the solid media in ordinary use they can not be cultivated. Special solid media suitable for their cultivation and composed of silicic acid and inorganic salts have been described by Winogradsky and by Omeliansky. ${ }^{29}$

Other nitrite-forming bacteria have since been described by various observers, all of them more or less limited to definite localities. Some of these are similar to nitrosomonas in that they exhibit the flagellated, actively motile stage. In others, this stage is absent.

The nitrite-forming bacteria, apart from their great agricultural importance, claim our attention because of their unique position in relation to the animal and vegetable kingdoms. Extremely sensitive to the presence of organic compounds, they are able to grow and develop only upon media containing nothing but inorganic material; and this entirely without the aid of any substances comparable to the chlorophyll of the green plants. The source of energy from which this particular class of bacteria derive the power of building up organic compounds from simple substances is to some extent a mystery. The carbon which they unquestionably require for the building up of organic material may be, as Winogradsky believed, derived to a certain extent from ammonium carbonate. But it is also quite certain that they are capable of utilizing directly atmospheric $\mathrm{CO}_{2}$. In the absence of ehlorophyll or of any highly organized chemical compound, it seems likely that the energy necessary for the utilization of the carbon obtained in this simple form is derived from the oxidation of ammonia during the process of nitrification.

The conversion of nitrites into nitrates is carried on by other species of bacteria also discovered by Winogradsky. These bacteria are much more generally distributed than nitrosomonas and probably include a number of varieties. The organism described by Winogradsky is an extremely small bacillus with pointed ends. Capsules have occasionally been demonstrated. It may be cultivated upon aqueous solutions containing:

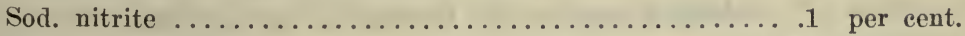

Potass. phosphate $\ldots \ldots \ldots \ldots \ldots \ldots \ldots \ldots \ldots \ldots \ldots \ldots . . \ldots 5$ " "

Magnesium sulphate $\ldots \ldots \ldots \ldots \ldots \ldots \ldots \ldots \ldots \ldots \ldots \ldots . . \ldots{ }_{0 . \ldots}^{6} 6$

Sodium earbonate..................... " " "

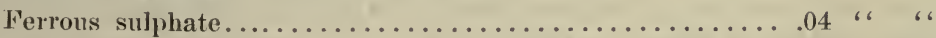

${ }^{29}$ Omeliansky, Cent. f. Bakt., II, 5, 1899. 
The development of the organism is slow and sparse, and is directly inhibited by the presence of organic matter. It is strongly inhibited by the presence of ammonia.

The Liberation of Energy by Bacteria.-Like all other living beings, bacteria in their metabolic processes liberate energy. It has been shown by several observers that slight quantities of heat are given off from actively growing eultures. The functions, furthermore, of reproduction, motility, and enzyme formation may be looked upon as forms of energy liberation. In addition to this, certain bacteria have been observed which may liberate energy in the form of light.

Light Production by Bacteria.--The production of light by bacteria is a power possessed chiefly by certain species inhabiting salt water. Thus, much of the phosphoreseence observed at sea, though more frequently due to Medusa and other invertebrate animals, is eaused by these bacteria. Numerous species which produce this phenomenon have been isolated, too many, and too unimportant, to be individually deseribed. All of them are aërobes and require highly complex food stuffs. They are elosely allied to the putrefactive bacteria, and in the sea are usually found upon rotting animal matter. ${ }^{30}$ The production of light seems directly dependent upon the free access of oxygen, since no light appears under anaërobie eonditions. Their luminous quality, moreover, is not a true phosphorescence, in that it does not depend upon previous illumination and develops as well in eultures kept in the dark as in those which have been exposed to light. ${ }^{31}$

The Formation of Pigment by Bacteria (Chromobacteria).-A large number of bacteria, when cultivated upon suitable media, give rise to characteristic colors which are valuable as marks of differentiation. For each speeies, the color is usually eonstant, depending, to a certain extent, upon the conditions of eultivation. In only a few of the pigmented bacteria is the pigment contained within the eell body, and in only one variety, the sulphur bacteria, does the pigment appear to hold any distinet relationship to nutrition. In most cases, the coloring matter is found to be deposited in small intercellular granules or globules. The absence of any relationship of the pigment to sunlight, as is the case with the chlorophyll of

${ }^{30}$ Pflügcr's Arch. f. Phys., xi, 1875.

${ }^{31}$ Fischer, Cent. f. Bakt., iii, 1888. 
the green plants, is indicated by the fact that most of the chromobacteria thrive and produce pigment equally well in the dark as they do in the presence of light. Among the most common of the pigment bacteria met with in bacteriological work are Staphylococcus pyogenes aureus, Bacillus pyocyaneus, Bacillus prodigiosus, and some of the green fluorescent bacteria frequently found in feces.

The chemical nature of these pigments has been investigated quite thoroughly and it has been shown that they vary in composition. Some of the pigments, like that of Staphylococcus aureus, are probably non-protein and of a fatty nature. ${ }^{32}$ They are insoluble in water but soluble in alcohol, ether, and chloroform. Because of their probable composition, they have been spoken of as "lipochromes." Other pigments, like the pyocyanin, which lends the green color to cultures of Bacillus pyocyaneus, are water soluble and are probably of protein composition. Pyocyanin may be crystallized out of aqueous solution in the form of fine needles. The crystals may be redissolved in chloroform. Aqueous solutions retain their color. Solutions in choloroform, however, are changed gradually to yellow.

The power of pigment production of various bacteria depends in each case upon cultural conditions. In most cases, this simply signifies that pigment is produced only when the microorganism, finding the most favorable environmental conditions, is enabled to develop all its functions to their fullest extent. Thus, a too high acidity or alkalinity of the culture medium may inhibit pigment formation. Oxygen is necessary for the production of color in some bacteria, since the bacteria themselves often produce the pigment only as a leuko-body which is then oxidized into the pigment proper. A notable example of this is the pigment of $\mathrm{B}$. pyocyaneus. In other cases, temperature plays an important rôle in influencing color production. Thus, Bacillus prodigiosus does not produce its pigment when growing in the incubator. By persistent cultivation in an unfavorable environment, colored cultures may lose their power of pigment production.

Sulphur Bacteria.-Wherever the decomposition of organic matter gives rise to the formation of $\mathrm{H}_{2} \mathrm{~S}$, in cess-pools, in ditches, at the bottom of the sea, and in stagnant ponds, there is found a curiously interesting group of microorganisms, the so-called sulphur

${ }^{32}$ Sclerceter, Cent. f. Bakt., xviii, 1895. 
or thiobacteria. Red, purple, and colorless, these bacteria all possess the power of utilizing sulphuretted hydrogen and by its oxidation into free sulphur obtain the energy necessary for their metabolic processes. The colorless sulphur bacteria, the Beggiatoa and Thiothrices, usually appear as threads or chains which, in media containing sufficient $\mathrm{H}_{2} \mathrm{~S}$, are usually well-stocked with minute globules of sulphur. If found upon decomposing organic matter, they often cover this as a grayish mold-like layer. The red sulphur bacteria, of which numerous species have been. described by Winogradsky, may appear as actively motile spirilla (thiospirillum) or as short, thick bacillary forms.

The physiology of all the sulphur bacteria, and especially of the colored varieties, is of the greatest interest in that these microorganisms are among the few members of the bacterial group which behave metabolically like the green plants. The higher organic substances play little or no part in the nutrition of these microorganisms. Strictly aërobic, the colorless thiobacteria are independent of sunlight, while the red and purple varieties exhibit their physiological dependence upon light by accumulating under natural conditions in well-lighted spots. Both varieties possess equally the power of oxidizing sulphuretted hydrogen as a source of energy. The sulphur is then stored as elemental sulphur within the bacterial body and when a lack of food stuffs sets in, the store of sulphur can be further oxidized into sulphurous or sulphuric anhydrides. With this sole source of energy, these bacteria are capable of flourishing aërobically, while an absence of $\mathrm{H}_{2} \mathrm{~S}$, even in the presence of organic food stuffs, leads to a rapid disappearance of their sulphur contents and an inability to develop.

In the case of the colored thiobacteria, the red pigment appears to fulfil, to some extent, a function comparable to that of the chlorophyll of the green plants.

Engelmann, ${ }^{33}$ who has studied this pigment spectroscopically, has found that besides absorbing the red spectral rays there is an absorption of rays on the ultra-red end of the spectrum. The absorption of the red rays between the lines $\mathrm{B}$ and $\mathrm{C}$ of the spectrum, and of violet rays at the line $F$, is the same as that of the absorption spectrum of chlorophyll, and it is in the zone of these rays that the physiological effects of cholorophyll are most active. In addition

ss Engelmann, Bot. Zeit., 1888. 
to these absorption bands, the bacteriopurpurin of the red sulphur bacteria shows absorption of the invisible ultra red rays of the spectrum.

Engelmann, with a microspectroscope, projected a spectrum into a microscopic field in which green algæ or, in the case under discussion, red sulphur baeteria had been placed. Other sources of light were, of course, excluded. By adding emulsions of strictly aërobic bacteria to such preparations, an accumulation of mieroorganisms was observed at those points in the spectrum at which most oxygen was liberated. In the case both of chlorophyll and of the red sulphur bacteria such areas of bacterial accumulation (in oxygen liberation) occurred in the zones of the absorption bands mentioned above.

\section{THE SO-CALLED “BACTERIOPHAGE', PHENOMENON}

During the last few years a phenomenon has been observed with bacteria which is likely to be of fundamental importance in the biological and immunological study of bacteriology.

The first observation upon this curious phenomenon was published by Twort in $1915 .{ }^{34}$ Twort was engaged in a search for a non-pathogenic filtrable virus on the very logical assumption that the few pathogenic filtrable virus which had been associated with a number of diseases probably represented isolated members of a large kingdom of microorganisms. His cultural and animal studies proving negative by the ordinary methods, he carried out experiments with glycerinated calf vaccine which he eultured on agar tubes. After variable periods of incubation such agar slants would exhibit watery areas and in those cultures in which micrococci grew, it was found that some of the colonies could not be sub-cultured, but if preserved, the originally normal culture would beeome glassy and transparent. Smears made from these glassy areas showed reddish staining granules by Giemsa. Plating of the condensation water of such slants resulted in colonies which usually after preservation beeame transparent, starting unlike most degenerative processes, from the edge of the colony. Pure eultures of pneumococei and other mieroorganisms when touched with small portions of the glassy colonies would become transparent at the point touched and this

${ }^{84}$ Twort, Lancet, December 4th, 1915. 
would gradually spread over the whole group. Such action was complete and rapid only with vigorously growing young cultures, but had little effect on dead cultures. The action took place best under aërobic conditions. If the transparent material was diluted and filtered and then added to an agar slant of similar organisms, growth was prevented. The transparent material did not grow on any medium and was destroyed in its activity at $60^{\circ}$. Its action on staphylococci grown from boils was very slight, and there was no action of the substance upon B. coli, streptococei, tubercle bacilli or yeasts. The nature of the material was left undecided by Twort. It was supposed that it might be a parasitic organism which destroyed the bacteria, perhaps an amoeba, but he thought that it was probably a substance derived from the bacteria themselves, since it occasionally reappeared in glassy eultures months after subculture. Twort investigated this peculiar oceurrence not only with vaccine virus, but also with Gram-negative baeilli obtained from the intestines of a dog suffering from acute distemper and some organisms derived from infantile diarrhea.

In 1917 D'Herelle $^{35}$ began the publication of a series of notes upon a phenomenon unmistakably identical with that of Twort. When he introduced two or three drops of a dysentery stool into about 20 c.c. of broth and filtered this through a Chamberland Candle and introduced a trace of this filtrate into a young Shiga bacillus culture, it caused a clearing up of the culture within a few hours, transplants remaining sterile. A trace of this dissolved culture placed into another young broth eulture of dysentery brought the same result, and in this way he made 935 successive passages of the lytic principle. His technique in detail was as follows: He made an emulsion of fecal material and filtered it through a filter candle. With a pipette he then transferred 1 c.c. of this filtrate into a small tube and inoeulated this material with a drop of a young dysentery or typhoid eulture. This tube is held for from 12 to 24 hours at a temperature of $22^{\circ}$, and is then placed for 2 or 3 hours at $37^{\circ}$. If the stool filtrate contains a lytic principle, the inoculated tube will be transparent at the end of this time, and the lytic principle can be transmitted in series from this tube. He assumed from his ability to keep this lytic activity going

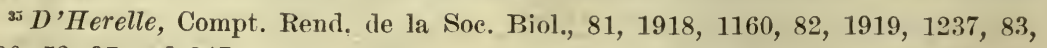
1920, 52, 97 and 247. 
through many generations that he was dealing with a living ultramicroscopic organism which fed upon the living bacteria and, therefore, spoke of it as a "bacteriophage."

A curious and important phase of the work was that this lytic principle was to some extent specific in that it acted only upon a single or a few closely related microorganisms.

In subsequent notes D'Herelle reported that he had produced such bacteriophage lytic principles against dysentery bacilli of Shiga and Flexner and "Y" type, against typhoid, paratyphoid "A" and "B," against enteritidis, hog cholera, coli, prodigiosus and some other Gram-negative bacilli. Without wishing to detail all his investigations, the principles may be summarized as follows: In all cases the lytic principle was filtrable and could be carried on from generation to generation as above described. Young actively growing cultures were necessary to transmit the lytic activity in series; it could not be transmitted by dead bacteria. The lytic principle and the bacilli were not killed by the same temperature. Whereas the Shiga bacillus and other organisms worked with were destroyed at or about $50^{\circ} \mathrm{C}$, the lytic principle resisted temperatures up to $65^{\circ}$. The lytic principle isolated from the feces might at first be feeble, but was considerably augmented in potency by cultures in series. Certain cultures were from the beginning endowed with bacteriolytic power against two or more different species and preserved this action against these species for a long time if cultivated only on one of them. For example, he isolated an active principle against the Shiga and typhoid bacillus from the stool of a dysentery convalescent. After a series of 1,000 passages, the successive cultures always being carried along on Shiga emulsions, there was still marked lytic action against typhoid bacilli. He never isolated two strains that were exactly alike. The intensity of action differed and one strain would from the beginning show lytic power against a number of intestinal bacilli. Others, again, would be active against only a single species. Another, again, might attack only two, the Flexner bacillus and paratyphoid "B."

D'Herelle was quite definitely convinced that he was dealing with an ultra-microscopic organism which perhaps was capable of developing in the intestines of man and animals at the expense of various bacteria, and could then be cultivated out of the body by the method he had developed. This was the reason for the name he applied to these principles, "bacteriophage," which implied that 
his ultra-microscopic organism was a parasite upon bacteria, adapted specifically in different eases to various organisms.

In 1920 Kabeshima $^{36}$ studied the D'Herelle phenomenon and interpreted it as being due to ferment action. He believes that D'Herelle's principle was a catalytic agent originating in the mucous membranes of the intestinal canal, which induced enzyme digestion of the organisms. In the cells of bacteria he assumes there exists a preferment which is activated by the catalyst and leads to autolysis of the organisms.

In 1920, also, Bordet and Cuica ${ }^{37}$ attacked the same problem from an entirely different standpoint. Bordet injected guinea pigs intraperitoneally three or four times with B. coli. One or two days after the last injection, the peritoneal exudate was examined and found to consist of an exudate rich in leucocytes in which still a few organisms were present. When a few drops of such an exudate were added to a normal B. coli culture, marked lytic powers were noticed. A small quantity of the exudate resulted in a clearing up of the normal broth culture, and a small amount of this original mixture would do the same if added to successive cultures of normal Colon bacilli. In other words, the lytic principle started in the peritoneal exudate of guinea pigs, was kept going through successive Colon cultures. This active principle, like that of D'Herelle, resisted heating to from $60^{\circ}$ to $65^{\circ}$, and this sterile exudate would still possess lytic powers for Colon cultures. It may be stated that in Bordet's experiments it was found that it was very difficult to start the principle in this way, and that only isolated Colon cultures lent themselves to successful experimentation. When a few drops of a culture cleared in this way were added to an agar slant freshly inoculated with normal Colon bacillus, no growth took place in those parts which had been touched by the fluid, and colonies which developed in or about this area soon became glassy and transparent. In the same way, Bordet often found that dissolved cultures were not entirely sterile on sub-culturing. A small number of colonies developed, some of which had an extremely irregular outline. These irregular colonies carried the lytic principle in subsequent generations. Bordet interpretes this phenomenon as the artificial produc-

${ }^{36}$ Kabeshima, Compt. Rend. de la Soc. Biol., 8.3, 1920.

${ }^{37}$ Bordet and Cuica, Compt. Rend. de la Soc. Biol., 88, 1920. 
tion of a variation in the cultures of Colon bacilli in which a variant producing an autolytic enzyme is developed, and this property of autolysin production is then inherited from culture to culture. Conceived in this way, the lytic principle would be regarded as the inheritance of an acquired characteristic.

In our own laboratory we have isolated a lytic principle by the D'Herelle technique described above, from the stool of a typhoid convalescent. It corresponds in most details to the lytic principles described by D'Herelle. It is filtrable, thermostable, and transmittible in series. It is both inhibitory and lytic, that is, a small amount of the original stool filtrate or any one of the subsequent generations will prevent growth of a heavily inoculated tube, or dissolve a young turbid broth culture. This lytic principle is only active against closely allied species of bacteria of the typhoid-paratyphoid and dysentery groups. In the earlier experiments this lytic principle did not appear active against paratyphoid "A" or " $\mathrm{B}$," but when retested recently, it was also active against these organisms. It has no action against cholera, B. pyocyaneous or B. coli, or against staphylococcus or pneumococcus.

Unlike the lytic principles described by D'Herelle, the one isolated by us has not increased in potency in the course of the generations through which it has been passed. It differs also from those described by D'Herelle in that it usually does not sterilize the dissolved culture completely. On sub-culturing a small number of colonies develop which are of two types: one a round typical typhoid colony, the other irregular in outline. The latter type was first described by Bordet in connection with the lytic principle that he was able to produce against $B$. coli by intraperitoneal injections of guinea pigs, and the bacilli composing these irregular colonies were shown by him to be the bearer of the lytic principle.

The typical colonies which develop from plating a dissolved culture, on restreaking, give only regular colonies. If, however, one of the irregular colonies are restreaked both regular and irregular colonies will develop. Daily restreaking of irregular colonies have failed to eliminate the typical normal colony in a series of 15 generations and the irregular colonies continued to carry the lytic principle. If one of these typical typhoid colonies is fished with broth, the broth will become cloudy after incubation. Broth fishings of the irregular colonies will, however, often remain clear after 12 to 18 hours incubation and the lytic principle is then present in 
the broth and can be transmitted in series from broth fishings of irregular colonies in the same way as from dissolved cultures.

If a number of irregular colonies are obtained on a streak plate, it will be found that on examining with the microscope or often with the naked eye, that there are minute transparent masses between the irregular colonies, which are referred to by most writers as "appearances." On examining the irregular lytic colonies under the microscope it will be found that the lytic colonies owe their irregular shape to the fact that their edges have faded out into these transparent appearances. All attempt to find a definite structure in the "appearances" by different methods of staining have failed, and we have no reason to believe that they are living. In our opinion the "appearances" represent the debris left when the susceptible bacilli in any culture are dissolved by action of the lytic principle.

The two most striking facts about these lytic agents from whatever source they are derived are: (1), that a single contact with the lytic agent is sufficient to divide a normal culture into two types of organisms, one the resistant type, the other the bearer of the lytic principle; (2), that the lytic principle could only be transmitted in series when young actively growing cultures were used. This suggested that once the dissolving action was started, the lytic agent in subsequent generations was derived from the bacterial cell itself. Experiments were consequently undertaken to isolate a lytic agent which would start the dissolving process without any interaction of the living animal body.

The early work of Twort in connection with vaccine virus suggested that tissue enzymes might be able to start the process. In consequence glycerine extracts of intestinal mucosa have been made and such extracts when added to young turbid typhoid or dysentery cultures in a dilution of 1-10 have had a dissolving action on the bacilli which is transmittible in series. Liver extracts prepared according to the method of Turro ${ }^{38}$ have given similar results.

Bordet has recently prepared an antilytic serum. ${ }^{39}$ In trying to duplicate his experiments, and testing the normal rabbit serum comparatively with the immune serum, it was found that in some instances the normal rabbit serum had a dissolving action when

Turro, Compt. Rend. de la Soe. Biol., February 12th, 1921.

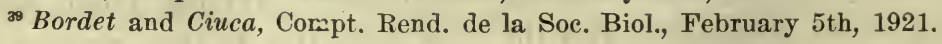


added in a dilution of 1-10 to young typhoid broth cultures. It seems, therefore, that this power of dissolving bacteria is the property of many different cells of the animal body and is also present in certain instances in the blood serum. Whether the lytic agent in the serum can be definitely identified with serum protease or lipase has not been determined. 


\section{CHAPTER V}

THE DESTRUC'TION OF' BAC'TERIA

\section{GENERAL CONSIDERATIONS}

No branch of bacteriology has been more fruitful in practical application than that which deals with the factors which bring about the destruction of microorganisms. Upon the study of this branch has depended the growth and the development of modern surgery.

The agents which affect bacteria injuriously are many, and are both physical and chemical in nature.

When a procedure completely destroys bacterial life it is spoken of as sterilization or disinfection, the term disinfection being employed more especially to designate the use of chemical agents. When the procedure destroys vegetative forms only, leaving the more resistant spores uninjured, it is spoken of as "incomplete sterilization." When an agent, on the other hand, does not actually kill the microorganisms, but merely inhibits their growth and multiplication, it is spoken of as an antiseptic. The term deodorant is indiscriminately applied to substances which mask or destroy offensive odors, and may or may not possess disinfectant or antiseptic value. Some deodorants act chemically on the noxious gases, destroying them.

\section{PHYSICAL AGENTS INJURIOUS TO BACTERIA}

The principal physical agents which may exert deleterious action upon bacteria are: drying, light, electricity, and heat.

Drying.-Complete desiccation eventually destroys most of the pathogenic bacteria, yet great differences in resistance to this condition are shown by various microorganisms. Ficker, ${ }^{1}$ who has made a systematic study of the influence of complete drying upon bacteria, concludes that the resistance of bacteria to desiceation is influenced

${ }^{1}$ Ficker, Zeit. f. Hyg., xxix, 1896. 
by the age of the culture investigated, the rapidity with which the withdrawal of moisture is accomplished, and the temperature at which the process takes place. Microorganisms like the gonococeus and the Pfeiffer bacillus are destroyed by drying within a few hours. The cholera vibrio dried upon a coverslip was found by Koch $^{2}$ to be killed within four hours; by Burkholtz, ${ }^{3}$ to survive about twenty-four hours. The spore-forms of bacteria are infinitely more resistant to this influence than are the vegetative forms, though they may be destroyed by rapid and complete drying in a desiceator.

It is self-evident that many diserepancies in the experimental results of various authors may depend upon the technique of investigation, since the degree of drying attained depends intimately upon the thickness and consistence of the material investigated, and upon the methods employed for desiccation.

Light.-Direct sunlight is a powerful germicide for all bacteria except a limited number of species like the thio- or sulphur bacteria, which utilize sunlight for their metabolic processes as do the green plants.

Koch ${ }^{4}$ has shown that exposure to sunlight will destroy the tuberele bacillus within two hours or less, the time depending upon the thickness of the exposed layers and the material surrounding the bacilli. Confirmatory researches have been published by Mignesco ${ }^{5}$ and others. The powerful disinfecting influence of sunlight upon bacteria suspended in water has been shown by Buchner. ${ }^{6}$ Observations in regard to the influence of sunlight upon anthrax spores have been made by Arloing, ${ }^{7}$ and similar observations upon a number of other mieroorganisms have been carried out by Dieudonne, Janowski, v. Esmarch, and many others. All these observers, while differing somewhat as to the time necessary for bacterial destruction, agree in finding definite and powerful bactericidal action of sunlight. Diffuse light, of course, is less active than direct sunlight. Aceording to Buchner, typhoid bacilli are inhibited by direct sunlight in one and one-half hours, by diffuse light in five hours. A remarkable statement is made by Arloing, who elaims to have found

${ }^{2}$ Koch, Arb. a. d. kais. Gesundheitsamt, iii, 1887.

s Burkholtz, Arb. a. d. kais. Gesundheitsamt, v, 1889.

"Koch, X Internat. Med. Congress, Berlin, 1890.

'Mignesco, Arch. f. Hyg., xxv, 1896.

- Buchner, Cent. f. Bakt., I, xi, 1892.

'Arloing, Compt. rend. de 1'acad. d. sci., c. 1885. 
that anthrax spores are more quickly destroyed by direct sunlight than are the vegetative cells. These findings require further confirmation.

It has been shown by various authors that the influence of sunlight is not to be attributed in any way to temperature, nor always to a direct action of the light upon the bacteria, but depends largely upon photochemical changes produced by the light rays in the media. Richardson ${ }^{8}$ and Dieudonné ${ }^{9}$ conclude that under ordinary aërobic conditions in fluid environment peroxide of hydrogen is formed under the influence of light. Novy and Freer ${ }^{10}$ believe that the bactericidal effects in fluids noticed as a result of exposure to light are too strong to, be explained by the formation of small quantities of peroxide of hydrogen, and attribute this action to organic peroxides formed under the described conditions, such as the peroxides of diacetyl, benzoylacetyl, and others. These views are somewhat strengthened by the fact that exclusion of oxygen from media markedly diminishes the bactericidal power of light. ${ }^{11}$ That the photochemical changes alone, however, do not explain this action follows from the fact that dried bacteria, not surrounded by media, are subject to a similar action. ${ }^{12}$

In analyzing sunlight in regard to its bactericidal power, it has been found by various observers that the most powerful action is exerted by the ultraviolet spectral rays, whereas the yellow, red, and ultra-red rays are practically innocuous. ${ }^{13}$

It is of importance to note that sunlight has been found also to have a strong attenuating influence ${ }^{14}$ upon some bacterial poisons, as shown by the experiments of Ferri and Celli upon tetanus toxin.

Electric light exerts a distinct bactericidal action when applied in strengths of 800 to 900 candle power for seven or eight hours. ${ }^{15}$

Röntgen or X-rays are said by Zeit, ${ }^{16}$ Blaise and Sambac, ${ }^{17}$ and

${ }^{8}$ Richardson, Jour. Chem. Soc., i, 1893, Ref. Deut. chem. Gesells., xxvi.

'Dieudonné, loc. cit.

${ }^{10}$ Novy and Freer, 3d Ann. Meeting Assn. Amer. Bacteriologists, Chicago, 1901.

${ }^{11}$ Roux, Ann. Inst. Past., ix, 1887.

${ }^{12}$ Dieudonné, loc. cit.

${ }^{13}$ Ward, Proc. Royal Soc., 52, 1893.

${ }^{14}$ Ferri and Celli, Cent. f. Bakt., I, xii, 1892.

15 Dieudonné, loc. cit.

${ }^{16}$ Zeit, Jour. Amer. Med. Assn., xxxvii, 1901.

${ }^{17}$ Blaise and Sambac, Compt. rend. de la soc. de biol., 1896. 
others to be without appreciable germicidal power. Rieder, ${ }^{18}$ on the other hand, has reported definite inhibition of bacterial growth after exposures of half an hour to X-rays.

Radium rays have a distinct inhibitory and even bactericidal power when applied at distances of a few centimeters for several hours. ${ }^{19}$

Electricity.-If we exclude the indirect actions of heat and electrolysis, it can hardly be said that the direct bactericidal action of electric currents has been satisfactorily demonstrated. Such action, however, has been claimed by d'Arsonville and Charrin, ${ }^{20}$ and by Spilker and Gottstein. ${ }^{21}$

Heat.-The most widely applicable and efficient physical agent for sterilization is heat.

The dependence of bacteria for growth and vitality upon the maintenance of a proper temperature in their environment, and the ranges of variation within which, bacteria may thrive, have been discussed in a preceding section, in which a table of so-called "thermal death points" has been given. In the method of expressing these values it was scen that two elements entered into the destruction of bacteria by heat, namely, that of the degree of temperature which is applied, and that of the time of application.

The prolonged application of moderately high temperatures, in other words, may in certain instances, accomplish the same result as the brief use of extremely high ones. In general, the death of bacteria following prolonged exposure to temperatures but slightly exceeding the optimum is due to the inability of the anabolic processes to keep pace with the accelerated katabolic processes, gradual attenuation resulting in death. At somewhat higher temperatures death results from coagulation of the bacterial protoplasm, and at still higher degrees of heat, applied in the dry form, direct burning of the bacteria may be the cause of their destruction.

Heat may be applied in the form of dry heat or as moist heat, these methods being of great practical value, but differently applicable according to the nature of the materials to be sterilized. The two methods, moreover, show a marked difference in efficiency,

${ }^{18}$ Rieder, Münch. med. Woch., 1898.

${ }^{10}$ Personal observations.

${ }^{20} \mathrm{D}$ 'Arsonville and Charrin, Compt. rend. de la soc. de biol.

${ }^{21}$ Spilker and Gottstein, Cent. f. Bakt., I, 9, 1891. 
temperature for temperature. For the recognition of this fact we are largely indebted to the early researches of Koch and Wolffhügel, ${ }^{22}$ and of Koch, Gaffky, and Loeffler. ${ }^{23}$

These observers were able to show that the spores of anthrax were destroyed by boiling water at $100^{\circ} \mathrm{C}$. in from one to twelve minutes, whereas dry hot air was efficient only after three hours' exposure to $140^{\circ} \mathrm{C}$. Extensive confirmation of these differences has been brought by many workers. An explanation of the phenomena observed is probably to be found in the changes in the coagulability of proteins brought about in them by the abstraction of water. Lewith ${ }^{24}$ working with various proteins, found that these substanees are coagulated by heat at lower temperatures when they contain abundant quantities of water, than when water has been abstracted from them. On the basis of actual experiment with egg albumin he obtained the following results, ${ }^{25}$ which illustrate the point in question:

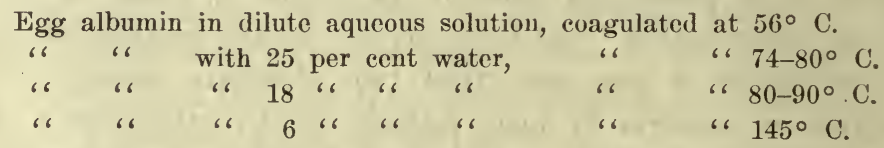

Absolutely anhydrous albumin, according to Haas, ${ }^{26}$ may be heated to $170^{\circ} \mathrm{C}$. without coagulation. It is thus clear that bacteria exposed to hot air may be considerably dehydrated before the temperature rises sufficiently to cause death by coagulation, complete dehydration necessitating their destruction possibly by actual burning.

Bacteria exposed to moist air or steam, on the other hand, may absorb water and become proportionately more coagulable.

The same principle, as Lewith points out, probably explains the great resistance to heat observed in the case of the highly concentrated protoplasm of spores.

Apart from the actually greater efficiency of moist heat when compared with dry heat of an equal temperature, an advantage of great practical significance possessed by moist heat lies in its greater

\footnotetext{
${ }^{22}$ Koch and Wolffhügel, Mitt. a. kais. Gesundheitsamt, I, 1882.

${ }^{23}$ Koch, Gaffky and Loeffler, ibid.

${ }^{24}$ Lewith, Arch. f. exp. Path. u. Pharm., xxvi, 1890.

${ }^{25}$ Lewith, loc. eit., p. 351.

${ }^{26}$ Haas, Prag. med. Woch., 34-36, 1876.
} 
powers of penetration. An experiment carried out by Koch and his associates illustrates this point clearly. Small packages of garden soil were surrounded by varying thicknesses of linen with thermometers so placed that the temperature under a definite number of layers could be determined. Exposures to hot air and to steam were then made for comparison, and the results were as tabulated $:^{27}$

\begin{tabular}{|c|c|c|c|c|c|c|}
\hline & \multirow{2}{*}{$\begin{array}{l}\text { Tempera- } \\
\text { tures. }\end{array}$} & \multirow{2}{*}{$\begin{array}{c}\text { Time of } \\
\text { Application. }\end{array}$} & \multicolumn{3}{|c|}{$\begin{array}{l}\text { Temperatures Reached within } \\
\text { THICKNESSES OF LINEN. }\end{array}$} & \\
\hline & & & $\begin{array}{c}\text { Twenty } \\
\text { Thicknesses. }\end{array}$ & $\begin{array}{c}\text { Forty } \\
\text { Thicknesses. }\end{array}$ & $\begin{array}{l}\text { One Hundred } \\
\text { Thicknesses. }\end{array}$ & \\
\hline Hot air.... & $130-140^{\circ} \mathrm{C}$ & 4 hours. & $86^{\circ}$ & $72^{\circ}$ & Below $70^{\circ}$ & $\begin{array}{c}\text { Incomplete } \\
\text { steriliza- } \\
\text { tion. }\end{array}$ \\
\hline Steam..... & $90-105.3^{\circ}$ & 3 hours. & $101^{\circ}$ & $101^{\circ}$ & $101.5^{\circ}$ & $\begin{array}{l}\text { Complete } \\
\text { steriliza- } \\
\text { tion. }\end{array}$ \\
\hline
\end{tabular}

This great penetrating power of steam is due presumably to its comparatively low specific gravity which enables it to displace air from the interior of porous materials, and also to the fact that as the steam comes in contact with the objects to be disinfected a condensation takes place with the consequent liberation of heat. When a vapor passes into the liquid state it gives out a definite amount of heat, which in the case of water vapor, at $100^{\circ} \mathrm{C}$., amounts to about 537 ealories. This brings about a rapid heating of the object in question. Following this process the further heating takes place by conduction, and it is, of course, well known that steam is a much better heat conductor than air. ${ }^{28}$

Moist heat may be applied as boiling water, in which, of course, the temperature varies little from $100^{\circ} \mathrm{C}$., or as steam. Steam may be used as live, flowing steam, without pressure, the temperature of which is more or less constant at $100^{\circ} \mathrm{C}$., or still higher efficiency may be attained by the use of steam under pressure, in which, of course, temperatures far exceeding $100^{\circ} \mathrm{C}$. may be produced, according to the amount of pressure which is used.

The spores of certain bacteria of the soil which can not be killed in live steam in less than several hours may be destroyed in a few

${ }^{27}$ Koch, Gaffky und Loeffler, loc. cif., p. 339.

${ }^{28}$ Gruber, Cent. f. Bakt., iii, 1888. 
minutes, or even instantaneously, in compressed steam at temperatures ranging from $120^{\circ}$ to $140^{\circ} \mathrm{C}^{29}$

In all methods of steam sterilization, it is of great practical importance, as v. Esmarch ${ }^{30}$ has pointed out, that the steam shall be saturated, that is, shall contain as much vaporized water as its temperature permits. Unsaturated, or so-called "super-heated steam" is formed when heat is applied to steam, either by passage through heated piping or over heated metal plates. In such cases the temperature of the steam is raised, but no further water-vapor being supplied, the steam exerts less pressure and contains less water in proportion to its yolume than saturated steam of an equal temperature. The super-heated steam, therefore, is heated considerably over its condensation temperature and becomes literally dried. In consequence, its action is more comparable to hot air than to saturated steam, and up to a certain temperature its disinfecting power is actually less than that of live steam at $100^{\circ} \mathrm{C}$. v. Esmarch, who has made a thorough study of these conditions, concludes that up to $125^{\circ} \mathrm{C}$., the efficiency of superheated steam is lower than that of live steam at $100^{\circ} \mathrm{C}$. Above this temperature, of course, it is again active as in the case of ordinary dry heat.

Practical Methods of Heat Sterilization.-Burning.-For objects without value, actual burning in a furnace is a certain and easily applicable method of sterilization. Flaming, by passage through a Bunsen or an alcohol flame, is the method in use for the sterilization of platinum needles, coverslips, or other small objects which are used in handling bacteria in the laboratory.

Hot air sterilization is carried out in the so-called "hot air chambers," simple devices of varied construction. The apparatus most commonly used consists of a sheet-iron, double-walled chamber, the joints of which, instead of being soldered, are closed by rivets. The inner case of this chamber is entirely closed except for an opening in the top through which a thermometer may be introduced, while the outer has a large opening at the bottom and two smaller ones at the top. A gas-burner is adjusted under this so as to play directly upon the bottom of the inner case. A thermometer is fitted in the top in such a way that it penetrates into the inner chamber. The air in the chamber is heated directly by

${ }^{29}$ Christen, Ref. Cent. f. Bakt., V, xiii, 1893.

${ }^{20}$ v. Esmarch, Zeit. f. Hyg., iv, 1888. 
the flame and by the hot air, which, rising from the flame, courses upward within the jacket between the two cases and escapes at the top. To insure absolute sterilization of objects in such a chamber, the temperature should be kept between $150^{\circ}$ and $160^{\circ} \mathrm{C}$. for at least an hour. In sterilizing combustible articles in such a chamber, it should be remembered that cotton is browned at a temperature of $200^{\circ} \mathrm{C}$. and over. This method is used in laboratories for the sterilization of Petri dishes, flasks, test tubes, and pipettes, and for articles which may be injured by moisture. Both heating and subsequent cooling should be done gradually to avoid eracking of the glassware.

Moist Heat.-Instruments, syringes, and other suitable objects may be sterilized by boiling in water. Boiling for about five minutes is amply sufficient to destroy the vegetative forms of all bacteria. For the destruction of spores, boiling for one or two hours is usually sufficient, though the spores of certain saprophytes of the soil have been found occasionally to withstand the moist heat at a temperature of $100^{\circ} \mathrm{C}$. for as long as sixteen hours. ${ }^{31}$ The addition of 1 per cent of sodium carbonate to boiling water hastens the destruction of spores and prevents the rusting of metal objects sterilized in this way. The addition of carbolic acid to boiling water in from 2 to 5 per cent usually insures the destruction of anthrax spores, at least, within ten to fifteen minutes.

Exposure to live steam is probably the most practical of the methods

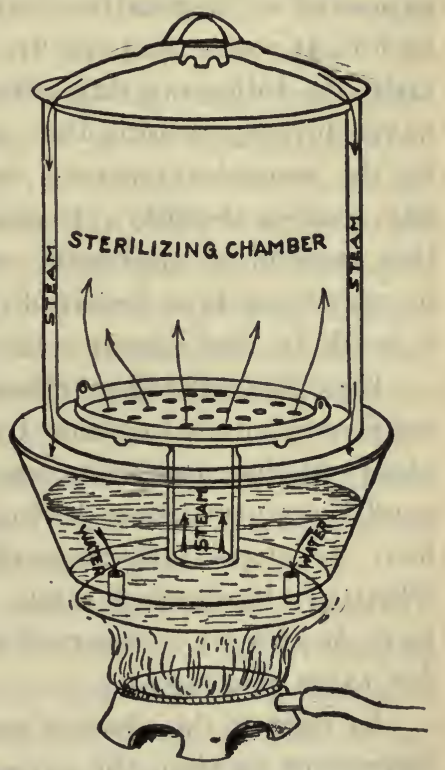

Fig. 8.-ArNold SteriLizer. of heat sterilization. It may be carried out by simple makeshifts of the kitchen, such as the use of potato-steamers or of wash-boilers. For laboratory purposes, the original steaming device introduced by Koch has been almost completely displaced by devices constructed on the plan of the so-called "Arnold" sterilizer (Fig. 8). In such an apparatus, water is poured into the reservoir

${ }^{31}$ Christen, loc. eit. 
$A$ and flows from there into the shallow receptacle $B$, formed by the double bottom. The flame underneath rapidly vaporizes the thin layer of water contained in $B$, and the steam rises rapidly, coursing through the main chamber $C$. Steam which eseapes through the joints of the lid of this chamber is condensed under the hood and drops back into the reservoir. Exposure to steam in such an apparatus for fifteen to thirty minutes insures the death of the vegetative forms of bacteria.

In the sterilization of media by such a device, the method of fractional sterilization at $100^{\circ} \mathrm{C}$. is employed. The principle of this method depends upon repeated exposure of the media for fifteen minutes to one-half hour on three succeeding days. By the first exposure all vegetative forms are destroyed. The media may then be left at room temperature, or at incubator temperature $\left(37.5^{\circ} \mathrm{C}\right.$.) until the following day, when any spores which may be present will have developed into the vegetative stage. These are then killed by the second exposure. A repetition of this procedure on a third day insures sterility. It must always be remembered, however, that this method is applicable only in cases in which the substance to be sterilized is a favorable medium for bacterial growth in which it is likely that spores will develop into vegetative forms.

Exceptionally the method may fail even in favorable media when anaërobic spore-forming bacteria are present. Thus, it has been observed that anaërobic spores, failing to develop under the aërobic conditions prevailing during the intervals of fractional sterilization, have developed after inoculation of the media with other bacteria, when symbiosis had made their growth possible. Tetanus bacilli have, in this way, occurred in cultures of diphtheria bacilli employed for toxin production.

In noting the time of an exposure in an Arnold sterilizer, it is important to time the process from the time when the temperature has reached $100^{\circ} \mathrm{C}$. and not from the time of lighting the flame.

The principle of fractional sterilization at low temperatures is applied also to the sterilization of substances which can not be subjected to temperatures as high as $100^{\circ} \mathrm{C}$. This is especially the case in the sterilization of media containing albuminous materials, when coagulation is to be avoided, or when both coagulation of the medium and sterilization are desired.

In such cases fractional sterilization may be practiced in simply constructed sterilizers, such as a Koch inspissator or, in the case 
of fluids, such as blood serum, by immersion in a water-bath at a temperature varying above $55^{\circ} \mathrm{C}$, according to circumstances. Exposures at such low temperatures may be repeated on five or six consecutive days, usually for an hour each day.

The use of steam under pressure is the most powerful method of heat-disinfection which we possess. It is applicable to the sterilization of fomites, clothing, or any objects of a size suitable to be contained in the apparatus at hand, and which are not injured by moisture. In laboratories this method is employed for the sterilization of infected apparatus, such as flasks, test tubes, Petri plates, etc., containing cultures. The device most commonly used in laboratories is the so-called autoclave, of which a variety of models may be obtained, both stationary and portable. The principle governing the construction of all of these is the same. The apparatus usually consists of a gun-metal cylinder supplied with a lid, which can be tightly closed by screws or nuts, and supplied with a thermometer, a safety-valve, and a steam pressure gauge. In the simpler autoclaves, water may be directly fillea into the lower part of the cylinder, and the objects to be sterilized supported upon a perforated diaphragm. In this case the heat is directly applied by means of a gas flame. In the more ela'iorate stationary devices, steam may be let in by piping it from the regular supply used for heating purposes. Exposure to steam under fifteen pounds pressure (fifteen in addition to the usual atmospheric pressure of fifteen pounds to the square inch) for fifteen to twenty mincites, is sufficient to kill all forms of bacterial life, including spores.

In applying autoclave sterilization practically, attention must be paid to certain technical details, neglect of which would result in failure of sterilization. It is necessary always to permit all air to escape from the autoclave before closing the vent. If this is not done, a poorly conducting air-jacket may be left about the objects to be sterilized, and these may not be heated to the temperature indicated by the pressure. It is also necessary to allow the reduction of pressure, after sterilization, to take place slowly. Any sudden relief of pressure, such as would be produced by opening the airvent while the pressure gauge is still above zero, will usually result in a sudden ebullition of fluid and a removal of stoppers from flasks.

The temperature attained by the application of various degrees of pressure is expressed in the following table: 


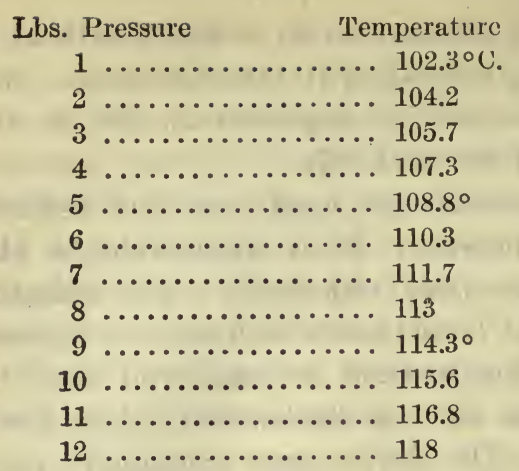

Lbs. Pressure

Temperature

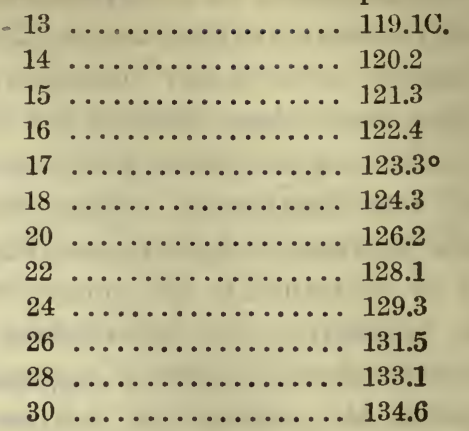

\section{CHEMICAL AGENTS INJURIOUS TO BACTERIA}

Since the time of Koch's ${ }^{32}$ fundamental researches upon chemical disinfectants, the known number of these substances has been enormously increased, and now embraces chemical agents of the most varied constitution. It is thus manifestly impossible to refer the injurious influence which these substances exert upon bacteria to any uniform law of action. The efficiency of a disinfecting agent, furthermore, is not alone dependent upon the nature and concentrations of the substance itself, but depends complexly upon the nature of the solvent in which it is employed, the temperature prevailing during its application, the numbers and biological characteristies of the bacteria in question, and the time of exposure. All these factors, therefore, must be considered in testing the efficiency of any given disinfectant. While it is true, furthermore, that all substances which in a given concentration exert bactericidal or disinfecting action upon a microorganism, will in greater dilution act antiseptically or inhibitively, no definite rules of proportion exist between the two values, which in each case must be determined by experiment.

Disinfectants Used in Solution.-The actual processes which take place in the injury of bacteria by disinfectants are to a large extent unknown. In the rase of strong acids, or strongly oxidizing substances, there may be destruction of the bacterial body as a whole by rapid oxidation. Other substances may act by coagulation of the bacterial protoplasm; others again by diffusion through the cell membrane are able to enter into rhemical combination with the

${ }^{82}$ Koch, Arb. a. d. kais. Gesundheitsamt, i, 1881. 
protoplasm and exert a toxic action. Again, in other cases, a difference in tonicity between cell protoplasm and disinfectant may tend to withdrawal of water from the bacterial cell and consequent injury of the microorganisn.

Among the inorganic disinfectants the most important are the metallic salts, acids, and bases, the halogens and their derivatives, and certain oxidizing agents like peroxide of hydrogen and permanganate of potassium.

It has been shown by Scheuerlen and Spiro, ${ }^{33}$ Krönig and Paul, ${ }^{36}$ and others, that in the case of the salts, acids, and bases, there is a distinct and demonstrable relationship between the disinfecting power of these substances and their dissociation in solution.

According to the theory of electrolytic dissociation, when bodies of this class go into solution they are broken up or dissociated into an electro-positive and an electro-negative ion. Thus, metallic salts are broken up into the kation, or positive metal, and into the anion, or negative acid radicle $\left(\mathrm{AgNO}_{3}=\mathrm{Ag}\right.$, + ion and $\mathrm{NO}_{3},-$ ion). In the case of the acids, ionization takes place into the hydrogen ions and the acid radicles, while in the case of the bases the dissociation occurs into the metal, on the one hand, and the OH group on the other. The degree of dissociation taking place depends upon the nature of the substance in solution, its concentration, and the nature of the solvent. Thus, in any such solution there appear three substances, the undissociated compound as such, its electro-negative ion, and its electro-positive ion, their relative concentrations depending upon an interrelationship calculable by definite laws. It goes without saying, therefore, that any chemical or physical reaction, taken part in by such a solution, may be participated in, not only by the dissolved undissociated residue as a whole, but by its separate ions individually as well. In the case of many disinfectants, the writers referred to above have been able to demonstrate a relationship between the degree of dissociation and the bactericidal powers. According to Krönig and Paul, double metallic salts, in which the metal is a constituent of a complex ion and in which the concentration of the dissociated metal-ions is consequently low, have very little disinfecting power. Thus potassium-silver-cyanide, which is a comparatively weak disinfectant, dissociates into the kation $K$ and the

${ }^{33}$ Scheuerlen und Spiro, Münch. med. Woch., 44, 1897.

${ }^{*}$ Krönig und Paul, Zëit. f. Hyg., xxv, 1897. 
complex anion $\mathrm{Ag}(\mathrm{CN})_{2}$, this latter further dissociating to a very slight degree only. The same writers conclude that the bactericidal action of mercuric chloride and of halogen combinations with metals is directly proportionate to the degree of dissociation. This consideration, moreover, explains why aqueous solutions of such substances are more active than are solutions in the alcohols or in ether, since it is well known that metallic salts are ionized in these substances to a much slighter degree than they are in water. ${ }^{35}$

On the other hand, the addition of moderate quantities of ethyl and methyl alcohol or acetones to aqueous solutions of silver nitrate or mercuric chloride, definitely increases the disinfecting action of such solutions. In the case of mercuric chloride, Krönig and Paul obtained the most powerful effects in solutions to which alcohol had been added in a concentration of 25 per cent. For this empirical fact a satisfactory explanation has not yet been found. Krönig and Paul suggest that low percentages of alcohol may facilitate the penetration of the disinfectant through the cell membrane and thus increase its efficiency, while high percentages of alcohol have the opposite effect, by decreasing the degree of dissociation. In this connection it has been suggested, however, that absolute and strong alcohols possibly act as desiccating agents, thus actually rendering the bacteria dry and less susceptible to deleterious chemical influences.

In the case of acids and bases the same authors have determined that the powers of disinfection of these substances are again directly proportionate to the degree of their dissociation: that is, to the concentration of the hydrogen or hydroxyl ions, respectively. The hydrogen ions are more powerfully active than the hydroxyl ions in equal concentration; acids, therefore, are more efficient disinfectants than bases.

A fact which appears to strengthen the opinion as to the relationship between bactericidal powers and dissociation, is that brought forward by Scheuerlen and Spiro, that the addition of $\mathrm{NaCl}$ to bichloride of mercury solutions reduces the disinfecting power of

${ }^{35}$ Water is the strongest dissociant known. Methyl alcohol has about one-half to two-thirds the dissociating power of water (Zelinsky, Zeit. f. physiol. Chemie, xx, 1896). Ethyl alcohol allows dissociation much less than methyl alcohol; ammonia allows dissociation to about one-third to one-fourth the extent of water. See Jones, "Elements of Physical Chemistry," p. 371. Macmillan, New York, 1902. 
such solutions, inasmuch as it diminishes the concentration of free ions. In practice, however, $\mathrm{NaCl}$ or $\mathrm{NH}_{4} \mathrm{Cl}$ is added to bichloride of mercury solutions, since these substances aid in holding in solution mercury compounds formed in the presence of alkaline albuminous material, blood serum, pus, etc.

The principles underlying disinfection have been still further elucidated by Chick, ${ }^{36}$ who showed that the rate at which bacteria were killed followed the definite mathematical expression for velocity of simple chemical reactions of the monomolecular type. It was found necessary to express the concentration of ionized antiseptics in terms of concentration of the active ion $(\mathrm{Hg})$ instead of total molecular concentration, thus adducing further verification of the work of Scheuerlen and Spiro, and Krönig and Paul. There was also found to be an unusually high temperature coefficient for disinfectant action, in the case of phenol the reaction velocity increasing eight times for a rise of $10^{\circ} \mathrm{C}$., while with metallic salts, the increase was about three-fold. The advantage to be gained by the use of warm solutions is, therefore, evident.

Halogens.*-In regard to the halogens, Krönig and Paul have shown that the germicidal power of this class of elements is inversely proportionate to their atomic weights. Thus, chlorine with the lowest atomic weight is the strongest disinfectant of the group. Next, and almost equal to this, is bromine. Iodine with a much heavier atomic weight than either of the former is distinctly less bactericidal.

Chloride of Lime.-Of the halogen compounds used in practice, the most important is chloride of lime or bleaching powder. As to the composition of this substance, there is some difference of opinion. It was formerly believed to be a mixture of calcium hypochlorite, $\mathrm{Ca}\left(\mathrm{ClO}_{2}\right)$, and of calcium chloride, $\mathrm{CaCl}_{2}$. The fact that the substance is not deliquescent, however, speaks against the presence of calcium chloride as such, and it is probable that it consists of a single compound with the formula $\mathrm{CaOCl}_{2}$. The action of acids or even of atmospheric $\mathrm{CO}_{2}$ upon this substance results in the liberation of chlorine. For instance:

$$
\begin{aligned}
& \mathrm{Ca}\left(\mathrm{Cl}_{2} \mathrm{O}\right)+2 \mathrm{HCl}=\mathrm{CaCl}_{2}+2 \mathrm{HClO} . \\
& 2 \mathrm{HClO}+2 \mathrm{HCl}=2 \mathrm{H}_{2} \mathrm{O}+2 \mathrm{Cl}_{2} .
\end{aligned}
$$

${ }^{36}$ Chick, Jour. of Hyg., 8, 1908, 92, and 10, 1910, 238.

* For consideration of the uses of chlorin and bleaching powder for the treatment of drinking water, see Chapter on "Water.", 
Hypochlorous acid may also decompose with the liberation of oxygen as shown in the following equation:

$$
2 \mathrm{HClO}=2 \mathrm{HCl}+\mathrm{O}_{2}
$$

It is conceivable that some of the disinfecting value of chloride of lime and hypochlorites in general is really due to the vigorous oxidizing action resulting from this decomposition. On the other hand, there is much evidence to show that chlorine may attack the protein molecule directly by replacing " $\mathrm{H}$ " in the amino groups, thus :

$$
-\mathrm{R}-\mathrm{CO}-\mathrm{NH}-\mathrm{R}-+\mathrm{Cl}=\mathrm{R}-\mathrm{CO}-\mathrm{NCl}-\mathrm{R}-+\mathrm{H}
$$

The chloramines thus formed seem to be toxic and result in the death of the bacteria. Bleaching powder is readily soluble in about twenty parts of water. Its bactericidal action depends on the hypochlorous acid formed. After water precipitation, an efficient dosage is 10 pounds to the million gallons. The high germicidal action of chloride of lime, together with its relatively low cost, suggest its use as a wound dressing. Solutions of calcium or sodium hypochlorite were found to be too irritating to be practicable, owing to the alkalinity of any available preparations. Recently, however, it has been possible to prepare neutral, and comparatively nonirritating solutions of sodium hypochlorite by several different methods.

Dakins Solution.-The following detailed descriptions of the preparation of Dakin's solution and its titration are taken from the Medieal War Manual, Number 6, Laboratory Methods of the United States Army, published from the Surgeon General's Office, and are given directly as printed in this Manual, because the method was standardized for Army use in this way during the war. The description which follows is taken verbatim from this Manual.

Preparation from Bleaching Powder.-Dakin's Original Method.-A strong solution of hypochlorite is prepared by decomposing 150 grams bleaching powder (about 25 to 35 per cent available ehlorine) with 105 grams dry sodium carbonate (122 grams monolyydrate $\left(\mathrm{Na}_{2} \mathrm{CO}_{3} \mathrm{H}_{2} \mathrm{O}\right)$ or 284 grams washing soda $\left(\mathrm{Na}_{2} \mathrm{CO}_{3} 10 \mathrm{H}_{2} \mathrm{O}\right)$. The mixture is very thoroughly shaken, both to make good contact and to render the precipitated ealcium earbonate granular and promote its settling. It is then allowed to stand quietly and after half an hour the clear liquid is siphoned off from the precipitate and filtered through paper or a cotton plug. 
A 10 c.c. portion is rapidly titrated with $\frac{\mathrm{N}}{2}$ boric acid solution (31 grams per liter), using powdered phenolphthalein as indicator (the usual alcoholic solution of phenolphthalein will not serve) in order to determine the amount of boric acid to be added to the rest of the filtrate. The end-point is the disappearance of the pink color. Each cubic centimeter of $\frac{\mathrm{N}}{2}$ boric acid required for the 10 c.c. sample calls for the addition of 3 grams boric acid per liter of filtrate. An excess of boric acid should be avoided, as it favors the liberation of hypochlorous acid and renders the solution less stable. It is best to add slightly less than the calculated amount. The concentrated solution thus prepared contains about 4 per cent of sodium hypochlorite, and before use should be diluted with about 7 parts of water and titrated with $\frac{\mathrm{N}}{10}$ thiosulphate to determine its precise hypochlorite concentration. It is then accurately diluted to the required strength (usually 0.5 to 0.45 per cent).

Preparation from Chlorine and Sodium Carbonate.-Chlorine may be obtained as the compressed gas in steel cylinders and is easily measured by a chlorine meter manufactured for the purpose. This is a stable, economical and convenient source of chlorine. A solution is prepared containing 15 grams of dry sodium .earbonate per liter (=17.6 grams monohydrate or 40 grams of washing soda). A measured quantity, 4.8 grams (or about 1600 c.c.) of ehlorine gas is allowed to run into the solution for each liter. Ten c.c. of the solution is then titrated. If the solution is too weak more chlorine is introduced. If the solution is too strong it should be diluted to a concentration of 0.5 per cent $\mathrm{NaOCl}$ with 1.5 per cent sodium earbonate solution, which at the same time serves to correct the unduly diminished alkalinity caused by the excess of chlorin introduced into the solution. If the final solution fails to give a momentary flash of color with alcoholic solution of phenolphthalein, it should be rejected. If the solution shows color with powdered phenolphthalein, it must be titrated with boric acid as described above, for preparation from bleaching powder, until this defect is corrected, or it must be discarded. The solution should be titrated for hypochlorite concentration every twenty-four to forty-eight hours and discarded when it falls below the desired lower limit (usually 0.45 per cent).

If a chlorin meter is not available, chlorin gas may be run into the 1.5 per cent carbonate solution through any improvised diffuser until the hypochlorite concentration has reached 0.5 per cent. The amount of ehlorin required to give a hypochlorite concentration of 0.5 per eent is approximately twice the amount required to eause decolorization of powdered phenolphthalein. It is therefore convenient to add powdered phenolphthalein and note the amount of chlorin required to cause its decolorization. When 
almost twice that amount of ehlorin has been introduced, frequent titrations of the hypochlorite content must be commenced to determine the proper point at which to stop the introduction of the chlorin.

Titration of Dakin's Solution.-To 10 c.c. of the Dakin solution, add approximately 5 c.e. of a 10 per cent solution of potassium iodid and 3 c.c. of glacial acetic acid. Iodin is liberated and dissolves in the excess of iodid present. Dilute with water to about 50 c.c. A standard $\frac{\mathrm{N}}{10}$ thiosulphate solution is then added from a burette until the solution is just rendered colorless. The number of cubic centimeters required to effect this result multiplied by the factor 0.0372 gives the percentage of sodium hypochlorite present in the Dakin solution.

Preparation of Standard ${ }_{10}^{\mathrm{N}}$ Thiosulphate Solution.-Dissolve exactly 24.82 grams of pure crystalline sodium thiosulphate in water and make up to exactly 1 liter. One c.c. of this standard solution is equivalent to 0.00372 gram of sodium hypochlorite.

Titration of Bleaching Powder.-Bleaching powders vary considerably in their "available chlorin" content, so that it is desirable to determine the available chlorine in each large batch. Bleaching powders with less than 20 per cent available chlorin should be rejected. Exceptional samples may contain as high as 35 per cent available chlorine.

The available chlorin content may be determined as follows: exactly 10 grams of bleaching powder made up of small samples from different parts of the jar, in order to obtain a representative sample, are well shaken with a liter of water. After standing about six hours the solution is filtered and a 10 c.c. sample of the filtrate is titrated in exactly the same manner as in the titration of Dakin's solution. In this case the number of cubic centimeters of decinormal thiosulphate required to decolorize, multiplied by the factor 3.55, gives the percentage of active chlorin in the bleaching powder.

The chlorin antiseptics in general, and particularly the hypochlorite type, have the disadvantage of exerting their disinfectant action over an exceedingly short space of time. The reaction between the hypochlorite solution and the proteins of the bacterial body or of the serum and pus in the wound, is almost instantaneous, and having taken place, no further toxic action is shown. It is, therefore, necessary in treating wounds with these solutions to repeat the application at frequent intervals, or else to apply through some sort of a continuous feed apparatus so that a fresh supply of the antiseptic is brought in contact with the wound at short intervals.

In order to overcome this disadvantage to some extent, Dakin 
prepared a number of different organic chlorin compounds which were soluble in oil, and which yielded up the chlorin rather slowly to the wound secretions, so that the action continued over a comparatively long time. Chloramine $T$ (1) and Dichloramine $T$ are the two most practicable compounds; these substances have the following formulae:
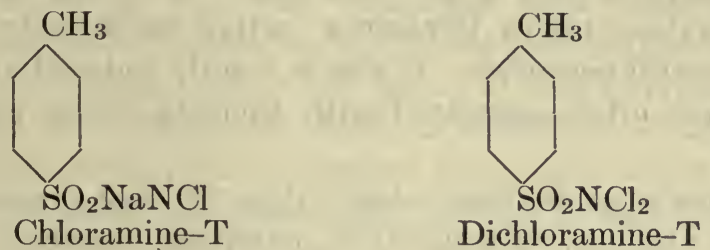

They contain chlorin replacing hydrogen in an amino group, and this chlorin is liberated slowly, in contact with protein material. They are used in solution in oil, either chlorinated paraffin oil or oil of eucalyptol, and are applied as a spray or on gauze.

Eusol.-The simple neutralization of calcium hypochlorite with boric acid renders it comparatively non-irritating, and under the name of eusol (2) this solution has been widely used.

For original articles on the use of Dakin's Solution, see below : ${ }^{37}$

TERChLORIDE OF IODIN $\left(\mathrm{ICl}_{3}\right)$ is an extremely strong disinfectant, being efficient for vegetative forms in solutions of 0.1 per cent in one minute and a 1 per cent solution destroying spores within a few minutes. ${ }^{38}$

Painting with Tincture of Iodin (10 per cent) is a simple and reliable method of sterilizing the skin. It is now used in many clinies in sterilizing the field of operation.

IoDoform $\left(\mathrm{CHI}_{3}\right)^{30}$ is weakly antiseptic in itself, but when introduced into wounds where active reducing processes are taking place-often as the result of bacterial growth-iodine is liberated from it and active bactericidal action results.

Peroxide of Hydrogen is formed by the action of dilute sulphuric

${ }^{37}$ Dakin, Brit. Med. Jour., August 28th, 1915. Carrel, Dakin, Daufresne, Dehelly and Dumas, Presse Médicale, October 11th, 1915. Dakin and Dunham, "Handbook of Antiseptics," New York, 1917. Lorrain Smith, Drennan, Rettie and Campbell, British Med. Jour., July 24th, 1915. See also Carrel and Dehelly, "Infeeted Wounds," Hoeber, N. Y. 1919.

${ }^{38}$ Behring, Zeit. f. Hyg., ix, 1891.

${ }^{20} v$. Behring, "Bekaempfung d. Infektions-Krankh.," Leipzig, 1894. 
acid upon peroxide of barium. It readily gives up oxygen and acts upon bacteria probably by virtue of the liberation of nascent oxygen. In the presence of organic matter, such as blood, pus, etc., associated with bacteria, $\mathrm{H}_{2} \mathrm{O}_{2}$ is quickly reduced and weakened. It is important that the $\mathrm{H}_{2} \mathrm{O}_{2}$ come in immediate contact with the bacteria. In practice, therefore, blood and pus should be removed from wounds when applying the $\mathrm{H}_{2} \mathrm{O}_{2}$ or a large excess of $\mathrm{H}_{2} \mathrm{O}_{2}$ should be used.

Permanganate of Potassium, acting probably in the same way, is a powerful germicide. It also is readily reduced by many organic substances often associated with bacteria, being rendered weaker thereby.

Among organic disinfectants those of most practical importance are the alcohols, formaldehyds, iodoform, members of the phenol group and its derivatives, carbolic acid, cresol, lysol, creolin, salicylic acid, certain ethereal oils, and, more recently introduced, organic silver salts such as protargol, argyrol, argonin, and others.

THE ALCOHOLS are but indifferent disinfectants. Koch ${ }^{40}$ in 1881 found that anthrax spores remained alive for as long as four months when immersed in absolute and in 50 per cent ethyl alcohol. On the other hand, while absolute alcohol possesses practically no germicidal powers, possibly because of the formation of a protecting envelope by the coagulation of the bacterial ectoplasm, or, as suggested above, by desiccation due to the abstraction of water, dilute alcohol in a concentration of about 50 per cent is distinctly germicidal, destroying the vegetative forms of bacteria in from ten to fifteen minutes or less. ${ }^{41}$ Attention has already been called to the fact that moderate additions of alcohol to aqueous solutions of mercuric chloride enhance the germicidal power of this disinfectant. Additions of ethyl and methyl alcohol to carbolic acid or formaldehyde solutions, on the other hand, progressively decrease the bactericidal activities of these substances. ${ }^{42}$

The value of boiling alcohol for the destruction of sporesespecially in the sterilization of catgut-has been investigated by Saul, ${ }^{43}$ who found that boiling in absolute ethyl, methyl, or propyl alcohol is practically without effect, while spores are destroyed

${ }^{40}$ Koch, Arb. a. d. kais. Gesundheitsamt, i, 1881.

${ }^{41}$ Epstein, Zeit. f. Hyg., xxiv, 1897.

42 Krönig und Paul, loc. cit.

${ }^{43}$ Saul, Archiv f. klin. Chir., 56, 1898. 
readily in boiling dilute alcohol, the most effectual being propyl alcohol of a concentration of from 10-40 per cent.

Carbolic ACID $\left(\mathrm{C}_{6} \mathrm{H}_{5} \mathrm{OH}\right)$, at room temperature, consists of colorless erystals which become completely liquefied by the addition of 10 per cent of water. In contradistinction to most inorganic disinfectants, the action of carbolic acid and other members of the phenol group is not in any way dependent upon dissociation. ${ }^{44}$ According t'o Beckmann ${ }^{45}$ and others, carbolic acid acts as a molecule and not by individual ions. The proof of this is brought out by the fact that the addition of $\mathrm{NaCl}$ to earbolic acid solutions, an addition which would tend to decrease the concentration of free ions, markedly increases the bactericidal powers of such solutions. On the other hand, as stated above, additions of alcohol progressively diminish the efficiency of the phenols.

Other members of this group of disinfectants are ORTHO-, META-, and PARACRESOL $\left(\mathrm{C}_{6} \mathrm{H}_{4} \mathrm{CH}_{3} \mathrm{OH}\right)$, isomeric compounds differing only in the position of the OH radicle. Tricresol is a mixture of these three. The cresols are relatively more powerfully germicidal than carbolic acid, but are less soluble in water. LYSOL is a substance obtained by the solution of coal-tar cresol in neutral potassium-soap. Dissolved in water it forms an opalescent easily flowing liquid. According to Gruber, ${ }^{46}$ its germicidal action is slightly greater than that of carbolic acid. Creolin, another combination of the cresols with potassic soap, forms with water a turbid emulsion. v. Behring ${ }^{47}$ expressed the relative germicidal powers of carbolic acid, cresol, and creolin for vegetative forms by the numbers $1: 4: 10$, in the order named.

Formaldehyd (H-COH), or methyl aldehyde, is a gas which is easily produced by the incomplete combustion of methyl alcohol. The methods of actually generating it for purposes of fumigation will be discussed in a subsequent paragraph. In aqueous solution this substance forms a colorless liquid with a characteristic acrid odor, and in this form is largely used as a preservative for animal tissues and as a germicide. It is marketed as "formalin," which is an aqueous solution containing from 35 to 40 per cent of the

\footnotetext{
${ }^{44}$ Scheuerlen und Spiro, Münch. med. Woch., 44, 1897.

${ }^{45}$ Beckmann, Cent. f. Bakt., I, xx, 1896.

${ }^{16}$ Gruber, Cent. f. Bakt, I., xi, 1892.

${ }^{47} v$. Behring, loc. eit., p. 111.
} 
gas and which exerts distinctly bactericidal action on vegetative forms in further dilutions of of from 1 to 10 to 1 to 20 (formaldehyd gas $1: 400$ to $1: 800$ ). Anthrax spores are killed in 35 per cent formaldehyde in ten to thirty minutes. ${ }^{48}$ Unlike the phenols, the addition of salt to formaldehyd solutions does not increase its efficiency, but similar to them, additions of ethyl and methyl alcohol markedly reduce its germicidal powers.

The Essential oils which are most commonly used in practicelargely as intestinal antiseptics-are those of cinnamon, thyme, eucalyptus, and peppermint. Omeltschenko ${ }^{49}$ believes that the employment of these oils in emulsions is illogical, inasmuch as their bactericidal powers depend upon their vaporization. He classifies the oils in decreasing order of their efficiency as follows: Oil of cinnamon, prunol, oil of thyme, oil of peppermint, oil of camphor, and eucalyptol.

The Flavine Dyes.-A number of dye stuffs have been shown to exert disinfectant action when applied to bacteria. The members of the acridine group are particularly active in this respect, and have been used to some extent practically. Benda ${ }^{50}$ first investigated one of these dyes, now known under the name of flavine or acriflavine, in connection with trypanosome infections, and recently Browning ${ }^{51}$ applied this substance to the antiseptic treatment of war injuries.

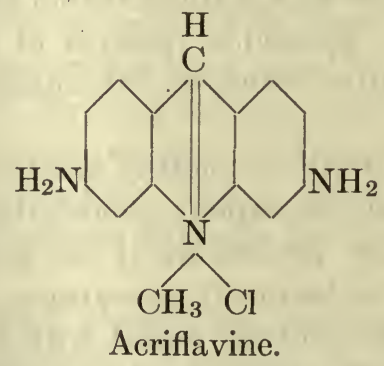

He believed that this dye, while being highly toxic to bacteria, was relatively harmless and non-irritating in its effect on the tissue cells.

${ }^{48}$ Krönig und Paul, loc. cit.

${ }^{49}$ Omeltschenko, Cent. f. Bakt., I, ix, 1891.

${ }^{50}$ Benda, Ber. Deutsch. Chem. Gesell, 45, 1787, 1912.

${ }^{51}$ Browning, Gulbsansen, Kennaway and Thornton, Brit. Med. Jour., Jan. 20th, 1917. 
Practical experience seems to have shown, however, that the healing of wounds that have been treated with acriflavine may be to a certain extent delayed by the action of the dye, which is, therefore, not entirely without effect on tissue cells. Proflavine, a closely related dye, is similar in its effects and less expensive in preparation.

Triphenylmethane Dyes.-Many of the dyes of this series, notably gentian violet, malachite green and brilliant green, have long been known to be highly toxic for bacteria.

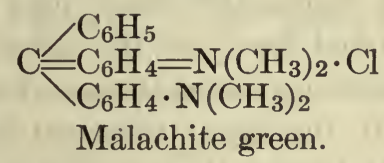

They are of especial interest since their toxic action seems to be directed almost entirely against Gram-positive bacteria, and sometimes a dilution one thousand times or more as strong as is necessary. to inhibit the growth of streptococci and staphylococci, will be needed for prevention of growth of the Colon bacillus. Since most of the common pyogenic organisms are Gram-positive, the use of these substances in wound dressings suggests itself. Practically, it has been found, particularly with brilliant green, that, while it acts as a very efficient antiseptic in wounds, here also the dye is not without effect on the tissue cells, and the granulations that are formed in its presence are not of the normal type. Concentrated solutions of crystal violet and brilliant green have been used for sterilizing the skin before operation, and are claimed to be more efficient for this purpose than tincture of iodin..$^{52}$

Methods of Testing the Efficiency of Disinfectants.-The efficiency of any given disinfectant depends, as we have seen, upon a number of factors, any one of which, if variable, may lead to considerable differences in the end result. Thus, as far as the bacteria themselves are concerned, it is necessary to remember that not only do separate species differ in their resistance to disinfectants, but that different strains within the same species may show such variations as well. This fact largely accounts for the widely varying reports made by different investigators as to the resistance of anthrax spores, and depends possibly upon temporary or permanent biological differences produced in bacteria by the conditions of their previous environment.

${ }^{52}$ Bonney and Browning, Brit. Med. Jour., May 18th, 1918. 
The numbers of bacteria exposed to the disinfectant, furthermore, is a factor which should be kept constant in comparative tests. The medium, moreover, in which bacteria are brought into contact with the disinfectant is a matter of great importance, inasmuch as either by entering into chemical combination with the disinfectant it may detract from its concentration or by coagulation it may form a purely mechanical protection for the microorganism. Thus bacteria which may be destroyed in distilled water or saltsolution emulsion with comparative ease, may evince an apparently higher resistance if acted upon in the presence of blood serum, mucus, or other albuminous substances. Temperature influences bactericidal processes in that most chemical disinfectants are more actively bactericidal at higher than at lower temperatures, a fact due most likely to the favorable influence of temperature upon all chemical reactions. ${ }^{53}$ As far as merely inhibitory or antiseptic values are concerned, however, the temperature least favorable for the reaction of the antiseptic is that which represents the optimum growth temperature for the microorganism in question and the inhibitory effects of any substance are less marked at this point than at temperatures above or below it.

The important influence exerted by the solvent in which the disinfectant is employed has already been discussed. For ordinary work it is customary to express absolute and comparative antiseptic and bactericidal values in terms of percentages based upon weight, and this, beyond question, is both simple and practical. For strictly scientific comparisons, however, as Krönig and Paul ${ }^{54}$ have pointed out, it is by far more accurate to work with ecuimolecular solutions.

Rideal and Walker ${ }^{55}$ have devised a method of testing disinfectants, in which an attempt is made to establish a standard for comparisons. They choose, as the standard, carbolic acid, and establish what they call the "carbolic-acid coefficient." This coefficient they obtain in the following way: the particular dilution of the disinfectant under investigation which will kill in a given time, is divided by the strength of carbolic acid which, under the same conditions, will kill the same bacteria in the same time. We quote

'3 $v$. Behring, "Bekaempf. der Infektions-Krankh., Infektion u. Desinfection," Leipzig, 1894.

${ }^{5}$ Krönig und Paul, loc. cit.

${ }^{55}$ Rideal and Walker, Jour. of the Sanitary Ins., London, xxiv. 
an example of such a test, given by Simpson and Hewlett, ${ }^{56}$ comparing formalin and carbolic acid.

BACILLUS PESTIS.

\begin{tabular}{|c|c|c|c|c|c|c|c|}
\hline \multirow{2}{*}{ Sample. } & \multirow{2}{*}{ Dilution. } & \multicolumn{6}{|c|}{ Time in Minutes. } \\
\hline & & 2.5 & 5 & 7.5 & 10 & 12.5 & 15 \\
\hline Formalin. & $\begin{array}{l}1 \text { in } 30 \\
1 \text { in } 40\end{array}$ & $\begin{array}{l}\text { growth } \\
\text { growth }\end{array}$ & $\begin{array}{l}\text { growth } \\
\text { growth }\end{array}$ & growth & & & \\
\hline Carbolic acid & $\begin{array}{l}1 \text { in } 100 \\
1 \text { in } 110\end{array}$ & growth & growth & $\begin{array}{l}\ldots \ldots \\
\ldots\end{array}$ & $\begin{array}{l}\ldots \ldots \\
\ldots \ldots\end{array}$ & $\begin{array}{l}\ldots \ldots \\
\ldots \ldots\end{array}$ & $\ldots \ldots$ \\
\hline
\end{tabular}

In the above table, formalin 1 in 30 killed in the same time as carbolic acid 1 in 110 . Thus the carbolic-acid coefficient of formalin in this test $={ }^{30} /{ }_{110}=.27$.

The Rideal-Walker method has been much used and is recommended by many workers. ${ }^{57}$

The most precise method of standardizing disinfectants is that now in use in the U. S. Public Health Service. It is a modification of the Rideal-Walker procedure devised by Anderson and McClintic. ${ }^{58}$

Stock 5 per cent solutions of the disinfectant in question and of the standard (phenol) are first prepared and a series of accurate dilutions made with distilled water using graduated pipettes. (To make 1:70 take 4 c.c. of stock and 10 c.c. distilled water; $1: 80=4$ c.c. of stock +12 c.c. distilled water $; 1: 90=4$ c.c. stock +14 c.c. distilled water $1: 500=2$ c.c. of stock +48 c.c. of distilled water. Complete dilution tables are given in their original article.) The series should include dilutions strong enough to kill B. typhosus in two and a half minutes and weak enough to fail to do so in fifteen minutes. If dilutions greater than 1-500 are required, a second 1 per cent stock solution is prepared. They adopted the following seale for their tests: Dilutions up to 1:70 should vary from the next in the series by a difference of 5 (i.e., 5 parts of water), and so on if higher solutions are necessary.

se Simpson and Hewlett, Lancet, ii, 1904.

${ }^{67}$ Sommerville, Brit. Med. Jour., 1904.

as Anderson and McClintic, Jour. of Inf. Dis., 1911, viii, 1. 


$\begin{array}{lll}\text { From } 1: 70 & \text { to } 1: 160 & \text { by a difference of } 10 \\ \text { From } 1: 160 & \text { to } 1: 200 & \text { by a difference of } 20 \\ \text { From } 1: 200 & \text { to } 1: 400 & \text { by a difference of } 25 \\ \text { From } 1: 400 & \text { to } 1: 900 & \text { by } a \text { difference of } 50 \\ \text { From } 1: 900 & \text { to } 1: 1800 & \text { by a difference of } 100 \\ \text { From } 1: 1800 & \text { to } 1: 3200 & \text { by a difference of } 200\end{array}$

Short wide test tubes 1 inch by 3 inches are used in making the test. These are placed in a rack in a water bath at $20^{\circ} \mathrm{C}$. Five c.c. of each dilution are measured into a series of these tubes beginning with the strongest specimen and rinsing the pipette once with each dilution before the 5 c.c. are measured out. For inoculation, a 24-hour broth culture of B. typhosus is prepared which has been transferred daily for at least 3 days. Before use it is shaken and filtered through sterile filter paper. The wide test tubes containing diluted disinfectant are inoculated with $1 / 10$ c.e. of this culture with a graduated pipette. The tip of the pipette is held against the side of the tube to insure accurate measurement and the tube immediately shaken to mix the bacteria thoroughly with the disinfectant. Test inoculations are made from this mixture at proper intervals into tubes containing 10 c.c. of standard extract broth of +1.5 acidity, using loops $4 \mathrm{~mm}$. in diameter. At least four such loops should be at hand, supported on a rack or wooden block so that a fan-tail Bunsen burner may be placed under each wire in turn. Each one is sterilized after a plant is made and allowed to cool while the other three are being used in order.

The test is conducted as follows: A row of ten wide tubes containing dilutions of the antiseptic is placed in the water bath at $20^{\circ} \mathrm{C}$. and time allowed for them to reach the temperature of the bath. They are then inoculated in order at intervals of exactly 15 seconds. Fifteen seconds after the last tube has been inoculated a subculture is made from the first tube of the series (i.e., 21/2 minutes after this first tube was inoculated) and from the other tubes in order at 15 -second intervals. Fifteen seconds after this first series of subcultures is completed, a second series of subcultures is begun which will give the result of a 5-minute exposure to the antiseptic and the subinoculations continued at 15 -second intervals until all dilutions have been tested for fifteen minutes. If the strength of the antiseptic is known approximately, subcultures of the lower dilutions for the longer periods may be omitted. It is convenient to have an assistant at hand to call time and to label 
the subcultures as soon as made. The tubes may, however, be placed in order in suitable racks without labeling. The subculture tubes are incubated for 48 hours at $37^{\circ}$ C. and those in which growth is observed are recorded positive.

\section{DETERMINATION OF THE CARBOLIC-ACID COEFFICIENT OF A DISINFECTANT.}

(Anderson and McClintic)

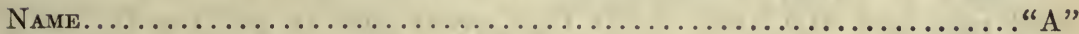

Temperature of Medication............................

Culture Used B. Typhosus................. 24-hr., Extract Broth, Filtered Proportion of Culture and Disinfectant............... 0.1 c.c. +5 c.c. Organic Matter, None; Kind, None; Amount, None.

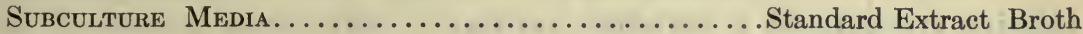
REACtion......................................... 1.5

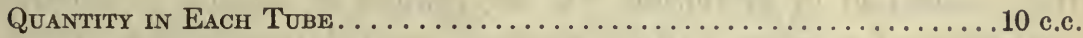

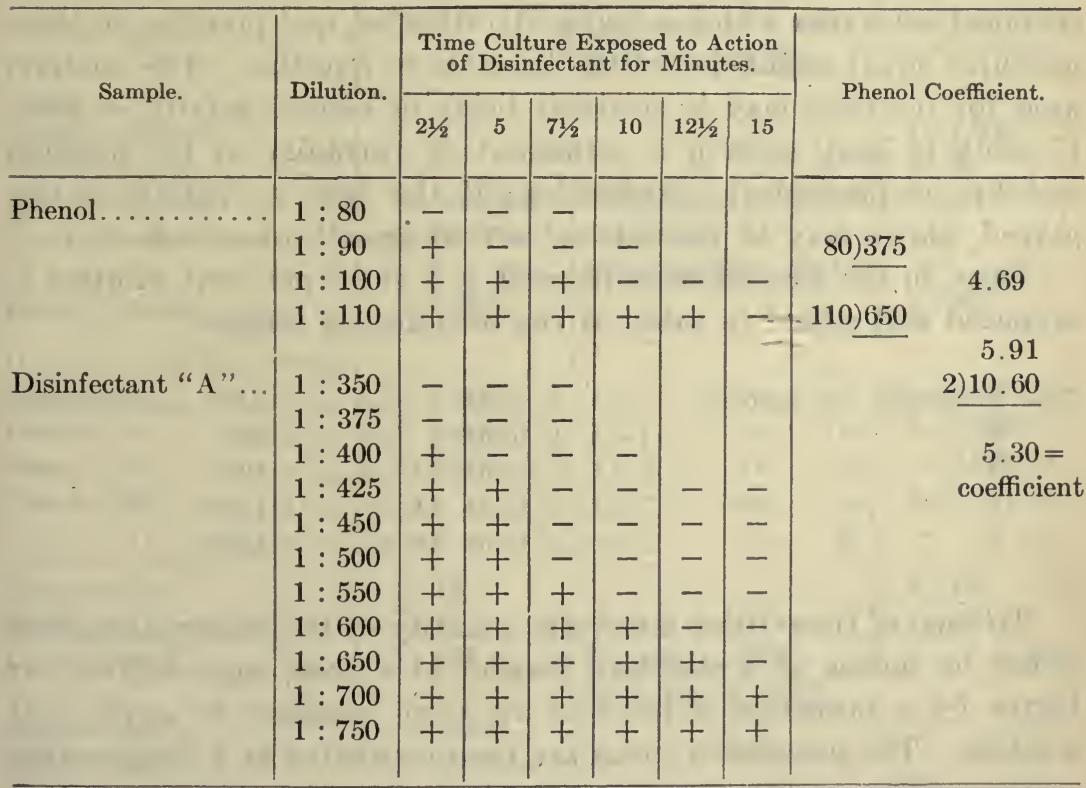

To obtain the coefficient the weakest dilution of the unknown antiseptic which kill in $21 / 2$ minutes is divided by the weakest dilution of phenol which kills in the same time. The same is done for the weakest strength that kills in 15 minutes and an average is taken. The results of such a test are shown in the table on page 104 . 
As only the $21 / 2$-minute and 15 -minute intervals are used in determining this result it seems unnecessary to make plants at the intervening periods except in special cases where more detailed information is desired.

The procedure may be modified by adding some organic substance such as killed bacteria to the diluted antiseptic. For many substances, e.g., bichloride of mercury, the antiseptic value in presence of organic matter is much lower than in watery solution. Anderson and McClintic insist that great care in making the dilutions and rigid adherence to a uniform technique are necessary to obtain consistent results in such tests.

Determination of Antiseptic Values.-The antiseptic or inhibitive strength of a chemical substance, sometimes spoken of as the "coefficient of inhibition," is determined by adding to definite quantities of a given culture medium, graded percentages of the chemical substance which is being investigated, and planting in these mixtures equal quantities of the bacteria in question. The medium used for the tests may be nutrient broth or melted gelatin or agar. If broth is used, growth is estimated by turbidity of the medium and by morphological examination; if the agar or gelatin is employed, plates may be poured and actual growth observed.

Thus, in the case of carbolic acid, a 5 or 10 per cent solution is prepared and added to tubes of the medium, as follows:

Tube 1 contains $5 \%$ carbolic

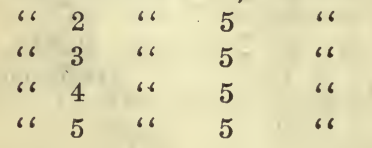

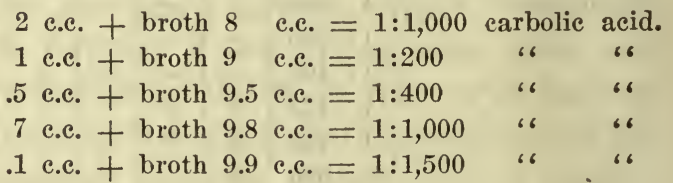

To each of these tubes a definite quantity of the bacteria is added either by means of a standard loopful of a fresh agar culture, or better by a measured volume of an even emulsion in sterile salt solution. The inoculated tubes are then incubated at a temperature corresponding to the optimum growth temperature for the microorganism in question. The tubes are examined for growth from day to day. From tubes containing higher dilutions, in which no growth is visible, transplants are made to determine the presence of living bacteria and to distinguish between inhibition or antisepsis and bacterial death or disinfection. 


\section{INHIBITION STRENGTHS OF VARIOUS ANTLSEPTICS.}

Adapted from FlügGe, Leipzig, 1902.

\begin{tabular}{|c|c|c|c|}
\hline . & Anthrax Bacilli. & Other Bacteria. & $\begin{array}{l}\text { Putrefactive Bac- } \\
\text { teria in Bouillon. }\end{array}$ \\
\hline Acids & & & \\
\hline Sulphuric............ & $1: 3,000$ & Chol. spir. $1: 6,000$ & \\
\hline Hydrochloric............ & $1: 3,000$ & $\begin{array}{l}\text { B. diph. } 1: 3,000 \\
\text { B. mallei } 1: 700 \\
\text { B. typh } 1: 500\end{array}$ & \\
\hline Sulphurous........... & $\ldots \ldots \ldots \ldots$ & Chol. spir. $1: 1,000$ & $1: 6,000$ \\
\hline Arsenous.... & & $\ldots \ldots \ldots \ldots \ldots$ & $1: 200$ \\
\hline Boric..... & $1: 800$ & $\ldots \ldots \ldots \ldots \ldots$ & $1: 100$ \\
\hline Alkalies & & & \\
\hline Potass. hydroxid ......... & $1: 700$ & $\begin{array}{l}\text { B. diphth. } 1: 600 \\
\text { Chol. spir. } 1: 400 \\
\text { B. typh. } 1: 400\end{array}$ & \\
\hline Ammon. hydroxid. & $1: 700$ & $\begin{array}{l}\text { Chol. spir. } 1: 500 \\
\text { B. typh. } 1: 500\end{array}$ & $x_{10}^{11}+1$ \\
\hline Calcium hydroxid........ & & $\begin{array}{l}\text { Chol. spir. } 1: 1,100 \\
\text { B. typh. } 1: 1,100\end{array}$ & \\
\hline SALTS & & & \\
\hline Copper sulphate......... & & $\ldots \ldots \ldots \ldots \ldots$ & $\begin{array}{l}1: 1,000 \\
1: 90\end{array}$ \\
\hline Mercuric chlorid. ........ & $1: 100,000$ & B. typhosus $1: 60,000$ & $1: 20,000$ \\
\hline Silver nitrate. . . . . . . . & $1: 60,000$ & $\begin{array}{l}\text { Chol. spir. } \\
\text { B. typhosus } 1: 50,000\end{array}$ & \\
\hline Potass. perman...... & $1: 1,000$ & $\ldots \ldots \ldots \ldots \ldots \ldots$ & $1: 500$ \\
\hline HaLOGENS AND Compounds & & & \\
\hline $\begin{array}{l}\text { Chlorin } . \ldots \ldots \ldots \ldots \ldots \\
\text { Bromin } . \ldots \ldots \ldots \ldots \ldots \ldots\end{array}$ & $\begin{array}{l}1: 1,500 \\
1: 1,500\end{array}$ & ${ }^{\ldots} \ldots \ldots \ldots \ldots \ldots$ & $\begin{array}{l}1: 4,000 \\
1: 2,000\end{array}$ \\
\hline Iodin . . . . . . . . . . . & $1: 5,000$ & $\ldots \ldots \ldots \ldots \ldots$ & $1: 5,000$ \\
\hline Potass. iodid........... & $\ldots \ldots \ldots \ldots$ & ............... & $1: 7$ \\
\hline ORganic Compounds & & & \\
\hline Ethyl alcohol......... & $1: 12$ & & $1: 10$ \\
\hline Acetic and oxalic acids... & $\ldots \ldots \ldots$ & B. diph. $1: 500$ & $1: 400$ \\
\hline Carbolic acid........... & $1: 800$ & $\begin{array}{l}\text { B. typh. } 1: 400 \\
\text { Chol. spir. } 1: 600\end{array}$ & \\
\hline Benzoic acid. & $1: 1,000$ & & 1 i. \\
\hline Salicylic acid............ & $1: 1,500$ & & \\
\hline $\begin{array}{l}\text { Formalin }(4 \% \text { formalde- } \\
\text { hydd } \ldots \ldots \ldots \ldots \ldots \ldots \ldots\end{array}$ & $\ldots \ldots \ldots$ & $\begin{array}{l}\text { Chol. spir. } 1: 20,000 \\
\text { Staphylo. } 1: 5,000\end{array}$ & $1: 1,000$ \\
\hline Camphor............... & $1: 1,000$ & & \\
\hline Thymol............... & $1: 10,000$ & & $1: 3,500$ \\
\hline Oil mentha pip........... & $1: 3,000$ & & (n) \\
\hline Oil of terebinth........... & $1: 8,000$ & 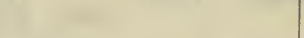 & \\
\hline Peroxide of hydrogen. & . & & $1: 2,000$ \\
\hline
\end{tabular}


BACTERICIDAL STRENGTHS OF COMMON DISINFECTANTS. AdAPted From FlügGe, LeIPZig, 1902.

\begin{tabular}{|c|c|c|c|c|}
\hline \multirow{2}{*}{ 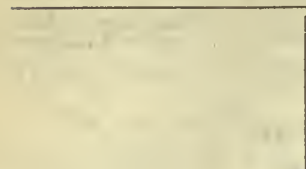 } & \multirow{2}{*}{$\frac{\begin{array}{c}\text { Strepto- and } \\
\text { Staphylo- } \\
\text { cocci. }\end{array}}{5 \text { Minutes. }}$} & \multicolumn{2}{|c|}{$\begin{array}{c}\text { Anthrax and Typhoid Bacilli. } \\
\text { Cholera Spirillum. }\end{array}$} & \multirow{2}{*}{ Anthrax Spores. } \\
\hline & & 5 Minutes. & 2-24 Hours. & \\
\hline Acids & & -5 & & \\
\hline Sulphuric.... . & $1: 10$ & $1: 100$. & $1: 1,500$ & $1: 50$ in 10 days \\
\hline Hydrochloric........ & $1: 10$ & $1: 100$ & $1: 1,500$ & $1: 50$ in 10 days \\
\hline Sulphurous........ & $\ldots \ldots \ldots$ & $\ldots \ldots \ldots$ & $\begin{array}{c}\text { (Typhoid } \\
1: 700)\end{array}$ & \\
\hline Sulphurous.... & . & $\cdots \cdots \cdots$ & $\begin{array}{r}1: 300(\mathrm{Gas} \\
10 \text { vol. } \%)\end{array}$ & \\
\hline Boric............ & $\ldots \ldots$ & $\ldots \ldots \ldots$ & $1: 30$ & $\begin{array}{l}\text { Conc. sol. incom- } \\
\text { plete disinfection }\end{array}$ \\
\hline Alkalies & & & & \\
\hline Potass. hydroxid. ... & $1: 5$ & $1: 300$ & & \\
\hline Ammon. hydroxid.... & $\ldots \ldots \ldots$ & $1: 300$ & & \\
\hline Calcium hydroxid.... & $\ldots \ldots \ldots$ & $x_{2}$ & $1: 1,000$ & \\
\hline SALTS & & 1 & & \\
\hline Copper sulphate.... . & $\ldots \ldots \ldots$ & $\ldots \ldots \ldots$ & $\ldots \ldots \ldots$ & $1: 20$ (5 days) \\
\hline Mercuric chlor....... & $\begin{array}{c}1: 10,000 \text { to } \\
1,000\end{array}$ & $1: 2,000$ & $1: 10,000$ & $1: 2,000$ (26 hours) \\
\hline Silver nitrate........ & $1: 1,000$ & $\ldots \ldots \ldots$ & $1: 4,000$ & \\
\hline Potass. permang...... & $1: 200$ & $\ldots \ldots \ldots$ & $\ldots \ldots$ & $1: 20$ (1 day) \\
\hline Chlorinated lime..... & $\ldots \ldots \ldots$ & $1: 500$ & $\ldots \ldots$ & $1: 20$ (1 hour) \\
\hline $\begin{array}{l}\text { HALOGENS AND COM- } \\
\text { POUNDS }\end{array}$ & & 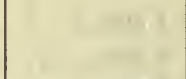 & 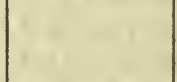 & \\
\hline Chlorin............ & 1 per cent & .1 per cent & $\ldots \ldots \ldots$ & 2 per cent (in $1 \mathrm{nr}$.) \\
\hline Trichlorid of iodin.... & $1: 200$ & $1: 1,000$ & $\ldots \ldots \ldots$ & $1: 1,000$ (in $12 \mathrm{hrs}$.) \\
\hline Organic Compounds & 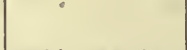 & & & \\
\hline Ethyl alcohol........ & $70 \%-15$ mins. & $70 \%-10$ mins. & $1: 2-300$ & Alcol. $50 \%$ for 4 \\
\hline Acetic and oxalic acids. & & & & $\begin{array}{l}\text { months without } \\
\text { killing } \\
\text { Koch. }{ }^{59}\end{array}$ \\
\hline Carbolic acid... & $1: 60$ & $\begin{array}{l}\text { Cholera } 1: 200 \\
\text { Typh. } 1: 50\end{array}$ & $1: 300$ & $\begin{array}{c}1: 20 \text { (4-45 days) } \\
\quad \text { (at } 40^{\circ} \text { in } 3 \text { hrs.) }\end{array}$ \\
\hline Lysol. . . . . . . . . & $1: 300$ & $1: 300$ & & \\
\hline Creolin............. & $\ldots \ldots$ & $1: 100$ & $1: 3,000$ & (10\% in 5 hrs.) \\
\hline Salicylic acid......... & $1: 1,000$ & 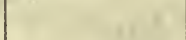 & & \\
\hline $\begin{array}{r}\text { Formalin }(40 \% \text { formal- } \\
\text { dehyde) } \ldots \ldots \ldots \ldots\end{array}$ & $1: 10$ & $1: 20$ & $1: 1,000$ & $1: 20$ (in $6 \mathrm{hrs}$.) \\
\hline Peroxide of hydrogen.. & Conc. & $1: 200$ & $1: 500$ & $\begin{array}{l}1: 100 \text { (in } 1 \mathrm{hr} \text { ). } \\
3: 100 \text { (in } 1 \mathrm{hr} \text { ) }\end{array}$ \\
\hline
\end{tabular}


The determination of the bactericidal or disinfectant value of a chemical substance upon spores may be carried out by a variety of methods. Koch, ${ }^{60}$ using anthrax spores as the indicator, dried the spores upon previously sterilized threads of silk. These were exposed to the disinfectant at a definite temperature for varying times, the disinfectant was then removed by washing in sterile water, and the threads planted upon gelatin or blood serum media and incubated. A serious objection to this method was pointed out by Geppert, ${ }^{61}$ who maintains that it is impossible by simple washing to remove completely the disinfectant in which the thread has been soaked. This author suggests that, whenever possible, the disinfectant, at the end of the time of exposure, should be removed by chemical means. In the case of bichloride of mercury, Geppert exposes emulsions of the bacteria to aqueous solutions of the disinfectant, and at the end of exposure precipitates out the bichloride of mercury with ammonium sulphide. In the case of a large number of disinfectants, however, this is not possible, and, when the thread method is used, removal of the chemical agent by washing must be practiced. Complete removal of the disinfectant is especially desirable, since spores previously exposed to these substances are more easily inhibited by dilute solutions than are normal spores. The spores may be dried upon the end of a glass rod, which, after exposure, is washed in distilled water or salt solution and then immersed in sterile broth. ${ }^{62}$

A simple method is that in which graded percentages of the disinfectant are added to the menstruum, blood, blood serum, broth, etc., in which the disinfectant is to be tested, and equal quantities of bacteria thoroughly emulsified in water or salt solution are added. Loopfuls of these mixtures are then planted from time to time in agar or gelatin plates upon which colony counts can afterward be made.

In all such tests, it is important to remember that the presence of organic fluids, blood serum, mucus, etc., considerably alters the efficiency of germicides, and whenever practical deductions are made, experimental imitation of the actual conditions should be attempted.

Gaseous Disinfectants for Purposes of Fumigation.-There are a large number of gaseous agents which are harmful to bacteria. Only

${ }^{60}$ Koch, Arb. a. d. kais. Gesundheitsamt, 1, 1881.

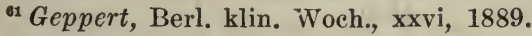

${ }^{6}$ Hill, Rep. Am. Pub. Health Assn., xxiv, 1898. 
a few, however, are of sufficient bactericidal strength to be of practical importance.

Oxygen, especially in the nascent state, may exert distinct bactericidal action upon some bacteria. That strictly anaërobic strains are inhibited by its presence has already been mentioned.

Ozone was shown by Ransome and Fullerton ${ }^{63}$ to exert considerable germicidal power when passed through a liquid medium in which bacteria were suspended, but was absolutely without activity when employed in the dry state.

Chlorine because of its powerful germicidal action was once looked upon with favor, but has been found quite inadequate from a practical point of view because of its injurious action upon materials, and its irregularity of action. Chlorine, too, is but weakly efficient unless in the presence of moisture. ${ }^{64}$

Sulphur dioxide or sulphurous anhydrid $\left(\mathrm{SO}_{2}\right)$, formerly much used for room disinfection, is no longer regarded as uniformly efficient for general use. The gas is produced by burning ordinary roll sulphur, conveniently in a Dutch oven. To be at all effective, water should be vaporized at the same time, since the disinfectant action is dependent upon the formation of sulphurous acid. The concentration of the gas should be at least 8 per cent of the volume of air in the room. For this purpose about three pounds of sulphur should be burned for every thousand cubic feet of space. It should be allowed to act for not less than twenty-four hours. The researches both of Wolffhügel ${ }^{65}$ and of Koch ${ }^{66}$ have shown that the gas is not sufficient for the destruetion of spores. Park ${ }^{67}$ believes that sulphur dioxid used in quantities of four pounds of sulphur to 1,000 eubic feet is of practical value for fumigating purposes in eases of diphtheria and the exanthemata. Sulphur dioxid fumigation is more effective than formaldehyd for the destruction of insects-fleas, lice and bedbugs-a matter of importance in epidemies of typhus fever, relapsing fever, plague, etc.

Of all known gaseous disinfectants by far the most reliable is formaldehyd. In all cases where formaldehyd fumigation is in-

\footnotetext{
63 Ransome and Fullerton, Rep. Public Health, July, 1901.

${ }^{64}$ Fischer and Proskauer, Mitt. a. d. kais. Gesundheitsamt, x, 11, 1882.

65 Wolffhügel, Mitt. a. d. kais. Gesundheitsamt, i, 1881.

${ }^{66}$ Koch, Mitt. a. d. kais. Gesundheitsamt, i, 1881.

6r Park, "Pathogen. Bact.,', N. Y., 1908.
} 
tended, clothing, bed-linen, and fabries should be spread out, cupboards and drawers freely opened. The cracks of windows and doors should be hermetically sealed with paper strips or by calking with cotton. In all cases moisture should be provided for, either in the generating apparatus or by a separate boiler.

Direct evaporation of formaldehyd from formalin solutions has been the principle underlying most of the methods. If such evaporation is attempted from an open vessel, however, polymerization of formaldehyd to the solid trioxymethylene occurs. To prevent this, Trillat $^{68}$ and others have constructed special autoclaves in which 20 per cent of calcium chlorid is added to formalin which is then vaporized under pressure.

The Trillat autoclave, as well as others constructed on the same principle, consists of a strong copper chamber of a capacity of about a gallon, fitted with a cover which can be tightly screwed into place. The cover is perforated by an outlet vent, a pressure gauge, and a thermometer. The whole apparatus is adjusted upon a stand and set over a kerosene lamp. Into the chamber is put about one-half to three-quarters its capacity of 40 per cent formaldehyd (commercial formalin) containing 15-20 per cent calcium chlorid. The solution used should be free from methyl alcohol, since this leads to the formation, with formaldehyd, of methylal, which is absolutely without germicidal action. for a room of about 3,000 cubic feet Trillat advises the continuance of the gas flow for about an hour. The method is not uniformly reliable.

A method which has found much favor is that in which glycerin - usually in a concentration of 10 per cent-is added to formalin. According to Schlossmann ${ }^{69}$ the presence of glycerin hinders polymerization. An apparatus in which this mixture is conveniently utilized is that of Lentz. Formalin with 10 per cent glycerin is placed in the copper tank and heated by a burner. This apparatus has been favorably endorsed by the War Department of the United States.

The so-called Breslau method of generating formaldehyd depends upon the evaporation of formaldehyd from dilute solutions. v. Brunn $^{70}$ elaims that where formalin in 30 to 40 per cent strength is evaporated, water vapor is generated more rapidly than formaldehyd is liberated, and a concentration leading to polymerization

* Trillat. Compt. rend. de l'acad. des se., 1896.

'Schlossmann, Münch. med. Woch., 45, 1898

${ }^{70} v$. Brunn, Zeit. f. Hyg., xxx, 1899. 
occurs. If, however, dilution is earried out until the formaldehyd in the solution is not more than 8 per cent, the generation of water vapor and formaldehyd take place at about equal speed and no concentration occurs. Schlossmann ${ }^{6 s}$ furthermore claims that polymerization in the vaporized formaldehyd does not oceur if sufficient water vapor is present-a prineiple which may also contribute to the efficiency of the Breslau method.

In practice, the apparatus devised by v. Brunn (Fig. 13) consists of a strong copper kettle of about $34 \mathrm{~cm}$. diameter by $7.5 \mathrm{~cm}$. height. This is completely closed except for two openings in the slightly domed top, one of which is the exit vent, the other, laterally placed, is for purposes of filling and is closed by a serew stopper. The tank is filled with a solution of formalin of a strength of from 8 to 10 per cent (commercial formalin 1:4). The apparatus permits the evaporation of large quantities of fluid in a short time (3 liters in one hour). When the lamp is left in a closed room care should be taken to fill it with a quantity of alcohol proportionate to the amount of fluid to be evaporated. This, according to v. Brunn, is about one-quarter of the volume of formalin solution used. By using 1.5 liters of 8 per cent formalin for each 1,000 cubic feet of space, this apparatus is said to yield a concentration of formaldehyd of about 25 grams to the eubic meter.

To do away with the use of liquid, a method has been devised which depends on principle upon the breaking up by heat of the solid polymer of formaldehyd (trixymethylene).

The apparatus (trade name, "Şchering's Paraform Lamp") as described by Aronson ${ }^{71}$ consists of a cylindrical mantle of sheet-iron placed upon a stand and supplied below with an alcohol lamp. Set into the top of the mantle is a small chamber, into which 1 gram tablets of trioxymethylene are placed. The alcohol lamp, so placed that the wicks project but slightlyto avoid overheating - is lighted, and the formalin generated passes out through slits in the upper case, mingling with the water vapor and other gases liberated by the alcohol flame. The more modern devices have waterboiler attachments to insure sufficient moisture. Two tablets are sufficient for the fumigation of about thirty-five cubic feet, and 2 c.c. of alcohol are filled into the lamp for each tablet. One hundred to one hundred and fifty tablets are usually enough for the ordinary room.

A simple method of generating formaldehyd is that which is known as the "lime method." In a wide shallow pan 40 ,per cent

${ }^{71}$ Aronson, Zeit. f. Hyg., xxv, 1897. 
formaldehyd solution(commercial formalin) is poured over quicklime $(\mathrm{CaO})$. The previous addition of concentrated sulphuric acid to the formalin, in proportions of one to ten, increases the speed of formalin liberation, and aids in limiting polymerization. One and one-half to two pounds (one-half to one kilogram) of quick-lime are used for every 500 c.c. of the formalin solution. The heat generated in the slaking of the lime produces volatilization of the formalin.

A modification of this method is that of Schering ${ }^{72}$ in which tablets of paraform and unslaked lime are together laid into a pan and warm water is poured over them.

A very simple method is the potassium permanganate method of Evans and Russell. ${ }^{73}$ This method depends upon the active reaction occurring when formalin and potassium permanganate are mixed. Permanganate is placed into a bucket and the formalin poured over it. The bucket should be large enough to prevent overflowing when the mixture foams. Special galvanized iron pails are made with funnel-shaped tops, and pails should be placed on a piece of iron sheeting or bricks to prevent overheating of the floor; 500 c.c. of commercial formalin and 250 grams of potassium per manganate should be used for every $1,000 \mathrm{cu}$. ft.

The room in which formaldehyd has been liberated is kept sealed, in the manner already described, for at least twelve hours, after which the windows and doors are opened. The odor which remains after formaldehyd fumigation may be removed by sprinkling with ammonia, or by the use of anyone of the various sorts of apparatus devised for the liberation of ammonia.

For the destruction of rodents, hydrocyanic acid gas is used in. the fumigation of ships and houses. This is of especial importance in controlling such diseases as plague. Recently Creel, Faget, and Wrightson, of the United States Public Health Service, have studied this method. They found hydrocyanic acid gas is more penetrating, more toxic, and more easily applied than either sulphur dioxid or carbon monoxid. $5 \mathrm{oz}$. of powdered potassium eyanid per 1,000 eu. ft. of space were sufficient to kill rodents. The gas is produced by dropping the potassium cyanid into sulphuric acid of a specific gravity of 1.84 , or commercial grade $66 \mathrm{~B}$. The gas is as effective

${ }^{72}$ Schering, Hyg. Rundschau, 1900.

${ }^{73}$ Evans and Russell, Rep. State Bd. Health, Maine, 1904. 
for insects as it is for rodents. The element of danger to human life must be always considered in carrying out such fumigation, but the writers referred to believe that there is no danger to men entering a place fumigated in this way 30 minutes or longer after the apertures have been opened. Subsequent experience in the fumigation of vessels has shown that longer periods of ventilation are necessary, depending on the conditions of moisture and air currents. In spite of rigorous precautions, loss of life has occasionally oceurred when cyanide is used as a routine procedure. For the fumigation of the hold of a ship, Creel, Faget, and Wrightson ${ }^{74}$ use an ordinary wooden barrel, into the top of which is placed a large galvanized iron funnel. The cyanide, in 5-gal. tins with top removed, may be attached to the funnel and dumped into the sulphuric acid by means of a rope attached to the tin, after the barrel has been lowered.

${ }^{74}$ Creel, Faget, and Wrightson, United States Public Health Reports, Vol. 30, No. 39, Dec. 3, 1915. 


\section{CHAPTER VI}

\section{METHODS USED IN THE MICROSCOPIC STUDY AND STAINING OF BACTERIA}

\section{MICROSCOPIC STUDY OF BACTERIA}

BACTERIA may be studied microscopically, in the living and unstained state, and, after the application of dyes, in colored preparations. For the manipulation of bacteria for such study, glass slides and coverslips of various design are used. These must be perfectly clean if the preparations are to be of any value. ${ }^{1}$

The Study of Bacteria in the Living State._Living bacteria may be studied in what is spoken of as the "hanging-drop" preparation. For this purpose a so-called hollow slide is employed, in the center of which there is a circular concavity about three-quarters of a centimeter to one centimeter in diameter. The preparation is manipulated as follows: if the bacteria are growing in a fluid medium, a drop of the culture fluid is transferred to the center of a cover-slip. If taken from solid media, an emulsion may be made in broth or in physiological salt solution, and a drop of this transferred to the cover-slip, or the bacteria may be emulsified in a drop of salt solution, or broth, directly upon the cover-slip. The concavity on the slide, having first been rimmed with vaseline, by means of a small camel's-hair brush, the cover-slip is inverted over

${ }^{1}$ Although the silicates of which glass is composed are extremely stable, nevertheless alkaline silicates which are said to separate out on the surface, together with grease and dirt left upon the glass by handling, during blowing and cutting, necessitate cleansing before use. This may be accomplished by a variety of methods. A simple one suitable for general application is as follows: (1) The slides and coverslips are thrown singly into boiling water and left there for half an hour; (2) wash in twenty-five per cent sulphuric acid; (3) rinse in distilled water; (4) wash in alcohol; (5) wipe with a clean cloth and keep dry under a bell-jar. Another methor convenient for rontine use is to immerse, after thorough washing in soap-suds and acid, in ninety-five per cent aleohol and to leave in this until the time of use. 
the slide in such a way that the drop hangs freely within the hollow space. The preparation is then ready for examination under the microscope.

Another method, known as the "hanging block method," devised by Hill, ${ }^{2}$ for the study of living bacteria in solid media is carried out as follows : nutrient agar is poured into a Petri dish and allowed to solidify. Out of this layer a piece about a quarter of an inch square is cut. This is placed on a sterile slide. The upper surface of the agar block is then inoculated with bacteria by surface smearing, and the preparation covered with a sterile dish and allowed to dry for a few minutes in the incubator. A sterile cover-slip is then dropped upon the surface of the block and sealed about the edges with agar. Block and cover-slip are then taken from the slide and fastened over a moist chamber with paraffin. The entire preparation can be placed upon the stage of a microscope. This method is especially designed for the study of cell-division.

Fig. 9.-Hanging-Drop Preparation.

Living bacteria may also be studied in stained preparations by the so-called "intravital" method of Nakanishi. Thoroughly cleaned slides are covered with a saturated aqueous solution of methylene-blue. This is spread over the slide in an even film and allowed to dry. After drying the slide should appear of a transparent sky-blue color. The microorganisms which are to be examined are then emulsified in water, or are taken from a fluid medium and placed upon a cover-slip. This is dropped, face downward, upon the blue ground of the slide. In this way bacteria may be stained without being subjected to the often destructive processes of heat or chemical fixation. According to Nakanishi, cytoplasm is stained blue, while nuclear material assumes a reddish or purplish hue.

The Study of Bacteria in Fixed Preparations.-Stained preparations of bacteria are best prepared upon cover-slips, the process consisting of the following steps: (1) Spreading on cover-slip; (2) drying in air; (3) fixing; (4) staining; (5) washing in water; (6) blotting; (7) mounting.

${ }^{2}$ Hill, Jour. of Med. Research, vii, 1902. 
(1) Smearing.-Bacteria from a fluid medium are transferred in a small drop of the fluid, with a platinum loop, to a cover-slip and carefully spread over the surface in a thin film. If taken from a solid medium, a small drop of sterile water is first placed upon the cover-slip and the bacteria are then in very small quantity carefully emulsified in this drop with the platinum needle or loop and spread in an extremely thin film.

(2) The film is allowed to dry in the air.

(3) When thoroughly dried, fixation is carried out by passing the preparation, film side up, three times through a Bunsen flame, at about the rate of a pendulum swing. Fixation by heat in this manner is most convenient for routine work, but is not the most delicate method, inasmuch as the degree of heat applied can not be accurately controlled. The other methods which may be employed are immersion in methyl alcohol, formalin, saturated aqueous bichloride of mercury, Zenker's fluid, or acetic acid. If ehemical fixatives are used, they must be removed by washing in water before the stain is applied. If a preparation is made upon a slide instead of a cover-slip, passage through the flame should be repeated eight or nine times.

(4) Staining.-The dyes used for the staining of bacteria are, for the greater part, basic anilin dyes, such as methylene-blue, gentian-violet, and fuchsin. These may be applied for simple staining in 5 per cent aqueous solutions made up from filtered saturated alcoholic solutions, or directly by weight. They are conveniently kept in the laboratory as saturated alcoholic solutions. The strengths of some saturated solutions are as follows: ${ }^{3}$

Fuchsin (aqueous), 1.5 per cent.

Fuchsin (alcohol 96 per cent), 3 per cent.

Gentian-violet (aqueous), 1.5 per cent.

Gentian-violet (alcohol 96 per cent), 4.8 per cent.

Methylene-blue (aqueous), 6.7 per cent.

Methylene-blue (alcohol 96 per eent), 7 per cent.

The staining solution, in simple routine staining, is left upon the fixed bacterial film for from one-half to one and one-half minutes according to the efficiency of the stain used. Methylene-blue is the weakest of the three stains mentioned; gentian-violet the strongest.

(5) The excess stain is removed by washing with water.

'After Wood, “Chemical and Microseopical Diagnosis," Appendix. N. Y., 1909. 
(6) The preparation is thoroughly dried by a blotter or between layers of absorbent paper.

(7) A small drop of Canada balsam is placed upon the film side of the dry cover-slip, which is then inverted upon a slide. The preparation is now ready for microseopical examination.

The chemical prineiples which underlie the staining process are still more or less in doubt. ${ }^{4}$ Suffice it to say here that most of the dyes in common use by bacteriologists and pathologists are coal-tar derivatives belonging to the aromatie series, all of them containing at least one "benzolring" combined with what Michaelis terms a "chromophore group," chief among which are the nitro-group $\left(\mathrm{NO}_{2}\right)$, the nitroso-group $(\mathrm{NO})$, and the azo-group $(\mathrm{N}=\mathrm{N})$. Just what the actual process of staining consists in is a question about which various opinions are held, some believing that the phenomenon is purely chemical, in which a salt is formed by the combination of the dye and the protoplasm of the cells, others that there is no such salt formation, and that the process takes place by purely physical means. To support the latter view it is argued that certain substances like cellulose are stainable without possessing the property of salt formation, and that staining may often be accomplished without there being a chemical disruption of the dye itself. Michaelis sums up his views by stating that probably both processes actually take place. A dye stuff, as a whole, may enter into and be deposited upon a tissue or cell by a process which he speaks of as "insorption." In such a case the coloring matter may be subsequently extracted by any chemically indifferent solvent. On the other hand, a dye after being thus deposited upon or within a cell, may become chemically united to the protoplasm by the formation of a salt, and in such a case the eolor ean be removed only by agents which are capable of decomposing salts, such as free acids.

The staining power of any solution may be intcnsified either by heating while staining, by prolonging the staining process, or by the addition of alkalies, acids, anilin oil, and other substances which. will be mentioned in the detailed deseriptions of special staining methods.

${ }^{4}$ For comprehensive reviews of the subject, the reader is referred to dissertations such as those of Mann ("Physiol. Hist. Methods and Theory," Oxford, 1902) and of Michaelis ("Einführung in die Farbstoffehemie,", etc., Berlin, 1902). 
In addition to the 5 per cent aqueous solutions of the saturated alcoholic solutions of the dyes mentioned above, a few extremely useful stains for routine work are as follows:

\section{Loeffler's Alkalin Methylene-Blue:}

Saturated alcoholic methylene-blue................. 30 c.c.

1 to 10,000 Potassium hydrate in water................ 100 c.c.

\section{Carbol-Fuchsin: ${ }^{5}$}

Basic fuchsin ............................ 1 gram

Alcohol, absolute ........................... 10 e.c.

$5 \%$ Aqueous earbolic acid.................... 90 c.c.

To make up this stain mix 90 c.c. of 5 per cent aqueous solution of carbolic acid with 10 e.e. saturated alcoholic basic fuchsin.

It can also be made up by weighing out:

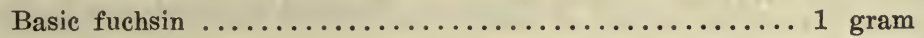

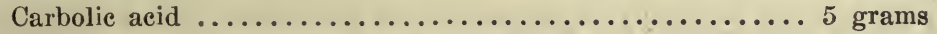

Dissolve in distilled water, 90 e.c. filter and add absolute alcohol, 10 e.e.

Toluidin-Blue Solution.-A very useful stain for general bacteriological work and for diphtheria bacilli.

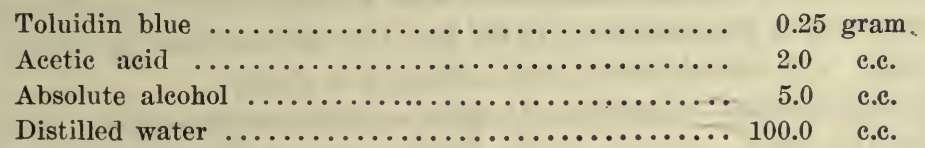

This ean be used alone when it gives a stain like Loeffler's methylene blue, but more clear, or else a counterstain of Bismarck brown ean be used.

Pappenheim-Saathof Methylgreen:

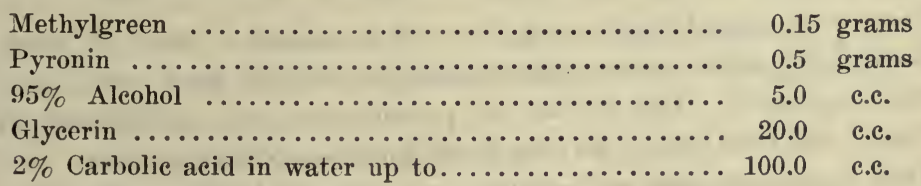

Stain 1 to 2 minutes, wash, blot. This is a splendid method of staining bacteria in general and it is particularly useful for the staining of phagocytes containing bacteria, as in gonococeus smears and in opsonic work.

saathof, Deut. med. woch., 1905. 


\section{SPECIAL STAINING METHODS}

Spore Stains.-Аввотт's Mетнор. ${ }^{6}$ - Cover-slips are smeared and fixed by heat in the usual manner.

Cover with Loeffler's alkaline methylene-blue and heat the stain until it boils, repeat the heating at intervals but do not boil continuously. Keep this up for one minute.

Rinse in water.

Decolorize with a mixture of alcohol eighty per cent 98 e.c. and nitric acid 2 c.c.; until all blue has disappeared.

Rinse in water.

Dip from three to five seconds in saturated alcoholic solution of eosin 10 e.c., and water 90 e.c.

Rinse in water, blot, and mount in balsam.

By this method the spores are stained blue, the bodies of the bacilli are stained pink.

MOELLER's Method. ${ }^{7}$ - Cover-slips are prepared as usual and fixed in the flame.

Wash in eholoroform for two minutes.

Wash in water.

Cover with five per cent chromic acid one-half to two minutes.

Wash in water. Invert and float cover-slip on carbol-fuchsin solution in a small porcelain dish and heat gently with a flame until it steams; continue this for three to five minutes. (This step can also be done by covering the cover-glass with earbol-fuchsin and holding over flame.)

Decolorize with five per cent sulphuric acid five to ten seconds.

Wash in water.

Stain with aqueous methylene-blue one-half to one minute. By this method spores will be stained red, the body blue.

Capsule Stains.-WELCH's METHOD. ${ }^{8}$ - Cover-slips are prepared as usual but dried without heat.

Cover with glacial acetic acid for a few seconds. Pour off acetic acid and cover with anilin water gentian-violet, renewing stain repeatedly until all acid is removed. This is done by pouring the stain on and off three or four times and then finally leaving it on for about three minutes.

Wash in two per cent salt solution and examine in this solution.

Hiss' Metrods. ${ }^{9}$ - (1) Copper Sulphate Method.-Cover-slip preparations

'Abbott, "Prin. of Bact.," Phila., 1905.

'Moeller, Cent. f. Bakt., I, x, 1891.

${ }^{8}$ Welch, Johns Hopkins Hosp. Bull., 1892, iii, 81.

'Hiss, Cent. f. Bakt., xxxi, 1902; Jour. Exper. Med., vi, 1905. 
are made by smearing the organisms in a drop of animal serum, preferably beef-blood serum.

Dry in air and fix by heat.

Stain for a few seconds with-

Saturated alcoholic solution of fuchsin or gentian-violet 5 c.e., in distilled water 95 c.e.

The cover-slip is flooded with the dye and the preparation held for a second over a free flame until it steams.

Wash off dye with twenty per cent aqueous copper sulphate solution.

Blot (do not wash).

Dry and mount.

By this method permanent preparations are obtained, the capsule appearing as a faint blue halo around a dark purple cell body.

Huntoon's Capsule Stain (applicable only to cultures, not to animal exudates).- - This depends upon the precipitating action of lactic acid on nutrose. Requires two solutions.

1. Diluent. -3 per cent solution of nutrose in distilled water; place in Arnold one hour, add a small amount of carbolic as preservative, and allow to settle.

2. Stain and fixative. -2 per cent earbolic, 100 e.e.; concentrated lactic acid, 0.5 c.c.; 1 per cent acetic acid, 1 c.c.; saturated alcoholic solution basic fuchsin, 1 e.c.; earbol fuchsin, 1 e.c.

As to the dye employed, most anything but methylene blue or Bismarck brown may be used. Methyl violet gives the most beautiful results but is not permanent and will not photograph. I have found the above mixture the best for classroom work.

Make a thin film, employing solution 1 as diluent. Dry in air. Do not fix. Cover with stain 30 seconds. Wash in water, dry, and examine.

Buerger's METHOD. ${ }^{10}$ - Cover-slip preparations are made by smearing in serum as in Hiss' method.

As the edges of the smear begin to dry, pour over it Zenker's fluid (without acetic acid) and warm in flame for three seconds.

(Zenker's fluid is composed of potassium bichromate $2.5 \mathrm{gm}$., sodium sulphate 1 gm., water 100 c.c., saturated with bichloride of mercury.)

Wash in water.

Flush with ninety-five per cent alcohol.

Cover with tincture of iodin, U. S. P., one to three minutes.

Wash with ninety-five per cent alcohol.

Dry in the air.

Stain with anilin water gentian-violet two to five seconds.

Wash with two per cent salt solutiori.

Mount and examine in salt solution.

${ }^{10}$ Buerger, Med. News, Dec., 1904. 
WADSWORTH'S METHOD. ${ }^{11}$-Wadsworth has devised a method of staining capsules which depends upon the fixation of smears with formalin. After such fixation capsules may be demonstrated both with simple stains and by Gram's method. The technique is as follows:

Smear preparations, made as usual, are treated as follows:

1. Formalin, 40 per eent, two to five minutes.

2. Wash in water, five seconds.

\section{Simple Stain.}

3. Ten per cent aqueuos gentian-violet.

4. Wash water, five seconds.

5. Dry, mount in balsam.
Differential Stain (Gram's Method).

3. Anilin gentian-violet, two minutes.

4. Iodin 'solution, two minutes.

5. Alcohol, 95 per cent, decolorize.

6. Fuchsin, dilute aqueous solution.

7. Wash water, two seconds.

8. Dry, mount in balsam.

It is important that the formalin be fresh and the exposure to water momentary. When decolorizing in the Gram method, strong alcohol only should be used. Wadsworth also found that encapsulated pneumococei could be demonstrated in celloidin sections of pneumonic lesions hardened in strong formalin. The lungs should be distended with the formalin or the lesions cut in very thin bits, hardened, dehydrated, embedded, and cut in the usual way. The celloidin sections may be fixed on the slides by partially dissolving the celloidin in alcohol and ether and setting the celloidin quickly in water before staining. Failure to obtain pneumococei encapsulated in such sections is usually due to improper or inadequate fixation in the formalin.

The differential method employed by Wadsworth for tissue staining is as follows:

1. Fix in formalin forty per cent, two to five minutes.

2. Wash in water.

3. Anilin gentian-violet, two minutes.

4. Iodin solution, two minutes.

5. Alcohol, ninety-five per cent, decolorize.

6. Eosin alcohol, counterstain.

7. Clear in oil of origanum.

8. Mount in balsam.

Flagella Stains.-All flagella stains, in order to be successful, necessitate particularly clean cover-slip preparations, best made from young agar cultures emulsified in sterile salt solution. Serupulous care should be exercised in cleaning the glassware used.

${ }^{11}$ Wadsworth, Jour. Inf. Dis., 1906. 
LOEFFler's METHOD. ${ }^{12}$-The preparation is dried in the air and fixed by heat. It is then treated with the following mordant solution:

Twenty per cent aqueous tannic acid............. 10 parts.

Ferrous sulphate aq. sol. saturated at room temperature... 5 parts.

Saturated alcoholic fuchsin solution............... 1 part.

This solution which should be freshly filtered before using, is poured over the eover-glass and allowed to remain there for one-half to one minute, during which time it should be gently heated, but not allowed to boil.

Wash thoroughly in water.

Stain with five per cent anilin water fuchsin or anilin water gentian-violet made slightly alkaline by the addition of one-tenth per cent sodium hydrate.

The stain should be filtered directly upon the cover-slip. Warm gently and leave on for one to two minutes. Wash in water. Mount in balsam.

VAN ERMengem's Method. ${ }^{13}$ - This method requires the preparation of three solutions.

(1) Twenty per cent tannic acid solution.........60 c.e.

Two per cent osmic acid solution............... 30 c.e.

Glacial acetic acid.................. drops

The cover-slip carrying the fixed preparation is placed in this solution for one hour at room temperature, or for five minutes at $100^{\circ} \mathrm{C}$. (boiling).

Wash in water.

Wash in absolute alcohol.

Immerse the cover-slip for one to three seconds in

(2) Silver nitrate, $0.25-0.5$ per cent solution.

Without washing, transfer to

(3) Gallic acid....................... $5 \mathrm{gm}$.

Tannic acid........................ 3 "

Fused potassium acetate................ 10 ،

Distilled water................................

Immerse in this for a few minutes, moving the cover-slip about.

Return to the silver nitrate solution until the preparation turns black.

Wash thoroughly in water.

Blot and mount.

Smitu's Modification of Pitfield's Metrod. ${ }^{14}$-A saturated solution of bichlorid of mercury is boiled and is poured while still hot into a bottle in which crystals of ammonia alum have been placed in quantity more than

${ }^{12}$ Loeffler, Cent. f. Bakt., I, vi, 1889.

${ }^{13}$ Van Ermengem, Cent. f. Bakt., I, xv, 1894.

${ }^{14}$ Smith, Brit. Med. Jour., I, 1901, p. 205. 
sufficient to saturate the fluid. The bottle is then shaken and allowed to cool. Ten c.c. of this solution are added to 10 e.c. of freshly prepared ten per cent tannic acid solution. - To this add 5 e.c. carbol-fuchsin solution. Mix and filter.

To stain, filter the above mordant directly upon the fixed cover-slip preparation. Heat gently for three minutes, but do not allow to boil. Wash in water and stain with the following solution:

Saturated alcoholic solution gentian violet............. 1 c.c.

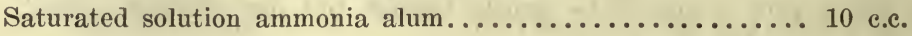

Filter the stain directly upon the preparation and heat for three or four minutes. Wash in water, dry, and mount in balsam.

Differential Stains.-GraM's METHOD. ${ }^{15}$ - By this method of staining, which is extremely important in bacterial differentiation, bacteria are divided into those which retain the initial stain and those which are subsequently decolorized and take the counterstain. The former are often spoken of as the Gram-positive, the latter as Gram-negative bacteria.

The reasons for the differential value of Gram's method are not entirely clear, but must, of course, depend upon peculiarities of the chemical constituents of the bacteria themselves. A considerable amount of work has been done on the subject which has not been entirely conclusive. A discussion of the subject may be found in Wells' "Chemical Pathology," Second Edition, page 105. Wells states that the results of Gram's method may be ascribed to the formation of an iodin-pararosanilin-protein compound in the Grampositive bacteria. Only dyes of this group, namely, gentian violet, methyl violet, etc., will form such combination. He quotes Bürgers ${ }^{16}$ as stating that trypsin will digest Gram-negative, but not Grampositive bacteria, and the gastric juice affects only a few of the Gram-positive species. It has also been suggested that the fatty substances in the bacterial bodies may have some relationship to the Gram-stain, and that the bacterial cell wall is the part most directly involved in the staining reaction. Benians ${ }^{17}$ has found that when Gram-positive bacteria are artificially disintegrated they lose their characteristic reaction and become Gram-negative, and this has been confirmed for tubercle bacilli in Wells' laboratory by

${ }^{15}$ Gram, Fortschr. d. Med., ii, 1884.

${ }^{16}$ Burgers and collaborators, Zeit. f. Hyg., 1911, $70^{\circ}$.

${ }^{17}$ Benians, Jour. of Pathol. and Bacter., 17, 1912, 199. 
Sherman. ${ }^{18}$ Wells' suggestion is that the iodin may render the cell membrane impermeable to alcohol.

Preparations are made on cover-slips or slides in the usual way.

The preparation is then covered with an anilin gentian-violet solution which is best made up freshly before use.

The staining fluid is made up, according to Gram's original directions, ${ }^{19}$ as follows:

Five c.e. of anilin oil are shaken up thoroughly with 125 c.c. of distilled water. This solution is then filtered through a moist filter paper.

To 108 c.c. of this anilin water, add 12 c.e. of a saturated alcoholic solution of gentian-violet. The stain acts best when twelve to twenty-four hours old, but may be used at once. It lasts, if well stoppered, for three to five days. A more convenient and simple method of making up the stain is as follows:

To 10 e.c. of distilled water in a test tube add anilin oil until on shaking the emulsion is opaque; roughly, one to ten. Filter this through a wet paper until the filtrate is clear. To this add saturated alcoholic solution of gentianviolet until the mixture is no longer transparent, and a metallie film on the surface indicates saturation. One part of alcoholic saturated gentianviolet to nine parts of the anilin water will give this result. This mixture may be used immediately and lasts two to five days if kept in a stoppered bottle.

Cover the preparation with this; leave on for 5 minutes. Pour off excess stain and cover with Gram's iodin solution for 2 to 3 minutes.

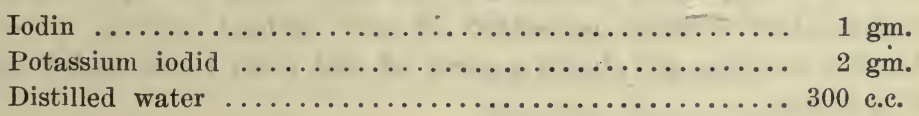

Decolorize with ninety-seven per cent alcohol until no further traces of the stain can be washed out of the preparation. This takes usually thirty seconds to two minutes, according to thinness of preparation.

Wash in water.

Counterstain with an aqueous contrast stain, preferably Bismarek brown, ${ }^{20}$ dilute fuchsin or safranin.

Paltauf's Modification. of Gram's Stain. ${ }^{21}$ - The staining fluid as prepared by this modification possesses the advantage of retaining

${ }^{18}$ Sherman, Jour. of Infec. Dis., 12, 1913, 249.

${ }^{10}$ Gram, loc. eit.

${ }^{20}$ To make up Bismarek brown solution, prepare a saturated aqueous solution of the powdered dye by heating. Cool and filter. Dilute 1 to 10 with distilled water.

${ }^{21}$ Sharnosky, Proc. N. Y. Pathol. Soc., Oct., 1909, n. s., ix, 5. 
its staining power for a longer period than does the anilin-water gentian-violet described in the original method.

The staining fluid is prepared as follows:

$$
\begin{gathered}
3-5 \text { c.c. anilin oil are added to } \\
90 \text { c.c. distilled water and } \\
7 \text { c.c. absolute alcohol. }
\end{gathered}
$$

This mixture is thoroughly shaken and filtered through a moist filter paper until clear. Then add:

Gruebler's gentian-violet $2 \mathrm{gm}$.

The fluid should stand twenty-four hours, during which a precipitate forms. This is filtered before use.

This gentian-violet solution retains its staining power for from 4 to 6 weeks. It is good only when a metallic luster develops on the surface.

It is used in the following way: Spreads on cover-slips or slides are dried and fixed as usual.

Then apply:

Anilin-water gentian-violet (as above), three minutes.

Gram's iodin solution, two minutes.

Absolute alcohol (with stirring), thirty seconds.

Counterstain, without washing in water, in aqueous fuchsin or in weak carbol-fuchsin.

Jensen's Modification of the Gram Stain.22-Jensen prepares his smears in the ordinary way. He uses a 5 per cent solution of methyl violet, "6B." This solution is stable. It is poured on the fixed smear for $1 / 4$ to $1 / 2$ minute. The methyl violet solution is poured off and, without washing, is eovered with Lugol's solution, and this is poured off and fresh Lugol added and left on for $1 / 2$ minute.

The iodine solution is now poured off and the preparation washed with $98 \%$ alcohol. When decolorized, the $98 \%$ alcohol is washed off with a few drops of absolute alcohol, and the preparation counterstained with a solution of neutral red consisting of 1 gram of neutral red in a liter of water to which 2 c.c. glacial acetic acid has been added.

Apparently the important point in Jensen's stain is that the preparation is never washed with water, and that the final washing off of the $98^{\circ}$ alcohol is done with a few drops of absolute.

We have not used this stain as a routine and do not know how reliable it is.

Sterling's Modification of Gram's Method.-2 e.c. anilin oil + 10 e.e. $95 \%$ alcohol. Shake and add 88 c.e. distilled water. 5 grams

${ }^{22}$ Jensen, Berl. klin. Woch., 49, 1912, 1163. 
gentian violet are ground in a mortar and the anilin solution added slowly while grinding. Filter. This solution keeps, and stains in one-half to one minute. ${ }^{23}$

Classification of the More Important Pathogenic Bacteria According to Gram's Stain.

Gram-positive.

(Retain the Gentian-violet.)

Micrococcus pyogenes aureus

Micrococcus pyogenes albus

Streptococcus pyogenes

Micrococcus tetragenus

Pneumococcus

Bacillus subtilis

Bacillus anthracis

Bacillus diphtheriæ

Bacillus tetanus

Bacillus tuberculosis and other acid-fast bacilli

Bacillus aërogenes capsulatus

Bacillus botulinus
Gram-negative:

(Take Counterstain.)

Meningococcus

Gonococeus

Micrococcus catarrhalis

Bacillus coli

Bacillus dysenteriæ

Bacillus typhosus

Bacillus paratyphosus

Bacillus fecalis alkaligenes

Bacillus enteritidis

Bacillus proteus (proteus)

Bacillus mallei

Bacillus pyocyaneus

Bacillus influenzæ

Bacillus mucosus capsulatus

Bacillus pestis

Bacillus maligni œdematis

Spirillum choleræ

Bacillus Koch-Weeks

Bacillus Morax-Axenfeld

Stains for Acid-Fast Bacteria.-These methods of staining are chiefly useful in the demonstration of tubercle bacilli. These bacteria because of their waxy cell membranes are not easily stained by any but the most intensified dyes, but when once stained, retain the color in spite of energetic decolorization with acid. For this reason they are known as acid-fast bacilli. The first method devised for the staining of tubercle and allied bacilli was that of Ehrlich.

${ }^{23}$ This is the routine method employed in our laboratory at present. In using Sterling's stain the time of staining can be abbreviated as follows:

Sterling's Gentian Violet.......... one minute

Iodin 
EHRLich Method. ${ }^{24}$ - This method is now rarely used. Cover-slip preparations are prepared as usual and fixed by heat.

Stain with anilin water gentian-violet, hot, three to five minutes, or twenty-four hours at room temperature.

Decolorize with thirty-three per cent nitric acid one-half to one minute.

Treat with sixty per cent alcohol, until no color can be seen to come off.

Counterstain with aqueous methylene-blue.

Rinse in water, dry, and mount.

Zienl-Neelson Method. ${ }^{25}$ - Thin smears are made upon coverslips or slides.

Fix by heat.

Stain in carbol-fuchsin solution as given on page 115. The slide or coverslip may be flooded with the stain, and this gently heated with the flame until it steams, or else the cover-slip may be inverted upon the surface of the staining fluid, in a porcelain dish or watch-glass, and this heated until it steams. This is continued for three to five minutes. Decolorize with either five per cent nitric acid, five per cent sulphuric acid, or one per cent hydrochloric acid for three to five seconds. The treatment with the acid is continued until subsequent washing with water will give only a faint pink color to the preparation.

Wash with ninety per cent alcohol until no further color can be removed. If, after prolonged washing with alcohol, a red color still remains in very thick places upon the smear, while the thin areas appear entirely decolorized, this may be disregarded.

Wash in water and counterstain in aqueous methylene-blue for one-half to one minute.

Rinse in water, dry, and mount.

By this method the tubercle bacilli are colored red, other bacteria and cellular elements which may be present are stained blue.

GabBet's Method. ${ }^{26}$-Gabbet has devised a rapid method in which the decolorization and counterstaining are accomplished by one solution. The specimen is prepared and stained with carbolfuchsin as in the preceding method. It is then immersed for one minute directly in the following solution:

Methylene-blue .......................... 2 gms.

Sulphuric acid 25 per cent (sp. gr. 1018)............ 100 c.c.

${ }^{24}$ Ehrlich, Deut. med. Woch., 1882.

${ }^{25}$ Ziehl, Deut. med. Woch., 1882; Neelson, Deut. med. Woch., 1883.

${ }^{28}$ Gabbet, Lancet, 1887. 
Then rinse in water, dry, and mount.

This method, while rapid and very convenient, is not so reliable as the Ziehl-Neelson method.

Pappenheim 's Method. ${ }^{27}$ - The method of Pappenheim is devised for the purpose of differentiating between the tubercle bacillus and the smegma bacillus. Confusion may occasionally arise between these two microorganisms, especially in the examination of urine where smegma bacilli are derived from the genitals, and less frequently in the examination of sputum where smegma bacilli may occasionally be mixed with the secretions of the pharynx and throat.

Preparations are smeared and fixed by heat in the usual way.

Stain with hot earbol-fuchsin solution for two minutes.

Pour off dye without washing and cover with the following mixture:

Corallin (rosolic acid) $\ldots \ldots \ldots \ldots \ldots \ldots \ldots \ldots \ldots \ldots \ldots \ldots .$.

Absolute alcohol ............................. 100 c.e.

Methylene-blue added to saturation

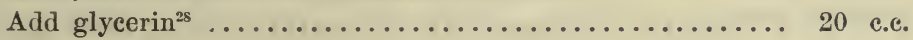

This mixture is poured on and drained off slowly, the procedure being repeated four or five times, and finally the preparation is washed in water. The combination of alcohol and rosolic acid decolorizes the smegma bacilli, but leaves the tubercle bacilli stained bright red.

BAumgarten's Method. ${ }^{29}$ - This method is recommended by the author for differentiation between the bacillus of tuberculosis and the bacillus of leprosy and depends upon the fact that the tubercle bacillus is less easily stained than Bacillus lepræ.

Smears are prepared and fixed by heat in the usual way.

Stain in dilute alcoholic fuchsin for five minutes.

Decolorize for twenty seconds in alcohol, ninety-five per cent, ten parts, nitric acid one part.

Wash in water.

Counterstain in methylene-blue.

Wash in water, dry, and mount.

${ }^{27}$ Pappenheim, Berl. klin. Woch.; 1898.

${ }^{28}$ The glycerin is alded after the other constituents have been mixer

${ }^{29}$ Baumgarten, Zeit. f. wissensch. Mikrosk., 1, 1884. 
The tubercle bacillus should be blue and the bacillus of leprosy red. Hermann's Stain for Acid Fast Bacteria: ${ }^{30}$

I. Crystal Violet $3 \%$ in alcohol.

II. Ammonium Carbonate $1 \%$ aqueous.

Mix one part of "I" with 3 parts of "II" just before using. Steam 3 minutes. Decolorize with $10 \%$ nitrie acid (or $5 \%$ sulphurie acid). Wash in alcohol, rinse and counterstain with Bismarck brown. The better contrast obtained with this stain is stated by Park to increase the ease with which tuberele bacilli can be found in sputum.

Special Stains for Polar Bodies.-These staining methods are designed to bring into view polar bodies as found, for instance, in the bacilli of diphtheria and plague.

Neisser's Method. ${ }^{31}$-Smear and fix in the usual manner.

Stain for two to five seconds in the following solution:

Methylene-blue .......................... $1 \mathrm{gm}$.

Absolute alcohol .......................... 20 c.c.

Glacial acetic acid.......................... 50 c.c.

Distilled water $\ldots \ldots \ldots \ldots \ldots \ldots \ldots \ldots \ldots \ldots \ldots \ldots, 1,000$ c.c.

Wash in water.

Counterstain in 0.4 per cent aqueous Bismarck brown solution for five seconds.

By this method polar bodies are stained blue, while the bacillary bodies are stained brown.

Carbol Thionin:

Saturated solution of thionin in $50 \%$ alcohol........... 10 c.c. $2 \%$ aqueous solutions of phenol................... 100 c.c. Stain one to three minutes.

See also Toluidin Blue solution given on page 115.

Polychrome Stains.-The various polychrome stains are of value to the bacteriologist chiefly for the staining of pus and exudates where the relation of bacteria to cellular elements is to be demonstrated. They are also extremely useful in the study of fixed specimens of protozoan parasites. There is a large number of these stains in use; a few only, however, can be given here. In principle,

${ }^{30}$ Quoted from Park and Williams, Pathogenic Microorganisms, Lea and Febiger, Phila., 1920.

"Neisser, Zeit, f. Hyg., xxiv, 1897. 
all these. stains depend upon a combination of eosin and methyleneblue, these elements staining not only as units, but acting together in combination. One and the same solution, therefore, contains at. least three elements which eolor the various struetures of the preparation selectively.

Jenner's Method. ${ }^{32}$ - This stain, because of its simplicity, is useful for routine use. It is made up as follows: Equal parts of eosin (Gruebler, "W. G.") one and two-tenths per cent aqueous solution, and methylene-blue (medicinal, Gruebler) one per cent aqueous solution, are mixed and allowed to stand for twenty-four hours. A coarse granular precipitate is formed which appears dark, with a metallic luster on its surface. This is separated by filtration and washed with distilled water until the filtrate appears almost clear.

To make up the stain 0.5 gram of the dry precipitate is dissolved in 100 c.e. of methyl aleohol.

In using the stain, preparations are not fixed, but simply dried in the air and immersed in the stain for one to two minutes. After this, wash in distilled water and examine.

Wright's Modification of Leishman's Method. ${ }^{33}$ - A one per. cent solution of methylene-blue (Gruebler) in five-tenths per cent solution of sodium bicarbonate in distilled water is steamed in a sterilizer at $100^{\circ} \mathrm{C}$. for one hour. After this has cooled, a one-tenth per cent aqueous solution of eosin (Gruebler, W. G.) is added until a metallic scum appears on the surface of the mixture. (About five parts of eosin solution to one of methylene-blue is necessary.) The precipitate which forms is collected by filtration, dried, and a saturated solution then made in methyl alcohol. This is filtered and diluted with one-quarter its bulk of methyl aleohol.

To stain, cover the dried preparation with the stain for one to one and one-half minutes. Dilute by dropping upon the stain distilled water from a pipette until a metallie film appears upon the top. Leave this on for three to fifteen minutes. Wash in distilled water.

GIEMSA's METHOD. ${ }^{34}$ - The method of Giemsa is really a modification of the Romanowsky method. It is widely applieable, being of great value in the staining of the Spirochæte pallida, Vincent's

${ }^{32}$ Jenner, Lancet, i, 1889.

${ }^{33}$ Wright, Jour. Med. Researeh, ii, 1902.

${ }^{34}$ Giemsa, Cent. f. Bakt., I, xxxvii, 1904. 
spirilla, protozoa, and Negri bodies. The stain has been modified several times by its originator, the following being the formula.given by him in 1904: The substance referred to as azur II and purchasable under that name, consists of pure methylenazur chloralhydrate combined with an equal quantity of methylene-blue chloralhydrate. The substance referred to as azur II-eosin is a combination of this substance with eosin.

The staining fluid is made up as follows : ${ }^{35}$

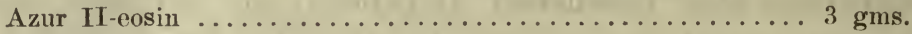

Azur II ............................. 3 gms.

This mixture is thoroughly dried over sulphuric acid in a desiccator, finely powdered, and rubbed through a fine sieve. It is then dissolved in 250 gms. of C. P. glyeerin (Merck), at $60^{\circ} \mathrm{C}$. To this is added methyl alcohol (Kahlbaum) 250 c.c., previously warmed to $60^{\circ} \mathrm{C}$. This mixture is well shaken and allowed to stand at room temperature for twenty-four hours. The mixture is now ready for use.

For use 10 c.c. of distilled water are poured into a test tube and one to two drops of a one per cent potassium carbonate solution are added. Ten drops of the staining solution described above (one drop to the c.c.) are mixed with this slightly alkaline water. The preparation which is to be stained is fixed in methyl alcohol, dried, and covered with the diluted staining solution. For the staining of protozoa and exudates containing bacteria, ten to fifteen minutes are sufficient. For the staining of Negri bodies or Spirochæte pallida, one or more hours of staining should be employed. After staining, wash in running tap water and blot.

Wood's MEтнор. ${ }^{36}$ - Wood has devised a simple staining method based on the principles of the Giemsa stain, in which azur II and eosin may be used in separate solutions. Preparations are fixed in strong methyl alcohol for five minutes and are then stained in a 0.1 per cent aqueous solution of eosin until the preparation is pink. The eosin is then poured off and the preparation is covered with a 0.25 per cent aqueous solution of azur II for one-half to two minutes. Following this, it is washed in tap water and dried by blotting.

${ }^{35}$ It is best not to attempt to make up the undiluted staining fluid, since this is purchasable under the nume of "Giemsa Lösung fïr Romanowsky Färbung."

${ }^{36}$ Wood, Med. News, 83, 1903. 
When an intense stain is desired, the solution of eosin and azur II may be flooded over the preparation together, using an excess of azur II. They are then left on from five to ten minutes. At the end of this time washing and drying as before complete the process.

The Staining of Bacteria in Tissues.-The preparation of tissue for bacterial staining is, in general, the same as that employed for purposes of cellular studies, in histology. For bacteriological studies the most useful are alcohol and Zenker's fluid. Other fixations, such as that by formalin, or Mueller's fluid, give less satisfaction. In other respects the details of dehydration and embedding are the same as those used in histological studies, except that it is desirable that the tissues should be handled rather more carefully than is necessary for ordinary pathological work, and the changes from the weaker to the stronger alcohols should be made less abruptly. ${ }^{37}$

Embedding in paraffin is preferable to celloidin, although the latter method is not unsuccessful if carefully carried out. The chief disadvantages of celloidin are the retention of color by the celloidin itself and the consequent unclearness of differentiation. It is also easier to cut thin sections from paraffin blocks than from those prepared with celloidin.

When staining tissue sections for bacteria, it is most convenient to carry out the process with the section attached to a slide. For celloidin sections this may be accomplished by means of ether vapor. For paraffin sections it is necessary to cover the slide with an extremely thin layer of a filtered mixture of equal quantities of egg albumin and glycerin, to which a small crystal of camphor or a drop or two of carbolic acid has been added. The sections are then floated upon a slide so prepared, and set away in the thermostat for four or five hours.

LOEFFLER's METHOD. ${ }^{38}$ - Stain in alcoholie methylene-blue solution five to fifteen minutes, or in Loeffler's alkaline methylene-blue solution one to twentyfour hours.

Wash in one to one-thousand acetic acid solution for about ten seconds.

Treat with absolute alcohol by pouring the alcohol over the preparation for ten to twenty seconds.

Clear with xylol.

Mount in balsam.

${ }^{37}$ For details of such work reference should be had to the standard textbooks on pathological technique, notably the very excellent one of Mallory and Wright.

${ }^{38}$ Loeffler, Mitt. a. d. kais. Gesundheitsamt, ii, 1884. 
When celloidin sections are stained in this way ninety-five per cent alcohol should be substituted for the absolute. A number of other staining solutions may be used in the same way, aqueous fuchsin or aqueous gentian-violet yielding good result.

Nicolle advises the use of a ten per cent aqueous solution of tannic acid for a few seconds after washing with the acetic acid.

Sections may also be stained by placing them over night into a dilute Giemsa solution (one drop to each c.c. of distilled water). When so stained the sections must not be run through the weaker alcohols but must be rapidly differentiated in absolute alcohol.

Method of Staining Gram-Positive Bacteria in Tissue Sections.Celloidin Sections.-After fixing section to the slide by pressure with a filter paper or by ether vapor, cover with anilin-water gentian-violet five minutes.

Pour off excess of stain and cover with Gram's iodin solution for two minutes. Decolorize with ninety-five per cent alcohol until no more color comes out.

Stain quickly with eosin-alcohol (ninety-five per cent alcohol to which enough eosin has been added to give a transparent pink color; about 1:15). Clear in eoșin-oil of origanum (oil of origanum, 25 c.e. and eosin alcohol, as above, about 3 e.c.). Blot and mount in balsam.

Paraffin Sections.-Stain with anilin-water gentian-violet five to ten minutes.

Wash in water.

Cover with Gram's iodin solution one minute.

Wash in water.

Decolorize with absolute alcohol until no more color comes out.

Clear in xylol.

Mount in balsam.

Gram-Weigert Method. ${ }^{39}-($ For celloidin sections.) -Stain for one-half hour in the following freshly filtered solution:

Carmine ......................... $3-5$ grams.

Saturated aqueous solution of lithium earbonate...... 100 c.c.

Dehydrate in ninety-five per cent alcohol.

Stick section to slide witl ether vapor.

Stain in anilin-water gentian-violet for five to fifteen minutes (or in a saturated solution of aqueous erystal violet diluted with water one to ten, five to fifteen minutes).

Wash in physiological salt solution.

Cover with Gram's iodin solution one to two minutes.

Wash in water and blot.

${ }^{30}$ Weigert, Fortschr. d, Med., v, 1887. 
Decolorize with anilin oil until no more color comes off.

This both decolorizes and dehydrates.

Treat with xylol. Mount in balsam.

Method for Gram Positive and Gram Negative Organisms in 'Tissues, Recommended by Pappenheimer.

Fixation: Zenker preferred. Formalin or alcohol.

Paraffin sections, $5 \mathrm{mu}$ or less.

Staining:

1. Stirling's Gentian violet- 5 minutes.

2. Gram's iodin -1 minute.

3. Aniline oil-or aniline oil-xylol. Decolorize to pale violet.

4. $100 \%$ alc. Few seconds only.

5. Distilled water.

6. Aqueous saffranin. $1 / 2 \% 30$ seconds.

7. Distilled water.

8. Blot. $100 \%$ ale. Few seconds only.

9. Clear in xylol.

GRAM-WEIGERT METHOD (for paraffin sections).-Preferably Zenker's fixation:

1. Stain sections lightly in alum-hematoxylin.

2. Wash in running water.

3 . Four per cent aqueous solution soluble in water, five minutes to onehalf hour.

4. Wash in water.

5. Aniline methyl-violet, one-half to one hour.

6 . Wash off with water.

7. Lugol's solution, one to two minutes.

8. Wash off with water.

9. Blot with filter-paper and dehydrate and clear in several changes of aniline and xylol, equal parts, or in aniline oil alone.

10. Wash off with xylol.

11. Mount in xylol-colophonium.

Mallory's Eosin and Methylene-Blue Stain.-For paraffin sections after Zenker's fixation only:

1. Stain paraffin sections in a 5 per cent aqueous solution of eosin for twenty minutes or longer. Sometimes it is advisable to get a deeper eosin stain by placing the sections in the paraffin oven for fifteen to twenty minutes.

2. Wash in water to get rid of excess of eosin.

3. Stain in Unna's alkaline methylene-blue solution, diluted $1 / 4$ or 5 with water, for ten or fifteen minutes.

4. Wash in water.

5. Differentiate and dehydrate in a dish of 95 per eent alcohol, to which 
has been added a few drops of a 10 per cent solution of colophonium in absolute alcohol, keeping the section in constant motion, so that the decolorization shall be uniform. Control the result under the microscope. When the pink color has returned to the section and the nuclei are still a deep blue, finish the dehydration quickly with absolute alcohol.

6. Xylol.

7. Xylol balsam.

Method of Staining for Tubercle Bacilli in Sections..$^{40}$-Paraffin Sections. - Stain in carbol-fuchsin solution hot for five minutes (or better cold, for twenty-four hours).

Wash in water.

Decolorize and countrrstain in Gabbet's methylene-blue sulphuric acid mixture for one minute.

Wash in water.

Dehydrate in absolute alcohol.

Clear in xylol.

Mount in balsam.

Celloidin Sections. ${ }^{41}$-Stain lightly in alum he.natoxylin.

Wash in water.

Dehydrate in ninety-five per cent alcohol.

Attach the slide by ether vapor.

Stain with steaming earbol-fuchsin two to five minutes.

Wash in water.

Wash with Orth's acid alcohol (alcohol ninety per cent., 99 c.c.; conc. $\mathrm{HCl}, 1$ c.c.) one-half to one minute.

Wash in water several changes.

Treat with ninety-five per cent alcohol until red color is entirely gone.

Blot and cover with xylol until clear. Mount in balsam.

Method of Staining Actinomyces in Sections.-Mallory's Method. ${ }^{42}$ -1. Stain deeply in saturated aqueous eosin ten minutes.

2. Wash in water.

3. Anilin gentian-violet two to five minutes.

4. Wash in normal saline solution.

5. Weigert's iodin solution (iodin 1, KI 2, and water 100 parts) one minute.

6. Wash in water and blot.

7. Clear in anilin oil.

8. Xylol several changes.

9. Mount in balsam.

${ }^{10}$ Mallory and Wright, "Pathol. Tech.," p. 413.

${ }^{41}$ After Mallory and Wright.

${ }^{12}$ Mallory and Wright, "Pathol. Tech.," 1904. 


\section{CHAPTER VII}

THE PREPARATION OF CULTURE MEDIA

\section{GENERAL TECHNIQUE}

THE successful cultivation of bacteria upon artificial media requires the establishment of an environment which shall be suitable in regard to the presence of assimilable nutritive material, moisture, and osmotic relations. These requirements are fulfilled in the composition of the nutrient media described in another section, media which are to some extent varied according to the special requirements of the bacteria which are to be cultivated. If cultivation, furthermore, is to have any value for scientific study of individual species, it is necessary to obtain these species free from other varieties of microorganisms, that is, in pure culture, and to protect such cultures continuously from contamination with the other innumerable species which are everywhere present:

The technique which is employed for these purposes has been gradually evolved from the methods originally devised by Pasteur, Koch, Cohn, and others.

Bacterial cultivation is carried out in glassware of varied construction, the forms most commonly employed being test tubes of various sizes, Erlenmeyer flasks, the common Florence flasks, and Petri dishes. All glassware, of course, must be thoroughly cleansed before being used.

Preparation of Glassware.-The cleansing of glassware may be accomplished by any one of a number of methods. New glassware may be immersed in a one per cent solution of hydrochloric or nitric acid in order to remove the free alkali which is occasionally present on such glass. It is then transferred to a one per cent sodium hydrate solution for a few hours, and following this is washed in hot running water.

In the case of old glassware which has contained culture media, sterilization in the autoclave is first carried out, then the glassware is boiled in five per cent soda solution or in soapsuds. After this, 
thorough mechanical cleansing is practiced, and the glassware may be treated by acid and alkali followed by running water, as given above. These last steps, however, are not essential, thorough washing in hot water after the soapsuds or soda solution being usually sufficient to yield good results. Other workers have recommended immersion of the glassware after mechanical cleansing in five per cent to ten per cent potassium bichromate solution in twenty-five per cent sulphuric acid. This is followed by thorough washing in hot running water, and drying.

Clean flasks and test tubes are then stoppered with cotton, which has been found to be a convenient and efficient seal against the bacteria of the air, catching them in the meshes of the fibers as in a filter. The technique of the stoppering or plugging of glass receptacles is important, in that, when poorly plugged, sterility is not safeguarded, and the purpose of culture study is defeated.

In almost all laboratories in this country non-absorbent cotton or "cotton batting" is used for the plug. In a few of the German laboratories, the absorbent variety is employed. The disadvantages of the latter, espccially in the case of fluid media, are obvious. The plugs should fit snugly, but not so tightly that force is necessary to remove them. Care should be taken, furthermore, that no creases are left between the surface of the glass and the periphery of the plug; for these, if present, may serve as channels for the entrance of bacteria. The plugging itself is carried out by tearing a small piece of cotton, about $2 \times 2$ inches, from the roll, folding over one of its corners, and, applying the smooth end of a glass rod to the folded portion, gently pushing it into the mouth of the tube.

After plugging and before media are introduced into the tubes and flasks, these should be sterilized. This is best done in one of the "hot-air sterilizers," by exposing the tubes for one hour to a temperature of $150^{\circ} \mathrm{C}$. If greater speed is desired exposure to $180^{\circ}$ to $190^{\circ} \mathrm{C}$. for half an hour is usually safe. If by mistake, however, the temperature is allowed to rise above $200^{\circ} \mathrm{C}$., a browning of the cotton plugs occurs and the glassware is apt to be stained by the burning of the fat and other organic material derived from the cotton. Petri dishes after cleansing are fitted together, and then sterilized in the hot-air chamber at $150^{\circ} \mathrm{C}$. for one hour.

Glassware so prepared is ready for the reception of media.

Ingredients of Culture Media.- The food requirements of bacteria have been discussed in another section. From what has there 
been said, it is apparent that artificial culture media must, to a certain extent, be adjusted to the peculiarities of individual bacteria. In the cases of the more strictly parasitic microorganisms growth can be obtained only by the most rigid observance of special requirements. For the large majority of pathogenic bacteria, however, routine or standard media may be employed, which, while slightly more favorable for one species than for another, are sufficiently general in their composition to permit the growth of all but the most fastidious varieties.

The basis of many of our common media is formed by the soluble constituents of meat. These substances are best obtained by macerating 500 grams of lean beef in 1,000 c.c. of distilled water. The mixture is allowed to infuse in the ice chest over night, and then strained through cheese-cloth. To this infusion are added the other required constituents in the manner given in the detailed instructions below. The soluble constituents of meat, however, may also be procured in a simpler way by the use of the commercial meat extracts, such as that of Liebig. These extracts are dissolved in quantities of five grams to the liter, and other constituents are added to this nutrient basis.

Though simpler to make, the meat-extract media are less favorable for the cultivation of the more delicate organisms than are the media made directly from fresh meat. Nevertheless, they suffice for the cultivation of the large majority of the more saprophytic pathogenic microorganisms and hold an important place in laboratory technique.

The ingredients and methods used in various laboratories in the preparation of such standard media should be, as much as possible, uniform, in order that confusion in results may be avoided; for, as is well known, the biological characteristics of one and the same bacterial species may vary considerably if grown on media differing in their composition.

A committee of the American Public Health Association, ${ }^{1}$ appointed in 1897 for the sake of standardizing the methods of preparation of media, recommended that the following rules should govern the choice of ingredients :

1. Distilled water should be used in all cases.

(This is not neces-

${ }^{1}$ Rep. Com. of Amer. Bact, to Com. of Amer. Pub. Health Assn. Meeting, Philadelphia, Sept., 1897. 
sary for routine laboratory media if the constitution of the tap water is known and controlled from time to time.)

2. The meat used should be fresh, lean beef (when veal or chicken is substituted the change should be stated).

3. The pepton used should be Witte's pepton, dry, made from meat. ${ }^{2}$

4. Only C. P. NaCl should be used.

5. For alkalinizing C. P. sodium hydrate should be used in normal solutions.

6. For acidification C. P. hydrochloric acid in normal solution should be used.

7. When glyeerin is used, this should be of the neutral redistilled variety.

8. The agar-agar employed should be of the finest grade of commercial thread agar.

9. The gelatin should be the commercial sheet gelatin washed as free as possible of acid and impurities.

10. Chemicals and carbohydrates which are used should be as nearly chemically pure as possible.

Titration of Media.-Next in importance to the actual composition of media is the adjustment of their reaction. Bacteria are highly susceptible to varations in the acidity and alkalinity of media, excessive degrees of either may completely inhibit development or moderate varations may lead to marked modifications of cultural charácteristics. It is necessary, therefore, to adjust the reaction both for the sake of favoring growth and in order to insure uniformity of growth characters. This is accomplished by titration which is best carried out according to the recommendations of the committee mentioned above.

Older Method of Direct Titration.-The color iñdicator employed for the titration is a five-tenths per cent solution of phenolphthalein in fifty per cent alcohol. The chief advantage of this indicator over others is due to the fact that it indicates the presence of organic acid and acid compounds in its reaction. For actual titration $\frac{N}{20}\left(\frac{1}{20}\right.$ normal $)$ solutions of sodium hydrate or of hydrochloric acid are used. Since media in the process of preparation are usually acid, the $\mathrm{NaOH}$ solution is the one most frequently

${ }^{2}$ Since the war many domestic peptons have been introduced and ean be used with success. 
needed. Five c.c. of the medium to be tested is measured accurately in a carefully washed pipette and transferred into a porcelain evaporating dish. To this are added 45 e.c. of distilled water. The mixture is thoroughly boiled for three minutes over a free flame. The boiling drives off $\mathrm{CO}_{2}$, giving the true neutral point, and approximates the conditions prevailing during the further sterilization of the medium from which the 5 c.c. have been taken. After boiling, 1 c.e. of the phenolphthalein is added. If the medium is acid, no eolor is present; if alkaline, a pink or red color appears. The $\frac{\mathrm{N}}{2}$ alkali or acid solution is allowed to drop into the dish from a graduated burette. When the neutral point is approached in an acid solution, each drop of sodium hydrate added brings forth at first a deep red, which, however, upon slight stirring with a clean rod, completely disappears. ${ }^{3}$ The end reaction is reached when a faint but elear and distinct pink color remains in the fluid after' stirring.

When titrating alkaline media, the addition of the phenolphthalein produces a red color in the hot medium which gradually fades upon the addition of $\frac{\mathrm{N}}{10} \mathrm{HCl}$, becoming colorless at the end point of titration. Titration should be done quickly and in a hot solution. From the result of the titration the eomputation for the neutralization of the entire bulk of the medium can be made by a simple arithmetical process as illustrated in the following example:

Let us suppose that we have used:

2.5 c.e. of $\frac{\mathrm{N}}{2} \mathrm{NaOH}$ to neutralize

then 2.5 c.c. of $\frac{\mathrm{N}}{1} \mathrm{NaOH}$ will neutralize

and 25 c.c. of $\frac{\mathrm{N}}{1} \mathrm{NaOH}$ will neutralize 1,000 c.c., or one liter.

The adjustment of the reaction of media is largely determined by the particular uses for which the media are designed. For examinations in the practice of sanitation, such as analyses of water, ice, and milk, etc., the American Public Health Association recommends a standard reaction of +1 per cent (the plus sign is used to indicate acidity, the minus alkalinity; +1 per cent is the expression used to indicate that one per cent of $\frac{\mathrm{N}}{1}$ sodium hydrate solution would be required to neutralize the medium or 10 c.c. to the liter). For general work with pathogenic bacteria, the most favorable reaction for routine media is slight alkalinity, neutrality, or an acidity not exceeding. +1 per cent.

\footnotetext{
${ }^{3}$ See standard textbooks on volumetric analysis.
} 


\section{The Colorimetric Method of Titration}

The method given above is not an accurate one because, in the titration of culture media, we are dealing with solutions which contain considerable quantities of materials, such as peptone, proteins, phosphates, etc., which have an action which is spoken of technically as that of a "buffer." This term signifies the power of these substances to oppose changes in reaction. The degree of "buffer" action, as shown by a number of writers, but recently in connection with bacteriological work particularly by Clark and Lubs, ${ }^{4}$ is proportionate to the concentration of the constituents, and the consequence of the action is that volumetrically proportionate amounts of acid or alkali added to such solutions do not change the reaction in the same proportions. Curves found in the paper of Clark and Lubs eited above, will make this sufficiently clear. In consequence, when we titrate 5 cubic centimeters of the medium as in the old method, to the neutral point of phenolphthalein, we could bring the entire media to the neutral point of phenolphthalein by adding proportionate amounts of acid or alkali, but we cannot foretell the final hydrogen ion concentration attained in the medium by adding fractions of this total amount. It is plain, therefore, that to make an accurate adjustment of media it would be better to apply either an electrode method or a colorimetric method to the medium, adjusting to a standard of known hydrogen ion concentration. The potentiometer methods have been considerably simplified, and have been applied by men like Clark and Lubs and others who are thoroughly versed in the handling of such instruments. For routine laboratory work colorimetric methods have been introduced which, as used by others and by us, have checked up quite accurately with potentiometer measurements.

Before describing the method at present in use, it will be best to say a few words about the nomenclature used at present for the expression of hydrogen ion concentrations.

The Meaning of $\mathrm{P}_{H}$. The hydrogen ion concentration of pure water is 0.0000001 . This is more simply expressed as $1 \times 10^{-7}$. Since, in pure water, hydroxyl ions are equal in concentration, to the hydrogen ions, and the hydroxyl ions are also $1 \times 10^{-7}$, and this is the neutral

4 Clark and Lubs, Jour. of Bacter., 2, 1917, 1, 109, 191. 
point. Solutions in which the hydrogen ion concentration is less than $1 \times 10^{-7}$ (and the hydroxyl, therefore, more than $1 \times 10^{-7}$ ) are alkalin, and vice versa. This method of expression offers certain difficulties to the plotting of curves, and it has been found simpler for such purposes to plot curves according to the logarithms of the expressions used above. Sörensen ${ }^{5}$ has initiated a method of doing this, by introducing the symbol $\mathrm{P}_{H}$ which signifies the logarithm of the reciprocal of the hydrogen ion concentration, expressed as above, thus, $\mathrm{P}_{H}$ equals $\log$ of $\frac{1}{[\mathrm{H}]}$.

Thus, if:

$$
\begin{aligned}
\mathrm{H} & =2 \times 10^{-4}, \\
\mathrm{P}_{H} & =\log \cdot \frac{1}{2 \times 10^{-4}} \\
& =\log 5000=3.7 .
\end{aligned}
$$

Another way of getting at this is as follows:

Supposing that,

$$
\begin{aligned}
& {[\mathrm{H}]=2 \times 10^{-6}} \\
& \mathrm{P}_{H}=\text { log. of reciprocal, }
\end{aligned}
$$

or,

Now,

$$
\mathrm{P}_{H}=\log \cdot\left(\frac{1}{2 \times 10^{-6}}\right) \text {. }
$$

$$
\begin{gathered}
\log .1=0, \\
\log .2=0.3, \\
\log \cdot 10^{-6}=-6 .
\end{gathered}
$$

Therefore:

$$
\begin{aligned}
\text { or, } \quad \mathrm{P}_{H}=\log \cdot \frac{1}{2 \times 10^{-6}}, \\
\quad \mathrm{P}_{H}=0-[+0.3-6] \text { or } 5.7 .
\end{aligned}
$$

The following table gives the relationship of the old method of expression to the new :

${ }^{5}$ Sorensen, Biochem. Zeit., 1909, 21, 131, 201. 
Hydrogen Ion Concentration $\mathrm{P}_{H}$ Value

\begin{tabular}{|c|r|}
\hline $1 \times 10^{-1}$ & 1 \\
$1 \times 10^{-2}$ & 2 \\
$1 \times 10^{-3}$ & 3 \\
$1 \times 10^{-4}$ & 4 \\
$1 \times 10^{-5}$ & 5 \\
$1 \times 10^{-6}$ & 6 \\
$1 \times 10^{-7}$ & 7 \\
$1 \times 10^{-8}$ & 8 \\
$1 \times 10^{-9}$ & 9 \\
$1 \times 10^{-10}$ & 10 \\
\hline
\end{tabular}

Indicators are substances, usually weak organic acids or bases which change in color when subjected to changes of reaction, that is, to changes of hydrogen or hydroxyl ion concentrations. A table which we take from a pamphlet prepared by the Army Medical School, will give the color reactions at various $\mathrm{P}_{H}$ measurements for a number of common indicators.

Color Reactions in Solutions of Different Hydrogen Ion Concentration

\begin{tabular}{|l|c|c|c|c|c|c|c|c|}
\hline True acidity & $10^{-3}$ & $10^{-4}$ & $10^{-5}$ & $10^{-6}$ & $10^{-7}$ & $10^{-8}$ & $10^{-9}$ & $10^{-10}$ \\
\hline Methyl orange & $\begin{array}{c}\text { Rose } \\
\text { red }\end{array}$ & $\begin{array}{c}\text { Orange } \\
\text { red }\end{array}$ & Yellow & - & $\leftarrow$ & $\leftarrow$ & $\leftarrow$ & $\leftarrow$ \\
\hline Rosolic acid & Yellow & $\rightarrow$ & $\rightarrow$ & $\rightarrow$ & Rose & Red & $\leftarrow$ & $\leftarrow$ \\
\hline Congo red & Blue & Violet & $\begin{array}{c}\text { Reddish } \\
\text { violet }\end{array}$ & Orange & $\begin{array}{c}\text { Orange } \\
\text { red }\end{array}$ & $\leftarrow$ & $\leftarrow$ & $\leftarrow$ \\
\hline Litmus & Red & $\rightarrow$ & $\rightarrow$ & $\begin{array}{c}\text { Reddish } \\
\text { violet }\end{array}$ & Violet & Blue & $\leftarrow$ & $\leftarrow$ \\
\hline Phenolphthalein & $\begin{array}{c}\text { Color- } \\
\text { less }\end{array}$ & $\rightarrow$ & $\rightarrow$ & $\rightarrow$ & $\rightarrow$ & $\rightarrow$ & Red & $\leftarrow$ \\
\hline
\end{tabular}

Clark and Lubs particularly have been responsible for introducing the method at present in use in bacteriological laboratories, based upon the preparation of solutions which can be used as standards for the colorimetric measurements of culture media. The solutions must be very carefully prepared, and should, whenever possible, be controlled by potentiometer measurements; however, with proper care 
and the use of pure substances by a reliable worker, this may, with reasonable safety, be omitted. The principle of making the dilutions is that carefully measured amounts of molecular solutions of acids and alkalis are mixed in series so that each successive tube shall contain a definite hydrogen ion concentration. These tubes are the standard. Clark and Lubs, in the first of the series of papers noted above, cite tables of mixtures for such purposes, with a range of $\mathrm{P}_{H}$ extending, from 1 to 10 . For the exact composition of all of these mixtures, the reader is referred to the original paper of Clark and Lubs, page 25. For the routine of the ordinary laboratory bacteriology, only the fourth section of this table is necessary. This is as follows:

$\mathrm{KH}_{2} \mathrm{PO}_{4}-\mathrm{NaOH}$

\begin{tabular}{|l|l|l|l|}
\hline 5.8 & 50 c.c. $\mathrm{M} / 5 \mathrm{KH}_{2} \mathrm{PO}_{4}$ & 3.72 c.c. $\mathrm{M} / 5 \mathrm{NaOH}$ & Dilute to 200 c.c. \\
6.0 & 50 c.c. $\mathrm{M} / 5 \mathrm{KH}_{2} \mathrm{PO}_{4}$ & 5.70 c.c. $\mathrm{M} / 5 \mathrm{NaOH}$ & Dilute to 200 c.c. \\
6.2 & 50 c.c. $\mathrm{M} / 5 \mathrm{KH}_{2} \mathrm{PO}_{4}$ & 8.60 c.c. $\mathrm{M} / 5 \mathrm{NaOH}$ & Dilute to 200 c.c. \\
6.4 & 50 c.c. $\mathrm{M} / 5 \mathrm{KH}_{2} \mathrm{PO}_{4}$ & 12.60 c.c. $\mathrm{M} / 5 \mathrm{NaOH}$ & Dilute to 200 c.c. \\
6.6 & 50 c.c. $\mathrm{M} / 5 \mathrm{KH}_{2} \mathrm{PO}_{4}$ & 17.80 c.c. $\mathrm{M} / 5 \mathrm{NaOH}$ & Dilute to 200 c.c. \\
6.8 & 50 c.c. $\mathrm{M} / 5 \mathrm{KH}_{2} \mathrm{PO}_{4}$ & 23.65 c.c. $\mathrm{M} / 5 \mathrm{NaOH}$ & Dilute to 200 c.c. \\
7.0 & 50 c.c. $\mathrm{M} / 5 \mathrm{KH}_{2} \mathrm{PO}_{4}$ & 29.63 c.c. $\mathrm{M} / 5 \mathrm{NaOH}$ & Dilute to 200 c.c. \\
7.2 & 50 c.c. $\mathrm{M} / 5 \mathrm{KH}_{2} \mathrm{PO}_{4}$ & 35.00 c.c. $\mathrm{M} / 5 \mathrm{NaOH}$ & Dilute to 200 c.c. \\
7.4 & 50 c.c. $\mathrm{M} / 5 \mathrm{KH}_{2} \mathrm{PO}_{4}$ & 39.50 c.c. $\mathrm{M} / 5 \mathrm{NaOH}$ & Dilute to 200 c.c. \\
7.6 & 50 c.c. $\mathrm{M} / 5 \mathrm{KH}_{2} \mathrm{PO}_{4}$ & 42.80 c.c. $\mathrm{M} / 5 \mathrm{NaOH}$ & Dilute to 200 c.c. \\
7.8 & 50 c.c. $\mathrm{M} / 5 \mathrm{KH}_{2} \mathrm{PO}_{4}$ & 45.20 c.c. $\mathrm{M} / 5 \mathrm{NaOH}$ & Dilute to 200 c.c. \\
8.0 & 50 c.c. $\mathrm{M} / 5 \mathrm{KH}_{2} \mathrm{PO}_{4}$ & 46.80 c.c. $\mathrm{M} / 5 \mathrm{NaOH}$ & Dilute to 200 c.c. \\
\hline
\end{tabular}

The method of procedure in using these solutions is as follows: From the solutions made above a colorimetric scale is prepared. All glassware must be very carefully cleansed, and it should be remembered that some of the cheaper glassware obtained in laboratories at the present time often seems to give off considerable amounts of alkali. Whatever method of cleaning is used, the final thorough rinsing must be done thoroughly with redistilled water. 10 c.c. each of the respective series of standard mixtures is placed into each test tube, and to it 10 drops of the indicator are added. For the range from 6.8 to 8.4 , which is sufficient for all ordinary pathogenic work, the indicator used is phenol-sulfon-phthalein, or phenol red in concentration of 0.02 per cent aqueous solution. If ranges from 6 to 7.6 are desired, brom-thymol blue in a concentration of 0.04 per cent may be used, and when ranges just above 8 are desired, cresol red in concentration of 0.02 per cent is recommended. 
A series of tubes so prepared, each of which contains 10 c.c. of each of the graded mixtures, with indicator added, represents a colorimetric scale against which the media can be standardized.

This standardization is now carried out as follows, and can be utilized for any media which are not too highly colored and not turbid: Into a thoroughly cleaned test tube 2 c.c. of the medium are measured and this diluted with 8 c.c. of redistilled water. 10 drops of indicator are then added, and after thoroughly mixing, a color reading is taken against the scale. If the reaction is too acid, as is usually the case, add $\frac{\mathrm{N}}{20} \mathrm{NaOH}$ from a burette or graduated pipette, a drop at a time, until the color matches that of the standard tube. By calculating from the amount of weak alkali added, the total quantity of media is then brought to the desired $\mathrm{P}_{H}$ with $\frac{\mathrm{N}}{1} \mathrm{NaOH}$. In the titration of agar, Clark and Lubs recommend that the broth be titrated and adjusted before the addition of the agar, in order to avoid possible colloidal changes between the agar and the indicator, and that the agar be added after the adjustment is made. It is possible, however, without excessive error, to carry out the titration of media containing agar in the same way as outlined above, adding cold water to the hot agar, and making the comparison at once at a temperature of $35^{\circ}$ to $40^{\circ}$ before the mixture has jelled. In solutions like bacterial media, which have a certain amount of color or some turbidity, a so-called "comparator" may be used in the form of a wooden box painted black with four holes for test tubes and a slit in front and behind, so that it can be looked through against a source of light. The arrangement of this is given in the following cut:

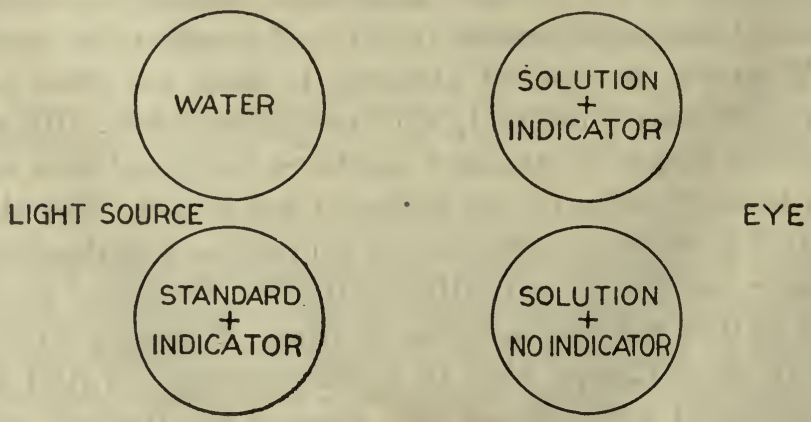

Arrangement for Reading Titration. 
Methods of Clearing Media.-Clearing with Eggs.-When culture media are prepared from substances containing no coagulable protein, it is often necessary, for purposes of clearing, to add the whites of eggs, and then to heat for forty-five minutes in the Arnold sterilizer. In the following detailed descriptions, the direction "clear with egg" has been given whenever such a. step is deemed necessary. The exact technique of such a procedure is as follows:

In a small pot or pan, the whites of several eggs (one or two eggs to each liter of medium) are beaten up thoroughly with a little water (20 c.c.). This egg white is then poured into the medium, which, if hot, as in the case of melted agar or gelatin, must first be cooled to about $50^{\circ}$ to $55^{\circ} \mathrm{C}$. The mixture is then thoroughly shaken and steamed in the Arnold sterilizer for thirty minutes. At the end of this time, the flask containing the medium is removed from the sterilizer and thoroughly shaken so as completely to break up the coagulum which has formed. It is then replaced and allowed to steam for another fifteen minutes. At the end of this time the medium between the coagula should be clear. It is now ready for filtration through cotton.

Filtering Media through Cotton.-The filtration of media after clearing, either by the addition of eggs or by the coagulation of the proteins originally contained in it, is best done through absorbent cotton. A small spiral, improvised of copper wire is placed as a support in the bottom of a lerge glass funnel. A square piece of absorbent cotton is then split horizontally giving two squares of equal size. Ragged edges and incisures should be avoided. These two layers of cotton are then placed in the funnel, one piece above the other in such a way that the direction of the fibers of the two layers is at right angles one to the other. They are then gently depressed into the filter with the closed fist. The edges of the cotton are made to adhere to the sides of the funnel by allowing a thin stream of tap water to run over them, while smoothing them against the glass with the hand.

The medium, when poured into such a filter, should be poured along a glass rod at first, to avoid running down the sides or bursting the filter. After filtration has begun, the filter should be kept as full as possible. The first liter or so which comes through may not be clear, but the filter gains in effieiency as the coagulum settles into the fibers of the cotton, and the first yield may be sent through a second time. Filtration of agar or gelatin is best done in a warm 
room with windows and doors closed, and the filter covered with a lid, to avoid too rapid cooling. The funnel and filter should be warmed just before use.

Filtering through Paper.-Many media may be efficiently cleared by filtration through close filter paper without the aid of coagula.

The Tubing of Media.-Most of the media described in the foregoing section are used in test tubes. In order to fill these tubes, the media are best poured into a large glass funnel to which a glass discharging tube has been fitted by means of a short piece of rubber tubing. Upon this is placed a thumb cock. The plug is then removed from the test tube by catching it between the small and ring fingers of the right hand and the glass outlet is thrust deeply into the test tube, in order to prevent the medium from touching the upper portion of the test tube where the cotton plug will be lodged. About 7 to 8 c.c. are put in each test tube.

Sterilization of Media.-By Heat.-Media which contain neither sugars, gelatin, glycerin, nor animal serum may be sterilized in the autoclave at fifteen pounds pressure for fifteen minutes to half an hour. Media which contain these or other substances subject to injury from the high temperature, must be sterilized by the fractional method, i.e., by twenty minutes' exposure in the live steam sterilizer (Arnold, Fig. 8, page 83) on each of three consecutive days. During the intervals between sterilizations, they should be kept at room temperature or in the incubator, to permit the germination of spores which may be present. Media containing animal serum or other albuminous solutions which are to be sterilized without coagulation, may be sterilized in water baths, or in hot-air chambers, at temperatures varying from $60^{\circ}$ to $70^{\circ} \mathrm{C}$., by the fractional method. In such cases five or six exposures of one hour on succeeding days should be employed.

By Filtration.-It is often desirable in bacteriological work to free fluid from bacteria. This is frequently necessary for the sterilization of blood-serum or exudate fluids, or for obtaining toxins free from bacteria. For these purposes a large variety of filters are in use. Those most commonly employed are of the Chamberland ${ }^{6}$ or Berkefeld type, which consist of hollow candles made of unglazed porcelain or diatomaceous earth. Both these types are made in various grades of fineness, upon which depend both the speed of

- Pasteur and Chamberland, Compt. rend. de l'acad. des sci., 1884. 
filtration and the efficiency. They are made in various forms and models, some of which are shown in the accompanying figures. In

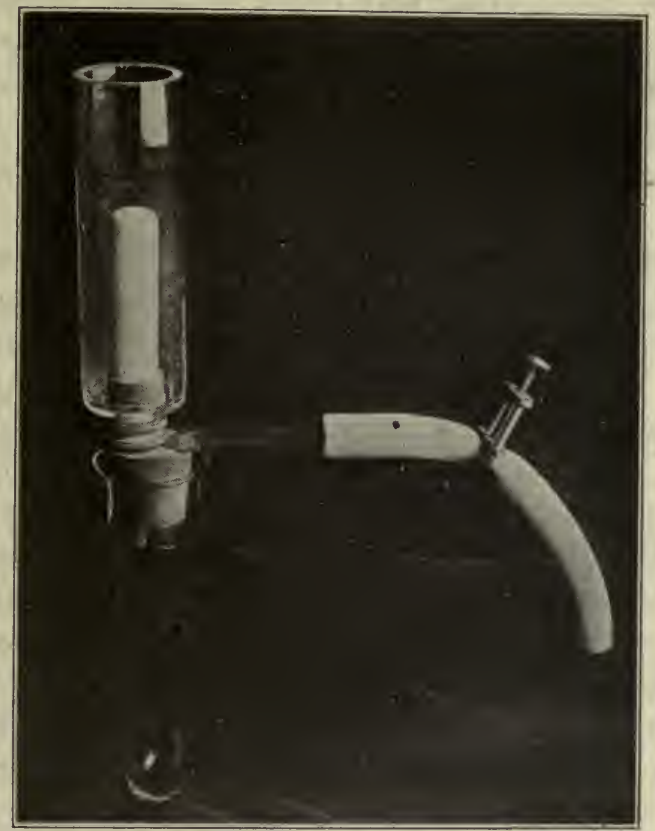

Fig. 10.-Berkefeld Filter.

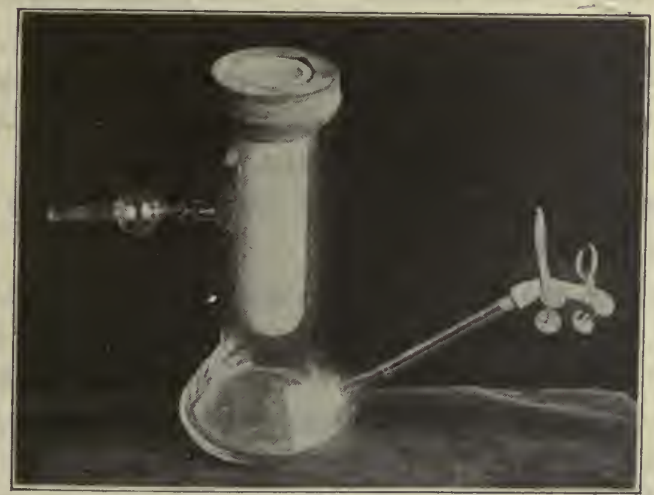

Fig. 11.-Reichel Filter.

most of the methods of filtration commonly employed the fluid which is to be filtered is sucked through the walls of the filter, cither by 
a hand suction-pump or by some form of vacuum-pump attached to an ordinary water-tap.

The hollow eandle-filter may either be firmly fitted into a cylindrical glass chimney and surrounded by the fluid which is to be filtered, or else the candle may be comnected to the collecting flask with sterile tubing and suspended freely in the fluid. Perfect filters of these types will hold back any of the bacteria known to us at present.

Filters before use must be sterilized. The candles themselves are subjected to $150^{\circ} \mathrm{C}$. in the hot-air sterilizer for one hour. The glassware and washers necessary for setting up the apparatus may be sterilized by boiling. In order that filters may be repeatedly used with good result, it is necessary that they should be carefully cleaned from time to time. This is best done in the following way:

Filters through which fluids from living cultures have passed are first sterilized in the Arnold steam sterilizer. Their exterior is then carefully cleaned with a fine brush. Following this a fivetenths per cent solution of potassium permanganate is passed through them and this again removed by sucking through a five per cent solution of bisulphite of soda. This last is washed out by sending a considerable quantity of distilled water through the filter, which is then dried and sterilized by heat.

The suction necessary for filtration through these filters is usually applied by means of the ordinary suction-pump attached to a running faucet.

Slanting of Media.-Solid media which are to be used in slanted form in test tubes should be inclined on a ledge (easily improvised of glass tubing) at the proper slant, after the last sterilization. Agar, the medium most frequently employed in this way, should be left in this position until hard.

Indicators.-We cannot in this place go into details concerning the theory of indicators. For this we may refer the reader to the recent treatise by Clark on the "Determination of Hydrogen Ions." ? According to Ostwald, indicators are acids or bases whose undissociated molecules "have a different color from that of their dissociation products." This conception has been somewhat modified by more recent work, in that color changes are found to be associated

${ }^{2}$ Clark, W. Mansfield, Determination of Hydrogen Ions, Williams and Wilkins Co., Baltimore, 1920. 
with tautomeric rearrangements of the original substances, and the state of these substances in a dissociated or undissociated form determines the color. The degree of dissociation as determined by the hydrogen ion coneentration brings about a predominance of one or the other tautomeric compound, and the color depends upon which one of these compounds is associated with the color. Chapter three of Clark's book goes into this matter with sufficient thoroughness for bacteriological work.

In cultural work not many indicators are needed. For the actual titration of media, the necessary indicators are given in another section. For actual addition to culture media, we may restrict ourselves to a limited number of useful indicators.

Litmus is the indicator most commonly used in former years, is a product obtained from a species of lichen, and is obtained by oxidation of the orcin contained in this plant. Asolitmin is the indicator substance in the litmus, chemically complex and not completely analyzed.

The litmus solutions used in the preparation of media are best made up as follows: Litmus in substance-Merck's purified, or Kaulbaum's-is dissolved in water to the extent of 5 per cent. The solution is made by heating in an Arnold sterilizer for about one to two hours, shaking occasionally. The solution is then filtered through paper and sterilized. It should be kept sterile, as molds will grow in it otherwise.

A standard litmus solution, which is marketed for laboratory purposes, known as "Kubel and Tiemann's" solution, may be used.

Andrade indicator is made up as follows:

0.5 per cent aqueous acid fuchsin.................... 100 e.e.

Normal $\mathrm{NaCH}$........................ 16 c.c.

The red color fades out gradually; the indicator should be yellow after standing two or three hours. If it remains red or reddish, 1 c.c. more of normal $\mathrm{NaOH}$ may be added.

Acid production turns this indicator red, due to the neutralization of the alkali present and the liberation of the acid color base. The medium in which Andrade indicator is used, must be adjusted to the neutral point of this indicator which is equivalent to $\mathrm{P}_{H} 7.2$. The reaction of the medium is right for this indicator, when the medium containing 1 per cent of the indicator is red when hot, and colorless when cold. 
China Blue Indicator.-Make up 1 per cent solution of China blue, heat almost to boiling in the water bath, add, drop by drop, normal $\mathrm{NaOH}$ until completely decolorized. Use 1.5 per eent concentration in medium. This indicator seems to be much less toxic than some of the others, and has been used with success particularly by Teague, to whom we are indebted for these practical details.

Neutral red an indicator formerly used extensively by the Germans in the proportions of 1 c.c. of staturated aqueous solution to 100 c.c. of culture medium. It has been particularly used in connection with the colon-typhoid group, in that typhoid bacilli do not change the color, whereas $\mathrm{B}$. coli decolorizes it.

The list of indicators might be very much increased, but there would be little point in it since most of these indicators are not being used at the present time.

\section{ACTUAL STEPS IN THE PREPARATION OF NUTRIENT MEDIA}

Meat Extract Broth.-To 1000 c.c. of distilled or clear tap water, add 5 grams or 0.5 per cent of Liebig's meat extract, 10 grams or 1 per cent of Witte or any other reliable brand of pepton, and 5 grams or 0.5 per cent of common salt, $\mathrm{NaCl}$.

The ingredients are mixed together in a suitable vessel and heated with stirring over a free flame. When the pepton and meat extract are completely dissolved, the vessel is removed from the flame. The medium is titrated by the colorimetric method described above and adjusted to the desired reaction. It is advisable to make the reaction about two points (on the hydrogen ion seale) more alkaline than the final reaction should be, since the heat of the autoclave usually increases the acidity of the medium. Using Liebig's meat extract and Digestive Ferment's pepton, we have found that the addition of 10-12 e.c. of normal $\mathrm{NaOH}$ to every liter of extract broth usually brings the reaction, before autoclaving, to 7.6, making it about 7.4 after autoclaving, which is the optimum for most pathogenic bacteria. After the addition of the alkali, the broth is autoclaved for thirty minutes at fifteen pounds pressure. The medium is then filtered, the reaction checked and after tubing and sterilization, is ready for use. It is not necessary in most cases to add eggs to extract broth, since it is easy to obtain clear without this step.

Meat Infusion Broth.-Infuse 500 grams $^{8}$ of lean meat (veal or beef) in 1000 c.c. of distilled or tap water for twelve to twenty-four hours in the ice-box. Strain through wet cheese cloth, squeezing the meat as dry as

${ }^{8}$ Roughly 1 pound (11/4 lbs.). 
possible. Make up the volume to 1000 c.c. Add 1 per cent pepton and 0.5 per eent salt. Heat over the free flame until the pepton is dissolved, stirring from time to time. Solution usually takes place so rapidly that the loss of water through evaporation is negligible. If desired, however, the medium can be measured after this preliminary heating, and the loss in volume made up by the addition of water. Titrate the medium and bring it to the desired reaction using the colorimetric method. Meat infusion media are usually considerably more acid than meat extract media. The addition of about 20 c.c. normal $\mathrm{NaOH}$ per liter in the ease of meat infusion broth usually brings the reaction to about 7.4 after autoclaving. The change in reaction after autoclaving is sometimes considerable, and must be carefully checked. After the addition of the alkali, the medium is autoclaved for thirty minutes at fifteen pounds pressure. The broth is now filtered through paper or cotton, and usually comes through clear without any further trouble. After tubing and sterilization, the medium is ready for use.

"Hormone Medium." 9-A curious observation has been made recently which seems to indicate that the filtration of media removes from them certain substances which considerably enhance their nutritive value for bacteria.

These substances, for want of a better name, have been spoken of as hormones, and the hormone media, which are used pretty generally, and which we have found to posisess unusual advantages over ordinary culture media, and which, consequently, we are using almost altogether in our routine work, are made as follows, the description given being that of Huntoon: ${ }^{10}$

The basis for "hormone media" is beef heart instead of the customary beef or veal. It is important that the hearts are fairly fresh. The heart muscle is cut up in the usual way ard after the removal of fat and large vessels, put through the meat grinder. The chopped meat is weighed and 1 liter of water added for every 500 grams of meat. One per cent pepton and 0.5 per cent salt are added directly, and $1 \mathrm{egg}$ (whole) added for each liter of medium. If the bouillon is to be used for broth, 1 per cent gelatin is added immediately. If the bouillon is to be used as a basis for agar, it is not necessary to add the gelatin. The 3 per cent agar, finely cut up, is added to the other ingredients.

When all the ingredients have been placed in the same pot, the mixture is heated over the free flame until it reaches a temperature of about $70^{\circ} \mathrm{C}$. and meat begins to turn brown. 25 c.c. of normal $\mathrm{NaOH}$ are then added per liter. The pot is placed in the Arnold and allowed to cook for $1 \frac{1}{2}$ to 2 hours. At the end of this time, a firm clot has usually formed and the broth or agar can be decanted.

${ }^{\circ}$ Cole and Lloyd, Journal Path. and Bact. vol. 21, 1916.

${ }^{10}$ Huntoon, F. M., Jour. of Inf. Dis., 23, 1918, 169. 
The medium is then titrated accurately and brought to the desired reaction. 'The medium usually gets slightly more acid on autoclaving, so that it is better to adjust to about 2 points more alkaline on the hydrogen ion scale, than the final reaction desired. It is important, in making hormone media, never to filter in any way. Cotton, paper, and cheese cloth filters are equally undesirable. If a firm clot is obtained after the first heating, there is usually no difficulty in obtaining a clear medium. On the first autoclaving a second precipitate usually forms, from which the clear medium can easily be decanted. It is best to check up the reaction after the final sterilization. The Hormone media ean also be cleared with the Sharpless centrifuge.

Sugar-free Broth.-Make one liter of infusion broth ${ }^{11}$ according to the directions given. Inoculate with a young culture of $\mathrm{B}$. coli communis. Incubate for twenty-four to forty-eight hours. The bacteria will ferment and thus destroy any sugar (mono-saccharide) which may be present in the broth, and thus render the medium sugar-free and acid.

Arnold for one hour to kill the B. coli.

Titrate and adjust.

Arnold again for thirty minutes.

Filter through paper until clear.

This sugar-free medium is used as a basis for fermentation reactions. The different sugars are added in 1 per cent concentrations and the medium is then sterilized for three successive days in the Arnold, since the higher temperatures of the autoclave tend to split the more complex sugars into the simpler ones.

Glycerin Broth.-To ordinary, slightly acid or neutral meat infusion broth, add six per cent of C. P. glycerin. Sterilize by fractional method.

Calcium Carbonate Broth.-This medium is designed for obtaining mass cultures of pneumococcus or streptococcus for purposes of immunization or agglutination.

To 100 c.e. of meat infusion broth in small flasks, add one per cent of powdered calcium carbonate, and one per cent of glucose. It is a wise precaution to sterilize the dried calcium carbonate in the hot-air chamber before using. Small pieces of marble may be used as suggested by Bolduan.

Method for the Preparation of Nutrient Broth ${ }^{12}$ (Avery) (for Streptococcus Agglutination).-One pound of lean chopped beef allowed to infuse in a liter of tap water over night in the ice-box. The unfiltered meat infusion boiled for 30 minutes, filtered through paper, and the loss by evaporation made up by the addition of water. One per cent peptone (Fairchild) and 0.2 per cent sodium phosphate $\left(\mathrm{Na}_{2} \mathrm{HPO}_{4}\right)$ are now added. The mixture

${ }^{11}$ It is not necessary to filter the infusion if it is to be used for making sugar free broth.

${ }^{12}$ We may add that this method of growing streptococeus for agglutination purposes does not always work out successfully-but is the best we know of so far. 
is allowed to boil for 20 minutes and the reaction is adjusted to the desired $\mathrm{P}_{H}$. (About 0.2 of a $\mathrm{P}_{H}$ is allowed for change in the reaction during sterilization. For pneumococens work the optimus $\mathrm{P}_{H}$ is 7.8 ; in the adjustment, therefore, before sterilization, the reaction is set at $\mathrm{P}_{H}$ 8.) The broth is sterilized in the Arnold sterilizer for 20 minutes on three successive days. For streptococeus work the final reaction should be $P_{I I} 7.4$.

Pepton-Salt Solution (Dunham's solution):

1. Distilled water 1,000 c.c.

Pepton (Witte) $\ldots \ldots \ldots \ldots \ldots \ldots \ldots \ldots \ldots \ldots, 10 \mathrm{gms}$.

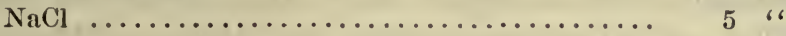

2. Heat until ingredients are thoroughly dissolved.

3. Filter through filter paper until perfectly clear.

4. Tube or store flasks.

Sterilize by discontinuous method.

Nitrate Solution:

1. Distilled water $\ldots \ldots \ldots \ldots \ldots \ldots \ldots \ldots \ldots \ldots \ldots \ldots$. 1,000 c.c.

Pepton $\ldots \ldots \ldots \ldots \ldots \ldots \ldots \ldots \ldots \ldots \ldots \ldots \ldots \ldots \ldots$. 10 gms.

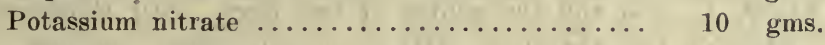

2. Heat until ingredients are thoroughly dissolved.

3. Filter through filter paper until perfectly clear.

4. Tube or store flasks.

Sterilize by discontinuous sterilization.

Uschinsky's Protein-Free Medium. ${ }^{13}$-To one liter of distilled water add:

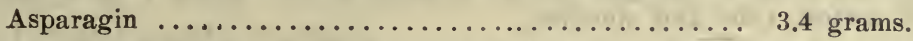

Ammonium lactate ...................... 10 "

Sodium chlorid ........................ 5

Magnesium sulphate ..................... 0.2 "

Calcium chlorid ....................... 0.1 "

Potassium phosphate ................... 1.0

When these substances are thoroughly dissolved, add 40 c.c. of glycerin. Tube and sterilize.

Meat Extract Gelatin.-Gelatin is best made in the Arnold and should at no stage be autoclaved.

One per cent pepton, 0.5 per cent salt, 0.5 per cent meat extract in one liter of water are dissolved in the Arnold. When these ingredients have been dissolved, 15 to 18 per cent of finest French sheet gelatin ${ }^{14}$ are added, and the mixture kept in the Arnold until the gelatin is completely dissolved.

${ }^{13}$ Uschinsky, Cent. f. Bakt., 1, xiv, 1893.

${ }^{14}$ The acidity and consistence of the different commercial gelatins vary considerably and care should be taken in selecting a uniform and suitable brand, such as Hesterberg's gold label gelatin. It is advisable, when working during the summer or in hot climates, to add 18 per cent. 
The medium is then titrated and adjusted. After cooling, the whites of two eggs are added and the media is put in the Arnold for forty-five minutes. The medium is filtered, tubed and sterilized by the fractional method. All unnecessary heating of gelatin is to be avoided.

Meat Infusion Gelatin.-Meat infusion gelatin is made in the same way as the above except that fresh meat infusion is substituted for the meat extract.

Meat-Extract Agar.-To 1 liter of distilled or tap water add:

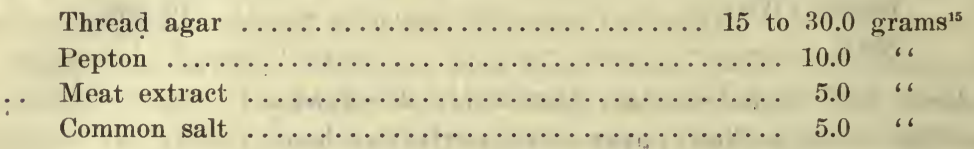

Put in autoclave, 15 pounds' pressure, for 15 minutes. The agar can also be dissolved over the free flame, but this takes a long time and is not necessary. However, if so done make up loss by evaporation.

Take out of autoclave, and adjust to desired reaction.

Cool to $60^{\circ} \mathrm{C}$. and add the whites of two eggs. Stir thoroughly.

Heat either in the autoclave, fifteen pounds, thirty minutes; or if no autoclave is available, thirty minutes in the Arnold. If the heating is done in the Arnold, take out after half an hour, stir and replace for fifteen minutes more.

Titrate, and adjust again if reaction has changed.

If a correction in reaction is made, heat again for ten minutes, filter through cotton, tube and sterilize.

Meat Infusion Agar. ${ }^{16}$ - In making meat infusion agar it is best to prepare two solutions, one consisting of the meat infusion to which the pepton and salt are added, the other an aqueous solution of agar which is dissolved in the autoclave. The ingredients are added in double strength to each solution so that when they are finally combined, the desired concentrations are obtained.

Solution (1) 500 grams of lean meat in 500 c.e. of distilled or tap water are infused over night in the ice-box. The infusion is then strained through cheese cloth and all the juice squeezed out of the meat. The infusion is measured and the volume made up to 500 c.e. Two per cent pepton or 10 grams, and 1 per cent or 5 grams salt are now added to the 500 e.c. infusion and the mixture is heated over the free flame until it reaches a temperature of about $50^{\circ} \mathrm{C}$. and the pepton dissolves. This solution is then

${ }^{15}$ Amount of agar varies according to stiffness desired.

${ }^{16}$ While titrating care should be taken that the medium does not solidify along the sides of the vessel. Glycerin agar is made by adding 6 per cent C.P. glycerin to meat extract or meat infusion agar. 
titrated and sufficient alkali added to make the reaction about two or three points more alkaline than the desired final reaction of the medium.

Solution (2) to 500 c.e. of water add the quantity of agar that will give the right concentration for the final volume (1 liter). For instance, if a 3 per cent agar is desired, add 30 grams of agar to 500 c.c. of water and dissolve in the autoclave at fifteen pounds pressure for fifteen minutes. When the agar is dissolved, cool it down to $50^{\circ} \mathrm{C}$. and add to it Solution (1). Mix thoroughly and titrate again. Usually the addition of the agar solution does not change the reaction. Add the whites of two eggs well beaten with a little water and autoclave for thirty minutes at fifteen pounds pressure. If no autoclave is available, the medium may be put in the Arnold for forty-five minutes. The autoclave, however, is more satisfactory. It is best to check the titration again after autoclaving.

The medium is now filtered, tubed and sterilized.

LACTOSE-LitMus-Agar (Wurtz).-This is ordinary meat extract agar which is adjusted to $\mathrm{P}_{H} 7.5$ to 7.8 , to which 1 per cent of lactose is added, and enough litmus to give it a bluish purple color when cooled. Instead of litmus, 1 per cent of the Andrade indicator can be used. If the latter indicator is used, the reaction of the medium must be brought down to $P_{\text {I }}$ 7.2. The medium containing 1 per cent of the indicator, if the reaction is correct, will be dark red when hot and colorless when cold. After the addition of the sugar and the indicator, it is best to sterilize by the intermittent method on three successive days.

The red color fades out gradually, the indicator should be yellow after standing 2 or 3 hours. If it remains red or reddish, 1 or 2 c.c. more of normal $\mathrm{NaOH}$ should be added.

The above description of the basic media, broth and agar, details chiefly the methods in general use until a few years ago. The recognition that bacteria grow more luxuriantly upon media containing considerable quantities of protein-split products, has resulted in the utilization of trypsinized culture media which has been found to possess considerable advantages for the cultivation of delicate organisms like the meningococei, ete. A simple formula for the production of trypsinized agar is the following, taken from the directions of Gordon: ${ }^{17}$

TrYPagar. - T'o one pound of ehopped meat, free from fat, add 1 liter tap water and make faintly alkalin to litmus with 20 per cent $\mathrm{NaOH}$ solution. Heat in double boiler at $75^{\circ}$ to $80^{\circ}$ for five minutes. Cool to $37^{\circ}$ and add 0.5 gram trypsin. (Fairchild's preparation. For other brands the amount must be determined by experiment.) Incubate for five to six hours. Test for pepton as follows: Take 5 e.c. of the liquid, add 5 e.c. $\mathrm{N} / 1 \mathrm{NaOH}$ and 1 c.c. dilute $\mathrm{CuSO}_{4}$. $\mathrm{A}$ pink color indicates that trypsinization is

${ }^{17}$ Gordon, Br. Med. Journal 2, 1916, 678. 
complete-a bluish purple shade, that it is incomplete. If test is satisfactory, slightly acidify the broth with glacial acetic acid and bring slowly to boiling point and boil gently for ten minutes and filter through paper. Add 2 per eent agar and 0.5 per cent salt, autoclave to dissolve agar, and proceed from this point as usual, elearing with egg and setting to $\mathrm{P}_{H} 7.5$, or any desired reaction.

Vedder Starch Agar.-Beef infusion agar is prepared without salt and pepton. This is adjusted to $\mathrm{P}_{H} 6.8,10$ grams of eorn starch is added to each liter, and the mixture is heated in the autoclave for 20 minutes at 15 pounds. It is tubed and sterilized. This medium has been recommended by Vedder for the cultivation of gonococcus.

Welch's Modification of Guarnieri's Medium. ${ }^{18}$ - This meaium is made on a meat infusion basis, according to the directions given for the preparation of meat-infusion agar. It contains 5 grams of agar, 80 grams of gelatin, 5 grams of $\mathrm{NaCl}$, and 10 grams of pepton to one liter. It should be adjusted to a neutral reaction. It is used for stab cultures and is designed chiefly for pneumococeus cultivation and storage.

Dorsett Egg Medium.-This medium is chiefly useful for the cultivation of tubercle bacilli.

1. Carefully break eggs and drop the contents into a wide-mouthed flask. Break up the yolk with a sterile platinum wire, and shake up the flask until the whites and yolks are thoroughly mixed.

2. Add 25 c.c. of distilled water to every four eggs; strain through sterile eloth.

3. Pour 10 c.e. each into sterile test tubes and slant in an inspissator and expose to $73^{\circ} \mathrm{C}$. for four to five hours on two days.

4. On the third day, raise the temperature to $76^{\circ} \mathrm{C}$.

5. The sterilization may be finished by a single exposure to $100^{\circ} \mathrm{C}$. in the Arnold sterilizer for fifteen minutes. Before inoculation, add two or three drops of sterile water to each tube.

Lubenau's GLycerin-EGG.-To 1 liter of veal broth containing 2 per cent pepton, 5 per cent of glycerin is added. Neutralize this to litmus, and to every 200 c.c. add 10 fresh eggs. The mixture is thoroughly stirred, and when homogeneous, is tubed, slanted and inspissated, as in the case of other egg media.

Petroff's Medium.-I. Meat Juice. 500 grams of beef or veal are infused in 500 c.e. of a 15 per cent solution of glycerin in water, in a cool place. After 24 hours the meat is squeezed in a sterile press and the infusion collected in a sterile beaker.

II. Eggs. The shells of the eggs are sterilized by 10 minute immersion in 70 per cent alcohol. They are broken into a sterile beaker, well mixed

${ }^{18}$ Welch, Bull. Johns Hopkins Hosp., 1892, vol. 3, p. 127. 
and filtered through sterile gauze. One part of meat juice is added to two parts of egg by volume.

III. Gentian Violet. 1 per cent alcoholic solution of gentian voilet is added to make a final proportion of $1: 10,000$.

The three ingredients are well mixed. The medium is tubed and inspissated as usual.

Petroff recommends for sputum the following technique: Equal parts of sputum and 3 per cent sodium hydroxid are shaken and incubated at $38^{\circ}$ C. for 15 to 30 minutes, the time depending on the consistency of the sputum. The mixture is neutralized to litmus with hydrochloric acid and centrifugalized. The sediment is inoculated into the medium described above. Pure cultures are obtained in a large proportion of cases.

Synthetic Media for the Tubercle Bacillus. - A considerable number of synthetic media have been made for the growth of the tubercle bacillus. The purpose of these media is to omit complex protein substances as much as possible. The one we give below is according to the formula used by Petroff with success.

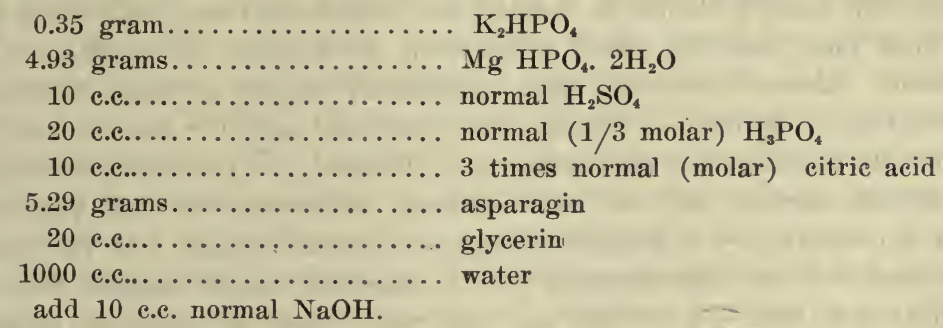

Potato Media.-Large potatoes are selected, washed in hot water, and scrubbed with a brush. They are peeled, considerably more than the cuticle being removed. The peeled potatoes are washed in running water, following which cylindrical pieces are removed with a large apple corer. The cylinders are cut into wedges.

Since the reaction of the potato is normally acid, this should be corrected by washing the pieces in running water over night, or, better, by immersing them in a one per cent solution of sodium carbonate for half an hour.

The pieces are then inserted into the large variety of test tubes known as "potato tubes." (See Fig. 21, c.) In the bottom of the tubes a small amount of water (about 1 c.c.) or a small quantity of moist absorbent cotton should be placed in order to retard drying out of the potato. The tubes are sterilized by fractional sterilization, twenty minutes to half an hour in the Arnold sterilizer on three successive days.

Ротато Bвотн.--Petroff has used extract of potatoes in fluid media for the growth of tubercle bacilli. There are many different ways of preparing the potato extract. The best way is to finely grind thoroughly, or grate the 
potatoes and soak them in tap water for from 12 to 24 hours, using about 500 grams to a liter of water. This mixture can be filtered, or, better, heated before filtration. It may be used as an ingredient with or without glycerin in ordinary broth or agar, or ean be used with pepton and salt added, as an independent culture medium.

Glycerin Potato.-In preparing glycerin potato the potato wedges are treated as above, and are then soaked in a ten to twenty-five per cent aqueous glycerin solution for one to three hours. A small quantity of a ten per cent glycerin solution should be left in the tubes. In sterilizing these tubes, thirty minutes a day in the Arnold after the sterilizer is hot, will sterilize without altering the glycerin.

Milk Media.-Fresh milk is procured and is heated in a flask for fifteen minutes in an Arnold sterilizer. It is then set away in the ice chest for about twelve hours in order to allow the cream to rise. Milk and cream are then separated by siphoning the milk into another flask. It is rarely necessary to adjust the reaction of milk prepared in this way, since, if acid at all, it is usually but slightly so. If, however, it should prove more than 1.5 per cent acid, it should be discarded or neutralized with sodium hydrate. The milk may then be tubed either with or without the addition of an indicator. Litmus gives the most satisfactory results in milk, but at the present time is diffieult to obtain. The Andrade indicator ean be used also, but usually discolors the milk somewhat. However, if fractional sterilization is carefully carried out, the milk becomes yellowish, but acid production shows up clearly by a distinct reddening of the medium. If the milk is to be used for the differentiation of the anaërobic bacilli isolated from war wounds, it is best not to add any indicator, since the type of coagnlum formed is a differential characteristic, and coagulation does not take place readily when Andrade is present.

Sodium Oleate Agar (For Influenza Bacilli).-Avery ${ }^{19}$ has found that sodium oleate will enhance the development of influenza bacilli, and at the same time will inhibit many of the Gram-positive organisms commonly found in sputum.

A neutral solution of Kahlbaum's sodium oleate in water is prepared and sterilized in the autoclave. Human or rabbit blood is defibrinated, centrifuged, the serum removed, and the volume made up to the original with broth. 1 e.c. of the red blood cell suspension and 5 c.c. of the 2 per cent sodium oleate solution are added to 94 e.c. of agar at $80^{\circ}$ to $90^{\circ} \mathrm{C}$. The agar is preferably a 2 per cent hormone agar with a reatcion of $\mathrm{P}_{H}$ 7.4.

Serum Media.-Loeffler's Medium.-Beef blood is collected at the slaughter house in high cylindrical jars holding two quarts or more. It is desirable that attempts should be made to avoid contamination as much as

${ }^{19}$ J. A. M. A. 1918, vol. 71, 2050. 
is feasible by previously sterilizing the jars, keeping them covered, and exercising care in the collection of the blood.

The blood is allowed to coagulate in the jars, and should not be moved from the slaughter house until coagulated. All unnecessary shaking of jars should be avoided. As soon as the coagulum is fully formed, adhesions between the clot and the sides of the jar should be carefully separated with a sterile glass rod or wire. The jars are then set away in the ice chest for 24 to 36 hours. At the end of this time clear serum will be found over the top of the clot, and between the clot and the jar. This should be pipetted off, preferably with a large pipette of 50 to 100 c.e. capacity, or siphoned. off with sterile glass tubing, and transferred to sterile flasks.

To three parts of the clear serum is then added one part of a one per cent glucose beef infusion or veal infusion bouillon. The mixture is filled into tubes, preferably the short test tubes commonly used for diagnostic diphtheria cultures. The tubes are then placed in a slanting position in the apparatus known as an inspissator (see p. 71). This is a doublewalled copper box covered by a glass lid, eased in asbestos, and •surrounded by a water jacket. It is heated below by a Bunsen flame. Together with the tubes a small open vessel containing water should be placed in the inspissator to insure sufficient moisture. The temperature of the inspissator is now raised to $70^{\circ}-75^{\circ} \mathrm{C}$, , care being taken that the rise of temperature takes place slowly. The temperature is maintained at this point for two hours, and the process is repeated, for the same length of time, at the same temperature, on six successive days, preferably without removing the tubes from the inspissator at any time. It is also possible, though less regularly yielding good results, to sterilize in the inspissator for one day, following this on the second and third days by exposure for thirty minutes to $100^{\circ} \mathrm{C}$. in the Arnold steam sterilizer. In doing this, the Arnold should be very gradually heated, at first without outer jacket, this being lowered only after thorough heating has taken place.

Serum-Water Media for Fermentation Tests.-For the determination of the fermentative powers of various microorganisms for purposes of differentiation, Hiss has devised the following media in which the cleavage of any given carbohydrate is indicated, not only by the production of an acid reaction, but by the coagulation of the serum proteins.

Obtain clear beef serum by pipetting from clotted blood in the same way as this is obtained for the preparation of Loeffler's blood-serum medium. Add to this two or three times its bulk of distilled water, making a mixture of serum and water in proportions of one to two or three. Heat the mixture for fifteen minutes in an Arnold sterilizer at $100^{\circ} \mathrm{C}$. to destroy any diastatic ferments present in the serum. Add one per cent of a five per cent aqueous litmus solution (the variation in the different litmus preparations as obtained in laboratories necessitates a careful addition of 
an aqueous litmus solution until the proper color, a deep transparent blue, is obtained, rather than rigid adherence to-any quantitative directions). With many batches of serum, it will be found that the addition of two or three times its bulk of distilled water is not a sufficient dilution to prevent coagulation. It will often be found necessary to add four or five volumes of distilled water to one volume of serum. One per cent of the Andrade indicator may be substituted for the litmus. Add to the various fractions of the medium thus made one per cent respectively of the sugars which are to be used for the tests.

For the preparation of inulin medium, made in this way for pneumococcusstreptococcus differentiation, it is necessary to sterilize the inulin dissolved in the water to be added to the serum in an autoclave at high temperature (15 pounds for 15 minutes) in order to kill spores before mixing with the serum. The serum-water media are sterilized by the fractional method at $100^{\circ} \mathrm{C}$., at which temperature they remain fluid.

Chocolate Media (Park and Williams).- It has recently been observed that for the cultivation of organisms like the influenza bacillus, meningococeus, and a number of other of the more delicately growing bacteria, an excellent medium can be made up in the following way: Agar or broth are made up as usual, and to them added defibrinated rabbit, beef, horse or human blood in proportions of from 5 per cent to 10 per cent by volume. This mixture is then heated gradually up to about $75^{\circ}$, until the blood begins to coagulate and assume a dark brown chocolate like color. The broth or agar ean first be adjusted to the desired reaction, but it is likely that any excess alkali or acidity is corrected by the proteins which are added. The medium can be tubed, or, in the ease of agar, plated or slanted, as it is, after distribution of the blood throughout the medium by shaking.

In the case of broth the medium can be filtered through paper while hot, and sterilized subsequently by filtration and fractional heating. Such filtrate consists of a elear brownish fluid on which influenza bacilli and other organisms grow with enormous speed. The speed with which influenza bacilli grow on this medium, and its almost complete freedom of hemoglobin, but very much increased cholestrin and other lipoid contents, have lead us to believe that it is not the hemoglobin particularly which is needed for influenza bacillus enltivation.

\section{Special Media for Colon Typhoid Differentiation}

Conradi-Drigalski Medium.20_Original directions.

(a) Three pounds of meat are infused in two liters of water for twelve hours or more. Strain, boil for one hour and add 20 gms. Witte's pepton, 20 gms. of nutrose, $10 \mathrm{gms}$. of $\mathrm{NaCl}$; boil one hour and filter. Add 60 gms.

${ }^{20}$ Conradi-1)rigalski, Zeit, f. Hyg., xxxix, 1902, 
of agar. Boil for three hours (or one hour in an autoclave) until agar is dissolved. Render weakly alkaline to litmus paper, filter, and boil for half an hour more.

(b) Litmus solution: 'Two hundred and sixty e.c. of litmus solution are boiled for ten minutes. (The litmus solution used by Conradi and Drigalski is the very sensitive arueous litmus recommended by Kubel and Tiemann, and purchasable under the name.) After boiling, 30 grams of chemically pure lactose are added to the litmus solution. The mixture is then boiled for fifteen minutes, and if, a sediment has formed, is carefully decanted.

(c) Add to the hot lactose mixture to the hot agar solution; mix well and, if necessary, again adjust to weak alkaline reaction, litmus paper being used as an indicator. To this mixture add 4 c.c. of a hot, sterile ten per cent solution of sodium carbonate, and 20 c.c. of a freshly made solution of erystal violet (c. p. Höchst), 0.1 gram in 100 c.c. of sterile distilled water.

Surface smears are made upon large plates. - These are incubated twenty-four hours. Typhoid colonies are small, blue, and transparent. Colon colonies are large, red, and opaque.

Endo's Medium. ${ }^{21}$-1. Prepare one liter of meat infusion three per cent agar, containing 10 grams of pepton and 5 grams of $\mathrm{NaCl}$.

2. Neutralize and clear by filtration.

3. Add 10 c.c. of $10 \%$ sodium carbonate to render alkaline.

4. Add 10 grams of chemically pure lactose.

5. Add 5 c.e. of alcoholic fuchsin solution, filtered before using. Endo in his original contribution does not mention the strength of this fuchsin solution, which, however, should be saturated. This colors the medium red.

6. Add 25 c.c. of a $10 \%$ sodium sulphite solution. This again decolorizes the medium, the color not entirely disappearing, however, until the agar is cooled.

7. Put into test tubes, 15 c.c. each, and sterilize.

The medium should be kept in dark. Plates are poured and surface smears made. The typhoid colonies remain colorless, while those of coli become red.

The preparation of Endo's medium presents difficulties due to the varying purity of sodium sulphite. Kastle and Elvove ${ }^{22}$ recommend the use of anhydrous sodium sulphite instead of the crystallized variety. Harding and Ostenberg ${ }^{23}$ add sodium sulphite solution to a measured amount of .5 per cent fuchsin to determine the propor-

${ }^{21}$ Endo, Cent. f. Bakt., xxxv, 1904.

${ }^{22}$ Kastle and Elvove, Jour. Inf. Dis., xvi, 1909.

${ }^{23}$ Harding and Ostenberg, Jour. of Inf. Dis., xi, 1, 1909. 
tions which give the greatest delicacy of reaction as tested with formaldehyd. The proportions so determined are then added to the hot 3 per cent agar.

Although Endo described his medium as dependent upon the formation of acid by the bacteria, this is not so. Acids give no coloration of the sulphite-fuchsin mixture. Indeed this mixture is used by chemists under the name of Schiff's reagent as a test for aldehyds. Acids decolorize the red caused by aldehyds, and this accounts for the frequent late discoloration of red colon colonies on prolonged cultivation. The medium is red when hot, and colorless when cold, because the compound between sulphite and fuchsin dissociates in the hot solution.

Robinson and Rettger's Modification of Endo. ${ }^{24}$-This seems to be at present the most useful modification of Endo available.

1. To 1 liter of water add 25 grams of agar, 10 grams of pepton, and 5 grams of meat extract. Dissolve the agar, meat extract and pepton. Bring to $\mathrm{P}_{H}$ 6.8, and heat in autoclave for $1 / 2$ hour at 15 pounds. Filter through cotton.

To this add 10 c.c. of a 10 per cent sodium carbonate solution. Heat. for a few minutes, and add 1 per cent lactose. The fuchsin sulphite indicator is then added in the form of 5 c.c. of saturated alcoholic fuchsin, and 10 c.c. of a 10 per cent solution of sodium bisulphite. This is tubed and sterilized. Krumwiede recommends preparing the medium by adjusting the reaction to $\mathrm{P}_{H} 8.5$ in the first place, relying upon sterilization and the addition of the bisulphite to bring the reaction to the desired end point.

The best results are obtained by adding the lactose, fuchsin and bisulphite just before use, and this can be done most conveniently if the agar basis is bottled in 100 c.e. amounts. The final reaction of the medium should be $\mathrm{P}_{H}$ 8. A more acid reaction favors the diffusion of the indicator. In our own laboratory we have found that the addition of these amounts of fuchsin and sodium bisulphite to the medium inhibit the growth of typhoid in some instances. We have obtained equally good differentiation by using 0.25 per cent fuchsin instead of 0.5 per cent, and 0.5 per cent sodium bisulphite instead of 1 per cent.

Kendall's Modification of Endo's Medium. ${ }^{25}-1.5$ per cent meat extract agar is prepared, and the reaction adjusted faintly alkaline to litmus by the addition of $\mathrm{NaOH}$. This agar is stored in small flasks and it is usually convenient to keep flasks containing 100 c.e. each. Just before use, 1 per cent of lactose is added, and then decolorized fuchsin solution, as in Endo's

${ }^{24}$ Robinson and Rettger, Jour. of Med. Res., 24, 1916, 363.

${ }^{25}$ Kendall, Boston Med. \& Surg. Jour. 
medium. Add about 1 c.c. of decolorized fuchsin solution, made up as above by mixing roughly prepared 10 per cent sodium sulphite with saturated alcoholic fuchsin. (The proportions of fuchsin and sulphite are sometimes difficult to adjust, possibly by reason of impurities in the sulphite due to formation of sulphate. The instructions given by most workers at present are to use 10 c.c. of a 10 per cent aqueous solution of sodium sulphite, and to add to this 1 c.c. of a 10 per cent solution of fuchsin in 96 per cent alcohol.) When these flasks containing the various ingredients are hot they are red or pink, but when plates are poured and allowed to harden, the medium should be either colorless or very faintly pinkish. It is best to pour a number of plates rather thickly and then allow them to dry with the covers off. Inoculations from the feces suspension are then made by surface smear, with a bent glass rod. Colon colonies are pinkish and red; typhoid colonies, smaller and grayish.

In concluding the description of some of the most important typhoid isolation media, we would like to add that a great deal seems to depend upon the habit-acquired skill which the individual worker attains. None of these stool isolation media are ordinarily successful at once in the hands of anyone, and a certain amount of practice must be attained before one can judge of the usefulness or uselessness of a medium.

Brilliant Green Agar for Typhoid Isolation.-Krumwiede has" recently devised a brilliant green agar with which he has had excellent results. ${ }^{26}$

The basis is an extract agar like that used for Endo's medium:

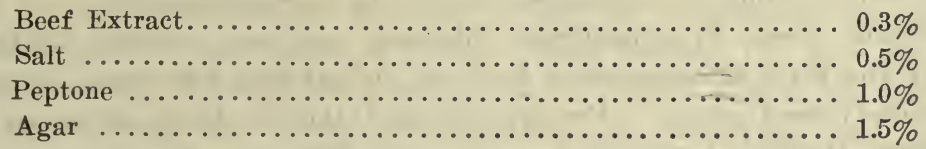

(Domestic peptones are satisfactory.)

Dissolve in autoclave; clear and filter. A clear agar is essential. The final reaction of the medium is to be neutral to ${ }^{27}$ Andrade's indicator, which in terms of phenolphthalein is $0.6-0.7 \%$ acid (normal $\mathrm{HCl}$ ) or $\mathrm{P}_{H}$ 7.2. It is more convenient to have the reaction set slightly alkaline to litmus at the time of preparation and to acidify each bottle as used. The agar is bottled in 100 c.c. amounts and autoclaved. When needed, the bottles are melted and the volume of each corrected (if necessary) to an approximate 100 c.c.. Add to each bottle:

${ }^{26}$ We are indebted to $D r$. Krumwiede for a preliminary account of this method.

${ }^{27}$ Andrade's Indicator : 0.5 per cent aqueous acid fuchsin......... 100 e.c.

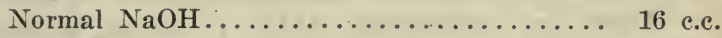

The dye is slowly ( 2 hours) alkalinized to the color-base; the red tint is restored by acids. 
One per cent Andrade Indieator.

$\Lambda$ cid to bring to neutral point of the indicator. ${ }^{28}$

One per cent Lactose. ${ }^{29}$

0.1 per cent Glucose.

Brilliant Green in 0.1 per cent aqueous solution.

Two dilutions of dye are used in routine plating, corresponding to 1-500,000 and $1-330,000$ in terms of solid dye ( 0.2 c.e. and 0.3 c.c. of 0.1 per cent solution per 100 c.c. of agar). The sample of dye which Krumwiede has used is from Bayer, but he has also tested and found equally satisfactory samples from Grübler and Höchst. 0.1 gram of dye is accurately weighed on a foil, washed with boiling $\mathrm{H}_{2} \mathrm{O}$ into a 100 c.e. volumetric flask and made up to the mark when cool. The flask should be clean and neutral (by test). Fresh solutions vary in activity (see standardization tests); they keep about a month.

Each bottle is mixed and poured into six plates only (a thick layer of agar gives the most characteristic colonies). Plates are left uncovered until agar has "jellied"; porous tops are used; dry plates are essential to avoid diffusion.

Standardization: The agar must have proper "balance." The reaction is important; sediment reduces the activity of the dye and light colored media are better than darker ones. Different lots of agar with the same dye solution act ununiformly; a new batch or a new solution must be tested.

Any variation in the composition of the media necessitates a readjustment of dye concentration; this statement cannot be over-emphasized.

Brilliant green, in appropriate dilutions, not only inhibits all Grampositive and many Gram-negative bacteria, but exhibits differential action on the colon-typhoid group. Paratyphoid and the B. lactis aërogenes are untouched, typhoid is restrained only at low dilutions, while dysentery and the other colon group are extremely susceptible. The typhoid colony on this medium is characteristic. Looking through the plate against a dark surface, in oblique light the colony has a snowflake appearance; the edge delicately serrate. With artificial light and a hand lens, the texture is that of a coarse woolen fabric. Acid production from the trace of glucose may tinge the colony. The colony is large.

Brilliant Green-Eosin Agar. ${ }^{30}$-Meat infusion agar is prepared and titrated to +1 to phenolphthalein. 'The following substances are added:

${ }^{28}$ An agar is neutral to Anilrade when, hot, the color is a deep red, but fades completely on cooling. This is determined by cooling 3 or 4 c.e. of acidified hot agar in a serum tube under the tap and arjusting accordingly.

29 These are conveniently added from one sterile solution coniaining $20 \%$ lactose and $2 \%$ dextrose, 5 c.c. to 100 of agar gives the requisite concentration.

so Teague and Clurman, Jour. of Infec. Dis., 18, 1916, 647. 


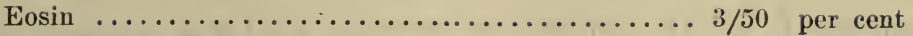

Brilliant green $\ldots \ldots \ldots \ldots \ldots \ldots \ldots \ldots \ldots \ldots \ldots \ldots \ldots$ per cent

Saccharose ......................... 1 per cent

Lactose .......................... 1 per cent

The typhoid colonies on this medium are large and have a grayish pink color. Most strains of B. coli do not grow upon it; the colonies of B. coli that do develop have deep red centers. Meyer and Stickel $^{31}$ claim that better results are obtained with the brilliant green-eosin medium if peptic digest agar is substituted for the meat infusion agar. They set their reaction at $\mathrm{P}_{H}=7$ to 6.8 .

Malachite-Green Bouillon (Peabody and Pratt)..$^{32}$-To 100 c.e. of beef infusion broth add 10 c.c. of one per cent solution of malachite green Höchst 120 , made with sterile water. This is tubed.

This medium is used as an enriching fluid. One drop of the suspected material (emulsified stool) is added to each tube and after incubation for eighteen to twenty-four hours inoculations may be made upon plates.

Peabody and Pratt found a reaction of .5 per cent acidity to phenolphthalein most favorable.

Lead Acetate Agar for the Differentiation of Paratyphoid " $A$ " and "B." ${ }_{3}$ -One drop of a ten per cent solution of neutral lead acetate is added to every 4 c.c. of agar. This is the original procedure of Burnet and Weissenbach. Krumwiede recommends the cooling of the agar to $60^{\circ}$, then adding enough of a 0.25 per cent basic lead acetate solution to bring the concentration to 0.05 per cent. The agar is tubed, and Burnet and Weissenbach recommend inoculating with a fine needle in several places between the agar and the walls of the tube. Typhoid and paratyphoid "B" bacilli-blacken the medium, while paratyphoid "A" leaves it unchanged. B. Enteritidis and Typhi Murium behave like paratyphoid "B."

Bile Medium.34-(Recommended for blood cultures by Buxton and Coleman.) The medium is prepared as follows:

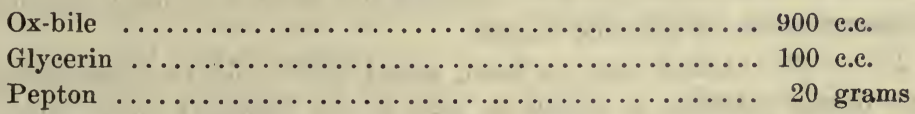

.t into small flasks containing quantities of about 100 e.c. each and sterilized fractional sterilization.

Jackson's Lactose-Bile Medium. ${ }^{35}$-This medium is used for isolating $\mathrm{B}$.

${ }^{31}$ Meyer, K. F., and Stickel, J. E., Jour. of Infec. Dis., 23, 1918, 48.

32 Peabody and Pratt, Boston Med. and Surg. Jour., clviii, 7, 1908.

${ }^{33}$ Burnet and Weissenbach, C. R. de la Soc de Biol., vol. 78, 1915, p. 565.

${ }^{34}$ Conradi, Deut. med. Woch., 32, 1906.

${ }^{35}$ Jackson, "Biol. Studies of Pupils of W. T. Sedgwick,' 1906, Univ. Chieago ?ress. 
typhosus and B. coli from water, and serves as a valuable enriching medium in isolating them from other sources. Jackon and Melia ${ }^{36}$ found that in this medium B. typhosius and B. coli outgrow all other microorganisms and eventually B. typhosus will even outgrow B. coli.

It consists of sterilized undiluted ox-bile (or a ten per cent solution of dry, fresh ox-bile) to which is added one per cent pepton and one per cent lactose. It is filled into fermentation tubes and sterilized by the fractional method.

MacConkey's Bile-Salt Agar:!

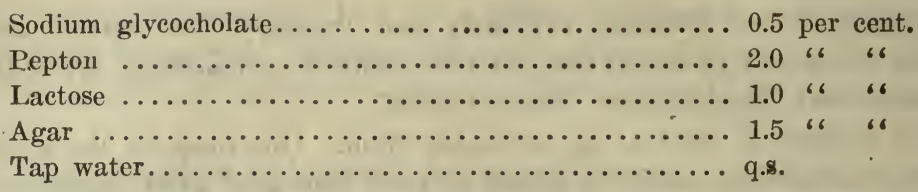

The agar and pepton are dissolved and cleared and the lactose and sodium glycocholate added before tubing. The $\mathrm{B}$. typhosus produces no change; B. coli, producing acid, eauses precipitation of the bile salts.

Neutral-Red Medium.-To 100 c.c. of a one or two per cent glucose agar add 1 c.c. of a saturated aqueous solution of a neutral-red.

The medium is used in tubes, stab or shake cultures. The typhoid bacillus produces no change, while members of the colon group render the medium colorless by reduction of the neutral-red and produce gas.

Bariekow's Medium. ${ }^{37}$-To 200 c.c. of cold water, add 10 grams of nutrose and allow to soak for one-half to one hour. Pour this into 800 c.c. of boiling water, and heat for twenty minutes in an Arnold sterilizer at $100^{\circ} \mathrm{C}$. Filter through cotton and to the opalescent solution of nutrose add 5 grams. of $\mathrm{NaCl}, 10$ grams of lactose, and sufficient aqueous litmus solution to give a pale blue color. ${ }^{38}$

Russell's. Double Sugar Agar. ${ }^{39}$-Russell has devised a simple medium for quick identification of typhoid bacilli.

A $2 \%$ or $3 \%$ extract agar is used, about $0.8 \%$ acid to phenolphthalein. Enough litmus solution is added to give it the ordinary deep blue. The reaction is then adjusted with sodium hydrate until neutral to litmus. Finally $1 \%$ lactose and $0.1 \%$ glucose (dissolved in a small amount of hot water) are added, the medium is carefully sterilized in the Arnold sterilizer and slanted. Inoculations are made by surface streak and stab.

${ }^{36}$ Jackson and Meiia, Jour. Inf. Dis., vi, 1909.

s7 Barsiekow, Wien. klin. Rund., xliv, 1901.

${ }^{38}$ Filtration may be done through paper, but takes a long time.

so Russell, Jour. Med. Research, xxv, 1911, 217. 
Sharper results are obtained with this medium if 1 per cent of the Andrade indicator (described above), is substituted for the litmus. When Andrade is used, the final reaction of agar should be about $\mathrm{P}_{H}$ 7.2. It is best to standardize the reaction against the particular solution of Andrade used. The reaction of the medium is satisfactory when the mixture containing 1 per cent of the indicator is red when hot, and colorless when cold. Typhoid and paratyphoid $\mathrm{A}$ and $\mathrm{B}$, and dysentery, on this medium show colorless growths on the slant, the butts, however, where partial anaërobiosis exists, show a deep red color, due to acid fermentation. Typhoid may be distinguished from paratyphoid $\mathrm{A}$ and $\mathrm{B}$ by the fact that typhoid, since it ferments glucose without the production of gas, forms no gas bubbles in the butt, whereas, there is gas formation with paratyphoid $A$ and $B$. Typhoid and dysentery give the identical reaction on Russell's medium colorless growth on the slant, and acid formation without gas in the butt, and must be distinguished by the motility test. There is no reliable way of distinguishing between paratyphoid $\mathrm{A}$ and $\mathrm{B}$, unless lead acetate is added to the medium (see below). Krumwiede recommends the addition of 1 per cent saccharose to the 1 per cent lactose, and 0.1 per cent glucose as a means of ruling out some of the "paratyphoid-like" intermediates that ferment saccharose more rapidly than lactose. The non-pathogenic types fermenting lactose, or lactose and saccharose, which are present in 1 per cent concentrations, produce acid on the slant, as well as in the butt, with the formation of gas, and are thus easily eliminated.

Russell Double Sugar Agar with Lead Acetate. ${ }^{40}$-The best basis for this medium is an infusion agar which has been rendered sugar free and adjusted to $\mathrm{P}_{H} 7.4$, or neutrality to Andrade indicator. To this medium add 1 per cent Andrade indicator, tube and sterilize in quantities of 5 e.e. to each tube.

Make up a solution containing 20 per cent lactose and 2 per cent glucose. Sterilize.

Make up a solution of 0.25 basic lead acetate. Sterilize.

To each tube of the agar add 0.25 c.e. of the double sugar solution and 1 c.e. of the lead acetate solution.

Add both the solutions to the agar at $60^{\circ}$ C. under sterile precautions and slant.

Typhoid bacilli eause a brown color near the surface of the stab. Paratyphoid " $A$ " and dysentery do not eause any browning. Paratyphoid " $B$ " and other members of the group cause browning. (The volume of agar per tube is not given in the article, but the sugar percentage works out for 5 c.e.)

Kligler, Jour. of Experimental Medicine, September, 1918. 


\section{Special Media for the Isolation of Cholera Spirilla}

Dieudonné Medium. ${ }^{41}$ - Fo 70 parts of ordinary 3 per cent agar made neutral to litmus, add 30 parts of a sterile mixture of defibrinated beef blood and normal sodimm hydrate.

This is sterilized by steam before being added to the agar. 'This alkali agar is poured into plates and allowed to dry several days at $37^{\circ}$, or 5 minutes at $60^{\circ}$. The material to be examined is smeared on the surface of these plates with a glass rod.

Aronson's Medium for Cholera Stool Isolation. ${ }^{22}$-This medium is prepared as follows: 35 grams of agar are added to 1 liter of tap water and soaked over night.

Add 10 grams of meat extract, 10 grams of pepton, 5 grams of sodium chloride and heat in steam sterilizer from 4 to 5 hours. The particles are allowed to settle by letting the hot agar stand, and the clear supernatant agar poured into flasks to hold 100 c.c. each.

The following solutions are previously made and sterilized for $1 / 2$ hour in the Arnold:

1. 10 per cent solution of sodium carbonate

2. 20 per cent solution of cane-sugar

3. 20 per cent solution of dextrin

4. saturated solution of basic fuchsin

5. 10 per cent solution of sodium sulfite (sterilized by being brought to a boil)

To 100 c.c. of agar add 6 c.c. of the 10 per cent solution of sodium carbonate and heat for 15 minutes at $100^{\circ} \mathrm{C}$. The agar, because of the alkalinity, becomes brown and cloudy. While hot, add 5 c.c. of the 20 per cent solution of cane-sugar, 5 c.c. of the 20 per cent solution of dextrin, 0.4 c.c. of the saturated solution of basic fuchsin, and 2 c.c. of the 10 per cent sodium sulfite solution. The flask is allowed to stand to let the coarser particles settles, and plates are poured with the clear supernatant fluid. The principle of this medium, like Dieudonnés, depends upon the ability of cholera spirilla to grow on very alkalin media and upon their ability to split polysaccharites with acid and aldehyde formation.

Cholera strains, recently from the human body, give. large red colonies in from 15 to 20 hours, whereas, the colon colonies are smaller and colorless. Teague and Travis ${ }^{43}$ found that strains of cholera spirillum that had been out of the human body for some time did not yield red colonies promptly, but they obtained excellent results even with these cultures, if they added 0.25 per cent nutrose to Aronson's medium.

${ }^{4}$ Dieudonné, Cent. f. Bakt., 1., orig., 1909.

2 Aronson, Deut. med. Woch., 41, 1915, 1027.

${ }^{43}$ Teague and Travis, Jour. of Infec. Dis., 18, 1916, 601. 
Teague and Travis Medium for the Cholera Spirillum.44-They prepare their medium as follows:

Two pounds of chopped beef are soaked in 2 liters of distilled water in the ice box over night. The fluid is squeezed out, heated in the Arnold, filtered through filter paper and made neutral to litmus with sodium hydrate, and is heated again. After being allowed to cool, it is inoculated with colon bacillus, and incubated for 2 or 3 days to make it sugar free. Agar is then prepared from it by adding 1 per cent pepton, 0.5 per cent sodium chlorid and clearing with egg. The reaction is adjusted to -0.5 phenolphthalein, and after the agar has been cleared and filtered, 0.25 per cent nutrose is added. A stock aqueous solution of 3 per cent bluish eosin is kept on hand in the dark, also a 1 per cent stock' solution of Bismarck-brown. The Bismarck-brown solution must be made up in water containing 10 per cent of lactose, because it is entirely soluble to 1 per cent in distilled water alone.

To 50 c.e. of this nutrose agar add 1 per, cent saceharose, 1 c.e. of the 3 per cent eosin solution, and 2 c.e. of the 1 per cent Bismarek-brown solution. After this mixture has been shaken until the stains are uniformly distributed, pour plates. These plates are uncovered and placed on a shelf, face down, in the incubator for 20 minutes to remove excess of moisture before smearing. On this medium the differentiation of the cholera colonies is striking, with large and with red centers, while the colon colonies are uniformly pink.

Rabbit's Blood for Ducrey Bacillus Cultivation.-Rabbits are bled from the heart with a sterile syringe and about $1 \frac{1 / 2}{2}$ to 2 e.c. placed into small test tubes. The blood is allowed to clot and inactivated at $56^{\circ}$ for $1 / 2 \mathrm{hour}$. This makes an excellent medium for the cultivation of the Ducrey bacillus, for streptococei and some other organisms. It is also excellent for the preservation of streptococei and pneumococei in a virulent condition.

The preparation of Anaërobic Tissue Tubes for the Cultivation of Spirochaëtes and other Anaërobes.-This is the Tarrozi and Smith method of using tissue for anaërobic purpose, adapted by Noguchi for the cultivation of various spirochaetes. The proportions of broth, serum and agar are adapted to the particular purpose for which it is to be used. It is necessary, therefore, in this place only to describe the best method of putting up the tissue tubes. High, narrow test tubes are used, about 7 to 8 inches in length, with a diameter not larger than that of a Wassermann tube. One-half inch tubes are convenient. A rabbit is rapidly killed by ether anesthesia. It is best to bleed him from the carotid at the same time, in order not to waste the blood. The animal is immediately opened with sterile precautions. It is best to dissect off the fur and wash the abdominal wall with alcohol before opening the abdomen. The abdomen is then opened carefully and widely, so that the organs ran be easily reached withont unnecessary poking about.

"Teague and Tratis, Jour, of Infec. Dis., 18, 1916, 601. 
The intestines are pulled aside so as to uncover the kidneys. With a fresh set of instruments, the hilum of the kidney is now grasped and the kidney rapidly separated from its capsule and passed through the flame before being placed into a sterile Petri plate. The spleen may be removed in the same way.

The kidney and spleen should then be eut up with the utmost precautions of sterility. Work in a dustless place with the windows closed and several Bunsen flames going elose to the field of operation. An assistant slightly raises the cover of the Petri plate and the bacteriologist, working with sterile forceps and a sterile old knife which ean be constantly flamed, euts the kidney in pieces of appropriate size against the bottom of the plate. In placing these bits of tissue into tibes, the stopper of the sterilized tube is pulled, and the tube heated around its lips and upper one-half inch. The tissue is then rapidly passed into the flame, thrust into the mouth of the tube, the cotton stopper flamed and inserted. The tube is then given a rapid flip with the hand, which sends the tissue to the bottom. When these tubes are filled with broth and aseitic fluid or agar, they should be incubated before use and the unsterile ones discarded.

In most of Noguehi's work, and some of our own, paraffin oil was used over the tops of these tubes. The sealing properties of this, however, are not what they were formerly supposed to be. Air passes through this paraffin oil, and if sealing is desired it is much better to heat the upper empty part of the tubes thoroughly, and thrust in a paraffin stopper. The top of the fluid also can be covered with melted paraffin which will solidify in the incubator.

Cooked Meat Medium, Robertson, ${ }^{45}$ for the Cultivation of Anaërobes.250 grams of beef heart are minced and ground in a mortar. Add 250 e.e. of tap water, heat slowly, cook thoroughly, neutralize to litmus with $\mathrm{NaOH}$, tube and sterilize in autoclave.

The simplest method of making cooked meat media which gives satisfactory results with the majority of the anaërobic bacilli, is the following. A few pieces of chopped meat (not necessarily heart) are placed in the bottom of the tube, enough infusion broth, $\mathrm{P}_{H}$ 7.8, is added so that there are about 3 c.e. clear broth over the meat. The medium is ready for use after autoclaving. The reaction usually becomes more acid on autoclaving in the presence of the meat fragments. The optimum for the most anaërobic bacilli is $\mathrm{P}_{H}=7.4$.

Enriching Substances Used in Media.-For the cultivation of microorganisms which are sensitive to their food environment, it is often necessary or advisable to add to the ordinary media enriching substances, which empirical study has shown to favor the growth

${ }^{45}$ Robertson, Jour. of Path. and Bacter., January, 1916, 20, No. 3. 
of the organism in question. The substances most commonly used for such enrichment are glucose, nutrose (sodium caseinate), glycerin, sodium formate, and unsolidified animal proteins. As animal and blood serum and whole blood must frequently be used in this way, an understanding of the methods employed in obtaining these substances is necessary.

Method of Obtaining Blood and Blood Media.-Blood serum from beef and sheep may be collected in the manner recommended for the collection of such serum in the preparation of Loeffler's medium, pipetted into test tubes, and sterilized in the fluid state by exposure to temperatures ranging from $60^{\circ}$ to $65^{\circ} \mathrm{C}$., for one hour upon six consecutive days. It is not a simple matter to sterilize serum in this way and requires much time and care.

The method most commonly employed, in laboratories which have access to hospitals, for obtaining clear serum depends upon the collection of exudate or transudate fluids by sterile methods directly from the pleural cavity, the abdominal cavity, or the hydrocele cavity. Sterile flasks or test tubes are prepared and the fluid is allowed to flow directly out of the cannula into these. It is necessary to avoid carbolic acid or other disinfectants in sterilizing instruments and rubber tubing used during the operation. These should be brought into the ward in the water in which they have been boiled and not in strong antiseptic solutions, as is frequently done. The fluid so obtained may be incubated and the contaminated tubes discarded. The serum may then be added, in proportions of one to three, to sterile broth or melted agar.

Agar thus used is melted and cooled to $60^{\circ} \mathrm{C}$., or below. Onethird of its volume of warmed exudate fluid is added, and the plates are poured.

Serum may be rendered free of bacteria by filtration through a Berkefeld or Pasteur-Chamberland filter. This is an effectual method, but requires much time and care.

Whole blood may be obtained for cultural purposes by bleeding rabbits or dogs or other animals directly from a blood-vessel into tubes of melted agar. In the case of a rabbit, after the administration of an anesthetic (ether), an incision is made directly. over the trachea, and, by careful section, the carotid artery is isolated, lying close to the side of the trachea. Blood may be collected and hemolysed by the gradual addition of the smallest amount of ether which will completely hemolyse the amount treated. This may be 
kept in stoppered sterile bottles and added to agar as desired. This is particularly useful in preserving blood for routine work on meningococcus carriers.

Methods of bleeding animals are briefly described above.

\section{Selective Action of Dye Stuffs}

In deseribing the selective media for typhoid bacilli we have seen that malachite green and crystal violet have been found to exert a certain amount of selective action upon the typhoid and colon groups. The selective influence of various dyes has been recently again studied by Churchman. Churchman ${ }^{46}$ found that the addition of gentian violet in dilutions of $1: 100,000$, to media, inhibited some bacteria, while others grew luxuriantly in its presence. Extremely interesting, both practically and theoretically, is his observation that upon such gentian violet media bacteria fall into two groups. Those which grow on gentian violet correspond in a general way to the Gram-negative bacteria; those which fail to develop on these media correspond roughly with the Gram-positive species. One strain of the enteritidis group could not be eultivated on gentian violet, and this was found to differ from the others also in its agglutination tests.

Signorelli ${ }^{47}$ claims that dahlia is useful in differentiating true cholera strains from similar spirilla. The true cholera strains grew with colored colonies, while others remain colorless, in his experiments.

Krumwiede and Pratt ${ }^{48}$ were unable recently to confirm the elaims of Signorelli. However they fully confirm the findings of Churchman both as to the selective action of gentian violet and in regard to the classification of bacteria into two groups corresponding to their reaction to the Gram stain. They state that among Gramnegative bacteria a strain is occasionally found which will not grow on the gentian violet media, differing in this respect from other members of the same species. They find also that the reaction is quantitative.

The streptococcus-pneumococcus group, according to their in-

46hurchman, Jour. Exp. Med., 16, 1912 ; also Churchman and Michael, ibid.

${ }^{47}$ Signorelli, Centralbl. f. Bakt., Orig. 56, 1912.

${ }^{48}$ Krumwiede and Pratt, Centralbl. f. Bakt., Orig. 68, 1913; and Proc. N. Y. Path. Soc., xiii, 1913. 
vestigations, differs from other bacteria in being able to grow in the presence of quantities of violet which inhibit other Gram-positive species. Dysentery bacilli show variations. They found, however that, in addition to gentian violet, Hoffman violet, crystal violet, dahlia violet, fuchsin, rosanilin and methyl violet will inhibit Grampositive but not Gram-negative bacteria in dilutions of from 1 to 5000 to 1 to 50,000 . 


\section{CIIAPTER VIII}

METHODS USED IN THE CULTIVATION OF BACTERIA

\section{INOCULATION OF MEDIA}

THE transference of bacteria from pathological material to media, or from medium to medium, for purposes of cultivation, is usually accomplished by means of a platinum wire or loop. The platinum wire used should be thin and yet possess a certain amount of stiffness and be about two to three inches in length. This is fused into the end of a glass rod six to eight inches long. It is an advantage, though not necessary, to use rods of so-called "sealing-in" glass which, having the same coefficient of expansion as platinum, does not crack during sterilization. For work with fluid media, the wire should be bent at its free end so as to form a small loop which will pick up a drop of the liquid. For the inoculation of solid media and the making of stab cultures, a straight "needle" or wire should be used. Other shapes of these wires and spatulæ from heavy wire have been devised for various purposes and are easily improvised as occasion demands.

When making a transfer from one test tube to another, the tubes should be held between the thumb and first and second fingers of the left hand. (See Fig. 12.) The plugs are then removed by grasping them between the small and ring fingers and ring and middle fingers of the right hand, first loosening any possible adhesions between glass and plugs by a slight twisting motion. The platinum wire is held meanwhile by the thumb and index fingers of the right hand in the manner of a pen. The wire is heated red hot in a Bunsen flame, and is then passed into the culture tube without being allowed to touch the glass. It is held suspended within the tube for a few seconds to permit of cooling before touching the bacterial growth. The wire is then allowed to touch lightly the surface of the growth and a small amount is picked up. It is then removed from the tube without allowing it to 
touch the sides of the glass, and is passed into the tube which is to be inoculated. If the tube contains a slanted medium, such as agar, a light stroking motion from the bottom of the slant to its apex will deposit the bacteria upon the medium evenly along a central line. The needle may also be plunged downward into the substance of the nutritive material so that in the same tube both surface growth and deep growth may be observed. If a stab culture is to be made in unslanted agar or in gelatin, the needle is simply plunged straight downward as nearly as possible along the axis of the medium. If a fluid medium is being inoculated, the wire should be introduced only into the upper part of the liquid and the bacteria gently rubbed into emulsion against the side of the glass. The

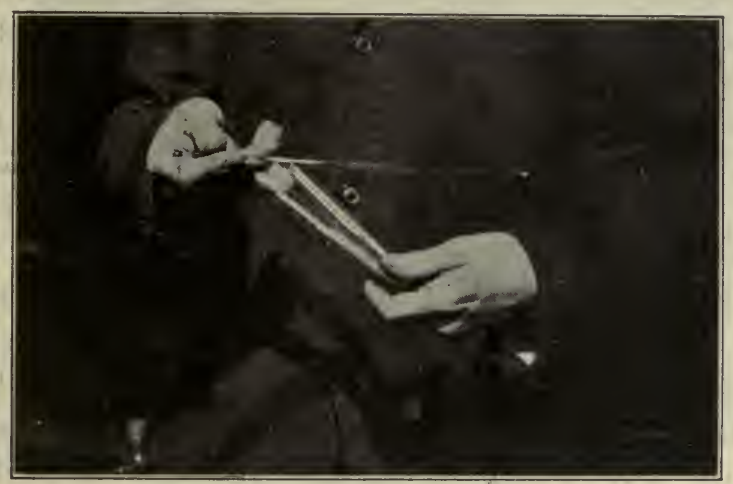

Fig. 12.-Taking Plugs from Tubes before Inoculation.

needle is then removed from the tube, the stopper carefully replaced, and the platinum wire immediately sterilized in the flame. This sterilization of platinum needles after they have been in contact with bacteria should become second nature to those working with bacteria, since an infraction against this rule may give rise to serious and widespread consequences. In burning off platinum needles it is well to remember that a part of the glass rod, as well as the wire itself, is introduced into the tubes and may become contaminated. and for this reason the rod itself, at least in its lower two or three inches, should be passed through the flame as well as the wire. As an extra precaution against contamination, the lips of test tubes and flasks and the protruding edges of cotton plugs may be passed through the flame and singed. 


\section{THE ISOLATION OF BACTERIA IN PURE CULTURE}

It is obvious that in many eases where hacteria ale cultivated from water, milk, pathological material, or other sources, many species may be present in the same specimen. It is likewise obvious that scientific bacteriological study of any bacterium can be made only if we obtain this particular species entirely apart from others, in what is known as "pure culture." The earliest methods for accomplishing this were the methods of Pasteur and of Cohn who depended upon the power of one species to outgrow all others, if cultivated for a sufficient length of time in fluid media. This method, of course, was inadequate in that it was often purely a matter of chance which one of the mixture of species was finally obtained by itself. A later method, by Klebs, depends upon serial dilution, in test tubes of fluid media, by which the eventual transference of one germ only, to the last tube was attempted. Such methods, none of them of great practical value, have been entirely displaced by those made possible by Koch's introduction of solid media which may be rendered fluid by heat.

The methods now employed for the isolation of bacteria depend upon the inoculation of gelatin or agar, when in the melted state, the thorough distribution of the bacteria in these liquids by mixing, and the subsequent congealing of these media in thin layers. By this means the individual bacteria, distributed in the medium when liquid, are held apart and separate when the medium becomes stiff. The masses of growth or "colonies" which develop from these single isolated microorganisms are discrete and are descendants of a single organism, and can be transferred, by means of a process known as "colony-fishing," to fresh sterile culture media.

Plating.- The first method employed by Koch for bacterial isolations was one that consisted in the use of simple plates of glass, about $3 \times 4$ inches in size, mounted upon a leveling stand. A wooden triangle, supported upon three adjustable serew-feet, formed the base of this apparatus. Upon this was set a covered crystallizing dish which could be filled with ice water. Upon the top of this rested the sterilized plates under a bell jar. By screwing up or down upon the supports of the triangle, leveling of the plate could be achieved and controlled by a spirit-level placed at its side. The inoculated gelatin was poured upon the plate and spread and rapidly 
cooled and hardened by the cold water contained in the erystallizing dish.

The original method of Koch has been modified considerably and the method universally employed at present depends upon the use of circular covered dishes, the so-called Petri dishes. These obviate the necessity of a leveling stand and prevent contamination of the plate when once poured. Each Petri dish plate consists of two circular glass dishes; the smaller and bottom dish has an area of 63.6 square centimeters; the larger is used as a cover for the smaller, and forms a loosely fitting lid. The plates when fitted together are sterilized and thus form a closed cell which, if properly handled, may remain sterile indiefinitely.

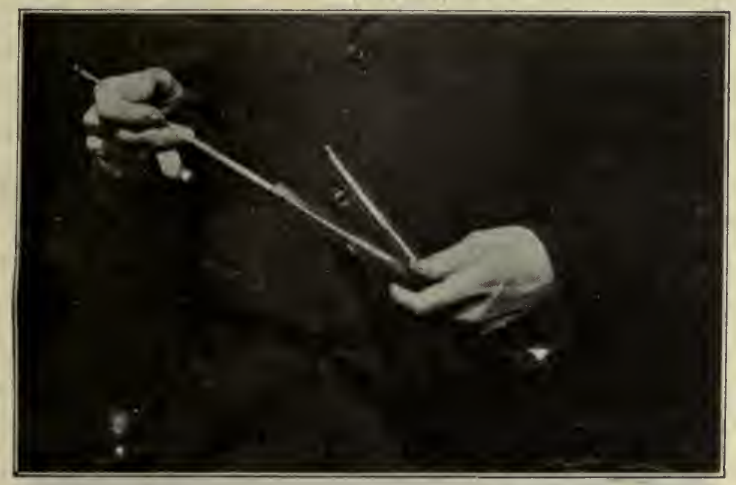

Fig. 13,-Inoculating.

The technique for making a pour plate for the purpose of isolating bacteria from mixed culture is as follows :

The actual "pouring" of plates is preceded by the preparation of usually three graded dilutions of the material to be examined. For this purpose three agar or gelatin tubes are melted and, in the case of the agar, are cooled to a temperature of about $42^{\circ} \mathrm{C}$. in a water bath. A platinum loopful of the material to be examined is transferred to one of these tubes. The bacteria are then thoroughly distributed throughout the melted gelatin or agar by alternately depressing and raising the plugged end of the tube, giving it a rotary motion at the same time. This thoroughly distributes the bacteria throughout the medium without allowing the formation of air-bubbles. Two loopfuls of this mixture are then transferred to the second tube and a similar mixing process is repeated. This 
second tube contains the bacteria in much greater dilution than the first and the colonies which will form in the plate poured from this tube, will be farther apart.- A third dilution is then made by transferring five loopfuls of the mixture in the second tube to the third. This again is mixed as before. The contents of the tubes are then poured into three sterile Petri dishes. The pouring should be done with great care. The cover of the dish is raised along one margin simply far enough to permit the insertion of the end of the test tube, the plug of which has been removed and the lips passed, with a rotary movement, through the flame. The medium is poured into the dish without the lips of the tube being allowed to touch either the bottom or the cover of the dish. The eover is then replaced and the medium allowed to harden.

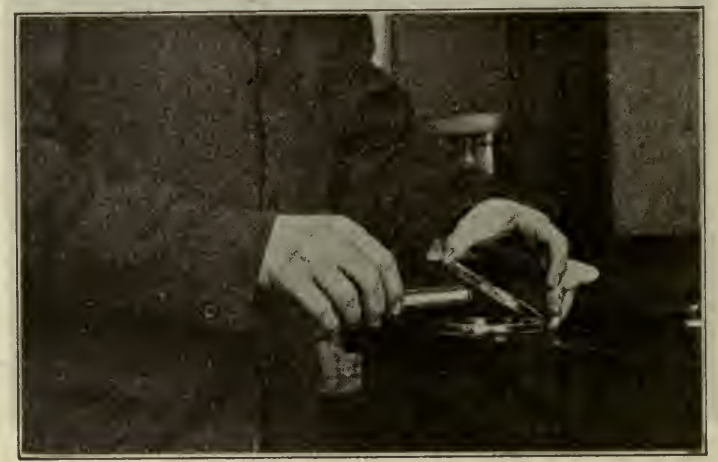

Fig. 14.-Pouring Inoculating Medium from Petri Dish.

When agar has been used, the dishes may be placed in an incubator at $37^{\circ} \mathrm{C}$. It is well to place the plates upside down in the ineubator. This prevents the eondensation water, squeezed out of the agar during hardening, from collecting on its surface, and forming channels for the diffuse spreading of bacteria. The same end may be attained by the use of Petri plates provided with porous earthenware lids, as suggested by Hill. Simple inversion of the plates, however, usually suffices. When gelatin has been used, the plates are allowed to remain in a dark place at room temperature or in a special thermostat kept at $22^{\circ}-25^{\circ} \mathrm{C}$.

Colonies in agar, kept at $37.5^{\circ} \mathrm{C}$., usually develop in eighteen to twenty-four hours; those in gelatin or agar at room temperature in from twenty-four to forty-eight hours, depending upon the species 
of bacteria which are being studied. Often in the second dilution, more frequently in the third, the colonies will be found well apart and can then be "fished." The process of "colony-fishing" is one which requires practice and should always be done with care, for upon its success depends the purity of the sub-culture obtained. Colonies should never be fished under the naked eye, no matter how far apart and discrete they may appear, since not infrequently close to the edge of or just beneath a larger colony there may be a minute colony of another species which may be too small to be visible to the naked eye, but which, nevertheless, if touched by accident will contaminate the sub-culture.

For proper "fishing," the Petri plate with cover removed, should be placed upon the stage of the microscope and examined with a low power objective, such as Leitz No. 2 or Zeiss AA. The sterilized platinum needle, held in the right hand, is then carefully directed into the line of focus of the lens, while the small finger of the hand is steadied upon the edge of the microscope stage. When the point of the needle is clearly visible through the microscope, it is gently depressed until it is seen to touch the colony and to carry away a portion of it. The needle is then withdrawn without again touching the nutrient medium or the edges of the glass or the lens, and transferred to a tube of whatever medium is desired. In this way, individuals of one colony, descendants of a single bacterium of the original mixture-are carried over to the fresh medium.

Esmarch Roll Tubes. ${ }^{1-A}$ simple method of obtaining separate colonies is that devised by von Esmarch and known as "roll-tube"' eultivation. Tubes of melted gelatin are inoculated with various dilutions of the bacterial mixture and, while still liquid, are laid in an almost horizontal position upon a block of ice, which has been grooved slightly by means of a test tube filled with hot water. The test tube containing the gelatin, after being placed in this groove, is rapidly revolved by passing the fingers of the right hand across it, while its base is earefully steadied with the left hand. If the revolving is carried out with sufficient speed, the gelatin will harden in a thin layer on the inner surface of the tube. The colonies will develop in this layer and may be "fished" by means of a platinum wire with bent point introduced into the tube. This method is useful for certain purposes, but is too inconvenient for routine work. It is now rarely used.

${ }^{1}$ Esmarch, Zeit. f. Hyg., i, 1886. 
Separation of Bacteria by Surface Streaking.-When it is necessary to isolate bacteria like the gonocoecus Bacillus influenzæ, the pneumococcus, and others, which, because of great sensitiveness to environment and possibly a preference for free oxygen, are not readily grown in pour plates, it is often advantageous first to pour plates of suitable media, allow them to harden, and then gently smear over their surfaces dilutions of the infectious material, usually in three or four parallel streaks.

Upon such plates, if dilutions have been properly made, and this is only a question of judgment based upon an estimation of the numbers of bacteria in the original material, discrete colonies of the microorganisms sought for may develop, and can be "fished" in the usual manner.

The media most favorable for the cultivation of various microorganisms will be discussed in the sections dealing with the individual species.

Barber Pipette Method for the Isolation of Single MicroORGANISMS.-Although we consider this one of the very important methods of bacteriological study, we shall give no extensive description of the apparatus or the manner of using it, because both are too complicated to permit of satisfactory use from text book deseription. The principle of the method depends upon a specially prepared mechanical stage adjusted to a compound microseope on which there is a moist chamber closed with a large coverglass, on the bottom of which drops of fluid. containing bacteria ean be placed. A very fine glass pipette, manipulated by a specially constructed pipette holder and with a rubber tubing attached, is governed by observation through the microseope and by means of it small drops of the fluid are taken up, an attempt being made to obtain a single microorganism in these drops. With a little practice this ean be accomplished, and these separate drops in which the individual bacteria can be seen swimming about, are made to suspend from the bottom of the cover slip, closing the top of the moist chamber. The apparatus can be understood and worked only by practice and suitable instruction, together with a study of the description given by Barber in his article in the Philippine Journal of Science. ${ }^{2}$ The apparatus is made by a number of firms under the name of the

${ }^{2}$ Barber, Philippine Jour. of Science, Sec. B, Vol. 9, No. 4, August, 1914. 
Barber Single Cell Apparatus, and no description that could be given in a text book would be of sufficient value to be allowed to take up space.

\section{ANA ËROBIC METHODS}

We have seen in a preceding chapter (p. 28) that many bacteria, the so-called anaërobes, will develop only in an environment from which free oxygen has been excluded.

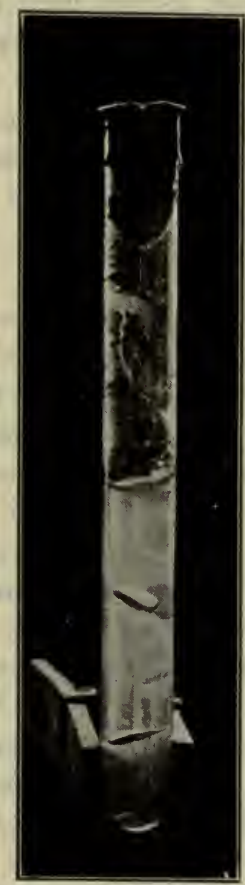

Fig. 15.-Deep Stab Cultivation of AnA ËROBIC BACTERIA.

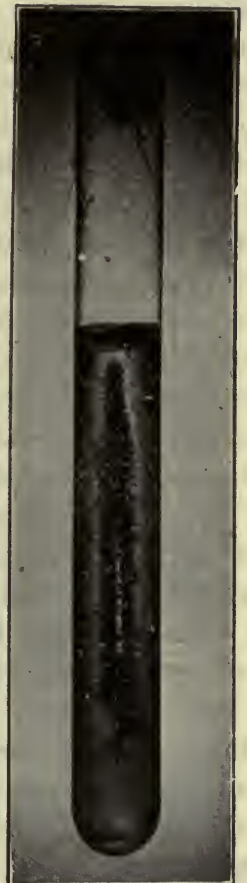

Fig. 16.-Deep Stab Cultivation of AnAËRoBIC BACTERIA.

The exclusion of oxygen for purposes of anaërobic cultivation may be accomplished by a variety of methods, depending upon a few simple principles which have been applied, either separately or in combination, by many workers.

The earliest methods depended upon the simple exclusion of air by meehanical deviees. In other methods, the oxygen of the air is displaced by inert gases (hydrogen), and others again depend 
upon the oxygen-absorbing qualities of alkaline solutions of pyrogallol.

Cultivation by the Mechanical Exclusion of Air.-Koch succeeded in growing anaërobic bacteria upon plates by simply dropping upon the surface of the inoculated agar or gelatin a flat piece of sterile mica. This method, however, rarely succeeds in sufficiently excluding the air.

Liborius' MeтноD. ${ }^{3}$ - This method consists in the use of deeply filled tubes of agar or gelatin, from which all oxygen has been removed by boiling for fifteen minutes or more. It is advantageous, as has been pointed out in the section on anaërobiosis, that media used for this purpose should contain carbohydrates in some form, preferably glucose. After boiling, the tubes are rapidly transferred to ice water so that as little oxygen as possible may be absorbed during the hardening of the medium. The tubes are then inoculated by deep stabs. After inoculation, the medium may be covered with a thin layer of agar, gelatin, or oil (albolin), and further sealed with sealing-wax to prevent oxygen-absorption.

This method may be utilized for the isolation of anaërobes (as in the original method of Liborius) by inoculating the medium just before it solidifies. The tubes may be gently shaken in order to distribute the bacteria throughout the medium and then rapidly cooled. In this case colonies which develop may be seattered throughout the deeper layers of the agar or gelatin, and may be "fished" after breaking the tube.

Esmarch's METhOD. ${ }^{4}$-Von Esmarch has applied the principles of his roll-tube to the cultivation of anaërobic bacteria. Gelatin tubes are inoculated as above and roll-tubes prepared. The tubes are then set into cold water to prevent melting of the thin gelatin layer and the interior of the tube is filled with melted gelatin.

Roux's MEтноD. ${ }^{5}$ - Anaërobic bacteria are cultivated by sucking the inoculated gelatin or agar into narrow tubes, which are then closed at both ends by fusing in the flame. After growth has taken place the tubes are broken and the organism recovered by "fishing."

Fluid Media Covered with OrL.-Erlenmeyer flasks or other vessels are partially filled with glucose-bouillon over which a thin layer of albolin or other oil is allowed to flow. The oxygen is driven out of the liquid by vigorous boiling for fifteen minutes or more.

It should be remembered whenever using this or similar methods that a layer of fluid oil does not form an impermeable seal. By covering an alkaline pyrogallol solution with oil it can easily be shown that oxygen

'Liborius, Zeit. f. Hyg., i, 1886.

"Von Esmarch, loc. cit.

B Roux, Ann. Past., i, 1887. 
slowly diffuses through the oil into the medium below. In using paraffin oil on anaërobic cultures it must be remembered that liquid oil is a very incomplete seal and that solid paraffin or any other solid seal is much more efficient.

The simple exclusion of air, also, is the principle underlying the cultivation of anaërobic bacteria in the closed arm of a Smith fermentation tube.

Wright's Mетнор. ${ }^{6}$ - Wright has described a simple and excellent method for the cultivation of anaërobic bacteria in fluid media. The apparatus necessary is easily improvised with the materials at hand in any laboratory. A short piece of glass tubing, constricted at both ends and fitted at each end with a small piece of soft-rubber tubing, is inserted into a test tube containing nutrient broth. The upper end of the inserted glass tubing is connected by the rubber with a pipette passed through the cotton plug in the tube. The entire apparatus, plus broth, may be sterilized after being put together. When a trarsplant is made, the fluid in the test tube is inoculated as usual. The fluid is then sucked up into the glass tubing until this is completely filled. A downflow of the fluid is then prevented by placing the finger over the pipette through which the suction has been made or by constricting a small piece of rubber tubing attached to the upper end of the pipette. The entire system of tubes is then pushed downward in such a way that both pieces of rubber tubing, attached to the ends of the little glass chamber, are kinked. The entire apparatus may then be incubated. Growth of anaërobic bacteria takes place within the airtight chamber formed by the short glass tubing within the test tube. The fluid in the test tube, outside of this chamber, usually remains clear.

When cultivation has been successful, the bacteria may be obtained either for morphological

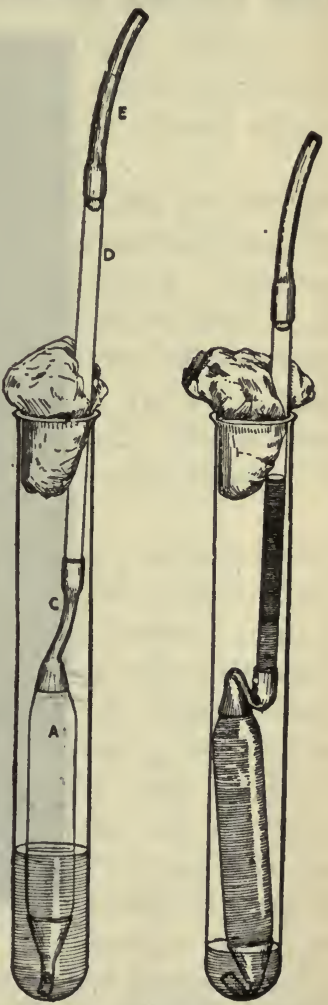

Fig. 17.-Wright's Method of Anä̈robic Cultivation in Fluid Media. study or for further cultivation, by simply allowing the fluid to flow out of the little ail-tight chamber back into the test tube. The method is simple and usually successful.

${ }^{6}$ Wright, J. П. Quoted from Mallory and Wright, "Path. Technique,' Phila., 1904. 
Methods Based upon the Displacement of Air by Hydrogen.-The principle of air-displacement by hydrogen, first utilized by Hauser, ${ }^{7}$ has been widely applied to the cultivation of anaërobic bacteria. In substance it consists of the conduction of a stream of hydrogen through an air-tight chamber in which plates or tubes containing inoculated media have been placed.

For the production of hydrogen, the most convenient apparatus is the Kipp hydrogen generated from zine and sulphuric acid and this may be

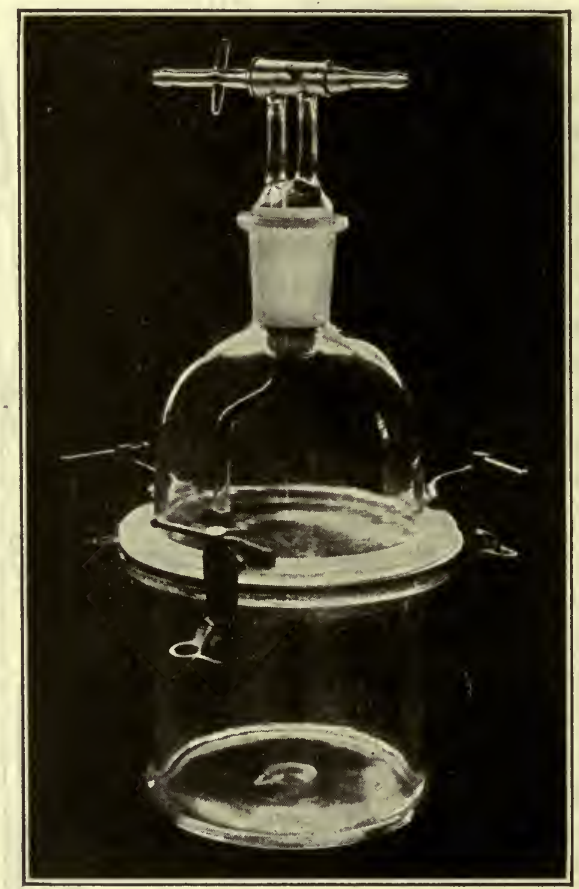

Fig. 18.-Novy JAR.

passed through a series of Woulfe-bottles, containing solutions of lead acetate and of pyrogallic acid, to remove traces of sulphuretted hydrogen and of oxygen, respectively, of Lugol's solution to absorb traces of acid vapor, and of one with a silver-nitrate solution to take up any hydrogen arsenide.

For the preparation of anaërobic conditions where very rigid anaërobiosis is necessary, nitrogen may be used, which ean be bought in tanks from commercial firms.

\footnotetext{
'Hauser, “Ueber Fäulnissbakterien,’' 1885.
} 
For anaërobic cultivation upon solid media, the inoculated tubes or plates are placed in an apparatus such as the Novy jar. This is connected with the hydrogen apparatus and hydrogen allowed to flow through it for five or ten minutes, and the stop-cocks then closed.

In applying the hydrogen method to fluid media, flasks containing the broth are fitted with sterile, tightly fitting rubber stoppers perforated by two holes, through which glass tubes are passed. One of these tubes, the inlet, passes below the surface of the liquid. The other one, the outlet, extends only a short distance below the stopper and is always kept above the surface of the medium. The flasks are inoculated and hydrogen is passed through the medium so that it enters the long tube, bubbles up through the fluid, and leaves by the short tube. The broth may be covered with a thin layer of liquid paraffin or albolin.

The Use of Pyrogallic Acid Dissolved in Alkaline Solutions for Oxygen Absorption.-Buchner ${ }^{8}$ has applied the principle of ehemical absorption for the removal of oxygen to the cultivation of anaërobic bacteria. This has been made use of in a number of different ways. The method is based upon the fact that alkaline solutions of pyrogallol possess the power of absorbing large quantities of free oxygen. At first such solutions are of a light straw-color, which becomes dark brown as oxygen is absorbed. The absorption of all the oxygen in the environment may be assumed when there is no further deepening of the brown color.

Buchner first utilized this principle by placing a small wire or glass holder within a large test tube, dropping dry pyrogallol (pyrogallic acid) into the bottom of this tube, then running thirty per cent sodium hydrate solution into it, and inserting within this large tube a smaller test tube containing the inoculated culture medium. The large tube was then tightly closed with a rubber stopper. In this way, the air space surrounding the smaller tube was rendered oxygen free. An excellent little trick with which to employ the Buchner tube method is to pack lightly over the dry pyrogallie acid in the bottle a small piece of absorbent cotton. This prevents the immediate solution of the pyrogallic acid, and allows one time to pour in the $\mathrm{KOH}$ solution, insert the smaller tube inside the Buchner tube, and tightly insert the rubber stopper in place before solution and oxygen absorption has begun.

A simple modification of the preceding method of Buchner has been devised by Wright. ${ }^{9}$ Stab-cultures of gelatin or agar in test tubes are made in the usual way. The cotton stopper closing the tube is then thrust into the tube to such a depth that its upper end lies at least $1 \mathrm{~cm}$. below the mouth of the tube. A small quantity of sodium or potassium hydrate solution

${ }^{8}$ Buchner, Cent. f. Bakt., I, iv, 1888.

- Wright, Jour. of the Boston Soc. of Med. Sci., Dec., 1900. 
in which some pyrogallic acid has been dissolved, is then allowed to flow on to the cotton of the plug and the mouth of the tube is immediately sealed by a tightly fitting rubber stopper. The eotton stopper in these eases must be made of absorbent cotton; 1.5 to 2.5 e.e. of the pyrogallic acid solution is usually sufficient for test tubes of ordinary size.

For cultivation of anaërobic bacteria upon agar slants, a simple technique

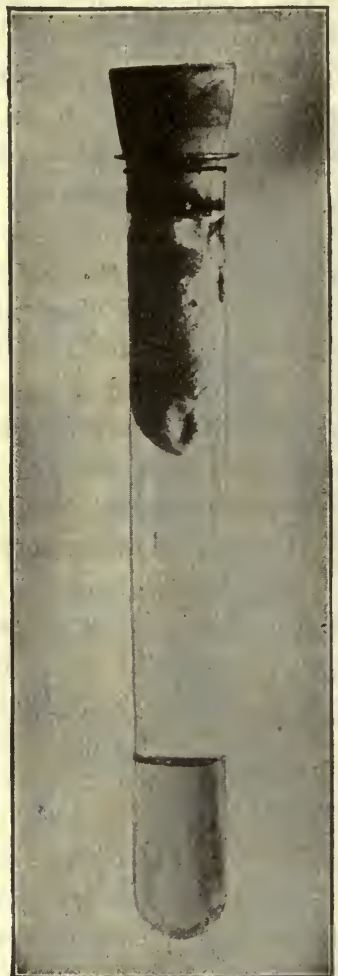

Fig. 19.-Wright's Method of ANä̈robic CultivaTION BY THE USE OF PYROgallic Acid Solution.

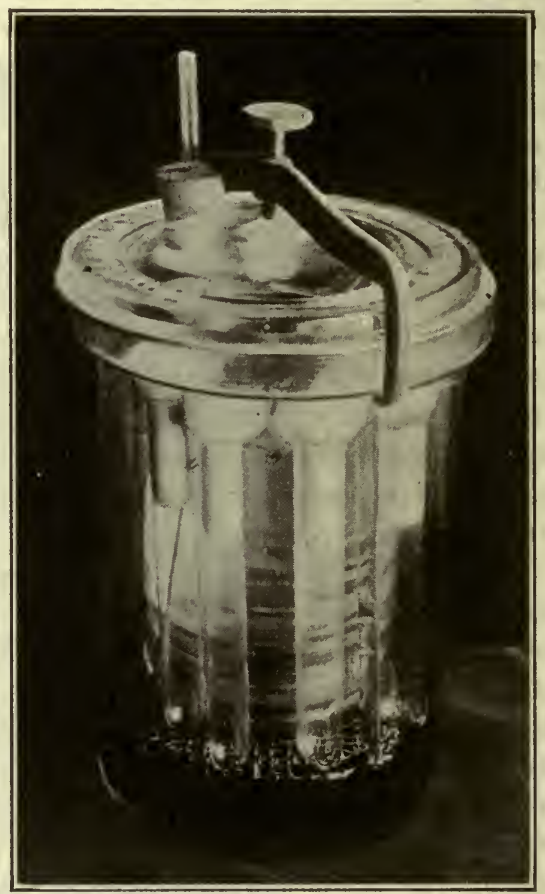

Fig. 20.-Jar for ANaËrobic CULTIVATION.

may be applied and easily improvised in the laboratory as follows: the tube of slant agar is inoculated with the infectious material in the usual way. It is then, with stopper removed, inverted into a tumbler or beaker containing about a gram of dry pyrogallic acid. A small quantity of a five per cent or three per cent sodium hydrate solution is then run into the tumbler and this is covered with a thin layer of liquid paraffin or albolin before the pyrogallic acid has been completely dissolved. In this way, com- 
pletely anaërobic conditions are obtained in the tube and the growth of anaërobes takes place upon the surface of the slant.

For the cultivation of anaërobes in Petri dishes, for purposes of separation, a combination of the pyrogallic acid method and the hydrogen displacement methods is often employed. For this purpose the jars devised by Novy and by Bulloch are extremely convenient.

In using the Novy jar, the inoculated plates are set upon a wire frame resting about an inch above the bottom of the jar. The cover is then tightly set in place and the air in the jar exhausted by means of a suction pump. The arrangement of the double stop-cock in the top renders it possible now, by simply turning this, to admit hydrogen from a Kipp generator into the jar. The process of alternate exhaustion and admission of hydrogen may be several times repeated.

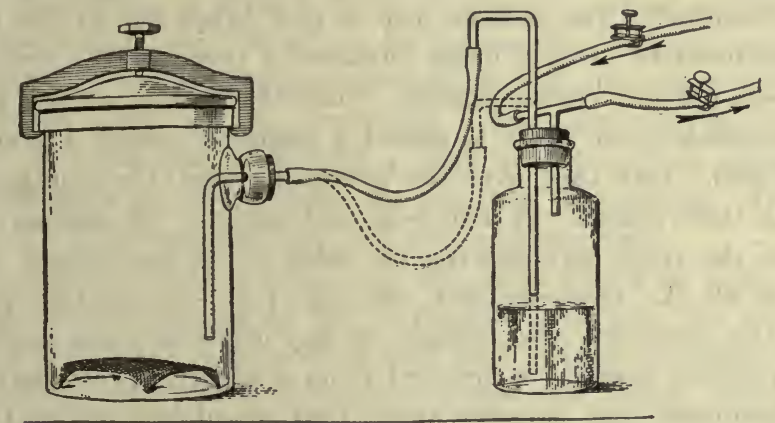

Fig. 21.-Apparatus for Combining the Methods of Exhaustion, Hydrogen Replacement and Oxygen Absorption.

A combination of air exhaustion, oxygen absorption, and hydrogen replacement may be practiced in jars such as that shown in Fig. 21. Dry pyrogallic acid is placed in the bottom of the jar and the cover tightly fitted. An opening in the side of the jar connects its interior with a bottle containing sodium or potassium hydrate solution. Through the stopper of this bottle pass two glass tubes, one of them of such length that it ean be puslied down into the alkaline solution, or pulled upward above the level of the fluid. By this means the $\mathrm{KOH}$ can be sucked into the jar after a vacuum has been produced with the exhaustion pump. The other glass tube is short, passing just through the stopper and at the top made in the form of a $\mathrm{T}$, one arm of the $\mathrm{T}$ being connected with a Kipp hydrogen generator, the other with a suction-pump.

A simple method for the separation of anaërobes: in plates without the use of hydrogen or of specially construeted jars, may be carried out as follows: ${ }^{10}$ The apparatus used consists of two eireular glass dishes, fitting

${ }^{10}$ Zinsser, Jour. Exp. Med., viii, 1906. 
one into the other as do the halves of a Petri dish, and similar to these in every respect except that they are higher, and that a slightly greater space is left between their sides when they are placed together. The dishes should be about three-fourths to one inch in height, they need be of no particular diameter, although those of about the same size as the usual Petri dish are most convenient. An important requirement necessary for the success of the method is that the trough left between the two plates, when put together, shall not be too broad, a quarter of an inch being the mosit favorable.

Into the smaller of these plates the inoculated agar is poured exactly as into a Petri dish in the ordinary aërobic work. Prolonged boiling of the agar before plating is not essential. When the agar film has become sufficiently hard on the bottom of the smaller dish, the entire apparatus is inverted. The smaller dish is now lifted out of the larger, and placed, still inverted, over a moist surface-a towel or the wet surface of the table-to prevent contamination. Into the bottom of the larger dish, which now stands open, there is placed a quantity (about 3 grams) of dry pyrogallic acid. Into this, over the pyrogallic acid, the smaller dish, still inverted, is then placed. A five per cent solution of sodium hydrate is poured into the space left between the sides of the two dishes, in quantity sufficient to fill the receiving dish one-half full. While this is gradually dissolving the pyrogallic acid, albolin, or any other oil (and this is the only step that requires speed), is dropped from a pipette, previously filled and placed in readiness, into the same space, thus completely sealing the chamber formed by the two dishes.

If these steps have been performed successfully, the pyrogallic solution will at this time appear of a light brown color, and the smaller plate, with its agar film, will float unsteadily above the other. Very rapidly, as the pyrogallic acid absorbs the free oxygen in the chamber, this plate is drawn down close to the other, and the acid assumes a darker hue, which remains without further deepening even after three or four days' incubation.

We have described a considerable number of methods of anaërobic eultivation which have been in use. Following our general purpose, however, of emphasizing the methods that we ourselves have found most useful, we will deseribe in the following paragraphs the methods which we are using as routine for anaërobic work in our own laboratory, and which we believe are the most practical.

For anaërobic cultivation on agar slants, we use the Buchner tube method as deseribed above.

For spirochaete cultivation, ete., we use the Noguchi method of narrow deep agar or broth tubes with tissue at the bottom, sterile 
oil at the top, placed into anaërobic jars, or incubated without jars according to purpose.

For anaërobic jar methods, we hardly ever use hydrogen, employing one of the two following:

Jars may be of any size or shape, provided they are well constructed strong museum jars with well fitting lids, preferably ground glass surfaces on lid and top of jar, and with one perforation in the top. Stoppers and glass tubing are fitted into the top of the jars with Major's cement, and recemented every time the jars are used. Glasis tubing which communicates with the exterior through the cemented joints is drawn out in the flame, along its course connecting with the suction pump, and elosure is effected by sealing in these narrow places with the flame.

The fitting of the lid for closure is accomplished by a thick layer of sculptors' plastocene, a smooth layer of which is placed on the top of the jar, and another on the lips of the lid before the lid is put in place. It is useful to have a vacuum gauge cemented into the lid so that the functioning of the suction pump can be controlled. This, however, is not absolutely necessary. A small tube containing a weak methylene blue solution in 1 per cent glucose broth is placed inside the jar with the cultures. Decolorization of this on the following morning will prove anaërobiosis.

I. One method which can be used for Petri plates or tubes consists in placing a suitable amount of dry pyrogallic acid into the bottom of the jar. The cultures are then inserted and next to them is placed an envelope of thick brown paper, such as used by commercial houses, which is torn so as to be open at the top and to form a sort of elongated bag or cornucopia. The glass top of the lid is then connected with the suction pump before it is placed on the jar, the suction pump started while an assistant holds the lid ready for placement on the jar. A suitable amount of 20 per cent $\mathrm{KOH}$ is then poured into the envelope and the lid immediately placed in position on the top of the jar, pushed down on all sides so that the plastocene flattens out between the lid and the jar. The suction immediately begins to draw the air out of the jar, before the $\mathrm{KOH}$ solution works its way through the envelope and begins to dissolve the pyrogallol. The suction is then continued for a suitable length of time, and while the pump is still going a Bunsen burner is placed under a narrow place in the connection glass tube and this is sealed in the flame. This method if successfully carried out, gives almost complete anaërobiosis.

II. The second method is that of McIntosh and Fildes, ${ }^{11}$ which is, in our opinion, the best anaërobic method for application to the

${ }^{11}$ McIntosh and Fildes, Lancet, 190, 1916, 768. Methylene blue added in sufficient quantity to 10 c.c. of a 2 per cent dextrose alkaline broth to give a distinct blue color, is the most convenient anaërobic indicator. It depends on 
growth of tubes and plates in anaërobic jars. It depends upon the removal of oxygen by the oxidation of hydrogen under the influence of palladinized asbestos wool. Pfuhl had previously used the catalytic action of platinum sponge for anaërobic cultivation in broth tubes. McIntosh and Fildes adopted and improved upon this method. The principle of the method is to suspend in an air tight jar a bit of asbestos wool impregnated with platinum or, better, with palladium black. Hydrogen is then allowed to pass in until no oxygen remains. We have used this method in our own laboratory with great success where a number of the workers compared it with other methods, and it has been used successfully at the Rockefeller Institute by Olitsky and others.

An ordinary museum jar, such as those used for anaërobic work, is the ressel employed. The lid is perforated for the passage of hydrogen, and from the bottom of the lid there is suspended a bit of the impregnated asbestos wool, inclosed in a small cage of copper wire. About 0.25 gram of the asbestos wool is the amount recommended by MeIntosh and Fildes.

"The palladium asbestos (about 40 per cent) is made by weighing out 0.25 gram of asbestos wool, placing it in a small evaporating dish, and adding 1.5 e.c. of a 10 per cent solution of palladium ehlorid. The wool is then molded into a flat mass about one inch square, and the dish gently heated until the wool is dry. Since the palladium chlorid is difficult to dissolve, the addition of a little concentrated hydrochloric acid may be necessary. The palladium chlorid is then reduced by heating the impregnated wool, first in a smoky gas flame until it is coated with a layer of carbon, and then in a blow-pipe. The palladium asbestos should now be able to light a jet of hydrogen which is made to impinge on it."

For ordinary purposes the palladium asbestos can be bought. For closure of lids upon the jars, we have used throughout plastocene, the material used by sculptors for molding.

In using the jars the culture tubes are placed inside the jars and the lid rim and the jar rim are covered with plastocene. The copper gauze, with the asbestos wool, is detached and held over a flame until red hot, and is then rapidly put in place, elosing the jar. Through the perforation with proper connections made (best a glass stopper connection) hydrogen is allowed to flow in very slowly, best from a liquid hydrogen cylinder, with careful regulating check valve. It is very necessary to take careful

the fact that in the absence of oxygen the reducing action of an alkaline solution of dextrose changes the methylene blue to the colorless luecomethylene blue. On exposure to the oxygen of the air, the leucomethylene blue is oxidized back to the colored compound. 
precautions that the hydrogen is not allowed to enter too fast, or else explosion may follow, and it is best to make some arrangement like that used in our laboratory by Dr. Teague where the hydrogen is first let into a glass chamber over water, and allowed to flow in very gradually by graded pressure. The main thing is to so work the apparatus that a very slow jet of hydrogen is made to flow in elose to the palladinized asbestos, while this is still lot. A small film of water will begin to form on the sides of the tube, and this is continued until a negative pressure has distinctly developed in the jar. Judgment concerning the amount of hydrogen that should be. let in ean be attained with practice. Closure of the jar may be then made more firm with paraffin or any of the other ordinary methods of making such jars air tight. Plastocene in sufficient amounts has usually served our purpose.

We do not illustrate this method because illustrations rarely help an experienced bacteriologist to any great degree, and it is best to see the apparatus work in some laboratory where it is in use. Many modifications are possible, but the principle is easy to apply in a great many different ways.

By this method it is possible to decolorize methylene blue tubes put into the jars, completely over night, and we believe that this method properly applied gives a practically perfect anaërobiosis.

The Use of Fresh Sterile Tissues as an Aid to Anaërobic Cultivation.-The addition of small pieces of fresh sterile tissue (rabbit or guinea-pig) to culture tubes, either solid or fluid, greatly favors the growth of anaërobic bacteria. By such a method anaërobes can be made to develop even when other precautions for the establishment of anaërobiosis are imperfectly observed. This was noticed first by Theobald Smith and by Tarozzi and has become an extremely useful reënforcement to other methods. It has been utilized most extensively by Noguchi of recent years in his technique for the cultivation of various treponemata. The simplest way to apply this method is to place a piece of freshly excised rabbit kidney, testicle, or spleen into the bottom of a high test tube $(20 \mathrm{~cm}$.) and then pouring over it the culture fluid. Kidney or other tissues are more suitable for this purpose than liver tissue since the latter is not easily obtained in a sterile condition, bacteria often getting into it during life through the portal circulation. The action of the tissues depends upon its great reducing power.

Partial Oxygen Tension for the Growth of Bacteria.-In 1916 Wherry and Oliver ${ }^{12}$ found that partial anaërobiosis was favorable

${ }^{12}$ Wherry and Oliver, Lancet-Clinic, 115, 1916, 306. 
for the growth of gonococcus. Cohen and Markle ${ }^{13}$ applied this to meningococcus and found again that partial anaërobiosis favored growth. It was later found by Wherry and Erwin, ${ }^{14}$ as well as by Cohen and Fleming ${ }^{15}$ that the growth of a number of different bacteria was favored when the air was partially replaced by carbon dioxide. Gates ${ }^{16}$ eonfirmed this, as did Kohman. ${ }^{17}$ We have tried this out in our own laboratory and, like other observers, have found that, in the case of meningococcus and also with the influenza bacillus, growth is definitely stimulated by replacing about 10 per cent of the air with carbon dioxide. Both Kohman, Gates and others believe that the principle underlying this is due to the effect of the carbon dioxide on the reaction of the medium. As the organisms grow they produce acid which displaces equivalent amounts of dissolved carbon dioxide, in consequence of which the acidity is not materially increased.

\section{THE INCUBATION OF CULTURES}

After bacteria have been transferred to suitable culture media, it is necessary to expose them to a temperature favorable to their development. In the ease of many saprophytes, the ordinary room temperature is sufficiently near the optimum to obviate the use of any special apparatus for maintaining a suitable temperature; in the case of most pathogenic bacteria, however, the body temperature of man, $37.5^{\circ} \mathrm{C}$., is either a necessary requirement for their growth, or at any rate favors speedy and characteristic development.

For the purpose of obtaining a uniform temperature of any required degree, the apparatus in general use is the so-called incubator or thermostat. This may be adjusted for gelatin cultivation at 20 to $22^{\circ} \mathrm{C}$, or for agar, broth, or other media at $37.5^{\circ} \mathrm{C}$.

Incubators, while varying in detail, are all constructed upon the same principles. They consist of double-walled copper chambers, which are fitted with a set of double doors, the outer being made of asbestos-covered metal, the inner of glass. The space between the two walls is filled with water, which, being a poor heat con-

${ }^{13}$ Cohen and Markle, Abst. of Bacter., 2, 1918, 10.

${ }_{14}^{14}$ Wherry and Erwin, Jour. of Infee. Dis., 22, 1918, 194.

${ }^{15}$ Cohen and Fleming, Jour. of Infee. Dis., 23, 337, 1918.

${ }^{16}$ Gates, Jour. of Exper. Med., 29, 325, 1919.

${ }^{17}$ Kohman, Jour, of Bacter., 4, 1919. 
ductor, tends to prevent rapid changes of temperature within the chamber as the result of changes in the external environment. Both walls are perforated above by openings to admit thermometers into the interior and one wall is perforated so that a thermo-regulator may be inserted into the water jacket. The under surface of the chamber is heated by a gas flame, the size of which is automatically regulated by the thermo-regulator.

A number of thermo-regulators are on the market, all of them constructed upon modifications of the same principle. One of the most efficient of those in common use is that shown in Fig. 22. This consists of a long tube of glass fitted with a metal cap through which an inlet tube $(A)$ projects

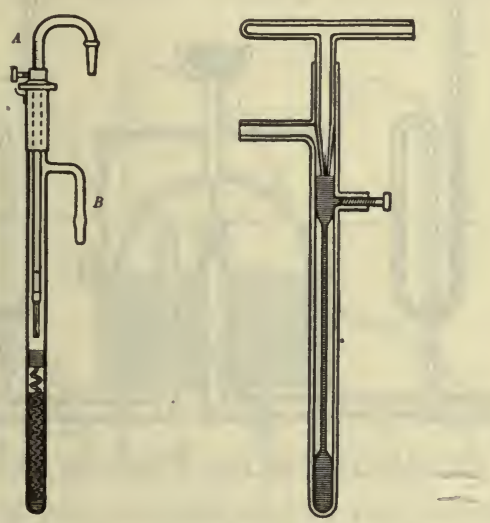

Figs. 22, 23.-Thermo Regulator.

into the interior. Slightly below the middle of the tube there is a glass diaphragm separating its interior into two compartments. In the middle of the diaphragm an aperture leads into a spiral of glass which projects into the lower compartment. The lower compartment is filled with ether and mercury. The lower end of the inlet tube $(A)$ has a wedge-shaped slit. The gas from the supply pipe passing through the tube $(A)$ is conducted through the slit-like opening in its lower end into the inner chamber and passes out to the burner through the elbow $(B)$. When the temperature is raised, the ether and mercury in the lower chamber expand and the mercury rises in the upper chamber, gradually restricting the opening through the $\mathrm{V}$-shaped slit in the inlet tube. Thus the gas supplied to the burner is diminished, the flame reduced, and the temperature again falls. The temperature ean be arbitrarily adjusted by raising or lowering the inlet tube. A scale at the upper end of the inlet tube allows exact adjustment. Complete 
shutting off of the gas is prevented by a small circular opening placed in the inlet tube just above the slit.

Another cheaper and simpler thermo-regulator is shown in Fig. 24. This consists of a long tube open at the top and fitted about $1 \frac{1 / 2}{2}$ inches from the top with two hollow glass elbows. One of these elbows remains open, the other, situated on a slightly lower level, is closed by a brass screw-cap. The tube is filled with mercury to a point slightly above the level of the elbow containing the screw-cap. The height of the mercury can thus be increased or decreased by screwing in or out upon the eap. Into the upper end of the tube there is fitted another device which consists of a T-shaped system of glass tubes, one arm of the $\mathrm{T}$ being open and the other closed, the perpendicular leg of the $\mathrm{T}$ tapering to a minute opening at the bottom.

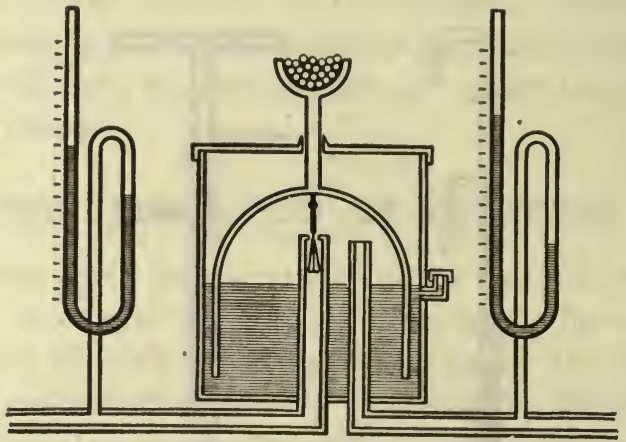

Fig. 24.-Moitegsier Gas Pressure Regulator.

The gas passes into one arm of the $\mathrm{T}$ down through the tapering leg and into the space immediately above the mercury. It then passes out through the open elbow of the main tube. As the mercury rises, it gradually diminishes the space between its surface and the small opening in the tapering tube above it, finally completely shutting off the gas from this source. Gas ean now pass only through a minute hole perforating the vertical leg of the $\mathrm{T}$ an inch above its end. The flame decreases and the temperature again sinks.

Since gas pressure in laboratories is apt to vary, it is eonvenient to interpose between the gas supply and thermo-regulator some one of the various forms of gas-pressure regulators. The use of these is not absolutely necessary but aids considerably in the maintenance of a constant temperature. The one most commonly employed is the so-called Moitessier apparatus. This consists of a eylindrical metal chamber within which there is fitted an inverted metal bell. Glycerin 
is poured into the cylinder to the depth of about two inches. An inlet pipe conducts gas into the open space between the top of the glycerin and the bell. From the top of the bell is suspended a conical piece of metal which hangs free in the outlet pipe. As the gas pressure under the bell increases, this is raised and the opening of the outlet pipe is gradually diminished by the cone. Thus the relation between the pressure in the inlet pipe and the actual quantity of gas passing through is equalized. A cup connected to the top of the bell through the roof of the cylinder by a bar can be filled with birdshot and the pressure against the gas can thus be modified to conform with existing conditions.

Colony Study.-Cultures are usually incubated for from twelve
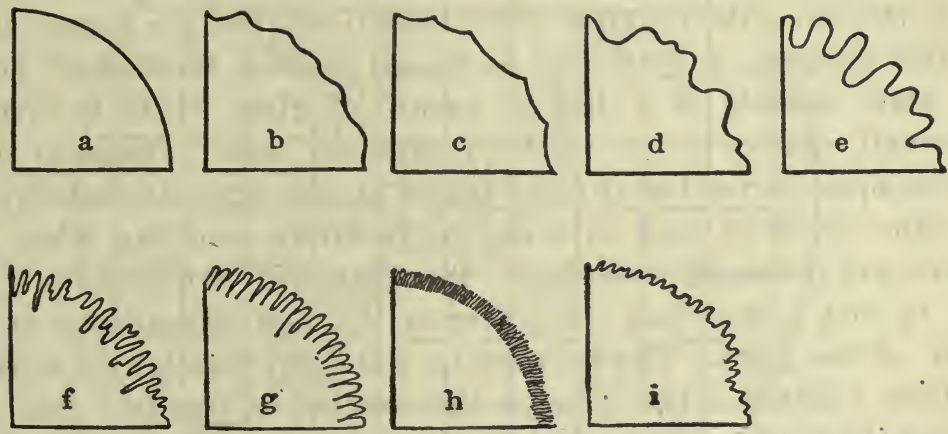

Pig. 25.-Variations in the Conformation of the Borders of Bacterial Colonies.

to forty-eight hours. Considerable aid to the recognition of species is derived from the observation of both the speed of growth and the appearance of the colonies. It is therefore necessary to proceed in the study of developed colonies in a systematic way. The development of colonies should be observed in all eases both upon gelatin and upon agar. In forming any judgment about colonies, the acidity or alkalinity, and the special constitution of the media should be taken into account. The colonies are carefully examined with a hand lens and with the low power (Leitz No. 2, Zeiss AA, Ocular No. 2) of the microscope. The colonies should be observed as to size, outline, transpareney, texture, color, and elevation from the surface of the media. Much information, also, can be obtained by observing whether a colony appears dry, mucoid, or glistening, like a drop of moisture. By a careful observation of these points, definite 
differentiation, of course, can not usually be made, but much corroborative evidence can be obtained which may guide us in the methods to be adopted for further identification and for a final summing up of species characteristic as a whole.

The Counting of Bacteria.-It is often necessary to determine the number of bacteria per c.c. contained in water, milk, or other substances. For this purpose definite quantities of the material to be analyzed are mixed with gelatin or agar and poured into Petri plates. The exact dilutions of the suspected material must largely depend upon the number of germs which one expects to find in it. The plates, if prepared with gelatin, are allowed to develop at room temperature for twenty-four to forty-eight hours. If agar has been used, they are usually placed in the incubator at $37.5^{\circ} \mathrm{C}$. At the end of this time, the colonies which have developed are enumerated. For this purpose, a Petri dish is placed upon a Wolffhügel plate. This plate consists of a disk or square of glass which is divided into small squares of one square centimeter each. Diagonal lines of these squares running at right angles to each other are subdivided into nine divisions each in order to facilitate counting when the colonies are unusually abundant. The Petri dish is placed upon the plate in such a way that the center of the dish corresponds to the center of the plate. The colonies in a definite number of squares are then counted. The greater the number of squares that are counted the more accurate the estimation will be. When the growth is so abundant that only a limited number of squares can be counted, these should be chosen as much as possible from different parts of the plate, and in practice one counts usually six squares in one direction and six at right angles to these, so as to preclude errors arising from unequal distribution. The final calculation is then made by ascertaining the average number of colonies contained in each square centimeter. If standard Petri dishes have been used, this is multiplied by 63.6 , the number of squares in the area of the dish, and then by the dilution originally used.

Thus if twelve squares have been counted with a total number of one hundred and forty-four colonies-the average for each square is twelve. Twelve times 63.6 is 763.2 , which represents the total number of colonies in the plate. Now if 0.1 c.c. of the original material (water or milk) has been plated, this material may be assumed to have contained $10 \times 763.2$, or 7,632 bacteria to each cubic centimeter. 
If dishes of an unusual size are employed, the square area must be ascertained by measuring the radius and multiplying its square by $\pi\left(\pi \times \mathrm{R}^{2}=\right.$ area $)(\pi=3.141592)$.

The number of bacteria in a given volume of a suspension can be estimated by a variety of methods without cultivation. The one most commonly used is the method developed by Wright, which consists in mixing a small measured amount of the bacterial suspension with an equal volume of a red blood cell suspension in which

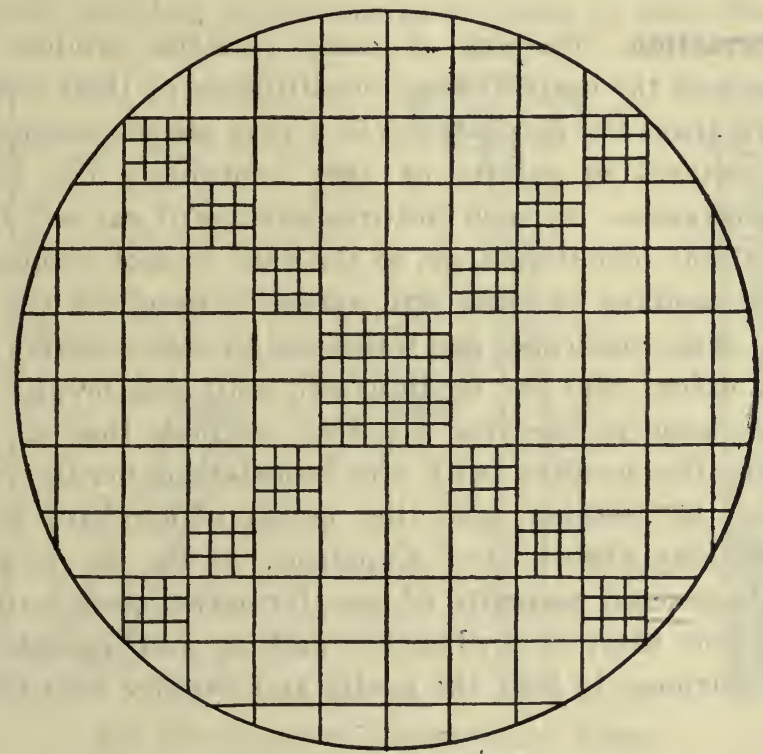

Fig. 26.-Wolffhügel Counting Plate.

the number of erythrocytes per cubic millimeter are known. Smears are made and the relative number of bacteria and red blood cells per one or two hundred fields are counted. A simple calculation can then be made. This method has been described in the section dealing with opsonin technique.

Another useful technique is the direct counting of dilutions of the bacterial suspension, unstained or stained with methylene blue, in a modification of the Thoma Zeiss counting chamber, known as the Helber chamber. 


\section{CHAPTER IX}

METHODS OF DETERMINING BIOLOGICAL ACTIVITIES OF BACTERIA ANIMAL EXPERIMENTATION

Gas Formation.-Bacteria of many varieties produce gas from the protein and the carbohydrate constituents of their environment.

Gas formation ean be observed in a very simple manner by making stab cultures in gelatin or agar containing the fermentable nutrient substances. In such cultures bubbles of gas will form along the track of the inoculation, or, in the case of such semisolid media as the tube medium of Hiss, will spread throughout the tube. In the case of some anaërobes gas formation in stab cultures will occur to such an extent that the medium will split and break. It should be borne in mind in carrying out such methods that air is readily earried into the medium with the inoculating needle or loop by splitting of the medium, also that media which have been stored in the cold may absorb air. Expansion of the air in such tubes may simulate small amounts of gas formation and lead to error. It is advisable, therefore, whenever making stab inoculations with the above purpose, to heat the media and rapidly cool them before use.

A more accurate method of gas determination is by the use of fermentation tubes, such as those devised by Smith. The gas which is formed collects in the closed arm of the fermentation tube and may be quantitatively estimated. The fermentation, with gas production, of certain substances such as carbohydrates, may be determined by adding these materials in a pure state to the media before inoculation with organisms.

In the ease of carbohydrates this method has proved of great differential value, since the power of splitting specific carbohydrates with gas production is a species characteristic of great constancy for many forms of bacteria.

Analysis of Gas Formed by BaCteria.-Carbon Dioxide.-For the estimation both qualitatively and roughly quantitatively of car- 
bon dioxide produced by bacteria, cultures are grown in fermentation tubes containing sugar-free broth (see page 150) to which one per cent of pure dextrose, lactose, saccharose, or other sugars has been added. The tubes are incubated until the column of gas formed in the closed arm no longer increases (twenty-four to fortyeight hours). The level of the fluid in the closed arm is then accurately marked and the column of gas measured.

The bulb of the fermentation tube is then completely filled with $\frac{\mathrm{N}}{5} \mathrm{NaOH}$ solution, the mouth closed with a clean rubber stopper, and, the bulb inverted several times in order to mix the gas with
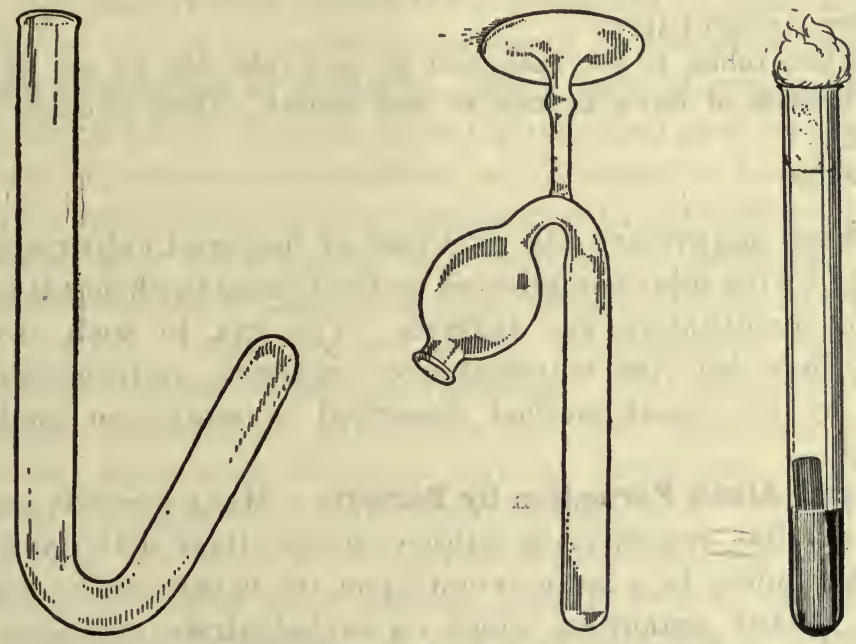

Fig. 27.-Types of Fermentation Tubes.

the soda solution. The tube is then again placed in the upright position, allowing the gas remaining to collect in the closed arm. The gas lost may be roughly estimated as consisting of $\mathrm{CO}_{2}$.

Hydrogen.-The gas remaining, after removal of the $\mathrm{CO}_{2}$ in the preceding -experiment, at least when working with carbohydrate solutions, may be estimated as hydrogen. When allowed to collect near the mouth, further evidence of its being hydrogen may be gained by exploding it with a lighted match.

Hydrogen Sulphid $\left(\mathrm{H}_{2} \mathrm{~S}\right.$, Sulphuretted hydrogen).--In alkaline media, sulphuretted hydrogen, if formed, will not collect as gas, but will form a sulphid with any alkali in the solution. For the estimation of the formation of hydrogne sulphid, bacteria are cul- 
tivated in a strong pepton solution to which 0.1 c.c. of a one per cent solution of ferric tartrate or lead acetate has been added. The addition of these substances gives rise to a yellowish precipitate in the bottom of the tubes. If, on subsequent inoculation, the bacteria produce $\mathrm{H}_{2} \mathrm{~S}$, this precipitate will turn black. The solution recommended by Pake for this test is prepared as follows:

1. Weigh out 30 grams of pepton and emulsify in 200 c.c. of tap water at $60^{\circ} \mathrm{C}$.

2. Wash into a liter flask with 80 c.c. tap water.

3. Add sodium chlorid 5 grams and sodium phosphate 3 grams.

4. Heat at $100^{\circ} \mathrm{C}$. for 30 minutes, to dissolve pepton.

5. Filter through paper.

6. Fill into tubes, 10 c.c. each, and to each tube add 0.1 c.c. of a one per cent solution of ferric tartrate or lead acetate. These solutions should be neutral.

7. Sterilize. ${ }^{1}$

ACCURATE QUANTITAVE GAS ANALYSES of bacterial cultures can be made only by the more complicated methods used in chemical laboratories for quantitative gas analysis. The gas, in such cases, is collected in a bell jar mounted over mercury, and subjected to analysis by the usual method described in works on analytical chemistry.

Acid and Alkali Formation by Bacteria.-Many bacteria produce acid or alkaline reactions in culture media, their activity in this respect depending to a large extent upon the nature of the nutrient material. Many organisms which on carbohydrate media produce acid will give rise to alkali if cultivated upon media containing only proteins.

Information as to the production of acid or alkali can be obtained by the addition of one of a variety of indicators to neutral media. The indicators most often employed for this purpose are litmus, neutral red, China blue and the so-called Andrade indicator. Andrade consists of 100 c.c. of a $0.5 \%$ aqueous solution of acid fuchsin to which 16 c.c. of accurately normal $\mathrm{NaOH}$ has been added. Changes in the color of these indicators show whether acids or alkalis have been produced.

Great help in differentiation is obtained by adding chemically pure carbohydrates to media to which litmus has been added, and

'Quoted from Eyre, “'Bact. Technique,'” Phila., 1903. 
then determining whether or not acid is formed from these substances by the microorganisms. These tests have been of special importance in the differentiation of the typhoid-colon groups of bacilli.

Quantitative estimation of the degree of acidity or alkalinity produced by bacteria may be made by careful titration of definite volumes of the medium before and after bacterial growth has taken place.

The variety of acid formed by bacteria depends largely upon the nature of the nutrient medium. The acids most commonly resulting from bacterial growth are : lactic, acetic, oxalic, formic, and hippuric acids. Qualitative and quantitative estimation of these acids may be made by any of the methods employed by analytical chemists.

Indol Production by Bacteria.-Many bacteria possess the power of producing indol. Though formerly regarded as a regular accompaniment of protein decomposition, later researches have shown that indol production is not always coexistent with putrefaction processes and occurs only when pepton is present in the pabulum.

Indol formation by bacteria is determined by the so-called nitrosoindol reaction. Organisms are grown in sugar-free pepton broth or in the pepton-salt bouillon of Dunham. (See page 151.) Media containing fermentable substances are not favorable for indol production since acids interfere with its formation. The cultures are usually incubated for three or four days at $37^{\circ} \mathrm{C}$. At the end of this time, ten drops of concentrated sulphuric acid are run into each tube. If a pink color appears, indol is present, and we gather the additional information that the microorganism in question has been able to form nitrites by reduction (e.g., cholera spirillum). If the pink color does not appear after the addition of the sulphuric acid alone, nitrites must be supplied. This is done by adding to the fluid about 1 c.c. of a 0.01 per cent aqueous solution of sodium nitrite. The sodium nitrite solution does not keep for any length of time and should be freshly made up at short intervals.

VANILlin Test. ${ }^{2}$-An excellent test for indol is the so-called vanillin test. To 5 c.c. of the culture add 5 drops of 5 per cent vanillin solution in 95 per cent alcohol, and 2 c.c. of concentrated hydrochloric acid or sulphuric acid. If indol is present, an orange color develops within 2 or 3 minutes. Tryptophane gives a reddish

'Steensma, Zeit. f. Physiol, Chem., 47, 1906; Nelson, Jour, of Biol. Chem., 24, 1916. 
violet which grows deeper when the medium is heated or allowed to stand.

Phenol Production by Bacteria.-Phenol is often a by-product in the course of protein cleavage by bacteria. To determine its presence in cultures, bacteria are cultivated in flasks containing about 50-100 c.c. of nutrient broth. After three to four days' growth at $37^{\circ} \mathrm{C}$., 5 c.c. of concentrated $\mathrm{HCl}$ are added to the culture, the flask is connected with a condenser, and about 10-20 c.c. are distilled over.

To the distillate may be added 0.5 e.e. of Millon's reagent (solution of mercurous nitrate in nitric acid), when a red color will indicate phenol; or 0.5 c.e. of a ferric chloride solution, which will give a violet color if phenol is present.

Reducing Powers of Bacteria.-The power of reduction, possessed by many bacteria, is shown by their ability to form nitrites from nitrates. This is easily demonstrated by growing bacteria upon nitrate broth (see page 151). Bacteria are transferred to test tubes containing this solution and allowed to grow in the incubator for four or five days. The presence of nitrites is then chemically determined. ${ }^{3}$

In bacteriological work, 4 e.c. of the culture fluid are poured into a clean test tube, and to it are gradually added 2 c.c. of the mixed test solutions. A pink color indicates the presence of nitrites, the intensity of the color being proportionate to the amount of nitrite present.

The reducing powers of bacteria may also be shown by their ability to decolorize litmus, methylene-blue, and some other anilin dyes, which on abstraction of oxygen form colorless leukobases.

${ }^{3}$ We are indebted to Dr. J. P. Mitchell, of Stanford University, for the following technique for nitrite tests:

I. Sulphanilic Acid.-Dissolve $0.5 \mathrm{~g}$. in 150 c.c. of acetic acid of Sp. Gr. 1.04 . (Acetic acid of 1.04 prepared by diluting 400 c.c. of conc. of sp. gr. 1.75 with 700. c.c. of water.)

II. A-Naphthylamin.-Dissolve $0.1 \mathrm{gr}$. in 20 c.c. of water, boil, filter (if necessary), and to clear filtrate add 180 c.c. of acetic acid, Sp. Gr. 1.04.

The solutions are kept separate and mixed in equal parts just before use.

In carrying out the test, put 2 c.c. of each reagent in a test tube and add substance to be tested. (In ordinary water analysis use 100 c.c.) Cover tube with watch glass and set in warm water for 20 minutes. Observe presence or absence of pink color promptly. Always run a blank on the distilled water used for rinsing to avoid errors due to nitrites in the water, or in the air of the laboratory. 
Enzyme Action.4-The action of the enzymes produced by bacteria may be demonstrated by bringing the bacteria, or their isolated ferments, into contact with the proper substances and observing both the physical and chemical changes produced. In obtaining enzymes free from living bacteria, it is convenient to kill the cultures by the addition either of toluol or of chloroform. Both of these substances will destroy the bacteria without injuring the enzymes. Enzymes may also be obtained separate from the bodies of the bacteria by filtration.

Proteolytic Enzymes.-The most common evidences of proteo-
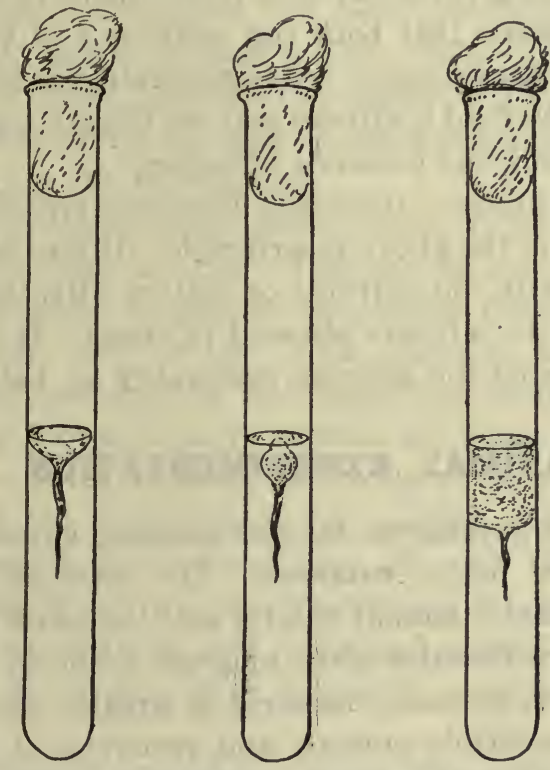

Fig. 28.-Types of Gelatin Liquefaction by Bacteria.

lytic enzyme action observed in bacteriology are the liquefaction of gelatin, fibrin or coagulated blood-serum, and the peptonization of milk. This may be observed both by allowing the proper bacteria to grow upon these media, or by mixing sterilized eultures with small quantities of these substances. ${ }^{5}$ The products of such a reaction may be separated from the baeteria by filtration and then tested for pepton by the biurct reaction.

${ }^{4}$ See also pp. 54 et seq.

'Bitter, Archiv f. Hyg., v. 1886. 
Proteolytic ${ }^{6}$ enzymes may also be determined by growing the bacteria upon fluid media containing albumin solutions, blood serum, or milk serum, then precipitating the proteins by the addition of ammonium sulphate (about 30 grams to 20 c.c. of the culture fluid) and warming between 50 to $60^{\circ} \mathrm{C}$. for thirty minutes. The precipitate is then filtered off, the filtrate made strongly alkalin with $\mathrm{NaOH}$, and a few drops of copper sulphate solution added. A violet color indicates the presence of pepton-proving proteolysis of the original albumin.

Diastatic Enzymes.-The presence of diastatic ferments may be determined by mixing broth cultures of the bacteria with thin starch paste. It is necessary that both the cultures and the starch paste be absolutely free from sugar. After remaining in the incubator for five or six hours, the fluid is filtered and the filtrate tested by methods used for determining the presence of sugars.

Inverting Ferments.--Inverting ferments are determined by a procedure similar to the above in principle. Dilute solutions of cane sugar are mixed with old cultures or culture filtrates of the respective bacteria and the mixture allowed to stand. It is then filtered, and the filtrate tested for glucose, preferably by Fehling's solution.

\section{ANIMAL EXPERIMENTATION}

In the study of pathogenic microorganisms, animal experimentation is essential in many instances. The virulence of any given organism for a definite animal species and the nature of the lesions produced are characteristics often of great value in differentiation. Isolation, moreover, of many bacteria is greatly facilitated by the inoculation of susceptible animals and recovery of the pathogenic organism from the heart's blood or from the lesions produced in various organs. That investigations into the phenomena of immunity would be absolutely impossible without the use of animal inoculation is, of course, self-evident, for by this method only can the action of bacteria in relation to living tissues, cells, and bodyfluids be observed.

The animals most commonly employed for such observations are guinea-pigs, white mice, white rats, and rabbits. The method of inoculation may be either subcutaneous, intrapleural, intraperitoneal,

- Hankin and Westbrook, Ann. Past., vi., 1892. 
intravenous, or subdural, etc. It must be borne in mind always that the mode of inoculation may influence the course of an infection no less than does the virulence of the microorganism or the size of the dose.

Inoculations are made with some form of hypodermic needle fitted to a syringe. The most convenient syringes are the all-glass Luer or the Debove syringes, which, however, are expensive. Any form of sterilizable syringe may be used. In making inoculations the hair of the animal should be clipped and the skin disinfected with carbolic acid or alcohol.

Subcutaneous inoculations are most conveniently made in the abdominal wall, where the skin is thin. After clipping and sterilizing, the skin is raised between the fingers of the left hand and the needle plunged in obliquely so as to avoid penetrating the abdominal wall and entering the peritoneum.

In making intraperitoneal inoculations, great eare must be exercised not to puncture the gut. This can be avoided by passing the needle first through the skin in an oblique direction, then turning it into a position more vertical to the abdomen and perforating the muscles and peritoneum by a very short and carefully executed stab.

Intravenous inoculations in rabbits are made into the veins running along the outer margins of the ears. The hair over the ear is clipped and the animal held for a short time head downward so that the vessels of the head may fill with blood. An assistant holds the animal firmly in a horizontal position, the operator grasps the tip of the ears with the left hand, and carefully passes his needle into the vein in the direction as nearly as possible parallel to its course.

Mice are usually inoculated under the skin near the base of the tail. They may be placed in a jar over which a cover of stiff wire-gauze is held. They are then grasped by the tail, by which they are drawn up between the side of the jar and the edge of the wire cover, so that the lower end of the back shall be easily accessible. The skin is then wiped with a piece of cotton dipped in carbolic solution and the needle is inserted. Great care must be exercised to avoid passing the needle too close to the vertebral column. Mice are extremely delicate, and any injury to the spine usually causes immediate death.

With proper care mice or rats may be easily injected intravenously if a sufficiently fine needle is used. There are four super- 
ficially placed veins running along the tail, which stand out prominently when rubbed with cotton moistened with xylol. Into these the injections are made.

When inoculating rats or guinea-pigs with Bacillus pestis the Kolle vacination method is used. The skin is merely shaved and a loopful of the culture vigorously rubbed into the shaven area.

The various forms of animal holders which have been devised are rarely necessary in bacteriological work unless working unassisted, immobilization of the animals being easily accomplished by the hands of a skilled assistant.

Autopsies upon infected animals must be carefully made. The animals are tied, back down, upon pans fitted in the corners with clamps for the strings. They are then moistened either with hot water or with a weak solution of carbolic acid, so that contamination by hair may be avoided. A median cut is made, the skin is carefully dissected back, and the body cavities are opened with sterile instruments. Cultures may then be taken from exudates, blood, or organs under precautions similar to those recommended below for similar procedures at autopsy upon man.

Inoculated animals should be, if possible, kept separate from healthy animals. Rabbits and guinea-pigs are best kept in galvanized iron-wire eages, which are fitted with floor-pans that can be taken out and cleaned and sterilized. Mice may be kept in battery jars fitted with perforated metal covers. The mice should be supplied with large pieces of cotton upon batting since they are delicately susceptible to cold.

The Bleeding of Animals.-Animals are bled for the purpose of obtaining either corpuscles, defibrinated blood or serum.

In order to obtain small amounts of blood, that is about 5 or 10 c.c., from rabbits, the ear is shaved and had best be immersed in warm water for a few moments in order to expand the vessels. Gentle rubbing with alcohol is also advantageous. The rabbit is then held with head hanging downward, and a broad needle of the Hagedore needle type is thrust into the vein and withdrawn. The drops can be caught directly in a centrifuge tube, or in the culture media for which it is intended. All blood media should be incubated for 24 hours and the contaminated tubes discarded.

A better method is to take blood from rabbits and from guinea pigs directly from the heart. If this is skillfully done the animals can be repeatedly bled without being killed. For taking blood for 
complement in Wassermann reactions, this is among the best methods since large guinea pigs can be alternately bled and rested. Both in rabbits and guinea pigs, bleeding directly from the heart is easily accomplished after a little practice. The anterior thorax of the animal is clipped and painted with tincture of iodin and the operator in feeling for the third interspace close to the sternum had best paint the tips of his fingers with iodin. A twenty-two gauge needle about two inches long is then attached to a syringe and passed downward in the third left interspace close to the sternum, slight suction being exercised at the same time. There is not much purpose in describing this in detail since it can be taught only by practice.

Both rabbits and guinea pigs ean be bled from the earotid. The animal is anesthetized as above, and the carotid laid bare. It is found very close to the trachea, in rabbits lying almost in contact with the trachea, and a little behind it. It is carefully separated from the vagus nerve, and tied off in its distal portion. The thread with which it is tied can be used to handle it thereafter. A sterile glass cannula can be thrust into the artery and the blood taken through this, or else, as we prefer to do it, the side of the artery is picked up with a very fine forceps and held with one hand while it is cut across with a sharp scissors. In this way the blood can be directed straight into a wide mouth flask without being allowed to come in contact with anything until it hits the inside of the flask.

Larger animals, like sheep, goats, horses, are. easily bled by plunging a sterile needle into the external jugular vein which runs in these animals from a line just behind the angle of the lower jaw to the sterno-clavicular junction.

The blood can be run directly into media as for blood agar, blood broth and chocolate medium.

If serum is desired, it can be run into containers of various kinds slanted and allowed to clot in the ice-box.

If defibrinated blood is desired, the blood can be taken directly into sterile flasks containing pieces of broken glass or beads and attenuated before clot. Such blood can be kept in the ice-chest and added to media subsequently.

Blood can be also preserved for culture purposes by the addition of just enough ether to hemolyze it, and added to media in this form. The ether is evaporated off. 


\section{CHAPTER $\mathrm{X}$}

THE BACTERIOLOGICAL EXAMINATION OF MATERIAL FROM PATIENTS AND AN OUTLINE OF THE BACTERIAL FLORA OF THE NORMAL HUMAN BODY

Tecinical procedures for the examination of specimens of exudates, stools, sputum, ete., in various conditions are given in appropriate places in the text dealing with the individual diseases. In this chapter we wish to discuss briefly general principles of bacteriological examination which will be useful in properly collecting and handling materials which are sent to the laboratory for diagnosis or which the bacteriologist takes from the patient himself.

In making bacteriological examinations of material taken from living patients, or at autopsy, the validity of result is as fully dependent upon the technique by which the material is collected, as upon proper manipulation in the later stages of examination.

Material taken at autopsy should be, if possible, directly transferred from the cadaver to the proper culture media. If cultures are to be taken from the liver, spleen, or other organs, the surface of the organ should first be seared with a hot scalpel and an incision made through the capsule of the organ in the seared area, with the same instrument. The platinum needle can then be plunged through this incision and material for cultivation be taken with little chance of surface contamination. When blood is to be transferred from the heart, the heart muscle may be incised with a hot knife, or else the needle of a hypodermic syringe may be plunged through the previously seared heart muscle and the blood aspirated. The same end can be accomplished by means of a pointed, freshly prepared Pasteur pipette. In taking specimens of blood at autopsy it is safer to take them from the arm or leg, by allowing the blood to flow into a broad, deep eut made through the sterilized skin, than from the heart, since it has been found that post-mortem contamination of the heart's blood takes place rapidly, probably through the large veins from the lungs. Exudates from the pleural cavities, the peri- 
cardium, or the peritoneum may be taken with a sterilized syringe or pipette. Under all circumstances it should be remembered that cultures taken from blood or tissues of the cadaver will be contaminated, unless cultures are taken within a few hours after death. Bacteria get into the circulation and multiply throughout the body with astonishing speed after death.

Materials collected at the bedside or in the operating-room should be transferred directly to the proper media or else into sterile test tubes and so sent to the laboratory. When the material is scanty, it may be collected upon a sterile cotton swab, which should be immediately replaced in the sterilized containing tube and sent to the laboratory.

Syringes, when used for the collection of exudates or blood, should be of some variety which is easily sterilizable by dry heat, or boiling. Most convenient of the forms in common use are the all-glass "Luer" syringe, or the cheaper "Sub-Q" model. Instruments which can be sterilized only by chemical disinfectants should not be used. When fluids are collected for bacteriological examination, such as spinal fluid, ascitic fluid, or pleural exudates, it is convenient to have them taken directly into sterilized centrifuge tubes, since it is often necessary to concentrate cellular elements by centrifugalization. By immediate collection in these tubes, the danger of contamination is avoided.

Examination of Exudates.-Pus.-Pus should first be examined morphologically by some simple stain, such as gentian-violet, and by the Gram stain. It is convenient, also, to stain a specimen by Jenner's stain, in order to show clearly the relation of bacteria to the cells. Such morphological examination not only furnishes a guide to future manipulation, but supplies a control for the results obtained by cultural methods. Specimens of the pus are then transferred to the proper media, and pour-plates made or streaks made upon the surface of previously prepared agar or serum-agar plates.

A guide to the choice of media is often found in the result of the morphological examination. In most cases, it is well also to make anaërobic cultures by some simple method. (See page 179 et seq.)

The colonies which develop upon the plates should be studied under the microscope, and specimens from the colonies transferred to cover-glasses and slides for morphological examination and to the various media for further growth and identification. Animal inoculation and agglutination tests must often also be resorted to. A 
knowledge of the source of the material may furnish considerable aid in making a bacteriological diagnosis, though great caution in depending upon such aid is recommended.

If the morphological examination shows Gram-positive micrococci, as in staphylococcus boils, any ordinary properly made agar will suffice.

If streptococci are present in the Gram stain, it will be useful to employ blood agar plates without glucose.

When the pus is gonorrheal, ascitic agar plates with glucose should be used, and the pus transferred directly from the patient to the plate and incubated before it chills. In the case of pus from abrasions of the skin, furuncles or boils that arouse any suspicion of anthrax a careful search for Gram-positive bacilli should be made with the Gram stain, and the characteristic colonies looked for on ordinary agar plates.

When plentiful leucocytes are present and the pus shows no organisms in smear, this should not discourage culture since it is not unusual to obtain colonies on culture when nothing can be found by smear.

In the examination of peritoneal, pericardial, or pleural exudates it is often advantageous to use the sediment obtained by centrifugalization. A differential count of the cells piesent may be of aid in confirming the bacteriological findings. Morphological examination and cultural examination are made as in the case of pus. Specimens should also in these cases be stained for tubercle bacilli. Whenever morphological examinations of such fluids are negative, no bacteria being found, and especially when among the cellular elements the lymphoeytes preponderate, the search for tubercle bacilli should be continued by means of animal inoculation. Guinea-pigs should be inoculated intraperitoneally with specimens of the fluid. The animals will usually die within six to eight weeks, but can be killed and examined at the end of about six weeks if they remain alive. The chances for a positive result are considerably increased if the fluid is set away in the ice-chest until a clot has formed and the animals are inoculated with the material from the broken-up clot.

Spinal Fluid.-Normal spinal fluid is a clear, colorless fluid which contains not more than ten to twelve cells per cubie millimeter. Anything above this should be regarded as suspicious. When clear spinal fluid is brought to the laboratory it is always well to shake up the specimen and do a direct count. 
It is of value also to do a globulin reaction on such clear fluids, which is easily done by Noguchi's butyric acid method as follows:

To 0.2 c.c. of spinal fluid add 0.5 c.e. of a 10 per cent butyric acid solution in physiological salt solution. Boil the mixture and add 0.1 e.c. of normal sodium hydrate, and boil again. A flocculent precipitate forms in positive reactions.

Clear specimens of fluid of this kind should be examined with an intelligent understanding of the nature of the case. Syphilitic spinal fluids are almost always clear, but the cells are increased to 100 or more per cubic millimeter. The cells consist mainly of lymphocytes. Low counts may bè encountered in tabes and general paresis. The determination of these facts will be valuable in connection with the subsequent Wassermann reaction or colloidal gold reaction on these fluids, and with bacteriological examination.

In infantile paralysis or acute poliomyelitis, the spinal fluid is usually clear. The cells here are increased from the beginning. According to Peabody, Draper and Dochez ${ }^{1}$ during the early days of the disease, 80 or more per cent of the cells may be polynuclear. After 72 hours, however, the mononuclears preponderate. The cell count may go up even in the prodromal period. The highest cell count is usually found in the first week, gradually coming down until the fourth week of the disease. The globulin reaction is usually highest in the second and third week, but the writers mentioned above found a percentage of cases in which the cell counts were normal. These facts are given because they should be taken into consideration, together with bacteriological examinations.

Tuberculous fluids are entirely clear, or but slightly turbid. When the suspicion of tuberculosis exists, the fluid should be handled as sterilely as possible, and allowed to stand in the ice-chest until a little, white, thread-like clot appears in the center, which sinks to the bottom of the tube. It is in this clot that tubercle bacilli can be found by careful search. It is smeared on the slide and stained by the usual methods. If enough fluid is available, the residue should be injected into one or two guinea pigs in as large quantities as can be obtained. The cells in tuberculous fluid are chicfly lymphocytes.

Acute meningitis is most commonly caused by the meningococcus, pneumococcus, streptococcus, less commonly by influenza bacilli and

${ }^{2}$ Peabody, Draper and Dochez. 
other organisms. Such fluid may range from slight turbidity to thick purulence. The cells in such fluid consist almost entirely of polynuclear leueocytes during the acute stages. Smears should be made immediately and stained by Gram. If Gram-positive organisms are present, they ean immediately be recognized, and eultures taken accordingly. In epidemic meningitis the Gram-negative meningococci will be found mostly intracellular, but some also extracellular. Sometimes a very prolonged search must be made before any meningococci can be found, because these organisms readily undergo autolysis. In the fluid from an acute case of meningitis in which many polynuclear leucocytes are present, and no organisms ean be found, it is pretty safe to suspect epidemic meningitis, since we have on a number of occasions encountered fluids of this kind in which no organisms could be seen. Cultures should, in all eases, be taken even when the organisms are found, since this may be of value in determining the meningococcus type, in finding out whether the particular meningococcus is agglutinable in the polyvalent serum used, and the collection of the type of meningococci which are present in an epidemic is a part of the bacteriologist's contribution to the successful production of sera. The cultures are best taken on plates of hormone agar with 0.5 per cent glucose, and hemolyzed blood or ascitic fluid added.

Pneumococci, streptococci, influenza bacilli, etc., may be cultivated by appropriate methods.

The cytological character of the fluid and the relationship of cells to bacteria should always be determined since this may have a certain amount of prognostic significance.

Examination of Urine.-Bacteriological examination of the urine is of value only when specimens have been taken with sterile catheters, and eare has been exercised in the disinfection of the external genitals. This is particularly important in the female. Many of the numerous finds of bacillus coli in urine are unquestionably due to defective methods of collecting material. Urine should be centrifugalized and the sediment examined morphologically and pour-plates and surface smears made upon the proper media. If necessary, animal inoculation may be done. In examining urine for tubercle bacilli, special care should be taken in staining methods so as to differentiate from Bacillus smegmatis. When the question is one of infection of one kidney alone the specimens must of course be obtained by ureteral catheterization. 
Examination of Feces.-Human feces contain an enormous number of bacteria of many varieties. Klein, ${ }^{2}$ by special methods, estimated that there were about $75,000,000$ bacteria in one milligram. of feces. It has been a noticeable result of all the investigations upon the feces, that although enormous numbers can be counted in morphological specimens, only a disproportionately smaller number can be cultivated from the same specimen. This is explicable upon the ground that special culture media are necessary for many of the species found in intestinal contents and upon the consideration that many of the bacteria which are present in the morphological specimen are dead, showing that there are bactericidal processes going on in some parts of the intestinal tract, possibly through the agency of intestinal secretions, bile, and the action of the products of metabolism of the hardier species present. By far the greater part of the intestinal flora consists of members of the colon group, bacilli of the lactis aërogenes group, Bacillus fæcalis alkaligenes, Bacillus mesentericus, and relatively smaller numbers of streptococci, staphylococci, and Gram-positive anaërobes. Many other species, however, may be present without being necessarily considered of pathological significance. Certain writers have recently laid much stress upon a preponderance of Gram-positive bacteria in specimens of feces, claiming that such preponderance signifies some form of intestinal disturbance. Herter ${ }^{3}$ has recently advanced the opinion that the presence of Bacillus aërogenes capsulatus in the intestinal canal is definitely associated with pernicious anemia. This is discussed in another section. The determination of these bacilli in the stools is made both by morphological examination by means of Gram stain and by isolation of the bacteria. Such isolation is easily done by the method of Welch and Nuttal. ${ }^{4}$ A suspension of small quantities of the feces in salt solution is made and 1 c.c. of the filtered suspension is injected into the ear vein of a rabbit. After a few minutes the rabbit is killed and placed in the incubator. After five hours of incubation, the rabbit is dissected, and if the Welch bacillus has been present in the feces, small bubbles of gas will have appeared in the liver from which the bacilli may be cultivated in anaërobic stab cultures.

'Klein, Ref. Cent. f. Bakt., I, xxx, 1901.

"Herter, "Common Bacterial Infections of the Digestive Tract,' N. Y., 1907.

'Welch and Nuttal, Bull. Johns Hopkins Hosp., 1892, 111, 81. 
Bacteriological examination of feces is most often undertaken for the isolation of Bacillus typhosus, dysentery, cholera, etc. These methods are diseussed in detail in the chapters dealing with the diseases. See also section on media.

The determination of tuberele bacilli in stools is difficult and of questionable significance, in that they may be present in people suffering from pulmonary tubereulosis as a consequence of swallowing sputum or infected food, and in that there may be other acidbacilli, such as the timothy bacillus, present. Perhaps the most reliable method is to treat a suspension of the feees with 5 per cent antiformin over night, centrifugalize thoroughly, wash the sediment, and inject into guinea pigs.

Blood Cultures.-The diagnosis of septicemia can be positively made during life only by the isolation of bacteria from the blood. Such examinations are of much value and are usually successful if the technique is properly carried out. A large number of methods are recommended, the writers giving, however, only the one which they have found successful and simple for general use.

The blood is taken by preference from the median basilic vein of the arm. If, for some reason (both forearms having been used for saline infusion), these veins are unavailable, blood may be taken from the internal saphenous vein as it turns over the internal malleolus of the ankle joint. The skin over the vein should be prepared before the specimen is taken by painting with iodin, as for a surgical operation. The syringe which is used should be of some sterilizable variety (the most convenient the Luer model), which is easily manipulated and does not draw with a jerky, irregular motion. Its capacity should be at least 10 e.c. It may be sterilized by boiling for half an hour, or preferably, when all-glass syringes are used, they may be inserted into potato-tubes and sterilized at high temperature in the hot-air chamber. Before drawing the blood, a linen bandage is wound tightly about the upper arm of the patient in order to cause the veins to stand out prominently. When the veins are plainly in view, the needle is plunged through the skin into the vein in a direetion parallel to the vessel and in the direction of the blood-stream. After perforation of the skin, while the needle is groping for the vein, gentle suction may be exerted with the piston. Great eare should be exereised, however, that the piston is not allowed to slip back, and air be, by accident, forced into the vessel. In most eases no suction is necessary, the pressure of the 
blood being sufficent to push up the piston. After the blood has been drawn, it should be immediately transferred to the proper media. Epstein has recently recommended the mixture of the blood with sterile two per cent ammonium oxalate solution in test tubes, by which means the clotting is prevented; and transfers can be made more leisurely to culture media. While this method is convenient

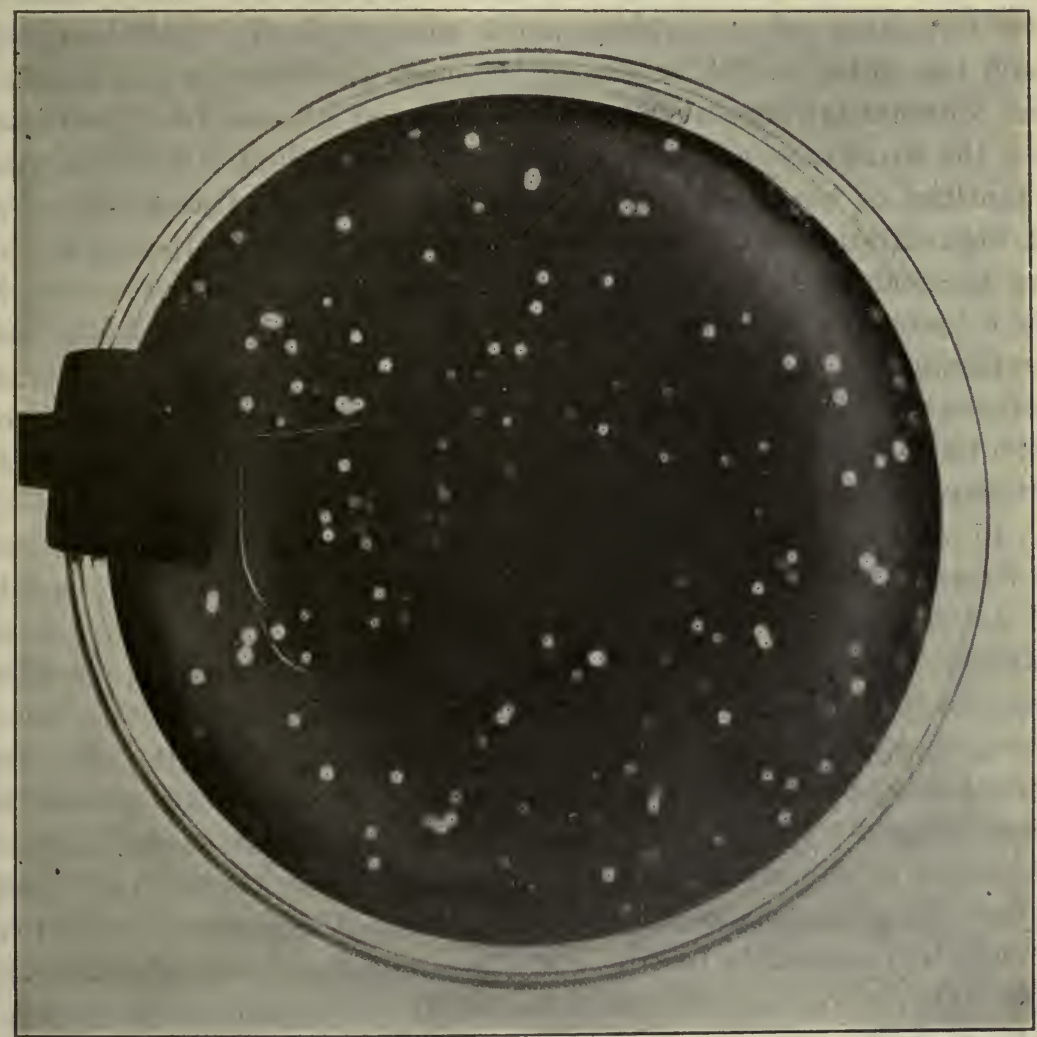

Fig. 29.-Blood-Culture Plate Showing Streptococcus Colonies. Note halo of hemolysis about each colony.

in cases where blood must be taken at some distance from the laboratory, it is preferable, whenever possible, to make cultures from the blood immediately at the bedside.

The choice of culture media for blood cultures should, to a certain extent, be adapted to each individual case. For routine work, it is best to employ glucose "hormone" agar and glucose-meat- 
infusion broth. At least six glucose-agar tubes should be melted and immersed in water at $45^{\circ} \mathrm{C}$. Before the blood is mixed with the medium, the agar should be cooled to $41^{\circ}$ in order that bacteria, if present, may not be injured by the heat. The blood is added to the tubes in varying quantities, ranging from 0.25 to 1 c.c. each, in order that different degrees of concentration may be obtained. Mixing is accomplished by the usual dipping and rotary motion, the formation of air-bubbles being thus avoided. The mouth of each test tube should be passed through the flame before pouring the contents into the plates. Three flasks of glucose broth, containing 100 to 150 c.c. of fluid each, should be inoculated with varying quantities of blood-at least one of the flasks containing the blood in high dilution. The most stringent eare in the withdrawal and replacement of the cotton stoppers should be exercised. ${ }^{5}$ The writers have found it convenient to use, in place of one of these flasks, one containing, in addition to the glucose, $1 \mathrm{gm}$. of powdered calcium carbonate. This insures neutrality, permitting pneumococci or streptococci, which are sensitive to acid, to develop and retain their vitality.

In making blood cultures from typhoid patients, Buxton and Coleman ${ }^{6}$ have obtained excellent results by the use of pure ox-bile containing ten per cent of glycerin and two per cent of pepton in flasks. The writers have had no difficulty in obtaining typhoid cultures by the use of slightly acid meat-extract broth in flasks containing 200 or more c.c. to which comparatively little blood has been transferred. The failure of a proper blood culture service in most hospitals is due, we believe, to the fact that blood cultures are taken by the interne staff, and worked out by the bacteriologist. It is of the utmost importance, in our opinion, that a single individual should be responsible for the entire examination from beginning to end. This is to avoid the great possibility of contamination in blood culture work.

Anärobic Blood Cultures.-These cultures may be taken by mixing blood in deep tubes with glucose-aseitic agar, covering with albolene and putting into Novy jars.

In estimating the results of a blood culture, the exclusion of

'Small Florence flasks are preferable to the Erlenmeyer flasks usually employed.

'Buxton and Coleman, Am. Jour. of Med. Sci., 1907. 
contamination usually offers little difficulty. If the same microorganism appears in several of the plates and flasks, if colonies upon the plates are well distributed within the center and under the surface of the medium, and if the microorganisms themselves belong to species which commonly cause septicemia, such as streptococeus and pneumococcus, it is usually safe to assume that they emanated from the patient's circulation. When colonies are present in one plate or in one flask only, when they are situated only near the edges of a plate or upon the surface of the medium, and when they belong to varieties which are often found saprophytic upon skin or in air, they must be looked upon with suspicion. It is a good rule to look upon all staphylococeus albus cultures skeptically.

Sputum.-In examining sputum, sufficient emphasis cannot be placed upon the necessity of collecting the sputum in a proper way. The sputum collected by patients in the ordinary sputum cup consists to a very large extent of material obtained from the mouth and throat. If a successful examination of sputum is to be made, the patient should be taught to rinse out his mouth thoroughly, and the sputum collected directly after a cough. It is very little to ask for this amount of care, if the examination is really worth making at all. Sputum so collected should not be left in the ward, but should be sent to the laboratory immediately. Smears should be made on such sputum, with an intelligent idea of what is desired.

For pneumoeoccus type examination, the sputum is thoroughly washed and intraperitoneally injected into mice, according to the detailed directions given for typing in the chapter on pneumococcus.

For influenza bacillus examinations, thin smears of the sputum should be stained by Gram and dilute carbol fuchsin. The characteristic grouping of influenza bacilli is of considerable help. Plates of chocolate agar are then streaked.

Sputum for tuberculosis examination is thinly smeared and stained by carbol fuchsin or Hermann's stain. When it is desired to carry the examination beyond this in negative examinations, washed sputum can be injected into guinea pigs, or the sputum can be antiforminized, washed and then injected. Also, the antiforminized sediment can be examined by stain preparation.

Direct culture of tubercle bacillus sputum ean be made by treating with sodium hydrate and plating upon Petroff's gentian-violetegg medium, by the method described in detail in the section on tuberculosis. 
Throat Smears and Throat Examinations.-The bacteriologist, if possible, should take these specimens himself, or the physician taking them should take them only with a clear illumination of the throat, taking his specimen from the exact spot where the lesion is supposed to be located.

For diphtheria examination, the specimen is taken with a sterile swab, and plated directly upon Loeffler's medium. This should be incubated without delay, and the swab sent to the laboratory with the culture. The method has been standardized and is deseribed in the section of diphtheria.

For Vincent's angina examination, smears should be taken and stained, best by strong gentian violet, such as used in the Gram stain, and the smear searched for the characteristic spirilla, and fusiform bacilli. If the patient is in the laboratory, it is best to make a dark field examination.

Examination of Lesions on the Genitatia.-Lesions suspicious of primary syphilitic nature should be gently washed, the superficial pus removed, and only exudate from the bottom of the lesion taken. If necessary, the lesion can be gently scraped and serum, mixed with as little blood as possible, used. No examination for treponema pallidum is equal to the dark field examination. It is important to use only thick slides. A drop of the exudate is placed on the slide and a cover-slip dropped on it. Then a drop of oil is placed on the bottom of the slide, over the preparation and on the top of the cover-slip, and the preparation is placed on the dark field condenser. In doing this, care should be taken to avoid air bubbles in the oil.

When suspicion of chaneroid exists, the material should be inoculated immediately into tubes of coagulated and inactivated sterile rabbit's blood, and incubated according to the method of Teague.

\section{Bacteria Habitually Inhabiting the Normal Human Body}

In studying bacteria in disease, it is of considerable importance to have a clear idea of the morphological and eultural eharacteristics of forms which are frequently encountered in different parts of the human body under normal eonditions.

Various eavities of the body which communicate with the external world, always contain considerable numbers of bacteria representing a large variety of species. Some of these may be habitual saprophytes associated with that particular part of the body, others 
may be accidental and temporary invaders, members of pathogenic groups which, either because of the reduced virulence of the strains or the increased resistance of the individual are not capable under the circumstances of eausing their specific infection.

It is such conditions which may lead to many erroneous etiological conelusions and which render the investigation of the causation of diseases in the mouth, intestines and other locations extremely difficult. It is perhaps best to diseuss this subjeet from the point of view of the individual locations studied.

Bacteria in the Normal Mouth and Pharynx.-The mouth and pharynx are habitually the habitat of numerous bacteria. Saliva itself is not a good culture medium, and, indeed, may, according to some investigators, show very slight inhibitory or even bacterieidal powers. But these, at best, are not very potent, and the saliva thus is a basis for a fluid medium whieh furnishes water as a solvent and a reaction suitable for a great many different bacteria.

Sloughing epithelium, deeayed teeth and gums, food particles, etc., furnish suitable nutrition. Catarrhal inflammation which is rarely entirely absent to some degree or in some place in the adult human being, favors the lodgment of bacteria upon the membranes and reduces the resistance of the tissues.

In spite of these facts, it is surprising that the frequent accidental injury of the gums and oral and pharyngeal mucous membranes so rarely leads to serious infection, and ends so readily. This is a faet which has not as yet been adequately explained.

Staphylococci can almost always be isolated from the mouth. They are usually of the Albus variety, but not infrequently staphylococeus Aureus also ean be found.

Of the streptococci the Viridans is almost always present. The isolation of a "viridans" from inflammatory processes of the mouth and throat, therefore, has very little true significanee, unless it is isolated from a closed process, such as a tooth abseess, or unless other strong corroborative evidence ean be adduced. The Hemolyticus variety is less frequently found in the normal mouth, but may be present without eausing disease. However, the isolation of a hemolyticus from an inflamed tonsil or pharynx is much more likely to mean that there is an etiologieal relationship, and it is of eourse well known that many of the severe inflammations in this location are of hemolytieus origin.

In examirations made many years ago by the writer, 30 per cent 
of people examined harbored pneumococci in their mouths, at one time or another, in the course of the cold months. Since then, the typing of the pneumococcus has made it possible to show that the pneumococci most frequently present in the mouth belong to Group IV. In the investigations of Dochez and Avery which are described in another place, it was found that this type caused only about 9.8 per cent of pneumonias, but was found with considerable frequeney in normal mouths. The other and more virulent types may be found in the normal mouth, as well, but are more apt to represent recent contact with pneumonia cases or a transitory carrier state. This, at least, is suggested by the writers mentioned above, though probably a definite, conclusive statement cannot be made concerning it at the present time.

Of the non-pathogenic Gram-positive cocei the Micrococcus Candicans and occasional pigment forming micrococei are not infrequent.

Micrococcus Tetragenus is very often an inhabitant of the mouth and, as a matter of fact, one sees it most frequently in routine work in Loeffler's cultures taken for the purpose of diphtheria diagnosis.

Of Gram-negative micrococei there is a considerable variety which, without being pathogenic, may be cultivated from the mouth and throat and add no little confusion to meningococcus carrier examinations. Most common among these are the Micrococcus Catarrhalis, which is described in another section, and may be distinguished from the meningococcus by its heavier growth, its growth at room temperature and its failure to produce fermentation of dextrose and maltose. The Micrococcus Flavus, which frequently has led to error in similar work, is a pigment forming Gram-negative coccus often found in the throat, which grows at room temperature, and in most cases agglutinates spontaneously in normal horse serum. Another which forms very dry colonies, the Micrococcus Pharyngis siccus, is often isolated, but easily recognized. In addition to this, Elser and Huntoon have described three different chromogenic groups of similar organisms often found in the mouth and throat. These probably do not exhaust all the possible Gram-negative micrococci that can be isolated from this locality, but it is really only of importance to make sure in human examination whether one is dealing with a true meningococcus, with a micrococcus catarrhalis, or with other saprophytes.

True meningococci are of course often found in normal or slightly inflamed throats during the carrier state, which is discussed at con- 
siderable length in another place. As discussed there, these organisms when they are present are usually located high up in the pharynx near its roof, and successful search for carriers depends very largely upon care of reaching the light spot with the swab.

Of bacilli, the mouth contains a large variety at different times. Few of these, however, are confusing from the bacteriologist's point of view, except some of the diphtheroids. The pseudo-diphtheria bacillus, or Bacillus Hoffmanni, may be present without having any relationship to disease. It is described in another section. The other larger and more irregular diphtheroids are not uncommon, and are easily distinguished from true diphtheria bacilli by their appearance and cultural characteristics.

Chain-forming Gram-positive bacilli and large obviously saprophytic varieties may be present in very dirty mouths, but offer no bacteriological difficulties.

Of the Gram-negative bacilli, Proteus, Lactis Aerogenes, and special members of the Friedländer group may be present. We have known one man who habitually had a Friedländer culture in his mouth, without ever suffering any harm from its presence.

The fusiform bacillus described in another section in connection with Vincent's angina, is almost always present between the gums and the teeth in mouths that are dirty, with carious teeth or where there is some inflammation of the gums themselves. It is an observation that we make almost every year with our students, that, if a platinum. loop is passed between the base of the tooth and the gums, and smears taken from a number of students, the bacteria usually associated with Vincent's angina, spirochætes and fusiform bacilli, can be seen in one or another of the cases examined.

Spirilla and spirochætes are almost habitually present. The Spirillum Milleri, named after Miller, who has made valuable studies upon mouth bacteria, is a small true spirillum, easily cultivated, and not easily confused with other morphologically similar organisms. Miller cultivated three of four varieties of mouth spirilla.

True treponema (Noguchi's classification), are almost always present in locations like those described for the fusiform bacilli, and even on the mucous membranes, especially when small spots of necrosis or inflammation occur. Most frequently discussed among these are the large spirochæte, associated with Vincent's angina, the Spironema Vincenti. There are, likewise, present very frequently the treponema macrodentium and microdentium, classified thus by Noguchi. These 
organisms are best observed under the dark field, but can also be stained in smear if strong gentian violet or carbol fuchsin are used. It is important to note that morphologically the macrodentium is very similar to the treponema pallidum, and in the dark field examination of syphilitic lesions of the mouth and throat, this similarity must be carefully taken into account. We have seen eases in which we were unwilling to make a definite diagnosis on these findings alone. It is our belief that whenever extensive necrosis of the tissues of the mouth and pharynx occur in consequence of other infection or or injury, the necrotic tissues are apt to be invaded by fusiform bacilli. and spirochætes, which in subsequent examination dominate the bacteriological picture. We believe, however, that in the large majority of these cases, perhaps including the clinical picture spoken of as Vincent's angina, the treponemata and fusiform bacilli are secondary to the primary etiological factors, such as those mentioned. These organisms are anaërobic. We believe that the early contention of Tunnicliff that the spirochætes and fusiform bacilli found in Vincent's Angina are different stages of the same organism, is not generally accepted to-day.

The normal mouth is also apt to contain occasional members of the Leptothrix and Streptothrix groups. One of these, the Leptothrix innominata of Miller, is supposed to be characteristic of the mouth flora. It may appear as a large Gram-positive bacillus form which is believed by some writers to be a true bacillus, rather than a leptothrix, and is spoken of as the Bacillus Maximus Buccalis (Miller).

Bacteria in the Nose and Accessory Sinuses.-That the nasal mucosa should be a favorable site for the deposit of numerous microorganisms follows from the fact that air is constantly passing in and out during respiration. The varieties of bacteria to be found in the nose, therefore, may belong to any that happen to be present in the inhaled air.

The subject of the bacteriology of the nose deserves more attention than has been given to it during recent years, for infections of the nasal sinuses and the conditions which lead to them, are being recognized of the utmost importance upon general health. Earlier investigators claimed that the passage of bacteria in the air to the deeper respiratory organs is very largely arrested by a sort of filtering action in the nose. Thomson and Hewlett ${ }^{7}$ found that in

'Thomson and Hewlett, Baumgarten's Jahresb., 12, 1896, 767. 
animals, the tracheal mucus, as well as the mucous membrane of the posterior portions of the healthy nose, are usually sterile, although the vestibulium nasae is usually heavily contaminated. When the nasal cavity and the septum were artificially inoculated with Bacillus prodigiosus, the organisms disappeared in about two hours. These observers believed that the healthy nasal mucus is not bactericidal, but does not favor growth. They examined air which passed through the nose, and found that in the case of air which contained over 20 mould spores and 9 bacteria per cubic centimeter, these organisms almost entirely disappeared in the passage of the air through the nose. Hilderbrandt ${ }^{8}$ has previously obtained similar results in Baumgarten's laboratory. Wright ${ }^{9}$ also has made similar investigations, and showed that between $3 / 4$ to $\frac{4}{5}$ of the bacterial flora of the inspired air was held back in its passage through the nose.

Among the most interesting studies along these lines are those of Neumann ${ }^{10}$ who studied the nasal secretions of over 200 people, of which about 111 were supposedly normal. Neumann found in normal noses a very large number of different microorganisms. The percentage findings of various bacteria were as follows:

Pseudo-diphtheria (probably including diphtheroids) -98 to 100 per cent Micrococcus albus-98 per cent

Micrococcus aureus-30 per cent

Streptococcus lancelatous (probably pneumococcus) -4 per cent

Friedländer bacilli-6 per cent

Micrococcus citreus-12 per cent

Colon bacilli-12 per cent

Streptococcus-2 per cent

Molds-20 per cent

Sarcinae-6 per cent

Lactis aerogenes-4 per cent

Yeasts-2 per cent

Neumann mentions other microorganisms in addition to these, but the figures given are sufficient to show that the normal nose may contain almost any of the known organisms including a great many of the non-pathogenic forms in air, of which the bacteriologist dealing with disease knows very little as a rule. Calamida and

${ }^{8}$ Hilderbrandt, Baumgarten's Jahresb., 4, 1888, 378.

'Wright, quoted from Baumgarten's Jahresb., 5, 1889.

${ }^{10}$ Neumann, Zeit. f. Hyg., 40, 1902, 33. 
Bertarelli ${ }^{11}$ also earried out interesting studies on the normal bacterial flora of the accessory nasal sinuses. Working first with dogs, they found that in 20 dogs of various ages, the frontal sinuses were always sterile. In a single case they isolated an organism which resembled the Colon bacillus.

In 8 dogs the ethmoidal sinuses were sterile. In 16 of 20 dogs the maxillary sinuses, antrum of Highmore, were sterile. In the others they found various cocci. When they inoculated the nasopharynx of dogs with cultures of B. prodigiosus, pyocyaneus, and subtilis, and killed them 8 to 24 hours later, 3 of them showed entirely sterile accessory sinuses and sterile middle ear. One animal gave a positive culture of prodigiosus in the antrum, frontal sinuses, and the ear. In 3 others, only the antrum was infected. Two of the animals treated with $B$. pyocyaneus retained sterile sinuses. Of 6 treated with Subtilis, 4 were entirely sterile. It is interesting to note that the animals that were infected, were those which were killed only 8 to 10 hours after inoculation. Of those which were killed between 18 and 24 hours after inoculation, all but one were sterile. They examined 12 fresh human eadavers within a few hours after death, never later than 20 hours. In all but one of these all accessory sinuses of the nose were sterile, and in this one a nonpathogenic Staphylococeus Albus was found. Kuster, who has summarized work on the nasal flora in the Kolle and Wassermann, on the basis of a study of the literature as well as his own investigations, comes to the conclusion that we cannot speak of a characteristic nasal flora, that practically all of the organisms with which man can come in contact through the air may settle there for a shorter or longer period. In a healthy nose, however, few organisms can gain a permanent foothold, largely because of unsuitable cultural conditions and of the action of leucocytes and the secretions.

Bacteria in the Tissues Themselves.-Recent work has given definite evidence that even the tissues themselves may not be sterile in normal human beings. This has led, we believe, to a certain amount of error in etiological conclusions when blood cultures and culfures from normal or slightly diseased lymphatic tissues have been taken, and diphtheroid and various coecus forms isolated. According to the experiments done by $\Lambda$ dami $^{12}$ there is a constant

${ }^{11}$ Calamida and Bertarelli, Ziet. f. Bakt., I Orig., 32, 1902.

${ }^{12}$ Adami, Jour. A. M. A., Dec., 1899, 
entrance of bacteria into the portal circulation from the intestines. These are very largely disposed of in the liver, but it may well be that the liver does not always eliminate all the bacteria from the portal circulation, and that some of these then lodge in other tissues and become latent there.

The latency of bacteria in the healthy body can no longer be questioned. We have long known that treponema pallidum the spirochætes that infect mice, and many trypanisomes can remain present for a long time in the circulation and in the tissues of animals and man without giving rise to characteristic symptoms or even to any symptoms. We have, ourselves, found pallida in the testes of rabbits four months after inoculation without there having been the slightest tissue reaction, and in human syphilis this latency is well recognized. That tetanus spores may remain latent in the spleen and other organs of guinea pigs under certain experimental conditions, has been shown by the Italian observer, Canfora, ${ }^{13}$ and recently we have seen a very convincing example of latency of streptococci in the tissues of the hand. A very severe hemolytic streptococcus lesion subsided under surgical treatment, and four months later a purely cosmetic secondary operation was undertaken at a time when there was not the slightest trace of infection, and hemolytic streptococei were again isolated from the tissues at this operation. There was, incidentally, no sign of infection of the wound, which healed uneventfully.

The investigations of Torrey and others have shown that from lymph nodes, the seat of various non-bacterial conditions, such as sarcoma, Hodgkin's disease, etc., many varieties of diphtheroids may be isolated, and Rosenau has reported a number of blood culture results in which diphtheroids and cocci were isolated from the blood in the presence of febrile conditions which obviously were not due to the particular organisms isolated.

Not much can be said about this problem of latency at the present time because little is known about it, but the possibility should be kept in mind, and should cause great conservatism whenever isolations from the tissues are made, and the etiological question is raised.

The Bacteriology of the Intestinal Tract.-More than any other part of the body, the intestinal canal has a specific flora of its own. This varies at different ages, with health and disease, and is to

${ }^{13}$ Canfora, Cent. f. Bakt., 45, 1908. 
a considerable extent dependent upon diet. Also, many of the bacteria that cause specific diseases of the intestinal canal, such, for instance, as the typhoid bacillus, the paratyphoid bacilli, the dysentery group, and some of the doubtfully pathogenic organisms like the Morgan bacilli, are very closely related in morphology and cultural reactions to non-pathogenic and saprophytic inhabitants of the bowel. In no type of bacteriological work, therefore, is it more necessary to have an intelligent understanding of the bacterial species that are likely to be found without pathogenic significance.

Furthermore, the intestinal canal is a large test tube from which bacterial products can be absorbed in sufficient amounts to cause severe illness. In it, different kinds of food supply nutritive material which may favor one or another species, and various conditions of aërobiosis and anaërobiosis may prevail. It is more than likely, therefore, that many so-called cases of intestinal poisoning, formerly loosely spoken of as ptomain poisoning, may be caused by substances formed within the intestine by bacterial action upon the food, rather than upon the relatively smaller amount of fermentative and putrefactive products taken in with partially decomposed food.

The intestinal canal of the child at birth is sterile. The meconium of such children has been found by many investigators to be free from bacteria. But this does not last very long. Within a few hours after birth, infection takes place, and from then until death, the intestinal canal is constantly the seat of a voluminous and varied bacterial life. Kendall, ${ }^{14}$ who has written much on this subject, and in his book has brought together much of the information, gathered from the researches of Escherich, ${ }^{15}$ Herter, ${ }^{16}$ and his own investigations, has classified the different stages of the bacterial flora in man, as follows:

1. Bowel at birth, sterile.

2. First to the third day a period of "adventitious bacterial infection."

After this time there is the period of establishment of the characteristic infantile intestinal flora which gradually changes as the diet

${ }^{14}$ Kendall, Bacteriology, General, Pathological and Intestinal, Lea \& Febiger, Phila., 1916, p. 580.

${ }^{15}$ Escherich, Darmbakterien des Sauglings, Stuttgart, 1886, p. 9.

${ }^{16}$ Herter, The Common Bacterial Infections of the Digestive Tract, Harvey Lect., 1906-1907. 
approaches more and more that of the adult, into the characteristic flora of the adult.

In the earliest days during the stage of "adventitious infection," when the child is getting its first bacteria from the air and subjects with which its mouth comes in contact, the bacterial flora is determined largely by accident.

When the child begin to take food, it is of great importance for the determination of the bacterial flora, whether it is being breast fed or being fed on artificially modified cows' milk.

In breast fed children, the upper part of the small intestine will usually contain enterococcus, strèptococcus lacticus, and a general predominance of the coccoid form. Lower down toward and behind the ileocecal valves, the $B$. lactis aërogenes and the Colon bacilli appear. In the lower parts of the cecum and the rectum, the anaërobic Bacillus bifidus of Tissier and similar anaërobes predominate, and many proteolytic bacteria may be present.

In contrast to this, in artificially fed infants, the bowel is relatively richer in the Colon group, and the B. aërogenes type; B. mesentericus and other anaërobic spore formers will be present in considerable numbers, and in the lower bowel the B. bifidus types are largely replaced by Colon bacilli, B. acidophilus and similar organisms. Very early there may be also present in children a curious little tetanus-like organism spoken of as Bienstock's B. putrificus.

Tissier, who has done a great deal of work on this problem, described the flora of a five year-old child in which the gradual transition from the milk to the mixed diet was taking place as follows. We quote from Kuster. ${ }^{17}$ "Constant fundamental flora, B. bifidus, enterococcus, Colon bacillus, B. acidophilus. Variable adventitious organisms, B. perfringens, cocci, and a number of other Gram-negative bacilli, together with some yeasts."

As adult life is attained, there is a gradual relative increase of organisms of the Colon type, which eventually constitute about 75 per cent of the intestinal bacteria.

In the normal adult the stomach is usually sterile. The duodenum contains a few cocci and Gram-positive and negative bacilli, not of the Colon type. There is a gradual numerical increase of bacteria downward. In the jejunum, or upper ileum, the Colon types begin

${ }^{17}$ Kuster, Kolle and Wassermann, 2nd Edition, Vol. 6, p. 469. 
to grow numerous, and in the cecum and colon, which are the seat of the greatest bacterial activity, the flora consist chiefly of Colon types, B. mesentericus, a few anaërobic spore formers of the Welch bacillus type, and Gram-positive cocci.

All who have studied this subject have found that diet has a definite and important bearing upon the intestinal flora and that definite changes may be brought about in the bacterial contents of the bowel by purposefully adjusting the diet. The studies of Herter $^{16}$ and of Kendall, ${ }^{14}$ particularly, have been contributed to our knowledge of this subject. Herter has laid particular stress upon the importance of the Welch bacillus and its subvarieties upon intestinal putrefaction. In this he is not entirely in agreement with Rettger $^{18}$ and others who believe that the Welch bacillus attacks proteins but slightly, being chiefly concerned with carbohydrate fermentation. Herter has produced indicanuria in dogs by feeding large amounts of meat, and found that with such feeding the colon and ileum contained considerable numbers of anaërobic bacilli. He also believes that this bacillus is particularly concerned with a chronic putrefactive activity which takes place in the large intestine, in the course of which anaërobic bacilli produce butyric acid. In consequence of this, there may be a considerable intestinal irritation and carbohydrate intolerance. Considerable anuria may also be a consequence. Other writers like Friedman ${ }^{19}$ believe that constipation favors the increase of these putrefactive organisms. Simonds ${ }^{20}$ has made an exhaustive study of the relationship of the Welch bacillus group to intestinal conditions, and has reviewed the literature extensively. He summarizes his studies on this problem as follows: "In the case of gas bacillus diarrhea, the presence of an excess of carbohydrates in the intestinal content brings about conditions in the lower ileum and first part of the colon which are particularly conducive to the growth of B. Welchii. The absence of lactic acid producing bacteria, as pointed out by Kendall, renders conditions still more favorable to the multiplication of these organisms. They, therefore, rapidly increase in numbers, produce irritating butyric acid, and are swept on in excessive numbers into the lower bowel. The number of spores produced will be measurably

${ }^{18}$ Rettger, Jour. of Biol. Chem., 2, 1906, 71.

${ }^{10}$ Friedman, Transac. of Chicago Pathol. Soc., 1901, cited from Simonds.

${ }^{20}$ Simonds, Monograph of Rock. Institute, No. 5, 1915. 
proportional to the number of bacilli which reach the lower part of the bowel; hence, the excessive number of spores of Bacillus Welchii in the stools in cases of gas bacillus diarrhea." Simonds' results substantiate the work of Kendall and Day ${ }^{21}$ to the effect that children and adults with diarrhea who showed large numbers of gas bacilli in the stools are made worse by feeding sugars, and that prompt improvement results when the diet is changed to one largely composed of protein. An absence of lactic acid by the feeding of butter-milk still further aids in eliminating the Welch bacillus. Kendall has shown by prolonged experimentation on monkeys, dogs and cats that feeding with cows' milk, to which sufficient lactose has been added to simulate breast milk, produces a bacterial flora in such animals which approaches that of the normal nursing infant. The stools take on an acid reaction, and organisms like B. bifidus and the Enterococcus begin to predominate. In order to bring this about, he states, it is necessary to continue the feeding for considerable periods. Kendall divides the pathological cases in which it can be reasonably suspected that abnormal bacterial conditions of the intestinal tract play a causative part, into those which are due to the action of the bacteria upon proteins, and those in which it is chiefly a matter of carbohydrate fermentation. In the case of the abnormal proteolytic processes, there may be a liberation of substances like histamin and other toxic amines, and there may even be the formation of specific toxins such as those which have been recently produced by Bull and others from the Welch bacillus. Abnormal carbohydrate splitting may result in hyperacidity and in stasis of the bowel, and secondary putrefaction in consequence of this.

It is due to studies like those of the writers mentioned above that we may hope to be able to exert considerable therapeutic influence upon abnormal intestinal conditions by altering the flora of the intestine, on the one hand by controlling the diet, and on the other hand by inoculating with, or, in other words, feeding bacteria of a type which may correct the condition that exists.

A detailed study of these therapeutic measures cannot be given in this place. They are treated of in articles like those of Coleman and Shaffer, ${ }^{22}$ in Kendall's book from which we have quoted freely,

${ }^{21}$ Kendall and. Day, Boston Med. and Surg. Jour., 1911, 741 and 1912, 753.

${ }^{22}$ Coleman and Shaffer, Archiv. Inter. Med., Vol. 4, 1909. 
and in the publications of Herter, Kendall, Rettger, Torre ${ }^{23}$ and others.

\section{Bacteria Important Because of Their Frequent Presence} IN the Intestinal Channel

Bacillus Acidophilus. ${ }^{24}$-A Gram-positive bacillus which easily undergoes granular degeneration and grows under conditions of acidity, not supported by most other bacteria. It is closely related to the Bacillus bulgaricus, does not form spores, and is closely related to a group of similar organisms which have not been sufficiently studied to be conclusively classified. (See Kendall, Jour. Med. Res., 1910, 22, and Rahe, Jour. Infec. Dis., 15, 1914, 41.) Its isolation can, according to Kendall, best be accomplished by inoculating the original material into dextrose broth containing 0.25 per cent acetic acid. Two or three transfers on a medium like this after intervals of several days will give pure cultures. Does not liquefy gelatin, has been associated by Escherich with acute diarrhea in children. Does not produce gas.

Bacillus Acidophilus Aerogenes.-Described by Torrey and Rahe ${ }^{25}$ which closely resembles the Bacillus acidophilus, except that it produces gas in mono-, di- and some polysaccharids.

Bacillus Bifidus of Tissier.-This is a strictly anaërobic bacillus. It was described by Tissier in 1900. (See also, Noguchi, Jour. Exper. Med., 12, 1910, 182.) It is spoken of as Bifidus because it will often show a bifid branching at the ends, a condition in which it is not shown in smears from the intestinal contents. It is found early in the stools of nursing children. It produces considerable amounts of acid, but no gas from carbohydrates. Morphologically this organism is often described as Gram-positive, but, as a matter of fact, many members are Gram-negative, and often the bacilli themselves may be Gram-negative with Gram-positive granules scattered through them.

Bacillus Mesentericus.-This is a Gram-positive, aërobic, spore bearing bacillus which is active proteolytic and concerned with putrefaction in the intestinal channel. The organism is more closely

${ }^{23}$ Torrey, Jour. of Infec. Dis., 16, 1915, 72.

${ }^{24}$ Moro, Wien. klin. Woch., 5, 1900 ; Finkelstein, Deut. med.. Woch., 22, 1900.

${ }^{25}$ Torrey and Rahe, Jour. of Infec. Dis., 17, 1915, 437. 
related to the common hay bacillus or Subtilis. It is actively motile, forms spores and grows with great ease on the simplest media. It differs from the ordinary Subtilis bacillus in that it does not ferment dextrose. Kendall ${ }^{26}$ has shown that symbiosis of the B. Mesentericus with the Colon bacillus in milk will produce a greatly increased metabolism of both.

Bacillus Putrificus of Bienstock. ${ }^{27}$-This is a Gram-positive anaërobic bacillus which forms end spores, and, therefore, morphologically resembles the tetanus bacillus. It is identical probably with Klein's bacillus cadaveris sporogenes. It is capable of producing powerful proteolytic cleavage in milk, cheese, etc. Bienstock believes that it is present constantly in normal feces.

${ }^{26}$ Kendall, Boston Med. and Surg. Jour., 163, 1910.

${ }^{27}$ Bienstock, Archiv. f. Hyg., 36, 335, 1899, and 39, 390, 1901. 


\section{SEGTION II}

\section{INFECTION AND IMMUNITY}

\section{CHAPTER XI}

\section{FUNDAMENTAL FACTORS OF PATHOGENICITY AND INFECTION}

WHEN microorganisms gain entrance to the animal or human body and give rise to disease, the process is spoken of as infection.

Bacteria are ever present in the environment of animals and human beings and some find constant lodgment on various parts of the body. The mouth, the nasal passages, the skin, the upper respiratory tract, the conjunctivæ, the ducts of the genital system, and the intestines are invariably inhabited by numerous species of bacteria, which, while subject to no absolute constancy, conform to more or less definite characteristics of species distribution for each locality. Thus the colon organisms are invariably present in the normal bowel, Döderlein's bacillus in the vagina, Bacillus xerosis in many normal conjunctivæ, and staphylococeus, streptococcus, various spirilla, and pneumococcus in the mouth. In contact, therefore, with the bodies of animals and man, there is a large flora of microorganisms, some as constant parasites, others as transient invaders; some harmless saprophytes and others capable of becoming pathogenic. It is evident, therefore, that the production of an infection must depend upon other influences than the mere presence of the microorganisms and their contact with the body, and that the occurrence of the reaction-for the phenomena of infection are in truth reactions between the germ and the body defenses-is governed by a number of important secondary factors.

In order to cause infection, it is necessary that the bacteria shall gain entrance to the body by a path adapted to their own respective cultural requirements, and shall be permitted to proliferate after gaining a foothold. Some of the bacteria then cause disease by 
lapid multiplication, progressively invading more and more extensive areas of the animal tissues, while others may remain localized at the point of invasion and exert their harmful action chiefly by local growth and the elaboration of specific poisons.

The inciting or inhibiting factors which permit or prohibit an infection are dependent in part upon the nature of the invading germ and in part upon the conditions of the defensive mechanism of the subject attacked.

Bacteria are roughly divided into two classes, saprophytes and parasites. The saprophytes are those bacteria which thrive best on dead organic matter and fulfill the enormously important function in nature of reducing by their physiological activities the excreta and dead bodies of more highly organized forms into those simple chemical substances which may again be utilized by the plants in their constructive processes. The saprophytes, thus, are of extreme importance in maintaining the chemical balance between the animal and plant kingdoms. Parasites, on the other hand, find the most favorable conditions for their development upon the living bodies of higher forms.

While a strict separation of the two divisions cannot be made, numerous species forming transitions between the two, it may be said that the latter, class comprises most of the so-ealled pathogenie or disease-producing bacteria. Strict saprophytes may eause discase, but only in eases where other factors have brought about the death of some part of the tissues, and the bacteria invade the necrotic areas and break down the proteins into poisonous chemical substances such as ptomains, or through their own destruction give rise to the liberation of toxic eonstituents of their bodies. It âs necessary, therefore, that bacteria, in order to ineite disease, should belong strictly or facultatively to the class known as parasitic. It must not be forgotten, however, that the terms are relative, and that bacteria ordinarily saprophytie may develop parasitic and pathogenic powers when the resisting forees of the invaded subject are redueed to a minimum by chronic constitutional disease or other eauses.

Organisms that are parasitic, however, are not neeessarily pathogenic, and there are certain more or less fundamental requirements which experience has taught us must be met by an organism in order that it may be infectious (or pathogenic) for any given animal; and by infectiousness is meant the ability of an organism 
to live and multiply in the animal fluids and tissues. For instance, an organism which is shown not to grow at the body temperature of warm-blooded animals may safely be assumed not to be infectious for such animals; and experience is gradually teaching us that strictly aërobic organisms, those thriving only in the presence of free oxygen and not able to obtain this gas in available combination from carbohydrates, can also be safely excluded from the infectious class. We have also learned that anaërobic organisms, although infectious when gaining entrance to tissues not abundantly supplied with blood, are practically unable to multiply in the blood stream and give rise to generalized infection.

The pathogenic microorganisms differ very much among themselves in the degree of their disease-inciting power. Such power is known as virulence. Variations in virulence occur, not only among different species of pathogenic bacteria, but may occur within the same species. Pneumococci, for instance, which have been kept upon artificial media or in other unfavorable environment for some time, exhibit less virulence than when freshly isolated from the bodies of man or animals. It is necessary, therefore, in order to produce infection, that the particular bacterium involved shall possess sufficient virulence.

Whether or not infection oceurs depends also upon the number of bacteria which gain entrance to the animal tissues. A small number of bacteria, even though of proper species and of sufficient virulence, may easily be overcome by the first onslaught of the defensive forces of the body. Bacteria, therefore, must be in sufficient number to overcome local defenses and to gain a definite foothold and carry on their life processes, before they can give rise to an infection. The more virulent the germ, other conditions being equal, the smaller the number necessary for the production of disease. The introduction of a single individual of the anthrax species, it is claimed, is often sufficient to cause fatal infection; while forms less well adapted to the parasitic mode of life will gain a foothold in the animal body only after the introduction of large numbers.

The Path of Infection.-The portal by which bacteria gain entrance to the human body is of great importance in determining whether or not disease shall occur. Typhoid bacilli rubbed into the abraded skin may give rise to no reaction of importance, while the same microorganism, if swallowed, may cause fatal infection. Conversely, virulent streptococei, when swallowed, may cause no 
harmful effects, while the same bacteria rubbed into the skin may give rise to a severe reaction.

Animals and man are protected against invasion by bacteria in various ways. Externally the body is guarded by its coverings of skin and mucous membranes. When these are healthy and undisturbed, microorganisms are usually held at bay. While this is true in a general way bacteria may in occasional cases pass through uninjured skin and mucosa. Thus the Austrian Plague Commission found that guinea-pigs could be infected when plague bacilli were rubbed into the shaven skin, and there can hardly be much doubt of the fact that tubercle bacilli may occasionally pass through the intestinal mucosa into the lymphatics without causing local lesions.

Even after bacteria of a pathogenic species, in large numbers and of adequate virulence, have passed through a locally undefended area in the skin or mucosa of an animal or a human being by a path most favorably adapted to them, it is by no means certain that an infection will take place. The bodies of animals and of man have, as we shall see, at their disposal certain general, systemic weapons of defense, both in the blood serum and the cellular elements of blood and tissues which, if normally vigorous and active, will usually overcome a certain number of the invading bacteria. If these defenses are abnormally depressed, or the invading microorganisms are disproportionately virulent or plentiful, infection takes place.

Bacteria, after gaining an entrance to the body, may give rise merely to local inflammation, necrosis, and abscess formation. They may, on the other hand, from the local lesion, gain entrance into the lymphatics and blood-vessels and be carried freely into the circulation, where, if they survive, the resulting condition is known as bacteremia or septicemia. Carried by the blood to other parts of the body, they may, under favorable circumstances, gain foothold in various organs and give rise to secondary foci of inflammation, necrosis, and abscess formation. Such a condition is known as pyemia. The disease processes arising as the result of bacterial invasion may depend wholly or in part upon the mechanical injury produced by the process of inflammation, the disturbance of function caused by the presence of the bacteria in the capillaries and tissue spaces, and the absorption of the necrotic products resulting from the reaction between the body cells and the microorganisms. To a large extent, however, infectious diseases are characterized by the symptoms result- 
ing from the absorption or diffusion of the poisons produced by the bacteria themselves.

Bacterial Poisons.-It was plain, even to the earliest students of this subject, that mere mechanical capillary obstruction or the absorption of the products of a local inflammation were iusufficient to explain the profound systemic disturbances which accompany many bacterial infections. The very nature of bacterial disease, therefore, suggested the presence of poisons.

It was in his investigations into the nature of these poisons that Brieger ${ }^{1}$ was led to the discovery of the ptomains. These bodies, first isolated by him from decomposing beef, fish, and human cadavers, have found more extended discussion in another section. Accurately classified, they are not true bacterial poisons in the sense in which the term is now employed. Although it is true that they are produced from protein material by bacterial action, they are cleavage products derived from the culture medium upon the composition of which their nature intimately depends. The bacterial poisons proper, on the other hand, are specific products of the bacteria themselves, dependent upon the nature of the medium only as it favors or retards the full development of the physiological functions of the microorganisms. The poisons, produced to a greater or lesser extent by all pathogenic microorganisms, may be of several kinds. The true toxins, in the specialized meaning which the term has acquired, are soluble, truly secretory products of the bacterial cells, passing from them into the culture medium during their life. They may be obtained free from the bacteria by filtration and in a purer state from the filtrates by chemical precipitation and a variety of other methods. The most important examples of such poisons are those elaborated by Bacillus diphtheriæ and Bacillus tetani. If cultures of these bacteria or of others of this class are grown in fluid media for several days and the medium is then filtered through porcelain candles, the filtrate will be found toxic often to a high degree, while the residue will be either inactive or comparatively weak. Moreover, if the residue possesses any toxicity at all, the symptoms evidencing this will be different from those produced by the filtrate.

There are other microorganisms, however, notably the cholera spirillum and the typhoid bacillus, in which no such exotoxins are formed. If these bacteria are cultivated and separated from the eul-

\footnotetext{
${ }^{1}$ Brieger, “Die Ptomaine," Berlin, 1885 and 1886.
} 
ture fluid by filtration, as above, the fluid filtrate will be toxic to only a very slight degree, whereas the residue may prove very poisonous. In these cases, we are dealing, evidently, with poisons not secreted into the medium by the bacteria, but rather attached more or less firmly to the bacterial body. Such poisons, separable from the bacteria only after death by some method of extraction, or by autolysis, were termed by Pfeiffer endotoxins. The greater number of the pathogenic bacteria seem to act chiefly by means of poisons of this class. The first to call attention to the existence of such intracellular poisons was Buchner, who formulated his conclusions from the results of experiments made with a number of microorganisms, notably the Friedländer bacillus and Staphylococeus pyogenes aureus, with dead cultures of which he induced the formation of sterile abscesses in animals and symptoms of toxemia. The conception of "endotoxins," received its clearest and most definite expression in the work of Pfeiffer ${ }^{2}$ on cholera poison.

Some clarity of conception, based on visual perception, may possibly be gained by comparing some of the products of pathogenic bacteria with bacterial pigments and with insoluble interstitial or intercellular substance, which may be seen accompanying bacteria in cover-glass preparations. Soluble toxic secretions are to be compared to such pigments as the pyocyanin of Bacillus pyocyaneus, which is so readily soluble in culture media; endotoxins proper, to pigments confined to the bacterial cell, or at least, when secreted, being insoluble in culture media, such for instance as the well-known red pigment of Bacillus prodigiosus, which may often be seen free among the bacteria in irregular red granules like carmine powder. That bodies such as this latter might be extruded from pathogenic bacteria and not be soluble in the usual culture fluids, is not improbable, and the fact that more or less insoluble interstitial substances are not infrequent among bacteria is well known.

In all bacterial bodies, after removal of toxins and endotoxins, a certain protein residue remains which, if injected into animals, may give rise to localized lesions such as abscesses or merely slight temporary inflammations. The nature of this residue has been carefully studied, especially by Buchner, who has named it bacterial protein and he believes the substance to be approximately the same in all bacteria, without specific toxic action, but with a general ability to exert a

${ }^{2}$ Pfeiffer, Zeit. f. Hyg., xl, 1892. 
positive chemotactic effect on the white blood cells, thereby causing the formation of pus. The nature of the bacterial proteins is by no means clear, and it is still in doubt whether the separation of these substances from the endotoxins can be upheld.

A number of bacteria may give rise to both varieties of poisons. Thus, recently, Kraus has claimed the discovery of a soluble toxin for the cholera spirillum and Doerr for the dysentery bacillus, both of which microorganisms were regarded as being purely of the endotoxin-producing type.

It is plain, moreover, that occasionally it may be very difficult to distinguish between a soluble toxin and an endotoxin. In the filtration experiment recorded above, it might well be claimed that the toxicity of the filtrate, when not very strong, may depend upon an extraction of endotoxins from the bodies of the bacteria by the medium. The final test, in such instances, lies in the power of true toxins to stimulate in animals the production of antitoxins; for, as we shall see later, the injection of true soluble toxins into animals gives rise to antitoxins, whereas the formation of such neutralizing bodies in the serum or plasma does not, it is claimed, follow the injection of endotoxins.

We could spend much time in analyzing the literature on the exotoxin and endotoxin, and this, of course, would be important were we attempting in this book to cover completely immunological problems. When all is said and done, however, the present status of the question is as follows: Certain bacteria, like the diphtheria bacillus, the tetanus bacillus, B. botulinus, some of the anaërobes of surgical infections, etc., produce secretory products during life which are highly toxic, can be obtained during the life of the culture by simple filtration, and which incite, in carefully treated animals, specific neutralizing substances, or antitoxins, which neutralize the action of the toxin, roughly according to the law of multiples. These antitoxins in the serum, therefore, can be shown definitely to prevent the injury of the animal by the toxin.

In many bacteria such soluble toxins cannot be demonstrated. Older bacterial culture filtrates and the bodies of the bacteria may be highly toxic, as in the case of typhoid, cholera, and practically all the Gram-negative organisms, but these toxic substances are derived either from direct extraction of the bacterial bodies, or, as claimed by some, may represent split products produced in the course of decomposition or cleavage of the bacterial protein. These 
substances, ealled by Pfeiffer endotoxins, do not produce antitoxins. It is doubtful, in our minds, whether they may be regarded as strictly specific in all cases.

In addition to these poisonous substances, the writer, with Kuttner and Parker, ${ }^{3}$ has recently obtained non-specific toxic substances for a great many different bacteria (streptococei, typhoid bacilli, influenza bacilli, etc.) which appeared in cultures as early as 6 to 18 hours, could be obtained by filtration, and could also be obtained by washing young eultures on solid media with salt solution and filtering. These substances are not unlikely similar to those encountered by some other writers who have interpreted them as true exotoxins, but, as far as we can determine, they are neither specific nor antigenic. Nevertheless, they are regular in appearance, sufficiently potent to make rabbits very sick, though rarely to kill them, and must be taken into account in all work in which the toxic substances of bacteria are studied by the usual methods. We ean speak of them, for want of more accurate definition, as bacterial " $\mathrm{X}$ " substances.

In resistance to chemical action and heat, the various poisons show widely divergent properties. As a general rule, most true soluble toxins are delicately thermolabile, they are destroyed by moderate heating, and deteriorate easily on standing. Their chemical nature is by no means clear, but, on precipitation of toxic solutions with magnesium sulphate, these poisons come down together with the globulins. The nature of the "endotoxins" is still less clearly understood. Most of them are far less liabile than the extracellular poisons. Some powerful intracellular poisons, like those of the Gärtner bacillus of meat poisoning and the poison attached to the bodies of typhoid bacilli may undergo exposure to even $100^{\circ} \mathrm{C}$. and still retain their toxic properties. The nature of each individual poison will be discussed in connection with its microorganism.

It should be remembered, moreover, by those studying bacterial poisons that recent investigations have shown that a number of bacteria (coli, influenza, ete.) may produee substanees either identical with or closely related to histamin and tyramin, on pepton media, after five or more days of growth.

'Zinsser, Parler and Kuttner, Transact. Soc. for Exp. Biol. and Med., Jan., 1921. 
The Mode of Action of Bacterial Poisons.-Close study of the toxic products of various microorganisms has shown that many of the bacterial poisons possess a more or less definite selective action upon special tissues and organs. Thus, certain soluble toxins of the tetanus bacillus and Bacillus botulinus attack specifically the nervous system. Again, certain poisons elaborated by the staphylococei, the tetanus bacillus, the streptococci, and other germs, the so-called "hemolysins," attack primarily the red blood corpuscles. Other poisons again act on the white blood corpuseles; in short, the characteristic affinity of specific bacterial poisons for eertain organs is a widely recognized fact.

In explanation of this behavior, much aid has been given by the researches of Meyer, ${ }^{4}$ Overton, ${ }^{5}$ Ehrlich, ${ }^{6}$ and others upon the causes for the analogous selective behavior of various narcotics and alkaloids. It seems probable, from the researches of these men, that the selective action of poisons depends upon the ability, chemical or physical or both, of the poisons to enter into combination with the specifically affected cells. From the nature of the combinations formed, it seems not unlikely that the physical factors, such as solubility in the cell plasma, may also play an important part.

Observations of a more purely bacteriological nature have tended to bear out these conclusions. Wassermann and Takaki, ${ }^{7}$ for instance, have shown that tetanus toxin, which specifically attacks the nervous system, may be removed from solution by the addition of brain substance. Removal of the brain tissue by centrifugation leaves the solution free from toxin. In the same way it has been shown that hemolytic poisons can be removed from solutions by contact with red blood cells, but only when the red blood cells of susceptible species are employed.

Similar observations have been made in the case of leukocidin, a bacterial poison acting upon the white blood cells specifically. ${ }^{8}$

That bacterial poisons injected into susceptible animals rapidly disappear from the circulation is a fact which bears out the view that a combination between affected tissue and toxin must take

- Meyer, Arch. f. exper. Pathol., 1899, 1901.

"Overton, "Studien üb. d. Narkose," Jena, 1901.

'Ehrlich, "Sauerstoffs-Bedürfniss des Organismus," Berlin, 1885.

"Wassermann und Takaki, Berl. klin. Woch., 1898.

${ }^{8}$ Sachs, Hofmeister's Beiträge, 11, 1902. 
place. Dönitz, for instance, has shown that within four to eight minutes after the injection of certain toxins, considerable quantities will have disappeared from the circulation. Conversely, Metchnikoff $^{10}$ has observed that tetanus toxin injected into insusceptible animals (lizards) may be detected in the blood stream for as long as two months after administration.

\footnotetext{
'Dönitz, Deut. med. Woch., 1897.

${ }^{10}$ Metchnikoff, "L'immunité dans les malad. infect.',
} 


\section{CHAPTER XII}

DEFENSIVE FACTORS OF THE ANIMAL ORGANISM

\section{GENERAL CONSIDERATIONS}

WE have seen that the mere entrance of a pathogenic microorganism into the human or animal body through a breach in the continuity of the mechanical defenses of skin or mucosa does not necessarily lead to the development of an infection. The opportunities for such an invasion are so numerous, and the contact of members of the animal kingdom with the germs of disease is so constant, that if this were the case, sooner or later all would succumb. It is plain, therefore, that the animal body must possess more subtle means of defense, by virtue of which pathogenic germs are, even after their entrance into the tissues and fluids, disposed of, or at least prevented from proliferating and elaborating their poisons. The power which enables the body to accomplish this is spoken of as resistance. When this resistance, which in some degree is eommon to all members of the animal kingdom, is especially marked, it is spoken of as "immunity."

From this it follows naturally that the terms resistance and immunity, as well as their converse, susceptibility, are relative and not absolute terms. Degrees of resistance exist, which are determined to a certain extent by individual, racial, or species peculiarities; and persons or animals are spoken of as immune when they are unaffeeted by an exposure or an inoculation to which the normal average individual of the same species would ordinarily suceumb. The word does not imply, however, that these individuals could not be infected with unusually virulent or large doses, or under particularly unfavorable circumstances. Thus, birds, while immune against the ordinary dangers of tetanus bacilli, may be killed by experimental inoculations with very large doses of tetanus toxin. ${ }^{1} \quad$ Similarly, Pasteur rendered

${ }^{1}$ Quoted from Abel, Kolle und Wassermann, "Handbuch," etc. 
naturally immune hens susceptible to anthrax by cooling them to a subnormal temperature, and Canalis and Morpurgo did the same with doves by subjecting them to starvation.

Absolute immunity is exceedingly rare. The entire insusceptibility of cold-blooded animals (frogs and turtles) under normal conditions to inoculation with even the largest doses of many of the bacteria pathogenic for warm-blooded animals, and the immunity of all the lower animals against leprosy, are among the few instances of absolute immunity known. ${ }^{2}$ Apart from such exceptional eases, however, resistance, immunity, and susceptibility must be regarded as purely relative terms.

The power of resisting any specific infection may be the natural heritage of a race or species, and is then spoken of as natural immunity. It may, on the other hand, he acquired either accidentally or artificially by a member of an ordinarily susceptible species, and is then called acquired immunity.

Natural Immunity.-SPecies Immunity.-It is well known that many of the infectious diseases which commonly affect man, do not, so far as we know, occur spontaneously in animais. Thus, infection with B. typhosus; the vibrio of cholera, or the meningococcus occurs in animals only after experimental inoculation. Gonorrheal and syphilitic infection, furthermore, not only does not occur spontaneously, but is produced experimentally in animals with the greatest difficulty-the consequent diseases being incomparably milder than those occurring in man. Other diseases, like leprosy, influenza, and the exanthemata, ${ }^{3}$ have never been sucessfully transmitted to animals.

Conversely, there are diseases among animals which do not spontaneously attack man. Thus, human beings enjoy immunity against Rinderpest, and, to a lesser degree, against chicken cholera.

Among animal species themselves great differences in susceptibility and resistance toward the various infections exist. Often-quoted examples of this are the remarkable resistance to anthrax of rats and dogs, and the immunity of the common fowl against tetanus.

The factors which determine these differences of susceptibility and resistance among the various species are not clearly understood. It has been suggested that diet in some instances may influence these relations, inasmuch as carnivorous animals are often highly resistant

${ }^{2}$ Lubarsch, Zeit. f. klin. Mediz., xix.

${ }^{8}$ With the possible exception of smallpox. 
to glanders, anthrax, and even tuberculous infections, to which herbivorous animals are markedly susceptible. ${ }^{4}$ It is likely, too, that the great differences between animals of various species in their metabolism, temperature, ete., may call for special cultural adaptation on the part of the bacteria. The fact that the bacillus of avian tuberculosis-whose natural host has a normal body temperature of $40^{\circ} \mathrm{C}$. and above-will grow on culture media at 40 to $50^{\circ} \mathrm{C}$., whereas $\mathrm{B}$. tuberculosis of man can not be eultivated at a temperature above $40^{\circ} \mathrm{C}$., would seem to lend some support to this view. The difference between warm- and cold-blooded animals has already been noted. The necessity for cultural adaptation, too, would seem to be borne out by the great enhancement observed in the virulence of certain microorganisms for a given species after repeated passage through individuals of this species.

Racial Immunity.- Just as differences in susceptibility and immunity exist among the various animal species, so the separate races or varieties within the same species may display differences in their reactions toward pathogenic germs. Algerian sheep, for instance, show a much higher resistance to anthrax than do our own domestic sheep, and the various races of mice differ in their susceptibility to anthrax and to glanders.

Similar racial differences are common among human beings. As a general rule, it may be said that a race among whom a certain disease has been endemic for many ages is less susceptible to this disease than are other races among whom it has been more recently introduced. The appalling ravages of tuberculosis among negroes, American Indians, and Esquimaux, bear striking witness to this fact. Conversely, the comparative inmunity of the negro from yellow fever, a disease of the greatest virulence for Caucasians, furnishes further evidence in favor of this opinion. It must not be forgotten, however, in judging of these relations, that the great differences in the customs of personal and social hygiene existing among the various races may considerably affect the transmission of disease and lead to false conclusions.

In so far as the statement made above is true, however, it seems to indicate that the endemic diseases have carried in their train a certain degree of inherited immunity.

"Hahn, in Kolle und Wassermann, vol. iv. 
In other cases $^{5}$-as in the instance of the malaria-immunity of negroes - the resistance seems to be acquired rather than inherited, for, as Hirsch was first to note, death from this disease occurred frequently among the children, while adult negroes were rarely attacked.

Differences in Individual Resistance.-In bacteriological experimentation with smaller test animals, a direct ratio may often exist between body weight and dosage in determining the outcome of an infection, provided the mode of inoculation has been the same and the virulence of the germ not excessive. It would seem, therefore, that among these animals the difference in resistance in the face of an artificial infection between individuals of the same race is very slight.

In higher animals, however, especially in the case of man, the existence of such apparent individual differences is a well-established fact, although in judging of them we must not forget that the conditions of infection are not subject to the uniformity and control which animal experimentation permits. Of a number of persons exposed to any given infection there are always some who are entirely unaffected and there are great variations in the severity of the disease in those who are attacked. In the absence of positive evidence in support of the direct inheritance of this individual immunity, the most reasonable explanation for such differences in resistance seems to lie in attributing them to individual variations in metabolism or body-chemistry. Depressions, for instance, in the acidity of the gastric secretion would predispose to certain infections of gastro-intestinal origin. Anatomical difierences, too, may possibly influence resistance. Thus, Birch-Hirschfeld believed that certain anomalous arrangements of the bronchial tubes predisposed to tuberculosis.

Instances of transient susceptibility induced by physical or mental overwork, starvation, etc., should hardly be classified under this heading, since the conditions in such cases correspond simply to experimental depression of natural species for race resistanec.

Acquired Immunity.-It is a matter of common experience that many of the infectious diseases occur but once in the same individual. This is notably the case with typhoid fever, yellow fever, and most of the exanthemata, and is too general an observation to require extensive illustration. A single attack of any of the diseases of this class

${ }^{5}$ Hahn, in Kolle und Wassermann, loc. cit. 
alters in some way the resistance of the individual so that further exposure to the infective agent is usually without menace, either for a limited period after the attack, or for life. Resistance acquired in this way is often spoken of as acquired immunity.

The protection conferred by certain diseases against further attack was recognized many centuries ago, and there are records which show that attempts were made in ancient China and India to inoculate healthy individuals with pus from small-pox pustules in the hope of producing by this process a mild form of the disease and its consequent immunity.

Pasteur, before all others, thought philosophically about the phenomena of acquired immunity, and, with adequate knowledge, realized the possibility of artificially bestowing immunity without inflicting the dangers of the fully potent infective agent. The first observation which, made by him, purcly accidentally, inspired the hope of the achievement of such a result, occurred during his experiments with chicken cholera. The failure of animals to die after inoculation with an old culture of the bacilli of chicken cholera, fully potent but a few weeks previously, pointed to the attenuation of these bacilli by their prolonged cultivation without transplantation. With this observation as a point of departure he carried out a series of investigations with the purpose of diseovering a method of so weakening or attenuating various incitants of disease that they could be introduced into susceptible individuals without endangering life and yet without losing their property of conferring protection. The brilliant results achieved by Jenner, many years before, in protecting against smallpox by inoculating with the entirely innocuous products of the pustules of cowpox furnished an analogy which gave much encouraging support to this prospect.

The experimental work which Pasteur carried out to solve this problem not only reaped a rich harvest of facts, but gave to seience the first and brilliant examples of the application of exact laboratory methods to problems of immunity.

\section{ACTIVE IMMUNITY}

Active Artificial Immunity.-The process of conferring protection by treatment with either an attenuated form or a sublethal quantity of the infectious agent of a disease, or its products, is spoken of as "active immunization." 
Whatever the method employed, the immunized individuals gain their power of resistance by the unaided reactions of their own tissues. They themselves take an active physiological part in the acquisition of this new property of immunity. For this reason, Ehrlich has aptly termed these processes "active immunization."

There are various methods by which this can be accomplished, all of which were, in actual application or in principle, discovered by Pasteur and his associates, and can be best reviewed by a study of their work.

Active Immunization with Attenuated Cultures.-In the course of his experiments upon chicken cholera, as mentioned above, Pasteur $^{6}$ accidentally discovered that the virulence of the bacilli of this disease was greatly reduced by prolonged eultivation upon artificial media. This was especially noticeable in broth cultures which had been stored for long periods without transplantation. By repeated injections of such cultures into fowl, he succeeded in rendering the animals immune against subsequent inoculations with lethal doses of fully virulent strains.

During the same year, 1880, in which Pasteur published his observations on chicken cholera, Toussaint ${ }^{7}$ succeeded in immunizing sheep against anthrax by inoculating them with blood from infected animals, defibrinated and heated to $55^{\circ} \mathrm{C}$. for ten minutes. Toussaint wrongly believed, however, that the blood which had been used in his immunizations was free from living bacteria. In repeating this work Pasteur showed that the protection in Toussaint's eases was conferred by living bacteria, the virulence of which had been reduced by their subjection to heat.

In following out the suggestions offered by these experiments, Pasteur $^{8}$ discovered that he could reduce the virulence of anthrax bacilli much more reliably than by Toussaint's method, by cultivating the organisms at increased temperatures $\left(42^{\circ}\right.$ to $43^{\circ}$ C.). By this process of attenuation he was able to produce "vaccines" of roughly measurable strength, with which he succeeded in immunizing sheep and cattle. A successful demonstration of his discovery was made by him at Pouilly-le-Fort, soon after, upon a large number of animals and before a commission of professional men.

- Pasteur, Compt. rend. de l'acad. des sci., 1880, t. xe.

"Toussaint, Compt. rend. de I'acad. des sei., 1880, t. xei.

${ }^{8}$ Pasteur, Chamberland et Roux, Compt. rend. de l'acad. des sci., 1881, t. xcii. 
It is a fact well known to bacteriologists that certain of the pathogenic microorganisms, when passed through several individuals of the same animal species, become gradually more virulent for this species. In his studies on the bacillus of hog cholera, Pasteur ${ }^{9}$ observed that when this microorganism was passed through the bodies of several rabbits it gained in virulence for rabbits, but became less potent against hogs. He succeeded, subsequently, in protecting hogs against fully virulent cultures by treating them with strains which had been attenuated by their passage through rabbits.

A further principle of attenuation for purposes of immunization was, at about this time, contributed by Chamberland and Roux, ${ }^{10}$ who reduced the virulence of anthrax cultures by growing them in the presence of weak antiseptics (carbolic acid 1:600, potassium bichromate $1: 5,000$, or sulphuric acid $1: 200)$. Cultivated under such conditions the bacilli lost their ability to form spores and became entirely avirulent for sheep within ten days. A similar result was later obtained by Behring ${ }^{11}$ when attenuating B. diphtheriæ cultures by the addition of terchlorid of iodin.

Active Immunization with Sublethal Doses of Fuldy Virulent BACTERIA.-The use of fully virulent microorganisms in minute quantities for purposes of immunization was first suggested by Chauveau, ${ }^{12}$ and is naturally inapplicable to extremely virulent organisms like B. anthracis. The principle, however, is perfectly valid, and has been experimentally applied by many observers, notably by Ferran ${ }^{13}$ in the case of cholera. A similar method proved of practical value in the hands of Theobald Smith and Kilborne ${ }^{14}$ in prophylaxis against the protozoan disease, Texas fever.

Active Immunization with Dead Bacteria.-Suggested by Chauveau, the method of active immunization with gradually increasing doses of dead microorganisms has been successfully employed by various observers, chief among whom are Pfeiffer, Brieger, Wright, and Wassermann. The method is especially useful against that class of bacteria in which the cell bodies (endotoxins) have been found to

${ }^{2}$ Pasteur, Compt. rend. de l'acad. des sci., 1882, t. xev.

${ }^{10}$ Chamberland et Roux, Compt. rend. de l'acar. des sci., 1882, t. xevi.

${ }^{11}$ Behring, Zeit. f. Hyg., xii, 1892.

${ }^{12}$ Chauveau, Compt. rend. de l'acad. des sci., 1881, t. xcii.

${ }^{13}$ Ferran, Compt. rend. de l'acad. des sci., 1895, t. ci.

${ }^{14}$ Th. Smith and Kilborne, U. S. Dept. of Agri., Bureau of Ani. Indust., Wash., 1893. 
be incomparably more poisonous than their extracellular products (toxins). From a practical point of view, the method is of the greatest importance in routine laboratory immunization against B. typhosus, Vibrio choleræ asiatiex, B. pestis, and a number of other bacteria. In the therapy of human disease, this method has recently come into great prominence, chiefly through the work of Wright, whose investigations will be more fully discussed in a subsequent section.

Active Immunization with Bacterial Products.-Many bacteria when grown in fluid media produce extracellular, soluble poisons which remain in the medium after the microorganisms have been removed by filtration or centrifugalization. Since the diseases eaused by such microorganisms are, to a large extent, due to the soluble poisons excreted by them, animals can be actively immunized against this class of bacteria by the inoculation of gradually inereasing doses of the specific poison or toxin. This method is naturally most suecessful against those microorganisms which possess the power of toxin formation to a highly developed degree. Most important among these are B. diphtherix and B. tetani. The first successful application of this principle of active immunization, however, was made by Salmon and Smith $^{15}$ in the case of hog cholera.

\section{PASSIVE IMMUNITY}

In Pasteur's basic experiments, as in those of the other scientists who followed in his footsteps, the methods of immunization were based upon the development of a high resistance in the treated subject by virtue of its own physiological activities. This process we have spoken of as "active immunization" and it is self-evident that a method of this kind ean, in the treatment of disease, be employed prophylactically only against possible infection, or in localized acute infections, or at the beginning of a long period of incubation before actual symptoms have appeared, as in rabies or in ehronic conditions in which the infection is not of a severe or acute nature.

A new and therapeutically more hopeful direction was given to the study of immunity when, in 1890 and 1892, v. Behring and his collaborators diseovered that the sera of animals immunized against

${ }^{15}$ Salmon and Smith, Rep. of Com. of Agri., Wash., 1885 and 1886. 
the toxins of tetanus ${ }^{16}$ and of diphtheria ${ }^{17}$ bacilli would protect normal animals against the harmful action of these poisons. The animals thus protected obviously had taken no active part in their own defense, but were protected from the action of the poison by the substances transferred to them in the sera of the actively immunized animals. Such immunity or protection, therefore, is a purely passive phenomenon so far as the treated animal is concerned, and the process is for this reason spoken of as "passive immunization."

Passive immunization of this description is practically applicable chiefly against diseases caused by bacteria which produce powerful toxins, and the sera of animals actively immunized against such toxins are called antitoxic sera. In the treatment of the two diseases mentioned above, diphtheria and tetanus, the respective antitoxic sera have reached broad and beneficial therapeutic application, and innumerable lives have been saved by their úse.

Passive immunization against microorganisms not characterized by marked toxin formation was attempted, even before Behring's discovery, by Richet and Hericourt, ${ }^{18}$ experimenting with cocei, and by Babes, ${ }^{19}$ in the case of rabies; and the underlying thought had been the basis of Toussaint's work upon anthrax. Microorganisms, however, which exert their harmful action rather by the contents of the bacterial cells than by secreted, soluble toxins, do not, so far as is known, produce antitoxins in the sera of immunized animals. The substances which they call forth in the process are directed against the invading organisms themselves in that they possess the power of destroying or of causing dissolution of the specific germs used in their production.

Such antibacterial sera are extensively used in the laboratory in the passive immunization of animals against a large number of germs, and are fairly effectual when used before, at the same time with, or soon after, infection. Their therapeutic use in human disease, however, has, up to the present time, been disappointing and their prophylactic and curative action has been almost invariably ineffectual or feeble at best, except when the antibacterial sera could be brought in direct contact with the germs, in closed cavities or localized lesions.

${ }^{16} v$. Behring and Kitasato, Deut. med. Woch., 49, 1890.

${ }^{17} v$. Bchring and Wernicke, Zeit. f. Hyg., 1892.

${ }^{18}$ Richet et Hericourt, Compt. rend. de l'acad. des sci., 1888.

${ }^{19}$ Babes et Lepp, Ann. de 1'inst. Pasteur, 1889. 
Thus, in epidemic meningitis, such sera have proved extremely useful in the hands of Flexner, when injected directly into the spinal canal.

\section{ANTIBODIES AND THE SUBSTANCES GIVING RISE TO THEM}

In the foregoing sections we have seen that the process of active immunization so changes the animal body that it becomes highly resistant against an infection to which it had formerly in many instances been delicately susceptible. In the absence of visible anatomical or histological changes accompanying the acquisition of this new power, investigators, in order to account for it, were led to examine the physiological properties of the body cells and fluids of immunized subjects. While it was reasonable to suppose that all the cells and tissues were affected by, or might have taken part in, a physiological change so profoundly influencing the individual, the blood, because of its unquestionably close relation to inflammatory reactions, and because of the ease with which it could be obtained and studied, elaimed the first and closest attention. The bactericidal properties or normal blood serum noted in 1886 by. Nuttall, ${ }^{20}$ v. Fodor, ${ }^{21}$ and Flügge, moreover, aided in pointing to this tissue as primarily the seat of the immunizing agents. It is an interesting historical fact, that, long before this time, the English physician Hunter had noted that blood did not decompose so rapidly as other animal tissues.

The study of the blood serum of immunized animals as to simple changes in chemical composition or physical properties has shed little light upon the subject. Beljaeff ${ }^{22}$ in a recent investigation found little or no alteration from the normal in the blood sera of immunized animals as to index of refraction, specific gravity, and alkalinity. Joachim $^{23}$ and Moll agree in stating that immune blood serum is comparatively richer in globulin than normal serum. Similar observations had been made by Hiss and Atkinson ${ }^{24}$ and others. Important and significant as these purely chemical observations are, they have helped little in explaining the nature of the processes going on in immune sera. The first actual light was thrown upon the mysterious phenomena

\footnotetext{
${ }^{20}$ Nuttall, Zeit. f. Hyg., i, 1886.

${ }^{21} v$. Fodor, Deut. med. Woch., 1886.

${ }^{22}$ Beljaeff, Cent. f. Bakt., xxxiii.

${ }^{23}$ Joachim, Pflügers Arehiv, xciii.

${ }^{24}$ Hiss and Atkinson, Jour. Exper. Med., v, 1900.
} 
of immunity by the investigations of Nuttall, ${ }^{25}$ v. Fodor, Buchner, and others, who not only demonstrated the power of normal blood serum to destroy bacteria, but also showed that this property of blood serum became diminished with age and was destroyed completely by heating to $56^{\circ} \mathrm{C}$. The thermolabile substance of the blood serum possessing this power was ealled by Buchner, ${ }^{26}$ alexin.

Soon after this work, Behring, in collaboration with Kitasato ${ }^{27}$ and Wernicke, ${ }^{28}$ in 1890 and 1892 , made further important advances in the elucidation of the immunizing processes by showing that the blood sera of animals actively immunized against the toxins of diphtheria and tetanus would protect normal animals against the poisons of these diseases. He believed, at the time of discovery, that such sera contained substances which had the power of destroying the specific toxins which had been used in the immunization. He called these bodies antitoxins. While Behring's first conception of actual toxin destruction soon proved to be erroneous, his discovery of the presence in immune sera of bodies specifically antagonistic to toxins was soon confirmed and extended, and stands to-day as an established fact.

Ehrlich, ${ }^{29}$ soon after Behring's announcement, showed that specific antitoxins could also be produced against the poisons of some of the higher plants antiricin, antikrotin, antirobin), and Calmette ${ }^{30}$ produced similar antitoxins against snake poison (antivenin). Stimulated by these researches, other observers have, since then, added extensively to the list of poisons against which antitoxins can be produced. Kempner $^{31}$ has produced antitoxin against the poison of Bacillus botulinus, and Wassermann, ${ }^{32}$ against that of Bacillus pyocyaneus. Antitoxin has been produced by Calmette ${ }^{33}$ against the poison of the scorpion, and by Sachs ${ }^{34}$ against that of the spider. Thus a large number of poisons of animal, plant, or bacterial origin have been found capable of causing the production of specific antibodies in the sera of animals into which they are injected.

\footnotetext{
${ }^{25}$ Nuttall, Zeit. f. Hyg., 1886.

${ }^{28}$ Buchner, Cent. f. Bakt., i, 1889.

${ }^{27}$ Behring und Kitasato, Deut. med. Woch., 1890, No. 49.

${ }^{28}$ Behring und Wernicke, Zeit. f. Hyg., 1892.

${ }^{29}$ Ehrlich, Deut. med. Woch., 1891.

${ }^{30}$ Calmette, Compt. rend. de la soc. de biol., 1894.

${ }^{31}$ Kempner, Zeit. f. Hyg., 1897.

3: Wassermann, Zeit. f. Hyg., xxii.

${ }^{3}$ Calmette, Ann. de l'inst. Pasteur, 1898.

${ }^{34}$ Sachs, Hofm. Beit., 1902.
} 
The formation of antitoxins directed against soluble poisons, however, did not explain the immunity acquired by animals against bacteria like Bacillus anthracis, the cholera vibrio, and others which, unlike diphtheria and tetanus, produced little or no soluble toxin. It was evident that the antitoxic property of immune blood serum was by no means the sole expression of its protective powers. Much light was shed upon this phase of the subject by the discoveries of Pfeiffer in 1894, who worked along the lines suggested by the investigations of Nuttall and Buchner. Pfeiffer ${ }^{35}$ showed that when cholera spirilla were injected into the peritoneal cavity of choleraimmune guinea-pigs, the microorganisms rapidly swelled up, became granular, and often underwent complete solution. The same phenomenon could be observed when the bacteria were injected into a normal animal together with a sufficient quantity of cholera-immune ${ }^{36}$ serum.

This process he observed microscopically by abstracting, from time to time, a small quantity of the peritoneal exudate and studying it in hanging-drop preparations. The reaction was specific in that the destructive process took place to any marked extent only in the case of the bacteria employed in the immunization.

Metchnikoff, ${ }^{37}$ Bordet, and others not only confirmed Pfeiffer's observation, but were able to show that the lytic process would take place in vitro, as well as in the animal body. The existence of a specific destructive process in immune serum was thus established for the vibrio of cholera and soon extended to other microorganisms. The constituents of the blood serum which gave rise to this destructive phenomenon were spoken of as bacteriolysins.

Following closely upon the heels of Pfeiffer's observation came the discovery of another specific property of immune serum by Gruber and Durham. ${ }^{38}$ These workers noticed that certain bacteria, when brought into contact with the serum of an animal immunized against them, were clumped together, deprived of motility, and firmly agglutinated. They spoke of the phenomenon as agglutination and of the substances in the serum giving rise to it as agglutinins.

The list of antibodies was again enlarged by Kraus, ${ }^{39}$ who in 1897

\footnotetext{
${ }^{2}$ Pfeiffer, Zeit. f. Hyg., xviii, 1894.

${ }^{38}$ Pfeiffer und Isaeff, ibid.

${ }^{37}$ Metchnikoff, Ann. de l'inst. Pasteur, 1895.

${ }^{35}$ Gruber und Durham, Münch. med. Woch., 1896.

${ }^{39}$ Kraus, R., Wien. klin. Woch., 32, 1897.
} 
showed that precipitates were formed when filtrates of eultures of cholera, typhoid, and plague bacilli were mixed with their specific immune sera. He called the substances which bestowed this property upon the sera precipitins.

The treatment of the animal body, therefore, with bacteria or their products gives rise to a variety of reactions which result in the presence of the "antibodies" described above. Extensive investigation has shown, however, that the power of stimulating antibody production is a phenomenon not limited to bacteria and their products alone. Antitoxins, we have already seen, may be produced with a variety of poisons of plant and animal origin. Sensitizing, agglutinating and precipitating effects may, likewise, be produced by the use of a large number of different substances. Chief among these, because of the great aid they have given to the theoretical investigation of the phenomena of immunity, are the red blood cells. Bordet ${ }^{40}$ and, independently of him, Belfanti and Carbone ${ }^{41}$ showed in 1898 that the serum of animals repeatedly injected with the defibrinated blood of another species exhibited the specific power of dissolving the red blood corpuscles of this species. This was the first demonstration of "hemolysis" - a phenomenon which, because of the ease with which it can be observed in vitro, has much facilitated investigation.

The knowledge that specific "cytotoxins" or cell-destroying anti-bodies could be produced by injection of red blood cells naturally suggested the possibility of analogous reactions for other tissue cells. It was not long, therefore, before Metchnikoff ${ }^{42}$ and, independently of him, Landsteiner ${ }^{43}$ succeeded, by repeated injections of spermatozoa, in producing a serum which would seriously injure these specialized cells. Von Dungern ${ }^{44}$ obtained similar results with the ciliated epithelium of the trachea. Since then a host of eytotoxins have been produced with the cells of various organs and tissues. Thus, Neisser and Wechsberg ${ }^{45}$ produced leucotoxin (leucocytes); Delezenne, ${ }^{46}$ neurotoxin and hepatotoxin; Surmont, ${ }^{47}$ pancreas cytotoxin; and Bogart and Bernard, ${ }^{48}$ suprarenal cytotoxin.

40 Bordet, Ann. de l'inst. Pasteur, 1898.

${ }^{41}$ Belfanti et Carbone, Giornale della R. Acad. di Torino, July, 1898.

${ }^{42}$ Metchnikoff, Ann. de l'inst. Pasteur, 1898.

${ }^{43}$ Landsteiner, Cent. f. Bakt., i, 25, 1899.

${ }^{44} v$. Dungern, Münch. med. Woch., 1899.

${ }^{45}$ Neisser und Wechsberg, Zeit. f. Hyg., xxxvi, 1901.

${ }^{46}$ Delezenne, Ann. de I'inst. Past. 1900; Compt. rend. de l'acad. des sci., 1900.

${ }^{47}$ Surmont, Compt. rend. de la soc. de biol., 1901.

18 Bogart et Bernard, ibid., 1891. 
One of the most interesting of the cytotoxins, moreover, is nephrotoxin -produced by the treatment of animals with injections of emulsions of kidney tissue.

In all cases it was supposed by those first working with these bodies, that the injection of the sera of animals previously treated with any particular tissue substance would produce speeifie injury upon the organs homologous to the ones used in immunization. It need hardly be pointed out how very important such phenomena would be in throwing light upon the degencrative pathological lesions oceurring in disease. As a matter of fact, however, sera so produced have been shown to be speeific for certain organs in a limited sense only. The question of specifie cytotoxins has been of especial importance in the case of nephritis, where Ascoli and Figari ${ }^{49}$ and others have suggested an autonephrotoxin as the basis of the pathology of this disease. In the hands of Pearce and others, however, the strict specificity of nephrotoxin could not be upheld and the subject is still in the experimental stage.

Recent experiments by Pearce $^{50}$ suggest that at least a part of the local injury to organs exerted by such "cytotoxie" sera may not be due to a specific action upon the organ cells so much as upon the hemaglutinating action of the sera causing embolism and necrosis.

It is a fact also that most cytotoxic sera are usually hemolytic as well. It is not easy to decide, therefore, how much of the action upon the organs is due to their true cytotoxic properties and how much is attributable to the concomitant action upon blood cells. The extravagant hopes at first based upon eytotoxin investigation, especially in regard to the problem of malignant tumors, have been disappointed, and much is still obscure in regard to the cytotoxins which calls for further researeh.

The many points of similarity existing between bacterial toxins and digestive ferments, by animal inoculation, suggested to several observers, the possibility of producing antibodies against, the latter. As a result, a number of antiferments have been obtained, chief among which are antilab. (Morgenroth $\left.{ }^{51}\right)$, antipepsin $\left(\right.$ Sachs $\left.^{52}\right)$, antisteapsin $\left(\right.$ Schütze $\left.^{53}\right)$, and antilactase (Schütze).

The stimulation of antibody formation in the sera of animals is a consequence, therefore, of the injection of a large variety of substances-some of them poisonous, some of them entirely innocuous.

49 Ascoli and Figari, Berl. klin. Woch., 1902.

"n Pearce, Jour. Exper. Med., viii, 1906.

${ }^{61}$ Morgenroth, Cent. f. Bakt., 1899.

${ }^{62}$ Sachs, Fort. d. Med., 1902.

${ }^{53}$ Schütze, Deut. med. Woch., 1904; Zeit. f. Hyg., 1905. 
The substances possessing this power have been conveniently named antigen or antibody-producers by German writers. The term antigen -though etymologically wrong, nevertheless is convenient and has crept into general usage. It signifies simply a substance which can stimulate the production or formation of an antibody. Such substances, so far as is known, belong to the group of proteins and are derivatives of animal or plant tissues. Being proteins, all antigens are colloids. Recently, however, some crystalloidal substances have been described as possessing antigenic properties. 


\section{CHAPTER XIII}

\section{TOXINS AND ANTITOXINS}

The Toxin-Antitoxin Reaction.-Apart from the therapeutic possibilities disclosed by the discovery of antitoxins, new light of inestimable value was thrown by these observations upon the biological processes involved in immunization. The most vital problem, of course, which immediately thrust itself upon all workers in this field, was the question as to the nature of the reaction in which toxin was rendered innocuous by antitoxin.

The simplest conception of this process would be an actual destruction of the toxin by its specific antitoxin, and it is not unnatural, therefore, that this was the view which, for a short time, found favor with some observers. Roux, and more particularly Buchner, ${ }^{1}$ however, under the sway of cellular pathology, advanced the opinion that the antitoxins in some way influenced the tissue cells, rendering them more resistant against the toxins. Antitoxin, according to this theory, did not act directly upon toxin, but affected it indirectly through the mediation of tissue cells. Ehrlich, ${ }^{2}$ on the other hand, conceived that the reaction of toxin and antitoxin was a direct union, analogous to the chemical neutralization of an acid by a base-an opinion in which Behring soon joined him.

The conception of toxin destruction received unanswerable refutation by the experiments of Calmette. ${ }^{3}$ This observer, working with snake poison, found that the poison itself (unlike most other toxins) possessed the property of resisting heat even to $100^{\circ} \mathrm{C}$., while its specific antitoxin, like other antitoxins, was delicately thermolabile. He noted, furthermore, that non-toxic mixtures of the two substances, when subjected to heat, regained their toxie properties. The natural inference from these observations could

'Buchner, "Schutzimpfung,', ete., in Penzoldt u. Stinzing, “Handbuch d. spez. Therap. d. Infektkrank.,"' 1894.

${ }^{2}$ Ehrlich, Deut. med. Woch., 1891.

${ }^{3}$ Calmette, Ann. de l'inst. Past., 1895. 
only be that the toxin in the original mixture had not been destroyed, but had been merely inactivated by the presence of the antitoxin, and again set free after destruction of the antitoxin by heat. A similar observation, made soon after by Wassermann ${ }^{4}$ and in the case of pyocyaneus toxin and antitoxin, fully supported the results of Calmette.

An ingenious proof of the direct action of antitoxin upon toxin was obtained by Martin and Cherry. ${ }^{5}$ It was found by them that very dense filters, the pores of which had been filled with gelatin, permitted toxin to pass through under high pressure, while the presumably larger antitoxin molecule was held back. Through such filters they forced toxin-antitoxin mixtures, under a pressure of fifty atmospheres, at varying intervals after mixing. They found that, if filtered immediately, all the toxin in the mixtures came through, but that, as the interval elapsing between mixing and filtration was prolonged, less and less toxin appeared in the filtrate, until, finally, two hours after mixing, no toxin whatever passed through the filter. Besides demonstrating the direct action of antitoxin upon toxin, this work of Martin and Cherry showed that the element of time entered into the toxin-antitoxin reaction, just as it enters into reactions of known chemical nature. The absolute nonparticipation of the living tissue cells in these reactions was demonstrated by Ehrlich himself. Kobert and Stillmarck ${ }^{6}$ had shown that ricin possessed the power of causing the red blood cells of defibrinated blood to agglutinate in solid clumps, a reaction which could easily be observed in vitro. Ehrlich, ${ }^{7}$ who had obtained antiricin in 1891 by injecting rabbits with increasing doses of ricin, found that this antibody possessed the power of preventing the hemagglutinating action of ricin in the test tube. By a series of quantitatively graded mixtures of ricin and antiricin, with red blood cells as the indicator for the reaction, he succeeded in proving not only that the toxin-antitoxin neutralization was in no way dependent upon the living animal body, but that definite quantitative relations existed between the two substances entirely analogous to those which, according to the law of multiple proportions, govern reactions between different substances of known chemical nature.

Wassermann, Zeit. f. Hyg., xxii, 1896.

'Martin and Cherry, Proc. Royal Soc., London, Ixiii, 1898.

- Kobert und Stillmarck, Arb. d. phar. Inst. Dorpat, 1889.

'Ehrlich, Fort. d. Med., 1897. 
Similar quantitative results were subsequently obtained by Stephens and Myers ${ }^{8}$ for cobra poison and its antitoxin, by Kossel ${ }^{9}$ for the toxic eel blood serum, and by Ehrlich ${ }^{10}$ for the hemolytic tetanus poison known as tetanolysin.

The introduction of the test-tube experiment into the investigation of these reactions permitted of much more exact observations, and by this means, as well as by careful, quantitatively graded, animal experiments, the further facts were ascertained that toxin and antitoxin combined more speedily in concentrated than in dilute solutions, and that warmth hastened, while cold retarded, the reaction-observations ${ }^{11}$ which in every way seem to bear out Ehrlich's conception of the chemical nature of the process.

Ehrlich's Analysis of Diphtheria Toxin.-Shortly after the discovery and therapeutic application of diphtheria antitoxin, it became apparent that no two sera, though similarly produced, could have exactly the same protective value. It was necessary, therefore, to establish some measure or standard by which the approximate strength of a given antitoxin could be estimated. Von Behring ${ }^{12}$ attempted to do this for both tetanus and diphtheria antitoxins by determining the quantity of immune sera which, in each case, was needed to protect a guinea-pig of known weight against a definite dose of a standard poison. He ascertained the quantity of standard toxin-bouillon which would suffice to kill a guinea-pig of 250 grams, and called this quantity the "toxin unit." This unit was later" more exactly limited by Ehrlich, who, considering the element of time, stated it as the quantity sufficient to kill a guinea-pig of the given weight in from four to five days.

Appropriating the terminology of chemical titration, v. Behring spoke of a toxin-bouillon which contained one hundred such toxin units in a cubic centimeter, as a "normal toxin solution" ("DTN" $\mathrm{M}^{250 ")}$, and designated as "normal antitoxin" a serum capable of neutralizing, cubic centimeter for cubic centimeter, the normal poison. ${ }^{13}$ A cubic centimeter of such an antitoxic serum was suffi-

${ }^{8}$ Stephens and Myers, Jour. of Path. and Bact., 1898.

' Kossel, Berl. klin. Woch., 1898.

${ }^{10}$ Ehrlich, Berl. klin. Woch., 1898.

${ }^{11}$ Knorr, Fort. d. Med., 1897.

${ }^{12} v$. Behring, Deut. med. Woch., 1893.

${ }^{13} \mathrm{DTN}^{1} \mathrm{M}^{250}$ signifies: D, Diphtheria; $\mathrm{TN}^{1}$, Normal Toxin solution; $\mathrm{M}^{250}$, Meerschweinchen or guinea-pig weighing 250 grams. 
cient, therefore, to neutralize one hundred toxin units, and was spoken of as an "antitoxin unit." In the experiments of v. Behring, toxin and antitoxin had been separately injected. Ehrlich ${ }^{14}$ improved upon this method by mixing toxin and antitoxin before injection, thereby obviating errors arising from differences which may have existed in the depth of injection or rapidity of absorption.

In order, however, that any such method of standardization of antitoxin may be practically applicable, it is necessary to produce either a stable toxin or an unchangeable antitoxin. This Ehrlich achieved for antitoxin by drying antitoxic serum in vacuo and preserving it in the dark, at a low temperature and in the presence of anhydrous phosphoric acid. By the use of such a stable antitoxin, various toxins may be measured and other antitoxic sera estimated against these.

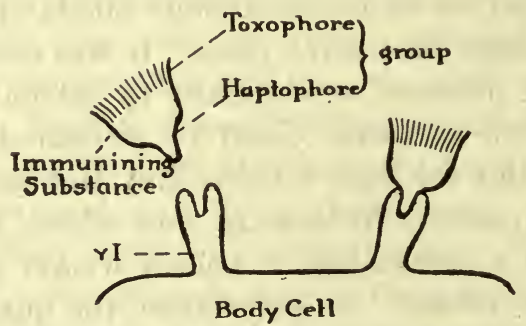

Fig. 30.-Toxin and Body Cells.

Given thus a constant antitoxin, the standardization of toxins would be a comparatively simple matter were the poison obtainable in a perfectly pure state. Unfortunately for the ease of measurement, however, this is not the case. The problem is rendered difficult by a number of complicating factors, many of which have been brought to light by Ehrlich ${ }^{15}$ in his laborious researches into the quantitative relationship between the two reacting bodies.

As previously stated, it had been noted by Ehrlich and others that toxin solutions would deteriorate with time; that is, a toxinbouillon which was found soon after production to contain, say, eighty toxin units in each cubic centimeter, would, after four or five months, be found to contain but forty units in the same gross quantity. It had lost, therefore, in this case, just one-half of its toxic power. In spite of this loss, however, Ehrlich found that such

${ }^{14}$ Ehrlich, Kossel und Wassermann, Deut. med. Woch., 1894.

${ }^{15}$ Ehrlich, Klin. Jahrbuch, vi, 1897; Deut. med. Woch., 1898. 
bouillon had retained its full original power of neutralizing antitoxin. If the reaction was purely one of chemical neutralization, there seemed to be but one explanation of this. The toxin molecule must contain two separate atom groups. One of these must possess the power of binding antitoxin and be stable; this he designates as the "haptophore" or "anchoring" group. The other, the one by which the toxin molecule exerts its deleterious action, must be more easily changed or destroyed; this he calls the "toxophore" or "poison" group. In the altered toxin-bouillon in which a part of the poisonous action has been lost while the antitoxin-neutralizing power is intact, the toxophore group of some of the toxin must have been changed or destroyed. Such altered toxin he speaks of as "toxoid."

In support of this hypothesis and for the purpose of perfecting the methods of standardization, Ehrlich was led to determine, for a large variety of specimens of diphtheria toxin, the precise quantity, in cubic centimeters, which was necessary to neutralize exactly one unit of his standard antitoxin. This he accomplished by making a series of toxin-antitoxin mixtures, in each of which the quantity of antitoxin was exactly one unit, while the amount of toxin was gradually increased. These mixtures were injected into guinea-pigs of 250 grams weight. It is self-evident that in such an experiment the mixtures containing the smaller quantities of toxin would have no effect upon the guinea-pigs. Soon, however, a mixture would be reached in which toxin would be sufficiently in excess of antitoxin to produce the symptoms of slight poisoning, as evidenced in local edema, rise of temperature, etc. The largest quantity of toxin which could be added without producing such symptoms was then regarded as exactly neutralizing the antitoxin unit. This quantity of toxin Ehrlich speaks of as "Limes zero" (Limes = threshold) or, briefly, " $\mathrm{L}_{0}$."

For instance:

One antitoxin unit +0.6 e.e. toxin...... No symptoms of poisoning.

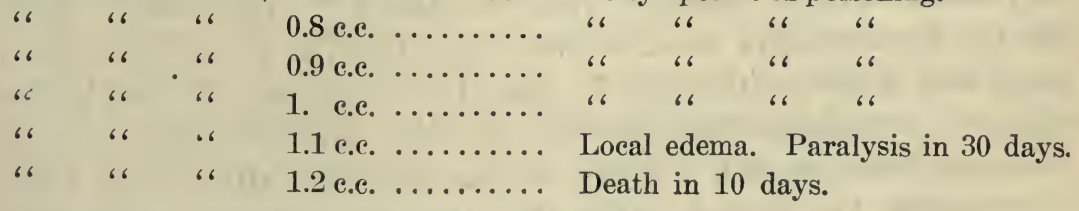

In this example, $\mathrm{L}_{0}$, therefore, equals 1 c.c. 
It is obvious, therefore, that because of the great difficulty in estimating the very slightest evidences of toxic action in guinea-pigs, a more exact method of standardizing the poisons against antitoxin would be to determine how much toxin would be required to neutralize one antitoxin unit and still be sufficiently in excess to cause the death of a guinea-pig of 250 grams in four to five days. This would then correspond to the action of one toxin unit, unmixed with antitoxin. A priori it would seem that this value (expressed by Ehrlich as "Limes death" or " $\mathrm{L}_{+}$") must simply be Lo plus one toxin unit. This, however, was found not to be the ease. Thus, in the example given, in which $\mathrm{T}$ (the toxin unit-the quantity of the bouillon killing a guinea-pig of 250 grams in four to five days) was equal to 0.01 c.c., $\mathrm{L}_{0}$ (the quantity of toxin completely neutralizing onc antitoxin unit) was found to be 1 c.c. or $100 \mathrm{~T}$. In this same poison, however, $\mathrm{L}_{+}$(the quantity of toxin necessary both to neutralize one antitoxin unit and yet to be sufficiently in excess of neutralization to kill a guinea-pig of 250 grams in four or five days) was not found to be merely $\mathrm{L}_{0}+1 \mathrm{~T}$; but on actual experiment proved to be $\mathrm{L}_{0}+101 \mathrm{~T}$.

Expressed graphically, the conditions may be stated as follows:

$T=.01$ e.c. of the toxin bouillon.

$L_{+}$(neutral. of 1 antitox, unit yet killing $\left.1 \mathrm{pig}\right)=2.01$ c.c. of $201 \mathrm{~T}$.

$L_{0}$ (complete neutral. of 1 antitox. unit) $=1$. e.c. or $100 \mathrm{~T}$.

Difference $=1.01$ c.c. or $101 T$.

Ehrlich, at first, endeavored to explain this surprising phenomenon on the basis of toxoids. He argued that the toxoids formed by deterioration of toxin might be conceived as possessing three different degrees of affinity for antitoxin. If their affinity for antitoxin were equal to, or more marked than, that of the toxin itself, they could have no influence upon the dose $\mathrm{L}_{+}$. If, however, their affinity for antitoxin were weaker than that of toxin, each fresh toxin unit added to the dose $\mathrm{L}_{0}$ would, first uniting with antitoxin, replace a corresponding quantity of these nontoxic substances of weaker affinity, and $\mathrm{L}_{+}$would be reached only after all of these " epitoxoids," as Ehrlich called them, had been replaced, and toxin became free in the mixture. 
Thus, in analyzing our example, we have:

100 tox.-antitox. +100 epitox.-antitox. $=L_{0}$; add $1 T$, and we have 101 tox.-antitox. +99 epitoxoid-antitoxin +1 epitoxoid free; add $101 T$ and we have 200 toxin-antitoxin +100 epitoxoid free $+1 T$ free $=I_{+}$.

Two facts, however, led Ehrlich to abandon the opinion that epitoxoid was merely a variety of toxoid. He found, in the first place, that the stated relations between $\mathrm{L}_{0}$ and $\mathrm{L}_{+}$were true for perfectly, fresh toxin-bouillon in which little or no deterioration had taken place. He observed, furthermore, that in old, altered toxin bouillon, while $\mathrm{T}$ was very much affected, the quantity needed to kill a pig constantly increasing, and the number of actual fatal doses in $\mathrm{L}_{0}$ constantly decreasing (by reason of toxoid formation), $\mathrm{L}_{+}$remained practically unchanged.

Simply stated, this means that the epitoxoids or substances which have weaker affinity for antitoxin than toxin itself are already present in fresh bouillon and are not increased with time. For this reason, Ehrlich has separated these substances from toxoids. $\mathrm{He}$ calls them "toxon" and believes them to be, like toxin, primary secretory products of the diphtheria bacilli. The toxoids themselves, Ehrlich believes, are of two kinds, those with a stronger affinity for antitoxin than toxin itself (protoxoids), and those whose affinity for antitoxin is equal to that of toxin. These latter he calls "syntoxoids.',

The toxon (epitoxoid originally), as Ehrlich believes, has a haptophore or "binding" group similar to that of toxin, but a different toxophore or "poisoning" group. Qualitatively it has been shown to differ from toxin in that, lacking the power to produce acute symptoms, it causes gradual emaciation and paresis in animals.

That this difference in the poisonous action of toxin and toxon is not merely a quantitative difference, referable to small quantities of toxin, was proved by Dreyer and Madsen, ${ }^{16}$ who showed that if they made a toxin-antitoxin mixture in which after injection the only evidence of incomplete neutralization lay in the emaciation and final paralysis of the test animals, the quantity of such a mixture could be increased five- and tenfold, without producing the true toxin symptoms in animals. These authors, too, claim to have been

${ }^{16}$ Dreyer und Madsen, Zeit. f. Hyg., xxxvii, 1901. 
able to immunize against toxin with such mixtures, thereby proving the identity of the haptophore groups of the two substances. The importance of this observation will become more evident in connection with the section on the "side-chain theory."

Method of Partial Absorption of Toxin.-Ehrlich ${ }^{17}$ has gathered more exact data in support of his views from what he terms the "Method of Partial Absorption", of toxin by antitoxin.

In order to understand this method clearly, it is necessary to remember that Ehrlich ${ }^{18}$ believes the union of toxin with antitoxin to take place according to the chemical laws of valeney. Just as in $\mathrm{H}_{2} \mathrm{O}$ oxygen has an atomic valeney of 2 for hydrogen, so, in the fully neutralized toxin-antitoxin compound, he believes antitoxin to have a valency of 200 for toxin. It would require, according to this, $200 \mathrm{~T}$ or toxin molecules to satisfy the affinities of one antitoxin molecule. ${ }^{19}$

This belief is based upon the following consideration: In determining the $\mathrm{I}_{0}$ dose, or fully neutralized toxin-antitoxin union, Ehrlich, as well as Madsen, found that the number of $\mathrm{T}$ units contained in such a dose was almost regularly a factor of one hundred, recurring again and again as $25,33,50,75$, etc. This pointed to more or less regularity in the deterioration of toxin into toxoid, and to a more or less regular relation of toxin to toxon. Now, as we have seen before, if we could procure a perfectly pure toxin, the $\mathrm{L}_{0}$ dose plus one toxin unit would give us the $\mathrm{L}_{+}$dose; that is, one toxin unit in excess of full neutralization would suffice to kill a guinea-pig of 250 grams in four to five days. Since a perfectly pure toxin, however, has not been obtainable up to the present time, it is clear that the number of pure toxin bonds contained in $\mathbf{L}+$ must be less than the actual number of neutralizing units in the combination, a part of the antitoxin being bound by toxon and toxoid. The actual values obtained for the number of $\mathrm{T}$ units in $\mathrm{L}_{+}$has never exceeded 200, and has usually been more than 100 , the highest value ascertained by Madsen being 160 . Given, therefore, a combining value which, being a multiple of one hundred, is often more than one hundred, but in an obviously impure state has never reached 200 , it is most likely that 200 represents the actual value sought for.

“ Ehrlich, "Gesammelte Arbeiten zur Immunitätsforsch.,' Berlin, 1904.

${ }^{18}$ Ehrlich, Deut. med. Woch., 1898.

${ }^{18}$ Ehrlich, "Schlussbetrachtungen," Nothnagel's System. 
Assuming, therefore, upon the foregoing considerations, that the valency of antitoxin for toxin is 200, Ehrlich carries out his experiments in the following way:

Given a toxin, the unit ( $\mathrm{T}$ ) of which is 0.024 c.c., he first determines the $\mathrm{L}_{+}$dose which, tested against the standard antitoxin unit, in this case is 2.05 c.e. But 2.05 c.c. $=85 \mathrm{~T}$. (or $2.05 \div .024)$ units. By mixing the $\mathrm{L}_{+}$dose of toxin and antitoxin in such a way that the quantity of antitoxin is gradually increased, while the toxin remains always $\mathrm{L}_{+}$, and determining upon animals the amount of free toxin contained in each mixture, the following table may be constructed : $:^{20}$

\begin{tabular}{|c|c|c|c|c|c|c|c|c|c|}
\hline 0 a & titc & unit & presenting & 0 & lenc & $+L_{+}=85$ & free & $T$ & units. \\
\hline .1 & 66 & ، & “ & 20 & “ & $+L_{+}^{+}=85$ & "6 & “" & “6 \\
\hline .25 & 66 & “" & "6 & 50 & "، & $+L_{+}=60$ & "“ & "“ & “6 \\
\hline .8 & 6 & “6 & "6 & 160 & "6 & $+L_{+}=10$ & 6 & 16 & 6 \\
\hline .9 & 66 & "6 & “6 & 180 & 66 & $+L_{+}=3.5$ & 66 & "6 & 6 \\
\hline
\end{tabular}

It is plain that the substances with the strongest affinity for antitoxin must be bound first by the antitoxin. This does not diminish the toxic value of the mixture; and these are the protoxoids. Next are bound syntoxoids and toxins, and, finally, the toxons. It is plain that, by this method, the constitution of any given toxin may be ascertained, and Ehrlich has constructed, on the basis of these observations, what he terms his toxin spectrum. Minor differences of toxicity and affinity for the antibody have caused him, by the partial saturation method described, still further to divide toxin into proto-, deutero-, and trito-toxin.

His spectra graphically describe the constitution of any given toxic bouillon and trace its deterioration as follows:

Ehrlich's opinions as to the constitution of toxin have borne important practical fruit in allowing him to develop a system of standardization of anti-toxin which will be considered in the next chapter, but his theoretical conceptions as described above are not accepted as truly representing the conditions at the present day. His assumption of the complexity of toxic filtrates, that is, toxin, the various toxoids, and toxons, results from strict adherence to the belief that the reaction is analogous to that taking place between strong acids and strong bases. The first to seriously throw doubt upon his theoretical conceptions on the basis of experiment, were Arrhenius and Madsen.

${ }^{20}$ Example taken from Ehrlich, Deut. med. Woch., 1898. 
Modern theories of solution maintain that substances in solution are broken up into their atoms or atom-groups, known as ions. Thus, $\mathrm{NaCl}$ in solution would be "dissociated" into its $\mathrm{Na}$ ion and its $\mathrm{Cl}$ ion, the completeness of the dissociation depending upon the concentration of the solution. A solution of $\mathrm{NaCl}$, therefore, contains, according to this view, three substances, NaCl undissociated and free ions of $\mathrm{Na}$ and $\mathrm{Cl}$, the relative quantities of the three present in any given solution being calculable, and depend upon a law known as the law of mass-action of Culdberg and Waage. These free ions are the elements, therefore, which are active in the formation of further chemical combination. When a strong acid, in solution, acts upon a base, say $\mathrm{HCl}$ upon ammonia $\left(\mathrm{NH}_{3}\right)$, strong acid having the property of quite complete dissociation in relatively concentrated solutions, little or no ammonia would remain unbound. A weak acid, like boric acid, however, not being as completely dissociated, would leave some ammonia uncombined even after more quantitatively sufficient boric acid had been added. Arrhenius and Madsen, on the basis of careful researches into the reaction between tetanolysin and its antibody, believe that toxin and antitoxin possess weak chemical avidity for each other, their interaction being comparable to that taking place between a weak acid and a base. Toxinantitoxin solutions, therefore, would contain the neutral compound, but at the same time uncombined toxin and antitoxin. The qualities which Ehrlich ascribes to toxon, they believe, are due to the unbound toxin present in such mixtures. In careful studies in which they inhibited the hemolytic action of ammonia by gradual addition of boric acid, they were able to show complete parallelism between the conditions governing this neutralization and those concerned in their tetanus experiments. Their explanation has the advantage of great simplicity over that of Ehrlich's and also the fact that it takes into account the laws of dissociation which always takes place in solutions in which the union oi two substances occurs. We cannot enter into the matter at greater length in this place, and must refer the reader to more extensive works on immunity. ${ }^{21}$ Objections to the ideas of Arrhenius and Madsen have been brought forward by Nernst, Bordet and others, largely on the basis that toxin-antitoxin are probably colloidal in nature, and that the laws of dissociation in colloidal reactions are not, as yet, clear.

${ }^{21}$ See Zinsser, Infection and Resistance, MacMilian \& Co., New York, 1917; Bordet, Immunité, Masson, Paris, 1920. 
We do know, however, from recent work, that antigen-antibody complexes can dissociate, and the work of Loeb has shown that reactions of proteins are, after all, in many ways strictly analogous to ordinary chemical reactions between less complexly constituted substances. Moreover, direct experimentation, such as that of Landsteiner on agglutinated typhoid bacilli, Gay and Chickering's work on the dissociation of antibody from precipitates, Huntoon's work on the dissociation of antibody from sensitized pneumococci, as well as observations made on the dissociation of diphtheria toxin from toxin-antitoxin mixtures in connection with active immunization in diphtheria, have now shown pretty definitely that dissociation of these substances in the animal body and in the test tube may take place. In the light of these newer researches the ideas of Arrhenius and Madsen, must eventually be reexamined.

Bordet, Landsteiner and others have brought out another point of view which might explain the quantitative relations which exist in toxin-antitoxin mixtures as worked out by Ehrlich. They dwell particularly upon the analogy of these reactions with colloidal reactions, assuming that when antitoxin is mixed with toxin in amounts insufficient to completely neutralize, the units of antitoxin are not taken up by a corresponding fraction of the total toxin present, leaving a part of the toxin absolutely free, but that, on the contrary, the antitoxin is equally distributed over all the toxin units present, leaving all of them partially saturated. This would not sharply neutralize a part of the toxin, leaving another part entirely free to exert its activity, but would partially neutralize all the toxin. It is more or less analogous, as Bordet brings out, to different degrees of coloration which are produced when starch absorbs varying quantities of iodine. They compare it to an adsorption phenomenon, rather than to a true chemical reaction.

More or less in harmony with this view is the Dansyz effect, ${ }^{22}$ which is as follows: When diphtheria toxin is added to its anti-toxin in two fractions, a definite period elapsing between the addition of the first and the second fraction, much more toxin remains free than when the total quantities are mixed at once. This view has been interpreted in Ehrlich's sense by Von Dungern, but by a very forced process of reasoning. We may assume with considerable confidence at the present time, that, while the Ehrlich method of

2s Dansyz, Ann. de l'Inst. Past., 16, 1902. 
standardization is still the most useful basis for therapeutic unit determination, the theoretical considerations upon which it is founded can no longer be accepted as conclusive.

This, however, need not prevent us from discussing the sidechain theory which has no direct relationship to his views on toxins, but offers a very ingenious explanation for the mysterious problem of antibody formation and specificity.

The Side-Chain Theory.-We have seen that the extensive researches of Ehrlich into the nature of the toxin-antitoxin reaction led him to believe that the two bodies underwent chemical union, forming a neutral compound. The strictly specific character of such reactions, furthermore, diphtheria antitoxin binding only diphtheria toxin, tetanus antitoxin only tetanus toxin, etc., led him to assume that the chemical affinity between each antibody and its respective antigen depended upon definite atom groups contained in each.

Ehrlich $^{23}$ had, in 1885, published a treatise in which he discussed the manner of cell-nutrition and advanced the opinion that in order to nourish a cell, the nutritive substance must enter directly into chemical combination with some elements of the cell protoplasm. The great number and variety of chemical substances which act as nutriment led him to believe that the highly complex protoplasmic molecules of cells,were made up of a central atom-group (LeistungsKern) upon which depended the specialized activities of the cell, and a multiplicity of side chains (a term borrowed from the chemistry of the benzol group), by means of which the cell entered into chemical relation with food and other substances brought to it by the circulation. If we illustrate graphically by the chemical conception from which the term side chain was borrowed, in salicylic acid, the formula given, the benzol ring represents the "Leistungs-Kern,"

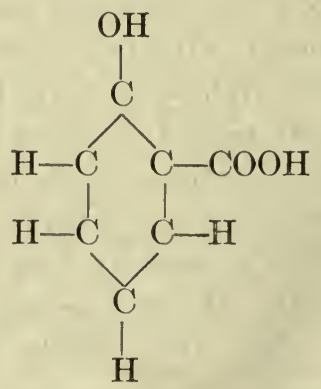

${ }^{23}$ Ehrlich, "Das Sauerstoffbedürfniss des Organismus," Berlin, 1885. 
or radicle, while $\mathrm{COOH}$ and $\mathrm{OH}$ are side chains by means of which a variety of other substances may be brought into relation with the "radicle," for instance, as in methyl salicylate.

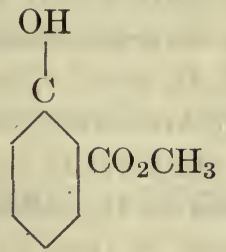

Just as nutritious substances are thus brought into workable relation. with the cell by means of the atom-groups corresponding to side ehains, so Ehrlich believes toxins exert their deleterious action only because the cells possess side chains by means of which the toxin can be chemically bound. These side chains, Ehrlich in his later work ealls "receptors." The receptors or side chains present in the cells and possessing by chance specific affinity for a

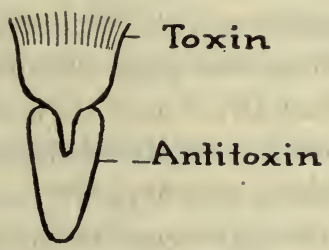

Fig. 31.-Toxin and Antitoxin.

given toxin, are, by their union with toxin, rendered useless for their normal physiologieal function. By the normal reparative mechanism of the body these receptors are probably east off and regeneraied. Regenerative processes of the body, however, do not, as a rule, stop at simple replacement of lost elements, but, according to the hypothesis of Weigert, ${ }^{24}$ usually tend to overeompensation. The receptors eliminated by toxin absorption are not, therefore, simply reproduced in the same quantity in which they are lost, but are reproduced in excess of the simple physiological needs of the cell. Continuous and increasing dosage with the poison, consequently, soon leads to such exeessive production of the particular receptive atom-groups that the cells involved in the 
process become overstocked and cast them off to circulate freely in the blood. These freely circulating receptors-atom-groups with specific affinity for the toxins used in their production-represent the antitoxins. These, by uniting with the poison before it can reach the sensitive cells, prevent its deleterious action.

The theory of Ehrlich, in brief, then, depends upon the assumptions that toxin and antitoxin enter into chemical union, that each toxin possesses a specific atom-group by means of which it is bound to a pre-existing side chain of the affected cell, and that these side chains, in accordance with Weigert's law, under the influence of repeated toxin stimulation, are eventually overproduced and cast off by the cell into the circulation.

It stands to reason that this theoretical conception would be vastly strengthened were it possible to show that such receptors or toxin-binding atom-groups actually pre-existed in the animal body, and such support was indeed given by the experiments of Wassermann and Takaki. ${ }^{25}$ These observers succeeded in showing that tetanus toxin could be rendered innocuous if, before injection into animals, it was thoroughly mixed with a sufficient quantity of the fresh brain substance of guinea-pigs. Similar observations were independently made by Asakawa, ${ }^{26}$ and variously confirmed. Kempner and Schepilewsky ${ }^{27}$ showed a similar relation to exist between brain tissue and botulismus toxin, and Myers ${ }^{28}$ brought proof of analogous conditions in the case of suprarenal tissue and cobra poison.

\footnotetext{
${ }^{25}$ Wassermann und Takaki, Berl. klin. Woch., 1898.

${ }^{26}$ Asakawa, Cent. f. Bakt., 1898.

${ }^{27}$ Kempner und Schepilewsky, Zeit. f. Hyg., 1898.

${ }^{28}$ Myers, Cent. f. Bakt., i, 1899.
} 


\section{CHAPTER XIV}

\section{PRODUCTION AND, TESTING OF ANTITOXINS}

\section{DIPHTHERIA ANTITOXIN}

IN spite of the great advances in our theoretical knowledge of antibodies, gained during the last three decades, extensive therapeutic application has been made of the antitoxins only. Preeminent among these from a practical point of view are the antitoxins against diphtheria and tetanus toxin. For diphtheria, careful statistical studies have demonstrated, beyond doubt, the therapeutic value of the serum treatment. Biggs and Guerard, in a general statistical review, arrived at the conclusion that the death rate of diphtheria had been reduced fifty per cent by the use of antitoxin. Approximately the same estimate is made by Dieudonné ${ }^{1}$ who studied almost 10,000 treated cases.

Production of Diphtheria Antitoxin.-The methods for producing diphtheria antitoxin vary only in minor technical details. The first requisite for successful antitoxin production is the possession of a strong toxin. The various means of obtaining this are outlined in the section on diphtheria toxin. The toxin used should be of such potency that less than 0.1 c.c. will kill a guinea-pig of 250 grams weight in four to five days. ${ }^{2}$

For experimental purposes, goats or sheep may be used for immunization; for antitoxin production on a large scale, horses have been found to be the most useful animals. The horses should be young, four to six years old, vigorous, and healthy. It is advisable that they be subjected to the mallein test to exclude possible infection with glanders.

The toxin injections are made subcutaneously. Because of the differences in susceptibility noted in various horses, it is advisable that the first doses of toxin should be either very small or weakened

${ }^{1}$ Dieudonné, Arb. a. d. kais. Gesundheitsamt, 1895 and 1897.

"Park, "Pathog. Bacteria and Protozoa," N. Y., 1908. 
by ehemicals or heat, or combined with antitoxin. In the Pasteur Institute in Paris, the small initial dose of toxin ( 0.5 c.c.) is mixed before injection with an equal quantity of Lugol's solution (iodinpotassium iodid solution).

Park $^{3}$ advises an initial dose of 5,000 toxin units (about 20 c.c. of toxin) combined with 100 units of antitoxin. The same amount is given with the second and third doses of toxin. The intervals are from five days to a week, determined by eomplete subsidence of the reaction (temperature). The doses are increased until, at the end of two or three months, more than ten times the original dose is given (50,000 units).

Horses vary greatly in the strength of antitoxin which they will produce. At the end of three or four months in favorable animals one cubic eentimeter of serum may contain 250 to 800 antitoxin units. Further immunization will often increase the antitoxin output to 1,000 and more units to the cubic centimeter of serum. Park states that none of the horses used by him has ever yielded 2,000 units to the cubic centimeter. The same writer advises a three months' period of rest from immunization at the end of every nine months. Given such resting periods, some horses have continued to furnish high-grade antitoxin for from two to four years.

In order to obtain serum from horses, a sharp cannula is introduced into the jugular vein. After leading the horse into a specially constructed stall, its head is slightly deflected and pressure is made upon the jugular vein below the point into which the needle is to be plunged. Compression ean also be made by surrounding the neck of the horse elose to the shoulders with a leather strap over a pad laid directly upon the vein. The vein becomes visible along the lower margin of the neck in a line running from the angle of the jaw to the edge of the scapula. The skin, of course, is previously shaved and sterilized. The eannula is then plunged into the vein, either with or without previous incision through the skin, and, through a sterile rubber tube, the blood is allowed to flow into high class eylinders or slanted Erlenmeyer flasks. In this way, large quantities of blood may be obtained and, aceording to Kretz, ${ }^{4}$ as much as six liters may be taken at a time at intervals of a month,

${ }^{3}$ Park, loc. eit., p. 212.

" Kretz, in "Handb. der Techn. u. Meth. d. Immun.," Kraus and Levaditi, vol. ii, 1908. 
without injuring the animal. Ligature of the vein after bleeding is unnecessary.

The cylinders and flasks are allowed to stand for two or three days at or below $10^{\circ} \mathrm{C}$. At the end of this time, the serum may be pipetted or siphoned away from the elot and stored in the refrigerator. In order to diminish the chanees of contamination, five-tenths per cent of carbolie aeid or four-tenths per eent of tricresol may be added.

Antitoxin is fairly stable and if kept in a cool, dark place, may remain active, with but slight deterioration, for as long as a year. Kept in a dry state, in vacuo, over anhydrous phosphoric acid, by the method of Ehrlich, it retains its strength indefinitely.

Standardization.-Antitoxin units being measured in terms of toxin, uniformly of measurement necessitates the possession by the various laboratories of a uniform toxin. Antitoxin being more stable than toxin, uniformity of toxin is obtained by means of a standard antitoxin distributed from a eentral laboratory: This was first done by Ehrlich in Germany, and is now done for the United States by the Public Health and Marine Hospital Service laboratories. Bottles of the distributed antitoxin are marked with the number of units contained in each c.c. Dilutions of this are mixed with varying quantities of the toxin to be tested, the mixtures are allowed to stand for 15 minutes to permit union of the two elements, and injections into guinea-pigs of 250 grams weight are made. Thus the $\mathrm{L}_{+}$dose of the toxin is determined. (The $\mathrm{L}_{+}$dose [p. 260] is the quantity of poison not only suffieient to neutralize one antitoxin unit, ${ }^{5}$ but to contain an excess beyond this sufficient to kill a guineapig of 250 grams in 4 to 5 days. $\mathrm{L}_{+}$is chosen rather than $\mathrm{L}_{0}$, the simple neutralizing dose, because of the difference between toxins in their contents of toxoid and toxon. ${ }^{6}$ )

The $\mathrm{L}_{+}$dose of the toxin having thus been determined, this

The older definition of a unit of diphtheria antitoxin is the quantity of antitoxin sufficient to protect a guinea-pig of 250 grams against 100 times the fatal dose of diphtheria toxin. This, however, holds true only if we are dealing with normal toxins and antitoxins as at first devised by Behring. In the conditions under which the measurements are made at present, however, this definition nust be revised as follows: A unit of antitoxin is that amount of antitoxin which will save the life of a guinea-pig if injected together with an $\mathrm{I}_{+}$dose of the toxin.

'Madsen, in Kraus u. Levaditi, “Handbuch," etc., 1907. 
quantity is mixed with varying dilutions of the unknown antitoxin. ${ }^{7}$ Thus, given an antitoxin in which 300 to 400 units to the c.c. are suspected, dilutions of $1: 200,1: 250,1: 300$, etc., are made. One c.c. of each of these is mixed with the $\mathrm{L}_{+}$dose of the toxin, and the mixtures are injected into guinea-pigs of about 250 grams. If the guinea-pig receiving $\mathrm{L}_{+}$plus the 1:250 dilution lives and the one receiving $L_{+}$plus the $1: 300$ dilution dies in the given time, we know that the unit sought must lie between these two values, and further similar experiments will easily limit it more exactly. The possibility of error in carrying out such measurement is much diminished by the use of larger quantities of dilutions higher than those given. Four c.e. is the volume usually injected.

Since 1902, the production and sale of diphtheria antitoxin has been regulated by law in the United States. From time to time, antitoxin is bought in the open market and examined at the hygienic laboratories of the United States Public Health and Marine Hospital Service. Antitoxic serum which contains less than two hundred units to each cubic centimeter is not permitted upon the market.

In a previous section we have seen that Hiss and Atkinson ${ }^{8}$ and others have shown an increase in the globulin contents of blood serum of immunized animals. It has been shown, furthermore, that the precipitation of such serum with ammonium sulphate carried down in the globulin precipitate all the antitoxic substances contained in the serum. Upon a basis of globulin precipitation, Gibson ${ }^{9}$ has recently perfected a method of concentrating and purifying diphtheria antitoxin for therapeutic use. This procedure, as carried out at the New York Department of Health, is, in principle, as follows :

The serum, as taken from the horse, is heated to $56^{\circ} \mathrm{C}$. for twelvr hours. This converts about half of the pseudoglobulin into euglobulin, the antitoxin remaining in the pseudoglobulin fraction. ${ }^{10}$ It is then ${ }^{11}$ precipitated with an equal volume of a saturated ammonium sulphate solution. After two hours, the precipitate is caught in a filter and redissolved in a quantity of water corresponding to

'Dönitz, “Die Werthbem. der Heilsera,' in Kolle u. Wassermann.

${ }^{8}$ Hiss and Atkinson, Jour. Fxper. Med., v, 1900.

- Gibson, Jour. of Biol. Chem., i, 1906.

10 Ir. Banzhaf, personal conmunication.

${ }^{11}$ Gibson and Collins, Jour. of Biol. Chem., iii, 1907. 
the original quantity of serum. After filtration, this solution is again precipitated with saturated ammonium sulphate solution and the precipitate again filtered off. The precipitate is then treated with a saturated solution of sodium chloride of double the volume of the original serum. This is allowed to stand for about twelve hours. At the end of this time the antitoxin-containing globulin is in solution and is pipetted away from the precipitate and filtered. This salt-solution extract is then precipitated with twenty-five hundredths per cent acetic acid. The resulting precipitate of globulin is thoroughly dried by pressure between filter papers and placed in a parchment dialyzer. Dialysis with running water is continued for seven to eight days, after neutralization with sodium carbonate, in order to remove the sodium chloride. At the end of this time, the globulin solution remaining in the dialyzer is filtered through a Berkefeld candle for the purpose of sterilization, after the addition of 0.8 per cent sodium chlorid. According to Gibson, this method produces a yield of antitoxin which equals about fourfifths of the original quantity but is concentrated five- to seven-fold. The method has more recently been modified as follows:

After heating to $56^{\circ} \mathrm{C}$, as above, and cooling, ammonium sulphate is added to the serum to thirty per cent saturation. This brings down all the euglobulins. This is then filtered and the filtrate, which contains the pseudoglobulins with the antitoxin, is again precipitated with ammonium sulphate in a concentration of fifty-four per cent of saturation. The precipitate is then separated on a paper, pressed to dryness, and directly dialyzed. ${ }^{12}$

Park and Thorne ${ }^{13}$ have found that the use of such coneentrated antitoxin is, therapeutically, equally efficient as the unconcentrated, and possesses the advantage of less frequently giving rise to the secondary reactions in skin and mucous membranes occasionally noticed after the use of ordinary antitoxin, and referable, probably, to some other constituent of the horse serum.

Diphtheria antitoxin is therapeutically used in doses ranging from 3,000 to 20,000 units. For prophylactic immunization of healthy individuals, about 500 units should be used.

See also the chapter on diphtheria for Schick reaction and active immunization with toxin-antitoxin mixtures.

${ }^{12} \mathrm{Dr}$. Banzhaf, personal communication.

${ }^{13}$ Park and Thorne, Amer. Jour. Med. Sci., Nov., 1906. 


\section{TETANUS ANTITOXIN}

Production of Tetanus Antitoxin.-The production of tetanus antitoxin is, in every way, analogous to that of diphtheria antitoxin. It is necessary in the first place to produce a powerful tetanus toxin. The methods of procuring this will be discussed in the section upon tetanus toxin. Suffice it to say here that the most satisfactory method of obtaining toxins consists in cultivating the bacilli upon veal broth containing five-tenths per cent to two per cent sodium chlorid and one per cent pepton. It has been advised, also, that the broth should be neutralized by means of magnesium carbonate rather than with sodium hydrate. The bacilli are cultivated for eight to ten days at incubator temperature and the broth filtered rapidly through Berkefeld filters. The toxin may be preserved in the liquid form with the addition of five-tenths per cent carbolic acid, or may be preserved in the dry state after precipitation with ammonium sulphate.

It is necessary to determine the strength of the poison. This is done according to $\mathrm{v}$. Behring ${ }^{14}$ by determining the smallest amount of toxin which will kill a white mouse of twenty grams weight within four days. This is most easily done by making dilutions of the toxin ranging from $1: 100$ to $1: 1,000$, and then injecting quantities of 0.1 c.c. of each of these dilutions subcutaneously into white mice. In this way, the minimal lethal dose is ascertained.

For the actual production of antitoxin, horses have been generally found to be the most favorable animals. The horses should be healthy and from five to seven years old. The first injection of toxin administered to these animals should be attenuated in some way. Various methods for accomplishing this have been in use. In America, the first injection of about ten to twenty thousand minimal lethal doses ${ }^{15}$ (for mice of twenty grams weight) is usually made subcutaneously together with sufficient antitoxin to neutralize this quantity. In Germany, v. Behring uses, for his first injection, a much larger dose of toxin to which about 0.25 per cent of terchlorid of iodin has been added. Immediately after an injection, the animals will usually show a reaction expressed by a rise of temperature,

${ }^{14} v$. Behring, Zeit. f. Hyg., xii, 1892; Deut. med. Woch., 1900.

${ }^{15}$ According to Park the "horses receive 5 c.c. as the initial dose of a toxin of which 1 c.c. kills 250,000 grams of guinea-pig, and along with this a sufficient amount of antitoxin to neutralize it.', 
refusal of food, and sometimes muscular twitching. A second injection should never be given until all such symptoms have completely subsided. This being the ease, after five to eight days double the original dose is given together with a neutralizing amount of antitoxin or with the addition of terehlorid of iodin. $\Lambda$ gain after five to eight days, a larger dose is given and thereafter, at similar intervals, the quantity of toxin is rapidly increased. In America the neutralizing antitoxin is omitted after the third or fourth injeetion; in v. Behring's laboratory the quantity of terchlorid of iodin is gradually diminished. The increase of dosage is often controlled by the determination of the antitoxin contents of the animal's blood serum. The immunization is increased until enormous doses (500 c.c.) of a toxin in which the minimal lethal dose for mice is represented by 0.0001 c.c., or less, is borne by the horse without apparent harm.

The antitoxic serum is then obtained by bleeding from the jugular vein, as in the ease of diphtheria antitoxin. It may be preserved in the liquid state by the addition of five-tenths per cent of carbolic acid or four-tenths per cent of tricresol.

Standardization.- The universal prophylactic use of tetanus antitoxin has, as in the ease of diphtheria antitoxin, necessitated its standardization. A variety of methods are in use in different parts of the world. In the following deseription the American method only will be considered as laid down under the law of July, 1908, and based upon the work of Rosenau and Anderson ${ }^{16}$ at the United States Hygienic Laboratories at Washington.

In conjunction with a committee of the Society of American Bacteriologists, these authors have defined the unit of tetanus antitoxin as follows:

The unit shall be ten times the least amount of serum necessary to save the life of a 350 gram guinea-pig for ninety-six hours against the official test dose of standard toxin. The test dose consists of 100 minimal lethal doses of a precipitated toxin preserved under special conditions at the hygienic laboratory of the Public Health and Marine Hospital Service. (The minimal lethal dose is in this ease, unlike v. Behring's minimal lethal dose, measured not against 20 gram mice, but against 350 gram guinea-pigs.)

In the actual standardization of tetanus antitoxin, as in that of

${ }^{16}$ Rosenau and Anderson, Pub. Health and Mar. Hosp. Serv. U. S., Hyg. Lab. Bull. 43, 1908. 
diphtheria antitoxin, the $\mathrm{L}_{+}$dose of toxin is employed. The $\mathrm{L}_{+}$ dose is, however, in this ease, defined as the smallest quantity of tetanus toxin that will neufralize one-tenth of an immunity unit, plus a quantity of toxin sufficient to kill a 350 gram guinea-pig in just four days. At the Hygienie Laboratory at Washington, a standard toxin and antitoxin are preserved under special conditions, and standard toxin and antitoxin, arbitrary in their first establishment, are kept constant by being measured against each other from time to time. In measuring the antitoxic serum thus preserved, at the Hygienic Laboratory, a mixture of one-tenth of a unit of antitoxin and 100 minimal lethal doses of the standard toxin must contain just enough free poison to kill the guinea-pig in four days. This $\mathrm{L}_{+}$dose of the standard toxin is given out to those interested commercially or otherwise in the production of antitoxin.

In measuring an unknown antitoxic serum against this $\mathrm{L}_{+}$dose of toxin, a large number of mixtures are made, each eontaining the $\mathbf{L}_{+}$dose of the toxin and varying quantities of the antitoxin. Dilutions must always be made with 0.85 per cent salt solution and the total quantity injected into the animals should always be brought up to 4 c.e. with salt solution in order to equalize the conditions of coneentration and pressure. The mixtures are then kept for one hour at room temperature in diffused light. After this they are subcutaneously injected into a series of guinea-pigs weighing from 300 to 400 grams. The following example of a test is taken from the article by Rosenau and Anderson quoted above.

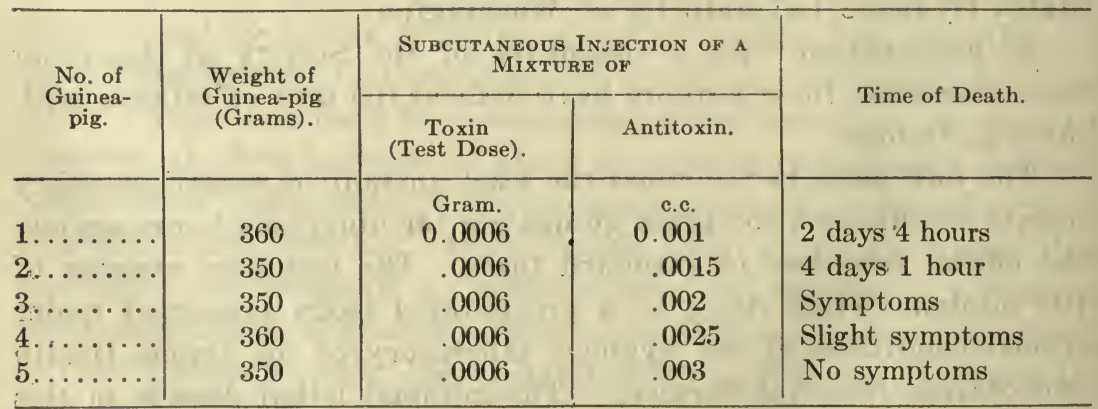

In this series the guinea-pig, receiving 0.0015 c.e. of the antitoxin, died in approximately four days; 0.0015 c.c. therefore represents one-tenth of an immunity unit.

In therapeutically employing antitoxin for prophylactic purposes, above 1,500 units should be employed. 


\section{CHAPTER XV}

SENSITIZING ANTIBODIES. (PHENOMENA OF LYSIS, AGGLUTINA'IION, PRECIPITATION, ETC.)

Alexin and Sensitizing Antibodies.-In the immediately preceding sections, we have dealt solely with immunity as it occurs where solüble toxins play an important part and in which antitoxins are developed in the immunized subject. There are many species of pathogenic bacteria, however, which stimulate the production of little or no antitoxic substance when introduced into animals, and the resistance of the immunized animal can not, therefore, be explained by the presence of antitoxin in the blood.

v. Fodor, ${ }^{1}$ Nuttall, ${ }^{2}$ Buchner, ${ }^{3}$ and others had in 1886 and the years following, carried on investigations which showed that normal blood serum possessed the power of killing certain of the pathogenic bacteria. Nuttall, working under the direction of Flügge, made the important discovery that this bactericidal power became gradually diminished with time, and could be experimentally destroyed by exposure of the serum to a temperature of $56^{\circ} \mathrm{C}$. for one-half hour. Buchner, who confirmed and extended the observations of Nuttall, called this thermolabile substance upon which the bactericidal character of the serum seemed to depend "alexin."

Our knowledge of the bactericidal action of serum was, soon thereafter, extensively increased by the discovery, by Pfeiffer and Isaeff, ${ }^{4}$ that cholera spirilla injected into the peritoneal cavity of a cholera-immune guinea-pig were promptly killed and almost completely dissolved. The same phenomenon could be observed when the spirilla, mixed with fresh immune serum, were injected into the peritoneum of a normal guinea-pig.

The processes observed by Pfeiffer as taking place intraperi-

${ }^{1} v$. Fodor, Deut. mel. Woch., 1886.

${ }^{2}$ Nuttall, Zeit. f. Hyg., 1886.

Buchner, Cent. f. Bakt., 1889.

- Pfeiffer und Isaeff, Zeit. f. Hyg., 1894. 
toneally were soon shown by Metchnikoff, ${ }^{5}$ Bordet, ${ }^{6}$ and others to take place, though to a lesser extent, in vitro. Bordet, furthermore, observed that the bacteriolytic digestive power of such immune serum, when destroyed by heating, or after being attenuated by time, could be restored by the addition to it of small quantities of normal blood serum. It could, in other words, be "reactivated" by normal serum. From this observation Bordet drew the conclusion that the bactericidal or bacteriolytic action of the serum depended upon two distinct substances. The one present in normal serum and thermolabile, he conceived to be identical with Buchner's alexin. The other, more stable, produced or at least increased in the serum by the process of immunization, he called the "sensitizing substance." This substance, he believed, acting upon the bacterial cells, rendered them vulnerable to the action of the alexin. Without the previous preparatory action of the "sensitizing substance" the alexin was unable to act. Without the cooperation of alexin, the "sensitizing substance" produced no visible effects.

Bordet's interpretation of the phenomenon of lysis differs essentially from that of Ehrlich, in that both active serum components are conceived by him, though independent, to act directly upon the bacterial cell. A few years later, Bordet was able to show that exactly analogous conditions governed the phenomenon known as "hemolysis" or disintegration of red blood cells.

It had been known for many years that in the transfusion of blood from an animal of one species into an animal of another species, injury was done to the red corpuscles which were introduced. Observed in the test tube, the red cells in the heterologous serum were seen to give up their hemoglobin in the fluid, the mixture taking on the red transparency characteristic of what is known as "laked" blood. Buchner, ${ }^{7}$ in his alexin studies, had shown that the blood-cell destroying action of the normal serum was subject to the same laws as the bactericidal power of similar serum, in that it was destroyed by heating, and he assumed that both the bacteriolytic and the hemolytic action of normal serum were due to the same "alexin." Metchnikoff, 8 moreover, had pointed out the striking analogy between the two phenomena as early as 1889 .

s Metchnikoff, Ann. de 1'inst. Pasteur, 1895.

Bordet, ibid., 1895.

"Buchner, Arch. f. Hyg., xvii, 1893; Waremberg, Arch. d. méd. exper., 1891.

${ }^{8}$ Metchnikoff, Ann. de 1'inst. Pasteur, 1889. 
Bordet $^{9}$ now observed that the blood serum of guinea-pigs previously treated with the defibrinated blood of rabbits developed marked powers of dissolving rabbits' corpuscles, and that this hemolytic action could be destroyed by heating to $56^{\circ} \mathrm{C}$., but "reactivated" by the addition of fresh normal serum. He liad thus produced an immune hemolysin, just as Pfeiffer had produced immune bacteriolysin, and had demonstrated the complete parallelism which existed between the two phenomena.

A practical test-tube method was thus given for the investigation of the lysins, just as a practical test-tube method for antitoxin researches had been developed by Ehrlich in his ricin-antiricin experiments.

The path of investigation thus pointed out by Bordet was soon explored in greater detail by Ehrlich and Morgenroth. ${ }^{10}$ The reasoning which Fhrlich had applied in explaining the production of antitoxins was thought, by these observers, to be equally applicable to the phenomena of bacteriolysis and hemolysis.

Since the thermolabile substance or alexin, renamed by Ehrlich "complement," was already present in normal serum and had been shown to be little, if at all, increased during the process of immunization, this substance could have but little relation to the changes taking place in the animal body as immunity was acquired. The more stable serum-component, however, the "substance sensibilisatrice" of Bordet, or, as Ehrlich now called it, the "immune body," was the one which seemed specifically called forth by the process of active immunization. Ehrlich argued, therefore, that when bacteria or blood cells were injected into the animal, certain atom-groups or chemical components of the injected substances were united to other atom-groups or "side chains" of the protoplasm of the tissue cells. These "side chains" or receptors, then reproduced in excess and finally thrown free into the circulation, constituted the "immune body." The immune body, therefore, he concluded, must possess atom complexes which endow it with specific chemical affinity for the bacteria or red blood cells used in its production. This contention was supported by Ehrlich and Morgenroth by an ingenious series of experiments.

Having in their possession, at that time, the blood serum of a

'Bordet, Ann. de l'inst. Pasteur, t. xii, 1898.

${ }^{10}$ Ehrlich und Morgenroth, Berl. klin. Woch., 1, 1899. 
goat immunized against the red blood cells of a sheep, they inactivated it (destroyed the complement or alexin) by heating to $56^{\circ}$ C. The serum then contained only the "substance sensibilisatrice" or immune body. To this inactivated serum they added sheep's red corpuscles, without obtaining hemolysis. Having left the inactive serum and the sheep's corpuscles in contact with each other for some time, they separated them by centrifugalization. To the supernatant fluid, they now added sheep-blood corpuscles and normal goat serum (complement) and found that no hemolysis took place. The immune body had apparently gone out of the serum. The red cells which had been in contact with the serum and separated by the centrifuge were then washed in salt solution and to them complement was added in the form of fresh normal serum. Hemolysis

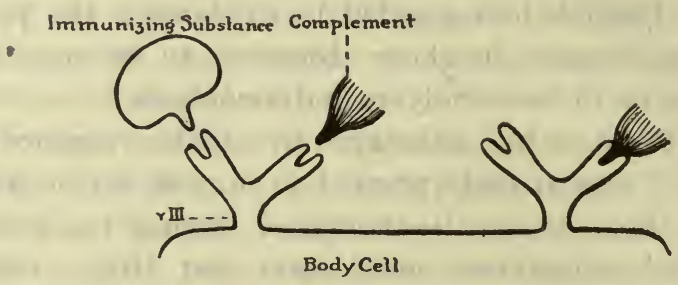

Fig. 32.-Ehrlich's Conception of Celi-Receptors, giving Rise to Immune Bodies.

occurred. It was plain, therefore, that the immune body of the inactivated serum had gone out of solution and had become attached to the red blood cells, or, as Ehrlich expressed it, the immune body by means of its "haptophore" atom-group had become united to the corpuscles. In contrast to this, if normal goat serum (containing complement only) was added to sheep corpuscles and separated again by centrifugalization, the supernatant fluid was found to be still capable of reactivating inactivated serum (immune body). This he interpreted as proving that the complement was not bound to the corpuscles directly.

If the three factors concerned-corpuscles, immune body, and complement-were mixed and the mixture kept at $0^{\circ} \mathrm{C}$., no hemolysis occurred; yet, centrifugalized at this temperature, immune body was found to have become bound to the corpuscles, the complement remaining free in the supernatant fluid. If the same mixture, however, was exposed to $37^{\circ} \mathrm{C}$., hemolysis promptly occurred. 
From this, Ehrlich concluded that complement did not directly combine with the corpuscles, but did so through the intervention of the immune body. This immune body, he reasoned, possessed two distinct atom-groups or haptophores; one, the cytophile haptophore group, with strong affinity for the red blood cell; the other, or complementophile haptophore group, with weaker avidity for the complement. Because of this double combining power, Ehrlich speaks of the immune body as "amboceptor." These views are graphically represented in Figs. 132 and 133.

Thus, according to Ehrlich and his pupils the alexin, or complements, acts upon the antigen indirectly only through the "zwischenkoerper" or "amboceptor." These views of Ehrlich have been described in more or less detail because they form an excellent

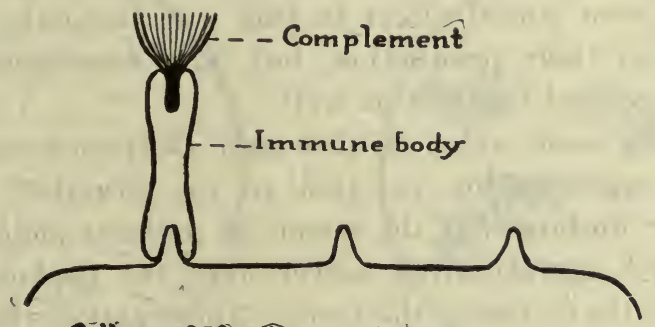

\section{Cell used for immunizing}

Fig. 33.-Complement, Amboceptor or Immune Body. and Antigen or Immunizing Substance.

introduction to a study of these phenomena. We do not believe, however, that they are tenable at the present time. It was Bordet particularly who has pointed out many of the uncertainties of the amboceptor point of view. The only thing that we ean say with certainty is that alexin or complement do not go into union with the unsensitized antigen, but that the action of the alexin upon the antigen is made possible only by preliminary sensitization, or, in other words, by union with the specific antibody. This complex then is amenable to alexin action. It is neither necessary, nor is it justifiable on the basis of experimental fact, to assume that the sensitizing antibody is an amboceptor or a sort of bridge between the antigen and the alexin. The difference is a fundamental one, and all the facts are on the side of the Bordet view, as far as we can see. It is better to refer to the substances involved as sensitizer and alexin, instead of as amboceptor and complement. As we shall 
see, we believe that the sensitizing antibody, whether in the proeess of lysis, agglutination, precipitation, etc., is in all cases the same substance.

Agglutination.--Although Metchnikoff ${ }^{11}$ and Charrin and Roger ${ }^{12}$ had noticed peculiarities in the growth of bacteria when cultivated in immune sera, which were unquestionably due to agglutination, the first recognition of the agglutination reaction as a separate function of immune sera was the achievement of Gruber and Durham. While investigating the Pfeiffer reaction with $B$. coli and the cholera vibrio, Gruber and Durham ${ }^{13}$ noticed that if the respective immune sera were added to bouillon cultures of these two species, the cultures would lose their turbidity and flake-like clumps would sink to the bottom of the tube, the supernatant fluid becoming clear. Gruber, at the same time, called attention to the fact that immune sera would affect in this way not only the microorganism used in their production, but, to a less energetic extent, other closely related bacteria as well.

Widal, very soon after Gruber and Durham's announcement, applied the agglutination reaction to the practical diagnosis of typhoid fever, finding that the serum of patients afflicted with this disease showed agglutinating power over the typhoid bacillus at early stages in the course of the fever. The reaction, thus practically applied to clinical diagnosis, was soon shown to be of great importance in its bearing on bacteriological species differentiation. Since animals immunized against a definite species of bacteria acquire in their sera specific agglutinating powers for these bacteria and at best only slight agglutinating powers for other species, immune sera can be used extensively in differentiating between bacterial varieties.

Agglutination may be observed microscopically or macroscopically. Bacteria brought into contact with agglutinating serum in the hanging drop rapidly lose their motility, if motile, as in the case of typhoid bacilli, and gather together in small clumps or masses. The microscopic picture is striking and easily recognized and the reaction takes place with varying speed and completeness, according to the strength of the agglutinating serum.

\footnotetext{
"Metchnikoff, "Etudes sur l'immunité,", IV Memoir, 1891.

${ }^{12}$ Charrin et Roger, Compt. rend. de la soc. de biol., 1889.

${ }^{13}$ Gruber und Durham, Münch. med. Woch., 1896.
} 
As the reaction approaches completeness, the clumps grow larger, individual microorganisms become more and more scarce, finally leaving the medium between clumps entirely clear. While the elumping of a motile organism suggests that motility has something to do with the coming together in clumps, it nevertheless has no relation whatever to agglutination, motile and non-motile organisms alike being subject to the reaction.

Macroscopically observed, in small test tubes or capillary tubes, agglutination evidences itself by the formation of flake-like masses which settle into irregular heaps at the bottom, leaving the supernatant fluid clear, in distinct contrast to the even flat sediment and the clouded supernatant fluid of the control. Macroscopically, too,
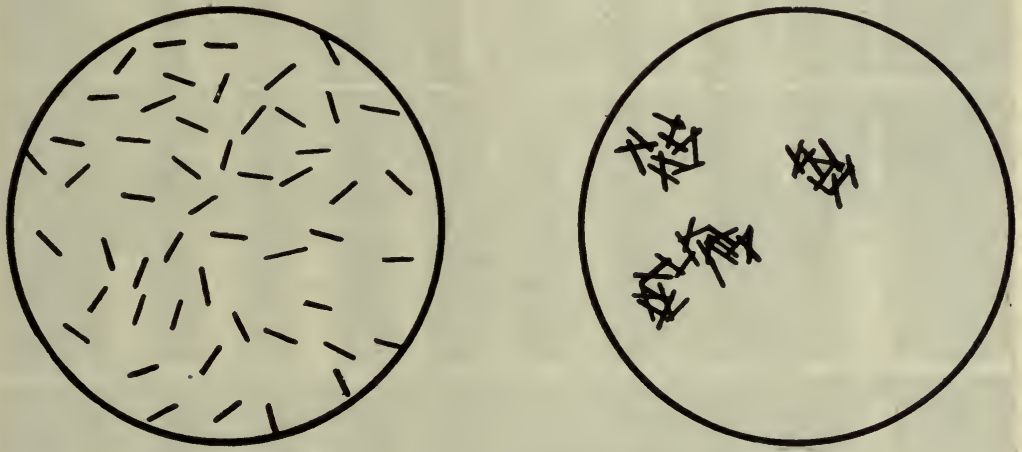

Fig. 34.-Microscopic Agglutination Reaction.

agglutination is evidenced when bacteria are grown in broth to which immune serum has been added. Instead of evenly clouding the broth, the microorganisms develop in clumps or chains.

Another phenomenon probably produced by agglutinins is the so-called "thread-reaction" of Pfaundler. ${ }^{14}$ This consists in the formation of long convoluted threads of bacterial growth in the hanging drop of dilute immune serum after twenty-four hours. Very strict specificity is attributed to this reaction by Pfaundler.

Agglutinins act upon dead as well as upon living bacteria. For the microscopic tests bacterial emulsions killed by formalin were introduced by Neisser.

Ficker ${ }^{15}$ has recently succeeded in preparing an emulsion of

${ }^{14}$ Pfaundler, Cent. f. Bakt., xxiii, 1898.

${ }^{15}$ Ficker, Berl. klin. Woch., 1903. 
typhoid bacilli, which is permanent and may be kept indefinitely, and may be employed for macroscopic agglutinations. ${ }^{16}$

Attention has been called by various workers to a source of error in all these methods, known as pseudo-clumping. ${ }^{17}$ The causes for such elumping not due to agglutinins seem to lie in the presence of blood cells in the serum or excessive acidity of the culture

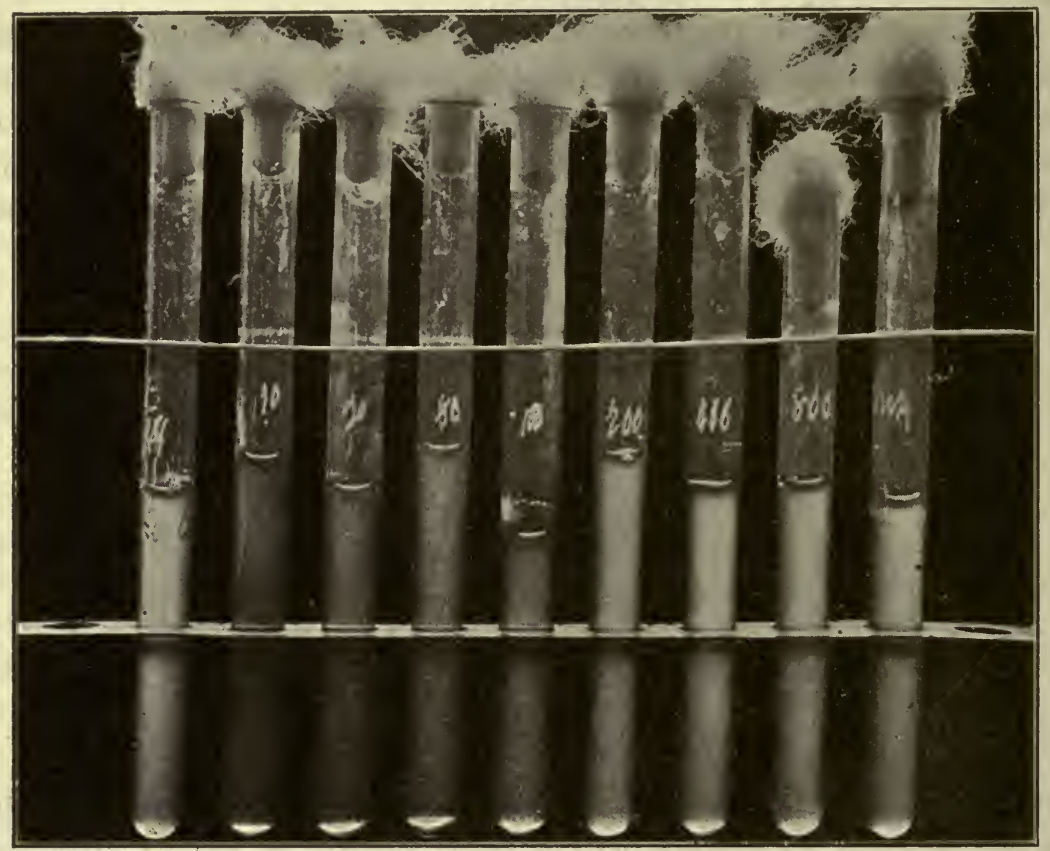

Fig. 35.-Macroscopic Agglutination. Dilutions from 1 to 10 in to 1 to 1,000 . The first tube contains a $1: 20$ control with the bacteria and normal serum Agglutination complete in the tubes marked 10,20,50, 100 .

medium. ${ }^{18}$ In fact, agglutination of bacteria by acids in definite concentration can bë carried out and seems to depend directly upon the hydrogen ion concentration.

While the microscopic methods are more suitable for clinical-

${ }^{16}$ Exact method of production of "Ficker's Diagnosticum", is a proprietary secret.

17 Savage, Jour. of Path. and Bact., 1901.

${ }^{18}$ Biggs and Park, Amer. Jour. of Med. Sci., 1897; Block, Brit. Med. Jour., 1897. 
diagnostic purposes, because of the smaller amounts of blood required, the macroscopic tests are far preferable for the purposes of bacterial differentiation and research. Greater exactitude of dilution is possible when dealing with larger quantities; microscopic unevenness in the bacterial emulsion does not become a source of error; and positive and negative reactions are more sharply defined.

Nature of Agglutinins.-Gruber and Durham, ${ }^{19}$ the discoverers of agglutinins, at first advanced the opinion that the agglutinins were identical with the immune body concerned in the Pfeiffer reaction, which by injuring the bacteria rendered them susceptible to the alexins. Pfeiffer ${ }^{20}$ and Kolle ${ }^{21}$ claimed, however, that by the addition of cholera vibrio to immune serum, the agglutinins could be completely absorbed, or used up, while bacteriolytic substances still remained. The same authors demonstrated that immune serum, preserved for several months, would lose its agglutinins without a corresponding loss of bacteriolytic power. It has also been shown since then, by these and other authors, that the agglutinins and the bactericidal substances are in no way parallel in their development, and that strongly agglutinating sera may be extremely weak in bactericidal substances and vice versa. We ourselves are not at all sure that this proves sufficiently that agglutinins and bacteriolysins are distinct substances. There are many reasons to believe that it requires a considerably more powerful sensitization to produce agglutination than it does to make an antigen amcnable to alexin action, or the action of leucocytes, and also the actual agglutination or clumping depends upon environmental conditions in which viscosity of the menstruum, the presence of electrolytes, and perhaps also the condition of the antibody in the serum, namely, whether or not it is relatively free or is bound up with serum proteins, play a part. Bordet has shown very definitely that elumping does not take place without the presence of electrolytes. If bacteria are sensitized heavily, or, in other words, allowed to absorb antibody, and then washed and resuspended in distilled water, they do not agglutinate. A small amount of salt solution added to such a mixture brings about rapid agglutination. In this, as well as in the phenomena of acid agglutination, the clumping of the bacteria

${ }^{19}$ Loc. eit.

${ }^{20}$ Pfeiffer, Deut. med. Woch., 1896.

${ }^{21}$ Pfeiffer und Kolle, Cent, f. Bakt., xx, 1896. 
is entirely analogous to the elumping of colloidal suspensions of any kind, and if we consider that bacterial suspensions are in many other respects similar to colloidal suspensions, namely, in their negative charge and their wandering to the positive pole in neutral solutions, in kataphoresis experiments, it becomes apparent that the actual clumping or agglutination is a purely physical phenomenon, determined by the colloidal equilibrium of the bacteria in suspension.

Altogether, we ourselves are inclined to regard the so-called agglutinins as identical with other sensitizers, and the actual clumping as a colloidal precipitation of organisms that have been altered in their suspension equilibrium as a result of the union with the antibodies together with some serum protein.

Agglutinated bacteria ${ }^{22}$ are not killed by the act of agglutination and are often as virulent as non-agglutinated cultures. Metchnikoff assigned to them a secondary role in relation to protection, but it is quite likely from more recent observations, that they do participate distinctly in the protective mechanism. Recent work by Bull seems to indicate that bacteria are agglutinated in vivo as a sort of preliminary step to phagocytosis.

The agglutinins, furthermore, unlike the bactericidal substances in sera, remain active after exposure to temperatures of over $55^{\circ} \mathrm{C}$., some of them withstanding even $65^{\circ}$ to $70^{\circ}$, and can not be reactivated by the subsequent addition of normal serum. These facts definitely preclude the participation in the reaction of the alexin or complement.

Production of Agglutinins.-Just as normal sera contain small quantities of bactericidal substances, so do they contain agglutinins in small amount. In a general way these "normal agglutinins" have the same nature as the immune agglutinins, and their presence is probably traceable to the various microorganisms parasitic upon the human and animal body.

As a matter of fact, the blood serum of new-born guinea-pigs hardly ever contains agglutinin for B. coli, while that of adults acts upon these bacilli in dilutions of $1: 20 .^{23}$ Similarly, infants show lower normal agglutinating values than adults. ${ }^{24}$

Agglutinins may be produced in the sera of animals by the intro-

22 Mesnil, Ann. de l'inst. Pasteur, 1898.

${ }^{23}$ Kraus und Löw, Gesell. d. Aerzte, Wien, 1899.

${ }^{24}$ Pfaundler, Jahrb. f. Kinderheilk., Bd.-50. 
duction of microorganisms subcutaneously, intravenously, or intraperitoneally. The intravenous method seems to give the most abundant and speedy results. ${ }^{25}$ The formation of agglutinins is a reaction to the body substances of the bacteria themselves, rather than to their toxic products. Thus agglutinins are produced in response to the introduction of dead bacteria and soluble extracts of cultures. Pathogenicity ${ }^{26}$ does not influence agglutinin formation to any great extent, non-pathogenic as well as pathogenic giving rise to these substances in serum. As a rule, however, agglutinins are more easily produced against a virulent than against fully virulent strains of bacteria of the same species.

Agglutinins can be produced with all the known bacteria, but great difficulty may be experienced in producing them with capsulated organisms such as the pneumococcus mucosum and the Friedländer bacillus, since the capsule seems to insulate such bacteria against reactions with serum. It is possible to agglutinate such capsulated bacteria often only by the method of Porges, the preliminary destruction of the capsule with weak acid and heat. As a rule, the agglutinins appear in the blood of animals three to six days after the introduction of bacteria. From the third to the sixth day they rapidly increase to a maximum at the seventh to thirteenth day. They then fall off rapidly until they reach a level at which they remain for a long period without very considerable change. Curves to illustrate these phases have been construeted by Jorgensen and Madsen. ${ }^{27}$

The Reaction between Agglutinin and Agglutinin-Stimulating Substances (Agglutinogen). - The fact that agglutinin can be removed from, or absorbed out of, serum by the specific bacilli which have led to its formation indicates that there is in the act of agglutination a combination between the agglutinin and the agglutinin-stimulating substance (agglutinogen). It is likely that this combination is of a chemical nature, since, as we have mentioned, agglutinins result from the injection of bacterial extracts as well as from the introduction of living bacteria. The probability that the process follows chemical laws of combination is furthermore strengthened by the work of $\mathrm{Joos}^{28}$ and others, who have demonstrated that definite

${ }^{25}$ Hoffmann, Hyg. Rundschau, 1903.

${ }^{26}$ Nicolle, Ann. de 1'inst. Pasteur, 1898.

${ }^{27}$ Jorgensen and Madsen, Festschrift, Kopenhagen, 1902.

${ }^{28}$ Joos, Zeits. f. Hyg., xxxvi, 1901. 
quantitative relations exist between the agglutinin-stimulating substances and the agglutinins. Every agglutination reaction, therefore, will vary in its degree of completeness with the quantities of agglutinin and agglutinogen, a fact which makes it neeessary, especially for clinical tests, to preserve a certain uniformity in the quantity and density of the bacterial culture or emulsion employed.

SPECIFICITY.-From the very beginning, Gruber and Durham ${ }^{29}$ had claimed specificity for the agglutination reaction, and in this sense it was clinically utilized by Widal for the diagnosis of typhoid fever. It was noticed, however, even by these earliest workers, that the serum of an animal immunized against one microorganism would often agglutinate, to a less potent degree, other closely related species. Thus, the serum of a typhoid-immune animal may agglutinate the typhoid bacillus in dilutions of $1: 1,000$, and the colon bacillus in dilutions as high as $1: 200$; while the agglutinating power of normal serum for the colon baeillus rarely exceeds $1: 20$. The specificity of the reaction for practical purposes, thus, is not destroyed if proper dilutions is carried out, the degree of agglutinin formation being always far higher for the specific organism used in immunization than it is for allied organisms. The specific immuneagglutinin in such experiments is speken of as the chief agglutinin (hauptagglutinin), and the agglutinins formed parallel with it, as the partial agglutinin (metagglutinin), terms introduced by Wassermann. Hiss has spoken of these as major and minor agglutinins. The relative quantities of the speeifie chief agglutinin and partial agglutinins present in any immune serum depend upon the individual cultures used for immunization, and the phenomenon is probably dependent upon the fact that certain elements in the complicated bacterial cell-body may be common to several speeies and find common receptors in the animal body. Whenever an immune serum agglutinates a number of members of the group related to the specific organism used for its production, the reaction is spoken of "group agglutination."

The partial agglutinins (metagglutinins) have been extensively studied by Castellani ${ }^{30}$. and others, by a method spoken of as the "absorption method." This consists in the separate addition of bacterial emulsions (agglutinogens) of the various species concerned

${ }^{29}$ Gruber und Durham, loc. eit.

${ }^{\text {y }}$ Castellani, Zeits. f. Hyg., xl, 1902. 
in a group agglutination, to the agglutinating serum. In this way, specific and partial agglutinins can be separately removed from the immune serum by absorption-each by its corresponding agglutinogen. In such experiments all agglutinins will be removed by the organisms used for immunization, a partial removal only resulting from the addition of allied strains. This method has thrown much light upon the intimate relations existing between members of various bacterial species, and has been particularly valuable in the study of the typhoid-colon-dysentery group. It is important to mention, however, that "groups" as determined by agglutination tests do not always correspond to classifications depending upon morphological and cultural characteristics.

An interesting phenomenon of great practical importance, which has been noticed by a number of observers, and which may often be encountered in routine agglutination tests, -is the frequent failure of a strongly agglutinating serum to produce agglutination if used in concentration, while in dilutions it produces a characteristic reaction. This has been explained theoretically by what is known as the "proagglutinoid zone." It is assumed that agglutinins may deteriorate as do toxins and be converted into substances which are capable of combining with agglutinogen without causing agglutination. Such substances, as we will see in discussing Ehrlich's views on the structure of agglutinins, may have a stronger affinity for agglutinogen than the agglutinins themselves, and are, therefore, termed "proagglutinoids." In strongly agglutinating sera these proagglutinoids may be present in considerable quantities and prevent the combination of agglutinin with agglutinogen. In dilution, this proagglutinoid action would naturally become weaker and of no actual significance in obscuring the reaction.

Agglutination, like other immune phenomena, is a manifestation of broad biological laws and not limited to bacteria. Thus, as hemolysins are produced by the injection of red blood cells, so hemagglutinins, or substances which clump together red blood cells, are similarly formed.

The theoretical considerations concerning the nature of agglutinins are discussed below, together with a similar section on the precipitins.

Precipitation.-R. Kraus, ${ }^{31}$ of Vienna, demonstrated that the sera of animals immunized aaginst B. pestis, B. typhosus, and Vibrio

${ }^{31}$ Kraus, Wien. klin. Woch., 1897. 
choleræ, when mixed with the clear filtrate of bouillon cultures of the respective organisms, produce-macroscopically visible precipitates. These precipitates occurred only when filtrate and immune serum were homologous, i. e., when the animal from which the serum had been obtained had been immunized by the same species of microorganism as that which was used in the test; it was for this reason Kraus spoke of them as "specific precipitates." It was evident, therefore, that during the process of active immunization with these organisms, a specific antibody had been produced in the serum of the treated animal, which, because of its precipitating quality, was named "precipitin." This peculiar reaction was soon found to hold good, not only for the bacteria used by Kraus, but also for other bacteria, few failing to stimulate the production of specific precipitins in the sera of immunized animals. The phenomenon of precipitation, however, is not limited to bacterial immunization, but has been found, like the phenomena of agglutination and lysis, to depend upon biological laws of broad application. Thus, Bordet $^{32}$ found that the blood serum of rabbits treated with the serum of the chicken gave a specific precipitate when mixed with chicken serum. Tchistovitch ${ }^{33}$ demonstrated a similar reaction with the sera of rabbits treated with horse and eel sera. By the injection of milk, Wassermann, ${ }^{34}$ Schütze, ${ }^{35}$ and others produced an antibody which precipitated the casein of the particular variety of milk employed for immunization. The reaction was thus applicable to many albuminous substances. These substances, because of their precipitin-stimulating quality, are called "precipitinogens."

Nature of Precipitins.-The precipitins, like the agglutinins, may be inactivated by heating to from $60^{\circ}$ to $70^{\circ} \mathrm{C}$., and can not be reactivated by the addition of normal serum or by any other known method. Such inactivated precipitin, however, while unable to produce precipitates, has not lost its power of binding the precipitinogen. This is shown by the fact that the inactivated precipitin, when mixed with precipitinogen, will prevent subsequently added fresh precipitin from eausing a reaction. From these facts the conclusion has been drawn that precipitin, like toxin, is built up of

${ }^{32}$ Bordet, Ann. de l'inst. Pasteur, 1899.

${ }^{\text {s3 }}$ Tchistovitch, Ann. de 1'inst. Pasteur, 1899.

${ }^{34}$ Tassermann, Deut. med. Woch., 29, 1900.

${ }^{35}$ Schütze, Zeit. f. Hyg., 1901. 
two atom groups, ${ }^{36}$ a stable haptophore and a labile precipitophore group. By the destruction of the latter, an inactive, yet neutralizing substance is produced which is spoken of as "precipitoid." The precipitoids, like protoxoids, have a higher affinity for precipitinogen than the unchanged precipitin, and thus are able to prevent the action of these.

Our own opinion would rather incline toward regarding the precipitins as identical in structure with sensitizer or amboceptorbeing in fact "albuminolysins" in the sense of Gengou. This problem is too complex to be discussed in detail in a summary of immunity as brief as the one here presented.

Specificity.-The specificity of precipitins is a question of the greatest importance, since, as we shall see, these bodies have been used extensively for the differentiation of animal proteins. In regard to bacterial precipitins it may be said that, just as in agglutination, there is in precipitation, a certain degree of "group reaction." The precipitin obtained with a colon bacillus, for instance, will eause precipitation with culture-filtrates of closely allied organisms, though in a less marked degree. According to Kraus, such confusion may be easily overcome by the proper use of dilution and quantitative adjustment, similar to that used in agglutination tests. Norris $^{37}$ found that the precipitates given by immune sera with the filtrates of the homologous bacteria were invariably heavier than those given with allied strains and that the latter could be eliminated entirely by sufficient dilution.

Specificity becomes of still greater importance in the forensic use of the precipitin reaction introduced by Uhlenhuth, ${ }^{38}$ Wassermann and Schütze, ${ }^{39}$ and Stern. ${ }^{40}$ These authors found that the precipitin reaction furnished a means of distinguishing the blood of one species from that of another. Thus, blood spots, dissolved out in normal salt solution, could be recognized by this reaction as originating from man or from an animal, even after months of drying and in dilutions as high as $1: 50,000$. Since the value of this test depends entirely upon the strict specificity of the reaction, this question has

${ }^{36}$ Kraus und v. Pirquet, Cent. f. Bakt., Orig. Bd. xxxii.

${ }^{3 \pi}$ Norris, Jour. Inf. Dis., i, 3, 1904.

3s Uhlenhuth, Deut. med. Woch., xlvi, 1900; vi and xvii, 1901.

${ }^{39}$ Wassermann und. Schïtze, Berl. klin. Woch., vi, 1901.

*n Stern, Deut. med. Woch., 1901. 
been studied with especial care, notably by Nuttall. ${ }^{41}$ All who have investigated the subject find the only important source of confusion in the blood of the anthropoid apes. The specificity of the reaction, too, has been found to depend very closely upon the amount of precipitin in the serum employed. If a highly immine serum is insufficiently diluted, the reaction loses much of its specific value. ${ }^{42}$ This source of error is easily eliminated in practice by careful control and titration of the sera used for the tests.

Unlike agglutinins, precipitins have, so far, not been demonstrated in normal sera. ${ }^{43}$

Theoretical Considerations Concerning Agglutinins and Precipitins.-We have seen that Ehrlich evolved his theories of antibody formation from his early views upon the absorption of nutritive

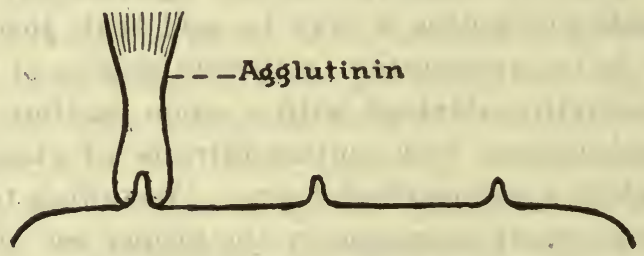

\section{Cell used for immunizing}

Fig. 36.-Ehrlich's Conception of the Structure of Agglutinins and Precipitins.

substances by the body cells, and we have followed, in more or less detail, the steps of his reasoning as he developed his hypothesis in its application to the antitoxic and the lytic substances. There still remained the agglutinins and precipitins, bodies which because of their individual characteristics can be classed neither with the group of antitoxic, nor with that of the lytic substances. These two antibodies, while by no means identical, possess the common characteristics of being more thermostable than the bacteriolytic substances, and of being insusceptible to reactivation by normal serum. It is plain, therefore, that both agglutinating and precipitating reactions take place without the co-operation of complement. The substances which give rise to precipitins and agglutinins, more-

\footnotetext{
${ }^{41}$ Nuttall, Brit. Med. Jour., i, 1901; ii, 1902.

${ }^{42}$ Kister und Wolff, Zeit. f. Medizinal-Beamte, 1902.

${ }^{43} \mathrm{~K}$ raus, loc. eit., and Norris, loc. cit.
} 
over, are not of the relatively simple soluble character of the toxins, but are intrinsic portions of complex albuminous molecules, comparable to and often identical with the true nutritive substances. For these reasons Ehrlich believes that the cell-receptors for the various substances which give rise to agglutinins and precipitins are neither of the simple structure of the toxin receptor, nor of the double-haptophore nature of the bacteriolytic receptors, but contain a single haptophore group for the anchorage of the ingested material and at the same time a constantly attached zymophore group or ferment by means of which the anchored substance is transformed preparatory to its absorption by the cell protoplasm. For the sake of clearness, this form of receptor may be compared to a bacteriolytic or hemolytic amboceptor with a permanently attached and inseparable complement.

Three forms of receptors, then, are proposed by Ehrlich in explanation of all known varieties of antibodies. The first, the simplest side chains of the body cells, he calls "receptors or haptines of the first order." These, overproduced and cast off, constitute the antitoxin and antiferments. Next "haptines of the second order" are the receptors planned both for the anchorage and further digestion of antigens. These, free in the circulation, are the precipitins and agglutinins. "Haptines or receptors of the third order" are merely able to anchor a suitable substance, but exert no further action upon it until re-enforced by the complement normally present in the serum. These, free in the circulation, with a chemical group having avidity for the antigen, and another complementophile group, are the amboceptors or immune bodies of bacteriolytic, cytolytic, and hemolytic sera.

We cannot, in a general work of this kind, go into a detailed discussion of the many complex problems involved in regard to the structure of antibodies. Again we will refer the reader to the larger works on this subject. It is our opinion, however, that the above views of Ehrlich can no longer be maintained in the light of present knowledge.

Bordet, ${ }^{44}$ points out that the conception of Ehrlich rests upon the basis of a number of undemonstrated hypotheses. He asserts, and with justice, that it has never been shown beyond question that

${ }^{44}$ Bordet, Résumé of Immunity in Bordet's "Studies in Immunity," transl. by Gay, Wiley \& Sons, 19009. 
the antibodies, free in the serum, are identical with the receptors of the body cells upon which the antigen originally acts.

In regard to agglutinins, Ehrlich, as we have seen, believes that it is the agglutinin itself which, first uniting with its antigen by its haptophore group, then causes clumping by its zymophore group. Now, as a matter of fact, Bordet ${ }^{45}$ has shown that it is not the agglutinin itself which agglutinates, but that agglutinin with its antigen forms a complex which is then agglutinable by the salt present in the solution. This conclusion seems borne out by the later work of Gengou, ${ }^{46}$ Landsteiner and Jagic, ${ }^{47}$ and others, who have shown that bacteria which have absorbed other substances, such as uranium compounds, colloidal silicic acid, etc., are subsequently agglutinable by salts. In consequence, from these and other observations, Bordet concludes that it is neither necessary nor accurate for the explanation of these phenomena, to assume the conditions conceived by Ehrlich, but that the phenomenon of agglutination consists primarily of the union of the antibody with its antigen in a colloidal suspension, and that the actual subsequent agglutination is a purely secondary phenomenon which depends possibly upon a change in the physical properties of the emulsion-upon "its colloidal stability." A similar condition he assumes for precipitins.

Without being able in the limited space available to go into a detailed discussion of the large volume of work which has appeared on this subject, we may say that it is our opinion at present that the evidence largely points in the direction indicated by Bordet, namely, that the essential feature of all these reactions is the specific union of an antigen with its antibody, that thereby the physical or chemical condition of the antigen is so changed that it now becomes less stable and is agglutinated or precipitated by such physical influences as, for instance, the presence of an electrolyte. The work of Neisser and Friedmann ${ }^{48}$ has shown that bacteria that have absorbed agglutinin are agglutinated by concentrations of salt far less than is necessary to agglutinate or precipitate the normal bacteria.

Our own opinion, set forth in a number of experimental studies, would go even further than this. We incline to the belief that all

4. Bordet, Ann. de 1'inst. Pasteur, 1899.

${ }^{46}$ Gengou, Annal. Past., 1904.

${ }^{47}$ Landsteiner und Jagic, Wien. klin. Woch., iii, 1904.

${ }^{48}$ Neisser and Friedmann, Münch. med. Woch., 1904, li. 465-827. 
antibodies, including the so-called amboceptors or sensitizers that take part in the phenomena of lysis and bactericidal action are essentially of one type; that the fundamental phenomenon is the union of the antigen with the specifie antibody or its "sensitization;" that by such sensitization the antigen is now rendered on the one hand more easily agglutinable or precipitable, on the other may be rendered more amenable to the action of the alexin or complement or to phagocytosis. The agglutination and precipitation phenomena, moreover, are merely evidences of the fact that these substances are in eolloidal suspension and are influenced by agencies which produce precipitations in such suspension. It is interesting to note in this connection, also, that bacteria in neutral suspension - carry negative charges which ean be weakened by sensitization with serum and weakened or reversed by the addition of acid. These points tend to strengthen such a point of view.

The degree of acidity necessary to reverse the normal negative charge of bacteria corresponds roughly to that at which growth is inhibited. This has led us to speculate whether or not vitality of bacteria and the negative charge may be related.

Facts Concerning Alexin or Complement.-Muir and Browning claim that, on the filtration of serum, amboceptor or immune body will pass through the filter, whereas alexin or complement is held back.

This retention of complement by filters occurs only when new filters are used, and this is probably due to adsorption or complement by the finely divided substances which make up the filter and not due to retention because of the large size of the complement molecule.

Alexin can be inactivated by shaking as well as by heat when diluted 1:10 and shaken for about 20 minutes in salt solution. According to Gramenitski it is spontaneously partially reactiviated on standing.

Alexin is dependent upon the total volume of the mixture in which it acts, i.e., upon eoneentration, the same actual quantity of complement acting more strongly in higher than in lower concentrations, this not being true of amboceptor or sensitizer which acts in direct proportion to its actual quantity independent of the concentration.

Alexin is inhibited by hypertonic salt solution and can be preserved in 15-25 per cent salt concentration for weeks in the icebox, 
resuming its activity when diluted to isotonicity with distilled water. Removal of salt by dialysis or other means of globulin precipitation divides the complement into two fractions, the globulin fraction and the albumin fraction, neither of which will act alone, but which together possess the properties of undivided eomplement. The globulin fraction attaches directly to the sensitized cells and is therefore spoken of by German investigators as "mid-piece." The albumin fraction acts upon the sensitized cells only after attachment of the globulin fraction and is therefore spoken of as "end-piece." It is seen, therefore, that a great many of the properties of alexin make it secm rather likely that this substance is quite similar to ferments in its action.

The Fixation of Complement by Precipitates.-It has been found . by Gengou ${ }^{49}$ and eonfirmed by Moreschi, Gay, ${ }^{50}$ and others, that when the serum of an animal immunized with the serum of another species or with a forcign albumin is mixed with a solution of the substance used in the immunization, the precipitate formed will remove eomplement from the mixture. In other words, preeipitates formed by the reaction of precipitin with its antigen will fix complement. This is of great importance in complement-fixation tests; for because of insufficient washing, the blood eells used in producing the hemolytic amboceptor, may, from the presence of serum, give rise to a precipitin as well as a hemolysin. In the test done subsequently, a precipitin reaction may take place and by thus removing complement may give a false result. The absorption of complement by such precipitates takes place when the two reacting factors, the precipitin and its antigen, are in dilution-so high a visible preeipitate ean not be observed. This fact, together with others too complieated to be diseussed in this place, have led us to the belief that the so-called precipitins are true sensitizers, exerting toward unformed proteins the same function that the so-called sensitizer or amboeeptor exerts toward cellular formed antigens. (See p. 293.)

\section{Quantitative Relationship Between Amboceptor and Complement.} -Morgenroth and Sachs ${ }^{51}$ have succeeded in showing that within certain limits an inverse relationship exists between these two bodies. If for a given quantity of red blood cells a certain quantity of

\footnotetext{
${ }^{49}$ Gengou, Ann. Past., 1902.

${ }^{5 n}$ Gay, Cent. f. Bakt., I, xxix, 1905.

"1 Morgenroth und Sachs, "Gesammel. Arb. für Immunitätsforschung." Berlin, Hirschwald, 1904.
} 
amboceptor and complement suffices to produce complete hemolysis, reduction of either the complement or the amboceptor necessitates an increase of the other factor. As amboceptor is increased, in other words, complement may be reduced and vice versa. This result is of great importance in arguing against the original conception of Ehrlich in supposing these substances to act together unit for unit.

Deviation of the Complement (Complement-Ablenkung).-It was noticed by Neisser and Wechsberg ${ }^{52}$ that in mixing together bacteria, inactivated bactericidal immune serum (immune body), and complement in the test tube, a great excess of immune body hindered rather than helped bactericidal action. As the amount of immune body in the mixture was carried beyond the experimental optimum, bactericidal action became less and less pronounced, and was finally completed suspended. They explain this by assuming that free immune body, uncombined with complement, has a greater affinity for the bacterial receptor than the immune body combined with complement. The complement is consequently diverted and prevented from activating the amboceptor attached to the bacterial cell. Graphically, the conditions may be illustrated as follows:

The above theory of Neisser and Wechs-
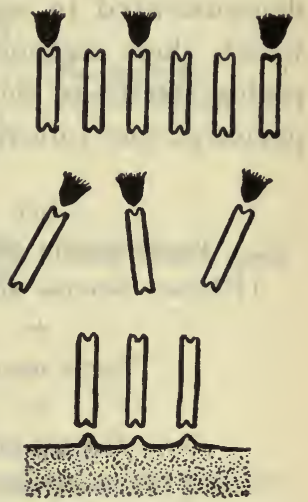

Fig. 37.-Neisser and Wechsberg's ConCEPTION OF COMPLEment Deviation. berg is here stated simply because of the wide discussion it has aroused. In the light of our present knowledge concerning the relations between antigen, amboceptor, and complement, their conception is obviously erroneous. The phenomenon of Neisser and Wechsberg is probably a "zone" phenomenon, namely, an occurrence which depends upon the fact that the complete or incomplete union of colloidal substances depends to a very great extent upon the relative concentrations of each, and too high a concentration of the anti-serum in experiments, such as those of Neisser and Wechsberg, may result in' incomplete union. Thus, it is possible in many colloidal precipitation phenomena to show that too high a concentration of one or the other reacting colloid will result in failure of precipitation, and in some cases, even

s2 Neisser und Wechsberg, Münch. med. Woch., xviii, 1901. 
when precipitation has taken place, dispersion will again occur, if one or the other component is added in excess. These phenomena are frequently observed in agglutination and precipitation reactions where the highest concentrations of serum will produce less precipitate, or perhaps none at all when greater dilutions produce heavy precipitation.

Fixation of the Complement.-Bordet and Gengou ${ }^{53}$ in 1901, devised an ingenious method of experimentation by which even very small quantities of any given immune body (amboceptor) can be demonstrated in serum. The term "fixation of complement," by which their method of investigation is now generally known, explains itself, as the steps of experimentation are followed. They prepared the following mixtures:

(a)

Bacteriolytic amboceptor

(Plague immune serum, heated)

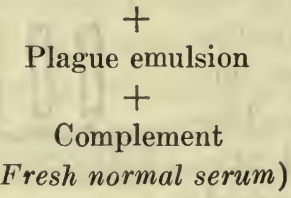

(b)

Normal serum, heated

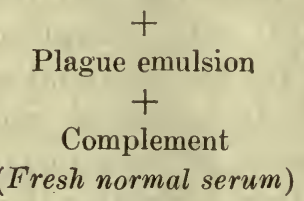

To both of these after five hours was added

Hemolytic amboceptor

(Heated hemolytic serum)

$+$

Red blood cells

Results :

(a) showed no hemolysis.

(b) showed hemolysis + .

The conclusion to be drawn from this was that in (a) the presence of immune body had led to absorption of all the complement. In (b), there being no bacteriolytic immune body to sensitize the bacteria and enable them to absorb complement, the latter substance was left free to activate the subsequently added hemolytic amboceptors. The Bordet-Gengou phenomenon has been extensively used by Wassermann and Bruck, ${ }^{54}$ Neisser and Sachs, ${ }^{55}$ and others to

\footnotetext{
${ }^{53}$ Bordet et Gengou, Ann. de 1'inst. Pasteur, 1901.

${ }^{64}$ Wassermann und Bruck, Med. Klin., 1905.

${ }^{55}$ Neisser und Sachs, Berl. klin. Woch., xliv, 1905, and i, 1906.
} 
demonstrate the presence of immune bodies in various sera. (See p. 315.)

It should be noted that this method, if valid, must presuppose the identity of the hemolytic and bactericidal complement in the activating serum.

Complement fixation will be more extensively discussed in the section dealing with the Wassermann reaction.

1.

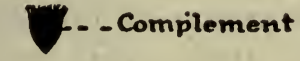

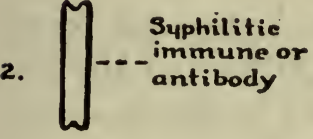

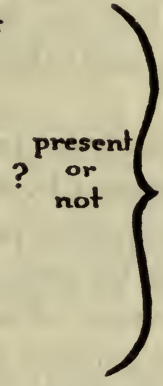

Together at

$37.50 \mathrm{c}$.

for onehour

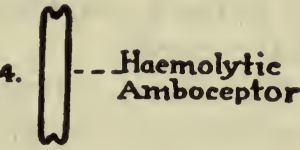

3.

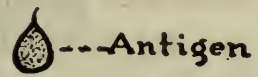

If (2) present, no haemolysis.

If. (2) not present, haemolysis.

Fig. 38.-Schematic Representation of Complement Fixation in the BordetGengou Reaction, as Conceived by Ehrlich. (This scheme is given because it aids in understanding the process, but must not be taken to represent the true manner of union in which the complements react.)

The Specificity of Hemolysins.-In the sections preceding we have seen that the blood cells of one animal, injected into an animal of another species, give rise to a hemolytic substance in the blood serum of the second animal, which is strictly specific for the variety of cells injected. Such hemolysins, when produced in one animal against blood cells of another species, are spoken of as heterolysins. In studying the nature of hemolysis, Ehrlich and Morgenroth ${ }^{56}$ now discovered that hemolysins could also be produced if an animal were injected with red blood cells of a member of its own species. Such hemolytic substances they called isolysins. In their experiments they injected goats with the washed red blood corpuscles of other goats and found that the serum of the recipient developed the power of causing hemolysis of the red blood cells of the particular goat whose blood had been used for injection. It did not, however, possess the

${ }^{56}$ Ehrlich und Morgenroth, Berliner klin. Woch., xxi, 1900. 
power of producing hemolysis in the blood of all goats, nor did it produce hemolysis with the red corpuscles of its own blood. It is thus shown that the specificity of the hemolysins extends even within the limits of species, and is, to a certain extent, an individual property.

The production of autolysins, that is, of substances in the blood serum which will produce hemolysis of the individual's own corpuseles, has, so far, been unsuccessful.

Ehrlich and Morgenroth, in the course of these experiments, furthermore succeeded in showing that the injection of isolysins into animals produced antiisolysins, and that these again were strictly specific.

The almost universal failure of autolysin production has found no satisfactory explanation. It is supposed by Ehrlich and Morgenroth that the failure of autolysin production may be due to a lack of suitable receptors in the animal for its own cells.

The clinical significance of the presence of isolysins and possibly of autolysins in human beings is too evident to require much discussion. A practical and extremely interesting result which these investigations have yielded is that of Donath and Landsteiner, ${ }^{57}$ who discovered an autolysin in the blood serum of patients suffering from paroxysmal hemoglobinuria. In these cases the sensitizing substance or amboceptor appeared to be absorbed by the red blood cells only at low temperatures-probably in the capillaries during exposure to the cold, and hemolysis subsequently resulted in the blood stream by the action of complement. These observations have been confirmed by other writers, but the phenomenon is surely not present in all cases of paroxysmal hemoglobinuria.

Isoagglutinins and blood typing in human beings are discussed in a subsequent section.

${ }^{57}$ Donath und Landsteiner, Münch. med. Woch., xxxvi, 1904. 


\section{CHAPTER XVI}

THE TECHNIQUE OF SERUM REACTIONS

Obtaining Serum from Animals and Man.-To obtain blood serum from man, the blood may be taken from the finger or the ear, either into a sterile centrifuge tube or into a Wright capsule. When taken into a centrifuge tube, the blood is allowed to clot and the serum separated by centrifugation. Larger quantities of blood may be taken with a syringe from the median basilic vein and either slanted in sterile test tubes in the ice chest or put into centrifuge tubes and centrifugalized. In bleeding small laboratory animals, a number of methods may be employed, depending upon the quantity of serum required.

The animals most frequently used for laboratory purposes are rabbits. To obtain small quantities of serum from rabbits, the animals may be bled from the marginal vein of the ear. The animal is strapped upon a tray and underneath it is placed a rubber bag filled with warm water. This is advised by Wadsworth to facilitate the flow of blood. The tray is then placed upon an easel so that the animal's head hangs downward. The skin over the ear vein is shaved and sterilized, and a Hagedorn needle plunged into the vein. The blood is eaught in test tubes or centrifuge tubes.

When larger quantities of blood are desired it may be taken from the earotid artery. In rabbits, the carotid may be found lying just lateral to the trachea and deeply placed, and must be carefully separated from the pneumogastric nerve by blunt dissection. The distal end of the artery is then tied off and the proximal end temporarily elosed with a small clamp. The artery is then raised out of the wound on a knife or forceps handle and, with sharp-pointed scissors, a small incision is made into but not through the vessel. A small glass cannula is now introduced and tied into place by a thread. To this cannula a small rubber tube fitted with a pinch-cock should have been attached, the whole being sterilized. Recently we have dispensed with the eannula, simply holding the vessel up with a pointed forceps. A larger yield of serum will be obtained 
if, after coagulation, the clot is separated from the glass with a sterile platinum wire.

In obtaining blood from larger animals, horses, sheep, etc., a cannula may be introduced into the jugular or internal saphenous veins. The skin is shaved and sterilized and a rubber tourniquet placed about the neck or thigh, as the ease may be, in order to eause the vein to stand out. A small incision may be made through the skin over the vein, but is not necessary. A eannula, with rubber tubing attached, is then plunged into the vein and the blood eaught in sterile high cylindrical jars, allowed to clot, and placed in the refrigerator. The serum is taken off after twenty-four to fortyeight hours with sterile pipettes.

Agglutination Tests.-For the determination of the agglutinating power of serum it is necessary to make suitable dilutions of the serum, and to prepare an even emulsion of the microorganisms to be tested. The test may be made microseopically or macroscopically. The microscopic test is the one in general use in the diagnosis of typhoid fever, and is occasionally applied to some other diseases. In its application to typhoid fever it is usually spoken of as the Gruber-Widal reaction.

Twelve- to eighteen-hour broth cultures of the typhoid bacillus, grown at incubator temperature, may be used. It is preferable, however, to use an emulsion of a twelve to twenty-four hour old agar culture in physiological salt solution ( 0.85 per cent). The salt-solution emulsion is made by adding about 10 c.c. of normal salt solution to the fresh agar slant culture, carefully detaching the culture from the surface of the agar with a flexible platinum wire, and pipetting off the emulsion thus made. With some microorganisms it is sufficient simply to allow the larger clumps to settle and to pipette off the supernatant turbid emulsion. With other microorganisms, the tendeney to form elumps makes it necessary to resort to further methods of securing an even distribution of the bacteria. This may be done either by sucking the emulsion in and out through a narrow pipette held perpendicularly against the bottom of a watch glass, as in Wright's technique for the opsonic test (see section on opsonins, p. 339), or by carefully rubbing the clumps against the watch glass with a stiff platinum wire. In the case of the tubercle bacillus not even this suffices, but it becomes necessary to grind the moist bacillary masses in a mortar before emulsifying. With the tubercle bacillus, too, it is preferable to use salt solution at 1.5 per cent eoncentration.

The serum dilutions are obtained by first making a one to ten dilution of serum with normal salt solution. The serum used for 
this purpose is freed from red blood corpuscles by centrifugation. From the 1 to 10 diluton any number of higher dilutions may be made, by mixing given parts of the 1 to 10 dilution with normal salt solution; thus one part of a 1 to 10 dilution plus an equal quantity of salt solution gives a dilution of 1 to 20 . One part of one to ten dilution plus two parts of normal salt solution gives one to thirty, etc. It must not be forgotten that, when equal parts of the serum and bacillary emulsion have been mixed, each one of these dilutions is doubled.

In making the micrascopic agglutination test, equal quantities of serum dilution and bacterial emulsion are mixed upon a cover-slip. The mixture may be made either by measuring out a drop of each substance with a standard platinum loop, depositing them close together on the cover-slip, and mixing; or equal quantities may be sucked up, each to a given mark, in a capillary pipette, mixed by suction in and out, and deposited upon the cover-slip. The coverslip is inverted over a hollow glass slide, the rim of which has been greased with vaseline. The drop is then observed through a (Leitz) No. 7 lens, ocular No. 3.

Macroscopic agglutination, preferable for exact laboratory research, is made in narrow test tubes measuring about $0.5 \mathrm{~cm}$. in diameter and about $5 \mathrm{~cm}$. in length.

Equal quantities, usually 1 c.c. each, of serum dilution and emulsion are mixed. A series of tubes is prepared, in each subsequent one of which the dilution is higher. These mixtures may be placed in the incubator for a few hours and then kept at room temperature. After removal from the incubator agglutination is in some instances hastened by transference to the ice chest. When agglutination takes place in these tubes, clumps of bacteria may be seen to form, which settle to the bottom of the tube, very much like snow-flakes. The surface of the sediment is heaped up and irregular. The supernatant fluid becomes entirely clear. When the reaction does not occur the sediment is an even, granular one with a flat surface, and the emulsion remains turbid.

Instead of using test tubes as described above, Wright has suggested the use of throttle pipettes of comparatively large diameter into each of which at least three or four different dilutions can be sucked with a nipple, a small air bubble being left between the mixtures. By sealing the distal end of these pipettes in a flame the various dilutions are kept at a distance from each other, and 
the pipettes may be set on end in a tumbler and observed just as are the test tubes (Fig. 42, p. 340). The special methods of carrying out agglutination tests with pneumococei have been deseribed on p. 460 .

Precipitin Tests.-In an earlier section on precipitins we have seen that precipitates are formed when clear filtrates of bacterial extracts or of both eultures are mixed with their specific immune sera. Such precipitin reactions are not limited to the realm of bacteria, but have a broad biological significanee, in that specific precipitating sera may be produced with proteins of varied source.

For carrying out a precipitin test, the following reagents are required:

1. A specific precipitating antiserum (antibacterial or antiprotein);

2. A bacterial filtrate or protein solution.

Production of Precipitating Antisera. ${ }^{1}$ - Antibacterial preeipitins may be produced in animals by a variety of methods. Animals, preferably rabbits, are injected with cultures of the bacteria in gradually increasing quantities. Five or six injections are given at intervals of from five to six days, the dosage and mode of administration being adapted in each case to the pathogenicity of the mieroorganisms in question. Myers ${ }^{2}$ elaims that specific precipitin for pepton in the eulture media may be formed which may lead to error. This could not be confirmed by Norris. ${ }^{3}$ The immunized animals should be bled about 7 to 12 days after the last injection.

Precipitating antisera against protein solutions are prepared by similar methods. Two or three injections, however, usually suffice. The sera or protein solutions used should be sterile. This may be accomplished by filtration through small porcelain filters. Injections into animals may be made subeutaneously, intraperitoneally, or intravenously. The subcutaneous route has no advantages unless the substances to be used are contaminated. It is far easier to produce precipitating sera against proteins like horse serum, egg albumin, ete., than it is to produce them against bacterial substances. This is due probably to the fact that bacterial bodies contain rela-

${ }^{1}$ R. Kraus, Wien. klin. Woch., 1897; Norris, Jour. Inf. Dis., 1 and 3, 1904.

= Myers, Lancet, ii, 1900.

${ }^{3}$ Norris, loc. cit. 
tively little soagulable protein, and the quantity injected, even with the largest tolerable doses of bacteria, may contain but very small amounts of true protein forming antigen.

The animals are weighed from time to time, and if considerable loss of weight ensues, the intervals should be increased. Doses from 2 to 5 c.c. should be given. In giving the later injections the danger of anaphylaxis must be remembered. A single injection of a large quantity has occasionally yielded a precipitating serum of considerable strength, ${ }^{4}$ but this method is not usually successful. Injections are made at intervals of from five to seven days. Seven to twelve days after the last injection the animals may be bled from the ear, and a preliminary test made to ascertain the precipitating value of the serum. If this is insufficient, more injections may be made. Bleeding should be done 7 to 12 days after the last injection. Such sera may be preserved in the dark and at a low temperature. If a preservative is added, Nuttall prefers chloroform to the phenols, because of occasional turbidity produced by these. Fornet and Müller ${ }^{5}$ and others have recommended rapid methods of precipitin formation by injecting relatively large amounts of the antigen daily, for three or four successive days, bleeding on the fifth or sixth day thereafter. This method has been followed by a number of workers subsequently, and is often successful, sera of considerable titers being obtained. In our experience, however, this method has not shown itself to be entirely advantageous, since it is rare that a very potent serum is so produced, and also animals bleed within a week after repeated large doses, may show in their serum not only antibodies but also residues of antigen, the two substances not united within the animal body, but gradually uniting and forming precipitates after the serum has been obtained and stored.

This phenomenon of the simultaneous presence of antigen and antibody in the circulating blood has been variously explained, the view formerly held being that of von Dungern, who believed that every injected antigen contained partial antigens, a, b, e, etc., each of which produced its partial antibody, A, B, C, which, by being present in unequal proportions, gave rise to the simultaneous presence of perhaps a and $B$, etc. Our own opinion is $^{6}$ that the

4Michaelis, Deut. med. Woch., 1902.

5 Fornet and Müller, Zeit. f. Hyg., 66, 1910.

- Zinsser and Young, Jour. Exper. Med., 17, 1913. 
failure of union in the circulating blood of the living animal is due to actions analogous to those of protective colloids, and represent a protective mechanism which represents the sudden union of antigen and antibody in the circulation.

Precipitating antisera for tests should be clear. If turbid, the sera should be filtered through small Berkefeld or porcelain candles.

Preparation of Bacterial Filtrates and Protein Solutions for Precipitin Tests.-Bacteria may be grown in nutrient broth having an initial reaction of neutrality or five-tenths per cent acidity to phenolphthalein. The cultures are incubated for times varying from a week to several months, and are then filtered through porcelain or Berkefeld candles until perfectly clear. Bacterial extracts may also be made by emulsifying agar cultures in salt solution, placing at $37.5 \mathrm{C}$. in the incubator for a week or longer, and filtering. More rapid extraction of bacteria may be accomplished by repeated, rapid freezing and thawing of salt-solution emulsions, by shaking in the shaking machine or by centrifugalizing, rubbing up the sediment with dry salt, and the addition of distilled water to isotonicity.

Protein solutions to be tested should be made in salt solution. When dealing with blood stains, as in doing the test for forensic purposes, the stains should be dissolved in salt solution, an approximate dilution of one in five hundred being aimed at. This solution if turbid should be filtered through a small porcelain filter. It should be clear and colorless, show a faint cloud on boiling with dilute acetic acid, and show distinct froth when shaken.

When the reaction is to be done for determining the nature of meat (detection of horse-meat substitution for beef, etc.), about 20 to 40 grams of the suspected meat are macerated in a flask, and covered with 100 c.e. of salt solution. This mixture is allowed to infuse at room temperature for three to four hours, and is then placed in the refrigerator for twelve hours or more. At the end of this time 2 c.c. are shaken into a test tube. If profuse frothing ${ }^{7}$ appears, the extract is ready for use. It is then filtered clear, either through paper, or through a Buchner or Nutsche filter. Berkefeld filters may also be used. The solution is then diluted until the addition of concentrated $\mathrm{HNO}_{3}$ produces only a slight even turbidity.

${ }^{7}$ P. Th. Müller, "'Technik. d. serodiagnos. Methoden.', 
Before use the reaction of the meat extract should be tested, and if neessary adjusted to neutrality or slight acidity or alkalinity.

In the actual test with bacterial filtrate, the procedure is as follows: In a series of narrow test tubes, the following mixtures are made:
Tube 1. Antibacterial serum .5 c.c. + bacterial filtrate 1. c.c. "2. Normal serum .5 c.c. + bacterial filtrate 1 . c.c.
"3. Antibacterial serum .5 c.c. + salt solution 1. e.e.
" 4. Salt solution .5 e.c. + bacterial filtrate 1 . e.c.

Place the tubes in the incubator at $37.5^{\circ} \mathrm{C}$. Tube 1 only should show a haziness which develops into distinct eloudiness or a flocculent precipitate within one hour. Tubes 2,3 , and 4 should remain clear.

In testing an unknown protein with serum of an animal immunized with the protein sought for, the technique of the test is as follows:

1. 0.1 c.c. immune serum +2 c.c. unknown protein solution.

2. 0.1 c.c. immune serum +2 c.c. known protein solution of variety suspected (similarly diluted).

3. 0.1 c.c. immune serum +2 c.c. protein solution of different nature (similarly diluted).

4. 0.1 c.c. immune serum +2 c.c. salt solution.

5. 2 e.c. unknown protein solution.

If the test is positive a precipitate appears in tubes 1 and 2 , but not in any of the others. The precipitate should appear within 15 to 20 minutes.

Bactericidal and Bacteriolytic Tests.-The bactericidal and bacteriolytic powers of serum may be tested either in the animal body or in the test tube. The in vivo test is known as Pfeiffer's phenomenon. This depends upon the fact that bacteria, when injected into the peritoneal cavity of a guinea-pig, together with a homologous immune serum, undergo dissolution.

As practiced, the test finds a double application. It may be done to determine the bacteriolytic power of a given serum against a known microorganism, or for the identification of a particular microorganism by means of its susceptibility to lysis in a known immune serum. 
1. Determination of the bacteriolytic power of serum against a known microorganism in vivo:

A number of dilutions of the serum are made with sterile neutral bouillon or salt solution, ranging from 1 in 20 to 1 in 500, or higher. It is convenient to make a first solution of 1 in 20 . One c.c. of this mixed with 4 c.c. of broth will give 1 in 100 . One c.c. of the 1 in 100 dilution with 1 c.c. of broth, 2 c.c. of broth and 4 e.c. of broth will give 1 in 200,1 in 300 , and 1 in 500 respectively. Into one cubic centimeter of each of these dilutions there is placed one platinum loopful of a twenty-four-hour agar eulture of the microorganism against which the serum is to be tested. Into another test tube is placed 4 c.c. of broth, without serum, and with one loopful of the microorganisms. The mixtures are thoroughly emulsified in each case by rubbing the bacteria against the sides of the tube with the platinum loop.

Intraperitoneal injections into guinea-pigs are then made of 1 c.c. of each of the serum-dilution-bacterial-emulsions. A control guineapig (better two or three) receives 1 c.e. of the broth emulsionone-fourth as many bacteria, therefore, as the animals receiving the serum dilutions.

Before making the injections, areas on the lateral abdominal walls of the guinea-pigs are shaved, and small incisions made through the skin, down to the muscular layers. The needle of the syringe is then introduced perpendicular to the skin until it has penetrated the peritoneum, and then carefully slanted to avoid puncturing the gut. The animals need not be strapped down during this procedure and afterward may be allowed to run about.

After one-half hour, and again after one hour has elapsed, a drop of peritoneal exudate is removed from each guinea-pig and examined in the hanging drop for granulation and swelling of the bacteria. The method of obtaining the peritoneal exudate is as follows: Small

Fig. 39.-Capiliary Pipette for Removal of Exudate in Doing the Pfeifferer Test.

glass tubing is drawn out into capillary pipettes, the ends of the capillaries being again drawn to fine points in a small yellow flame.

${ }^{8}$ P. Th. Müller, "Technik d. serodiagnos. Methoden," Jena, 1909. 
A number of such pipettes should be prepared before the test is begun. The guinea-pig is then held down upon a table, either by an assistant or by the left hand of the operator, and the point of the pipette pushed through the cut in the abdominal wall into the peritoneum by a sharp, quick thrusting motion. A column of peritoneal fluid will run into the glass tubing by capillary attraction; this can then be blown out upon a cover-slip for hanging-drop examination or may be blown upon a slide, smeared, and examined after staining. The reaction is regarded as positive if within thirty minutes to an hour the peritoneal exudates of the animals receiving immune sera contain only swollen or disintegrated microorganisms, while in that of the control animals only well-preserved and undegenerated bacteria are found. In dealing with typhoid bacilli and cholera spirilla, in connection with which the test is most often used, active motility in the controls is of much help. Should there be extensive degeneration of the bacteria in the exudate of the control animals the test is of no value.

2. Identification of a microorganism by observing its susceptibility to lysis in a known immune serum in vivo:

The technique for this test is practically the same as that of the preceding except that in this case we require a potent known immune serum and normal serum for control. It is necessary, furthermore, that by previous tests we should know the degree of dilution in which the immune serum will cause complete bacteriolysis of the microorganism used in its production. Thus, if we are employing a typhoid immune serum and are about to test by this method an unknown Gram-negative bacillus, we must know the titer of the serum for the typhoid bacillus itself.

Mixtures are then made of dilutions of this serum and definite quantities of the microorganism to be tested. It is best, always, to employ from ten to one hundred times the amount of immune serum which suffices to froduce lysis with its homologous microorganism. Thus, if the serum has been found to be active in dilutions of $1: 1,000$, it is employed in the test in dilutions at $1: 1,000$, $1: 100$, and $1: 10$. These dilutions are then injected into guinea-pigs in quantities of 1 e.e. together with the bacteria to be tested, and control guinea-pigs are injected with undiluted normal serum mixed with the bacteria and with salt solution and the bacteria. The cxudates are then observed in the same way as in the preceding experiment. 
Bactericidal Reactions in the Test Tube.-Bactericidal reactions in the test tubes may be made by mixing in small sterile test tubes, definite quantities of the bacteria with inactivated serum and complement, the latter in the form of unheated normal serum. The mixtures, diluted with equal volumes of neutral broth or salt solution, are set away for a definite time three to four hours in an incubator at $37.5^{\circ} \mathrm{C}$., and equal quantities from all the tubes are then inoculated into melted agar at $40^{\circ} \mathrm{C}$., and plates are poured. Control plates must be made in each case with mixtures of similar quantities of bacteria in salt solution, and similar quantities of bacteria in normal serum. By colony counting after the plates have developed, it is then possible to estimate the degree of bacterial destruction in any of the given dilutions.

In actually carrying out the test, dilutions of the inactivated serum are first made, ranging from 1:10 to 1:1,000 and over. An emulsion of bacteria from a twenty-four-hour agar slant is then made in salt solution, or a twenty-four-hour broth culture properly diluted may be used. Complement is obtained by taking fresh normal rabbit serum and diluting it with salt solution $1: 10$ or $1: 15$. Into a series of test tubes, then, 1 c.c. of each of the serum dilutions is placed, and to each tube is added 0.5 c.c. of the diluted fresh normal rabbit serum (complement). To these mixtures the bacteria are then added. In adding the bacterial emulsion to these tubes, the writers have found it more accurate to discard the use of the platinum loop and to measure the bacterial emulsion in a marked capillary pipette such as that used in the opsonin test. (See page 340, Fig. 42.) The controls are set up in a similar way, all of them containing a similar quantity of bacterial emulsions, one control containing 1.5 c.c. of salt solution, another control containing 1 e.c. of salt solution +0.5 c.c. of the diluted complement, and the third control containing inactivated normal serum 1 c.c. +0.5 c.c. of diluted complement. Definite quantities of these mixtures, taken with a standard loop, or preferably with a capillary pipette, are plated in agar immediately after mixing.

After incubation for two or three hours similar quantities are again measured into tubes of melted agar with the capillary pipette. With a little practice, great accuracy in these measurements can. be acquired. The inoculated agar tubes are very thoroughly mixed, and plates are poured. At the end of twenty-four hour's' incubation, 
an enumeration of the colonies in the various plates is made and the results are compared.

\section{BACTERICIDAL TEST IN VITRO}

(To Determine the Bactericidal Power of a Typhoid Immune Serum against TYPHOID BACILLI).

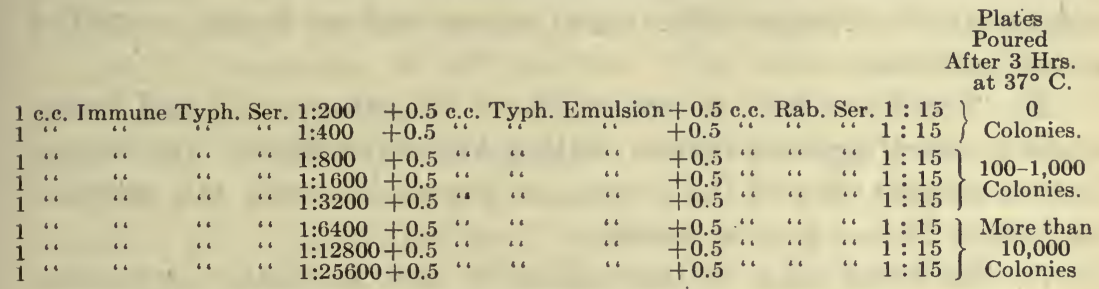

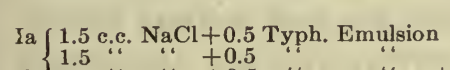

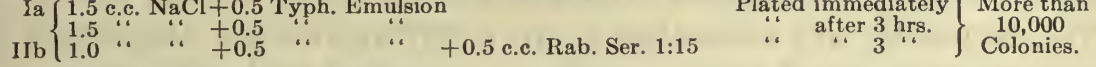

The in vitro bactericidal tests have been employed, practically, chiefly in the diagnosis of typhoid fever by Stern and Korte. ${ }^{9}$ While the serum of normal individuals shows practically no bactericidal power for typhoid bacilli, the sera of typhoid patients may be actively bactericidal in dilutions as high as $1: 50,000$.

Protection tests in mice, etc., are described under the "standardization" of pneumococcus and streptococcus serum in the chapters dealing with these organisms.

Hemolytic Tests.-Determination of the hemolytic action of blood serum, bacterial filtrates, and of a variety of other substances, such as tissue extracts and animal and plant poisons, is frequently made in bacteriological laboratories. Familiarity with the methods of carrying out such tests is especially essential since hemolytic tests are also employed in determining other serum reactions, such as the "complement-fixation tests" discussed in another section.

For these tests it is necessary to prepare washed red corpuscles of the species of animal against which the hemolysins are to be tested, and to obtain these, blood may be taken in one of the following ways :

$A$. If small quantities of blood corpuscles are desired, the blood may be received into a sterile test tube into which a copper or other wire bent into a loop at the lower end has been introduced. This

${ }^{9}$ Stern und Korte, Berl. klin. Woch., 1904. 
is used to prevent elotting and to remove the fibrin. Immediately after receiving the blood into this tube, the wire is twirled between the fingers so that the blood is beaten by the wire as by an eggbeater. At the end of five minutes of continuous agitation, the fibrin adhering in a mass to the wire may be lifted out. The corpuscles are then washed and centrifugalized in several changes of salt solution to remove all traces of serum, and are finally emulsified in salt solution.

$B$. The blood may be taken into a centrifuge tube and immediately centrifugalized before elotting has taken place. The plasma is then poured off and the corpuseles are washed with salt solution, as before, to remove the serum.

$C$. The blood may be taken directly into a solution containing five-tenths per cent sodium ehlorid and one per cent sodium citrate. The corpuscles are concentrated by centrifugalization, the citrate solution is decanted, and corpuseles are washed with salt solution, as before, to remove the serum.

$D$. When large quantities of blood are desired, either from man or from an animal, the blood may be received directly into a flask into which a dozen or more glass beads or short pieces of glass tubing have been placed. The flask is shaken for five or ten minutes, immediately after the blood has been taken and, in this way, defibrination is accomplished.

Since, for comparative tests, it is necessary to establish some standard concentration of red blood cells, it is eustomary in these tests to employ a five per cent emulsion of corpuseles in salt solution. To obtain this, one volume of sediment of washed red blood cells is mixed with nineteen parts of 0.85 per cent salt solution. ${ }^{10}$ Sueh an emulsion, if kept sterile and in the refrigerator, will serve for hemolytic tests for from one to three days. An emulsion should not be used if the supernatant salt solution shows any transparent redness, as this indicates hemolysis.

If the substance in which hemolysins are to be determined is serum, this should be inactivated by exposure to $56^{\circ} \mathrm{C}$. in a water bath, and to each test, complement may be added in the form of fresh guinea-pig or rabbit's serum. No absolute rule for the quan-

${ }^{10}$ The method here given was formerly much employed. It is now the general practice, however, to use one volume of the actual sediment to nineteen volumes of salt solution. 
tity of complement to be used in these tests can be given. In each case the particular complement used should be titrated to determine the minimum quantity which will produce hemolysis of 1 c.e. of the sensitized cell suspension.

In the actual test, mixtures are made of the corpuscle emulsion, the inactivated immune serum, and complement in small test tubes and the volumes of the various tubes made equal by the addition of definite quantities of salt solution. The contents of the tubes are thoroughly mixed and the tubes put in the incubator or in a water bath at $37.5^{\circ} \mathrm{C}$. If complete hemolysis occurs, the fluid in the tube will assume a deep Burgundy red. If no hemolysis occurs, the fluid will remain uncolored and the corpuseles will settle out. Incomplete hemolysis will be evidenced by a lighter tinge of red in the tube and the settling out of a varying quantity of blood corpuscles.

In all hemolytic tests the time element is important. No hemolysis should be adjudged as incomplete unless at least one hour has elapsed.

\section{ISOAGGLUTININS}

In 1901 Landsteiner found that 22 individuals whose blood he studied could be divided into three groups with respect to isohemoagglutinins. It was found, in other words, that, analogous to the isolysins deseribed by Ehrlich and his co-workers in the ease of goats, human beings could exert specific hemoagglutinating action, and, in some cases, hemolytic action upon the corpuscles of other individuals. This is, of course, of the greatest importance in connection with transfusion tests. It is hardly worth while to go into detailed historic considerations in this place. Subsequent investigation has shown that there are four main isoagglutinating groups among human beings. The first classification of this kind was made by Jansky. Subsequently, a similar elassification was made by Moss, but unfortunately Moss reversed the tabulation in such a way that Jansky's group I became Moss's group IV, and vice versa. In America the Moss classification has become universal, and, for this reason, the table given below represents the Moss elassification. It must be borne in mind by all workers who control human transfusions on this tesst, however, that such a reversal of Moss and Jansky exists, and whenever report is rendered it must be made entirely . 
plain which classification is being referred to, and the serologist ought to see to it that no error arises from misunderstanding.

The table indicates also that the blood groups can be explained by the existence of two agglutinins, $a$ and $\beta$, and two agglutinogens, $A$ and $B$. How these may determine the reaction, the table makes clear.

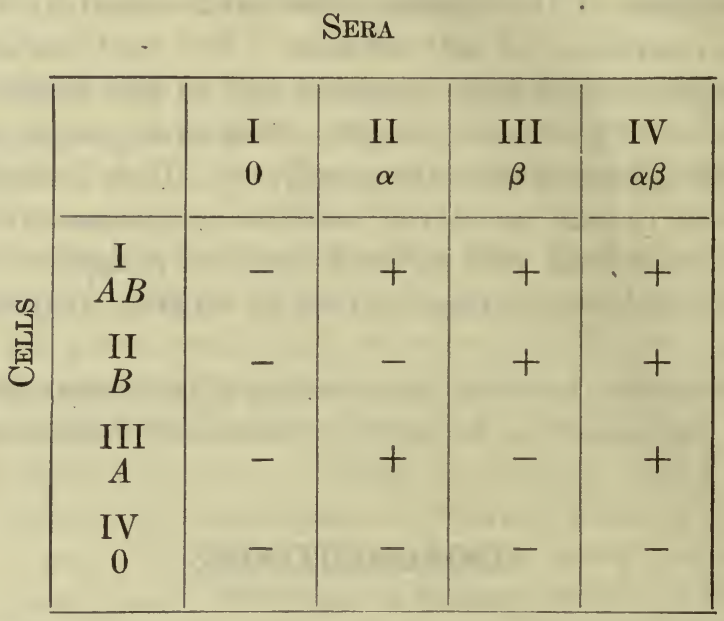

Group I constitutes about 8 per cent of human beings. This group is generally said to be eapable of reeeiving blood from group I, II, III, and IV, but may be used, as a donor only for group I. It is spoken of as a universal recipient, although it is best to avoid this and use the same group, if possible.

Group II constitutes about 40 per cent of all persons, and may be used as a donor for groups I and II, or may receive from group II and IV.

Group III, about 10 or more per eent, may be used as donor for group I and III, or may receive from groups III and IV.

Group IV, about 50 per eent of individuals, may receive from group IV only, but is spoken of as the "universal donor." However, recent writers, especially Unger, have warned against the use of the so-called "universal donor" since severe reactions have resulted following the use of group IV donors for people of another group.

In all eases it is always best to try to get a donor of the same. group. 
The tests may be done in a number of different ways; the most easily carried out, however, is the so-called slide agglutination which was introduced during the war.

In order to properly type an unknown blood, sera from group II and III should be available. A drop of each of these sera is put upon a slide and the corpuseles of the unknown blood added either in the form of a fraction of a drop of the blood taken directly from the finger or ear of the subject, or, better, a fraction of a drop of defibrinated blood or blood taken into about twice its volume of salt solution or sodium citrate solution. The preparation of the blood to be tested is so simple that nothing further need be said. The corpuscles so obtained are mixed with the types of sera of types II and III. By referring to the table it will be easily seen that, if:

The blood agglutinates in neither of the sera, the subject belongs to type IV.

If the cells agglutinate in type II, and not in type III serum, the subject belongs to type III.

If the corpuscles agglutinate in type III and not in type II serum, the subject belongs to type II.

If the corpuscles agglutinate in both sera, the subject belongs to type $\mathrm{I}$.

The blood types as described above are among the few serological reactions which are inheritable by Mendelian laws. They are not, however, present in the child at birth. According to recent studies by Unger, only about 25 per cent of new born infants have cells that can be agglutinated. And only about 13 per cent of new born children have isoagglutinins. Incompatibility between mother and child may occur.

The Determination of Antibodies in Sera by Complement Fixation.-The principle of complement fixation, discovered by Bordet and Gengou ${ }^{11}$ in 1901, has been utilized both in bacteriological investigations, and in practical diagnosis for the determination in serum of the presence of specific antibodies. The reaction depends upon the fact that when an antigen, i.e., a substance capable of stimulating the formation of antibodies, is mixed with its inactivated antiserum, in the presence of complement, the complement is fixed by the combined immune body and antigen and can no longer be found free in the

${ }^{11}$ Bordet and Gengou, Ann. de 1'inst. Pasteur, xv, 1901. 
mixture. If such a mixture is allowed to stand at temperature for an hour or more, and to it is then added an emulsion of red blood cells together with inactivated hemolytic serum, no hemolysis will take place, since there is no free complement to complete the hemoly tio system. If, on the other hand, the original mixture contains no antibody for the antigen used, the complement present is not fixed and is available for the activation of the hemolytic serum later added.

The reaction thus depends upon the fact that neither antigen ${ }^{12}$ alone, nor amboceptor (antibody) alone, can fix complement, but that this fixation is carried out only by the combination of antigen plus amboceptor. Any specific can be determined by this method, provided the homologous antigen is used; and vice versa, by the use of a known antibody a suspected antigen may be determined.

When testing immune sera for antibodies given rise to in man or animals by microorganisms which can be cultivated, either the whole bacteria or extracts of the bacteria may be used as an antigen.

For the diagnosis of syphilis by this method, in the so-called "Wassermann reaction," the antigen employed was originally obtained by the extraction of syphilitic organs, in which free syphilitic antigens, i.e., uncombined products of Spirochæte pallida, were assumed to be present.

It has been more recently shown, however, that the Wassermann reaction is not specific in any sense of the word and that suitable antigens can be produced by the alcoholic extraction of lipoids from the normal organs of many animals and man.

Bacterial extracts for complement-fixation can be made in various ways. The use of thick salt solution suspensions of the cultures themselves is not advisable because of the anticomplementary action of such suspensions. Good bacterial antigens can be produced by centrifugalizing them from salt solution suspensions and adding to about 20 mgms., 90 mgms. of common salt, rubbing up with a glass rod for an hour, and then adding distilled water to isotonicity. This is the method of Besredka. This method has been used with success by Miller and Zinsser in the case of tubercle bacilli for complementfixation in tuberculosis.

Wassermann and Bruck ${ }^{13}$ prepare bacterial antigen by emulsifying

12 Bordet and Gay, Ann. de l'inst. Pasteur, xx, 1906.

${ }^{13}$ Wassermann und Bruck, Med. Klinik, 55, 1905, and Deut. med. Woch., xii, 1906. 
growths of about ten agar slant cultures in 10 c.c. of sterile, distilled water. This is shaken for twenty-four hours in a shaking apparatus. At the end of this time 0.5 per cent of carbolic acid is added and the fluid cleared by centrifugalization.

The Wassermann Test for the Diagnosis of Syphilis. ${ }^{14}$ - The substances for the test are the following:

I. The Antigen.-In their original experiments, Wassermann and his collaborators made use of salt-solution extracts of the organs (chiefly of the spleen) of a syphilitic fetus. The tissue was cut into small pieces and to one part by weight of this substance, four parts of normal salt solution and 0.5 per cent of carbolic acid were added. This was shaken in a shaking apparatus for twenty-four hours, and after this the coarser particles removed by centrifugalization. The reddish supernatant fluid was used as the antigen and could be preserved for a long time in dark bottles in the ice chest.

Alcoholic extracts of syphilitic organs were subsequently used by a number of authors, syphilitic liver being extracted for twenty-four hours with five times the volume of absolute alcohol. This was filtered through paper and the alcohol evaporated in vacuo at a temperature not above $40^{\circ} \mathrm{C}$. About 1 gram of this material was then emulsified in 100 c.c. of salt solution to which 0.5 per cent of carbolic acid has been added.

It was soon found that the Wassermann antigen was a purely nonspecific substance, and since this discovery was made, there are few laboratories in which syphilitic organs are still used. It appears that lipoidal extracts from almost any tissue can be employed, and that fairly useful antigens can even be obtained with solutions of commercial lecithin and mixtures of commercial lecithin and sodium oleate. It is apparent, therefore, that in the Wassermann reaction an even suspension of lipoidal substances constitutes the antigen, and that the complement-fixing complex is made by these antigens in combination with some substance spoken of by Noguchi as "lipotrophic" in the syphilitic serum, which has probably no relation to true antibody. Our own work with treponema pallidum antigen would tend to confirm this, as well as the experience of Noguchi, Craig and Nichols, Kolmer, and others, who have found that a pure treponema pallidum extract gives reactions in only a few late tertiary cases, rumning not

${ }^{14}$ Wassermann, Neisser und Bruck, Deut. med. Woch., xix, 1906; Wassermann, Neisser, Bruck und Schucht, Zeit. f. Hyg., lv, 1906. 
at all parallel to the fixations obtained with non-specific lipoidal substances. Although we are, at the present writing, still in the dark as to whether the syphilitic antigen depends for its properties upon the lipoidal nature of the extracts or upon the size and disposition of the particles present in the extracts, we can still assert that the test is reliable and, with care in execution and interpretation, of enormous value in the diagnosis of syphilis. However, it is necessary to recognize that it is surely not a specific antigen-antibody reaction.

The antigens most eommonly in use today are prepared as follows :

1. Beef heart or guinea-pig heart muscle is finely chopped up and extracted in five times its volume of absolute alcohol. This mixture is kept 5 to 7 days in the incubator, being frequently shaken. It is then filtered and titrated. Human heart muscle may also be used.

2. Noguchi's Acetone Insoluble Lipoid Antigen. Fresh spleen is macerated and extracted for 5 to 7 days in the incubator in five times its volume of absolute alcohol, being frequently shaken. It is then filtered and evaporated to dryness with the aid of a fan. The sticky residue is taken up in a small quantity of ether and this ether solution poured into four times its volume of $\mathrm{C}$. P. acetone. The floccular precipitate which forms is collected and can be preserved under acetone. About 0.2 gram of this paste is dissolved in 5 c.c. of ether. This is shaken up with 100 c.c. of salt solution until the ether is evaporated. The resulting antigen is titrated.

3. Chloresterinized Antigen. According to the researches of Sachs and Rondoni, Browning and Cruikshank, and Walker and Swift, antigen can be made more delicate by the addition of cholesterin. Walker and Swift recommend that an alcoholic extract of human or guinea-pig heart be made up to a concentration of 0.4 per cent of cholesteriñ.

A large number of other antigens might be mentioned, but we think that the thrce mentioned above represent the most important, and in principle all of those at present in common use.

Before an antigen can be used for the actual test, it is necessary to determine the quantity which will furnish a valid result. The substances which are used as antigens often have the power, if used in too large quantity, of themselves binding complement. It is necessary, therefore, to determine the largest quantity of each given antigen which may be used without exerting an anti-complementary action, i.e., which will not inhibit in the presence of normal serum but which will at the same time inhibit hemolysis when syphilitic serum is used. 
This is done by mixing graded quantities of the antigen with a constant quantity of complement (0.1 e.e. of fresh guinea-pig serum), in duplicate sets, adding to each tube of one set 0.2 c.c. of a normal serum, and to the other 0.2 e.c. of a known syphilitic serum. These substances are allowed to remain together for one hour and then red blood corpuseles and inactivated hemolytic serum are added. The quantity which has given complete inhibition with the syphilitie serum, but absolutely no inhibition with normal serum, is the one to be employed in subsequent reactions. Before actual use, it is convenient to make a dilution of antigen in salt solution in such a way that 1 e.c. shall contain the amount required. Thus if 0.05 c.c. is wanted, mix 0.5 c.e. with 9.5 e.c. salt solution. Then 1 e.c. of this can be added to each tube in the test.

II. The Hemolytic Serum.-The hemolytic amboceptor, for the reaction, is obtained by injecting into rabbits the washed red blood corpuseles of a sheep. A 5 per cent emulsion of the corpuseles is made and of this 5 e.c., 10 c.c., 15 c.c., etc., are injected at intervals of five or six days. Three or four graded injections of this kind are usually sufficient to furnish a serum of adequate hemolytic power. The injections may be made intraperitoneally or intravenously. About nine or ten days after the last injection of corpuscles, the rabbit is bled from the carotid artery and the serum obtained by pipetting it from the clot.

It is best to have a hemolytic serum of high potency in order that the quantities used for the reaction may be as small as possible. This is desirable because of the fact that the serum may contain small amounts of precipitins for sheep's serum, due to insufficient washing of the corpuscles employed in the immunization.

It is necessary to carefully titrate the hemolytic serum. For the actual reaction most observers make use of two hemolytic units. A hemolytic unit is the quantity of inactivated immune serum which, in the presence of complement, suffices to cause complete hemolysis in 1 c.c. of a 5 per cent emulsion of washed blood corpuscles. It is the custom in most laboratories today to halve all the quantities, using 0.5 c.c. of the suspension instead of 1.0 e.c. and other ingredients accordingly. Noguchi ${ }^{15}$ has pointed out very clearly the dangers of not delicately adjusting the quantity of amboceptor used in the reac-

${ }^{15}$ Noguchi, Poc. Soc. for Exper. Biol. and Med., VI, 3, 1909. 
tion. He calls attention to the experiments of Morgenroth and Sachs ${ }^{16}$ who have shown that the relationship between complement and amboceptor necessary for hemolytic reactions is one of inverse proportions. In their own words, "in the presence of larger quantities of amboceptor, smaller quantities of complement suffice," and vice versa. Noguchi, in his work, has found that, while in the presence of one unit of amboceptor, 0.1 c.c. of guinea-pig's complement is required to produce hemolysis, by using four, eight, and twenty units of amboceptor, complete hemolysis is obtainable with one-third, one-fifth, and one-tenth of the 0.1 c.c. of complement, respectively. For this reason an excess of amboceptor might result in complete hemolysis in a test, if a small fraction of the complement were left unfixed by the syphilitic antibody. Another result of an excess of amboceptor would consist in a partial dissociation of the complement from its combination with the antigen-antibody compound. As Noguchi puts it, "a quantity of syphilitic antibody just sufficient to fix 0.1 c.c. of the complement against two units of the amboceptor is no longer efficient in holding back the complement from partial liberation against the influence exerted by more than four units of the amboceptor."

From these considerations it follows that the serum from rabbits immunized against sheep corpuscles must, in each case, be titrated in order to determine the hemolytic unit. For this purpose a number of mixtures are made in test tubes, containing each 0.1 c.c. of complement (fresh guinea-pig serum), 1 c.c. of a 5 per cent emulsion of sheep's corpuscles, and diminishing quantities of the inactivated hemolytic serum, thus :

$$
\left.\begin{array}{c}
1 \text { c.c. of } \\
\text { complement } \\
\text { fresh } \\
\text { guinea-pig } \\
\text { serum. }
\end{array}\right\}+\left\{\begin{array}{c}
1 \text { c.c. } \\
\text { of } 5 \text { per } \\
\text { cent } \\
\text { emul- } \\
\text { sion } \\
\text { sheep's } \\
\text { corpus- } \\
\text { cles. }
\end{array}\right\}+\left\{\begin{array} { c } 
{ \text { Inac- } } \\
{ \text { tivated } } \\
{ \text { hemo- } } \\
{ \text { lytic } } \\
{ \text { serum. } }
\end{array} \left\{\begin{array}{ll}
.01 & \text { c.c. }=\text { complete hemolysis } \\
.009 & \text { c.c. }=\text { complete hemolysis } \\
.005 & \text { c.c. }=\text { complete hemolysis } \\
.003 & \text { c.c. }=\text { complete hemolysis } \\
.001 & \text { c.c. }=\text { complete hemolysis } \\
.0009 & \text { c.c. }=\text { partial hemolysis } \\
.0005 & \text { c.c. }=\text { no hemolysis } \\
.0003 \text { c.c. }=\text { no hemolysis }{ }^{17}
\end{array}\right.\right.
$$

In the given case, 0.001 c.c. of the serum represents one unit, and 0.002 c.c., two units, is the quantity to be used for each test.

${ }^{16}$ Morgenroth und Sachs, in Ehrlich's "Gesammelte Arbeiten," ete., Berlin, 1904.

${ }^{17}$ In each tube the volume of the mixture should be made up to 5 c.e. with 0.85 per cent salt solution. 
III. The Complement.-The complement used in Wassermann reaction is fresh guinea-pig serum. This may be obtained in one of the following ways: A guinea-pig may be killed by an incision in the throat and the blood allowed to flow into a large Petri dish. This is set away in the ice chest until clear beads of serum have formed upon the surface, and these are then carefully removed with a pipette.

It is more economical to puncture the heart of large guinea-pigs with a needle attached to a syringe and withdraw 5 or 6 c.c. of blood without killing the animal. This can be transferred to a centrifuge tube and the serum obtained by centrifugation after clotting. Serum used as complement in the Wassermann reaction must be titrated each day before reactions are done. This is done by putting into a series of tubes 1.0 c.c. (or if half quantities are used, as with us, 0.5 c.c.) of the cell suspension sensitized with 2 units of amboceptor, and adding to these tubes varying quantities of guinea-pig serum. The guinea-pig serum is best diluted $1: 10$ in salt solution, and quantities ranging from 0.05 to 0.35 c.c. are added to the tubes. The unit is the amount in the tube which shows complete hemolysis at the end of an hour. The reactions are usually complete in about 30 minutes. Two units of the complement are used in the ordinary test. The titration of the complement is one of the most important steps in accurate work.

IV. The Sheep Corpuscles.-The sheep corpuscles for the actual reaction are obtained by receiving the blood in a small flask containing a sterile solution of a 0.5 per cent sodium citrate and 0.85 per cent sodium chloride, or into one containing glass beads or short pieces of glass tubing. In the former case, the citrate solution prevents clotting and the corpuscles may be washed free from the citrate solution and emulsified in salt solution before use in the test. In the latter case, it is necessary to shake the blood in the flask immediately after taking, and to continue the shaking motion for about ten minutes. The corpuscles are washed free from serum by at least 3 washings in salt solution. A 5 per cent suspension of the corpuscles is employed for the test, made by measuring the bulk of centrifugalized corpuscles and adding nineteen parts of sterile salt solution.

V. The Serum to be Tested for Syphilitic Antibody.-The serum of the patient is best obtained in the same way that blood is obtained for blood cultures. After.surgical precautions, a needle is plunged into the median basilic vein and 3 or 4 c.c. of blood are removed. Before 
use for the test, the patient's serum must be inactivated by heating in a water bath to $56^{\circ} \mathrm{C}$. for twenty minutes to half an hour.

The Test.-The actual test for antibody in a suspected serum is carried out in the following way: In a test-tube of suitable size, 2 units of the complement, 0.2 c.c. of the inactivated suspected serum, and the antigen, in quantity determined by titration, are mixed, and the total volume brought up to 3 c.c. with normal salt solution. This mixture is thoroughly shaken, and placed for one hour in a water bath or in the incubator at $37.5^{\circ} \mathrm{C}$. Recently it has been found that more delicate results are obtained when the fixation is allowed to take place in the refrigerator for three or four hours-the so-called "ice-box method." At the end of this preliminary incubation 1 c.e. of a 5 per cent emulsion of sheep's corpuscles, and two units of hemolytic amboceptor, determined by a titration of the inactivated hemolytic rabbit serum, as deseribed above, are added. This mixture is again placed at $37.5^{\circ} \mathrm{C}$. for one to two hours. If the antibody is present in the suspected serum, no hemolysis takes place. If absent, hemolysis is complete.

In our own work all tests are done in half the quantities of the original Wassermann. Hence only 0.1 c.c. of the patient's serum, and the antigen and complement as determined in titrations with 0.5 c.c. of the cells are mixed in a total volume of 1.5 c.c. At the end of the preliminary incubation, 0.5 c.c. of cells prcviously sensitized with 2 units of amboceptor are added.

No test is of use unless suitable controls are made. The controls set up should be as follows:

Control 1. For each serum tested the mixture described above, omitting antigen.

Controls 2 and 3 . The mixture made as in the test but with known syphilitic serum (2) with and (3) without antigen.

Controls 4 and 5 . The mixture made as in the test, but with normal serum (4) with and (5) without antigen.

Controls 6 and 7 . The hemolytic system, complement, blood cells and amboceptor, set up in order to show that the system is in working order (6) with and (7) without antigen. It is convenient to set the tubes in two rows in a rack, the front row containing antigen, the back row eontaining the same mixture without antigen.

In a positive test, the test itself, and Control 2, alone, should show inhibited hemolysis. The other tubes should show complete solution of the hemoglobin.

(See scheme, p. 324.) 
Modifications of the Wassermann Test.-Since the original formulation of the Wassermann reaction a great many modifications have been suggested by various workers, some of them being radical changes involving the altering of the hemolytic system; others, however, merely adding precautions here and there to increase the delicacy of the reaction. The literature on this subject is too voluminous to be completely covered. We indicate, therefore, some of the most important changes from the original that have been found valuable, and give in greater detail the methods as at present in use in our own laboratory.

Bauer has called attention that human serum contains a certain amount of natural hemolysin for sheep corpuscles. In his original modification, therefore, he does not use hemolytic rabbit serum as amboceptor. His modification as a whole cannot be accepted for general use because human sera do not contain a uniform amount of hemolysin for sheep cells, and some contain none whatever. However, the presence of natural amboceptor, so-called, in human sera is taken account of by many workers, and it is important to recognize this, since naturally it adds to the amboceptor added with sensitized cells and leads to a lack of uniformity in the dosage of amboceptor in individual tubes if included.

Noguchi has worked out a test in which the difficulties presented by the presence of normal sheep amboceptor are eliminated, in that he uses an antihuman hemolytic serum and human cells as the hemolytic system. It enables him also to use the cells of the patient or of any other human being, thus eliminating the necessity of getting fresh sheep cells. His tests are set up as follows:

Tube 1. 1 drop patient's serum + complement ( 0.1 c.c. of 40 per cent guinea-pig serum) + antigen.

Tube 2. 1 drop patient's serum + complement. (No antigen.)

Tube 3. 1 drop known syphilitic serum + complement + antigen.

Tube 4. 1 drop known syphilitic serum + complement. (No antigen.)

Tube 5. 1 drop known normal serum + complement + antigen.

Tube 6. 1 drop known normal serum + complement. (No antigen.)

Tube 7. Complement alone (for hemolytic system control).

To each tube then add 1.0 c.c. of the one per cent emulsion of human corpuscles. Shake mixtures thoroughly and incubate or place in water bath at $38-40^{\circ}$ C. for one hour. Then add to each tube 2 units of antihuman amboceptor (serum of rabbit immunized with human cells) and replace in water bath for one hour. At the end of this time in a positive test there will be no hemolysis in Tubes 1 and 3 while all the other tubes will show hemolysis. 
The method of Noguchi is still used by a few investigators, but is not at present in common use, though we have no doubt that if systematically followed it would develop as quite satisfactory.

The tests are done in our own laboratory with the original sheep cell-antisheep serum hemolytic system. They are done in half quantities, titrations being made with 0.5 c.c. of a 5 per cent emulsion of washed sheep cells. Each day the complement (fresh guinea-pig diluted $1: 10$ ) is titrated with cells sensitized with 2 units of stock amboceptor. Fresh amboceptors are titrated from time to time so that a reasonable constancy is obtained. The hemolytic system is kept as constant as possible from day to day. The unit (minimal hemolytic amount) of a new specimen of amboceptor is determined by titrating

\section{SCHEME FOR WASSERMANN TEST.}

ADAPTED TO ORIGINAL WASSERMANN SYSTEM AFTER SCHEME OF NOGUCHI.

\begin{tabular}{|c|c|c|c|c|c|}
\hline & $\begin{array}{l}\text { Test with Unkno } \\
\text { Serum. }\end{array}$ & & $\begin{array}{c}\text { Test with Known } \\
\text { Positive Syphilic } \\
\text { Serum. }\end{array}$ & $\begin{array}{c}\text { Test with Known } \\
\text { Negative Normal } \\
\text { Serum. }\end{array}$ & $\begin{array}{c}\text { Test without Serum } \\
\text { to Control Efficiency } \\
\text { of Hemolytic } \\
\text { System. }\end{array}$ \\
\hline 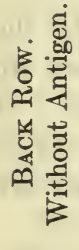 & $\begin{array}{l}\begin{array}{c}\text { Serum .2 c.c. } \\
+ \\
\text { Complement } \\
.1 \text { c.c. } \\
+ \\
\text { Salt sol. 3. c.c. } \\
2 .\end{array}\end{array}$ & 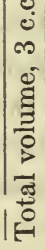 & $\begin{array}{l}\text { Serum .2 c.c. } \\
+ \\
\begin{array}{c}+ \\
\text { Complement } \\
.1 \text { c.c. } \\
+ \\
\text { Salt sol. } 3 . \text { c.c. } \\
4 .\end{array}\end{array}$ & $\begin{array}{c}\text { Serum .2 c.c. } \\
+ \\
\text { Complement } \\
.1 \text { c.c. } \\
+ \\
\text { Salt sol. } 3 \text {. c.c. } \\
6 .\end{array}$ & $\begin{array}{c}\text { Complement } \\
.1 \text { c.c } \\
+ \\
\text { Salt sol. 3. c.c. } \\
\text { 8. }\end{array}$ \\
\hline 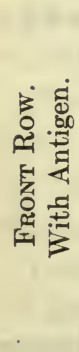 & $\begin{array}{l}\text { Serum .2 c.c. } \\
+ \\
\text { Complement } \\
.1 \text { c.c. } \\
+ \\
\text { Antigen } \\
\text { (required amount } \\
\text { in 1 c.c. salt sol.). } \\
\text { Salt sol. 2. c.c. } \\
\text { 1. }\end{array}$ & 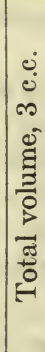 & 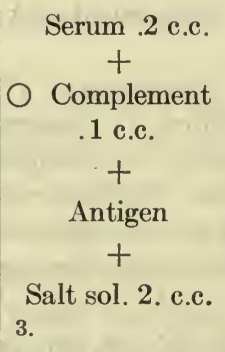 & 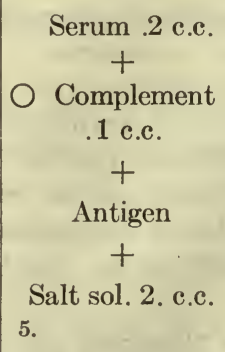 & 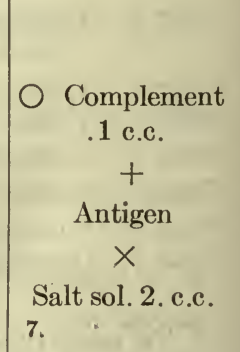 \\
\hline
\end{tabular}

$\mathrm{O}=$ test tube.

Place in water bath at $40^{\circ} \mathrm{C}$. for one hour, then add to all tubes red blood cells and amboceptor. These are previously mixed so that 2 c.c. contains the equivalents of 1 c.c. of a 5 per cent emulsion of sheep corpuscles and 2 units of amboceptor. Again expose to $40^{\circ} \mathrm{C}$. If the serum tested is positive, tubes 1 and 3 should show no hemolysis, all the other tubes showing complete hemolysis in one hour. 
it on a number of successive days with 0.5 c.c. of 5 per cent cells and 0.5 c.c. of 10 per cent guinea-pig sera, readings being made at the end of a half hour. When complement is then subsequently titrated with 2 such amboceptor units and 0.5 c.c. of 5 per cent red cells, it is usually found that the minimal hemolytic dose of complement lies between 0.2 and 0.25 c.c., and in the actual tests twice this minimal hemolytic dose of complement is used with the 2 units of amboceptor. The daily titration of complement frequently shows marked variations even though the complement is obtained from a number of guinea-pigs. By always using twice the amount which just lakes with 2 units of amboceptor, one keeps the actual amount of complement as nearly as possible constant.

Obviously one can titrate the strength of the reaction by varying the amount of any one of the three ingredients which primarily enter into it-the antigen, the patient's serum, or the complement, and all three of these systems have been proposed and used with success by different workers. The method which is most commonly used is that of Citron and consists of using diminishing quantities of both antigen and patient's serum. Citron in addition to the main tubes uses one additional tube containing one-half the amount of patient's serum and one-half the amount of antigen used in the main tube, and he expresses his results as follows:

If both tubes show complete inhibition.................... +++ If the main tube shows complete inhibition and the half dose tube almost complete ............................. ++ If the main tube shows complete inhibition and the second tube

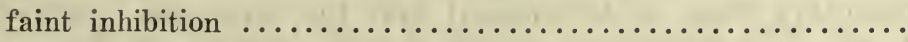
If the main tube shows incomplete inhibition and the second tube none or little

If the main tube shows very faint inhibition and the second tube

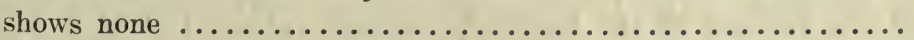

",$++++ "$ ",$+++ "$ and " ++ " are regarded as conclusive results; "+" as a probable positive; " \pm " as suspicious merely.

The Determination of Antigen by Complement Fixation.-The principles underlying the preceding tests for the determination of suspected antibodies may be equally applied to the determination of suspected antigen. In the former case it was necessary to bring the serum to be tested into contact with the antigen specific for the suspected antibody, in the presence of complement, and at a suitable temperature. At the end of an hour the mixture was tested for free 
complement by the addition of hemolytic amboceptor and red blood cells. In testing for antigen, the procedure is reversed, in that the serum or other substance (bacterial extract) to be tested is brought into contact with an antibody specific for the aritigen, in the presence of complement; and at the end of an hour at suitable temperature, free complement is again determined by hemolytic reaction as before.

When dealing with bacterial antigen, it is necessary, therefore, to prepare a highly potent immune serum against the bacteria which contain the specific antigen which is sought.

Thus in testing for typhoid-bacillus antigen in the serum of a patient, the substances required are as follows:

1. Complement: obtained from fresh guinea-pig serum. It is best to titrate the complement when possible, using for the test double the quantity necessary to produce complete hemolysis of 1 c.c. of a five per cent emulsion of blood cells, in the presence of two units of amboceptor.

2. Hemolytic amboceptor: rabbit serum hemolytic for sheep corpuscles. Inactivated and titrated as for Wassermann test.

3. A five-per-cent emulsion of sheep corpuscles in salt solution, prepared as for Wassermann test.

4. A highly potent typhoid antiserum obtained from an immunized rabbit. In this case the smallest quantity of the immune serum which will cause the fixation of complement in the presence of an emulsion or extract of known typhoid bacilli is determined by experiment.

It is best to rub up the centrifugalized bacteria with dry salt, adding distilled water to isotonicity. When the extract is made, its anticomplementary dose is determined and the minimum quantity which in the presence of known antityphoid serum will fix complement. These preliminary titrations are analogous to those described as preliminary to the Wassermann test. When these quantities have been determined, an amount of the bacillary extract (about 1-3 or 1-4 of the anticomplementary dose) is chosen for the actual tests. It is well, also, in such tests to determine the amount which will fix complement in the presence of a known normal serum, since occasional presence of antibodies against some bacteria in normal serum may otherwise confuse the test. The quantities of antigen and complement must then be chosen for the test in such proportions that no fixation will occur with normal serum.

5. Serum from the patient, inactivated at $56^{\circ}$ C. for twenty minutes. 
In the actual test a series of tubes are prepared each of which contains :

1. Complement, the determined quantity.

2. Antiserum, the determined quantity.

3. Diminishing quantities of the inactivated serum to be tested for antigen beginning with 1 c.c.

Salt solution is added for dilution to 3 c.c.

These substances are left together at $37^{\circ}$ to $40^{\circ} \mathrm{C}$. for one hour and then the required quantities of amboceptor and red cells are added. The reaction is controlled by tubes containing the same ingredients without the typhoid antiserum. In a positive test there will be no hemolysis in the tubes containing the patient's serum.

Protein Differentiation by Complement Fixation.-That the technique of complement fixation was applicable to the determination of specific protein antigen-such as human or animal blood-was shown by Gengou ${ }^{18}$ in 1902 . The principles worked out by him have been practically applied by Neisser and Sachs ${ }^{19}$ and others to the forensic differentiation of animal proteins and these tests are said to be more delicate and reliable than precipitation tests made for the same purpose.

The substances necessary for the reaction are as follows:

1. Complement, titrated as above.

2. Hemolytic amboceptor as above.

3. A five-per-cent emulsion of sheep corpuscles as above.

4. Specific antiserum.

This is obtained from a rabbit immunized with the protein for which the test is to be made; viz.: human or animal blood serum. This must be titrated. In order to do this, diminishing quantities of the antiserum are mixed in a series of tubes with the determined quantity of complement, and the antigen which is to be tested for, i.e., the homologous serum with which the antiserum has been produced. Since the test should be sufficiently delicate to determine 0.0001 c.c. of the antigen, this quantity is added to each tube. The actual titration is as follows: ${ }^{20}$

${ }^{18}$ Gengou, Ann. de l'Inst. Pasteur, 1902.

${ }^{19}$ Neisser und Sachs, Berl. klin. Woch., 1905 and 1906. See also Citron, in Kraus and Levaditi "Handbuch," ete.

${ }^{20}$ Citron, loe. eit. 
1. Antiserum, $0.1+$ homologous serum $.0001+$ complement

2. Antiserum, $0.05+$ homologous serum $.0001+$ complement

3. Antiserum, $0.02+$ homologous serum $.0001+$ complement

4. Antiserum, $0.01+$ homologous serum $.0001+$ complement

(Antiserum and homologous serum each brought to 1 c.c. volume with salt solution.)

These tubes are incubated for one nour and hemolytic amboceptor and red blood cells are added. The smallest quantity of antiserum which has completely inhibited hemolysis is the "unit," and one and a half to two times this quantity is used for the test.

5. A solution of the blood spot or other material to be tested prepared as for precipitin test. (See page 306.)

For the actual test the following mixtures are made in a series of tubes, each of which contains:

1. Complement

2. Antiserum $\}$ quantity determined by titration.

3. Diminishing quantities of the substance in which the antigen is suspected, ranging from 0.1 e.c. downward to 0.0001 c.c.

Salt solution is added as a diluent up to 3 c.c. and the tubes are placed in the incubator or water-bath at $37.5^{\circ}$ to $40^{\circ} \mathrm{C}$. At the end of this time red blood cells and amboceptor are added as before.

The tubes are controlled by a series containing all the above ingredients except the antiserum.

\section{SACHS-GEORGI REACTION FOR SYPHILIS * '}

\section{(Direct Precipitation)}

Preparation of Extract.-A beef heart is freed from fat and endocardium, cut up finely and ground in a mortar. It is then shaken with 5 volumes of 95 per cent alcohol and a few glass beads in a shaking machine for 5 hours, allowed to stand at room temperature over night, filtered through ordinary filter paper next morning, then placed in the ice-box for at least two days, when it is again filtered through ordinary filter paper, and is now ready for use. It must first be titrated against a standard extract on a number of sera to determine the optimum dilution and cholesterinization. For this, the alcoholic extract is diluted with 1,2 , and 3 parts of alcohol, and to fractions of each of these dilutions, $0.3,0.45,0.6$ and 0.75 per cent of

* For this description we are indebted to our associate, Dr. Fred'k. Parker, Jr., who has given particular attention to this reaction. 
a 1 per cent alcoholic solution of cholesterin is added. These various portions are then diluted with 5 parts of saline as described below, and set up against a standard extract. At least two such extracts should be used in each test.

Extract Dilution.-The alcoholic cholesterinized extract is diluted with 5 parts of saline as follows: The required amount of extract is placed in an Erlenmeyer flask; to it is rapidly added from a burette an equal volume of saline. It is shaken gently and allowed to stand 10 minutes, then the remaining 4 volumes of saline are rapidly run in. It is again shaken and is ready for use.

Serum.- Serum should be as fresh as possible. Three or four days is not too old. A slight degree of hemolysis does not interfere. Before use in the test, it is heated for $1 / 2$ hour at $55^{\circ}$ to $56^{\circ} \mathrm{C}$. and should not be used sooner than 3 hours after heating. Sachs and Georgi ${ }^{21}$ recommend that spinal fluids should be used undiluted in amounts of 1 c.c. and 0.5 c.c.

Saline.-0.85 per cent sodium chlorid in distilled water. Should be sterile and as fresh as possible.

Test. -0.1 c.c. serum is diluted with 0.9 c.c. saline and to this is added 0.5 c.c. extract dilution. On each serum a control should be set up consisting of 0.1 c.c. serum +0.9 c.c. saline +0.5 c.c. of 95 per cent alcohol, diluted $1: 6$ with saline. Each extract dilution should be controlled by a tube containing 0.5 c.c. extract dilution +1 c.c. saline.

The tubes are thoroughly shaken and placed in the incubator at $37.5^{\circ}$ C. for 20 hours. A preliminary reading may now be made; then the tubes are placed at $14^{\circ}$ to $18^{\circ} \mathrm{C}$., or in the ice-box for 20 hours, and the final and decisive readings are taken.

The reactions present appearances similar to macroscopic bacterial agglutination or precipitin reactions, and are read with the naked eye, the positives showing varying amounts of precipitates and the negatives remaining opalescent as at the beginning of the test. Suspicious tests are centrifuged at moderate speed for a few minutes, and are proved positive or negative by the fact that in the positives after centrifuging a few definite white compact floceuli can be shaken from the bottom of the tube, whereas the negatives, at most, show a slight grayish sediment which disperses on shaking. The serum controls should remain clear. If a precipitate does occur, the serum is unsuitable and another specimen must be obtained. The extract controls should remain diffusely opalescent, and should show no precipitate.

${ }^{21}$ Sachs-Georgi, Med. Klinik, No. 33, 1918, 805; Parker and Haigh, unpublished. 


\section{CHAPTER XVII}

\section{PHAGOCYTOSIS}

THE studies on immunity which we have outlined in the preceding sections have dealt entirely with the phenomena occurring in the reaction between bacteria or bacterial products and the body fluids. These studies, we have seen, have formed the basis of a theoretical conception of immunity formulated chiefly by the German school of bacteriologists under the leadership of Ehrlich, Pfeiffer, Kruse, and others. Parallel with these developments, however, investigations on immunity have been earried on which have brought to light many important facts concerning the participation of the cellular elements of the body in its resistance to infectious germs.

The inspiration for this work and the greater part of the theoretical considerations which have been based upon it, have emanated from Metchnikoff ${ }^{1}$ and his numerous pupils at the Pasteur Institute in Paris. The phenomenon which these observers have studied in great detail and upon the occurrence of which they have based their conceptions of immunity, is known as phagocytosis.

It is well known that among the lowest unicellular animals the nutritive process consists in the ingestion of minute particles of organic matter by the cell. The rhizopods, which may be found and studied in water from stagnant pools or infusions, when observed under the microseope, may be seen to send out short protoplasmic processes, the pseudopodia, by means of which they gradually flow about any foreign particle with which they come in contact. If the ingested particle is of an inorganic nature and indigestible, it will be again extruded after a varying period. .If, however, the ingested substance is of a nature which can be utilized in the nutrition of the protozoon, it is rapidly surrounded by a small vacuole within which it is gradually dissolved and becomes a part of the cellular protoplasm. This digestion within the unicellular organism is probably due to a proteolytic enzyme ${ }^{2}$ which acts in the presence of a weakly alkaline

'Metchnikoff, "'L'Immunité dans les maladies infectueuses."

${ }^{2}$ Mouton, Ann. de l'inst. Pasteur, xvi, 1902. 
reaction. This has been shown by the actual extraction, from amebæ, of a trypsin-like ferment.

As we proceed higher in the scale of the animal kingdom, we find that this power of intracellular digestion, while not uniformly an attribute of all the body cells, is still well developed and a necessary physiological function of certain cells which have retained primitive characters. In animals like the cœlenterata, in which there are two cell layers, an entoderm and an ectoderm, the ectodermal cells have lost the power of intracellular digestion, while the entodermal cells are still able to ingest and digest suitable foreign particles. It is only as we proceed to animals of a much higher organization that the function of cell ingestion of crude food is entirely removed from the process of general nutrition. Nevertheless; in these animals also, the actual cell ingestion of foreign particles occurs, but it is now limited entirely to a definite group of cells. In the higher animals and in man, this function of phagocytosis is limited to the white blood cells of the circulation, or leucocytes, to certain large endothelial cells lining the serous cavities and blood-vessels, and to cells of a rather obscure origin which contribute to the formation of giant cells within the tissues. A convenient division of these phagocytic cells is that into "wandering cells" and "fixed cells." The wandering cells are the polymorphonuclear leucocytes, called "microphages" by Metchnikoff, and certain large mononuclear elements or "macrophages." Fixed cells, also called macrophages by Metchnikoff and possessing the power of ameboid motion, include the cells lining the serous cavities, and the blood and lymph spaces. The small lymphocytes, so far as we know, have no phagocytic functions.

In studying the cellular activities which come into play whenever foreign material of any description gains entrance into the animal body, a definite reaction on the part of the phagocytic cells may be observed. When we inject into the peritoneal cavity of a guinea-pig a small quantity of nutrient broth, and examine the exudate within the cavity from time to time, we can observe at first a diminution from the normal of the cells present in the peritoneal fluid. This may be due either to an injury of the leucocytes by the injected substance, or to an actual repellent influence which the injected foreign material exerts upon the wandering cells. ${ }^{3}$ Very soon after this, however, the exudate becomes extremely rich in leucocytes, chiefly of the polymorphonuclear

${ }^{3}$ Pierrallini, Ann. de 1'inst. Pasteur, 1897. 
variety, the maximum of the reaction being reached about eighteen to twenty-four hours after the injection. After this, there is a gradual diminution in the leucocytic elements until the fluid in the peritoneal eavity again reaches its normal condition. It is plain, therefore, that the presence of the foreign material in the peritoneal cavity has, after a primary repellent action upon the phagocytes, attracted them in large numbers to the site of the foreign substance. Such repelling or attracting influences upon the leucocytes are spoken of as negative or positive chemotaxis. The reasons for chemotaxis are not well understood. In the case of bacteria, which chiefly interest us in the present connection, chemotactic attraction or repulsion is intimately dependent upon the nature of the microorganism, and very probably has a definite relationship to its virulence. Whether or not the principles of chemotaxis may serve to explain the hypo- and hyper-leucocytoses, observed and diagnostically utilized in clinical medicine, is by no means positive. It is likely, however, that the two phenomena are closely associated. Levaditi ${ }^{4}$ believed that he obtained some evidence that negative chemotaxis may take place within the blood-vessels when he noticed that the intravenous injection of cholera spirilla into immunized guinea-pigs resulted in an immediate disappearance of leucocytes from the circulating blood, and their accumulation in the internal organs. On the other hand, this may possibly be more logically explained by a concentration of both bacteria and leucocytes in the capillary system of such an organ as the liver, as it is known that injected bacteria rapidly disappear from the general circulation, but may be demonstrated in the various organs for some time after injection.

We have seen, therefore, that the invasion of the animal body by foreign material, living or dead, is followed by a prompt response on the part of the phagocytic cells. In the case of bacteria, when these are deposited in the subcutaneous areolar tissues, the inflammatory reaction which follows brings with it an emigration of microphages (polynuclear leucocytes) from the blood-vessels-and these are the so-called pus cells. When the injection of bacteria is intraperitoneal, after a primary diminution, there is an increase of leucocytes in the peritoneal cavity which soon results in the formation of a copious turbid exudate. If the pus of an abscess or the exudate from an infected peritoneum is examined microscopically; it will be seen that many of the microphages have taken bacteria into their cytoplasm.

"Levaditi, Presse méd., 1900. 
That fully virulent living bacteria ean be so taken up has been variously proven. The phagocytosis is, therefore, not simply a removal of the dead bodies of bacteria previously killed by the body-fluids, but represents an actual attack upon living and fully virulent microorganisms. That the ingested bacteria are often alive after ingestion is proved by the fact that the injection of exudate containing, so far as can be determined, only intracellular bacteria, has, in several instances, been found to give rise to infection.

After the bacteria have remained for some time within the cytoplasm of the leucocyte, vacuoles may be seen to form about them, similar to those mentioned in discussing the digestive processes of amebæ. If the preparations are, at this stage or later, stained with a one-per-cent solution of neutral red, it will be found that the bacteria, colorless under normal conditions, will be stained pink, an evidence of their beginning disintegration: At a later stage in the process of intracellular digestion, the bacteria will lose their form, and appear swollen, granular, and vacuolated, and finally will be no longer distinguishable. If, on the other hand, the ingestion of bacteria brings about the death of a leucocyte, the neutral red will not stain the bacteria, the digestive vacuoles will not form, and the leucocyte itself will disintegrate.

It must not be forgotten, however, that not all microorganisms are equally susceptible to phagocytosis. Some may resist ingestion more energetically than others by agencies not fully understood. Others again, like the tubercle bacillus and the anthrax bacillus for instance, may, after ingestion, oppose great difficulties to intracellular digestion.

To a certain extent, moreover, the variety of the bacterium determines the variety of phagocyte attracted to the point of invasion. In the cases of most of the bacteria of acute diseases, the microphages or polymorphonuclear leucocytes are the ones upon which the brunt of the battle devolves. Other invaders, like the Bacillus tuberculosis, blastomyces, and others, find themselves opposed chiefly by the macrophages. Cells of animal origin, such as the dead or injured cells of the animal's own body or the cells of other animals artificially introduced, are ingested by macrophages. This is true also of many parasites of animal nature.

It is clear, thus, that the process of phagocytosis is a universal response on the part of the body to the invasion of foreign particles of dead material, of alien cells, and of living microorganisms. It remains to be shown upon what basis this process may be regarded as an essential feature in protecting the body against infection. 
The numerous researches of Metchnikoff have brought out the important fact that phagocytosis is regularly more active in cases in which the infected animal or human being eventually recovers. In animals, furthermore, which show a high natural resistance against any given microorganism, phagocytosis is decidedly more energetic than it is in animals more susceptible to the same incitant. Thus, experimenting with anthrax infection in rats, Metchnikoff was able to show that, in these animals, a decidedly more rapid and extensive phagocytosis of anthrax bacilli takes place than in rabbits and guineapigs and other animals which are delicately susceptible to this infection. While different interpretations have been attached to this phenomenon, its actual occurrence may be accepted as a proven fact.

In his later investigations, furthermore, Metchnikoff was able to show that a direet parallelism existed between the development of immunity in an artificially immunized animal and the phagocytic powers of its white cells. He showed that rabbits artificially immunized to anthrax, responded to anthrax infection by a far more active phagocytosis than did normal, fully susceptible animals of the same species.

It is quite impossible, in the space allotted, to recount the many similar experiments by which the accuracy of these observations has been eonfirmed. While few bacteriologists at the present day harbor any doubt as to the truth of these contentions, the fundamental differences between the conclusions drawn from these various phenomena by the school of Metchnikoff and by that of the German workers may be clearly stated as follows: Metchnikoff believes that phagocytosis is the cardinal factor which determines immunity, while Pfeiffer and others maintain that the determining factors upon which recovery or lethal outcome depends, lie in the fluids of the body, the serous exudate and its contents of immune body and complement, while the phagocytosis occurring coincidently, is merely a means of removal of the bacteria after the outcome has already been decided.

In the further developments of his theory, Metchnikoff has claimed that the immune body and complement-the presence of which in blood serum and exudates he by no means overlooks-are derivatives of the leucocytes.

The immune body or "fixator," as Metchnikoff has named it, has been shown by Wassermann and Takaki ${ }^{5}$ to be most plentiful in the

${ }^{5}$ Wassermann und Takaki, Berl. klin. Woch., 1898. 
spleèn, lymph nodes, ånd bone marrow of animals-all of them organs in which large collections of leucocytic elements are found. Metchnikoff's opinions as to the leucocytic origin of the complement, or "cytase," have found support in the experiments of Levaditi, ${ }^{6}$ who was able to demonstrate the absence of complement in blood plasma,i.e., where no destruction of leucocytes had taken place-and in those of Cantacuzène, ${ }^{\top}$ who showed that cholera-immune guinea-pigs would succumb to intraperitoneal injection of these bacteria when the diapedesis of leucocytes had been prevented by the administration of opium.

The chapter of phagocytosis in its relation to bacterial immunity is by no means closed. The problems involved in it are intricate and will require much further study. The subsequent sections upon opsonins, aggressins, and upon leucocyte extract, incorporate the more recent studies which may be said to have followed logically in the footsteps of Metchnikoff's work.

- Levaditi, Presse méd., 1900.

' Cantacuzène, Ann. de 1'inst. Pasteur, 1897. 


\section{CHAPTER XVIII}

OPSONINS AND VACCINE THERAPY. LEUCOCYTIC SUBSTANCES. NON-SPECIFIC PROTEIN THERAPY. VIRULENCE

\section{OPSONINS}

Although the theories of immunity are, as we have stated, generally classified as the humoral and the cellular or phagocytic theories, the separation has never, even in the minds of the warmest partisans, been an absolute one. Thus, Buchner and his successors looked for the origin, first, of alexin, then of antibody, in the leucocytes, and Metchnikoff attributed to immune serum the quality of stimulating the leucocytes (stimulins) to increased phagocytosis. The serum, according to Metchnikoff, acted, not directly upon the bacteria, in the nature of bactericidal or lytic substances, but rather upon the leucocytes, preparing or arming these for the fray. Denys and Leclef ${ }^{1}$ were the first definitely to oppose this view. These authors, on the basis of experiments done upon streptococcus immunity in rabbits, came to the conclusion that the serum aided phagocytosis rather by its action upon the bacteria than by its influence upon the leucocytes.

Wright $^{2}$ in 1903 and 1904 undertook a systematic study of the relation of the blood serum to phagocytosis, in a series of careful experiments. Using his own modifications of the technique of Leishman, ${ }^{3}$ he first determined the direct dependence of phagocytosis upon some substance contained in the blood serum. He further proved conclusively that this serum component acts upon the bacteria directly and not upon the leucocytes, is bound by the bacteria, and renders them subject to phagocytosis. The presence of these substances in sera, furthermore, which appear entirely free from bactericidal or lytic bodies, and the thermolabile character of the substances $\left(60^{\circ}\right.$ for ten or fifteen minutes destroys them) seemed to exclude their identity with the immune bodies of other authors.

\footnotetext{
${ }^{1}$ Denys et Leclef, La cellule, xi, 1895.

${ }^{2}$ Wright and Douglas, Proc. Royal Soc. London, ixxii, 1904.

${ }^{3}$ Leishman, Brit. Med. Jour., i, 1902.
} 
Beeause of their action in preparing the bacteria for ingestion by the leueocytes, he named those bodies "opsonins" (ö $\psi \omega \nu \in \hat{\imath} v$, to prepare food).

Neufeld and Rimpau ${ }^{4}$ soon after, and independently of Wright, described similar substances in the blood serum of streptococcus and pneumococeus immune animals, which they called bacteriotropins. Because of their greater thermostability it is not yet possible to identify these bacteriotropins absolutely with the opsonins.

The importance of these opsonic substances in immunity was shown by $\mathrm{W}^{\prime}$ ight $^{5}$ in a series of experiments in which he determined that in persons ill with staphylococcus or tubercle-bacillus infections, the phagocytic powers were relatively diminished toward these microorganisms, but could be specifically increased upon active immunization with dead bacteria or bacterial products.

The results of Wright have been confirmed and elaborated by numerous workers.

The diminished power of leucocytes to take up bacteria without the cooperation of serum was demonstrated, after Wright, by Hektoen and Ruediger, ${ }^{6}$ who worked with gradually increasing dilutions of serum. The contention of the Wright school, however, that leucocytes are entirely impotent for phagocytosis without the aid of serum, can not be regarded as proven, in face of the work of Löhlein ${ }^{7}$ and others who have observed phagocytosis on the part of washed leucocytes.

The specificity of opsonins and their multiplicity in a given serum were shown mainly by the work of Bullock and Atkin, ${ }^{8}$ Hektoen and Ruediger, ${ }^{9}$ and Bullock and Western. ${ }^{10}$ These authors showed that the opsonic substances in sera could be absorbed out of the sera, one by one, by treatment with various species of bacteria, a procedure analogous to the method of absorption used in the study of agglutinins.

The increase of phagocytic power demonstrated by Wright in immune sera naturally led to the question whether this depended

${ }^{4}$ Neufeld und Rimpau, Deut. med. Woch., xl, 1904.

- Wright and Douglas, Proc. Roy. Soc., London, lxxiv, 1905.

'Hektoen and Ruediger, Jour. Inf. Dis., ii, 1905.

${ }^{7}$ Löhlein, Ann. de l'inst. Pasteur, 1905 and 1906.

${ }^{8}$ Bullock and Atkin, Proc, Roy. Soc., London, Ixxiv, 1905.

'Heltoen and Ruediger, loc. cit.

${ }^{10}$ Bullock and Western, Proc. Roy. Soc., loc. cit. 
merely upon an increase of the normal opsonins or whether the newly formed immune opsonins were entirely different substances. The greater thermostability of the opsonins in immune sera seemed, at first, to support the latter view. Dean, ${ }^{11}$ however, showed that not all of the normal opsonins are thermolabile and that, by absorption experiments, bacteria treated with normal sera could be prevented from taking up opsonins from immune sera. These facts seem to point strongly toward the identity of normal and immune opsonic substances.

Further study of the opsonins has led to numerous other questions regarding their structure, their relation to other. immune bodies, etc., which are largely still in the stage of controversy, and for which the original monographs must be consulted.

The controversial questions may be briefly reviewed as follows:

As stated above, Wright believed originally that the bodies discovered by him in normal sera, the "normal opsonins," in other words, were distinct bodies that could not be identified with either the complement or antibodies present in serum. Neufeld and Hüne, ${ }^{12}$ Levaditi and Inmann, ${ }^{13}$ and others, on the other hand, maintain that the opsonic action of normal serum, at least, is intimately related to the complement contents of such serum.

They base this contention not only upon the thermolability of normal opsonins, but also upon the fact that opsonin may be removed from normal serum at the same time as complement by the method of complement fixation, detailed in another section (see pp. 295 and 315$).^{14}$

The contention of Wright that the thermostable opsonic substances of immune serum are distinct bodies, not identical with the amboceptors, is supported by the work of Hektoen, ${ }^{15}$ Neufeld and Töpfer, ${ }^{16}$ and others. The problem, however, can by no means be regarded as finally settled, since other workers, notably Levaditi, are inclined to identify the immune opsonins with lytic amboceptors.

As to the structure of the opsonic substances, moreover, differ-

${ }^{11}$ Dean, Proc. Roy. Soc., London, lxxvi, 1905.

${ }^{12}$ Neufeld and Hüne, Arb. a. d. kais. Gesundheitsamt, xxv.

${ }^{13}$ Levaditi and Inmann, Compt. rend. de la soc. de biol., 62, 1907.

${ }^{14}$ Levaditi, Presse médicale, 70, 1907.

${ }^{15}$ Hektoen, Jour. of Inf. Dis., iii, 1906.

${ }^{16}$ Neufeld und Töpfer, Cent. f. Bakt., xxxviii, 1905. 
ences of opinion still exist. Hektoen and Ruediger ${ }^{17}$ who have investigated the question attribute to opsonins a complex constitution. They believe them to possess a thermostable haptophore group and a thermolabile "opsonophore" gioup and that heating beyond a definite temperature converts the opsonins into opsonoids by destruction or alteration of the "opsonophore" group. This view is not shared by all workers and has been disputed by Bullock and Atkin. ${ }^{18}$

The Technique of Wright.-The three factors necessary for the performance of an opsonic test are (1) the blood serum to be tested; (2) an even emulsion of bacteria, and (3) leucocytes.

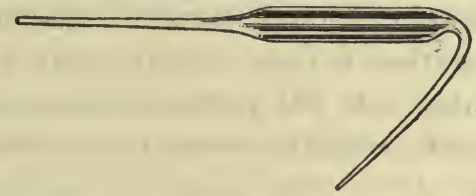

Fig. 40.-Wright's Capsule for Collecting Blood.

(1) Blood serum is obtained by bleeding from the finger and receiving the blood into glass capsules (Fig. 40). These are sealed at both ends; the blood is allowed to elot; and the separation of serum is hastened by a few revolutions of a eentrifuge.

(2) The bacterial emulsion is obtained by rubbing up a few loopfuls of a twenty-four-hour slant agar eulture with a little physiological salt solution ( 0.85 per cent) in a watch glass. A very

Fig. 41.-Pipette for Opsonic Work.

small amount of salt solution is used at first and more is gradually added, drop by drop, as the emulsion beeomes more even. The final breaking up of the smaller clumps is best aceomplished by eutting off very squarely the end of a capillary pipette, placing it perpendicularly against the bottom of the watch glass, and sucking the emulsion in and out through the narrow chink thus formed. (Fig. 41.)

${ }^{17}$ Hektoen and Ruediger, Jour. of Inf. Dis., ii, 1905.

${ }^{18}$ Bullock and Atkin, Proc. Royal Soc., lxxiv, 1905. 
Emulsions of tubercle bacilli are more difficult to make. The bacilli filtered off in the manufacture of old tubereulin are commonly used. These are washed in salt solution on the filter, and are then scraped off and sterilized. They are then, in a moist condition, placed in a mortar and thoroughly ground into a paste. While grinding, salt solution 1.5 cent) is gradually added until a thick emulsion appears. This emulsion may be diluted and larger clumps separated by centrifugalization.

(3) The leucocytes are obtained by bleeding from the ear or finger directly into a solution containing eighty-five hundredths per cent to one per cent of sodium chlorid and five-tenths to one and five-tenths per cent of sodium eitrate. Ten or fifteen drops of blood to 5 or 6 c.c. of the solution will furnish sufficient leucocyfes for a dozen tests. This mixture is then centrifugalized at moderate speed for five to six minutes. At the end of this time, the corpuscles at. the bottom of the tube will be covered by a thin grayish pellicle,

Fig. 42.-Pipette with Three Surstances, Corpuscles, Bacteria and Serum, as First Taken Up.

the buffy coat, consisting chiefly of leucocytes. These are pipetted off with a eapillary pipette (by careful superficial seratching movements over the surface of the buffy coat).

There being, of course, no absolute seale for phagocytosis, whenever an opsonin determination is made upon an unknown serum, a parallel control test must be made upon a normal serum. This normal is best obtained by a "pool" or mixture of the sera of five or six supposedly normal individuals.

The three ingredients-scrum, bacterial emulsion, and leucocytes -having thus been prepared, the actual test is earried out as follows: Capillary pipettes of about six or seven inches in length and of nearly even diameter throughout, are made. These are fitted with a nipple and a mark is made upon them with a grease-pencil about 2 to $3 \mathrm{~cm}$. from the end (Fig. 42). Corpuseles, bacteria, and serum are then successively, in the order named, sucked into the pipette up to the mark, being separated from each other by small airbubbles. Equal quantities of each having thus been secured, they are mixed thoroughly by repeatedly drawing them in and out of the pipette upon a slide. The mixture is then drawn into the pipette; 
the end is sealed; and incubation at $37.5^{\circ}$ is carried on for an arbitrary time, usually fifteen to thirty minutes. ${ }^{19}$ The control with normal serum is treated in exactly the same way. After incubation the end of the pipette is broken off, the contents are again mixed, and smears are made upon glass slides in the ordinary manner of blood smearing. Staining may be done by .Wright's modification of Leishman's stain, by Jenner's, or by any other of the usual blood stains. In these smears, then, the number of bacteria contained in each leucocyte is counted. The contents of about eighty to one hundred cells are usually counted and an average is taken. This average number of bacteria in such leucocytes is spoken of as the "phagocytic index." The phagocytic index of the tested serum, divided by that of the "normal pool" (control) serum, gives the "opsonic index."

Another method of estimating the opsonic content of a given blood serum has been contributed by Simon, Lamar, and Bispham. ${ }^{20}$ These authors employed dilutions both of the patient's serum and of normal serum ranging from one in ten to one in one hundred. With these dilutions, they carry out opsonic experiments with bacterial emulsions and washed leucocytes in the same way as this is done in the Wright method, except that they recommend the employment of thinner bacterial emulsions than are usually employed in the former method. In examining their slides, they do not estimate the number of bacteria found within the leucocytes, but rather the percentage of leucocytes which actually take part in the phagocytic process, ${ }^{21}$ i.e., which contain bacteria.

By the same method of dilution, they determine what they have called "the opsonic coefficient of extinction," a phrase which is used to express the degree of dilution of the serum at which no further phagocytosis takes place. They claim for their methods the more delicate determination of variations in opsonic power. The method has not been sufficiently used to permit the expression of an opinion as to its value.

The Vaccine Therapy of Wright.-In connection with his more theoretical work upon opsonins, Wright has laid much stress upon

${ }^{19}$ For the purpose of incubation, specially constructed water baths, marketed under the name of "opsonizers," may be used.

20) Simon, Lamar, and Bispham, Jour. Exp. Med., viii, 1906.

${ }^{21}$ Simon and Lamar, Johns Hopkins Hosp. Bull., xvii, 1906. 
the value of active immunization in the treatment of infectious diseases. Beginning his work with staphylocoecus and tubercle-bacillus infections, he has extended his methods, with the aid of many collaborators, to gonococcus, streptoeoceus, pneumococcus, and a number of other bacterial infections. In all these cases, when possible, he uses for therapeutic purposes a so-called "autogenous vaccine" which is made with the bacteria isolated from the patient himself. In the case of tubercle-bacillus infections, he uses for treatment the new-tuberculin-bacillary-emulsion of Koch. The production of vaccine is, according to Wright, as follows:

Production of Vaccines.-After isolation of the organisms from the patient, cultures are made with a view of obtaining considerable amounts of bacterial growth. In making vaccines with poorly growing organisms, large surfaces must be inoculated. Organisms are best grown for this purpose upon the surface of agar or glucose agar (the enrichment of the agar with sugar or acetic fluid, etc., depending upon the cultural requirements of the organism in question), in square eight-ounce medicine bottles laid upon their sides. This furnishes a large area for inoculation. After sufficient growth has taken place upon the agar, two or three cubic centimeters of sterile rormal salt solution are introduced into the bottles with a sterile pipette. With this the growth is gently washed off the surface of the agar, more salt solution gradually being added as necessary. The emulsification may be facilitated by gently scraping the growth off the medium by means of a flexible platinum loop. This thick bacterial emulsion is then pipetted out of the bottles, during which process an equalization of the emulsion can be attained by repeated sucking in and out with the pipette. The emulsion is then placed in a sterile test tube which may then be drawn out at its open end into a capillary opening. It is a point of practical importance that, in preparing such capsules out of a test tube, a few inches of air space should be left above the surface of the emulsion, so that expansion during heating may not blow out the top of the glass tube. $\Lambda$ dozen or so of sterile glass beads may be put into these tubes in order to aid in emulsification. Shaking the beads in such a tuhe will help in breaking up small clumps of bacteria.

The emulsion is then standardized; that is, a numerical estimation of bacteria per cubic centimeter must be made. This standardization is best done before sterilization, because during the latter 
process a number of bacteria may be broken up, and, while unrecognizable morphologically, are, nevertheless, represented in the emulsion by their products. The standardization may be accomplished by highly diluting a definite volume of the emulsion, planting plates with definite quantities of the dilution, and counting colonies. Wright prefers, as more exact, an enumeration of the bacteria against red blood cells. This is done in the following way:

A little of the emulsion is placed in a watch glass and from it, with a pipette as used in the estimation of the opsonic index, one volume is taken and is mixed with an equal volume of blood from the finger and two or three volumes of salt solution. The salt solution is added in order to dilute the red cells so that they can be conveniently counted and to prevent clotting. These substances are thoroughly mixed in a pipette and spread upon a slide as in making a blood smear, and as even and uniform a smear as possible should be made. They are then stained either by Jenner's or Wright's blood stain.

The preparations are examined with an oil-immersion lens. In order to limit a definite microscopic field, it is convenient to use an Ehrlich diaphragm, or else, in lieu of this, to mark a circle with a blue pencil upon the lens of the eye-piece. The red blood cells and bacteria, in a number of these fields, are counted and the ratio between them is estimated. Knowing the number of red blood cells to the cubic millimeter in the particular blood employed, by previous blood count, and knowing that equal volumes of blood and of bacterial emulsion have been used in the mixture, it is easy from this ratio to ascertain the number of bacteria contained in a cubic millimeter of the original emulsion. Thus, for instance, if in an average of twenty fields bacteria are to red blood cells as two is to one, and the blood employed contains five million red blood cells to each cubic millimeter, then a cubic millimeter of our emulsion contained ten million bacteria, and a eubic centimeter one thousand times as many.

Special centrifuge tubes with graduated narrow tips at the bottom have been suggested by Hopkins for vaceine standardization. Bacteria centrifugalized up to a certain mark represent a definite number per cu. $\mathrm{mm}$. when taken up in a given volume of salt solution.

The vaccine, thus standardized, is sterilized at $60^{\circ} \mathrm{C}$. for one hour for several days. Its sterility is then controlled by culture and animal inoculation. 
From this stock emulsion small quantities may be drawn off and diluted for therapeutic use.

The initial dose given by Wright in staphylococcus infections, in which the method has been most frequently employed, varies from fifty to one hundred millions of bacteria. In working with the tubercle bacillus, the ordinary tuberculin dosage is adhered to.

Wright, in his work, makes use of the opsonic index in order to estimate ehanges in the resistance of the patient against the given infection. In other words, he bases his judgment as to whether the patient is improving or not, upon the opsonic power of the patient's serum. In following the opsonic index of a patient during systematic treatment with vaccine, Wright has found definite changes upon the basis of which he constructs a eurve of opsonic power. Immediately after the injection of vaccine, he finds that there is a brief period during which the opsonic power of the patient is depressed below its original state. This he calls the negative phase. The length of time occupied by this negative phase depends both upon the condition of the patient and upon the size of the dose given. It is usually completed within twenty-four hours. After this, there is a gradual rise in the opsonic power, at first rapid, later more slow, until a maximum is reached after a varying number of days. This period of rise represents the positive phase. The second inoculation with vaccine should, according to Wright, be made when the opsonic power is again beginning to sink after the highest point of the positive phase.

The facts of Wright's investigations have been given in the preceding without much critieal consideration. Those features which concern themselves with the proof of the opsonic properties of normal and immune serum have been of the greatest scientific importance and a great deal of benefit has accrued from the renewed attention turned by him toward methods of active immunization in human beings. Vaceine therapy in many conditions has come to stay, although some of the very extravagant earlier claims have had to fall down. It is also pretty certain at present that the opsonic index measurements as a guide to treatment are of very little value and may even mislead. Prophylactic vaccination in various diseases is dealt with in the chapters on the individual infections.

Leucocytic Substances.-In the sections upon Phagocytosis and Opsonins, we have discussed the protective action exerted by the living leucocytes against bacterial infection and the relation of these 
cells to the blood serum; furthermore, that, while our knowledge of the blood serum, as developed at present, shows that phagocytes may be aided by this in the ingestion of bacteria, the subsequent digestion of the germs, and possibly the neutralization or destruction of their intracellular poisons, is, as far as we know, largely accomplished by the unaided phagocytic cell. It is an obvious thought, therefore, that, in the struggle with bacterial invaders, the leucocytic defenders might be considerably re-enforced if they were furnished, as directly as possible, with a further supply of the very weapons which they were using in the fight with Hiss ${ }^{22}$ conceived the plan of injecting into infected subjects the substances composing the chief cells or all the cells usually found in exudates, in the most diffusible form and as little changed by manipulation as possible; and he also assumed that extracts would be more efficacious than living leucocytes themselves, since if diffusible they would be distributed impartially to all parts of the body by the circulatory mechanism.

The method of obtaining these substances as used both in animal experiments and in the treatment of human subjects is at present as follows:

Rabbits, preferably of 1,500 grams weight or heavier, receive intrapleural injections of aleuronat. This is prepared by making a three per cent solution of starch in meat-extract broth, without heating, and adding to this, after the starch has gone into thorough emulsion, five per cent of powdered aleuronat. This is thoroughly mixed, boiled for five minutes, and filled into sterile potato tubes, 20 c.c. into each tube. Final sterilization is done preferably in an autoclave. The rabbit injections are carried out by injecting 10 c.c. into each pleural cavity in the intercostal spaces at the level of the end of the sternum, in the anterior axillary line, great eare being exerted to avoid puncturing of the lungs. The rabbits are left for twenty-four hours, at the end of which time a copious and very cellular exudate will have accumulated in the pleural cavities. This is removed, after killing the animals with chloroform, by opening the anterior chest wall under rigid precautions of sterility, and pipetting the exudate into sterile centrifuge tubes. Immediate centrifugalization before clotting can take place then permits the decanting of the supernatant exudate fluid. To the leucocytic sediment is then added about 2 c.c. of sterile distilled water, and the

${ }^{22}$ Hiss, Jour. Med. Res., N. S., xiv, 3, 1908. 
emulsion is thoroughly beaten up with a stiff bent platinum spatula. Smears are now made on slides, stained by Jenner's blood stain, and examined for possible bacterial contamination. It is well also to take cultures. Sterile distilled water is then added to each tube, about twenty volumes to one volume of sediment, and the tubes are set away in the ineubator for eight hours. At the end of this time the sterility is again controlled as above, and further extraction in the refrigerator continued until the extract is used.

Experiments by Hiss and by Hiss and Zinsser showed that leucocytic extracts injected into animals infected with various organisms exerted a distinct though not very powerful therapeutic effect. They have also had a certain degree of beneficial effect in human beings suffering from various infections. However, it is our present opinion, based chiefly upon the researches of one of us with Tsen, ${ }^{24}$ that the leucocytic substances act in more or less the same way as do other non-specific proteins, in that they produce an increased leucocytosis and perhaps, as pointed out by Jobling and Petersen in another connection, may lead to an increased discharge of the blood of various proteolytic and other enzymes.

That bactericidal substances can be extracted from leucocytes by various methods has been repeatedly shown by Schattenfroh, Pettersen, Korschun, and others. ${ }^{25}$ The researches of Pettersen as well as, more recently, the work of Zinsser, have shown that these "endolysins," as Petterson has called them, have a structure quite different from that of the serum bacteriolysins in that they are not rendered inactive by temperatures under $80^{\circ} \mathrm{C}$., but, when once destroyed by higher temperatures, can not be reactivated either by the addition of fresh serum or of unheated leucocyte extracts. The last-named authors, moreover, have shown that these endocellular bactericidal substances are not inereased by immunization, the quantity present in each leucocyte being probably at all times simply sufficient for the digestion of the limited number of bacteria which ean be taken up by the individual leucocyte.

${ }^{23}$ Hiss, Jour. Med. Res., N. S., xiv, 3, 1908.

${ }^{24}$ Zinsser and Tsen, Jour. of Immun., 2, 1917.

${ }^{25}$ Schattenfroh, Arch. f. Hyg., 1897; Pctterson. Cent. f. Bakt., I, xxxix, 1905; and ibid., xlvi, 1908; Korschum, Ann. de l'Inst. Pasteur, xxii, 1908; Zinsser, Jour. Med. Res., xxii, 3, 1910. 


\section{Non-SPECIFiC Protein 'THerapy}

A very surprising development of the last ten years has been the observation that profound physiological reactions accompanied by occasional therapeutic benefit in infectious diseases has followed the intravenous injection of bacterial and other proteins which apparently had no specific relationship to the nature of the infectious process. The first observations were more or less accidental, incident to attempts by various writers to treat diseases like typhoid fever by the intravenous injection of typhoid bacilli. Ichiwaka, Kraus, Gay and others injected sensitized and unsensitized typhoid bacilli intravenously into patients suffering from typhoid fever, observing a sudden drop of temperature with chill, and frequent benficial effects on the course of the disease. It was soon found that similar results could be obtained in these diseases with colon bacilli, paratyphoid bacilli, etc. Holler ${ }^{26}$ obtained striking results in typhoid fever by injecting deutero-albumose. Other proteins and proteose substances were subsequently used by many observers, and important studies on the theoretical effects of the injection of such substances have been made by Jobling and Petersen. ${ }^{27}$ It can be regarded as quite definite that these reactions are entirely nonspecific. The substances used have been typhoid vaccine, primary and secondary albumoses, gonococcus and other bacterial vaccines, normal serum, leucocyte extracts, etc., etc. The method has been applied to a great many definite infections, such as typhoid fever, general sepsis, pneumonia, gonorrheal infections, and to arthritis and dermatological lesions, ete. Miller ${ }^{28}$ has used typhoid vaccines in typhoid fever and other conditions, and, in general, concludes that there can be little doubt that in a limited number of cases rapid and sometimes permanent beneficial results, are obtained after a preliminary slight rise of temperature and subsequent drop, often with a chill. He also concludes that if an amount just sufficient to incite a chill is used, that is, if the dosage is carefully controlled, the treatment is without danger. He has given 2,000 intravenous injections of typhoid vaccine in the Cook County Hospital, without serious consequences, except for the development of delirium tremens

${ }^{26}$ Holler, Beit. z. Frank. u. Inkeft., 6, 1917, cited from Miller, Jour. A. M. A., 76, Jan., 1921.

${ }^{27}$ Jobling and Petersen, Jour. Exper. Med., 20, 1914.

${ }^{28}$ Miller, loc. cit. 
in some alcoholics. He, however, carefully selected his cases. As Petersen $^{29}$ concludes, non-specific therapy has produced definite results, though the eventual determination of its definite value cannot yet be made. It is in the experimental stage, and according to Petersen ${ }^{29}$ concludes, non-specific therapy has produced definite results, though the eventual determination of its definite value cannot yet be made. It is in the experimental stage, and according to Petersen, "its usefulness and ultimate range" cannot yet be fully judged.

The effects of the injection, as analyzed by Petersen from his own studies and a study of the literature, are as follows: After injection of the more powerful and active substances, there is at first a chill, sweating, and a definite rise of temperature; there is a leucopænia followed by leucocytosis, lowering of the blood pressure, and changes in the blood, such as increase in fibrinogen, a rise of enzyme curve and an increase in blood sugar and antibodies. The less active substances produce some temperature, a slight chill and other symptoms mentioned, to a lesser degree. The beneficial effects may perhaps be to some extent explained by the increase of leucocytes and of enzymes, and Petersen makes a point of the fact that if the method is to exert beneficial effects, it is probably necessary to use it early in the disease. We are not in any position at present either to recommend or further comment upon the method, but it is an important problem for laboratory experimentation and for careful clinical application in the hands of men trained in experimental studies.

The Problem of Virulence.-An extremely obscure chapter in our knowledge of the reaction of animals and man against infection is the one dealing with the questions of varying pathogenicity between different bacterial species and between different races of the same microorganism. We know that certain bacteria may be injected into an animal or human being in considerable quantities, without producing anything more than the temporary local disturbance following the subcutaneous administration of any innocuous material. Other bacteria, on the other hand, such as the bacillus of anthrax or the bacillus of chicken cholera, injected in the most minute dosage, may give rise to a rapidly fatal septicemia. Within the same species, furthermore, fluctuations in virulence may take place

${ }^{29}$ Petersen, Jour. A. M. A., 76, January, 1921. 
which may depend upon a variety of influences which have been discussed in another section and need not be recapitulated. Suffice it to say that variations in the susceptibility of inoculated subjects do not, in any way, furnish a sufficient explanation for these phenomena.

In an effort to cast light upon this subject, Bail, following in the footsteps of his predecessors, Kruse, ${ }^{30}$ Deutsch and Feistmantel, ${ }^{31}$ has formulated his so-called "aggressin-theory."

Bail $^{32}$ was first led to the formulation of his theory by extensive researches which he had made in conjunction with Petterson $^{33}$ into anthrax immunity. He had noted, as others before him had, that animals, highly susceptible to anthrax, often possessed marked bactericidal powers against this bacillus. When such animals, whose serum should surcly be capable of bringing about the death of, at least, a few hundred anthrax bacilli, were injected with doses far less than this number they nevertheless succumbed rapidly and the bacilli multiplied enormously in their bodies. He argued from this that the injected microorganisms must possess some weapon whereby they were enabled to counteract the protective forces of the animal organism. In an anthrax-immune animal, as a matter of fact, no proliferation of bacteria took place and the injected germs were rapidly disposed of by the protective forces, foremost of which was phagocytosis.

The theory of Bail $^{34}$ contains the following basic principles: $:^{35}$

Pathogenic bacteria differ fundamentally from non-pathogenic bacteria in their power to overcome the protective mechanism of the animal body, and to proliferate within it. They accomplish this by virtue of definite substances given off by them, probably in the nature of a secretion, which acts primarily by protecting them against phagocytosis. These substances (referred to by Kruse as "Lysins") were named by Bail, "Aggressins." The production of aggressins by pathogenic germs is probably absent in test-tube cultures, or, at any rate, is greatly depressed under such conditions,

${ }^{30}$ Kruse, Ziegler's Beiträge, xii, 1893.

${ }^{31}$ Deutsch und Feistmantel, "Die Impfstoffe in Sera," Leipzig, 1903.

${ }^{32}$ Bail, Cent. f. Bakt., I, xxvii, 1900, and xxxiii, 1902.

s3 Bail und Petterson, Cent. f. Bakt., I, xxxiv, 1903; xxxv, 1904; xxxvi, 1904.

${ }^{34}$ Bail, Arch. f. Hyg., lii, 1905; liii, 1905; Wien. klin. Woch., xvii, 1905.

${ }^{85}$ Bail und Weil, Wien. klin. Woch., ix, 1906; Cent. f. Bakt., I, xl, 1906; xlii, 1906. 
but is called forth in the animal body by the influences encountered after inoculation.

These aggressins can be found, according to Bail, in the exudates about the site of inoculation in fatal infections. He obtained them, separate from the bacteria, by the centrifugation and subsequent decanting of edema fluid, and pleural and peritoneal exudates.

Two experimental observations are brought by Bail in support of the truth of his contentions. In the first place, he was able to show that fatal infection could be produced in animals by the injection of sublethal doses of bacteria, when these were administered with a small quantity of "aggressin." He inferred from this that the injected aggressin had paralyzed the onslaught of phagocytic and other protective agencies, and had thus made it possible for the bacteria to proliferate.

The second experimental support of Bail's theory consists in the successful immunization of animals with aggressin. Animals were treated with aggressive exudates, from which all bacteria had been removed by centrifugalization and which had been rendered sterile by three hours' heating to $60^{\circ} \mathrm{C}$. and addition of 0.5 per cent phenol. Animals so treated were not only immune themselves, but contained a substance in their serum which permitted the passive immunization of other untreated animals. Bail explained this by assuming the production of anti-aggressins in the treated subjects. His experiments and those of his pupils were conducted with the typhoid and dysentery bacilli, the bacilli of chicken cholera and of plague, the cholera spirillum, and various micrococci. According to whether a microorganism is capable of producing an aggressin and consequently of invading the animal body, he divides bacteria into "pure parasites," "half parasites," and "saprophytes."

The theory. of Bail has been attacked chiefly. by Wassermann and Citron, ${ }^{36}$ Wolff, ${ }^{37}$ and Sauerbeck. ${ }^{38}$ The eriticism which these investigators make of Bail's views has succeeded in placing the "aggressin" theory in doubt. It is claimed by them that much of the "aggressive" character of Bail's exudates is due to their containing liberated bacterial poisons (endotoxins). This they have maintained both because the sterile "aggressin" exudates could be shown to possess a considerable degree of toxicity and because the

\footnotetext{
so Wassermann and Citron, Deut. med. Woch., xxviii, 1905.

s7 Wolff, Cent. f. Bakt., I, xxxviii, 1906.

ss sauerbeck, Zeit. f. Hyg., lvi, 1907.
} 
aggressive action could be duplicated by aqeous extracts of bacteria. Citron, ${ }^{39}$ was able to show, by the Bordet-Gengou method of complement fixation, that the exudates of Bail contained quantities of free bacterial receptors, which, in taking up immune body, would neutralize any destructive power on the part of the infected animal.

The writer in conjunction with Dwyer ${ }^{40}$ has done certain experiments which seem to indicate that Bail's aggressin may be in the nature of anaphylatoxin. The addition of such anaphylatoxin to bacteria will convert a sublethal into a lethal dose, as will Bail's aggressin, and in principle the manner of production is the same. The nature of the immunity produced in animals by Bail's method of treatment is less easily explained and less exposed to adverse criticism. Whatever may be the truth about the possession of offensive weapons on the part of bacteria, it is certainly a fact that microorganisms differ much in their powers of defense against destruction by the cells in sera of the animal body. Virulent bacteria are not destroyed by serum or agglutinated or taken up by leucocytes as easily as are the non-virulent. In some cases there seems to be no morphological clue to the reason for this. In other cases, like pneumococei, Friedländer bacilli and others, there is a bacterial capsule which seems to insulate these organisms against attack. Many bacteria lose their capsules in the non-virulent stage on culture media, but form them within the animal body in the process of infection. Again, bacteria rendered non-virulent by cultivation on artificial media may become virulent, inagglutinable, and more resistant to phagocytosis when cultivated on immune sera or passed through the animal body.

Thus the power to invade depends possibly upon a combination of offensive properties and defensive qualities on the part of the bacteria. Added to this, some of us believe that the reaction between lytic antibodies and the bacterial protein may produce toxic substances which poison the animal body, prevent positive chemotaxis, and thereby aid the invader.

Again, there are microorganisms like the treponema pallidum in syphilis where adaptation between invader and host seems to be of such a nature that an indifferent reaction against the invading organism only is set up.

${ }^{39}$ Citron, Cent. f. Bakt., I, xl, 1905; xli, 1906 ; and Zeit. f. Hyg. lii, 1905.

4) Zinsser and Dwyer, Proceedings of the Soc. for Exper. Biol. and Med., 1914, $\mathrm{xi}, 74-76$. 


\section{CHAPTER XIX}

\section{HYPERSUSCEPTIBIIITY}

THE phenomena now grouped together under the heading of anaphylaxis and hypersusceptibility have but recently become the subject of systematic experimentation. Nevertheless, manifestations now recognized as belonging to this eategory had not escaped the attention of a number of the earlier workers in immunity.

Although the development of scientific knowledge of hypersusceptibility has concerned itself particularly during the last fifteen years with hypersusceptibility phenomena as they apply to antigenic substances, we must deal with the subject a little more comprehensively than this at the present day, and call attention to the fact that clinicians had for many years noticed so-called idiosyncrasies against drugs of various kinds, morphin, strychnin, arsenic, etc., etc., and against many things which cannot be regarded at the present time as antigens in the sense in which this term is used in immunological literature.

By hypersusceptibility in general, then, we mean that a certain individual, human being or animal, suffers injury from the administration of a substance which, in similar amounts and methods of administration, does not exert any injurious action upon normal members of the same species.

In some cases, such as pollen hypersensitiveness and some other food idiosyncrasies, the hypersusceptibility may be congenital, that is, inherited by the individual without traceable previous contact with the substance to which he is sensitive.

In most instances, however, some form of previous physiological contact with the particular substance seems to be necessary for the development of the hypersensitive state.

Owing to the varied manifestations of specific hypersusceptibility, it has been necessary to classify the phenomena of this nature into two main subgroups. In doing this we follow the classification of Doerr. ${ }^{1}$ Doerr has grouped together all phenomena of hypersus-

${ }^{1}$ Doerr, Kolle and Wassermann Handb., 2, 1913, 947. 
ceptibility under the general term of allergy, which means altered reaction. Under this heading he classifies, $A$, those forms of hypersusceptibility in which the inciting substance is, as far as we know at the present time, non-antigenic, and, $B$, that form of allergy in which the inciting substance is a known antigen.

We ourselves would rather defirie these subdivisions as, $A$, those forms of allergy in which the mechanism of the hypersusceptibility cannot be shown to be due to an antigen-antibody union, and, $B$, those forms in which an antigen-antibody union within the body can be proved to be responsible.

The difference is a slight one, but may, in the future, perhaps become a fundamental one, for our recent studies on the tuberculin reaction make us believe that the conception of the word "antigen" will necessarily change in the course of the next few years of investigation.

We do not see any particular purpose in altering the Doerr classification to one suggested by $\mathrm{Coca}^{2}$ in which the general term "hypersensitiveness" is subdivided into true anaphylaxis and allergy, the term "allergy" here being confined to the reactions in which no true antigen-antibody reaction can be determined. In fact, we believe that this would be harmful, in that Coca thereby implies that there is a general identity of mechanism underlying the manifestations which he classifies together as "allergy," some of which are certainly open to justified differences of opinion.

We will first deal with those forms of hypersusceptibility in which truly antigenic substances are involved, and, for this form, we may reserve the term of True Anaphylaxis.

Anaphylaxis.-As early as 1893, Behring ${ }^{3}$ and his pupils ${ }^{4}$ had noticed that animals, highly immunized against diphtheria toxin, with high antitoxin content of the blood, would occasionally show marked susceptibility to injections of small doses of the toxin.

The phenomena observed by them was interpreted as an increased tissue susceptibility to the toxin, and Wassermann, reasoning on the basis of Ehrlich's side-chain theory, formulated the conception that the increased susceptibility was due to toxin receptors, increased in number by immuniza-

${ }^{2}$ Coca, Tice's System of Medicine, Vol. 3, 1920.

${ }^{3}$ Behring, Deut. med. Woch., 1893.

"Knorr, Dissert., Marburg, 1895; Behring und Kitashina, Berl. klin, Woch., 1901 . 
tion, but not yet separated from the cells that had produced them; the cells thereby becoming more vulnerable to the poison. In the same eategory belongs the observation of Kretz, who noticed that normal guinea-pigs did not show any reaction after injections of innocuous toxin-antitoxin mixtures, but that marked symptoms of illness often followed such injections when made into immunized guinea-pigs. Other phenomena which are now regarded, a posteriori, as probably depending upon the principles involved in anaphylaxis, are the tuberculin and mallein reactions, fully described in another place, and the adverse effects often following the injections of antitoxins in human beings, conditions spoken of under the heading of "serum sickness." The last-named condition has been made the subject of an exhaustive study by v. Pirquet and Schick. ${ }^{5}$

That the injection of diphtheria antitoxin in human beings is often followed, after an incubation time of from three to ten days, by exanthematous eruptions, urticaria, swelling of the lymph glands, and often albuminuria and mild pulmonary inflammations, has been noticed by many clinicians, who have made extensive therapeutic use of antitoxin. It was recognized early that such symptoms were entirely independent of the antitoxic nature of the serum, but aepended upon other constituents or properties peculiar to the antitoxic serum. Moreover, symptoms of this description were by no means regular in patients injected for the first time, but seemed to depend upon an individual predisposition, or idiosyncrasy. v. Pirquet and Schick, however, noticed that in those injected a second time, after intervals of weeks or months, the consequent evil effects were rapid in development, severe, and occurred with greater regularity.

The fundamental observations from which our present knowledge of anaphylaxis takes its origin are those made in 1898 by Hericourt and Richet, ${ }^{6}$ who observed that repeated injections of eel serum into dogs gave rise to an increased susceptibility toward this substance instead of immunizing the dogs against it. Following up the lines of thought suggested by this phenomenon, Portier and Richet ${ }^{7}$ later made an interesting observation while working with actino-congestin-a toxic substance which they extracted from the tentacles of Actinia. This substance in doses of 0.042 gram per kilogram produced vomiting, diarrhea, collapse, and death in dogs. If doses considerably smaller than this were given in quantities sufficient to cause only temporary illness, and several days allowed to elapse, a second injection of a quantity less than one-quarter or one-fifth of the ordinary lethal dose would cause rapid and severe symptoms and often death. Similar

'Pirquet and Schick, 'DDie Serum Krankheit,' monograph, Leipzig and Wien, 1905.

- Hericourt and hichet, Compt. rend. de la soc. de biol., 53, 1898.

${ }^{7}$ Portier and Richet, Compt. rend. de la soc. de biol., 1902; Richet, Ann. de 1 'inst. Pasteur, 1907 and 1908. 
observations were made soon after this by Richet with mytilo-congestin, a toxic substance isolated from mussels. In these experiments there remained little doubt as to the fact that the first injection had given rise to a wellmarked increased susceptibility of the dogs-for the poison used.

It was Richet who first applied to this,phenomenon the term "anaphylaxis" (ảvá against, $\phi v \dot{\lambda} \alpha \xi_{\iota s}$ protection), to distinguish it from immunization or prophylaxis.

Soon after Richet's earlier experiments, and simultaneously with his later work, Arthus $^{8}$ made an observation which plainly confirmed Richet's observations, though in a somewhat different field. The observation of Arthus is universally spoken of as the "phenomenon of Arthus."

He noticed that the injection of rabbits with horse serum (a substance in itself withont toxic properties for normal rabbits) rendered the rabbits delicately susceptible to a second injection made after an interval of six or seven days. The second injection-even of small doses-regularly prcduced severe symptoms and often death in these animals.

An observation very similar to that of Arthus was made by Theobald Smith $^{9}$ in 1904. Smith observed that guinea-pigs injected with diphtheria toxin-antitoxin mixtures in the course of antitoxin standardization, would be killed if after a short interval they were given a subcutaneous injection of normal horse serum.

The fundamental facts of hypersusceptibility had thus been observed, and Otto, ${ }^{10}$ working directly upon the basis of Smith's observation, earried on an elaborate inquiry into the phenomenon. Almost simultaneously with Otto's publication there appeared a thorough study of the condition by Rosenau and Andersion. ${ }^{11}$

The researches of Otto, and Rosenau and Anderson, besides confirming the observations of previous workers, brought out a large number of new facts. They showed conclusively that the action of the horse serum had no relationship to its toxin or to its antitoxin constituents, that the "sensitization" of the guinea-pigs by the first injection became most marked after a definite incubation time of about ten days. Sensitization was accomplished by extremely small doses (one one-millionth in one ease, usual doses $1 / 250$ to 1 c.e.). Rosenau and Anderson, furthermore, excluded hemolysin or precipitin action as explanations of the phenomena, and proved that hypersusceptibility was transmissible from mother to offspring, and that it was specific - animals sensitized with horse serum not being sensitive to subsequent

${ }^{8}$ Arthus, Compt. rend. de la soc. de biol., 55, 1903.

'Th. Smith, Jour. Med. Res., 1904.

1" Otto, "'Leuthold Geilenkschrift,' 1905.

${ }^{11}$ Roscnau and Anderson, Hyg. Lab. U. S. Pub. Healt'ı and Marine Hosp. Serv. Bull., 29, 36, 1906, 1907. 
injections of other proteins. These authors, Vaughan ${ }^{12}$ and Wheeler, Nicolle, ${ }^{13}$ and others, furthermore, showed that the reaction was by no means limited to animal sera, but was elicited by proteins in general, pepton, egg albumin, milk, the extract of peas, and bacterial extracts.

These observations, together with those of many other workers which we must omit for the sake of conciseness, were the fundamental ones. In the time immediately following this first work, many theories of anaphylaxis were advanced and many faulty ideas conceived, justifiable in the light of the knowledge available at that time, but no longer tenable as more precise analyses followed. Such, in our opinion, are the earlier ideas of Gay and Southard, ${ }^{14}$ and that of Besredka, ${ }^{15}$ both of which depended chiefly upon the premise that the substance which sensitized in the first injection was not the same as that which incited the harmful effect at the second or subsequent injections. Into the same category at the present time belong the earlier views of Wolf-Eisner, ${ }^{16}$ and in a less definite way, the theories of Vaughan and Wheeler. ${ }^{17}$ The latter, however, have had an important influence upon subsequent developments which will be referred to below. Von Pirquet and Schick, ${ }^{18}$ from the beginning, maintained the analogy of the anaphylactic phenomena to other immune reactions, and believed that the reaction was dependent essentially upon an antigen-antibody union; and similar views were held by Rosenau and Anderson, ${ }^{19}$ from the beginning. In order to make the subject clear, however, without unnecessarily lengthening its discussion, we must abandon the historic method of treatment, and define the various elements that enter into the reaction more systematically.

Wells ${ }^{20}$ has, in our opinion, most concisely laid down the criteria which must be met in the light of our present knowledge, in order that a condition may be regarded as one of true anaphylaxis. They are,

12 Vaughan, Assn. Am. Phys., May, 1907.

${ }^{13}$ Nicolle, Ann. de 1'Inst. Pasteur, 2, 1903.

${ }^{14}$ Gay and Southard, Jour. Med. Res., May, 1907.

${ }^{15}$ Besredka and Steinhardt, Ann. de l'Inst. Past., 1907.

${ }^{16}$ Wolf-Eisner, Berl. klin. Woch., 1904.

${ }^{17}$ Vaughan and Wheeler, Jour. Inf. Dis., 4, 1907.

${ }^{18}$ Von Pirquet and Schick, Die Serum Krankheit, Vienna, 1905.

${ }^{10}$ Rosenau and Anderson, Hyg. Lab. U. S. Pub. Health and Marine Hosp. Serv. Bull., 29, 36, 1906 and 1907.

${ }^{20}$ Wells, Physiological Reviews, 1, No. 1, January, 1921. 
with a few commentaries and slight modifications, as follows: "The observed toxicity of the injected material must depend upon sensitization of the animal, that is, the substance must not produce similar symptoms in the non-sensitized animal" (of the same species). "It should be possible to demonstrate passive sensitization with the serum of a sensitized animal." This we would modify somewhat since, of course, in the early stages of developing hypersusceptibility, an animal, though sensitive, may have but slight or even no demonstrable antibodies in his serum. It would perhaps be more accurate to say that it must be possible to produce passive sensitization to an antigen by the administration of serum which contains antibodies to this antigen. If dealing with guinea pigs, it should be possible by the Dale Method, to demonstrate typical reactions as described below with the uterus of the sensitized guinea pig. After recovery from anaphylactic shock, a condition of desensitization should be apparent if quantitative conditions are taken into account. This is not all that Wells says about it, and we have somewhat modified it for our own purposes, but, in general, these criteria are the chief ones which must be met, and they all boil down to the statement that, in order to be considered true anaphylaxis, it must be shown that the mechanism of whatever reaction that may occur is one that is fundamentally based upon the meeting of an antigen with its homologous antibody. Further limitations of this, as to the site and manner of such meeting will be described below.

The Anaphyiactic Antigen.-We may save much discussion for the purposes of this particular book by saying that substances with which true anaphylaxis can be produced are all, as far as we know, protein in nature. No conclusive proof has ever been brought that lypoids or carbohydrates can act as antigens, and work with proteinsplit products has not been sufficiently satisfactory. Racemized proteins have been shown by Ten Broeck ${ }^{21}$ not to exert antigenic action for anaphylaxis. Wells, himself, states that he has always obtained negative results with protein cleavage products, but says that Fink $^{22}$ in his own laboratory has occasionally obtained slight anaphylactic reactions with proteose fractions obtained from coagulated egg-white by hydrolysis with steam under pressure, for those parts of the proteose solution which were precipitated by

${ }^{21}$ Ten Broeck, Jour. Biol. Chem., 17, 1914, 369.

${ }^{22}$ Fink, Jour. Inf. Dis., 25, 1919, 97. 
complete and three-fourths saturation with ammonium sulphate. It is interesting to note that these two fractions also incited antibodies, by which precipitin and complement fixations could be obtained, a matter which, in this case, is of considerable importance. For further discussion of other claims of non-protein anaphylactic antigens, we refer the reader to the article by Coca and the one by Wells. We may summarize here by saying that, as far as we know at the present time, anaphylactic antigens differ in no way from other antigens, and that no substance at the present time has been proven to be an anaphylactic antigen (in the sense in which we define the term above), with which antibody formation in animals has failed. In other words, any substance that can incite the formation of true antibodies may also be an anaphylactic antigen.

The Methods of Sensitization.-Experimental sensitization may be active or passive, and differs to some extent according to the species of animal under observation. The early observations were chiefly made on guinea pigs.' Guinea pigs can be actively sensitized by a single injection of various amount. When dealing with animal sera such as horse serum quantities of anywhere from 0.1 to 1 e.c. are most suitable. Minute amounts, however, will suffice, and Rosenau and Anderson ${ }^{23}$ succeeded in one case in sensitizing with one one-millionth of c.c. of horse serum. If so sensitized, the animals become hypersusceptible at varying periods, hardly ever in less than six days, the ideal time for reinjection ranging between two and three weeks, somewhat dependent upon the amount given. Various statements have been made as to the relationship of the incubation time and the initial dose given to guinea pigs. The ideal time for injection is that at which the maximum amount of antibody has been formed on the cells with the minimum amounts of circulating antigen and antibody in the blood. The statement of Coca is probably right, that, in general, the small amounts injected into guinea pigs require a relatively longer incubation period, but extremely large amounts (5-10 c.c.) may have a similar effect. The method of administration, to some extent, governs the incubation period in the same way that it governs the speed of antibody formation. However, the administration of the antigen to guinea pigs and other animals may be carried out in any way, except by feeding, and even feeding may result in a certain amount of hypersusceptibility

${ }^{23}$ Rosenau and Anderson, loc. cit. 
under unusual or pathological conditions (abnormal permeability of the intestinal mucosa).

When sensitizing guinea pigs with bacterial proteins or pollen and some other vegetable proteins, it is necessary to inject anywhere from six to ten times on consecutive days, and testing about three weeks after the last injection.

In dogs active sensitization is hard to obtain by one injection. Single doses of 2 to 3 c.c. of normal horse serum are usually followed by sensitization in three weeks, at least such results seem to have been obtained with some regularity by Simonds ${ }^{24}$ and others. Such sensitization, however, is not as acute and severe as that generally observed in guinea pigs under similar conditions. Weil, ${ }^{25}$ however, obtained acute shock in dogs by giving two sensitizing injections within a few days, and testing with large quantities of serum after two and three weeks.

Rabbits are difficult to sensitize with a single dose under any circumstances, but may easily be sensitized by repeated injection.

The lower monkeys are extremely difficult to sensitize under any circumstances. ${ }^{26}$

Our knowledge of sensitization in man is of course based entirely upon clinical observation. And it is held, especially by one investigator of anaphylaxis, that man cannot be sensitized. This, however, does not seem to us to be tenable, and anaphylaxis in man is not an uncommon observation as manifested by serum sickness, immediate skin reaction and accidents observed especially among asthmatics treated with foreign protein for one or another clinical reason. Fatal acute shock in man, however, is fortunately rare.

Passive Sensitization.-It is this phenomenon of passive sensitization which has thrown the most important light upon the process. It was first demonstrated by Nicolle, ${ }^{27}$ by Otto,${ }^{28}$ and by Gay and Southard, ${ }^{29}$ all of whom showed that the hypersusceptible state could be passively transferred to normal animals by injecting them with the serum of anaphylactic animals. In such experiments the scrum of the anaphylactic animal is first injected in quantities of

${ }^{24}$ Simonds, Jour. Infee. Dis., 19, 1916.

${ }^{25}$ Weil, Jour. Immun., 2, 1917, 429.

${ }_{26}$ Zinsser, Proe. Soe. Exper. Biol. and Med., 18, 1920, 57.

${ }^{27}$ Nicolle, Ann. de 1'Inst. Past., 2, 1903.

${ }^{28}$ Otto, Munch. med. Woch., 1907.

${ }^{29}$ Gay and Southard, Jour. Med. Res., May, 1907. 
0.5 c.c. or preferably more, and twenty-four hours later an injection of the specific antigen-that is, the protein used for sensitizationis given. The animals so treated show typical symptoms of hypersusceptibility and often die.

Simultaneous inoculation of the two substances, either mixed or injected separately, does not produce the same effect. A fact, observed by Otto, is that the serum of guinea-pigs who have been given the sensitizing or first injection will confer passive anaphylaxis on the eighth or tenth day after injection, before the animals themselves show evidenees of being actively hypersensitized. It is also true that occasionally the serum of antianaphylactie animals will possess the power of conferring passive anaphylaxis.

It is by means of the passive method of sensitization that the relations between anaphylaxis and antibodies have been most successfully studied. Doerr and Russ ${ }^{30}$ showed that the power of a serum to convey anaphylaxis passively depended directly upon its contents of specific antibody. It was then' shown by Nicolle, ${ }^{31}$ Otto, ${ }^{32}$ and others, that a sharp reaction ean be produced by this method only when a distinct interval not less than four to six hours, was allowed to lapse between the injection of the antibodies and the injection of the antigen. This may be taken as an axiom for all eases in which the antigen is an unformed protein in solution.

It was also shown by Weil $^{33}$ that passive sensitization could be conferred by the injeetion of precipitates formed in the test tube between an antiserum and its antigen, a thing which we ean now well understand in view of the knowledge we have of the dissociation of antibody from such precipitates.

Anaphylaxis may be transmitted passively by inheritance. Thus the young of anaphylactic guinea-pigs show hypersusceptibility, irrespective of whether the mother became hypersusceptible before or after the beginning of pregnancy. Such anaphylaxis has no reference to the condition of the father, and is not transmitted by the milk.

The nature of these anaphylactie antibodies has aroused mueh discussion. By many observers they are regarded as speeial

${ }^{30}$ Doerr and Russ, Ztschr. f. Immunitätsforsch., 1909, iii.

${ }^{31}$ Nicolle, Bull. de 1'Inst. Past., 1907, v.

${ }^{32}$ Otto, Das Theobald Smithsche Phaenomenon, ete., von Leuthold Gedenkschrift, 1905, i.

33 Weil, Jour. of Immunol., 1, 1916, 19. 
anaphylactic antibodies, separate from precipitins, opsonins, etc., etc.; Friedberger ${ }^{34}$ himself, from the beginning, identified them with precipitins. The direct quantitative relationship between precipitating antibodies and the power to convey passive sensitization, described by Doerr and Russ ${ }^{35}$ would point in the same direetion as would the above mentioned experiments of Weil. Sinee we have variously expressed in the preceding pages our own opinion that all antibodies developed against a single antigen are one and the same substance, we have no hesitation in stating that we think that the so-called sensitizing antibody is identieal with the sensitizing antibody which is formed to the antigen, rendering it amenable to agglutination or precipitation or complement-fixation.

Where Does the Reaction Occur.-As we have seen, when the antigen and antibody are injected simultaneously or within a very short period of one another, no anaphylactic symptoms occur. The study of this interval has gradually led to the recognition that the anaphylactic reaction, whatever it may be, takes place upon the body cells and that the interval in passive sensitization is necessitated by the time required for the anchoring of the antibodies to the cells of the tissues. Experiments by Pearce and Eisenbrey ${ }^{36}$ (1910) showed definitely that a hypersusceptible dog remained sensitized even when his entire blood volume was substituted with that of a normal dog. The prineiple has been made especially clear by the introduction of direct methods of observation of the smooth muscle cells of animals, by Schultz ${ }^{37}$ and by Dale, ${ }^{38}$ a method which has been particularly developed by Weil. ${ }^{39}$ It seems fairly clear from this work and a volume of other researches which cannot be reviewed here, that acute protein anaphylaxis as we see it in guineapigs and other laboratory animals is due to the direct reaction between antigen and a specific antibody when this reaction takes place upon the body eells and not in the blood stream. Just how much influence the reaction within the blood stream can exert or

${ }^{34}$ Friedberger, with Hartoch, Zeit. f. Immunit., 3, 1909.

${ }^{35}$ Doerr and Russ, Zeit. f. Immunit., 3, 1909.

${ }^{36}$ Pearce and Eisenbrey, Congr. Am. Phys. and Surg., 1910, viii.

${ }^{37}$ Schultz, Jour. Pharmacol. and Exper. Therap., 1910, i.

${ }^{28}$ Dale, Jour. Pharmacol. and Exper. Therap., 1913, iv.

${ }^{39}$ Weil, Jour. Med. Research, 27, 1913; 30, 1914; Proc. Soc. Exper. Biol. and Med., 1914, xi, 86. 
whether it takes any important part in the phenomena is at present a matter of considerable doubt.

We can feel safe, therefore, in stating very definitely that the site of the anaphylactic reaction, that is, the place at which the union between antigen and antibody occurs in the production of the various symptoms of anaphylactic injury is upon the cells. That, in other words, the important reaction which determines the train of symptoms which we call anaphylaxis occurs when the antigen goes into relationship with antibodies which are still in some way united to tissue cells, or are, in the jargon of immunology, "sessile" upon the cells.

Whether or not any injury may occur when antigen meets antibody in the circulation is still an open question. There are experiments on record by Friedemann ${ }^{40}$ in which he obtained reactions in rabbits by the simultaneous intravenous injection of antigen and antibody, and similar occasional occurrences have been observed by Brion, Scott and ourselves. But these reactions are neither regular in occurrence, nor are they ever very severe. As a matter of fact, as we have shown, the union of antigen and antibody in the circulation is inhibited probably by colloidal protection, and this may be regarded as being very likely a protective mechanism. As a matter of fact, Weil and others have shown that a sufficient amount of antibody in the circulation may even protect the cells to some extent against anaphylactic shock, and it is interesting to note in this connection that very large doses of protective antiserum are necessary to bring about this result, a circumstance which is again easily explained by the inhibition of union between circulating antigen and antibody.

Concerning the possibility of the formation of poisonous substances in the circulation, produced by the union of antigen-antibody, a question which is involved in Friedberger's theory of anaphylaxis, we will have more to say in a subsequent paragraph.

Symptoms of ANaphylaxis.-Anaphylaxis differs in its symptomatology and pathology according to the species of animal in which shock is produced. There are certain fundamental systemic reactions which are common to all species, but in each species that has been observed, there are particular localization of the immediate and severe changes which lead to acute death. As general symptoms

${ }^{40}$ Friedemann, Zeit. f. Immunit., 2, 1909. 
we may enumerate drop in blood pressure, fall of temperature, diminution of leueocytes, increased flow of chyle, and certain metabolie disturbanees, the identity of which in different animal species have not been worked out.

In guinea pigs, as first demonstrated by Auer and Lewis, the typical lung inflation which leads to respiratory death is due to spasms of the muscles of the bronchioles.

In rabbits, acute death is not respiratory, but is a circulatory one, and has been shown by Coca ${ }^{41}$ to be due to spasms of the muscular coats of the arterioles of the pulmonary circulation, the rabbit's lung during shock development remarkably increased pressure against the passage of perfusion fluid.

In dogs, aeute anaphylactic symptoms have been localized in the liver by Manwaring ${ }^{42}$ and others. This peculiar physiologieal difference in various animals in reaction to the same general mechanism of injury has been difficult to understand, but recent observations of Coea, Simonds, Huber and Koessler ${ }^{43}$ and others have been eorrelated by Wells into what seems to us a very rational and likely explanation. Wells calls attention to the fact that acute death in guinea pigs is due to spasm of the bronchial museles, and that anatomically the guinea pig has a very high development of musculature in the bronchii, the smaller bronchioles being "practically nothing but muscular tubes." Similarly, Coea's findings in relation to the pulmonary circulation of rabbits coineides with the histological demonstration that the pulmonary arteries of the rabbit show a marked muscular development. Simonds has shown that the hepatie veins of dogs differ from those of all other animals in having a highly developed musculature, and it would seem, as Wells points out, as though the localization of acute changes in different organs in the various animals were dependent upon fortuitous differences in the anatomical distribution of the smooth muscle. He also points out, as further evidence, that fatal reactions in man have occurred only or most frequently in persons suffering from chronic pulmonary conditions, chiefly asthma; and Huber and Koessler have shown that asthmatic people develop a hypertrophy of the bronchial musculature which in its final histology is elosely analogous to that of guinea pigs.

${ }^{41}$ Coca, Jour. Immunol., 4, 1919, 219.

42 Manwaring, Jour. of Immunol., 2, 1917, 517.

${ }^{43} \mathrm{Huber}$ and Koessler, Arch. Int. Med., 1921. 
This explanation of Wells seems to us eminently logical. We would add to it only the following consideration. Acute death may well be caused directly by the acute spasm of smooth muscle tissue, and the acute pathological manifestations may be dependent upon the distribution of such muscle. This does not, however, exclude the likelihood that severe anaphylactic injury may be caused in other cells in the body as well, but these, not being able to react by acute contraction, or by any other pathological alteration that can cause acute and sudden death, may still be injured severely without there being an immediately noticeable effect.

Anti-ANAPHYlaxis.-When sensitized animals recover from anaphylactic shock, they do not react to a subsequent injection of the same substance made within a reasonable interval.

This desensitization or "antianaphylaxis" as Besredka and Steinhardt have called it, appears immediately after recovery from the second injection. Antianaphylaxis may also be produced if animals which have received the first or sensitizing dose are injected with comparatively large quantities of the same substance during the preanaphylactic period-or, as it is sometimes spoken of, during the anaphylactic incubation time. This injection should not be done too soon after the first dose, but rather toward the middle or end of the preanaphylactic period.

If given within one or two days after the sensitizing injection, anaphylaxis will develop, nevertheless. The desensitized condition is a purely transitory state. Besredka and Steinhardt believe that it lasts a long time, while Otto found guinea-pigs immunized in the above manner to lose their antianaphylaxis within three weeks.

It is not at all necessary to actually shock an animal to desensitize it. The doses may be given gradually, either in small fractions or slowly by means of high dilution, as by the method of Friedberger, and gradual desensitization thereby accomplished without noticeable harm. This is of great practical importance.

Desensitization in the ordinary sense probably means a gradual saturation of the sessile antibodies with antigen. It can be demonstrated not only in the living animal but upon the sensitive uterus in the Dale apparatus.

Another form of partial protection against anaphylaxis, by the injection of large amounts of specific antiserum has been mentioned above. The mechanism of this is obvious.

Desensitization by injection into the rectum or by feeding has 
been accomplished but since the absorption of unchanged antigen by these routes is ordinarily slight, little hope can be expected in this direction for practical purposes.

There are certain forms of protection against anaphylactic shock produced by the injection of foreign sera, and other proteins and processes non-specific as far as the particular anaphylactic mechanism is concerned, but there is too little positive knowledge about these to permit us to diseuss them here.

ANAPhYlatoxin Theories, ETC.-Vaughan and Wheeler ${ }^{44}$ early suggested that anaphylaxis might be a poisoning produced as follows. Antibodies are formed by the first injection, which on subsequent reaction with the antigen administered in the second injection, lead to poisonous protein-split products. A similar idea was advanced by Wolf-Eisner. ${ }^{45}$ Proceeding from this general concept, Friedberger, whose extensive experimental work may be found in many articles in the Zeitschrift f. Immunitätsforschung, elaborated a theory of anaphylaxis which may be summarized in the following way: When the antigen and antibody meet in the circulation, the union of the two renders the antigen amenable to complement action, and the action of the alexin or complement upon this complex splits off from it a poison which he ealls "anaphylatoxin." He succeeded in producing poisonous substances in vitro by treating specific precipitates as well as sensitized and unsensitized bacteria with alexin. Injection of these substances into guinea pigs caused acute death, analogous in symptoms to anaphylaxis. Friedberger and Hartoch ${ }^{46}$ showed that there was a diminution of alexin in the serum of animals suffering from acute shock in the course both of active and of passively transmitted anaphylaxis. He showed that the intravenous injection of substances which inhibited complement action in vitro, such as, for instance concentrated salt solution, would diminish and sometimes prevent shock in sensitized animals, a phenomenon, however, which the writer with Lieb and Dwyer ${ }^{47}$ showed to be dne to diminution of the irritability of smooth musele eaused by hypertonic salt. It was subsequently shown, however, that similar poisons could be produced from boiled as well as from normal bacteria, that they could be produced by the treatment of fresh guinea pig serum

44 Vaughan and Wheeler, Jour. Infec. Dis., 4, 1917.

${ }^{45}$ Wolf-Eisner, Berl. klin. Woch., 1904.

${ }^{40}$ Friedberger and Hartoch, Zeit. f. Immunität., 1909, 3.

"Zinsser, Lieb and Dwyer, Jour. Exper. Biol. and Med., 12, No. 8, 1915. 
with kaolin, barium sulphate. The literature of this subject is extensive. The most important contributions recent have been made by Bordet, Moldovan and Doerr, ${ }^{48}$ and Novy and DeKruiff. ${ }^{49}$ All of these investigations tend to show that guinea pig serum can acquire strongly toxic properties when treated with any form of substance in fine suspension, whether this be living or dead bacteria, or protozoa, or indifferent matcrials such as agar, kaolin, ete. Furthermore, the direct injection of very dilute solutions of agar, ete., into guinea pigs and rabbits may cause fatal symptoms closely resembling anaphylaxis. Moreover, it has been shown by a number of worker's that blood taken from guinea pigs and rabbits, either defibrinated or centrifuged and reinjected before the clotting was complete, (oould exert similar toxic action. We believe that these anaphylatoxin phenomena are of very great importance, that they represent a phenomenon dependent upon very delicate adjustment of the colloidal conditions prevailing in the eireulating blood, and demonstrate the possible dangers accruing from the disturbance of such conditions, a matter which has been particularly emphasized by Jobling and Petersen. ${ }^{50}$ But, we do not believe that the anaphylatoxin phenomena bear a direct relationship to the processes that we may classify under true anaphylaxis. The demonstration of the cellular localization of the mechanism which causes true anaphylactic shock has, we believe, amply demonstrated this.

SERum Sickness.-The injection of foreign proteins into human beings, especially in the form of horse serum as in antitoxin treatment, causes a train of symptoms which are classified together as serum sickness. We will not go into the history of serum sickness, however interesting, and refer the reader to larger works on the subject, the summaries of anaphylaxis mentioned above, as well as the book of Von Pirquet and Schick, "Die Serum Krankheit," Vienna, 1905. A peculiarity of this condition is that it may follow the first injection of horse serum or any other foreign protein, as well as subsequent ones.

After first injection, the incubation period may last as long as twelve days, or longer, although it may be considerably shorter than this. Coca states that in 24 to 48 per eent of all eases the incubation

${ }^{48}$ Moldovan and Doerr, Zeit. f. Immunität., 7, 1910.

40 Novy and DeKruiff, Jour. A. M. A., 68, 1917, 1524.

so Jobling and Petersen, Jour. Exper. Med., 19, 1914, No. 5. 
period after first injection is less than eight days, while in about 14 per cent it is longer than twelve days.

The symptoms of serum sickness usually consist of an eruption at the site of injection, which ordinarily comes on quite early, some time before any general eruption is noticed. It often takes an urticarial form, and becomes general after several days. There is usually some fever, occasionally albuminuria, and there may be joint manifestations. The joint manifestations are often of a peculiar nature, with slight tenderness, but considerable stiffness, and very little, if any, objective symptoms of the joints, and we remember distinctly a case in which it was difficult to tell whether or not the patient, who had received tetanus antitoxin ten days before, was developing tetanus or not. The case turned out to be one of serum sickness. Serum sickness may also be accompanied by leucopaenia, and, according to Weil, ${ }^{51}$ by drops in blood pressure and decreased coagulability of the blood. The latter manifestations, as Wells points out, bring it still closer to true anaphylaxis.

When a human being is being treated for the second time with horse serum at intervals longer than two or three weeks, the resemblance to true anaphylaxis is still greater, and as Doerr points out, the procedure is more dangerous and will vary in its manifestations, according to the length of time elapsing between the two injections. If the injections are not very much more than a month apart, there may be, according to Von Pirquet and Schick, an immediate reaction, which takes the character of severe serum disease. At the point of injection there may be swelling and edema, within twenty-four hours, with general symptoms such as those described above but rather more severe, within one or two days. If the injections are months and years apart, the onset, while usually more rapid than at the first injection, is still likely to occur more rapidly than when the antigen is given the first time.

Van Pirquet and Schick, from the beginning, believed that serum sickness was due to the reaction of antigen which had not yet disappearcd from the circulation of the patient at a time when antibodies were already being actively formed.

Opinions as to whether serum sickness is to be regarded as true anaphylaxis or not, seem to differ at the present time. Coca particularly seems to hesitate about incorporating these phenomena into

"Weil, Jour. Immunol., 2, 1917, 399. 
those of true anaphylaxis. We cannot go into the controversial literature in this place, but may set down our own opinion that we think that the time interval between observation of symptoms and injection, on first administration, the speeding up of symptoms in cases of second and third injections, the nature of the symptoms, and the relationship between serum sickness and antibody formation in the patient, as pointed out by C. W. Wells, ${ }^{52}$ as well as more recently by MacKensie and Longeope, ${ }^{53}$ together with the phenomena of desensitization of patients, leave little room for doubt that this peculiar condition is fundamentally of an anaphylactic nature.

It is not so easy to include in true anaphylaxis the apparently inherited sensitiveness to foreign proteins most frequently observed as food idiosyncrasies. Whether or not these belong into the category of anaphylaxis, the future must reveal.

Skin Reactions.-There are two kinds of skin reaction, one which can be obtained, for instance, in horse serum sensitive people by the injection of minute quantities, 0.1 to 0.01 c.c. of horse serum intracutaneously injected. In these eases, within a few minutes to onehalf hour, a growing urticarial wheal begins to appear which fades again within an hour or longer. This reaction has been variously used to determine whether or not patients possessed a high degree of sensitiveness just before the administration of therapeutic sera. We have recently experimentally studied the relationship of such immediate skin reactions with generalized anaphylaxis in guinea pigs, and have found that the two phenomena correspond with considerable accuracy, namely, that guinea pigs give definite immediate skin reactions during the periods at which they are highly sensitive to intravenous injections, and that recovery from severe anaphylactic shock desensitized them not only to general anaphylaxis but to the skin reaction as well.

There, is another form of skin reaction typified by the tubereulin reaction and the typhoidin reaction in which very little manifest change occurs during the first two hours, but in which after twelve to twenty-four hours, definite inflammatory swellings occur at the point of injection with the occasional development of a little central neucrosis and hemorrhage, and true cell injury. Such reactions fade only after three or four days or longer, and are similar in

${ }^{62}$ Wells, $C$. W., Jour. Infec. Dis., 16, 1915.

${ }^{53}$ MacKensie and Longcope, Med. Sec. N. Y. Academy Med. April, 1921. 
many ways to the toxin skin reactions as observed in the Schick test with diphtheria toxin. The relationships of these latter reactions to anaphylaxis has been much questioned and with justice. A recent analysis by ourselves in the case of the tuberculin reaction has shown that these reactions are not anaphylactic in the ordinary sense, but are brought about by substances probably proteose in nature which among other things, differ from the true anaphylactic antigens in being more permeable than these, and penetrating into cells. We refer the reader to our article in the J. of Exp. Med., unpublished, probably 1921.

Methods of Testing and Desensitization in Individuals about to be InJected with Antitoxic or Antibacterial Sera.-A considerable degree of practical importance attaches to the anaphylactic phenomena in connection with the administration of sera. When small doses of tetanus or diphtheria antitoxin are to be administered for the first time, it is generally unnecessary to precede this with a skin test. Although we should always advise that this is done in asthmaties or in people who have suffered:from chronic coughs or the prolonged pulmonary inflammations that are apt to oceur in children following measles, influenza or whooping cough. This is necessary for the reasons stated above and pointed out by Huber and Koessler, which demonstrated the hypertrophy of smooth muscles in the bronchioles of such people.

When intravenous serum injections are to be made, as in the treatmient of pneumonia and meningitis, it is best to precede the injection with a skin test. Such tests have been done more frequently than anywhere else, we believe, at the Rockefeller Hospital, where 0.02 e.e. of a 1:10 dilution of horse serum is injected intraeutaneously with a similar injeteion of sterile salt solution as control. In unsensitive people the wheal of the injection disappears rapidly. But in sensitive ones it will begin to increase after five or ten minutes, and within four hours may show a large red erythematous, urticaria-like area.

In such cases, careful desensitization should be practiced. The precautions to be taken are twofold. One consists of a preliminary attempt at desensitization, the other in slow administration by dilution of the serum during the injection of the therapeutic dose.

The desensitization is best aceomplished by the method of Besredka $\mathrm{a}^{54}$ which consists in the gradual injection of progressively

${ }^{64}$ Besredka, Bull. de l'Inst. Past., 6, 1908, 826. 
increasing doses of the antigen, in this case horse serum, over an interval of a number of hours. Cole ${ }^{55}$ states that it is safe to begin with a subeutaneous injection of 0.025 c.c. of serum, and at one-half hour intervals thereafter, giving further doses, doubling the amount. each time. Adverse symptoms should of course lead to still greater care and a lengthening of the interval. If by this careful method finally 1 e.e. has been given at a dose without adverse symptoms, 0.1 c.c. may be given intravenously, and continued every one-half hour with double the dose. Cole recommends continuing this careful procedure until about 25 c.c. total of the serum has been given. He then waits about four days, and gives the remainder of the dose.

It stands to reason that no absolute rules for such a procedure can be given and that after the general principle has been understood, the individual physician must be guided by close observation of the patient and familiarity with the symptoms to be expected.

The adverse symptoms to be expected are immediate respiratory distress, rapidity of the pulse, and there may be coughing.

In finally giving the serum, in such eases, in the larger quantities, it should be diluted with sterile salt solution by at least one-half, and may be so given by gravity that the first 10 e.c. should occupy about ten minutes.

Hay Fever, Urticaria, ETC.-The clinical condition spoken of as hay fever, asthmatic attacks ealled forth by apparent hypersusceptibility to certain kinds of dust, the emanation of animals, etc., etc., cannot at present be classified clearly with true anaphylaxis because of certain apparent differences from ordinary anaphylactic phenomena which eannot be ignored. Dunbar ${ }^{56}$ at first regarded the susceptibility to pollen or hay fever as due to a toxin. This, however, is not tenable on present experimental evidence. The toxin idea is not tenable because of the fact that normal insusceptible individuals do not react to many times the amounts to which the hay fever patients respond. Numerous studies upon the inheritability of the hay fever tendency seemed to show that a tendency to hypersensitiveness of this kind may be hereditary, an observation which in the train of a number of other investigators has recently been corroborated by Cooke and Vander Veer. ${ }^{57}$ They seem to believe

${ }^{55}$ Cole, Monograph of the Rock Inst., 1917, No. 7.

${ }^{5}$ Dunbar, cited from Doerr, Kolle and Wassermann Handbuch, Eilit. 2.

${ }^{57}$ Cooke and Vander Veer, J. of Immun. I, 1916, 201. 
"that the tendency is inherited as a dominant characteristic." The question of whether or not hay fever is a phase of protein sensitization, then, cannot be answered either affirmatively or negatively at the present time. It is accompanied by cutaneous sensitiveness to pollen extracts and, according to Cooke and Vander Veer, subcu-. taneous injections of such extracts into sensitive individuals may produce a general eruption. On the basis of its heredity, Cooke and Vander Veer and Coca ${ }^{58}$ remove the hay fever complex from the class of protein anaphylaxis. Coca claims in addition that pollen extracts are not antigenic in the ordinary sense. of the word, but in this he is mistaken, since Mrs. Parker in our laboratory recently proved that extracts made by us, as well as the ones used by Cooke and Vander Veer, could be used as anaphylactic antigens if the methods of sensitization and of test were sufficiently delicate.

Drug Idiosyncrasies.-A bnormal sensitiveness to many drugs belonging to almost all chemical classes of substances, inorganic and organic, has been noted for years by clinicians. Among them are morphin, strychnin, atropin, salicylates, halogens, and their compounds, salvarsan, etc. These substances are obviously not antigenic in the ordinary sense. The hypersusceptibility is generally specific at least for the chemical group, and it seems to be a fact that the reaction elicited in the individual does not represent exaggerated symptoms of the physiological effects of the drugs, but are, in a general way, alike, whatever the drug used. The symptoms usually come on rapidly, within a few hours or days, and consist in various kinds of skin rashes, and fever. In such cases, where salvarsan, iodin, ete., preparations have been used, there may be marked and rapidly developing local inflammatory effects at the point of inoculation. Drug idiosyncrasies cannot be transmitted passively, and, so far, no conclusively successful experiments on artificial hypersensitization of animals with these substances have been made. There is only one exception to this, namely the experiments of Swift ${ }^{59}$ with salvarsan in which active sensitization of guinea pigs with salvarsan-guinea pig serum mixtures resulted in apparently successful, though inconclusive, results.

There is no adequate explanation at the present time for drug idiosyncrasies. The most reasonable, suggestion which has been made, however, is the one that drugs enter into some form of com-

${ }^{58}$ Coca, Tice's Practice of Medicine, Vol. 2, 1920.

${ }^{59}$ Swift, J. Exp. Med. 24, 1916, 373. 
bination with the serum proteins of the host, which are then so altered in their antigenic properties that they can act as antibody inciting or, in other words, antigenic substances. Wher horse serum or other animal sera for instance are treated with iodin, an iodinprotein combination is formed, which represents an antigenic alteration of the original serum protein. Animals injected with such an iodized horse serum will produce antibodies which are, to some extent, specific for this iodin-protein combination. It is not impossible that this is the fundamental basis for drug allergy, but there are very strong arguments against this assumption, particularly the failure up to date to produce such conditions uniformly in animals by active or passive sensitization. It would seem to us futile at the present time to make either positive or negative statements in this respect.

Anaphylaxis in Infectious Disease.-There ean be little doubt about the fact that both general and localized hypersensitiveness play an important rôle in infectious disease. Whenever an infection becomes subacute or chronic, the body may become sensitized to the coagulable protein in the bacterial body. This we have ourselves shown with typhoid protein, and with tuberculous guinea pigs and tuberculo-protein; and, in the case of tuberculosis, our experiments done with the Dale method corroborated the previous experiments of Baldwin, ${ }^{60}$ Krause $^{61}$ and others who worked by the intravenous method. Thus, in all infectious diseases which last any length of time, we can count upon anaphylactic phenomena to participate in the general symptomatological and pathological picture. This subject is still very much a matter of experimental investigation, and a discussion of it would carry us too far afield in the present connection.

Delayed skin reactions, like the typhoidin, tuberculin, ete., reaction, are, we believe, phenomena of specific sypersensitiveness to substanees of a somewhat less complex molecular structure than the proteins, substances which do not produce antibodies in the usual sense; these substances are more diffusible than are the true proteins, can get inside the cells, and their reaction with the cells, then, is an intracellular one in which the intervention of sessile antibodies is not necessary. For a further analysis of this relationship and the experimental basis for the statements we make, we refer the reader to our unpublished article mentioned above. 


\section{SEGTION III}

\section{PATHOGENIC MICROORGANISMS}

\section{CHAPTER XX}

\section{AN INTRODUCTION TO THE STUDY OF INFECTIOUS DISEASES}

\section{THE RELATIONSHIP OF BACTERIOLOGY TO THE CLINIC AND TO PUBLIC HEALTH}

THE problem of infectious diseases is peculiar in that, more than any other branch of medicine, it calls for the intimate cooperation of the laboratory worker, the clinician and the sanitarian. It is a subject in many phases of which problems of engineering are important, in which food production plays a part, and to which the educational and sociological agencies of the community as a whole can contribute very materially. The eventual suppression of infectious diseases cannot be accomplished by any one of the agencies mentioned, or by all of them together without the cooperation of the public in general.

In many respects the study of infectious diseases is the most logical branch of medicine at the present time. In many of these conditions we are familiar with the causative agents, we know the manner in which they gain entrance to the body, where they lodge and multiply, where and how they form their secondary foci, what manner of poisons they produce, and what reactions they call forth in the living animal tissues. We can often isolate the bacteria from the bodies of the sick and the dead; we can recognize them when we isolate them in locations outside the body; we can study their biological activities, and their poisons both apart from the animal body and in animal experimentation. Moreover, both in animals and man we can study directly the reactions of the body to invasion, and the agencies by which the invaders are destroyed.

For these reasons, the first requirement for a thorough clinical 
comprehension of infectious discase as it occurs in man, is a fundamental biological knowledge of the bacteria themselves, their actions upon artificial media, the characteristics by which they ean be differentiated, the conditions under which they grow and produce poisons, and the reactions which they or their poisons elicit in animals. Of course there are many points in such a chain of reasoning which investigation has not yet cleared up, and there is, especially, much uneertainty and half-knowledge in regard to some of the most important phases of the chemical and immunological reactions which take place between the invaders and the animal body. Moreover, it is quite likely that many of our theories concerning the fundamental principles which govern such reactions are defective. But without, at least, a knowledge of the biological facts available, the physician who is confronted by a human infection is working very largely in the dark. It is the task of the specialized laboratory worker to prepare this material and submit it to the clinician so that we may use it in the premises of his reasoning.

Considered in this way, an infection in an animal or a human being resolves itself into a balance between the forees of infection, on the one hand, and the injuries and resisting mechanisms of the infected subject, on the other.

We may consider the entrance of a foreign living being into the tissues of a higher animal or plant as a process in which a struggle for existence is initiated. The invading microorganism must take its nourishment from the invaded host, abstracting thereby materials needed by the host, and, in the course of its multiplication and digestive processes, it not only injures the host mechanically by local accumulation, but also by the remote action of the substances which it produces in the course of its metabolism, many of which are toxic. The host, if not overwhelmed, responds by reactions which express themselves both in local morphological changes in places where direct contact with the bacteria is established, and by remote systemic reactions incited by absorption not only of the toxic derivatives of the bacteria, but also to some extent of the products of the local struggle in which proteolytic destruction, necrosis, ete., are involved. The symptom complex, therefore, which we recognize as disease results in part from injury, but to a larger extent represents the manifestations of the defense reactions of the host. When a physician makes a diagnosis of a partieular infection by history and physical examination, he does so on the basis of his own observa- 
tions and of those of his clinieal predecessors, which have taught him that a certain kind of bacterial invader elicits characteristic reactions on the part of the host. In many cases, however, microorganisms of entirely different biological classes may elicit clinical pictures of great similarity, because of analogous methods of invasion, like degrees of virulence and similar selective distribution. Pneumonias caused by pncumococei and Friedländer bacilli may show very little clinical difference for these reasons, and septicemic invasions of the blood by a variety of microorganisms may not be clinically differentiable. On the other hand, one and the same species of microorganism may cause widely divergent clinical pictures under conditions of varying balance between the virulence of the invader and the resistance of the host. A streptococcus of low virulence in a vigorous subject may for instance cause only a localized abscess, whereas, if the virulence is enhanced and the subject very susceptible, the localized symptoms may be negligible and a septicemia with secondary localizations and fatal in outcome may result.

The accurate diagnosis of an infectious disease, therefore, depends first of all upon a clinical understanding of the reactions of the human body with the different microorganisms that can invade it, a knowledge of the manner in which the different forms can enter the body, how they progress and are distributed, where in the body they are apt to accumulate, how great a degree of fluctuation in virulence and toxicity can be expected from them, what poisons they produce and what the pharmacological action of these poisons is. While such information may often, and must frequently suffice to make the diagnosis, yet a knowledge of the manner in which blood cultures, urine cultures, stool cultures, throat cultures, etc., can be made, is necessary to affirm the diagnosis in relatively clear cases and to determine it in doubtful cases; and this implies not only a knowledge of the preceding facts, but also both the knowledge and the technical ability supplied by the trainea bacteriologist.

Moreover, the dependence of clinical understanding of infectious diseases upon bacteriological knowledge is not a bit less intimate than is that of preventive measures or sanitation.

For purposes of prevention, every infected individual or every carrier of a pathogenic organism may be regarded as the potential source for infection of other human beings. 
Some diseases will of necessity remain sporadic because the infection of a new individual ean be brought about only by unusually depressed resistance or by accidentally enhanced virulence on the part of the causative agent. And in some diseases transmission requires conditions of contact which are not an ordinary feature of intercourse between the members of communities. Diseases, thus, which under ordinary conditions of eivilized life may occur more or less frequently as sporadic scattered cases, may become epidemic only when life in army cantonments, in crowded and unsanitary city quarters, subject to poverty, filth and neglect, produces community susceptibility and facilitates transmission. Such, as we shall see, is the case with many respiratory infections, especially the pneumonias.

Other diseases, on the other hand, are characteristically epidemic because the ordinary virulence of the microorganisms is such that practically all normal human beings may be regarded as susceptible and because the most important avenues of invasion are open in the course of normal community association. Such diseases are plague, smallpox, cholera, influenza and, to a less extreme degree, the enteric fevers.

For this reason, the ultimate basis of sanitation in infectious diseases depends upon close observation of the possible sources of infection so that they may be circumscribed before broadeast dissemination has taken place; it involves the routine safeguarding of ordinary community life in such a way that the avenues of transmission for the various possible invaders may be controlled, and finally it necessitates attention to the maintenance of the resistance of the community as a whole, both by the hygiene of every day life, the prevention of undue lowering of resistance of large groups by economic or other hardships, or, as in smallpox and typhoid fever, by the artificial reenforeement of community resistance by methods of immunization.

The source of infection lies invariably in direct or indirect transmission of microorganisms from a human or animal source. Every case of infection represents the possible origin of many others, and necessitates surrounding the patient with all the safeguards appropriate to the type of infection from which he is suffering, based on knowledge of the manner in which the particular disease is transmitted. If the diseased individual were the only problem, the task would be relatively easy. As we shall see, however, in the 
subsequent chapters, a great many irfectious agents may live saprophytic lives in and upon the bodies of human beings, who themselves are not suffering from the disease. Such individuals are known as carriers, and the carrier problem has infused difficulties into sanitary procedure which, in some cases, it is almost impossible to combat. Thus, every community has in it a definite percentage of typhoid and paratyphoid carriers; many individuals carry meningococei, diphtheria bacilli and virulent pneumococei and streptococei; there are quite surely carriers of poliomyelitis and scarlet fever, and it is not impossible that the carrier state can exist for a number of other diseases in which we have not yet been able to prove the condition by actual experiment.

In addition to the case and the carrier, a source of great danger are unrecognized mild cases. Typhoid fever may take a very mild form, especially in vaccinated pcople; the atypical cases of poliomyelitis which occur in the course of every epidemic and may occur in interepidemic periods may not be recognized until secondary cases occur; and in many adults who possess a relatively high immunity, diphtheria infection of the throat may be so mild that no suspicion of the disease is aroused. It is in connection with such occurrenees that the diagnostic acumen of the practicing physician is especially important, and it is in the recognition of the atypical cases and in the early diagnosis of the ordinary cases, that the practitioner represents the first line of defense against epidemic outbreaks.

It is for this part of the protective campaign that we need reporting systems, so that early cases may be centrally collected and charted. Organizations for epidemiological survey to trace the early cases to their sources, and laboratory units to affirm the diagnosis, seareh out the possible carriers and trace the infection, if possible, to contaminated food or water. Here, too, are necessary arrangements for isolation and hospitalization which involve the bacteriological control by which may be determined when it is safe to release the patient for free association with his fellows.

The transmission of infectious disease may be either by direct contact from person to person, by indirect contact through materials that have passed from the sick or the carrier to the new victim by food and water and by conveyance through the agency of insects. These factors will be considered in detail in connection with every individual disease, and it is quite clear that, in order to properly 
safeguard a community, it is neeessary to know where in the body of the sick or the carrier the organisms are to be found; how they may leave the body; what the viability of the infectious agent is in nature; how long it can live under different conditions of environment in the interval between its leaving one body and entering the next, and by which channels it most easily infects. Also, the habits of insects that ean carry disease must be studied.

To interrupt the chain of transmission from source to victim, also, there must be a routine organization for the safeguarding of water, food and other agencies which in crowded communities are always in danger of contamination. And in special cases it may involve emergency measures of personal hygiene, engineering problems and insect destruction.

In thinking of infectious diseases from the point of view of sanitation it is well to carry them in one's mind in the tentative elassification based upon means of transmission, since for each partieular subdivision of this kind, preventive measures will fall into a definite common plan of procedure.

Thought of in this way, all the infectious diseases fall into four groups :

(1) The first of these consists of those transmitted by the respiratory channels. This means that the infectious virus leaves the case, convalescent or earrier, with the saliva or mucus of the upper respiratory passages and enters the new victim by the same channels. On this basis, the respiratory group would consist in the common cold, the pneumonias, influenza, scarlet fever, measles, smallpox, chickenpox, mumps, whooping cough, tuberculosis, diphtheria, meningitis, poliomyletis and a few others.

In dealing with such diseases, the task is to suppress conditions which would make it possible for a wholesale distribution of sputum, for close contact between individuals in sleeping quarters, the avoidance of crowding in homes, schools, institutions, etc., ventilation, dust prevention, and, in short, to diminish as much as possible the opportunities for close individual contact in elosed spaces. In this group of discases, prevention is most difficult because it is quite obvious that contacts need not be of long duration, and that close association in publie velicles, and in the ordinary course of business and social intereourse may suffice for transmission. In these diseases, particularly, when there is universal susceptibility, as in the case of influenza or smallpox, or some of the exanthemata in the 
case of children, epidemic spread is almost roavoidable if unsanitary, close association exists. Fortunately, in many of the diseases so transmitted, namely, pneumonia, meningitis, diphtheria and a number of others, the normal resistance. of the human being is relatively high and epidemic occurrence takes place only when unusual conditions prevail.

(2) The intestinal group consisting very largely of typhoid and the paratyphoid fevers, the dysenteries, cholera, the food poisonings, and the simple diarrheas. In this group sanitation, apart from isolation of the recognized case, focuses upon a constant vigilance in regard to the distribution of dejecta from human beings, for every infection signifies a sanitary defect in the transmission of the contents of the bowels of one human being to the mouth of another by any one of a variety of routes. Sanitation here requires proper sewage disposal, the care of privies and latrines, and careful control of water and food supplies, since naturally large epidemics can be most easily brought about in this group by the contamination of the daily diet of the community. In these diseases, wholesale infection with water and milk is constantly diminishing, as we are improving in our sanitary organizations. But contact epidemies from person to person becoming relatively more prominent. Recent studies are tending, for instance, to show more and more the increasing importance of contact infection in typhoid fever. Schule ${ }^{1}$ studying the reports of the German Laboratory at Trier for 1918, states that of the typhoid cases occurring in the district controlled by this laboratory during that year, 60 per cent were due to contact with cases, 5 per cent were due to contact with carriers and only 1 per cent were due to water and milk each. Of 5,889 cases analyzed at this laboratory, 71 per cent were contact cases.

The manner of contact may vary, and Gay ${ }^{2}$ summarizes in the following way, the manner in which this may take place:

1. Fingers or utensils-mouth.

2. Fingers-food-mouth.

3. Fomites-fingers-food-mouth.

4. Flies-food-mouth.

5. Fingers-flies-food-mouth.

In the case of the first route, more or less direct contact with a case is implied. In each of the five routes it is easy to draw

${ }^{1}$ Schule, Mil. Surgeon, 45, 1919, 268.

${ }^{2}$ Gay, Typhoid Fever, Macmillan Company, N. Y., 1918. 
vertical lines across the dashes and see where and how sanitary interference may interrupt the progress of transmission. Thus, in the prevention of the intestinal infections, the municipal and state authorities must care for the water supplies and sewage disposal plants ; the administrative public health authorities must be supplied with a reporting system for the early recognition and demarkation of foci, and must epidemiologically attempt to trace existing cases to their sources, following this with carrier examinations of suspected small groups; physicians must act as the first line of defense in regard to early diagnosis, intelligent, immediate isolation and the collection of the first significant epidemiological information for the use of the health authorities; city cleaning departments and other agencies must aid in preventing fly breeding, in garbage disposal, etc., and last but not least, general educational campaigns must elicit the intelligent cooperation of the public by supplying the simple information which is necessary for individual protection. As a matter of fact, if the public realized that most cases of typhoid and the paratyphoid fevers could be suppressed at the present time by a regular hand washing after defecation and before meals, the problem would be largely solved.

(3) The third group comprises diseases in which intimate contact between infectious material and the external surface of the body of the new victim is necessary. In some of these diseases transmission can take place without a visible break in the skin, as perhaps in plague, rabies, syphilis and some others, where the lesion through which the virus can enter may be so microscopical in size that no visible trauma is apparent. In others, such as the pyogenic infections, glanders, anthrax and the anaërobic infections, trauma of some kind is usually necessary. Few of these diseases can ever become epidemic to any great extent in the ordinary sense of the word. Some of them, however, like the venereal diseases may be regarded as so plentifully endemic, owing to the nature of their transmission, that we may look upon the present condition of communities as subject to a constant subacute epidemic state. Venereal diseases are a special sanitary group which requires individual treatment which we cannot enlarge upon in this place.

(4) In the fourth group are those diseases which are transmitted by insects. Such are malaria, yellow fever and dengue fever, transmitted by different species of mosquitoes, African sleeping sickness, pappataci fever, transmitted by flies, Rocky Mountain spotted fever 
and African relapsing fever, transmitted by a species of tick, European and Balkan relapsing fever, and kala-azar transmitted by bed bugs, and typhus fever, trench fever and some varieties of relapsing fever transmitted by lice, and plague largely transmitted by fleas.

In the sanitation of these diseases it is quite obvious that, in addition to our knowledge of the pathogenic microorganisms which cause the disease, we must study carefully the habits of insects, their seasonal occurrence, their nocturnal or diurnal habits, their methods and places of breeding, the distances which they can travel, the manner in which they acquire the parasites and the manner in which they can be suppressed.

Based upon an understanding of the conditions outlined above, moreover, is the science of epidemiology which, in its turn, can indirectly contribute a great deal, both to the solution of the problems of bacteriology and to those of transmission. For, by epidemiological surveys, by a careful study of the manner of spread of disease from group to group, and from place to place, the explosiveness with which it appears and relationship of cases to personal contact, water or milk supplies, etc., many deductions can be made which have important bearing in guiding bacteriological investigation and preventive measures. As to preventive measures, nothing is so important in the suppression of an epidemic disease as the rapid circumscription of the initial focus, a thing which can be accomplished only by prompt epidemiological survey, backed up by accurate bacteriological diagnosis. Such results can be achieved only when communities have efficient organizations for the prompt reporting of communicable diseases, for the systematic charting of the cases and for intelligent and experienced study of such charts. Indeed, studies of this nature alone may often make possible a causal classification of the epidemic before its bacteriological nature is accurately known. For epidemics will vary in the respects mentioned above, very largely according to whether they are water borne, distributed by milk or other food, or whether they are spread by contact or by insects.

It is probable that small water epidemics from individual rural house supplies may still be quite frequent and simulate contact epidemics. The larger water epidemics of typhoid, cholera, etc., will become, as we have stated, more and more infrequent as water supplies are more carefully supervised, but when they do occur, 
their onsets and courses will be characteristic to a degree which makes it possible for an experienced epidemiologist to suspect the water simply by a study of the incidence of cases in time and place. To some extent, of course, this depends upon whether the water is polluted by a single introduction of large quantities of sewage or whether contamination is continuous over a longer period. In both cases, however, a large number of the people living in the area of the water supply will be infected during a relatively short period of time. The rise of the curve which can be constructed from the cases, therefore, will be steep and rapidly reach a peak. Classical instances of this are the cholera epidemic in Hamburg and an epidemic of typhoid fever in an American city which is described in the section on typhoid fever. The incidence of the cases in places will be sharply limited by the distribution of the water supply and examination of the water will reveal colon bacilli.

In milk epidemics, a still more explosive rise of the cases will appear and in such cases as the Stamford epidemic described by Trask, a definite connection between the milk route and the distribution of cases may be traced. On the basis of such suspicion, the bacteriologist can investigate the milk and attempt a determination of recent intestinal disease or the carrier state in milk handlers. Also, it is stated by many who have studied these epidemies that milk epidemics are apt to claim the largest numbers of victims among women and children.

Contact epidemics will proceed by a more insidious course. In such epidemics it is extremely important to gather together careful data concerning the earliest cases observed and to attempt to trace the cases to some association at a common meal, a restaurant or at some other common source of food. Sawyer traced a contact epidemic to the carrier state in a ship's cook by a simple epidemiological study of the individual cases which all led by separate trails to the same ship's galley. We have, ourselves, traced cases in this way to company kitchens in military units. In large communities contact epidemics may trail along for long periods of time and when association is indiscriminate it may be necessarily impossible to establish accurate relationships. In some contact epidemics, such as those occurring in the Allied Armies in France when intestinal diseases appeared in large numbers, the rise of the curve simulated that of a water epidemic very largely because the indiscriminate distribution of unprotected dejecta over large areas 
of recently occupied territory, the prevalence of flies, and the crowding of large masses of men in small areas made frequent contact unavoidable. Such conditions, however, are not likely to happen to any extent at ordinary times, and contact epidemies are usually limited in area and distribution, and careful epidemiological study of three or four cases may lead to the original focus which can then be determined by prompt laboratory investigation. 


\section{CHAPTER XXI}

\section{THE STAPHYLOCOCCI (MICROCOCCI)}

THE power to incite purulent and sero-purulent inflammations and localized abscesses in man and animals is possessed by a large variety of pathogenic bacteria. Most infections, in fact, in which the relative virulence of the incitant and the resistance of the infected subject are so balanced that temporary or permanent localization of the infectious process takes place are apt to be accompanied by the formation of pus. The large majority of acute and subacute purulent processes, however, are caused by the members of a welldefined group of bacteria spoken of as the pyogenic cocei. Among these, pre-eminent in importance, are the "staphylococci" or "micrococci."

Many of the earlier investigators of surgical infections had seen small round bodies in the pus discharged from abscesses and sinuses and had given them a variety of names. Careful bacteriological studies, however, were not made until 1879 and the years immediately following, when Koch, Pasteur, Ogston, ${ }^{1}$ and others not only described morphologically, but cultivated the eocei from surgical lesions of animals and man. Of fundamental importance are the studies published by Rosenbach ${ }^{2}$ in 1884, in which the technical methods of modern bacteriology were brought to bear upon this subject for the first time. The group of staphylococci-so named from their growth in irregular, grape-like clusters-is made up of several members, by far the most important of which, pathologically, is the Staphylococeus pyogenes aureus.

\section{STAPHYLOCOCCUS PYOGENES AUREUS}

Morphology and Staining.-This microorganism, the most frequent cause of abscesses, boils, and many surgical suppurations, is a spherical coccus having an average diameter of about 0.8 micra,

${ }^{1}$ Ogston, Brit. Med. Jour., 1881.

${ }^{2}$ Rosenbach, "'Microorganismen bei Wundinfektion,' 1884. 
but varying within the extreme limits of 0.4 to 1.2 micra. Any considerable variation from the average size, however, is rare. The perfectly spherical character may not develop, whenever, as is usually the case, two or more are grouped together, unseparated after cell cleavage. In this case, adjacent cocci are slightly flattened along their contiguous surfaces.

Examined in smears from cultures or pus, the staphylococei may appear as single individuals, in pairs, or, most frequently, in irregular grape-like clusters. Occasionally, short chains of three or four may

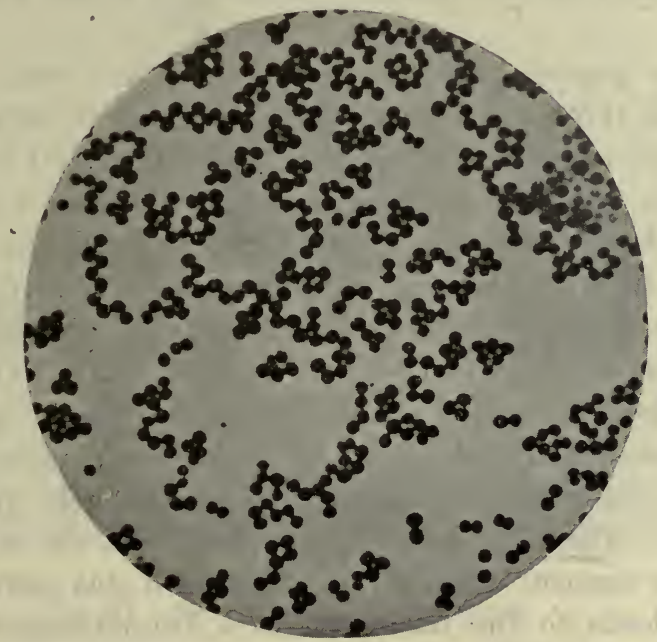

Fig. 43.-Staphylococcus pyogenes aureus. (After Gunther.)

be seen. In very young eultures in fluid media, the diplococcus form may predominate.

The staphylococci stain with all the usual basic aqueous anilin dyes, and, less intensely, with some of the acid dyes. Stained by the method of Gram, they retain the anilin-gentian-violet. Gram's method of staining is excellently adapted for demonstration of these cocci in tissue sections.

Although exhibiting marked Brownian movements in the hanging drop, staphylococci are non-motile and possess no flagella. They are non-sporogenous and form no capsules.

Cultural Characters.-Staphylococci grow readily upon the usual laboratory media. The simpler media, made of meat extract, are quite as efficient for their cultivation as are the freshly made meat- 
infusion products. The optimum temperature for staphylococcus cultivation lies at or about $35^{\circ} \mathrm{C}$., though growth readily takes place at temperatures as low as $15^{\circ} \mathrm{C}$., and as high as $40^{\circ} \mathrm{C}$. Slow but denfiite growth has been observed at a temperature as low as $10^{\circ} \mathrm{C}$.

While development is most characteristic and luxuriant under aërobic conditions, staphylococci are facultatively anaërobic on suitable media. They grow readily in an atmosphere of hydrogen.

As to the reaction of media, staphylococcus develops most favorably upon those having a slightly alkalin titer. Moderately increased alkalinity or even moderate acidity of media does not inhibit growth.

On gelatin plates, growth occurs readily at room temperature, forming within thirty-six to forty-eight hours, small, shining, pin-head shaped colonies, appearing, at first, grayish-white, and later assuming a yellowish hue, which intensifies into a light brown and often a bronze color as the colony grows older. The intensity of the color differs considerably in different races of staphylococci. Liquefaction of the gelatin occurs, and, shallow, saucer-shaped depressions are formed about the colonies after forty-eight hours or more. These zones of fluidification grow larger as the colonies grow, finally becoming confluent. Microscopically, the colonies themselves, before liquefaction has destroyed their outline, are round, rather finely granular, with smooth edges. They are not flat, but rise from the surface of the medium as the segment of a sphere. In gelatin stab cultures in tubes, fluidification leads to the formation of a funnel-shaped depression, with, finally, complete liquefaction of the medium and sedimentation of the bacteria. Liquefaction of gelatin by the staphylococcus is due to a ferment-like body elaborated by it, which is spoken of as "gelatinase." This substance can-be obtained apart from the cocci by the filtration of cultures. ${ }^{3}$ It is an extremely thermolabile body.

On agar plates the characteristics of the growth, barring liquefaction, are much like those on gelatin. Colonies do not show a tendency toward confluence, remaining discrete, and show a rather remarkable difference in the size of the colonies occurring upon the same plate. Upon slanted agar in tubes, rapid growth occurs, at first grayish-white, but soon covering the surface of the slant as a glistening, golden-brown layer.

In broth; growth is rapid, leading to a gencral, even clouding of

${ }^{3}$ Loeb, Cent. f. Bakt., xxxii, 1902. 
the medium, and giving rise, after forty-eight or more hours, to the formation of a thin surface pellicle. As growth increases, the bacteria sink to the bottom, forming a heavy, mucoid sediment. The odor of old cultures is often peculiarly acrid, not unlike weak butyric acid.

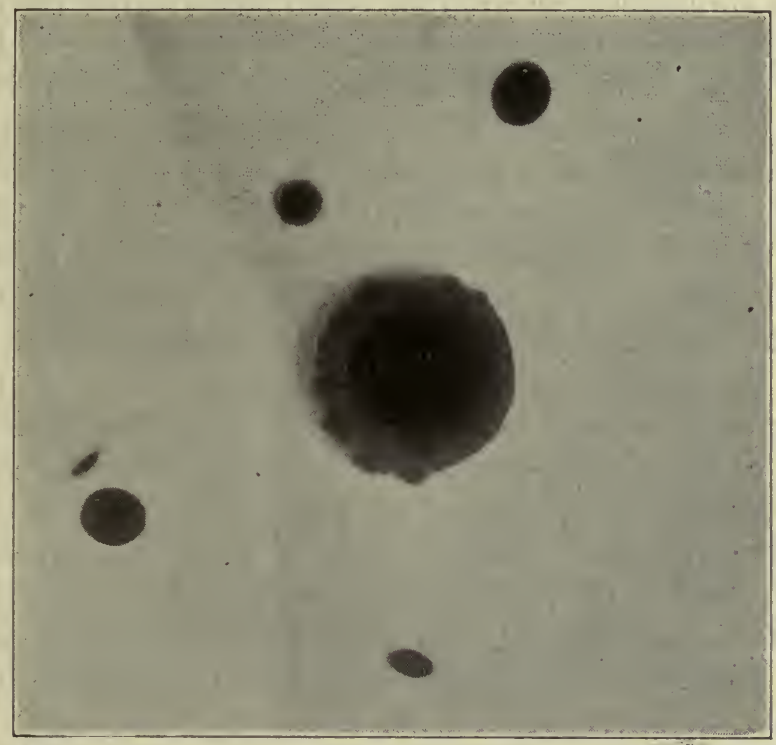

Fig. 44.-Staphylococcus colonies.

In milk, staphylococcus causes coagulation usually within three or four days, with the formation of lactic and butyric acids.

On potato, growth is abundant, rather dry and usually deeply pigmented.

Upon coagulated animal sera, rapid growth takes place and eventually slight liquefaction of the medium occurs.

In nitrate solutions, reduction of the nitrates to nitrites is caused.

In Dunham's broth, indol is not formed. Bayne-Jones and Zinninger have studied 115 strains of various staphylococei in all kinds of media suitable for the production of indol, and have not found a single indol producer.

In media containing the carbohydrates-dextrose, lactose, or saccharose-acidification takes place with the formation chiefly of lactic, butyric, and formic acids. There is no gas formation, however. 
In protein media free from sugars, the staphylococcus produces alkali.

The reducing action of staphylococcus is shown by decolorization in cultures of litmus, methylene-blue, and rosanilin. ${ }^{4}$

Pigment Formation.-Differentiation between the various members of the staphylococcus group is based largely upon the formation of pigments. These pigments, so far as we know, seem to be species characteristics. Thus, Staphylococcus pyogenes aureus is recognized primarily by its production of a yellowish-brown pigment, varying in different strains from a pale brown hue to a deep golden yellow. Prolonged cultivation upon artificial media may lead to a diminution in the depth of color produced. ${ }^{5}$ It appears only when cultivation is carried on under freely aërobic conditions, anaërobic cultivation resulting in unpigmented colonies. The coloring matter is insoluble in water but soluble in alcohol, chloroform, ether, and benzol. ${ }^{6}$ According to Schineider, the pigment belongs to the elass of "lipochromes" of fatty pigments, and is probably composed of carbon, oxygen, and hydrogen, without nitrogen. Treatment with concentrated sulphuric acid changes it to a green or greenish-blue. ${ }^{8}$ Neisser ${ }^{9}$ states that the pigment of staphylococei is excreted into the media by the organisms but does not diffuse because it is not soluble in water. Differences in pigment have been the basis of differentiations within the micrococcus group as we shall see below.

Resistance.-Although not spore formers, staphylococei are more resistant to heat than many other purely vegerative forms. The thermal death point given for Staphylococcus pyogenes aureus by Sternberg ${ }^{10}$ lies between $56^{\circ}$ and $58^{\circ} \mathrm{C}$., the time of exposure being ten minutes. The same author states that, when in a completely dried state, the coccus is still more resistant, a temperature of from $90^{\circ}$ to $100^{\circ} \mathrm{C}$. being required for its destruction. Against low temperatures, staphylococci are extremely resistant, repeated freezing often failing to sterilize cultures.

Desiccation is usually well borne, staphylococei remaining alive

* Fr. Müller, Cent. f. Bakt., xxvi, 1899.

'Flügge, "Die Microorg.,", etc.

'Migula, "System d. Bakt.,', Jena, 1897.

"Schneider, Arb. a. d. bakt. Inst., Karlsruhe, 1, vol. i, 1894.

${ }^{8}$ Fischer, "Vorles. über die Bakt.," Jena, 1903.

' Neisser, Kolie and Wassermann, 2nd Ed. vol. 4, p. 369.

${ }^{10}$ Sternberg, "'Textbook," etc., N. Y., 1901, p. 375. 
for six to fourteen weeks when dried upon paper or cloth. ${ }^{11}$ On slant agar, staphylococci may be safely left for three or four months without transplantation, and remain alive. ${ }^{12}$

The resistance of staphylococei to chemicals, a question of great surgical importance, has been made the subject of extensive researches, notably by Lübbert, ${ }^{13}$ Abbott, ${ }^{14}$ Franzott, ${ }^{15}$ and many others. According to Lübbert, inhibition of staphylococcus growth is attained by the use of boric acid 1 in 327 , salicylic acid 1 in 650 , corrosive sublimate 1 in 80,000 , carbolic acid 1 in 800 , thymol 1 in 11,000. Staphylococei are killed by corrosive sublimate 1 in 1,000 in ten minutes, by carbolic acid 1 per cent in 35 minutes, 3 per cent in 2 minutes (Franzott). Ethyl alcohol, ${ }^{16}$ even when absolute, is not very efficient as a disinfectant. Nascent iodin, as split off from iodoform in wounds, is extremely powerful in destroying staphylococci.

Pathogenicity.-Separate strains of Staphylococcus pyogenes aureus show wide variations in relative virulence. The most highly virulent are usually those recently isolated from human suppurative lesions, but no definite rule can be formulated in this respect. The virulence of a given strain, furthermore, may be occasionally enhanced by repeated passages through the body of a susceptible animal. Prolonged cultivation upon artificial media is liable to decrease the virulence of any given strain, though this is not regularly the case. There are, moreover, unquestionably, many staphylococci constantly present in the air, dust, and water, which although morphologically and culturally not unlike the pathogenically important species, may be regarded as harmless saprophytes.

The susceptibility of animals to staphylococcus infection is, likewise, subject to extreme variations, depending both upon differences between species and upon fortuitous individual differences in susceptibility among animals within the same species. Animals on the whole are less susceptible to staphylococcus than is man. Among the ordinary laboratory animals, rabbits are most suseeptible to this microorganism. Mice, and especially the white Japanese mice, show

\footnotetext{
${ }^{11}$ Deslongchamps, Paris, 1897.

${ }^{12}$ Passet, Fort. d. Med., 2 and 3, 1885.

${ }^{13}$ Lübbert, "Biol. Untersuch.,", Würzburg, 1886.

${ }^{14}$ Abbott, Medical News, Phila., 1886.

${ }^{15}$ Franzott, Zeit. f. Hyg., 1893.

${ }^{16}$ Hanel, Beit. z. klin. Chir., xxvi.
} 
considerable susceptibility. Guinea-pigs possess a relatively higher resistance. ${ }^{17}$

Subcutaneous or intramuscular inoculation of a susceptible animal usually results in the formation of a localized abscess with much pus formation and eventual recovery. Intraperitoneal inoculation is more often fatal. Intravenous inoculation of doses of 0.5 c.c., or more, of fresh broth cultures of virulent staphylococci usually leads to pyemia with the production of secondary abscesses, located chiefly in the kidneys and the heart and voluntary muscles, but not infrequently in other organs as well. In the kidney they occur as small foci, situated most often in the cortex, composed of a central, necrotic pus cavity, surrounded by a zone of acute inflammatory exudation. Staphylococcus lesions form histologically the typical "acute abscess." Not infrequently the pyemic condition is accompanied by suppurative lesions in the joints. Intravenous injections of virulent staphylococci preceded by injury to a bone is often followed by the development of osteomyelitis. Mechanical or chemical injury of the heart valves preceding intravascular staphylococcus inoculation may result in localization of the infection on or about the heart valves, leading to "malignant endocarditis." In producing experimental lesions in rabbits all varieties of staphylococcus infection may be obtained by suitable methods of injection. If, for instance, a rabbit is given one-half to 1 c.c. of a young broth culture, from which the clumps have been gently centrifuged down, into the ear vein, a rapid fatal septicemia will result with organisms in the heart's blood, but no secondary localization or abscess formation. If, however, staphylococcus cultures containing clumps are gently centrifuged, the supernatant fluid taken off, and small clumps injected in not too large amounts (and the amounts must be adjusted to the virulence of the culture) the animal will pass through a protracted illness, with secondary abscess formation in kidneys, liver and other organs, in which emboli have becn formed, a condition simulating accurately pyemia in human beings. The pyemic conditions following staphylococcus inoculation usually lead to chronic emaciation and death after an interval dependent upon the relative virulence of the microorganism, the amount injected, and the resistance of the infected subject.

${ }^{17}$ Terin, Ref. in Lubarsch und Ostertag, Ergebnisse, 1896; Lingelsheim, "'Aetiol. d. Staph. Inf.," ete., Wien, 1900. 
As above stated, the susceptibility of man to spontaneous staphylococcus infection is decidedly more marked than is that of animals. The form of infection most frequently observed is the common boil or furuncle. As Garı'é, ${ }^{18}$ Büdinger, ${ }^{19}$ Schimmelbusch, ${ }^{20}$ and others have demonstrated by experiments upon their own bodies, energetic rubbing of the skin with virulent staphylococeus cultures may often be followed by the development of a furuncle. Subcutaneous inoculation of the human subject invariably gives rise to an abscess. The organisms are apparently present on the skin of human beings with great frequency, and it is not unlikely that in the course of daily life, they may be rubbed into hair follicles and sweat glands, and be present constantly on some part of the body, prepared for immediate invasion of an abrasion or other accident furnishes the opportunity. A simple and frequent disease, furunculosis, is, nevertheless, a condition about the pathogenesis of which we are considerably in the dark. Reductions of general resistance, especially those accompanying overwork, indoor occupations, and faulty diet, seem to be concerned in furnishing the proper conditions for invasion by the ever present staphylococci. General metabolic diseases, such as nephritis and especially diabetes, render the individual abnormally susceptible. 'In certain instances it has been suggested, especially by Wright, that reduction in coagulation time of the blood might influence this state of affairs.

Staphylococcus lesions of the skin are characteristic in that, after an induration, there occurs a central softening with the formation of liquid pus. It is an important observation, confirmed by much experience, that if incision is practiced in the indurated and inflamed tissue before the process has come to a central head, infection is usually spread, perhaps by the opening of adjacent lymphatics. Therefore, there is much judgment required in treating even these simple lesions. Faulty surgical interference may easily convert a simple furuncle into a dangerous carbuncle.

Common among staphylococcus skin infections is paronychia, or infection of the nail bed on the fingers. This may often lead to troublesome extension up the fingers and into the hands.

${ }^{18}$ Garré, Beit. z. klin. Chir., x, 1893.

${ }^{19}$ Bïdinger, Lubarseh und Ostertag, Ergebnisse, ete., 1896.

${ }^{20}$ Schimmelbusch, Ref. by Büdinger. 
Especially dangerous are boils about the nose and lips, and not infrequently infections in these locations may extend rapidly, and cause fatal septicemia.

Impetigo contagiosum, a skin disease consisting of boil-like inflamed papules and occurring particularly in young children, is caused by staphylococci.

In suppurative lesions of the bones, or osteomyelitis, staphylococei are the most frequent causative agents. This may result, after compound fracture, by infection from without, or not infrequently staphylococci will lodge in the site of mechanical injury of bone or fracture, reaching the focus through the circulation. The lesions produced in bone may consist of slow localized abscesses, or may extend along the medullary canal of the entire bone.

In addition to these most common lesions, staphylococci may cause abscesses in almost any part of the body. In cases in which resistance if low and the staphylococci particularly virulent, septicemia may follow in any of these. Unlike the rapid, acute septicemia death, however, which is likely to ensue when similar general infection with streptococci takes place, staphylococcus generalization is apt to lead to secondary foci in kidneys, liver and other organs. This leads to the condition of pyemia in which an irregular septic temperature with frequent chills are characteristic. Blood culture in such cases will give a clue to the nature of the infection.

Ascending infections of the genito-urinary tract, eystitis and pyelonephritis, may be caused by staphylococci.

Staphylococcus empyema and peritonitis are not particularly common, but may occur.

Puerperal sepsis, while not as commonly a staphylococcus infection, as it is a streptococcus lesion, may occur.

By some writers staphylococei have been held responsible for rheumatism, but there is no convincing evidence of this.

Staphylococci may also appear in meningitis. It is a curious fact that occasionally a very low grade staphylococcus may get into the meninges, and cause a very slow and apparently mild meningitis. We have seen one such case caused by a staphylococcus albus recover, and another which died after a prolonged illness in which the organisms were repeatedly isolated from the spinal fluid, and, at autopsy, in which the origin was a cerebellar abscess.

Prolonged chronic infection with staphylococei may give rise to the so-called amyloid changes in liver, spleen and kidneys. 
Toxic Products.-Endotoxins.-The dead bodies of staphylococci injected into animals may occasionally give rise to abscess formation, and, ${ }^{21}$ if in sufficient quantity, may cause death. To obtain the latter result, however, large quantities are necessary, the endotoxic substances within the dead cell body of these microorganisms being probably neither very poisonous nor abundant. ${ }^{22}$

That dead cultures of Staphylococcus aureus exert a strong positive chemotaxis for leucocytes was shown beyond question by the experiments of Borissow. ${ }^{23}$

IIemolysins.-In $1900 \mathrm{Kraus}^{24}$ noticed the hemolytic action of staphylococci growing upon blood-agar plate cultures. Neisser and Wechsberg ${ }^{25}$ then showed that this hemolytic substance, secreted by the staphylococcus, could be demonstrated in filtrates of bouillon cultures. Such hemolysins are produced by Staphylococcus aureus, and, to a lesser degree, by Staphylococcus albus. The quantity produced varies enormously with different strains and seems to be roughly proportionate to the virulence of the particular microorganism, though exceptions to this rule are not uncommon. Absolutely avirulent races do not, so far as we know, produce hemolysins. Relationship of hemolysin formation to virulence, however, is not by any means as regular as at first supposed. A great many staphylococei may be isolated from human lesions which produce absolutely no hemolysin. The culture medium most favorable to the formation of these substances is, according $t$ ( $)$ Neisser and Wechsberg, a moderately alkalin beef bouillon. Cultivated at $37.5^{\circ} \mathrm{C}$., the bouillon contains the maximum amount of hemolytic substance between the eighth and fourteenth day, and this may be separated from the bacteria by filtration through Berkefeld or Chamberland filters. We have not ourselves attempted to confirm these investigations, but in the particular respect of lateness of concentration in the cultures the staphylococeus hemolysin seems to differ distinctly from that produced by streptococci, in which the optimum for a large yield is early, within the first twelve or fourteen hours of cultivation.

The hemolytic action may be observed by the general technique for determining hemolysis (given on page 311 ). It is important to

${ }^{21}$ Schattenfroh, Arch. f. Hyg., xxxi, 1887.

${ }^{22} v$. Lingelsheim, "'Aetiol. u. Therap. d. Staph. Krank.," Wien, 1900.

${ }^{23}$ Borissow, Zieglers Beitr., xvi, 1894.

${ }^{24}$ Kraus, Wien. klin. Woch., iii, 1900.

${ }^{25}$ Neisser und Wechsberg, Zeit. f. Hyg., xxxvi, 1901. 
wash the red blood corpuscles used for the experiments, since many animals normally possess small quantities of antihemolysin in their blood-sera (man and horse especially). ${ }^{26}$ The red blood corpuscles of rabbits, dogs, and guinea-pigs are extremely susceptible to the action of the staphylo-hemolysin. Those of man are less easily injured by it. The hemolytic action takes place, as $\operatorname{Todd}^{27}$ and others ${ }^{28}$ have shown, not only in vitro, but in the living animal as well.

The staphylo-hemolysin is comparatively thermolabile. According to Neisser and Wechsberg, heating it to $56^{\circ}$ C. for twenty minutes destroys it. According to some other authors, however, higher temperatures $\left(60^{\circ}\right.$ to $80^{\circ}$ C.) are required. Reactivation of a destroyed staphylo-hemolysin has so far been unsuccessful. The fact that antistaphylolysin is occasionally present in normal sera has been mentioned above. This antibody is most abundant in the blood of horses and of man. Artificially antistaphylolysin formation is easily induced by subcutaneous inoculation of staphylolysin into rabbits.

Leucocidin.-In 1894, Van de Velde ${ }^{29}$ discovered that the pleural exudate of rabbits following the injection of virulent staphylococei, showed marked evidences of leucocyte destruction. He was subsequently able to show that the substance causing the death and partial solution of the leucocytes was a soluble toxin formed by the staphylococcus, not only in vivo, but in vitro as well; for cultures of Staphylococcus pyogenes aureus, grown in mixtures of bouillon and blood 'serum, contained, within forty-eight hours, marked quantities of this "leucocidin."

Other workers since Van de Velde have evolved various methods for obtaining potent leucocidin. Bail ${ }^{30}$ obtained it by growing virulent staphylococcus in mixtures of one-per-cent glycerin solutions and rabbit serum. Neisser and Wechsberg ${ }^{31}$ advise the use of a carefully titrated alkalin bouillon. To obtain the leucocidin free from bacteria, the cultures are passed through Chamberland or Berkefeld filters, after about eight to eleven days' growth at $37^{\circ} \mathrm{C}$, at which time the contents in leucocidin are usually at their highest point.

The action of leucocidin upon leucocytes may be observed in vivo

${ }^{26}$ Neisser, Deut. med. Woch., 1900.

${ }^{27}$ Todd, Trans. London Path. Soc., 1902.

${ }^{28}$ Kraus, Wien. klin. Woch., 1902.

${ }^{20} \mathrm{~V}$ an de Velde, La Cellule, $\mathrm{x}, 1894$.

${ }^{30}$ Bail, Arch. f. Hyg., xxxii, 1898.

${ }^{31}$ Neisser und Wechsberg, Zeit. f. Hyg., xxxvi, 1901. 
by the simple method of Van de Velde, of injecting virulent staphylococci intrapleurally into rabbits and examining the exudate. Bail advises the production of leucocytic intrapleural exudates by the use of aleuronat and following this after twenty-four hours by an injection of leucocidin-filtrate. In vitro the phenomenon may be observed by direct examination of mixtures of leucocytes and leucocidin in the hanging drop on a warmed stage, or by the "methylene-blue method" of Neisser and Wechsberg. This method is based upon the fact that living leucocytes will reduce methylene-blue solutions and render them colorless, while dead leucocytes have lost this power. Leucocidin and leucocytes are allowed to remain in contact for a given time and to them is then added dilute solution of methyleneblue. If the leucocytes have been actively attacked by leucocidin, no reduction takes place. This method is particularly adapted for quantitative tests.

All staphylococcus strains do not produce leucocidin to the same degree. Almost all true Staphylococcus pyogenes aureus cultures produce some of this toxin, but one strain may produce fifty- and a hundred-fold the quantity produced by another. Staphylococeus pyogenes albus gives rise to this substance but rarely, and then in small quantity.

Leucocidin seems to be similar to the soluble toxins of other bacteria. It is rapidly destroyed by heat at $58^{\circ} \mathrm{C}$., and deteriorates quickly in culture fluids at incubator temperatures. It is distinct from staphylohemolysin as shown by differences in thermostability.

Soon after Van de Velde's discovery of leucocidin, Denys and Van de Velde ${ }^{32}$ produced an antileucocidin by treating rabbits with pleural exudate containing leucocidin. Neisser and Wechsberg ${ }^{33}$ later confirmed these results and showed that among staphylococci, leucocidin is not specific, the toxin of all strains of Staphylococcus aureus and albus examined being neutralizable by the same antileucocidin. Antileucocidin is often found in the normal sera of horses and man. ${ }^{34}$

Leucocidin should not be confounded with "leucotoxin," a substance obtained in serum by treatment of animals with leucocytes, a true "cytotoxin," having no eonnection whatever with the staphylococcus.

${ }^{32}$ Denys et Van de Velde, I.a Cellule, xi, 1895.

${ }^{33}$ Loc. eit.

${ }^{34} V$ an de Velde, Presse médicale, i, 1900. 
Staphylococci, besides the toxic substances already mentioned, give rise to gelatinase, spoken of in the section upon cultivation, and to a proteolytic ferment by means of which albuminous media (Loeffler's serum) may be slightly digested.

Immunization.-Animals ean be rendered actively immune by repeated inoculations with carefully graded doses of living or dead staphylococeus cultures. $^{35}$ The production of antistaphylolysin and of antileucocidin in the sera of animals so treated, has been alluded to in the preceding seetions. The sera of such actively immunized animals possess distinct protective power when administered to other animals, slightly before or at the same time with an inoculation of staphylococei. They do not, however, exhibit very high bacterieidal power in vitro, the protective properties depending probably upon their opsonie contents. ${ }^{36}$

Agglutinins have been demonstrated in staphylococeus immune sera by a number of authors, and have been of some slight value in differentiating between the several groups of staphylococci. ${ }^{37} \mathrm{~A}$ rather surprising result of these researches has been the recognition that immune sera obtained with pathogenic staphylococci will agglutinate other pathogenic staphylococei, whether belonging to the group of Staphylococcus pyogenes aureus or that of Staphylococcus pyogenes albus, but will not agglutinate any of the non-pathogenic members of either group. ${ }^{38}$ In view of the recent studies on the antigenic elassification of streptococei and pneumococci, a reexamination of these relationships within the staphylococcus group should be undertaken. It is more than likely that this group is a heterogeneous one, and that, for purposes of intelligent experimentation in serum and vaccine therapy, an antigenic classification should be attempted.

Active immunization of human beings suffering from staphylococeus infections has been extensively practiced by Wright, in connection with his work on opsonins. There can be no question about the fact that the opsonie substances in the blood are inereased by the injection of dead staphylococci. The procedure is of therapeutic value in subacute and chronic cases. The work of Hiss on the use

${ }^{25}$ Richet et Hericourt, Compt. rend. de l'acad. des sei., evii, 1888.

Kolle und Otto, Zeit. f. Hyg., xli, 1902.

"Proscher, Cent. f. Bakt., xxxiv, 1903; v. Lingelsheim, "Aetiol. u. Therap. d. Staphyl.,", etc., Wien, 1900.

Proscher, Deut. med. Woch., xi, 1903. 
of leucocyte extracts in animals infected with Staphylococeus pyogenes aureus has given encouragement for such treatment in human beings. A number of staphylococeus infections in man have, been successfully treated with leucocyte extracts by Hiss and Zinsser.

Passive immunization with anti-staphylococeus sera has not been a therapeutic success. Extensive work has been done especially by German investigators in animal experimentation, but in most cases it has been found that serum injections were of little use if administered after the infection had been established. Injection into the test animals before infection or at the same time with infection sometimes gave favorable results. In man, passive immunization has not been eneouraging, although we believe that hope of some benefit in this direction should not be eompletely abandoned until the antigenic classification of the staphylococci has been attempted.

Hooker ${ }^{39}$ has recently reported a number of cases in which he has transfused into patients suffering from staphylococcus septicemia the blood of donors immunized with staphylocoecus vaccines. This procedure of course would he applicable only to subacute cases, but in the procedure of Hooker there seems to be promise.

Staphylococcus Pyogenes Albus.-This coceus differs from Staphylococcus pyogenes aureus simply in the absence of the golden yellow coloration of its cultures. Morphologically, culturally, and pathogenically, it is in every way identical with the staphylococeus described in the preceding section, but its toxin- and enzyme-producing powers in general are less developed than those of the aureus variety. Its close biological relationship to aureus is furthermore demonstrated by its agglutination in Staphylococeus pyogenes aureus immune sera.

Staphylococcus Epidermidis Albus.-The Staphylococeus epidermidis albus described by Welch is merely one of the nonpathogenic varieties of Staphylococcus pyogenes albus and possibly does not deserve separate classification. It may give rise to unimportant abscesses.

Staphylococcus Pyogenfs Citreus.-Staphylococcus pyogenes (itreus produces a bright yellow or lemon-colored pigment of distinctly different hue from that of Staphylococeus pyogenes aureus.

- Hooker, An. of Surgery, Nov., 1917, p. 513. 
It may be pyogenic and in every way similar to Staphylococcus pyogenes aureus, but is less often found in connection with pathological lesions than either of the preceding staphylococci.

A large number of staphylococci, differing from those described above in one or another detail, have been observed. They are of common occurrence and are met with chiefly as contaminations in the course of bacteriological work. Few of these have any pathological significance and none of them are toxin-producers, so far as we know. Many of them differ, furthermore, in their inability to liquefy gelatin. All of them belong more strictly to the field of botany than to that of pathological bacteriology.

Atypical pathogenic staphylococei have been described by a number of observers. Thus Weichselbaum ${ }^{40}$ isolated a staphylocoecus from a case of malignant endocarditis which could not be cultivated at room temperature, and grew only in very delicate colonies. Veillon, ${ }^{41}$ moreover, has described a strictly anaërobic staphylococcus cultivated from the pus of an intra-abdominal abscess.

Micrococcus Tetragenus.-In 1881 Gaffsky $^{42}$ discovered a micrococcus which occurs regularly in groups of four or tetrads. He

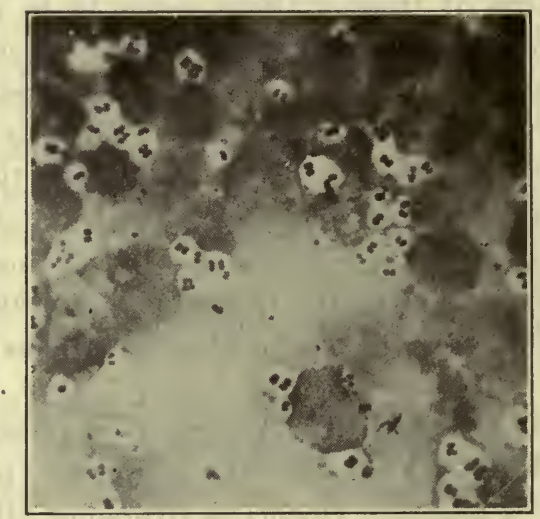

Fig. 45.-Micrococcus tetragenus.

first isolated it from the pus discharged by tuberculous patients with pulmonary lesions. Observed in smear preparations from pus,

40 Weichselbaum, Baumgarten, Jahresb., 1899, Ref.

${ }^{41}$ Veillon, Compt. rend. soc. de biol., 1893.

${ }^{42}$ Gaffsky, Mitteil. a. d. kais. Gesundheitsamt, i, 1881. 
the tetrads are slightly larger in size than the ordinary staphylococcus, flattened along their adjacent surfaces, and surrounded by a thick halo-like capsule. Preparations from cultures often lack these capsules. The micrococeus is easily stained by the usual basic anilin dyes. Stained by Gram's method, it is not decolorized, retaining the gentian-violet.

Cultivation.-Micrococcus tetragenus grows on the ordinary laboratory media, showing a rather more delicate growth than do the staphylococci.

On agar, the colonies are at first transparent, later they become grayish-white, but are always more transparent than are staphylococcus cultures.

On gelatin, growth is rather slow and no liquefaction takes place. Broth is evenly elouded.

On potato there is a white, moist growth which shows a tendency to confluence.

Milk is coagulated and litmus milk indicates acid formation.

Pathogenicity.-Micrococeus tetragenus is especially pathogenic for Japanese mice, which succumb within three or four days to subcutaneous inoculation. ${ }^{43}$ Gray mice, rats, guinea-pigs, and rabbits are less susceptible, showing only a localized reaction at the point of inoculation. The organism has occasionally been isolated from spontaneous abscesses observed in domestic animals.

In man, this microorganism is usually found without any particular pathogenic significance, in sputum or saliva. In isolated cases, however, it has been described as the sole incitant of abscesses.

Bezançon $^{44}$ has isolated Micrococcus tetragenus from a case of meningitis. A single case of tetragenus septicemia is on record, reported in 1905 by Forneaca. ${ }^{45}$

In America, this microorganism has not been frequently observed in connection with disease. It is often found, however, in considerable numbers, in smears of sputum which are being examined for pneumococei or tubercle bacilli.

ENTERococcus.-This is a capsulated streptococeus found frequently in the intestines of infants, and first described by Escherich and Tavel. It is a pleomorphic organism which may appear in the

${ }^{43}$ Müller. Wien. klin. Woch., 17, 1904.

${ }^{4+}$ Bezançon, Semaine méd., 1898.

${ }^{45}$ Forneaca, Rif. med., 1903. 
stools as a diplococcus or in short chains. Even in the diplococcus form, the individuals may be of different size and shape, some of them oval, rather than round. It may assume diplobacillus-like forms.

It is Gram-positive, produces neither gas nor indol, and grows well on ordinary broth and agar. It is aërobic, but may grow under anaërobic conditions.

Its relationship to intestinal disease in children has been suspected but never conclusively proven. Mice are susceptible to inoculation, rabbits and guinea-pigs less so. 


\section{CHAPTER XXII}

\section{THE STREPTOCOCCI}

AMONG the pyogenic cocci, there is a large and important group of organisms which multiply by division in one plane of space only, and thus give rise to appearances not unlike chains or strings of beads. The term streptococcus or chain-coccus is, therefore, a purely morphological one,which includes within its limits microorganisms which may differ from each other considerably, both as to cultural and pathogenic properties. Thus, cocei which form chains may be isolated from water, milk, dust, and the feces of animals and man. These may have little but their morphological appearance in common with the pyogenie streptococei which are so important as the incitants of disease. The interrelationship between streptococei from different sourees, however, is by no means fully understood, and we are foreed at present to content ourselves with the recognition of a large morphological group, in no individual case taking the pathogenic or more special cultural characteristics for granted.

Of paramount importance among the streptococci are those which possess the power of giving rise to disease processes in animals and in man, and which, because of their frequent association with suppurative inflammations, are roughly grouped under the heading of Streptococcus pyogenes.

The same researches upon surgical infections which led to the discovery of the staphylococei laid the basis for our knowledge of the streptococci. The fundamental studies of Pasteur and Koch ${ }^{1}$ were followed, in 1881, by the work of Ogston, ${ }^{2}$ who was the first to differentiate between the irregularly grouped staphylococei and the chain-cocei.

Pure cultures of streptococei were first obtained by Fehleisen ${ }^{3}$ in 1883 and by Rosenbach ${ }^{4}$ in 1884 . The thorough and systematic

${ }^{1}$ Koch, “Untersuch. über Wundinfektion," etc., 1878.

${ }^{2}$ Ogston, Brit. Med. Jour., 1881.

'Fehleisen, "Aetiol. d. Erysipelas," Berlin, 1883.

'Rosenbach, 'Mikroorg. bei Wundinfektion,', ete., Wiesbaden, 1884. 
researches of the last-named authors, together with those of Passet, ${ }^{5}$ were of special influence in placing our knowledge of the pathogenic properties of streptococci upon a scientific basis.

Morphology and Staining.-The individual streptococcus is a spherical microorganism measuring from 0.5 micron to 1 micron in diameter. Since the line of cleavage of cocci, when in chains, is

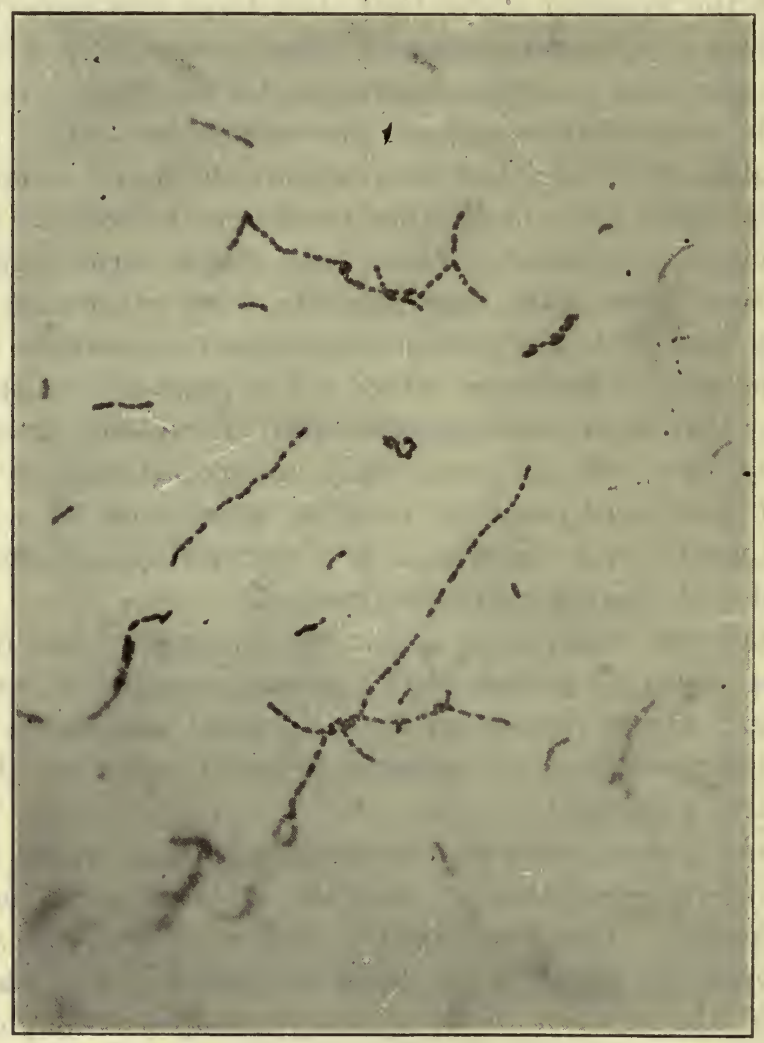

Fig. 46.-STREPTOCOCCUS PYOGENES.

perpendicular to the long axis of the chain, adjacent cocci often show slight flattening of the contiguous surfaces, forming, as it were, a series of diplococci arranged end to end. As a general rule the streptococei pathogenic for man, when grown upon favorable media, have a tendency to form chains made up of at least eight

'Passet, “'Untersuch. über die eitrigen Phlegm.,'” ete., Berlin, 1885. 
or more individuals, while the more saprophytic, less pathogenic varieties are apt to be united in shorter groups. Upon this basis a rough morphological distinction has been made by v. Lingelsheim, ${ }^{6}$ who first employed the terms streptococeus "longus" and "brevis." A differentiation of this kind can hardly be relied upon, however, since the length of chains is to some degree dependent upon cultural and other environmental conditions. Species which exhibit long and tortuous chains, when grown upon suitably alkalin bouillon, or ascitic broth, may appear in short groups of three or four, or even in the diplo form; when cultivated upon solid media or unfavorable fluid media.

It has often been noticed that some streptococei will form capsules. ${ }^{7}$ We are not referring by this to the so-called "streptococcus mucosus" of Schottmüller, which is now spoken of as "pneumococcus mucosus" or Type III, but ordinary and probably true hemolytic streptococei may on occasion form capsules which are noticeable in smears from infected animals and in early cultures made upon media rich in animal fluids, but may be lost on subsequent transplantation to simpler media. The capsule here is an attribute of virulence. It has been particularly noticed in connection with the so-called streptococeus epidemicus isolated from milk, to which we will refer in subsequent paragraphs.

Streptococei do not form spores, are non-motile, and do not possess fiagella.

An astonishing change in the size and appearance of streptococei may be noticed under different conditions of cultivation. Streptococci which have grown under anaërobic or partially anaërobic conditions will often show chains of the organisms of minute size. Indeed, we have seen, interspersed with chains of the ordinary appearance, individual chains composed of organisms almost as small as the globoid bodies of Noguchi. It is these small individuals which appear under anaërobic conditions that have been responsible for Rosenau's ideas concerning the etiological role of streptococei in poliomyelitis. Again, in old cultures there may be either at the ends or even in the middle of the chains, large, swollen individuals, almost as big as small yeast cells. When these are first encountered

\footnotetext{
'v. Lingelsheim, "Aetiol. u. Therap. d. Streptok. Infek.", Beit. z. Exp. Ther., Hft. 1, 1899

'Pasquale, Zieglers Beit., xii; Bordet, Ann. de l'Inst. Pasteur, 1887; Schottmüller, Münch. med. Woch., xx, 1903; Hiss, Jour. Exp. Med., vi, 1905.
} 
by an inexperienced bactericiogist, he may assume for some time that his eulture has been contaminated. These swollen individuals may probably be interpreted as involution forms.

Streptocoeci are easily stained by the usual anilin dyes. Stained by the method of Gram, the pyogenic streptococei are not decolorized and invariably retain the gentian-violet. Certain speeies found in stools and deseribed as Gram-negative, are rare and are nonpathogenic. Others of the "Streptoeoccus brevis" variety, and purely saprophytic, may stain irregularly by the Gram method.

Cultivation.-The pyogenic streptoeocei are easily eultivated upon all the richer artifieial media. While meat extract-pepton media may suffice for certain strains, it is usually better to employ those media which have the beef or veal infusion for a basis. For the cultivation of more delieate strains of streptocoeei, especially when taken directly from the animal or human body, it is well to add to the media animal albumin in the form of whole blood, blood serum, or aseitic or pleural transudates. Glucose, added in proportions of one to two per eent, likewise renders media more favorable for streptococcus cultivation. Prolonged cultivation of all races upon artificial media renders them less fastidious as to eultural requirements. The most favorable reaction of media for streptococeus eultivation is moderate alkalinity (two-tenths to five-tenths per cent alkalinity to phenolphthalein). Growth may be readily obtained, however, in neutral media or even in those slightly acid. The optimum temperature for growth is at or about $37.5^{\circ}$. Above $43^{\circ}$ to $45^{\circ} \mathrm{C}$., development eeases. At from $15^{\circ}$ to $20^{\circ} \mathrm{C}$, growth, while not energetie, still takes place, an important point in the differentiation of these microorganisms from pneumococci. While the free access of oxygen furnishes the most suitable environment for most races of streptococci, complete anaërobiosis does not prevent development in favorable media. Strictly anaërobic streptococei have been eultivated from the human intestinal tract by Perrone $^{8}$ and others.

In alkalin bouillon at $37.5^{\circ} \mathrm{C}$, pyogenic streptococei grow rapidly, form long and tortuous chains, and have a tendeney to form flakes which rapidly sink to the bottom. Diffuse elouding occurs rarely and is a characteristic rather of the shorter so-called Streptococcus brevis. When sugar has been added to the broth the rapid formation of lactic acid soon interferes with extensive development.

${ }^{8}$ Perrone, Ann. de l'inst. Pasteur, xix, 1905. 
This may be obviated, especially when mass cultures are desired, without sacrifice of the growth-increasing influence of the glucose; by adding to the sugar-broth one per cent of sterile powdered $\mathrm{CaCO}{ }^{9}$

In milk, Streptococeus pyogenes grows readily with the formation of acid, followed, in most cases, by coagulation of the medium.

On agar-plates at $37.5^{\circ} \mathrm{C}$, growth appears within eighteen to twenty-four hours. The colonies are small, grayish, and delicately opalescent. They are round with smooth or very slightly corrugated or lace-like edges, and rise from the surface of the medium in regular ares, like small droplets of fluid. Microscopically they appear finely granular and occasionally, under high magnification, may be seen to be composed of long intertwining loops of streptococcus chains, which form the lace-like edges. When ascitic fluid or blood serum has been added to agar, growth is more energetic and the colonies correspondingly more rapid in appearance and luxuriant in development. In glucose-ascitic-agar, acid formation from the sugar causes coagulation of albumin with the consequent formation of flaky white precipitates throughout the medium. ${ }^{10}$

In gelatin stab-cultures growth takes place slowly, appearing after twentyfour to thirty-six hours as a very

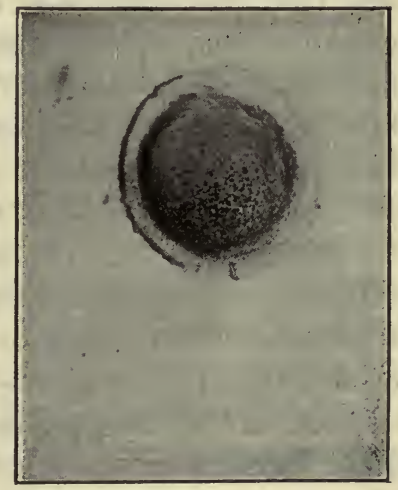

Fig. 47.-STREPtococcus coLONY ON SERUM AGAR. thin white line, or as disconnected little spheres along the line of the stab. The colonies on gelatin plates are similar in form to those on agar, but are usually more opaque and more distinctly white. The gelatin is not liquefied by the pyogenic streptococei, though eertain of the more saprophytic forms may occasionally bring about slow fluidification.

On Loeffler's coagulated blood serum, growth is rapid and luxuriant and may show a slight tendeney to confluence if the medium is very moist. Good chain formation takes place on this medium.

Upon potatoes, growth is said not to take place. ${ }^{11}$

On media containing red blood cells, most pathogenic streptococei

\footnotetext{
'IIiss, Jour. Exp. Med., vi, 1905.

${ }^{10}$ Libman, Medical Record, lvii, 1900.

${ }^{11}$ Frosch und Kolle, in Flügge, "'Die Mikroorganismen,' 1891.
} 
cause hemolysis and decolorization. It is useful to remember this when examining blood-culture plates, for here the yellow transparent halo of hemolysis and decolorization surrounding the colonies may aid in differentiating these bacteria from pneumococci. This is of especial importarce, since many streptococei, when cultivated directly out of the human blood, do not exhibit chain formation, but appear as diplococci.

In the inulin-serum media of Hiss, ${ }^{12}$ streptococei do not produce acid and coagulation. The so-called Streptococeus mucosus, a capsule-bearing, inulin-fermenting microorganism, is very probably a sub-species of the pneumococcus (see later section). A very important differential characteristic of the streptococci as a class is the fact that they are not bile soluble. This distinguishes them sharply from the pneumococci. The test is carried out by the method of Neufeld described in the chapter on pneumococci. Ox bile or a ten per cent solution of taurocholate of sodium is added to a young broth culture in proportions of one part of bile or taurocholate to nine parts of the culture.

Resistance.-Streptococci on the ordinary culture media, without transplantation and kept at room temperature, usually die out within ten days or two weeks. They may be kept alive for much longer periods by the use of the calcium-carbonate-glucose bouillon, if the cultures are thoroughly shaken and the powdered marble thoroughly mixed with the bouillon from time to time. ${ }^{13}$ Preservation at low temperatures $\left(1^{\circ}\right.$ to $2^{\circ} \mathrm{C}$.), in the ice chest, considerably prolongs the life of cultures. Virulence is preserved longest by frequent transplantation upon albuminous media. In sputum or animal excreta, streptococci may remain alive for several weeks. Streptococei like pneumococci may be preserved alive and with virulence unchanged by drying in the frozen condition by the method described by Swift, and referred to on page 456 . For ordinary purposes preservation in tubes of defibrinated rabbit's blood in the ice-chest is the most practical method.

Streptococei are killed by exposure to a temperature of $54^{\circ} \mathrm{C}$. for ten minutes. ${ }^{14}$ Low temperatures, and even freezing, do not destroy some races.

12 Hiss, Jour. Exp. Merl., vi, 1905.

${ }^{13}$ Hiss, loc. cit.

${ }^{14}$ Sternberg, "'Textbook of Bact.,', 2đ ed., 1901; Hartmann, Arch. f. Hyg., vii. 
The action of various chemical disinfectants has been thoroughly investigated by v. Lingelsheim, ${ }^{15}$ who reports among others the following results: Carbolic acid 1:200 kills streptococei in fifteen minutes. In the same time, bichloride of mercury is efficient in a dilution of $1: 1,500$, lysol in a dilution of $1: 200$, peroxide of hydrogen $1: 35$, sulphuric acid $1: 150$, and hydrochloric acid $1: 150$. Inhibition is exerted by carbolic acid 1:550, and by bichloride of mercury 1:65,000. Exposure to direct sunlight kills streptococei in a few hours.

\section{CLASSIFICATION OF STREPTOCOCCI}

Since the first discovery of the chain forming cocei there has been much confusion concerning their classification.

The Gram-positive cocci of this morphological group are widely distributed in nature and vary very markedly in minor cultural characteristies and virulence, so that a unification of the group, as it has been possible with many other organisms, has been practically impossible. The earliest observers were forced to abandon their separation of the streptococci of erysipelas from other streptococci because of the work of Marbaix ${ }^{16}$ and others, who produeed erysipelas in rabbits with streptococci from non-erysipelatous lesions, after enhancement of their virulence. V. Lingelsheim ${ }^{17}$ proposed a purely morphological differentiation of "longus" and "brevis"; the former class including the streptococei usually found in pyogenic lesions with tendency to form chains of six or more links, the latter designating the short-chained varieties, including the less virulent streptococci. This classification, however, is not tenable because of the dependence of chain formation upon reaction, consistency, and nutritive qualities of the media employed for cultivation, and upon the influence of animal fluids if the microorganisms are taken direct from lesions. Schottmüller, ${ }^{18}$ in 1903 , proposed a classification based both upon morphology and the appearance of cultures upon. human blood agar. By this method he divided streptococci into two main

${ }^{15} v$. Lingelsheim, “Aetiol. u. Therap. d. Streptoc. Inf.," ete., Beit. z. Exper. Therap., Hft., 1, 1899.

${ }^{16}$ Marbaix, loe. eit. 1899.

${ }^{17}$ v. Lingelsheim, "Aetiol. u. Therap. đ. Streptokok. Krankh.," etc., Berlin,

${ }^{18}$ Schottmüller, Münch. med. Woch., 1903. 
groups as follows: I. Streptococcus longus or haemolyticus, consisting of the more virulent varieties, with tendency to form long chains, and producing hemolysis upon blood media. II. Streptococcus mitior or viridans, including less virulent strains, with usually shorter chainformation, and producing green, non-hemolyzing colonies upon blood media. A third group, Streptococcus mucosus, will receive special consideration in a separate section, and is probably more closely related to the pneumococei.

Another nomenclature has recently been suggested by Smith and Brown. ${ }^{19}$ These workers disapprove of the use of the name "streptococcus viridans" for the less hemolytic streptococei because many of them actually produce little or no green color on blood agar, and all of them did produce more or less hemolysis. They describe two types of streptococci with reference to hemolysis, calling the less hemolytic the "Alpha" type, and the markedly hemolytic the "Beta" type. A third type, "Camma," produces neither discoloration nor hemolysis. They describe them as follows:

Type Alpha.-As observed after forty-eight hours incubation the change produced may be described as a somewhat greenish discoloration and partial hemolysis immediately surrounding the colony, forming an indefinitely bounded zone, 1 to 2 millimeters in diameter, and surrounded by a second narrow clearer and not discolorized partially hemolyzed zone.

Type Beta.-Streptococei of this type produce hemolyzed zones on horse blood agar plates, radically different from those of the Alpha type. They produce clear transparent, completely hemolyzed colorless zones, 2 to 4 millimeters in diameter after forty-eight hours.

Type Gamma.-By this they mean streptococeus strains which grow within and on blood agar plates without the production of perceptible hemolysis or discoloration. Such strains are those described by Mandelbaum as streptococens saprophyticus and correspond to some of the Rosenow types.

Smith and Brown ${ }^{19}$ call attention to the great care which is necessary in regard to details of cultivation for observing streptococei for their hemolytic properties on blood plates. They contradict Ruediger's assertion that the formation of acid in such plates may give rise to the greenish coloration. There is no essential difference in result between plates made of defibrinated horse, rabhit

${ }^{19}$ Smith and Brown, Jour. Med. Res., 31, 1914, 455, and Monograph of Rock Inst., No. 9, January, 1919. 
or human blood. Meat infusion agar is more favorable than meat extract agar. The most favorable acidity is in the neighborhood of 1 per cent acid to phenolphthalein. The presence of dextrose in the medium produces a marked effect, and acid hemolysis may result in some of the so-called Gamma types. Very small amounts of dextrose in blood agar, however, may lead to partial or complete inhibition of hemolysis by the hemolytic Beta types. The reason that the Alpha types do not produce green zones in sugar-free media is explained by Smith and Brown as depending upon their scant growth in such media. Under anaërobic conditions Alpha streptococci and pneumococei simulate Beta type zones.

This broad classification into the hemolyticus ana viridans is the practical basis on which bacteriologists at the present time deal with the classification of pathogenic streptococci. Since, however, bacteria, morphologically and often culturally indistinguishable from those responsible for disease processes, can be found present on mucous membranes of the mouth, intestines, etc., in many normal persons without apparently doing the slightest harm, a great deal of effort has been spent in attempts to discover whether cultural characteristics could in any way be related to pathogenic properties. This has led to a great many investigations on the classification of various streptococci by carbohydrate fermentation. Such studies have been made by Gordon, ${ }^{20}$ Houston, ${ }^{21}$ Andrewes and Horder, ${ }^{22}$ Broadhurst, ${ }^{23}$ Winslow and Palmer, ${ }^{24}$ Hopkins and Lang, ${ }^{25}$ and Holman. ${ }^{26}$

Gordon found ten different fermentation reactions among twenty pyogenic streptococci examined, and forty-eight different fermentation reactions among two hundred streptococci isolated from saliva. Other work by Andrewes and Horder and by Buerger ${ }^{27}$ confirms the irregularity of the fermentation reactions within this group.

${ }^{20}$ Gordon, Rep. Med. Off. to Local Gov't Bd., Great Britain, 1902-3, 32 p. 421; ibid; 1903-4, 33, p. 388.

${ }^{21}$ Houston, Rep. Med. Off. to Local Gov't Bd., 1903-4, 33, p. 472.

${ }^{22}$ Andrewes and Horder, Lancet, 2, 1906, 708.

${ }^{23}$ Broadhurst, Jour. Infec. Dis., 1912, 10, 272.

${ }^{24}$ Winslow and Palmer, Jour. Infee. Dis., 7, 1910, 1.

${ }^{25}$ Hopkins and Lang, Jour. Infee. Dis., 15, 1914, 63.

${ }^{28}$ Holman, Jour. Med. Res., 34, 1916, 377.

${ }^{27}$ Andrewes and Horder, Lancet, 1906; Buerger, Jour. Exp. Med., ix, 1907. 
Andrewes and Horder suggest the following elassification:

(1) Streptococcus pyogenes. Grows in long chains and ferments lactose, saccharose, and salicin; does not coagulate milk. Streptococei which cause suppurative lesions or severe systemic infections belong to this group.

(2) Streptococcus mitis. A saprophytic type found frequently in the mouth which shows the same cultural characteristics as the streptococcus pyogenes, but grows in short chains.

(3) Streptococcus anginosus. Found frequently in throats of scarlet-fever patients which differs from the pyogenes only in coagulating milk.

(4) Streptococcus salivarius. A short-chain type which ferments lactose, saccharose, and raffinose, and coagulates milk. Streptococci of this type are found frequently in the mouth, but are rarely pathogenic.

(5) Streptococcus fecalis. A short-chain type which ferments lactose, saccharose, and mannite. This type is found normally in the intestine, and is oceasionally pathogenic.

(6) Streptococcus equinus. A short-chain type which does not ferment lactose. Found in horse dung and never pathogenic.

Quantitative determinations of the amount of acid formed in various sugars by different races have also been made by Winslow and Palmer $^{28}$ and others, but have led to no satisfactory classification.

Studies by Hopkins and Lang seem to show that the streptococei found in most human infections may be differentiated from the ordinary saprophytic types by the fact that they ferment lactose and salicin; but fail to ferment raffinose, inulin, or mannite. According to their results, the usual saprophytic types found in the mouth either fail to ferment salicin or ferment raffinose or inulin, whereas the usual fecal types ferment mannite. They also found in infection mannite fermenters which were apparently of fecal origin. Streptococci which gave the same fermentative reaction as the mouth saprophytes were, however, frequently found in malignant endocarditis.

Holman combined, or tried to eombine classification by the Gordon carbohydrate fermentations and by the blood agar medium, and somewhat simplified the difficulties. He recognized a hemolytic and a non-hemolytic group under each of which he classifies eight fermentative subgroups. The practical importance which he attaches to his investigations is that streptococei from similar sources

פs Jour. of Inf. Dis., No. viii, 1910, 1. 
very often fall into identical groups. He believes that by his method many of the air streptococei could be traced to their sources, and that similar relation between source and eultural characteristies could be found for streptococei from milk, from the mouth, from the intestinal tract, and from animal tissues. He concluded that individual groups of streptococci, however, were not specific in their disease production.

Cultural classification beyond the differentiation into hemolyticus and viridans, therefore, has not helped a great deal in characterizing the streptocoeci from the pathological point of view. It has been very important, therefore, to study the serological relationships of this group, a problem which, apart from its importance in pure classification, has fundamental bearing upon the questions of diagnosis and serum therapy. The earlier work of Arouson, ${ }^{29}$ Marmorek ${ }^{30}$ and others showed that, although the serum produced with any race of pyogenic streptococei frequently exerted considerable agglutinative and protective action against other strains, these relationships were variable and that antigenically the group was probably polyvalent. There was much confusion at first concerning these matters, but there has never been much doubt about the fact that sera produced with single strains or with only a few could not be relied upon to react with any chance strain obtained from a case. It was not until quite recently that more light has been shed upon this problem.

In order to deal clearly with the problem, it will be necessary to consider separately the viridans group and the hemolyticus group. The antigenic relationship between the two groups has been studied by Kinsella ${ }^{31}$ who finds that there is no absolutely sharp antigenic line between viridans and hemolyticus strains, but that sera immune to hemolytic streptococei were, in a definitely limited way, related to non-hemolytic antigens. On the other hand, sera immune to nonhemolytic strains gave no positive reactions with any of the hemolytic antigens. His work, however, was limited in the number of sera used, and one of his conclusions was that the hemolytic streptocoeci are probably not heterogeneous, but what he calls "unique." Kinsella and Swift ${ }^{32}$ also attempted to classify non-

${ }^{29}$ Aronson, Deut. med. Woch., 29, 1903, 439.

${ }^{30}$ Marmorek, Berl. klin. Woch., 31, 1902, 299.

${ }^{31}$ Kinsella, Jour. Exper. Med., 28, 1918, 181.

${ }^{32}$ Kinsclla and Swift, Jour. Exper. Med., 28, 1918, 169. 
hemolytic streptococei by means of complement fixation. In this work they used a number of strains and a large number of organisms, and found that the group was very heterogeneous. They developed in their work an interesting theory as to the inter-relationship of these heterologous groups. This is not sufficiently well founded to permit our going into it. It is, however, certain from their work, and from that of others, that the group of viridans is probably as antigenically heterologous as are the so-called Type IV pneumococci.

When we turn to the hemolytic group we find that a more hopeful state of affairs seems to be developing. There seemed to be from the beginning a certain amount of immunological grouping in organisms of this type. Baginsky and Sommerfeld ${ }^{33}$ found that agglutinins were present in scarlet fever serum which seemed to be specific for other strains isolated from the throats of scarlet fever patients, and this observation was confirmed by Moser and Von Pirquet. ${ }^{34}$ A similar apparent similarity between strains isolated from the blood of smallpox cases was found by De Waele and Sugg. ${ }^{35}$ Neufeld ${ }^{36}$ suggested that there was a relationship between agglutinability and virulence. Kinsella and Swift ${ }^{37}$ studying this problem in 1918 came to the conclusion that with complement fixation methods, at least, the hemolytic variety of streptococeus is homogeneous, consisting of members that are almost identical. More recent work, however, by Dochez, Avery and Lancefield ${ }^{38}$ seems to have shown that by means of agglutination it may be possible to classify the hemolytic streptococci into immunological sub-groups. They define four biological types which check up both by agglutination reactions and by protection experiments. They encountered at least two other types, and indications that more existed. Their work seems well founded on experimental fact, and has been recently confirmed to this extent that Bliss, working with the streptococei obtained from the throats of scarlet fever cases found that the organisms from such sources seemed to fall into a single group. The specificity of streptococci has also been recently studied by

${ }^{33}$ Baginsky and Sommerfeld, Berl. klin. Woch., 37, 1900, 588.

${ }^{34}$ Moser and Von Pirquet, Cent. f. Bakt., I, 1903, 34, 560.

${ }^{35} \mathrm{De}$ Waele and Sugg, Arch. Internat. pharm. et therap., 12, 1904, 205.

${ }^{26}$ Neufeld, Zeit. f. Hyg., 40, 1902, 54.

${ }^{37}$ Kinsella and Swift, Jour. Exper. Med., 28, 1918, 877.

${ }^{3}$ Dochez, Avery and Lancefield, Jour. Exper. Med., 30, 1919, 179. 
Tunnicliff. $^{39}$ She has given particular attention to absorption tests, and has found, in agreement with Bliss, that the hemolytic streptococci from scarlet fever form a distinct group, these organisms removing opsonins and agglutinins for these cocci, whereas, erysipelas eocci have no such effect. With scarlet fever streptococci in a sheep, Tumnicliff produced a serum which agglutinated hemolytic streptococci in dilutions of 1 to 2000, and opsonized them in dilutions of 1 to 360 . She found that this serum protected mice against the hemolytic streptococei isolated from scarlet fever patients, but not from erysipelas, mastoiditis and influenza. This protective power was rapidly lost by the sheep serum, but was restored by the addition of normal sheep serum. Her further work indicates that, like the scarlet fever cocci, the erysipelas cocci form a distinct group, also.

As we shall see, the difficulty with these investigations has largely been technical in that the spontaneous agglutination of the hemolytic streptococci makes it very difficult to carry out reliable agglutination work. These difficulties will be alluded to in connection with agglutination.

Virulence and Pathogenicity.-Different races of pyogenic streptococci show considerable variations in virulence, and there are few organisms, pathogenic both for animals and man, which show comparable fluctuations in virulence. The character or severity of the lesion in man gives little evidence as to the virulence of the organism for animals. Such differences are, to a certain extent, dependent upon inherent individual characteristics, but are rather more likely to be the consequences of previous environment or habitat. Prolonged cultivation upon artificial media usually results in the reduction of the virulence of a streptococcus, while an originally low or reduced virulence may often be much enhanced by repeated passage of the streptococei through animals. It is noteworthy, however, that while the passage of a streptococcus through rabbits will usually enhance its virulence for susceptible animals in general, repeated passages through mice may increase the virulence for these animals only, even occasionally depressing the virulence for rabbits.

Among the domestic animals, those most susceptible to experimental streptococcus infection are white mice and rabbits. Guineapigs and rats are less easily infected, and the larger domestic animals,

30 Tunnicliff, Jour. of the A. M. A., 75, 1920, 1339. 
cattle, horses, goats, cats, and dogs, are relatively refractory. Al. most complete immunity toward streptococeus infections prevails among birds.

The nature of the lesions following experimental animal inoculation depends upon the manner of inoculation, the size of the dose given, and most of all upon the grade of virulence of the inoculated germ. Subcutancous inoculations, according to the virulence of the inoculated material, may result in a simple localized abscess, differing from a staphylococcus abscess only in the more serous nature of the exudate and the frequent occurrence of edema, or in a severe general septicemia with a hardly noticeable local lesion. Subcutaneous inoculation of mice may result in general sepsis followed by death within thirty-six to forty-eight hours, or less, with the presence of streptococei in the heart's blood and the viscera. Intrapleural or intraperitoneal inoculation of susceptible animals with virulent streptococci leads usually to a peculiarly hemorrhagic form of exudate, due both to the diapedesis caused by the violent inflammatory process, and to the hemolysis of the red cells by the streptococcic hemolysins. Inoculation of rabbits at the base of the ear with virulent streptococci may result in the formation of a lesion indistinguishable histologically from erysipelas in man. ${ }^{40}$ Marbaix $^{41}$ has shown that such erysipeloid lesions could be produced in rabbits by streptococei from various and indifferent sources, provided that the virulence of each strain could be sufficiently enhanced.

Intravenous inoculation of rabbits with virulent cultures usually results in a rapidly fatal septicemia. An animal which has died of a streptococeus infection usually shows serosanguineous edema about the point of inoculation, multiple hemorrhagic spots upon the serous membranes, and congestion of the viscera. The microorganisms can almost invariably be found in the heart's blood, in the spleen, and in the exudate about the inoculated area. Microscopically, when the process has lasted sufficiently long, parenchymatous degeneration of all the organs may be observed. In the more chronic infections articular and periarticular lesions may occur. ${ }^{42}$

Spontaneous streptococcus disease seems to occur among some of the larger domestic animals.

40 Fehleisen, loc. eit.; Frankel, Cent. f. Bakt., vi.

${ }^{41}$ Marbaix, La Cellule, 1892.

${ }^{42}$ Schütz, Zeit. f. Hyg., iii ; Hiss, Jour, Med, Res., xix, 1908. 
Thus, in horses, mules and donkeys, there is an acute contagious disease of the upper air passages, colloquially known as "strangles" (in Cerman, Druse) due to the streptococens equinus, a non-hemolysing, salicin fermenting strain. The etiological significance of this organism was first recognized by Schüt ${ }^{43}$ and has been subsequently confirmed. The disease attacks chiefly young animals, is characterized by fever and general systemic depression, with subsequently an acute catarrh of the nasal and pharyngeal mucosa, with local glandular swelling. Often the submaxillary gland may be involved, and pneumonia may ensue. In cattle, streptococei are most frequently associated with the inflammations of the udders. Streptococcus mastitis may seriously effect the quality and quantity of the milk, and perhaps has very definite relationship to disease in human beings, a matter to which we will refer in a subsequent paragraph. Occasionally, post-partum uterine inflammation in cows may be caused by streptococci.

In chickens there oceurs a form of septicemia caused by a streptococeus which is described by Moore ${ }^{44}$ as being an anaërobe which does not liquefy gelatine, does not coagulate milk, forms acid in alkalin broth and grows in flaky masses like a streptococeus hemolyticus in broth cultures. Moore does not state whether it produces hemolysis or not. The disease has a sudden onset, and is usually fatal. It is apparently transmitted from chicken to chicken.

Among the smaller laboratory animals, occasional streptococeus infections may be observed in rabbits. Recently an epidemic disease among white mice due to streptococcus was studied by Kutscher. ${ }^{45}$ As a rule, however, streptococcus disease is by far more rare among animals than it is among human beings.

Streptococcus INFEctions IN MAN.-In man, a large variety of pathological processes may be caused by streptococei and here again the nature of the infection, whether definitely localized or generally distributed, depends upon the relationship existing between the virulence of the incitant and the resistance of the subject.

Superficial cutaneous infections are frequently caused hy streptococei and these in the milder eases may be similar to the localized abscesses caused by staphylococci. In severe cases, however, infec-

${ }^{43}$ Schutz, Arch. f. Thier, Heilk., 14, 1889, 172.

${ }^{44}$ Moore, Pathology, ete., of Infec. Diseases in Animals, MacMillan, New York, 1916.

${ }^{45}$ Kutscher, Cent. f. Bakt., xlvi. 
tion is followed by rapidly spreading edema, lymphangitis, and severe systemic manifestations with the development of a grave cellulitis, often threatening life and requiring energetic surgical interference.

The particular significance of streptococei in surgically infected wounds and the effect of their presence upon therapeutic procedures is considered in another section dealing with the bacteriology of infected wounds.

Suppurations of bone may be caused by streptococci, and constitute a severe form of osteomyelitis. Such lesions when occurring in the mastoid bone are not infrequently secondary to streptococcus otitis and may lead to a form of meningitis which is in most cases fatal.

Streptococcus meningitis fortunately does not occur very often, but when it does occur is usually fatal. Most cases are probably secondary to such lesions as otitis and mastoiditis mentioned above, but occasionally primary streptococcus meningitis may occur in the course of bronchopneumonia. We have seen a number of cases in which streptococei were associated in the spinal fluid with influenza bacilli.

As mentioned above, erysipelas is a streptococeus hemolyticus disease. It was first isolated from such lesions by Fehleisen ${ }^{46}$ who believed that the organism obtained by him was a particular variety, specific in erysipelas. He named it, for this reason, streptococcus erysipelatis. Subsequently, however, it was shown by Marbaix ${ }^{47}$ and Petruschky ${ }^{48}$ and others that erysipelas-like lesions could be produced in animals with streptococei from many other sources. The production of the peculiar erysipelas lesion seems to depend, on the one hand upon the relative virulence of the strain and upon the infection of the lymphatics of the skin. The streptococci in this disease are, according to MacCallum, ${ }^{49}$ located in the crevices of the tissues and the lymph channels of the skin. A peculiarity is that, unlike streptococcus lesions in other places, in this disease the inflammatory exudate consists very largely by mononuclear cells.

${ }^{46}$ Fehleisen, Aeriol. d. Erysipelas, Berlin, 1883.

${ }^{47}$ Marbaix, La Cellule, 1892.

${ }^{48}$ Petruschky, Zeit. f. Hyg., 13.

${ }^{49}$ MacCallum, Textbook of Pathology, Second Edition, 1920; Monograph of the Rock. Inst., No. 10, 1919. 
This disease is particularly dangerous in infants in whom it may spread over the entire surface of the body.

It was furmerly supposed that a number of different forms of acute enteritis might be due to streptococci, but this has never been positively demonstrated, and is doubtful. However, streptococcus infections of the walls of the intestines by passage into the submucosa may occasionally oceur.

The inflammation which is known as Ludwig's angina is usually of a streptococcus hemolyticus nature. In this condition the origin is usually from a focus in the teeth, tonsils or pharynx, or perhaps a peritonsular abscess, and consists of a violent, acute inflammation of the areolar tissues of the submaxillary region of the neck.

Superficially, infections through the skin, through abrasions and injuries are among the most frequent and dangerous infections caused by the hemolytic streptococcus. These are particularly frequent among surgeons and pathologists, and their course and outcome is determined entirely by the relationship between virulence of the strain and resistance of the individual. Given a sufficiently virulent strain, a very minute abrasion or injury with the inoculation of a small quantity of the organisms only, may suffice to result in a rapid and fatal infection. We know of one autopsy infection in which a minute pin prick through a rubber glove, hardly visible to the eye, resulted, within twelve hours, in high fever, and within twenty-four hours, in delirium with little red lymphatic lines running up the arm from the source of the lesion. In such cases, the local lesion may look relatively innocent, since, with sufficient virulence, the point of inoculation may show nothing more than a small red swelling which is soggy and edematous and looks quite different from the slower processes, with central abscess formation, caused by the less virulent streptococci and staphylococci.

Puerperal sepsis is one of the most dangerous of the infections caused by the hemolytic streptococei, but with the advent of more perfect obstetrical methods, this grave infection of the uterus is becoming more and more rare.

From all of the streptococcus lesions a general infection of the blood stream may arise, if the infection proceeds with sufficient virulence. Although both the viridans and the hemolyticus strains may be responsible for such septicemic conditions, the most frequent and dangerous ones are those caused by the hemolyticus. We will devote a separate paragraph to a description of the viridans 
septicemia accompanying endocarditis. Hemolyticus septicemia, secondary to wound infection, puerperal septicemia or other lesions, are usually initiated by grave symptoms of fever, chill and general depression, and the organisms can be isolated in blood cultures taken in agar plates or in hormone broth. It is an important thing to remember that in all of these septicemias the presence of the organisms in the blood may not signify that they are actually multiplying in the blood. It is, in our opinion, more likely that the organisms at first simply enter the blood stream from the lesion, and are destroyed in the circulation. If, during this period, the focus can be surgically cleaned out, rapid recovery may follow. We have seen cases, in which the surgical lesion was accessible to recover promptly after operation, although blood cultures taken before had shown numerous organisms in the blood stream. In cases dead of streptocococcus septicemia, the organisms may be cultivated from all the organs and from the heart's blood.

In dealing with other streptococcus lesions in man, it will be convenient for us to differentiate sharply between those caused by the hemolyticus and those due to the viridans strains. Since normal human beings harbor hemolytic streptococei in the mouth and pharynx in a large percentage of individuals examined, it is natural that inflammations of the upper respiratory tract should frequently be due to streptococci. Thus, the hemolytic streptococci are frequently the causative agents in pharyngitis, and are often associated with the more severe forms of follicular tonsilitis. Some of these tonsillar infections may be accompanied by high fever, and severe illness, and the local inflammation may be so severe that it cannot be clinically differentiated, with certainty, from diphtheria. From such throat infections, septicemia does not often follow, but occasionally severe generalized infections may ensue. MacCallum describes the case of a physician, one of our colleagues, who had repeated attacks of throat infection caused by streptococci. In one of these he developed glands of the throat which were incised and streptococcus pus was found. A year later there was a sudden recurrence with a rapidly developing general septicemia, and a scarlatiniform rash. He died within a few days. In connection with this, it is interesting to note that we have seen three or four cases which occurred among soldiers in France who, in the course of severe streptococeus throat infections developed rashes that were difficult to distinguish from those of scarlet fever. Just what the 
explanation of these eruptions is, we cannot say. They occur in fatal septicemias of many varieties, but are particularly associated with fatal streptococeus infection.

In scarlet fever, hemolytic streptococei are invariably found associated with and probably the causative agents of the severe angina that regularly accompanies this disease. This was observed by Loeffler in 1884, and has been confirmed, since then, by many observers. The regularity of these findings has been such that it has led to the assumption that the hemolytic streptococci might be etiologically related to scarlet fever itself. This is a problem which has been much discussed, but cannot be thoroughly gone into without an extensive review of the literature. We may say that the general weight of evidence is at the present time distinctly against such an assumption, and that it is most likely that the streptococci in scarlet fever are the most regular and severe secondary invaders. Yet, it must be admitted that no conclusive denial of the possibility can be made on the basis of experimental evidence. The hemolytic streptococci not infrequently invade the blood stream of scarlet fever patients in the course of the disease. Baginsky and Sommerfeld $^{50}$ found them in the blood at autopsy, and Anthony ${ }^{51}$ and others have confirmed this. Anthony found them in the heart's blood of ten out of eighteen autopsies. An important fact first noted by Moser and Von Pirquet ${ }^{52}$ is the apparent antigenic identity of strains of hemolytic streptococci isolated from different cases of scarlet fever. Bliss has recently confirmed this by the newer and more reliable methods of agglutination developed by Dochez and Avery. ${ }^{53}$

In diphtheria and in smallpox the hemolytic streptococei are frequently found as secondary invaders. Gay ${ }^{54}$ who has carefully analyzed the literature on streptococcus infection, cites a number of observers who have found the organisms in the blood streams of fatal cases of smallpox, and in some of those that recovered.

It has long been known that the dangerous bronchopneumonias which occur in the course of measles and influenza may be of strep-. tococcus origin. Fyre ${ }^{55}$ has made particular studies of this condition

so Baginsky and Sommerfeld, Berl. klin. Woeh., 27, 1900.

"Anthony, Jour. Infee. Dis., 6, 1909, 332.

52 Moser and Ion Pirquet, \%eit. f. Bakt., 34, 1903, 560 and 714.

"s Jochez and Avery, and Lancefield, Journ. Exp. Merl., vol. 30, 1919, P. 179.

5t Gay, Jour. of Lab. and Clin. Med. 3, 1918, 721.

${ }^{55}$ Eyre, Jour. of Bacter. and Pathol., 14, 1910, 160. 
in measles bronchopneumonias, and for both measles and influenza much information has been gathered during the late war. It appears that both measles and influenza, as well as perhaps some other mild infections of the upper respiratory tract, render the individual tremendously susceptible to secondary infection. Under conditions such as those developed in the camps during the war, these streptococcus bronchopneumonias may become epidemic, probably by reason of the generalized interchange of mouth organisms among susceptible individuals crowded together under camp conditions. Epidemiologically, it is of interest to note that under these conditions the carrier rate of hemolytic streptococci reaches high percentages. Irons and Marine, ${ }^{56}$ at Camp Custer, found 70 per cent of the individuals examined to be carriers of these organisms, and Levy and Alexander ${ }^{57}$ in certain regiments found 89 per cent to be carriers. Given, at the same time, extensive outbreaks of measles and influenza, the conditions for widespread secondary streptococcus pneumonias are established. It is not impossible that widespread ward infection may occur, in that patients who enter hospitals with measles and influenza, even without being carricrs of hemolytic streptococci, may pick them up in the hospitals from adjacent beds, from doctors or from nurses, possibilities which indicate the great importance of prompt removal of cases developing pneumonias from measles and influenza wards, the careful hygiene of the mouths and throats of such cases, isolation of beds by screening, and the wearing of masks by doctors and nurses, not so much for their own protection as for that of the susceptible patient. MacCallum, Cole and Dochez ${ }^{58}$ made careful studies of the streptococcus pneumonias occurring at some of the camps. The pathological facts resulting from this study are reported by MacCallum in the 10th Monograph of the Rockefeller Institute, issued in 1919. According to him, the streptococci seem to extend downward into the smaller bronchioles, giving rise to intensive inflammations in the air passages, then extending into the network of lymphaties surrounding the bronchioles and the pleura. There was a rapid production of pleurisy and empyema, with hemorrhage about the bronchioles and a very curious infiltration of the alveolar walls themselves with leucocytes chiefly

${ }^{56}$ Irons and Marine, Jour. A. M. A., 70, 1918, 687.

${ }^{57}$ Levy and Alexander, Jour. A. M. A., 1918, 1827, Vol. 70.

${ }^{58}$ MacCallum, Cole and Dochez, Jour. A. M. A., 70, 1918, 1146. 
of the mono-nuclear variety. The appearance of these processes, according to MacCallum, is quite different from that seen in the ordinary forms of bronchopneumonia.

\section{EPIDEMIC SORE THROAT DUE TO MILK INFECTION}

Sore throat epidemics traceable to milk have been observed in England since 1875. The onset of these cases is usually accompanied by sudden chilliness, with muscular soreness, headache and nausea. The cases are strikingly similar to the milder forms of influenza. The first carefully observed epidemic in this country occurred in Boston in 1911, and was epidemiologically studied by Winslow. ${ }^{59}$ There were 48 fatal cases in the Boston epidemic. Since that time a number of similar epidemics have been described, one of the most extensive being that which took place in Chicago in 1911 and was studied by .Capps and Miller, ${ }^{60}$ and by Davis and Rosenow. ${ }^{61}$ In the Chicago epidemic there were 10,000 cases, hardly any of which came from the west side of the eity. The relationship to the milk supply was carefully studied. Of 622 cases investigated, 87 per cent or 537 used milk from a certain dairy, and 79 per cent of the fatal cases used the same milk. People taking milk from this dairy were fourteen times more numerous than those getting it from other sources. Of 153 nurses in a certain hospital using the milk, 80 per cent got the disease, while of 721 in other hospitals, only 4.8 per cent eame down. There was a coincident epidemic of sore throats among the employees of the dairy where bovine mastitis was found in the cows. In fact, almost 5 per cent of the cows of this dairy had mastitis, and streptococci were isolated from the milk of a cow and from the throat of a girl on the same farm. Davis and Rosenow describe the organisms isolated from these cases. In all of them they found a streptococcus which produced large colonies on blood agar, larger than the ordinary hemolytic organisms. There was moderate hemolysis, and the organism was virulent for guinea pigs, mice and rabbits. Capsules were developed on animal passage. They believed their organism, which they called streptococcus epidemicus, to be a distinct species. However, Davis, in a subsequent

${ }^{59}$ Winslow, Jour. Infec. Dis., 10, 1912, 73.

(c) Capps and Miller, Jour. A. M. A., 58, 1912, 1848.

${ }^{61}$ Davis and Rosenow, Jour. A. M. A., 58, 1912, 773. 
paper $^{62}$ declares the organism to be indistinguishable from other hemolytic streptococci.

Viridans InfEctions.-The non-hemolytic or so-called green streptococei are, as stated above, more likely to be associated with the less severe and subacute lesions. These organisms are usually harmless saprophytes.

They are associated frequently with tooth abscesses, may be present in middle ear disease and infections of the accessory sinuses of the nose. They are not infrequently found in the tonsillar crypts and have been known to eause mild forms of tonsillitis.

The viridans is frequently associated with subacute vegetative endocarditis. In such cases the organisms cause relatively firm vegetations, usually on the mitral, but sometimes also on the aortic valves, extending occasionally along the walls of the auricle. The organisms can be readily cultivated from the blood stream where they may remain for weeks and months. Often, in such cases the resistance of the patient is unusually high for a long time, the bacteria probably being disposed of in the blood stream, but are passed either constantly or intermittently into the blood stream from the infected valves. Death may ensue after weeks or months. Death usually follows by a gradual wearing down of the patient's resistance. Cerebral or renal embolism may occur, and joint conditions may develop secondarily.

It has been suggested that the frequent association of rheumatism and cardiac lesions may be due to the fact that the green streptococei are responsible for both conditions. An enormous amount of rather confusing work has been done upon the etiology of rheumatism from this point of view. It is a fact that many coceus infections, and, for that matter, other forms of general infection, may lead to secondary joint lesions in both animals and man. Poynton and Paine $^{63}$ in 1900 described a diplococeus not very dissimilar from viridans strains which they obtained from eight eases of acute rheumatic fever, and with which they claimed that they could produce lesions in rabbits which they considered typical of rheumatism. The organism was recovered from the blood and pericardial fluids of their patients. They describe them as minute diplococci, grown best in acid media under anaërobic conditions, but capable of growth

${ }^{62}$ Davis, Amer. Jour. Pub. Health, 8, 40, eited after Gay, loc. cit.

63 Poynton and Paine, Lancet, London, 1900, 860 and 932. 
on the surface of ordinary media. Recently, Rosenow ${ }^{64}$ reported the isolation of streptococcus viridans from the joints of seven cases of articular rheumatism. He also claimed that he could produce non-suppurative arthritis, endocarditis and pericarditis in rabbits with these cultures. He described the organisms as intermediate in character between the ordinary viridans and the hemolyticus. These elaims have led to a great deal of subsequent experimentation and speculation. There is no question about the fact that viridans strains have often been isolated from cases of rheumatism, but it is not unlikely that equal care in similar attempts would have suceeded in isolating these ubiquitous organisms from many other groups of disease. Swift and Kinsella ${ }^{65}$ have made a particularly careful study of this problem, and, though working with great care and experience, obtained the viridans from only 8.3 per cent of 58 cases. In no case did they obtain the organisms from the joints themselves, and we ourselves can attest to the fact that the large majority of direct cultures taken from rheumatic joints prove quite sterile. While, therefore, the rôle of the viridans in endocarditis is clear, its relationship to rheumatic fever is entirely unproven at the present writing.

The same uncertainty exists concerning its association with another disease which is frequently classified with the two diseases mentioned, namely, chorea. A number of writers, notably Quigley, ${ }^{66}$ have found the viridans in the blood of chorea cases, but nothing more definite than this has been noted.

Rosenow, ${ }^{67}$ too, has recently claimed that there was an association between viridans-like streptococei and poliomyelitis. In the special section on poliomyelitis in this book we will deal with the globoid or coccoid bodies isolated by Flexner and Noguchi. ${ }^{68}$ Some two or three years, after these observations were made, Rosenow and Towne, ${ }^{69}$ as well as Mathers, ${ }^{70}$ reported that they had found streptococei of the viridans group in the central nervous system of cases of poliomyelitis. Rosenow and Wheeler ${ }^{71}$ grew these cocci

\footnotetext{
64 Rosenow, Jour. A. M. A., 60, 1123 and 61, 1947 and 2007.

${ }^{65}$ Swift and Kinsella, Arch. Inter. Med., 19, 1917, 381.

${ }^{6}$ Quigley, Jour. Infec. Dis., 22, 1918, 198.

${ }^{67}$ Rosenow, Jour. Infec. Dis., 22, 1918, 379.

Filexner and Noguchi, Jour. Exper. Med., 18, $1913,461$.

${ }^{69}$ Rosenow and Towne, Jour. Med. Research, 36, 1917, 175.

"Mathers, Jour. A. M. A., 67, 1916, 1019.

"Rosenow and Wheeler, Jour. Infec. Dis., 22, 1918, 281.
} 
under anaërobic conditions, and found that minute forms developed, small enough to be filtered, and they believed that it was these anaërobic minute forms that represented the coccoid bodies of Noguchi. It is very difficult to give any conclusive judgment on this matter. Bull could not confirm Rosenow's work, and showed that the organisms isolated by him were neither uniform in eultural behavior, nor had they any specificity as regards the production of lesions in animals. The weakest parts of Rosenow's work are his animal experiments in which he produced lesions in guinea pigs, dogs and monkeys with these cultures; none of these animals have been found susceptible to poliomyelitis virus derived directly from the filtered brain tissues of human cases. Mistakes are quite likely to occur since cocei similar to the Rosenow types may be found in a considerable number of rabbits, and even in monkeys dead of a variety of conditions, unless cultures are taken before or very soon after death.

Personally we do not believe that Rosenow is right.

The Question of Tissue Specificity of the Viridans.-Rosenow, in the course of the work cited above, isolated viridans streptococei not only from poliomyelitis brains and rheumatic cases, but also from gastric ulcers, and in the course of this work he developed the theory that individual strains of these organisms may aequire a specific affinity for particular tissues. His idea is that organisms isolated from rheumatic lesions, gastric lesions, ete., will selectively lodge in analogous tissues on animal injection or on gaining entrance to another individual. Other observers have not been able to confirm this claim, and, indeed, it is very unlikely that sueh a thing occurs. At any rate, no definite proof has been brought, and the weight of evidence is against his claim. It is an interesting thought, yet a dangerous one to spread broadeast, since it has influenced clinical thinking to an extent not warranted by experimental fact. In the specific localization of organisms in the tissues, it seems to us much more likely that tissue factors are paramount, such as perhaps specific hypersusceptibility, or local reduction of resistance. Such a thought is indicated by the work of Faber ${ }^{72}$ who sensitized joints with extracts of streptococci, subsequently producing lesions in these joints by intravenous injection of the microorganisms themselves. None of these ideas, however, have been proven, and the entire question remains one of the difficult, unsettled problems.

${ }^{72}$ Faber, Jour. Exper. Med., 22, 1915, 615. 
Toxic Products.-In spite of extensive researches by many investigators upon the nature of the poisons produced by streptococei, our understanding of these substances is still very incomplete. The grave systemic symptoms so often accompanying comparatively slight streptococcus lesions argue strongly for the production by these microorganisms of a powerful diffusible poison. Toxic filtrates of streptococcus cultures have indeed been obtained by Roger, ${ }^{73}$ Marmier, ${ }^{74}$ Baginsky and Sommerfeld, ${ }^{75}$ Marmorek, ${ }^{76}$ and many others; but these have in no case been comparable in potency to the soluble toxins of diphtheria or of tetanus. When injected into young guinea-pigs in sufficient quantity, these filtrates produce rapid collapse and death.

Aronson $^{77}$ in 1902 found that filtrates from streptococcus cultures made in various ways were practically without toxicity, although he occasionally obtained toxic symptoms by giving very large doses of two to four-day cultures. The residue of streptococcus body substances was not toxic at all. Simons ${ }^{78}$ in 1904 found a similar lack of toxicity of the bodies of the organisms, but claimed that filtrates of broth cultures two to nine days old produced emaciation and diarrhea in rabbits. He claimed that it was necessary to grow the organisms in the presence of animal exudates or leucocytes, a claim which corresponds to Marmorek ${ }^{79}$ ideas. Braun ${ }^{80}$ found some toxicity of filtrates of streptococcus cultures for rabbits. More recently, Clark and Fenton ${ }^{81}$ cultivated hemolytic streptococci upon Locke's solution containing defibrinated blood and 0.5 per cent glucose. They claimed that 0.5 to 1 c.c. of the filtrates of such cultures killed rabbits with considerable regularity.

We ourselves have made similar studies of streptococeus poisons and were unable to confirm the claim of Clark and Fenton though following the very same method. We have found, however, that cultures of various hemolytic streptococei made upon hormone broth, and similar broth with defibrinated blood, grown for from

${ }^{73}$ Roger, Rev. de méd., 1892.

${ }^{74}$ Marmier, Ann. de l'inst. Pasteur, ix, 1895, p. 533.

${ }^{75}$ Baginsky und Sommerfeld, Berl. klin. Woch., 1900.

${ }^{76}$ Marmorek, Berl. klin. Woch., 1902.

${ }^{77}$ Aronson, Berl. klin. Woch., 1902, 39.

${ }^{78}$ Simons, Cent. f. Bakt., 35, 1904.

${ }^{79}$ Marmorek, Ann. de 1'Inst. Past., 16, 1902, 169.

${ }^{80}$ Braun, Cent. f. Bakt., 61, 1912, 383.

${ }^{81}$ Clark and Fenton, Jour. A. M. A., 71, 1918, 1048. 
eighteen to twenty-four hours under partially anaërobic conditions, contained definite toxic substances. Filtered through Berkefeld candles and injected into rabbits in quantities ranging from 3 to 6 cubic centimeters, sickness consisting of general muscular weakness, respiratory difficulty, and in about 8 per cent of the eases death ensued. Similar toxic substances could be obtained by repeatedly washing young agar growths of streptococei in salt solution and filtering immediately. We do not regard these as specific exotoxins, but believe them to be entirely similar to substances obtained in the same way from young influenza cultures, typhoid, dysentery, meningococcus and prodigiosus cultures. The work on these substances is not yet completed. They are not antigenic and not specific, and repeated injection into rabbits in sublethal amounts produces chronic emaciation and usually death after five or six injections. They may well have important bearing on the symptoms of streptococcus diseases. They are certainly neither specific exotoxins nor endotoxins, and, for the present, we speak of them, for lack of a better term, as " $\mathrm{X}$ " substances. ${ }^{82}$

STReptococcus Hemolysin.-The production of hemolysin by streptococci has been mentioned briefly in connection with our section on classification, since this property has become one of the important criteria of differentiation. The substance responsible for the laking of red blood corpuscles, being a secretion of the organisms, comparable to the true toxins of some other bacteria, has been made the subject of a considerable number of investigations. The observation of this phenomenon was first made by Marmorek ${ }^{83}$ in 1895. Marmorek believed that there was a direct relation between virulence and hemolytic power. Other investigators, however, notably Schottmüller, ${ }^{84}$ believed, from the beginning, that the hemolytic power was a constant characteristic of certain strains, unchangeable by experimental enhancement or reduction of the virulence. As mentioned before, the streptococeus hemolysins may be conveniently observed by cultivation of the organisms on blood agar plates. The criteria for media; blood and reaction have been mentioned above. The hemolysins may also be obtained in liquid media by the filtration of young liquid cultures. Apparently it is

${ }^{82}$ Zinsser, Jour. of Immunol., 5, 1920, No. 3, p. 265; Zinsser, Parker and Kuttner, Transac. Soc. Exp. Med. and Biol., Dec., 1921.

${ }^{83}$ Marmorek, Ann de l'Inst. Past., 1895.

${ }^{84}$ Schottmüller, Mun. med. Woch., 1903. 
advantageous that media for this purpose should contain ascitic fluid or serum. The first to obtain streptolysin in this was Besredka. ${ }^{85}$ The nature of the filter used for this purpose makes a considerable amount of difference, and it is stated by De Kruif and Ireland ${ }^{86}$ that the Maassen filters are the most suitable ones, and that even with these considerable amounts of hemolysin are lost in filtration. According to Besredka, Braun ${ }^{87}$ and others, the streptolysin is not a very stable substance, and for this reason it is necessary to filter the cultures at a point of optimum growth. The same observation has been made by M'Leod ${ }^{88}$ who obtained the best results by filtering serum broth cultures after sixteen to eighteen hours' growth. A careful experimental study of the substance has recently been made by DeKruif and Ireland in the paper cited above. They paid particular attention to the correlation of streptolysin production to the logarithmic growth curve of the organisms. The broth used by them in most of their experiments consisted of a 2 per cent pepton beef infusion broth, brought to a of 7.8 with $P_{H}$ sodium carbonate. To this broth, following the methods of M'Leod and others, animal serum in various concentration was added. The best yields were obtained with sheep serum at a concentration of 20 per cent, although similar concentrations of human and rabbit serum gave good yields.

By avoiding filtration and using the high-power centrifuge, the ordinary loss of hemolysin incident to filter adsorption can be avoided. The hemolytic effects can then be titrated against various types of red corpuscles by adding the supernatant fluid or filtrate in graded quantities to 0.5 c.c. of a 2.5 per cent suspension of washed cells.

De Kruif and Ireland's results, in the main, confirm the observations of others, namely, that the hemolysin appears early in the cultures, and when the broth flasks are seeded with young agar cultures the peak may be reached at from seven to eight hours. When the crest of the growth curve has been passed, the lysin begins to decrease and may disappear completely in fourteen hours.

According to Braun, the streptolysin is extremely sensitive to heat. Six hours exposure at $37^{\circ}$ seemed to destroy it. Six hours

85 Besredla, Ann. de 1'Tnst.Past., 15, 1901, 880.

s6 DeKruif and Ireland, Jour. Infee. Dis., 26, 1920, 285.

si Braun, Cent. f. Bakt., 62, 1912, 383.

ss II'Leod, Jour. Pathol, and Bacter., 16, 1912, 321. 
at room temperature caused decided reduction, but, kept in the ice-box for this period, reduction was slight only.

The substance is not apparently specific for any one variety of cell, but as produced from most strains it may effect the red cells of human beings, of mice and rabbits of sheep and of horses, indiscriminately, though perhaps with quantitative differences. Both Besredka and Braun failed to produce an anti-lysin for the streptococcus substance. The nature of the anti-lytic properties possessed by some normal sera is not entirely clear. Since it is an extremely heat stable substance, being but incompletely destroyed at $100^{\circ} \mathrm{C}$., it has been suggested that it could not be an antibody in the ordinary sense of the work, but may consist of lypoidal substances like cholestrin in the blood stream.

Streptolencocidin.--In 1905 Ruediger $^{89}$ observed that filtrates of virulent streptococci would prevent the phagocytosis of nonvirulent streptococci by leucocytes. The same thing was affirmed by Hektoen. ${ }^{90}$ Recently, Nakayama ${ }^{91}$ has made more detailed studies of streptoleucocidin. He finds that, as a general rule, hemolytic and non-hemolytic streptococci, when non-virulent, and easily taken up by phagocytosis, under the influence of normal serum, do not produce leucocidin. Virulent strains, however, which are not phagocytable produce leucocidin in demonstrable quantities. In cultures grown on 10 per cent serum broth, the largest quantity of leucocidin was produced in from ten to twenty-four hours, after which production fell off. The largest quantities were obtained in broth containing goat serum and horse serum. There was a definite relationship between the volume of production and virulence. The substance was rendered inactive by heating at $58^{\circ}$ to $60^{\circ}$ for thirty minutes. It was unstable on preservation, and could not be reactivated by the addition of small quantities of fresh culture fluid. Normal serum and leucocytic extract possess some anti-leucocydal power, a property which is destroyed by heating at $70^{\circ}$ for thirty minutes.

He mentions that immunization with streptococci did not produce anti-leucocydal properties, but injections with the leucocydal culture fluids were successful. The streptoleucocidin seemed to be distinct

${ }^{89}$ Ruediger, Jour. A. M. A., 44, 1905, 198.

.90 Hektoen, Jour. A. M. A., 46, 1906, 1407.

${ }^{91}$ Nakayama, Jour. Infec. Dis., 27, 1920, 86. 
from the streptolysin, and the antibody against it did not neutralize streptoleucocidin.

Nakayama used the following method for the determination of leucocidin, a method which is given because it can be applied to tests for similar substances from many organisms. His procedure was as follows: Leucocytes were obtained by injecting aleuronat as usual. When rabbits were used the aleuronat was injected into the pleura. After twelve hours, the animals were bled, and the exudate withdrawn after opening the chest, and mixed with equal amounts of 1.5 per cent sodium eitrate solution to prevent coaggulation.

A solution of methylene blue is made as follows: 1 c.e. methylene blue, 20 c.e. absolute alcohol, and 29 c.e. distilled water.

Varions leucocytic suspensions are made up with 0.9 per cent salt solution, and the mixtures made up to 2 c.c. There are progressively fewer leucocytes in the successive mixture. Two drops of methylene blue solution are then added to each tube and the mixture covered with a layer of liquid paraffin. The tube is then put in the ineubator at $37^{\circ}$ for two hours. If reduction oceurs, the solution becomes colorless; if no reduction occurs, the color remains green. This would make the minimum quantity of leucocytes which cause a reduction of the methylene blue. Then, to test the leucocidin different quantities of the leucocydal fluid are added to twice the minimum quantity of leucocytic suspension which has caused reduction in the above test. These mixtures are then incubated for one and one-half hours at $37^{\circ}$; and at the end of this period, two drops of methylene blue are added and the tubes again covered with liquid paraffin. Again they are incubated for two hours and readings are made. If the tube contains a green color, it indicates that something has prevented reduction.

Antibodes and Immunization.-For reasons not wholly understood at present, recovery from streptococcus infection does not to any marked degree produce immunity against these bacteria. Active immunity may, however, be produced in rabbits, goats, horses, and other domestic animals by treatment with gradually increasing doses of streptococeus cultures. ${ }^{92}$

In carrying out such immunizations it is necessary to use for the first injection attenuated or dead bacteria. Attenuation may be accomplished by moderate heating or by the addition of chemicals (terchloride of iodin). Neufeld ${ }^{93}$ advises, for the first injection in immunizing rabbits, the use of ascitic-broth cultures killed by heat-

${ }^{92}$ Koch und Petruschky, Zeit. f. Hyg., xxiii, 1896.

${ }^{93}$ Neufeld, Zeit. f. Hyg., xliv, 1903. 
ing to $70^{\circ} \mathrm{C}$. This is followed, after ten days, by a second injection of a small quantity of fully virulent coeci. Following this, injections are made at intervals of ten days with constantly increasing doses. Modifications of these general principles are employed in most laboratories.

The sera of animals so treated contain no demonstrable antitoxic or antihemolytic substances. ${ }^{94}$ It has been claimed that these sera exerted demonstrable bactericidal power both in vitro and in vivo. Personally, we doubt the validity of this claim considerably, since bactericidal action upon Gram-positive eocei by body fluids unaided by leucocytes, is not likely to occur. In all such experiments we believe errors have been made, due to the agglutination of organisms and the subsequent diminution of colonies when the tests were made by plating methods. The opsonic powers of such sera, however, cannot be doubted, and enhancement of phagocytosis has been demonstrated, both in vitro and in vivo. In fact, it was with such sera that Denys made his important observations on the importance of serum in phagocytosis, showing that the immune bodies in the serum acted upon the bacteria and not upon the leucocytes.

The protective value of streptococcus immune sera for infected animals is considerable, reaching often a potency hardly explicable by the demonstrable bactericidal or opsonic power, and thereby suggesting some other active factor not understood as yet. ${ }^{95}$ Aronson ${ }^{96}$ has produced immune sera by the treatment of horses with a streptococcus derived from a case of scarlatina, 0.0004 c.c. of which sufficed to protect mice from ten times the fatal dose of a streptococcus culture. These high protective values, however, are obtained only when the serum injections are given simultaneously with the bacteria. Given four or six hours after infection, much higher dosage must be employed and protective results are much less regular in occurrence.97 Other antistreptococcic sera have been produced by Denys, Menger, Tavel, and others, all showing more or less marked potency in protecting animals. ${ }^{98}$

${ }^{94}$ Lingelsheim, Zeit. f. Hyg., x, 1891.

פ5 Denys et Marchand, "Mécanisme de l'immunité," ete., Brussels, 1896.

A Aronson, Berl. klin. Woch., xxxii, 1896 ; ibid., xlii and xliii, 1902 ; ibid., viii and $\mathrm{ix,} 1905$.

${ }^{7}$ Denys, "Le Sérum antistreptoc.," Louvain, 1896; Tan de V'elde, Ann. de 1'Inst. Pasteur, 1896.

9s Denys et Marchand, Bull. de l'acad. roy. de méd. de Belgique, J898; Menger, Berl. klin. Woch., 1902; Tavel, Corr.-Bl. f. Schw. Aertze. 
Since these sera, while in a general way potent against all streptococci, have been found protective chiefly against the specific microorganism employed for their production, Van de Velde, ${ }^{99}$ Denys, Aronson, and others had advised the immunization of the animal with a large variety of streptocoecus races, derived from many different human sourees. The resulting "polyvalent" serum is more apt to exert equally high protective powers against all streptocoeeus infections. The therapeutic value of such sera in the treatment of human infections is still sub judice. Undeniably favorable reports are published each year in increasing number, but are by no means regular or comparable to the results obtained in diphtheria with diphtheria antitoxin. Nevertheless, in mild cases or in those in which the lesions have been distinetly loealized, the sera seemed to be suffieiently useful to justify their use and necessitate their standardization. The recent antigenic elassifieation of streptococei by Dochez and Avery may considerably alter our proeedures in regard to the polyvalence of serum. It may become possible in the future to type the infecting streptoeocei and to use a corresponding serum.

Standardization of sera in regard to their protective power is aecomplished by the methods first devised by Marx ${ }^{100}$ for the standardization of swine-plague serum, and depends upon the ability of the serum to protect animals against a measured dose of virulent streptoeocci. Aronson ${ }^{101}$ designates as a "normal serum" one of which 0.01 c.c. will protect a mouse against ten to one hundred times the fatal dose of virulent streptocoeci. One e.c. of this serum equals one serum unit. Comparisons by animal experiment with this standard serum approximately determine the value of other sera.

In titrating the proteetive power of sera, it is of course necessary to remember, as we have stated above, that streptococei fall into antigenic groups and a serum produced with one strain or a mixture of strains may oceasionally have no action whatever upon a given strain from a ease. The methods of testing protective power on mice are becoming so important in all kinds of bacteriological researeh, that we think it important to insert some of the technical details as described by Dochez, Avery and Laneefield in the paper

${ }^{29}$ Van de Velde, Areh. de méd. expér., 1897.

${ }^{10 M}$ Marx, Deutsche thieräizt. Woch., vi, 1901.

${ }^{101}$ Aronson, Berl. klin. Woch., xliii, 1902; Otlo, Arb. a. 1. königl. Inst., ete., Frankfurter a M., Heft 2, 1906. 
quoted above. ${ }^{102}$.In order to do protection experiments properly, it is necessary, in the first place, to produce powerful serum; in the second place, it is important to raise the virulence of the test strain to a high point. We may state from our own experience that not all streptococci can be raised to the same degree of potency for mice. Dochez and Avery raised the virulence of a number of strains, by continuous mouse passage, to a point at which 0.000001 to 0.00000001 c.c. of a broth culture would kill the animals in twentyfour to forty-eight hours. Workers in our own laboratory have occasionally been able to do this with individual strains, but most strains could not be raised to a virulence approaching this. The organisms are best grown in ascitic broth. 0.5 c.c. of serum is injected intraperitoneally, according to the technique of Neufeld, twenty-four hours before injection of the organisms. On the following day, a series of virulence controls is inoculated intraperitoneally with appropriate quantities of organisms, and the serum animals are injected, intraperitoneally, with doses of twenty-four-hour cultures ranging from 0.001 to 0.00000001 c.e. (though these quantities must be adapted to the known virulence of the strain). Animals that survive for a period of five days are considered by Dochez and Avery as sufficiently protected. The following protocol, taken from the paper by Dochez, Avery and Lancefield, illustrates the method.

\section{PROTOCOL I}

In this protocol is shown the titration of the serum of a sheep immunized against Strain 23. The culture employed for infection was an 18-hour broth culture of No. S 23, which had received eighteen passages through white rats and mice. Each mouse had received 0.5 c.c. of immune serum 24 hours previous to infection.

\begin{tabular}{c|c|c|c}
\hline \multicolumn{2}{c|}{ Virulence Control } & \multicolumn{2}{c}{ Protective Power of Serum S 23} \\
\hline $\begin{array}{c}\text { Dose of Culture. } \\
\text { c.c. }\end{array}$ & Result. & $\begin{array}{c}\text { Dose of Culture. } \\
\text { c.c. }\end{array}$ & Result. \\
\hline$\ldots \ldots$ & $\ldots \ldots \ldots \ldots$ & 0.001 & S. \\
$\ldots \ldots$ & $\ldots \ldots \ldots$ & 0.0001 & D. in 4 days \\
0.00001 & D.* in 24 hours' & 0.000001 & S. \\
0.000001 & D.* in 24 hours & 0.0000001 & S. \\
0.0000001 & D.* in 24 hours & 0.0000001 & S. \\
\hline
\end{tabular}

* In the tables D. indicates died, S. survived.

${ }^{102}$ Dochez, Avery and Lancefield, Jour. Exper. Med., 30, 1919, 179. 
Leucocyte extracts ${ }^{103}$ have been employed in various forms of streptocoecus infections of man, with suecess in many eases. F'avorable results have been obtained with these extracts in eases of erysipelas.

The agglutinins found in streptococeus immune sera are usually most active toward the race of bacteria employed in the immunization. Other streptocoeei are also agglutinated, but in relatively higher concentration. While a specifie group reaction is useful in differentiating streptococei from other species, agglutination ean not be relied upon to differentiate individual streptococei from one another (Hiss). It has been found that a serum produced with a streptocoecus from one source contained a higher agglutinating value for some other, streptococcus than for the one employed in its production. Agglutinins may be produced by treating animals with dead as well as with the living streptococei. The teehnique of streptococeus agglutination is difficult unless we are dealing with strains which grow with even clouding in fluid media. The frequent spontaneous elumping in broth cultures necessitates the use of a special technique. The most simple of these methods is the one in which calcium-carbonateglucose broth is used for cultivation. ${ }^{104}$ Growing in this medium and thoroughly shaken once a day, the streptococei are found evenly divided in the supernatant fluid after the settling out of the calciumcarbonate powder.

This method, however, is not often suceessful. In fact, a constantly reliable method for the agglutination of hemolytic streptococei has not yet been devised.

The method upon which recent antigenic study has been based, has depended upon the use of a speeial sugar free broth containing buffers, since, in acid cultures, the elumping of streptococei is particularly noticeable. This method has been developed particularly by Krumwiede and by Dochez and Avery.

The special broth for these purposes is made from heart musele which is carefully selected so that fat is avoided, and instead of using sodium chloride, a sufficient quantity of a balanced phosphate mixture is added to give the required salt eoneentration, and to adjust the hydrogen ion to a $P_{H}$ of 7.4. The hemolyticus is grown on this for twenty-four hours, at which time it usually produces

${ }^{103}$ Hiss, Jour. Med. Res., xix, 1908.

${ }^{104}$ Hiss, Jour. Exp. Med., vii, 1905. 
a $\mathrm{P}_{\mathrm{H}}$ of 7.2. The organisms are then centrifuged down, are washed once or twice in the stock broth of a $\mathrm{P}_{\mathrm{H}}$ of 7.4 prepared as above. They are then resuspended in the same medium in approximately the original eoneentration of the culture, and this suspension is used for agglutination. This method has given sufficiently reliable results, and, although we have tried a great many other procedures, such as grinding the organisms in salt, shaking them for long periods, etc., we have not been able to obtain homogeneous mixtures better adapted for agglutination tests. For the present we would recommend the method of Dochez and Avery as perhaps the most useful, but it cannot be regarded as entirely satisfactory as yet. (For preparation of the medium see chapter on media.)

Passive therapeutic immunization with polyvalent antistreptococcus serum has not yet given reliable results. In view of the recent work on pneumococcus sera we believe it should be subjected to careful clinical trial especially in cases of spreading cellulitis and streptococcus pneumonias and empyemas which do not yield to the ordinary forms of treatment.

\section{THE PROBLEM OF MUTATION}

The many minor differences in morphology and cultural characteristics which have been noticed among the streptococci have lead to many assumptions of possible mutations in this group. It is, of course, well known that changes in the length of chains in the production of green color by viridans strains, and the degree of hemolysis can be observed in streptococei grown in the laboratory for a long time. These changes, however, may be regarded as analogous to minor changes produced under conditions of artificial cultivation in many other groups, such as the typhoid bacilli and others, but do not imply a fundamental change or true mutation. Burger and Wyntenberg $^{105}$ claimed that passage through white mice converted a capsulated hemolytic streptococeus into what they took to be a pneumococcus. Rosenow ${ }^{106}$ has been the most prominent worker along these lines, and in 1912 claimed that he had transformed a capsulated streptococcus into a typical streptococcus by cultivation

${ }^{105}$ Burger and Wyntenberg, Jour. Infec. Dis., 4, 1907, 609.

${ }^{108}$ Rosenow, Jour. Infec. Dis., 14, 1914, 1. 
on agar, and later he claims that by means of animal passage he had transformed a number of viridans strains into pneumococcus, and a number of hemolyticus strains into viridans. Similar claims have been made by Davis. ${ }^{107}$ It is very difficult to comment upon these results. Broadhurst ${ }^{108}$ also observed changes in fermentation reactions produced in streptococei when they were subjected to growth in fresh milk, saliva and extracts of fresh tissues, but she draws conservative conclusions, and changes such as those noted by her, might well be minor ones in their biological significance. The fundamental changes noted by Rosenow particularly would serve to disturb considerably the methods of classification now employed. The subject is so important that the greatest conservatism in critical evaluation of these claims must be exercised. There are many sources of error, such as the possibility in animals of injecting one organism and getting another one out, and the many possibilities of contamination, that further work must be done along these lines before judgment can be finally given. We, ourselves, have put viridans organisms into rabbits in open celloidin agar capsules, by a method described by Raymond and ourselves ${ }^{109}$ and have, after four months, recovered the identical unchanged organisms that we put in, although they had been in the rabbit as long as four months. It is our opinion at the present time that such fundamental mutations do not take place, and that it will take a great deal of very careful and accurate work before such claims ean be seriously considered.

${ }^{107}$ Davis, Jour. Infec. Dis., 12, 1913, 386.

${ }^{108}$ Broadhurst, Jour. Infec. Dis., 17, 1915, 277.

${ }^{109}$ Zinsser and Raymond, Transac. Soc. Exper. Med. and Biol., January, 1921. 


\section{CHAPTER XXIII}

THE COMMON COLD, THE PNEUMOCOCCUS AND A CONSIDERATION OF THE PNEUMONIAS

\section{THE COMMON COLD}

In dealing with infections of the respiratory tract of man, it is impossible to avoid referring briefly to a condition, which, though mild, is perhaps the most common of all such infections, is unquestionably transmitted from person to person, and yet is etiologically entirely obscure. The condition is of great importance because of the great loss of economic efficiency which wholesale infection of a population with the common cold entails, and because of the fact that the catarrhal inflammation of the nose, throat and upper bronchii, which accompanies the cold, prepares a site for the lodgment and multiplication of influenza bacilli, pneumococei, streptococei, diphtheria bacilli, perhaps meningococei, and other organisms that may lead to more serious disease. Also, the sneezing, coughing and expectoration of individuals suffering from colds results in the promiscuous distribution of bacteria lodged, by chance, in the respiratory passages of such people. Carriers of virulent organisms of various kinds, such as diphtheria bacilli, meningococei, streptococei, pneumococeus types I, II, and III, etc., begin, in the course of their colds, to distribute the virulent organisms they carry to others. They not only spread the virus that has given them the "cold," but scatter a spray which contains the virulent organisms to which they themselves are immune, and, therefore, not only directly infect susceptible contacts, but transmit to them directly a condition which will make it possible for these virulent organisms to lodge in their mucuous membranes, and perhaps cause the secondary more serious diseases. A circuit of carrier distribution is so started, and it has been variously proved that during the colder months of the year, when colds abound, the carrier rate of all respiratory diseases increases. If we consider that in any large group of people there may be three or more per cent of meningococcus carriers, similar 
percentages of pneumococcus, streptococcus carriers, a fraction of a per cent of virulent diphtheria carriers, and that in this group'a number of the carriers begin to cough, spit and hawk, the percentage of all these will go up, and susceptible contacts will not only contract the cold, but will get the specific disease. These facts have been noted in the case of meningococci by Bassett-Smith, ${ }^{1}$ for diphtheria bacilli by Moss, ${ }^{2}$ and the writer has frequently noted this with pneumocoeci and streptococei in army camps.

It is thus seen that the common cold offers indirect problems of great importance, and that the fluctuations of the bacterial flora of the mouth, nose and throat, incident to the common colds, are of interest entirely apart from the problems of specific etiology of the cold itself.

As to the etiology of so-called colds, little is definitely known. It is uneertain whether it is caused by a single or by a variety of infectious agents. Though streptococei, pneumococei, and numerous other organisms have been described as possible causative agents, to none of these can etiologieal importance be conclusively attached. Kruse $^{3}$ in 1914 published work which suggests that the cold may be due to a filterable virus. He succeeded in transmitting the condition to human beings with filtered mueus. Similar work by Foster ${ }^{4}$ in 1917 has seemed to bear out Kruse's contention. Foster went so far as to believe that he had cultivated the filterable virus by the anaërobic methods utilized by Noguehi and others in treponema eultivation, and described in another place. An experiment done by Hopkins in our laboratory suggests similarly the participation of a filterable virus, but it must be remembered that experiments during the season of colds on human beings are fraught with many possibilities of error, and many recent workers have failed to obtain positive results. The etiology of the common cold, therefore, is in doubt, and awaits further elueidation.

Meanwhile, the sanitary importance of the condition must not be underestimated, and the principles of prevention are prefectly plain, although they offer almost insuperable difficulties to successful enforeement.

${ }^{1}$ Bassett-Smith, Lancet, 194, 1918, 290.

${ }^{2}$ Moss, Guthrie and Gelien, Transac. 15th Internat. Congress, Hygiene, Washington, 1913.

${ }^{3}$ Kruse, Munch. Med. Woch., 61, 1914, 1547.

${ }^{4}$ Foster, Jour. Infec. Dis., 21, 1917, 451. 
The striking power of the cold is probably not very great, and direct or indirect contact, relatively close in time and space, seems to be necessary for transmission. The gravity of the malady itself is so slight that it is difficult to impress upon individuals the necessity for care, and the very grave influence upon general respiratory epidemiology cannot be made clear to those not professionally interested.

It is our belief that general rigid attention to the prevention of colds in schools, hospitals, military units, and other closely associated groups of people would indirectly exert a very considerable effect upon the general respiratory sick rate.

Prevention depends upon impressing these facts upon the public and laying stress upon the great danger of severe secondary disease.

The avoidance of close contact, sleeping in the same beds, avoidance of the kissing of children, protecting companions from contamination by coughing and sneezing, disinfection of handkerchiefs, etc., may prevent the disease from going through families as is so often the case.

Children in the initial stages of severe colds should be excluded from school for a day or two. Periodical disinfection of nose and throat with 20 per cent argyrol solution has a definite effect on preventing transmission, in our opinion.

Of especial importance. is the attention to colds during the existence of epidemics of diphtheria, measles, poliomyelitis, meningitis, influenza, and the epidemic pneumonias that may take place in army camps. Under such conditions the common cold may be the main "catalysing agent," as it were, which keeps the more serious disease active. At such times people with increased mucous secretions who cough, spit and distribute mucus with handkerchiefs and hands, are a sufficiently grave menace to call for rigid public health measures. That these cannot be successfully enforced in the general population of cities, seems plain. But they can be controlled in factories, schools, military organizations, hospitals, asylums, and perhaps, under certain conditions, in places of amusement, and innumerable ideal opportunities for spread can thus be eliminated.

\section{THE PNEUMOCOCCUS AND PNEUMONIA}

The opinion that lobar pneumonia is an infectious disease was held by many far-sighted clinicians long before the actual bacterio- 
logieal facts had been ascertained. This idea, so well founded upon the nature of the clinical course of the disease, with its violent onset and equally rapid deferveseence, led many of the earlier bacteriologists to make it the subject of their investigations - a subject made doubly diffieult by the abundant bacterial flora found normally in the upper respiratory passages, and by the fact, which is now recognized, that lobar and other pneumonias are by no means always eaused by one and the some microorganisms.

Coeei of various deseriptions and eultural characteristies were isolated from penumonia eases by Klebs, ${ }^{5}$ Koch, ${ }^{6}$ Günther, ${ }^{7}$ Talamon, ${ }^{8}$ and many others, which, however, owing to the insufficient differential methods at the command of these investigators, eannot positively be identified with the mieroorganism now known to us as Diplococcus pneumoniæ or the pneumococeus. Although thus unsuccessful as to their initial object, these early investigations were by no means futile, in that they gave valuable information regarding the manifold bacterial factors involved in acute pulmonary disease and incidentally led to the diseovery by Friedländer ${ }^{9}$ of B. mucosus eapsulatus.

A tabulation is given in the monograph published on acute lobar pneumonia by Avery, Chickering, Cole and Dochez. ${ }^{10}$ " Among 529 cases diagnosed from the clinical and pathologic features as acute lobar pneumonia, the following were the etiologic agents concerned:

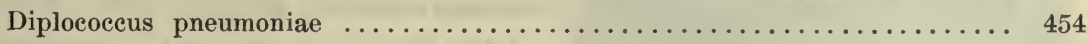

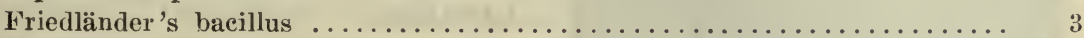

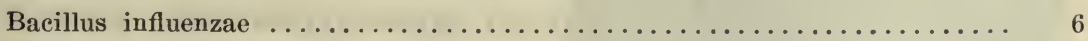

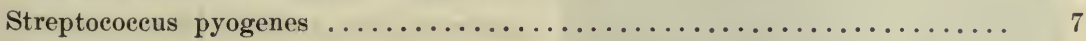

Streptococeus mucosus $\ldots \ldots \ldots \ldots \ldots \ldots \ldots \ldots \ldots \ldots \ldots \ldots \ldots \ldots \ldots \ldots \ldots \ldots$

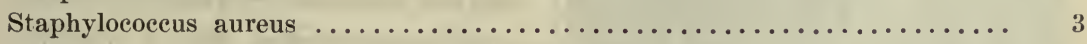

Cases of mixed infection with combinations of Staphylococcus aureus, Fried-

länder's bacillus, B. influenzae, Streptococeus pyogenes, and Strepto-

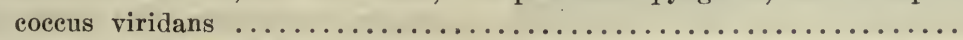

Undetermined (Most of them occurring before accurate methods for determining the etiologic agent had been devised $\ldots \ldots \ldots \ldots \ldots \ldots \ldots .49$

Total

${ }^{5}$ Klebs, Arch. f. exp. Path., 1873.

'Koch, Mitt. a. d. kais, Gesundheitsamt, Bd. 1.

'Günther, Deut. med. Woch., 1882.

- Talamon, Progr. méd., 1883.

'Friedländer, Virchow's Arch., lxxxvii.

10 Avery, Chickering, Cole and Dochez, Monograph of The Rockefeller Inst. for Med. Res., No. 7, Oct. 16, 1917. 
Communications upon lance-shaped cocei found in saliva, and capable of producing septicemia in rabbits, were published almost simultaneously by Sternberg ${ }^{11}$ and by Pasteur ${ }^{12}$ in 1880 . These workers, beyond reasonable doubt, were dealing with the true pneumococeus, but did not in any way associate the microorganisms they described with lobar pneumonia. The solution of this problem was reserved for the labors of $\mathrm{A}$. Fränkel ${ }^{13}$ and Weichselbaum ${ }^{14}$ who published their results, independently of each other, in 1886, demonstrating beyond question that the pneumococcus is the etiological factor in a large majority of cases of lobar pneumonia.

Recent studies by Neufeld and Haendel, and in this country by Cole and his coworkers at the Rockefeller Hospital, have shown that in the pneumococci we are dealing not with a single organism, but with a group. In this grouping three specific types have been described, named respectively, types I, II, and III, and a heterogeneous collection of organisms classified for convenience together as type IV. In a subsequent section, under the main heading of "Immunity and Specific Therapy," this phase of the pneumococeus

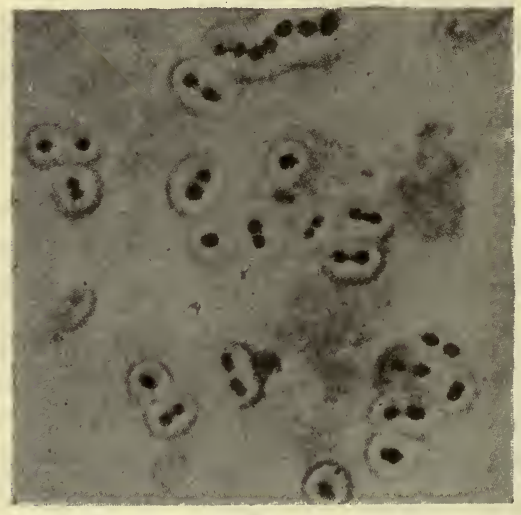

Fig. 48.-Pneumococci, Grown on LOefFler's Serum. (Capsule stain by gentian-violet-potassium-carbonate method.) problem is discussed at greater length.

Morphology and Staining.The morphology of the pneumococcus is, in general, one of the most valuable guides to its identification.

When typical, the pneumococcus is a rather large, lancetshaped coccus, occurring in pairs, and surrounded by a definite and often wide eapsule, which usually includes the two approximated cocei without a definite indentation opposite their lines of division. The pneumococci may, however, occur singly or in short chains, and even fairly long chains are not infrequently

\footnotetext{
11 Sternberg, Nat. Board of Health Bull., 1881.

${ }^{12}$ Pasteur, Bull. de l'acad. de méd., 1881.

${ }^{13}$ A. Fränkel, Zeit. f. klin. Med., x, 1886.

${ }^{14}$ Weichselbaum, Med. Jahrbücher, Wien, 1886.
} 
met with under artificial cultural conditions. This may be chiefly due to the cultural conditions or may be a prominent characteristic of ecrtain strains. Apparently the capsules of organisms making up the chains are continuous; wavy indentations are usually present, however, in the capsule of chains, and at times distinct divisions are observed.

The chief variations from the typical morphology consist either in the assumption of a more distinctly spherical coccus type, or in an elongation approximating the bacillary form. Under certain conditions of artificial cultivation a distinct flattening of the organisms, particularly of those making up chains, may be seen, and even the impression of a longitudinal line of division, characteristic of many streptococcus cultures, is not infrequently gained.

The capsules under certain conditions, especially in artificial media, may be absent or not demonstrable, and in certain strains capsules apparently may not be present under any conditions. Practically any of the described variations may dominate one and the same culture under different or even apparently the same conditions of cultivation, and all grades may oceur in eapsule development, from its typical formation through all variations, to its total and apparently permanent absence.

The presence or absence of eapsules depends, to a large extent, upon the previous environment of the pneumococci under observation. The most favorable conditions for the development or preservation of the pneumococcus capsule are found in the body fluids of man and animals suffering from pneumococcus infection. For instance, capsules may be demonstrated with ease by the usual capsule-staining methods in the blood, serum, and inflammatory exudate of the infected rabbit and white mouse. Capsules may be equally well marked in the fresh sputum of pneumonia patients, especially in the early stages of the disease and in the exudate accompanying such pneumococcus infections as meningitis, otitis media, and empyema. In sputum and the exudates of various localized infections, the organisms are, however, frequently degenerated or under chemical conditions unfavorable for capsule staining, and satisfactory results are not then easily obtained. The same is often true of the serapings from lungs of patients dead of pneumonia, "ven in the stage of red hepatization.

In artificial cultivation, if the nutrient medium is not milk or does not contain serum, capsules cannot usually be demonstrated by 
the ordinary methods of preparing and staining. Capsules may, however, with much regularity be demonstrated on pneumococci, in agar, broth, or on almost all, if not all, artificial media, irrespective of the length of time the organisms have been under artificial cultivation if beef or rabbit serum is used as the diluent, when they are spread on the cover-glass for staining. ${ }^{15}$

The pneumococcus is non-motile and possesses no flagella. Spores are not formed. Swollen and irregular involution forms are common in cultures more than a day old.

The pneumococeus is stained readily with all the usual aqueous anilin dyes. Stained by the method of Gram, it is not decolorized. Special methods of staining have been devised for demonstration of the capsule. The ones most generally used are the glacial acetic-acid method of Welch ${ }^{16}$ and the copper-sulphate method of Hiss. ${ }^{17}$

Huntoon's capsule stain described in the section on staining is the easiest one to apply successfully on material from cultures. It is not adapted to use on pathological material. More recently Buerger ${ }^{18}$ has devised a more complicated method for staining capsules, for which he claims differential value. (For methods see section on Technique, p. 117.)

For simple staining of pneumococci in tissue sections, the GramWeigert technique is excellent. For demonstration of the capsules in tissue sections, Wadsworth ${ }^{19}$ has described a simple method.

Cultivation.-The pneumococeus being more strictly parasitic than many other bacteria, presents greater difficulties in its cultivation. On meat-extract media growth does not take place with regularity. On those media, however, which have beef or veál infusion for their basis, growth ean be obtained with considerable regularity, although such growth may be sparse and delicate.

Growth takes place most regularly at a temperature of $37.5^{\circ} \mathrm{C}$. Development does not usually occur below $25^{\circ}$ nor above $41^{\circ} \mathrm{C}^{20}$ At ordinary room temperature, $18-22^{\circ} \mathrm{C}$., the temperature used for gelatin cultivation, growth either does not take place at all or is exceedingly slow and unenergetic.

${ }^{15}$ Hiss, Cent. f. Bakt., xxxi, 1902; Jour. Exp. Med., vi, 1905.

${ }^{16}$ Welch, Johns Hopk. Hosp. Bull., xiii, 1892.

${ }^{17}$ Hiss, Cent. f. Bakt., xxxi, 1902; Jour. Exp. Med., vi, 1905.

${ }^{13}$ Buerger, Medical News, lxxxviii, 1904.

${ }^{19}$ Wadsworth, "Studies by the Pupils of W. T. Sedgwick," Chicago, 1896.

${ }^{20}$ A. Frankel, Dent. med. Woch., xiii, 1886. 
Aërobic and anaërobic conditions both permit the growth of pneumococcus, there being very little difference in speed or extent of growth along the course of deep stab cultures in favorable media. The most favorable reaction of media for the cultivation of this microorganism is a $\mathrm{P}_{\mathrm{H}}$ of 7.6 to 7.8. Slight acidity, however, if not exceeding eight-tenths per cent, does not materially hamper development.

The broth or agar basis for pneumococcus media must be earefully made, both in regard to nutrient contents and reaction. Ordinary meat extract media are not usually rich enough, and even carelessly made meat infusion broth may fail to grow pneumococci. In our laboratory we have come to use the "hormone" broth and agar media almost entirely for pneumococcus and streptococcus work.

The growth of pneumococci on all media may be considerably enhanced by the addition to these media of animal or human serum or whole blood. Additional substances which, among others, unquestionably have a favorable influence upon pneumococcus growth, are glucose, nutrose, and glycerin. The addition of the latter substances to the media, however, probably because of acid formation, hastens the death of pneumococcus cultures. An increase of the amount of pepton used for the preparation of media is desirable for the cultivation of this microorganism; two to four per cent of pepton may be found advantageous.

Transfer of recently isolated penumococeus from broth culture to broth culture necessitates rather heavy inoculation, since frequent failure is experienced when only a loopful or so is transferred. Cole mentions that about 0.1 c.c. should be transferred for every 5 c.c. of broth.

In suitable nutrient broth, growth is rapid, and within twentyfour hours leads to slight clouding of the fluid. This clouding, as a rule, eventually disappears as the microorganisms, sinking to the bottom of the tube or disintegrating, leave the fluid more or less clear. In broth, pneumococei have a tendency to form short chains. When glucose has been added to the broth, growth is more rapid and profuse, but considerable acid formation causes the cultures to die out rapidly. It is possible, however, to employ glucose as a growth-enhancing element in broth cultures without interfering with the viability of the cultures by adding small quantities (one per cent) of sterile, powdered calcium carbonate. This method of cultivation in broth is especially 
adapted to the production of mass cultures for purposes of immunization or agglutination. ${ }^{21}$ The addition of ascitic fluid or blood serum to broth, in the proportion of one to three, makes an extremely favorable medium in which growth is rapid and profuse.

Upon agar plates, pneumococcus growth is not unlike that of streptococcus. The colonies are small, round, and slightly more transparent than those of the streptococci. They appear more moist than streptococeus colonies and often are more flat. Microscopically examined, the colonies are finely granular, with dark centers and slightly corrugated lighter-colored peripheral areas. Under high magnification no such intertwining convolutions can be seen as those noticed under similar magnification in streptococcus cultures. The addition of animal albumin to agar results in the more rapid development, larger size, and deeper opacity of the colonies.

Agar stab cultures show growth within twenty-four to thirty-six hours, which takes place with equal thickness along the entire course of the stab. There is nothing distinctive in these cultures to differentiate them from similar streptococeus cultures.

In gelatin plate and stab cultures at $22^{\circ} \mathrm{C}$., growth, as a rule, does not take place. This, however, is not true of all races of penumococci. Occasionally strains are met with which will grow fairly abundantly in gelatin at a temperature of $22^{\circ} \mathrm{C}$. When the gelatin is rendered sufficiently firm to bear $25^{\circ}$ to $26^{\circ} \mathrm{C}$. without melting, growth appears slowly and sparsely as minute, grayish-white, transparent colonies. The gelatin is not liquefied.

Growth upon milk is profuse, resulting in the production of acid and coagulation of the medium. Races are encountered in which this is suppressed and coagulation in milk is absent or long delayed.

Upon potato, thin, gray, moist growth occurs, hardly visible and indistinguishable from an increased moisture on the surface of the medium. This is not a favorable medium.

Upon Loeffler's coagulated blood'serum, the pneumococcus develops into moist, watery, discrete colonies which tend to disappear by a drying out of the colonies after some days, differing in this from streptococcus colonies, which, though also discrete, are usually more opaque and whiter in appearance than those of the pneumococcus and remain unchanged for a longer time. This medium, as will be seen, is useful in differentiating pneumococci from the so-called Streptococcus mucosus.

${ }^{21}$ Hiss, Jour. Exp. Med., vii, 1905. 
Upon mixtures of whole rabbit's blood and agar, the pneumococcus grows well, and forms, after four or five days, thick, black surface colonies, not unlike sun blisters on red paint. These colonies are easily distinguished from those of streptococci, and are of considerable differential value. ${ }^{22}$

Pneumococcus colonies on blood plates may cause a slight halo of hemolysis and methemoglobin formation with a zone of greenish color about the colony after 48 hours or longer in the incubator.

The hemolysin formation by pneumococcus is slight, but quite definite. It occurs late, rarely sooner than 48 hours, but is mentioned because errors of diagnosis through ignorance of this might occur. Cole $^{23}$ has particularly studied the hemotoxins of this organism.

Guarnieri $^{24}$ has recommended a medium with a pepton-beefinfusion basis rendered semisolid by mixtures of agar and gelatin. A modification of this medium has been described by Welch ${ }^{25}$ and has been much employed. Cultivation within eggs and upon egg media ${ }^{26}$ has been used. Wadsworth ${ }^{27}$ has recommended a medium composed of ascitic fluid to which agar has been added-sufticient to give a soft, jelly-like consistency. He observed prolonged viability and the preservation of the virulence on this medium.

For the purpose of differentiating pneumococei from streptococei, Hiss $^{28}$ devised a medium of becf serum one part, and distilled water two parts, to which is added one per cent of inulin (c. p.), and enough litmus to render the medium a clear, transparent blue. By fermentation of the inulin the pneumococcus acidifies this mixture, causing coagulation of the serum. Streptococei do not ferment inulin.

Since inulin fermentation is a very important differential characteristic of the pneumococcus, it is necessary to say a few words about the irregularity with which it occurs. Occasionally, failure to ferment inulin is due to the fact that the particular strain of pneumococcus does not grow well in the inulin medium made up by the older method of Hiss. The addition of one per cent of pepton to this medium has been suggested by Bürger and is a distinct improvement. It may

\footnotetext{
${ }^{22}$ Hiss, loc. eit.

${ }^{23}$ Cole, Jour. Exp. Med., 1914, 20, 346 and 363.

${ }^{24}$ Guarnicri, Att. dell' Acarl. di Roma, 1883.

2n Welch, Johns Hopk. Hosp. Bull., iii, 1892.

${ }^{26}$ Sclavo, Riv. d'Igiene, 1894.

${ }^{27}$ Warlsworth, Proc. N. Y. Path. Soc., 1903.

${ }^{2 s}$ Hiss, Jour. Exp. Med., vi, 1905.
} 
happen, however, that occasional strains will react irregularly on different preparations of inulin. These irregularities must be taken into consideration when this test is used for differential purposes.

Isolation.-For the isolation of pneumococei from mixed eultures or from material containing other species, such as sputum, surface smears of the matcrial are made upon plates of neutral glucose-agar, glucose-serum-agar, or blood agar. According to the number of bacteria present in the infected material, it may be smeared directly upon the plate, or diluted with sterile broth before planting. After incubation for twenty-four hours, the pneumococcus eolonies are easily differentiated from all but those of streptococeus. With practice, however, they may be distinguished from these also, by their smoother edges and greater transpareney and flatness.

The easiest way to isolate pneumococci from mixed culture and especially from material from patients like sputum, pulmonary exudate, ete., is injection jnto white mice. When sputum is used the sputum should be washed by gently rinsing in successive wateh glasses or pipette plates containing salt solution or broth. It can be injected directly into a white mouse, intraperitoneally, or, if very stringy or dry, ean be rubbed in a mortar with a little broth before injection. Great care must be taken not to inject too much material. The details of this method are given in connection with clinical considerations in a subsequent paragraph. If virulent pneumococci are present, death will occur within 24 hours, or thereabouts. Pneumococei will be found in pure eulture in the heart's blood and in large numbers in the peritoneal exudate.

Pneumococcus Trpes.-As stated above, Neufeld and Haendel ${ }^{29}$ in 1910 found that pneumococei were by no means all alike, serologically. Although all the true pneumococei have morphological and eultural characteristics which would appear to classify them as a single species, it was found that within this apparently homologous group there were sharp serological differentiations. Dochez and Gillespie ${ }^{30}$ not only confirmed the work of Neufeld and Haendal, but made a careful study of pneumococcus types as they oceurred in America, both by agglutination reactions and by protection tests on mice. They isolated a large number of pneumococeus strains, and immunized animals with them. The sera of these animals were now

${ }^{29}$ Neufeld and Haendel, Arb. a. 1. k. Gsndhtsamte, 34, 1910, 293.

3n Dochez and Gillespie, Jour. of the A. M. A., 61, 1913, 727. 
examined for cross-agglutination with the various strains and for their protective powers on mice. It was found that the pneumococei they studied fell into very sharp classes. The surprising thing about their results is that the distinctions between the types seem to be so sharp that very little "group" reaction occurs.

Their classification, which has been many times confirmed and which may be accepted as representing, in a tentative way, the conditions existing in the United States at any rate, divides pneumococei into four main types, numbered accordingly, I, II, III, and IV.

Types I and II are morphologically and culturally typical pneumococci, and represent well circumseribed species, sharply classifiable by the fact that members of type I agglutinate only, and are protected against only, by type I serum, and members of type II will react similarly only with type II serum. Type III represents what was formerly spoken of as the Streptococcus Mucosus, but which is included in the pneumococci because of its inulin fermentation, bile solubility, and pathogenic properties which are quite similar to those of the pneumococci.

By type IV is meant a heterogeneous group which comprises all of the true pneumococei which cannot be serologically placed into the other three types. This fourth group is merely a matter of convenience of nomenclature, since few of these organisms are related to each other. They repreesnt a sort of attic into which unclassifiable pneumococci have been thrown for the present, until they can be sorted out. Olmstead ${ }^{31}$ has studied a considerable number of so-called type IV organisms, and has found that smaller subgroups could be established by serological classification. But so many different subgroups were found that, for the present, no practical results have come out of these attempts to systematize group IV. The difficulties in this group are similar to those encountered in connection with streptococcus viridans.

Although type II is a very sharply defined variety, there are certain subgroups within type II, studied particularly by Avery. ${ }^{32}$ They consist of atypical members of group II which are agglutinated by type II serum, with diminished intensity. All of them will be agglutinated with the concentrated serum, but when dilutions of 1 to 20 or over are used, they fail to agglutinate. Avery has defined three such subgroups of type II which he calls subgroups II $a$, II $b$,

\footnotetext{
${ }^{31}$ Olmstead, Jour. of Immunol., 2, 1917, 425.

${ }^{32}$ Avery, Jour. of Exper. Med., 22, 1915, 804.
} 
and IIx. All of these are proven members of type II because they all agglutinate in concentrated type II serum. All but II $x$ are protected against by type II serum. Absorption of type II serum with a type II organism removes the antibodies for all the subgroups. Absorption of the anti-pneumococcus type II serum with any one member of a subgroup removes only the antibodies for that particular subgroup.

That the three subgroups are specifically different from each other and from type II, is determined by Avery by the fact that any given subgroup is not agglutinated by antisera made with organisms of the other two subgroups. The same is true of protection reactions. Also, no one of them absorbs the antibodies for the other subgroups from serum.

Subgroups "A" and "B" have immunity reactions identical, each within its respective group. Subgroup II $x$, however, consists of heterogeneous strains in which each strain seems to produce its character istic antibodies specific only for the strain used.

In addition to these types, there are unquestionably a number of other types in different parts of the world. Thus, Lister ${ }^{33}$ working in South Africa, found three homogeneous types, A, B, C, his types C and $\mathrm{B}$ corresponding to types I and II of the American classification, respectively; his type A being not so far identified with any of our American types.

The importance of this discovery of the serological pneumococcus group in serum therapy is obvious, and its bearing on epidemiology is dealt with in another section.

Pneumococcus (streptococcus) Mucosus.-First definitely described by Howard and Perkins ${ }^{34}$ in 1901, and subsequently carefully studied by Schottmüller, ${ }^{35}$ who isolated it from cases of parametritis, peritonitis, meningitis, and phlebitis. It has since been described by many as the incitant of lobar pneumonia and of a variety of other lesions and as often an apparently harmless inhabitant of the normal mouth. Morphologically, though showing a marked tendency to form chains, on solid media it often appears in the diplococcus form. It is enclosed in an extensive capsule, which appears with much regularity and persistence. Though very similar in appearance, therefore, to pneumococei, these bacteria do not appear in the typical lancet shape. Upon solid media they show a tendency to grow in transparent moist

\footnotetext{
${ }^{33}$ Lister, Pub. South African Inst. for Med. Res., 1916, No. 8.

${ }^{34}$ Howard and Perkins, Jour. Med. Res., 1901, N. S., i.

${ }^{35}$ Schottmüller, Münch. med. Woch., xxi, 1903.
} 
masses. The regularity with which this microorganism ferments inulin medium, make it probable that it is more accurate to place it with the group of pneumococei than with that of streptococci. ${ }^{36}$

Most of the organisms of this group show the common characteristies of the pneumococei and are soluble in bile. Occasional strains, such as one studied by Dochez and Gillespie, neither ferment inulin nor are bile soluble. Rarely, too, does it cause hemolysis. From the various studies earried out upon this group it must be concluded that while perfectly distinct in its formation of a heavy mucoid colony and capsulation, this group is more closely related to the pneumococci than to the true streptococei. As would be expected from its capsulation, its virulence is very powerful and serum reactions are not easily carried out. A further discussion of the immune serum reactions with this organism is included in this chapter, page 462.

Resistance.-On artificial media, the viability of the pneumococcus is not great. Cultures upon agar or bouillon should be transplanted every third or fourth day, if the cultures are kept within an incubator. In all media in which rapid acid formation takes place, such as glucose media, the death of cultures may occur more rapidly. In media containing albumin and of a proper reaction, preservation for one or even two weeks is possible. The longer the particular race has been kept upon artificial media, the more profuse is its growth, and the greater its viability, both qualities going hand in hand with diminishing parasitism. The length of life may be much increased by preservation at low temperature, in the dark, and by the exclusion of air. In calcium carbonate broth and kept in the ice-chest, cultures may often remain alive for months.

Neufeld has succeeded in keeping pneumococci alive and virulent, by taking out the spleens of mice dead of pneumococcus infection and preserving them in a Petri dish in a desiccator, in the dark and cold. In this way, the organisms can be eultivated from the spleen, and will be found virulent for longer periods than in eulture media. The best way to get such cultures back is by injecting a suspension of the desiccated spleen, in broth, into a mouse, and recovering the pneumococcus from the heart's blood.

In sputum the viability of pneumococei seems to exceed that observed in eulture. The studies of Guarnieri, ${ }^{37}$ Bordoni-Uffreduzzi, ${ }^{38}$

\footnotetext{
${ }^{36}$ Hiss, Jour. Exp., Med., 1905; Buerger, Cent. f. Bakt., I, xli, 1906.

${ }^{37}$ Guarnieri, Atta della R. Acad. Med. di Roma iv, 1888.

${ }^{38}$ Bordoni-Uffreduzzi, Arch. p. 1. Sc. med. xv, 1891.
} 
and others have shown that pneumococci slowly dried in sputum may remain alive and virulent for 1 to 4 months, when protected from light; and as long as nineteen days when exposed to diffused light at room temperature. Experiments by Ottolenghi ${ }^{39}$ have confirmed these results; the virulence seems, in Ottolenghi's experiments, to have become considerably attenuated before death of the cocci. Recent studies by Wood, ${ }^{40}$ whose attention was focused chiefly upon pneumococcus viability in finely divided sputum-in a condition in which inhalation transmission would be possible-have shown that pneumococci survive for only about one and one-half hours, under ordinary conditions of light and temperature. Exposed to strong sunlight, pneumococci die off within an hour.

Low temperatures slightly above zero are conducive to the prolongation of life and the preservation of virulence.

The resistance of the pneumococcus to heat is low, $52^{\circ} \mathrm{C}$. destroying it in ten minutes. ${ }^{41}$ To germicidal agents, carbolic acid, bichlorid of mercury, permanganate of potassium, etc., the pneumococcus is sensitive, being destroyed by weak solutions after short exposures.

The disinfection of sputum, difficult because of the protective coating of the secretions about the bacteria, has been recently studied by Wadsworth. ${ }^{42}$ The conclusions reached by this writer indicate that pneumococci in exudates are most rapidly destroyed by twenty per cent alcohol, other and stronger disinfectants being less efficient, probably because of slighter powers of diffusion.

Differentiation of Pneumococcus from Streptococcus.-Pneumococci and streptococci which do not differ in morphology from their classic types can usually be differentiated from each other and identified by their morphological characters without difficulty; but it is equally true that certain cultures of these organisms, either at the time of their isolation or after cultivation on artificial media, approach the type of the other so closely that it may be impossible to identify them by their morphology alone. When such morphological variations occur there are no constant cultural or pathogenic characters as yet demonstrated which distinguish between these organisms.

This lack of distinct cultural differences between pneumococei and streptococci has not infrequently led to confusion, and that uncer-

${ }^{39}$ Ottolenghi, Cent. f. Bakt., xxv, 1889.

40 Wood, Jour. Exp. Mel., vii, 1905.

${ }^{41}$ Sternberg, Cent. f. Bakt., xii, 1891.

${ }^{42}$ Wadsworth, Jour. Inf. Diseases, iii, 1906. 
tainty should exist and mistakes be made in identification is not surprising when one considers the characters usually depended upon to distinguish pneumococci from streptococci. Chief among these, as has just been implied, are the morphological features which are, in the case of pneumococci, a slightly lancet or elongated form rather than the more typical coccus form characteristic of the streptococci, and an arrangement of such cocei in pairs rather than in chains; added to these features is the possession of a more or less well-defined capsule. All of these characters are subject to variation or may be absent. Compared with the morphological, the cultural characters are variable and of minor importance. The pneumococcus colonies on coagulated blood serum and on agar are moister and flatter, and the freshly isolated pneumococcus is usually unable to develop readily or at all on gelatin at below $22^{\circ} \mathrm{C}$.

The distinctness of the capsule of the pneumococcus in the body fluids of man and animals and on blood serum, milk, or serum agar, has been depended upon as the chief distinguishing and diagnostic character. Nevertheless, instances have been reported of distinct capsule formation by organisms which had either been previously identified as Streptococcus pyogenes, or at the time of the isolation could not be definitely identified as belonging to this group or to the pneumococei, but were considered intermediate in character. ${ }^{43}$

${ }^{43}$ Brief Deseription of Organisms Reported as Capsulated Streptococci.-Bordet (Bordet, Ann. de l'inst. Pasteur, 1897, xi, p. 177), working with an organism previously identified as Streptococeus pyogenes, described such capsule formation occurring in the peritoneal exudate of infected rabbits.

Schuetz' (Schuetz, Cent. f. Bakt., Ref. 1, 1887, p. 393) Diplokokkus der Brustseuche der Pferde, Poels and Nolen's (Poels und Nolen, Ford. d. Med., iv, 1886, p. 217) streptococcus of contagious pneumonia of cattle, and especially the organism described by Bonome (Bonome, Ziegler's Beit., viii, 1890, p. 377) as Streptococcus der meningitis cerebrospinalis epidemica, may all be looked upon as organisms differentiated on insecure grounds from either pneumococeus or streptococcus. The first two of these organisms, however, are said to be decolorized by Gram's method, and as suggested by Frosch and Kolle (Frosch und Kolle, Flügge's " Mikro-organis.,", ii, 1896, p. 161), in the case of Schuetz' organism may belong to a group intermediate between Fraenkel's diplococcus and the chicken-cholera group.

Tavel and Krumbein (Tavel und Krumbein, Cent. f. Bakt., xviii, 1895, p. 547) describe a streptococeus with a capsule, which was isolated from a small abscess on the finger of a child. Capsules were also present in the artificial cultures, and although ordinarily remaining uncolored, could be stained by Loeffler's flagella stain. This organism was said to be differentiated from Fraenkel's diplococcus and also in general from streptococeus (pyogenes) by a rapid and 
There are oceasions, then, both within the animal body and in artificial cultivations, when it is practically impossible to distinguish

rich growth on gelatin, agar, and potato. A pellicle was formed on broth. The organisms forming this pellicle had capsules, but those in the deeper portions of the broth generally lacked it.

In 1897 Binaghi (Binaghi, Cent. f. Bakt., xxii, 1897, p. 273) deseribed a capsulated streptococeus isolated from a guinea-pig dead of a spontaneous peribronchitis and multiple pulmonary abscesses. In the pus were found some diplococei and short chains (four to six) surrounded by a cupsule, shown by staining with carbol fuchsin. This organism he proposes to call Streptococcus capsulatus.

Le Roy des Barres and Weinberg in 1899 (Le Roy des Barres et Weinberg, Arch. d. méd. expér., xi, 1899, p. 399) published an account of a streptococcus with a capsule. This was isolated from a man who had apparently been infected from a horse which had died of an acute intestinal disorder. The patient neglected the infection and died. Diplococei and short chains furnished with a capsule were found in the subcutaneous tissue at the area of infection. The blood, liver, and spleen also contained these organisms. The capsule in all the preparations remained uncolored, but the authors say that its existence was not to be doubted. Ascitic broth inoculated from the peritoneal exudate of a rabbit dying from the infection gave streptococei in extremely long chains and surrounded by capsules. These were not so distinct as in the case of the organisms in the original smear preparations. All fluid media (bouillon, milk, and ascitic broth) were said to be strongly acid after twenty-four hours. These authors report that Achard and Marmorek have assured them that they have seen capsulated streptococci, and that Marmorek showed them some preparations in which one of his streptococci presented the same characters as that isolated by them.

Although Le Roy des Barres and Weinberg have used the term encapsulated, they believe that it would perhaps be more prudent to eall their organism streptocoque aureole, since they were not able to define this capsule by staining it.

Howard and Perkins (Howard and Perkins, Jour. Med. Res., 1901, iv, p. 163) have lately deseribed an organism, probably of the foregoing type, which was present in a tubo-ovarian abscess and in the peritoneal exudate, the blood, and some of the organs of a woman dying in the Lakeside Hospital, Cleveland, Ohio. The organisms were biscuit-shaped cocei in pairs, usually arranged in chains of four, six, eight, or twenty elements, and surrounded by a wide and sharply staining capsule. In the artificial cultures special capsule stains, it was noted, failed to stain any definite area, but numerous small deeply stained granules were to be seen within the halo, especially near its outer border. Howard and Perkins propose for the group composed of the streptococei of Bonome, Binaghi, and their own organism, the name Streptococeus mucosus. Streptococei isolated from cases of epidemic sore-throat have also shown capsules (p. 421).

Reference to the original descriptions of these various capsulated streptococei will show that, with the exception of a rather poorly staining eapsule, the majority of these organisms are separated from the typical Streptococeus pyogenes or from the pneumococeus by exceedingly slight and unstable morphologica? and cultural characters. This is true of the difference in their pathogenic action in animals. 
definitely between some races of pneumococci and races of streptococci. This difficulty is especially heightened when the pneumococcus has become non-virulent, and at the same time no very typical morphology or capsule formation is to be determined and a tendency to chainformation is marked. Cultures of pneumococei in such condition eannot readily be distinguished morphologically from streptococcus cultures.

Under these circumstances recourse must be had to a careful biological study of the organism in question. The following are the criteria mainly relied upon at present for the differentiation of these two groups.

Pneumococci ferment inulin, if cultivated in inulin-serum-water medium. Acid formation from the inulin results within two days or more in coagulation of the serum and reddening of the litmus. Streptococci, because of their inability to attack the inulin, leave the medium unchanged. ${ }^{44}$

Cultivated on whole-blood-agar, streptococci usually cause hemolysis, pneumococci usually do not. ${ }^{45}$ In eontradistinction to Streptococcus viridans which does not hemolyze, pneumococei have a tendency on these media to form the black, dry, paint-blister colonies. ${ }^{46}$

Neufeld,$^{47}$ in 1900 , noticed that normal rabbits' bile added in quantities of 0.1 c.c. to each one or two cubic centimeters of a pneumococcus broth culture caused lysis of the bacteria, rendering the culture fluid transparent and clear. This does not occur with streptococci, and has been used to differentiate the two species. According to Libman and Rosenthal, ${ }^{48}$ great reliance may be placed upon this method.

The most convenient reagent for use in the Neufeld bile test is a 10 per cent solution of sodium taurochlorate in physiological salt solution. This should be sterilized or kept on ice. One-tenth volume of such a solution produces prompt lysis in a broth culture of pneumoeocei.

Decisive differential importanee may be attached to the agglutinations of these microorganisms in immune sera (see p. 462).

The permanency of the various types in the pneumococcus-strepto-

${ }^{44}$ Hiss, Cent. f. Bakt., xxxi, 1902 ; Jour. Exp. Med., vi, 1905.

${ }^{45}$ Schottmüller, Münch. med. Woch.

${ }^{46}$ Hiss, Jour. Exp. Med., vii, 1905.

${ }^{47}$ Neufeld, Zeit. f. Hyg., 1901.

${ }^{48}$ Libman and Rosenthal, Proc. N. Y. Path. Soc., March, 1908. 
coccus group is still open to question. E. C. Rosenow ${ }^{49}$ has recently reported that he has transmuted typical pneumococci into typical hemolytic streptococei by methods which he has not as yet fully described, but among which were animal passage, growth in symbiosis with bacillus subtilis, and growth in an atmosphere of oxygen. The pneumococci when first altered took on the characteristics of the streptococcus viridans, later of the so-called streptococeus rheumaticus, and finally of streptococcus hemolyticus. Together with cultural characteristics the pathogenicity of these various strains for rabbits changed. The pneumococcus produced acute sepsis, the streptococcus viridans caused endocarditis, the streptococcus rheumaticus periarticular or serous arthritis, and hemolyticus suppurative arthritis. In intermediate stage the organisms quite regularly caused myositis. Although he was able to transmute these types one into the other in both directions, Rosenow believes that the cultural characteristics of each type correspond to a fairly definite type of pathogenicity in animals and man. This work has not as yet appeared in detail and has not been confirmed.

Toxic Products of the Pneumococcus.-Our knowledge of pneumococcus poisons is still very imperfect. Attempts to obtain soluble toxins by the filtration of cultures have been practically unsuccessful. G. and F. Klemperer, ${ }^{50}$ Mennes,${ }^{51}$ Pane,${ }^{52}$ Foa and Carbone, ${ }^{53}$ and others failed to obtain pneumococcus filtrates of any degree of toxicity, though working with highly virulent strains. The feeble toxin so obtained produced no antitoxin.

The general failure to procure strong soluble poisons from cultures, gives weight to the assumption that the most potent toxic products of pneumococci are in the nature of endotoxins and closely bound to the cell-bodies themselves. This assumption is borne out by the more recent experiments of Macfadyen. ${ }^{54}$ This author obtained acutely poisonous substances from pneumococci by trituration of the organisms after freezing, and extracting them with a one 1:1,000 caustic potash solution. With the filtrates of these extracts he was able to cause rapid death in rabbits and guinea-pigs by the use of doses not

\footnotetext{
49 Rosenow, J. A. M. A., 1913, 1xi, 2007.

${ }^{50} G$. and $F$. Klemperer, Berl. klin. Woch., xxxiv and $\mathrm{xxxv}, 1891$.

${ }^{51}$ Mennes, Zeit. f. Hyg., xxv, 1897.

s2 Pane, Rif. med., xxi, 1898.

${ }^{53}$ Foa und Carbone, Cent. f. Bakt., x, 1899.

Macfadyen, Brit. Med. Jour., ii, 1906.
} 
exceeding 0.5 to 1 c.c. He found, furthermore, a striking parallelism between the degree of toxicity and the virulence of the extracted culture. Cole, ${ }^{55}$ too, in recent studies, inclines to the belief that the poisons of the pneumococcus are in the nature of endotoxins and has produced toxic substances by salt solution and bile extraction of the organisms.

Cole $^{56}$ states that when the cells of pneumococci are dissolved with bile salts the solution becomes hemolytic and toxic. This hemolytic substance is easily destroyed by heat, by tryptic digestion, and is partially lost in filtration through Berkefeld filters. He believes that there is some relationship between the virulence of the organisms and these substances. An interesting fact, furthermore, is that the cholestrin inhibits such toxic effects, but anti-pneumococcus serum is only partially effective. Cole differentiates this endocellular hemotoxin from another substance that converts hemoglobin into methemoglobin. The latter substance is produced only by the living cells, and depends upon the presence of oxygen.

In addition to these substances, there are very soluble materials that appear in culture media in which the pneumococcus is grown, during the life of the organisms, and which can be detected by specific precipitin reactions. These substances, as we shall see, have been found by Avery and Dochez ${ }^{57}$ to be present in the blood and urine of pneumonia patients. It is questionable whether or not they exert toxic action in the infected body.

Virulence and Pathogenicity.-The virulence of pneumococei is subject to much variation, depending upon the length of time during which it has been cultivated. It has been mentioned above that under conditions such as those prevailing in dried sputum or blood the virulence of pneumococei may be preserved for 'several weeks. Ordinarily, the virulence diminishes as the cocci adapt themselves to life upon artificial media. Upon media containing animal albumin, such as ascitic fluid or blood agar, this attenuation is less rapid than upon the simple meat-infusion preparations.

The maintenance of virulence, according to Kirkbride, ${ }^{58}$ is greatly aided by making transfers from broth to broth at intervals not

${ }^{55}$ Cole, Harvey Lecture, N. Y., Dec., 1913.

${ }^{56}$ Cole, Jour. of Exper. Med., 16, 1912, 644.

${ }^{57}$ Avery and Dochez, Proc. Soc. Exper. Med. and Biol., 14, 1916, 126.

${ }^{58}$ Kirlbride, Paper read before the Amer. Assoc. Pathol, and Bacter., New York, April, 1917. 
longer than eight hours. Apparently cultures grown for as long as twenty-four hours diminish in virulence much more rapidly. To some extent this observation is in keeping with Chesney's ${ }^{59}$ statement that growth is best obtained when the transfers are made from broth at the period of maximum growth rate.

Swift has recently succeeded in keeping organisms of the pneumococcus and streptococcus varieties alive and virulent for a long time by centrifugating broth cultures, taking off the supernatant fluid, and drying the residue in a frozen condition in vacuo. This material can be kept for a very long time without death of the bacteria, and without appreciable loss of virulence.

In the blood of rabbits dead of a pneumococeus infection, taken directly into sterilized tubes, sealed and kept in the dark, Foa ${ }^{60}$ has been able to preserve the virulence of pneumococei for as long as forty-five days. Preservation in the spleen of animals dead of pneumococcus infection, as practiced by Neufeld, has been mentioned above. Whether or not the virulence of pneumococci is attenuated or enhanced by sojourn within the human body during disease is uncertain. The attenuation of virulent pneumococei on artificial media may be hastened, according to Fränkel, ${ }^{61}$ by cultivation of the organism at or above a temperature of $41^{\circ} \mathrm{C}$.

The virulence of attenuated cultures may be rapidly enhanced by passage of the organisms through the bodies of susceptible animals. The virulence of strains may be so enhanced that one one-millionth of a c.c. will kill a mouse.

Among the domestic animals white mice and rabbits are most susceptible. Guinea-pigs, dogs, rats, and cats are much more resistant. Guinea-pigs can be given astonishingly large doses of pneumococei without injury. Birds are practically immune. Kyes who has studied pneumococcus infection in birds particularly has shown that the fixed tissue cells of the liver, spleen and lungs destroy the organisms by prompt and effective phagocytosis. His attempts at treating pneumonia with immune chicken serum, suggested by these observations, will be referred to below.

The results of pneumococcus inoculation into susceptible animals vary according to the size of the dose, the virulence of the introduced bacteria, the mode of administration, and the susceptibility of the

${ }^{58}$ Chesney, Jour. of Fxper. Med., 24, 1916, 387.

${ }^{60} \mathrm{Foa}$, Ztsehr. f. Hyg. iv, 1888.

${ }^{81}$ Fränkel, Deut. med. Woch., 13, 1886. 
subject of the inoculation. Subcutaneous inoculation of virulent pneumococei into mice and rabbits usually results in an edematous exudation at the point of inoculation, which leads to septicemia and death within twenty-four to seventy-two or more hours. When the dose has been extremely small or the culture unusually attenuated, a localized abscess may be the only result. Intravenous inoculation is usually more rapidly fatal in these animals than the subcutaneous method. Intraperitoneal inoculation in rabbits results in the formation of a rapidly spreading peritonitis in which the exudates are apt to be accompanied by a deposit of fibrin, and to lack the transparent red color so often caused by the hemolyzing streptococci. With very virulent strains, these differences are less marked. In almost all of these infections death is preceded by septicemia and the microorganisms can be recovered from the heart's blood of the victims.

The production in animals of lesions comparable to the lobar pneumonia of human subjects has long been the aim of many investigators. Wadsworth, ${ }^{62}$ recognizing that such lesions probably depended upon the partial immunity which enabled the infected subjects to localize the pneumococcus processes in the lungs after infection by way of the respiratory passages, succeeded in producing typical lobar pneumonia in rabbits by partially immunizing these animals and inoculating them intratracheally with pneumococci of varying virulence. Lamar and Meltzer ${ }^{63}$ produced lobar pneumonia in dogs in 1912 by injecting cultures in the bronchi and blowing them into the finer bronchioles with air. Similar experiments have been made by Winternitz and Hirschfelder. ${ }^{64}$

The most striking parallelism between experimental animal infection and pneumonia as it occurs in man has recently been obtained by Cecil and Blake. Using Macaceus and other species of monkey, they injected small amounts, 0.1 to 0.2 e.c. of virulent pneumococcus cultures directly into the trachea of these animals, and, after an incubation time of a day or slightly longer, they obtained typical lobar pneumonias. This work will be further spoken of below.

${ }^{62}$ Wadsworth, Amer. Jour. Med. Sei., May, 1904.

${ }^{63}$ Lamar and Meltzer, Jour. Exp. Med., xv, 1912.

6. Winternitz and Hirschfelder, Jour. Exp. Med., xvii, 1913. 


\section{PNEUMOCOCCUS INFECTIONS IN MAN, AND CLINICAL- BACTERIOLOGICAL CONSIDERATIONS}

In man the most frequent lesion produced by the pneumococeus is acute lobar pneumonia. About 90 per cent of all eases of this disease are caused by the pneumococeus, the remainder being due to streptococci, influenza bacilli, and other organisms, the relative frequency of which, in this disease has been given in an earlier section of this chapter. The relative frequency of the various pneumococcus types in lobar pneumonia in and about New York is given as follows by Avery, Chickering, Cole and Dochez, ${ }^{85}$ from whose work the following table is taken.

\section{TABLE 2-INCIDENCE OF TYPES OF PNEUMOCOCCUS IN LOBAR PNEUMONIA}

\begin{tabular}{|c|c|c|c|}
\hline \multirow{2}{*}{ Type of Pneumococcus. } & \multicolumn{3}{|c|}{ INCIDENCE } \\
\hline & No. Cases. & the & Per Cent. \\
\hline I. . . . . . & 151 & & 33.3 \\
\hline II $\ldots \ldots \ldots \ldots \ldots \ldots$ & 133 & & 29.3 \\
\hline $\mathrm{II} a \ldots \ldots \ldots \ldots \ldots \ldots \ldots \ldots$ & 6 & & 1.3 \\
\hline $\mathrm{II} b \ldots \ldots \ldots \ldots \ldots \ldots \ldots \ldots$ & 4 & & 0.9 \\
\hline 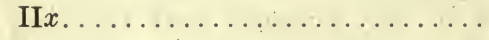 & 9 & : & 2.0 \\
\hline III $\ldots \ldots \ldots \ldots \ldots \ldots \ldots$ & 59 & 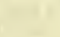 & 13.0 \\
\hline IV $\ldots \ldots \ldots \ldots \ldots \ldots \ldots \ldots \ldots$ & 92 & & 20.3 \\
\hline
\end{tabular}

This table may be contrasted with the following, also the result of work by the above named authors, which shows the distribution of the different types in the mouths of normal individuals, a point which has considerable importance in connection with the problem of autoinfection with which we will deal in greater detail in speaking of the epidemiology of the disease.

There has been a great deal of discussion concerning the route by which infection of the lung comes about after the pneumococeus has entered a susceptible subject. The difficulties experienced by many observers in infecting animals by direct instillation of pneumococei into the lungs, have inclined many observers to assume that

${ }^{65}$ Avery, Chickering, Cole and Dochez, Monograph of the Rock. Inst., No. 7, Oct. 16, 1917. 
in most pneumonias infection of the blood, or bacteriemia, precedes pulmonary lodgment. The recent experiments of Blake and Cecil, ${ }^{66}$ however, have, it seems to us, shown pretty definitely that the failure of other observers to produce direct infection of the lungs experimenally, were due very largely to failure to choose the most favorable animals for this purpose, and, therefore, failing to obtain the balance between pathogenicity of the organism and resistance of the subject which is necessary to determine the localization of the pneumococci in the pulmonary alveola.

TABLE 3-DISTRIBUTION OF DIFFERENT TYPES OF PNEUMOCCOCUS IN MOUTHS OF NORMAL PERSONS *

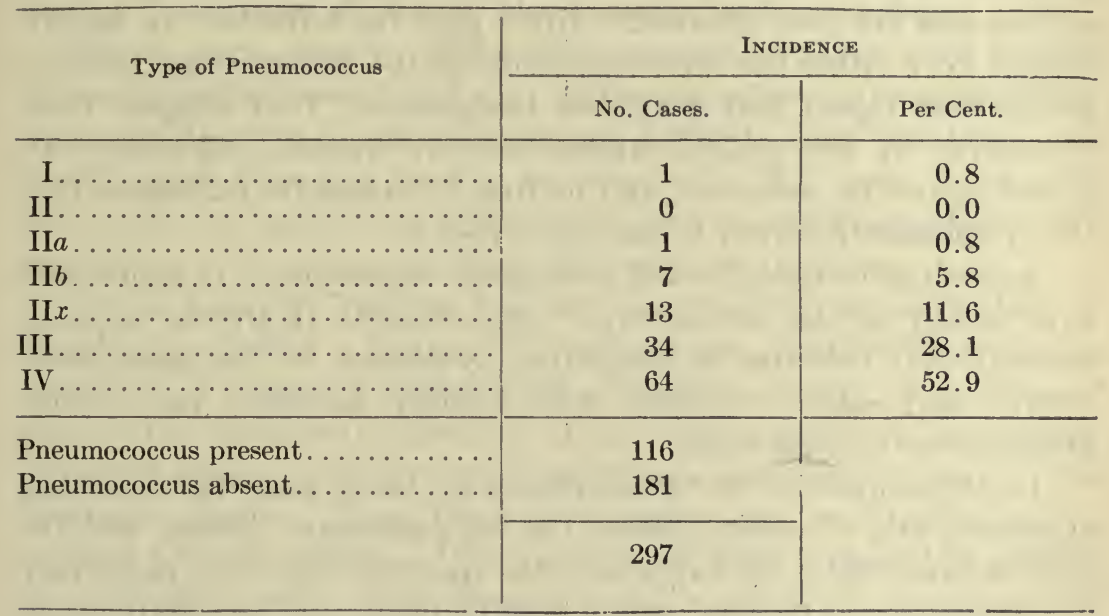

Blake and Cecil succeeded in producing various types of pneumonias in the lower monkeys (Macaccus Syrichtus, etc.) by injecting small amounts of virulent organisms directly into the trachea with a fine needle. When they injected 0.1 c.c. of an eighteen-hour broth culture of virulent pneumococcus they usually obtained definite symptoms within twenty-four hours, at which time the monkeys showed rapid respiration and positive blood cultures. Such monkeys often died within eight to twelve days, with typical pneumonic autopsy findings, often with actual fibrinous pleurisy and the classical anatomical changes of human pneumonia. These experiments seem to represent a fairly accurate analogy to the human disease.

${ }^{66}$ Blake and Cecil, Jour. Exper. Med., 31, 1920, 403, 455, 599, 518, 657, 685; Jour. Exper. Med., 32, 1920, 1, and 401. 
Winternitz, Smith and Robinson ${ }^{67}$ have, however, recently suggested another possibility. They have called attention to the fact that there is a rich plexus of lymphatics about the trachea, within the submucosa. They believe that the nature of the tracheal and bronchial submucosa, with its ciliated mucous epithelium, renders it extremely difficult for bacteria and other materials to reach the lung by this open route under normal conditions, and call attention to the great difficulty which has been encountered in attempts to produce disease by mere inhalation without pulmonary irritation or injury of the respiratory tract. Their idea is that infection of the lung is accomplished by the entrance of the bacteria into the Jymphatics surrounding the trachea through some injury to the mucosa and are then afforded a direct path for infection. In experimental work either the infecting needle or the catheter may produce preliminary injury and lymphatic inoeulation. They support thcir contention by inoculating rabbits both by tracheal injection with a needle, and by catheters, and finding in successful infections that the lymphatics referred to were involved.

According to Cole, ${ }^{68}$ when pneumonia is secondary to septicemia it is usually of the lobular type. Experiments of Meltzer seem to indicate that infection is facilitated by closure of the small bronchioles, and cold or ehilling may possibly stimulate the mucous glands so as to plug these.

In the course of the development of pneumonia the infecting organisms are, of course, located in the pulmonary alveolæ and the smaller bronchioles, and appear in the sputum. Since the possibility of specific serum therapy which will be dealt with in detail below have made it desirable not only to determine whether the disease is caused by the pneumococeus, but also have necessitated our knowing which particular type is responsible in the individual case, all pneumonia cases should be typed whenever possible.

Typing from Sputum.-A technique for this has been developed by the workers mentioned above and the technique of Dochez and Avery, with no essential changes, but a few additional remarks, is as follows: Since it is important not to confuse the lung invader with adventitious pneumococei present in the mouth, the collection of the sputum is an important part of the technique. Failure to

${ }^{67}$ Winternitz, Smith and Robinson, Bull. Johns Hopkins Hosp., 31, 1920, 63.

${ }^{68}$ Cole, Harvey Lecture, New York, Dec. 13, 1913. 
exercise care in this respect has lead to many Type IV reports when the pneumonia was actually caused by I, II, or III. The special sputum for typing should be collected in a separate eup, and not in the general sputum eup into which the patient has been spitting all day. The patient should rinse his mouth thoroughly with salt solution, bicarbonate of soda solution, or perhaps weak alcohol, and a specimen of sputum should be immediately obtained by coughing. It is collected in a sterile Petri dish or clean cup, and should not be allowed to stand around in the ward for any length of time, but should be sent to the laboratory, or stored in the ice chest.

A Gram stain should be made of the sputum as a preliminary survey. A capsule stain may also be made with advantage.

In the rare cases in which mice are not available, the sputum should be plated upon blood agar plates for subsequent agglutination or single colonies by the microscopic method. But this is not desirable, and mice can usually be procured. We have found gray mice quite as suitable as white mice, and in outlying laboratories an extensive use of mouse traps will solve the problem.

As soon as the sputum has been received in the laboratory it should be washed. A small bit of sputum, preferably from the center of a clump, is lifted with a platinum needle into three or four Petri dishes containing sterile salt solution or broth, and gently swabbed about. The thorough washing is extremely important for clean-cut results. Some times the sputum can be directly sucked up into a sterile syringe. If this cannot be done, it should be ground in a sterile mortar with about 1 to 2 c.c. of sterile broth or salt solution added gradually. About 0.5 c.c. of this is intraperitoneally injected into a mouse. Great care should be exercised not to inject too much since massive inoculation may give contaminating organisms a chance, and many mice have been wasted in this way. In the mouse the pneumococcus, if virulent, outgrows most other organisms. However, occasionally mice will die of streptococcus infection and may show streptococeus, influenza and oecasionally staphylococcus infection, but this is relatively infrequent.

The mouse will appear very sick or die between ten and twentyfour hours. When the mouse is either in extremis or dead it should be immediately autopsied.

Pin the mouse down on a small board, carefully dissect off the skin and, with sterile instruments, open the peritoneum. 
Make a smear of the peritoneal exudate and stain by Gram. If the pneumococeus is pure in the peritoneum wash out the peritoneal exudate with a small nipple pipette, with about 3 or 4 (.c. of sterile salt solution into a centrifuge tube. Make a smear from the heart's blood and also take a culture on a blood agar slant from the heart's blood with sterile instruments.

Centrifuge the exudate previously removed gently at low speed for a few moments to throw down leucocytes and larger particles. Remove the turbid supernatant fluid which should have the maximum turbidity of a well grown broth culture of pneumococei, into another centrifuge tube and throw down the organisms at high speed. Resuspend the sediment in enough salt solution to give a turbid suspension of proper concentration for agglutination reactions. With this material agglutinations are set up in small tubes as follows: The table presents the routine method advised by the workers mentioned above.

\section{TABLE 1-DETERMINATION OF PNEUMOCOCCUS TYPES BY AGGLUTINATION $^{69}$}

\begin{tabular}{|c|c|c|c|c|}
\hline $\begin{array}{l}\text { Pneumococcus. } \\
\text { Suspension, } 0.5 \text { c.c. }\end{array}$ & $\begin{array}{l}\text { Serum I } \\
(1: 20) \\
0.5 \text { c.c. }\end{array}$ & $\begin{array}{l}\text { Serum II } \\
\text { (Undiluted) } \\
0.5 \text { c.c. }\end{array}$ & $\begin{array}{l}\text { Serum II } \\
(1: 20) \\
0.5 \text { c.c. }\end{array}$ & $\begin{array}{c}\text { Serum III } \\
(1: 5) \\
0.5 \text { c.c. }\end{array}$ \\
\hline Type I $\ldots \ldots \ldots \ldots \ldots \ldots \ldots$ & ++ & - & - & - \\
\hline Type II . . . . . . . . . . . . . . & - & ++ & ++ & - \\
\hline Sub-groups $\mathrm{II} a, b, x \ldots \ldots \ldots \ldots$ & - & + & - & - \\
\hline Type III . . . . . . . . . . & - & - & - & ++ \\
\hline Type IV. . & - & - & - & - \\
\hline
\end{tabular}

Incubation for 1 hour at $37^{\circ} \mathrm{C}$.

Circumstances may arise, as in the recent war, where it has been practically impossible to obtain enough mice to carry out the above technique in all cases. In such cases we ourselves have often successfully typed from colonies grown on blood agar plates by microscopic methods, in the unsuccessful cases having typed subsequently from blood cultures. Avery has recently advised the use of a medium in which the pneumococcus is apt to outgrow other organisms. It depends upon the use of the following medium:

To 90 c.c. of a suitable meat infusion broth of a $P_{H}$ of 7.8 , add 5 e.c. of a sterile 20 per cent solution of dextrose to bring it to a

${ }^{60}$ This table taken from Avery, Chickering, Cole and Docher, loc. cit. 
concentration of 1 per cent and 5 e.c. of defibrinated rabbit's blood. These substances, sterile, must be mixed in tubes containing about 5 c.c. each and must not be resterilized after mixture.

The sputum is very carefully washed in sterile salt solution. We prefer to do the washing for this particular technique in successive test tubes of sterile broth. After about three washings, the sputum is gently ground in the last broth tube with a glass rod, and about 0.5 to 1 c.c. of this inoculated into tubes of the medium given above. Growth is allowed to take place for from five to eight hours, and when pneumococei have appeared in large numbers in the supernatant fluid, the red cells are thrown down by centrifugation, and agglutinations done on the supernatant fluid as above.

In all such typings it must not be forgotten that agglutination reactions must be accompanied by a bile test on the materials used in order to make sure that the organism is a pneumococeus.

Typing by Protection Experiment.-The protection experiment is not very practical for elinical use where speed is required. It is, however, important that the technique of specific mouse protection should be well known to the bacteriologist. This technique will be given in greater detail in connection with the determination of the potency of immune serum in a subsequent section.

Typing by Precipitins.-In broth cultures the pneumococcus gives up an antigenic substance which is soluble to the broth which has been made use of by Blake ${ }^{70}$ for typing purposes. The method is useful when cultures or peritoneal exudates from mice are too heavily contaminated for clear agglutination tests. The fluid to be examined, either culture or peritoneal exudate, is centrifuged at very high speed to throw down all the organisms, and the clear supernatant fluid taken off for precipitation experiments. It is then mixed in quantities of 0.5 c.c. with equal quantities of serum specific for the three main types of pneumococci. Type $I$ serum is used in a 1-10 dilution, Type II, undiluted, 1-10, and Type III, 1-5. In this connection Dochez and Avery ${ }^{71}$ have made an interesting observation which is of great theoretical as well as practical importance. They have shown that a soluble antigenic substance which gives specific precipitin reactions with anti-pneumococeus serum, appears in the urine of over 60 per cent of pneumonia eases. The substance may

70 Blake, Jour. Exper. Med., 26, 1917, 67.

${ }^{71}$ Dnches and Avery, Jour. Exper. Med., 26, 1917, 477. 
be excreted within the first day after the onset of the disease, and may continue in the urine during early convalescence. Since it is almost always present in septicemia, it may have some value in sizing up a case in this way.

The substance ean be demonstrated by direct precipitation of the clear urine against specific pneumococeus sera. A curious thing about the substance is that it can be obtained in concentrated urine in the following way. 25 c.c. of the urine is boiled with weak acetic acid and the proteins removed by filtration. The filtrate is then precipitated with 95 per cent alcohol, rapidly dried, and redissolved in small amounts of salt solution up to 3 c.c. This solution reacts specifically with the serum.

Blood Cultures in Pneumonia.-During the course of pneumonia, pneumococcus septicemia is common. Frankel ${ }^{72}$ in 1902 stated that he believed in most, if not all cases of pneumonia the organisms are present in the blood stream at some stage of the disease. Proschaska, in a carefully repeated culturing of ten unselected cases, obtained the organisms in every one of them. The older literature, if carefully reviewed, shows positive blood cultures in about 25 per cent of the eases. In the Rocikefeller Hospital where systematic blood cultures were done, among other things, it is stated by Cole that in 448 cases of lobar pneumonia, the pneumococei were obtained by blood culture in 30.3 per cent. When blood cultures were repeatedly made at frequent intervals the positive findings were obtained in 50 per cent.

In taking blood cultures it is important that plenty of blood is taken and inoculated. The culture is taken from the basilic vein with a sterile syringe as follows: At least 5 or 10 e.c. of blood should be added to flasks of hormone glucose broth, of a $\mathrm{P}_{\mathrm{H}}$ of 7.6 or 7.8, containing not less than 100 c.c. of broth. Hormone glucose agar plates should at the same time be made, and graded quantities of blood can be added to successive plates in order that one may obtain a numerical estimate of the number of organisms per c.c. Growth is often delayed, and no negative report should be finally turned in for at least three days. Cole and others have attached great prognostic significance to blood cultures. Cole believes that the development of a septicemia is of very serious prognostic significance, and the typing of the organisms from the blood culture

${ }^{22}$ Frankel, “v. Leyden Festschr.,", 1902. 
is important in this respect since the mortality of type II cases in the blood in his experience is 73.4 per eent, and of type III cases in the blood, 100 per cent, whereas, in type IV cases a mortality of only 52.3 per cent, and the low percentage, 26 per cent, in type I blood culture cases, he attributes to the effect of serum treatment.

Aside from lobar and lobular pneumonia, the pneumococeus may cause a number of other types of infection in human beings either subsequent to a preliminary pulmonary infection, or primary in nature.

The most common complications of pneumococcus infection of the lung are empyemia, endocarditis, and pericarditis, meningitis and arthritis. Meningitis may occur as a primary disease especially in children without previous traceable pneumonia. The same may be said of arthritis.

Empyema was a very frequent and fatal complication of the war pneumonias, and pneumococci can be easily obtained by ordinary cultural methods from puncture fluid. It has recently been suggested that empyema is more apt to follow in cases which have been treated with serum, perhaps because of its effect in localizing the infection. This point has not been settled, but it would seem to us that the only manner in which such a result could eventuate would be by just such localizing effect, and this would mean that had not the serum localized the infection the outcome might have been fatal, a consideration which all the more persuades us of the wisdom of treating type I cases with serum whenever possible. Pneumococcus meningitis, whether primary or secondary, is a very fatal disease from which recovery is rare. Direct serum treatment should always be tried if it is a type I case, and intravenous treatment to forestall a possible septicemia should also be applied.

Pneumococcus peritonitis occurs particularly in children.

The pneumococcus also causes a very severe form of corneal ulceration which presents great difficulties to successful therapy. The bacteriologist confronted with severe ulcerative infections of the eye, in which ulcerations are especially localized on the cornea, should search particularly for pneumococei.

Typing from all these various lesions should be done if for no other reason in the interest of statistical knowledge. From the statistics furnished by the Rockefeller Hospital studies, as well as additional information which the past five years have furnished over all the United States and Europe, it seems fair to assume that 
type III is probably the most dangerous of the organisms, followed closely by types II and I, in the order named, and that type IV organisms have, as a rule, a less alarming prognosis.

\section{ANTIBODY FORMATION, IMMUNITY, AND SPECIFIC THERAPY}

Recovery from a spontaneous pneumococcus infection confers immunity for only a short period. Two and three attacks of lobar pneumonia in the same individual are not unusual, and it is uncertain whether even a temporary immunity is acquired in such infections. Our recent knowledge of types has made it seem not impossible that successive attacks of pneumonia may be due to consecutive infection with different types of organisms, thus leaving open the possibility of the acquisition of prolonged immunity. But this seems doubtful in view of the fact that Chickering ${ }^{73}$ and others have seen individuals who have had four or five attacks within a relatively short time. The point, at any rate, is by no means settled. Active immunization of laboratory animals may be carried out by various methods. The method usually followed is to begin by injecting attenuated ${ }^{74}$ or dead bacteria or bacterial extracts. Subsequent injections are than made with gradually increasing doses of living, virulent microorganisms. Great care in increasing the dosage should be exercised since the loss of an animal after two or three weeks' treatment by a carelessly high dose of pneumococci is not unusual. Wadsworth centrifugalizes freshly grown pneumococcus cultures and to the pneumococcic sediment adds a definite quantity of concentrated salt solution. At the end of 12 hours, the pneumococci are dead and considerable destruction of the cell-bodies has taken place. Dilution with water until the solution equals 0.85 per cent $\mathrm{NaCl}$ now prepares the emulsion for inoculation. The sera of animals immunized with pneumococci contain active bactericidal substances.

Specific agglutinins in pneumoeoccus immune sera were first thoroughly studied by Neufeld ${ }^{75}$ and since then have been made the

${ }^{73}$ Chickering-Discussion on pneumonia-N. Y. Academy of Medicine, April, 1920.

${ }^{74}$ Radzïewsky, Zeit. f. Hyg., xxxvii, 1901; Neufeld, Zeit. f. Hyg., xi, 1902.

${ }^{75}$ Neufeld, loc. cit. 
subject of extensive studies by Wadsworth, ${ }^{76}$ Hiss,${ }^{77}$ and many others. For the sake of obtaining plentiful growth for agglutination purposes, Hiss has recommended cultivation in 1 per cent glucose broth with the addition of small amounts of sterile ealcium carbonate to absorb acid formed from the glueose. Pneumococei do not regularly agglutinate in diluted immune sera and agglutinations are best studied in suspensions of more concentrated immune serum. Agglutination begins at the end of about 15 minutes, and can be studied both by formation of elumps and by the sediment. See preceding paragraphs on typing.

Specific precipitating antibodies have been demonstrated in pneumococeus immune serum by Neufeld, ${ }^{78}$ Wadsworth, ${ }^{79}$ Hiss, ${ }^{80}$ and others, the organism for such tests being brought into solution either with bile or with concentrated salt solution. Such sera also contain powerful opsonic substanees, or, as Neufeld and Rimpau ${ }^{81}$ prefer to call them, "bacteriotropins." It scems most likely that such phagocytosis-aiding substances are most powerfully concerned in protection and cure. Clough ${ }^{82}$ has reported an increased of opsonins at the time and crisis, and Dochez ${ }^{83}$ has shown that protective substances may appear in the serum at or soon after the time of crisis. The outcome of a case according to Cole depends very largely on the virulence of the organism and on the ability of the body first to limit the local infection and to prevent the invasion of the blood with the organisms. In this process, of course, the protective and opsonic bacteriotropic substances would play a most important part.

\section{PASSIVE IMMUNIZATION AND USE OF PROTECTIVE SERA}

The history of attempts to produce sera for passive immunization in man is extensive. Washburn, ${ }^{84}$ Mennes, ${ }^{85}$ Pane $^{86}$ and many others in the past have succeeded in protecting animals with such sera, but

\footnotetext{
${ }^{76}$ Wadsworth, loe cit.

${ }^{77}$ Hiss, Jour. Exp. Med., vii, 1905.

${ }^{78}$ Neufeld, Zeit. f. Hyg., 1902, xi.

${ }^{79}$ Wadsworth, loc. eit.

${ }^{80}$ Hiss, Jour. Exp. Med., vii, 1905.

${ }^{81}$ Neufeld and Rimpau, Deut. med. Woch., 1904.

${ }^{82}$ Clough, Johns Hopkins Hosp. Bull., Oet., 1913.

${ }^{83}$ Dochez, Jour. Exp. Med., 1913.

s4 Washburn, Brit. Med. Jour., 1897.

${ }^{85}$ Mennes, Zeit. f. Hyg., 1897.

${ }^{s 6}$ Pane, Rif. med., 1897.
} 
with irregular results. The rational beginning based on the recognition of different pneumococeus types was made by Neufeld and Haendel in Germany, and carried to a considerable degree of success by Cole and his associates at the Rockefeller Hospital in New York. By the immunization of horses with the various types of pneumococci mentioned above, considerable success has attended the use of sera produced with Type I, and less success but great promise, that of sera produced with Type II. The injection of considerable quantities of the homologous sera intravenously at least aids in sterilizing the blood stream, and upon this the eventual outcome of many cases may depend.

The actual method of producing serum at the present time depends upon the injection of horses with, at first, killed cultures and then living pneumococei. Horses are used as in other serum production, and all the preliminary precautions against glanders and tetanus taken. The pneumococci used are, of course, typed, since, as we shall see below, type I serum is the only one that so far has yielded at least hopeful results. The organisms are grown on suitable broth, and young cultures are injected. For the early injections the organisms are killed by heating from $56^{\circ}$ to $60^{\circ}$. The method of Cole is to inject daily for six days the killed bacteria thrown down from 50 c.c. of a 12-hour broth culture. A rest of a week is then given, and the serum of the horse tested for agglutination. A second series of dead culture injections is then carried out, and again an interval allowed. Again a test is made, and if agglutination is as high as 1-200, and 0.2 c.c. protects a mouse against 0.1 c.c. of a virulent culture, the serum could be used, but Cole states that this is not often the case. After the second series of dead culture injections, living bacteria are injected; three injections containing bacteria from 2.5 c.c. of the original culture are then given. The actual methods of injecting horses will vary with individual experience in different places, but in all cases the principle is the old one of first injecting the living cultures with great care not to infect the horse, and bleeding determined by preliminary test.

The horses are bled in the usual way, and the serum obtained. The serum is taken up, stored, and handled as in the case of other protective sera.

Recently a great deal of very interesting work has been done upon the relative purification of pneumococcus anti-sera from horse protein by attempts to isolate the antibodies from whole serum. Gay and Chickering ${ }^{87}$ precipitated dissolved pneumococeus antigen with anti-

${ }^{87}$ Gay and Chickering, Jour. Exper. Med., 21, $1915,389$. 
serum, thus carrying down the antibodies. They then exiracted these precipitates with weak sodium carbonate at $42^{\circ}$, and in the supernatant fluid found protective antibodies which agglutinate pneumococci. Huntoon has recently perfected this method of dissociation of antibody from its antigen in a way that promises practical usefulness. He treats large amounts of pneumococci with an excess of antibody, at $3712^{\circ}$. After throwing down the pneumococci with a centrifuge, he now washes them with physiological salt solution, at almost the freezing point in order to remove traces of serum, and then treats them at a temperature of about $40^{\circ}$ with weak sodium bicarbonate solution, a treatment which, as Landsteiner and others have shown with other organisms, dissociates antibodies in large amounts from the antigenantibody complex. These antibody solutions, if the volume of final solvent is either at or about one-fourth the original serum volume, is protective in approximately the same degree as the original sera, and is almost or perhaps completely protein free. These antibody solutions of Huntoon are at the present time being used experimentally and promising results have already been obtained. Their intravenous injection produces an initial chill, probably due to the non-specific reaction caused, in our opinion, by traces of bacterial protein in the solution. The cases which we have seen reported, show, however, that subsequently a specific reaction seems to occur which promises to render them perhaps an improvement upon treatment with whole serum.

Standardization of Pneumococcus Serum.-After a considerable amount of discussion as to which of the antibody reactions should be used for pneumococcus serum standardization, it has been generally accepted that standardization by mouse protection is the most reliable method. The pioneer work on such standardization was done largely by Neufeld. Recently, it has been developed by various pneumococeus workers, Wadsworth and Kirkbride, ${ }^{88}$ the workers at the Rockefeller Hospital, and a number of the manufacturers of pneumococcus seruin. The standardization depends upon the amount of serum necessary to protect a white mouse of 20 grams weight against a standard virulent culture.

One of the most important points in the standardization is to use a culture of very great and accurately known virulence. This can be produced by passage through mice. The virulence of the organism

ss Wadsworth and Kirkbride, Jour. Exp. Med., 25, 1918. 
used should be so great that 0.000001 e.c. of an 18-hour broth culture will kill a mouse in 48 hours. Broth dilutions are then prepared in such 2 . way that 0.5 c.c. of each dilution contains varying quantities of the pneumococeus culture ranging from 0.2 c.c. to 0.0000001 c.c. These dilutions should be freshly made in order that the number of organisms in the tube shall not be materially changed by growth or death before the tests are made. With each of these dilutions, then, 0.2 c.c. of the serum to be tested is mixed in a syringe and the mixture immediately injected intraperitoneally into the abdominal wall just above the groin. In some laboratories the amount of serum used for these standard tests is 0.1 instead of 0.2 c.c. The following is a typical protocol taken from a pretection experiment by Dochez and Avery, which illustrates the method:

\begin{tabular}{|c|c|c|c|c|c|c|}
\hline \multirow{2}{*}{$\begin{array}{l}\text { Dose of } \\
\text { Culture }\end{array}$} & \multicolumn{3}{|c|}{67 (Group I). } & \multicolumn{3}{|c|}{ A69 (Group II). } \\
\hline & Controls. & $\begin{array}{l}\text { Serum } I \text {, } \\
0.2 \text { c.c. }\end{array}$ & $\begin{array}{l}\text { Serum II, } \\
0.2 \text { c.c. }\end{array}$ & Controls. & $\begin{array}{c}\text { Serum I, } \\
0.2 \text { c.c. }\end{array}$ & $\begin{array}{l}\text { Serum II, } \\
0.2 \text { c.c. }\end{array}$ \\
\hline 0.1 c.c. & $\begin{array}{c}\text { Dead } \\
17 \text { hrs. }\end{array}$ & $\begin{array}{c}\text { Survived } \\
\text { " }\end{array}$ & $\begin{array}{c}\text { Dead } \\
41 \text { hrs. }\end{array}$ & & $\begin{array}{l}\text { Dead } \\
18 \text { hrs. }\end{array}$ & $\begin{array}{c}\text { Dead } \\
18 \text { hrs. }\end{array}$ \\
\hline 0.01 & $17 \mathrm{hrs}$. & "“ & $25 \mathrm{hrs}$. & & $18 \mathrm{hrs}$. & Survived \\
\hline 0.001 & $41 \mathrm{hrs}$. & “6 & 41 hrs. & & $18 \mathrm{hrs}$ & 16 \\
\hline 0.0001 & $41 \mathrm{hrs}$. & “6 & $41 \mathrm{hrs}$. & $\begin{array}{c}\text { Dead } \\
18 \mathrm{hrs} .\end{array}$ & & $" 6$ \\
\hline 0.00001 & 96 hrs. & “ & $41 \mathrm{hrs}$. & $18 \mathrm{hrs}$. & & “ \\
\hline 0.000001 & 48 hrs. & or & $72 \mathrm{hrs}$. & $18 \mathrm{hrs}$. & & "6 \\
\hline
\end{tabular}

(The quantities in c.c. representing dose of culture refer to 18 hour broth cultures.)

The rule laid down for sera by Cole and his co-workers is that only sera should be employed which in doses of 0.2 c.e. protects against doses of 0.1 c.c. of a culture of the above description. Variations in these standards are set up in other laboratories, and eonstant changes are taking place in this phase of the work, but the above will sufficiently illustrate the principle applied.

Methods of Serum Treatment and Results.-Hope of success with serum treatment depends upon early diagnosis and immediate undelayed typing of the organism, since, as we shall see, the type I serum alone is at the present time thought hy everyone to exert definite beneficial action. 
Since the serum is given intravenously it is important to be very careful in estimating whether or not the patient is horse serum sensitive. Inquiry as to previous serum injections must be made, and intradermal skin tests with horse serum are done by injecting, intracutancously, with a tuberculin syringe, a small amount, anywhere from 0.05 to 0.02 e.c. of a $1-10$ horse serum dilution. If the injection is properly made, in a sensitive subject within anywhere from five to thirty minutes a large urticaria-like wheal will arise, which will remain for one-half to two hours, gradually disappearing. In such eases great care should be exercised in administering the serum and attempts made at desensitization by the Besredka method, that is, gradual injection of increasing amounts. This is not absolutely reliable, but probably is of great help in most cases. It is best to begin with slow subcutaneous injection of about 0.02 c.c. of horse serum, best diluted in a total quantity of 5 c.c. of salt solution. This can be repeated, gradually increasing the dose to one c.c. in three or four instillations if no untoward symptoms appear. Even in these preliminary injections it is best to inject very slowly, and to be sure that the needle does not enter a small venule, leaving about an hour between injections. It is difficult to lay down definite rules for quantity and manner of injection, since in each individual case an experienced worker should. feel his way gradually in the case. Gradual desensitization until a large intravenous injection can be given may consume 24 hours or more during which time the cumulative doses may furnish a considerable fraction of the therapeutic dose.

When the time for actual injection comes, the serum is diluted with equal parts of sterile salt solution. This permits one to inject the substance more slowly. A special gravity apparatus for slow injection has been devised for this purpose by Cole and his co-workers, but with great care the injection can be done directly with a large syringe. The serum mixture should, of course, be brought to body temperature before injection. The important point is that the injection of the first 10 c.c. of the serum should occupy at least ten minutes, and this is the critical time for the development of anaphylactic symptoms during which the patient must be carefully watched. Any signs of respiratory difficulty, sudden changes in the pulse, etc., should be an indication for immediate cessation of the injection, which can be begun again when the patient has returned to normal.

The total dosage advised by Cole and his co-workers is about 90 to 100 c.c. Reinjection is a matter of judgment, and these workers advise 
that the treatment should be vigorously continued by reinjections every 8 or 10 hours, as often as it seems advisable.

Serum disease which occasionally follows is similar to that which follows diphtheria antitoxin injections.

In discussing the results of serum treatment no final judgment can be given. There have been all kinds of extravagant claims both as to usefulness and uselessness of the serum. Cole's judgment we consider to be entirely objective and unprejudiced in this matter, and he states from his own experience that he has obtained no results with any serum except type I. With this serum, however, he believes that there has been a definite drop in the mortality, to about 8 per cent as contrasted with a normal mortality of 20 per cent, or more, in untreated type I cases. In general, this opinion scems to have been borne out by other observers who have used the method. It is of the greatest importance that in judging of results of such treatment a discrimination should be made between cases that have been treated promptly in the early stages of the disease, and those in which the treatment has been delayed, for, as every bacteriologist knows, when dealing with the pathogenic Gram-positive cocci, that no amount of serum will save an experimental animal when once an extensive septicemia has been established.

\section{EPIDEMIOLOGY OF PNEUMONIA}

Pneumonia is endemic in most well-populated centers of the world, but seems to be particularly frequent in the temperate zones. The disease is present sporadically at almost all times of the year, but is particularly frequent during the colder months, usually reaching its annual peak in this latitude during February or March. It is not commonly an epidemic disease, but may become so under conditions of crowding, and wholesale exposure to wet and cold, incident to military life, or the life in mining camps, etc. Wherever, in other words, very close association of limited groups of people takes place under conditions of poor hygiene and crowding with coincident hardships of various kinds, pneumonia epidemics are apt to occur. The most extensive epidemies which have occurred within the last 20 years are those which took place in the South African mining districts, in Panama, and during the World War in the camps and among the armies at the front. Pneumonia of all kinds in ordinary times accounts for about 
10 per cent of the death rate, but under conditions like those occurring during the war, a much larger percentage of all deaths are due to pneumonias. The Surgeon General estimates that in the year 1918 the total number of deaths chargeable to respiratory disease (and this means with very few exceptions, death by pneumonia of one kind or another) was 39,701 , out of a strength of 2,518,499 men, which amounts to a death rate of 15.75 per thousand, and 82 per cent of all deaths occurring in the Army during this year.

In order to discuss the epidemiological and preventive problem concerned with pneumonia with intelligence, it will be necessary to discriminate between the so-called "primary" pneumonias and "secondary" pneumonias.

Inflammations of the lung may be caused by a variety of bacteria. However, for the purposes of considering the epidemiology of these diseases we need take into account only those caused by various pneumococei, the hemolytic streptococei, and influenza bacilli. The characteristics of an epidemic will vary considerably according to whether the majority of the cases are typical lobar pneumonias, coming on without previous illness, or whether most of the cases represent pulmonary infection, secondary to a preceding attack of influenza or to measles. Typical lobar pneumonia is almost regularly a pneumococeus infection and this type of the disease is by far less fatal than the other. Tho secondary pneumonias may be caused by many different organisms. Even in the same community, cases occurring at about one and the same time, may be caused by various pneumococci, or streptococci, the majority of the cases being due to organisms most prevalent in that particular place. Such epidemics of secondary pneumonia are the types which are most apt to develop in times of war or under other abnormal community conditions, and this type is far more fatal than is the typical lobar pneumonia.

Primary Pneumonias.-That pneumonia was a communicable disease was recognized by Johannesen and other clinicians as early as the middle of the last century. This point of view, however, was not generally accepted until quite recently. One of the difficulties that has stood in the way of a more general belief in the communicability of the disease has been the fact that many normal individuals harbor in mouth and throat pneunococei which, until recent years, were indistinguishable from the organisms found in the lungs in pneumonia. It was taken for granted, therefore, that the entrance of pneumococci into 
the upper respiratory passages could not, in itself, produce pneumonia, and that when the disease occurred, it was in most cases due to autoinfection, owing to unusual depression of resistance in an individual in whose mouth the pneumococcus happened to be present. Moreover, there seemed to be many instances of relationship between unusual exposure to cold and wet, and the occurrence of pneumonia, while it was rarely possible to trace definitcly the origin of a case to exposure to a previous one.

Recent recognition that there are a number of different types of pneumococei, investigations which were begun by Neufeld and Haendel, ${ }^{89}$ and followed out more particularly in this country by Dochez and Avery, ${ }^{90}$ has furnished us with an entirely new set of facts for the understanding of pneumonia epidemies. The types of pneumococei, as worked out by these writers, have been tabulated in another place. This tentative subdivision into types has made it possible to determine, in the first place, whether or not the ordinary mouth types are identical with those found in the lungs during pneumonia, and have also permitted us to determine whether the type present in any particular case was identical with that found in a preceding case or in a closely associated contact. Following up this trail, workers at the Rockefeller Hospital have found that over 50 per cent of the mouth organisms found in normal human beings in and about New York city belong to the heterogeneous type IV group, whereas, over 80 per cent of lobar pneumonias are due to types I, II, and III. The obvious inference from this reversed percentage is that lobar pneumonia is in most cases caused by organisms transmitted to the victim from an extraneous source, and that autoinfection with the patient's own mouth organisms cannot be regarded as a very common occurrence.

It should not be concluded from this, however, that type IV is unimportant as a causative agent in the disease, since the more recent statistics gathered during the war show that a considerable percentage of eases in different localities may be caused by this group. However, since this group is composed of many apparently unrelated members, we ean not obtain further light upon the epidemiological conditions, under such circumstances, at the present time.

It has also been shown by Stillman ${ }^{91}$ and others that the more

${ }^{89}$ Neufcld ami Haendcl, Arb. a. d. k. Gsndhtsante, 1910, 34, 293.

30) Dochez and Avery, Prce. Soc., Exper. Med. and Biol., 14, 1916-17, 126.

${ }^{91}$ Stillman, Jour. Exper. Med., 26, 1917, 513. 
virulent types I, II, and III, may disappear from the mouths of convalescents within three or four weeks, and sometimes sooner, and be supplanted at such times by the less virulent normal type IV strains. Stillman has also shown that individuals associated with pneumonia patients may frequently harbor organisms of the same type as those infecting the patients, and he has found organisms corresponding to the patients' type in the dust of the siekroom. It seems unquestionable, therefore, that there may be carriers of virulent pneumocoeei entirely analogous to the earrier states developed with meningoeoeei and other organisms.

While autoinfeetion, therefore, cannot be completely excluded, it seems probable that the origin of most eases of lobar pneumonia is best explained by the aequisition of a virulent pneumococcus strain, either directly from a case or from a carrier, with a depression of resistance due to cold, exposure, etc., coincident with the presenee of this virulent strain. In the light of these facts it is clear that our sanitary point of view in regard to pneumonia must be materially changed. We can now understand why localized epidemics have been so often observed in institutions, war hospitals, and other crowded communities, and can justly evaluate the importance of the transmission factor in the spread of this disease. In outlining sanitary procedures for any disease it is of the utmost importance that such a thorough understanding of the relative importance of transmission and the suseeptibility faetor should be acquired. It is never possible to carry out all desirable measures of prevention completely, and it is, therefore, necessary to know definitely upon which factors the greatest stress must be laid in planning the sanitary campaign.

In all communicable diseases the two factors which influence spread are, in the first place, the transmission of the virulent organisms, and, in the second place, the suseeptibility of the recipient. When transmission becomes general and community susceptibility is normally high, as in plague, typhoid, cholera, ete., epidemics are bound to spread rapidly. There are diseases like those mentioned above, as well as smallpox, measles, scarlet fever, and influenza, in which the susceptibility of the normal, previously unexposed individual is so great that hardly anyone sufficiently exposed, will escape. It is plain that in such diseases sanitary measures must be aimed particularly at the prevention of transmission, with, wherever possible, artificial immunization of the community. There are other infections, however, chief among which we believe is pneumonia, in which the resistance of normal 
human beings is comparatively high. The disease will not occur in an individual simply because he has received the virulent organisms by the proper route, from a case or a carrier, but, in addition to this, there must be coincident hygienic defects which temporarily depress his resistance. A temporary coincidence of two factors, therefore, transmission of the organisms and increased susceptibility, must occur, and it is plain that in such diseases epidemic spread cannot take place to any extensive degree unless both of these factors, widespread transmission and depression of resistance, become generalized. In such cases, therefore, while proper safeguards against dissemination of the organisms must be developed, yet the efforts of the sanitarian should focus particularly upon measures by which the normal resistance of the community is maintained.

As a matter fact, pneumonia epidemics do not occur as a rule in well nourished and housed communities. The epidemic form of the primary disease develops only under such eonditions as those prevailing in army camps during the cold weather, when men are crowded together in sleeping quarters, and are developing colds and coughs, and are, at the same time, exposed to unusual conditions of life, cold, wet, unaccustomed food and hard work. Exceptions to this are, of course, epidemics like those that have occurred in Panama and South Africa, but in these cases the community in which the disease was prevalent consisted very largely of tropical negroes, whose greater susceptibility to pneumococcus infection is well known.

In a number of epidemics in which we have had the opportunity of studying cases, it was quite apparent that the susceptibility factor was the determinative one in individual instances. Surveys showed that, while it frequently happened that a number of cases came from the same tent, the infections were often of different bacterial types. While direct transmission from one case to another often seemed to be circumstantially proved, in only a few instances at a certain camp in which these studies were made, were such cases associated with a single type. On the other hand, a certain regiment which was ordered to the shooting range during a very wet spell, marching in the rain and camping on wet ground, developed 26 pneumonias within 16 days. Analysis showed that these cases were caused by all four pneumococcus types without particular relationship between contacts and types. On the other hand, a considerable number of men were found, in this same regiment, at that time, to be carriers of virulent pneumococci and streptococei without coming down with the disease. 
From evidence like this, we conclude that in the sanitation of pneumonia it would be dangerous to lay too great proportionate stress upon mere transmission, but to remember that the average resistance to pneumococcus infection of the lung is fairly high among human beings, and that sanitary precautions must include a very rigid attention to the factors of warmth, ventilation in sleeping quarters, adequate food, dryness of feet, and avoidance of overwork. In communities like those of South Africa, the prevention of transmission alone cut short the epidemic, but we have already pointed that the susceptibility factor was unusually high in these communities.

It will rarely be necessary for sanitarians working in civilized. communities to be called upon to prevent epidemics of primary pneumonia. They will develop under such conditions as those prevailing in military camps, and which might well be imagined as possible in badly managed industrial communities, schools, labor camps, etc., where laborer's are forced to sleep in ill-ventilated barracks-are crowded during working hours, or in mines, and crowded institutions. Such conditions may occur among civilian populations at times of famine, and penury incident to war. Primary pneumonia epidemics will occur only when erowding, coincident with generalization of mild respiratory infections increases the distribution of bacteria and when, at the same time, the community suffers from insufficient shelter and is perhaps under-nourished and overworked. The most important factor in the prevention of such outbreaks, therefore, is attention to the ventilation of sleeping quarters, sufficient number of blankets on beds, dry feet, warm and plentiful food, and opportunities for reasonable rest. If this is combined with isolation of coughing and sneezing individuals, at least during indoor life, if spitting is stopped and careful supervision of the cleansing of eating utensils, sterilization of handkerchiefs, etc., is enforced, such epidemics should yield readily.

Cases which have been diagnosed as lobar pneumonia should be reportable, like other infectious diseases. This has already been introduced by a number of health departments. In hospitals pneumonia cases should be treated as communicable, the cases isolated, at least by maintaining proper distance between beds, screening between beds, and care in the collection and disposition of sputum and other secretions. Care of eating utensils and general cleanliness should be carried out with proper consideration of the possibilities of communication that have been spoken of above. In view of the probability of persistence of the carrier state for four weeks or longer after con- 
valescence, great care in mouth disinfection and control of this feature before patients are returned to their homes should be practiced.

Secondary Pneumonias.-In Secondary pneumonias we are dealing with an entirely different sanitary problem. While pneumonia may be secondary to a large number of different diseases, the only ones which are of distinct epidemiological importance in this eonnection are influenza and measles. There is no epidemic of measles or influenza in which there are not, at the same time, a considerable number of pneumonias, and these pneumonias are more apt to take the form of the lobular or broncho-pneumonic type. In both of these diseases there is a certain amount of inflammation of the bronchial mucous membranes which seems to render the patient particularly susceptible to secondary infection with virulent pneumococei and streptococei. The peculiar susceptibility of patients with measles and influenza to pneumonia cannot be explained purely on the basis of the mild bronchial inflammation which may be considered distinctly an integral part of these diseases themselves. There is a depression of resistance to pulmonary infection which is quite out of proportion to that which accompanies many other conditions in which bronchitis and catarrhal inflammation of the upper respiratory tract are common. Measles epidemics are fortunately uncommon in urban communities, but may assume dangerous proportions in army camps, institutions, schools, etc. The mortality of uncomplicated measles is low, but the high mortality which so often accompanies epidemics of measles is almost entirely a pneumonia mortality. In one such epidemic which occurred at Camp Wheeler during the early stages of our entrance into the War, the mortality of measles pneumonias was 29 per cent. Sanitary measures under such conditions include, of course, those aimed at the prevention of the primary disease, as well as attempts at preventing the secondary pneumonias with which we are here particularly concerned. But for the saving of life, the sanitary attention to the prevention of the secondary complications is by far the more important of the two. With the prevention of the primary disease we deal with in the chapter on Measles, but a few drops may be said in this place concerning the important measures which should be taken during measles epidemies to prevent the occurrence of secondary pneumonias. The principles of such measures are twofold, in the first place to prevent the case which is coughing and spitting from transferring its mouth streptococci and pneumococei to others. There should be the 
most careful attention to the cleanliness of the mouth of measles patients, both for the reasons mentioned, as well as in order to discourage the lodgment of virulent organisms in the patient himself. Doctors and nurses should wear gauze masks when in close contact with the patient as much for the protection of the patient as for their own. A measles patient should never be allowed to remain in the same ward with pneumocoecus or streptococcus eases, and as soon as a measles case develops a severe bronchitis or pneumonia, he should be removed from the measles ward into a separate ward or room, since he has now become an active danger to other measles cases. In the measles ward itself beds should be screened one from the other, and there should be at least five feet between beds. It is of great importance that measles cases should be put to bed and kept warm and protected from catching cold as soon as the suspicion of the disease is definite, and similar eare should be taken during the course of convalescence.

In the case of influenza the conditions are similar. In the section on influenza it will be seen that this disease, in its pure form, is relatively mild and has a very low mortality. During the second and third waves of an epidemic, however, practically all influenza cases show some degree of respiratory infection, and the susceptibility to pneumonia is so great during this stage that not only is the percentage of pneumonias very high, but the mortality is appalling. During the year 1918 when the second great influenza wave struck the American Army, influenza was charged, by the Surgeon General's Report, with 688,869 admissions among the American troops for the year, the disease and its complications causing 23,007 deaths. 82 per cent of all the deaths occurring in the Army were due to acute respiratory disease. When we consider, as we shall, in the chapter on Influenza, that all the respiratory deaths chargeable to influenza are really deaths from secoudary infection, and not to influenza itself, the appalling importance, and not to influenza itself, the appalling importance of the secondary pneumonias from a sanitary point of view becomes apparent. The preventive measures that ean be taken in guarding against secondary pulmonary infection are chiefly indirect ones, but are of great importance. Among the most important are care of the patient himself. Studies by Swift, Harlow Brooks and others during the war have shown that immediate care in bed, as soon as the first suspicion of diagnosis of influenza is made, is of the utmost value in preventing the development of secondary infection. The greatest care should be taken of the 
mouth of influenza patients, brushing of the teeth and cleansing of the mouth with sodium bicarbonate solution, or salt solution gargle to which 20 per cent or 30 per cent alcohol can be added. The patient should be carefully guarded from infection by the doctors and nurses who should wear gauze masks for this purpose. This is not primarily in our minds a precaution to protect the physicians and nurses, but rather the other way around. Dangers of transmission of pneumomococei and streptococei from bed to bed should be guarded against as above in the case of measles. The pneumonias which occur during an influenza epidemic are very rarely due to influenza bacillus infection of the lung alone. The fatal disease is caused by a large variety of organisms, including the various pneumococci, the streptococei and some others. In any one particular place the majority of eases may be due to one or another of these organisms, this depending somewhat upon the bacteria which happen to be most prevalent in this community, and are passed from mouth to mouth under conditions of generalized respiratory transmission, occurring in this place. Thus, MacCallum and Cole studied a secondary pneumonia epidemic in which hemolytic streptococci were responsible for most of the cases, but usually the pneumonias following in the train of influenza are not of a single type, but caused by any virulent member of the lung invading group of bacteria that happened by chance to lodge on the mucous membranes of the subject rendered susceptible by his primary disease.

Thus, after an influenza epidemic has started, sanitary measures aimed at the prevention of the fatal secondary infections must focus upon the transmission factor entirely.

Prophylactic Vaccination against Pneumococcus Infection.-The value of prophylactic vaccination against pneumonia is not yet definite. Wright ${ }^{92}$ was the pioneer in this work in 1911, but since he did not know about type differentiation, the value of his work is limited. The first hopeful experiments were made by Lister ${ }^{93}$ in South Africa, who typed his pneumococci by the usual agglutination method, having his own types $\mathrm{A}, \mathrm{B}$, and $\mathrm{C}$ ( $\mathrm{B}$ and $\mathrm{C}$ corresponding respectively to II and $\mathrm{I}$ of our classification), made salt solution suspensions of the organisms, injecting at first six to seven billion, intravenously. Later he resorted to subcutaneous inoculations, giving three doses of two billion

${ }^{92}$ Wright and Morgan, Lancet, 1, 1914, 1 and 87.

${ }^{93}$ Lister, Pub. South African Inst. f. Med. Res., 1916, No. 8. 
each at weekly intervals. His results were distinctly encouraging, though inconclusive. Cecil and Austin ${ }^{94}$ did some experiments at Camp Upton in which they vaceinated 12,519 men against types I, II, and III, and their results also were encouraging, but again unconvincing. The problem is sufficiently advanced to encourage wholesale use, if conditions exist under which careful comparison'with unvaccinated controls can be made. It cannot, however, be regarded as definitely established as a prophylactic measure in the same way that we may regard typhoid vaccination. At times of epidemic it should be employed on as large a scale as possible, whenever possible, since only in this way will we be able to judge of its value eventually. The experiment can be done without harm, since the use of the vaccine is contra-indicated only in acutely sick individuals, in pulmonary tuberculosis and nephritis, in patients with chronic heart disease, and Cecil states also in pregnant women.

The vaccines which so far have been used consist of culture suspensions in salt solution killed by heating at $56^{\circ} \mathrm{C}$., for one-half hour, and standardized either by counting against red blood cells or by means of the nephelometer. 0.3 per cent tricresol may be added to preserve the suspensions which are made up so that about one thousand million pneumococei are contained in 1 c.c. The dosage advised by Cecil is three billions for the first dose, six billions for the second and nine billions for the third.

Lipo-vaccines which consist of pneumococcus suspensions in olive oil and other vegetable oil mixtures have been used, but the method of preparing these vaccines has not been satisfactorily perfected.

${ }^{94}$ Cecil and Austin, Jour. Exper. Med., 28, 1918, 19. 


\section{CHAPTER XXIV}

INFLUENZA-THE DISEASE AND ITS EPIDEMIOLOGY

THE INFLUENZA BACILLUS AND OTHER ORGANISMS OF THE HEMOPHILIC GROUP

The Disease.-In no disease is it so difficult to discuss the etiological and epidemiological problems as it is in influenza since clinical recognition is not sharp and is fraught with many uncertainties. In time of epidemics there are certain characteristies of onset, sequence of symptoms, and similarity of course which make the diagnosis relatively simple. But in interepidemic periods it is not such an easy matter, and the ordinary diagnosis of grippe or influenza is at such times very largely a matter of likelihood, rather than actual recognition.

As will be seen, it is not possible at the present time to base the diagnosis of true influenza upon the isolation of the Pfeiffer bacillus, although this had almost become the habit until the outbreak of the last epidemic. We cannot in this place undertake to describe in detail the clinical manifestations of this disease. For accurate descriptions and historical considerations of both the clinical and epidemiological problems, we may refer the reader to Leichtenstern's book published in Nothnagel's System, to Thompson's Annals of Influenza (London, 1852 ) and to the more recent treatises in various medical text books. ${ }^{1}$ We wish to emphasize, however, that in our own experience in the last epidemic, together with a study of the clinical records of previous outbreaks, we have become convinced that pure, uncomplicated influenza is a very mild disease in which respiratory symptoms may be either entirely lacking, or may be extremely mild. This is an important fact to remember in connection with etiological studies, since a very large part of the bacteriological work done on this phase of influenza has unfortunately had to be carried out on cases occurring late in epidemies when seeondary respiratory infection had beeome almost universal. The pure, uncomplicated cases are found in considerable numbers only in the early stages of epidemic outbreaks.

${ }^{1}$ See also Zinsser, "Etiology and Epidemiology of Influenza," in the Oxford Medical Papers, 1921. 
The characteristies of such cases are as follows : the onset is almost regularly abrupt. Typical cases become ill suddenly, without premonition. More rarely there are a few days of general tired feeling and malaise, but this is unusual. The first symptoms consist in headache, feverishness, loss of appetite, pains in the back and somatic muscles, particularly in the calves of the legs; sometimes suffusion and burning of the eyeballs, and often mild sore throat. The temperature rises to anywhere from $101^{\circ}$ to $104^{\circ}$, and this condition continues for two or three days, when the patient gradually returns to normal, but is left quite exhausted. Very oceasionally, skin rashes appear in the form of erythematous patches, not at all uniform in appearance and difficult to characterize dermatologically. The spleen is usually not enlarged. The leucocytes range from 5,000 to 9,000 .

Such cases, if they develop respiratory complications at all, suffer from nothing much more than a mild laryngitis or bronchitis, which appears toward the third or fourth day and rapidly subsides. The first 200 or 300 eases which we saw during the last epidemic, among American soldiers in France, developed practically no respiratory symptoms whatever and no focal lesions anywhere. The disease was so brief and mild that it was not recognized as influenza at first, and was spoken of as "three-day fever." The morbidity at such times is high, the mortality practically nil.

As we study the literature of past epidemics and the observations made on the last epidemic in different places, we find that this experience has been universal. In the 1898 epidemic, Heyfelder speaks of Siberian fever, which was at first looked upon as malaria, and noted the absence of catarrhal symptoms in the respiratory organs. Just before this, the epidemic outbreak in Constantinople was spoken of as "dengue fever," and similar, unrecognized, mild cases characterized the beginnings of the epidemic in Petrograd. During the war-epidemic of 1917 and 1918, Vaughan and Palmer also noted the uncertain and mild characters of the first cases at Camp Oglethorpe (in March, 1918) and in Italy, San Pictro suggested sand-fly fever as a possible diagnosis.

These considerations may seem irrelevant in a book of this character, but are important in indicating that etiological investigations on influenza must include a careful study of eases of this kind. In the later periods of the first epidemic waves, as well as in the seeond and third waves, the overwhelming majority of the eases, hecause of secondary infection, are respiratory in character, an important 
characteristic of the basic influenza being the susceptibility which it creates to secondary respiratory infection. The possibilities of error in etiological investigation are, therefore, obvious.

Etiology of Influenza.-In 1892, Pfeiffer described the influenza bacillus which he isolated from the sputum of typical eases but failed to find in normal controls. The organisms were present in large numbers in the sputum of early cases, and in those with pulmonary complications which came to autopsy; if the bacterial contents of the respiratory passages were examined from the pharynx progressively downward to the lung, the influenza bacilli were found in increasing numbers as the examination proceeded toward the smaller bronchi and bronchioles. Since that time, an enormous amount of investigation has been carried out in regard to the relationship of the influenza bacillus to the disease, but we cannot as yet state definitely whether or not it is the actual cause. During the 1889 epidemic and in the years following, much etiological research was done and Huber, Baümler, Kretz, Kruse, Pfuhl and many others isolated influenza bacilli with great frequency from all the various lesions associated with the disease. In the interepidemic periods influenza bacilli were found with less and less frequency in respiratory infections, but still were found to be present in a great many individuals even when they did not suffer from clinical influenza. It is difficult to come to any definite conclusions on the etiological considerations involved in influenza at the present time, and it is quite impossible to detail the enormous amount of work that has been done since 1889 on this problem. There are few organisms that appear in the respiratory tract that have not been thought of by someone as possibly causing influenza, but most observers have confined themselves either to the confirmation or refutation of Pfeiffer's claim concerning the specific significance of the small Gramnegative bacillus which he discovered. We feel confident that if the disease is a bacterial disease at all, no other bacteria need be seriously considered.

It seems best to us to summarize as briefly as possible the evidence bearing on the etiological problem in influenza, referring the reader for details to the books mentioned to the numerous articles that have appeared on the subject in the last three or four years, and to our own summary mentioned above, in the Oxford Series.

In the first place, let us reiterate that correct judgment of etiological work in this disease must be based upon the recognition that 
influenza, in its pure and uncomplicated form, consists of the mild, systemic, febrile disease outlined above. It is the causation of this basic condition which constitutes the true etiological problem. The point is to decide whether the influenza bacillus initiates this condition, or whether, like pneumococci, streptococci, and other bacteria, it may not be a secondary invader, preliminary to which there may have been infection by some other agent, perhaps a filtrable virus, which opened the path for the secondary invasion.

In favor of regarding the influenza bacilli as the primary cause are: The frequent isolation of bacilli from the throats of the earliest cases; the frequency with which these organisms have been isolated from all varieties of early and late complications; the distribution of the influenza bacilli in the bronchial trees in fatal cases, and their discovery in many cases in pure culture at autopsy; the wide distribution of the organisms throughout communities at times of epidemic, and their gradually diminishing frequency in normal and diseased respiratory passages as epidemics fade into the past.

The recent discovery by Parker in our laboratory of the powerful poisons which can be obtained from young influenza cultures, has further given strength to the possibility that the profound prostration and systemic symptoms in an influenza patient might be due to absorption of such poison from even a small focus in the throat.

Recently, also, Cecil and Blake carried out a series of significant experiments in which they increased the virulence of influenza bacilli by passing them through mice and inoculated these cultures into Philippine monkeys by swabbing them into the nasal mucosa and injecting them into the trachea. They obtained acute respiratory disease, with prostration and subsequent respiratory symptoms, bronchitis, etc., in these animals. The experiments are not conclusive, since, of course, it was impossible to say that the disease which they produced was analogous to influenza in the human being. Cecil also has carried out similar experiments on human volunteers. The idea underlying the experiments was the assumption that the virulence of the strains used must be raised to a certain pitch before simple introduction into the nose can lead to typical disease. In his human experiments, Cecil obtained moderate, local and systemic symptoms which suggested very mild influenzal attacks. None developed temperature. Other investigators who paid less attention to the virulence of the strains, particularly Wahl, White and Lyall, Bloomfield, Rosenow and McCoy and others, have obtained entirely negative results. 
Against the assumption of the etiological importance of the influenza bacillus, are the occasional failures to find these organisms in early cases, the frequent presence of the bacilli in the throats of normal individuals suffering from no typical influenza, and their presence in interepidemic periods in many pathological conditions observed in clinical influenza; their frequent presence as complicating invaders in whooping cough, measles, etc., and the antigenic multiplicity of strains isolated at times of epidemic. The absence of the organisms in most early blood cultures, also, should be added to the negative evidence.

Investigations of antibodies in the serum of cases that are sick permit of no etiologic conclusions, since secondary invasion might lead to antibody formation, whatever the primary cause.

Protection experiments with vaccines have been absolutely inconclusive.

All of this evidence must be considered in connection with recent experiments upon the possibility of causation by a filtrable virus in influenza, a problem upon which much work has been done, and which we must, therefore, include in our summary as follows:

Filtrable Virus and Influenza.-The idea that a filtrable virus might be the etiological agent in influenza is suggested by the characteristics of the pure uncomplicated mild cases described above, the extreme infectiousness of the disease, and the negative character or, at least, the lack of uniformity of the bacteriological findings in early eases. In consequence, a considerable number of workers have attacked the problem from this point of view. In 1918, Nicolle and Lebailly ${ }^{2}$ reported on studies made on the filtration of influenzal virus and its inoculation into animals and man. They filtered the blood and nasal secretions of uncomplieated grippe cases, and instilled them into conjunctival saes and nasal cavities of several monkeys (Macaceus Sinicus) and into a number of human volunteers. They obtained symptoms within about six days in several monkeys, and in two men, but were unable to carry the infection into a second generation. They conclnded that the filtrates of secretions in influenza are virulent, and ean infect human beings and certain monkeys by nasal and conjunctival inoculation.

Very soon after this, Dujarric de la Riviere ${ }^{3}$ filtered blood from

${ }^{2}$ Nicolle and Lebailly, Ann. de l'Inst. Past., 33, 1919, 385.

${ }^{3}$ Dujarric de la Riviere, Compt. Rend. Acad. des Sciences, 167, 1918, 406. 
four influenza cases and injected it into himself. He became definitely ill with influenza-like symptoms.

Lister and Taylor ${ }^{4}$ in 1919 carried out a series of filtration experiments which may be summarized as tending to refute the idea that the influengal virus is filtrable. In the same year, Bradford, Bashford and Wilson ${ }^{5}$ claimed that they had produced disease in animals with filtrates of the blood, sputum and other exudates from influenza patients, and had grown from this a minute filter passing body. 'This work was later withdrawn by the writers themselves. Claims of the filtrability of the influenzal virus on the basis of experiments carried out by filtration of influenzal secretions and inoculation into monkeys and man were also made in 1919 by Leschke, ${ }^{6}$ Fejes, ${ }^{7}$ and others. A reivew of most of the German etiological work is to be found in the Centralblatt für Bakteriologie, (Ref., 68, 1919, p. 401). Selter, ${ }^{8}$ failing to find influenza bacilli with any regularity in supposedly typical cases, filtered the nasopharyngeal mucus and gargle water of patients in the early stages of the disease, and sprayed it into his own throat and that of a woman assistant, both of them inhaling the spray. In both of them, after 17 to 20 hours, he claims, mild influenza resulted. Yamanouchi, Sakami and Iwashima ${ }^{9}$ carried out more extensive experiments in 1919. If one could accept these experiments without question, the filtrability of the virus of influenza would be an established fact. In their first experiment they emulsified the sputum of 43 influenza patients in Ringer's solution. Part of this they filtered. The unfiltered emulsion they injected into the noses and throats of 12 healthy people. The filtrate of the same emulsion was similarly injected into the noses and throats of 12 other healthy people, 6 of whom had had influenza. All of the 24, except the ones who had had influenza recently, eame down with an influenza-like malady after an incubation of two or three days. Following this, they injected the filtrate of blood of influenza patients into the noses and throats of 6 more healthy people, with similar positive results. Filtrates of sputum were inoculated into 4 healthy people, and 4 others received filtrates of blood of influenza patients, subcutaneously. All except one, who

${ }^{4}$ Lister and Taylor, Pub. South Afric. Inst. Meil. Res., April 30th, 1919, No. 12.

5 Bradford, Bashford and Wilson, Lancet, 1, 1919, 169.

${ }^{6}$ Leschke, Berl. klin. Woch., 11, 1919.

${ }^{7}$ Fejes, Dent. med. Woch., 1919, 653.

${ }^{8}$ Selter, 1)ent. merl. Woch., 1918, 932.

'Yamanouchi, Saliami and Iwashima, Lancet; 1, 1919, 971. 
had had influenza, came down after two or three days. Finally, pure cultures of Pfeiffer, and mixed cultures of Pfeiffer bacilli with pneumococci, staphylococei and streptococci were injected into the noses and throats of 14 healthy people who had not had influenza. No symptoms followed these injections. More recently, Olitsky and Gates ${ }^{10}$ reported upon experiments they had made in the 18 months previous to publication, beginning during the epidemic wave of 1918 . They intratracheally inoculated secretions from influenza patients in the early stages, into rabbits. After a short period these rabbits developed fever, leucopenia, minute pulmonary hemorrhage, and pulmonary edema and emphysema. The rabbits did not ordinarily die of the disease, but when they killed them in the active stages and filtered the material from these pulmonary lesions, they were able to continue producing such disease in rabbits with the filtrate. They proved by careful experimentation that the symptoms and lesions they obtained were not due to bacteria in the ordinary sense of the word. It is impossible at the present time to comment conclusively on these results, but the evidence is submitted to show that there is a considerable amount of evidence at the present time which should make one conservative in definitely claiming etiological relationship for the influenza bacillus.

\section{EPIDEMIOLOGY OF INFLUENZA}

As stated before, the etiological and diagnostic difficulties in connection with influenza are such that records of the disease are less apt to be reliable than would be similar records of smallpox, diphtheria, ete. However, a great deal is known about past epidemies which have been described with sufficient accuracy to permit us to recognize them definitely as true influenza epidemics. A great deal has been written in the past and is available in the works of Caienus of Griefswald (1579), Jaeques Pons of Lyon (1596), Sydenham (1675), Slevogt (Jean, 1712), Haygrath and Hamilton (1775), Pringle and Huxham, Massin (Strassburg, 1858) and many others. Leichtenstern, who has written a vary thorough treatise on influenza tabulates the great influenza epidemies of the world as follows:

Less extensive outbreaks seem to have prevailed in different parts of the world between 1709 and 1912.

${ }^{10}$ Olitsky and Gates, Jour. Exper. Med., 33, 1921, No. 2 and 3. 
Between 1729 and 1733 the disease, traveling from Russia westward, spread over Europe in two great waves, one in 1729 and the other in 1732.

Another epidemic started on the shores of the Baltic in 1742.

In $1757-1758$

$1761-1762$

1767

Epidemics occurred of which we have but poor geographical records.

Of the epidemic of 1742 , Friedrich states that all but about onetenth of the entire population of Germany was attacked.

From 1781 to 1782 an epidemic supposed to have started in China spread through Siberia to Russia and thence to Europe.

Another traveled approximately the same route in 1788 .

The same thing oecurred between 1799 and 1803 .

In 1827 there was an outbreak in Europe less extensive than most of the others.

Between 1830 and 1833 there were two or three pandemic waves, the first one supposedly originating in China.

Other outbreaks, again traveling from East to West, occurred in 1836 and 1847. During this epidemic the Prussian Army is said by Friedrich to have been attacked in its entire personnel.

These brief data, which bring us up to the pandemic of 1889 , are condensed chiefly from Leichtenstern. ${ }^{11}$

The characteristies of influenza epidemies are summarized by Leichtenstern in a manner which can be accepted as roughly describing the actual facts on the basis of experience with the last warepidemic. 1. The disease appears in true pandemic waves; 2 , it travels with tremendous speed over the globe; 3 , it is characterized by sudden mass infection; 4 , it is rapidly burnt out after several weeks in one locality; 5 , it is independent of season or weather; 6 , it begins at first with an enormous morbidity and a relatively slight mortality; 7 , it is but slightly influenced by age, sex, or occupation.

The second characteristic which we have mentioned, namely, that the disease seems to originate in one particular part of the world and from there spreads out (such foci having been also described as existing in Asia (Netter), China (Pearson), etc.), is at the present time somewhat in dispute, Frost ${ }^{12}$ and others believing that the last two epidemies probably started in several places at once.

${ }^{11}$ Leichtenstern, Influenza in the 19th Century, 2nd Edit., Sticker, Leipzig, 1912.

${ }^{12}$ Frost, U. S. Public Health Serv. Rep., No. 550, August 15th, 1919. 
The pandemic of 1889 probably started in the East where it is quite likely that an outbreak of so-called "dengue fever"' in Constantinople in 1888 formed one of the earliest manifestations. Late in. 1888 and in early 1889, it seems to have appeared synchronously in Greenland, in Russia and in Siberia. Heyfelder ${ }^{13}$ saw cases in Bokhara in May, 1889, and wrote of its enormous westward speed of travel. In October it reached Petrograd, and in November entered Germany. It swept westward through France, Austria and Italy, reaching Spain in early December, New York and London by the middle of December, and by this time had also reached the United States from the other side, having traveled eastward as well as westward from its origin.

It is quite certain at the present time that influenza is spread by direct and indirect contact. The tempestuous suddenness with which it attacked whole communities formerly gave rise to the opinion that it was spread by other means, such as air, dust, etc., but studies of the last two epidemics seem to contradict this. It does not travel more rapidly than human communication, as shown during the 1889 epidemic by Parsons, ${ }^{14}$ Friedrich, ${ }^{15}$ and others. Communities that are out of touch with infected populations by reason of lack of communication (islands, mountain tops, etc.), usually remain uninfected. Examples of this were noted during the 1889 epidemic on the Island of Borkum, and on the Senlis mountain. In large cities the epidemies burn themselves out within a relatively short time, while in country communities where communication is slower and the population is scattered, it travels more slowly and lasts longer. According to the studies of Abbott of the epidemic in Massachusetts in January, 1890, it was shown that from the 4 th of January to the 10th of February, there were about 800,000 cases, that is, about 40 per cent of the population, and the disease had practically burnt itself out in this short period. In London, the epidemic appeared in December, attained a death rate of 28.1 per thousand, during February, and began to decline in March. During the last pandemic similar facts were observed, although the state of war, necessitating the transportation of large bodies of men from one part of the world to another, rendered tracing of the correlationship of influenza and travel routes extremely

${ }^{13}$ Heyfelder, Wien. klin. Woch., 1890, 3, 11.

${ }^{14}$ Parsons, Local Government Board Report, London, 1893.

${ }^{25}$ Friedrich, Arb. a. d. k. Gesundh., 1894, Bd. 9. 
difficult. However, MacNeal ${ }^{16}$ who has written up the progress of the epidemic among American troops in France cites a number of cases where a true connection between ship communication and the outbreak of influenza could be observed. Hospital outbreaks, such as the one described by Foster and Cookson, ${ }^{17}$ and prison outbreaks, such as the one described by Stanley ${ }^{18}$ for the San Quentin Prison, very definitely prove the paramount importance of contact infection. Stanley shows definitely that the disease was brought in by an infected prisoner, and that prisoners in contact with this one and with other infected inmates contracted the disease, while those who were isolated in other buildings or not in particularly close contact with others, were spared. As to the suddenness with which the disease attacks a great many people in a community, the chief point which would make it seem that contact might be entirely responsible, this can probably be explained by the extreme infectiousness, the large percentage of susceptibles, and the fact that people in the early stages of the milder forms of influenza are up and about and are freely mingling with their fellows. An epidemic is rarely recognized in a large community until two weeks or longer after the first eases have appeared. Parsons in his studies of the epidemies in England in 1889 and 1890, calls attention to the fact that influenza is not more rapid in its spread and epidemic onset than was smallpox in the days before vaccination. $\mathrm{He}$ also has found evidence that shows that in localities where the outbreak seemed particularly explosive, this could often be traced to meetings of crowds at conventions or other organizations at times just preceding the beginnings of the epidemies. The explosiveness of the outbreaks during the last epidemic was evident, particularly, in military communities.

Influenza epidemies are always followed by secondary and tertiary waves during which the disease after a definite lapse of time seems to return often in a more dangerous form. This has been noted in almost all carefully studied epidemics.

After the epidemics of 1729 and 1730, secondary waves occurred in 1732 and 1733. After the 1780 to 1781 epidemic, another series of waves followed in 1782 . The 1788 epidemic was followed by

\footnotetext{
${ }^{16}$ MacNeal, Areh. of Inter. Med., 23, 1919, 657.

${ }^{17}$ Fioster and Coolison, Lancet, 2, 1918, 585.

${ }^{1 s}$ Stanley, U. S. Pub. Health Serv. Rep., No. 19, May 9th, 1919.
} 
secondary waves that lasted as long as 1800 , and similar observations were made during the epidemies of 1836 and 1837. The most careful studies of such waves were made by Parsons during the 1889 epidemic. He divided this epidemic into the first wave which began in England in the winter of 1889 to 1890, a second wave in the spring of 1891, and a third in the winter of 1891 to 1892. Frost has similarly studied the American epidemics, and has come to analogous conclusions. Brownlee ${ }^{19}$ has attempted to establish a law of periodicity for the intervals between pandemies, and for the intervals between several waves of each outbreak. In general, his studies seem to show that there is an approximate period of ten years between large epidemics, and that a period of about thirtythree weeks intervenes between individual waves. We cannot go into these purely statistical facts in the present work, but refer the reader to papers by Brownlee, and the more recent paper by Raymond Pearl. ${ }^{20}$

Secondary and tertiary waves have eertain characteristies which it is important to note. In contrast to the relatively mild onset of the primary waves, these later waves are marked by greater severity of the cases, and almost universal secondary infection. The disease takes on a much more dangerous respiratory form. The mortality becomes progressively higher during these waves than during the original outbreak. Leichtenstern, also agrees that, in the secondary and tertiary waves, that the morbidity is lower and the mortality much higher. Similar observations have been made by Wutzdorff ${ }^{21}$ for the 1889 epidemic.

The secondary epidemic waves do not travel with the same speed and to the same extent as do the first waves. Cases are more scattered and the period of prevalence is more prolonged. These waves never stop abruptly, but play out, in gradually diminishing ripples, into subsequent years. Also, according to Leichtenstern, these secondary and tertiary waves do not seem to take their origins from a single place, but erop up here and there from many scattered foci. As Netter says, "they have appeared in separate, synchronous or successive explosions, without connection between various reappearances in different places, as this was possible during the first appearanees in 1889.",

19 Brownlee, Lancet, 2, $1919,856$.

20 Pearl, R., U. S. Pub. Health Serv. Rep., No. 548, August 8th, 1919.

${ }^{21}$ Wutzdorff, Arb. a. d. k. Gesundh., 9, 1894, 414. 
What is at the bottom of this suecession of waves, it is hard to say. The most natural explanation would be that there is a short lived immunity, conferred by the original attack, and that the second and third waves appear at times when the infectious agent is still widely distributed while the immunity has waned. It is very difficult to get at the facts, but serious attempts are being made, particularly by Frost and others. Studies by Jordan and Sharp ${ }^{22}$ at the Great Lakes Training Station indicate that no marked immunity existed twelve to fifteen months after the first attack. This, too, seems to be the conclusion reached by Frost who states that in Baltimore those persons who were attacked during the 1918 to 1919 epidemic, showed no relative immunity during the epidenic of 1920. It would seem in general that an almost universal infection of a community with the first mild disease conferred a short lived immunity. As a consequence of this, the epidemic would burn itself out and wane. Gradual return of susceptibility in the course of the subsequent period of months, not, however, bringing the community back to the very low universal resistance which it was characterized by before, would now create a soil in which reintroduction of the infectious agent could produce many cases, but in which spread would be less rapid and extensive. Such a view, however, must be regarded as purely tentative, and it is hoped that a final study of the statistics gathered during the great war epidemic will clear this matter up.

It is a difficult question to decide where the last war epidemic began. After the 1889 epidemic, it seems that the disease may have remained endemic in a great many different parts of the world. It may have been so universally distributed that we eannot speak, in this ease, of a definite focus in China or Turkestan, as this was done in past epidemies. Before 1889, the world was not so continuously traveled over by large numbers of people. There was less travel by railroad communication, steam-ship lines, ete. It may be that this development of eivilization has completely altered the epidemic conditions prevailing in regard to influenza. It has been suggested in the ease of the last epidemic however, that it eame from the East, as did previous outbreaks. MeNalty in an article in Nelson's System states that the disease was prevalent in March, 1918, in China, and that in April cases appeared on a Japanese ship in a Chinese port. Frost who has given particular attention to this

${ }^{22}$ Jordan and Sharp, Jour. of Infee., Dis., May, 1920, p. 463. 
question, on the other hand, finds that the roots of the epidemic go far deeper than this, since his studies of statistics of respiratory diseases in the United States seem to show that as early as December, 1915, and January, 1916, there oceurred, in New York and Cleveland, sudden and considerable rises in mortality from respiratory diseases. In January, 1916, influenza was reported in twenty-two cities of the Union. These epidemies were mild and attracted little attention.

During the winter of 1917, many so-called eases of influenza occurred in Europe among French and British troops. In the winter of 1917, similar cases, supposedly influenza, appeared in many American camps. MacNeal states that in November and December, 1917, and January, 1918, there were many eases of so-called influenza in the American Expeditionary Forees. The disease appeared without question at Camp Oglethorpe in Mareh, 1918, a month before it was recognized in any numbers in Europe, almost at the same time at which it seems to have appeared in Spain.

It cannot be said with definiteness just where the last epidemic began, but if we summarize the evidence available at the present time, it would seem that Frost is probably right in that it did not begin in a single place, as previous epidemics are said to have begun, but started in a great many different places almost at the same time.

Like other epidemies it appeared in successive waves, the first wave probably beginning in 1917 in some places, in the spring of 1918 in others. There was an interval, and then in September and October of 1918 the second wave had gathered its full velocity. This was the really fatal wave all over the world. The mortality was enormous. Pearl estimates that in the United States alone, deaths from influenza were not less than 550,000 and this is approximately five times the number $(111,179)$ of American soldiers officially stated to have lost their lives from all eauses in the war. In the Surgeon General's report it is stated that influenza, with its complications, is charged with 688.86 admissions of American and native troops for the year 1918, and caused 23,007 deaths, practically 82 per cent of all deaths in the Army being attributed to respiratory diseases.

Morphology and Staining.-The bacillus of influenza ${ }^{23}$ (Pfeiffer bacillus) is an extremely small organism, about 0.5 micron long by

${ }^{23}$ Pfeiffer, Deut. med. Woch., ii, 1892; Zeit. f. Hyg., xiii, 1892 ; Pfeiffer und Beck, Deut. med. Woch., xxi, 1893. 
0.2 to 0.3 micron in width. They are somewhat irregular in length, but show rounded ends. They rarely form chains. They are nonmotile, and do not form spores.

Influenza bacilli stain less easily than do most other bacteria with the usual anilin dyes, and are best demonstrated with 10 per cent aqueous fuchsin ( 5 to 10 minutes), or with Loeffler's methylene-blue (5 minutes). They are Gram-negative, giving up the anilin-gentian voilet stain upon decolorization. Occasionally slight polar staining may be noticed. Grouping, especially in thin smears of bronchial secretion, is characteristic, in that the hacilli very rarely form threads or chains, usually lying together in thick, irregular clusters without definite parallelism.

Isolation and Cultivation.-Isolation of the influenza bacillus is not easy. Pfeiffer ${ }^{24}$ succeeded in growing the bacillus upon serumagar plates upon which he had smeared pus from the bronchial

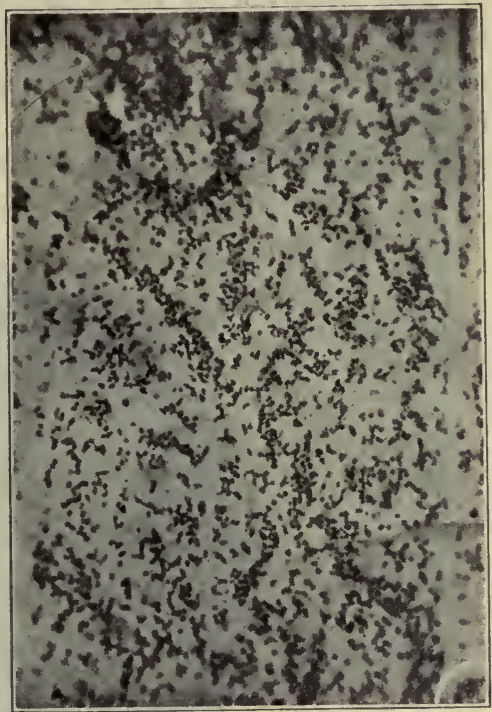

Fig.49.-Bacillus Influenz ; Smear from pure culture on blood agar. secretions of patients. Failure of growth in attempted subcultures made upon agar and gelatin, however, soon taught him that the success of his first cultivations depended upon the ingredients of the pus earried over from the sputum. Further experimentation then showed that it was the blood, and more particularly the hemoglobin, in the pus which had made growth possible in the first cultures. Pfeiffer made his further cultivations upon agar, the surface of which had been smeared with a few drops of blood taken sterile from the finger. Hemoglobin separated from the red blood cells was found to be quite as efficient as whole blood. Whole blood taken from the finger may be either smeared over the surface of slants or plates, or mixed with the melted meat-infusion agar. In isolating from sputum, only that secretion should be used which 
is coughed up from the bronchi and is uncontaminated by microorganisms from the mouth. It may be washed in sterile water or bouillon before transplantation, to remove the mouth flora adherent to the outer surface of the little clumps of pus. The blood of pigeons or that of rabbits may be substituted for human blood.

For isolation of the organisms on plates from sputum or other sources, the best medium to use is the Avery sodium oleate blood medium described in the section on technique. The sodium oleate in this medium inhibits a great many of the contaminating organisms and seems to favor the growth of influenza bacilli.

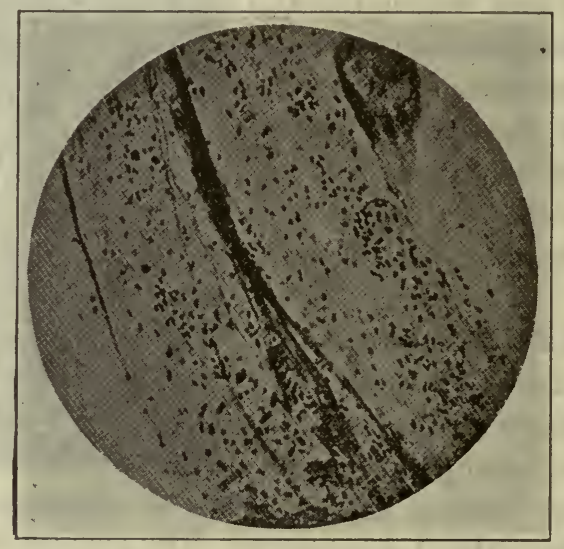

Fig. 50.-Bacillus Influenze; Smear from sputum. (After Heim.)

In place of this medium, blood agar plates can be used, rabbit's blood being, in our opinion, somewhat more favorable than other varieties of blood. It is best prepared in small lots by puncturing a rabbit's heart with a sterile needle and transferring the blood directly to tubes of melted agar.

For further cultivation, the best medium is a chocolate agar made as described in the section on media, in which a 5 or 10 per cent rabbit's blood agar with a $\mathrm{P}_{\mathrm{H}}$ of 7.8 is heated to about $90^{\circ} \mathrm{C}$. (but not boiled), and plates are prepared by shaking this brownish discolored medium, pouring it into plates or tubes and letting it cool before all the blood has settled to the bottom. For fluid cultivation, chocolate broth may be similarly prepared and filtered through cotton or paper while hot. This makes a transparent strawcolored fluid on which influenza bacilli grow with great speed and 
luxuriance. In the hands of J. T. Parker in this laboratory, this method has given excellent results and it is by means of such a medium that she has produced the toxic substances referred to below. The fact that this fluid medium does not contain much, if any, hemoglobin, but is very rich in lipoidal substances, makes us believe that possibly the nutritive substances in blood necessary for the growth of this bacillus may consist in the lipoidal contents of the blood eells, rather than the hemoglobin. This, too, is confirmed by recent observation of Avery. ${ }^{25}$

The general view, however, is the one which attributes to the hemoglobin in the blood the nutritive function.

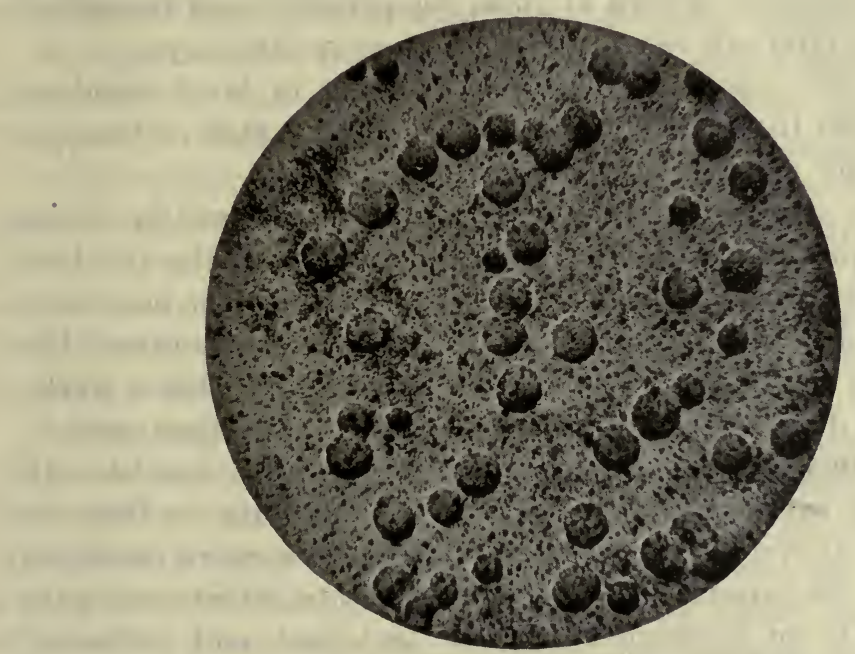

Fig. 51.-Colonies of Influenza Bacillus on Blood Agar. (After Heim.)

A more luxurious growth of influenza bacilli may be obtained by eultivation in jars in which about $1 / 10$ the volume of air has been replaced by $\mathrm{CO}_{2}$.

For preservation of laboratory cultures of influenza bacilli, the best medium is fresh, defibrinated rabbit's blood, kept at room temperature in the dark. In this way, laboratory strains after several generations of cultivation outside the body can be kept alive for weeks and even months. They will not keep well either in the iee-box or in the incubator.

${ }^{25}$ Avery, Proc. Soc. Exper. Biol. and Med., 18, 1921, 6. 
Other substances which, added to neutral or slightly alkalin agar, have been used for the cultivation of influenza bacilli are the yolk of eggs ${ }^{26}$ (not confirmed) and spermatic fluid. ${ }^{27}$ None of these, however, are as useful as the blood media. Symbiosis with staphylococei, ${ }^{8}$ too, has been found to create an environment favorable for their development.

Influenza bacilli do not grow at room temperature. Upon suitable. media at $37.5^{\circ} \mathrm{C}$. colonies appear at the end of eighteen to twenty-four hours, as minute, colorless, transparent droplets, not unlike spots of moisture. These never become confluent. The limits of growth are reached in two or three days. To keep the cultures alive, tubes should be stored at room temperature and transplantations done at intervals not longer than four or five days.

Biology.-The bacillus is aërobic, growing in broth-blood mixtures only upon the surface, hardly at all in agar stab cultures, and not at all under completely anaërobic conditions.

As it does not form spores, it is exeeedingly sensitive to heat, desiceation, and disinfectants. Heating to $60^{\circ} \mathrm{C}$. kills the bacilli in a few minutes. In dried sputum they die within one or two hours. They are easily killed even by the weaker antisepties. Upon culture media the baeilli, if not transplanted, die within a week or less, the time depending to some extent upon the medium used.

Toxin Formation.-The opinion in former years has been that the poisonous substances produced by the influenza bacillus were in the nature of endotoxins and a great many observers noted toxic symptoms on the injection of whole cultures into rabbits and guinea pigs. There is no question about the fact that such eultures in quantities of a cubie centimeter or more can exert powerful poisonous action. In our laboratory, Julia T. Parker ${ }^{29}$ recently showed that culture filtrates of young influenza bacilli would kill rabbits in doses of from 1.5 e.e. upward. The best poisons are produced by eultivating the organisms on broth of $\mathrm{P}_{\mathrm{H}}$ of 7.8 , with 5 to 10 per cent defibrinated rabbit's blood. They were also produced actively in the chocolate broth produced by the filtration of such rabbit's blood broth as described above. The nature of these poisons is uncertain. The symptoms in the rabbits consist in marked prostration,

\footnotetext{
${ }^{26}$ Nastjukoff, Cent. f. Bakt., Ref., xix, 1896.

${ }^{2 \pi}$ Cantani, Cent. f. Bakt., xxii, 1897.

${ }^{28}$ Grassberger, Zeit. f. Hyg., xxv, 1897.

${ }^{29}$ Parker, Jour. A. M. A., 72, 1919, 476.
} 
flattening out on the bottoms of the cages, muscular weakness and death in a considerable percentage of the cases within two to six hours. A characteristic feature is the incubation time which is regularly between forty-five minutes and one hour and one-half. These poisons have been studied in parallel series with similarly produced streptococcus and typhoid filtrates by Zinsser, Parker and Kuttner ${ }^{30}$ and belong to a class of substances probably non-specific and non-antigenic, described by us as " $\mathrm{X}$ " substances, the exact nature of which is at present uncertain.

Influenza Bacilli not Associated with Epidemic Influenza.The question of the etiological relationship of the influenza bacillus to the epidemic disease has been sufficiently discussed above. It is an important fact that the influenza bacillus is a common respiratory invader of man in the interepidemic periods, and without any apparent relationship to the typical epidemic disease. Subsequent to the epidemic of 1889 , its wide distribution was established by a multitude of investigations. Pfeiffer ${ }^{31}$ noted its frequent presence in tuberculous processes in his early studies, and this observation was confirmed by many others. It is especially frequent in bronchiectatic cavities. Boggs ${ }^{32}$ reported a number of such cases in which influenza bacilli were present symbiotically in the cavity fluids in cases that showed negligible symptoms. A number of observers have isolated influenza bacilli from the blood in cases that had died of other conditions. Jaehle ${ }^{33}$ obtained the bacilli from the heart's blood at autopsy from two scarlet fever cases. He also found the organisms in blood culture at autopsy in fifteen eases of measles. In one of these cases the influenza bacillus was present when the only other influenza bacillus lesion present was a tonsillar infection. He also found the organisms in the blood five times in nine cases of chickenpox, and twice in twenty-four cases of whooping cough. He found them in the respiratory passages in fifteen cases of diphtheria. Wynekoop ${ }^{34}$ has made similar studies, especially in connection with lesions of the larynx, and in chronic laryngitis he found the organisms in pure culture. He described a special form of severe tonsillitis due to the influenza bacillus. Some of these were clinically

${ }^{30}$ Zinsser, Parker and Kuttner, Jour. Exper. Biol. and Med., 18, 1920, 49.

${ }^{\text {s1 }}$ Pfeiffer, Deut. med. Woch., 18, 1892, 28.

${ }^{32}$ Boggs, 130, 1905, 902.

${ }^{83}$ Jaehle, Zeit. f. Heilkd., 22, 1901, 190.

अ Wrekoop, Jour. A. M. A., 40, 1903, 574. 
mistaken for diphtheria. Madison ${ }^{35}$ eollected from the literature thirty eases in which influenza bacilli were present in the blood during life.

Influenza bacilli in the meninges have been described by Wollstein $^{36}$ and others and we have seen them in a number of eases in they were associated in this location with streptococei. Influenza bacillus meningitis is not uneommon in children.

In diseases of the cavities of the skull, the antrum of Highmore, the frontal sinuses etc., influenza bacilli may be chronically present and have been held responsible for intermittent attacks of asthma.

Wollstein has made an extensive study of the presence of influenza bacilli in children at the Babies Hospital in New York. In the interepidemic periods, Wollstein has found them present frequently in bronchopneumonia, less frequently in cases of lobar pneumonia. In thirteen cases of bronchopneumonia at autopsy she found the organism three times. Six times she found them in connection with tuberculosis. Other workers, as well as Wollstein, have frequently found the organisms in whooping cough where they were reported as being almost regularly present after the disease was well established. In the lungs in measles, scarlet fever and diphtheria their presence is very frequent. Wollstein states that she has found the organisms rarely in the throats of healthy infants, and that whenever it was present, it seemed to have a definitely aggravating influence upon the existing disease.

The carrier state may persist after infection with influenza bacilli for long periods. During epidemies this seems to be particularly important as found by the investigations of Pritchett and Stillman ${ }^{37}$ and of $\mathrm{Opie}^{38}$ and his collaborators.

Varieties of the Influenza Bacillus.-A great many investigators have reported organisms almost identical with the influenza bacillus which, however, they have regarded as suffieiently different to be considered as distinct types.

It is the opinion of the workers at the New York Department of health, moreover, that the Gram-negative, hemophilic organisms found in trachoma must be regarded also as belonging to the general influenza bacillus type, and cannot be sharply separated.

${ }^{35}$ Madison, Amer. Jour. Med. Sc., 139, 1910, 527.

${ }^{36}$ Wollstein, Jour. Exper. Med., 1905, 7, 335.

${ }^{87}$ Pritchett and Stillman, Jour. Exper. Med., 29, 1919, 259.

${ }^{\text {ss }}$ Opie, et al., Surgeon General's Rep., Jour. A. M. A., 72, 1919, 168. 
The recent epidemic has given much opportunity for bacteriological and serological study upon influenza bacilli from many different types of lesion, from many different parts of the world. The result of these studies has been confusing. Studies of Valentine and Cooper ${ }^{39}$ have shown that agglutination reactions do not indicate hemogenicity of the influenza group. In a large number of isolations and agglutinin reactions, they found that very few of the strains fell into antigenically identical groups. Of ten autopsy strains isolated by them, all strains seemed to be distinct. Of seventy-three miscellaneous strains, no two strains were identical. Two strains from different individuals were found to be identical, but in a family in which there were six cases of influenza, all the different races were distinct. It would seem that either influenza bacilli were composed of an infinite number of antigenic varieties or that the agglutinin reaction in these particular organisms, because of their small size, is not a suitable test for biological relationship. For the present, the biological subdivision of the influenza bacilli into well defined groups cannot be regarded as settled.

Dr. Anna Williams ${ }^{40}$ has recently studied hemoglobinophilic bacilli isolated from the eye in cases of trachoma. She believes that trachoma is probably caused by bacteria of this group. At first an acute infection or acute conjunctivitis occurs. Later when chronic productive inflammation supervenes the clinical picture is that of trachoma.

Experimental infection of animals reveals susceptibility only in monkeys. Pfeiffer and Beck ${ }^{41}$ produced influenza-like symptoms in monkeys by rubbing a pure culture of the bacillus upon the unbroken nasal mucosa. Intravenous inoculation in rabbits produced severe symptoms, but the bacilli do not seem to proliferate in these animals, the reaction probably being purely toxic. Cultures killed with chloroform may produce severe transient toxic symptoms in rabbits. ${ }^{42}$ Immunity produced by an attack of influenza, if present at all, is of very short duration.

Pseudo-Influenza Bacillus.-In the broncho-pneumonic proc-

${ }^{39}$ Valentine and Cooper, Rep. 84, Dept. Health, City of N. Y., 1919, December.

40 Mr. Anna Williams, Irter. Congress of Hygiene and Demography, Washington, 1912 .

${ }^{\star 1}$ Pfeiffer und Beck, Deut. med. Woch., xxi, 1893.

${ }^{42}$ Pfeiffer, loc eit. 
esses of children, Pfeiffer ${ }^{43}$ found small, non-motile, Gram-negative bacilli, which he was forced to separate from true influenza bacilli because of their slightly greater size, and their tendency to form threads and involution forms. These microorganisms are strictly aërobic and grow, like true influenza bacilli, only upon blood media. They are differentiated entirely by their morphology upon twentyfour-hour-old blood-agar cultures. Wollstein, ${ }^{44}$ who has made a careful study of influenza-like bacilli, both culturally and by agglutination tests, has come to the conclusion that these bacilli are so similar to the true influenza organisms that the term pseudo-influenza should be discarded. Strains of similar bacilli isolated from cases of

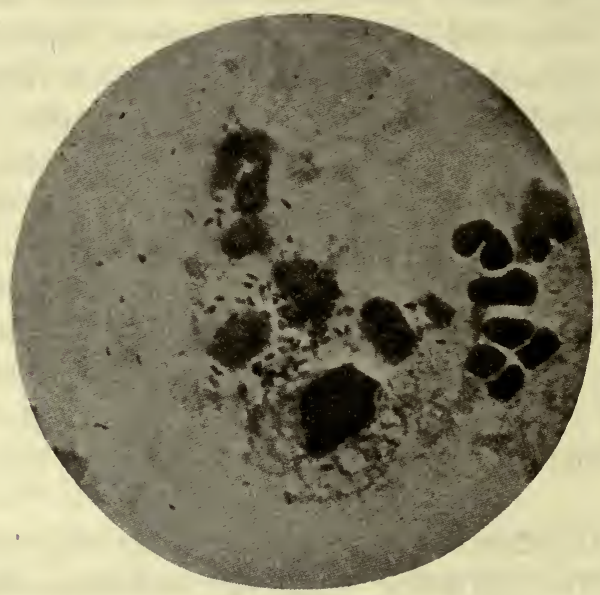

Fig. 52,-Косh-Weeks Bacillus.

pertussis, while differing from the others in some of their characteristies, could not properly be maintained as distinct species.

Koch-Weeks BaCILlus.-Koch, ${ }^{45}$ in 1883 , Weeks ${ }^{46}$ and Kartulis, in 1887, described a small Gram-negative bacillus found in connection with a form of acute conjunctivitis which occurs epidemically. The bacillus is morphologically similar to $\mathrm{B}$. influenzæ, but is generally longer than this and more slender. The bacilli grow only at incubator temperature. Recent studies by Anna Williams at the

${ }^{43}$ Pfeiffer, Zeit. f. Hyg., xiii, 1892.

44 Wollstein, Jour. Exp. Med., viii, 1906.

${ }^{45}$ Koch, Arb. a. d. kais. Gesundheitsamt, iii ; Cent. f. Bakt., 1, 1887.

${ }^{46}$ Weeks, N. Y. Eye and Ear Infirmary Rep., 1895; Arch. f. Augenheilk., 1887. 
New York Department of Health seem to indicate that the KochWceks bacillus may be merely a variety of the true influenza bacillus.

Bacillus of Pleuko-Peneumonia of Rabits. -This is a small Gramnegative bacillus, described by Beck, not unlike that of influenza. These microorganisms are slightly larger than the Pfeiffer bacili and grow upon ordinary media eren without animal sera or hemoglobin.

Bacillus murisepticus aNd Bacillus RHUSiopathis.-While morphologieally similar to the microorganisms of this group, these bacilli are culturally easily separated because of their luxuriant growth on simple media. The last two mieroorganisms are more elosely related to the groups of the bacilli of hemcrrhagic septicemia. 


\section{CHAPTER XXV}

BORDET-GENGOU BACILLUS, WHOOPING COUGH AND MORAX-AXENFELD BACILLUS, ZUR NEDDEN'S BACILLUS, DUCREY BACILLUS

\section{BORDET-GENGOU BACILLUS}

("Microbe de la Coqueluche," Pertussis bacillus, Bacillus of whoopingcough.)

Whooping cough is endemic in most cold countries and, on occasion, in schools, infant asylums and other places where children are crowded, may assume epidemic proportions. Occasional cases may occur in adults, though they are rare. The disease seems to occur sporadically all through the year in large centers, but is more common during the winter. According to Rosenau ${ }^{1}$ susceptibility is pretty general and he states that the disease causes at least 10,000 deaths a year in the United States. Indeed, Rosenau who has analyzed the statistics for the United States for the year 1910, states that whooping cough caused almost as many deaths as scarlet fever. It is the pulmonary complications that follow on the initial infection, however, which, in this disease, are responsible for the deaths and it is the subacute and chronic inflammations of the lung which lead to prolonged illness and pave the way for tuberculosis and other secondary infections. For sanitary purposes, an arbitrary incubation time of about two weeks has been regarded as probably nearest the facts. During the incubation time, the infectiousness does not seem to be great, the most contagious period being the first few days of the actual disease when the Bordet-Gengou bacilli are brought up in large numbers. It is at this time only that pure cultures of the organisms can be obtained. Very soon after the onset, influenza bacilli and other secondary invaders are found. Transmission is probably by direct and indirect contact as in other

${ }^{1}$ Rosenau, Preventive Medicine and Hygiene, D. Appleton and Co., New York, 1921. 
respiratory infections, and the general infectiousness is very high, since susceptible children definitely exposed do not often escape. After the disease has set in, the patient may remain infectious for others all through the disease and long into convalescence. It is best to keep children isolated for several weeks after the cough has subsided. It has been possible to reproduce conditions simulating the disease in monkeys and in dogs, and there is a suspicion that the disease may be transmitted by domestic animals, especially dogs. Though this is not absolutely certain, Rosenau and other sanitarians advise care in this regard.

Prevention consists in carly diagnosis and isolation, exclusion from school and absolute avoidance of close contact with other children. Quarantine should continue, as stated above, for several weeks after the cough has completely subsided.

In 1900 Bordet and Gengou ${ }^{2}$ observed in the sputum of a child suffering from pertussis a small ovoid bacillus which, though similar to the influenza bacillus, showed a number of morphological characteristics which led them to regard it as a distinct species. As they were at first unable to cultivate this organism, their discovery remained questionable until 1906, when cultivation succeeded and the biology of the microorganism was more fully elucidated.

Morphology.-The morphology of this organism is described by them as follows: The organism in the sputum, early in the disease, is scattered in enormous numbers indiscriminately among the pus

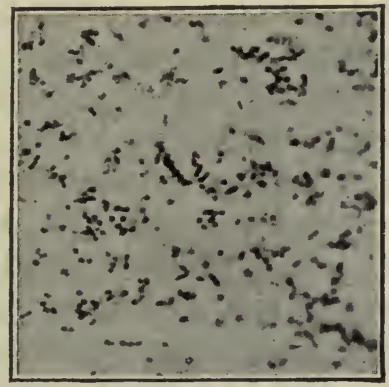

Fig. 53.-Bordet-Gengou Bacillus.

cells, and at times within the cells. It is extremely small and ovoid, and frequently is so short that it resembles a micrococeus. Often its poles stain more deeply than the center. In general, the form

Bordet et Gengou, Ann, de l'inst. Pasteur, 1906. 
of the organisms is constant, though occasionally slightly larger individuals are encountered. They are usually grouped separately, though oceasionally in pairs, end to end.

Compared with the influenza bacillus in morphology, the bacillus of pertussis is more regularly ovoid and somewhat larger. It has, furthermore, less tendency to pleomorphism and involution.

Staining.-The Bordet-Gengou bacillus may be stained with alkaline methylene-blue, dilute carbol-fuchsin, or aqueous fuchsin solutions. Bordet and Gengou recommended as a staining-solution carbolated toluidin-blue made up as follows:

Toluidin-blue

$5 \mathrm{gms}$.

Alcohol

100 c.c.

Water

500 c.c.

Allow to dissolve and add 500 c.c. of 5 per cent carbolic acid in water. Let this stand one or two days and filter.

Stained by the method of Gram, the bacillus of Bordet and Gengou is decolorized.

Cultivation.-Early attempts at cultivation made by the discoverers upon ordinary ascitic agar or blood agar were unsuccessful. They finally obtained successful cultures from sputum by the use of the following medium :

One hundred grams of sliced potato are put into 200 e.c. of 4 per cent glycerin in water. This is steamed in an autoclave and a glyccrin extract of potato obtained. To 50 c.c. of this extract, 150 c.c. of 6-per-cent salt solution and 5 grams of agar are added. The mixture is melted in the autoclave and the fluid filled into test tubes, 2 to 3 c.c. each, and sterilized. To each tube, after sterilization, is added an equal volume of sterile defibrinated rabbit blood or preferably human blood, the substances are mixed, and the tubes slanted.

On such a medium, inoculated with sputum, taken preferably during the paroxysms of the first day of the disease, colonies appear, which are barely visible after twenty-four hours, ${ }^{3}$ plainly visible after forty-eight hours. They are small, grayish, and rather thick. After the first generation the organisms grow with markedly greater luxuriance and speed. On the potato-blood medium after several generations of artificial cultivation, they form a grayish glistening layer which, after a few days, becomes heavy and thick, almost

3 Wollstein, Jour. Exp. Med., xi, 1909. 
resembling the growth of typhoid bacilli. In these later generations, also, they develop readily upon plain blood agar or ascitic agar and in ascitic broth or broth to which blood has been added. In the fluid media they form a viscid sediment, but no pellicle.

Culturally, the bacillus varies from B. influenzæ in growing less readily on hemoglobin media than the latter, on first cultivation from the sputum. Later it grows much more heavily on such media and shows less dependence upon the presence of hemoglobin than does B. influenzæ. It also grows rather more slowly than the influenza bacillus. It is strictly aërobic and in fluid cultures is best grown in wide flasks with shallow layers of the medium.

The Bordet-Gengou bacillus grows moderately at temperatures about $37.5^{\circ} \mathrm{C}$., but does not cease to grow at temperatures as low as $5^{\circ}$ to $10^{\circ} \mathrm{C}$. On blood agar and in ascitic broth it may remain alive for as long as two months (Wollstein).

Pathogenicity.-As regards the pathogenicity and etiologicai specificity of this organism for whooping-cough, no positive statement can as yet be made. The fact that it has been found in many cases in almost pure cultures during the early paroxysms, renders it likely that the organism is the specific cause of the disease. Mallory and Horner ${ }^{4}$ have found bacilli appearing to be Bordet and Gengou's organisms lying between the cilia in the tracheal epithelium of whooping cough cases that have come to autopsy. However, in early cases true influenza bacilli have been often found, and these latter seem to remain in the sputum of such patients for a longer period and in larger numbers than the bacillus of Bordet and Gengou. Endotoxins have been obtained from the cultures of the bacilli by Bordet and Gengou by the method of Besredka. ${ }^{5}$ The growth from slant cultures is washed up in a little salt solution, dried in vacuo, and ground in a mortar with a small, measured quantity of salt. Finally, enough distilled water is added to bring the salt into a solution of 0.75 per cent and the mixture is centrifugalized and decanted. One to two c.c. of such an extract will usually kill a rabbit within twenty-four hours after intravenous inoculation. Subcutaneous inoculation produces non-suppurating necrosis and ulceration without marked constitutional symptoms.

Inoculation of monkeys with the bacilli themselves by the respiratory path has failed to produce the disease.

"Mallory and Horner, Journ. Med. Res. xxvii, 1912, p. 115.

"Bordet, Bull. de la Soc. Roy. de Brux., 1907. 
Specific agglutinins may be obtained in immunized animals which prove absolutely the distinctness of this organism from Bacillus infiuenzx. ${ }^{6}$ In the serum of afflieted children the agglutination is too irregular to be of value.

Specific complement fixation with the serum of patients is reported by Bordet and Gengou, but failed in the hands of Wollstein.

\section{MORAX-AXENFELD BACILLUS}

In 1896 Morax $^{7}$ described a diplo-bacillus, which he associated etiologically with a type of chronic conjunctivitis to which he applied the name "conjonctivite subaigue." Soon after this, a similar microorganism was found in cases corresponding to those of Morax by Axenfeld. ${ }^{8}$ The condition which these microorganisms characteristically produce is a catarrhal conjunctivitis which usually attacks both eyes. The inflammation is especially noticeable in the angles of the eye, most severe at or about the caruncle. There is rarely much swelling of the conjunctiva and hardly ever ulceration. The condition runs a subacute or chronic course. Its diagnosis is easily made by smear preparations of the pus which is formed with especial abundance during the night.

Morphology.-In smear preparations from the pus, the microorganisms appear as short, thick bacilli, usually in the form of two placed end to end, but not infrequently singly or in short chains. Their ends are distinctly rounded, their centers slightly bulging, giving the bacillus an ovoid form. They are usually about two micra in length.

They are easily stained by the usual anilin dyes, and, stained by the method of Gram, are completely decolorized.

Cultivation.-The Morax-Axenfeld bacillus can be cultivated only upon alkalin media containing blood or blood serum.

It grows poorly, or not at all, at room temperature.

Upon Loeffler's blood serum, colonies appear after twenty-four to thirty-six hours as small indentations which indicate a liquefaction of the medium. Axenfeld states that eventually the entire medium

Wollstein, loc. cit.

'Morax, Ann. de l'inst. Pasteur, 1896.

${ }^{8}$ Axenfeld, Cent. f. Bakt., xxi, 1897. 
may become liquefied. Upon serum agar delicate grayish drop-like colonies are formed which are not unlike those of the gonococcus. hours.

In ascitic bouillon general clouding occurs within twenty-four

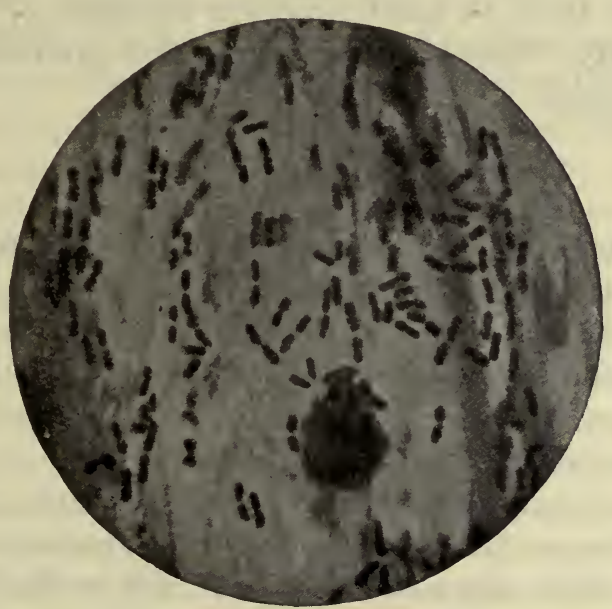

Fig. 54.-MoraX-Axenfeld Diplo-Bacillus.

Pathogenicity.-Attempts to produce lesions in the lower animals with this bacillus have been universally unsuccessful. Subacute conjunctivitis, however, has been produced in human beings by inoculations with pure cultures.

\section{ZUR NEDDEN'S BACILLUS}

In ulcerative conditions of the cornea, Zur Nedden has frequently found a bacillus to which he attributes etiological importance.

The bacillus which he has described is small, usually less than one micron in length, often slightly curved, and generally found singly. It may be found in the diplo form but does not form chains. It is stained by the usual dyes, often staining poorly at the ends. Stained by Gram's method it is decolorized. The bacillus is non-motile.

Cultivation.-It is easily cultivated upon the ordinary laboratory media. Upon agar it forms, within twenty-four hours, transparent, slightly fluorescent colonies which are round, raised, rather coarsely granular, and show.a tendency to confluence. 
Gelatin is not liquefied.

Milk is coagulated.

Upon potato, there is a thick yellowish growth.

Upon dextrose media, there is acid formation, but no gas.

The bacillus forms no indol in pepton solutions.

Pathogenicity.-Corneal ulcers have been produced by inoculation of guinea-pigs.

\section{BACILLUS OF DUCREY}

The soft chancre, or chancroid, is an acute inflammatory, destructive lesion which occurs usually upon the genitals or the skin surrounding the genitals. The infection is conveyed from one individual to another by direct contact. It may, however, under conditions of surgical manipulations, be transmitted indirectly by means of dressings, towels, or instruments.

The lesion begins usually as a small pustule which rapidly ruptures, leaving an irregular ulcer with undermined edges and a necrotic floor which spreads rapidly. It differs clinically from the true or syphilitic chancre in the lack of induration and in its violent inflammatory nature. Usually it leads to lymphatic swellings in the groin which, later, give rise to abscesses, commonly spoken of as "buboes."

In the discharges from such lesions, Ducrey, ${ }^{9}$ in 1889 , was able to demonstrate minute bacilli to which he attributed an etiological relationship to the disease, both because of the regularity of their presence in the lesions and the successful transference of the disease by means of pus containing the microorganisms.

Morphology and Staining.-The Ducrey bacillus is an extremely small bacillus, measuring from one to two micra in length and about half a micron in thickness. It has a tendency to appear in short chains and in parallel rows, but many of the microorganisms may be seen irregularly grouped. It is not motile, possesses no flagella, and does not form spores.

Stained by the ordinary anilin dyes, it has a tendency to take the color irregularly and to appear more deeply stained at the poles. By the Gram method, it is decolorized. In tissue sections, it may be demonstrated by Loeffler's methylene-blue method, and in such preparations has been found within the granulation tissues forming

'Ducrey, Monatschr. f. prakt. Dermat., 9, 1889. 
the floor of the ulcers. In pus, the bacilli are often found within leucocytes.

Cultivation and Isolation.-Early attempts at cultivation of this bacillus were universally unsuccessful in spite of painstaking experiments with media prepared of human skin and blood serum. In 1900 , Besançon, Griffon, and Le Sourd ${ }^{10}$ finally succeeded in obtaining growths upon a medium containing agar to which human blood had been added. They were equally successful when dog's or rabbit's blood was substituted for that of man. Since the work by these authors, the cultivation by similar methods has been carried out by a number of investigators. Coagulated biood, which has been kept for several days in stcrile tubes, has been found to constitute a favorable medium. Freshly clotted blood cannot be employed, probably because of the bactericidal action of the serum. Serum-agar has occasionally been used with success, but does not give results as satisfactory as those obtained by the use of the whole blood.

The best method of obtaining pure cultures upon such media consists in puncturing an unruptured bubo with a sterile hypodermic needle and transferring the pus in considerable quantity directly to the agar. If possible, the inoculation of the media should be made immediately before the pus has had a chance to cool off or to be exposed to light. When buboes are not available, the primary lesion may be thoroughly cleansed with sterile water or salt solution, and material scraped from the bottom of the ulcer or from beneath its overhanging edges with a stiff platinum loop. This material is then smeared over the surface of a number of blood-agar plates.

Upon such plates, isolated colonies appear, usually after fortyeight hours. They are small, transparent, and gray, and have a rather firm, finely granular consistency. The colonies rarely grow larger than pinhead size, and have no tendency to coalesce. At room temperature, the cultures die out rapidly. Kept in the incubator, however, they may remain alive and virulent for a week or more.

On the simpler media, glucose-agar, broth, or gelatin, cultivation is never successful. On moist blood-agar and in the condensation water of such tubes, the bacilli have a tendency to grow out in long chains. Upon media which are very dry, they appear singly or in short chains.

During recent years interest has again been aroused in the

1n Besançon, Griffon, et Le Sourd, Presse méd., 1900. 
chancroidal lesions because of the apparent relative frequency of such lesions among venereally infected soldiers in Europe. We are informed by Walker that during the post-armistice periods of the existence of American troops in France, the proportion of chancroids to other venereal infections rose quite beyond the ordinary relative proportion of this variety of infection, apparently for the reason that prophylaxis as practiced had less effect upon chancroidal infection than it did upon the syphilitic and gonorrheal infections. Since there had apparently developed in the minds of genito-urinary specialists, a certain amount of skepticism regarding the rôle played by the Ducrey bacillus at this time, the matter was reinvestigated in our laboratory by Teague and Deibert. ${ }^{11}$ They developed a method for direct diagnostic cultivation of Ducrey bacilli from chancroidal lesions which has so much practical value that it will be well to quote it in considerable detail. The method as described by them is as follows: A rabbit is bled from the heart with a sterile 20 c.c. syringe and the blood is distributed in amounts of 1 c.c. in small test tubes, a little larger than the ordinary Wassermann tube. The blood is allowed to clot at room temperature and is then heated for five minutes at $55^{\circ} \mathrm{C}$. It can thus be preserved in the ice-box or can be used immediately. Equally good results can be obtained when the tubes are kept in the ice-box for three to four days before use without heating.

Pieces of stiff iron wire, gauge 18 , about $51 / 2$ inches long are bent upon themselves at one end for about $1 / 8$ inch. Ten or twelve of these wires are placed in a 6-inch test tube and are heated in the dry sterilizer. The patient removes the dressing and a bit of the pus is picked up with the bent end of the wire, the latter having been first rubbed gently over the base of the ulcer or under its undermined edge. The pus is then transferred to a tube of clotted blood and distributed in the serum by passing the wire around the clot. A second tube is prepared in the same way. After twenty-four hours incubation at $37^{\circ} \mathrm{C}$. the serum around the clot is thoroughly stirred with a platinum loop and a smear is made. Examination with the oil-immersion lens shows characteristic chains of small Gramnegative bacilli, sometimes in pure culture, sometimes in mixed culture. The organism is usually so characteristic that such an examination is sufficient basis for a positive diagnosis. Even when anti-

${ }^{11}$ Teague and Deibert, Jour. of Urology, 4, 1920, 543. 
septic power or ointments have been applied, repeated positive eultures have been obtained by finding a bit of pus free from drug. It is not even necessary to wash the ulcer before taking cultures.

At the time of the publication of their first paper, Teague and Deibert had cultured by the above method, 274 sores. In most cases these were indiscriminately cultured, even in many cases when no clinically characteristic picture was apparent. Of these 274 sores, 140 yielded positive Ducrey cultures. Of the 134 negative cases, satisfactory notes were obtained of only 69 , and from these notes it is apparent that 42 of these 69 negative cases at least were not chancroidal but primary syphilitic lesions. It seems to Teague fair to assume that by this method probably over 90 per cent of true chancroids can be diagnosed, and it is so simple that the physician in the clinic can take the cultures as directed and send them to the laboratory. Isolations can subsequently be made by inoculating blood agar plates from the clotted blood tubes after 24 hours. The nutricnt agar should have a $\mathrm{P}_{\mathrm{H}}$ of 7.2 or 7.3 , and the agar must be neither too stiff nor its surface too dry. Teague's results not only furnish a simple method for the determination of mixed infection, but also reaffirm the etiological importance of the Ducrey bacillus in chaneroids.

As to prophylactic treatment, the recent experience seems to indicate that warm water and soap very thoroughly applied is probably more effective in the prophylaxis of this type of infection, than are the specific methods used for venereal prophylaxis.

Pathogenicity.-Besançon, Griffon, and Le Sourd, and others, have succeeded in producing lesions in man by inoculation with pure cultures. Inoculation of the lower animals has, so far, been entirely without result. 


\section{CHAPTER XXVI}

MICROCOCCUS INTRACELLULARIS MENINGITIDIS (MENINGOCOCCUS) AND EPIDEMIC CEREBROSPINAL MENINGITIS

INFECTIOUs processes in the meninges may be caused by many different microorganisms.

Meningitis may be primary or secondary. Secondary meningitis may often occur during the course of pneumonia, when pneumococci, carried to the meninges by the blood stream, give rise to a usually fatal form of the disease. More rarely a similar process may occur as a secondary manifestation of typhoid fever or influenza. Meningitis may also result secondarily by direct extension from suppurative lesions about the skull, such as those occurring in diseases of the middle ear or frontal sinuses or after compound fractures. In such cases the invading organisms are usually staphylococci, streptococei, or pneumococci.

Isolated cases of meningeal infection with B. coli, B. paratyphosus, Bacillus pestis, and Bacillus mallei have been reported. A frequent more chronic form of the disease is caused by Bacillus tuberculosis.

Primary acute meningeal infection, however, is due chiefly to two microorganisms, Micrococcus intracellularis meningitidis, and the pneumococcus.

A tabulation of the comparative frequency with which the various microorganisms are found in the meninges has been attempted by Marschal. ${ }^{1}$ This author estimates that about 69.2 per cent of all acute cases are due to the meningococcus, 20.8 per cent to Diplococcus pneumoniæ, and the remaining 10 per cent to the other bacteria mentioned.

The cases caused by the pneumococcus and the other less frequent incitants usually occur sporadically. When the disease occurs in epidemic form, it is almost always due to the meningococcus.

${ }^{1}$ Marschal, Diss. Strassburg, 1901, Quoted from Weichselbaum, in Kolle und Wassermann, "Handbuch." 
Diplococcus intracellularis meningitidis was first seen in meningeal exudates by Marchiafava and Celli ${ }^{2}$ in 1884 . These authors not only described accurately the morphological characteristics now recognized, but also called attention to the intracellular position of the microorganism and to its gonococcus-like appearance. They failed, however, to cultivate it.

Observations confirmatory of the Italian authors were, soon after, made by Leichtenstern. ${ }^{3}$ Cultivation and positive identification as a separate species was not accomplished, however, until Weichselbaum, ${ }^{4}$ in 1887, reported his observations upon six cases of epidemic cerebrospinal meningitis in which he not only found the cocci morphologically, but was able to study their biological characteristics in pure culture. The researches of Weichselbaum were soon confirmed and extended by elaborate studies ${ }^{5}$ which left no doubt as to the specific relationship between the microorganism cultivated by him and the clinical condition.

Morphology and Staining.--Stained in the spinal fluid from an infected patient, the meningococcus bears a striking similarity to the gonococcus. The microorganisms appear intra- and extracellularly, usually in diplococcus groups, sometimes as tetrads, or even in larger agglomerations. The individual diplo-forms are flattened on the sides facing each other, presenting somewhat the biscuit-form of the gonococcus. The variation in size of the cocci in the same smear is a noticeable feature and of some diagnostic importance. This dissimilarity in size is noticeable also in cultures, which, especially when older than twenty-four hours, contain forms double or even triple the size of the average coccus. These may possibly be involution forms.

The meningococcus is non-motile and non-spore forming. It stains easily with all the usual aqueous anilin dyes. Its behavior toward Gram's stain was long a subject of controversy, owing to the error of Jaeger, ${ }^{6}$ who claimed to have found it Gram-positive. There is no question now, however, that the cocci decolorize by Gram's method when this is earefully carried out.

${ }^{2}$ Marchiafaya and Celli, Gaz. degli ospedali, 8, 1884.

${ }^{3}$ Leichtenstern, Deut. med. Woch., 1885.

"Weichselbaum, Fort. d. Med., 1887.

${ }^{5}$ Councilman, Mallory, and Wright, Special Rep. Mass. Board of Health, 1898, Albrecht und Ghon, Wien. klin. Woch., 1901.

' Jaeger, Zeit. f. Hyg., xix, 1895. 
In spinal fluid satisfactory preparations may be obtained by staining in Jenner's blood stain. Councilman, Mallory, and Wright ${ }^{7}$ were the first to notice that, when stained with Loeffler's methyleneblue, meningococcus stains irregularly, showing metachromatic granules in the center of the cell bodies. These granules can be demonstrated more clearly with the Neisser stain employed for similar demonstration in the case of $\mathrm{B}$. dipththerix and have some value in differentiating meningococeus from gonococeus.

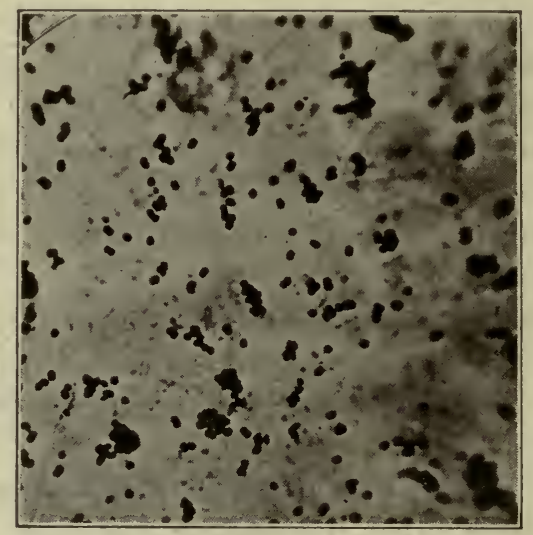

Fig. 55.-Meningococcus Pure Culture.

It is important to remember that meningococei in spinal fluid undergo solution very readily, a solution which is probably an autolysis, with the result that spinal fluid which may be full of polymorphonuclear leucocytes, contains very few recognizable organisms. This readiness of meningococei to go into solution will be spoken of below in connection with problems of cultivation.

Cultivation of the Meningococcus.-The meningococeus is peculiar in that there is considerable difference in the ease with which separate strains can be made to grow upon artificial media. Some meningococci grow readily upon all meat infusion culture medià. They may even grow upon some meat extract media, but growth upon these is never profuse. It is never well to rely upon media to which no enriching substance has been added, or that have not been especially made for meningococeus cultivation when attempts are made at first

'Councilman, Mallory, and Wright, Rep. Mass. State Bd. of Health, 1898. 
isolation from human material. After the bacteriologist is familiar with the individual strains, he may at times carry his strains on the simpler media, meat infusion agar and broth.

Growth is more luxuriant and rapid upon media to which animal protein in the form of blood serum or ascitic fluid has been added. Coagulated serum is not liquefied. For cultivation of the meningococcus directly from the human body it is wise to use the richer serum or blood media. Agar to which whole rabbit's blood has been added

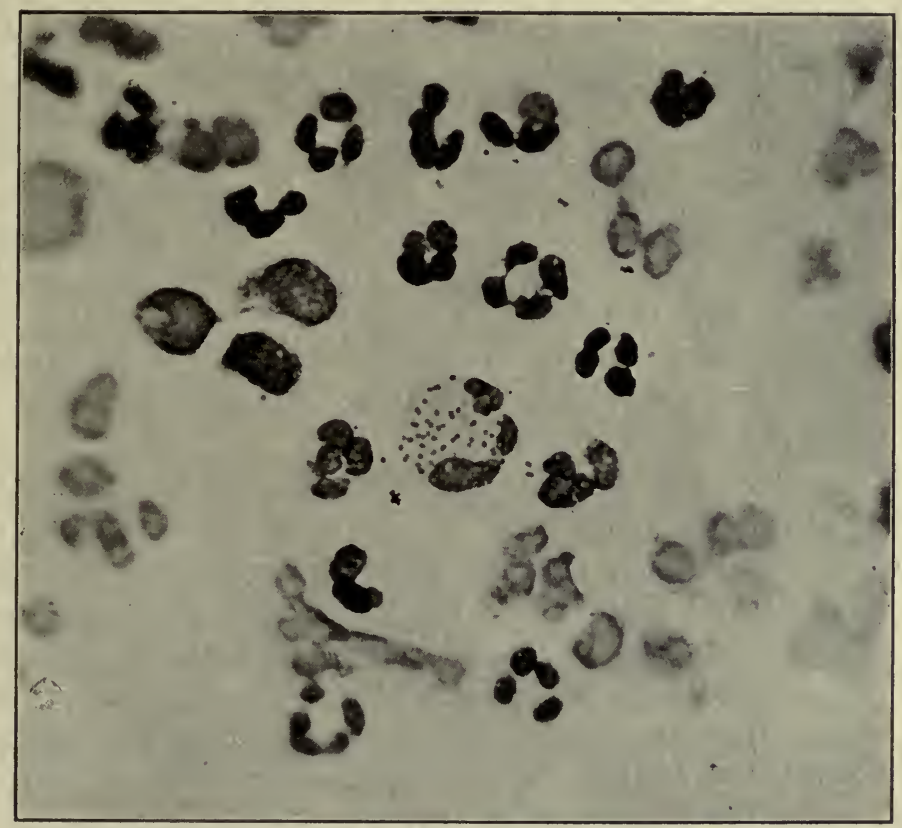

Fig. 56.-Meningococcus in Spinal Fluid.

forms an excellent medium, both for cultivation and for keeping the organism alive. Loeffler's blood serum is less favorable. It is advisable, too, when cultivating directly from spinal fluid, to plant rather large quantities ( 1 to 2 c.c.), since many of the cocei in the exudate will fail to develop colonies, possibly because of their prolonged exposure either to the body fluids or to their own products in a closed space.

Upon broth, growth is slow and takes place chiefly upon the surface, the sediment consisting mainly of dead bacteria. Glucose added to agar or to broth renders the medium more favorable for rapid 
growth, but, owing to acid formation, tends to cause a more rapid death of the culture. In flasks of broth containing glucose one per cent, and $\mathrm{CaCO}_{3}$ one per cent, however, cultures have been kept alive for as long as fourteen months (Hiss). On milk, growth takes place without coagulation of the casein. Potatoes are not a favorable medium, though growth occasionally takes place.

While slight alkalinity or acidity does not inhibit, the most favorable reaction of media is a $\mathrm{P}_{\mathbf{H}}$ of 7.4 to 7.6 .

Oxygen is necessary for development. Complete anaërobiosis, while not absolutely inhibitory, is extremely unfavorable, unless proper carbohydrates be present.

Recently, work by Wherry and Erwin, ${ }^{8}$ as well as by Gates, ${ }^{9}$ has shown that the growth of the meningococcus is definitely stimulated by replacing about ten per cent of the air by carbon dioxide. The plates are placed into a closed jar, into which freshly produced carbon dioxide is allowed to pass, and the jars are then sealed and incubated. This matter has been discussed in a previous section on partial oxygen tension in bacterial cultures. We have confirmed this in our laboratory, and find that the colonies grow larger and growth is more rapid under the partial $\mathrm{CO}_{2}$ atmosphere.

While growth may take place at temperatures ranging from $25^{\circ}$ to $42^{\circ} \mathrm{C}$., the optimum is $37.5^{\circ} \mathrm{C}$. It is an important aid to the recognition of true meningococei that they never grow at ordinary room temperature. Apart from the remarkable viability displayed upon calcium-carbonate broth, the average length of time during which the meningococcus will remain alive without transplantation is rather short. Recently isolated cultures grown on agar or serum-agar may die within two or three days. Accustomed to artificial cultivation through a number of generations, however, the cultures become more hardy and transplantation may safely be delayed for a week or even longer. Albrecht and Ghon ${ }^{10}$ have kept a culture alive on agar for one hundred and eighty-five days. It is a strange fact that after prolonged artificia! cultivation some strains of meningococcus may gradually lose their growth energy and finally be lost because of their refusal to develop in fresh transplants.

It is our belief that this phenomenon which hitherto we have been at a loss to explain, may have some connection with the so-called

${ }^{8}$ Wherry and Erwin, Jour. of Infec. Dis., 22, 1918, 194.

${ }^{\circ}$ Gates, Jour. Exper. Med., 29, 1919, 325.

${ }^{10}$ Albrecht und Ghon, Wien. klin. Woch., 1901. 
"bacteriophage" phenomena discussed in a separate section. Since the extensive investigation of the autolytic properties acquired by bacteria under certain conditions, there has been no investigation of the meningococcus problem from this point of view. We ourselves have often found meningococcus cultures to lose their viability under conditions which, retrospectively, we suspect now may have been due to this kind of development. It is also not unlikely that the extensive autolysis of the organism in the spinal fluid may have a similar significance. Storage is best carried out at incubator temperatures. At room temperatures or in the ice chest, the diplococcus dies rapidly. ${ }^{11}$

Special Meningococcus Media.-For the routine cultivation of meningococcus, there are certain media which are better than others and which are, therefore, described in this section.

As a basis for meningococcus media, we like to use hormone agar or hormone broth, or trypagar or trypsinized broth as described in the section on media. To these basic media enriching substances are added. The necessity for these enriching substances may have a more complex cause than the simple addition of nutrition, since, as Lloyd has suggested, the occasional first growth on simple media of meningococci directly from the human body, may depend upon the presence of a certain amount of "vitamine" furnished by the animal fluids present in the exudate. The most convenient substances to add to these media are blood in one form or another. Many different varieties of blood are favorable, and human, horse, or rabbit blood can be most conveniently used. The blood may be defibrinated and added directly in quantities of about five per cent, and if agar for plating is used, melted agar is mixed with the blood and thoroughly shaken just as the plates are poured. Laked blood is very convenient, and may be prepared by mixing whole blood with about four parts of sterile distilled water. This laked blood may be kept and mixed with the agar just before pouring the plates, after the agar has been cooled below $50^{\circ}$. The blood may also be laked in ether and in this way can be kept sterile for a long time before being added to the basic medium. Blood serum and ascitic fluid ean be used, but do not seem to give as good results as does laked or whole blood. The addition of one-half to one per cent of glucose is always favorable. The special pea-powder-blood-agar

${ }^{11}$ A very thorough biological study of meningococeus and related organisms has recently been male by Elser and II untoon (Jour. Med. Res., N. S. vol. xv, 1909), which may be consulted for a more detailed deseription of cultural characteristics. 
used by the British during the war is not described in detail because we believe that for ordinary laboratory work its production is too complicated, without offering sufficient advantages over other media. To summarize, we, therefore, recommend for plating media, carrier work, and isolation from spinal fluid, hormone or trypagar with a $\mathrm{P}_{\mathrm{H}}$ of 7.4 to 7.5 , containing one-half per cent glucose, to which about 5 or 10 per cent of defibrinated or laked blood is added just before the plates are poured.

For the storage of stock cultures, Vedder's starch agar described in the section on media has been used with satisfaction.

Gordon ${ }^{12}$ and others also have used coagulated egg media in slants for storage of stock cultures with good results.

Egg-yolk Medium for the Storage of Meningococcus Cultures. ${ }^{13}$ T'he egg-yolk used may be the yolks of eggs, the whites of whieh have becn used for clearing media. One volume of the egg-yolk is mixed with one-half volume of physiological salt solution. The yolk and salt are thoroughly mixed, tubed and slanted. The slants are then inspissated in the usual way. This can be done in an autoclave by bringing the temperature up gradually without letting out the air, until 14 pounds pressure has been reached, and then maintaining this for twenty minutes. Great care should be taken to prevent bubbles in the medium. The tubes should be plugged with paraffin, since water of condensation is necessary to make the medium useful for storage.

For fermentation reactions, solid or fluid media with various sugars and litmus indicator, may be used. Gordon, whose experience in this kind of work has been extensive, used for his fermentations a liquid medium of simple pepton water with one per cent blood serum and the sugar to be investigated, added. Elser and Huntoon ${ }^{14}$ employed among other things, for their extensive fermentation work, sheep serum water, ascitic broth, and broth made with nutrose.

Resistance.-The meningococcus is killed by exposure to sunlight or to drying within twenty-four hours. ${ }^{15}$ It is extremely sensitive to heat and cold and by the common disinfectants is killed in high dilutions and by short exposures. At $0^{\circ} \mathrm{C}$. it usually dies within two or three days.

${ }^{12}$ Gordon, Flack and Hines, Mert. Res. Com., Speeial Report Series, No. 3, London, 1917.

${ }^{13}$ Directions of Major Foster from Gordon's Laboratory.

${ }^{14}$ Elser and Huntoon, Jour. Med. Res., 20, 1909, 371.

${ }^{15}$ Councilman, Mallory, and Wright, Boston, 1898; Albrecht and Ghon, loc. cit. 
A special study of the resistance of meningococci to various dye stuffs has been made by Binger. ${ }^{16}$ An interesting result of these investigations was that Binger found that methylene-blue had a specific inhibiting action upon meningococeus and gonococeus at dilutions too low to inhibit other pathogenic microorganisms, and that this inhibitory action was not interfered with by the presence of the protein in spinal fluid or other exudates.

Toxic Products of the Meningococcus.-No soluble exotoxin has ever been conclusively isolated from meningococcus cultures. A substance which causes acute symptoms in rabbits within an hour can be recovered from young broth cultures of meningococei, and from filtered washings from meningococcus cultures on agar. These substances are analogous to the so-called " $\mathrm{X}$ " substances which one of the writers with Kuttrier and Parker ${ }^{17}$ has described, which are non-specific, can be obtained from many different microorganisms and are nonantigenic. That they are a very real and important substance in connection with meningococei we are persuaded to believe by the fact that those who immunize horses for serum production with meningococci find that it is necessary to wash the agar cultures once before injecting. into horses, otherwise severe symptoms occasionally result.

A number of investigators have found that cultures that have been kept in broth long enough for a certain amount of extraction or autolysis to occur, yield toxic products which are in general identical in their action to that of whole meningococci injected in analogous quantities. This has been the experience of Flexner, ${ }^{18}$ Kraus and Doerr, ${ }^{19}$ and others. Extracts of meningococci made with salt solution, weak sodium hydrate, etc., kill guinea pigs within 24 hours, with symptoms of general intoxication, peritoneal exudates, and often pleural exudates. Intravenous injection of sufficient quantities of such extracts or of dead meningococci may kill rabbits. No reliable or constant results with such substances have been obtained, but it is quite definite that the bodies of meningococci, like the bodies of typhoid and colon bacilli, are toxic for animals by what is generally spoken of as an endotoxin action.

${ }^{16}$ Binger, Jour. Infec. Dis., 25, 1919, 277.

${ }^{17}$ Zinsser, Kuttner and Parker, Proc. Soc. Exper. Biol. and Med., November, 1920.

${ }^{18}$ Flexner, Cent. f. Bakt., 43, 1907.

${ }^{10}$ Kraus and Doerr, Wien. klin. Woch., 1908. 
Types of Meningococci.-Until 1909 it was believed that the meningococcus group, was homogeneous, and that no essential difference between individual members of the group existed. In this year, Dopter ${ }^{20}$ found that some of the meningococci isolated from cases which occurred in Paris and environment, could be distinguished by specific agglutination reactions from the ordinary or normal type. This para-meningococcus, as Dopter called it, opened the way for investigations aimed at the serological classification of the group, and, as was to be expected, it was found that there were a considerable number of different meningococcus sub-types. Wollstein ${ }^{21}$ confirmed Dopter's work and found, among other things, that the various parameningococcus strains were not wholly homologous, and suggested their possible further subdivision. Gordon ${ }^{22}$ examined a large number of meningococci from cases occurring among British and Canadian troops, and found that all the organisms studied by him could be divided into four definite types. He used not only the agglutination reaction, but controlled them with absorption tests. Tulloch, ${ }^{23}$ following up Gordon's work on a considerable material, found that, out of 356 cocci investigated, 234 gave specific results with the four type sera used by Gordon's laboratory. He found that, with remarkably few exceptions, the organisms responsible for the outbreaks among British troops were comprised in the four Gordon types. He did, however, find some organisms in the nasopharyngeal cultures of earriers which, though closely resembling meningococci, did not react with any of the type sera. There was some question, however, in his mind as to whether these represented true virulent meningococci. An important result of Gordon's investigations was to show that very many of the organisms obtained from carriers belong to one of the four types known to exist in actual cases of meningeal infection.

In America, Flexner ${ }^{24}$ and his associates have investigated the group relationships of the meningococei very carefully, and their results indicate that there are probably two main types, the normal and the para-meningococcus of Dopter; and, in addition to this, a considerable number of heterogeneous intermediate types which are related to each other and to the fixed types more or less in the same

${ }^{20}$ Dopter, Coompt. Rend. de la Soc. de Biol., 67, 1909, 74.

${ }^{21}$ Wollstein, Jour. Exper. Med., 20, 1914.

${ }^{22}$ Gordon, Brit. Med. Res. Commit. Reports, Londor, 1915 and 1917.

${ }^{28}$ Tulloch, Jour. Royal Medical College, February, 1918, p. 9.

${ }^{24}$ Flexner, Bulletin, Rock. Inst. for Med. Res., 1917. 
way, but somewhat more closely than are the different members of the viridans group of streptococei, a point which makes it plain that a diagnostic or curative serum, to be truly polyvalent, must be produced with many different representatives of organisms isolated from cases. The correspondence of the different types, as named in various countries, is as follows:

Gordon's type $I=$ para-meningococcus

Gordon's type II = normal meningococeus

Gordon's types III and IV = intermediate or irregular strains, of which there are a considerable number of different ones, shading into each other, serologically.

As far as the prevalence of type is concerned, no definite rule can be established at present. In the extensive investigations of Gordon and his co-workers, it was found that the earliest cases were mostly his type I, later eame his type II, especially in the London district, and after March type IV cases began to appear, but no type III cases were noticed until July.

Agglutination.-Immunization of animals by repeated inoculations of meningococcus ${ }^{25}$ results in the formation in the blood serum of agglutinins. Kolle and Wassermann ${ }^{26}$ obtained from horses a serum which had an agglutinating value of $1: 3,000$ for the homologous strain, and of as much as 1:500 for other true meningococeus strains. Similar experiments by Dunham ${ }^{27}$ and others have proved the unquestionable value of agglutination for species identification of this group. Great differences may, however, exist between individual races in their agglutinability in the same immune serum.

Kutscher has recently called attention to the fact that strains which cannot be agglutinated in specific sera at $37^{\circ} \mathrm{C}$. will often yield positive results when subjected to $55^{\circ} \mathrm{C}$., a fact of some practical importance if confirmed.

Elser and Huntoon ${ }^{28}$ have shown that in the serum of infected human subjects agglutination of some strains takes place in dilutions as high as $1: 400$.

The Production of Agglutinating Sera for Meningococcus Determination in. Laboratories.-For this purpose, rabbits are best

25 Albrecht and Ghon, Wien. klin. Woch., 1901.

${ }^{26}$ Kolle und Wassermann, Deut. med. Woch., 15, 1906.

${ }^{27}$ Dunham, Jour. Inf. Dis., 11, 1907.

${ }^{28}$ Elser and IIuntoon, loc. eit. 
employed. Amoss has found that young rabbits are more satisfactory than older ones for this purpose, and he uses rabbits weighing between 1500 and 1800 grams. He grows his meningococci on glucose agar slants, and washes up the growth in salt solution. 0.001 of a culture is inoculated as the first dose. For the rapid production of agglutinating sera, he injects his rabbits for three succeding days, giving a rest of five days, and then another course of three days injection. He bleeds the animal two or three days after the second course of inoculation. For ordinary purposes, the slow method of three or four day intervals, about five or six injections, with bleeding eight or nine days after the last injection, may be used. Among English workers, Hine ${ }^{29}$ injects culture suspensions grown on 25 per cent hemoglobin serum agar, killed at $65^{\circ}$ and brought to a standard opacity. 0.5 per cent carbolic acid is added for preservation. He standardizes all his suspensions by opacity comparisons against suspensions of freshly precipitated barium sulphate. He compares by diluting his suspension in a test tube of similar dimensions as the standard tube, until the image of a small flame is just visible in the same distance from the flame as in the case of the standard tube. With such suspensions he immunizes rabbits, beginning with an injection of two doses of five hundred million cocei at an interval of one hour. Six days later he gives three million cocci, and, if the serum is satisfactory on the eighth day later, he bleeds. This method was satisfactory in the hands of Hine, with types I and III. With the other types he has had to give larger and more frequently repeated doses. In all such immunization, experience and judgment, with frequent titration of samples of the rabbit serum taken from the ear, are necessary.

Nicolle ${ }^{30}$ at the Pasteur Institute uses for immunization, powdered meningococcus antigen prepared from growth on agar slants by suspension in salt solution, centrifugation and drying. Hine also recommends the use of rabbits ranging from 800 to 1500 grams.

Agglutination Technique with Meningococci.-Agglutination of meningococci present considerable difficulties because of the relative inagglutinability of many meningococcus cultures. This is a peculiarity of these organisms which has necessitated much investigation and many technical modifications. Hine has found that allowing the

${ }^{29}$ II ine, Med. Res. Committee, Spec. Rep., Series 3, No. 3, p. 99.

so Nicolle. 
diluted earbolic saline suspension to stand for twenty-four hours, increases agglutinability, and recommends this technique if time permits. Tulloch ${ }^{31}$ has called attention to a number of precautions both in handling of the cultures and the serum for agglutination which seem to us sufficiently important to note. He recommends the use of standardized suspensions of the meningococci as described by Hine, and recommends great caution in the nature of the medium on which the cultures are grown. He advises getting rid of the condensation water in the slants before washing off the growth, owing to the possibility of alkalin or acid reactions in this condensation water. The strength of the phenol in standard suspensions should never be more than 0.5 per cent. Also he warns against getting any of the agar into the suspension because he believes that it may act under certain eircumstances as a protective colloid.

On the basis that moderate heat increases the agglutinability of organisms like the typhoid bacillus and meningococei, the workers in the British Central Laboratory used the routine method described under carrier determination, that is, incubating the serum culture agglutination mixtures in a water bath at $55^{\circ}$, for varying periods, usually twelve hours before the final readings are made. Hot air ovens at $55^{\circ}$ are not good substitutes because of the great evaporation which, according to Hine, occasionally leads to spontaneous agglutination. (See also section on Carriers.)

Agglutinin Absorption Experiments for Meningococcus Typing. ${ }^{32}$ A thick suspension of the meningococci to be examined, quantity 0.5 c.c., is mixed with the various monovalent type sera, 0.5 e.c., dilutions 1 to 25, in saline. Similar serum dilutions were set up without suspensions. The tubes are thoroughly shaken, set in the water bath at $37^{\circ}$ for one hour, and then at room temperature overnight. The tubes are then centrifugalized at high speed until the supernatant fluid is clear. The supernatant fluids of the tubes containing the suspensions, as well as the fluids similarly treated without suspension, now represent serum dilutions of 1 to 50 .

Each suspension-absorption tube now has a serum control which has been exposed to the same temperature in the same dilutions without meningococei. From each set of two tubes now other tubes are made, into which $0.4,0.2,0.1$, and 0.05 c.c. are taken, and with salt

${ }^{31}$ Tulloch, Royal Army Med. Jour., February, 1918.

${ }^{32}$ The description given is that given by the British Medical Research Committee, loc. cit. 
solution the volume of all of these tubes is brought up 0.4 c.c. To each of the tubes now 0.4 c.c. of the homologous meningococcus suspension is added, giving serum dilutions ranging from 1 to 100 , to 1 to 800 . These are now incubated at $55^{\circ}$ for twenty-four hours, as in the first agglutination. The agglutinating titers of the absorbed sera are now compared with those of the un-absorbed, and diminutions of titer are noted.

Animal Pathogenicity.-Animals are not very susceptible to infection with Diplococcus meningitidis. Subcutaneous inoculation is rarely followed by more than a local reaction unless large quantities are used. White mice are rather more susceptible than other species. Intraperitoneal and intravenous inoculation of sufficient quantities usually results in the death of mice, rabbits, guinea-pigs, and dogs. Occasional strains have been found to possess a not inconsiderable degree of toxicity for rabbits, grave symptoms or even death following intravenous injection of but moderate quantities without any traceable development of the microorganisms in the organs of the animals.

Similar observations have been made by Albrecht and Ghon, ${ }^{33}$ who succeeded in killing white mice with dead cultures. It would seem, therefore, that the effect of this coccus upon animals depends chiefly upon the poisonous substances contained in the bacterial bodies (endotoxins). Lepierre ${ }^{34}$ has obtained the meningococcus toxin by alcohol precipitation of broth cultures.

Weichselbaum himself succeeded in producing meningeal suppuration and, in one case, brain abscess, by subdural inoculation of dogs. Councilman, Mallory, and Wright produced a disease in many respects similar to the human disease by intraspinous inoculation of a goat. More recently, Flexner ${ }^{35}$ has succeeded in producing in monkeys a condition entirely analogous to that occurring in human beings.

\section{THE DISEASE IN MAN}

The disease produced in man consists anatomically in a suppurative lesion of the meninges, involving the base and cortex of the brain and the surface of the spinal cord. The nature of the exudate may vary

\footnotetext{
${ }^{33}$ Albrecht und Ghon, loc. eit.

${ }^{34}$ Lepierre, Jour. de phys. et de path. gén., v, No. 3.

${ }^{\text {s }}$ Flexner, Journ. of Exp. Med., 1906.
} 
from a slightly turbid serous fluid to that of a thick fibrinous exudate. In chronic cases encephalitis and dilatation of the ventricles may take place. Apart from their presence in the meninges and in the nasopharynx, meningococci have not been satisfactorily demonstrated in any of the complicating lesions of the diseasc. Reports of their presence in the conjunctivæ, in the bronchial secretions from broncho- or lobar pneumonia, and in otitis media, have been reported but are not very eommon.

The occurrence of this microorganism in the circulating blood of meningitis cases has been definitely proved by Elser, ${ }^{36}$ who found it in ten cases.

In the discussions on epidemiology, below, we will see that Herrick and others claim that the meningococcus is probably, in the majority of cases, in the blood before it reaches the meninges, making its way to the central nervous system by way of the blood stream rather than directly along the lymphaties at the base of the skull. It seems fair to assume from blood culture evidence that this certainly happens in many eases even though it may not be the rule. During epidemics, also, there are occasional cases in which a general septicemia due to meningococei occurs, without ever giving rise to symptoms pointing to meningeal involvement. These cases are always violent in course, usually fatal and accompanied by a profuse petechial rash.

\section{BACTERIOLOGICAL MANAGEMENT OF THE MENINGITIS CASE AND SERUM TREATMENT}

In the light of our present knowledge of the bacteriology and serum treatment of epidemic meningitis, a considerable responsibility rests with the bacteriologist. The difference between recovery and death may depend directly upon the speed with which a bacteriological diagnosis is made and a proper management of the serum treatment. When a case of suspicious fever in which slight stiffness of the neck, and a developing Kernig sign is associated with the other indications of an acute infection, the first step must consist of lumbar puncture.

A sterile lumbar puncture needle is thrust into the spinal canal, a little to one side of the third or fourth lumbar space, and the fluid

${ }^{36}$ Eilser, Jour. Med. Res., xiv, 1906. 
which is always under some pressure, is taken directly into a centrifuge tube. This fluid must then be examined as above indicated in the technical section on spinal fluid, and the diagnosis made. If possible, a smear should be made at the bed side, and an immediate Gram stain done with the first drop of fluid that flows. In this way, it may be possible to inject the first dose of serum immediately after the withdrawal of the diagnostic fluid.

Examination of Spinal Fluid.-The spinal fluid of meningococeus cases is slightly turbid in the very early periods, becoming increasingly purulent, with large numbers of polymorphonuclear leucocytes. In some cases the fiuid which has been very purulent may clear up considerably, and then become purulent again, a matter probably dependent upon sacculation in parts of the subarachnoid space. The fluctuations in the nature of the spinal fluid under intraspinous serum treatment will be spoken of in another place. A certain amount of prognostic information can be obtained from the spinal fluid in that in severe cases that are not doing well, there will be a considerable number of organisms, extracellular. Ordinarily, the majority of the meningococci are intracellular. Such spinal fluid should be taken into sterile centrifuge tubes, brought to the laboratory without delay, slides smeared from the sediment, and stained by Jenner and by Gram. It is important to remember that because of the extensive autolysis of meningococci in the fluid, it may under circumstances be very difficult to find meningococci. In such cases, if prolonged search has failed to reveal organisms, our experience has taught us to assume that purulent fluid from a case of an acute meningitis in which there are a preponderance of polymorphonuclear leucocytes without organisms is probably "meningococcus" in origin. Streptococcus and pneumococcus fluids invariably show Gram-positive cocci.

The fluid should be cultured upon blood agar plates, the medium prepared as described above. It is well to inoculate the plates heavily since the viable organisms present, even in acute fluids, may be relatively few in numbers. To be on the safe side it is sometimes well too, to place a portion of the fluid in the original centrifuge tube in the incubator for three or four hours before inoculating media from it.

The typing of meningococei derived from spinal fluid is desirable since the preliminary injection of polyvalent serum upon first diagnosis may be advantageously followed by the injection of type sera, 
homologous to the organisms found in the patient. This method is impracticable on a large scale since so many types, shading into each other, are possible in this disease. However, the method is used to a considerable extent in France.

As stated in the section on the manner of entrance of the meningococci into the subarachnoid space, it is a question now under discussion whether the organisms travel along the lymphatics to the base of the skull directly, or whether bacteriemia precedes meningeal infection. It is well, always, in cases of early meningitis, to take blood cultures. In taking blood cultures it is best to inoculate hormone glucose broth flasks containing not less than 100 c.c. of culture fluid and to make a number of glucose hormone agar plates with varying amounts of blood. The presence of meningococci in the blood is, of course, an indication for intravenous as wèll as intraspinous injection of serum, a procedure which is in our opinion advisable in all cases, since it is quite likely that meningococcus septicemia, constant or intermittent is a regular feature of the pathology of the disease.

Serum Therapy of Meningitis.-During recent years, attempts have been made to treat epidemic meningitis by injections, subcutaneous and intraspinous, of meningococcus-immune serum. Wassermann, ${ }^{37}$ in 1907, reported results of such treatment in one hundred and two patients, with a recovery of 32.7 per cent. The serum, manufactured by Wassermann and his associates, was obtained from horses immunized with cultures of meningococeus and with toxic meningococcus extracts. More recently Flexner and Jobling ${ }^{38}$ have used a similar serum in the United States with apparently excellent results. The serum, in Flexner's cases, as in the technique first used by Jochmann, is injected intraspinously after a quantity of spinal fluid had been withdrawn. The cases treated by Flexner and Jobling's method have now reached large numbers, both in this and foreign countries and the value of the serum as a therapeutic agent seems firmly established.

The Serum.-In America polyvalent serum is used almost universally. Horses, as for other serum production, are the animals employed. The cultures with which the horses are immunized must be many containing representatives of the normal

a7 Wassermann, Deut. med. Woch., 39, 1907.

${ }^{38}$ Flexner and Jobling, Jour. Exper. Med., x, 1908. 
and para-types, and a considerable number of intermediates, if possible, to represent individuals from various parts of what we may call the spectrum of intermediate agglutination types. The choice of cultures is perhaps the most important single feature in serum production, and those who undertake to produce serum should be constantly receiving cultures from various parts of the country, isolated from cases, checking them up with their serum product, and adding them to their immunizing collection, if they are not represented by antibodies in the polyvalent serum. It is still a question of demanding some research, whether or not a definite limitation of the number of strains used for immunization would be of advantage, since the use of too many different strains may keep down the agglutination value of the serum of the immunized horse. The cultures injected into the horse are grown on agar, and once washed in salt solution before injection. Various routines for the injection of horses have been devised, the most useful method at the present time consisting of injecting on two or three consecutive days, giving rests of seven or eight days, between courses of injection. By this method, antibody production may be speeded up. It is unnecessary here, however, to go into the details of the actual technical procedures and measurements used in the production of serum. These methods are constantly changed and can be learned only by taking part in the process in a well equipped producing laboratory.

Serum must be standardized before it can be marketed. This has been a very difficult matter and a number of suggestions have been made. Flexner and Jobling ${ }^{39}$ first attempted standardization by opsonin contents. Complement fixation has been recommended by some writers, but the usual method at the present time is that of agglutination. As a general rule Flexner states that the polyvalent sera are ready for use when they agglutinate the normal and para-types in dilutions of 1:1500 or 1:2000. Such sera should also agglutinate intermediate strains in dilutions of 1:200 and upward.

Administration of Serum.-The most important single consideration in serum treatment of meningitis is the early recognition of the case and avoidance of delay in starting the specific treatment. Failure of serum treatment can probably in most cases be referred

${ }^{39}$ Flexner and Jobling, Jour. Exper. Med., 10, 1908. 
to delay. Lumbar puncture, therefore, should be done as early as the first suspicion is aroused, and, if meningococei are found, the injection of serum should follow as rapidly as possible.

It is probably best, in the long run, to inject serum immediately upon obtaining a turbid fluid in a case in which the clinical suspicion points strongly to epidemic cerebrospinal meningitis.

The technique of serum injection consists in first withdrawing spinal fluid by tapping the eanal with a sterile needle and allowing the fluid to flow out, of course without suction, holding a centrifuge tube directly over the butt of the needle. The flow is allowed to continue until the drops begin to come quite slowly, that is, a drop every ten or twenty seconds, and then the serum is injected, either by gravity or with a syringe through the same needle. It is important that the serum at body temperature shall enter the canal very slowly, and, for this reason, the gravity method is advised. A gravity arrangement can easily be constructed by attaching about eighteen inches of catheter tubing, sterilized, to the end of the needle with a small sterile funnel at the other end. The withdrawal of large amounts of fluid suddenly sometimes eauses trouble, the patient breathing rapidly, and showing symptoms of threatened collapse, but this is rare, and a little judgment in withdrawing fluid which has been under considerable pressure too rapidly will usually guard against accident. During the injection of the serum, the patient should be carefully watched, since occasionally alarming symptoms may arise from too rapid increase of internal pressure. The physician must be on the alert for such symptoms and immediately discontinue the injection for the time being. Flexner ${ }^{40}$ advises 10 minutes for the injection of the entire amount of serum used.

The dosage of serum should, to some extent, depend upon the amount of fluid withdrawn, and the amount injected should usually be less by several centimeters than the amount withdrawn. The average dose for an adult should be about 30 c.c., though more may be given when large quantities of fluid have been withdrawn, and when the case is very carefully watehed by an experienced man. Sophian ${ }^{41}$ has recommended controlling the withdrawal of spinal fluid and the injection of the serum by blood pressure measurements.

\footnotetext{
40 Flexner, Bulletin, Rock. Inst. for Med. Res., 1917.

${ }^{41}$ Sophian, Epidemic Cerebrospinal Meningitis, St. Louis, 1913, p. 54.
} 
Sudden drops of blood pressure in either case, should lead to caution, and perhaps interruption of the procedure.

Repetition of the injections is as important as the initial injection, as far as cure is concerned. The action of the serum may be compared somewhat to the action of anti-serum in a Pfeiffer reaction in a guinea pig's peritoneum. Thus, probably some bacteriolysis and considerable stimulation to phagocytosis by opsonic action may result. The spinal fluid shows changes in that the numbers of organisms are diminished and the extracellular ones disappear. Purulent spinal fluid may become clearer and may even become entirely free from organisms or leucocytes. There is probably a certain amount of poison neutralization by the serum. Repetitions of the doses, therefore, must be governed to some extent by the progress of the case, clinical conditions pointing to changes in the meningeal inflammation, and observation of the spinal fluid. One injection a day for three to six days usually controls a case that is treated with sufficient promptness.

In addition to the intraspinous administration, it is wise to inject from 30 to 50 c.c. intravenously, preceding this by withdrawal of blood for blood culture, and being governed as to repetition by subsequent blood culture control.

Everyone dealing with meningitis during an epidemic must remember that occasionally meningococcus septicemia cases occur which never show meningeal infection. We have mentioned these in another place, but believe that more attention should be given them, since they are very apt to be fatal, either without meningitis, or subsequently followed by a violent meningeal involvement. Such cases displayed the clinical picture of a general severe septic infection with usually a profuse eruption in which petechial spots not unlike those of typhus fever may cover the entire body. There is an irregular septic temperature with a high leucocytosis and sometimes delirium. Blood culture will diagnose these cases and vigorous intravenous serum treatment would be indicated.

Effects of Serum Treatment.-The mortality of meningitis in the days before serum was used varied between 60 and 80 per cent. Higher mortalities have been noted in individual epidemics. The average for many different parts of the world fluctuates about 70 per cent. Since serum treatment was begun just before the year 1906, a great many statistical studies have been made which are of course subject to great error, owing to the fact that the treated 
cases must have included a great many treated too late to permit any kind of treatment to be effective. Flexner's statisties of eases under serum treatment show that, of 1211 eases, analyzed, those treated between the first and third day (199) showed a mortality of 18.1 per cent, those treated between the fourth and seventh day (346) showed a mortality of 27.2 per cent, and those treated later than the seventh day (666) showed a mortality of 36.5 per cent. The following table taken from a paper by Flexner, published by the Rockefeller Institute as a Bulletin in 1917, gives similar comparative mortality statistics reported by different observers.

COMPARATIVE MORTALITY REPORTED BY VARIOUS OBSERVERS 42

\begin{tabular}{l|r|r|r|r|r|r}
\hline \multicolumn{1}{c|}{ Treatment Begun. } & $\begin{array}{c}\text { Flexner, } \\
\text { Per Cent. }\end{array}$ & $\begin{array}{r}\text { Netter, } \\
\text { Per Cent. }\end{array}$ & $\begin{array}{r}\text { Dopter, } \\
\text { Per Cent. }\end{array}$ & $\begin{array}{c}\text { Christo- } \\
\text { manos, } \\
\text { Per Cent. }\end{array}$ & $\begin{array}{c}\text { Levy, } \\
\text { Per Cent. }\end{array}$ & $\begin{array}{c}\text { Flack, } \\
\text { Per Cent. }\end{array}$ \\
\hline Before third day....... & 18.1 & 7.1 & 8.2 & 13.0 & 13.2 & 9.09 \\
From fourth to seventh day & 27.2 & 11.1 & 14.4 & 25.9 & 20.4 & \\
After seventh day....... & 36.5 & 23.5 & 24.1 & 47.0 & 28.6 & 50.00 \\
\hline
\end{tabular}

Altogether, then, it seems quite clear that serum treatment has made a tremendous differenee in the mortality from this otherwise so fatal disease.

\section{OTHER GRAM-NEGATIVE MICROCOCCI WHICH IMST BE DIFFERENTIATED FROM MENINGOCOCCI}

Micrococci Catarrhalis.-This organism is more particularly described in a separate section below. It is one of the common organisms which may confuse carrier examinations because of its frequent presence in the nose and throat of normal human beings. Its fermentation reactions are described in the table from Elser and Huntoon ${ }^{43}$ also given below. The organisms are slightly larger than meningocoeei, grow readily on the simplest media, the colonies are larger, thicker, opaque and white, and have a tendency to dryness quite distinct from the dew-drop like appearance of meningococeus colonies, and do not agglutinate in specifie serum. They show a

${ }^{42}$ Flexner, Bulletin of the Rock. Inst. for Med. Res., 1917.

${ }^{43}$ Elser and Huntoon, Jour. Med. Res., 20, 1909, 371. 
tendeney to spontancous agglutination in salt solution and in horse serum.

Micrococcus Flavus.-A common inhabitant of the normal throat which grows easily on simple media and may be grown at room temperature at or below $25^{\circ}$, temperatures at which the meningococcus eeases to grow. It is always important to expose suspected cultures at room temperature in the dark. A yellowish pigment is formed by the eultures, but often does not come out for several days. The very young colonies may very closely resemble meningococcus colonies, but are easily distinguished in sub-cultures, especially when the growth is forty-eight or more hours old. There are a considerable number of chromogenic organisms closely related to the Flavus. Elser and Huntoon describe three chief chromogenic groups, one of which has a greenish gray or greenish yellow appearance by reflected light, with an opacity that approximates the meningococcus colony. The second group is the one most closely resembling Lingelsheim's M. Flavus.

Their third chromogenic group also makes a greenish yellow pigment, and, except for this, is very similar to the M. catarrhalis. A curious fact has been noted by Elser and Huntoon, namely, that some of their chromogenic organisms were easily distinguishable from meningococcus colonies at first isolation, but in the course of artificial cultivation they lost some of their original characters and their power to produce pigment, and gradually approximate the appearance of meningococcus, at least as it appears in strains long isolated.

The Flavus group gives pernaps most diffieulty in meningococcus carrier examinations, since the young colonies of these organisms may look very much like the young meningococcus cultures. The chief points of differentiation, apart from sugar fermentation, which confirm them, are: The fact that Flavus eolonies will grow out at room temperature on slants of simple media; that they begin to form pigment after forty-eight hours or so, and that they will agglutinate in normal horse serum in dilutions often as high as 1 to 50 , and in the meningococeus sera, indiscriminately, often as high as 1 to 100. Meningococci do not agglutinate in salt solution spontaneously, unless under the conditions mentioned above as noted by Hine, and under the influence of abnormal acid or alkalin reactions. In all series in which the specific agghtination test is used for the determination of a meningococcus, therefore, normal horse serum 
tubes should be set up in dilutions ranging up to 1 to 50 at least, in order to exclude organisms of the Flavus type.

Micrococcus Pharyngis Siccus.-This organism deseribed by Lingelsheim $^{44}$ is a Gram-negative diplococcus often found in the normal pharynx, and is recognized by its dry, crenated colonies on simple media. According to Elser and Huntoon, it sediments spontaneously in salt solution and this, together with the fact that the colonies are formed in a way almost impossible to break up, makes it easy, according to these observers, to distinguish it from the meningococeus. It is a little more difficult to distinguish from M. Catarrhalis, but can be easily separated from this organism by means of the fermentation test.

Diplococcus Crassus.-This is the organism that Kutscher ${ }^{45}$ described as probably identical with the so-called "Jaeger" variety of meningococcus. According to Kutscher and von Lingelsheim, this organism has a tendency to wander from the normal pharynx into the central nervous system in cases of meningitis of other origin. Lingelsheim claims to have found it in the fluids of traumatic meningitis and tubereulous meningitis. It has the peculiarity that the cultures are said to be composed of Gram-negative and Grampositive organisms some of the cocci retaining the Gram-stain. According to Von Lingelsheim, the colonies are smaller and more rompact than meningococcus colonies, and it will grow at room temperature.

FERMENTATION REACTIONS OF GRAM NEGATIVE DIPLOCOCCI

\begin{tabular}{c|r|c|c|c|c|c|c}
\hline \multicolumn{1}{c}{ Strains Tested. } & Strains & $\begin{array}{c}\text { Dex- } \\
\text { trose. }\end{array}$ & $\begin{array}{c}\text { Mal- } \\
\text { tose. }\end{array}$ & $\begin{array}{c}\text { Levu- } \\
\text { lose. }\end{array}$ & $\begin{array}{c}\text { Sacch- } \\
\text { arose. }\end{array}$ & $\begin{array}{c}\text { Lac- } \\
\text { tose. }\end{array}$ & $\begin{array}{c}\text { Gal- } \\
\text { actose. }\end{array}$ \\
\hline Meningococcus............. & 200 & + & + & 0 & 0 & 0 & 0 \\
Pseudomeningococcus........ & 6 & + & + & 0 & 0 & 0 & 0 \\
Gonococcus............... & 15 & + & 0 & 0 & 0 & 0 & 0 \\
Mierococcus catarrhalis....... & 64 & 0 & 0 & 0 & 0 & 0 & 0 \\
Micrococcus pharyngis siccus... & 2 & + & + & + & + & 0 & 0 \\
Chromogenic group I........ & 28 & + & + & + & + & 0 & 0 \\
Chromogenic group II........ & 11 & + & + & + & 0 & 0 & 0 \\
Chromogenic group III....... & 9 & + & + & 0 & 0 & 0 & 0 \\
Jaeger meningococcus, Kral.... & 1 & + & + & + & + & + & + \\
Diplococcus crassus, Kral...... & 1 & + & + & + & + & + & + \\
\end{tabular}

Table taken from Elser and Huntoon, loc. cit.

${ }^{44}$ Lingelsheim, Klin. Jahrb., 15, 1906.

${ }^{45}$ Kutscher, Kolle and Wassermann, Vol. 4, Second Edition, p. 603. 
Diplococcus Mucosus.-A form of Gram-negative diplococeus, the description of which we take from Elser and Huntoon. Its eolonies may resemble meningococcus eolonies on aseitic agar. They are said to differ from the meningoeoceus colonies by being more mueoid, resembling the colonies of the $\mathrm{B}$. capsulatus mueosus. The colonies have a tendeney to confluence and the above writers say that the luxurianee of its growth on serum free media helps to tell it from the meningoeoccus. It also grows at room temperature, and shows capsules with capsule stains.

\section{EPIDEMIOLOGICAL PROBLEMS IN MENINGITIS}

Although sporadic eases of meningitis may oceur in a eommunity for a considerable number of years after an epidemic is over, and the disease may, therefore, be regarded as, to some extent, endemie in all crowded eities, it is ehiefly important for its epidemic oeeurrenee. In 1905 a reeognizably deseribed epidemic oeeurred in Switzerland. Since that time epidemies have been reasonably frequent, especially at times of war, when they appeared among armies in barracks and mobilization camps. During the many continental campaigns in the time of Napoleon, outbreaks oceurred in the various armies, and secondary epidemies among the eivilian population in many eities followed in the train of these. In Ameriea, a number of limited epidemies oecurred in the States along the Eastern sea board during the early half of the 19th eentury, and in these eivilian epidemies the disease particularly selected children and young adults. Extensive civilian epidemies occurred in different parts of the world in the early years of the 20th eentury. In 1903 the disease appeared in East Prussia and spread to other parts of Germany from there. In 1904 and 1905 it appeared in New York City, and the adjacent country, on an extensive seale causing the death of 3,000 people and altogether about 7,000 cases in New York City alone. In the summer following its appearance in New York, it extended to Canada, and in the years sinee then, small outbreaks and sporadic cases have appeared with gradually decreasing frequency all through the more thickly populated parts of North Ameriea.

During the late war, there was little meningitis among the European armies until an extensive outbreak oecurred among the 
Canadian troops on Salisbury Plains. The increase of cases among these troops took place in February, 1915, and after this time the disease began to appear in the overseas expeditionary troops, although among these no extensive epidemic occurred at any time. Among American troops the disease was most prevalent in 1917 and 1918 among the troops gathered in the cantonments in the United States. According to the epidemiological studies of Vaughan and Palmer" ${ }^{46}$ in the camps in 1918, meningitis showed "of all diseases, the greatest excess over the disease in civilian communities." Vaughan estimates that meningitis was forty-times as frequent in the Army as in civilian life. The highest morbidity occurred at Camp Jackson where it reached a rate of 25.1 per thousand, and a death rate of 7.05. Next to pneumonia, it was the most serious disease occurring in the camps. In the Surgeon General's report for 1918, the disease stood fifth as a eause of death for enlisted men in the United States and Europe, with a case mortality of 34.8 per cent.

During the Army epidemies there was a definite racial difference in that, according to Surgeon General Ireland's report, the admission rate for colored troops in the United States was 2.44, whereas, it was only 1.2 for whites, and the death rate for colored troops was 0.98 against 0.41 for whites.

As to seasonal prevalence, meningitis usually develops in the late autumn and winter months, the largest case rates being coincident with the cold and wet weather, when a basic eatarrhal inflammation of the upper respiratory tract, creates favorable conditions for the lodgment of organisms and for the general distribution of saliva by coughing, sneezing and spitting. During the war, the highest admission rates in the United States usually fell into the months of November, December and January, which is the time of the highest case rate for most respiratory epidemies. Yet cases will usually trail along through the hot weather.

Meningitis epidemies, therefore, will oceur chiefly in the temporate zones during the winter months at times when, during the prevalence of generalized respiratory disease, large numbers of people are crowded in close quarters, under conditions which render attention to hygiene and sanitation difficult. The reasons for this will become apparent as we study the manner of transmission.

The meningococeus does not survive easily outside the body, and

${ }^{46}$ Vaughan and Palmer, Jour. Lab. and Clin. Med., 4, 1919, 647. 
rapidly dies out in dust, or even in sputum, under conditions of low temperature, deficient moisture and competition with other microorganisms. As far as we know, it is not carried by any of the domestic animals and, therefore, the origin of infection lies in the secretions of cases and of carriers. The microorganisms are found in the noses and throats of the sick, sometimes in the secre- tions of the eye where a meningococcus conjunctivitis may exist. With the secretions of these mucous membranes it reaches the outer world. The meningococci may be present in cases for a long time after convalescence, and, as we know, they are present in a considerable percentage of people who have never had meningitis, with whose secretions the organisms may be constantly transferred to contacts. Transmission probably occurs by close contact between carrier or case and new host, through the nasopharynx, where the organisms lodge and multiply. From this lodgment they pass into the meninges, either directly along the lymphatic channels to the base of the skull, or perhaps indirectly by way of the general circulation. The former route is the one favored by most observers. However, during the early periods of the war, a few cases were reported by British clinicians, in which blood culture was positive before symptoms of meningitis had developed, and during these army epidemics, we, as well as others, saw occasional cases of general meningococcus septicemia which died without ever developing meningitis. Incidentally, it may be stated that these cases develop a generalized rash which, in some of its stages, is not unlike that of typhus fever. The writer recalls a case in which he made a probable diagnosis of typhus fever which in the light of subsequent experience seems to him to have possibly been a case of meningococcus septicemia. Positive blood culture in such cases will differentiate between the two diseases. Herrick ${ }^{47}$ studied this phase of the problem at Camp Jackson in 1918 with great care, and came to the conclusion that in 50 per cent of the cases early blood culture will reveal general infection before elinical evidences of meningeal invasion are apparent. This observation is of the greatest importance, indicating the desirability of early blood culture work for doubtful diagnosis, and also throwing light upon the wisdom of intravenous serum therapy combined with the intraspinous injections.

Infection of a healthy individual from a case is of very rare

${ }^{4 \tau}$ IIerrick; Arch. Inter. Med., 21, 1918, 541. 
vecurence, and sine there are in every epidemic a very much larger number of carriers than of cases, the carrier is the chicf epidemiological problem. As far as infection of new individuals from patients is concerned, experience during the New York epidemic showed only two or three eases of infection of doctors and nurses, although the hospitals in the city were constantly handling consider-: able numbers of the sick. In our epidemiological experience with the army, the actual tracing of one case to a preceding one was also relatively very rare. This does not mean that the greatest precautions should not be taken to prevent such transmission in hospitals and sick room. But the epidemiological emphasis lies with the carrier.

This rareness of transmission from cases to the healthy is in our opinion due to the peculiar conditions of susceptibility that prevail in relation to meningitis, and the fact that the number of people with whom the sick come in contact is relatively small.

The susceptibility of man to meningitis is a curious one, different in some aspects from susceptibility relations to almost all other infections, except perhaps poliomyelitis. In the general population there seems to be a great variability in individual susceptibility to infection with the meningococcus, a variation which can be traced to no determinable cause. Unlike pneumonia, temporary fluctuations in well being, produced by respiratory disease, malnutrition, exposure to cold, etc., do not seem to play a determining rôle. The disease indiscriminately picks out individuals here and there, some of them in the most robust health, strong and hardy, while sparing associates who may be feeble and run down. It is obvious that some individuals are normally resistant and will not come down, in spite of considerable exposure, while others are delicately susceptible. The difference may possibly have been produced fortuitously by the fact that some individuals may have been carriers at one time or another, and have become, thereby, spontaneously immunized. It is difficult to get at this question experimentally, and there are no serum or other reactions which we can apply at the present time, by which we can discriminate between the susceptible and the nonsusceptible of a community. There is no available method, moreover, by which we can distinguish between virulent and non-virulent strains of meningococei.

The Carrier Problem.-As stated above, the meningococcus carrier probably is the source of infection in most of the cases that 
develop during an epidemic. Earlier carrier work has lost value to a considerable extent, owing to the fact that the criteria of meningococcus identification of which we are now more thoroughly informed were neglected in these early studies. The flora of the nose and throat contains many Gram-negative diplococei, mentioned above under the heading of identification, some of which were mistaken in this earlier work for true meningococci. Micrococcus Catarrhalis, Microcoecus Flavus, and a number of other similar microorganisms probably represent a definite percentage of the earlier statistics. The criteria of meningococcus determination have been discussed in a special section above, and these, in general, were applied in the extensive meningococeus carrier work which was done during the years of the war, especially by British and American bacteriologists. The studies of Bassett-Smith, ${ }^{48}$ Gordon, ${ }^{49}$ Mathers and Herrold, ${ }^{50}$ show that in the American camps under conditions of ordinary life and weather, there may be anywhere from two to five per cent of meningococcus carriers. Mathers and Herrold at the Great Lakes Naval Training Station examined over 15,000 men, finding over 4 per cent to be carriers and between 1 and 2 per cent to be chronic carriers. Their work also showed that the carrier rate is higher among those taking care of cases, and that over 38 per cent of those recovering from the disease may remain carriers during convalescence for variable periods. Contacts showed a carrier rate of 36.7 per cent during a period in which the general carrier rate in the camps (15,000 men examined) was slightly over 4 per cent. The hospital Corps showed a carrier rate of 13.5 per cent. It is natural that there have been many endeavors to establish relationship between new cases and contact with carriers. This line of investigation has not been conclusive, owing to the great difficulties incident to such investigation. The transmission of respiratory organisms may take place during a very brief contact, in conversation, close association in barracks, moving picture shows, public conveyances, sleeping quarters, etc., and the innumerable associations of this kind established by each man in the course of a day, makes it almost impossible to trace them with accuracy. Among the most interesting studies made in this connection are those of

${ }^{48}$ Bassett-Smith, Lancet, 194, 1918, 290.

${ }^{49}$ Gordon, Med. Res. Com., Spee. Rep. Ser., No. 3, London, 1917.

${ }^{50}$ Mathers and Herrold, Jour. Infect. Dis., 22, 1918, 523. 
Glover, ${ }^{51}$ who swabbed the throats of a considerable number of men in overcrowded barrack rooms, in the course of sanitary supervision during which the spacing between beds was among the many precautionary measures taken. It will be seen here that sanitary measures, including the spacing out in sleeping quarters, brought about a very considerable drop in the carrier rate, with coincident diminution of cases of meningitis. Meleney and Ray ${ }^{52}$ traced fourteen out

EFFECTS OF "SPACING OUT" ON "SEVERELY OVER CROWDED" BARRACK-ROOMS *

\begin{tabular}{|c|c|c|c|c|c|}
\hline Unit. & $\begin{array}{c}\text { Date of } \\
\text { First } \\
\text { Swabbing. }\end{array}$ & $\begin{array}{l}\text { Percentage } \\
\text { Carrier } \\
\text { Rate before } \\
\text { Spacing. } \\
\text { Out. }\end{array}$ & $\begin{array}{l}\text { Period } \\
\text { Spaced } \\
\text { Out } \\
\text { Approxi- } \\
\text { mately. }\end{array}$ & $\begin{array}{c}\text { Date of } \\
\text { Second } \\
\text { Swabbing. }\end{array}$ & $\begin{array}{l}\text { Percentage } \\
\text { Carrier } \\
\text { Rate after } \\
\text { Spacing } \\
\text { Out. }\end{array}$ \\
\hline No. $1 \ldots \ldots \ldots \ldots$ & Sept. 29 & 22.0 & 8 weeks & Dec. 6 & 2.0 \\
\hline No. $2 \ldots \ldots \ldots \ldots$ & Oct. 2 & 28.0 & 6 weeks & Nov. 23 & 7.0 \\
\hline One room of No. $2 \ldots$ & Oct. 2 & 38.5 & 6 weeks & Nov. 23 & 4.5 \\
\hline No. $4 \ldots \ldots \ldots \ldots$ & Oct. 26 & 28.0 & 5 weeks & Nov. 30 & 4.5 \\
\hline
\end{tabular}

*Glover's Table

of twenty-four cases which occurred in an American camp to contact with carriers, and found parallelism between the incidence of cases and the rise of the carrier rate. These examples, however, are exceptional and it is relatively rare that a definite relationship of this kind can be established. It is a fact that carrier rates are high in such camps during the cold months, and in connection with the general spread of respiratory disease, and that, at such times, the incidence of the disease increases, and it is absolutely logical to assume that the new cases arise by contact with the carriers. It is of importance, however, to recognize that the tracing of the case to the individual from whom he has been infected, at times when high carrier rates exist, is not often possible, and comprehension of this must considerably influence the measures instituted for the control of the epidemic.

It is our belief that the extensive carrier examinations made during epidemics and the wholesale isolation of carriers, were relatively ineffective during the war, and that it is far better to bend all one's energies upon a general improvement of the respiratory

${ }^{51}$ Glover, Jour. Hyg., 17, 1918, 367.

${ }^{52}$ Meleney and Ray, Jour. Inf. Dis., 23, 1918, 317. 
sickrate with the reduction of carriers, focusing the carricr $\mathrm{cx}$ aminations upon the small epidemiologically determined intimate group from which the case has come, rather than making wholesale carrier examinations of carriers of meningococcus through whole regiments and divisions.

Carrier Determination.-The bacteriological analysis of a carricr is not a simple procedure, and implies the proper control of a great many conditions which necessitate special description.

To obtain the material properly the swab must be taken from high up in the pharynx, behind the soft palate. A general swabbing of the pharynx and throat is not sufficient. The best swabs for this purpose are made by the West tube method, as follows: A cotton swab is fixed on the end of a copper wire, about eighteen centimeters long, and this is inserted in a glass tube, bent upward at the swab end, in such a way as to permit passage upward behind the soft palate. The swab is placed into the tube, both ends plugged with cotton, and is so sterilized. For large scale work it is sufficient to take copper wire swabs, sterilize them in a box, and bend them up carefully with the finger, being careful not to touch the cotton, just before use. Swabbing through the nose has also been practiced, but we do not believe that it is as efficient as the method described above. The swab must be taken with the patient facing the light. A tongue depressor is used, and the swab inserted so as to pass behind the soft palate. The copper wire is then thrust forward so that the swab emerges from the tube and touches the posterior and upper pharyngeal wall: Slow motion to and fro brings the cotton in contact with the sides of the upper pharynx. The swab is then immediately passed over the surface of the plate medium. It is best not to carry the inoculated swab back to the laboratory, but to plate it directly upon removing the material from the patient.

The media employed are various, but for ordinary use a glucosehormone-agar, $P_{\mathbf{H}}$ of 7.4 with addition of 1-10 defibrinated or hemolyzed human or rabbit's blood, is best. The plates thus inoculated should be kept warm and immediately taken to the laboratory where they are incubated. The British prefer trypagar to the hormone agar as the basic medium for such work.

After eighteen to twenty-four hours incubation, the plates are examined and the colonies suspected of being meningocoeci are fished. This is not a matter which ean be taught by book. The colonies are of small, rounded appearance, the recognition of which 
is a matter of judgment. Every bacteriologist confronted with the problem should immediately plant plates of the medium which he is going to use, with spinal fluid or with cultures recently isolated, and familiarize himself with the colonies on this medium, at various stages of growth. In spite of our not inconsiderable experience, we would do this ourselves. Plates that are too thickly covered with colonies are of no use.

On blood medium, the true meningococcus colonies do not produce any change in the blood. They are slightly translucent and look somewhat stringy. They are homologous and slightly glistening. They are practically indistinguishable from young colonies of $\mathbf{M}$. Flavus which is also a Gram-negative diplococcus, but which is easily distinguished subsequently by the fact that it will grow at and below $25^{\circ}$ C., produces a yellow pigment on further cultivation, and has a tendency to agglutinate spontaneously in normal horse serum.

Having ringed the suspicious colonies, some of them are now picked and stained by Gram. Our habit is to take up part of a colony which we strongly suspect of being meningococcus, plant part of it immediately, and from the rest make the Gram stain, since plating after taking material for Gram stain may increase the chances for contamination. This point, however, is not of very great importance.

The suspicious colonies are now planted upon blood agar slants, the medium being made up as for the plates. These slants should not be used directly from the ice-box, but should be warmed. Dried media must not be used.

If two tubes can be inoculated, one should be kept at room temperature. Growth in the incubator for about twelve hours gives sufficient growth for further identification.

Gram stains are now made from the tubes.

If the morphological and staining properties are proper, agglutination is carried out.

Diagnostic Agglutination.-The organisms are emulsificd in isotonic salt solution. Agglutination may be done against type sera, or against polyvalent serum. When large numbers of cases are examined, as in times of epidemic, it is best first to agglutinate in polyvalent serum, preferably one in which the agglutinin titre for a great many different meningococcus strains has been controlled. As a general rule, the polyvalent sera used in this country will show specific agglutinations for practically all meningococcus 
strains in dilutions of 1 to 100. For this reason, 1 to 100 was the dilution adopted for such work in the American Army laboratories.

One-half c.c. of the bacterial emulsion is mixed with 0.5 c.c. of the polyvalent serum. A control of a similar amount of the culture suspension in 1 to 50 normal horse serum must always be made to guard against spontaneous agglutination. It is always well also, to run a tube with a known meningococeus.

Since meningococci show a certain amount of resistance to agglutination, Gordon has recommended the method in general use during the war, that is, placing the tubes in a water bath at $50^{\circ}$ for twelve to eighteen hours. Evaporation must be guarded against.

Olitsky has recommended saving time by growing the organisms in normal horse serum broth directly from the colonies on the plate, discarding all those that grow in a granular form.

We, ourselves, have used a method that we have not published because we have not had a chance to use it on material on a large scale, which depends upon the thread reaction. Dilutions of polyvalent sera are made in broth tubes, so that the final concentration is 1 to 100 . The colony is directly inoculated into this, and in the case of true meningococci grow in granular form. A similar inoeulation is made in control tubes of 1 to 50 normal horse serum. These procedures sometimes save time.

Nicolle in France makes his diagnosis by another method, in that he uses, instead of a dilution of serum, the serum in concentrated form, checking it up with the bacterial suspension, and noting the speed of agglutination. In such concentrations he often gets rapid agglutination of true meningococci in the concentrated serum.

After the preliminary identification has been made, typing of the meningocococus may be desirable by agglutination against type serum.

Carriers occasionally will develop meningitis some time after they have been recognized as carriers, showing that the organisms may remain in the nasopharynx for some time, without penetrating, insusceptible individuals. We know of a number of cases in which this seems to have occurred. Gordon mentions a number of cases in which "meningismus" developed among carriers, namely, carriers complained of severe headache, pains in the back of the neck, slight fever up to $102^{\circ}$, and slight Kernig. One ease he mentions had been in contact for a few hours with a case of cerebro-spinal meningitis, which died within twenty-four hours. It was swabbed 
and found to be negative. A month later, he went to a military hospital with the symptoms above enumerated, and the swab from his nasopharynx revealed meningococcus, but curiously not of the same type as that of the case with which he had been in contact. He mentions other similar cases.

An interesting point comes up in regard to whether or not the presence of meningococcus in the nasopharynx leads, in itself, to a catarrhal inflammation. Flügge in the early days of meningococcus carrier investigation believed that the carrier state was usually associated with local inflammations. Gordon, however, finds that, in general, there was no nasopharyngeal catarrh associated with the earrier state. But he also finds that cases with tonsiliar or pharyngeal inflammations were much more difficult to free from meningococcus than others. The same he says is true of convalescents, a point which indicates the importance of bringing the mucous membranes to normal in connection with the cure of carriers.

The question of how we are to deal with meningococcus carriers in times of epidemic is a difficult one. Local treatment of the nose and throat has been tried with antimeningococeus serum, with astringent solutions, and various disinfectants, without encouraging result. Sprays of Dichloramine $\mathrm{T}$ and other chlorin preparations have been tried, also, in our opinion, without marked success. During the war the British constructed rooms of about one thousand cubic feet capacity, along the sides of which steam pipes were placed at about the height of a man's waist, and jets were fitted to them in such a way that a spray of steam could be ejected. These sprays were connected with bottles containing 1 to 2 per cent chloramine, or 0.5 per cent zinc sulphate. The carriers were put into these inhaling rooms for from fifteen to twenty minutes a day, during which they inhaled the medicated spray through their nostrils. By this method, they claimed to clear up all but the most resistant cases of so-called pure meningococcus carriers. In general, it may be said that cases in which only a few meningococcus colonies form on the plates, clear up rather readily, and that the others in which the cultures are almost pure are extremely resistant to any kind of treatment. Our own impression from some experience with the various methods would lead us to conclude that the best treatment for a carrier would be careful attention to the nasopharynx, with an attempt to bring it back to normal as far as the condition of the mucous membrane is concerned, correction of tonsillar, adenoid, 
or septum defects, cessation from smoking or other habits that irritate the mucous membrane, and outdoor life, especially in the sunlight, with sea baths if available. Specific antiseptic treatment in general seems to us to have been a failure as far as the handling of large numbers of men is concerned.

The virulence of meningococei is a matter that is very difficult to determine because of our inability to produce invasive infections with regularity in any known laboratory animal. So far, extensive attempts to determine the virulence of standardized injections into mice have not succeeded. Death in most laboratory animals is due to the toxic effects and not by invasion. This is a very unfortunate circumstance, inasmuch as our failure to be able to distinguish between virulent and non-virulent strains makes it impossible for us to tell a dangerous carrier from one who is relatively harmless, as we can in the case of diphtheria carriers. All we can do at the present time is to regard as dangerous any. carrier whose meningococcus agglutinates in a polyvalent serum. Those with.strains which neither agglutinate nor absorb with the polyvalent serum at our disposal, if culturally they seem to be true meningococei, must be regarded as suspicious. 


\section{CHAPTER XXVII}

\section{DIPLOCOCCUS GONORRHEE (GONOCOCCUS), MICROCOCCUS CAT'ARRHALIS, AND OTHER GRAM-NEGATIVE COCCI}

\section{DIPLOCOCCUS GONORRHCE}

NeIsser, ${ }^{1}$ in 1879 , described diplococci which he had found regularly in the purulent secretions of acute cases of urethritis and vaginitis and in the acute conjunctivitis of the new-born. His researches were purely morphological, as were the numerous ccnfirmatory investigations which rapidly followed his announcement.

Cultivation of this diplococcus, now usually spoken of as gonococcus, was not definitely successful until 1885, when Bumm ${ }^{2}$ obtained growth upon tubes of coagulated human blood serum. Bumm was not only able to keep the organisms alive by transplantation in pure culture, but produced the disease by inoculation of his cultures upon the healthy urethra.

Morphology and Staining.-The gonococcus is usually seen in the diplococcus form, the pairs being characteristically flattened along the surfaces facing each other. This gives the cocci a peculiar coffee-bean or biscuit shape. The size of the diploforms is about 1.6 micra in the long diameter, about 0.8 micron in width. Stained directly in gonorrheal pus from acute cases, the microorganisms are found both intra- and extra-

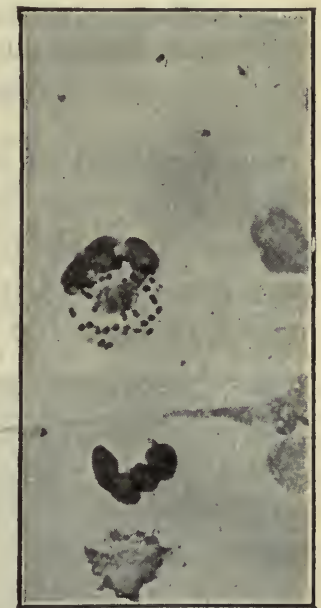

Fig. 57.-GoNoRrheal PUS From URETHRA, Showing The Cocci WITHIN A LEUCOCYTE. cellularly, a large number of them crowded characteristically within the leucocytes. They are never found within the nucleus. The phagocytosis which produces this picture has been shown by Scholtz ${ }^{3}$

${ }^{1}$ Neisser, Cent. f. d. meil. Wiss., 1879.

${ }^{2}$ Bumm, "Beitr. z. Kenntniss des Gonococeus,' Wiesbarden, 1885.

${ }^{3}$ Scholtz, Arch. f. Dermat., 1899. 
and others to take place in the free secretions, not in the depth of the tissues. The intracellular position, which is of considerable diagnostic importance, is lost to a great extent in secretions from chronic cases. In smears made from pure cultures the arrangement in groups of two may often be less marked than in pus, clusters of eight or more being common.

The gonococcus is non-motile and does not form spores. It is easily stained with aqueous anilin dyes. Methylene-blue alone, or eosin followed by methylene-blue, give good results. An excellent picture is obtained with the Pappenheim-Saathof stain consisting of

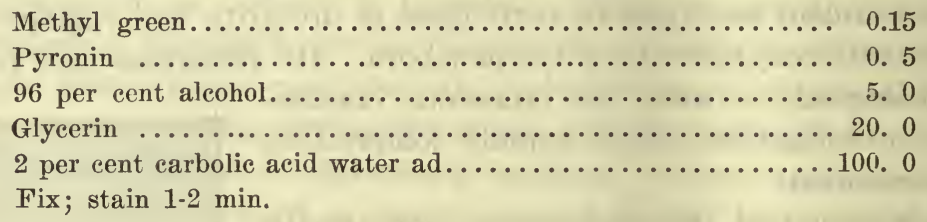

Gram's method of staining, however, is the only one of differential value, gonococcus being Gram negative. The Gram stain applied to pus from the male urethra, while not absolutely reliable, is, for practical purposes, sufficiently so to make a diagnosis. In exudates from the vagina or from the eye the morphological picture is not so reliable,

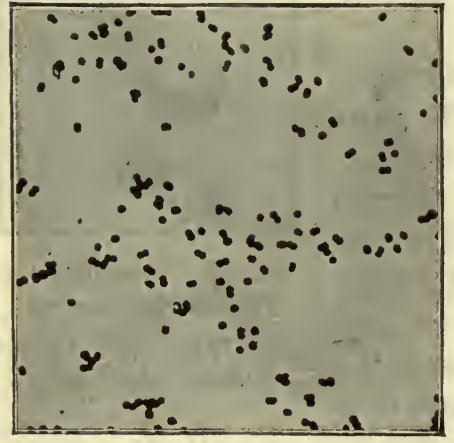

Fig. 58.-Gonococcus. Smear from pure culture. owing to the frequent presence in these regions of other Gram-negative cocci. The great scarcity of gonococci in very chronic discharges necessitates thorough cultural investigation; negative morphological examination in such cases can not be regardea as conclusive. ${ }^{4}$

Cultivation.-The gonococcus is delicate and difficult to cultivate. Bumm $^{5}$ obtained his first growths upon human blood serum which had been heated to partial coagulation.

The medium most commonly used at the present day was introduced by Wertheim, ${ }^{6}$ and consists of a mixture of two or three parts of

\footnotetext{
${ }^{4}$ Heiman, Medical Record, 1896.

' Bumm, Deut. med. Woch., 1885.

'Wertheim, Arch. f. Gynäkol., 1892.
} 
meat infusion-agar with one part of uncoagulated human ascitic fluid, hydrocele fluid, or blood serum. The agar is melted and cooled to $45^{\circ}$ before the serum is added. The mixture may then be slanted in the test tube or poured into a Petri plate. One per cent of glucose may be added. Cultures in fluid media may be obtained by similar additions of serum to meat-infusion-pepton-broth. Whole rabbit's blood added to agar, or the swine-serum-nutrose medium of Wassermann ${ }^{7}$ may occasionally be used with success.

Plates may be made by smearing for enrichment a drop of blood from the finger over the surface of agar in the manner of Pfeiffer's method for influenza-bacillus cultivation. Inoculations from gonorrheal material are best made by surface smearing upon plates, since the gonococcus grows best in the presence of free oxygen. Growth becomes more luxuriant after prolonged cultivation upon artificial media. The most favorable reaction of media is neutrality or slight acidity.

When the gonococcus has been successfully cultivated from pus upon media without serum additions, the success has probably been due to the substances carried over in the pus. The ease of cultivation differs considerably with different strains of gonococci. Some grow very heavily after first isolation, but the majority show a very delicate growth even on rich ascitic glucose agar. After several generations of growth on artificial media, however, the organism develops with increasing ease and on simpler media. It may eventually be cultivated on plain agar, especially when this is made of veal infusion. Recently a medium upon which gonococci after first cultivation can be grown with ease has been recommended by Edward B. Vedder. ${ }^{8}$ The medium consists of a 1.5 to 1.75 per cent agar made with beef infusion neutral to phenolphthalein, and after clearing, 1 per cent of corn starch added. The corn starch is best added after grinding with a little agar to avoid clumps, this then being poured into the bulk of the agar and thoroughly mixed. The medium should be sterilized at not over $15 \mathrm{lbs}$. pressure to avoid changes in the starch. Recently we have isolated several strains of gonococci which grew very heavily on simple media without ascitic fluid in the second culture generation.

The gonococcus will develop sparsely under anaërobic conditions

"Wassermann, Berl. klin. Woch., 1897.

(Fifteen c.c. swine-serum, 35 c.c. of water, 3 c.c. glycerin, with two per cent nutrose. The nutrose is dissolved by boiling and the solution sterilized. This is then added to agar, in equal parts, and used in plates.)

${ }^{8}$ Vedder, Jour. Infec. Dis., May 15, 1915, xvi, 385. 
but has marked preference for aërobiosis. The optimum temperature is $37.5^{\circ} \mathrm{C}$. Growth ceases above $38.5^{\circ}$ and below $30^{\circ}$.

Upon suitable media colonies appear as extremely delicate, grayish, opalescent spots, at the end of twenty-four hours. The separate colonies do not tend to confluence and have slightly undulated margins. Touched with a platinum loop their consistency is found to be slimy. In fluid media, growth takes place chiefly at the surface.

Types of the Gonococcus. - As in the case of so many other organisms, it has been found that the Gram-negative diplococei which cause gonorrheal infections are not a single type, but must be regarded as representing a group, including many closely related, but antigenically differentiable subgroups. Such subgrouping of species formerly regarded as homogeneous has been a very natural development of the more intensive study of serum reactions, incident to diagnostic agglutination and complement fixation, and to the control of specific therapy. Torrey ${ }^{9}$ and Teague and Torrey ${ }^{10}$ in 1907 showed that the gonococcus group is not homologous, but that agglutination and agglutinin absorption divided this group into at least three separate subtypes. Agglutinin absorption seemed to show more types than complement fixation,. a matter which we ourselves would rather expect at the present day because of the almost universal experience that complement fixation reactions are not as strictly specific as agglutination. Torrey's claims have been to some extent misquoted in the past ten years, in that he himself never supposed that his ten strains represented the entire gonococcus group. In 1910 Watabiki ${ }^{11}$ made a similar study of the gonococcus group, and studying a limited number of strains, confirmed the heterogeneous nature of the group by referring to them as "comparative but not distinctive differences between individual strains." In $1915^{\circ}$ Louise Pearce ${ }^{12}$ made a comparison between gonococci isolated from adult males and from the vulvovaginitis of children. She came to the conclusion that strains from these two sources constituted fairly definite serologically distinct groups, that at least there was a relative distinction between the two types. This, in view of the important sanitary problem involved in these infections in children, would be of great importance if confirmed. We will refer to it again below. More

'Torrey, Jour. Med. Res., 16, 1907, 329.

${ }^{10}$ T'eague and Torrey, Jour. Med. Res., 17, 1907, 223.

${ }^{11}$ Watabiki, Jour. Infec. Dis., 1910, 7, 159.

${ }^{12}$ Pearce, Jour. Med. Res., 21, 1915, 289. 
recently, Hermanies ${ }^{13}$ studied 85 gonococcus strains from various sources, using for cultivation the partial tension method of Wade W. Oliver. He concluded that gonococci fall into distinct types, with little relationships to each other. Agglutinins produced by one type cannot be absorbed by strains of other types, and his 85 strains fell into six distinct groups. At the time of the present writing, Torrey is again working on the same subject, since the conflicting evidence of these researches and earlier work shows clearly that the subject cannot be regarded as closed. The work Torrey is doing with Buckell is not yet completed, and it would, therefore, be rash to make a final statement concerning his opinion, but, since he has devoted a considerable amount of time and energy to this subject, and since his bacteriological judgment is unusually sound, we quote as follows from advanced information he has courteously furnished us in a personal communication. Torrey's impressions at the present time are that it is not possible to demonstrate definite groups, such as the three fixed pneumococcus types, or even groupings, comparable to that established for the meningococcus and discussed in another section. A study of 50 strains from many sources and from different parts of the world by agglutination and agglutinin absorption, has shown that there are certain generalized strains which possess antigenic properties common to a considerable part of the "whole" species. On the other hand, there are a large number of variants among recently isolated strains which show no serological relationship to one another as far as specific agglutination is concerned, and which quite often show some specific relationship to one or more of the specific strains. These-variants overlap and show such intergradations that it is not possible to subdivide them into definite groups. It has also been noticeable in his work that some of the original strains which he studied as long as fourteen years ago, and which at that time seemed to be unrelated to each other, are now beginning to show some specific relationships. All the older strains show greater relationships than do similar groups of recently isolated ones. Torrey has not been able to confirm the contentions of Louise Pearce that there is a recognizable distinction between vulvovaginitis strains from children and those from adult males.

Recognition of the Gonococcus.-Speaking of sugar fermentations, Torrey has come to the conclusion that, taken together with typical colony formation, morphology, staining reactions and absence of growth on ordinary laboratory media, sugar fermentations form the most

${ }^{13}$ IIermanies, Jour. Infee. Dis., 28, 1921, 133. 
reliable methods of recognition of the gonococcus. Of 60 strains examined by him, all except one fermented glucose, and none of them fermented maltose, thus differentiating from meningococcus. The one exception was an old strain, all the recent ones fermenting true to type.

A gonococcus can, therefore, be recognized bacteriologically by the Gram-negative diplococcus form, the typical colony formation on ascitic agar, its failure to grow on simpler media or at room temperature, its ability to split dextrose and failure to split maltose.

Resistance.-Recent cultures of gonococcus, if not transplanted, usually die out within five of six days at incubator temperature. At room temperature they die more rapidly.

The resistance of the gonococcus to light and heat is slight. A temperature of $41^{\circ}$ to $42^{\circ}$ kills it after a brief exposure. Complete drying destroys it in a short time. Incompletely dried, however, and protected from light (gonorrheal pus) it may live, on sheets and clothing, for as long as eighteen to twenty-four hours. ${ }^{14}$

It is easily killed by most disinfectant solutions ${ }^{15}$ in high dilution and seems to be almost specifically sensitive to the various silver salts, a fact of therapeutic importance.

Pathogenicity.-Gonorrheal infection occurs spontaneously only in man. True gonorrheal urethritis has never been experimentally produced in animals. In human beings, apart from the infection in the male and female genital tracts, and in the conjunctivæ, the gonococcus may produce cystitis, proctitis, and stomatitis. It may enter the circulation, giving rise to septicemia, ${ }^{16}$ to endocarditis and arthritis. Isolated cases of gonorrheal periostitis and osteomyelitis have been reported. ${ }^{17}$

The acute infections of the genito-urinary passages are often followed by prolonged chronic infection, which, though quiescent, may for many years be a source of social danger. In children, especially females, the infection is not rare, and may as ume epidemic characters, traveling from bed to bed in institutions. Such hospital epidemics can be stopped only by the most rigid isolation. This is more specifically dealt with in the paragraphs on Sanitary Considerations, below.

While inoculation of animals has never resulted in active proliferation of the gonococcus upon the new host, local necrosis, suppuration,

${ }^{14}$ Heiman, Medical Record, 1896.

1.5 Schaeffer und Steinschneider, Kong. Deut. Dermat. Geselis., Breslau, 1894.

${ }^{16}$ Review of cases of Gon. Septicemia, Faure-Beaulieu, Thesis, Paris, 1906.

${ }^{17}$ Ullmann, Wien, med. Presse, 1900. 
and temporary systemic reactions have been produced by subcutaneous and intraperitoneal inoculation. A toxin has been isolated by Nikolaysen ${ }^{18}$ by extraction from the bacterial bodies with distilled water or sodium hydrate solutions. It was found to be resistant to a temperature of $120^{\circ}$ and to remain potent after complete drying. The same author found that the isolated toxin and dead cultures were fully as toxic for animals as living cultures, 0.01 gram killing a white mouse.

Specific injury to the nervous system by injections of gonococcus toxin has been reported by Moltschanoff. ${ }^{19}$

The secretion of a true soluble toxin by the gonococcus, asserted by Christmas, ${ }^{20}$ is denied by Wassermann, ${ }^{21}$ Nikolaysen, ${ }^{22}$ and others. Christmas, ${ }^{23}$ and, more recently, Torrey, ${ }^{24}$ have reported successful active immunization of animals by repeated injections of whole bacteria. Torrey and others apparently have successfully treated human cases by injections of the serum of immunized animals.

Antibodies to Gonococcus.-Patients infected with gonococci seem to produce antibodies against the organisms. Although in the ordinary gonorrheal urethritis, or vaginitis, it is relatively simple to make the diagnosis by finding gonococci in the discharges, diagnosis may be difficult in cases of gonorrheal rheumatism, or endocarditis, when isolation of the bacteria fails or when the connection between the local venereal disease and the general condition is obscure. Various serological diagnostic methods have been attempted, and of recent years the complement-fixation test has been found to be very useful. The method has been especially developed by Archibald McNeil, at the New York Department of Health. It consists in making a polyvalent antigen, using the 10 Torrey strains which are kept in stock transplants on glucose ascitic agar. It has been found that the best medium for antigen production is an agar made of "bob veal." For the production of antigen, stock cultures are transplanted on "bob veal" agar, without salt, glucose or ascitic fluid, the reaction carefully adjusted to an acidity of 0.1 per cent to 0.2 per cent. Twenty-four hour growths on this medium are scraped off and emulsified in neutral sterile distilled water. The emulsion is autolyzed one hour in a water bath at $56^{\circ}$ and heated

${ }^{18}$ Nikolaysen, Cent. f. Bakt., 1897.

${ }^{19}$ Moltschanoff, Münch. med. Woch., 1899.

${ }^{20}$ Christmas, Ann. de l'Inst. Pasteur, 1897.

${ }^{21}$ Wassermann, Zeit. f. Hyg., xxvii, 1897.

${ }^{22}$ Nikolaysen, Fort. d. Med., xxi, 1897.

${ }^{23}$ Christmas, loc eit.

${ }^{24}$ ' Correy, Jour. Amer. Merl. Assn., xlvi, 1906. 
one hour at $80^{\circ} \mathrm{C}$. It is then filtered through a sterile Berkefeld filter. The filtrate is aseptically bottled and sterilized three days at $56^{\circ}$, half an hour each day. It is then made isotonic and is ready for titration.

Vaccine therapy in systemic gonorrheal infection has been tried and is promising, though as yet unconvincing. The vaccine, if possible, should be made with the organism isolated from the patient, for reasons described above. Passive immunization with the serum of gonococcusimmune animals has also been attempted, but records on it at present are not sufficiently complete to permit definite judgment.

\section{SANITARY CONSIDERATIONS IN CONNECTION WITH GONOCOCCUS INFECTION}

Of the three prevalent venereal infections, those caused by the gonococcus are probably the most common. For more exhaustive statistical studies of the prevalence of these diseases the reader is referred to such books as those of Pusey, Morrow, and the larger text-books of hygiene, such as that of Rosenau. Although it has been well known that gonorrhea was extremely common, the astonishing prevalence among young men of draft age was revealed during the late war when the figures of the Surgeon General ${ }^{25}$ show that about 5.6 per cent of the men who came into the military service were infected with a veneral disease. Considering that these diseases, in their early detectable acute stages, do not last very long, that many cases still apparent to a slight degree must surely be missed in physical examinations of large numbers of men, it seems to indicate that the estimate by many authorities of a prevalence of venereal disease in civilian life as high as 10 per cent may be very near the truth: The percentage of gonorrhea to other venereal diseases is probably pretty well exemplified by the percentage of these diseases for the entire army during 1918, during which, according to the Surgeon General's report, there were 44,213 cases of syphilis, 16,173 cases of chancroid, and 167,475 cases of gonococcus infection, a total of 227,861 cases of venereal disease. The rate for syphilis was 17.56 per 1000 , for chancroid 6.42 , for gonorrhea 66.50 .

One of the great dangers in connection with gonorrheal infection has been the relative indifference of the public to these diseases. In the past, there has been a remarkable lack of appreciation of the seriousness of the infection, which actually, in its economic and sociological impor-

${ }^{25}$ Rep. of Surgeon General, U. S. A., 1919, Vol. 1, p. 956. 
tance, is equal to, if not more serious, than syphilis. The gonococcus is primarily infectious for the genital organs, but may also infect the eye, and in its secondary manifestations cause disease of the prostate, epidydimis and bladder of the male, of the fallopian tubes and ovaries of the female. In both it may and often does cause sterility. Invading the blood stream, it may cause endocarditis, and not infrequently an acute and subacute arthritis which is characterized by its frequent localization in single joints or the bursæ about joints, and cause periarticular inflammation. It may, but rarely does attack other organs.

A most important consideration is the difficulty of complete cure. A male who has contracted gonorrhea may seem to be completely cured, but if a posterior urethritis has occurred, the organisms may remain viable and capable of infecting others for a great many years. Individuals who, therefore, seem to have been cured for years, may still cause infection upon marriage, a fact which is the most frequent cause of gynecological lesions in women. It goes without saying that even the most careful bacteriological examination of such individuals may often fail to reveal the gonococci, even though they may be present.

Infection with gonococcus is almost invariably by sexual contact, though the organism may remain viable on wearing apparel, bed clothing, towels, hands, etc., for brief periods, especially if protected from light and drying, and others may be infected in this way. The danger of self-infection of eyes by people who are suffering from an acute discharge is a frequent one, and physicians and nurses, especially, are liable to such infection.

Gonorrheal infection of the eye is one of the most serious infections that can occur in this organ. Ophthalmia of the new born may be due to other organisms, but is almost invariably caused by the gonococcus. It is acquired by the child in the course of delivery, from the secretions of the mother, and if not attended to, may lead to blindness. The importance of this infection may be estimated from the following figures quoted by Rosenau from Kerr, who states that in the United States and Canada 23.9 per cent of 351 admissions to schools for the blind in 1910 the blindness was the result of gonorrheal infection.

Fortunately, the method introduced by Credé has, to a very large extent, done away with this accident. Credé, many years ago, introduced the method of instilling a 2 per cent silver nitrate solution into the conjunctival saes of every child at birth. Since his time other silver salts have been in use, the most popular ones at the present time being protargol, 5 per cent solution, and argyrol, 20 per cent solution, which 
are dropped into the eye at birth. It is extremely important that this should be done properly and the entire conjunctival sac bathed in the fluid. The method is so important that it is regarded as a matter of very serious and inexcusable omission, if, under any circumstances, in dealing with any class of the population, the physician managing a childbirth fails to carry out this measure as soon as feasible after birth.

Another very important gonorrheal problem is the vulvovangitis which occurs in children. In our own experience, this infection has occurred most often in connection with the children's wards in hospitals. The condition has, however, been observed in schools and in small family groups where children were infected by sleeping in the same beds with adults. In hospitals the disease may spread in epidemics, and from bed to bed, with an ease that is astonishing when one considers the delicate life of the gonococcus outside the body. It has often been extre nely difficult to stop such bed to bed infection, in spite of the most rigid precautions. Epidemics are so difficult to arrest, and the consequences for the child so grave from many points of view, that it has become the custom in all well-managed hospitals to delay the admission of female children to the general children's ward until vaginal smears have been made and examined for gonococci. It is in our opinion extremely important that when such smears are made, they should be taken not only from the visible secretion, but should be taken from high up in the vagina through a small Kelly speculum, with good illumination. When there is danger of spread and a case has been inadvertently admitted, only the greatest care in avoiding indirect contact from bed to bed can stop it. As a matter of routine in children's wards, there should be separate thermometers, unless all thermometers are very carefully sterilized, thermometers should be kept in weak carbolic solutions and washed with alcohol before use; the sterilization of diapers and towels should be attended to, nurses handling cases with discharge should wear gloves, and there should be no common use of towels and washing utensils. Great attention should be given to the scouring of bath tubs, and bed linen, night clothes, etc., should be sterilized by boiling.

Public Health Management of Venereal Diseases.-During the past ten years there has been a very wholesome increase of interest in venereal disease prevention. There are certain general fundamental principles which apply to all venereal discases equally. In the first place, it is necessary to look upon venereal infections as preventable 
diseases. It has been unfortunate that the sanitary and moral issues have been so closely interwoven in these diseases, that it has been impossible to create the free discussion and spread the information necessary to obtain the cooperation of the public in these matters. Without public education and cooperation, large scale public health results cannot be achieved. In our opinion, one of the most important factors that have prevented earlier progress in the prevention of venereal disease has been the ignorance of the public in regard to these matters. It has been especially wrong that women of the marriageable age have been kept in ignorance about facts concerning these infections, an ignorance which has often left them absolutely at the mercy of chance. Accurate and clear information, free of sensationalism, will do more eventually to reduce the venereal rate than any other single factor.

It is not the function of a book of this kind to go into the very complex problems of general sex education, and the moral issues involved. We will restrict ourselves entirely to the purely sanitary phases of the problem. Chief among these are:

1. Diagnosis.-Education and knowledge of the seriousness of these infections should lead to a gradual attraction of patients, away from quacks, to reliable clinics and physicians. The development of diagnostic clinics by departments of health, the improvement of clinical facilities in large cities, and the better understanding by physicians, as a whole, of the sanitary importance of these relatively simple infections, must lead to more accurate diagnosis and proper-instruction of the patient.

2. Reporting of Venereal Diseases.-In a great many communities at the present time gonorrheal infections, as well as other venereal diseases, are regarded, like other communicable diseases, as subject to report. There are many reasons why such reporting systems will meet with objections, and will for many years be unsatisfactory. This, too, we believe is a matter of education, and the fact that it will fail for the present is no reason why the principle should not be upheld. Eventually we believe it will be accepted as a sensible and necessary step. These diseases are communicable to others during certain stages, and when they are regarded primarily as possibilities for the spread of disease and the public stress is not laid purely on the moral issue, objections to reporting will cease. In our opinion the chief objection that has been raised against the reporting of these diseases is the permanent record, apparent disgrace and perhaps opportunity for blackmail which is opened by the public registration of an individual in this way. 
When we consider, on the other hand, these dangers as balaneed against the danger of the uncontrolled circulation among their fellows of individuals capable of infecting others, there seems very little choice in our minds between the two evils. Moreover, we believe it would be possible to develop a system of reporting whereby the reported individual could have the record destroyed when he could bring a certificate of cure from a responsible clinic or physician. This, we believe, would add a further inducement to proper care and cure. At any rate, we believe that the prompt report of cases, following them up from municipal health bureaus, and prompt destruction of the record when the individual has been cured, will greatly aid in this matter.

3. Hospitalization.-It will probably be impossible to hospitalize all infectious cases of venereal diseases because, unfortunately from the public-health point of view, these patients are not incapacitated during their most infectious stages. We are able to confine a case of smallpox with or without consent, but diseases that in their remote possibilities are responsible for far greater injury and unhappiness, are permitted to walk about and follow their own devices, through the course of their illnesses. The eventual ideal would consist in making physicians responsible for the isolation of cases which came under their care and to hospitalize those who could not be taken care of in their own quarters. Hospitalization in separate hospitals would confer so great a stigma that it would probably be impossibie. It might also be impossible to admit these cases into general hospitals in spite of special arrangements. We do not ourselves believe that compulsory hospitalization could be enforced at the present time. It should be looked upon, however, as an attempt worth making, as soon as education and general public cooperation has reached a point at which success would seem at least not totally out of question. It is our opinion that the sooner the attitude toward these diseases is made one purely of sanitary principles, and the more purely moral factors are allowed to take care of themselves under the influence of increased civilization and sense of community responsibility, the sooner these ends may be accomplished.

While it is of course quite impossible to do justice to as fundamentally important a problem as the sanitary control of venereal diseases in a section of this kind, it has seemed to us of great importance to at least point out to physicians and bacteriologists who may read this book, the enormous responsibility that falls upon them whenever they are in a position to deal with cases of this kind. 
Prophylaxis of Gonorrhea.-As practiced in the United States army stations during the war ${ }^{26}$ this consisted in injecting about 10 c.c. of a 2 per cent protargol into the urethra - enough to thoroughly distend it, with a glass hand syringe, holding it there with the syringe in place, for one-half minute. The procedure is twice repeated. Its success depends very largely upon early application after intercourse. As to general efficiency in regard to gonorrhea we are not in a position as yet to submit reliable statistics.

\section{MICROCOCCUS CATARRHALIS}

Micrococcus catarrhalis is a diplococcus described first by $\mathrm{R}$. Pfeiffer, ${ }^{27}$ who found it in the sputum of patients suffering from catarrhal inflammations of the upper respiratory tract. It was subsequently. carefully studied by Ghon and H. Pfeiffer. ${ }^{28}$ According to these authors the pathogenic significance of the micrococcus is slight, though occasionally it may be regarded as the causative factor in catarrhal inflammations. Its chief claim to attention, however, lies in its similarity to the meningococcus and the gonococcus, from neither of which it can be morphologically distinguished. It is decolorized by Gram's stain, appears often in the diplococcus form, and has a tendency, in exudates, to be located intracellularly. Not unlike the two microorganisms mentioned, too, it shows but slight pathogenicity for animals.

Differentiation from gonococcus is extremely simple in that Micrococcus catarrhalis grows easily on simple culture media and shows none of the fastidious cultural requirements of the gonococcus.

From meningococcus the differentiation is less simple and, because of the presence of both microorganisms in the nose, is of great importance.

Distinction between the two is made entirely upon cultural characteristics and agglutination reactions. Culturally, Micrococcus catarrhalis grows more heavily than meningococcus upon the ordinary culture media. The colonies of Micrococcus catarrhalis are coarsely granular and distinctly white in contradistinction to the finely granular, grayish meningococcus colonies. ${ }^{29}$ Micrococcus catarrhalis will

${ }^{26}$ Bayard Clark, Medical Times, April, 1919.

${ }^{27}$ Flügge, “'J)ie Mikroorg.,', 3i ed., 1896.

${ }^{28}$ Ghon und II. Pfeiffer, Zeit. f. klin. Med., 1902.

${ }^{29}$ Ghom und Pfeiffer, loc, eit. 
develop at temperatures below $20^{\circ} \mathrm{C}$, while meningococcus will not grow at temperatures below $25^{\circ} \mathrm{C} .^{30}$

Dunham, ${ }^{31}$ who has recently made a comparative study of meningococcus and other Gram-negative diplococci from the nose and throat, states that while some of the supposed Micrococcus catarrhalis cultures are easily distinguished from meningococcus simply by the characteristics of their growths upon two-per-cent glucose agar, others offer great difficulties to differentiation. He recommends as a differential medium a mixture of sheep serum and bouillon containing 1 per cent of glucose. Upon this medium all true meningococci produce acid, but no coagulation, within twenty-four hours. Cultures from the nose and throat, however, produce acid and coagulation, or else produce an alkaline reaction.

${ }^{30}$ Weichselbaum, in Kolle und Wassermann, Bd. iii, p. 269.

${ }^{31}$ Dunham, Jour. Inf. Dis., 1907. 


\section{CHAPTER XXVIII}

\section{BACILLUS DIPHTHERIA, BACILLUS HOFFMANNI, AND BACILLUS XEROSIS}

\section{BACILLUS DIPHTHERIÆ⿸厂}

Since 1821, when Bretonneau of Tours published his observations, diphtheria has been an accurately recognized clinical entity. Our knowledge of the disease in the sense of modern bacteriology, however, begins with the first description of Bacillus diphtheriæ by Klebs in 1883 . Klebs ${ }^{1}$ had observed in the pseudomembranes from diphtheritic throats, bacilli which in the light of more recent knowledge we can hardly fail to recognize as the true diphtheria organism. His work, however, was purely morphological and, therefore, inconclusive. One year after this announcement, Loeffler ${ }^{2}$ isolated and cultivated an organism which corresponded in its morphological characters to the one described by Klebs. He obtained it from thirteen clinically unquestioned cases of diphtheria, and, by inoculating it upon the injured mucous surfaces of animals, succeeded in producing lesions which resembled closely the false membranes of the human disease. His failure to find the bacillus in all the cases he examined, his finding it, in one instance, in a normal throat, and his inability to explain to his own satisfaction some of the systemic manifestations of the infection which we now know to be due to the toxin, caused him to frame his conclusions in a tone of the utmost conservatism. The second and third publications of Loeffler, ${ }^{3}$ however, and the inquiry into the nature of the toxins produced by the bacillus, published in 1888 by Roux and Yersin, ${ }^{4}$ eliminated all remaining doubt as to the etiological relationship existing between this organism and the disease.

Innumerable observations, both clinical and bacteriological, by other workers, have, since that time, confirmed the early investigations, and it is to-day a scientific necessity to find the bacillus of Klebs and

${ }^{1}$ Klebs, Verh. d. 2. Kongr. f. inn. Medizin, Wiesbaden, 1883.

${ }^{2}$ Loeffler, Mittheil. a. d. kais. Gesundheitsamt, 1884.

${ }^{3}$ Loeffler, Cent. f. Bakt., 1887 and 1890.

${ }^{4}$ Roux and Yersin, Ann, de l'inst. Pasteur, 1888 and 1889. 
Loeffler in the lesion before a diagnosis of "diphtheria" (an properly be made.

Morphology and Staining.-While Bacillus diphtherix presents certain characteristic appearances which facilitate its recognition, it is, at the same time, subject to a number of morphological variations with all of which it is important to be familiar. These variations are, to a limited extent, dependent upon the age of the culture and upon the constitution of the medium on which it has been grown. These factors, however, do not control the appearance of the organism with any degree of regularity, and any or all of its various forms may occur in one and

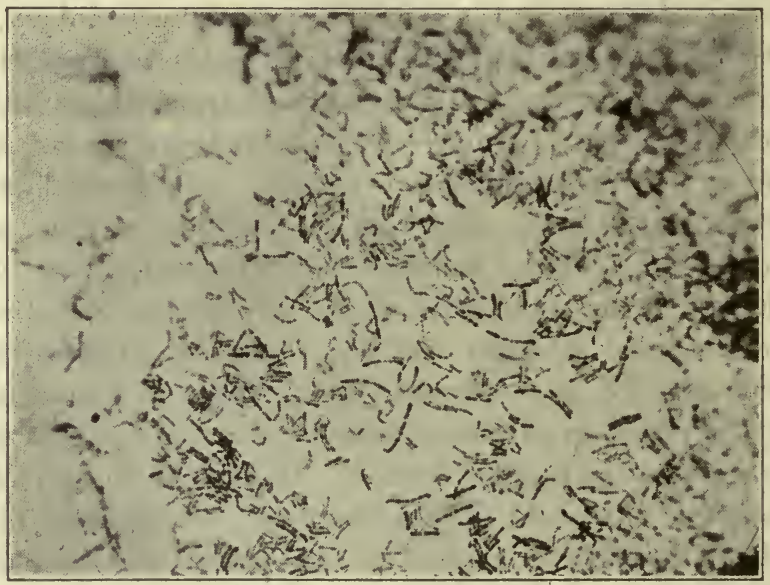

Fig. 59.-Bacillus Diphtheria.

the same culture. It is likely that these different appearances represent stages in the growth and degeneration of the individual bacilli, but there does not seem to be any just reason for believing that, as several observers have stated, there is definite correlation between its microscopic form and its biological characteristics, such as virulence, toxicity, etc.

The bacilli are slender, straight, or slightly curved rods. In length they vary from 1.2 micra to 6.4 micra, in breadth from 0.3 to 1.1 . As seen most frequently when taken from the throat they are about 4 to 5 micra in length. They are rarely of uniform thickness throughout their length, showing club-shaped thickening at one or both ends. Occasionally they may be thickest at the center and taper toward the extremities. When thickened at one end only, a slender wedge-shape 
results. Such forms are usually straight, of smaller size than their neighbors, and are more often stained with great miformity. These are spoken of by Beck ${ }^{5}$ as the "ground type," and assumed, for insufficient reasons, to be the young individuals. Branched forms have been described by some investigators. They are rare and probably to be regarded as abnormal or involution forms due to unfavorable environment.

The organisms stain with the aqueous anilin dyes. A characteristic irregularity of staining which is of great aid in diagnosis is best obtained with Loeffler's "alkalin methylene-blue." (For preparation see section on Staining, p. 115.) Stained with this solution for five to ten minutes many of the bacilli appear traversed by unstained transverse bands which give them a striped or beaded appearance. The longer individuals often have a strong resemblance to short chains of streptococci. Others may appear unevenly granular. In cultures which are about eighteen hours old, many of the bacilli may show deeply stained oval bodies situated most frequently at the ends. These are the socalled "polar" or "Babes-Ernst" bodies. ${ }^{6}$ Special stains have been devised for the demonstration of these appearances. One of these was originated by Neisser, ${ }^{7}$ who claims for it differential value in distinguishing these organisms from pseudodiphtheria and xerosis bacilli.

His method requires two solutions:

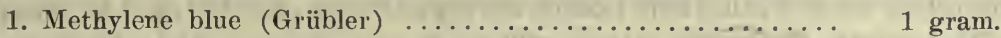

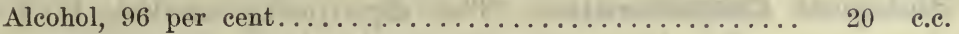

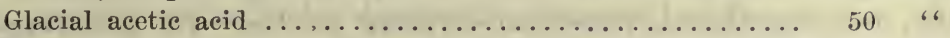

Water ............................. $950 \ldots$

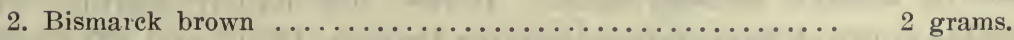

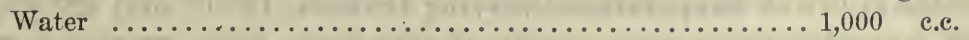

The cover-slip preparation, after having been fixed, is stained with solution No. 1 for one to three seconds. It is then washed in water and immersed for from three to five seconds in solution No. 2. With this stain the bodies of the bacilli appear brown, the polar granules blue.

Our own choice of a stain is toluidin blue. A staining solution can be made up as follows according to the original formula, we believe, of

${ }^{5}$ Beck, in Kolle und Wassermann, ii, p. 773.

'Babes, Zeit. f. Hyg., Bd. v, 1889.

'Neisser, Zeit. f. Hyg., xxiv, 1897. 
Trincas, and quoted from the First Volume of the Kolle and Wasserman Handbook.

Toluidin blue

0.25 gram

Alcohol

5

e.c.

Two per cent acetic acid.

Staining with this for one minute gives very much the same picture as Loeffler's alkalin-methylene-blue, but rather a clearer contrast between polar bodies and cytoplasm, and makes an eminently satisfactory stain.

The significance of the polar bodies is not well understood. Their discoverer, Ernst, regarded them as bodies analogous to the spores of other organisms. The ease with which they are stained, however, and the low temperatures to which the bacteria succumb make this appear very unlikely. A more probable interpretation seems to be that of Escherich ${ }^{8}$ who regards them as chromatic granules.

Stained by Gram's method, the diphtheria bacilli retain the gentianviolet.

In stained smears from the throat or from cultures a characteristic grouping of the bacilli has been observed. They lie usually in small clusters, four or five together, parallel to each other, or at sharp angles. Two organisms may often be seen attached to each other by their corresponding ends while their bodies diverge to form a "V" or " $\mathrm{Y}$ " shape.

Biological Characteristics.-The diphtheria bacillus is a nonmotile, non-flagellated, non-spore-forming aërobe. Its preference for oxygen is marked, but it will grow in anaërobic environment in the presence of suitable carbohydrates. It does not liquefy gelatin. The bacillus grows at temperatures varying between $19^{\circ} \mathrm{C}$. and $42^{\circ} \mathrm{C}$., the most favorable temperature for its development being $37.5^{\circ} \mathrm{C}$. Temperatures above $37.5^{\circ}$, while not entirely stopping its growth, impede the development of its toxin.

Resistance.-The thermal death point of this organism is $58^{\circ} \mathrm{C}$. for ten minutes, according to Welch and Abbott. Boiling kills it in about one minute. Low temperatures, and even freezing, are well borne. Desiccation and exposure to light are not so fatal to this organism as to most of the other pathogenic bacteria. Sternberg ${ }^{9}$ has found it alive in dried bits of the pseudomembrane after fourteen weeks. It is

${ }^{8}$ Escherich, "Aetologie, etc., d. Diphth.," Wien, 1894.

'Sternberg, "Manual Bac., p. 455. 
easily killed by chemical disinfectants in the strengths customarily employed. $\mathrm{H}_{2} \mathrm{O}_{2}$ seems especiallv efficacious in killing the organisms rapidly.

Cultivation.-The diphtheria bacillus grows readily on most of the richer laboratory media. It will grow upon media made of meat extract, but develops more luxuriantly on all those which have a meat infusion as their basis. While it will grow upon both acid and alkalin media, it is sensitive to the extremes of both, the most favorable reaction for its development being probably about 0.5 per cent alkalinity expressed in terms of $\mathrm{N} / 1 \mathrm{NaOH}$. Animal proteins added to the media, in the form of blood serum, ascitic fluid, or even whole blood, increase greatly the rapidity and richness of its growth. Horse serum is supposed by some to be especially favorable. ${ }^{10}$

Loeffler's Medium.-The most widely used medium for the cultivation of this bacillus is the one devised by Loeffler. This consists of:

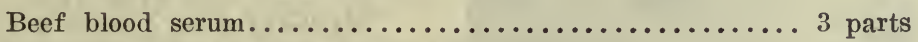

One per cent glucose meat-infusion bouillon........... 1 part

The mixture is coagulated at $70^{\circ} \mathrm{C}$. in slanted tubes and sterilized at low temperatures by the fractional method. Upon this medium the diphtheria bacillus in twelve to twenty-four hours develops minute, grayish-white, glistening colonies. These enlarge rapidly, soon outstripping the usually accompanying streptococci. The medium seems to possess almost selective powers for the bacillus and, for this reason, it is especially valuable for diagnostic purposes.

Meat-infusion Agar.-Upon slightly alkalin meat-infusion agar the bacillus develops readily, though less so than on Loeffler's serum. Organisms which have been on artificial media for one or more generations may grow with speed and luxuriance upon this medium. When planted directly from the human or animal body upon agar, however, growth may occasionally be slow and extremely delicate. Colonies on agar appear within twenty-four to thirty-six hours as small, rather translucent, grayish specks. The appearance of these colonies is quite characteristic and easily recognized by the practiced observer. Surface colonies are irregularly round or oval, showing a dark, heaped-up, nucleus-like center, fringed about by a loose, coarsely granular disk. The edges have a peculiarly irregular, torn appearance which distinguishes them readily from the sharply defined streptococcus colonies.

${ }^{10}$ Michel, Cent. f. Bakt., 1897. 
For these reasons agar is the medium most commonly used for purposes of isolation.

The addition of dextrose 1 per cent, nutrose 2 per cent, or glycerine 6 per cent, renders agar more favrable for rapid growth, but unfits it for the preservation of cultures, the organism dying out more rapidly,

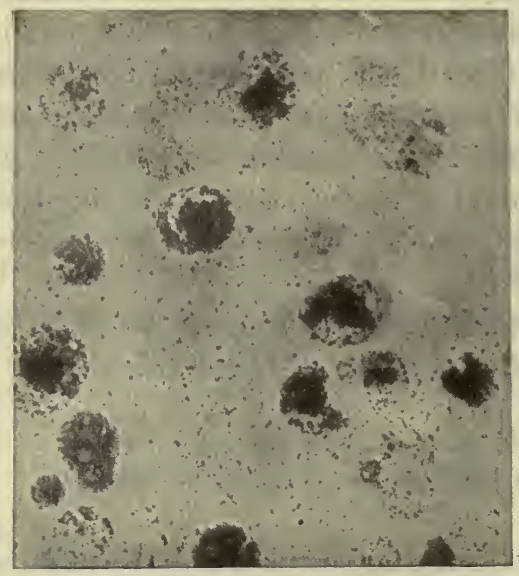

Fig. 60.-Colonies of Diphtherie on Glycerine Agar. probably because of acid formation.

Meat-infusion Broth.-Upon beef or veal broth the diphtheria bacillus grows rapidly, almost invariably forming a pellicle upon the surface,-another expression of its desire for oxygen. The broth remains clear. Broth tubes with such growth, therefore, have a characteristic appearance.

Meat-infusion gelatin is a favorable medium for the Klebs-Loeffler bacillus, but growth takes place slowly because of the low temperature at which this medium must be kept. Gelatin is not fluidified.

Milk is an excellent medium, and for this reason may even occasionally be a vehicle of transmission. There is no coagulation of the milk.

Upon potato, B. diphtheriæ will grow only after neutralization of the acid. It is, at best, however, a poor nutrient medium.

Upon the various pepton solutions the bacillus of diphtheria produces no indol.

Many special media have been recommended for the cultivation of this organism. The most important of these are the modification of Loeffler's serum devised by Beck, ${ }^{11}$ the horse-blood-fibrin cake used by Escherich, and Wassermann's ascitic-fluid-nutrose-agar, called by him "Nasgar." None of these has sufficient advantages over the simpler media, however, to make its substitution desirable.

Isolation.-Cultures are taken from throats upon Loeffler's blood serum. These are permitted to grow at $37.5^{\circ} \mathrm{C}$. for from elghteen to twenty-four hours. At the end of this time about 5 c.c. of bouillon

${ }^{11}$ M. Beck, Kolle und Wasscrmann; Brit. Meti. Jour. 
are poured into the tubes and the growth is gently emulsified in the broth with a platinum loop. 'Two or three loopfuls of this emulsion are then streaked over the surface of glucose agar, serum agar, or nutrose agar. After twenty-four hours' incubation these plates show characteristic colonies which can be easily fished and again transferred to Loeffler tubes or any other suitable medium.

Diagnosis.-Cultures from suspected throats are taken on Loeffler's blood serum medium and incubated at $37.5^{\circ} \mathrm{C}$. for 12 to 18 hours. At the end of this time morphological examination by staining with Loeffler's alkalin methylene blue and by some polar body stain like that of Neisser is carried out. Occasionally direct smears from the throat may show the bacilli, but it is rarely possible to make a satisfactory diagnosis in this way.

Williams has pointed out that in throat cultures in which the diphtheria bacilli are few in number it is of advantage to inoculate a tube of ascitic broth with the mixed culture. The diphtheria bacilli will appear in eighteen to twenty-four hours as a pellicle on the surface. A portion of this pellicle may then be plated on ascitic agar and isolated in pure culture from the colonies. This, however, is not necessary for routine examinations. The important point is to take cultures as early as possible on fresh and moist Loeffler's medium, avoiding the dried tubes so often passed out from old stock at drug store stations. It is important to smear from the actually involved areas, examining the throat with good illumination, and to have the tubes incubated without delay, instead of carrying them about for hours after inoculation. Such cultures, examined by an experienced man should give positive diagnoses in almost all of the actual cases. In the diagnosis of children and in carrier work it is important to take nasal as well as throat cultures.

Pathogenicity.-Bacillus diphtheriæ causes a more or less specific local reaction in mucous membranes, which results in the formation of the so-called "pseudo-membranes." When these are characteristically present, infection with this bacillus should always be suspected. It should be remembered, however, that membranous inflammation is not necessarily present in all cases. We have seen positive cultures in a considerable number of people, especially in relatively insusceptible individuals, in whom the throat showed nothing more than severe congestion and catarrhal inflammation. The consequent disease depends, in part, upon the mechanical disturbance caused by the local inflammation and, in part, upon the systemic poisoning with the toxin which the 
bacilli produce. Although the diphtheria bacillus has been found after death in the spleen and liver, we have no data which would justify the assumption that a true diphtheria-septicemia may occur during life. It is probable that in those cases which Baginsky ${ }^{12}$ has called the septicemic form of diphtheria, bacillus diphtheriæ has merely opened a path by which accompanying streptocncci have gained access to the lymphatics and the blood stream. The most frequent sites of diphtheritic inflammations are the mucous membranes of the throat, larynx, and nose. They have also been found in the ear, upon the mucous membrane of the stomach and the vulva, and upon the conjunctiva and the skin. According to Loeffler, Strelitz, ${ }^{13}$ and others, the bacillus may, by extension from the larynx, give rise to a true diphtheritic bronchopneumonia.

Thus, the localized injury due to the very violent inflammatory reaction elicited by the diptheria bacilli at their point of lodgment which, in the large majority of cases, is in the upper respiratory tract, especially pharynx and tonsils, are the immediately noticeable and visible changes in the disease of human beings. In attempting to make a diagnosis of these by inspection, the clinician should remember that the pseudomembranous inflammation characterized by its adherence to the submucosa and the bleeding points it leaves on being scrapped, is not pathognomonic of the diphtheria bacilli, but means simply a very violent inflammatory reaction and that, while this condition is most commonly caused by the diphtheria bacillis, a great many other severe injuries or inflammations may give rise to very similar appearances. Thus, a very severe streptococcus infection of the throat may simulate a diphtheritic membrane and escharotics or other chemical or mechanical injuries may give rise to simpler lesions. Another point of considerable clinical importance is the fact that diphtheritic inflammation of the throat may often be associated with other concomitant infectious processes. Streptococcus infections superimposed upon a local diphtheria infection, somewhat changes the appearance, both of the local lesion and of the general clinical picture and is apt to lead to a very much greater severity of the illness. Another common experience is to find, at the site of the inflammation, an ulcerative process, smears from which on staining with Gram's gential violet or carbolfuchsin will show a typical picture of Vincent's angina with the fusiform bacilli and the spirilla characteristic of this infection. Owing to a number of mis-

${ }^{12}$ Baginsky, "Lehrbuck d. Kinderkrankheiten."'

${ }^{13}$ Streiitz, Arch. f. Kinderheilk., 1891 
takes we have made, it has become our rule whenever we see a case of Vincent's angina to take cultures on Loeffer's medium as for diphtheria diagnosis, since we have on two or three occasions found almost pure cultures of diphtheria bacilli taken from the depths of such anginal ulcers. The systemic symptoms in diphtheria are not always severe since, as we shall see, adults are apt to be particlly protected by the presence of diphtheria antitoxin in their blood and in consequence their disease may be both locally and systemically so mild that diphtheria might not be seriously considered on purely clinical evidence.

The mechanical injury may actually lead to death. This is particularly due in the care of children in whom extension downward with membrane formation in the larynx may lead to laryngeal obstruction, necessitating tracheotomy or intertubation. While layrngeal obstruction was often the cause of death in children in former years, it has fortunately been rendered much less frequent by antitoxin treatment, by the greater vigilance of doctors and their ability to make an early diagnosis. The responsibility of the physician in this regard is a grave one, since early diagnosis and consequent antitoxin treatment is the only way to prevent such accidents.

The general symptoms of diphtheria are due to the action of the toxin absorbed from the lesion. These consist in increased temperature, rapid pulse, headache, muscular pains, etc. In severe infections there may be erythematous eruptions. Death in diphtheria may occur as a consequence of secondary bronco-pneumonia, but in uncomplicated cases is usually attributed to circulatory failure. According to experiments done by MacCullum ${ }^{14}$ in the perfusion of the heart with diphtheria toxin, the toxin does not seem to act directly upon the heart, in spite of the fact that there seems to be definite evidence that the heart is involved in diphtheria. According to the same investigator, there dose not seem to be sufficient gross or microscopic changes in the hearts of people dead of diphtheria to explain death. It is MacCallum opinion's that Passler and Romberg were probably right in stating that the effect of the poison is "chiefly upon the vasomotor control of the blood vessels." Injuries to other organs are apparent in albuminuria due to acute interstitial nephritis, and cloudy swelling of parenchyma cells in other organs. In view of the marked changes in the suprarenal bodies in guinea pigs treated with diphtheria toxin, these organs have been especially investigated in diphtheria, and though usually little or no

${ }^{14}$ MacCallum, Textbook of Pathology. 
change has been found, MacCallum states that "the changes in the adrenals are likely to be more intense than in most of the other organs," showing hemorrhages and cellular degeneration. This may have direct bearing on the abnormal fall in blood pressure. There is a marked action on the part of the diphtheria toxin upon nerve tissues, both neurons and cells. This results in post-diphtheritic paralyses which occur particularly in cases that have been insufficiently or not at all treated with antitoxin.. The paralyses are particularly frequent in the areas supplied by the cranial nerves and among the most common forms are paralysis of the muscles of the soft palate of the larynx and the ocular muscles. Paralysis, however, may also appear in other parts of the body. We have seen several cases where a sore throat was not suspected of being diphtheria until late paralysis had occurred.

For the usual laboratory animals the diphtheria bacillus is highly pathogenic. Dogs, cats, fowl, rabbits, and guinea-pigs are susceptible. Rats and mice are resistant. False membranes, analogous to those found in human beings, have been produced in many animals, but only when inoculation had been preceded by mechanical injury of the mucosa. Small quantities ( 0.5 to 1 c.c.) of a virulent broth culture, given subcutaneously to a guinea-pig, may produce the greatest symptoms and within six to eight hours the animal may show signs of great discomfort. Death occurs usually within thirty-six to seventy-two hours. Upon autopsy the point of inoculation is soggy and serosanguineous exudate; neighboring lymph-nodes are edematous. Lungs, liver, spleen, and kidneys are congested. There may be pleuritic and peritoneal exudates. Pathognomonic is a severe congestion of both suprarenal bodies. The gastric ulceration recently described by Rosenau and Anderson ${ }^{15}$ may occur, but are by no means regularly found (two out of fifty in our series). ${ }^{16}$

Determination of Virulence.-When diphtheria or diphtheria-like bacilli are isolated from the throats of patients not showing typical clinical diphtheria, or from healthy individuals suspected of being carriers, it is important to determine whether these organisms are toxin producers. The usual criterion is their virulence for guinea-pigs. Two c.c. of a forty-eight-hour broth or ascitic broth culture are injected subcutaneously into a normal guinea-pig. This dose will kill the pig in three to five days if the culture is virulent. A control injection should

${ }^{15}$ Roscnau and Anderson, Jour. Inf. Dis., iv, 1907.

${ }^{16}$ Zinsser, Journ. Med. Res., xvii, 1907. 
always be made into another pig of the same weight, which has received an injection of antitoxin (at least 250 units) twelve to twenty-four hours previously. Recently Neisser has suggested that the intracutaneous injection of the suspected bacilli may be used for the determination of virulence. This has the advantage of economy, as several tests can be carried out on the same pig. The method as applied by Zingher and Soletsky ${ }^{17}$ has been to use the following modification of Neisser's method: Two guinea-pigs of about $250 \mathrm{gr}$. are used for the test. The abdominal wall is prepared by shaving or plucking out the hair. A twenty-four-hour pure culture on Loeffler's medium is emulsified in 20 c.c. of normal salt solution and 0.15 c.c. of this suspension is injected intracutaneously at a corresponding site into each of the two guineapigs. One of these animals is given at the same time an intracardial injection of about 250 units of antitoxin, or preferably is prepared by an intraperitoneal injection of antitoxin twenty-four hours before the tests are made. Six cultures may be tested in this way on two animals. Virulent strains produce a definitely circumscribed local infiltrated lesion, which shows superficial necrosis in two to three days. In the control pig the skin remains normal. This method, in the hands of Zingher, gives results parallel to those obtained with the subciutaneous tests.

Diphtheria Toxin. ${ }^{18}$-Animals and man infected with B. diphtheriæ show evidences of severe systemic disturbances and even organic degenerations, while the microorganism itself can be found in the local lesion only. This fact led even the earliest observers to suspect that, in part at least, the harmful results of such an infection were attributable to a soluble and diffusible poison elaborated by the bacillus. The actual existence of such a poison or toxin was definitely proved by Roux and Yersin ${ }^{19}$ in 1889 . They demonstrated that broth cultures in which B. diphtheriæ had been grown for varying periods would remain toxic for guinea-pigs after the organisms themselves had been removed from the culture fluid by filtration through a Chamberland filter.

Methods of Production of Diphtheria Toxin.-While toxin can be produced with almost all of the virulent diphtheria bacilli, there is great variation in the speed and degree of production, dependent upon the strain of organisms employed and upon the ingredients and reac-

\footnotetext{
${ }^{17}$ Zingher and Soletsky, Jour. Inf. Dis., 1916, xvii, 54. .

${ }^{18}$ Loeffler, Cent. f. Bakt., 1887.

${ }^{19}$ Roux and Yersin, loc. eti.
} 
tion of the medium upon which they are grown. Most laboratories possess one or several strains of bacilli which are empirically known to be especially potent in this respect. One of the most extensively used, not only in this country but in Europe as well, is the strain known as "Culture Americana," or "Park-Williams Bacillus No. 8," an organism isolated by Dr. Anna Williams of the New York Department of Health in 1894. Throughout more than ten years of cultivation this bacillus has retained its great power of toxin production.

Because of the severity of cases of diphtheria in which the diphtheria bacilli were associated with streptococci, many observers were led to believe that the presence of streptococci tended to increase the toxinproducing power of B. diphtheriæ. Experiments by Hilbert, ${ }^{20}$ Theobald Smith, ${ }^{21}$ and others seem to have given support to this view.

The medium most frequently employed for the production of toxin is a beef-infusion broth. There are minor differences of opinion as to the most favorable constitution of this medium for the production of toxin. All agree, however, in recognizing the importance of pepton, without which, according to Madsen, ${ }^{22}$ no satisfactory toxin has yet been produced. This is added in proportions of from 1 to 2 per cent. The presence of sugars in the medium is not desirable in that it leads to acid production; L. Martin ${ }^{23}$ removes the sugars from the meat by fermentation with yeast. Smith ${ }^{24}$ accomplishes the same purpose with B. coli. According to Park and Williams, ${ }^{25}$ however, this is superfluous, the quantity of sugar present in ordinary butcher's meat not being sufficient to exert unfavorable influence.

Experience has shown that a primary alkalin reaction offers the most favorable conditions for toxin production. In all cultures of B. diphtheriæ in non-sugar free broth, there is, at first, a production of acid and, while this continues, there is, as Spronk ${ }^{26}$ has shown, little or no evidence of toxin elaboration. Park and Williams, ${ }^{27}$ in an inquiry into the question of reaction, came to the conclusion that the best results are obtained with a broth to which, after neutralization to

${ }^{20}$ Hilbert, Zeit. f. Hyg., xxix, 1898.

${ }^{21}$ Smith, Medical Rec., May, 1896.

${ }^{22}$ Madsen, Krausund Levaditi, "Handbuch d. Technic,"' etc., 1907.

${ }^{23}$ L. Martin, Ann. de l'inst. Pasteur, 1897.

${ }^{24}$ Th. Smith, Jour. Exp. Med., iv, 1899.

${ }^{25}$ Park and Williams, Jour. Exp. Med., 1897.

${ }^{26}$ Spronk, Ann. de 1 'inst. Pasteur, 1895.

${ }^{27}$ Parls and Williams, Jour. Exp. Med., 1897. 
litmus, $\mathrm{N} / 1 \mathrm{NaOH}$ is added in an amount of 7 c.c. to the liter. In such a medium the largest yield of toxin is obtained after about five to eight days' growth at a temperature of $37.5^{\circ} \mathrm{C}$.

Free access of oxygen to the culture medium during the growth of the organisms has been found to be of great importance. Roux obtained this by passing a stream of oxygen through the bouillon. The supply is quite sufficient for practical purposes, however, if the medium is distributed in thin layers in large-necked Erlenmeyer flasks.

Chemical Nature and Physical Properties of Diphtheria Toxin.-The chemical composition of diphtheria toxin is not known. Brieger and Frankel, ${ }^{28}$ by repeated precipitation with alcohol, succeeded in extracting from toxic bouillon a white, water-soluble powder which possessed most of the poisonous properties of the broth itself. This, in solution, gave many of the useful protein reactions, but differed from proteins in failing to coagulate when boiled and in not giving precipitates when treated with magnesium sulphate, sodium sulphate, or nitric acid. It was believed by them to be closely related to the albumoses, bodies representing intermediate phases in the peptonization of albumins. Similar results have been obtained by Wassermann and Proskauer, ${ }^{29}$ Brieger and Boer, ${ }^{30}$ and others. Uschinsky, ${ }^{31}$ on the other hand, has disputed the protein nature of toxins in general, having produced diphtheria toxin by growing the organism upon a medium entirely free from albuminous bodies. Uschinsky believes that the protein reactions observed by others may be due to ingredients of the precipitates other than the toxin. It is not impossible, however, that the organisms may have produced protein substances by synthesis from the simpler substances in Uschinsky's medium. The production of toxin from such a medium, therefore, is not a conclusive argument against the protein nature of toxins. Accurate chemical isolation and analysis of diphtheria toxin have not yet been accomplished.

Diphtheria toxin is destroyed, ${ }^{32}$ when in the fluid form, by temperatures of $58^{\circ}$ to $60^{\circ} \mathrm{C}$. In the dry state, it resists a temperature of $70^{\circ} \mathrm{C}$. and over, without change. Light and free access of air produce rapid deterioration. Sealed, protected from light, and kept at almost freezing

${ }^{28}$ Brieger und Frankel, Berl. klin. Woch., xi_xii, 1889.

${ }^{29}$ Wasscrmann und Proslaur, Deut. med. Woch., 1891, p. 585.

${ }^{30}$ Brieger und Bocr, Deut. med. Woch., 1896, p. 783.

${ }^{31}$ Úschinsky, Cent. f. Bakt., xxi, 1897.

${ }^{32}$ Roux et Yersin, loc. eit. 
point, the toxin remains stable for long periods. Electrical currents passed through toxic broth have little or no effect upon it.

Epidemiology of Diphtheria. ${ }^{33}$ - There is no disease in which sanitary control can accomplish so much as in diphtheria because we are furnished in this instance not only with accurate and simple methods of bacteriological diagnosis, of cases and carriers, but we have available a specific susceptibility test by means of which we can pick out the susceptibles in any group, as well as methods of prophylactic protection which allow a choice between a speedy and a slow procedure, both of them of proven value. In this disease, sanitary measures have made tremendous strides and greatly reduced both morbidity and death rate since the introduction of bacteriological methods. Newsholm ${ }^{34}$ has studied death rates in diphtheria, and found that in earlier years, great epidemics of diphtheria used to spread through the great cities. There was such an epidemic in London in 1874 . In 1872 he states that the death rate from diphtheria and croup in Boston was 35 per 100,000, but in 1881, it was close to 218 per 100,000 . Epidemic waves seem to have recurred at five- and ten-year intervals, but even in the interepidemic years in cities generally, the death rate seems to have ranged anywhere from 20 to 60 per 100,000 . It was, and is always endemic all over the world, is somewhat more prevalent in colder climates where upper respiratory inflammations are more common, and, for some unknown reason, has been relatively more common in rural than in urban communities. Quite naturally, the disease has always been particularly a school disease among children. From studies by Schick, ${ }^{35}$ $\mathrm{Hahn},{ }^{36}$ and others, it appears that the new-born child is endowed with a certain amount of diphtheria antitoxin by the mother, probably through the placenta, to some degree perhaps transmitted through the colostrum. This relative immunity fades at the end of the first year of life and is gradually reacquired so that the most susceptible years are between one and perhaps nine or ten years of age. In the army, with an age group ranging from twenty to thirty years, it was found, during the recent war, that about 10 per cent of the personnel was Schick positive or, therefore, susceptible. For more accurate susceptibility statistics we must await more extensive measurements and statistical studies of Schick tests.

${ }^{33}$ See Zinsser, Nelson's Loose-Leaf Medicine, 9, 205.

${ }^{34}$ Newsholm, Epidemic Diphtheria, London, 1898.

${ }^{35}$ Schick, Ueber Diphtherimmunitat., Wiesbarlen, 1910.

${ }^{36}$ Iahn, Dent, meil. Woch., 38, 1912, 1366. 
The striking distance of diphtheria is not very large, but the organism is relatively resistant to the ordinary influences of exterior corporal circumstances, and may live for considerable lengths of time in mucus or saliva deposited on heating utensils, playthings, pencils, handkerchiefs, etc. In dried bits of membrane the bacilli may live for many weeks as Loeffler has shown.

Diphtheria is transmitted from one individual to another directly or indirectly by contact or droplet infection-as in coughing, etc. It has been found that individuals may retain virulent diphtheria bacilli in nose and throat for long periods after recovery from the disease. These are the so-called "diphtheria carriers."

The problem of diphtheria carriers has become one of considerable importance and has been given special prominence of recent years by the studies of Von Scholly, Moss, and Nuttall and Graham Smith. Anderson, Goldberger and Hachtel ${ }^{37}$ studied 4,039 healthy people in the city of Detroit, and found that 0.928 per cent harbored bacilli identical morphologically with the Klebs-Loeffler bacillus. This figure is rather lower than those of some other investigators, but would indicate, as the writers stated, that there were from 5000 to 6000 diphtheria carriers in the city of Detroit.

Of 19 cultures isolated from 19 of the carriers, only 2 were virulent, which would indicate that only 0.097 per cent of the people examined carried organisms capable of producing disease. An interesting further point is that the bacillus Hoffmanni was present in at least 41.9 per cent of over 2000 individuals examined, and that 47 cultures, morphologically identified as Bacillus Hoffmanni, were avirulent. This would confirm the impression gained, we believe, by most experienced laboratory workers that a true Hoffmanni can be distinguished with considerable certainty from a Klebs-Loeffler bacillus by morphological examination alone, and that its significance is probably that of a frequently present saprophyte of the throat and pharynx. The studies of Goldberger, Williams and Hachtel also indicate that in examining for diphtheria carriers it is best not to confine oneself either to the nose or throat, but that cultures should be taken from both places in every case.

Carriers naturally increase in crowded communities in the course of cold weather, when nasopharyngeal catarrhs are common, and, we have seen as high as 17 per cent carriers in a military unit in which diphtheria

${ }^{37}$ Goldberger, Williams and Hachtel, Bull. No. 101, of the Hygienic Laboratories, of the U. S. Public Health Service. 
and other respiratory diseases were prevalent soon after they had to be crowded together during Transatlantic transportation. The carrier state in kitchen personnel is particularly important, and attention should be paid to these groups in a community whenever the source of infection is being traced. Indirect and direct contact with carriers is probably the most common method by which the disease is kept going in modern communities. Less important, though still of some significance, is food transmission and milk epidemics have not been uncommon. Also, these will mean infection of the milk by a milk handler, who is a carrier or suffering from a mild diphtheria. However, in one instance diphtheria bacilli were isolated from the inflamed udders of a cow.

The carrier problem thus becomes the most important epidemiological feature. The carriers may either be temporary or chronic. Convalescent diphtheria cases usually get rid of their bacilli spontaneously within two, three, or four weeks. Healthy individuals exposed to cases or carriers as a rule do not keep their organisms more than either a few days or a few weeks, depending perhaps to some extent upon the condition of the mucous membranes. A small percentage remain chronic carriers and, in isolated instances, it has seemed almost impossible to free these individuals of their diphtheria bacilli. They are, however, such definite menaces that prolonged isolation and vigorous attempts at cure must be made for the protection of the community. In all such cases, virulence tests should be made, since prolonged isolation implies so much interference with the life of the affected individual that it would be quite improper to confine a person unless we were sure that the organisms carried by him were capable of transmitting the disease.

Many different methods have been employed for the freeing of carriers. Not a single one of them, however, has been permanently successful. Practically all the ordinary throat antiseptics, peroxide of hydrogen, permanganate of potassium, iodin and glycerin, weak formaldehyd, various hypochlorite solutions have been used, sorre of them with occasional success, but without regularity in any of them. Acriflavine and other dyes have been applied and implantation of staphylococci or some of the acidophilic bacteria upon the throat in the hope of driving out the diphtheria bacilli by bacterial competition have been tried and have usually failed. The spraying of the throat with pyocyanase was in vogue a few years ago, but cannot be said to have brought encouraging results. It is probable that quite the most impor- 
tant thing is to correct pathological conditions of the nose and throat by the correction of a deviated septum or the removal of tonsils and adenoids, whenever necessary. Added to this, sunshine, cleanliness and the cure of chronic catarrhal inflammation are probably more important than any kind of antiseptic treatment.

The detection of carriers can easily be carried out on a large scale by a relatively small force of bacteriologists. It requires a large supply of sterile swabs and Loeffler's medium, and wholesale cultures can be taken on a group as large as entire infantry regiments at war strength without too great an expenditure of energy.

The prevention of diphtheria for all these reasons falls into a very logical system of procedure. Whenever diphtheria breaks out in a school, institution, military unit, factory unit, etc., etc., the first thing to do is to make a thorough inspection and immediately institute precautions against the transmission of mucus from one individual to another. This will imply supervision of the kitchen, food preparation, dish washing, prohibition of spitting, isolation of individuals who are coughing and hawking or suffering from severe catarrhal inflammations; cleanliness of sleeping quarters and mess halls, inspection of the entire group, and the taking of throat cultures; segregation of those that show positive cultures; especially attention in this regard, and repeated culturing of kitchen personnel and food handlers; Schick reactions upon the entire group, with prophylactic immunization (preferably by the active method), of those with positive Schick reactions; subsequent attention to chronic carriers. These precautions are simple and applicable with minor modifications to any kind of group in which diphtheria appears.

Shick Reaction.-The studies of Roemer and others have shown that the blood of the majority of normal adults contains a small amount of diphtheria antitoxin. This normal antitoxin probably accounts for resistance of many individuals to diphtheria. Its presence may be very easily detected by means of the Schick reaction. A standardized diphtheria toxin is diluted in normal saline so that 0.1 c.c. of the solucontains $1 / 50$ M.L.D. for a guinea-pig. This amount is injected intracutaneously. If the blood of the subject has less than $1 / 30$ unit of antitoxin per cubic centimeter, a positive reaction appears in twentyfour to thirty-six hours, which consists in a slight infiltration of skin surrounded by a red areola, 1 to $2 \mathrm{~cm}$. in cliameter. A negative reaction indicates that sufficient natural antitoxin is present to protect the individual against diphtheria, although he may nevertheless harbor the 
bacilli as a carrier. It has been found unnecessary to give prophylactic injections of antitoxin to individuals with a negative Schick reaction. Park and Zingher ${ }^{38}$ have found that negative reactions were obtained in 93 per cent of the new-born, and became less frequent up to the second or fifth year, when 37 per cent were negative. In older children negative reactions were more frequently met with, and about 90 per cent of adults were negative. A pseudoreaction which appears earlier than the true reaction and which disappears in twenty-four to forty-eight hours is occasionally seen in individuals who have natural antitoxin. This is due to sensitiveness to some of the proteins used in the injection, and may be reproduced in the same individual by the injection of autolysate of diphtheria bacilli or sometimes by the injection of broth media. The most satisfactory method for detection of the pseudoreaction is to make a control injection of $1 / 50$ M.L.D. of a toxin which has been heated at $80^{\circ} \mathrm{C}$. for five minutes. This heating destroys the toxin, but leaves uninjured the substances which produce the pseudoreaction.

The Schick reaction has been extensively used in the last six years, especially on large bodies of troops and on school children, and has been found eminently satisfactory, though, of course, as with all other biological reactions, exceptions are noted and difficulties encountered. The New York Department of Health and other health departments are now putting out materials for the Schick reaction in packets so arranged that the necessary toxin is inclosed in capillary tubes and can be blown out into a mixed amount of salt solution with a rubber bulb. A part of this mixture can then be heated to $80^{\circ}$ and an injection with a control done on the upper arm of subjects as indicated.

Notes on Specific Therapy.-Specific therapy in diphtheria is, of course, the main thing in clinical control. It must be remembered here as in all other forms of specific therapy that success depends as much upon the time at which the diagnosis is made as it does upon the manner of treatment. Therefore, in speaking of specific therapy, it is important to emphasize the necessity for early diagnosis. It is in this particular that the responsibility of the physician is greatest, and every severe sore throat in a child should be immediately cultured and, though not as imperative in adults, it is not a bad rule to culture all throats by the Loeffler method. We have discovered a number of cases in that way, especially in military sanitation, which would not have been suspected clinically. The procedure is easy, consumes no time and requires so

${ }^{28}$ Parls and Zingher, Jour. A .M. A., 65, 1915, 2216. 
little skill that even in outlying districts the physician can easily carry out the steps himself with a simple equipment.

In order to understand the practical principles of diphtheria antitoxin treatment, it is necessary for the physician to remember chiefly two basic facts. One is the observation by Schick and others that even though diphtheric toxin does not enter so rapidly into combination with the tissues of the nervous system, as does tetanus toxin, it, nevertheless, is bound to some extent and that the antitoxin probably does not reach poison that is already combined with tissue elements. It is probable that the injury once done is irretrievable, at least to a great extent, and that the antitoxin is chiefly effective against the circulating poison before such cellular attachment has been established. Experiments of Park and his co-workers have shown that if rabbits are given 10 lethal doses of diphtheria toxin, they can be saved by relatively small doses of antitoxin if this is given just before or with the poison. As the time between injection of the poison and the injection of the antitoxin grows longer, rapidly increasing doses of antitoxin are necessary and if an hour or more has elapsed, no amount of antitoxin will save. The second basic point is the one brought out by the measurements of Henderson Smith and others which show that antitoxin subcutaneously injected, is but slowly absorbed and reaches its maximum concentration in the blood not much before seventy-three hours after injection.

The deductions to be made from these considerations are, first, that early diagnosis must be made, that it is essential to get the antitoxin in as early as possible, and that when the injection is made, it is better to give a sufficient amount at the first dose than to dribble it along in insufficient amounts with intervals of many hours between doses. These observations impose upon the physician great responsibilities of judgment, since in cases seen late in the disease, with very severe symptoms of intoxication, it may be necessary to resort to intramuscular or intravenous injections of the antitoxin.

The dosage of antitoxin must vary according to severity of the case, the stage at which it is seen, and the age of the patient. In severe cases, 10,000 to 20,000 units should be injected.

If intravenous injection is resorted to, precautions against the occurrence of anaphylaxis must of course be taken, but in view of the relatively slight danger of death from horse serum injections in man, the risk of anaphylaxis in cases in which intravenous test is actually indicated, is probably much less than the risk of delaying the introduction of antitoxin into the blood. If skin tests can be done beforehand, 
they should be done. They take very little time and give the physician a signal of possible danger. When such intravenous injections are actually made in sensitive subjects, it seems advisable to dilute the serum 50 per cent with sterile salt solution, in order to render slow injection easier so that the injection of each cubic centimeter may occupy at least one minute.

Prophylactic Immunization in Diphtheria.-There are two chief methods of prophylaxis in diphtheria the first and older consists in injecting 500 to 1000 units of antitoxin subcutaneously. This is carried out on contacts with positive Schick reactions whenever speed of immunization is desired. It is simple and the principles underlying it are obvious, but the immunization is short-lived and constitutes a sensitization of the subject with horse serum which naturally is undesirable under modern conditions.

There is another method of prophylactic immunization which is rapidly growing in importance, and in this country is being at the present time very actively worked upon by Park and his collaborators at the New York Department of Health. This method consists in the active immunization of individuals with overneutralized mixtures of toxin and antitoxin. The possibility of developing this method is really suggested in the original procedure adopted by the New York Department of Health for the production of diphtheria antitoxin in horses, namely, the partial neutralization of the first toxin injections by antitoxin. It was suggested some years ago by Theobald Smith that some such method might be applicable for prophylactic immunization in human beings, and subsequently the method was developed by the work of Behring. ${ }^{39}$ Behring made his first studies on animals, showing that immunization in monkeys, guinea pigs, asses, etc., could be accomplished by such mixtures. His own first mixtures were so balanced that the toxic action on guinea pigs was practically nil. Subsequently Schreiber 40 and others studied the antitoxin production in human beings. It was found that the results of immunization were noticeable in about two to three weeks, and that 2 to 3 injections of properly balanced mixtures would suffice to give a sufficient degree of immunity to protect against ordinary spontaneous infection. Park and Zingher ${ }^{41}$ originally recommended three injections at intervals of six to seven days,

${ }^{30}$ Behring, Deut. med. Woch., 39, 1913.

${ }^{40}$ Schreiber.

${ }^{41}$ Park and Zingher, Jour. A. M. A., 5, 1915, 2216; Park and Zingher, Jour. Amer. Pub. Health, 6, 1916, 431. 
consisting of 1 c.c. each of a toxin-antitoxin mixture so balanced that there were about $4 \mathrm{~L}+$ doses per cubic centimeter, and that the mixture contained about 85 per cent of an $\mathrm{L}+$ dose per unit of antitoxin. They added to these doses a billion killed diphtheria bacilli in order to produce a certain degree of anti-bacterial immunity. With such treatment Schick reactions could be rendered negative within a period of a few months. A few individuals did not seem to respond by the development of antitoxin and remained Schick positive.

Recently, the method has been much perfected and it is being intensively studied by Park and his force of workers at the present time. The mixtures as recommended at the present writing are such that each human dose of 1 c.c. contains at least $3 \mathrm{~L}+$ doses of toxin, so neutralized with antitoxin that 5 human doses (5 c.c.) injected into a guinea pig of 300 grams permits survival for at least fourteen or fifteen days, but causes paralysis and death thereafter, while 1 c.c. of the actual human dose produced no ill effects. From Drs. Krumwiede and Banzhof, we have the details of the N. Y. Health Department requirements which have been accepted by the United States Public Health Department as standards at the present moment. It must not be forgotten, however, that by the time this book appears slight changes may have been made since we are awaiting the publication of recent studies from the Department of Health.

We are indebted to the Director of the Hygienic Laboratory of the United States Public Health Service in Washington, Dr. George W. McCoy, for the following suggestions issued by the Public Health Service. We include these matters because accidents have occurred of recent years by mistakes in the preparation of the mixtures and even deaths have been recorded. We take from the memorandum sent us by Dr. McCoy, the following:

The toxin and antitoxin used should be at least three months old from the date of planting and date of bleeding, respectively. The L+ doses have been found with reference to the United States standard antitoxin. The unitage of the antitoxin to be used should be determined against this toxin so that one unit of the antitoxin to be used, when mixed with the $\mathrm{L}+$ dose of this toxin should permit survival for ninety-six hours of 25 per cent of the guinea pigs injected. When a tentative mixture of the product has been made, no further toxin shall be added. Changes made should consist not in the addition of toxin, but in the reduction of toxicity by the addition of antitoxin, if necessary. Approximately one unit of antitoxin is, therefore, added to start with, 
for each $\mathrm{L}+$ dose in the bulk toxin, and with each addition of antitoxin to the mixture in bulk, it shall be immediately thoroughly shaken for twenty minutes. After each addition to the mixture, or after the original mixing, the preparation should stand at least twenty-four hours in order to allow combination to take place before tests on guinea pigs are made. Six guinea pigs are then injected receiving, respectively, $0.1,0.2,0.5,1.0,2.0$, and 5.0 c.c. If all six guinea pigs die in seventytwo hours, add 0.1 the former antitoxin used. mix and retest. Continue this until the 5 c.c. pig survives ninety-six hours and at this point the mixture is probably ready for filtering. After filtering, the mixture is preserved until at least one-half of the 5 c.c. guinea pigs on each bulk bottle survive ten days, but show definite diphtheritic paralysis thereafter. The human dose is 1. c.c., and three doses a week apart are usually given.

Bacteria Similar to Bacillus Diphtheriæ.-BaciluUs HoFFMANNI (Pseudodiphtheria bacillus).--Hoffmann-Wellenhoff, ${ }^{42}$ in 1888, and, at almost the same time, Loeffler, ${ }^{43}$ described bacilli which they had cultivated from the throats of normal persons and in several instances from those of diphtheritic persons, which were in many respects similar to true B. diphtheria, but differed from this chiefly in being non-pathogenic for guinea-pigs. These organisms were at first regarded by some observers as merely attenuated diphtheria bacilli. More recent investigations, however, prove them to be unquestionably a separate species, easily differentiable by proper methods. They differ from B. diphtheriæ in so many important features, moreover, that the term "pseudodiphtheria bacillus" is hardly an appropriate one for them.

Morphology.-Bacillus Hoffmanni is shorter and thicker than Bacillus diphtheria. It is usually straight and slightly clubbed at one end, rarely at both. Stained with Loeffler's blue it occasionally shows unstained transverse bands; unlike B. diphtheriæ, however, these bands hardly ever exceed one or two in number at most. In many cultures the single transverse band gives the bacillus a diplococcoid appearance.

Staining.-Stained by Neisser's or Roux's method, no polar bodies can be demonstrated. The bacillus forms no spores, is non-motile, and possesses no flagella.

Cultivation.-On the usual culture media B. Hoffmanni grows more luxuriantly than B. diphtheriæ, developing even in first isolations from

${ }^{42}$ Hoff mann-Wellenhoff', Wien. med. Woch, iii, 1888.

${ }^{43}$ Loeffler, Cent. f. Bakt., ii, 1887. 
the human body upon the simple meat-extract media. On agar plates its colonies are larger, less transparent, and whiter than are those of true diphtheria bacilli. In fluid media there is even clouding and less tendency to the formation of a pellicle than with B. diphtheriæ. A positive means of distinction between the two is given by the inability of B. Hoffmanni to form acid upon various sugar media. The differentiation on a basis of acid formation was first attempted by Cobbett ${ }^{44}$ and has been recently worked out systematically by Knapp, ${ }^{45}$ and con-

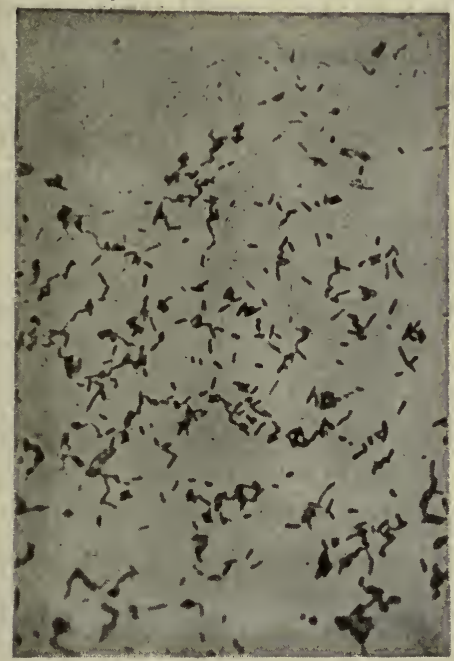

Fig. 61.-Bacillus Hoffmanni.

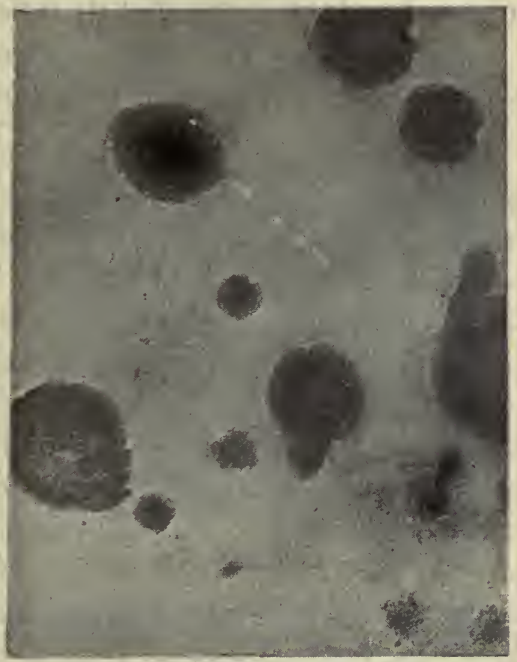

Fig. 62.-Colonies of Bacilli HofFManNi on Agar.

firmed by various observers. ${ }^{46}$ The results of this work, carried out with the serum-water media of Hiss, to which various sugars were added, show that B. Hoffmanni forms acid upon none of the sugars used, while B. diphtheriæ acidifies and coagulates media containing monosaccharids and several of the more complex sugars, as given in the diagram in the section following, dealing with B. xerosis.

Differentiation can finally be made on the basis of animal pathogenicity, B. Hoffmanni being entirely innocuous to the ordinary labora-

${ }^{44}$ Cobbett, Cent. f. Bakt., 1898.

s5 Krapp, Jour. Med. Res., vii, 1904.

${ }^{46}$ Graham Smith, Jour, of Hyg., vi, 1906; Zinsser, Jour. Med. Res., xvii, 1907. 
tory animals. B. Hoffmanni forms no toxins, and animals immunized with it do not possess increased resistarice to $\mathrm{B}$. diphtheriæ.

Bacillus Xerosis.- In 1884, Kutschert and Neisser ${ }^{47}$ described a bacillus, isolated from the eyes of patients suffering from a form of chronic conjunctivitis known as xerosis. This bacillus, which, morphologically, is almost identical with B. diphtheriæ, they believed to be the etiological factor of the disease. The frequency with which it has been isolated from normal eyes, precludes this etiological relationship, and it may safely be regarded as a harmless parasite which may indeed be more abundant in the slightly inflamed than in the normal conjunctiva.

Morphology.-B. xerosis resembles B. diphtheriæ closely. It is occasionally shorter than this, but on the whole no absolute morpho-

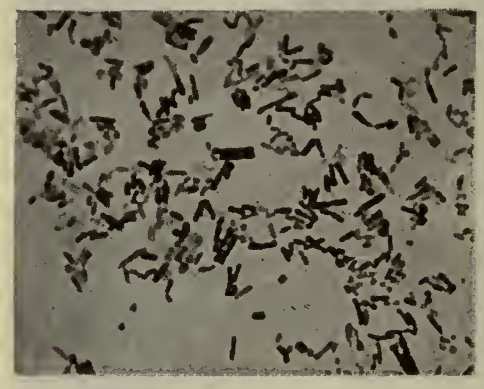

Fig. 63.-Bacillus Xerosis. logical differentiation between the two is possible. It forms no spores and is non-motile. Polar bodies may occasionally be seen.

Cultivation.-On Loefler's blood serum, on agar, glycerin agar, and in broth, its growth is very similar to that of B. diphtheriæ, but more delicate throughout. It cannot easily be cultivated upon the simple meatextract media, nor will it grow on gelatin at room temperature. Its colonies on glycerin or glucose agar are microscopically identical with those of B. diphtheriæ.

Differentiation.-It differs from B. diphtheriæ distinctly in its acidifying action on sugar media. These relations were first worked out by Knapp for various sugars and the alcohol mannit, and have been extensively confirmed by others. See table on page 585 .

A reference to the table shows that differentiation may be made by the use of two sugars - saccharose and dextrin. B. diphtheriæ forms acid from dextrin, not from saccharose; B. xerosis from saccharose, not from dextrin; B. Hoffmanni does not form acid from either.

B. xerosis is non-pathogenic to animals and forms no toxin.

The Diphtheroid Bacilli.-In addition to the bacteria mentioned above, there is a large group of microorganisms spoken of as the diphtheroid bacilli, largely because of their morphological resemblance to the diphtheria bacillus. For this group, Lehman and Neumann have

${ }^{47}$ Kutschert und Neisser, Deut. med. Woch., xxiv, 1884. 
suggested the term corynebacterium. The characteristics of this group are a morphological similarity to the diphtheria bacillus, that they are Gram-positive, non-motile, often show metachromatic granules and have no spores. It is not, at the present writing, possible to formulate a classification of these organisms. They are apparently very numerous and have been isolated from a great many different sources, both

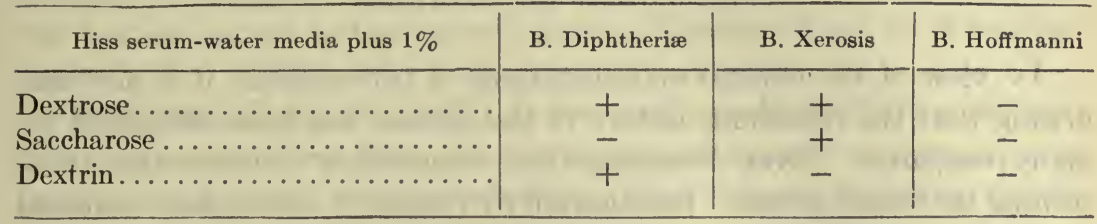

in connection with the human body and in nature. Recently Bunting and Yates have claimed that an organism of this group has etiological connection with Hodgkin's disease. Studies by many other workers, notably by Bloomfield and Fox; and studies going on in our own laboratory show that organisms very similar to these strains can be isolated from the skin, from the lymph nodes of healthy and diseased people, from ascitic fluid in varying eonditions, and from supposedly sterile tissues. They are frequently present in the nasal mucus and in the throat, and are so ubiquitous that any association of them with specific disease must be very conservatively approached. According to the investigations of many men who have studied the flora of the nasopharynx, it seems that organisms belonging to the general group of diphtheroid bacilli are the most common saprophytes habitually present in this part of the normal human body.

Very similar to this group are the bacilli of pseudo-tuherculosis oris, isolated from necrotic lesions in the kidneys of sheep by Preisz and Nocard.

It is impossible at present to do more than indicate that the "diphtheroid bacilli" are a large heterogeneous group, held together by morphological and superficial cultural similarity and largely consisting of saprophytes and probably harmless parasites on the human and animal body.

Recently, Mallory and Frederic Parker have isolated diphtheroid bacilli from middle ear lesions secondary to scarlet fever which seem to deserve more than ordinary attention because of their apparent production of a powerful toxic substance. These organisms are now being studied in our laboratory by Dr. Parker. 


\section{CHAPTER $\cdot$ XXIX}

\section{THE TUBERCLE BACILLUS}

Is view of the clinical manifestations of tuberculosis, it is not surprising that the infectious nature of the disease has been suspected for many centuries. Even Fracastor had remarkably modern ideas concerning its transmission. Inoculation by means of tuberculous material was first successfully accomplished by Klencke, in 1843 , and, more elaborately, by Villemin, ${ }^{1}$ in 1865 . It was not until 1882 , however, that Koch ${ }^{2}$ succeeded in isolating and cultivating the tubercle bacillus. Baumgarten ${ }^{3}$ had previously seen the bacillus in tissue sections, but his researches were limited to purely morphological observations. Koch, in addition to demonstrating the bacillus in tuberculous tissues from various sources, produced characteristic lesions in guinea-pigs and other animals by infecting them with pure cultures, and established beyond doubt the etiological relationship of the hacillus to the disease.

Morphology.-Tubercle bacilli appear as slender rods, 2 to 4 micra in length, 0.2 to 0.5 micra in width. Their ends are usually rounded. The rods may be straight or slightly curved; their diameters may be uniform throughout; more often, however, they appear beaded and irregularly stained. The beaded appearance is due to different causes. Unstained spaces may occur along the body of the bacillus, especially in old cultures. These are generally regarded as vacuoles. The bodies of the bacilli, on the other hand, may bulge slightly here and there, often in three or four places, showing oval or rounded knobs which stain with great depth and are very resistant to decolorization. These thickenings were formerly regarded as spores, but in view of the fact that the bacilli are not more resistant against heat and disinfectants than other vegetative forms, this interpretation is probably incorrect. The bacilli are said to possess a cell membrane which confers upon them their resistance

${ }^{1}$ Villemin, Gaz. hebdom., 1865.

${ }^{2}$ Koch, Berl. klin. Woch., 1882 ; Mitt. a. d. kais. Gesundheitsamt, 1884.

${ }^{3}$ Baumgarten, Virchow's Arch., Ixxxii. 
against drying and entrance of stains. This membrane gives a cellulose reaction and is believed to contain most of the waxy substances which can be extracted from the cultures.

Various observers, notably Nocard and Roux, ${ }^{4}$ Mafucci, ${ }^{5}$ and Klein, ${ }^{6}$ have demonstrated branched forms of the tubercle bacillus. These observations, variously extended and confirmed, make it probable that Bacillus tuberculosis is not a member of the family of schizomycetes, but belongs rather to the higher bacteria, closely related to the actinomyces.

Staining.-Tubercle bacilli do not stain easily with the ordinary anilin dyes; to these they are made permeable only by long exposure or by heating of the staining solution. Once stained, however, the dye is tenaciously retained in spite of treatment with alcohol and strong acids. For this reason, this bacillus, together with some other bacteria to be mentioned later, is spoken of as "acid-fast." The acid-fast nature of the bacillus seems to depend upon the fatty substances contained in it, ${ }^{7}$ and has furnished the basis for differential staining methods. All the staining methods devised for the recognition of the tubercle bacillus thus depend upon the use of an intensely penetrating staining solution, followed by vigorous decolorization which deprives all but the acid-fast group of their color. Counterstains of any of the weaker dyes may then be used to stain the decolorized elements. One of the first of the staining solutions to be of practical use was the anilin-water-gentianviolet solution of Ehrlich ${ }^{8}$ (11 c.c. saturated alcoholic gentian-violet to 89 c.c. 5 per cent anilin water). This dye, although of sufficient penetrating power, has the disadvantage of deteriorating rapidly and has in practice been almost entirely displaced by Ziehl's ${ }^{9}$ carbol-fuchsin solution. (Fuchsin $1 \mathrm{gm}$. in 10 c.c. alcohol absolute, added to 90 c.c. 5 per cent carbolic.) This staining solution is the one now in general use and is employed as follows: Thin smears, on slides or cover-slips, are covered with the dye and gently heated. In the case of cover-glasses, these may be floated, face downward, in staining dishes filled with the dye. The dye is allowed to act for about three minutes, steaming but not allowed to boil. At the end of this time the preparation is washed

${ }^{4}$ Nocard et Roux, Ann. de 1'inst. Pasteur, 1887.

sMafucci, Zeit. f. Hyg., ii.

'Klcin. ('ent. f. Bakt., 1890.

${ }^{7}$ bicnstock, Fort. 1. Med., 1886; Weyl, Deut. med. Woch., 1891.

"Ehrtich, Dent. mel. Woch., 1882; Weigert, Deut. med. Woch., 1855.

'Zichl, Deut. med. Woch., 1883; Neelsen, "Lehrb, d. allg. Path.,' 1894. 
either with 5 per cent nitric acid, 5 to 20 per cent sulphuric acid, or 1 per cent hydrochloric acid, until most of the red color has disappeared (a few seconds), and the preparation appears pale pink. This results in decolorization of all microorganisms with the exception of members of the acid-fast group. Thorough washing in 80 to 95 per cent alcohol is now employed to complete the decolorization. The preparation is then rinsed in water and counterstained with 1 per cent aqueous methylene-blue.

Tubercle-bacillus staining has been further simplified by Gabbett, ${ }^{10}$ who combines decolorization and counterstaining. In this method preparations are stained with Ziehl's carbol-fuchsin as in the preceding; they are then rinsed in water and covered with a solution containing methylene-blue 1 gram, concentrated sulphuric acid 25 grams, and distilled water 100 c.c. This is allowed to act for from two to four minutes, at the end of which time all elements in the preparation except the acid-fast bacilli will be decolorized and counterstained.

Another excellent stain for tubercle bacilli, which has the advantage of greater clearness of contrast over the carbol-fuchsin stain is that of Hermann in which Crystal Violet is used. It is described in the section on staining.

Tubercle bacilli in very young culture are often not acid-fast and it is not always possible to demonstrate acid-fast bacilli in pus from cold abscesses in sputum, in serous exudates, and in granulomatous lesions of the lymph nodes which can be shown by animal inoculation to be tuberculous. Much ${ }^{11}$ demonstrated in such material Gram-positive granules which lay singly, in short chains or in irregular clumps, and which he believed to be non-acid-fast tubercle bacilli. He found similar granules in cultures of tubercle bacilli which showed on further incubation numerous acid-fast bacillary forms. His work has been repeatedly confirmed, and there seems little doubt but that these granules are really tubercle bacilli. Their demonstration is not, however, of great diagnostic value, as other bacilli form granules of the same appearance. Small rods and splinters are also found which stain by Gram's method, but not by carbol-fuchsin. ${ }^{12}$

To find "Much's granules," smears or sections are steamed in a solution of methyl violet B.N. (10 c.c. of saturated alcoholic solution of the dye in 100 c.c. of (listilled water containing 2 per cent phenol).

${ }^{10}$ Gabbett, Lancet, 1887 .

${ }^{11}$ Much, Berỉ. klin. Woch., 1908, xlv, 700.

${ }^{12}$ Liębermeister, Deutsche med. Woch., 1909, xxxv, 1324. 
They are then treated with Gram's iodine solution one to five minutes; 5 per cent nitric acid one minute; 3 per cent hydrochloric acid ten seconds; absolute alcohol and acetone equal parts, until decolorized. The granules may be stained by other modifications of Gram's method. Weiss ${ }^{13}$ has devised a combination stain. One part of Much's methyl violet is mixed with three parts of Ziehl's carbol-fuchsin and filtered; slides are stained for twenty-four to forty-eight hours in the mixture. They are then decolorized as in Much's method and counterstained with Bismarck brown or safranin 1 per cent. Both acid-fast and Grampositive forms are stained by this method and in the red may be seen blue-black granules.

While the acid-fast group of bacteria is composed of a number of organisms to be mentioned later, a few only of these offer difficulties of differentiation from the tubercle bacillus. Those to be considered practically are the bacillus of leprosy and that of smegma. The latter bacillus, because of its distribution, is not infrequently found to contaminate feces, urine, or even sputum, and it is sometimes desirable to apply to suspected specimens one or the other of the stains devised for the differentiation of the smegma bacillus from Bacillus tuberculosis. The one most frequently employed is that of Pappenheim. ${ }^{14}$ The preparations are stained in hot carbol-fuchsin as before; the carbol-fuchsin is then poured off without washing and the preparation immersed in solution made by saturating a 1 per cent alcoholic solution of rosolic acid with methylene-blue and adding 20 per cent of glycerin. In such preparations tubercle bacilli remain red, smegma bacilli appear blue.

Stained by Gram, tubercle bacilli retain the gentian-violet.

When tubercle bacilli are very sparsely present in sputum and other material it may be impossible to find them by direct examination, and often the only method of finding them will be animal inoculation. However, a number of methods have been devised by which the bacilli may be concentrated in such a way that they may be found even when a few only are present. One of these is to add peroxide of hydrogen to the sputum. By this the mucus is dissolved out and the solid particles settle or may be centrifugalized. A method very commonly employed to-day is that which depends on the use of "antiformin." This is a preparation used extensively for the cleansing of vats in breweries. It

${ }^{13}$ Weiss, Berl. klin. Woch., 1909, xlvi, 1797.

${ }^{14}$ Pappenheim, Berl. klin. Woch., 1898. 
is described by Rosenau ${ }^{15}$ as consisting of equal parts of liquor sodæe chlorinatæ and a 15 per cent solution of caustic soda. The formula for liquor sodæ chlorinatæ he gives as:

Sodium carbonate ........................... 600

Chlorinated lime .......................... 400

Distilled water .......................... 4,000

If sputum is poured into a 10 to 15 per cent solution of antiformin and allowed to stand for several hours, most of the other elements of the sputum, cells, and bacteria, will dissolve out, and acid-faşt bacilli be left in the residue. 'Strangely enough they are not killed by this process and if sufficiently washed may be cultivated or can produce lesions in guinea-pigs.

Isolation and Cultivation.-Tubercle bacilli are not easily cultivated. Their slowness of growth precludes isolation by plating. The first isolations by Koch ${ }^{16}$ were made upon coagulated blood serum from tuberculous tissue.

Isolation from tuberculous material may be aided by inoculation into guinea-pigs. These animals will withstand the acute infection produced by the contaminating organisms and succumb later (four to six weeks) to tuberculosis. The bacilli may then be obtained by cultivations from lymph nodes or other foci which contain only tubercle bacilli. When isolation from sputum is attempted whether directly or by means of animal inoculation, the sputum may be rendered comparatively free from contaminating bacteria by washing. The sputum is rinsed in running water to free it from pharyngeal mucus. It is then washed in eight or ten changes of sterile water. The material selected is taken from the center of the washed mass, if possible from the flakes of caseous material visible in such sputum.

For the isolation of tubercle bacilli from sputum and other materials in which contaminating bacteria of other species are present, Petroff ${ }^{17}$ has devised an excellent method which has been tried out and used with suceess in our laboratory. The principles on which Petroff's method rests are, first of all, the bactericidal power of 3 per eent sodium hydroxid on non-acid-fast bacteria, and the selective action of dyes like gentian violet on bacterial growth, as first practically utilized by Churchman.

${ }^{15}$ Rosenau, "'Preventive Medicine and Hyigene," D. Appleton, N. Y., 1913; Uhlenhuth, Berl. klin. Woch., No. 29, 1908.

${ }^{16}$ Koch, loc eit.

${ }^{17}$ Petroff, Johns Hopkins Hosp. Bull., vol. xxvi, No. 294, August, 1915, p. 276. 
The medium used by Petroff is made as follows:

I. Meat Juice. Five hundred grams of beef or veal are infused in 500 c.c. of a 15 per cent solution of glycerin in water, in a cool place. After twenty-four hours the meat is squeezed in a sterile press and the infusion collected in a sterile beaker.

II. Eggs. The shells of the eggs are sterilized by ten minute immersion in 70 per cent alcohol. They are broken into a sterile beaker, well mixed and filtered through sterile gauze. One part of meat juice is added to two parts of egg by volume.

III. Gentian Violet. One per cent alcoholic solution of gentian violet is added to make a final proportion of $1: 10,000$.

The three ingredients are well mixed. The medium is tubed and inspissated as usual.

Petroff recommends for sputum the following technique: Equal parts of sputum and 3 per cent sodium hydroxid are shaken and incubated at $38^{\circ}, \mathrm{C}$. for fifteen to thirty minutes, the time depending on the consistency of the sputum. The mixture is neutralized to litmus with hydrochloric acid and centrifugalized. The sediment is inoculated into the medium described above. Pure cultures are obtained in a large proportion of cases.

Petroff's methed has been applied by him to feces, in which the problem is made more difficult by the presence of many spore-formers which resist sodium hydroxid. Feces is collected and diluted with three volumes of water, and then filtered through several thicknesses of gauze. The filtrate is saturated with sodium chlorid and left for half an hour. The floating film of bacteria is collected in a wide-mouthed bottle and an equal volume of normal sodium hydroxid is added. This is shaken and left in the incubator for three hours, shaking every half hour. It is then neutralized to litmus with normal hydrochloric, eentrifugalized, and the sediment planted.

Once isolated, the bacilli are best grown on glycerin egg medium which is described in the section on Media. On this medium colonies of the human bacilli begin to appear after six or eight days as yellowish white moist crust-like flakes.

On blood serum at $37.5^{\circ} \mathrm{C}$., colonies become visible at the end of eight to fourteen days. They appear as small, dry, scaly spots with corrugated surfaces. After three or four weeks, these join, covering the surface as a dry, whitish, wrinkled membrane. Coagulated dog serum is regarded by Theobald Smith ${ }^{18}$ as a favorable media for the growth of tubercle bacilli.

1x Th. Smith, Jour. Fxp. Merl., iii, 1898. 
Slants of agar, to which whole rabbit's blood has been added in quantities of from 1 to 2 c.c. to each tube, make an excellent medium.

Cultivation methods were simplified by the discovery by Roux and Nocard that glycerin facilitates cultivation. Upon glycerin-agar (glycerin 3 to 6 per cent), at $37.5^{\circ} \mathrm{C}$., colonies become visible at the end of from ten days to two weeks.

Glycerin bouillon (made of beef or veal with pepton 1 per cent, glycerin 6 per cent, slightly alkaline)

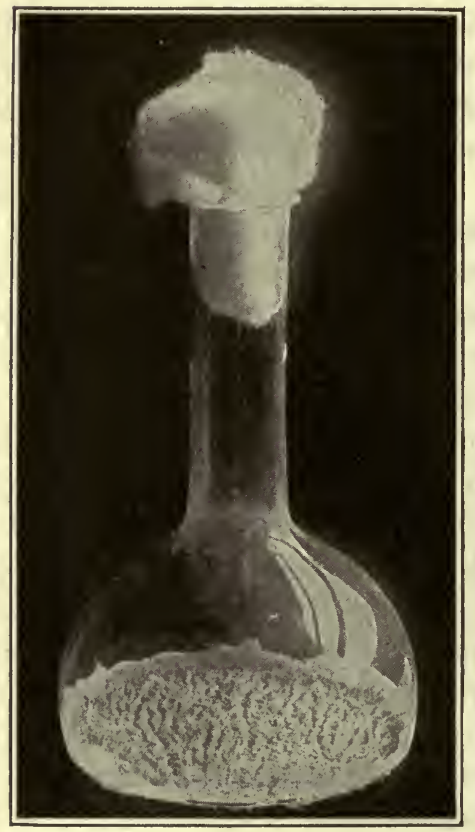

Fig. 64.-Culture of Bacillus

TUBerculosis IN Flask OF GLyCERIN BouilLon. is a favorable. medium. It should be filled, in shallow layers, into widemouthed flasks, since oxygen is essential. Transplants to this medium should be made by carefully floating flakes of the culture upon the surface. In this medium the bacilli will spread out upon the surface, at first as a thin, opaque, floating membrane. This rapidly thickens into a white, wrinkled, or granular layer, spreading over the entire surface of the fluid in from four to six weeks. Later, portions of the membrane sink. In old cultures, the membrane becomes yellowish. These cultures emit a peculiar aromatic odor. Cultures when first grown on solid media are a little difficult to start on glycerin broth. To accomplish this it is best to grew them for a few weeks on egg or glycerin egg slants containing considerable amounts of condensation water. At the end of this time the growth will have begun to grow over the surface of the condensation water, and from this pellicle a bit can be picked up with a bent loop, carefully removed without allowing it to immerse in the fluid and this carefully floated on the surface of the glycerin broth in the flask.

Glycerin potato forms a favorable culture medium for the bacillus. Hesse ${ }^{19}$ has devised a medium containing a proprietary preparation 
known as "Nährstoff Heyden," upon which tubercle bacilli are said to proliferate more rapidly than other bacteria. His method has yielded excellent results. It is prepared as follows:

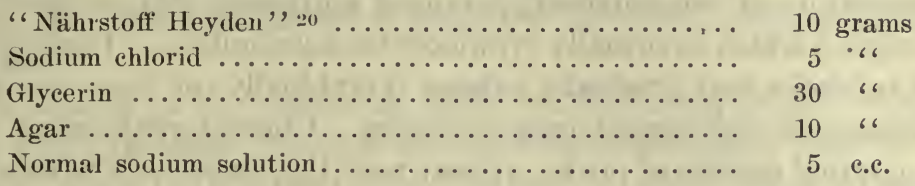

Aq. dest $\ldots \ldots \ldots \ldots \ldots \ldots \ldots \ldots \ldots \ldots \ldots \ldots, 1,000$ "

Biological Considerations.-The tubercle bacillus is dependent upon the access of oxygen. Its optimum temperature is $37.5^{\circ} \mathrm{C}$. Temperatures below $30^{\circ}$ and above $42^{\circ} \mathrm{C}$. inhibit its growth. In fluid media, the bacilli are killed by $60^{\circ}$ in fifteen to twenty minutes, by $80^{\circ}$ in five minutes, by $90^{\circ}$ in one to two minutes. They will withstand dry heat at $100^{\circ} \mathrm{C}$. for one hour. They are resistant to cold. The comparatively high powers of resistance of the bacillus are attributed to the protective qualities of the waxy cell membrane. ${ }^{21}$

The life of cultures, kept in favorable environment, is from two to eight months, varying to some extent with the nature of the culture medium. The viability of the bacilli in sputum is of great hygienic importance. In most sputum they may remain alive and virulent for as long as six weeks, in dried sputum for more than two months. ${ }^{22}$

Five per cent carbolic acid kills the bacilli in a few minutes. ${ }^{23}$ Used for sputum disinfection, where the bacilli are protected, complete disinfection requires five to six hours. Bichloride of mercury is not very efficient for sputum because of the formation of albuminate of mercury. Direct sunlight kills in a few hours.

Pathogenicity.-The tubercle bacillus gives rise in men and susceptible animals to specific inflammations which are so characteristic that a diagnosis of tuberculosis may be made by histological examination, even though tubercle bacilli themselves are not found. These foci, known as tubercles, were first studied in detail by Baumgarten, ${ }^{24}$ and, since then, have been the object of many pathological investiga-

20 " Nährstoff Heyden" is prepared in Germany. It is a white powder similar to nutrose.

${ }^{21}$ T'h. Smith, Jour. Exper. Med., 1899; Grancher et Ledoux-Lebard, Areh. de méd. expér., 1892; Galtier, Compt. rend. de l'acad. des sci., 1887.

${ }^{22}$ Schell und Fischer, Mitt. a. d. kais. Gesundheitsant, 1884.

${ }^{23}$ De Toma, Ann. di med., 1886.

${ }^{24}$ Baumgarten, Berl. klin. Woch., 1901. 
tions. The lesions are fundamentally alike wherever they occur, though in their detailed histological structure they may vary somewhat accord, ing to the tissue in which they appear. They begin as microscopic agglomerations of concentrically arranged epithelium-like, or epitheloid cells, around which eventually lymphocytes accumulate. These microscopic tubercles may gradually enlarge individually, or they may grow by coalescence with neightoring tubeicles. Characteristic giant cells, with marginal centers of nuclei, appear near the centers of the tubercles and in these giant cells the tubercle bacilli are usually found. As the tubercles grow in size, the central mass becomes necrotic. Fluid pus does not form, and the centers assume a grumous and friable condition which is generally described as caseous or cheesy. Such tubercles may result from the injection of dead bacilli, as well as living ones as Prudden and Hodenpyl have shown. The cheesy degeneration may be in part due to the toxic action of the substances of the tubercle bacilli and in part by pressure and lack of vascularization. It is astonishing how difficult it is to find tubercle bacilli histologically in such lesions. This may be due perhaps to the fact that, owing to degeneration, most of the tubercle bacilli have lost their acid-fastness. If tubercles heal, as they often do, they undergo a fibrinous change, are surrounded by connective tissue and, if central necrosis has begun at the time that healing sets in, calcification results. For more detailed descriptions we refer the reader the Text books of Pathology of Adami's, MacCallum's or Delafield and Prudden.

There has been a great deal of discussion concerning the manner in which tubercle bacilli enter the human and animal body in the course of spontaneous infection. A thorough discussion of this will be found in the recent book by Calmette, L'Infection Bacillaire de la Tuberculose (Masson, Paris, 1920), Chapter 8, p. 110. Calmette believes that when a tubercle bacillus "is deposited on the surface of the skin or a mucous membrane or is introduced into the healthy body by another route" it becomes the prey of leucocytes which carry it into the lymphatic circulation and into the blood. The leucocytic enzymes are not capable of digesting the organism and eventually the organism is deposited in the lymphatics when the leucocyte degenerates.

Tubercle bacilli may remain latent in the body, in lymph nodes, especially, for long periods. It appears that the point of entrance of the tubercle bacilli into the body may be through the tonsils, and secondarily thence through the lymphatics, then to other organs. Pulmonary infection may be either by direct inhalation or indirectly through the 
lymphatics. Calmette believes that actual direct infection of the lung by inhaled bacilli is relatively rare and bases this upon experimental evidence. This, however, is not in agreement with the bulk of evidence, and direct inhalation is probably the most common manner of invasion.

According to the researches of Bartel and many others, it appears that direct infection through the apparently uninjured mucous membrane of the intestinal tract may take place, and after such entrance the bacilli may be carried by the lymphatics and blood to the lungs and other parts of the body. Calmette states that, in all susceptible animals, man included, and in all its varieties of localization, tuberculosis in the large majority of cases originates in a primary infection of the lymphatics which takes its origin by entrance of the tubercle bacilli through the mucous membranes of the digestive tract, chiefly the mucous membranes of the mouth, pharynx and intestine. This is the extreme view, but one that is favored in addition to Calmette by Von Behring, Ravenel and others.

Opie ${ }^{25}$ as a result of recent studies, states that first infection with tuberculosis may occur either by way of the lungs or the gastro-intestinal tract, and the occurrence of one lesion tends to prevent the other.

In man, tuberculosis is most common in the lungs where it usually starts in the apices. The apical situation of early tubercles is not entirely explained. A number of theories have been advanced, most of them based on anatomical reasoning. In the lungs there may be a miliary distribution of tubercles or, by coalescence of these, large areas of consolidation may occur, which are then spoken of as phthisis. Extension to the pleura is common.

Although pulmonary infections constitute the very large majority of cases of tuberculosis in adult life, this is not strictly true of childhood. In statistics quoted by Calmette for Europe, it was found by Hamberger and Sluka ${ }^{26}$ that of 160 cases in children there were only 50 per cent pulmonary lesions. According to Dr. Holt's statistics for New York, however, of 119 autopsies of tuberculous children, pulmonary lesions were found in 99 per cent. In 1515 autopsies studied by Comby during fifteen years, involvement of the tracheo-bronchial lymph nodes was found in all. Aside from the pulmonary and lymphatic infections, tuberculosis may occur in practically all other parts of the body.

${ }^{25}$ Opie, Am. Rev. of Tuberculosis, vol. 4, 1920, p. 629.

${ }^{26}$ Hamburger and Sluka, eited from Calmette, loc. cit. 
Tuberculosis of the skin or lupus is a common disease. Involvement of the bones and joints may occur, and according to Fraser ${ }^{27}$ may in many cases occur without any previous tuberculosis. Tuberculous meningitis is not infrequent in children, and is always fatal. Calmette quotes Grunberg's studies of the comparative frequency of mortality of children from tuberculosis in 568 families. He analyzed 209 deaths by tuberculosis in children from birth to fifteen years, from birth to one year, 82 per cent were meningitic, 6 per cent were pulmonary and 3 per cent other forms of tuberculosis. At fifteen years, only 6 cases were meningitis, 7 pulmonary and 5 of other forms.

The liver may be the site of tubercles, and tuberculosis of the spleen has been observed though it is not particularly common. The kidneys and the genito-urinary system are frequently involved, and the suprerenal gland may be tuberculous, and in this case may lead to a condition spoken of as Addison's disease.

In the intestines themselves, various forms of tuberculosis have been described. It appears that the only parts of the body in which tuberculosis is not common are the muscle tissues themselves, and the wall of the stomach.

Rosenberger ${ }^{28}$ has reported finding tubercle bacilli in the circulating blood of all cases of human tuberculosis which he examined. This announcement aroused much interest and has led to many investigations by other workers. Rosenberger's results were obtained by morphological examination of smears of citrated blood taken from the patients, dried upon slides and laked with distilled water. Many other observers have failed to confirm Rosenberger's results. Anderson ${ }^{29}$ examined 47 cases in which tubercle bacilli were found in the sputum and one case of joint tuberculosis. In none of these 48 cases was he able to obtain tubercle bacilli, either by morphological examination nor by guinea-pig inoculation. Brem ${ }^{30}$ subsequently found that laboratory distilled water may frequently contain acid-fast saprophytes -a fact which may account in many cases for errors when morphological examination alone is relied upon and blood examined by the technique of Rosenberger. This, too, is suggested by the finding of acidfast bacilli in the blood of perfectly healthy individuals. Therefore, although the bacilli may be present in the blood in a certain number of

\footnotetext{
${ }^{2 \pi}$ Fraser, Jour. Exper. Med., No. 4, 16, 1912.

${ }^{28}$ Rosenberger, Am. Jour. of Med. Sc., exxxvii, 1909.

${ }^{29}$ Anderson, U. S. P. H. Service, Hygienic Lab., Bull. 57, 1909.

${ }^{30}$ Brem, Jour. A. M. A., liii, 1909.
} 
cases it does not seem likely that they are so distributed in anything like the high percentages found by Rosenberger. ${ }^{31}$

Although, therefore, in patients suffering from tuberculosis, the presence of the tubercle bacilli in the blood is generally slight or intermittent, there may be times when large numbers of tubercle bacilli are thrown into the blood stream, and, according to the manner and quantity thus distributed, secondary foci or general miliary tuberculosis may occur.

Bacillus tuberculosis (typus humanus) is pathogenic for guineapigs, less markedly for rabbits, and still less so for dogs. It is slightly pathogenic for cattle, a question spoken of more extensively below.

Secondary Infection in Tuberculosis.-An important consideration in the symptomatology and prognosis of pulmonary tuberculosis is the fact that on the basis of the chronic inflammatory condition of bronchii and alveoli in the neighborhood of tuberculous processes in the bronchiectatic cavities and perhaps in cavities communicated with bronchioles, masses of bacteria of various species may accumulate and habitually lodge. Staphylococci, streptococci, Gram-negative cocci, and frequently influenza bacilli may be present in such cases and materially contribute to the illness of the patient by superimposing acute and subacute inflammatory processes upon the tuberculous one.

\section{FREQUENCY AND TRANSMISSION}

In man, tuberculosis is the most common of diseases. Naegeli's statistics, based on a large series of autopsies, show not only the frequency of the disease, but its relation to age. Before one year of age he finds it very rare. From the first to the fifth year it is rare, but usually fatal. From the fifth to the fourteenth year, one-third of his cases showed tuberculosis; from the fourteenth to the eighteenth year, one-half of the cases. Between eighteen and thirty, almost all the cases examined showed some trace of tuberculous infection. Three-quarters of these were active, one-quarter healed. Two-fifths of all deaths occurring at these ages were due to tuberculosis. After the age of thirty, active lesions gradually diminished, healed lesions increased.

In 1900 it was stated that the average yearly mortality from tuberculosis in New York amounted to 6000, and that in Manhattan alone there were constantly 20,000 tuberculous persons. Cornet estimates

${ }^{31}$ Suzuki and Takaki, Centralbl. f. Bakt., lxi, 1911. 
that in 1894 the deaths in Germany from all other infectious diseases amounted to 116,705 , those from tuberculosis alone to 123,904. Similar statistics might be chosen from the health reports of any large city. While the disease is less common in rural districts than in large towns, the difference is not so striking as is generally supposed.

Kober ${ }^{32}$ states that, owing to active measures of prevention, the death rate from tuberculosis has been reduced from 326 per 100,000 in 1888 to 147.6 in 1913 , which he says means that, if the former rate of mortality had been continued "the number of deaths from this disease last year (1914) would have bcen 322,027 , instead of 143,000 ," meaning the saving of over 179,000 lives.

Although there has been much discussion concerning the different methods of infection, there seems to be very little doubt at the present time that inhalation is the most common means of human infection. In coughing, expectoration and sneezing, small droplets of fluid in which all kinds of microorganisms are found, are sprayed into the air, and these may be deposited upon the mucous membranes of people in close contact with the disease. The striking distance of such droplet infection is not very large, but is, as Kober points out, particularly dangerous because the bacilli thus enter the respiratory passages directly from body to body. In addition to this, the tubercle bacilli may remain alive in dust sufficiently dry to be blown about by draughts and winds. Although the bacilli are not spore bearers, their acid-fast nature renders them somewhat more resistant to desiccation and sunlight than are most other germs.

Next to direct inhalation, the most frequent method of transmission is probably through the digestive tract. Such infection may take place by direct contamination of food from the expectoration and saliva of consumptives or by indirect infection of food, and milk, through the agency of fingers, flies, etc.

Milk Infection.-The question of intestinal infection, however, is particularly important in connection with transmission of bovine tuberculosis to man through the agency of infected milk. The general public has probably very little idea of the frequency of tuberculosis in cattle. In a community supervised more closely than usual, the work of Public Health Service bacteriologists in Washington, revealed 6.72 per cent of samples of market milk infected with tubercle bacilli. This percentage is probably very much lower than that which would naturally be found in districts with a less well-developed dairy supervision, and in some

${ }^{22}$ Kober, Rep. No. 309, U. S. P. H. S. Reports, October, 1915. 
of the poorer farm districts of the country the cattle tuberculosis situation is actually appalling. The question of how frequently infection with bovine tubercle bacilli through the intestinal tract may occur is still a matter of some controversy.

$\mathrm{V}$. Behring expressed the belief that a large percentage of all cases of tuberculosis originated in childhood from infection through the intestinal tract. He determined that tubercle bacilli may penetrate the intestinal mucosa without causing lesions. Behring's contention caused a great deal of discussion, and the question he raised is intimately bound up with the problem of the virulence of bovine tubercle bacilli for human beings, as he assumes that the infection is due to the use of infected milk.

COMBINED TABULATION, CASES REPORTED AND OWN SERIES OF CASES

(From Park and Krumwiede, loc. cit.)

\begin{tabular}{|c|c|c|c|c|c|c|}
\hline \multirow{2}{*}{ Diagnosis. } & \multicolumn{2}{|c|}{$\begin{array}{c}\text { Adults, } 16 \text { Years } \\
\text { and Uver. }\end{array}$} & \multicolumn{2}{|c|}{$\begin{array}{l}\text { Children, } \\
5 \text { to } 16 \text { Years. }\end{array}$} & \multicolumn{2}{|c|}{$\begin{array}{c}\text { Children } \\
\text { Under } 5 \text { Years. }\end{array}$} \\
\hline & Human. & Bovine. & Human. & Bovine. & Human. & Bovine. \\
\hline Pulmonary tuberculosis. . . . . . . . . . . & 568 & $1 ?$ & 11 & - & 12 & - \\
\hline Tuberculous adenitis, axillary or inguinal & 2 & - & 4 & - & 2 & - \\
\hline Tuberculous adenitis, cervical......... & 22 & 1 & 33 & 20 & 15 & 20 \\
\hline Abdominal tuberculosis. . . . . . . . . . & 15 & 3 & 7 & 7 & 6 & 13 \\
\hline $\begin{array}{l}\text { Generalized tuberculosis, alimentary ori- } \\
\text { gin } \ldots \ldots \ldots \ldots \ldots \ldots \ldots \ldots \ldots \ldots \ldots \ldots\end{array}$ & 6 & 1 & 2 & 3 & 13 & 10 \\
\hline Generalized tuberculosis............ & 28 & - & 4 & 1 & 28 & 5 \\
\hline $\begin{array}{l}\text { Generalized tuberculosis, including men- } \\
\text { inges, alimentary origin ............ }\end{array}$ & - & - & 1 & - & 3 & 8 \\
\hline 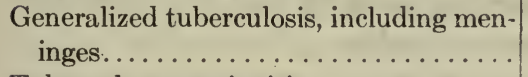 & 4 & - & 7 & - & 45 & 1 \\
\hline Tuberculous meningitis............ & - & - & 2 & - & 14 & 2 \\
\hline Tuberculosis of bones and joints. & 18 & 1 & 26 & 1 & 21 & - \\
\hline Genito-urinary tuberculosis. . . . . & 11 & 1 & 1 & - & - & - \\
\hline Tuberculosis of skin . . . . . . & 1 & - & 1 & - & 1 & - \\
\hline Miscellaneous Cases & & & & & & \\
\hline Tuberculosis of tonsils. . . . . . . . . . & - & - & - & - & - & - \\
\hline Tuberculosis of mouth and cervical nodes & - & 1 & - & - & - & - \\
\hline Tuberculous sinus or abscesses. . . . . . . & 2 & - & - & - & - & - \\
\hline Sepsis, latent bacilli.............. & - & - & - & - & 1 & - \\
\hline Totals... & 677 & 9 & 99 & 33 & 161 & 59 \\
\hline
\end{tabular}

Mixed or double infections, 4 cases. 
The problem is plainly of the greatest importance, and for this reason has been diligently investigated during the last few years. The only reliable method of approaching it has been to isolate the tulercle bacilli from diseased human beings and determine for each case whether the organism obtained belonged to the human or bovine tyre. These types can be differentiated definitely by cultural characteristics and pathogenicity; and it is not likely that the type changes during the sojourn in the human body. Granted this permanence of tyre, it is naturally of much value in revealing the source of an infecticn, to determine whether or not a human being is harboring a bacillus of the human type or one of the bovine type.

One of the most valuable contributions made to this problem during the last three years is that of Park and Krumwiede. ${ }^{33}$ The above tabulation is taken from their paper and represents a summary of their own cases and those reported by others.

From this table it is evident that out of a total of 1042 cases, 101 only were bovine in origin and over 50 per cent of these occurred in children under five years of age. Fifty-one out of the 59 cases occurring in the 161 infants were directly or indirectly traced to alimentary infection.

It seems reasonably accurate, therefore, to state the case as follows: Human adults are relatively insusceptible to bovine infection. Such infection can take place, but is unusual. Below sixteen years of age the human race is relatively more susceptible and up to this age the danger of milk infection is unquestionably great, this source accounting for about one-third of the cases. Below five years the danger is greatest. This table alone should form sufficient evidence to silence absolutely any doubts as to the dangers of milk infection and remove any objections to the most rigid sanitary control of milk supplies.

On the other hand, it also shows that Behring's original claims were far too sweeping and can not be upheld.

Kober ${ }^{34}$ also calls attention to the fact that one must not be deceived into believing that childhood is the only really dangerous age for infection with tuberculosis, and quotes the results of a French committee which, in a small group carefully investigated, found 64 cases in which the disease was transmitted from husband to wife, 43 cases in which it was transmitted from wife to husband, 38 cases transmitted from brother

${ }^{33}$ Park and Krumwiede, Jour. of Med. Res., Oct., 1910.

s Kober, Repr. No. 309, U. S. P. H. S. Reports, October, 1915. 
to sister, 19 from mother to child, 16 from other relatives, and in 33 cases it was traced to people who were not relations, but with whom the patient had been in communication. He also quotes Zasetsky who reports the case of a tuberculous woman who, in the course of eleven years married three husbands who had been previously healthy. The first one died of tuberculosis seven years after marriage, the second three years later, and the third at the time of the report had the disease, the wife in the meantime having died of tuberculosis.

The fact that tubercle bacilli may be conveyed in dust has been indicated above, but there are other means by which habitual inhalation of dust may favor the spread of tuberculosis, namely, by virtue of the irritant properties of inhaled dust in predisposing the lung to infection. Attention to the dangers of trades in which dust is an habitual environmental factor has been particularly emphasized by Winslow and Greenberg. Sommerfeld, whom we quote from Kober, made a statistical study in which he showed that in the population of Berlin, the average tuberculosis death rate was 4.93 per 1000 . The rate in the non-dusty trades, was 2.39 , and in the dusty trades 5.2. He also states that the analysis of tuberculosis in the towns in Vermont where granite and marble "cutting is carried out showed a tuberculosis rate of 2.2 per 1000 against a rate of 1.3 for the whole state, and Röpke is stated by the same writer to have shown that the mortality from tuberculosis of the population in the large cutlery center, Solingen, in Germany was reduced from 5.4 per 1000 in 1885 , to 1.8 in 1910 , by measures aimed at the control of the dust in work rooms. A recent study by Drury ${ }^{35}$ shows that polishers and grinders in axe factories are subject to a death rate from pulmonary tuberculosis considerably above that of others in the same mill. In the two decades from 1900 to 1919 , the polishers and grinders showed a death rate of 19 per 1000 as against 6.5 of the entire mill population, and between 1 and 2.4 of the general population of the district.

In regard to the predisposing factors to tuberculous infection, many phases enter into the problem in this disease which exert a much less direct or perhaps negligible influence in connection with other infections. There can be no question about the fact that poverty, with its coincident crowding in living quarters, close personal contact at night, insufficient warmth, and particularly undernutrition and low fat diet, play a rôle

${ }^{35}$ Drury, Pub. Health Reeports, U. S. P. H. S., Feb., 1921, Vol. 36, No. 5. 
of immense importance in tuberculosis. In no disease is prevention so intimately influenced by general sociological and economic improvement as in tuberculosis.

Wernicke ${ }^{36}$ in a study of the relationship of diseases to social conditions shows an almost direct relationship between the provision of air space and parks in cities to tuberculosis. The statistics of the influence of the war upon tuberculosis have not yet become available for study, but it is important to note that at the present writing we are informed by sanitarians who have returned from Europe that the sanitary problem in the European States is very largely one of tuberculosis, and that the effects of prolonged undernutrition, especially upon children during the war years has resulted in an enormously increased tuberculosis rate.

The question of the inheritance of tuberculosis has frequently been raised, and a large literature on this subject has accumulated, but an analysis of this literature seems to show that inheritance must be regarded as predisposition rather than as a method of direct infection. Children of tuberculous parents are likely to be more susceptible to tuberculosis and, of course, are exposad to tuberculosis more intimately during the early years of life than are children of normal parents. There is no direct proof that tuberculosis is transmitted from mother to the fœtus:

Prevention.-As to preventive measures, we must refer the reader to special books on the subject since the problem is too large to be dealt with briefly with anything like completeness. The following summary of preventive measures is based largely upon the conclusions reached in Kober's discussion of this subject:

We may assume, as premises for prevention, that tuberculosis can be transmitted at all periods of life and that foci acquired in youth may be arrested but light up under conditions of general undernutrition, malnutrition, etc., etc., in later years. Infection may be direct from person to person, indirect through contaminated food, fomites, flies; through dust, and in childhood through milk from infected cattle. The most common manner of acquiring tuberculosis is by inhalation and, next to that, probably tliruugh the digestive tract.

The most important factor in the prevention of tuberculosis is education. This must elucidate the methor of infection and the importance of the economic and sociological factors as they effect habits of food, sleep and fresh air.

${ }^{36}$ Wernicke, quoted from Kober. 
Direct infection must be prevented by compulsory notification, care and disinfection of expectorations, isolation, at least as far as the possibilities of sputum infection are concerned, in the home, in hospitals, etc., with introduction of pocket sputum flasks and the other simple measures by which a well-controlled tuberculosis patient can avoid infecting others. The actual prevention in deed, as well as word, of expectoration in public places, the protection of public drinking places, introduction of individual cups and public cleanliness in general; especial supervision of these conditions in places of public lodgment and public amusement in schools and public conveyances; introduction of vacuum cleaning, etc.

Attention in regard to the marriage of tuberculous people.

Supervision of dairies and the marketing of milk by governmental grading, and control, with pasteurization of suspicious milk or milk not produced under the required conditions for grade "A" milk.

Public provision for the proper and humane care of tuberculous people in state and municipal sanatoria so arranged that the poor will regard them as havens of hope, rather than as penalties imposed for disease.

The public must be educated in knowing that tuberculosis is a curable disease, provided that the diagnosis is made early and clinical facilities must be so arranged in cities so that accurate diagnosis in the early stages may be made and proper fresh air and nutritional care instituted if necessary, at public expense. In our cities, roofs, playgrounds, parks, etc., should be provided for school children. Summer care of children living in the crowded districts must be developed on a more generous and more important scale. The nutrition of school children in the public schools must be supervised and subsidized so that no child in a civilized community should suffer at any time from under nutrition.

The prevention of tuberculosis is only in small part a medical problem and must rest in its last analysis on the prevention of the means of direct infection and the predisposing considerations in which the sociologist and the educator must play as important a part as the physician.

Chemical Analysis of Tubercle Bacilli. ${ }^{37}$-Diligent efforts by many investigators to isolate the specific toxins which lend tubercle bacilli their pathogenic properties have led to careful chemical analysis of the organisms. About 85.9 per cent of the bacillus consists of water; 20

${ }^{37}$ Hammerschlag, Cent. f. klin. Med., 1891; Weyl, Deut. med. Woch., 1891; De Schweinitz and Dorset, Jour. Amer. Chem. Soc., 1895; Hammerschlag, loc. cit. 
to 26 per cent of the residue can be extracted with ether and alcohol. This material consists of fatty acids and waxy substances (fatty acids in combination with the higher alcohols). The residue after alcoholether extraction is composed chiefly of nitrogenous constituents. These can be extracted with dilute alkaline solutions, and consist chiefly of nucleoproteins. After removal of the so-called nucleoproteins-that is the material which comes down on trcatment with dilute acetic acid in the cold, there remains a small amount of coagulable protein and, we have recently observed, small amounts of a substance that reacts like Bence-Jones protein. ${ }^{38}$ The final residue contains alcohol precipitable substances-proteoses and polypeptids that constitute the active substances of the tuberculin reactions. Cellulose is also found and is supposed to represent the framework of the cell membrane, and there is an ash rich in calcium and magnesium.

Toxins of the Tubercle Bacillus.-The Tuberculins.-Filtrates of bouillon cultures of Bacillus tuberculosis ${ }^{39}$ will occasionally produce slight emaciation when injected into guinea-pigs, and when administered to tuberculous subjects in sufficient quantity will give rise to marked increase of temperature. It is likely, therefore, that the tubercle bacillus actually secretes a soluble toxin. ${ }^{40}$

The chief toxic principles, however, of Bacillus tuberculosis are probably endotoxins or bacterial proteins, bound during cell life to the body of the bacillus. Dead bacilli will produce sterile abscesses when injected into animals. Prudden and Hodenpyl, ${ }^{41}$ Straus and Gamaleia, ${ }^{42}$ and others, ${ }^{43}$ moreover, have shown that the injection of dead and carefully washed cultures of this bacillus will produce lesions histologically similar to those occurring after infection with the living germs, and will often lead to marasmus and other systemic symptoms of poisoning.

The hope of actively immunizing with substances obtained from dead bacilli led Koch to employ various methods of extraction of cultures for the manufacture of tuberculin.

"Old Tuberculin"44 (Koch) ("T.A.K.").--The first tuberculin made

${ }^{88}$ Zinsser, Journ. exp. med., Nov., 1921.

${ }^{39}$ Straus and Gamaleia, Arch. méd. exp., 1891.

40 Denys, “Le Bouillon Filtré,", Louvain, 1905.

${ }^{41}$ Prudden and Hodenpyl, N. Y. Med. Jour., June, 1891; Prudden, ibid., Dec. 5.

${ }^{42}$ Straus and Gamaleia, loc. eit.

${ }^{43}$ Mafucci, Cent. f. allg. Path., 1890.

${ }^{44}$ Koch, Cent. f. Bakt., 1890; Deut. med. Woch., 1891. 
by Koch is produced in the following manner: Tubercle bacilli are grown in slightly alkaline 5 per cent glycerin-pepton bouillon for six to eight weeks. At the end of this time, growth ceases and the corrugated pellicle of tubercle bacilli, which during growth has floated on the surface, begins, here and there, to sink to the bottom. The entire culture is then heated on a water-bath at about $80^{\circ} \mathrm{C}$., until reduced to one-tenth of its original volume. It is then filtered either through sterile filter paper or through porcelain filters. The resulting filtrate is a rich brown, syrupy fluid, containing the elements of the original culture medium and a 50 per cent glycerin extract of the tubercle bacilli. While the glycerin is of sufficient concentration to preserve it indefinitely, 0.5 per cent phenol may be added as an additional precaution. Dilutions of this fluid are used for diagnostic and therapeutic purposes.

"New Tuberculin"45 (Koch) (TA, TO, TR).-Koch believed that the immunity resulting from treatment with the old tuberculin was purely an antitoxic immunity, devoid of all antibacterial action. The use of whole dead tubercle bacilli for immunization purposes, however, was impracticable; because, injected subcutaneously, they were not absorbed, and introduced intravenously they were deposited in the lungs and gave rise to lesions. Koch was led, therefore, to resort to more energetic extraction of the bacilli in the hope of procuring a substance which could be easily absorbed and would at the same time give rise, when injected, to antibodies more definitely bactericidal. By extracting tubercle bacilli with decinormal $\mathrm{NaOH}$, for three days, filtering through paper and neutralizing, he obtained his TA (alkaline tuberculin). This preparation seemed to fulfill some of the hopes of its discoverer, but had the disadvantage of often producing abscesses at the points of injection. Koch then resorted to mechanical trituration of the bacilli. The method he subsequently followed for tuberculin production is now extensively used, and is carried out as follows: ${ }^{46}$

Virulent cultures of tubercle bacilli are dried in vacuo and thoroughly ground in a mortar. Grinding is continued until stained preparations reveal no intact bacilli. (This is done by machinery in all large manufactories.) One gram of the dry mass is shaken up in 100 c.c. of sterile distilled water. This mixture is then centrifugalized at high speed. The supernatant fluid, known as TO (Tuberculin-Oberschicht), contains the water-soluble constituents of the bacillus, gives no precip-

${ }^{45}$ Koch, Deut. med. Woch., 14, 1897.

${ }^{46}$ Ruppel, Lancet, March 28, 1908. 
itate on the addition of 50 per cent glycerin, and has the same physiological action as the old tuberculin. The residue TR (Tuberculin-Rückstand), after pouring off TO, is again dried and ground up, and again shaken in water and centrifugalized. This process is repeated several times, and eventually, after three or four repetitions, all the TR goes into emulsion. The total volume of water used for these TR extractions should not exceed 100 c.c. All of the TR emulsions are then mixed together. This gives TR a precipitate with 50 per cent of glycerin, and is supposed by Koch to contain substances important in producing an antibacterial immunity. For purposes of standardization, the amount of solid substance in 5 c.c. of the TR is determined by evaporation in vacuo and drying. To the rest are added a little glycerin and formaldehyd and enough water to allow each cubic centimeter of the solution to contain 0.002 gram of solid material. Thus the culture and the medium remaining the same, fairly accurate standardization is possible.

"New Tuberculin-bacillary Emulsion." 47 -In 1901, Koch combined "TO" and "TR" by putting forth a preparation referred to as "Bazillenemulsion." This consists of an emulsion of pulverized bacilli $1: 100$ in distilled water. After several days of sedimentation to remove the coarser particles, the supernatant fluid is poured off and fifty per cent volume of glycerin is added to it for purposes of preservation. This preparation contains 5 milligrams of solid substance in each cubic centimeter.

Bouillon Filtré (Denys). ${ }^{48}$ - This preparation consists of the filtrate (through Chamberland filters) of 5 per cent glycerin-pepton-bouillon cultures of Bacillus tuberculosis. Phenol 0.25 per cent is added to insure sterility. The filtered bouillon corresponds to the unconcentrated old tuberculin of Koch, but, not having been heated, is supposed by Denys to contain important soluble and possibly thermolabile secretory products of the bacillus.

Tuberculoplasmin (Buchner and Hahn). ${ }^{49}$-Buchner and Hahn, by crushing tubercle bacilli by subjecting them to a pressure of 400 atmospheres, obtained a cell-juice in the form of an amber fluid, to which they attributed qualities closely analogous to those of TR.

Other tuberculins are those of Beraneck, ${ }^{50}$ highly recommended

${ }^{47}$ Koch, Deut. mer. Worh., 1901.

4s Denys, "Le Bouillon Filtré,', Louvain, 1905.

${ }^{49}$ Buchner und IIahn, Münch. med. Woch., 1897; Hahn, ibid.

5o Beraneck, Compt. rend. de 1'acad. des. sei., 1903. 
clinically by Sahli, ${ }^{51}$ that of Klebs, ${ }^{52}$ and the tubereulin produced from bovine tubercle bacilli by Spengler. ${ }^{53}$

Diagnostic Use of Tuberculin.-Subculancous•Use.-The preparation usually employed for diagnostic purposes is Koch's "Old Tuberculin" (Alttuberculin). This preparation is administered by hypodermic injection of small quantities obtained by means of dilutions. The dilutions are best made with a 0.5 per cent aqueous carbolic acid solution. In practice a 1 per cent solution is made by pipetting 0.1 c.c. of tuberculin into 9.9 c.c. of the 0.5 per cent carbolic solution. A cubic centimeter of this then contains 0.01 c.c. of tuberculin. One cubic centimeter of this solution added to 9 c.c. of 0.5 per cent carbolic acid gives a solution in which each cubic centimeter contains 0.001 c.c., or $1 \mathrm{~mm}$. of tuberculin.

The initial dosage in adults in Koch's ${ }^{54}$ early work, and as used by Beck $^{55}$ on a large number of patients, was $1 \mathrm{mgm}$. This, according to present opinions, is too high, and most clinicians to-day prefer 0.1 to 0.2 of a milligram. If after three or four days no reaction has occurred, a second dose of $1 \mathrm{~mm}$. is given.

The reaction itself is recognized chiefly by the changes in temperature. In a positive reaction the patient's temperature will begin to increase within six to eight hours after injection, rising sharply within a few hours to 0.5 or $1.5^{\circ}$ higher than the temperature before injection. It then sinks more gradually than it rose, the reaction usually being complete within thirty to thirty-six hours. With the temperature there may be nausea, a chill, rapid pulse, and general malaise. Locally visible tuberculous processes, such as lupus, lymph nodes, etc., may become tender or swollen, and if the tuberculosis is pulmonary, there may be coughing and increased expectoration. The temperatures of persons subjected to the test should be taken regularly for three or four days before tuberculin is used.

Ophthalmo-tuberculin Reaction.-Wolff-Eisner ${ }^{56}$ and, soon after him, Calmette, ${ }^{57}$ proposed a method of using tuberculin for diagnostic purposes by instillation into the conjunctival sac. In tuberculous

\footnotetext{
${ }^{51}$ Sahli, Corrbl. d. Schw. Aerzte, 1906.

5. Klebs, Cent. f. Bakt., 1896 ; Deut. med. Woch., 1907.

${ }^{5}$ Spengler, Deut. med. Woch., xxxi, 1904; xxxi and xxxiv, 1905.

${ }^{64}$ Koch, Deut. med. Woch., 1890.

${ }^{55}$ Beck, Deut. med. Woch., 1899.

${ }^{5}$ Wolff-Eisner, Berl. med. Gesell., May 15, 1907.

s7 Calmette, Acarl. des sei., June 17, 1907 .
} 
patients this process is followed by a sharp conjunctival congestion lasting from one to several days.

The preparation used for this purpose is produced in the following way:

"Old Tuberculin" is treated with double its volume of 95 per cent alcohol, the precipitate allowed to settle and the alcohol then filtered off through paper. The sediment is washed with 70 per cent alcohol until the filtrate runs clear, then pressed between layers of filter paper to remove excess of moisture, scraped into a dish, dried in vacuo over $\mathrm{H}_{2} \mathrm{SO}_{4}$, and broken up in a mortar under a hood.

Solutions of the powder are made in sterile normal salt solution, 1 per cent by weight, boiled and filtered. The solutions are used in strengths of 0.5 to 1 per cent, a drop of which is instilled into the conjunctival sac. 58

Cutaneous Tuberculin Reaction.-Von Pirquet ${ }^{59}$ has suggested the cutaneous use of tuberculin for diagnostic purposes. A 25 per cent solution of "Old Tuberculin" was first used. At present the undiluted substance is employed.

After sterilization of the patient's forearm, two drops of this solution are placed upon the skin about $6 \mathrm{~cm}$. apart. Within each of these drops scarification is done, and the skin between them scarificed as a control. Within twenty-four to forty-eight hours, in tuberculous patients, erythema, small papules, and herpetiform vesicles will appear. According to recent investigations, about 70 per cent of adults show a positive reaction. This diminishes its diagnostic value for adults.

Moro ${ }^{60}$ has modified this by making a 50 per cent ointment of tuberculin in lanolin and rubbing it into the skin without scarification.

Complement Fixation in Tuberculosis. $^{61}$-The problem of complement fixation for diagnostic purposes in tuberculosis has been very actively investigated of recent years. The most promising results have been reported with an antigen made by Besredka of a filtrate of an egg-meat-broth, upon which the tubercle bacilli had been grown for several weeks with a similar filtrate of cultures on a watery extract of potato with glycerin, used by Petroff, and with an antigen made by Miller and Zinsser by triturating dead tubercle bacilli with dry crystals

ss The conjunctival test is not in general use at the present time owing to possible dangers to the eyes.

${ }^{59}$ v. Pirquet, Berl. klin. Woch., xx, 1907; Med. Klinik, xl, 1907.

6o Moro, Münch, med. Woch., 1906, p. 216.

${ }^{61}$ H. R. Miller, Jour. Lab. \& Clin. Merl., 1916, i, 816. 
of $\mathrm{NaCl}$ and adding distilled water to isotonicity. Craig, Bronfenbrenner and the above-named writers have reported good results with these various antigens, and, although it is too early to say which will prove most useful, it is clear that complement fixation methods can aid in the diagnosis of active tuberculosis. We can, of course, judge concisely only of the method used in our laboratory, where Miller has followed carefully a considerable number of cases on which the method has been used. It would appear at present that about 70 per cent of the fixation results correspond with clinical findings.

The Tuberculin Test as Applied to Cattle.-In cattle, the symptoms of tuberculosis are not easily detected by methods of physical diagnosis until the disease has reached an advanced stage. In consequence, cows may be elements of danger without appearing in any way diseased. In consequence, routine examination of herds by the tuberculin test has become one of the necessary measures of sanitation. According to Mohler, ${ }^{62}$ an accurate diagnosis may be established in at least 97 per cent of the cases. It is natural that a good deal of objection to the test is encountered on the part of dairy farmers and cattle raisers, and it has been claimed that the cattle are injured by the test. There is, however, no scientific basis for this belief, if the test is carried out carefully and intelligently. As a matter of fact, the systematic use of the test would eventually be distinctly advantageous to the owners of the cattle themselves, since it has been shown that cows, even in the early stages of the disease, may expel tubercle bacilli, either during respiration or with the feces, and thus become a menace to healthy members of the herd.

The tuberculin test on cattle should be made as follows: (The directions given below are taken directly from the circular sent out from the Bureau of Animal Industry at Washington.)

1. Begin to take the rectal temperáture at $6 \mathrm{A.M} .$, and take it very two hours thereafter until midnight.

2. Make the injection at midnight.

3. Begin to take the temperature next morning at 6 A.M., and continue as on preceding day.

To those who have large herds to examine, or are unable to give the time required by the above directions, the following shortened course is recommended:

1. Begin to take the temperature at 8 A.M., and continue every 2 hours until 10 P.M. (omitting at 8 P.M., if more convenient); or take the temperature at 8 A.M., 12 м., and 10 P.M.

${ }^{62}$ Mohler, Pub. H. and Mar. Hosp. Serv. Bull., 41, 1908. 
2. Make the injection at 10 P.M.

3. Take the temperature next morning at 6 or 8 A.M., and every two hours thereafter until 6 or 8 Р.м.

Each adult animal should receive 2 c.c. of the tuberculin as it is sent from the laboratory. (The tuberculin sent out from the central laboratory at Washington is already diluted; 2. c.c. represents 0.25 c.c. of the concentrated "Old Tuberculin" of Koch.) Yearlings and two-year-olds, according to size, should receive from 1 to 1.5 c.c. Bulls and very large animals may receive three c.c. The injection should be made beneath the skin of the neck or shoulders behind the scapula, after washing the area with a weak carbolic acid solution.

There is usually no marked local swelling at the seat of the injection.

There are now and then uneasiness, trembling, and frequent passage of softened dung. There may also be slight acceleration of the pulse and of breathing.

The febrile reaction in tuberculous cattle following the subcutaneous injection of tuberculin begins from six to ten hours after the injection, reaches the maximum nine to fifteen hours after the injection, and returns to normal eighteen to twenty-six hours after the injection.

A rise of two or more degrees Fahrenheit above the maximum temperature observed on the previous day should be regarded as an indication of tuberculosis. For any rise less than this a repetition of the injection after four or six weeks is highly desirable.

It is hardly necessary to suggest that for the convenience of the one making the test the animals should not be turned out, but fed and watered in the stable. It is desirable to make note of the time of feeding and watering and of any temperature fall after watering.

The tuberculin should. not be used later than six weeks after the date on the bottle, nor if there is a decided clouding of the solution.

Therapeutic Uses of Tuberculin.-Tuberculin was first used therapeutically, by Koch, ${ }^{63}$ shortly after its discovery. Hailed with the most optimistic enthusiasm, its possibilities were overestimated and hopeless cases were treated unskillfully, with unsuitable dosage. The consequence was that harm was done, the method was attacked by Virchow and others and the new therapy fell into almost complete neglect. At present, the use of tuberculin has again been revived, but with greater caution and with a thorough understanding of its limitations. The tendency has been toward smaller dosage and the limitation of the

${ }^{63}$ Koch, Deut. med. Woch., iii, 1891. 
agent to early cases. No two institutions use tuberculin in exactly the same manner, and it is, therefore, impossible to do more than outline the general scheme of treatment. It must never be forgotten, however, that all forms of tuberculin treatment consist in an "active immunization" in which, for the time being, the toxemia of the patient is increased rather than neutralized. It is obvious, therefore, that only cases in which the process is not a very acute one, are at all suitable for treatment. The general principle of modern tuberculin therapy seems to lie in choosing doses so small that no marked general reaction shall follow. The preparations most frequently employed are Koch's "Alttuberculin," his "TR," his "Neu Tuberkulin-Bazillen Emulsion," and the Bouillon filtré of Denys. Initial doses of Alttuberculin range from 0.1 to 0.01 of a milligram. In case of complete absence of a reaction, the injection may be repeated, gradually increasing, about twice a week. The occurrence of a reaction should be the signal for a longer interval and a slower advance in the size of the dose.

The initial dose of "TR" is, as advised by Koch, ${ }^{64}$ about $0.002 \mathrm{mgm}$. This usually causes no reaction. The dose is doubled, at reasonable intervals, up to $1 \mathrm{mgm}$. After this, further increase is carefully gauged by the clinical indications. The maximum dose is about $20 \mathrm{mgm}$.

"Neu Tuberkulin-Bazillen Emulsion," 65 is begun with a dose of $0.001 \mathrm{mgm}$. Gradual increase as with the other preparations is then practiced. The maximum dose is about $10 \mathrm{mgm}$.

Bouillon filtré has been used chiefly by Denys ${ }^{66}$ who claims excellent results. Denys is very emphatic in advising the absolute avoidance of any reaction. He begins with a millionth or even the tenth of a millionth of a cubic centimeter of the bouillon and increases with extreme caution. His dilutions are made with glycerin broth.

Active immunization with tubercle bacilli of reduced virulence has been suggested and attempted at various times but, so far, without definite success. No strikingly favorable results can be justly claimed for any of these preparations.

Passive Immunization in Tuberculosis.-Numerous attempts have been made to immunize tuberculous subjects with the sera of actively immune

${ }^{84}$ Koch, Deut. med. Woch., xiv, 1897 .

${ }^{85}$ Bandelier and Roepke, "Lehrb. d. specifisch, Tub. Ther ${ }_{s}$," Würzburg, 1908; Koch, Deut. med. Woch., 1901.

"Denys, "Le Bouillon filtré,"' Louvain, 1905. 
animals. The most widely used method of producing such serum is that of Maragliano.

Maragliano's Serum. ${ }^{67}$-Maragliano believes that a toxalbumin is present in tubercle-bacillus cultures which is destroyed by the heating employed in the usual tuberculin production. He procures this substance by filtration of unheated cultures and precipitation with alcohol (tossina præcipitata). He furthermore makes an aqueous extract of the bacillary bodies. With these two substances he immunizes horses. He draws blood from these after four to six months of treatment. The serum is extensively used in Italy. Its value is, at present, very doubtful.

Marmorek's Serum. ${ }^{68}$-Marmorek claims that the poisons produced by Bacillus tubereulosis depend largely upon the medium on which it is grown. He adyanced the view in 1903 that the substances obtained in tuberculin were not the true toxins of the tubercle bacillus, that there was a marked difference between these and the poisons elaborated by a younger (primitive) phase of the bacillus as it occurs only within the animal body or on media composed of animal tissue. He consequently grows his cultures on a medium composed of a leucotoxic serum (produced by inoculating calves with guineapig leucocytes) and liver tissue. Such cultures, he claims, contain no tuberculin. To the sera produced by immunization with these cultures he attributes ligh curative powers.

We may say with considerable confidence at the present time that no method of passive immunization in tuberculosis has, up to the present, had any degree of success.

Bacilli Closely Related to the Tuberculin Bacillus.-The Bacillus of Bovine Tuberculosis.-Tuberculosis of cattle (Perlsucht) was studied by Koch ${ }^{69}$ in connection with his early work on human tuberculosis. Koch -did not fail to recognize differences between the reactions to infection in the bovine type of the disease and that of man. He attributed these, however, to the nature of the infected subject rather than to any differences in the infecting agents. This point of view met with little authoritative contradiction, until Theobald Smith, ${ }^{70}$ in 1898 , made a systematic comparative study of bacilli isolated from man and

${ }^{67}$ Maragliano, Perl. klin. Woch., 1899; Soc. de biol., 1897.

${ }^{68}$ Marmorek, Berl. klin. Woch., 1903; p. 1108; Med. Klinik, 1906.

${ }^{69}$ Koch, Arb. a. d. kais. Gesundheitsamt, 11, 1882.

${ }^{70}$ Th. Smith, Jour. Exp. Med., 111, 1898. 
from cattle and pointed out differences between the two types. The opinion of Smith was fully accepted by Koch ${ }^{71}$ in 1901.

Since that time, the question, because of its great importance to prophylaxis, has been the subject of many investigations, most of them confirming Smith's original work. Morphologically, Smith 72 found that the bovine bacilli were usually shorter than those of the human type and grew less luxuriantly than these upon artificial media. He determined, furthermore, that, grown upon slightly acid glycerin bouillon, the bovine bacillus gradually reduces the acidity of the culture medium until the reaction reaches neutrality or even slight alkalinity. Fluctuations after this do not exceed 0.1 to 0.2 per cent on either side of neutrality. In the case of the human bacillus, on the other hand, there is but slight reduction of the acidity during the first weeks of growth; after this acidity increases and, though subject to fluctuations, never reaches neutrality. This behavior is probably due to action exerted upon the glycerin, since on ordinary bouillon no such differences between the two varieties can be noticed. These observations of Smith were confirmed by Ravenel, ${ }^{73}$ Vagedes, ${ }^{74}$ and others.

The cultural differences between the two types have been studied with especial care by Wolbach and Ernst, ${ }^{75}$ and Kossel, Weber, and Heuss. $^{76}$ All of these observers bear out Smith's contention that luxuriance and speed of growth are much more marked in the human than in the bovine variety. Marked differences, furthermore, have been shown to exist in the pathogenic properties of these bacilli toward various animal species.

Guinea-pigs inoculated with the bovine type ${ }^{77}$ die more quickly and show more extensive lesions than those infected with human bacilli. The difference in the pathogenicity of the two organisms for rabbits is sufficiently striking to be of diagnostic value. The bovine bacilli usually kill a rabbit within two to five weeks; the human bacilli produce a mild and slow disease, lasting often for six months, and occasionally fail to kill the rabbits at all.

The practical importance of distinguishing between the two types,

71 Koch, Deut. med. Woch., 1901.

${ }^{72}$ Th. Smith, Jour. Exp. Med., 1905.

${ }^{73}$ Ravenel, Lancet, 1901; Univ. Penn. Med. Bull., 192.

${ }^{74}$ Vagedes, Zeit. f. Hyg., 1898.

75 Wolbach and Ernst, "Studies from the Rockefeller Inst.,' 11, 1904.

${ }^{76}$ Kossel, Weber, und Heuss, Arb. a. d. kais. Gesundheitsamt, 1904 and 1905.

${ }^{77} \mathrm{Smith}$, loc. eit., and Medical News, 1902. 
of course, attaches to the question as to whether the bovine and the human disease are mutually intercommunicable. This has been discussed in the preceding section dealing with the human type.

Summary of the Differentiation between Bovine and Human Tubercle Bacilli.-Morphologically the bovine bacillus is a little plumper and thicker than the human type, but this cannot be regarded as sufficiently constant to be reliable for differentiation. On glycerin broth, the final reaction in the case of human bacilli is considerably acid, the final reaction with the bovine is very slightly above the neutral point. The bovine bacillus does not grow as readily as the human and is not aided by the addition of glycerin to the media to the same extent as the human. The growth of the human hacillus is apt to be more luxuriant than that of the bovine, especially in earlier generations.

As to virulence, the bovine is much more virulent for all the ordinary laboratory animals than is the human. The difference is particularly marked in rabbits. Small doses of human bacilli inoculated into rabbits will kill them very late, and if quantities of less than 0.1 of a milligram are used intravenously, the rabbits may live for longer than two months, or may survive. Similar injection of the bovine type into rabbits kills with greater regularity and more extensive lesions, usually within two months.

The Bacillus of Avian Tuberculosis.-A disease resembling in many features the tuberculosis of man is not uncommon among chickens, pigeons, and some other birds. Koch was the first to discover in the lesions of diseased fowl, bacilli much resembling Bacillus tuberculosis. It was soon shown, however, by the studies of Nocard and Roux, ${ }^{78}$ Mafucci, ${ }^{79}$ and others, that the bacillus of the avian disease represented a definitely differentiable species.

Morphologically, and in staining characteristics, the bacillus is almost identical with that of the human disease. In culture, however, growth is more rapid and takes place at a temperature of $41^{\circ}$ to $45^{\circ} \mathrm{C} .80$ (the normal temperature of birds), while the human type is unable to thrive at a temperature above $40^{\circ}$.

The organisms grow more easily than do either the human or bovine bacilli. Colonies appear, on glycerin agar within a week and cultivation may also be successful on media without glycerin. It is char-

${ }^{78}$ Nocard et Roux, Ann. de 1'inst. Pasteur, 1887.

${ }^{79}$ Mafucci, eit. f. Hyg., xi.

${ }^{80} \mathrm{Mafucci}$, loc. eit. 
acteristic of the avian type that cultures on liquid media (glycerin broth) grow as readily within the liquid as on the surface and may even become homogeneous.

Guinea-pigs, very susceptible to human tuberculosis, are very refractory to infection with the avian type; while, on the other hand, rabbits which are resistant to the human type, succumb rapidly to infection with avian tuberculosis. ${ }^{81}$ Prolonged cultivation and passage through the mammalian body is said to cause these bacilli to approach more or less closely to the mammalian type. Conversely, Nocard ${ }^{82}$ claims to have succeeded in rendering mammalian tubercle bacilli pathogenic for fowl by keeping them in the peritoneal cavities of hens in celloidin sacs for six months.

Recently Koch and Rabinovitsch ${ }^{83}$ have isolated from the spleen of a young man dead of tuberculosis, a microorganism. which, culturally, morphologically, and in its pathogenic action upon birds, seemed to belong to the avian type. Löwenstein ${ }^{84}$ describes a similar organism cultivated from a human case which seems to be a transitional type. Observations of this order are, however, too few at the present time to be used as the basis of a definite opinion as to the relationship between the two varieties.

Tuberculosis in Cold-blooded Animals.-The bacillus isolated by Dubarre and Terre ${ }^{\$ 5}$ resembles Bacillus tuberculosis in morphology and in a certain degree of acid-fastness. It grows at low temperatures, $15^{\circ}$ to $30^{\circ} \mathrm{C}$. It is non-pathogenic for animals, but kills frogs within a month. Except for the acid-fastness it has little in common with Bacillus tuberculosis.

Similar acid-fast bacilli have been isolated from other cold-blooded animals (carp, frogs, turtles, snakes) by many observers.

There have been many attempts to show a close relationship between the tubercle bacilli of cold-blooded and those of warm-blooded animals. Moeller, Hansemann, Friedmann, Weber, Küster, and others have given this subject particular attention and it has gained especial interest because of the recent notorious claims of Friedmann that he had succeeded in obtaining, from turtles, a strain of acid-fast bacilli which could

81 Straus et Gamaleia, Areh. de méd. expér., 1891; Courmont et Dor, Areh. de mér. exp., 1891.

82 Norard, Ann. de 1 'inst. Pasteur, 1898.

${ }^{83}$ Koch und Rabinovitsch, Virch. Arch., Beiheft to Bd. 190, 1907.

${ }^{84}$ Lïuenstein, quoted from Koeh and Rabinovitsch, loe. eit.

${ }^{85}$ I) ubarre et Tcrre, Compt. rend. de la soc. de biol., 1897. 
be successfully used in actively immunizing human beings. In 1903 Friedmann ${ }^{86}$ described two cases of spontaneous infection of a salt-water turtle (Chelone corticata) with acid-fast bacilli, presenting lesions in the lungs which simulated pulmonary tuberculosis in the human being (cavity formation and miliary nodules). The organisms cultivated from these lesions presented much similarity to those of the human type and, according to Friedmann, ${ }^{87}$ unlike other acid-fast bacilli of cold-blooded animals, could be grown at $37.5^{\circ} \mathrm{C}$. As a possible human origin for the turtle infections Friedmann mentions that the attendant who fed these turtles suffered from a double pulmonary tuberculosis.

Upon inoculation into guinea-pigs localized lesions only were produced, and dogs, rats, and birds were immune. The implication of Friedmann's work is that his culture represented a human strain attenuated for man by passage through the turtle, although, as far as we are aware, no definite statement as to this was made.

Summarizing the work of many investigators (Weber, Taute, Küster, Allegri, Bertarelli, and others) Küster ${ }^{88}$ makes a statement which is, in essence, as follows: In the carp, in snakes, turtles, and frogs spontaneous "tuberculosis" may occur. The organisms which cause these diseases are specific for cold-blooded animals, similar in many respects to the tubercle bacillus of warm-blooded animals, but in the latter do not produce progressive disease. Human, bovine, and avian tubercle bacilli inoculated into cold-blooded animals can produce lesions which histologically simulate tuberculosis. These microorganisms can remain a year in cold-blooded animals without losing their pathogenicity for guinea-pigs. Mutation of the tuberele bacillus of warm-blooded animals into cold-blooded ones has not been proven.

For these reasons it is quite impossible to exclude, in the apparently positive work of Friedmann and others, the isolation of a true "coldblooded" type organism, rather than a mutation form originally of the warm-blooded type. What Friedmann's present claims in this respect are for his culture has not been stated as far as we know. The possibility of a positive immunizing value of organisms isolated from cold-blooded animals in human beings, though remote, is not out of question. The problem is so serious and important, and the experience

${ }^{86}$ Friedmann, D. Med. Woch., No. 2, Jan., 1903, 25.

${ }^{87}$ Friedmann, D. Med. Woch., No. 26, 464, 1903, and Centralbl. f. Bakt., I, xxxiv, 1903, also Zeitschr. f. Tuberkulose, iv, Heft 5, 1903.

88 Kolle und Wassermann's Handbuch, $2 d$ edition, v, 767. 
of many workers is, so far, so inconclusive that the time has not come for commercial exploitation and the cruel deceptions of false hopes. The subject, however, deserves carefully controlled further investigations.

Bacillus of Timothy.-Moeller isolated from timothy-grass and from the dust in haylofts acid-fast bacilli, like Bacillus tuberculosis. They grow rapidly on agar, soon showing a deep red or dark yellow color.

Bacillus butyricus (Butter Bacillus).--Slightly acid-fast bacilli resembling Bacillus tuberculosis have been isolated from milk and butter by Petri, ${ }^{89}$ Rabinovitsch, ${ }^{90}$ Korn, ${ }^{91}$ and others.

These bacilli are easily differentiated from Bacillus tuberculosis culturally. They are slightly pathogenic for guinea-pigs, but not for man.

Bacillus smegmatis and the bacillus of leprosy will be discussed in separate sections. The differentiation of these organisms by staining reactions has been discussed in the section on staining methods.

${ }^{89}$ Petri, Arb. a. d. kais. Gesunđheitsamt, 1897.

${ }^{90}$ Rabinovitsch, Zeit. f. Hyg., 1897.

${ }^{91}$ Korn, Cent. f. Bakt., 1899. 


\section{CHAPTER XXX}

\section{THE SMEGMA BACILI.US AND THE BACILLUS OF LEPROSY}

\section{BACILLUS SMEGMATIS}

IN 1884, Lustgarten ${ }^{1}$ announced that he had succeeded in demonstrating, in a number of syphilitic lesions, a characteristic bacillus, which he declared to be the etiological factor in the disease. The great importance of the subject of Lustgarten's communication caused numerous investigators to take up the study of the microorganisms found upon the genitals of normal and diseased individuals. As a result of these researches the presence of the Lustgarten bacilli upon the genitals of many syphilitics was confirmed; but at the same time bacilli, which in all essential particulars were identical with them, were found in the secretions about the genital organs and anus of many normal persons. The first to throw doubt upon the etiological significance of Lustgarten's bacillus, and to describe in detail the microorganism now recognized as Bacillus smegmatis, were Alvarez and Tavel. ${ }^{2}$ Similar studies were made soon afterward by Klemperer, ${ }^{3}$ Bitter, ${ }^{4}$ and others.

The smegma bacilli are now known to occur as harmless saprophytes in the preputial secretions of the male, about the external genital organs of the female, and within the folds of thighs and buttocks. They are usually found, in these situations, in clumps upon the mucous membrane, and occasionally in the superficial layers of the epithelium, intraand extra-cellularly.

Morphology.-The smegma bacilli are very similar to tubercle bacilli, but show greater variations in size and appearance than do the latter. In length the individuals may vary from two to seven micra. They are usually straight or slightly curved, but according to Alvarez and Tavel may show great polymorphism, including short comma-like forms, and occasional S-shaped spiral forms.

${ }^{1}$ Lustgarten, Wien. med. Woch., 47, 1884.

${ }^{2}$ Alvarez et Tavel, Arch. d. physiol. norm. et path., Oct., 1885.

${ }^{3}$ Klemperer, Deut. med. Woch., xi, 1885.

"Bitter, Virchow's Arch., ciii. 
They are not easily stained, and though less resistant in this respect than the tubercle bacillus, they yet belong distinctly to the group of acid-fast bacilli. Once stained by the stronger dyes, such as carbolfuchsin or anilin-water-gentian-violet, they are tenacious of the dye, though less so than tubercle bacilli.

The identification of the smegma bacillus by staining methods has become of practical importance since Fraenkel, ${ }^{5}$ Müller, ${ }^{6}$ and others have demonstrated the occasional presence of acid-fast bacilli, probably of the smegma group, in sputum, and in secretions from the tonsillar crypts and throat. The methods of differentiation which have been found most practical are those which depend upon differences in the retention of stain shown by these bacilli. While it may be stated as a general rule that the smegma bacilli are more easily decolorized than tubercle bacilli, it is nevertheless important that a control, as suggested by Wood, be made with known tubercle bacilli whenever a slide of suspected smegma bacilli is examined. For the actual differentiation an excellent method is that of Pappenheim, described in detail in the section on Staining, page 125. This method depends upon the fact that prolonged treatment with alcohol and rosolic acid decolorizes the smegma bacilli but not the tubercle bacilli. Coles ${ }^{7}$ has stated that smegma bacilli will resist Pappenheim's decolorizing agent for four hours at the most, while tubercle bacilli will retain the stain, in spite of such treatment, for as long as twenty-four hours.

The smegma bacilli have no pathogenic significance. They are found upon human beings as harmless saprophytes, and all attempts to infect animals have so far been unsuccessful. They are cultivated with great difficulty, first cultivations from man being successful only upon the richer media containing human serum or hydrocele fluid. After prolonged cultivation upon artificial media they may be kept alive upon glucose agar or ascitic agar. Their growth is slow; and the colonies, appearing within five or six days after inoculation, are yellowish white, corrugated, and not unlike tubercle-bacillus colonies.

${ }^{5}$ Fraenkel, Berl. klin. Woch., 1898.

'Müller, Deut. med. Woch., 1898.

${ }^{\top}$ Coles, Jour. of State Med., 1904. 


\section{LEPROSY BACILLUS LEPRE}

The bacillus of leprosy was first seen and interpreted as the etiological factor in the disease in 1879, by G. Armauer Hansen, 8 a Norwegian observer. Hansen found the bacilli in the tissues of the nodular lesions of patients, lying in small clumps, intra- and extracellularly, as well as in the serum oozing from the tissue during its removal. Hansen's observation was the fruit of over six years of careful study and as to his priority in making this discovery, there can be no doubt. Almost simultaneously with his publication, however, Neisser ${ }^{9}$ published similar results, obtained by him during a brief stay at Bergen, during the preceding summer. The bacilli described by these workers are now recognized as being unquestionably the cause of the various forms of the disease known as leprosy.

Morphology and Staining.-The leprosy bacillus is a small rod measuring about 5 to $7 \mu$ in length and has a close morphological resemblance to Bacillus tuberculosis, except in that it is less apt to display the beaded appearance and is slightly less slender than the latter. It is non-motile, possesses no flagella, and forms no spores.

Like tubercle bacilli, the leprosy bacilli belong to the class of so-called acid-fast bacteria, being stained with much difficulty; but when once stained they are tenacious of the color, offering considerable resistance to the decolorizing action of acids. It is necessary for differential diagnosis, however, to note that both the difficulty of staining and the resistance to decolorization are less marked in the case of this microorganism than in the case of Bacillus tuberculosis. It was this peculiar behavior to stains that caused the delay of several years in Hansen's publications, since he failed in obtaining good morphological specimens until the work of Koch upon bacterial staining had supplied him with proper methods. The bacillus is stained most easily with anilin-water-gentian-violet or with carbol-fuchsin solution. Stained by Gram's method, it is not decolorized and appears a deep blue. Differential staining by the Ziehl-Neelson method shows the bacillus stained red unless decolorization by means of the acid and alcohol are prolonged for an unusual time. A differentiation from tubercle bacilli by virtue of greater ease of decolorization is of value only in the hands of those having much experience with these bacilli, and follows no regular laws

${ }^{8}$ Hausen, Virch. Arch., 79, 1879.

- Neisser, Breslauer ärztl. Zeitschr., 20, 1879. 
of acid-strengths or time of application which can be generally applied by the inexperienced. In tissues, the bacilli are easily stained by the methods used for staining tubercle bacilli. The sections are left in the Ziehl carbol-fuchsin solution either from two to twelve hours at incubator temperature or for twenty-four hours at room temperature. Subsequent treatment is that employed in the case of tuberculous tissue sections (see p. 132).

Cultivation.-Cultivation of the leprosy bacillus has not met with success. Hansen and others who have approached the problem with a thorough knowledge of the microorganism, combined with a competent bacteriological training, have failed in all their attempts. The numerous positive results reported by observers have always lacked adequate confirmation. Recently, Rost, ${ }^{10}$ of the British Army Medical Corps, claimed success in cultivation of leprosy bacilli upon salt-free bouillon, his point of departure being the previous observation that salt-free media favored the growth of tubercle bacilli. His results have not been confirmed.

In 1909 Clegg ${ }^{11}$ succeeded in growing an acid-fast bacillus from leprous tissue, obtaining his results by inoculating leprous material upon agar plates upon which ameba coli had been grown in symbiosis with other bacteria. On such plates the acid-fast bacilli multiplied, and subsequently, pure cultures were obtained by heating the cultures to $60^{\circ} \mathrm{C}$., which destroyed the ameba colic and other bacteria. These results were confirmed by other workers and, soon after that, Duval ${ }^{12}$ not only succeeded in repeating Clegg's experiments, but obtained cultures of an acid-fast bacillus directly from leprous lesions without the aid of ameba. He first observed that the leprosy organism would multiply around a transplanted piece of leprous tissue upon ordinary blood agar tubes upon which influenza bacilli and meningococci were grown. He concluded that such growth depended upon chemical changes in the media and believed the formation of amino-acids essential for the initial growth. The method he subsequently described depended upon supplying these substances either by adding tryptophan to nutrient agar or by pouring egg albumen and human blood serum in Petri dishes, inspissating, at $70^{\circ} \mathrm{C}$., for three hours and, after inoculating with leprous tissue, adding a 1 per cent solution of trypsin. Indirectly the same result was obtained by employing culture media containing albu-

\footnotetext{
${ }^{10}$ Rost, Brit. Med. Jour. 1, 1905.

1. Clegg, Philippine Jour. of Sc., iv, 1909.

${ }^{12}$ Duval, Jour. Exp. Med., xii, 1910, and ibid., 15, 1912.
} 
minous substances and inoculating with bacteria capable of producing amino-acids from the medium. After leprosy bacilli had been grown on for several generations, they could easily be cultivated on agar slants without special additions or preliminary treatment.

In spite of extensive work upon this very important problem opinions are still divided as to the specific nature of the organisms cultivated by Clegg and by Duval. Animal experiments with these cultures have remained inconclusive. The cultures after prolonged preservation upon artificial media grow heavily, often lose their acid-fast characteristics, develop into streptothrix-like or diphtheroid forms and become markedly chromogenic, all these characteristics suggesting saprophytism.

In a recent communication, Duval ${ }^{13}$ states his opinion as follows: From 29 cases of leprosy, 22 successive cultivations of acid-fast bacilli were made; in 14 of them a chromogenic organism, similar to that of Clegg, was found. This grows either as a non-acid-fast streptothrix in subsequent cultivations or as non-acid-fast diphtheroid forms. From eight cases an organism distinctly different from the former was cultivated which grows only on specific media and by serological tests seems to give reaction which differentiates it from Clegg's organism. Duval believes that there is no reason to assume specific etiological relationship for the first organism mentioned. In the case of the second, he admits that not sufficient proof has been brought, but states his belief that its etiological significance is probable.

Pathogenicity.-Numerous attempts to transmit leprosy to animals by inoculation have been unsuccessful. Nicolle, ${ }^{14}$ however, has recently claimed successful experiments upon monkeys (macacus) in whom inoculation with tissue from infected human beings was followed, in sixty-two days, by the development of a small nodule at the site of inoculation, in which, upon excision, leprosy bacilli were found. In most cases, however, inoculation has given rise merely to a transient inflammatory reaction.

Among human beings, leprosy has been a widely spread disease since the beginning of history, and much evidence is found in ancient literature which testifies to a wide distribution of the disease long before the Christian era and throughout the Middle Ages. At the present day, leprosy is most common in the eastern countries, especially in India and

${ }^{13}$ Duval, Jour. of Inf. Dis., xi, 1912.

${ }^{14}$ Nicolle, Sem. médicale, 10, 1905. 
China. In Europe the disease is found in Norway, in Russia, and in Iceland. In other European countries, while the disease occurs, it is not at all common. In the United States, there are, according to Osler, three important centers of leprosy situated in Louisiana, in California, and among the Norwegian settlers in Minnesota. The disease is also present in several provinces of Canada. In all countries in which segregation of lepers is rigidly practiced, the disease is diminishing. In Norway, according to Hansen, proper sanitary measures have reduced. the number of lepers from 2870 in 1856, to 577 in 1900.

Clinically, the disease appears in two chief varieties, tubercular leprosy and the so-called anesthetic leprosy. In the former variety, hard nodular swellings appear, usually in the face, but often on other parts of the body as well. These lead to frightful disfigurement and are accompanied by a falling-out of hair and a loss of sensation in the affected areas. In the anesthetic form, there is usually at first pain in definite areas of the extremities and the trunk, which is soon followed by the formation of flat or slightly raised pigmented areas, within which there is absolute anesthesia with, later, atrophy and often secondary necrosis in the atrophied parts. The disease is usually chronic in its course.

The bacilli are found in large numbers in the cutaneous lesions. In the knobs of the nodular variety, they lie in clumps between the connective-tissue cells and within the large spheroidal cells which make up the nodules. They are found, also, in advanced cases, in the liver and in the spleen, lying within the cells, and, to a slighter extent, in the intercellular spaces. They have also been found within the kidneys, the endothelium of the blood-vessels, and in the testicles. ${ }^{15}$ In the blood, the bacilli have frequently been demonstrated, especially during the febrile attacks which occur during the disease. Westphal and Uhlenhut $^{16}$ have found the bacilli within the central nervous system, and these observers, as well as others, have found them lying within the substance of the peripheral nerves, thus explaining the anesthesia. A fact of importance to the question of transmission is the observation made by various observers, more especially by Sticker, that the bacilli are found with great regularity and in considerable numbers in the nasal secretions of persons suffering from the disease. Sticker is inclined to regard the nose as the primary path of infection. Whether or not this

${ }^{15}$ Sticker, Mïnch. med. Woch., 39, 1897.

${ }^{16}$ Westpleal und Uhtenhut, Klin. Jahrb., 1901. 
be true can not, at present, be decided. As a source of infection, however, the nasal mucus and, secondarily, the saliva, are certainly the vehicles by which large numbers of the bacilli leave the infected patient, and therefore, tend to spread the disease.

The contagiousness of leprosy is far less than is that of most other bacterial discases. Physicians and others who come into direct contact with large numbers of leprous patients, the ordinary precautions of cleanliness, rarely contract the disease. On the other hand, intimate contact with lepers without such precautions is the only possible means of transmission. The demonstration of leprosy bacilli in dust, soil, etc., must always be looked upon with suspicion, since, apart from actual human inoculation, there is no method of positively differentiating the bacilli from similar acid-fast organisms. Instances of transmission by contact are on record, not the least famous of which is the case of Father Damien, who contracted the disease while taking care of the lepers upon the island of Molokai. Hansen states that in his knowledge no case of leprosy can be found in which careful examination of the past history will not reveal direct contact with a previous case. Direct inoculation of the human being with material from a leprous patient has been successfully carried out by Arning, ${ }^{17}$ upon a Hawaiian criminal. In this case a piece of a leprous nodule was planted into the subcutaneous tissue of the left arm. One month after the inoculation, pain appeared in the arm and shoulder, and four and a half months later a typical leprosy nodule was formed. Four years after the inoculation, the patient was a typical leper.

Although our inability to cultivate the leprosy bacillus, and the lack of success attending animal inoculation, have made it impossible to study more closely the toxic action of this microorganism, there is, nevertheless, some evidence which points toward the production of a poisonous substance of some kind by the bacillus. Rost, ${ }^{18}$ who claims to have cultivated the bacillus, manufactured from his cultures, by the technique for the production of "Old Tuberculin," a substance which he called "leprolin," and which he employed therapeutically in the same manner in which tuberculin is employed in tuberculosis. As stated before the results of Rost still lack confirmation.

Of far greater importance, both in demonstrating the probability of the existence of a definite toxin as well as in indicating the close

"Arning, Vers. d. Naturfor. u. Aerzte, 1886.

${ }^{18}$ Rost, loc. eit. 
relationship between the leprosy bacillus and the Bacillus tuberculosis, are the investigations upon the action of tuberculin upon leprous patients. When tuberculin is administered to lepers, a febrile reaction occurs usually twenty-four or more hours after the administration. The fever differs from that produced by the use of the same substance in tuberculous patients in that it is of late occurrence and lasts considerably longer. At the same time, there may be marked redness and tenderness of the nodules. In isolated cases, Babes ${ }^{19}$ has noticed alarmingly high and prolonged fever together with systemic symptoms such as nausea, headache, and even unconsciousness, following the injection of tuberculin. The same writer claims to have extracted from the organs of lepers, which contained enormous numbers of bacilli, substances which showed an action similar to that of the tuberculin.

Until recently all therapeutic methods applied to leprosy have been discouraging. In 1914 Heiser reported on the treatment of 12 cases by intramuscular injections of Chaulmoogra oil. McDonald and Dean, Sir Leonard Rogers and others have used this oil and its derivatives since then with encouraging results. MeDonald and Dean report 186 cases treated from 1918 to 1919,25 of whom were discharged as "clinically and bacteriologically" free during this period. They used intramuscular injections of ethyl esters of the fatty acids of the Chaulmoogra oil. This form of treatment is being further studied by them and many others. For details we refer the reader to their communication in the U. S. Public Health Reports, August 20, 1920, Vol. 35. No. 34 .

\section{RAT LEPROSY}

Stefansky ${ }^{20}$ first observed this disease among rats in Odessa, and since then it has been observed in Berlin (Rabinovitsch ${ }^{21}$ ), in· London $\left(\right.$ Dean $^{22}$ ), in New South Wales (Tidswell ${ }^{23}$ ), and in San Francisco (Wherry ${ }^{24}$ and $\mathrm{McCoy}^{25}$ ). The disease occurs spontaneously among

${ }^{19}$ Babes, in Kolle und Wassermann, "Handbuch," ete., Erst. Ergånz. Bil., 1907.

${ }^{20}$ Stefansky, Centralbl. f. Bakt., xxxiii, 481.

${ }^{21}$ labinovitsch, Centralbl. f. Bakt., xxxiii, 577.

${ }^{22}$ Dean, Centralbl. f. Bakt., xxxiv, 222 ; Jour. Hyg., xeix.

${ }^{23}$ Tiaswell, eited by Brinkerhoff in "The Rat and Its Relation to Public Health,' 'Treas. Dept., Wash., 1910.

${ }^{24}$ Wherry, J. A. M. A., June 6, 1908.

${ }^{25}$ McCoy, Rep. U. S. P. H. and M. H. S., xxiii, 981. 
house rats and is characterized by subcutaneous induration, swelling of lymph nodes, with, later, falling out of the hair, emaciation, and sometimes ulceration. Its course is protracted and rats may live with it for six months or a year. When a rat suffering from this disease is dissected there is usually found, under the skin of the abdomen or flank, a thickened area which has the appearance of adipose tissue except that it is more nodular and gray and less shiny than fat. It is so like fat, however, that it is often overlooked by those unfamiliar with the condition. In this area acid-fast bacilli looking like the Bacillus lepræ are found in large numbers. These bacilli are also found in the lymph nodes and sometimes in small nodules in the liver and lung.

The disease can be transmitted experimentally from rat to rat and probably is transmitted naturally from rat to rat by the agency of fleas (Wherry, McCoy). Although clinically not exactly like human leprosy the condition is sufficiently like it to arouse much hygienic interest. The distribution of the disease in various parts of the world does not correspond with the distribution of leprosy. A peculiar feature of its distribution is the fact that in San Francisco, as the writer was told by McCoy, almost all the rats that suffered from this disease came from the district in which the retail meat business is located, known as "Butchertown." The organisms were made to multiply in vitro by Zinsser and Cary in plasma preparations of growing rat spleen. Chapin has succeeded in cultivating them by a method analogous to the trypsin-egg albumen method employed by Duval. In the experiments of Zinsser and Cary it was found that although the organisms may retain their acid-fast characteristics for many weeks within leucocytes they degenerate rapidly within the spleen cells, a fact which seems to have some bearing on the mechanism of resistance possessed by the body against acid-fast organisms. 


\section{CHAPTER XXXI}

BACILLI OF' T'HE COLON-TYPHOID-DYSENTERY GROUP

\section{THE COLON BACILLI}

THE bacilli belonging to this group of microorganisms, while presenting great differences in their pathogenic characteristics, possess many points of morphological and biological similarity which have made their differentiation extremely difficult. Among pathogenic bacilli, they are probably the ones most commonly encountered and because of the fact that some of them are specifically pathogenic, while others are essentially saprophytic and are pathogenic only under exceptional conditions, the necessity of accurate differentiation is a daily occurrence in bacteriological laboratories. It has been through the study of this group particularly that many of the modern differential methods of bacteriology have been developed.

The group includes the colon bacillus and its allies, the typhoid bacillus, the paratyphoid organisms, the several varieties of dysentery bacillus and numerous closely related species, and Bacillus fecalis alkaligenes. Closely related to the group though not properly within it, are Bacillus lactis aerogenes, B. acidi lactici, bacilli of the Friedländer or mucosus capsulatus group, and a number of less important subdivisions of this last group.

All bacilli of the group possess morphological characteristics which, although exhibiting slight differences, are insufficient to permit accurate morphological diagnosis. They are none of them spore-bearing. Stained by Gram's method they are decolorized.

Cultivated upon artificial media, they grow readily both at room and at incubator temperatures. None of them liquefies gelatin. Though showing, often, distinct differences in the speed and luxuriance of growth upon ordinary media, these differences are, nevertheless, too slight to become the basis of differentiation.

In order to distinguish between the individual members of this group, therefore, we are forced to a careful biological and cultural study. This is carried out by the observation of the cultural character- 
istics upon special media and by the study of serum reactions in specific immune sera. Our mainstays in the accurate differentiation of these bacilli are their fermentative actions upon carbohydrate media, and their agglutinating reactions in immune sera. These points will be taken up in the description of the individual microorganisms, and will again be summarized in the differential tables given at the end of the chapters dealing with this group.

\section{BACILLUS COLI COMMUNIS AND MEMBERS OF THE COLON BACILLUS GROUP}

Under the name of "colon bacilli" are grouped a number of varieties differing from one another in minor characteristics, but corresponding in certain cardinal points which stamp them as close relatives and amply warrant their consideration under one heading. While usually growing as harmless parasites upon the animal and human body, and capable of leading a purely saprophytic existence, they may, nevertheless, under certain circumstances become pathogenic and thus, both culturally and in their pathological significance, form a link between pure saprophytes like Bacillus lactis aërogenes, on the one hand, and the more strictly pathogenic Gram-negative bacilli of the paratyphoid, typhoid, and dysentery groups, on the other. As a type of the group we may consider in detail its most prominent and thoroughly studied member, Bacillus coli communis.

\section{BACILLUS COLI COMMUNIS}

This microorganism was seen and described by Buchner ${ }^{1}$ in 1885 . It was thoroughly studied in the years immediately following, by Escherich, ${ }^{2}$ in connection with the intestinal contents of infants.

Morphology.-Bacillus coil communis is a short, plump rod about 1-3 micra long, and varying in thickness from one-third to one-fifth of its length. Under varying conditions of cultivation, it may appear to be more slender than this or shorter and even coccoid in form. In stained preparations, it usually appears singly, but occasionally may be seen in short chains. It stains readily with the usual anilin dyes and decolorizes by Gram's method. Spores are not formed. It is motile, and flagella staining reveals eight or more flagella peripherally arranged. Its motility is subject to wide variations. Young cultures, in the first

${ }^{1}$ Buchner, Arch. f. Hyg., 3, 1885.

${ }^{2}$ Escherich, 'Die Darmbakt. des Säuglinngs,' Stuttgart, 1886; Cent. f. Bakt., $1,1887$. 
generation after isolation from the body, may be extremely motile, while old laboratory strains may show almost no motility. Independent of these modifying condition's, however, separate races may show individual characteristics as to motility, varying in range between a motility hardly distinguishable from Brownian movement and one which is so active as to be but little less than that of the typhoid bacillus. Ordinarily, the colon bacillus possesses a motility intermediate between these two extremes.

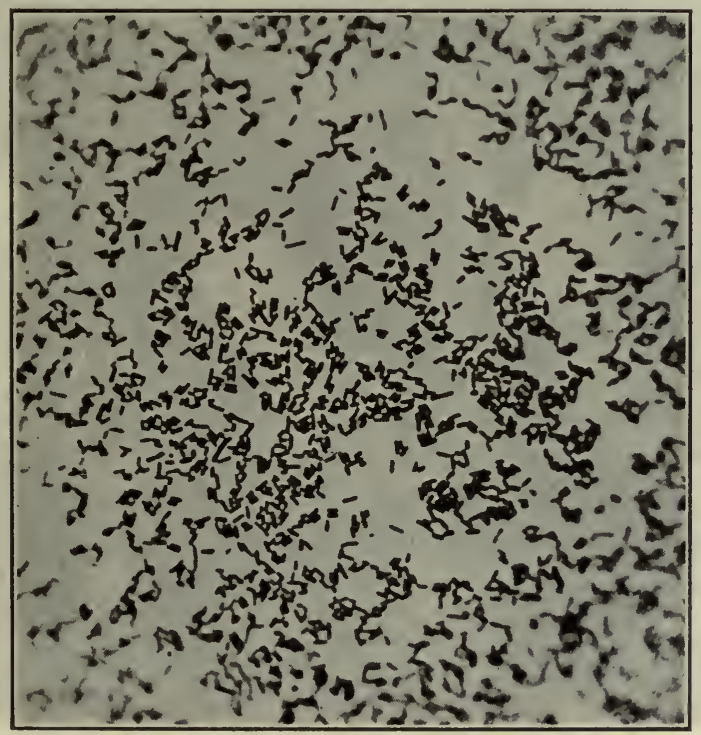

Fig. 65.-Baccillus Coli communis.

Cultivation.-The bacillus is an aërobe capable of anaërobic growth under suitable cultural conditions. It grows well on the simplest media at temperatures ranging from $20^{\circ}$ to $40^{\circ} \mathrm{C}$., but finds its optimum growth at about $37.5^{\circ} \mathrm{C}$. Upon broth it grows rapidly, giving rise to general clouding; later to a pellicle and a light, slightly slimy sediment. Within moderate ranges, it is not delicately susceptible to reaction, growing equally well on media slightly acid and on those of a moderate alkalinity.

Upon agar, it forms grayish colonies which become visible within twelve to eighteen hours, gradually becoming more and more opaque as they grow older. The deep colonies are dense, evenly granular, oval, or round. Surface colonies often show a characteristic grape-leaf 
structure, or may be round and flat, and show a definitely raised, glistening surface. Upon agar slants, growth occurs in a uniform layer.

On gelatin the colon bacillus grows rapidly, causing no liquefaction. Surface colonies are apt to show the typical grape-leaf formation. Deep colonies are round, oblong, and glistening. In gelatin stabs growth takes place along the entire line of inoculation, spreading in a thin layer over the surface of the medium.

On potato, growth is abundant and easily visible, within eighteen

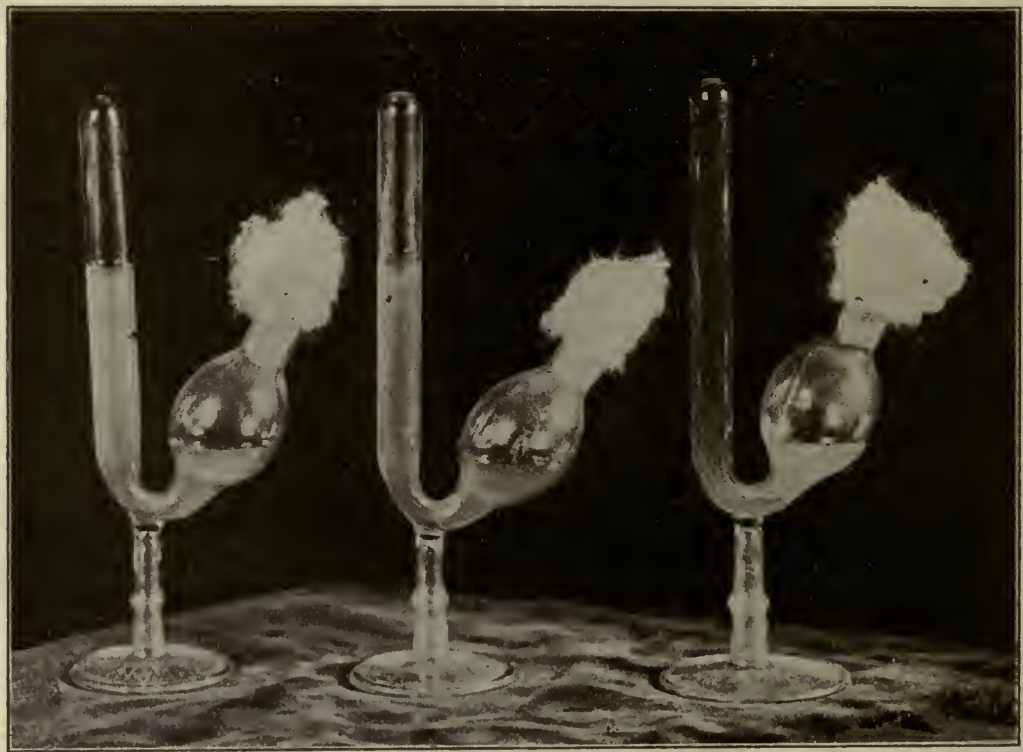

1

2

3

Fig. 66.-Baccillus coli communis, Grown in: 1. Dextrose, $2 . \quad$ Lactose, 3. Saccharose broth. The baccillus forms acid and gas from dextrose and lactose, not from saccharose. Note the absence of growth in the closed arm of the saccharose tube, in which no acid or gas is formed.

to twenty-four hours, as a grayish-white, glistening layer which later turns to a yellowish-brown, and in old cultures often to a dirty greenish-brown color.

In pepton solution indol is formed. In milk there is acidity and coagulation. In lactose-litmus-agar acid is formed, the medium becoming red, and gas-bubbles appear along the line of the stab inoculation.

In carbohydrate broth, gas is formed in dextrose, lactose, and mannit, but not in saccharose. - Levulose, galactose, and maltose are also fermented with the formation of acid and gas. 
Cultures of the colon bacillus are characterized by a peculiar fetid odor which is not unlike that of diluted feces. The acids formed by the colon bacillus from sugars are chiefly lactic, acetic, and formic. The gas it produces consists chiefly of $\mathrm{CO}_{2}$ and hydrogen. The bacillus grows well on media containing urine and on those containing bile. Upon the latter fact methods for the isolation of the colon bacillus from water and feces have been based.

Isolation of the colon bacillus from mixed cultures is most easily accomplished by plating upon lactose-litmus-agar, the Conradi-Drigalski medium, or the Endo medium after preliminary enrichment if necessary in bile or malachite-green broth. (In the case of feces such enrichment is superfluous.)

Distribution.--The colon bacillus is a constant inhabitant of the intestinal canal of human beings and animals. It is also found occasionally in soil, in air, in water, and in milk and is practically ubiquitous in all neighborhoods which are thickly inhabited. When found in nature its presence is generally taken to be an indication of contamination from human or animal sources. Thus, when found in water or milk, much hygienic importance is attached to it. Recently, Papasotiriu ${ }^{3}$ and independently of him, Prescott,${ }^{4}$ have reported finding bacilli apparently identical with Bacillus coli upon rye, barley, and other grains. They believe, upon the basis of this discovery, that Bacillus coli is widely distributed in nature and that its presence, unless it appears in large numbers, does not necessarily indicate recent fecal contamination. These reports, however, have not found confirmation by the work of others.

In man, Bacillus coli appears in the intestine normally soon after birth, at about the time of taking the first nourishment. ${ }^{5}$ From this time on, throughout life, the bacillus is a constant intestinal inhabitant apparently without dependence upon the diet. Its distribution within the intestine, according to Cushing and Livingood, ${ }^{6}$ is not uniform, it being found in the greatest numbers at or about the ileocecal valve, diminishing from this point upward to the duodenum and downward as far as the rectum. Adami ${ }^{7}$ and others claim that, under normal conditions, the bacillus may invade the cortal circulation, possibly by the

${ }^{3}$ Papasotiriu, Arch. f. Hyg., xli.

${ }^{4}$ Prescott, Cent. f. Bakt., Ref., xxxiii, 1903.

${ }^{3}$ Schild, Zeit. f. Hyg., xix, 1895; Lembke, Arch. f. Hyg., xxiv, 1896.

'Cushing and Livingood, "Contributions to Med. Sci. by Pupils of Wm. Welch,' Johns Hopk. Press, 1900.

'Adami, Jour. of Amer. Med. Assn., Dec., 1899. 
intermediation of leucocytic emigration during digestion. After death, at autopsy, Bacillus coli is often found in the tissues and the blood without there being visible lesions of the intestinal mucous membrane. ${ }^{8}$ It is probable, also, that it may enter and live in the circulation a few hours before death during the agonal stages.

The distribution of the colon bacilli in the human intestine at different periods of life, and under varying dietetic conditions has been considered in the section on the "normal flora of the intestinal canal," p. 223.

Extensive investigations have been carried out to determine whether or not the constant presence of this microorganism in the intestinal tract is an indication of its possessing a definite physiological function of advantage to its host. It has been argued that it may aid in the fermentation of carbohydrates. The question has been approached experimentally by a number of investigators. Nuttall and Thierfelder 9 delivered guinea-pigs from the mother by Cesarean section and succeeded in preserving them from infection of the intestinal canal for thirteen days. Although no microorganisms of any kind were found in the feces of these animals, no harm seemed to accrue to them, and some of them even gained in weight. Schottelius, ${ }^{10}$ on the other hand, obtained contradictory results with chicks. Allowing eggs to hatch in an especially constructed glass compartment, he succeeded in keeping the chicks and their entire environment sterile for seventeen days. During this time they lost weight, did not thrive, and some of them were moribund at the end of the second week, in marked contrast to the healthy, well-nourished controls, fed in the same way, but under ordinary environmental conditions. Although insufficient work has been done upon this important question, and no definite statement can be made, it is more than likely that the function of the Bacillus coli in the intestine is not inconsiderable if only because of its possible antagonism to certain putrefactive bacteria, a fact which has been demonstrated in interesting studies by Bienstock ${ }^{11}$ and others. ${ }^{12}$

Pathogenicity.-The pathogenicity of the colon bacillus for animals is slight and varies greatly with different strains. Intraperitoneal injections of 1 c.c. or more of a broth culture will often cause death in guinea-pigs. Intravenously administered to rabbits it may

${ }^{8}$ Birch-Hirschfeld, Ziegler's Beitr., 24, 1898.

- Nuttull und Thierfelder, Zeit. f. Physiol. Chemie, xxi and xxii.

${ }^{10}$ Schottelius, Arch. f. Hyg., xxiv, 1889.

${ }^{11}$ Bienstock, Arch. f. Hyg., xxix, 1901.

${ }^{12}$ Tisser and Martelly, Ann. de l'inst. Pasteur, 1902. 
frequently cause a rapid sinking of the temperature and death with symptoms of violent intoxication within twenty-four to forty-eight hours. Subcutaneous inoculation of moderate doses usually results in nothing more than a localized abscess from which the animals recover.

In man, a large variety of lesions produced by Bacillus coli have been described. It is a surprising fact that disease should be caused at all, in man, by a microorganism which is so constantly present in large numbers in the intestine and against which, therefore, it is to be expected that a certain amount of immunity should be developed. A number of explanations for this state of affairs have been advanced, none of them entirely satisfactory. It is probable that none of the poisonous products of the colon bacillus are absorbed unchanged by the healthy unbroken mucosa and that, therefore, the microorganisms are, strictly speaking, at all times, outside of the body proper. Under these circumstances, no considerable process of immunization would be anticipated. It is also possible that, whenever an infection with Bacillus coli does occur, the infecting organism is one which has been recently acquired from another host, having no specific adaptation to the infected body. Virulence may possibly be enhanced by inflammatory!processes caused by other organisms. Considering the subject from another point of view, colon-bacillus infection may possibly take place simply because of unusual temporary reduction of the resistance of the host. Whether or not altered cultural conditions in the intestine may lead to marked enhancement in the virulence of the colon bacilli cannot at present be decided. The opinion has been frequently advanced, however, without adequate experimental support.

Septicemia, due to the colon bacillus, has been described by a large number of observers. It is doubtful, however, whether many of these cases represent an actual primary invasion of the circulation by the bacilli, or whether their entrance was not simply a secondary phenomenon occurring during the agonal stages of another condition. A few unquestionable cases, however, have been reported, and there can be no doubt about the occurrence of the condition, although it is probably less frequent than formerly supposed. The writer has observed it on two occasions in cases during the lethal stages of severe systemic disease due to other causes. An extremely interesting group of such cases are those occurring in new-born infants, in which generalized colon-bacillus infection may lead to a fatal condition known as Winckel's disease or hemorrhagic septicemia. ${ }^{13}$ Prominent among disease processes

${ }^{13}$ Kamen, Ziegler's Beitr., 14, 1896. 
attributed to these microorganisms are various diarrheal conditions, such as cholera nostras and cholera infantum. The relation of these maladies to the colon bacillus has been particularly studied by Escherich, ${ }^{14}$ but satisfactory evidence that these bacilli may specifically cause such sonditions has not been brought. While it is not unlikely that under conditions of an excessive carbohydrate diet, colon bacilli, may aggravate morbid processes by a voluminous formation of gas, they do not, of themselves, take part in actual putrefactive processes. It is likely, therefore, that in most of the intestinal diseases formerly attributed purely to bacilli of the colon group, these microorganisms actually play but a secondary part. ${ }^{15}$

It is equally difficult to decide whether or not these bacilli may be regarded as the primary cause of peritonitis following perforation of the gut. Although regularly found in such conditions, they are hardly ever found in pure culture, being accompanied usually by staphylococci, streptococci, or other microorganisms, whose relationship to disease is far more definitely established. Isolated cases have been reported, however, one of them by Welch, in which Bacillus coli was present in the peritoneum in pure culture without there having been any intestinal perforation. ${ }^{16}$ Granting that the bacillus is able to proliferate within the peritoneum, there is no reason for doubting its ability of giving rise to a mild suppurative process.

Inflammatory conditions in the liver and gall-bladder have frequently been attributed to the colon bacillus. It has been isolated from liver abscesses, from the bile, and from the center's of gall-stones. Welch has reported a case of acute hemorrhagic pancreatitis in which the bacillus was isolated from the gall-bladder and from the pancreas.

In the bladder, Bacillus coli frequently gives rise to cystitis and occasionally to ascending pyonephrosis. No other microorganism, in fact, is found so frequently in the urine as this one. It may be present, often, in individuals in whom all morbid processes are absent. The condition is frequently observed during the convalescence from typhoid fever. It may disappear spontaneously, or cystitis, usually of a mild, chronic variety, may supervene.

Localized suppurations due to this bacillus may take place in all parts of the body. They are most frequently localized about the anus and the genitals, and are usually mild and amenable to the simplest surgical treatment.

14 Escherich, loc. cit.

${ }^{15}$ Herter, "Bact. Infec. of Digest. Tract," N. Y., 1907.

${ }^{16} \mathrm{Welch}$, Med. News, 59, 1891. 
For a consideration of the distribution of colon bacilli in the intestines of human beings at various ages and under modified dietary conditions the reader is referred to the section on the normal flora of the intestinal canal.

Poisonous Products of the Colon Bacillus.-The colon bacillus belongs essentially to that group of bacteria whose toxic action is supposed to be due to the poisonous substances contained within the bacillary body. Culture filtrates of the colon bacillus show very little toxicity when injected into animals; whereas the injection of dead bacilli produces symptoms almost equal in severity to these induced by injection of the live microorganisms. Corroborative of the assumption of this endotoxic nature of the colon-bacillus poison is the fact that, so far, no antitoxic bodies have been demonstrated in serum as resulting from immunization.

Dead colon bacilli have a very high toxicity for rabbits and some what less for guinea pigs.

Immunization with the Colon Bacillus.-The injection into animals of gradually increasing doses of living or dead colon bacilli gives rise to specific bacteriolytic, agglutinating, and precipitating substances.

The bacteriolytic substances may be easily demonstrated by the technique of the Pfeiffer reaction. In vitro bacteriolysis is less marked than in the case of some other microorganisms such as the cholera spirillum or the typhoid bacillus. Owing probably to the habitual presence of colon bacilli in the intestinal tracts of animals and man, considerable bacteriolysis may occasionally be demonstrated in the serum of normal individuals.

Agglutinins for the colon bacillus have often been produced in the sera of immunized animals in concentration sufficient to be active in dilutions of $1: 5000$ and over. The agglutinins are produced equally well by the injection of live cultures and of those killed by heat, if the temperature used for sterilization does not exceed $100^{\circ} \mathrm{C}$. It is ${ }^{17}$ a noticeable fact that the injection of any specific race of colon bacilli produces, in the immunized animal, high agglutination values only for the individual culture used for immunization, while other strains of colon bacilli, although agglutinated by the serum in higher dilution than are paratyphoid or typhoid bacilli, require much higher concentration than does the original strain. The subject has been extensively studied by a number of observers and illustrates the extreme individual specificity of the agglutination reaction. Thus a serum which will

${ }^{17}$ Wolff, Cent. f. Bakt., xxv, 1899. 
agglutinate its homologous strains in dilutions of one to 1000 will often fail to agglutinate other races of Bacillus coli in dilutions of $1: 500$ or $1: 600$.

The normal serum of adult animals and man will often agglutinate this bacillus in dilutions as high as $1: 10$ or $1: 20$ - a phenomenon possibly referable to its habitual presence within the body. Corroborating this assumption is the observation of Kraus and Löw, ${ }^{18}$ that the serum of new-born animals possesses no such agglutinating powers. The fact that agglutinins for the colon bacillus are increased in the serum of patients convalescing from typhoid fever or dysentery is probably to be explained, partly by the increase of the group agglutinins produced by the specific infecting agent, and partly by the invasion of colon bacilli, or the absorption of its products induced by the diseased state of the intestinal mucous membrane.

Varieties of the Colon Bacillus.-During the earlier days of bacteriological investigations, a large number of distinct varieties of colon bacilli were described, many of which may now be dismissed as based simply upon a temporary depression of one or another cultural characteristic of Bacillus coli communis, while others can be definitely included within other closely related, but distinct groups.

That secondary features, such as dimensions, motility, and luxuriance of growth upon various media, may be markedly altered by artificial cultivation is a common observation. It has not, however, been satisfactorily shown that cardinal characteristics, such as the formation of indol from pepton, or the power to produce gas from dextrose and lactose, can be permanently suppressed without actual injury or inhibition of the normal vitality of the microorganism. Such alteration is, in fact, contrary to experience, which demonstrates that whenever such changes do occur, they are purely temporary and a few generations of cultivation under favorable environmental conditions will regularly restore the organism to its normal activity.

Distinct and constant varieties of the Colon Bacillus or, at least, close biological relatives do occur. It is necessary to consider the organisms as a group for this reason, since, in sanitary work, it is of the utmost importance to recognize forms which should properly be classified under this category. It may be well, therefore, to reiterate the criteria for identification of the group established by the American Public Health Association Committee on Standard Methods of Water Analysis. ${ }^{19}$ This report defines the general characteristics of the group, as follows:

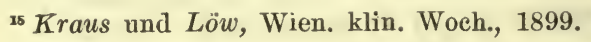


short bacillus form, failure of spore formation, facultative anaërobiosis, growth on gelatin without liquefaction in fourteen days, Gram-negative stain and fermentation of dextrose and lactose with gas formation. They add that there is a positive reaction with esculin.

The organisms which are placed under the Colon group in this report are the B. Coli communis described above, the B. Coli communior of Durham, the B. aërogenes of Escherich, and B. acidi-lactici of Hüeppe. The chief differential characteristics are as follows:

\begin{tabular}{l|c|c|c|c}
\hline & Dextrose. & Lactose. & Saccharose. & Dulcit. \\
\hline B. coli communis............... & + & + & - & + \\
B. coli communior................ & + & + & + & + \\
B. lact. aërogenes............... & + & + & + & - \\
B. lact. acidi................. & + & + & - & - \\
\hline
\end{tabular}

Individual descriptions of these organisms follow:

B. Coli Communior.- This organism first described by Durham was called Communior by him because of his belief that it was more abundant in the human and animal intestine than the Communis type. It possesses all the characteristics of the Colon group. It is a Gramnegative bacillus, motile, non-sporulating, and morphologically indistinguishable from the Communis variety. It does not liquefy gelatin, it produces indol from pepton, coagulates and acidifies milk, and grows characteristically upon agar and potato. It differs from B. coli communis in that it produces acid and gas from saccharose as well as from dextrose and lactose, whereas the former does not form acid or gas from saccharose. Several varieties have been described by Melia, and by Avery. The Melia type differs from the ordinary variety in not producing indol. The Avery type did not coagulate milk.

B. LACTIS Aërogenes.-Bacillus lactis aërogenes is the type of a group which is closely similar to the colon group and often distinguished from it with difficulty. It was first described in 1885 by Escherich ${ }^{19}$ who isolated it from the feces of infants. Since then it has been learned that this bacillus is almost constantly present in milk, and, together with one or two other microorganisms, is the chief cause of the ordinary souring of milk. Apart from its occurrence in milk, moreover, the bacillus is widely distributed in nature, being found in feces, in water, and in sewage.

${ }^{19}$ A. P. H. A. Standard Methods of Water Analysis, 1915. 
It is distinguished from the Colon bacillus chiefly by the fact that it is less motile, hardly ever forms chains, and, when cultivated upon suitable media, especially milk, it possesses a distinct capsule. It differs from other forms of the Colon group in not fermenting dulcite, and differs from B. acidi-lactici in fermenting saccharose. It ferments with gas production, dextrose, lactose, saccharose, mannite and raffinose. It produces indol, reduces nitrate, possesses either no motility, or is very slightly motile. It coagulates milk, and when grown on milk or lactose bile it often makes a stringy viscous culture. On agar and gelatin it makes heavy white colonies of a some-

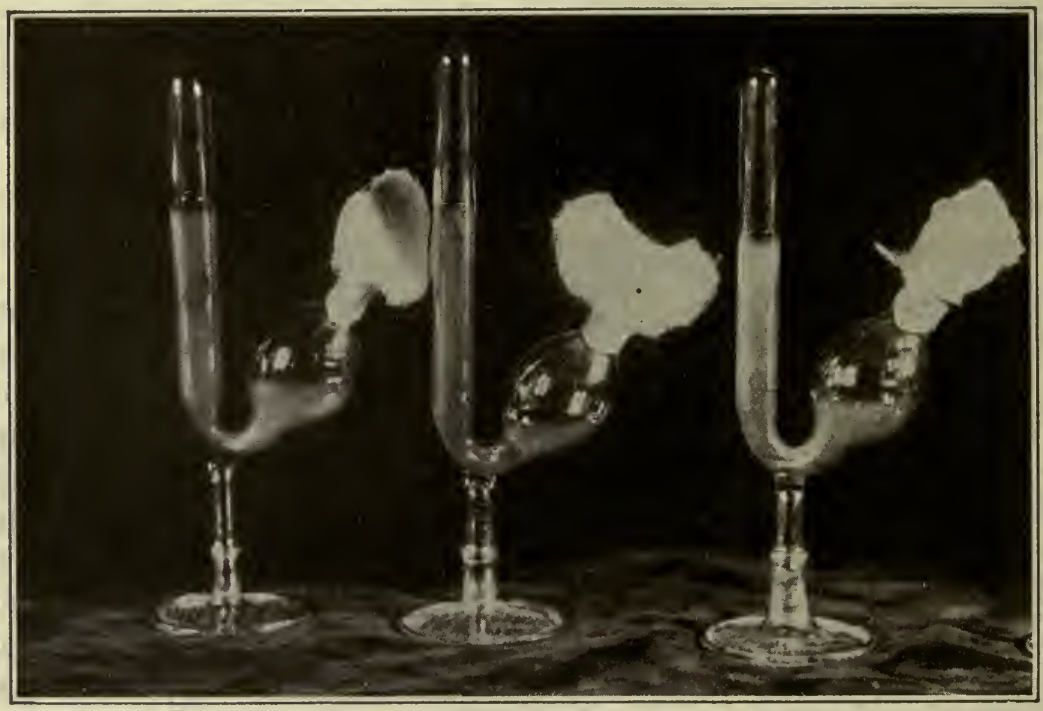

1.

3

Fig. 67.-Bactluds coli communior. Grown in: 1. Dextrose, 2. Lactose, 3. Saccharose broth.

what mucoid appearance, certainly more mucoid than most colon colonies. It does not liquefy gelatin. In broth it causes general clouding with later a pellicle, and a sour odor. It grows heavily on potato.

It is a facultative anaërobe and grows at room temperature.

Varieties have been described depending upon minor cultural characteristics which have no particular importance in this connection.

The pathogenicity of Bacillus lactis aërogenes for man is slight. Its chief claims to importance lie in its milk-coagulating properties and its almost constant presence in the human intestine. In infants, it may give rise to flatulence and it has been occasionally observed as the sole incitant of eystitis. Among such cases rare instances have been 
observed in which it has formed gas in the bladder (pneumaturia). When this occurs the urine is not ammoniacal but remains acid.

Different strains of this bacillus vary much in their pathogenicity for animals. Wilde claims that it is more pathogenic for white mice and guinea-pigs than is the bacillus of Friedländer. He speaks of it as the most virulent member of this group. Kraus, writing in Fluegge's "Mikroorganismen," rates its pathogenicity less high.

Closely related to this bacillus, as well as those of the Friedländer group, is an encapsulated bacillus isolated from a case of bronchopneumonia by Mallory and Wright, ${ }^{20}$ which is strongly pathogenic for mice, guinea-pigs, and rabbits.

B. ACIDI-LACTICI.-This organism, like the others, is a Gramnegative, non-liquefying, non-sporulating bacillus. Just like the B. aërogenes, it has no motility. It differs from the Colon bacilli proper in not fermenting dulcite. It differs from the Lactis aërogenes in failing to ferment saccharose. Like the Lactis aërogenes, it is nonmotile. - It forms indol, reduces nitrate, and coagulates milk.

It is commonly present in milk and may be present in water. It is often found in the intestinal canal but as far as we know has no pathogenic significance.

Bacillus Fecalis Alkaligenes.-In 1896 Petruschky $^{21}$ described a bacillus which is a not infrequent inhabitant of the human intestine, being found chiefly in the lower part of the small intestine and in the large intestine. This organism, which he called Bacillus fecalis alkaligenes, is of little pathogenic importance, although Neufeld states that he has seen a case of severe gastroenteritis in which the watery defecations contained this bacillus in almost pure culture. As a rule, however, this organism cannot be regarded as pathogenic, and is important chiefly because of the ease with which it may be mistaken for Bacillus typhosus.

Bacillus fecalis alkaligenes is an actively motile, Gram-negative bacillus, possessing, like the typhoid bacillus, numerous peritrichal flagella. On the ordinary culture media it grows like the typhoid bacillus. It does not coagulate milk. It produces no indol, and on sugar media in fermentation tubes produces no acid or gas. On potato, its growth, while somewhat heavier than that of the typhoid bacillus, is not sufficiently so to permit easy differentiation. It differs from Bacillus typhosus in that it produces no acid on any of the sugar media, and is therefore easily differentiated by cultivation upon Hiss serum-

${ }^{20}$ Mallory and Wright, Zeit. f. Hyg., 20, 1895.

${ }^{21}$ Petruschky, Cent. f. Bakt., I, ixix, 1896. 
water media or on pepton waters containing sugars. On the Hiss semi-solid tube-medium Bacillus fecalis alkaligenes, while clouding the medium throughout, grows most heavily on the surface, where, eventually, it forms a pellicle.

\section{BACILLI OF THE PROTEUS GROUP}

There are a great many other organisms which are similar to the Colon Bacillus in general appearance and superficial morphological and cultural characteristics, and which are found frequently associated with it in feces, water and sewage. It will be important for this reason to speak of them briefly. The most important of these is the Proteus Group, which is sharply separable from the Colon and allied bacteria by its gelatin liquefaction.

The bacilli of this group have little pathological interest, but are important because of the frequency with which they are encountered in routine bacteriological work. They may confuse the inexperienced because of a superficial similarity to bacilli of the colon-typhoid group. In form they may be short and plump or long and slender, staining easily with anilin dyes and decolorizing with Gram's method. They are actively motile and possess many flagella. Individuals stain irregularly, often showing unstained areas near the center. The so-called Bacillus proteus vulgaris described by Hauser ${ }^{22}$ in 1885 is the type of the group.

Bacilli of this group are widely distributed, being found in water, soil, air, and wherever putrefaction takes place. In fact, proteus is one of the true putrefactive bacteria possessing the power to cause the cleavage of proteins into their simplest radicles.

Bacillus proteus vulgaris grows best at temperatures at or about $25^{\circ} \mathrm{C}$. and develops upon the simplest media. It is a facultative anaërobe and forms no spores. In broth, it produces rapid clouding with a pellicle and the formation of a mucoid sediment.

In gelatin, the colonies are characteristically irregular, giving the name to this group.

Gelatin is Rapidly Liquefied.-Liquefaction, however, is diminished or even inhibited under anaërobic conditions.

On agar and other solid media, as well as upon gelatin before liquefaction has taken place, characteristic colonies are produced. From the central flat, grayish-white colony nucleus, numerous irregular streamers grow out over the surrounding media, giving the colony a stellate appearance. On potato, it forms a dirty, yellowish growth.

${ }^{22}$ Hauser, "Ueber Fäulniss-Bakt.," Leipzig, 1885. 
In mill; , there is coagulation and an acid reaction at first; later the casein is redissolved by proteolysis. Blood serum is often liquefied, but not by all races.

A great many really dissimilar bacteria have been described under the name of Proteus. The type of the group is the so-called Proteus vulgaris (Hauser, 1885). Other organisms spoken of as proteus are the Proteus mirabilis, which differs in slower gelatin liquefaction from vulgaris, the Proteus Zenkeri, which does not liquefy gelatin, the Proteus septicus, and the Bacillus Zopfi, a Gram-positive organism. A good many of these were formerly classified as of Bacterium termo. Closely related is the slow liquefying organism known as Bacillus cloacee, common in sewage.

There is no group which so urgently requires study as this, since organisms belonging here are so often found in the human body and human excreta. In urine we have encountered a non-gelatin liquefying Gram-negative bacillus belonging to this group which has given us much trouble in identification. As far as we can establish any general characteristics for the group at all, we may say that they are Gramnegative, non-spore-bearing, motile bacilli, which on the surface of gelatin plates show colonies characterized by spreading streamers, most of which liquefy gelatin, a few of which, however, do not. All of them ferment dextrose and saccharose with gas, but few of them attack lactose.

The pathogenic powers of proteus are slight. Large doses injected into animals may give rise to localized abscesses. In man proteus infections have been described in the bladder, in most cases, however, together with some other microorganism. The Urobacillus liquefaciens septicus described by Krogius was a variety of this group. Epidemics $^{23}$ of meat poisoning have been attributed to the proteus family by some observers. Thus Wesenberg ${ }^{24}$ cultivated a proteus from $^{25}$ putrid meat which had caused acute gastroenteritis in sixtythree individuals. Similar epidemics have been reported by Silberschmidt, ${ }^{25}$ Pfuhl, ${ }^{26}$ and others.

B. ClOACA.-This organism was first described by Jordan and is one of the commonest of the sewage bacteria. It is closely related to the Proteus organisms, but is less motile than they. It coagulates milk, and liquefies gelatin, but its gelatin liquefaction is not as active as that .

26 Schnitzler, Cent. f. Bakt., viii, 1890.

${ }^{24}$ Wesenberg, 'Zeit. f. Hyg., xxviii, 1898.

${ }^{25}$ Silberschmidt, Zeit. f. Hyg., xxx, 1899.

${ }^{26}$ Pfuhl, Zeit. f. Hyg., xxxv, 1900. 
of the Proteus group. It forms indol and produces acid and gas on dextrose and saccharose, but one of its chief characteristics is its slight action on lactose. Jordan states as one of its chief characteristics the relatively large proportion of $\mathrm{CO}_{2}$ formed as compared with hydrogen, the ratio being in some cases as high as 5 to 1 . Kendall, Day and Walker 27 have observed the same thing. The same investigators state that after three days' growth, even sugar broths become alkalin, owing to protein decomposition.

Recently certain stains of Proteus have become important because of their apparently specific agglutination in Typhus Serum (WeilFelix Reaction). See chapter on Typhus.

Were we following a purely biological order of presentation, we should now proceed to a description of the organisms belonging to the so-called Mucosus Capsulatus or Friedländer group. These bacilli are closely related to the Colon type, more particularly to the B. aerogenes variety, and have been regarded by some observers, notably Fitzgerald, as perhaps representing members of the Colon group which have acquired capsulation and virulence. In practice, however, these bacteria are rarely encountered under conditions where differentiation from Colon bacilli is necessary, and their heavy mucoid colonies and capsulated morphology renders their recognition relatively easy. It will be better, therefore, from the point of view of practical discussion, to proceed directly to the study of organisms of the typhoid and dysentery groups, since these are the ones which in medical and sanitary bacteriology, are associated in the human body, in water and sewage with members of the Colon group and which, therefore, present the most frequent differential problems.

${ }^{27}$ Kendall, Day and Walker, Jour. Amer. Chem. Soc., 1913, 35. 


\section{CHAPTER XXXII}

\section{BACILLI OF THE COLON-TYPHOID-DYSENTERY GROUP (Continued)}

\section{THE BACILLUS OF TYPHOID FEVER}

\section{(Bacillus typhosus, Bacillus typhi abdominalis)}

Trphoid FEVER, because of its wide distribution and almost constant presence in most communities, has from the earliest days been the subject of much etiological inquiry. A definite conception as to its infectiousness and transmission from case to case was formed as early as 1855 by Budd. ${ }^{1}$

But it was not until 1880 that Eberth ${ }^{2}$ discovered in the spleen and mesenteric glands of typhoid-fever patients who had come to autopsy, a bacillus which we now know to be the cause of the disease. Final proof of such an etiological connection was then brought by Gaffky, ${ }^{3}$ who not only saw the bacteria referred to by Eberth, but succeeded in obtaining them in pure culture and studying their growth characteristics.

Morphology and Staining.-The typhoid bacillus is a short rod from $1-3.5 \mu$ in length with a varying width of from .5 to $.8 \mu$. In appearance it has nothing absolutely distinctive which could serve to differentiate it from other bacilli of the typhoid-colon group, except that it has a general tendency to greater slenderness. Its ends are rounded without ever being club-shaped. Contrary to the descriptions of the earlier observers, typhoid bacilli do not form spores. They are actively motile and have twelve or more flagella peripherally arranged.

The bacilli stain readily with the usual anilin dyes. Stained by Gram's method, they are decolorized.

Cultivation.-Bacillus typhosus is easily cultivated upon the usual laboratory media. It is not delicately susceptible to reaction, but will grow well upon media moderately alkaline or asid. It is an aërobic and facultative anaërobic organism, when the proper nutriment is present. Upon agar plates growth appears within eighteen to twenty-

\footnotetext{
${ }^{1}$ Budd, "Intestinal Fever," Lancet, 1856.

${ }^{2}$ Eberth, Virch. Archiv., 81, 1880, and 83, 1881.

3 Gaffky, Mitt. a, d. kais. Gesundheitsamt, 2, 1884 ,
} 


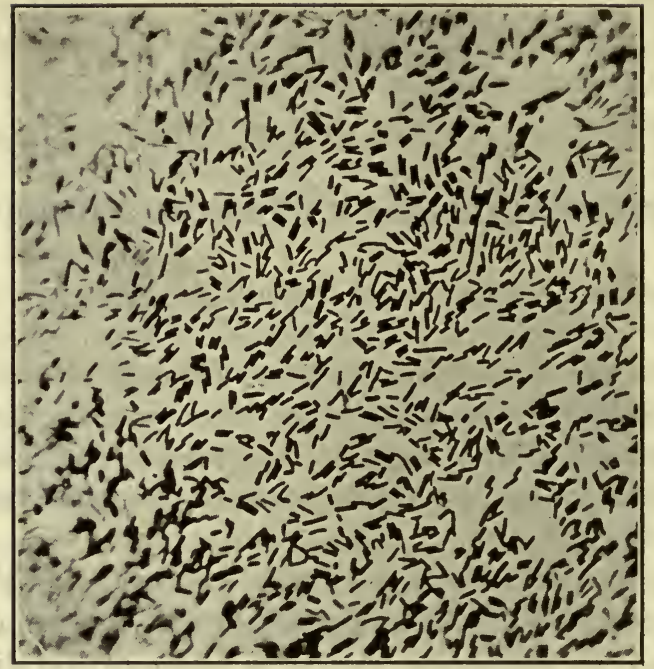

Fig. 68.-Bacillus TYPHosus, from twenty-four-hour culture on agar:

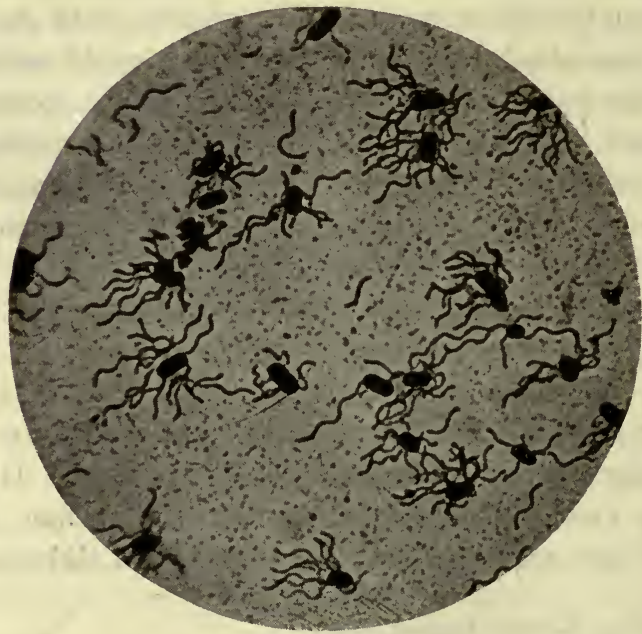

Fig. 69.-Bacillus typhosus, showing flagella. (After Fränkel and Pfeiffer.) 
four hours as small grayish colonies at first transparent, later opaque. Upon agar slants growth takes place in a uniform layer. There is nothing characteristic about this growth to aid in differentiation.

In broth, the typhoid bacillus grows rapidly, giving rise to an even clouding, rarely to a pellicle.

Upon gelatin, the typhoid bacillus grows readily and does not liquefy the medium. In stabs, growth takes place along the entire extent of the stab and over the surface of the gelatin in a thin layer. In gelatin plates the growth may show some differences from that of other members of this group, and this medium was formerly much used for isolation of the bacillus from mixed cultures. Growth appears within twenty-

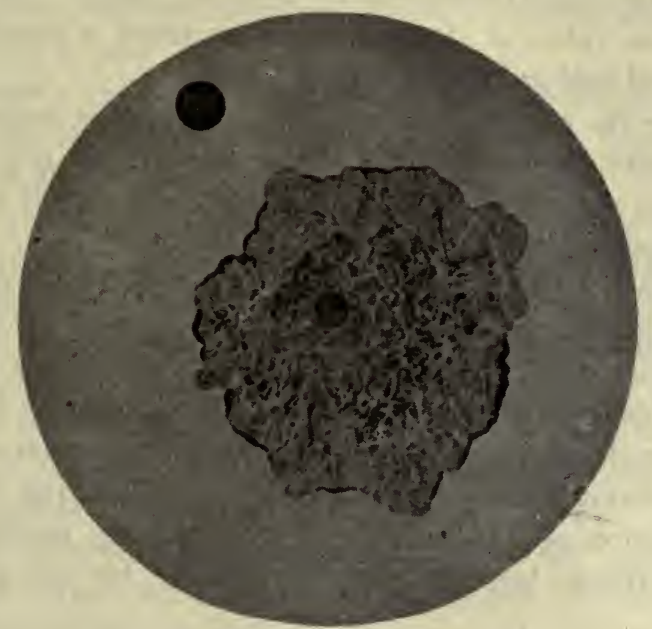

Fig. 70.-Surface Colony of Bacillus typhosus on Gelatin. (After Heim.)

four hours as small transparent, oval, round, or occasionally leaf-shaped colonies which are smaller, more delicate, and more transparent than contemporary colonies of the colon bacillus. They do not, however, show any reliable differential features from bacilli of the dysentery group. As the colonies grow older they grow heavier, more opaque, and lose much of their early differential value.

On potato the growth of typhoid bacilli is distinctive, and this medium was recommended by Gaffky ${ }^{4}$ in his early researches for purposes of identification. On it typhoid bacilli, after twenty-four to forty-eight hours, produce a hardly visible growth, evident to the naked eye only 
by a slight moist glistening, an appearance which is in marked contrast to the grayish-yellow or even brown and abundant growth of colon bacilli. If the potato medium is rendered neutral or alkaline, this distinction disappears, the typhoid bacillus growing more abundantly.

In milk, typhoid bacilli do not produce coagulation. In litmus-milk, during the first twenty-four hours, the color is changed to a reddish or violet tinge by the formation of acid from the small quantities of monosaccharid present. Later the color becomes deep blue owing to the formation of alkali.

In Dunham's pepton solution no indol is produced. According to Peckham, however, continuous cultivation in rich pepton media may lead to eventual indol formation by typhoid bacilli. This fact has no bearing on the value of the indol test, as indol is never have formed under the usual cultural conditions.

Tested for its power to form acid from sugars commonly used in differential tests, typhoid bacilli form acid, but no gas, on the monosaccharides, on mannit, maltose and dextrin, and neither acid nor gas on lactose and saccharose. (See Table, p. 718.)

In the Hiss tube medium (formerly employed extensively) the typhoid bacillus within eighteen to twenty-four hours produces an even clouding by virtue of its motility, but does not form gas. In contradistinction to this, dysentery bacilli grow only along the line of inoculation, while bacilli of the colon group move in irregular sky-rocket-like figures away from the stab, at the same time breaking up the medium by the formation of gas-bubbles. Some actively motile colon bacilli cloud the medium, but the ruptures caused by the gas are always evident.

The differentiation of the typhoid bacillus in pure culture from similar microorganisms by means of its growth upon media has been the subject of many investigations. It is neither practicable nor desirable to enumerate all the various media which have been devised and reported. The aim has been chiefly the differentiation of typhoid bacilli from the bacilli of the colon group, and most of the media have been devised with this end in view. (See section on Media.)

Rothberger ${ }^{5}$ devised a mixture of glucose agar to which is added 1 per cent of a saturated aqueous solution of neutral-red. Shakecultures or stab-cultures are made in tubes of this medium. The typhoid bacillus causes no changes in it, while members of the colon group, by

${ }^{5}$ Rothberger, Cent. f. Bakt., xxiv, 1898. 
reduction of the neutral-red, decolorize the medium and produce gas by fermentation of the sugar.

Utilizing the fact that bile-salts are precipitated in the presence of acids, MacConkey devised a medium composed of sodium glycocholate, pepton, lactose, and agar (the composition of this medium is given on page 164), in which Bacillus typhosus grows without causing such change, but distinct clouding results from the growth of the colon bacillus which; producing acid from the lactose, causes precipitation of the bile-salts.

On Wurtz's lactose-litmus-agar (see page 153) the typhoid bacillus produces no acid, but eventually deepens the purple color to blue; the colon bacillus produces acid and in stab-cultures gas bubbles and the color changes to red.

In Barsiekow's (see page 164) lactose-nutrose-litmus mixture the typhoid bacillus causes no change, while the colon bacillus produces coagulation and an acid reaction.

The differentiation of the typhoid bacillus from other similar organisms of the typhoid, dysentery, colon group, is based chiefly on growth. upon differential media in which the inability of the typhoid bacillus to form acid or gas from lactose has been the most commonly used basis for differentiation. Various indicators to show whether acid has been formed, added to such media will sharply separate this organism from the colon bacilli and their close relatives. Failure to produce gas with dextrose, differentiates it from the paratyphoid group. The reader is referred to the differential tables given on page 687 and 718 , for the basic reactions upon which cultural differentiation is made. The media most convenient for this purpose are, in plates, the ConradiDrigalski medium, the Endo medium, the Krumwiede brilliant green medium, or the Teague eosin-methylene-blue medium, all of which are described in the section on media; and, in tubes, some of the most convenient media are the Hiss semi-solid mentioned above, Barsiekow's medium, or the Russell double sugar agar. The Russell double sugar agar is particularly useful to give a quick index of differentiation, since it contains both lactose and glucose, and, whereas, the colon group give redness throughout, and a few gas bubbles, the typhoid gives no gas, a red butt due to its action in the depths of the stab on the glucose and an uncolored surface growth.

Final differentiation is best based upon specific agglutination.

${ }^{6}$ Winslow, Kligler and Rothenberg, Jour. of Bacter., 4, 1919, 426. 
Winslow, Kligler and Rothberg, ${ }^{6}$ on the basis of recent careful investigations, describe the typhoid bacillus as a Gram-negative, non-spore forming, actively motile rod which forms translucent irregular colonies on gelatin, and a colorless growth on potato. It produced strong and prompt acid, but no gas, on media containing the hexoses, maltose, mannit, sorbit, xylose (rapid or slow), and dextrin; it does not attack arabinose, rhamnose, or lactose; produces a slight initial reddening of litmus milk, which, after two weeks, reverts to neutrality or slight alkalinity. It does not form indol, nor liquefy gelatin, does not grow in asparagin-mannitol medium, does not reduce neutral red, and causes browning of lead acetate medium (irregular). It has low tolerance for acid, but high tolerance for malachite and brilliant green dyes. It has characteristic serum agglutination.

Differences Within the Typhoid Group.-Recent work has shown that not all typhoid bacilli are culturally alike, there being two distinct groups, one which ferments xylose rapidly, the other slowly. Since there are also antigenic differences it may be necessary in the future to speak rather of a typhoid group than of the typhoid bacillus.

Xylose fermentations of typhoid bacilli have recently been studied in more detail by Krumwiede, Kohn and Valentine, ${ }^{7}$ and by Morishima. ${ }^{8}$ The first-named authors inoculated 37 strains of typhoid bacilli into xylose broth and found that 29 of them produced acid within twentyfour hours, while 8 of the strains required from five to thirteen days for this result. Morishima, of this laboratory, obtained rapid and slow xylose fermenters from a single strain by repeatedly fishing different colonies on plates. An atypical strain has recently been described by Bull and Pritchett ${ }^{9}$ whose bacillus agglutinated in typhoid serum typically up to 1 to 20,000 , but which gave positive indol reactions.

Biological Considerations.- The typhoid bacillus is an aërobic and facultatively anaerobic organism growing well both in the presence and in the absence of oxygen when certain sugars are present, showing a slight preference, however, for well aërated conditions. It grows most luxuriantly at temperatures about $37.5^{\circ} \mathrm{C}$., but continues to grow within a range of temperature lying between $15^{\circ}$ and $41^{\circ} \mathrm{C}$. . Its thermal death point, according to Sternberg, is $56^{\circ} \mathrm{C}$. in ten minutes. It remains alive in artificial cultures for several months or even years if moisture is supplied. In carefully sealed agar tubes Hiss found the organisms alive after thirteen years. In natural waters it may remain alive as long

${ }^{7}$ Krumwiede, Kohn and Valentine, Jour. of Med. Res., 38, 1918, 89.

${ }^{8}$ Morishima, Jour, of Bacter., March, 1921.

${ }^{9}$ Bull and Pritchett, Jour. of Exper. Med., 24, 1916, 55. 
as thirty-six days, according to Klein. ${ }^{10}$ In ice, according to Prudden, ${ }^{11}$ it may reinain alive for three months or over. Against the ordinary disinfectants, the typhoid bacillus is comparatively more resistant than some other vegetative forms. It is killed, however, by $1: 500$ bichlorid or 5 per cent carbolic acid within five minutes.

Pathogenicity.-In animals, some early investigators to the contrary, typhoidal infection does not occur spontaneously and artificial inoculation with the typhoid bacillus does not produce a disease analogous to typhoid fever in the human being. Frankel ${ }^{12}$ was able to produce intestinal lesions in guinea-pigs by injection of the bacilli into the duodenum, and recovered the bacteria from the spleens of the animals after death, but the disease produced was in no other respect analogous to typhoid fever in the human being. It is probable that typhoid bacilli injected into animals do not multiply extensively and that most of the symptoms produced are due to the poisons liberated from the dead bacteria. In corroboration of this view is the observation that inoculation with dead cultures is followed by essentially the same train of symptoms as inoculation with live cultures. ${ }^{13}$ The injection of large doses into rabbits or guinea-pigs intravenously or intraperitoneally is usually followed by a rapid drop in temperature, often by respiratory embarassment and diarrhea. Occasionally blood may be present in the stools. According to the size of the dose or the weight of the animal, death may ensue within a few hours, or, with progressive emaciation, after a number of days, or the animal may gradually recover.

Welch and Blachstein ${ }^{14}$ have shown that typhoid bacilli injected into the ear vein of a rabbit appear in the bile and may persist in the gall-bladder for weeks. Doerr, ${ }^{15}$ Koch, ${ }^{16}$ Morgan, ${ }^{17}$ and more recently Johnston 18 have all confirmed this, the last named showing that the typhoid bacillus could not only remain latent for a long time in the gall-bladder of rabbits, but would appear in the blood stream with considerable regularity after the seventh or ninth day, and persist in the gall-bladder for as long as one hundred and

\footnotetext{
${ }^{10}$ Klein, Med. Officers' Report, Local Govern. Bd., London, 1894.

11 Prudden, Med. Rec., 1887.

12 Frankel, Cent. f. klin. Med., 10, 1886.

${ }^{13}$ Petruschky, Zeit. f. Hyg., xii, 1892.

14 Welch and Blachstein, Bull. Johns Hop. Hosp., ii, 1891.

15 Doerr, Centralbl. f. Bakt., 1905.

${ }^{16}$ Koch, Zeitschr. f. Hyg., 1909.

17 Morgan, Jour. of Hyg., 1911.

18 Johnston, Jour. of Med. Res., xxvii, 1912.
} 
twenty-five days. Gay and Claypole ${ }^{19}$ have been able to produce the carrier state in rabbits with great regularity by growing the typhoid cultures used for inoculation upon agar containing 10 per cent defibrinated rabbit's blood. Such cultures are not as readily agglutinated by immune serum as are those grown on plain agar, and it may well be that they have acquired a certain degree of resistance to the serum antibodies which renders them more competent to survive in the body of the rabbit. Gay has used rabbits inoculated with such cultures for the determination of the efficacy of his sensitized vaccines.

Typical typhoid fever simulating the disease in man has not been produced in any animals except in chimpanzees, by Metchnikoff and Besredka, ${ }^{20}$ who produced it in connection with their experiments on protective vaccination: They produced a disease almost identical with human typhoid by feeding cultures to chimpanzees.

Typhoid Fever IN MAN.-It is not within the province of a book of this kind to give an accurate clinical description of the disease as it occurs in man in all its details. The disease is one in which a wide range of variation may occur, and in which complications are various and manifold. We will, therefore, give only a brief account of the infection as it is relevant to bacteriological work. The organisms enter by mouth, with food, water or contact with fingers, direct or indirect, as described in the epidemiological section. Subsequently, the organisms, which pass through the stomach uninjured, multiply in the intestine, but cause no symptoms for anywhere from seven to fourteen days. During this time they probably begin to proliferate partly within the mucous membrane of the bowel, although there is little definite knowledge concerning this. The symptoms of the disease begin insidiously by gradual malaise, headache, loss of appetite, sleeplessness, and during the first week of the actual signs of infection, the organisms have probably penetrated or are penetrating into the lymphatics. At this time there is a swelling of the lymphoid nodules of the intestine and Peyer's patches, and there is a moderate catarrhal inflammation of the mucous membrane. At this time too the bacilli enter the blood stream and can be found in blood culture.

Though formerly regarded as primarily an intestinal disease, the disease is in truth at this time a bacteriemia, and it is not impossible that the intestinal lesions are as much due to the action of toxic products which are excreted in part through the intestinal wall, as they are due

${ }^{19}$ Gay and Claypole, Arch. of Inf. Med., Dec., 1913.

'20 Metchnikoff and Besredka, Ann. de l'Inst. Past., 1911, 25, 193. 
to the direct reaction caused in the intestine by local growth of the bacilli. Secondarily, the bacilli appear and can be cultivated from the spleen, the liver, and can be demonstrated in the sinuses and tissues of the lymphatic and retroperitoneal lymph nodes.

Typhoid Bacilli in the Blood during the Disease.-The investigations of many workers have shown that typhoid bacilli are present in the circulating blood of practically all patients during the early weeks of the disease. Series of cases have been studied by Castellani, ${ }^{21}$ Schottmüller ${ }^{22}$ and many others. More recently Coleman and Buxton ${ }^{23}$ have reported their researches upon 123 cases, and have at the same time analyzed all cases previously reported. Their analysis of blood cultures taken at different stages in the disease is as follows

Of 224 cases during first week, 89 per cent were positive.

Of 484 cases during second week, 73 per cent were positive.

Of 268 eases during third week, 60 per cent were positive.

Of 103 cases during fourth week, 38 per cent were positive.

Of 58 cases after fourth week, 26 per cent were positive.

The technique recommended by Coleman and Buxton for obtaining blood cultures is that recommended by Conradi, ${ }^{24}$ slightly modified. The blood is taken into flasks each containing about 20 c.c. of the following mixture:

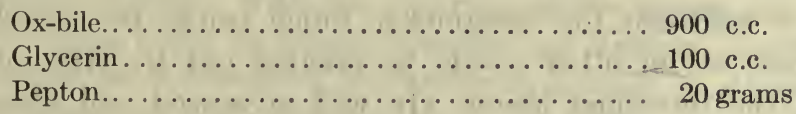

About 3 c.c. of blood are put into each flask. The ox-bile, besides preventing coagulation, may possibly neutralize the bactericidal substances present in the drawn blood. The flasks are incubated for eighteen to twenty-four hours, at the end of which time streaks are made upon plates of lactose-litmus-agar and the organisms identified by agglutination or by cultural tests.

European workers have generally preferred to make high dilution of the blood in flasks of bouillon, small quantities of blood, 1 to 2 c.c., being mixed with 100 to 150 c.c. of nutrient broth.

Epstein ${ }^{25}$ has reported excellent results from mixing the blood in

${ }^{21}$ Castellani, Riforma medica, 1900.

${ }^{22}$ Schottmüller, Deut. med. Worh., xxxii, 1900, and Zeit. f. Hyg., xxxvi, 1901.

2 'Coleman and Buxton, Am. Jour. of Med. Sci., 133, 1907.

${ }^{24}$ Conradi, Deut. med. Woch., xxxii, 1906.

${ }^{25}$ Epstein, Proc. N. Y. Path. Soc., N. S., vi, 1906. 
considerable concentration with 2-per-cent glucose agar and pouring plates.

The writers in hospital work have had equally good results with the bile medium and with broth in flasks, rather less uniform but still satisfactory results with the plating method. In general it may be said that any one of these methods carried out with reasonable accuracy may be satisfactorily employed.

Typhoid Bacilli in the Stools.-The examination of the stools for typhoid bacillus is performed for diagnostic purposes chiefly in obscure cases. It may, furthermore, furnish information of great hygienic importance. Thus Drigalski and Conradi ${ }^{26}$ have succeeded in isolating typhoid bacilli from the stools of ambulant cases so mild that they were not clinically suspected. It is by means of such examinations that the so-called typhoid-carriers are detected, a problem which is considered at length in the section dealing with epidemiology. Such cases have been known to harbor the bacilli for periods as long as several years.

The examination itself is fraught with difficulties, owing to the preponderating numbers of colon bacilli found in all feces and the difficulty of isolating the typhoid bacilli from such mixtures.

Reviewing the data collected by a number of investigators, it seems probable that the bacilli do not appear in the stools, at least in numbers sufficient for recognition, much before the middle of the second week, or, in other words, as pointed out by Hiss, about the time that the intestinal lesions are well advanced and ulceration is occurring. Thus Wiltschour ${ }^{27}$ could not determine their presence before the tenth day; Redtenbacher, ${ }^{28}$ in reviewing the statistics, states that in a majority of cases the bacilli first appear toward the end of the second week, and Horton-Smith ${ }^{29}$ could not find the bacilli before the eleventh day. Hiss, ${ }^{30}$ in an investigation of the same subject, obtained the following results:

First to tenth day, inclusive, twenty-eight cases examined; typhoid bacilli isolated from three; percentage of positive cases 10.7 per cent.

Eleventh to twentieth day, inclusive, forty-four cases examined; typhoid bacilli from twenty-two; percentage of positive cases 50 per cent.

${ }^{26}$ Drigalski and Conradi, Zeit. f. Hyg., xxxix, 1902.

${ }^{27}$ Wiltschour, Cent. f. Bakt., 1890.

${ }^{28}$ Redtenbacher, Zeit. f. klin. Med., xix, 1891.

${ }^{29}$ Horton-Smith, Lancet, May, 1899.

${ }^{30}$ Hiss, Med. News, May, 1901. 
Twenty-first day to convalescence, sixteen cases examined; typhoid bacilli isolated from thirteen; percentage of positive cases 81.2 per cent.

Stool Examination and Method of Typhoid Carrier Detection.Fecal carriers of typhoid bacilli may be detected by cultural methods applied either to specimens of feces or to duodenal contents, obtained by a tube passed through the stomach into the duodenum. The simplest method, of course, is direct examination of the feces. The duodenal tube, however, will probably be used considerably in the future, since in some cases it may be positive when stool cultures are negative. As a matter of fact, in the hands of Garbat ${ }^{31}$ and Nichols, ${ }^{32}$ the duodenal method seems to have given more regular results than the stool method.

Stool Examinations.- Stool material for typhoid examination should be fresh. Preserving stools for as long as twelve hours will diminish positive findings by 50 per cent. If large numbers are to be examined, it is a good plan to give mild, saline catharties in the morning, so that all specimens can be collected at about the same time. It is best to collect specimens by cotton swabs, on swab sticks thrust into tubes in which there are a few drops of salt solution to prevent drying. We have found that rectal swabbing, if properly carried out, may be a valuable method of collecting material. If it is absolutely necessary to ship stools some distance, the addition of 20 per cent glycerin is of advantage.

A suspension of about one part of feces to twenty-five parts of salt solution is made, thoroughly emulsified, and allowed to stand to allow the large particies to settle. With this material, surface smears are made with a glass rod upon plates of either Robinson and Rettger's modification of Endo's medium, or Krumwiede's brilliant green medium, as described in the section on media. It is of advantage to use the large plates. A bent glass rod is dipped into the emulsion and rubbed over the surface of a plate, beginning in the center, by passing in concentric eireles so that the entire plate is gently smeared. A second plate is inoculated in the same way, without redipping. It is sometimes well to make similar plates with a $1: 5$ dilution of the original suspension.

Plates for this purpose should be poured and allowed to dry on a laboratory desk for a few hours before use, and should be kept in the dark if Endo's medium is used. Great care in the accurate production and testing out of the media, should be taken as indicated in the section deseribing these media. The plates should either be inverted in the incubator, or else earthen-ware covers should be used. Large pieces of blotting paper inserted under the lid serve the same purpose.

${ }^{31}$ Garbat, The Typhoid Carrier Problem, Monographs of the Rock. Inst., in press.

${ }^{32}$ Nichols H. J., Jour. Exp. Med., xxiv, 1916, 497; Jour. of A. M. A., lxviii, 1917, 958. 
After eighteen hours growth, the plates should be examined for typical colonies. Suspicious colonies should be immediately inoculated upon tubes of Russell double-sugar medium. Slide agglutinations against 1:100 dilution of a high titer stock typhoid antiserum should be made for preliminary identification from suspicious colonies, of course together with morphological determination by smear and stain.

Much information ean be obtained after twelve more hours, by observations of the growth in the Russell double-sugar medium. From this tube, then, the growth can be emulsified in salt solution, and macroscopic agglutinations set up. This usually is sufficient to identify the organism, but it is always well to set up a few sugar fermentation tubes.

Duodenal examinations are made by means of the Einhorn duodenal tube, which is sterilized by boiling, and given to the patient the evening before the examination is to be made, about three hours after the last meal. We take our description chiefly from Garbat who has had considerable experience with this method. The patient, properly instructed, swallows the tube without gagging, with ease, and retains it throughout the night. On the following morning it has usually passed into the duodenum, and bile can be aspirated with a sterile 20 c.c. Luer syringe. Suction must usually be exerted, and Garbat recommends a well fitting syringe because such suction must often be strong. In about 5 per cent of Garbat's eases the tube remained in the stomach and more difficulty was experienced with the test. When there is difficulty in obtaining sufficient bile, the patient is made to sit up in bed with his head bent forward, pressing upward on the abdomen with the palms of his hands. Sometimes the flow of bile can be stimulated by a cold drink. The bile is handled bacteriologically on Endo plates.

Typhoid Bacilli in the Urine.-Careful investigation has revealed typhoid bacilli in the urine in about 25 per cent of all patients. Neumann ${ }^{33}$ discovered the bacilli in eleven out of forty-six and Karlinsky ${ }^{34}$ in twenty-one out of forty-four cases. Investigations by Petruschy, ${ }^{35}$ Richardson, ${ }^{36}$ Horton-Smith, ${ }^{37}$ Hiss, ${ }^{38}$ and others have confirmed these results. In general the bacilli have not been found before the fifteenth day of the disease, and examination of the urine, therefore, can be of little early diagnostic value. A series of seventy-five cases examined by Hiss before the fourteenth day of the disease did not once reveal typhoid bacilli in the urine. On the other hand, they have been found

${ }^{33}$ Neumann, Berl. klin. Woch., xxvii, 1890.

${ }^{34}$ Karlinsky, Prag. med. Woch., xv, 1890.

${ }^{35}$ Petruschy, Cent. f. Hyg., xxiii, 1898.

${ }^{36}$ Richardson, Jour. Exp. Med., 3, 1898.

${ }^{37}$ Horton-Smith, Lancet, May, 1899.

${ }^{38}$ Hiss, Med. News, May, 1901. 
to be present for weeks, months, and, in isolated cases, for year's after convalescence, the examination thus having much hygienic importance. They are probably present in about 12 per cent of cases during the early days of convalescence. In most of these, albumin is present in the urine in considerable quantities. The bacilli usually appear and disappear with the albuminuria.

An obstinate cystitis caused by typhoid bacilli may follow in the path of typhoid fever. Such cases have been reported by Blumer,39 Richardson, ${ }^{40}$ and others. Suppurative processes in the kidneys are less frequent. It is noteworthy, also, that in the course of, and following, typhoid fever Bacillus coli is often present in the urine. This may obstinately persist for considerable periods after convalescence.

For examination of the urine for typhoid bacilli, specimens should be taken with aseptic precautions and planted directly into equal volumes of broth. Direct plates on Endo can also be made, or had better be made at the same time. It is relatively easy under such circumstances to obtain the organism if present.

Typhoid Bacilli in the Rose Spots.-Neufeld ${ }^{41}$ obtained positive results in thirteen out of fourteen cases. According to his researches and those of Fränkel, ${ }^{42}$ the bacilli are localized not in the blood, which is taken when the rose spots are incised, but are crowded in large numbers within the lymph spaces.

Typhoid Bacilli in the Sputum.-In rare cases typhoid bacilli have been found in the sputum of cases complicated by bronchitis, bronchopneumonia, and pleurisy. Such cases have been reported by Chantemesse and Widal, ${ }^{43}$ Fränkel, ${ }^{44}$ and a number of others. Empyemia, when it occurs in connection with such cases, is usually accompanied by a mixed infection. From a hygienic point of view the spread of typhoid fever by means of the sputum is probably of rare occurrence.

Suppurative Lesions Due to Typhoid Bacillus.-In the course of typhoid convalescence or during the latter weeks of the disease, suppurative lesions may occur in various parts of the body. The most frequent localization of these is in the periosteum, especially on the long bones, and in the joints. A considerable number of such lesions have

\footnotetext{
${ }^{39}$ Blumer, Johns Hopk. Hosp. Rep., 5, 1895.

${ }^{40}$ Richardson, loc. cit.

${ }^{41}$ Neufeld, Zeit. f. Hyg., xxx, 1899.

${ }^{42}$ F'ränkel, Zeit. f. Hyg., xxxiv, 1909.

${ }^{43}$ Chantemesse and Wilal, Arch. de physiol. norm. et path., 1887.

${ }^{44}$ Fränkel, Deut. med. Woch., xv and xvi, 1899.
} 
been described by Welch, Richardson, ${ }^{*}$ and others. They usually take the form of periosteal abscesses, often located upon the tibia, occurring either late in the disease or months after convalescence, and are characterized by very severe pain. Osteomyelitis may also occur, but is comparatively rare. Subcutaneous abscesses and deep abscesses in the muscles, due to this bacillus, have been described by Pratt. ${ }^{45}$ Synovitis may also occur.

Meningitis, due to the typhoid bacillus, occurs occasionally, usually during convalescence from typhoid fever. A case of primary typhoid meningitis has been reported by Farnet. ${ }^{46}$

Peritoneal abscesses, due to the typhoid bacillus, have been reported. The writer ${ }^{47}$ has reported a case in which typhoid bacilli were found free in the peritoneal cavity during typhoid fever without perforation of the gut.

Isolated instances of typhoid bacilli in abscesses of the thyroid and parotid glands and in brain abscesses have been observed.

Typhoid Fever without Intestinal Lesions.-A number of cases have been reported in which typhoid bacilli have been isolated from the organs after death or from the secretions during life of patients in whom the characteristic lesions of typhoid fever have been lacking. Most of these cases must be regarded as true typhoid septicemias. In some cases the bacilli were isolated from the spleen, liver, or kidneys; in others, from the urine or the gall-bladder. In a case observed by Zinsser the bacilli were isolated from an infarct of the kidney removed by operation. In this case the clinical course of the disease had pointed only toward the existence of an indefinite fever accompanied by symptoms referable to the kidneys. The Widal test, however, was positive. A summary of such cases, together with several personally observed, has been given by Flexner. ${ }^{48}$

Poisons of the Typhoid Bacillus.-Investigation of the toxic products of the typhoid bacillus has occupied the attention of a large number of workers. The first to do experimental work upon the subject was Brieger ${ }^{49}$ soon after the discovery and cultivation of the bacillus. That toxic substances can be obtained from typhoid cultures is beyond question. There is, however, a definite difference of opinion

\footnotetext{
* Richardson, Jour. Boston Soc. Med. Sci., 5, 1900.

45 Pratt, Jour. Boston Soc. Med. Sci., 3, 1899.

${ }^{46}$ Farnet, Bull. de la soc. méd. des hôp. de P., 3, 1891.

${ }^{47}$ Zinsser, Proc. N. Y. Path. Soc., 1907.

48 Flexner, Johns Hopk. Rep., 5, 1896.

49 Brieger, Deut. med. Woch., xxvii, 1902.
} 
as to whether these poisons are so-called endotoxins only, or whether they are in part composed of soluble toxins comparable to those of diphtheria and tetanus, following the injection of which antitoxic substances may be formed.

The evidence so far seems to bear out the original contention of Pfeiffer, ${ }^{50}$ who first advanced the opinion that the poisonous substances are products of the bacterial body set free by destruction of the bacteria by the lytic substances of the invaded animal or human being. These poisons, when injected into animals for purposes of immunization, in Pfeiffer's experiments, did not incite the production of neutralizing or antitoxic bodies, but of bactericidal and lytic substances. That these endotoxins constitute by far the greater part of the toxic products of the typhoid bacillus can be easily demonstrated in the laboratory, by the simple experiment of filtering a young typhoid culture (eight or nine days old) and injecting into separate animals the residue of bacilli and the clear filtrate respectively. In such an experiment there will be little question as to the overwhelmingly greater toxicity of the bacillary bodies as compared with that of the culture filtrate. On the other hand, if such cultures, especially in alkaline media, are allowed to stand for several months and the bacilli thus thoroughly extracted by the broth, the toxicity of the filtrate is found to be greatly increased.

Nevertheless, more recent experiments by Besredka, ${ }^{51}$ Macfadyen, ${ }^{52}$ Kraus and Stenitzer, ${ }^{53}$ and others have tended to show that, together with such endotoxic substances, typhoid bacilli may produce a true toxin which is not only obtainable by proper methods from comparatively young typhoid cultures, but which fulfills the necessary requirement of this class of poisons by producing in treated animals a true antitoxic neutralizing body.

The typhoid endotoxins may be obtained by a variety of methods. Hahn ${ }^{54}$ has obtained what he calls "typhoplasmin" by subjecting them to a pressure of about four hundred atmospheres in a Buchner press. The cell juices so obtained are cleared by filtration. Macfadyen has obtained typhoid endotoxins by triturating the bacilli after freezing 1896.

${ }^{50}$ Pfeiffer, Deut. med. Woch., xlviii, 1894; Pfeiffer und Kolle, Zeit. f. Hyg., xxi,

${ }^{51}$ Besredka, Ann. de l'inst. Pasteur, 1895, 1896.

${ }^{52}$ Macfadyen and Rowland, Cent. f. Bakt., I, xxx, 1901; Macfadyen, Cent. f. Bakt., I, 1906. 1907.

${ }^{53}$ Kraus und Stenitzer, Quoted from "Handb. d. Tech.," etc., 1, Fischer, Jena,

${ }^{54}$ Hahn, Münch. med. Woch, xxiii, 1906. 
them with liquid air and extracting in 1:1000 potassium hydrate. Besredka obtained toxic substances by- emulsifying agar cultures of bacilli in' salt solution, sterilizing them by heating to $60^{\circ} \mathrm{C}$. for about one hour, and drying in vacuo. The dried bacillary mass was then ground in a mortar and washed in sterile salt solution which was again heated to $60^{\circ} \mathrm{C}$. for two hours. The remnants of the bacterial bodies settle out and the slightly turbid supernatant fluid contains the toxic substances.

Vaughan ${ }^{55}$ has obtained poisons from typhoid bacilli by extracting at $78^{\circ} \mathrm{C}$. with a 2 -per-cent solution of sodium hydrate in absolute alcohol. In this way he claims to separate by hydrolysis a poisonous and a non-poisonous fraction. He claims, moreover, that this poisonous fraction is similar to the poisons obtained in the same way from Bacillus coli and the tubercle bacillus, and other protein substances, believing that the specific nature of such proteids depends upon the non-toxic fraction.

A simple method of obtaining toxins from typhoid bacilli is carried out by cultivating the microorganisms in meat-infusion broth, rendered alkaline with sodium hydrate to the extent of about 1 per cent. The cultures are allowed to grow for two or three weeks and then sterilized by heating to $60^{\circ} \mathrm{C}$. for one hour, and allowed to stand for three or four weeks at room temperature. At the end of this time the cultures may he filtered through a Berkefeld or Pasteur-Chamberland filter and will be found to contain strong toxic substances.

The accounts concerning the thermostability of the various toxins obtained are considerably at variance. In general, corresponding with other endotoxins, observers agree in considering them moderately resistant to heat, rarely being destroyed at temperatures below $70^{\circ} \mathrm{C}$. We have ourselves often boiled typhoid suspensions without destroying their toxicity for guinea pigs.

Intravenous inoculation of rabbits with typhoid endotoxins, if in sufficient quantity, produces, usually within a few hours, a very marked drop in temperature, diarrhea, respiratory embarrassment, and death. If given in smaller doses or by other methods of inoculation-subcutaneous or intraperitoneal-rabbits are rendered extremely ill, with a primary drop in temperature, but may live for a week or ten days and die with marked progrèssive emaciation, or may survive. Guineapigs and mice are susceptible to the endotoxins, though somewhat less so than rabbits.

In unpublished experiments we have perfused the isolated guinea pig heart with typhoid extracts for prolonged periods without killing it, 
showing that the poison does not act upon the normal heart muscle directly.

Zinsser, Parker and Kuttner ${ }^{55}$ have recently shown that in broth cultures of the typhoid bacillus as young as five to six hours, a mildly toxic substance is formed which can be recovered in filtrates, and which, injected into rabbits intravenously, gives rise to definite symptoms, after an incubation time of an hour or more. This substance is not specific in that it is formed by many other different bacteria similarly grown, and is not antigenic in all probability. It can also be obtained by washing young agar growths repeatedly in salt solution, and filtering. Whether or not this substance plays a part in the disease cannot be stated at the present time.

\section{IMMUNITY AND ANTIBODIES}

Animals may be actively immunized by the injection of typhoid bacilli in gradually increasing doses. In actual practice, this is best accomplished by beginning with an injection of about 1 c.c. of broth culture heated for ten minutes at $60^{\circ}$ in order to kill the bacilli. After five or six days, a second injection of a larger dose of dead bacilli is administered; at similar intervals, gradually increasing doses of dead bacilli are given and finally considerable quantities of a living and fully virulent culture may be injected without serious consequences to the animal. While this method is convenient and usually successful, it is also possible to obtain satisfactory immunization by beginning with very small doses of living microorganisms, according to the early method of Chantemesse and Widal, ${ }^{56}$ and others.

Such active immunization, successfully carried out upon rabbits and guinea-pigs, within a short time after the discovery of the typhoid bacillus, was believed to depend upon the development of antitoxic substances in immunized animals. This point of view, however, was not long tenable, and was definitely disproven by the investigations of Pfeiffer and Kolle ${ }^{57}$ in 1896 . These investigators, as well as a large number of others working subsequently, have shown that there are present in the blood serum of typhoid-immune animals and human beings, bacteriolytic, bactericidal, and agglutinating substances, and to a lesser extent, precipitating and opsonic bodies but no true antitoxins.

${ }^{55}$ Zinsser, Parker and Kuttner, Proc. of the Soc. Exper. Med. and Biol., Meeting, Nov., 1920.

${ }^{56}$ Chantemesse and Widal, Ann. de l'inst. Pasteur, 1892.

${ }^{57}$ Pfeiffer und Kolle, Zeit. f. Hyg., xxi, 1896. 
One attack of typhoid fever protects against subsequent infection. Accurate statistics upon the matter have been difficult to obtain, however, because histories of the disease are apt to be indefinite, and until recently, no proper differentiation was made between true typhoid fever and the paratyphoid group. However, taking into consideration these possibilities of error, the estimations made by various clinicians who have studied the subject, indicate that a second attack of typhoid fever occurs in not more than from 0.7 to 4 per cent of all cases. Two to 3 per cent represents a fair average of all estimates made. When typhoid fever does occur for the second time, it is usually of a milder type than the first attack, though, according to V. Vaughan, Jr., this is not always the case.

Circulating antibodies disappear from the typhoid convalescent usually within the first seven months after recovery. Permanent immunity cannot, therefore, be explained upon the basis of serum antibodies. The ultimate cause for permanent immunity, in all diseases in which it occurs, must be regarded as depending upon the physiological unit, namely, the tissue cell. It is likely that individuals who have passed through an infection of this nature, thereafter retain a capacity. to react more rapidly and effectively to small quantities of introduced antigen. A case in point is the well-known experiment of Wassermann, who immunized a number of rabbits to typhoid bacilli until a highly agglutinin titer was produced. He kept these rabbits until their blood had returned to normal and no agglutinins could be found. Subsequently he reinoculated them with typhoid bacilli, at the same time giving a number of normal control rabbits similar injections. The previously treated rabbits responded with a rapid and powerful antibody production in contrast to the slower antibody curve of those that had received the typhoid antigen for the first time. Recent observations by Moon on revaccination of previously vaccinated people, have given analogous results; and it appears from this that a person, once immunized, is capable of reacting with much greater promptness than a normal individual. Our own idea would be somewhat as follows:

When the typhoid bacillus enters the bowel of the infected subject it begins to proliferate and gradually enters into the lymphatic system. As a consequence, a small amount of antigen is gradually introduced into the circulation, reaches the cells and stimulates antibody production. In the normal individual this reaction is a slow one, and the typhoid bacilli multiply with a speed disproportionate to the appearance of antibodies. In the previously immunized individual or in the person who has had the disease, the first absorption of small amounts of antigen 
is followed by a tissue reaction (a part of which is evident as antibody production) so rapid that the protective processes are developed with sufficient potency to check the infection before it has reached a phase at which symptoms become apparent.

Bactericidal and Bacteriolytic Substances.-The bacteriolytic substances in typhoid-immune serum may be demonstrated either by the intraperitoneal technique of Pfeiffer or in vitro. In the former experiment a small quantity of a fresh culture of typhoid bacilli is mixed with the diluted immune serum and the emulsion injected into the peritoneal cavity of a guinea-pig. Removal of peritoneal exudate with a capillary pipette and examination in the hanging drop will reveal, within a short time, a swelling and granulation of the bacteria-the so-called Pfeiffer phenomenon. The test in vitro, as recommended by Stern and Korte, ${ }^{58}$ may be carried out by adding definite quantities of a fresh agar culture of typhoid bacilli to progressively increasing dilutions of inactivated immune serum together with definite quantities of complement in the form of fresh normal rabbit or guinea-pig serum. At the end of several hours' incubation at $37.5^{\circ} \mathrm{C}$. definite quantities of the fluid from the various tubes are inoculated into melted agar and plates are poured to determine the bactericidal action. Careful colony counting in these plates and comparison with proper controls will not only definitely demonstrate the presence of bactericidal substances in the immune serum, but will furnish a reasonably accurate quantitative estimation. (For technic of these tests see page 307.)

Although normal human serum contains in small quantity substances bactericidal to typhoid bacilli, moderate dilution, 1:10 or $1: 20$, of such serum will usually suffice to eliminate any appreciable bactericidal action. The bactericidal powers of immune serum, on the other hand, are often active, according to Stern and Korte, in dilutions of over $1: 4000$. The specificity of such reactions gives them a considerable degree of practical value, both in the biological identification of a suspected typhoid bacillus in known serum and in the diagnosis of typhoid fever in the human patient by the action of the patient's serum on known typhoid bacilli. In the publication of Stern and Korte, quoted above, it was found that typhoid patients during the second week often possess a bactericidal power exceeding 1:1000, whereas the blood of normal human beings was rarely active in dilutions exceeding 1 : 50 or 1: 100. While scientifically accurate the practical application of bactericidal determinations for diagnosis

${ }^{58}$ Stern und Korte, Berl. klin. Woch., x., 1904. 
presents considerable technical difficulties and gives way to the no less accurate and much simpler method of agglutination.

Agglutinins.-Agglutinins are formed in animals and man inoculated with typhoid bacilli, and in the course of typhoid fever. It was, in fact, while studying the typhoid bacillus that the agglutinins were first discovered by Gruber and Durham.

In animals, by careful immunization, specific typhoid agglutinins may easily be produced in sufficient quantity to be active in dilution of $1: 10,000$, and occasionally even $1: 50,000$ or over. In the blood of typhoid patients, the agglutinins may often be found in dilutions. of $1: 100$ and over. It is interesting to note that irrespective of the agglutinin contents of any given serum, there may occasionally be noted differences in the agglutinability of various typhoid cultures, a point which is practically important in the choice of a typhoid culture for routine diagnosis work. Weeny ${ }^{59}$ has called attention to the fact that bacilli which do not readily agglutinate when directly cultivated from the body, may often be rendered more sensitive to this reaction by several generations of cultivation upon artificial media. Walker has noted ${ }^{60}$ a loss of agglutinability if the bacilli are cultivated in immune serum. A similar alteration in the agglutinability of typhoid bacilli was noted by Eisenberg and Volk ${ }^{61}$ when they subjected the microorganism to moderate heat or to weak acids such as $\frac{\mathrm{N}}{4} \mathrm{HCl}$.

Morishima ${ }^{62}$ has recently studied the same phenomenon, and has confirmed the observations of Eisenberg and Volk, ${ }^{63}$ and others. He has also shown, however, that if organisms are cultivated in anti-serum for a sufficiently long time, their preliminary inagglutinability will eventually, after twenty to seventy-five days, revert to almost normal agglutinability.

Practical application of agglutination to bacteriological work is found in the identification of suspected typhoid bacilli, and in the diagnosis of typhoid fever.

When it is desired to determine whether or not a given bacillus is a typhoid bacillus, mixtures may be made of young broth cultures, or preferably of emulsions of young agar cultures in salt solution, with dilutions of immune serum. The tests are made microscopically in the hanging-drop preparation or, preferably, macroscopically in

${ }^{59}$ Weeny, Brit. Med. Jour., 1889.

${ }^{60}$ Walker, Jour. of Path. and Bact., 1892; Totsuka, Zeit. f. Hyg., xlv, 1903.

${ }^{61}$ Eisenberg und Volk, Zeit., f. Hyg., xlv, 1903.

${ }^{62}$ Morishima, Jour. of Bacter., March, 1921.

${ }^{63}$ Eisenberg and Volk, Erbeg. der Immunit. Exper. Ther. Bakt. u. Hyg., 1913, 73. 
small test tubes. In all cases it is desirable first to determine the agglutinating power of the serum when tested against a known typhoid culture. (For detailed technique, see chapter on Technique of Serum Reactions, page 302, 282.)

In scientific investigations, specific agglutinations in high dilutions of immune serum constitute very strong proof of the species of the microorganism and may often furnish much information as to the biological relationships between similar species. It is found in immunizing animals with any given strain of typhoid bacilli, that there are formed the "chief" or "major" agglutinins which are specific and active against the species used in immunization, and the "group" or "minor" agglutinins, active also against closely related microorganisms. The following extract from a table will serve to illustrate this point in the case of typhoid and allied bacilli.

\begin{tabular}{|c|c|c|c|c|c|}
\hline \multirow{2}{*}{ 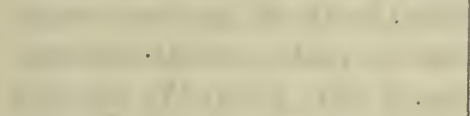 } & \multicolumn{5}{|c|}{ Highly Immune Typhoid Serum. } \\
\hline & $1: 100$ & $1: 2.50$ & $1: 500$ & $1: 1000$ & $1: 2500$ \\
\hline B. typh. & + & + & + & + & + \\
\hline B. paratyph. (Schottmüller). & + & + & + & - & - \\
\hline B. enteritidis............... & + & - & - & - & - \\
\hline B. coli communis.............. & + & - & - & - & - \\
\hline
\end{tabular}

The sera of most adult normal animals and human beings usually contain a small amount of agglutinin for these bacilli. Immunization with the typhoid bacillus, while increasing chiefly the agglutinin for this bacillus itself, also to a slighter extent increases the group agglutinins for other closely allied species. That these group agglutinins are separate substances and not merely a weaker manifestation of the action of the typhoid agglutinin itself upon these other microorganisms, may be demonstrated by the experiments of agglutinin absorption. (See section on Agglutinins, page 288.)

In the clinical diagnosis of typhoid fever, the phenomenon of agglutination was first utilized by Widal. ${ }^{64}$ This observer called attention to the fact that during the last part of the first or the earlier days of the second week of typhoid fever, as well as later in the disease and in convalescence, the blood serum of patients would cause agglutination of typhoid bacilli in dilutions of $1: 10$, or over, whereas the serum, of normal individuals usually exerted no such influence. Upon this basis

${ }^{64}$ Widal, Bull. de la soc. méd. des hôpit., vi, 1896; Widal et Sicard, Ann. de l'inst. Pasteur, xi, 1897. 
he recommended, for the diagnosis of the disease, the employment of a microscopic agglutination test carried out by the usual hanging-drop technique. The reaction of Widal is, at present, widely depended upon for diagnostic purposes and although not universally successful, owing to irregularities in agglutinin formation in some patients and because of differences in agglutinability of the cultures employed, it is nevertheless of much value. The fact that the recent work of Hooker and of Weiss has shown that typhoid bacilli differ in antigenic properties, and may on the basis of agglutination and agglutinin absorption be divided into a number of groups, is not of sufficient practical importance to necessitate the use of a variety of strains since the atypical antigenic ones are relatively rare. Original conclusions as to the dilutions of the serum which must be employed, have, however, necessarily been modified. Owing to the fact that Gruber, ${ }^{65}$ Stern, ${ }^{66}$ Fränkel, ${ }^{67}$ and a number of others have found that occasionally normal serum will give rise to agglutination of typhoid bacilli in dilutions exceeding $1: 10$, it has been found necessary, whenever making a diagnostic test, to make several dilutions, the ones most commonly employed being $1: 20,1: 40,1: 60$, and $1: 80$. The wide application of the method has given rise to the development of a number of technical procedures, all of them devised with a view toward simplification. In ordinary hospital work, it is most convenient to keep on hand upon slant agar, a stock typhoid culture, the agglutinability of which is well known. From this stock culture, fresh inoculations upon neutral bouillon should be made each day, so that a young broth culture may always be on hand to furnish actively motile, evenly distributed bacteria. These bouillon cultures may be grown for from six to eight hours at incubator temperature or for from twelve to eighteen hours at room temperature. The temperatures at which the broth cultures are kept must depend, to a certain extent, upon the peculiarities of the typhoid bacillus employed, since some strains are more actively motile and furnish a more suitable emulsion if kept at a temperature lower than $37.5^{\circ} \mathrm{C}$. A false clumping in the broth cultures due to a too-high acidity of the bouillon or a tooprolonged incubation must be carefully guarded against. It is also possible to use for this test an emulsion of typhoid bacilli prepared by rubbing up a small quantity of a young agar culture in salt solution. Uniformity in the preparation of broth cultures or of emulsions should be observed, since the quantitative relationship between typhoid bacilli

${ }^{65}$ Gruber, Verhand. Congr. f. inn. Med., Wiesbaden, 1896.

${ }^{66}$ Stern, Cent. f. inn. Med, xlix, 1896.

${ }^{67}$ Fränkel, Deut. med. Woch., ii, 1897. 
and agglutinins will markedly affect the completeness or incompleteness of the reaction. In high dilutions an excess of typhoid bacilli may bring about complete absorption of all the agglutinins present, without agglutinating all the microorganisms.

The blood of the patient to be used for a Widal test may be obtained in a number of ways. The most convenient method is to bleed the patient from the ear or finger into a small glass capsule, in the form of that used in obtaining blood for the opsonin test, or into a small centrifuge tube. About 0.5 to 1 c.c. is ample. These capsules or tubes, after clotting of the blood, may be placed in the centrifuge which in a few revolutions will separate clear serum from clot. The dilutions of the serum are then made. It is best to use sterile physiological salt solution as a diluent, but neutral broth may be used. The dilutions may be made either by means of an ordinary blood-counting pipette or by means of a capillary pipette upon which a mark with a grease pencil, made about an inch from the tip, furnishes a unit of measure, and upon which suction is made by means of a rubber nipple. It is convenient to have at hand a small porcelain palette such as that used by painters, in which the various cup-like impressions may be utilized to contain the various dilutions. Dilutions of the serum are made, ranging from $1: 10$ to $1: 50$. A drop of each of these dilutions is mixed with a drop of the typhoid culture or emulsion upon the center of a cover-slip and the coverslip inverted over a hollow slide. A control with normal serum and with the same culture should always be made and also one with the culture alone to exclude the possibility of spontaneous clumping. Mixture with the typhoid culture, of course, each time doubles the dilutions so that, for instance, a drop of serum dilution $1: 10$, plus a drop of the typhoid culture, gives the final dilution of $1: 20$. The preparation may be examined with a high power dry lens or an oil immersion lens. In a positive reaction, the bacilli, which at first swim about actively, singly or in short chains, soon begin to gather in small groups and lose much of their activity. Within one-half to one hour, they will be gathered in dense clumps between which the fluid is clear and free from bacteria, and only upon the edges of the agglutinated masses may slight motility be observed. The degree of dilution and the time of exposure at which such a reaction may be regarded as of specific diagnostic value have been largely a matter of empirical determination. It is generally accepted at present that complete agglutination within one hour in dilutions from $1: 40$ to $1: 60$ is definite proof of the existence of typhoid infection. Exceptions, however, to this rule may occur. Agglutinations of typhoid bacilli in dilutions of $1: 40$, and over, have occasionally 
been observed in cases of jaundice and of tuberculosis, and these conditions must occasionally be considered, though their importance was formerly exaggerated.

The method of making the Widal test from a drop of whole blood dried upon a slide, is not to be recommended since accuracy in dilution by this method is practically impossible.

As stated above, the agglutinin reaction rarely appears in typhoid fever before the beginning of the second week. It may continue during convalescence for as long as six to eight weeks and occasionally, in cases where there is a chronic infection of the gall-bladder, a Widal reaction may be present for years after an attack.

For very exact work, even in clinical cases, the microscopic agglutination method may be replaced by macroscopic agglutination, according to the technique described in another section (page 303.)

In order to avoid both the necessity of keeping alive typhoid cultures for routine agglutination tests and also to preclude the danger of infection by the use of living culture, Ficker ${ }^{68}$ has recommended typhoid bacilli killed by formalin. This method has no advantages for practical purposes and in scientific bacteriological work it is, of course, not to be considered in comparison with the more exact methods.

The more recently introduced general practice of vaccination in typhoid fever has added a complicating factor to diagnostic agglutination. Individuals so vaccinated develop agglutinins in consequence of the inoculations, which may persist for six months or more, and even after they have disappeared from the blood stream, various non-typhoid febrile conditions may induce their appearance in the circulation for reasons not well understood. In consequence of this, it is necessary, before drawing conclusions concerning the meaning of a Widal reaction, to be thoroughly informed concerning the vaccination history of the patient and the time which has elapsed since the vaccination was done. A certain amount of reliable information may be obtained even in such cases by the study of the quantitative changes in the agglutinins in the patient's blood by the comparative method of Dreyer as in use in the United States Army.

This method, however, is, in our opinion, not sufficiently useful or simple to be recommended for ordinary clinical use. In the Dreyer method, standardized suspensions of Bacillus typhosus or the paratyphoid types are used. To obtain these, cultures of the bacilli are grown for about two weeks by daily transplant in broth, a procedure

${ }^{68}$ Ficker, Berl. klin. Woch., xlvii. 1903. 
which is supposed to increase agglutinability. After this, it is planted in flasks in broth, allowed to grow overnight, and 0.1 per cent formalin is added. ${ }^{69}$ This formalinized culture is placed in the refrigerator and shaken frequently during four or five days. It is then standardized for opacity against an arbitrary standard kept on hand, and prepared in the Army Medical School. Necessary dilutions are made with physiological salt solution to which 0.1 per cent formalin has been added.

The suspension is then standardized for agglutinability against a known agglutinating immune serum. For this purpose incubation at $55^{\circ}$ for two hours is a method used for the final reading. It is easy, from this test, then, to determine the agglutinability factor of the new suspension.

The example cited in the Army Medical School War Manual, No. 6, is as follows: If the dilution of the solution in which the standardized suspension is agglutinated is 1 to 6400 , while that of the new suspension is 1 to 3200 , then the factor of the new bacterial suspension is one-half.

By the use of such suspension it is clear that comparative titrations of the rise and fall of agglutinins in the patient's serum can be made, and information obtained which may have considerable importance in deciding whether the appearance of agglutinins in the patient is due to previous vaccination, or is present in response to a fresh infection.

Precipitins.-The investigations of Kraus, ${ }^{70}$ by which the precipitins were discovered, revealed specific precipitating substances, among others, also in typhoid immune sera. Since Kraus' original investigation, these substances have been studied by Norris ${ }^{71}$ and others. ${ }^{72}$

Opsonins.-A number of observers have shown that opsonins specific for the typhoid bacillus are formed in animals immunized with these organisms. Opsonins are formed also in patients suffering from typhoid fever, but exact opsonic estimations in all these cases are extremely difficult because of the rapid lysis which these bacteria may undergo both in the serum, and intracellularly, after ingestion by the leucocytes. Klein ${ }^{73}$ has attempted to overcome this difficulty by working with dilutions of serum, at the same time using comparatively thick bacterial emulsions and exposures to the phagocytic action not exceed-

${ }^{69}$ U. S. Medical War Manual No. 6. Lea \& Febiger, 1919.

${ }^{70}$ Kraus, Wien. klin. Woch., xxxii, 1897.

${ }^{71}$ Norris, Jour. of Inf. Dis., I, 3, 1904.

72 Barker and Cole, 22d Ann. Session, Assn. of Amer. Phys.; Wash., 1897.

${ }^{73}$ Klein, Bull. Johns Hopkins Hosp., 1907. 
ing ten minutes. Chantemesse ${ }^{74}$ has claimed that the opsonic index of typhoid patients was increased after treatment with a serum obtained by him from immunized horses, and Harrison ${ }^{75}$ has reported similar results in patients treated by a modification of Wright's method of active immunization. Klein claims to have demonstrated that in typhoid-immune rabbits, after five injections, the opsonic contents of the blood were increased to an equal extent as the bactericidal substances. He concludes from this interesting observation that it may well be that the opsonins are quite as important in typhoid immunity as are the latter substances.

For diagnostic purposes in typhoid fever the estimation of the opsonic index, so far, has not been proven to be of great value.

\section{SANITARY CONSIDERATIONS IN TYPHOID FEVER}

Typhoid fever is a disease which has been constantly diminishing in frequency in civilized countries during the last one hundred years, but is still a very formidable cause of death rate and disability. The morbidity rates and death rates for typhoid fever vary considerably in different communities according to the extent to which sanitary supervision of water supplies, garbage and sewage disposal, etc., have been developed. In general, the United States has been considerably behind most European communities. In a table given by Gay ${ }^{76}$ a comparison of mortality averages per 100,000 population, comparing a group of over $31,000,000$ people compiled from the statistics of the 33 largest European cities, with $21,000,000$ people representing the populations of 57 of the largest American cities, the European mortality average was 6.5, and the American 19.59. In similar compilations taken by Gay largely from the report of the New York State Department of Health for 1914, it is shown that there has been a progressive decrease since 1910, running parallel to increased attention to water supplies and general sanitation. For more extensive figures on the prevalence of typhoid fever the reader is referred to the above-mentioned compilation of Gay. He states that in 1900 there were about 350,000 cases of typhoid fever in the United States as estimated by Whipple, who at the same time calculates that the cost to the community of these cases must have been approximately $\$ 212,000,000$. Since, as we shall see, it has been variously shown during the last ten years that typhoid

${ }^{74}$ Chantemesse, 14th Internatl. Cong. for Hyg., Berlin, 1907.

${ }^{75}$ Harrison, Jour. Royal Army Med. Corps, 8, 1907.

${ }^{76}$ Gay, F. P., Typhoid Fever, Macmillan Company, New York, 1918. 
fever is a distinctly preventable disease, amenable perhaps not to complete eradication on account of the difficulties of the carrier problem, but certainly readily subject to material diminution, much of this suffering and economic loss would seem unnecessary.

Infection with typhoid fever always means that intestinal contents of a case or a carrier have come into direct or indirect contact with something ingested by the patient. Since this is true, we may best begin the description of the circle of infection from man to man by first considering the manner in which the organism leaves the body of the patient and the carrier.

In the patient the typhoid bacillus begins to accumulate in the intestines during the later stages of the incubation time, and at this time will begin to appear in the feces. The organisms increase in the intestines from this time on, being distributed in very considerable numbers after the second week, and decreasing only towards the end of the disease, remaining present, however, throughout convalescence and sometimes, as we shall see, for months or years thereafter. During the second and third or later weeks, the organisms appear in the urine. It is generally stated that about 30 per cent of typhoid cases will show the organisms in the urine, but it seems likely that this is too low an estimate. Raubitschek, by precipitating considerable quantities of urine with ferric chlorate succeeded in finding the bacilli in 100 per cent of his cases in the earlier stages of the disease, and it is not at all unlikely that in slight numbers, and perhaps intermittently, they may appear in the urine of all typhoid cases. Other routes of distribution from the patient, such as suppurations, sputum, etc., are occasionally mentioned, but may be dismissed as of no practical sanitary importance.

Since the recognized typhoid case is usually well guarded from a sanitary point of view, the greater danger of typhoid infection lies in the mild, atypical, unrecognized case and in the carrier. Atypical, mild cases will probably become more and more frequent as typhoid vaccination becomes a more generalized habit. Such a case may show nothing more than a very slight febrile movement, with intestinal disturbances and diarrhea. Unless typhoid fever is particularly looked for and suspected, many of these cases may never be put upon typhoid precautions and the resulting possibilities of spread are obvious.

More important from the sanitary point of view, under the conditions of modern community life, however, is the typhoid carrier.

TyPHOID CARRIERs.-The great importance of the typhoid carrier in the spread of the disease has led to extensive studies of the problem in many countries during the last ten years. We may mention par- 
ticularly the studies of Conradi and Drigalski ${ }^{77}$ the paper of Sacquepée, ${ }^{78}$ the summary given by Kutscher ${ }^{79}$ in the Second Edition of the Kolle and Wassermann Handbook, the summary of Gay in the book mentioned above, and the article by Garbat, not yet published, but about to appear as one of the Monographs of the Rockefeller Institute. The first definite suggestion of the danger of typhoid infection emanating from convalescents long after the disease itself had been cured, came from Koch. ${ }^{80}$ He based this opinion at first upon purely epidemiological evidence, but in 1904 Drigalski ${ }^{81}$ began to isolate bacilli from individuals who were apparently in complete health. Sacquepée ${ }^{78}$ classifies typhoid carriers chiefly into convalescent carriers who become free of the bacilli within three months after the termination of their disease, and chronic carriers who continue to harbor the bacilli for many years, and perhaps permanently. In addition to this, there are a certain number of so-called healthy carriers in whom no history of their ever having had the disease can be arduced.

The distinction between a temporary carrier and a chronic carrier must, of course, be to a certain degree arbitrary, but in general it may be said that in most typhoid cases the organisms disappear from the urine and feces within from six weeks to three months after recovery. Sacquepée classifies as chronic carriers only those in which the organisms are still present three months after complete recovery. After this period, the length of time to which the carrier may persist is variable, depending upon whether or not chronic lesions are established. These will be discussed below. The frequence with which chronic carriers following typhoid fever occur may be gathered from the table compiled by Gay and published in the book mentioned above.

If we consider that the figures presented in this table must necessarily represent underestimates because of the technical difficulties attending the discovery of small numbers of typhoid bacilli, it becomes apparent that the number of potential foci for infection in a community is enormous. Gay estimates, on a basis of a 5 per cent minimum, that we have 7500 added annually to the carriers present in the United States. On this basis about 0.2 per cent to 0.3 per cent of the general population may be assumed to be carriers.

According to the foci upon which the carrier state depends, typhoid

77 Conradi and Drigalski, Zeit. f. Hyg., 34, 1902, 283.

${ }^{78}$ Sacquepée, Bull. de l'Inst. Past., 8, 1910, 521 and 689.

${ }^{79}$ Kutscher, Kolle and Wassermann Handbuch, 2d Edition, Fischer, Jena, 1913.

${ }^{80}$ Koch, Ver. a. d. Militarsanitätswesen, H. 21, 1902.

${ }^{81}$ Drigalski, Cent. f. Bakt., 35, 1904, 776. 
carriers have been subdivided by a number of writers into intestinal carriers, gall-bladder carriers, and liver or bile duct carriers. We will see how the newer methods of duodenal tube examination have made these distinctions between carriers possible.

PERCENTAGES OF CHRONIC TYPHOID CARRIERS FOUND BY VARIOUS INVESTIGATORS IN A STUDY OF CONVALESCENT CASES *

\begin{tabular}{|c|c|c|c|}
\hline Author. & Date. & $\begin{array}{l}\text { Number of } \\
\text { Cases. }\end{array}$ & $\begin{array}{l}\text { Percentage } \\
\text { Carriers for } \\
3 \text { Months } \\
\text { and More. }\end{array}$ \\
\hline Lentz. . . . . . . . . . . . . . . . & 1905 & 400 & 3.0 \\
\hline Conradi.................... & 1907 & 400 & 0.5 \\
\hline 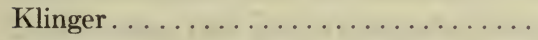 & 1907 & 482 & 1.7 \\
\hline 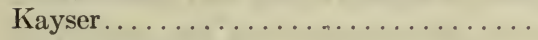 & 1907 & 101 & 3.5 \\
\hline Semple and Greig $\ldots \ldots \ldots \ldots \ldots \ldots$ & 1908 & 86 & 11.6 \\
\hline 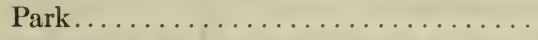 & 1908 & 68 & 5.9 \\
\hline 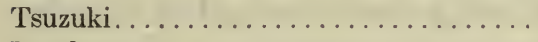 & 1910 & 51 & 5.8 \\
\hline Bruckner................... & 1910 & 316 & 3.8 \\
\hline Stokes and Clarke............... & 1916 & 810 & 1.85 \\
\hline
\end{tabular}
1918.

* Table taken from Gay, F. P., Typhoid Fever, MacMillan \& Co., New York,

By far the most common localization of typhoid bacilli in the body of the carrier is the gall bladder. In speaking of the sequelæ of typhoid fever we have seen that cholecystitis is almost always related to a preceding attack of typhoid fever. As a matter of fact in the course of typhoid fever the organisms are always present in the gall bladder. This was noted by Chiari ${ }^{82}$ as early as 1894 , by Pratt, ${ }^{83}$ and by many others. Longcope is quoted by Gay to have taken bile cultures as a routine in suspected typhoid deaths at the Pennsylvania Hospital; and found typhoid bacilli in all positive cases. In the gall bladder apparently the organisms find a protected nidus where they can persist for years. If gall stones are formed later, typhoid bacilli can often be isolated from them. We ourselves have reported a case in which we isolated the organisms from gall stones seventeen years after the attack of typhoid.

That liver-duct carriers, however, may exist independently of gallbladder infection has been shown by such cases as the one cited by Garbat in the essay mentioned above. He speaks of two patients 
who, during typhoid convalescence, manifested gall-bladder symptoms. Direct culture of the bile by means of the duodenal tube method showed typhoid bacilli in "A," but not in "B." In both, the gall bladder was removed and a pure culture of typhoid bacilli obtained from both gall bladders. At the time of operation, the negative culture in "B" was explained by the fact that a large stone was fixed in the cystic duct which completely occluded the passage. The bile from "B," before operation had come directly from the liver, and had not entered the gall bladder which was in this case, the only site of infection. After operation however, typhoid bacilli completely disappeared from "B," where the bile that had come from the liver had been found sterile by the original duodenal culture, but in "A," in spite of the complete removal of the gall bladder and cystic duct, repeated duodenal cultures remained positive. Similar cases have been reported in the literature, but none which seem quite as convincing as a proof for the existence of the true liver carrier as these instances reported by Garbat.

The manner in which typhoid bacilli get into the gall bladder has occupied the attention of a number of investigators. According to Küster ${ }^{84}$ and a more recent report by Garbat ascending infection of the gall bladder from the duodenal is a possibility, though it is probably not the most common method of infection. Nichols 85 too has admitted the possibility of such a process, although no one believes that this is very common. The fact also that, according to Blumenthal ${ }^{86}$ Laubenheimer ${ }^{87}$ and others, colon bacilli are very commonly found in the gall bladder, gives support to the possibility of ascending infection. The opinion, however, that the bile is hematogeneously infected by way of the hepatic circulation in most cases is generally accepted.

The existence of pure intestinal carriers has been suggested by Kraus and others, and in addition to the cases cited by Kraus, there is one by Garbat in which duodenal cultures were repeatedly negative, whereas the feces remained positive. Cholecystectomy on this case did not relieve the carrier condition. The intestinal carrier type, according to Kraus ${ }^{88}$ may be associated with chronic intestinal ulcerations, chronic appendicitis, etc., but is unquestionably extremely rare, a large majority of carriers being due to actual gall-bladder infection.

Chronic urinary carriers are less common than chronic feces carriers.

${ }^{84}$ Kü ter, Beitr. f. Klin. d. Infkrankh, etc., 7, 1918, 98.

${ }_{85}$ Nichols, Jour. of the A. M. A., 68, 1917.

${ }^{86}$ Blumenthal, Arch. f. klin. Med., 88, 1907, 509.

${ }^{87}$ Laubenheimer, Zeit. f. Hyg., 58, 1909.

${ }^{88}$ Kraus, Wien. klin. Woch., 27, 1914, 1443. 
Yet, when they occur, they are of much greater danger to others because of the more indiscriminate distribution of urine. According to Garbat ${ }^{89}$ about 6.8 per cent of all typhoid cases show typhoid bacilluria for one or two months after the fever has disappeared. In such cases often the organisms are discharged intermittently, and for this reason repeated examination is necessary. Chronic urinary carriers have been reported by Prigge, ${ }^{90}$ Houston, ${ }^{91}$ and others, and are usually associated with some pathological lesion of the genito-urinary tract. In a case reported by Mayer and Ahreiner 92 there was a pyonephrosis, and in other cases, cystitis, or other inflammations of the bladder, ureter, and kidney have been found.

By so-called healthy carriers are meant individuals who harbor typhoid bacilli in the stools and in whom no history of typhoid infection at any time in their lives can be obtained. We know more about typhoid fever than we did formerly, and we think that it is quite clear to most students of the disease that a negative history of this kind, especially if obtained from people who have lived vigorous, active, physical lives, must be very unreliable. Extremely mild cases of typhoid fever, while not common, do occur, and it is not impossible that an individual with an unusual resistance may have been ill for a few weeks, perhaps with a little fever at sometime without going to a doctor. On the other hand, Scheller ${ }^{93}$ in examining a group of people, in connection with the investigations made of a mild epidemic, found a considerable number of temporary carriers who did not develop the disease. These people had taken milk infected from a carrier, 18 of a total of 44 acquiring the organisms without getting sick, while 32 of the same group actually got sick. It is not impossible, therefore, that individuals associated with typhoid cases and during epidemics may become temporary carriers.

Carriers may increase enormously in the course of epidemics especially if these epidemics take place among the large groups of vaccinated people. Such conditions prevailed among the Allied and probably among the German Armies during the war, when the enormously increased opportunities for fecal transmission produced incident to active warfare, with open latrines, unprotected kitchens, unlimited fly breeding, and defective scattered small water supplies, made sanitary control impossible. Hundreds of thousands of men suffered from diarrheas and

${ }^{89}$ Garbal, Jour. A. M. A., Nov., 1916, 1493.

${ }^{90}$ Prigge, Ílin. Jahr., 22, 1909-1910, 245.

${ }^{91}$ Houston, with Irwin, Lancet, 1, 1909, 311.

92 Mayer and Ahreiner, cited from Gay, loc. cit.

${ }^{93}$ Scheller, Cent. f. Bakt., Erste Abt., Orig., 45, 1908, 385. 
mild intestinal disease, without, or with very slight febrile manifestations, and the investigations of many bacteriologists, as well as our own, show that a considerable percentage of these people were actually infected with organisms of the typhoid, paratyphoid, and dysentery groups.

As to the relative importance of the typhoid carrier in the morbidity of typhoid fever, it is very difficult to adduce accurate data. It is pretty safe to say that the carrier is growing relatively more important, will in the future probably be the chief source of typhoid morbidity in well sanitated communities, and is the only stumbling block which will probably prevent the complete eventual eradication of the disease. Of recent years, as water, milk, and food supplies are coming more and more directly under the vigilant eyes of health authorities, the estimates of the percentage of cases due to carriers, as contrasted with other sources of infection, is growing larger and larger.

Pathological Consequences of the Carrier State.-Perhaps the most common sequelum of the chronic carrier state is cholelithiasis. According to Exner and Heyworski 94 typhoid bacilli have a particular property of decomposing the bile salts, giving rise to a precipitation of cholesterin, and Dörr ${ }^{95}$ experimentally produced small concretions in the gall bladder of infected animals. Typhoid bacilli have often been found in gall stones. Furthermore, obstruction of the bile and stagnation due to inflammatory processes may be indirectly responsible for stone formation.

It is probable that typhoid carriers possess an especially high resistance to second attacks, higher even than that of the ordinary individual who recovers without developing the carrier state. Küster reports that of 800 chronic carriers observed in the military hospital at Cologne during two and one-half years, not a single clinical disturbance attributable to the typhoid bacillus could be determined. The occurrence of cystitis, pyelitis and renal stones in typhoid carriers is not particularly common.

Occasionally, typhoid carriers may possess-agglutinins and other antibodies in the blood higher than normal. Lentz examined a number of chronic carriers and found positive Widals in 10 out of 11; however, only in dilutions of $1: 20$. Gaethgens ${ }^{96}$ found both agglutinins and opsonins higher in chronic carriers than in normal people, but Schone ${ }^{97}$

${ }^{94}$ Exner and Heyworski, Wien. klin. Woch., 1908, 7.

${ }_{95} \mathrm{Dör}$, cited from Küster, loc. cit.

${ }^{96}$ Gaethgens, Deut. med. Woch., 1907, 1337.

${ }^{97}$ Schone, Munch. med. Woch., 1908, 1063. 
found no increase in complement fixation. In general, we would not hope for very much light from serological investigations upon the question of whether or not an individual was a carrier, but would immediately proceed to bacteriological examination, either by feces, urine, or duodenal examination.

The Treatment and Cure of Typhoid Carriens.-The great importance of the typhoid carrier from the epidemiological point of view has led to innumerable medical and surgical attempts at cure. That there can be no doubt about the possibility of cure in most cases, by surgical gall-bladder extirpation, is, of course, certain. It will be necessary in the future, however, especially on the basis of the recent work of Nichols, Garbat and some German observers, to precede such operations by fecal and duodenal examinations, and it must be remembered that there will always be a certain percentage of cases which are liver-duct carriers, in contrast to gall-bladder foci, which will not clear up. Also, the operation is not without danger, with a certain amount of mortality, and, of course, cannot be applied generally upon the enormous numbers of carriers that exist.

According to Garbat and others, attempts to cure by other means necessarily depend to a very large extent upon early diagnosis of the carriers before the condition has become stubbornly chronic. But in all cases, cure by other than surgical means has been discouraging. Many different methods have been attempted. Vaccination with the ordinary typhoid vaccines has given discouraging results in the hands of Park and many others, although much was hoped from it at first. Irwin and Houston ${ }^{98}$ claimed to have cured a urinary carrier by vaccination, but Houston and Thomas ${ }^{99}$ failed in other cases. Petruski 100 in 1902 claimed that vaccination during the course of the disease might prevent the development of the carrier state. But, on the whole, vaccination has not brought the results that have been hoped for it, and we are rather reluctant to believe that on a theoretical basis it is encouraging, since the organisms in the chronic carrier are physiologically outside the body, and not in contact with antibodies or leucocytes.

Medicinal treatment has been tried with many agents, but without much success. Discouraging results have been obtained with urotropin, methylene blue, saliciyates, iodin and arsenic preparations. Conradi 101 in 1910 tried chloroform with apparently successful results in rabbits

${ }^{98}$ Irwin and Houstom, Iancet, 1, 1909, 154.

${ }^{99}$ IIoustom and Thomas, Cent. f. Bakt. Ref., 45, 1910, 390.

${ }^{100}$ Petruski, cited from Küuster, loc. cit.

${ }^{101}$ Conradi, Centralb. f. Immunit., 7, 1910, 158. 
experimentally converted into typhoid carriers. Bully 102 tried this treatment upon human carriers, giving 0.5 c.c. of chloroform in capsules four times a day for twenty days, without any results. Neosalvarsan has been tried without effect. The only encouraging reports we can find in an extensive review of the literature to date are the recent ones of Kalberlah ${ }^{103}$ who administered tincture of iodine together with animal charcoal, and those of Geronne ${ }^{104}$ who similarly combined charcoal with thymol. None of these methods have, however, been sufficiently confirmed to encourage great hope.

The Typhoid Bacillus in Transit from Source to Victim.The typhoid bacillus which reaches the outer world in the feces and urine of carriers and cases is fortunately not a very resistant organism. It requires moisture and a favorable temperature approaching $37.5^{\circ} \mathrm{C}$. for multiplication, and suitable nutritive material. These conditions being unfavorable, it is.subjected to a rapid diminution in concentration by dilution, and dies out with relative speed. In sewage and feces, moreover, it is subject to rapid destruction in the competition with the more hardy plebeians with which it comes in contact. In feces the organisms will live for very variable periods according to temperature and conditions governing decomposition. They may be destroyed within a day or two, and in cesspools, etc., where they are immediately mixed with large numbers of decomposing feces, they live for probably not longer than a few days under any conditions. If feces are frozen, that is, deposited in the open in the winter, the organisms may live throughout the winter and enter water sheds, etc., with the thaw. In water, as a rule, they do not live more than a few days or perhaps a week, and according to Rosenau they live longer in clean than in contaminated water. In sewage their life is short. Freezing does not kill them. According to the investigations of Gärtner ${ }^{105}$ the typhoid bacilli could be found in the flowing water of the Paris water supply after a day and one-half. In all statements of this kind it must be remembered by the sanitarian that no absolute rules can be set up, since we know that the inability of all microorganisms like the typhoid bacillus depend very delicately upon temperature, nutrition, the presence of other bacteria, moisture, heat, light reaction, etc. We have ourselves seen typhoid bacilli alive and viable after many years of sealing in agar cultures preserved in the dark and in a cool place, and, while in nature

102 Bully, Zeit. f. Hyg., 61, 1911, 29.

${ }^{103}$ Kalberlah, Med. Klinik., 1915.

${ }^{104}$ Geronne, Berl. klin. Woch., 1915.

${ }^{105}$ Gärtner, Klin. Jahrb., 9, 1902. 
the organisms disappear with relative speed, no rule can be set up. Klein ${ }^{106}$ claims to have found typhoid bacilli alive in natural waters for as long as thirty-six days.

Since the organisms can remain in the soil for limited periods of time, unwashed vegetables, salads, etc., are dangerous, and, as we shall see, oysters grown near sewage outlets, may also be sources of infection.

Channels of Transmission.-Suspicion of typhoid infection by means of water supplies dates back to the early writing of the English physician, Budd, in 1856, who not only believed that sewage contaminated water conveyed the disease, but suggested that the origin of this pollution lay in human feces. Since that time, of course, bacteriological investigation and sanitary water purification on a large scale has indisputably proven the danger of water supplies in this respect. In a few cases, direct proof of typhoid bacilli in the water supply has been brought, but, as a rule, indirect proof has had to be adduced, since the rapid dilution, the usual lateness of water investigation after the occurrence of cases, and the many agencies which lead to the destruction of typhoid bacilli in water supplies, has made it extremely difficult to find the organisms in the water. Indirect evidence, however, has been sufficiently convincing in that colon bacillus tests have revealed massive human feces contamination of water to which typhoid infection could be epidemiologically traced. Also, in many localities the direct diminution of typhoid fever in a community after purification of the water supply has left little room for doubt. Thus, in Schüder's ${ }^{107}$ investigations of 640 epidemics, 72 per cent were directly traceable to water. Water was unquestionably in former years the most important means of the conveyance of typhoid fever when it occurred in definite epidemics, and whenever typhoid cases occur in any considerable number in cities and towns, the water supply must first be excluded as a source of infection. It must also always be taken into consideration when typhoid fever occurs in country districts where small well supplies are the chief sources of drinking water. In Schüder's statisties, 110 of his 640 epidemics could be indirectly traced to milk. Gay ${ }^{76}$ states that in the rural communities typhoid fever has remained more or less stationary during the last ten years, while, in the cities, owing probably to water-supply supervision, it has been diminishing progressively. The methods of examining water under such circumstances will be detailed in the section on water, where emphasis will also be placed

${ }^{106}$ Klein, Medical Officers Report, Local Govern. Board.

${ }^{107}$ Schüder, Zeit. f. Hyg. u. Infec., 38, 1901, 343. 
upon tne fact that bacteriological water examinations of this kind must always be associated with careful sanitary survey of the water shed and engineering examination of the purification plant, if there is one available. It is important for the sanitarian to remember that, while water epidemics are constantly diminishing as large scale water purification becomes more and more universal, there are still occasional epidemics in which accidents have occurred to ordinarily properly functioning purification plants. 'Such an epidemic was recently reported from Salem, Ohio, ${ }^{108}$ where in September and October of 1920 , following a rainy period, enteritis of unknown origin afflicied about one-half the population. Subsequent investigation showed that 3 cases of typhoid fever had occurred in early September; and typhoid fever reports began in late October and early November. Investigation of the water supply revealed pollution probably due to the contamination of one of the gravity lines connecting a group of wells with the reservoir. Aside from the earliest cases mentioned above, the first cases appeared about October 1st, and reached a peak of 54 new cases on November 1st, which was the highest daily number of the epidemic. Up to November 20 th, a total number of 785 cases, with 12 deaths occurred. Recently, we have heard of another epidemic which occurred in a California town, where a small explosive outbreak of typhoid fever occurred owing to accident to the water supply followed by direct pumping from the river, for one day, necessitated by repairs. The considerable and unexceptional diminution of typhoid fever in all cities where water supply purification plants have been installed, may be found tabulated in such books as Rosenau's Hygiene, Mason's book on water supply, and others.

Milk may act as a distributor of typhoid fever either by direct infection of the milk from milk handlers who are carriers, or from bottles that are returned from houses where typhoid fever or typhoid carriers exist. A considerable number of milk epidemics have been traced beyond doubt, and have usually been characterized by an explosive onset and by the fact that the majority of the patients were women and children. Milk is an excellent culture media for the typhoid bacillus, and an enormous increase of the organisms in the milk between contamination and consumption may occur without visible changes in the milk. Uncooked vegetables, salad, radishes, etc., may be responsible for typhoid infection, and of recent years it has also been shown that oysters may

108 Jour. of the A. M. A., 75, 1920, 1498. 
be a source of danger. Conn ${ }^{109}$ was the first to suggest this, tracing an epidemic, which broke out, to this cause. Experiments by Foote ${ }^{110}$ showed that typhoid bacilli may be found alive in oysters, within three weeks after they had disappeared from the surrounding water. While there is very little question as to the possibility of this form of infection, it probably does not occur very often. In the investigations of Rosenau, Lumsden and Kastle ${ }^{111}$ it was found to be a negligible factor in the cases of typhoid fever occurring in the District of Columbia.

That fies play a very important rôle in the carrying of typhoid bacilli from feces to food, was suggested by Vaughan and by Veeder ${ }^{112}$ in 1898. Vaughan studied this particularly, and showed that in the Army camps in 1898 , flies flew directly from the latrines to the kitchen; in fact, he found hypochlorite of lime on the food, picked up by the flies in the latrines. During the late war, there can be very little question about the fact that the enormous morbidity of intestinal diseases which occurred in the Allied Armies at various times, and especially during the July offensive at Chateau Thierry, was caused by open latrines and flies, typhoid epidemics being avoided only by the universal vaccination of the Armies.

As water supplies, milk supplies, etc., are being supervised and, therefore, excluded as sources of typhoid infection, contact infection is becoming more and more important. As a matter of fact, the recent studies of typhoid morbidity seem to indicate that contact infection is growing to be the chief problem in the prevention of typhoid fever. Frosch who analyzed 978 cases, concluded that 65.6 per cent were contact infections, and Drigalski makes similar estimates. Such infections may be from individual to individual by close contact. They may be from cook and kitchen personnel, to raw food to consumer. Instances of such infection are frequent, the most famous one being that of "Typhoid Mary" who was recently made the subject of a special publication by Soper. ${ }^{113}$ This woman, a cook, worked for eight families in the course of ten years, during which time 7 outbreaks directly traceable to her occurred. Since that time her movements from place to place have usually been followed by circumscribed epidemics. Again,

${ }^{109}$ Conn, Medical Record, December, 1894.

${ }^{110}$ Foote, Medical News, 1895.

${ }^{111}$ Rosenau, Lumsden and Kastle, Pub. No. 52, Hyg. Lab. U. S. Pub. Health Serv., 1908

112 Vecder, Medical Record, 45, 1898.

113 Soper, Military Surgeon, 45, 1919, 1. 
contact infection may take place from fomites-fingers-food to mouth, that is, towels, bed clothing, underclothing, etc., and emphasizes the importance of sanitary paper towels, etc., in toilets.

Since in contact infection the case is of relatively little danger, largely because of the fact that danger of a case is so well recognized and precautions against transmission from such a source are easily taken, and have become matters of routine in well-regulated sick-rooms and hospitals, the interest in these infections centers upon the typhoid carrier.

The Prevention of Typhoid Fever. ${ }^{114}$-The measures which are necessary for the prevention of typhoid fever can be easily deduced from a consideration of the material in the foregoing paragraphs.

Of great importance is recognition of cases, hospitalization and isolation. During such hospitalization there should be careful attention to disinfection of discharges, sterilization of bedding, bed-pans, eating utensils, etc., etc. Patients should never be discharged from hospitals until the urine and feces have been found free from typhoid bacilli, and several examinations at intervals of two or three days should be negative before this is considered to be the case. . .

Attention to sewage disposal, water supplies, filtration and chlorination of water with constant supervision of such plants from both a bacteriological, chemical and engineering point of view.

Similar supervision of milk supply, with especial attention to the carrier state of the personnel of dairies and milk handlers.

Public health arrangements for the immediate epidemiological study of cases which occur and laboratory facilities for the tracing of carriers indicated by such epidemiological studies.

Eventual examination for the carrier state of food handlers, professional cooks, and exclusion from such professions of people found to be carriers.

Community measures for the suppression of fly-breeding places and flies, screening of kitchens, and the absolute elimination of open latrines of any kind.

The prevention of oyster culture near sewage outlets.

Finally, more and more attention must be given to generalized vaccination.

Vaccination and Specific Therapy in Typhoid Fever.-The failure to produce a soluble toxin from typhoid cultures has naturally so far

${ }^{114}$ See Rosenau's Hygiene-Also Zinsser-Prevention of Com. Dis. in Nelson's System, 1921. 
precluded the possibility of an antitoxin therapy, such as that which has been successful in diphtheria. In the light of our present knowledge of the poisonous products of the typhoid bacillus it seems but natural that attempts by earlier investigators to apply the principles of Behring's work to typhoid fever were doomed to fail. Attempts to employ specific bactericidal and bacteriolytic sera for therapeutic purposes in this disease have also been without favorable result.

Active Prophylactic Immunization.-We have seen that work by Pfeiffer and Kolle and later by many others has shown that it is comparatively easy to immunize animals actively against typhoid infection by the sytematic injection of graded doses, at first of dead bacilli, later of fully virulent live cultures. Attempts to apply these principles prophylactically have been made recently on a large scale by Wright and his associates upon English soldiers in South Africa, and by German observers in East Africa.

The first recorded experiment of this sort which was done upon human beings was that of Pfeiffer and Kolle, ${ }^{115}$ who in 1896 treated two individuals with subcutaneous injections of an agar culture of typhoid bacilli which had been sterilized at $56^{\circ} \mathrm{C}$. The first injection was made with $2 \mathrm{cmm}$. of this culture. Three or four hours after the injection the patient suffered from a chill, his temperature gradually rose to $105^{\circ} \mathrm{F}$., and there was great prostration and headache, but within twenty-four hours the temperature had returned to normal.

This experiment showed that such injections could be practiced upon human beings without great danger.

Simultaneously with the work of Pfeiffer and Kolle, Wright ${ }^{116}$ conducted similar experiments on officers and privates in the English army.

The actual number of persons treated directly or indirectly under Wright's ${ }^{117}$ supervision in an investigation covering a period of over four years comprised almost one hundred thousand cases. The methods employed by Wright have been modified several times in minor details; the principles, however, have remained consistently the same. In the first experiments Wright employed an agar culture three weeks old, grown at $37^{\circ} \mathrm{C}$., then sterilized at a temperature below $60^{\circ} \mathrm{C}$., and protected from contamination by the addition of five-tenths per cent of

${ }_{115}$ Pfeiffer und Kolle, Deut. med. Woch., xxii, 1896, xxiv, 1898.

${ }^{116}$ Wright, Lancet, Sept., 1896.

${ }_{117}$ Wright and Semple, Brit. Med. Jour., 1897; Wright and Leishmann, Brit. Med. Jour., Jan., 1900. 
carbolic acid. Later, Wright ${ }^{118}$ employed bacilli grown in a neutral 1-per-cent pepton bouillon in shallow layers of flasks.

Great importance is attached both to the virulence of the typhoid strain, which may to a moderate extent be standardized by passage through guinea-pigs, and to care in using low temperatures for final sterilization. The temperature recommended by Harrison, is $52^{\circ} \mathrm{C}$. after which the cultures are carbolized.

It is nevertheless extremely difficult to tabulate satisfactory statistics from a mass of experiments observed by a large number of individuals. On the whole, however, it seems fair to state that advantageous results followed the active immunization practiced by Wright. Wright's own estimation, in a careful attempt to present the subject fairly, gives a reduction of the morbidity from typhoid fever in the British army of 50 per cent, and a reduction of the mortality of those who became infected in spite of inoculations of 50 per cent also. It is not at all impossible that a number of different strains will have to be used eventually for the ideal vaccine, inasmuch as the antigenic differences which have been recently discovered would make it seem that no single strain can be expected to produce antibodies which would protect against all other strains. It is not impossible that some individual strain may combine the antigenic properties of the entire group. This, however, has still to be worked out.

The method of Pfeiffer and Kolle consists in the injection of saltsolution emulsions of fresh agar cultures sterilized at $60^{\circ} \mathrm{C}$. The results reported were in general favorable.

Extensive tests in the United States Army, observed by Russell, ${ }^{119}$ have removed any doubt which may have existed as to the efficacy of prophylactic typhoid vaccination. Russell's statistics show a steady decline of typhoid in the U.S. Army beginning with the introduction of compulsory vaccination in 1910. In 1913 there was but one case among over 80,000 men.

The method at present employed is as follows: The "Rawlings" strain of typhoid, obtained from Wright, is used. Eighteen-hour agar cultures in Kolle flasks are washed off with sterile saline to an approximate concentration of one billion to the cubic centimeter. The suspension is killed at $53^{\circ} \mathrm{C}$. for one hour and 0.25 per cent tricresol is added. Aërobic and anaërobic culture controls are made and a rabbit

${ }_{118}$ Wright, Brit. Med. Jour., 1901; Lancet, Sept., 1902; Brit. Med. Jour., Oct., 1903.

${ }^{119}$ Russell, Am. Jour. of Med. Sc., cxlvi, 1913. 
and mouse inoculated to insure sterility. For immunization 3 to 4 doses are given ranging in quantity from 500 million to one billion at seven- to ten-day intervals. The protection probably lasts about two years, though this is not certain.

Another point of importance in this connection has recently been raised by Metchnikoff and Besredka. ${ }^{120}$ They vaccinated chimpanzees with typhoid bacilli and found that when emulsions of the clear bacteria were used, protection was only slight. Better results were obtained - that is, apparently complete protection within eight to ten dayswhen living sensitized bacteria were injected (bacteria which had been exposed to the action of inactivated immune serum.) Broughton has applied this method to human beings. Gay ${ }^{76}$ has also prepared a sensitized dead typhoid vaccine which he has used in a considerable number of cases. It will take some time, however, before a statistical estimation of the superiority of this method over the older vaccination with dead bacteria will be possible.

Gay $^{76}$ sensitizes his bacilli with strong immune serum, precipitates with alcohol, dries and grinds them into powder, and uses weighed. amounts of the powder.

During recent years in France another form of vaccine has been used which has advantages which lead to its general adoption, if its immunizing value can be successfully proven. This is the so-called "lipovaccine." It consists of typhoid cultures grown on agar, taken up in salt solution, the sediment partially dried, and then shaken up with olive oil, the formula for which is not available. It can also be produced by grinding up the typhoid sediment with lanolin and shaking up this well-ground paste with sterile olive oil. Great difficulty has been encountered in the sterilization of this vaccine, a matter which still needs much investigation. The vaccine has the advantage of giving much diminished reactions, and it is claimed that three times the amounts given in the ordinary saline suspensions can be given without serious discomfort to the patient. There is probably slow absorption of this vaccine and it is claimed that a single dose, because of the slow absorption of the organisms, may be sufficient to vaccinate.

During the recent war, typhoid vaccination has thoroughly justified itself. We are not in possession at the present writing, of consolidated reports of all the European Armies, but the Surgeon General's report for the Uniter States Army, published in 1919, shows the magnificent results obtained by vaccination in American troops. During the pre-vaccination

${ }^{120}$ Metchnikoff and Besredka, Am. de l'inst. Past., 1911. 
days in the Civil War and the Spanish American War, the admission rates for typhoid fever were enormous. During the first year of the Civil War the annual admssion rate was 70.69 , with a death rate of 19.61 , and it is likely that, in addition to this, a large number of unrecognized cases occurred. During the Spanish American War and the Philippine Insurrection in the years 1898 to 1899 , the annual admission was 91.22 , and the death rate 9.67. During the last World War the method of vaccination used consisted in three inoculations at seven-day intervals, of the salt solution suspension triple vaccine, containing typhoid "Rawlings," paratyphoid A and B, the first dose containing one-half million bacilli, and the second and third containing a billion each. The typhoid rate was so low in the camps in the United States that a young man in the camp had 45 times less opportunity of getting typhoid fever than did the same age group in civilian life during the same period. Although approximately three million men passed through the camps during the course of 1918, the actual admission rate for the United States was 0.17. In Europe, in spite of the most terrific sanitary conditions in some of the battlefields during the summer, and with perhaps two million troops in France, there were only 488 cases with 88 deaths, and this, in spite of the fact, as we, ourselves, observed, that the opportunities for transmission were enormous in battle areas in which sanitation was practically impossible, and water supplies were bad and could not be corrected.

The question still remains as to how long typhoid vaccination can be regarded as efficient. There is no absolute information upon which opinions can be based. Vaccination is not a complete protection at any time, and a recently vaccinated individual may still occasionally contract the disease if he is injected with a large dose of virulent organisms. The protection, however, is very powerful and will prevent contraction of the disease from the ordinary chance infection. We should state on general information that repetition every two years ought to be sufficient for civilian purposes. For the armies in the field, we ourselves would favor a first vaccination with three doses as stated above, and single or double doses repeated every six months.

Specific Treatment of Typhoid Fever. ${ }^{76}$-Anti-sera against typhoid fever have been produced by a large number of workers, notably Chantemesse ${ }^{121}$ and Besredka ${ }^{122}$ both of whom used the serum of horses immunized with typhoid bacilli or "endo-toxin," so-called. Garbat

${ }^{121}$ Chantemesse, Prog. med., 7, 1989, 245.

122 Besredka, Ann. Inst. Past., 16, 1902, 918. 
and Meyer ${ }^{123}$ believed that an improvement of results could be obtained by mixing the sera of animals that had been immunized with sensitized bacteria and those treated with normal typhoid bacilli.

At the present time, however, practice has not sustained the hopes of a specific passive immunization in the treatment of typhoid fever.

Since 1893, various workers have tried to treat typhoid fever by injecting killed cultures or vaccines of typhoid bacilli. No results of importance were obtained until Ichikawa ${ }^{124}$ in 1914 began to inject dead typhoid bacilli, intravenously. Gay and Claypole ${ }^{125}$ and others have since taken up this method. The intravenous injection of vaccines in this way has given most astonishing results in that the injection has usually resulted in a violent reaction, with often a chill and sudden drop of temperature, and, subsequently, very often definite improvement of the cases. Although this method was at first regarded as specific by the writers mentioned above, Kraus ${ }^{126}$ in 1915 showed that he could produce similar reactions in typhoid patients with colon bacilli, as well as with typhoid bacilli, and similar observations were made by Lüdke ${ }^{127}$ and others. It is now quite clear that, whatever results are obtained by such treatment of typhoid patients, they cannot be regarded as specific reactions in the ordinary sense of the word.

${ }^{123}$ Garbat and Meyer, Zeit. f. exper. Path., 8, 1910, 1.

${ }^{124}$ Ichikawa, Mitteil. d. medic. Gessellsch. zu. Tokio, 1914, 28, H. 21.

125 Gay and Claypole, Arch. Inter. Med., 12, 1913, 613.

${ }^{126}$ Kraus, Wien. klin. Woch., 1915, 29.

${ }^{127}$ Lüdke, Munch. med. Woch., 1915, 321.

* For a thorough and clear treatment of the problem connected with typhoid fever, see Gay, Typhoid Fever, MacMillan and Company, New York, 1918. 


\section{CHAPTER XXXIII}

\section{BACILLI OF THE COLON-TYPHOID-DYSENTERY GROUP \\ (Continued)}

\section{BACILLI OF THE PARATYPHOID-ENTERITIDIS GROUP AND THE PARATYPHOID INFECTIONS}

\section{(Bacilli of Meat Poisoning and Paratyphoid Fever)}

There is an extensive group of Gram-negative bacilli which because of their morphology, cultural behavior, and pathogenic properties, are classified as intermediate between the colon and the typhoid types. The microorganisms belonging to this group have been described, most of them, within the last fifteen years, but few of them have been fully identified with one another. They have been variously designated as the "hog-cholera group," "the enteritidis group," the "paracolon group" or "paratyphoid group," because of the pathological conditions with which the chief members under investigation have been found associated.

Attempts to systematize the group by the comparative study of a large number of its members have been made, notably by Buxton ${ }^{1}$ and by Durham, ${ }^{2}$ and the work of these writers, based on cultural and agglutinative studies, has added materially to our knowledge of these organisms.

The microorganisms of this group are morphologically indistinguishable from the colon and typhoid bacilli. They are Gram-negative and possess flagella. Their motility is variable, but usually approaches that of the typhoid bacilli in activity. They correspond, furthermore, to the two other groups in their cultural characteristics upon broth, agar, and gelatin. On potato they vary, some of them approaching in delicacy the typhoid growth upon this medium, others more closely approximating the heavy brownish growth of $\mathrm{B}$. coli. Indol is rareiy formed by them, though this has not been absolutely constant in all descriptions. As a group, they are easily distinguished from Bacillus

${ }^{1}$ Buxton, Jour. Med. Res. N. S., iii, 1900.

${ }^{2}$ Durham, Jour. Exper. Med., v, 1901. 
typhosus on the one hand, and from Bacillus coli on the other, by the following simple reactions tabulated by Buxton. ${ }^{3}$

\begin{tabular}{|c|c|c|c|}
\hline & B. Coli. & Paratyph. etc. & B. Typhosus. \\
\hline Coagulation of milk. & + & - & - \\
\hline Production of indol. ................ & + & - & - \\
\hline Fermentation of lactose with gas....... & + & - & - \\
\hline Fermentation of dextrose with gas...... & + & + & - (ac.) \\
\hline Agglutination in typhoid-immune serum. & - & - & + \\
\hline
\end{tabular}

The simplest differentiation between the large groups can be made by fermentation tests as follows:

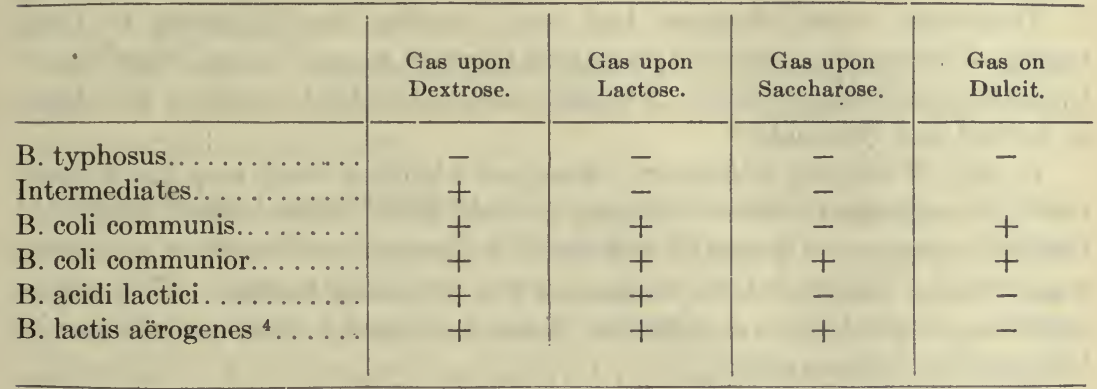

\section{${ }^{4}$ Jackson.}

Pathogenically, the bacilli of this "intermediate group" have attracted attention chiefly in connection with meat poisoning, and with protracted fevers indistinguishable from mild typhoidal infections.

General Survey of the Group.-In 1888, Gärtner ${ }^{5}$ described a bacillus which he isolated from the meat of a cow, the ingestion of which had produced the symptoms of acute gastrointestinal catarrh in 57 people. One of these died of the disease and the bacilli could be demonstrated in the spleen and blood of the patient.

This bacillus, called Bacillus enteritidis by Gärtner, was actively motile, formed no indol, but produced gas in dextrose media. Acute gastrointestinal symptoms could be induced by feeding the organisms to mice, guinea-pigs, rabbits, and sheep, and the bacilli could be recovered from the infected animals. The bacterial bodies themselves were found by Gärtner to be toxic, containing a poison which was extremely resistant to heat. Sterilized cultures showed the same pathogenic efferts as the living bacilli. Epidemics of meat poisoning sim-

${ }^{3}$ Buxton, loc. cit.

${ }^{5}$ Gärtner, Corresp. Bl. d. Aerzt. Vereins, Turingen, 1888. 
ilar to the one described by Gärtner, in which similar bacteria were isolated, were those described by Van Ermengem, ${ }^{6}$ occurring at Morseele in 1891, the one described by Holst, ${ }^{7}$ the Rotterdam epidemic described by Poels and Dhont, ${ }^{8}$ the one described by Basenau, and many others.

Bacillus Morseele of Van Ermengem, Bacillus bovis morbificans of Basenau, ${ }^{9}$ and bacilli isolated in similar epidemics by others, are, except for slight differences in minor characteristics, almost identical with Gärtner's microorganism.

In 1893, Theobald Smith and Moore ${ }^{10}$ noted a great similarity between the so-called hog-cholera bacillus, the bacilli of the Gärtner group, and Bacillus typhi murium isolated by Loeffler. These observers first used the term "hogcholera" group for the organisms under discussion.

In 1899 Reed and Carroll ${ }^{11}$ noted that Bacillus icteroides, associated by Sanarelli with yellow fever, was culturally similar to the bacillus of hog cholera.

Meanwhile, other observers had been isolating bacilli, similar to those spoken of above, from cases of protracted fevers in human beings, often closely simulating typhoid infections. The first cases of this kind on record were those of Achard and Bensaude. ${ }^{12}$

In 1897 , Widal and Nobecourt ${ }^{13}$ described a bacillus which they had isolated from an esophageal abscess following typhoid fever, which closely resembled Bacillus psittacosis of Nocard, ${ }^{14}$ and which, following a nomenclature previously suggested by Gilbert, ${ }^{15}$ they designated the paracolon bacillus. This microorganism, isolated from a parrot by Nocard, showed a close resemblance to bacilli of the Gärtner group.

There are a large number of apparently nonpathogenic organisms sometimes referred to as paratyphoid C, but better perhaps as "heterogeneous types," which are culturally identical with the paratyphoid but do not agglutinate in either paratyphoid A or B sera. An anti-serum produced with these types usually reacts with the homologous strain only. Such organisms have been studied by Krumwiede and many others, including ourselves. Strains recently isolated at this laboratory came from cases of nephritis, German measles, jaundice, and the stools of healthy soldiers, plated as a matter of routine.

In 1898, Gwyn ${ }^{16}$ reported a case at the Johns Hopkins Hospital, which pre-

${ }^{6}$ Van Ermengem, Bull, Acad. d. méd. de Belgique, 1892; "Trav. de lab. de Puniv. de Gand," 1892.

${ }^{7}$ Holst, Ref. Cent. f. Bakt., xvii, 1805.

8 Poels und Dhont, Holland Zeit. f. Tierheilkunde, xxiii, 1894.

${ }^{9}$ Basenau, Arch. f. Hyg., xx, 1894.

${ }^{10}$ Th. Smith and Moore, U. S. Bureau of Animal Industry Bull., vi, 1894.

${ }^{11}$ Reed and Carroll, Medical News, lxxiv, 1899.

${ }^{12}$ Achard and Bensaude, Bull. de la soc. d. hôpitaux de Paris, Nov., 1906.

${ }^{13}$ Widal et Nobecourt, Semaine méd., Aug., 1897.

${ }^{14}$ Nocard, Ref. Baumgarten's Jahresb., 1896.

${ }^{15}$ Gilbert, Semaine méd., 1895.

${ }^{16}$ Gwyn, Johns Hopkins Hosp. Bull., 1898. 
sented all the symptoms of typhoid fever, but lacked serum agglutinating power for Bacillus typhosus. From the blood of the patient, Gwyn isolated an organism, with cultural characteristics similar to those of the Gärtner bacillus, which he called a "paracolon bacillus." This bacillus was agglutinated specifically by the serum of the patient.

Cushing, ${ }^{17}$ in 1900 , isolated a similar microorganism from a costochondral abscess, appearing during convalescence from supposed typhoid fever.

In the same year, Schottmüller ${ }^{18}$ reported five cases from which similar bacilli were isolated. Careful cultural studies of the microorganisms here obtained showed that they could be divided into two similar, yet distinctly different types, one of them, the "Müller" organism (later "A" type), approaching closely to the typhoid type, especially in its growth upon potato; the other, the "Seeman" type (later "B" type) corresponding more closely to the Gärtner bacilli. Similar cases were reported by Kurth, ${ }^{19}$ Buxton and Coleman, ${ }^{20}$ Libman, ${ }^{21}$ and others.

The two types of organisms, paratyphoid A and B described by Schottmüller and studied by many other observers, can be culturally differentiated, though not without difficulty.

Differential Considerations.-The differentiation of the various organisms within the paratyphoid, enteritidis group is a very difficult matter. The paratyphoid "A" organisms split off rather easily from the rest of them, and seem to represent a fairly homologous group. The paratyphoid "A" organisms, as shown by Ford,22 and more particularly by Krumwiede and Kohn, ${ }^{23}$ differ from the rest of the organisms of this general group in not fermenting xylose. Also, they do not change lead acetate agar. Serologically, it may be said in a general way, though it is not absolutely true, that paratyphoid "A" represents an antigenically homologous group and that a serum produced with one member of the "A" group will, in a general way, possess antibodies against other "A" organisms. The remaining organisms of this paratyphoid group differ materially from each other and cannot be subdivided into final groups, as yet. However, a tentative grouping, based partly upon the sources from which they were obtained and partly upon fermentations and serological reactions, can be attempted, and a great deal of valuable work in this direction has been done by Theobald Smith and his co-

${ }^{17}$ Cushing, Johns Hopkins Hosp. Bull., 1900.

${ }^{18}$ Schottmüller, Deut. med. Woch., 1900; Zeit. f. Hyg., xxvi.

${ }^{19}$ Kurth, Deut. med. Woch., 1901.

${ }^{20}$ Buxton, and Coleman, Proc. N. Y. Pathol. Soc., Feb., 1902.

${ }^{21}$ Libman, Jour. Med. Res., N. S., iii, 1902.

${ }_{22}$ Ford, Med. News, June 17, 1905.

${ }^{23}$ Krumwiede and Kohn, Jour. Med. Res., 36, 1917, 509. 
workers, by Smith and Ten Broeck, ${ }^{24}$, by Krumwiede, Pratt and Kohn, ${ }^{25}$ and many others. The following fermentation chart indicates briefly a summary of the reactions of the more common members of this group, chiefly constructed according to the work of Krumwiede and his coworkers.

\begin{tabular}{|c|c|c|c|c|c|c|c|c|c|c|}
\hline & $\begin{array}{l}\text { Dex- } \\
\text { trose. }\end{array}$ & $\begin{array}{l}\text { Man- } \\
\text { nit. }\end{array}$ & $\begin{array}{l}\text { Lac- } \\
\text { tose. }\end{array}$ & Xylose & $\begin{array}{c}\text { Rham- } \\
\text { nose. }\end{array}$ & $\begin{array}{l}\text { Sac- } \\
\text { cha- } \\
\text { rose. }\end{array}$ & Dulcit & $\begin{array}{l}\text { Lead } \\
\text { Ace- } \\
\text { tate } \\
\text { Agar. }\end{array}$ & Indol. & $\begin{array}{l}\text { Motil- } \\
\text { ity. }\end{array}$ \\
\hline B. parat. "A" . . & $\oplus$ & $\oplus$ & - & - & + & - & slow & - & 1 & + \\
\hline B. parat. "B" . . & $\oplus$ & $\oplus$ & - & + & + & - & + & + & E. & + \\
\hline B. enteritidis..... & $\oplus$ & $\oplus$ & - & + & + & - & + & + & ‡ँ्ञ⿰⿺乚一匕 & + \\
\hline B. abortus equi. . & $\oplus$ & $\oplus$ & - & + & + & - & + & - & $\Rightarrow$ & + \\
\hline B. hog cholera... & $\oplus$ & $\oplus$ & - & + & + & - & irreg. & - & 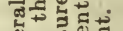 & + \\
\hline B. typhi murium. & $\oplus$ & $\theta$ & - & + & + & - & $\ldots$ & + & 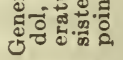 & + \\
\hline
\end{tabular}

$\oplus=$ acid and gas

- = negative $\quad+=$ acid, no gas.

This table brings out a very important point namely, that xylose fermentation is one of the sharply differential characteristics between paratyphoid "A" and all the rest of the group. Whether or not it is possible to differentiate by cultural study between the " $\mathrm{B}$ " organisms of man and those derived from different animal sources is questionable. We do not believe that it can be done reliably at the present time, though certain studies, by the writers named, on the quantitative relationship between reduction of fuchsin and fermentation of dulcite and arabinose would hold out some hope for future clarification of this difficult problem. These writers, however, also call attention to a fact noted in a less extensive way by us and by many other writers, namely, that agglutination and cultural reactions do not correlate in many instances within this group. This is a thing which Theobald Smith pointed out not only for this group but for other groups of bacteria some time ago, and would lead us to be slow to assume that either agglutination or even cultural similarity are always to be regarded as definitely signifying biological relationship.

This opinion, too, is confirmed by the extensive and careful investigations of Jordan which the reader interested in this subject may find in the series of papers published in the Journal of Infectious Diseases, Volumes 20, 21, 22, 23 and 26 . Jordan ${ }^{26}$ finds, for instance, that the

${ }^{24}$ Smith and Ten Broeck, Jour. Med. Res., 31, 1915, 503 and 547.

${ }^{25}$ Krumwiede, Partt and Kohn, Jour. Med. Res., 34, 1916, 34 and 355, pp. 55, 357 and 443 .

${ }^{26}$ Jordan, Jour. Infec. Dis., Vols., 20, 21, 22, 23 and 26. 
B. enteritidis group, in other words, the meat poisoning group of man, is culturally indistinguishable from other paratyphoid "B" organisms, but represents a distinctive agglutinative group, at least in the strains obtained by him.

Jordan adds in his last paper of 1920 that there is no escape from the conclusion that variations are common in the paratyphoid-enteritidis group, both as to fermentation and agglutination. He summarizes that the most permanent characteristics of the group are the fact that rhamnose is fermented by all the paratyphoids, but is not fermented by typhoid; that paratyphoid "A" neglects to ferment xylose, whereas all the other members of the group do ferment xylose. Added to this is the fundamental ability of the group as a whole, including the typhoid bacilli, to attack glucose. These fermentation characteristics are fundamental, are the least subject to change, and, according to Jordan, are more basically characteristic than are the agglutinative reactions, •

\section{PATHOGENICITY OF THE PARATYPHOID GROUP FOR ANIMALS}

We have already seen that organisms of this group cause a considerable number of different animal disease.

B. Hog Cholera.- - It was formerly believed that hog cholera was due to the bacillus which bears this name, and first described in this connection by Salmon and Smith, ${ }^{27}$ but since then it has been found that this disease is due to a filtrable virus. The constant presence of the organism in animals suffering from this disease, is, therefore, something of a mystery, but is probably due, as Dorset has suggested, to the fact that the organism is a constant inhabitant of the intestinal canal in hogs, and manages to get into the circulation as a consequence of the pathological conditions incident to hog cholera. It is worth noting that a similar association of organisms in blood cultures, etc., with diseases of which they are obviously not the primary etiological factor has been observed in other conditions, notably, for example, the Plotz bacillus in typhus fever.

B. Typhi Murium.- - In rodents diseases caused by the members of the paratyphoid group are common. We have already spoken of Loeffler's discovery of the B. Typhi Murium as the cause of an epidemic disease of mice, a condition which is of the greatest annoyance to breeders of mice for cancer research and other laboratory purposes.

${ }^{27}$ Salmon and Smith, Rep. of Com. of Agri., Wash., 1885 and 1886. 
The fact that this organism cannot with regularity be distinguished from the hog cholera bacillus and some other members of the paratyphoid "B" group, opens the question as to whether human beings can be infected by organisms derived from the disease in mice. In connection with attempts at the wholesale destruction of mice by infecting bait in traps with cultures, in the hope of starting an epidemic among them, human infections have been reported. Troomsdorf, ${ }^{28}$ was the first to publish such cases. Meyer ${ }^{29}$ reported an accidental laboratory infection from which he concluded that in man the disease could produce an acute and rather severe, but short-lived disease. Shibajama.30 has reported a number of cases which he carefully investigated. In all of them there was definite circumstantial evidence that the individuals had been exposed to infection with these organisms. In one of them food had been taken from a wooden dish in which mouse typhoid bacilli for the infection for bait had been kept. In another, a peasant woman accidentally mixed the mouse typhoid cultures with flour. In another, again, a number of people had eaten meat of a horse which had been fatally infected by accidental mixture with its food of mouse typhoid virus. In the last case, 34 people were infected; one of whom died. The symptoms were violent gastroenteritis, coming on within twentyfour hours after eating of the meat, and in many respects similar to the typical disease described by Gaertner.

B. Pestis Cavia.-The guinea pig disease caused by the B. Pestis Caviæ usually takes the form of what is commonly known as pseudotuberculosis. It may occur epidemically in laboratory guinea pigs and kill large numbers.

DANysz Type.-A definite group of the organisms, the so-called Ratin or Danysz group, produce epidemic diseases among rats. By German authors this Danysz group, described by the worker whose name they bear in 1900, are generally regarded as very close to the true Gaertner. bacillus. It is pathogenic for guinea pigs and mice, and can be transmitted to rats, as well as to these animals by feeding. There is, as in all other diseases of rodents caused by these organisms, a very much enlarged spleen, with inflammatory changes in the intestinal mucous membrane and necrotic foci in the organs.

The B. Abortus Equi, first described by Smith and Kilborne, has been found to be the cause of infectious abortion in horses, and deserves more detailed description as follows:

${ }^{28}$ Troomsdorf, Munch. med. Woch., 48, 1903.

${ }^{29}$ Meyer, Munch. med. Woch., 47, 1905.

${ }^{30}$ Shibajama, Munch. med. Woch., 54, 1907, 979. 
B. Abortus Equi and Infectious Abortion of Horses.-The infectious abortion of horses and cattle has interested veterinary bacteriological workers for a great many years, since, in both cases, the diseases have been the cause of much economic loss. There is, however, considerable confusion in the minds of general bacteriological workers concerning the various organisms that have been described in connection with these diseases and there seem to be two very distinct types associated with the condition in different animals, one of them belonging to the paratyphoid or hog cholera group, the other apparently having nothing to do with these organisms, biologically. We refer to them both in this place, however, because anyone looking up the B. Abortus in this book would desire to have a definite statement concerning the status of the bacteriology of the diseases in general. The idea that abortion of domestic animals was an infectious disease really originated with breeders and was first suggested to scientific workers by Flandrin in $1806 .^{31}$ Nothing positive from the bacteriological point of view came out of this until 1897 when Bang ${ }^{32}$ described an organism which he isolated from cows which had aborted. There seems to be little question about the ability of Bang's organism to cause this disease in cattle. The description of the Bang organism which is generally spoken of as B. Abortus Bovis will be found in another part of this book.

Soon after Bang's announcement, a British Commission investigated the same subject with a view of finding out whether the abortion on horse farms in England were due to the same bacillus, but they could not confirm this. Ostertag ${ }^{33}$ also investigated the same subject in 1900 in Germany, but could not confirm the original suggestion of Bang that his bacillus caused abortion in horses as well as in cattle. In 1903, Smith and Kilborne ${ }^{34}$ described a bacillus belonging definitely to the hog-cholera group which they had isolated from the vagina of horses that had aborted, and which they held responsible for the disease. De Jong ${ }^{35}$ confirmed these findings and was able to produce the disease in mares by intravenous injection. Numerous other investigations have been made since then, a recent one by Gminder ${ }^{36}$ confirming everything claimed by Smith and Kilborne in the beginning. Gminder isolated 113.

${ }^{31}$ Flandrin, quoted from Gminder, Arb. a. d. Reichsgesundheitsamte, 52, 1920,

32 Bang, Zeit. f. Tier. Med., 1, 1897, 241.

${ }^{3}$ Ostertag, quoted from Gminder, loc: cit.

${ }^{34}$ Smith and Kilborne, Bull. No. 3, U. S. Bur. Animal. Ind., U. S. Treas.

${ }^{35}$ De Jong, Cent. f. Bakt., 67, 1912, 148.

${ }^{36}$ Gminder, Arb. a. d. Reichsgesundheitsamte, 52, 1920, 113. 
similar organisins from aborting mares. He showed specific agglutinins and complement fixing bodies in the sera of these mares and produced abortion in laboratory animals, guinea pigs, rabbits and rats by intravenous injection and by feeding.

Gminder found the Smith-Kilborne bacillus most frequently, but also found organisms closer to the Gaertner enteritidis organisms, and others closely related to the paratyphoid "B" strains, and believes that abortion may be due to a number of other closely related organisms of the paratyphoid-enteritidis groups.

The B. Psittacosis belonging to this group was described by Nocard ${ }^{37}$ as the causative agent in a disease of parrots and is fatal for birds of many different species. Nocard succeeded in transferring it from one parrot to another by feeding.

Durham ${ }^{38}$ studied this organism and pointed out its close similarity to the Enteritidis organisms. The possibility of infection in man with this organism was mentioned by Nocard, and, though this question cannot be regarded as definitely settled at the present time, other apparent infections from parrot to man have been reported. Jackson has recently reported a small epidemic in a Pennsylvania town which he thought he could trace, by epidemiological study, to original infection from birds.

Organisms of this class have been regarded as causing disease in calves and cows and it has been shown that some of the bacteria mentioned above could cause diseases in cattle and horses by feeding experiments.

In judging of these animal diseases, it must not be forgotten that organisms belonging unquestionably to the paratyphoid group have often been found in the intestinal canals of normal calves, pigs and, in a few cases, in horses, and that it is not unlikely that the bacillus is pretty generally distributed in the animal kingdom as perhaps a normal or occasional inhabitant of the intestinal canal.

Bacillus of fowl Typhoid (B. Sanguinarium, Moore).-This organism, together with the B. Pullorum, has been difficult to classify because of several definite and apparently fundamental differences between it and other members of the group. It was isolated first by Moore ${ }^{39}$ from diseased fowl. The chief pathological condition in the fowl seems

${ }^{37}$ Nocard, eited from, Uhlenhuth and Hubener, Kolle and Wassermann Handb., 2d Edit. Vol. 3.

${ }^{38}$ Durham, Brit.. Med. Jour., 1898.

${ }^{39}$ Moore, 12th Annual Rep. Bur. Animal Industry, 1895. 
to consist of severe blood changes (anæmia), and it was spoken of, for some time, as Infectious Leukæmia of fowls. The organism is a Gram-negative bacillus, non-sporulating and uncapsulated, which differs from other members of the paratyphoid group chiefly in being non-motile and in not producing gas from dextrose. Smith isolated a similar organism from a diseased chicken in Rhode Island in 1894. The organism has been studied since then, particularly by Smith and Ten Broeck, ${ }^{40}$ by Rettger and Koser, ${ }^{41}$ and by Krumwiede and Kohn. ${ }^{42}$ To summarize, then, it is a short, Gram-negative bacillus which does not form spores, and does not liquefy gelatin. It does not produce gas in any of the carbohydrate media, but produces acid on glucose, mannite, maltose and xylose. It does not produce indol. An important point is that it produces acid upon rhamnose, an observation made by Krumwiede and Kohn, and this rhamnose reaction is one which this organism has in common with all other organisms of the paratyphoid-enteritidis group. Krumwiede and Kohn regard the rhamnose fermentation as an essential characteristic, differentiating both the aërogenic and anaërogenic members of this group from B. Typhosus. Smith and Ten Broeck first pointed out the close relationship of fowl typhoid to the group in which we are placing it, and showed that it was closely related to the typhoid bacilli by agglutination and agglutinin absorption.

B. Pullorum (Rettger ${ }^{43}$ ) Bacillus of White Diarrhea in Fowls. - This organism, like the one preceding it, has a very close antigenic relationship to B. Typhosus. Unlike B. Sanguinarium, it produces a small amount of gas in dextrose and mannite, though as Smith and Ten Broeck have shown, the gas producing property may be suppressed in certain strains, and again resumed on further cultivation. A non-gas producing strain forwarded by them to Krumwiede resumed its ability to produce gas subsequently. Like the preceding, it is non-motile, but B. Pullorum produces no visible changes in media containing maltose, dulcite and dextrin, while B. Sanguinarium produces acid in such media.

The B. Pullorum is the cause of a spontaneous epidemic disease in young chicks. It is important to note, also, that B. Pullorum like the B. Sanguinarium and other members of the paratyphoid group produces acid on rhamnose (Krumwiede and Kohn).

40 Smith and Ten Broeck, Jour. Med. Res., 31, 1915, 503 and 523.

${ }^{41}$ Rettger and Koser, Jour. Med. Res., 35, 1916-1917, 443.

${ }^{42}$ Krumwitde and Kohn, Jour. Med. Res., 36, 1917, 509.

${ }^{43}$ Rettger, Jour. Exper. Med., 19, 1914. 


\section{PATHOGENICITY OF THE PARATYPHOID GROUP FOR MAN}

Organisms of this class may be the causative agents of a number of clinically varying conditions of man. In general, it may be said that two main types of disease can be caused by this group, 1, that in which the disease simulates a mild or severe typhoid fever recognizable as different from true typhoid fever only by isolation and identification of the paratyphoid organisms; and 2, those which fall into the category of "meat poisoning" in which, after a very short incubation time, one to two days or even less, there are symptoms of gastroenteritis which may be mild, but more frequently are explosive and severe.

1. True paratyphoid fever, or a typhoid-like fever, may be caused by members of the paratyphoid "A" or "B" group.

The paratyphoid "A" group may be regarded as standing somewhat apart from the other microorganisms of this large and heterogeneous family in being quite sharply distinguishable from the others, both by cultural and agglutinative reactions. The organism, unlike other paratyphoid and enteritidis bacilli, fails to ferment xylose, as shown by Krumwiede and his coworkers, and fails to give a positive reaction on the lead acetate media. By agglutination reactions the organisms of the "A" type are found to be more homologous than others and a powerful "A" serum usually agglutinates "A" organisms in general, a condition which is quite unlike that existing among the " $\mathrm{B}$ " and other members of the group. Paratyphoid "A" is probably conveyed in exactly the same way as is typhoid fever, namely, by water, milk and direct and indirect contact, food contamination, carriers and the agency of flies.

The disease itself may take the form either of a very mild and shortlived enteric disturbance with slight fever, or it may take the form of a moderateiy severe typhoid fever with rose spots, enlargement of the spleen and positive blood culture. Paratyphoid "A" cases are not a very large percentage of the ordinary sick rate of communities, but occasionally small group epidemics have been studied; and during recent years in the United States, military epidemics have occurred. Upon the recent return of some American militia regiments from the Mexican Border, there appeared among them an epidemic of paratyphoid which consisted largely of the "A" type. We had the privilege of studying a good many of these cases, and found a most varied clinical picture. The severe cases were practically indistinguishable from typhoid fever, But there were at the same time cases of very mild fever with nothing but a little diarrhea, in which diagnosis was made only by 
stool culture and in a few cases by blood culture. A large number of the men of these regiments turned out to be paratyphoid " $\mathrm{A}$ " carriers.

Paratyphoid "B" is probably a more common disease than paratyphoid "A," and is more apt to be typhoid-like and severe. From the very distinct differences between the clinical manifestations of this disease and the ordinary case of so-called meat poisoning, it would appear that there must be a very definite human paratyphoid " $\mathrm{B}$ " organism which is conveyed by the same agencies and subject to the same epidemiological laws as typhoid fever. It is difficult to base this on bacteriological evidence since it is often impossible to find any cultural or agglutinative distinctions between organisms isolated from the human blood or bowel, and other bacilli which, from their sources and general reactions, would fall into the groups of hog cholera, enteritidis, etc. Numerous attempts have been made to classify these organisms according to source; one of the last attempts to correlate group with host-origin being that made by Krumwiede, Pratt and Kohn. ${ }^{44}$ Their results seem to indicate that reduction of fuchsin and quantitative differences in the fermentation of dulcite and arabinose may to some extent bring about a tentative correlation. But it cannot be said in any sense at the present time that we can sharply differentiate between those " $\mathrm{B}$ " types which invade human beings and produce the typical typhoidlike disease, and those which may originate in a disease of animals and be secondarily transferred to man.

Paratyphoid "B," then, occurs in man in exactly the same way and by the same agencies as typhoid fever, and produces a disease indistinguishable from typhoid, except by bacteriological methods.

In contradistinction to true typhoid the temperature reaction of this case may set in more abruptly and remain more irregular throughout the disease. Gastric symptoms, vomiting, and nausea are often more prominent than in typhoid fever and enlargement of the spleen is less regularly present. Owing to the low mortality of paratyphoid fever (in 120 cases observed by Lentz ${ }^{45}$ less than 4 per cent, and in many other smaller epidemics no deaths have occurred), we have remained relatively ignorant concerning the pathologic anatomy of the disease. Longcope ${ }^{46}$ observed a case, fatal after two weeks of illness, in which there was no enlargement of Peyer's patches and no sign of even beginning ulceration. Most other observers have also found less involvement of

${ }^{44}$ To be supplied.

${ }^{45}$ Lentz, Klin. Jahrb., xiv, 1914.

${ }^{46}$ Longcope, Amer, Jour. of Med. Sciences, cxxiv, 1902. 
the lymphatics of the bowel than is found in typhoid fever. During the disease the bacteria can often be cultivated from the blood, and the serum of the patient may agglutinate specifically paratyphoid strains. In this way the diagulosis can often be made. Libmann ${ }^{47}$ has isolated the organism from the fluid aspirated from the gall bladder in a case operated on for cholecystitis.

Meat Poisoning.-As stated in the beginning of this chapter, this disease and its etiological causation were first described by Gaertner in 1888. His observations were based upon an epidemic occurring in Germany in which 57 cases of more or less violent gastroenteritis occurred in a group of people who had eaten the meat of a condemned cow. From a fatal case and from the meat itself, Gaertner isolated the organisms which bear his name.

Since then, many similar observations have been made. Uhlenhuth and Hübener ${ }^{28}$ have made a very thorough study of the literature, to which the reader is referred for more extensive treatment of the subject. They have tabulated a large number of outbreaks which took place in Germany and the neighboring countries between the years 1885 and 1910. A considerable number of these were studied by competent bacteriologists and can be regarded as reliably reported. It appears that beef and pork are the most common sources of infection, whereas infection is also possible from horse meat and mutton. In the majority of cases the disease followed the ingestion of the meat of cattle that were diseased before being slaughtered. The fact that the organisms have appeared in the circulation of the animal before death and have perhaps accumulated in the meat that is eaten, may account for the acute nature of the disease and the severe toxic symptoms, since, as we know, the poisons of the bacterial bodies of organisms of this group may be quite powerful, may act directly upon the gastro-intestinal mucosa and may not be destroyed by considerable heating. It must not be forgotten, however, that organisms belonging to the paratyphoid " $\mathrm{B}$ " and enteritidis groups may be present in the intestinal canals of a large percentage of normal animals of the species mentioned and that to some extent invasion of the organs may be a post-mortem phenomenon. The studies of most observers, however, seem to indicate that the most severe infections occur when the animal was diseased before being killed. Again, there have been cases in which infection has been due to second-

${ }^{47}$ Libmann, Jour. of Med. Res., viii, 1902.

${ }^{48}$ Uhlenhuth and Hübener, Kolle and Wassermann Handb., 2d edit. Vol. 3. 
ary contamination of the meat either by a carrier or by another agency exactly as this may occur in typhoid transmission.

The clinical picture of this variety of paratyphoid infection is fundamentally different from the one just described. Ty pical bacillary meat poisoning comes on within less than twenty-four hours after the ingestion of the meat. Its onset is acute with a rapid rise in temperature and the accompanying systemic symptoms which this implies. There is usually great prostration, rapidity of the pulse and localized symptoms referable to the gastro-intestinal canal, namely, nausea, vomiting and painful diarrhea. Some of these cases have been compared with cholera in the severity of their courses. The mortality has not usually been very high, although in one epidemic it was as high as 7 per cent. The organisms may in these cases also be found in the blood stream by culture, but not as regularly as in the typical typhoid fever-like form. At death they may be found in the spleen and intestines.

From the epidemiological point of view, it is always important, when a group of persons after a traceable common meal is seized with acute gastroenteritis, to make an epidemiological survey, study the time and place of the common meal, follow up the subsequent histories of others who were present at this meal, and, if possible, secure for bacteriological study some of the meat, milk, etc., consumed.

Preventive Measures.-Measures for the prevention of the typhoidlike forms of paratyphoid fever are identical with those advised for the prevention of typhoid. The carrier problem is practically the same and it seems logical to assume that the percentage of carriers compared with that of typhoid carriers is approximately similar to the ratio of incidence between the two diseases.

In regard to the meat poisoning epidemics, preventive measures must be chiefly aimed at the sanitary control of slaughter houses and care in food preparation. Concerning the slaughter house survey, this must go farther than simply controlling the health of animals before slaughter, since, as we have seen above, many organisms of these types may be found in the normal intestines of animals. Though studies in this direction have not been made with sufficient extensiveness, it is still suggested by our general knowledge of this subject that an unduly long interval between evisceration and slaughter, especially in warm weather, may lead to invasion and multiplication, in the tissues, of organisms from the bowels, which may render the meat of a previously healthy animal, unsafe. Removal of the intestines promptly after slaughter would be the most important preventive measure that reasoning would indicate. 


\section{CHAPTER XXXIV}

\section{BACILLI OF THE COLON-TYPHOID-DYSENTERY GROUP (Continued)}

\section{BACILLARY DYSENTERY AND THE DYSENTERY BACILLI}

Although acute dysentery has been an extremely prevalent disease, occurring almost annually in epidemic form in some of the Eastern countries and appearing sporadically all over the world, its etiology was obscure until 1898 when Shiga ${ }^{1}$ described a bacillus which he isolated from the stools of patients suffering from this disease in Japan, and established with scientific accuracy its etiological significance. Since the discovery of Shiga's bacillus a number of other bacilli have been described by various workers, all of which, while showing slight biological differences from Shiga's microorganism, are sufficiently similar to it culturally and pathogenically to warrant their being classified together with it in a definite group under the heading of the "dysentery bacilli."

The manner in which Shiga made his discovery furnishes an instructive example of the successful application of modern bacteriological methods of etiological investigation. Many workers preceding Shiga had attempted to throw light upon this subject by isolations of bacilli from dysenteric stools, and by extensive animal inoculation. Shiga, following a suggestion made by Kitasato, approached the problem by searching for a microorganism in the stools of dysentery patients which would specifically agg'utinate with the serum of these patients. His labors were crowned with success in that he found, in thirty-six cases, one and the same microorganism which showed uniform serum agglutinations. Further, he found that this bacillus was not present in the dejecta of patients suffering from other diseases nor in those of normal men, and that when tested against the blood serum of such people it was not agglutinated.

Description of Shiga's Bacillus.-Shiga's bacillus is a short rod, rounded at the ends, morphologically very similar to the typhoid bacillus, and, like it, inclined to involution forms. The organism generally occurs singly, more seldom in pairs. It is decolorized by Gram's method of staining. With the

${ }^{1}$ Shiga, Cent. f. Bakt., xxiii, 1898; ibid., xxiv, 1898; Deut. med. Woch., xliii, xliv, and xlv, 1901. 
ordinary anilin dyes it stains easily, showing a tendency to stain with slightly greater intensity at the ends. The organism is an aërobe and facultative anaërobe. Although described at first by Shiga as being motile, its motility has not been satisfactorily proven, and most observers agree in denying the presence of flagella and affirming the complete absence of motility.

On agar the colonies are not characteristic, resembling those of the typhoid bacillus.

On gelatin, the colonies appear very much like typhoid colonies and the gelatin is not liquefied. On potato, the growth, like that of typhoid, is at first not visible, but after about a week turns reddish brown.

In broth, there is clouding, with moderate deposits after some days. No pellicle is formed.

Milk is not coagulated. Litmus milk shows a slight primary acidity, later again becoming alkaline and taking on a progressively deeper blue color.

Indol is not formed.

No gas is formed in media containing dextrose, lactose, saccharose, or other carbohydrate.

While not delicately susceptible to reaction, the bacillus prefers slightly alkaline media.

Shiga differentiated his organism from the typhoid bacillus chiefly by supposed differences in colony characters and by the agglutination reaction.

Following the work of Shiga, a large number of investigators turned their attention to the subject of dysentery, with the result that many new forms were discovered and at first a considerable amount of confusion prevailed.

Flexner ${ }^{2}$ in 1899 investigated dysentery in the Philippines, and isolated a bacillus which, he considered, corresponded to Shiga's organism.

Strong and Musgrave ${ }^{3}$ in 1900 described a bacillus isolated from dysentery cases in the Philippines which was essentially like that of Flexner.

Nearly simultaneously with the papers of Flexner and of Strong and Musgrave, Kruse ${ }^{4}$ published investigations of an epidemic of dysentery occurring in Germany. His observations were of the greatest importance and largely formed the starting point of the further advances which have been made in the etiology of dysentery.

Kruse's organism was described as forming colonies on gelatin and agar, practically like those of Bacillus typhosus. Like this bacillus, no gas was formed from grape sugar, and the growth in milk and on potato,

${ }^{2}$ Flexner, Phila. Med. Jour., vi, 1900, and Bull. Johns Hopkins Hosp., xi, 1900.

${ }^{3}$ Strong and Musgrave, Report Surg. Gen. of Army, Washington, 1900.

${ }^{4}$ Kruse, Deut. med. Woch., xxvi, 1900. 
and even in Piorkowski's urine gelatin, resembled that of Bacillus typhosus. According to Kruse, this organism was absolutely without motility.

In 1901 Kruse $^{5}$ contributed a second paper. In this, besides confirming his previous observations, he described another class of organism coming from cases which he designated as "pseudo-dysentery of insane asylums." In the case of one patient, and at two autopsies, he isolated organisms which he could not distinguish, morphologically or culturally, from the true dysentery bacillus, but which showed differences in their serum reaction. By careful study of the behavior of these bacilli in the serum of patients and in immune serum from animals he not only showed that they were different from the original cultures from cases of epidemic dysentery which, no matter what their source, were found to be alike, but that they showed differences among themselves and apparently fell into two or more varieties. One of these organisms culturally and by its serum reactions showed itself practically identical with one of the cultures he had received from Flexner.

Spronck ${ }^{6}$ in 1901 described an organism isolated in Utrecht from dysentery cases, which showed great similarity to the Shiga-Kruse organism; but, when tested in the serum of a horse immunized against true dysentery bacillus, showed practically no agglutination. He placed this organism in the group designated by Kruse as the "pseudodysentery bacilli." His communication is of importance, since it is the first reported instance in which any investigator had recognized and associated the so-called pseudo-dysentery bacilli with dysentery approaching the acute epidemic form in type.

Following this work a number of investigators, including Vedder and Duval, ${ }^{7}$ Flexner, and Shiga ${ }^{8}$ himself, published communications in which they claimed identity for the various forms previously described.

In 1902 Park $^{9}$ and Dunham described an organism which they found in a small outbreak of dysentery occurring in Maine. This organism differed from most of those previously described in that it was found to produce indol in pepton solutions.

In the same year Martini ${ }^{10}$ and Lentz published an article in which they attempted to differentiate various dysentery bacilli by means of

${ }^{5}$ Kruse, Deut. med. Woch., xxvii, 1901.

${ }^{6}$ Spronck, Ref. Baumgarten's Jahresber., 1901.

7 Vedder and Duval, Jour. Exp. Med., vi, 1902.

${ }^{8}$ Shiga, Zeit. f. Hyg., 41, 1902.

${ }^{9}$ Park and Dunham, N. Y. Univ. Bull. of Med. Sci., 1902.

${ }_{10}$ Martini und Lentz, Zeit. f. Hyg., xli, 1902. 
agglutination. This research is of importance in that it supported the work of Kruse and of Spronck, indicating a difference between the agglutinative character of the Kruse organism and the so-called "pseudodysentery" type, in which Flexner's organisms were included. It is of further interest, since it indicated a marked difference between Flexner's Philippine cultures and the Philippine culture of Strong, the Strong organism refusing to agglutinate not only in "Shiga" immune serum, but also in "Flexner" immune serum.

Simultaneously with this article Lentz ${ }^{11}$ published the results of comparative cultural researches with dysentery and "pseudo-dysentery" bacilli, in which he made the important observation that the original Shiga-Kruse bacilli dir not affect mannit, while the "pseudodysentery" bacilli, including Flexner's and Strong's Philippine cultures, fermented mannit, giving rise to a distinct acid reaction in the medium. The Flexner organisms and others of the "pseudo-dysentery" bacilli, however, fermented maltose, while the Shiga-Kruse type, as well as Strong's bacillus, left it unchanged at the end of forty-eight hours.

In January, 1903, Hiss and Russell ${ }^{12}$ described a bacillus ("Y") from a case of fatal diarrhea in a child, which by ordinary cultural test and absence of motility was found to resemble the Shiga-Kruse and Flexner bacilli. Immediately upon its isolation, it was found, however, to differ from the Kruse culture by its ability to ferment mannit. This observation was made independently of Lentz's work, which, at that time, had not become known in America. In the comparative study of Hiss and Russell on the fermentative abilities of various dysentery cultures, the serum water media (described on page 157) were used. By the use of these media, it was found that the Kruse culture, a culture of Flexner's bacillus from the Philippines, and Duval's "New Haven" culture fermented dextrose with the production of a solid acid coagulum, but did not affect mannit, maltose, saccharose, or dextrin. The culture of Hiss and Russell, on the other hand, fermented not only dextrose but also mannit with the production of acid and coagulation of the medium. Maltose, saccharose, and dextrin were not fermented. The "Y" bacillus, furthermore, was shown to differ entirely from the cultures of Shiga, Kruse, and "New Haven" in the serum of immunized animals. This serum had for bacillus " $\mathrm{Y}$ " a titer of $1: 500$ while the three other above-named organisms did not agglutinate in it at any dilution. In normal beef serum, the Hiss-Russell organism was found

${ }^{11}$ Lentz, Zeit. f. Hyg., xli, 1902.

12 Hiss and Russell, Med. News, Feb., 1903. 
to agglutinate as highly at $1: 320$, while the other three cultures gave no reaction in dilutions of over $1: 10$ or 20 .

Park and Carey, ${ }^{13}$ in March, 1903, described an epidemic of dysentery occurring in the town of Tuckahoe, near New York city, and isolated an organism which resembled the Shiga-Kruse bacilli in not fermenting mannit, but produced indol in pepton solution after five days. It corresponded in agglutination with the cultures "New Haven" and "Shiga" when tested in the serum of a goat immunized against the mannit-fermenting culture "Baltimore," i.e., did not react at 1 : 50, whereas Flexner's "Manila" and "Baltimore" cultures, Park and Dunham's "Seal Harbor" culture, and some New York cultures, all fermenting mannit, agglutinated up to two thousand dilution in the "Baltimore" serum.

The preceding review of a part of the literature, by which our knowledge of the dysentery bacilli was developed, demonstrates sufficiently that we have to deal in this group with a number of different microorganisms. This, as we have seen, was a fact first recognized by Kruse when he spoke of his true dysentery and his pseudo-dysentery strains. In spite of much confusion at first, the careful study of fermentation phenomena, of specific agglutinations, and, more recently, by Ohno ${ }^{14}$ and others of the bacteriolytic phenomena in immune sera, has made it possible to distinguish sharply between a number of groups.

Basing the grouping of these microorganisms upon a careful study of fermentations, Hiss ${ }^{15}$ has divided them as follows:

"Shiga"
"Kruse"
"New Haven"
"Y" (Hiss and R
"Seal Harbor"
"Diamond"
"Ferra"
"Strong" (type)
"Harris" (type)
"Gray"
"Baltimore"
"Wollenstein"

Ferment dextrose. Group I.

Ferment dextrose and mannit. Group II.

Ferments dextrose, mannit, saccharose. Group III.

Ferment dextrose, mannit, maltose, saccharose, dextrin. Fermentation of saccharose (as a rule) only after six days. Group IV.

It was noticed, it should be mentioned, however, that in the case of the

${ }^{13}$ Park and Carey, Jour. Med. Res., ix, 1903.

${ }^{14}$ Ohno, Philippine Jour. of Sci., 1, ix., 1906.

${ }^{15}$ Hiss, Jour. Med. Res., N. S., viii, 1904. 
BACILLI OF THE COLON-TYPHOID-DYSENTERY GROUP 705

"Y," "Diamond," and "Ferra" there was usually delayed acid fermentation of maltose, never any of dextrin.

In studying the agglutinative characters of these groups, furthermore, it was found that fermentation tests and agglutinations went hand in hand. The following table will i!lustrate the point: ${ }^{16}$

Serum of Rabbit Immunized against Group I. (Shiga's culture). Bacilli of Group I.:

"Shiga" (homologous)...................... 20,000

"Kruse"............................. 20,000

"New Haven"......................... 20,000

Bacilli of Group II.:

"Y"

"Ferra".............................. 200

"Seal Harbor"......................... 200

Bacilli of Group IV.:

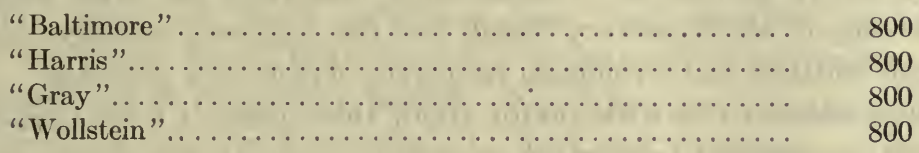

Serum of Rabbit Immunized against Group II. ("Y" culture; Hiss and Russell).

Bacilli of Group I.:

"Shiga" .......................... less than

"Kruse"............................... 100

"New Haven".............................. 100

Bacilli of Group II.:

"Y" (homologous) ....................... $\quad 6,400$

"Ferra" . . . . . . . . . . . . . . . . . . . . . . . . . . $\quad 6,400$

"Seal Harbor........................... $\quad 6,400$

Bacilli of Group IV.:

"Baltimore".............................. $\quad 1,600$

"Gray" ............................. 1,600

"Harris"............................... $\quad \mathbf{1 , 6 0 0}$

"Wollstein"........................... $\quad 1,600$

Serum of Rabbit Immunized against Group IV. ("Baltimore" culture).

${ }^{16}$ Hiss, Jour. of Med. Research, 13, N. S., viii, 1904. 
Bacilli of Group I.:

"Shiga"....................... less than 100

"Kruse"................................. 100

"New Haven".......................... 100

Bacilli of Group II.:

"Y".................................. 400

"Ferra"............................. 400

"Seal Harbor"........................... 400

Bacilli of Group IV.:

"Baltimore" (homologous)................... 3,200

"Harris"............................... 3,200

"Gray"............................ 3,200

"Wollstein"............................ $\quad 3,200$

We have described the above experiments of Hiss in considerable detail not because we believe they represent the final classification of the dysentery group, but because they illustrate the close antigenic relationship of the dysentery bacilli and the relatively close correlation between cultural and serological properties which they revealed.

There seems to be little doubt about there being a large number of different dysentery bacilli which vary from each other in minor characteristics, and it is very difficult to be sure whether one is dealing with permanent type differences or with temporary variations or suppressions. For this reason, it may be best to state definitely what the common characteristics are which belong to organisms of this group and into what main subdivisions they can be classified.

All the dysentery bacilli are Gram-negative bacilli, usually slender, but on occasion, especially in old cultures, short and plump, being morphologically indistinguishable with certainty from typhoid or similar bacilli. There are all of them non-motile and non-spore bearing. They do not liquefy gelatin. None of them produce gas on carbohydrate media, none of them produce acid on lactose.

They grow easily on the ordinary media, their colonies on agar and gelatin being like those of typhoid but apt to be more delicate. On broth they produce an even clouding and on differential media, like those of Endo, Conradi-Drigalski, etc., they grow like typhoid but rather more delicately.

The main types into which they can be divided are the Shiga type, the Flexner type, the Bacillus "Y" type of Hiss and Park and the Strong type. By many observers, notably Park, the Shiga type is the only one that is spoken of as the B. dysenteriæ, the other types being 
spoken of as the paradysentery bacilli. Differentiation between the four types can be made by sugar fermentations as follows:

\begin{tabular}{|c|c|c|c|c|}
\hline . & Dextrose & Mannite & Maltose & Saccharose \\
\hline B. dys. "Shiga".. & + & - & - & - \\
\hline B. dys. "Flexner".. & + & + & + & - \\
\hline B. dys. "Y" ${ }^{\prime} \ldots \ldots \ldots \ldots \ldots \ldots$ & + & + & - & - \\
\hline B. dys. "Strong"............... & + & + & - & + \\
\hline
\end{tabular}

$$
+=\operatorname{acid} \text { (no gas). }
$$

The Shiga bacillus also is the only one of these fixed types which does not produce indol. We do not believe, as we did before, that these types represent all possible variants of the dysentery bacilli. During the recent war epidemics, we ourselves, as well as many others, isolated non-motile, Gram-negative bacilli from the stools of mild dysentaroid cases which presented cultural peculiarities and did not agglutinate with the type sera, and many organisms have been described by various observers which do not fit in with any of the main sub-divisions.

Resistance of Dysentery Bacilli.-Not being spore bearers, dysentery bacilli are not very resistant to heat and chemicals. They are destroyed at $60^{\circ}$ within ten minutes, and the usual strength of the common chemical disinfectants kill them. Their resistance to the ordinary conditions in nature is, of course, the important feature of the epidemiology of the disease. Accordingly, a considerable amount of research has been done on these problems. We quote the following facts from Vincent and Muratet. ${ }^{17}$ In garden soil they have been known to live from six to fifteen days, and up to forty-nine days at a depth of 12 inches. In damp sand they have been known to live as long as thirty-nine days. Cultures in broth have lived for twenty-five days, and they have lived more than thirty days in dejecta buried in the soil, and on linen folded up. In ice, they may live for longer than a month. Exposure to sunlight of course destroys them rapidly. We would like to remark in connection with the viability of all organisms outside the animal body, that such statements must always be limited to the peculiar conditions of symbiosis, light, moisture, temperature, etc., existing under the particular conditions investigated. If we remember that non-spore bearing organisms, like the typhoid, the dysentery bacilli, etc., can be kept alive for very long periods if young agar growths are kept in sealed jars in a cool dark place, we may understand that under peculiarly favorable conditions

17 Vincent and Muratet, Military Med. Manual, Univ. of London Press, 1917. 
in nature, organisms have survived far beyond anything suggested by our knowledge of their general resistance under adverse conditions.

Poisonous Products of the Dysentery Bacilli.-The separate types of dysentery bacilli vary exceedingly in their powers to produce toxic substances. Of all the various types which have been described, the strongest poisons have been produced with bacilli of the Shiga-Kruse variety, less regularly active ones with bacilli of the Flexner and of the " $Y$ " type. In fact, investigations carried out with the Shiga bacillus have tended to show that the disease itself is probably a true toxemia, its symptoms being referable almost entirely to the absorption of the poisonous products of the bacillus from the intestine.

The earliest investigations, carried on chiefly upon rabbits, which are more susceptible to this poison than any other animals, showed that even small doses of cultures of this bacillus administered intravenously or subcutaneously would produce death within a very short time. Conradi, ${ }^{18}$ Vaillard ${ }^{19}$ and Dopter, and others, finding that toxic symptoms were almost as pronounced when dead cultures were given as when the living bacilli were administered, came to the conclusion that the poisons of this bacillus were chiefly of the endotoxin type. More recently Todd ${ }^{20} \mathrm{Kraus}^{21}$ and Rosenthal ${ }^{22}$ have claimed independently that they were able to demonstrate strong soluble toxins, similar in every way to diphtheria toxin. Kraus and Doerr, ${ }^{23}$ moreover, claim to have further corroborated this by producing specific antitoxins with these substances.

It is easy to obtain poisonous substances from dysentery cultures in considerable strength, both by extracting the bacilli themselves and by filtration of properly prepared cultures. It is therefore not unlikely that both types of poison are produced by the bacilli. Neisser and Shiga ${ }^{24}$ obtained toxins by emulsifying agar cultures in sterile salt solution, killing the bacilli at $60^{\circ} \mathrm{C}$., and allowing them to extract at $37.5^{\circ} \mathrm{C}$. for three days or more. The filtrates from such emulsions were extremely toxic. The simplest method of obtaining poisons from these bacilli is to cultivate them for a week or longer upon moderately alkaline meat-infusion broth. At the end of this time, the micro-

${ }^{18}$ Conradi, Deut. med. Woch., $190 \dot{3}$.

19 Vaillard et Dopter, Ann. de l'inst. Pasteur, 1903.

${ }^{20}$ Todd, Brit. Med. Jour., Dec., 1903, and Jour. of Hyg., 4, 1904.

${ }^{21}$ Kraus, Monatschr. f. Gesundheit, Suppl. II, 1904.

${ }^{22}$ Rosenthal, Deut. med. Woch., 1904.

${ }^{23}$ Kraus and Doerr, Wien. klin. Woch., xlii, 1905.

${ }^{24}$ Neisser and Shiga, Deut. med. Woch., 1903. 
organisms themselves may be killed by heating to $60^{\circ}$ and the cultures filtered. According to Doerr, ${ }^{25}$ the toxins may be obtained in the dry state by precipitation with ammonium sulphate and re-solution of the precipitate in water.

More recently, Olitsky and Kligler ${ }^{28}$ have repeated and extended the thorough study of the Shiga dysentery toxin made by Todd. ${ }^{27}$ These writer's differentiate definitely between a so-called exotoxin and an endotoxin. Their exotoxin they obtained by growing the Shiga bacilli for five days in alkalin-egg broth. Their endotoxin was produced by incubating agar growths in salt solution for two days and filtering. The exotoxin in small fractions of a cubic centimeter, after an incubation time of a few hours to four days, produces typical paralysis and severe nerve lesions in rabbits. This poison was killed by $75^{\circ} \mathrm{C}$. after one hour, and powerful neutralization was obtained with the serum of horses immunized with it. Their endotoxin, so-called, produces loss of weight and diarrhea in the animals, but no paralysis. In general, their results agree with those of Krause, Todd, Pfeiffer and Ungermann, ${ }^{28}$ and Bessau. ${ }^{29}$ They have produced far more potent toxins and more powerful antitoxins than previous workers.

The action of the dysentery toxin upon animals is extremely characteristic and throws much light upon the disease in man. The injection of a large dose intravenously into rabbits causes a rapid fall in temperature, marked respiratory embarrassment, and a violent diarrhea. This is at first watery, later contains large amounts of blood. If the animals live a sufficient length of time, paralysis may occur, the animal may fall to one side or may drag its posterior extremities. It is a remarkable fact that intravenous inoculation gives rise to intestinal inflammation of a severe nature, unquestionably due to the excretion of the poison by the intestinal mucosa and limited, usually, to the cecum and colon, rarely attacking the small intestine. Flexner, ${ }^{30}$ who has experimented extensively upon this question, believes it probable that most of the pathological lesions occurring in the intestinal canal of dysentery patients are referable to this excretion of dysentery toxin, rather than to the direct local action of the bacilli.

${ }^{25}$ Doerr, "Das Dysenterietoxin," Jena, 1907.

${ }^{26}$ Olitsky and Kligler, Jour. Exper. Med., 31, 1920, 19.

${ }_{27}$ Todd, Brit. Med. Jour., 2, 1903, 1456.

${ }^{28}$ Pfeiffer and Ungermann, Cent. f. Bakt., Orig., 50, 1909.

${ }^{29}$ Bessau, Cent. f. Back. 57, 1911, 21.

${ }^{30}$ Flexner, Jour. Exp Med., 8, 1906. 
Characteristic, of course, for the Shiga poison is the paralytic action which seems to be a specific phenomenon characteristic of this bacillus.

Immunization with Dysentery Bacilli.-The immunization of small animals, such as rabbits and guinea-pigs, against dysentery bacilli, especially those of the Shiga type, is attended with much difficulty, owing to the great toxicity of the cultures. Nevertheless, successful results may be accomplished by the administration of extremely small doses of living or dead bacilli, increased very gradually and at sufficient intervals. Horses may be more easily immunized. The serum of such actively immunized animals contains agglutinins in considerable concentration and of a specificity sufficiently illustrated in the preceding section dealing with the identification of the various species. For diagnostic purposes in human beings, the agglutination reaction, according to the technique of the Widal reaction for typhoid fever, has been utilized by Kruse ${ }^{31}$ and others. According to most observers, normal human serum never agglutinates dysentery bacilli in dilutions greater than one in twenty, while the serum of dysentery patients will often be active in dilutions as high as one in fifty.

Bactericidal substances have been demonstrated in the serum of immunized animals as well as in that of diseased human beings. These have been determined, in vitro, by Shiga, ${ }^{32}$ and, by the intraperitoneal technique of Pfeiffer, by Kruse. ${ }^{33}$ Bacteriolysis may take place in high dilutions of the serum, and has recently been used for the differentiation of the types of the dysentery bacilli by Ohno. ${ }^{34}$

True antitoxins in immune sera have been recently described by Kraus and Doerr. ${ }^{35}$

Todd has demonstrated that the mixture of such an immune serum with solutions of toxin and exposure of the mixture at $37.5^{\circ} \mathrm{C}$. for a half hour would produce almost complete neutralization of the poison, thus demonstrating that at least a large part of the beneficial action of the immune sera was due to a true antitoxic process. Because of the different varieties of dysentery bacilli, polyvalent serum has been recommended.

The results of Olitsky and Kligler are even better than this, in that they have succeeded in protecting rabbits against 1000 lethal doses of the poison with their antitoxic horse serum.

${ }^{31}$ Kruse, Deut. med. Woch., 1901.

${ }^{32}$ Shiga, Zeit. f. Hyg., xli.

${ }^{33}$ Kruse, Deut. med. Woch., 1903.

${ }^{34}$ Ohno, Philippine Jour. of Sci., vol, i, 1906.

${ }^{35}$ Kraus und Doerr, loc. cit. 


\section{DYSENTERY IN MAN}

The clinical term "dysentery" is a vague one and may signify violent diarrheal disturbances from almost any cause. Technically, the term dysentery should be restricted either to the amœbic variety or the bacillary. Bacterial dysenteries have been attributed to many different organisms besides the true dysentery bacilli, such as some of the paratyphoid bacilli, B. pyocyaneus, the Morgan bacilli, etc.

Endemic in a large part of the world, especially in the warmer climates, the disease most frequently occurs in epidemics of more or less definite localization, usually under conditions which accompany the massing of a large number of human beings in one place, such as those which occur in the crowded quarters of unsanitary towns, in institutions such as insane asylums, or in military camps. The mortality of such epidemics may be very large. According to Shiga, the disease in Japan frequently shows a mortality of over 20 per cent.

The disease in human beings usually begins as an acute gastroenteritis which is accompanied by abdominal pain and diarrhea. As it becomes more severe, the colicky pains and diarrhea increase, the stools lose their fecal character, becoming small in quantity and filled with mucus and flakes of blood. There is often severe tenesmus at this stage, and the bacilli are present in large numbers in the dejecta. Owing to the absorption of toxic products, symptoms referable to the nervous system, such as muscular twitching, may supervene, and if the disease is at all prolonged, there are marked inanition and prostration.

At autopsy in early stages there may be found only a severe catarrhal inflammation of the mucous membrane of the large intestine. In the later stages there are extensive ulcerations, and the bacteria are found lodged within the depths of the mucosa and submucosa. Occasionally they may penetrate to the mesenteric glands, but as far as we know there is no penetration into the general circulation.

Although this acute disease represents the typical picture of clinical dysentery, it must not be forgotten that bacteria of the dysentery types may cause very much milder intestinal inflammations and even simple diarrheas. "Y" bacilli and Flexner bacilli have often been isolated from such mild conditions, especially during the hot weather.

Epidemiology and Prevention.-Bacillary dysentery is not limited to any particular part of the world. Unlike the ameobic variety, it is probably just as common in temperate climates as it is in the tropical ones, though actual epidemic occurrence is probably a little more frequently observed in tropical communities where fly suppression and 
sewage and garbage disposal have not been developed to a satisfactory extent.

Dysentery epidemics also are more apt to occur during the hot and dry parts of the year when flies are prevalent. It is a mistake to think of the disease only as occurring in epidemics, since organisms of the dysentery group probably cause a great many sporadic cases and small group attacks of diarrheal diseases which in their clinical manifestations cannot be strictly classified as dysentery: Thus, numerous small group outbreaks have been studied in America and Europe, occurring either in cities sometimes, as in those studied by Kruse and others, in public institutions as insane asylums and orphanages, and in connection with such outbreaks a great many dysentery-like organisms have been described. At times, however, the disease has caused widespread epidemic outbreaks, and Castellani and Chalmers ${ }^{36}$ mention great epidemics which occurred in Europe in 1538, 1777, 1779, and 1834 . In its epidemic form it is particularly a disease of armies. Vincent and Muratet ${ }^{37}$ state that a destructive epidemic took place in the English Armies in 1415 after the battle of Agincourt. There were serious epidemics in the armies of the Allies during the Crimean War, during the American Civil War, during the Franco-Prussian War, the Russo-Turkish and the Russo-Japanese War. Considerable dysentery morbidity occurred in the South African War, and during the recent World War the disease was prevalent among all the armies fighting on the Eastern and Western fronts. In speaking of the dysentery morbidity under war conditions, it is always important to remember the almost insuperable difficulties which render accurate diagnosis of the disease almost impossible. Also, it is likely that large epidemics are rarely caused by a single dysentery type.

In $1905 \mathrm{Amako}^{38}$ studied the epidemics which occurred in the town of Kobe and from 743 cases isolated dysentery bacilli in 526. During this single epidemic he found 5 different types of dysentery bacilli, the first type being the typical Shiga bacillus, the second being a mannite fermenter, the fourth and fifth fermenting maltose and dextrin, the third having no effect upon maltose and dextrin but fermenting saccharose like the fourth and fifth. He found the first type in 108 cases, the second in 202 , the third type in 9 , the fourth in 169 , and the fifth in

${ }^{36}$ Castellani and Chalmes s, Text-book of Tropical Medicine.

${ }^{37}$ Vincent and Muratet. Dysentery, etc., Military Med. Manual, Univ. of London Press, 1917.

${ }^{38}$ Amako, Zeit. f. Hyg., 60, 1908, 93. 
16 cases. Such investigations amply prove that even local epidemics may be caused by a considerable number of different organisms. In one case, as a matter of fact, he found two different types in the same patient. Studying family epidemics at the same time, he usually found one and the same type in a single family, but in six families in which there were 25 patients he found two different types.

As we shall see, dysentery is transmitted by much the same agencies which are responsible for transmission of other intestinal diseases, typhoid, paratyphoid, etc., and in consequence, sanitary and other conditions which bring about one disease give rise to cases of the other. It is a noticeable feature of the outbreaks of intestinal disease which occurred in the Allied Armies during the late war that every outbreak of dysenteric maladies was accompanied by enormous numbers of mild diarrheal conditions. This is mentioned by Vincent and Muratet for the French and British Armies, and was noticed by us during the outbreak of similar conditions among the American troops in July, 1918. At such times the intestinal disturbances are almost universal among troops, taking, in most, the form of a mild temporary and recurring diarrhea, in others a more severe diarrhea with fever, and in others, again, severe symptoms of typical dysentery. Bacteriological analysis of large numbers of eases is next to impossible under such conditions, but even the limited number made during the late war, at least in the zone of the American Armies showed that all the known varieties of intestinal invaders, typhoid, paratyphoid and the various dysentery bacilli, played a rôle in the outbreaks. It is not at all impossible that many of the mild cases may have been true dysentery or even true typhoid, modified by increased natural resistance and by vaccination in the men. On the other hand, it is also quite likely, in fact is the view we favor, that the large majority of the mild cases which constituted perhaps 90 per cent of the total, represented infections by various other bacterial agencies originating in the massive infection of food and water with fecal organisms, the result of open latrines, limited water supplies and active fly transmission.

The most severe epidemics are probably those due to the Shiga bacillus. However, the Flexner bacilli have been known to cause considerable epidemics in southeastern Europe and in parts of Asia, in the Philippines, Japan, China and Ceylon. The Strong bacillus has been known to cause disease in the Philippines and the " $Y$ ". bacillus has been found rather frequently in milder outbreaks and in sporadic cases especially in the United States. The " $\dot{Y}$ " bacillus has been par- 
ticularly found in connection with cases of so-called infantile diarrhea, and it is not impossible that many cases of diarrhea in children may be due to this organism.

In many countries there seem to be endemic foci of dysentery. This has been particularly studied in France where outbreaks of dysentery seem often to have started in the central part in the neighborhood of Tours, and it is not unlikely that in our own country there may be scattered dysentery foci in the large cities of the north and in some parts of the south.

Dysentery may be conveyed by a number of different agencies. It is easy to understand that in a disease in which the movements become fluid and very frequent, and in which many mild unhospitalized cases may exist, the scattering of infectious material is very much more important epidemiologically than it would be in a disease like typhoid fever. In consequence, transmission from man to man by hands and indirect contamination of food, etc., is common during epidemics. The organisms may remain alive for a considerable period in the soil, as we have seen, and under camp conditions, infected latrines may contaminate water supplies and by the intervention of flies, scatter the organisms to the food. Fly transmission is of the utmost importance in dysentery among armies in the hot weather. It was probably the most important means of transmission during the American Army epidemic spoken of above. Large water epidemics, as described in the case of typhoid fever, are uncommon, although isolated ones have been described.

Dysentery carriers unquestionably exist. The organisms may persist for months in the intestinal canals of convalescents and have been described in the stools of individuals who give no history of having had the disease. The carrier problem in dysentery is a very difficult one because the isolation of small numbers of dysentery bacilli from stools is even more difficult than in the case of typhoid bacilli. It has been suggested that chronic carriers may harbor the organisms in the gall bladder, but this is not definite.

Shiga who has made extensive epidemiological studies on dysentery, expresses the opinion that the chief element in the spread of dysentery is the carrier. The first positive carrier examination was made by Conradi ${ }^{39}$ who isolated dysentery bacilli from the stools of three perfectly healthy children during the occurrence of a con-

${ }^{39}$ Conradi, Festschr. für Robt. Koch, Jena, 1903, cited from Lentz, Kolle and Wassermann, 2d ed., vol. 3. 
tact epidemic in Metz. Martha Wollstein ${ }^{40}$ found typical dysentery bacilli at autopsy in the intestines of children who had presented none of the symptoms of dysentery before death, and similar isolations were made by Duval and Shorer ${ }^{41}$ in connection with epidemics of summer diarrhea. Shiga claims that in every dysentery epidemic a great many contact cases can be traced epidemiologically. His belief is that it is contact which keeps the disease going almost entirely during the interepidemic periods in Japan, and that this is largely due to the carrier condition in healthy people, mild sporadic cases which clinically are diarrheal, and convalescents of typical dysentery cases. Water, he thinks, can play a part, but in his accounts of water epidemics this implies rather gross carelessness in the care of water. It was suggested during recent epidemics in the British Army that dried feces carried about by dust in sand storms may have contributed to the spread of epidemics.

Whether or not domestic animals may act as carriers is not certain, but it has been suggested that dogs may be spontaneously infected with bacillary dysentery and transmit the disease to human beings.

Castellani mentions that Kruse and Bowman ${ }^{42}$ have reported spontaneous bacillary dysentery in monkeys in which Flexner bacilli were isolated, and Messerschmidt" found " $Y$ " bacilli in the feces of healthy rabbits. Indirect transmission by means of food is of course, to be expected. A small epidemic occurring in a hospital in New York city and caused by the bacillus "Y," was indirectly traced to milk by ourselves. ${ }^{44}$

The length of time during which the bacilli may live in the soil has been mentioned as a source of danger by a number of writers, and Vincent and Muratet mention a ease which seems to us of considerable importance in dealing with army epidemies, since it indicates the danger of bringing new troops to old eamping grounds either in the course of advances over enemy territory or in the periodical use of cantonments. They state that dysentery had been common at the Chalons Military Camp in 1889. A year later, troops coming to this camp pitehed their tents and ditched them over the

${ }^{40}$ Wollstcin, Martha, Stud. from the Rock. Inst., 2, 1904.

${ }^{41}$ Dural and Shorer, Stud. from the Roek. Inst., 2, 1994.

42 Kruse and Bowman, eited from Castellani and Chalmers, loc. eit.

${ }^{43}$ Messerschmidt, eited from Lentz, loc. cit.

${ }^{44}$ Zinsser, Proc. N. Y. Path. Soc., 1907. 
site of the old latrines. Dysentery appeared among this particular troop unit, whereas, other troops remained free.

Preventive measures must center chicfly upon disinfection of dejecta, elosure of latrines, fly-proofing of latrines and kitchens, early recognition of suspicious eases with appropriate measures and enforeed cleanliness of food handlers and of all men before and after defecation. Care of water supplies and food supplies is of course indieated. It is also important to inquire into the possibility of recent intestinal disturbances however mild, among the kitchen personnel and others in contact with food. It is not uncommon in armies to find that company cooks are suffering from mild intestinal disturbances to which they, themselves, have paid little attention, but which may be sources of great danger to others.

Prophylactic Vaccination.-Many different methods of prophylactic vaceination have been attempted. Early attempts were discouraging because of the violent reactions obtained by the injection of killed broth cultures or even killed suspensions of agar growths.

Shiga ${ }^{45}$ carried out vaecination experiments very largely because he believed that the periodical appearance of epidemies in Japanindicated the acquisition of immunity by the community. He states, for instance, that in the thirty years preeeding 1908, two great epidemies passed through Japan from the south to the north. The epidemic remained in a single region from one to three years, rapidly reaching a maximum and gradually declining. After a period varying from ten to twenty years, these great epidemies reappeared and he believed that the interval could be explained by acquired immunity. Shiga's method consisted very largely in injecting, simultaneously, killed suspensions of agar eultures with specific serum (or in other words, sensitized cultures), following this first injection with killed agar suspensions without serum. This had the advantage, which sensitized cultures always have, of not giving the severe reactions which follow the injections of cultures alone. By these methods he vaceinated 10,000 Japanese in epidemic regions without very striking results as far as the morbidity is concerned, but with considerable lowering of the mortality.

Castellani inoculated 0.5 c.e. of a peptone water culture, killed by heating to $55^{\circ}$ for one hour, following this, after a week, with a second inoculation of 1 c.e. Most observers have worked with killed

${ }^{45}$ Shiga, Cent. f. Bakt., Orig., 42, 1909, 132. 
suspensions of agar cultures taken up in salt solution. The statistics of vaccinations done during the late war in isolated troop unit are not available at the present writing. Systematic dysentery vaccination was not, however, carried out in any of the European armies as far as we know. The entire subject is still in its experimental stages and no conclusive statements ean be made regarding it. As a matter of fact, judging from the immunological eonditions prevailing in the disease and the localization of the organisms, one would expect just what Shiga found, a reduction of the mortality without any considerable diminution of the morbidity.

Serum Treatment.-Sera, both monovalent and polyvalent, have been made by a large number of observers and extensive attempts at treatment have been carried out. Shiga himself used a multivalent dysentery serum in which he used the various types isolated in Japan. He obtained very encouraging results in thousands of cases in Japan, and believed that there was a very definite therapeutic advantage to be gained by use of the serum. Other reports have been conflicting. If dysentery serum is to be of great value, it probably will be most valuable in the Shiga types of the disease in which an exotoxin seems definitely to oceur. Sera like those produced by Olitsky and Kligler have not yet been used with sufficient extensiveness to warrant final judgment.

\section{THE MORGAN BACILLI}

In 1905 and 1906 Morgan $^{46}$ made a systematic study of the bacteriology of diarrheal diseases in infaney. He made a large number of isolations from stools of such children, and, among other things, isolated from a number of cases types of Gram-negative bacilli, obviously belonging into the paratyphoid-dysentery group which, however, did not correspond exactly with species previously described.

Morgan's bacillus I, he isolated from twenty-eight cases of infant diarrhea, out of fifty-eight examinations, and in seventeen of these it was the only lactose non-fermenting organism present. This organism was motile, produced acid and gas on glueose, but did not ferment mannite, duleite, lactose and saccharose. It differed from the hog cholera bacillus in producing an alkalin reaction on

${ }^{46}$ Morgan, H. de R., Brit. Med. Jour., 1, 1906, 908. 
litmus milk and in giving a powerful indol reaction. It produced neither acid nor gas on maltose and dextrin. It differed from most other indol producing organisms in that it produced this substance in glucose broth in the presence of excessive glucose, and by distillation Morgan proved that it produced indol and not skatolcarboxylic acid. Morgan's I bacillus is the only one of his bacilli in which pathogenicity can be considered as a possibility. He does not draw any definite conclusions in this regard. He is inclined, however, to believe that the No. I bacillus may have pathogenic properties.

COMPOSITE TABLE OF MOST IMPORTANT TYPES

\begin{tabular}{|c|c|c|c|c|c|c|c|c|c|c|c|}
\hline 7 & $\begin{array}{c}\text { Dex- } \\
\text { trose. }\end{array}$ & $\begin{array}{c}\text { Man- } \\
\text { nit. }\end{array}$ & $\begin{array}{l}\text { Mal- } \\
\text { tose. }\end{array}$ & $\begin{array}{l}\text { Lac- } \\
\text { tose. }\end{array}$ & $\begin{array}{l}\mathrm{Xy-} \\
\text { lose. }\end{array}$ & $\begin{array}{c}\text { Rham- } \\
\text { nose: }\end{array}$ & $\begin{array}{l}\text { Sac- } \\
\text { cha- } \\
\text { rose }\end{array}$ & $\begin{array}{c}\text { Dul- } \\
\text { cit. }\end{array}$ & $\begin{array}{l}\text { Lead } \\
\text { ace- } \\
\text { tate } \\
\text { agar. }\end{array}$ & Indol. & $\begin{array}{l}\text { Mo- } \\
\text { tility. }\end{array}$ \\
\hline B. coli com'is... & $\oplus$ & $\oplus$ & $\oplus$ & $\oplus$ & $x$ & $x$ & - & $\oplus$ & varies & + & + \\
\hline B. coli com'ior. . & $\oplus$ & $\oplus$ & $\oplus$ & $\oplus$ & $x$ & $x$ & $\oplus$ & $\oplus$ & varies & $\ldots \ldots$ & + \\
\hline B. acidi lactici. . & $\oplus$ & $\oplus$ & $\oplus$ & $\oplus$ & $x$ & $x$ & - & - & $\ldots$ & $\ldots \ldots$ & + \\
\hline B. lactis aerog. . & $\oplus$ & $\oplus$ & $\oplus$ & $\oplus$ & $x$ & $x$ & $\oplus$ & - & $\ldots$ & . & + \\
\hline B. parat. " $A$ ". . & $\oplus$ & $\oplus$ & $\oplus$ & - & - & + & - & slow & - & 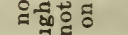 & + \\
\hline B. parat. "B". & $\oplus$ & $\oplus$ & $\ldots$ & - & + & + & - & + & + & పี & + \\
\hline B. enteritidis. . & $\oplus$ & $\oplus$ & $\ldots$ & - & + & + & - & + & + & $\Rightarrow$ 혼 & + \\
\hline B. abortus...... & $\oplus$ & $\oplus$ & $\cdots$ & - & + & + & - & + & - & పేర大 & + \\
\hline B. hog cholera.. & $\oplus$ & $\oplus$ & $\ldots$ & - & + & + & - & irreg. & - & 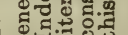 & + \\
\hline B. typhi murium & $\oplus$ & $\oplus$ & .. & - & + & + & - & + & + & $0 \pi=0$ & + \\
\hline B. typhosus.... & + & + & + & - & slow & - & - & slow & + & - & + \\
\hline B. dys. Shiga... & + & - & - & - & $x$ & $x$ & - & - & $x$ & - & - \\
\hline $\begin{array}{l}\text { B. dys. Flexner. } \\
\text { B. dys. "Y" }\end{array}$ & + & + & + & - & $x$ & $x$ & - & - & $? \times$ & + & - \\
\hline (Hiss-Park)... & + & + & - & - & $x$ & $x$ & - & $x$ & $x$ & + & - \\
\hline B. dys. Strong... & + & + & - & - & $x$ & $x$ & + & $x$ & $x$ & + & - \\
\hline B. faec.alkal.... & - & $x$ & $x$ & $x$ & $x$ & $x$ & $x$ & $x$ & $x$ & & + \\
\hline B. Morgan, No. 1 & $\begin{array}{c}\oplus+ \\
\text { slight } \\
\text { gas }\end{array}$ & - & - & - & $?$ & $?$ & - & - & $3 \times$ & + & + \\
\hline
\end{tabular}

Other organisms which he regards as possibly important he speaks of as his Nos. III and IV types, both of which resembled somewhat the Flexner type of dysentery. No. III he says agglutinated equally well with either Flexner or typhoid serum as if closely related to both. It differed from the Flexner bacillus in producing acid on sorbite.

His No. IV differs from the Flexner in not producing indol. The 
No. IV agglutinated in the Flexner serum, but not at all with typhoid serum.

It would be confusing to go more deeply into the Morgan group. It is important, however, to mention them since his work illustrates the possibility of there being a large number of insufficiently differentiated types of Gram-negative bacilli in the intestines of human beings. In view of the many researches of recent years in which the possibility of variants of unknown species in regard to sugar fermentations, agglutinability, etc., has been pointed out, it would seem proper to advise great caution in accepting as new species, organisms which differ from well-known types in only one or another characteristic. 


\section{CHAPTER XXXV}

BACILLUS MUCOSUS CAPSULATUS, RHINOSCLEROMA AND OZANA

\section{BACILLUS MUCOSUS CAPSULATUS}

(Bacterium pneumoniœ, Friedländer's bacillus, Pneumobácillus)

IN 1882 Friedländer ${ }^{1}$ announced the discovery of a microorganism which he believed to be the incitant of lobar pneumonia and which, in his original communications, he described as a "micrococcus."

A superficial morphological resemblance between Friedländer's microorganism and Diplococcus lanceolatus, now recognized as the most frequent cause of lobar pneumonia, led, at first, to much confusion, and it was not until several years later, owing to the careful researches of Fränkel" and of Weichselbaum, ${ }^{3}$ that the "micrococcus" of Friedländer was recognized as a short, encapsulated bacillus which occurred in lobar pneumonia exceptionally only. Similar bacilli were subsequently found by other observers, bacilli which, upon morphological grounds, are classified together as the "Friedländer group," or the "group of Bacillus mucosus capsulatus."

Morphology and Staining.-The Friedländer bacillus is a short, plump bacillus with rounded ends, subject to great individual variations as to size. Its average measurements are from 0.5 to 1.5 micra in width and 0.6 to 5 micra in length. Forms approaching both extremes may be met with in one and the same culture. The short, thick forms, frequently found in animal and human lesions, are almost coccoid and account for Friedländer's error in first describing the bacillus as a micrococcus. The bacilli may be single, in diploform, or in short chains. They are non-motile and possess no flagella. Spores are not formed. 1884.

${ }^{1}$ Friedländer, Virchow's Arch., lxxxvii, 1882; Fort. d. Med., i, 1883; ibid., ii.

${ }^{2}$ Fränkel, Zeit. f. klin. Med., x, 1886.

${ }^{3}$ Weichselbaum, Med. Jahrb., Wien, 1886. 
The bacillus is characteristically surrounded by a well-developed eapsule which is most perfectly demonstrated in preparations taken directly from some animal fluid, such as the secretion or exudate from infected areas. It is also seen, however, in smears made from agar or gelatin cultures. The capsule is usually large, twice or three times the size of the bacillus itself. When seen in chains or in groups, several bacilli may appear to be inclosed in one capsule. Prolonged cultivation on agar or gelatin may result in disappearance of the capsule. The bacillus is easily stained with the ordinary

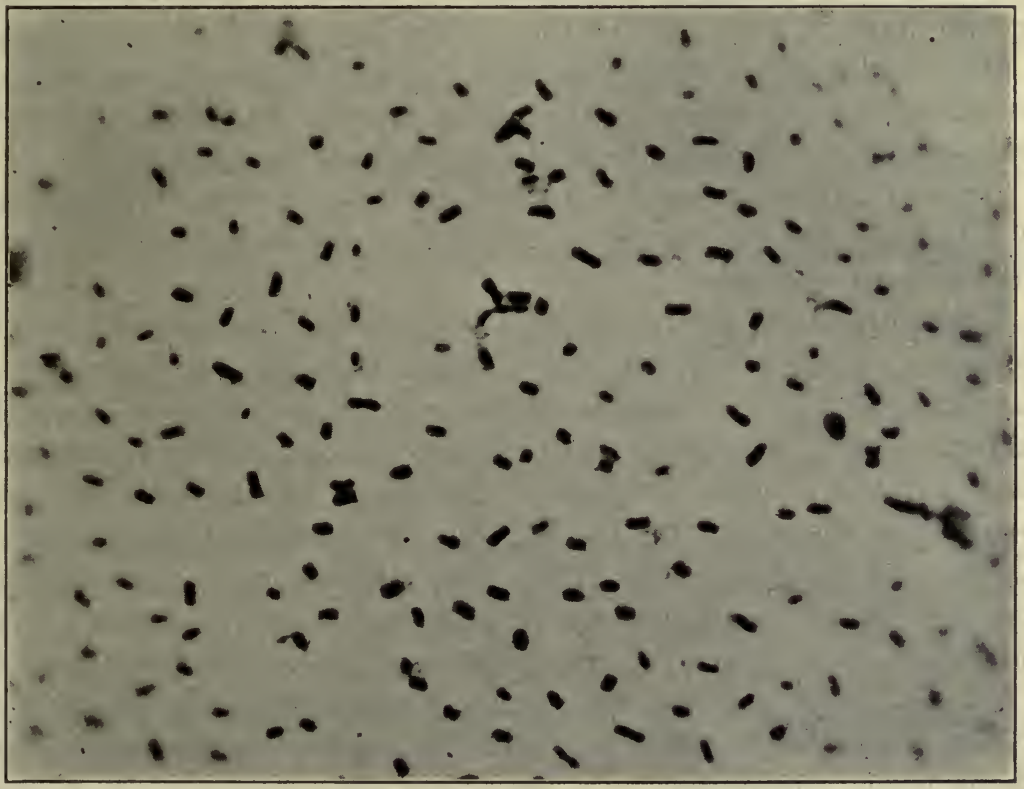

Fig. 71.-Bacillus mocosus Capsulatus.

dyes, but is decolorized when stained by the Gram-method. Capsules may often be seen when the more intense anilin dyes are employed. They are brought out with much regularity by any of the usual eapsule stains.

Cultivation.-B. mucosus capsulatus is easily cultivated. It grows readily on all the usual eulture media, both on those having a meatinfusion basis and on those made with meat extract. Growth takes place at room temperature $\left(18^{\circ}\right.$ to $\left.20^{\circ}\right)$ and more rapidly at $37.5^{\circ} \mathrm{C}$. A temperature of $60^{\circ} \mathrm{C}$. and over kills the bacilli in a short time. The thermal death-point according to Sternberg is $56^{\circ} \mathrm{C}$. Growth 
ceases below $10^{\circ}$ to $12^{\circ} \mathrm{C}$. Kept at room temperature and protected from drying, the bacillus may remain-alive, in eultures, for several months.

The bacillus is not very fastidious as to reaction of media, growing equally well on moderately alkaline or acid media. It is aërobic and facultatively anaërobic; growth under anaërobic conditions, however, is not luxuriant.

On agar, growth appears in the form of grayish-white mucus-like colonies, having a characteristically slimy and semi-fluid appearance. Colonies have a tendency to confluence, so that on plates, after three or four days, a large part of the surface appears as if covered with a film of glistening, sticky exudate, which, if fished, comes off in a tenacious, stringy manner. It is often possible to make a tentative diagnosis of the bacillus from the appearance of this growth.

In broth, there is rapid and abundant growth, with the formation of a pelliele, general clouding, and later the development of a profuse, stringy sediment.

Stab cultures in gelatin show, at first, a white, thin line of growth along the course of the puncture. Soon, however, rapid growth at the top results in the formation of a grayish mueoid droplet on the surface, which, enlarging, gives the growth a nail-like appearance. This nail-shape was originally described by Friedländer and regarded as diagnostie for the bacillus. The gelatin is not fluidified. As the culture grows older the entire surface of the gelatin tube may be covered with growth, flowing out from the edges of the nail-head. The gelatin acquires a darker color and there may be a few gas bubbles below the surface. Microscopically, colonies on gelatin plates have a smooth outline and a finely granular or even homogeneous consistency.

On blood serum, a confluent mueus-like growth appears.

On potato, abundant growth appears, slightly more brownish in color than that on other media.

In pepton solutions, there is no indol formation.

In milk, there is abundant growth and marked eapsule development. Coagulation oecurs irregularly.

In considering the general cultural characteristics of the Friedländer bacillus, it must not be forgotten that we are dealing with a rather heterogeneous group, the individuals of which are subject to many minor variations. Capsule development, lack of motility, inability to fluidify gelatin, failure to form indol, and absence of 
spores, are characteristics common to all. In size, general appearance, gas formation, and pathogenicity, individual strains may vary much, one from the other. Strong ${ }^{4}$ has studied various races as to gas formation and concludes that most strains form gas from dextrose and levulose, but that lactose is fermented by some only. About two-thirds of the gas formed is hydrogen, the rest $\mathrm{CO}_{2}$. Acid formation, according to Strong, is also subject to much variation among different races. Similar studies by Perkins ${ }^{5}$ show that most of the ordinary cultural characteristics of bacilli of this group are extremely variable and can not serve as a basis for differentiation. Reactions on sugars, however, are more constant. Perkins suggests the following tentative division classes on this basis:

I. All carbohydrates fermented with the formation of gas.

II. All carbohydrates, except lactose, fermented with the formation of gas.

III. All carbohydrates, except saccharose, fermented with the formation of gas.

Type I. corresponds to B. aërogenes (Migula), type II. to B. Friedländer or Bacterium pneumoniæ (Migula), and type III. to Bacillus lactis aërogenes.

Differentiation by means of serum reactions has not proved satisfactory. $^{6}$

Pathogenicity.-When Friedländer first described this microorganism, he assumed it to be the incitant of lobar pneumonia. Subsequent researches by Weichselbaum ${ }^{7}$ and others have shown it to be etiologically associated with pneumonia in about seven or eight per cent of all cases. The percentage in this country is probably lower. Such cases can often be diagnosed by the presence of the bacilli in the sputum, which is peculiarly sticky and stringy. Cases

${ }^{4}$ Strong, Cent. f. Bakt., xxv, 1899.

${ }^{5}$ Perkins, Jour. of. Infect. Dis., I, No. 2, 1904.

${ }^{6} \mathrm{~J}$. G. Fitzgerald, who has recently made a careful study of the mucosus capsulatus group has concluded that present methods do not permit a subdivision of these organisms into separate species. He ofters the following "tentative suggestion": "It is conceivable that mutations based on the necessity of maintaining a parasitic existence have caused Gram-negative bacilli found normally in the body elsewhere than in the intestinal tract to develop capsules for protection and a new group has arisen which we designate B. mucosus capsulatus; and the varieties B. aërogenes and B. acidi lactici connect the group with the non-encapsulated colon group."

${ }^{7}$ Weichselbaum, loc. cit. 
of Friedländer pneumonia are extremely severe and usually fatal. The bacillus has been found in cases of ulcerative stomatitis and nasal catarrh; in two cases of severe tonsillitis in children; in the pus from suppurations in the antrum of Highmore and the nasal sinuses (Fränkel and others), and in cases of fetid coryza (ozena), of which disease it is supposed by $\mathrm{Abel}^{8}$ and others to be the specific cause. Whether the ozena bacillus represents a separate species or not, can not at present be decided. The bacillus of Friedländer has been found in empyema fluid, in pericardial exudate (after pneumonia), and in spinal fluid. ${ }^{9}$ Isolated cases of Friedländer bacillus septicemia have been described. ${ }^{10}$ Being occasionally a saprophytic inhabitant of the normal intestine, it has been believed to be etiologically associated with some forms of diarrheal enteritis.

B. mucosus capsulatus is pathogenic for mice and guinea-pigs, less so for rabbits. Inoculation of susceptible animals is followed by local inflammation and death by septicemia. If inoculation is intraperitoneal, there is formed a characteristically mucoid, stringy exudate.

The question of immunization against bacilli of the Friedländer group is still in the stage of experimentation. Immunization with carefully graded doses of dead bacilli has been successful in isolated cases. Specific agglutinins in immune serum have been found by Clairmont, ${ }^{11}$ but irregularly and potent only against the particular strain used for the immunization.

\section{OTHER BACILLI OF THE FRIEDLÄNDER GROUP}

Bacillus of Rhinoscleroma.-This bacillus, described by $\mathrm{v}$. Frisch $^{12}$ in 1882 , is a plump, short rod, with rounded ends, morphologically almost identical with Friedländer's bacillus; it is nonmotile and possesses a distinct capsule. Although at first described as Gram-positive, it has been shown to be decolorized with this method of staining. It forms slimy colonies, has a nail-like appearance in gelatin stab cultures, and in pepton solutions produces no indol. It differs from B. mucosus capsulatus $\left(W^{2}\right.$ d de $^{13}$ ) in forming no gas in

${ }^{8}$ Abel, Zeit. f. Hyg., xxi.

${ }^{9}$ Jäger, Zeit. f. Hyg., xix.

${ }^{10}$ Howard, Johns Hopkins Hosp. Bull., 1899.

${ }^{11}$ Clairmont, Zeit. f. Hyg., xxxix.

12 Frisch, Wien. med. Woch., 1882.

${ }^{13}$ Wilde, Cent. f. Bakt., xx, 1896. 
dextrose bouillon, in producing no acid in lactose bouillon, and in never coagulating milk.

Pathogenicity.-The bacillus of rhinoscleroma is but moderately pathogenic for animals delicately susceptible to the bacillus of Friedländer. Rhinoscleroma, the disease produced by this bacillus in man, consists of a slowly growing granulomatous inflammation, located usually at the external nares or upon the mucosa of the nose, mouth, pharynx, or larynx. It is composed of a number of chronic, hard,

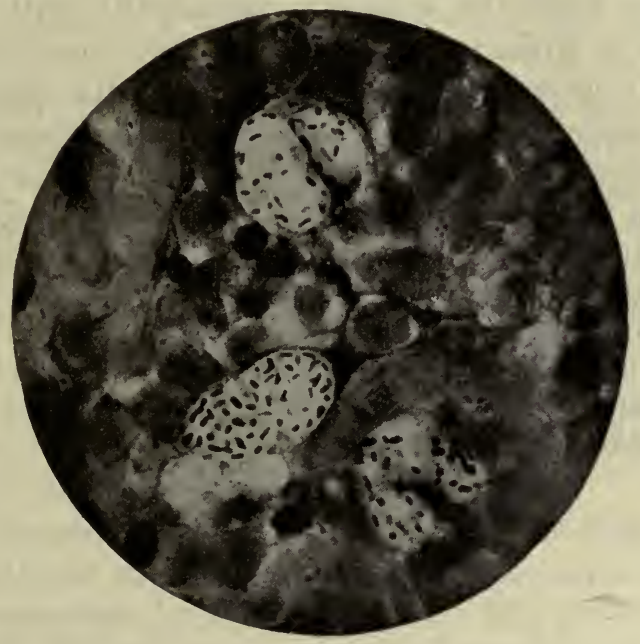

Fig. 72.-Bacillus of Rhinoscleroma. Section of tissue showing the microorganisms within Mikulicz cells. (After Fränkel and Pfeiffer.)

nodular swelïings, which, on histological examination, show granulation tissue and productive inflammation. In the meshes of the abundant connective tissue lie many large swollen cells, the so-called "Mikulicz cells." 14 The rhinoscleroma bacilli lie within these cells and in the intercellular spaces. They can be demonstrated in histological sections and can be cultivated from the lesions, usually in pure culture. Rhinoscleroma is rare in America. It is most prevalent in Southeastern Europe. The disease is slowly progressive and comparatively intractable to surgical treatment, but hardly ever affects the general health unless by mechanical obstruction of the air passages. 
B. Ozænæ.-The work of $\mathrm{Abel}^{15}$ and others has shown that ozena, or fetid nasal catarrh, is almost always assoeiated with a bacillus morphologieally and eulturally almost identieal with B. mucosus eapsulatus. The baeillus can not be definitely separated from the latter. Aceording to Wilde it forms no gas in dextrose bouillon and is less pathogenie for mice than B. Friedländer. Whether it is a separate species, or merely an atypieal form ehanged by environment, ean not be stated at present.

Perez Bacillus of Ozæna.-Perez ${ }^{16}$ in 1899 deseribed another mieroorganism which he eonneets etiologically with ozæna. The Perez baeillus is Gram-negative, pleomorphie, non-motile and noncapsulated. It grows easily on ordinary media, does not liquefy gelatin, and makes indol. Its eultures have a eharaeteristic fetid odor. Intravenously injected into rabbits it seems to produce a localized lesion in the nasal eavity on the turbinated bones. Hofer ${ }^{17}$ has also isolated it, but recent work leaves its importance as the eausative agent in doubt.

\footnotetext{
${ }^{15}$ Abel, Zeit. f. Hyg., xxi.

16 Perez, Animal de l'Inst. Past. 1899.

${ }^{17}$ Hofer, Wien. klin. Woch., vol. 26, pp. 1011 and 1628.
} 


\section{CHAPTER XXXVI}

THE ANAËROBIC BACILLI. TETANUS AND BACILLUS TETANI. BOTULISMUS AND THE BACILLUS BOTULINUS

LockJAW or tetanus, though a comparatively infrequent disease, has been recognized as a distinct clinical entity for many centuries. The infectious nature of the disease, however, was not demonstrated until 1884, when Carlo ${ }^{1}$ and Rattone succeeded in producing tetanus in rabbits by the inoculation of pus from the cutaneous lesion of a human case. Nicolaier, ${ }^{2}$ not long after, succeeded in producing tetanic symptoms in mice and rabbits by inoculating them with soil. In connection with the lesions produced at the point of inoculation, Nicolaier described a bacillus which may have been Bacillus tetani, but which he was unable to cultivate in pure culture. Kitasato, ${ }^{3}$ in 1889 , definitely solved the etiological problem by obtaining from cases of tetanus pure cultures of bacilli with which he was able again to produce the disease in animals.

Kitasato succeeded where others had failed because of his use of anaërobic methods and his elimination of non-spore-bearing contaminating organisms by means of heat. His method of isolation was as follows: The material containing tetanus bacilli was smeared upon the surface of agar slants. These were permitted to develop at incubator temperature for twenty-four to forty-eight hours. At the end of this time the cultures were subjected to a temperature of $80^{\circ} \mathrm{C}$. for one hour. The purpose of this was to destroy all non-sporulating bacteria, as well as aërobic spore-bearers which had developed into the vegetative form. Agar plates were then inoculated from the slants and exposed to an atmosphere from which oxygen had been completely eliminated and hydrogen substituted. On these plates colonies of tetanus bacilli developed.

Morphology and Staining.-The bacillus of tetanus is a slender bacillus, 2 to 5 micra in length, and 0.3 to 0.8 in breadth. The

${ }^{1}$ Carlo e Rattone, Giornale d. R. Acad. d. Torino, 1884.

${ }^{2}$ Nicolaier, Inaug. Diss., Göttingen, 1885.

Kitasato, Deut. med. Woch., No. xxxi, 1889. 
vegetative forms which occur chiefly in young cultures are slightly motile and are seen to possess ${ }^{4}$ numerous peritrichal flagella, when stained by special methods. After twenty-four to forty-eight hours of incubation, the length of time depending somewhat on the nature of the medium and the degree of anaërobiosis, the bacilli develop spores which are characteristically located at one end, giving the bacterium the diagnostic drumstick appearance.

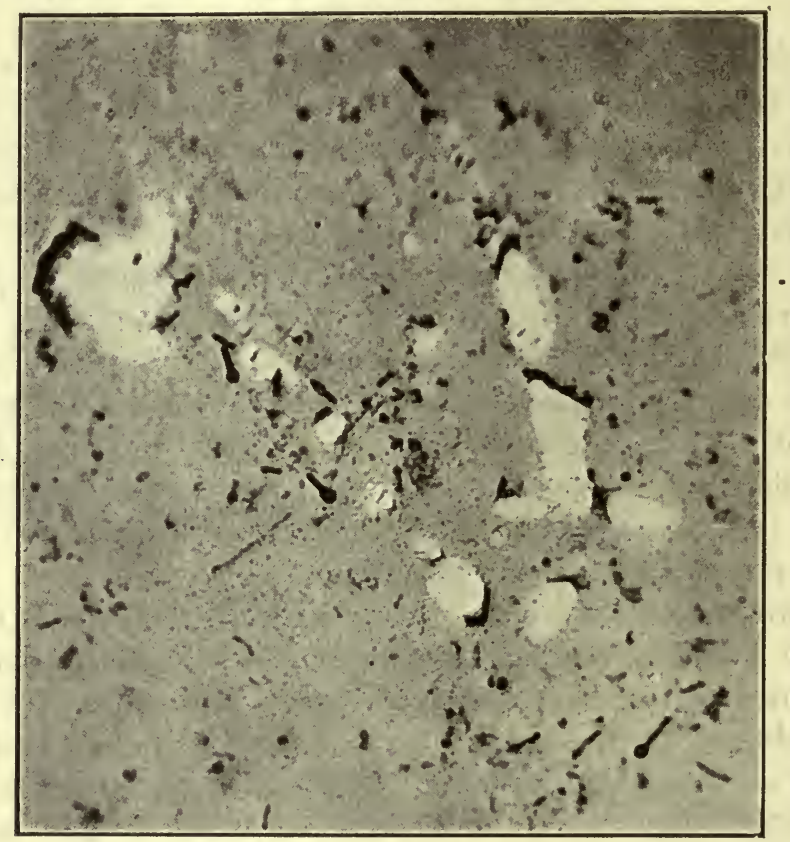

Fig. 73.-Bacillus tetani. Spore stain.

As the cultures grow older the spore-bearing forms completely supersede the vegetative ones. Very old cultures contain sporebearing bacilli and spores only.

The tetanus bacillus is easily stained by the usual anilin dyes, and reacts positively to Gram's stain. Flagella staining is successful only when very young cultures are employed.

Distribution.-In nature, the tetanus bacillus has been found by Nicolaier and others to occur in the superficial layers of the soil. The earth of cultivated and manured fields seems to harbor this

'Vottaler, Zeit. f. Hyg., xxvii. 
organism with especial frequency, probably because of its presence in the dejecta of some of the domestic animals.

Biological Characteristics.-The bacillus of tetanus is generally described as an obligatory anaërobe. While it is unquestionably true that growth is ordinarily obtained only in the complete absence of oxygen, various observers, notably Ferran ${ }^{5}$ and Belfanti, ${ }^{6}$ have successfully habituated the bacillus to aërobic conditions by the gradual increase of oxygen in cultures. Habituation to aërobic conditions has usually been accompanied by diminution or loss of pathogenicity and toxin-formation. Anaërobic conditions may likewise be dispensed with if tetanus bacilli be grown in symbiosis with some of the aërobic bacteria. The addition to culture media of suitable carbohydrates, and of fresh sterile tissue, has also been found to render it less exaeting as to mechanical anaërobiosis. ${ }^{7}$

Anaërobically cultivated, Bacillus tetani grows readily upon meatinfusion broth, which it clouds within twenty-four to thirty-six hours.

Upon meat-infusion gelatin at $20^{\circ}$ to $22^{\circ} \mathrm{C}$. the tetanus bacillus grows readily, growth beeoming visible during the second or third day. There is slow liquefaction of the gelatin.

On agar, at $37.5^{\circ} \mathrm{C}$, growth appears within forty-eight hours. Colonies on agar plates present a rather characteristic appearanee, consisting of a compact center surrounded by a loose meshwork of fine filaments, not unlike the medusa-head appearance of subtilis colonies. In agar stabs, fine radiating processes growing out in all directions from the central stab tend to give the eulture the appearance of a fluff of cotton. Milk is a favorable culture medium and is not coagulated.. On potato, growth is delicate and hardly visible.

The most favorable temperature for the growth of this bacillus is $37.5^{\circ} \mathrm{C}$. Slight alkalinity or neutrality of the culture media is most advantageous, though moderate acidity does not altogether inhibit growth. All the media named may be rendered more favorable still by the addition of one or two per cent of glucose, maltose, or sodium formate. ${ }^{8}$ In media containing certain carbohydrates, tetanus bacilli produce acid. In gelatin and agar, moderate amounts of gas are produced, consisting chiefly of $\mathrm{CO}_{2},{ }^{9}$ but with the admix-

${ }^{5}$ Ferran, Cent. f. Bakt., xxiv, No. 1.

- Belfanti, Arch. per le sci. med., xvi.

7 Th. Smith, Brown, and Walker, Jour. Med. Res., N. S., ix, 1906.

${ }^{8}$ Kitasato, Ztschr. f. Hyg. 1891.

$9 v$. Eisler and Pribram in Kraus and Levaditi, Handbuch, etc., Jena, 1907. 
tures of other volatile substances which give rise to a characteristically unpleasant odor, not unlike that of putrefying organic matter. This odor is due largely to $\mathrm{H}_{2} \mathrm{~S}$ and methylmereaptan.

The vegetative forms of the tetanus bacillus are not more resistant against heat or chemical agents than the vegetative forms of other microorganisms, Tetanus spores, however, will resist dry

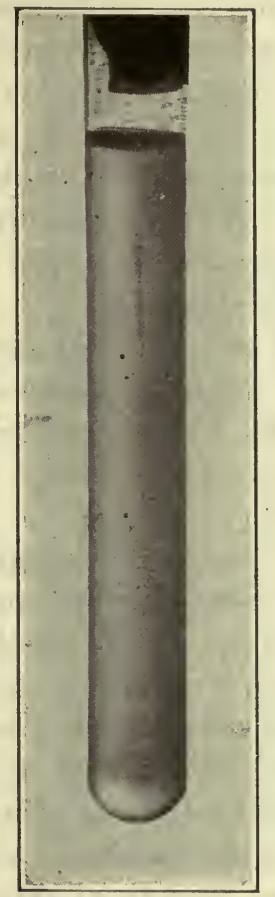

Fig. 74.-Young TeTanus Culture in Glucose Agar.

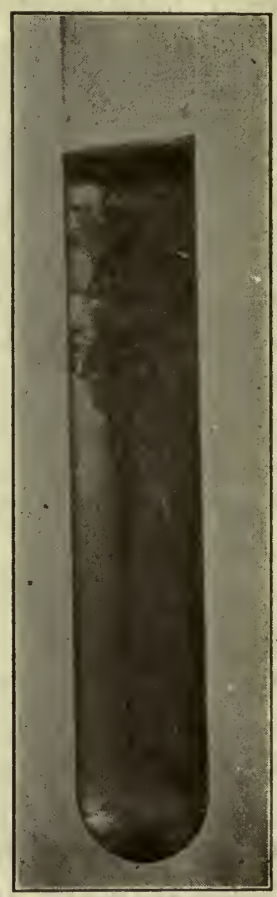

Fig. 75.-Older TeTanus Culture in GluCose Agar.

heat at $80^{\circ} \mathrm{C}$. for about one hour, live steam for about five minutes; five per cent carbolic acid kills them in twelve to fifteen hours; one per cent of bichlorid of mercury in two or three hours. Direct sunlight diminishes their virulence and eventually destroys them. Protected from sunlight and other deleterious influenees, tetanus spores may remain viable and virulent for many years. Henrijean ${ }^{10}$ has reported suecess in produeing tetanus with bacilli from a splinter of wood infected eleven years before.

${ }^{10}$ Henrijean, Ann. de la soc. méd. Chir. de Liège, 1891. 
Tetanus Toxin.-The pathogenicity of the tetanus bacillus depends entirely upon the soluble toxin which it produces. This toxin is produced in suitable media by all strains of virulent tetanus bacilli, individual strains showing less variation in this respect than do the separate strains of diphtheria bacilli. While partial aërobiosis does not completely eliminate toxin formation, anaërobic conditions are by far more favorable for its development.

The medium most frequently employed for the production of tetanus toxin is neutral or slightly alkaline beef-infusion bouillon containing five-tenths per cent $\mathrm{NaCl}$ and one per cent pepton. Glueose, sodium formate, or tincture of litmus may be added, but while these substances increase the speed of growth of the bacilli they do not seem to enhance the degree of toxicity of the cultures. Glucose is said even to be unfavorable for strong toxin development. It is important, too, that the bouillon shall be freshly prepared..$^{11}$ There does not seem to be any direet relationship between the amount of growth and the degree of toxicity of the cultures. Under anaërobic conditions in suitable bouillon and grown at $37.5^{\circ} \mathrm{C}$., the maximum toxin content of the cultures is reached in from ten days to two weeks. After this time the toxin deteriorates rapidly.

Tetanus toxin has been produced without resort to meehanical anaërobie methods by several observers, notably by Debrand, ${ }^{12}$ by eultivating the bacilli in bouillon in symbiosis with Bacillus subtilis. By this method, Debrand claims to have produced toxin which was fully as potent as that produced by anaërobic cultivation.

The tetanus toxin, in solution in the bouillon cultures, may be separated from the bacteria by filtration through Berkefeld or Chamberland filters. Since the poison in such filtrates deteriorates very rapidly, much more rapidly even than diphtheria toxin, various methods have been devised to obtain the toxin in the solid state. The most useful of these is precipitation of the poison out of solution by saturation with ammonium sulphate. ${ }^{13}$ Very little of the toxin is lost by this method and, thoroughly dried and stocked in vacuum tubes, together with anhydrous phosphoric acid, it may be preserved indefinitely without deterioration. The precipitate thus formed is easily soluble in water or salt solution, and therefore

${ }^{11}$ Vaillard et Vincent, Ann. de l'inst. Pasteur, 1891.

12 Debrand, Ann. de l'inst. Pasteur, 1890, 1902.

${ }^{13}$ Brieger und Cohn, Zeit. f. Hyg., xv. 
permits of the preparation of uniform solutions for purposes of standardization.

Brieger and Boer ${ }^{14}$ have also suceeded in precipitating the toxin out of broth solution with zine chloride. Vaillard and Vincent ${ }^{15}$ have procured it in the dry state by evaporation in vacuo.

Brieger and Cohn, ${ }^{16}$ Brieger and Boer, ${ }^{17}$ and others have attempted to isolate tetanus poison, removing the proteins from the ammonium sulphate precipitate by various chemical methods. The purest preparations obtained have been in the form of fine yellowish flakes, soluble in water, insoluble in alcohol and ether. Solutions of this substance have failed to give the usual protein reactions.

The toxin when in solution is extremely sensitive to heat. Kitasato ${ }^{18}$ states that exposure to $68^{\circ} \mathrm{C}$. for five minutes destroys it completely. Dry toxin is more resistant, ${ }^{19}$ often withstanding temperatures of $120^{\circ} \mathrm{C}$. for more than fifteen minutes. Exposure to direct sunlight destroys the poison in fifteen to eighteen hours. ${ }^{20}$

Interesting experiments as to the action of eosin upon tetanus toxin have been carried out by various observers. Flexner and Noguehi ${ }^{21}$ found that five per cent eosin added to the toxin would destroy it within one hour. This action is aseribed to the photodynamic power of the eosin.

Tetanus toxin is one of the most powerful poisons known to us. Filtrates of broth cultures, in quantities of $0.000,005$ c.c., will often prove fatal to mice of ten grams weight. Dry toxin obtained by ammonium sulphate precipitation ${ }^{22}$ is quantitatively even stronger, values of $0.000,001$ gram as a lethal dose for a mouse of the given weight not being uneommon. Brieger and $\mathrm{Cohn}^{23}$ succeeded in producing a dry toxin eapable of killing mice in doses of $0.000,000,05$ gram.

Different species of animals show great variation in their susceptibility to tetanus toxin. Human beings and horses are probably

${ }^{14}$ Brieger und Boer, Zeit. f. Hyg. xxi.

${ }^{15}$ Vaillard et Vincent, Ann. de l'inst. Pasteur, 1891.

${ }^{16}$ Brieger und Cohn, loc. cit.

${ }^{17}$ Brieger und Boer, Zeit. f. Hyg., xxi.

${ }_{18}$ Kitasato, Zeit. f. Hyg., x.

19 Morax et Marie, Ann. de l'inst. Pasteur, 1902.

${ }^{20}$ Fermi und Pernossi, Cent. f. Bakt., xv.

${ }^{21}$ Flexner and Noguchi, "Studies from Rockefeller Inst.," v., 1905.

${ }^{22}$ Brieger und Cohn., loc. cit.

${ }^{23}$ Brieger und Cohn, Zeit. f. Hyg., xv. 
the most susceptible species in proportion to their body weight. The common domestic fowls are extremely resistant. Calculated for grams of body weight, the horse is twelve times as susceptible as the mouse, the guinea-pig six times as susceptible as the mouse. The hen, on the other hand, is 200,000 times more resistant than the mouse.

After the inoculation of an animal with tetanus toxin there is always a definite period of incubation before the toxic spasms set in. This period may be shortened by increase of the dose, but never entirely eliminated. ${ }^{24}$ When the toxin is injected subcutaneously, spasms begin first in the museles nearest the point of inoculation. Intravenous inoculation, ${ }^{25}$ on the other hand, usually results in general tetanus of all the muscles. The feeding of toxin does not produce disease, the poison being passed through the bowel unaltered.

The harmful action of tetanus toxin is generally attributed to its affinity for the central nervous system. Wassermann and Takaki ${ }^{26}$ show that tetanus toxin was fully neutralized when mixed with brain substance. Other organs-liver and spleen, for instanceshowed no such neutralizing power. The central origin of the tetanic contractions was made very evident by the work of Gumprecht, ${ }^{27}$ who succeded in stopping the spasms in a given region by division of the supplying motor nerves.

The manner in which the toxin reaches the central nervous system has been extensively investigated, chiefly by Meyer and Ransora, and Marie and Morax. Meyer and Ransom ${ }^{28}$ from a series of careful experiments reached the conclusion that the toxin is conducted to the nerve centers along the paths of the motor nerves. Injected into the circulation, ${ }^{29}$ the toxin reaches simultaneously all the motor nerve endings, producing general tetanus. In this case too, therefore, the poison from the blood can not pass directly into the central nervous system, but must follow the route of nerve tracts.

These observations have been of great practical value in that they pointed to the desirability of the injection of tetanus antitoxin

${ }^{24}$ Courmont et Doyen, Arch. de phys., 1893

${ }^{25}$ Ransom, Deut. med. Woch., 1893.

${ }^{26}$ Wassermann und Takaki, Berl. kiln. Woch., 1898.

${ }^{27}$ Gumprecht, Pflüger's Arch., 1895.

${ }^{28}$ Meyer und Ransom, Arch., f. exp. Pharm. u. Path., xlix.

${ }^{29}$ Marie et Morax, Ann. de l'inst. Pasteur, 1902. 
directly into the nerves and the central nervous system in active cases.

Tetanolysin.-Tetanus bouillon contains, besides the "tetanospasmin" described above which produces the familiar symptoms of the disease, another substance discovered by Ehrlich ${ }^{30}$ and named by him "tetanolysin." Tetanolysin has the power of causing hemolysis of the red blood corpuseles of various animals, and is an entirely separate substance from tetanospasmin. It may be removed from toxic broth by admixture of red blood cells, is more thermolabile than the tetanospasmin, and gives rise to an antihemolysin when injected into animals.

Pathogenicity.-The comparative infrequency of tetanus infection is in marked contrast to the wide distribution of the bacilli in nature. Introduced into the animal body as spores, and free. from toxin, they may often fail to incite disease, easily falling prey to phagocytosis and other protective agencies before the vegetative forms develop and toxin is formed. The protective importance of phagocytosis was demonstrated by Vaillard and Rouget, ${ }^{31}$ who introduced tetanus spores inclosed in paper saes into the animal body. By the paper capsules the spores were protected from the leucocytes, not from the body fluids. Nevertheless, tetanus developed in the animals. The nature of the wound and the simultaneous presence of other microorganisms seem to be important factors in determining whether or not the tetanus bacilli shall be enabled to proliferate. . Deep, lacerated wounds, in which there has been considerable tissue destruction, and in which ehips of glass, wood splinters, or grains of dirt have become embedded, are particularly favorable for the development of these germs. The injuries of compound fractures and of gunshot wounds are especially liable to supply these conditions, and the presence in such wounds of the common pus eocei, or of other more harmless parasites, may aid materially in furnishing an environment suitable for the growth of the tetanus bacilli. Apart from its occurrence following trauma, tetanus has been not infrequently observed after childbirth, ${ }^{32}$ and isolated cases have been reported in which it has followed diphtheria and ulcerative lesions of the throat. ${ }^{33}$

${ }^{30}$ Ehrlich, Berlin. kl. Woch., 1898.

${ }^{31}$ Vaillard et Rouget, Ann. de l'inst. Pasteur, 1892.

${ }^{32}$ Baginsky, Deut. med. Woch., 1893.

${ }^{33}$ Foges, Wien. med. Woch., 1895. 
A definite period of incubation elapses between the time of infection with tetanus bacilli and the development of the first symptoms. In man this may last from five to seven days in acute eases, to from four to five weeks in the more chronic ones. Experimental inoculation of guinea-pigs is followed usually in from one to three days by rigidity of the muscles nearest the point of infection. This spastic condition rapidly extends to other parts and finally leads to death, which occurs within four or five days after infection.

Autopsies upon human beings or animals dead of tetanus reveal few and insignificant lesions. The initial point of infection, if at all evident, is apt to be small and innocent in appearance. Further than a general and moderate congestion, the organs show no pathological ehanges. Bacilli are found sparsely even at the point of infection, and have been but rarely demonstrated in the blood or viscera. Nicolaier succeeded in producing tetanus with the organs of infected animals in but eleven out of fifty-two eases. More recently, Tizzoni ${ }^{34}$ and Creite $^{35}$ have suceeded in cultivating tetanus bacilli out of the spleen and heart's blood of infected human beings.

The researches of Tarozzi ${ }^{36}$ and of Canfora ${ }^{37}$ have shown also that spores may be transported from the site of inoculation to the liver, spleen, and other organs, and there lie dormant for as long as fifty-one days. If injury of the organ is experimentally practiced and dead tissue or blood elot produced, the spores may develop and tetanus ensue. These experiments may explain cases of so-called cryptogenic tetanus.

In man tetanus may take either an acute or chronic form, the word "chronic" here meaning simply that the onset is less abrupt, the ineubation time longer, the symptoms slower in development and the prognosis more favorable. In the acute form, the incubation time ranges from three or four days to ten or fourteen days, the common very rapid cases taking about seven. In the so-called chronic form the incubation time may occasionally exceed a month. The first symptoms usually consist in headache and general depression; followed rather rapidly by difficulties in opening the mouth, due to spasms or trismus of the masseters. There is slight stiffness of the neck which makes it difficult for the patient to bring the

\footnotetext{
${ }^{34}$ Tizzoni, Ziegler's Beit., vii.

${ }^{35}$ Creite, Cent. f. Bakt., xxxvii.

${ }^{36}$ Tarozzi, Cent. f. Bakt. Orig. xxxviii.

${ }^{37}$ Canfora, Cent. f. Bakt. Orig. xlv.
} 
chin forward on the chest. Gradually there develops a spasm of the muscles of the cheeks which results in a drawing up of the tissues about the mouth, giving a curious and characteristic expression. Gradually the spasms extend to the trunk and back, with the development of opisthotonos after several days. Difficulty in swallowing may ensue, and there may be involuntary movements of urine and feces. The localization of the symptoms to some extent follows the location of the injury. Tetanus may occur in the new born, occasionally, developing soon after birth. For differential diagnosis, it is best to refer to books on general medicine and surgery.

Many types of atypical tetanus in untreated and in prophylactically treated cases have been reported, a description of which can be found in extenso in the volume on "The Abnormal Forms of Tetanus" by Courtois-Suffit and Giroux in the British Medical War Manuals, published in 1918. They speak of splanchnic tetanus characterized especially by the involvement of the muscles of deglutition and respiration, with great dysphagia. Simple cephalic tetanus in which the infection may be confined to the head, is a type in which dysphagic and paralytic symptoms are never present, and which result most frequently from wounds of the head. It may be characterized only by unilateral and bilateral trismus, or by contraction of muscles of the face. There is, however, a dysphagic form of this in which pharyngeal spasms precede trismus. Rarely they have noticed a so-called hydrophobic form in which convulsions accompany the spasms.

\section{Prophylactic Use of Tetanus Antitoxin}

The most important use for tetanus antitoxin which has been found hitherto, is its prophylactic administration. The methods of applying this have varied in different parts of the world and in different armies. That it is of great value was demonstrated by the almost immediate reduction of tetanus in wounded soldiers after the universal introduction of prophylactic tetanus antitoxin in all the armies in the field. The wounds which are particularly dangerous as far as tetanus is concerned are those in which there is considerable laceration, especially injury to bone, and in which dirt, and especially manured soil or soil from cultivated fields, and feces, are likely to be present. The growth of tetanus bacilli is favored by the presence of dead tissue and other infected organisms. Studies 
by members of the United States Public Health Serviee have shown that tetanus can be produced with regularity if staphylococcus infection is added to the infection with tetanus spores, and injury of tissue by the injection of small quantities of such substances as quinine, may start the growth of latent tetanus spores with subsequent development of the disease. Tetanus spores pass through the intestinal canals of animals and man without injury, and are distributed in the soil where they can live for almost unlimited periods. Wounds inflicted upon men in the field, especially by the blunt and ragged projectiles of high explosives, and by any injury passing through soil and filth covered clothing, through unwashed skin, furnish an ideal nidus for infection. In consequence, in practically all the allied armies every wounded man was given an injection of about 1,000 to 1,500 units of tetanus antitoxin as soon after the injury as he came under medical observation. In civilian life, the wounds that require similar prophylactic treatment are those inflected with much traumatism and under dirty conditions, especially those in which compound fractures are involved.

We refrain from giving any set rules for prophylactic treatment. The principles involved are that the injection of from 500 to 1,500 or even up to 5,000 units should be made subcutaneously as soon as possible after the injury. It should be remembered that the first injection may not be sufficient. The antitoxin gradually disappears in the course of about twelve days, and wounds that are slow in cleaning up or cases in which secondary interference, such as removal of sequestrum, resetting of bones, etc. becomes necessary, may call for a second injection after six to eight days, with due precautions to prevent anaphylaxis. In such cases, according to the judgment of the surgeon, second injections should become almost the rule since experience in the war has shown that after two injections tetanus is very rare in appearance.

Recently, very important advances in our knowledge of tetanus have been made by W. J. Tulloch ${ }^{38}$ who in 1917 showed that tetanus bacilli could be classified into at least three and perhaps four types by agglutination with anti-bacterial serum prepared by injection of the bodies of tetanus bacilli. The important point which arises in this connection is, of course, whether the toxins produced by these various agglutinative types differ either qualitatively or quantita-

${ }^{38}$ Tulloch, Journal of the R. A. M. C., Dec. 1917. 
tively. Subsequent investigations by Tulloch ${ }^{39}$ showed that of a large number of isolations from infections on the Western Front, his type I organisms were the most frequent, type II and type III next, and type IV the least common. It seemed, in these investigations, that the agglutinin titer of an antitoxic serum is no index to its antitoxic value; the agglutinating sera, even when of very high titer, does not bring out group reactions, but maintains a sharp separation of the types and that the types remain true even after prolonged cultivation. The types eould be demonstrated by opsonic as well as by agglutination experiments. Most important is the observation that the spasm-producing toxin is not specific to the types, although there may be quantitative differences in toxin production. Tulloch's investigations suggest that anti-bacterial properties in tetanus sera, if polyvalent, would aid considerably in the phagocytosis of the organisms, and, therefore, have a prophylactic and therapeutic value. His work, also, seems to indicate that small amounts of the tetanospasmin do not locally aid the growth of the tetanus bacillus to any considerable extent, an observation in distinct contrast with the earlier work on this subject. However, B. Welchii toxin and, to a smaller extent, that of Vibrion Septique, considerably aid the growth of tetanus, by, as he expresses it, "devitalizing" the tissues. This leads Tulloch to favor a procedure, advised by other observers on purely bacteriological grounds, namely the combining of antitoxins against the poisons of $\mathrm{B}$. Welchii and of Vibrion Septique with Tetanus antitoxin, in the prophylactic treatment of war wounds.

Even when this is done, however, he cautions against any feeling of false security which might lead to the neglect of surgical prophylaxis.

He emphasizes the great importance of early excision of the wound area. No particular dressings seem to make a great deal of difference, but thorough excision seems to have considerable influence on the development of tetanus and on the mortality.

The Treatment of Developed Tetanus.-To speak of the specific treatment of tetanus without saying a few words about the surgical treatment would be taking the risk of conveying a false impression. Therefore, though our business here is eoncerned largely with specific treatment, we wish to emphasize that surgical treatment must al-

39 Tulloch, Journal of Hygiene, vol. 18, 1919, p. 103. 
ways be carried out whatever method of serum therapy be employed. This must consist in thoroughly cleansing the wound, removal of foreign bodies, fragments of projectiles, clothing, gross dirt, etc., and, as the late war has shown, it is perhaps best whenever possible to carry out débridement or excision of the wound. From Tulloch's ${ }^{40}$ studies it would appear that no dressing is particularly superior to any other, and we doubt very much whether oxidizing agents, like the insufflation of oxygen, peroxides, etc., are of much use, because of the redueing powers of tissues.

As to specific serum treatment, it must be admitted that earlier results were very disappointing, and the mere subcutaneous injection even of large doses of tetanus antitoxin has usually been disappointing in the acute forms of the disease. This has perhaps been largely due to the fact that the injected antitoxin could have no possible influence on the toxin which had already become united with the substances of the nerve tissues. A great many modifications in the method of injection have been employed, such as injection directly into the central nervous system and into the nerve trunks, themselves. It may be stated that the relative acuteness of the tetanus infection very definitely influences the results of serum therapy. The following table taken from Etienne and copied from Courtois-Suffit and Giroux in the series of British War Manuals mentioned above, gives a general idea of the usefulness of serum therapy in this disease:

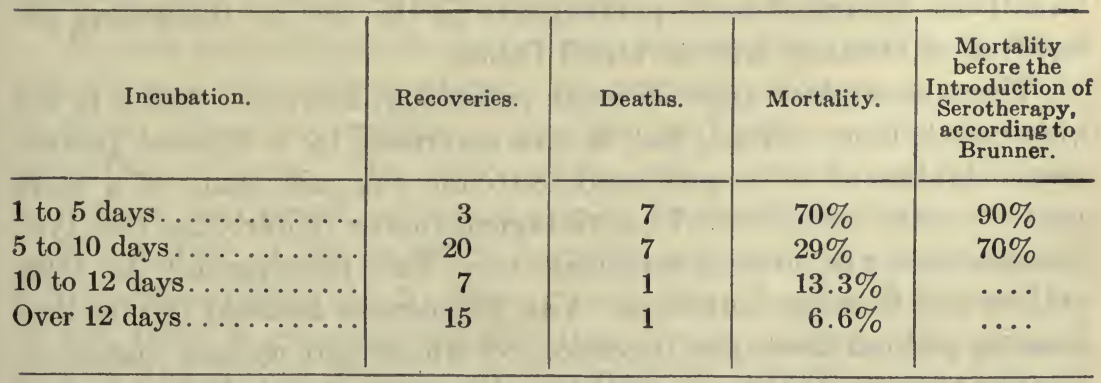

This table cited from Courtois-Suffit and Giroux, Military Medical Manuals, Univ. of London Press, London, 1918, page 193.

It would be useless to go into the various methods of administering tetanus antitoxin that have been tried, and we will confine

${ }^{40}$ T'ulloch, Jour. R. A. M. C., December, 1917, Jour. Hygiene, 18, 1919, 103. 
ourselves to the intraspinal method developed in this country by Park and Nicoll,41 and in France by-Doyen, and gradually coming into general use. As advised by Park and Nicoll, a spinal puncture is made and a moderate amount of spinal fluid taken out. Then, slowly by gravity from 3,000 to 5,000 units of tetanus antitoxin are injected until a total amount of 3 c.e. has been reached, the amount injected approximately replacing the amount of fluid withdrawn. At the same time, 10,000 units are given intravenously or intramuscularly. This procedure must be repeated according to indications.

For other forms of treatment such as carbolic acid injections, magnesium sulphate, etc., we must refer the reader to books on surgical therapy.

\section{BACILLUS BOTULINUS}

Meat poisoning was formerly regarded as entirely dependent upon putrefactive changes in infected meat, resulting in the production of ptomains or other harmful products of bacterial putrefaction. It was not until 1888 that certain of these cases were definitely recognized as true bacterial infections, in which the preformed poison probably aided only in establishing the infection. Gärtner, in that year, discovered the Bacillus enteritidis, a microorganism belonging to the group of the paratyphoid bacilli, and demonstrated its presence both in the infecting meat and in the intestinal tracts of patients. The characteristies of this type of meat poisoning have been discussed more particularly in the section describing the bacillus of Gärtner and its allied forms.

There is another type of meat poisoning, however, which is not only much more severe, but is characterized by a clinical picture more significant of a profound systemic toxemia than of a mere gastroenteric irritation. The etiological factor underlying this type of infection was first demonstrated by Van Ermengem, ${ }^{42}$ in 1896 , and named Bacillus botulinus. Van Ermengem isolated the bacillus from a pickled ham, the ingestion of which had caused disease in a large number of persons. Of the thirty-four individuals who had eaten of it, all were attacked, about ten of them very severely. Van Ermengem found the bacilli in large numbers lying between the muscle fibers in the ham, and was able to cultivate the same micro-

${ }^{41}$ Park and Nicoll, Jour. A. M. A., 63, 1914, 245.

${ }^{42}$ Van Ermengem, Cent. f. Bakt., xix, 1896; Zeit. f. Hyg., xxvi, 1897. 
organism from the stomach and spleen of one of those who died of the infection. The results of Van Ermengem have been confirmed by Römer, ${ }^{43}$ and others.

Morphology and Staining:-Bacillus botulinus is a large, straight rod with rounded ends, 4 to 6 micra in length by 0.9 to 1.2 micra in thickness. The bacilli are either single or grouped in very short chains. Involution forms are numerous on artificial media. The bacillus is slightly motile and possesses from four to eight undulated flagella, peripherally arranged. Spores are formed in suitable media, most regularly in glucose-gelatin of a distinctly alkaline titer. The spores are oval and usually situated near the end of the bacillus, rarely in its center. Spores are formed most abundantly when cultivation is carried out at $20^{\circ}$ to $25^{\circ} \mathrm{C}$, , and are usually absent when higher temperatures are employed.

The bacillus is easily stained by the usual aqueous anilin dyes, and retains the anilin-gentian-violet when stained by Gram. It is necessary, however, in carrying out Gram's stain to decolorize carefully with alcohol since overdecolorization is easily accomplished, leaving the result doubtful.

Cultivation.-The bacillus is a strict anaërobe. In anaërobic environment it is easily cultivated on the usual meat-infusion media. It grows most readily at temperatures about $25^{\circ} \mathrm{C}$., less luxuriantly at temperatures of $35^{\circ} \mathrm{C}$. and over.

The bacillus is delicately susceptible to the reaction of media, growing only in those which are neutral or moderately alkaline.

In deep stab cultures in one per cent glucose agar, growth is at first noticed as a thin, white column, not reaching to the surface of the medium. Soon the medium is cracked and split by the abundant formation of gas. On agar plates, the colonies are yellowish, opalescent, and round, and show a finely fringed periphery.

On gelatin, at $20^{\circ}$ to $25^{\circ} \mathrm{C}$., growth is rapid and abundant, and differs little from that on agar, except that, besides the formation of gas, there is energetic fluidification of the medium. On glucosegelatin plates, Van Ermengem describes the colonies as round, yellowish, transparent, and composed of coarse granules which, along the periphery in the zone of fluidification, show constant motion. The appearance of the surface colonies on glucose-gelatin plates is regarded by the discoverer as diagnostically characteristic.

${ }^{43}$ Römer, Cent. f. Bakt. xxvii, 1900. 
In glucose broth there is general clouding and large quantities of gas are formed. At $35^{\circ} \mathrm{C}$. and over, the gas formation ceases after four or five days, the broth becoming clear with a yellowish-white flocculent sediment. At lower temperatures this does not occur.

Milk is not coagulated and disaccharids and polysaccharids are not fermented.

The gas formed in cultures consists chiefly of hydrogen and methane. All cultures have a sour odor, like butyric acid, but this is not so offensive as that of some of the other anaërobic organisms.

The bacillus lives longest in gelatin eultures, but even upon these, transplantations should be done every four to six weeks, since the spores of this bacillus show less viability and resistance than do those of most spore-formers.

Isolation.-The isolation of $\mathrm{B}$. botulinus from infected material is often quite easy, since as Burke states, few other organisms may be present in the canned or pickled food products. Often anaërobic shake cultures in agar made directly, serve to isolate the organisms. In most contaminated material, however, she recommends inoculation from the original material into Von Ermengem's broth, inoculating quite richly, duplicate cultures being made and heated at $60^{\circ}$ for one hour, to destroy non-spore bearers. These cultures are then incubated at $28^{\circ}$ after which parts of them are filtered, and the filtrate in quantities of 1 c.c. injected into 250-gram guinea-pigs. If the guinea-pigs die within four days, the pigs are again tested with specific botulismus antitoxin. The presence of the organisms having been thus proven in the broth, isolation is now carried out by eareful anaërobic plating or shake cultures. We do not know whether the platinized asbestos method has been used in botulisinus work, but would recommend its use in eases, such as those mentioned.

Botulismus Toxin.-Botulismus toxin is produced under conditions of strict anaërobiosis on any media on which the organism will grow readily. According to Dickson ${ }^{44}$ the toxin is much more potent if the organisms are grown on an alkalin medium and in the dark. Von Ermengem ${ }^{45}$ obtained his best toxin by growing the organisms on a beef infusion broth to which he added 1 per cent

${ }^{44}$ Dickson, Monograph of the Rock. Inst., No. 8, July 31, 1918, (1a). Jour. of the A. M. A., 65, 1915, 492.

${ }^{45}$ Von Ermengem, quoted from Kolle and Wassermann, Second Edition. 
sodium ehloride, 1 per cent pepton and 2 per cent glucose. Leuchs ${ }^{46}$ used a pork infusion with 0.5 per cent sodium ehlorid, 1 per cent glucose and 1 per cent pepton. Landmann ${ }^{47}$ claimed that animal protein was necessary for good toxin production. According to Dickson this is not essential. He has produced toxin in media made from string beans and from peas, and found that, although an alkalin reaction is favorable, an acid reaction does not prevent toxin formation. According to Burke ${ }^{48}$ toxin is produced as readily at $37.5^{\circ}$ as it is at $28^{\circ} \mathrm{C}$. The toxin is destroyed at temperatures of about $80^{\circ}$. Thom, Edmonson and Giltner ${ }^{49}$ claim that their toxin was destroyed by ten minutes' heating at $75^{\circ} \mathrm{C}$. Von Ermengem's original report was that heating at $56^{\circ}$ for three hours killed it, as does heating at $80^{\circ}$ for one-half hour: According to Dickson, it is rapidly destroyed by exposure to sunlight and to air, but will maintain its virulence for six months if kept in the dark as it would be in preserved foods. It is not affected by drying and is insoluble in alcohol, ether and chloroform. Normal soda, 20 per cent by volume, is stated by Dickson to destroy it, though similar amounts of acid do not reduce its virulence in twenty-four hours.

Its potency is considerable. Dickson produced his strongest toxin in pork and beef infusions, but also obtained potent preparations in media of string beans, peas, green eorn, and less virulent toxin in media prepared from asparagus, artichokes, peaches, and apricots. Brieger and Kempner ${ }^{50}$ obtained a toxin of which $0.000,001$ of a c.c. would kill a 250-gram guinea-pig in four days, and (we quote from Diekson), Von Ermengem found in one of his outbreaks that 200 grams of the poisonous ham caused the death of one patient. He quotes another case in which a piece of preserved duck the size of a walnut was sufficient to cause a disease lasting six weeks, and in his own series, a patient died after tasting a small spoonful of spoiled corn, another died after "nibbling a portion of a pod of the spoiled string beans." A third was quite ill after tasting, but not swallowing a pod of beans. An important point is the claim that has been made by Von Ermengem and others that the organism will not produce toxin in the tissues. Injection of the bacilli alone

${ }^{46}$ Leuchs, Zeit. f. Hyg., 1910, 65, 55.

${ }^{47}$ Landmann, eited from Dickson, loc. cit.

${ }^{48}$ Burke, Jour. Bacter., 4, 1919, 555.

${ }^{49}$ Thom, Edmonson and Giltner, Jour. A. M. A.. Vol. 73, 1919, page 901.

${ }^{50}$ Brieger and Kempner, Deut. med. Woch., 23, 1897, 521. 
into suitable aninıals produces no botulismus, and intravenous injection or feeding of the bacilli alone, may not produce symptoms. The conclusion drawn by Von Ermengem was that the toxin could not be produced in the bodies of mammals.

The toxin is potent for monkeys, rabbits, guinea-pigs, cats, and various birds. Dickson found chickens highly susceptible, and also found that dogs were not as resistant as formerly thought to be. The most susceptible animals seem to be mice, guinea-pigs and monkcys; rabbits, eats, dogs and rats are relatively resistant.

In studying various isolations of the Bacillus; Dickson found that the strains isolated by him were not entirely homologous, and that the toxins produced by some of them were not neutralized by the antitoxin produced with others. Burke also found two types that produced heterologous toxin and claims that the strains could be easily identified by a toxin-antitoxin test.

Pathology.-In animals, according to Von Ermengem there is a general hyperemia of the organs and especially of the nervous system. Dickson has made a thorough review of the pathological work done by Von Ermengem, Vander Stricht,* Marinesco ${ }^{51}$ and others, ${ }^{52}$ summarizing the pathology as follows: In the central nervous system the meninges at the base of the brain, especially around the pons and the medulla, are usually more markedly congested than at the cortex, and there may be hemorrhage in the upper part of the cord and at the base of the brain. The lungs may be hyperemic, heart muscles flabby, but nothing characteristic. An important and regular lesion found by Ophuls ${ }^{53}$ was multiple thrombosis in both the arteries and veins of the central nervous system. Ophuls believes this is due to a certain vasodilation with slowing of the blood stream due to a powerful paralyzing effect of the poison on the unstriped muscles. The thromboses are particularly common at the base of the brain. Ophuls, too, differs from others in believing that the specific action of the toxin on the nerve cells themselves has been very much exaggerated in that his histological examinations of the brains of fatal cases did not bear out this earlier opinion.

Transmission and Occurrence of Botulismus.-Kempner and Pol-

* Stricht, Quoted from Van Ermengem, in Kolle and Wassermann.

51 Marinesco, Compt. rend. de l'acad. des sci., Nov., 1896.

${ }^{52}$ Kempner und Pollack, Deut. med. Woch., xxxii, 1897.

${ }_{53}$ Ophuls, with Wilbur, Arch. Int. Med., 14, 1914, 589. 
lack $^{54}$ in 1897 isolated B. botulismus from the intestines of a normal hog, but Dickson was unable to find the organism in the intestinal eanals of 250 grain-fed hogs in the slaughter houses of San Francisco. He also collected soil from gardens, but in a considerable series of specimens did not find organisms. Burke ${ }^{55}$ examined materials from many different localities in California where a number of epidemics had occurred, cultivating from large varieties of substances, such as water, hay, vegetables, fruits, manure from horses, hogs, chickens, etc. She obtained seven cultures of the B. botulinus from the following sources: Moldy cherries, leaves touched with droppings of insects, sprouts from bush-bean plants, manured bush beans, manure from a hog, and moldy hay. These, and other investigations seem to indicate that the $B$. of botulismus is quite common in nature, and may be present in the intestines of domestic animals, such as hogs. It may possibly be disseminated by insects, and may often be present on vegetables and fruits at the time that they are picked for canning.

The outbreaks first reported were largely due to meat and it is interesting to note that Van Ermengem's first isolations were from pickled meat, thus showing that ordinary salting or brine preservation does not kill the botulinus spores. The epidemies that have occurred are summarized in a monograph of Mayer, ${ }^{56}$ and more recently in an admirable study of the disease by Dickson, published in a Monograph of the Rockefeller Institute, ${ }^{57}$ and carried out at the Stanford University Pathological Laboratory. At the time of Mayer's publication (in 1913), 800 European cases had been observed since 1882, 200 of which had been fatal. Meat in general, or animal food had been the source of the disease; and the generally prevalent idea at that time was that meat was the chief source of danger. During recent years, however, the studies of Dickson and others have shown that vegetable foods, especially canned vegetables were equally as dangerous. Of sixty-four cases collected by Dickson for the United States during the past twenty-five years, fifty-four occurred in California. Wilbur and Ophuls ${ }^{58}$ reported an outbreak in 1914 due to the eating of canned beans. Since that time, a

${ }^{54}$ Kempner and Pollack, Deut. med. Woch., 23, 1897, 505.

${ }_{55}$ Burke, Jour. Bacter., 4, 1919, 541.

${ }^{56}$ Mayer, Deut. Vrtjschr. off. Gsndhtspflg., 35, 1913, 8.

${ }^{57}$ Dickson, Monograph of the Rock. Inst., No. 8, July 31, 1918.

${ }^{58}$ Wilhur and Ophuls, Arch. Int. Med., 14, 1914, 589. 
number of other cases have occurred in California and the rest of the United States, many of which have been studied by Dickson and his associates, most of them originating from canned com and string beans. Thom, Edmonson and Giltner, reported an outbreak occurred in 1919, traceable to canned asparagus.

Armstrong $^{59}$ carefully studied an outbreak in 1919 which was traced to ripe olives. It is impossible in this space to do justice to the large and valuable botulismus literature which has developed during the last few years, since the studies of Dickson and others have renewed the interest of laboratory workers in the disease. The mortality of the disease has been high, and for the United States generally, as stated by Diekson, it has been over 64 per cent.

Attention has also been recently called to the relationship of botulismus in man to the disease called limber neck in chickens. In Hillsboro, Oregon, Dickson states ${ }^{60}$ that fifty ehickens eame down after eating home-canned corn which had caused the death of a woman who tasted it. In Hornbrook, California, between fifty and one hundred chickens became paralyzed and died at the same time as the woman who cared for them died of "bulbar paralysis." In the San Jose district, eight chickens died after eating home-canned string beans which had caused the death of a woman; and seven chickens died in Fallbrook, California, after eating home-canned apricots which had killed five people. Dickson obtained the carcasses of some of the chickens of the San Jose and Hillsboro outbreaks, and from the gizzard of one of those which had eaten the canned corn and from the crop of three which had eaten canned beans, he obtained B. Botulinus. He also succeeded in producing symptoms by feeding chickens with the infected material obtained in this way. He described the symptoms as follows: The chickens refuse to eat, remaining quiet, and gradually develop weakness of the neck, wings and legs, finally drooping completely. In the experimental cases death occurred within twenty-four hours after feeding. This, apart from its diagnostic value and experimental importanee, may have considerable bearing on epidemiological studies; and limber neck in chickens should always be inquired into when human cases are observed or suspected.

Clinical Manifestations of Botulismus.-Botulismus is characteristic in its clinical manifestations and should not present great

59 Armstrong, Pub. Health Reports, 1919, 54, December.

${ }^{60}$ Dickson, Jour. Amer. Vet. Assoc., January, 1917. 
diagnostic difficulties, once the disease is suspected. Since the toxin is preformed before ingestion, the symptoms are not long in following the eating of infected food, coming on usually within twenty-four hours or less. Delays of two or three days, however, may occur, and should not throw out possible positive diagnosis. The earliest symptoms usually consist in a general weakness and lassitude, with fatigue and some headache. Characteristic is the frequent lack of any symptoms pointing to the gastrointestinal canal. Constipation is the rule. Very early in the disease, disturbances of vision may occur which are due to impairment of the muscles of the eye-ball. There is, particularly, involvement of the third cranial nerve, with blepharoptosis, mydriasis, impaired light reflex and diplopia. There may be photophobia. For a detailed discussion of the symptomatology, the reader is referred to the Monograph of Dickson. ${ }^{61} \mathrm{Im}$ pairment of the pharyngeal muscles may produce difficulties in swallowing with inability to chew, and sluggishness of the tongue with thickness of speech. Absence of temperature is an important feature, and in the early stages there is usually no fever and no change in the heart rate. Fatal cases usually end in death within three to seven days, due either to cardiac failure or terminal asphyxia. In discussing the differential diagnosis, Dickson mentions particularly, poliomyelitis, cerebrospinal syphilis, early stages of bulbar paralysis, belladonna poisoning and methyl alcohol poisoning. The last must be particularly thought of during the present period of drought.

Specific Therapy.-Potent antitoxins may be produced by the treatment of susceptible animals with toxin. Kempner ${ }^{62}$ in 1897 was the first to experiment on this extensively, using the Von Ermengem strain, and producing antitoxin in goats. The immunization of small laboratory animals is comparatively difficult, unless minute doses and attenuated toxin is used. The chief studies on these phases of the problem have been made by Forssman and Lunstrom $^{63}$ and by Leuchs. ${ }^{64}$ More recently, Dickson and Howitt ${ }^{65}$ have produced potent antitoxins in goats, though their products, they state, were not as powerful as those reported by Kempner and

${ }^{61}$ Dickson, Monograph of the Rock. Inst., No. 8, July 31, 1918.

${ }^{62}$ Kempner, Zeit. f. Hyg., 26, 1897, 481.

${ }^{63}$ Forssman and Lunstrom, Ann. de l'Inst. Past., 16, 1902, 294.

${ }^{64}$ Leuchs, Kolle and Wassermann Handb., Second Edition, 4, 939.

${ }^{65}$ Dickson and Howitt, Jour. A. M. A., 74, 1919, 718. 
some other observers. It is very important to note that in these experiments antitoxin produced against one series of strains had no appreciable effects upon the toxins of three other strains. This brings out the great importance of producing eurative sera by the use of toxins from a number of different strains. According to Dickson, there are at least two types which must be used. As to the therapeutic value, reports have been confusing. Dickson advises intravenous injection and states that his procedure would be as follows: The usually precautions for the administration of horse serum should be observed, and the patient tested for skin sensitiveness. If no such sensitiveness is found, the serum should be injected immediately into a vein at the rate of not more than 1 c.c. a minute. Comparatively large doses should be given, since the amount of toxin ingested may be quite large.

Prevention of Botulismus.-Deducing preventive measures from the facts cited in the above paragraphs, it would seem that, in the first place, all people in the habit of preparing eanned food should be thoroughly alive to the possibilities of contamination and know that $B$. botulinus spores may be present on fruit, vegetables, ete., before they are preserved. It should be well. understood that food may be contaminated with botulinus, without being changed in any way in its gross appearance, and that not even the slightest rancid odor which sometimes indicates its presence, need be apparent. The sterilization of canned food, sausages, preserved meat, etc., should be thoroughly attended to and no home-canned preparations be eaten under any circumstances unless cooked before eating. 


\section{CHAPTER XXXVII}

THE ANAEROBIC BACILLI (Continued)

\section{THE ANAËROBIC ORGANISMS ASSOCIATED WITH TRAU. MATIC INJURIES, WITH A CONSIDERATION OF THEIR IMPORTANCE IN WAR SURGERY, ${ }^{1}$ ALSO BACILLUS OF SYMPTOMATIC ANTHRAX}

THE anaërobie bacilli which infect wounds have been studied extensively during the last war, and the literature which has appeared on this subject since 1914, is voluminous: Unfortunately much that has been written is inaccurate, due to the fact that in many instances the work was carried on in poorly equipped laboratories and under difficulties. The two most important sources of confusion in this field are: the nomenclature and the impurity of cultures. During the war many previously described bacilli were rediscovered and given new names, and the literature is full of papers claiming or disclaiming that certain organisms isolated from wounds are identical with organisms described by earlier workers. Even such a well-known species as B. Welehii appears in the literature under four names.

It is now generally considered that in all probability most of the early descriptions of anaërobic bacilli cannot be relied upon, because of the extreme difficulty of isolating the anaërobes in pure culture. Many investigators even during the first year of the war were describing a mixture of two or more anaërobes when they thought they were dealing with a pure culture. It was not until the development of the newer anaërobic methods ${ }^{2}$ which made surfaee growths feasible that the purity of anaërobie cultures could be relied upon. It is only by repeated plating of these anaërobie spore-bearing bacilli, as emphasized repeatedly by English workers, that pure cultures can be obtained. The French workers have adhered for the most part to the older method of Veillon which consists of making varying dilutions of the material to be examined

${ }^{1}$ For the thorough revision of this group of organisms we are indebted to Miss Ann Kuttner, Instructor in this Laboratory, Zinsser.

${ }^{2}$ MacIntosh and Fildes, Lancet, 1, 1916, 768, April 8th. 
in deep agar shake tubes until isolated eolonies are obtained. The tube is then filed through at the level of the colony and the isolated colony can be fished. This is a laborious method and is umreliable. Barber $^{3}$ has developed a technique whereby he can isolate a single bacillus and has applied this method to the problem of the purifieation of the anaërobic bacilli.

The souree of anaërobic bacilli in war wounds can always be traced to the contamination of the wound either directly or indirectly with fecally contaminated soil. Most of the anaërobie bacilli have been shown to be normal inhabitants of the intestinal tract of man or animals, and are present in great numbers in cultivated ground. During the war the incidence of anaërobic bacilli in wounds and their relation to the development of gas gangrene was studied in detail.

In civilian practice gas gangrene is generally attributed to the presence of B. Welchii. In war wounds, however, it was found that although B. Welchii was isolated from the majority of the eases of gas gangrene, it practically never occurred in pure culture, and was usually associated, besides aërobes, with other anaërobic bacilli which in many instances proved to be more pathogenic than B. Welchii. The separation and identification of these anaërobie bacilli, and the development of methods for the eontrol of such infections were among the most important problems of bacteriologists during the war.

In this book only the most important and frequently oceurring anaërobic bacilli will be discussed. The reader is referred for more detailed description to the book of Weinberg and Seguin "La Gangrene Gazeuse," and to the Report of the British Medieal Research Committee. The anaërobie bacilli found in war wounds can be divided into two general groups, as first suggested by Von Hibler ${ }^{4}$ in his book on Anaërobes, published in 1908: the saechrolytic and the proteolytic.

The saccharolytic group ineludes as its most important members:

B. Welchii

Vibrion Septique

B. Oedematiens

B. Fallax

${ }^{3}$ Barber, Jour. Exper. Med., 32, 1920, 295.

"Von Hibler, "Untersuchungen über die pathog. anærob.," Jena, 1908. 
The proteolytic group includes:
B. sporogenes
B. histolyticus
B. putrificus

This classification, like most others, is not a rigid one. It merely means that members of the saccharolytic group have a much greater avidity for carbohydrates than have members of the proteolytic group.

It may be possible to demonstrate proteolytic activity of the organisms included in the saccharolytic group on special media from which all carbohydrate has been removed, but on the ordinary culture media the difference between the two groups is striking.

Members of the proteolytic group can be distinguished from the saccharolytic group by the fact that they liquefy coagulated horse serum. Organisms of the saccharolytic group fail to liquefy this medium even after prolonged incubation. In milk the saccharolytic bacilli produce acid and varying amounts of gas. The proteolytic bacilli digest milk with the production of alkali.

The organisms of the proteolytic group are not in themselves pathogenic, but complicate wounds by their intense proteolytic action. They are saprophytes, they have no power of invading the tissues and if present without members of the saccharolytic group usually do not interfere with the healing of the wound.

Whether the organisms of the saccharolytic group are saprophytes or not, is an open question. De Kruif ${ }^{5}$ concludes that B. Welchii cannot be classified as a pure saprophyte, because a twice washed bacillary emulsion in doses of 0.1 c.c. to 1 c.c. will kill a guinea pig. But in this case even the most careful intramuscular injection will kill some of the tissue at the point of inoculation, and with even a very small amount of dead tissue, B. Welchii can produce a toxin which has tremendous aggressive action and has the property of killing the tissue with which it comes in contact. This makes possible the further invasion of $\mathrm{B}$. Welchii.

B. Welchii.-B. Welchii is the organism most frequently found in gas gangrene. It was present in about 72 to 80 per cent of the cases of gas gangrene studied during the war, and has been consistently associated with civilian eases of gas gangrene. It is generally considered the most important etiological factor in this disease.

${ }^{5}$ De Kruif, Jour, Infec. Dis., 21, 1917, No. 6. 
On the other hand, it must also be stated that B. Welchii was frequently present in wounds which never developed gangrene. Taylor reports that $\mathrm{B}$. Welchii was found in 80 per cent of all wounds examined and that only 10 per cent of these developed gas gangrene. The development of gas gangrene depends on the virulence of the strain of B. Welchii, the amount of dead tissue present, and the anaërobic conditions in the wound. In war wounds B. Welchii was practically never present in pure culture. It was usually associated with aërobes and with other anaërobes of the saccharolytic and the proteolytic type.

B. Welchii was discovered independently in three countries. It was first discovered by Welch and Nuttall ${ }^{6}$ in 1892 , and called by them Bacillus aërogenes capsulatus, a name still used by the majority of English writers. In this country this organism is usually called B. Welchii. Welch isolated it from the blood and organs of a cadaver dead eight hours. In 1893 Fraenkel $^{7}$ isolated a similar organism in Germany from several cases of gaseous phlegmons, calling it B. Phlegmonis enephysematosae, but soon recognized that he was working with the same bacillus previously described by Welch. However, this organism is still referred to as the Fraenkel bacillus in German literature. In 1897, without having heard either of Welch's or Fracnkel's work, this organism was again described by Veilon and Zuber ${ }^{8}$ in France and called by them $B$. perfringens. B. Welchii, Bacillus aërogenes capsulatus, Fraenkel bacillus, B. perfringens are all names for the same organism.

B. Welchii is a short, square Gram-positive bacillus, occurring singly or in pairs. Chains are not formed as a rule. It is non-motile and has a capsule. It grows best under strictly anaërobic conditions, but its requirements for anaërobiosis are less rigid than those of tetanus. It grows well in media containing tissue such as cooked meat medium after simple boiling. With milk boiling is not always sufficient to obtain good growth, and it is best to put milk tubes in anaërobic jars. The majority of strains do not form spores readily. Alkaline sugar-free media rich in protein, such as alkaline egg, are necessary to demonstrate spore formation with the majority of strains. The spore of B. Welchii is large, oval, and central or

${ }^{6}$ Welch and Nuttall, Bull. Johns Hopkins IIosp., 3, 1892, 81.

${ }^{7}$ Fraenkel, Cent. f. Bakt., Bd. 13, 1893, 13.

${ }^{8}$ Veillon and Zuber, Arch. de Med. Exper., 10, 1898, 517. 
subterminal. B. Welchii is the most active fermenter in the saccharolytic group. It ferments all the common sugars with the production of large amounts of gas. Lactic and butyric are the two acids most frequently formed, the latter often giving eultures of B. Welchii a characteristic odor. Glucose agar is sometimes fragmented to such an extent that the plug is blown off the tube. Simonds ${ }^{9}$ has been able to divide the B. Welchii group into subdivisions depending on the ability to ferment either glycerine or inulin, or both, or neither, and this classification has been confirmed by Henry. ${ }^{10}$ In wounds, B. Welchii ferments the musele sugar producing gas in the tissues and for this reason is commonly called the "gas" bacillus. The crepitation thus produced is characteristic of gas gangrene and indicates the extent of the infection. The rapid fermentation of the lactose in milk gives a characteristic reaction in this medium which is diagnostic for B. Welehii. The acid clot torn by gas bubbles and the separation of the milk into coagulum and whey is easily recognized and is not given by other anaërobes. The inoculation of a mixed culture from a wound into milk makes possible the diagnosis of $\mathrm{B}$. Welchii within twelve to eighteen hours.

Opinions as to the ability of $\mathrm{B}$. Welchii to liquefy gelatin vary greatly. B. Welehii does not grow well on sugar-free gelatin and it is, therefore, difficult to draw conclusions as to its action on gelatin. B. Welchii as pointed out by Rettger in $1906,{ }^{11}$ never attacks proteins if earbohydrates are present and even in the absence of carbohydrate shows only a very slight proteolytic activity. No indol is formed from broth, and coagulated serum is not liquefied or blackened.

The hemolytic power and pathogenicity of different strains of B. Welchii vary greatly. B. Welehii is particularly pathogenic for guinea-pigs and pigeons, the latter being used in the standardization of B. Welchii toxin. B. Welchii in fatal eases usually invades the blood stream shortly before death, and ean usually be isolated from the blood after death. Spores are never formed in the animal body. Rabbits and mice are much less suseeptible. Agglutinin production in response to injections of $\mathrm{B}$. Welchii in rabbits and horses is extremely poor. Simonds obtained a serum in rabbits which agglutinated the homologous strain in a dilution of 1-80. Ten strains

${ }^{9}$ Simonds, Mon. Rock. Inst., No. 5, 1915.

1n Henry, Jour. Pathol. and Bacter., 21, 1917, 344.

${ }^{11}$ Rettger, Jour. Biol. Chem., 11, 1906, 71. 
of B. Welchii failed to agglutinate with this serum, and ten others agglutinated only in a dilution of 1-20. The agglutination reaction for the identification of anaërobes has so far proved unsatisfactory.

Toxin Production.-Klose in $1916^{12}$ reported the isolation of a toxin from B. Welchii prepared by growing B. Welchii for fourteen days in a 5 per cent glucose broth. The antitoxin produced by injections of this toxin only protected guinea-pigs against three lethal doses of $\mathrm{B}$. Welchii cultures. The antigenic properties of this toxin were too feeble to consider it a true toxin. The most important contribution to the bacteriology of B. Welchii was made in 1917 by Bull and Pritchett ${ }^{13}$ who were able to prepare a soluble toxin which, when injected into a suitable animal, produced a potent antitoxin possessing protective and curative properties against $B$. Welchii infections, in animals. One e.c. of antitoxin per 100 grams body weight injected subcutaneously protected guinea-pigs against 300 lethal doses of culture. ${ }^{14}$ The production of a powerful toxin ( 0.3 c.c. to 3 c.c. being the M. L. D. for a pigeon of $300 \mathrm{gr}$. injected intramuscularly) depended on the virulence of strain, a short incubation period, twenty-one to twenty-four hours, and the presence of fresh muscle and glucose in the broth. Bull and Pritchett found no variations in the ability of different strains of B. Welchii, irrespective of the source of the culture, to produce toxin. The toxin production of less active strains could be increased by raising the virulence of the culture by animal passage. Caulfield in a recent paper $^{15}$ finds that he does not get good toxin production unless the virulence of his strain is such that 0.02 c.c. of supernatant fluid of a young broth culture will kill a $300 \mathrm{gr}$. pigeon. Caulfield also emphasizes the importance of fresh muscle in the culture medium, although. DeKruif and the Hygienic Laboratory in Washington obtained good results by substituting chopped veal which can be autoclaved, for the fresh musele tissue. The most potent toxins, however, seem to be obtained by inoculating the infected muscle of a pigeon dying of a $\mathrm{B}$. Welchii infection directly into the medium to be used for toxin production, or, at most, allowing one short culture generation (ten hours) to intervene between the last animal passage and the inoculation of the broth for toxin. By preparing

12 Klose, Munch. med. Woch., Bd. 63, 1916, 723.

${ }^{13}$ Bull and Pritchett, Jour. Exper. Med., 26, 1917, 867.

${ }^{14}$ Bull and Pritchett, Jour. Exper. Med., 26, 1917, 867.

${ }^{15}$ Caulfield, Jour. Infec. Dis., 27, 1920, No. 2. 
a toxin in this way, Bengston of Hygienic Laboratory has been able to prepare a $\mathrm{B}$. Welchii antitoxin in which 1 e.c. of serum contains one unit, one unit neutralizing 1000 M. L. D. of B. Welchii toxin. ${ }^{16}$

In laboratory animals infected with pure cultures of 13. Welchii, the antitoxin gives complete protection. Protection in laboratory animals is also afforded by injections of antimicrobial sera prepared by injections of whole broth culture of B. Welchii by Weinberg and Seguin, but the antitoxin content of these sera has not been determined.

Bull and Pritchett in their original paper, state that the toxin produced by $\mathrm{B}$. Welchii is comparable to the toxins produced by Tetanus and Diphtheria, and judged by its antigenic properties it must certainly be classified as a true exotoxin. It differs from the classical toxins in that toxin production varies directly with the virulence of the strain and that it has no definite incubation period. B. Welchii antitoxin cannot protect against mixed infections where B. Welchii is associated with either Vibrion Septique or B. Oedematiens. In this case the animal always dies of the Vibrion Septique or B. oedematiens infection. However, since both these organisms occur in a smaller percentage of cases and have rarely been isolated from civilian cases of gas gangrene, B. Welchii antitoxin will probably prove of great value.

Isolation.-B. Welchii is a normal inhabitant of the intestinal tract of adults and may be found in the stools of infants. Simonds found B. Welchii present in eight out of nineteen stools of babies under one year of age. It can be easily isolated from stools by the following procedure: 5 e.c. of a fecal suspension in saline are inoculated into a tube of milk which has been freshly boiled and cooled. The tube is heated at $80^{\circ}$ for one hour to kill off the vegetative forms of the fecal flora, and is then incubated. The development of the "stormy fermentation" described above indicates the presence of $B$. Welchii. Purification is best completed by plating anaërobically from the milk culture.

Animal inoculation is also useful in the isolation of B. Welchii. The material suspected of containing $\mathrm{B}$. Welchii is injected intravenously into a rabbit. After five minutes the rabbit is killed and placed in the incubator for five to eight hours. At the end of this time, the animal is usually distended with gas. At autopsy gas

${ }^{16}$ Bengston, Hygienic Laboratory Bulletin, No. 122, 1920. 
bubbles will be found distributed throughout the organs, especially in the liver. B. Welchii if present, .can usually be isolated from the liver and the heart's blood. Cultures can also be identified by injecting them intramuseularly into two guinea-pigs, one normal, the other protected by a dose of $\mathbf{B}$. Welehii antitoxin. If both pigs die, and an anaërobic organism is isolated from the heart's blood, it indicates the presence of some other pathogenic anaërobe, not B. Welchii. If the normal pig dies with an anaërobic, capsulated, non-motile Gram-positive bacillus in the heart's blood, and the antitoxin pig survives, it is a fairly sure indication that the organism in question is $\mathrm{B}$. Welchii.

Vibrion Septique (Synonyms, Bacillus of Ghon and Sachs, and Bacillus III of Von Hibler).-Vibrion septique according to Weinberg and Seguin, occurred in 12 per cent of the wounds examined by them. Henry isolated it in 16 per cent of his cases. Before the war, cases of human gas gangrene due to vibrion septique alone were very few in number. Such eases were described by Ghon and Sachs, ${ }^{17}$ by Von Hibler, Gould, ${ }^{18}$ and by Muir and Ritehie. ${ }^{19}$ During the war, vibrion septique was usually associated with other anaërobes, notably B. Welchii. Weinberg and Seguin eite only one case of gas gangrene in which vibrion septique was the only anaërobe present.

Vibrion septique was first described by Pasteur $^{20}$ in 1877 who isolated it from the blood of a cow dead three days, and from the blood of a horse dead one day, both animals having supposedly died of anthrax. Pasteur called this organism a vibrion, although it is in reality a bacillus, because it is extremely motile in animal exudates and may look slightly curved when in motion. In 1881, Koch ${ }^{21}$ in studying the etiology of anthrax, isolated an organism which he called the baeillus of malignant edema. Koch considered his organism identical with Pasteur's vibrion septique, although the bacillus of malignant edema had marked proteolytic properties, which Pasteur did not mention in the description of vibrion septique. A great amount of confusion has arisen out of this controversy and the literature is full of papers diseussing whether or not Pasteur

${ }_{17}$ Ghon and Sachs, Cent. f. Bakt., 1 Abt. Orig., 48, 1909, 396.

${ }_{18}$ Gould, Annals of Surgery, 38, 1903, 481.

${ }^{19}$ Muir and Ritchie, Manual of Bacteriology, 2d Edit., Edinburgh, 1899.

${ }^{20}$ Pasteur et Jourhet, Bull. de l'Acad. Med., Scien., Vol. 6, 793.

${ }^{21}$ Koch, Mitt. a. d. k. Gesundheitsamt., 1, 1881, $53^{\circ}$. 
and Koch were working with the same organisms and with attempts to identify organisms isolated from wounds with one or the other of these bacilli. The majority of workers now consider that Pasteur was working with a strictly saccharolytic organism which is identical with what we call vibrion septique at the present time. The bacillus of malignant edema of Koch is thought by most investigators to belong to the proteolytic group and is fairly definitely identified with B. sporogenes.

Vibrion septique is a motile, slender Gram-positive bacillus with slightly rounded ends. It is a strict anaërobe. It forms spores readily in most media. The spore is oval, occurring either centrally or subterminally, and appears at the end of twenty-four to fortyeight hours. It has no capsule. It ferments the common sugars with the exception of saccharose. It produces a loose clot in milk in one to four days. Gelatin is liquefied, but coagulated serum is not attacked. Vibrion septique is hemolytic. It is always pathogenic for laboratory animals and guinea-pigs, mice, pigeons and rabbits are all susceptible. It invades the blood stream producing a septicemia. The occurrence of long filamentous forms in the livers of guinea-pigs dying of a vibrion septique infection is characteristic and is used in the identification of vibrion septique.

Robertson ${ }^{22}$ has recently divided the vibrion septique group into four serologieal types, based upon the agglutination reaction. Miss Robertson again stresses the necessity of minute care in purifying cultures and points out that impure eultures fail to agglutinate. It is difficult, however, to attach much importance to variations in agglutinin production of different strains, since there is no difference in toxin production, and since the antitoxin produced by the injection of the toxin of strains belonging to one serological type neutralizes the toxins produced by members of the other types. The agglutination reaction in the case of vibrion septique subdivides strains that agree in every other respect, and may in this instance be regarded as ultraspecific, as Miss Robertson suggests. She was able to obtain agglutinating sera with a titer of $1-25,000$.

Toxin Production.-A powerful soluble toxin is produced by all strains of vibrion septique and does not depend on the virulence of the culture. Aecording to Robertson as potent toxins are produced by old laboratory strains of vibrion septique as by recently

${ }^{22}$ Robertson, Jour. Bacter. and Pathol., 1920, 
isolated cultures. The toxin, like that of B. Welchii, has no incubation period. The toxin of vibrion septique often fails to produce death in guinea pigs when injected subcutaneously or intramuscularly, merely producing local necrosis. Toxin production is tested both in rabbits and guinea pigs by intravenous injection. 0.5 e.c. of toxin injected intravenously kills a guinea pig in five minutes. 0.1 to 1 c.c. injected into rabbits intravenously kills them without a latent period, with symptoms of respiratory disturbance, paralysis and convulsions. It is difficult to establish an M. L. D. for rabbits of the same weight owing to individual variation. In some instances, death is produced immediately in one rabbit, whereas another rabbit of the same weight will show severe symptoms followed by recovery.

Method of Producing Toxin.-The Hygienic Laboratory obtains a powerful toxin using a 0.2 per cent glucose broth containing 10 . per cent horse serum. Robertson recommends using liver of a pig dying of a vibrion septique infection with which to inoculate the broth, but the difficulties of obtaining a liver without gross contaminations are such that the former method is preferable. The broth is incubated twenty-four to forty-eight hours. Care should be taken in the selection of the filter since certain filters seem to hold back a large percentage of the toxin. This point is emphasized both by Weinberg and Seguin and by Robertson. Antitoxins are prepared by injecting the toxin into horses or sheep. The French standard requires that $1 / 1000$ c.c. of the antitoxin should neutralize two fatal doses of the toxin after thirty minutes incubation of the mixture at room temperature. The vibrion septique antitoxin is specific; it does not protect against B. oedematiens.

OCCURRENCE.-Vibrion septique has been isolated from milk. Heller $^{23}$ in an excellent summary of anaërobic infections in animals has shown that spontaneous infections by vibrion septique occur in sheep, horses, and hogs. Meyer ${ }^{24}$ in 1915 reported the isolation of typical vibrion septique from two cases of symptomatic anthrax in hogs. Cattle, according to Heller, are less susceptible to vibrion septique infections than the other animals mentioned. Herbivorous animals are subject to infections with vibrion septique, both followlowing and not following demonstrable wounds, whereas infections in man seem to occur only as the result of wounds.

${ }^{23}$ Heller, Jour. Infec. Dis., 27, 1920, 385.

${ }^{24}$ Meyer, Jour. Infec. Dis., 12, 1915, 458. 
Differentiation of Vibrion Septique and B. Chauvaei (Bacillus of symptomatic anthrax, Blackleg).-B. chauvaei was in no instance isolated from wound cultures, and has never been known to cause an infection in man. Vibrion septique, on the other hand, frequently infects animals and a bacteriological differentiation between vibrion septique and B. chauvaci must be made. These two organisms are closely related and very similar, and a reliable differentiation is difficult even for a bacteriologist familiar with anaërobic bacilli. Robertson distinguishes between $B$. chauvaei and vibrion septique by the fact that the former ferment saccharose and not salicin, whereas vibrion septique ferments salicin and not saccharose. Long snake-like filaments are demonstrable in smears from the liver of guinea-pigs dead of vibrion septique infection; these are entirely lacking in B. chauvaei infections. Vibrion septique is more pathogenic for laboratory animals and produces more gas in the tissues than B. chauvaei. B. chauvaei grows more slowly than vibrion septique. Vibrion septique is Gram-positive, whereas most investigators consider B. chauvaei Gram-negative. Protection tests with a known vibrion septique antitoxin ought to prove the most reliable way of identifying vibrion septique.

B. oedematiens.-Weinberg and Seguin claim to have isolated this organism in 34 per cent of the wounds examined by them. This is a higher proportion than that obtained by other workers. Henry found B. oedematiens in five out of fifty cases examined. B. oedematiens was isolated in 1915 by Weinberg and Seguin. In 1916 a similar organism was isolated by Sacquepée Sts $^{25}$ under the name "bacille de l'oedeme gazeuse malin." Later Sacquepée called this organism B. Bellonensis. B. Bellonensis and B. oedematiens are now considered to be the same organism by the majority of workers. According to Heller, B. oedematiens is closely related to but not identical with a bacillus discovered in 1894 by Novy and called by him B. oedematiens maligin II. ${ }^{26}$ B. oedematiens is a strict anaërobe. It is a large Gram-positive bacillus, resembling anthrax in appearance. It is practically non-motile. It forms chains in culture and often shows curved forms after two or three days. Filaments are not formed in the animal body. It forms oval subterminal spores readily in all media. It ferments most of the

${ }^{25}$ Sacquepée, Ann. de l'inst. Past., 30, 1916, 76.

${ }^{26}$ Novy, Zeit. f. Hyg., 17, 1894, 209. 
common sugars, ${ }^{27}$ and forms a loose elot in milk in three or four days. It liquefies gelatin, but does not attack coagulated serum.

Pathogenicity.-B. oedematiens is usually pathogenic, although Weinberg and Seguin report the isolation of two non-virulent strains, rabbits are less susceptible than guinea-pigs. Mice are also susceptible. It may or may not enter the blood stream. The lesion in the animal is characterized by a whitish gelatinous exudate and the absence of gas. The production of agglutinating sera with $B$. oedematiens has not been satisfactory, because B. oedematiens tends to agglutinate spontaneously. It is feebly hemolytic much less so than vibrion septique and $B$. perfringens.

Toxin.-B. oedematiens forms a soluble toxin. Different strains vary in their toxin production. With a potent strain $1 / 100$ c.e. of toxin injected intravenously kills a 300-400-gram guinea-pig in forty-eight hours. This toxin differs from those of vibrion septique and $\mathrm{B}$. Welchii in that it never kills acutely on intravenous injection. The toxin is prepared according to Weinberg and Seguin, by growing B. oedematiens in broth containing chopped veal for six to ten days.

Antitoxin.-Rabbits, sheep and horses have been used to produce antitoxic sera. Immunization is difficult and small doses must be used at first. Weinberg and Seguin prepared an antitoxin in a horse of such a titer that $1 / 10,000$ dilution neutralized two lethal doses (guinea-pig).

B. Fallax.-B. fallax was discovered during the war by Weinberg and Seguin. It is a much less important factor in gas gangrene than the members of the saceharolytic group already described. It is. usually associated with other pathogenic anaërobes. Weinberg and Seguin eite one ease in which it invaded the blood stream and caused death. It was isolated by Henry in three cases out of a series of fifty.

It is an anaërobic Gram-positive bacillus, resembling vibrion septique in appearance. It has a capsule and is slightly motile. It does not form spores readily in most culture media. Spores are formed on coagulated serum and are subterminal.

B. fallax coagulates milk very slowly. It does not liquefy gelatin or coagulated serum.

B. fallax is not hemolytic. It is only slightly pathogenic and soon becomes avirulent on artificial cultivation.

${ }^{2}$ Wolf, Jour. Pathol. and Bacter., 23, 1920, 254. 
Proteolytic Group.-The organisms of this group ean never produce gas gangrene without the presence of one or more bacilli of the saccharolytic group. The members of the proteolytic group digest milk without the formation of a clot and liquefy and often blacken coagulated serum. These two characteristics together with the fact that cultures of the proteolytic organisms usually have a very offensive odor, make it comparatively easy to distinguish them from the saccharolytic group. Sugars are fermented by the proteolytic type, but much less rapidly and with the production of less acid and gas than in the case of the saccharolytic group. The members of the proteolytic group produce spores readily in all media. None of these organisms are very pathogenic and produce no general picture of toxemia in spite of the tremendous liquefaction of tissue caused by them. The ferments ${ }^{23}$ of several of the proteolytic anaërobes have been isolated and have been found to split proteins to animo acids so rapidly that there is no time for the intermediate products to intoxicate the animals. The separation of the proteolytic anaërobes from the saccharolytic is extremely difficult. The members of the two groups are usually present together, and what will seem to be a pure culture of a saccharolytic organism if held for any length of time, will often show a contamination with a proteolytic organism. The best methods for separation of the two groups are: by rapid transplantation in sugar media, where the saccharolytic organisms outgrow the proteolytic, combined with frequent plating, or by animal inoculation. The latter is the most satisfactory. In the animal body after intramuscular injection, the more pathogenic organisms belonging to the saccharolytic group frequently invade the blood stream and may be isolated from the heart's blood.

B. sporogenes.-This organism was next to B. Welchii most frequently found in wound cultures. Weinberg and Seguin isolated it in 27 per cent of their cases. B. sporogenes was the anaërobe usually responsible for the foul odor of wounds. According to most author's, the pathogenicity of this organism is negligible. Weinberg and Seguin claim to have isolated a few toxic strains, but these may possibly have been mixed with members of the saccharolytic group. Heller has not found any proteolytic anaërobes that are pathogenic for animals.

${ }^{23}$ Blanc and Pozerski, Compt. Rend. Soc. Biol., 87, 1920, 29. 
B. sporogenes was definitely described by Metchnikoff' ${ }^{29}$ in 1908 . Whether B. sporogenes is identical or not with Koch's bacillus of malignant edema, will probably never be definitely settled. It is considered identical by many workers, although this is emphatically denied by others. B. sporogenes is a Gram-positive, anaërobic bacillus, actively motile, forming oval subterminal spores readily in all media and in the animal body. It is intensely proteolytic, liquefying gelatin and coagulated serum, and digesting and blackening meat. Most strains of B. sporogenes are not hemolytic. Occasionally a feebly hemolytic strain has been isolated. It does not produce a soluble toxin and is not pathogenie for laboratory animals unless injected in large quantities.

B. Histolyticus.-This organism was diseovered by Weinberg and Seguin and isolated by them from eight wound cultures. Like B. sporogenes, it is intensely proteolytic and is of interest chiefly because of the striking lesion it produces in the animal body. It is a Gram-positive anaërobic, motile bacillus with rounded ends. Sporulation takes place in all media, different strains varying in the time required for spore formation. The spores are large and oval, and occupy a terminal position. No gas is formed in cultures of B. histolyticus and no putrid odor develops. Gelatin and coagulated serum are liquefied. It does not produce a soluble toxin. It is not hemolytic. The injection of large doses, 2 to 3 e.c. intramuscularly into guinea pigs, of the whole culture digests the tissues so rapidly that at the end of twelve to twenty-four hours, the bone may be exposed. The picture is striking, one of the characteristics of $\mathrm{B}$. histolytic infection being that in spite of a tremendous local lesion, the animal appears well.

B. putrificus.-B. putrificus was occasionally found in putrid wounds. It was first discovered in 1884 by Bienstock and is characterized by a terminal oval spore. Bienstock isolated B. putrificus from the intestine of a cadaver. It is a Gram-positive anaërobe, motile, forming spores in all media. It is actively proteolytic, producing a foul odor. No pathogenie strains have been isolated. It has been studied by Tissier and Martally who found it in putrid meat. Klein worked with a similar organism which he called 13. sporogenes eadaveris. Hibler considers 3. putrificus and I3. sporogenes cadaveris the same.

${ }^{29}$ Metchnikoff, Ann. de l'Inst. Past., 22, 1908, 419. 
Identification of Anaërobes Present in Wound Cultures.-From the point of serum treatment of infected wounds, the prompt identification of the members of saccharolytic group, B. Welchii, B. oedematiens and Vibrion Septique, is most important. The process of purification and identification by cultural methods is at best slow, and Henry ${ }^{30}$ has, therefore, suggested the inoculation of the unknown material into immunized guinea-pigs as the quickest and most reliable method. The procedure he outlines is as follows: inoculate the unknown mixed culture into cooked meat medium, and incubate. The next day inoculate the supernatant fluid into milk, and inject intramuscularly into two immunized guinea-pigs, one pig having received a mixture of B. Welchii and Vibrion Septique antitoxin, the other a mixture of $\mathrm{B}$. Welchii and $\mathrm{B}$. oedematiens. The stormy fermentation of milk is diagnostic for $\mathrm{B}$. Welchii and this reaction takes place within twenty-four hours. If the pig that was protected against Vibrion Septique (the B. Welchii factor having been eliminated in both pigs) dies, it indicates the presence of some other pathogenic anaërobe, probably B. oedematiens. The diagnosis of B. oedematiens is further indicated if the guinea-pig that received the B. oedematiens combination of sera survives. If the animal inoculations come out in the opposite way, the presence of Vibrion Septique is indicated. The pathogenic organism can usually be isolated from the heart's blood of the animal that succumbs. By using a "filter" of protected guinea-pigs in this way, the pathogenic organisms can be separated out and the specific serum injected into the patient within forty-eight hours.

\section{THE COOPERATION OF SURGERY AND BACTERIOLOGY IN THE MANAGEMENT OF TRAUMATIC WOUNDS (WAR WOUNDS)}

The extensive experience gained by surgeons, during the war, in connection with infected wounds has developed a number of important bacteriological methods which are likely to remain as parts of the routine work of civil hospitals, especially those in which traumatic cases are handled.

It is not our intention to go into the various problems and controversies that have arisen among surgeons concerning the value of irrigation with Dakin's solution or with other antiseptics. This

${ }^{30}$ Henry and Lacy, Jour. Pathol. and Bacter., 32, 1920, No. 3. 
is not directly concerned with the question of bacteriological control, a matter which is desirable and seems eminently logical whatever method the surgeon chooses to use for the disinfection of the wound. It is, however, chiefly in connection with Dakin's solution irrigation that this method was developed by Carrel. The most complete treatise on the entire matter may be found in the book by Carrel and De Helly, "The Treatment of Infected Wounds," (Hoeber, New York, 1919).

The usual type of war wound or, for that matter, any kind of traumatic wound, presents conditions in regard to the possibilities of infection which are quite different from those ordinarily encountered in aseptic surgery. From the skin and clothing bacteria, both aërobic and anaërobic, are carricd by the projectiles or other foreign bodies into the tissues. Tissues are destroyed to a variable degree, and such devitalized tissues furnish an excellent medium for bacterial growth. There is always an interval or latent period between contamination of the wound and proliferation and penetration of the organisms. The duration of this latent period varies, but usually approximates six hours.

The immediate aim of treatment is the prevention or limitation of infection, and, for this reason, the rational method of determining whether this purpose is being accomplished and what the next procedure should be is bacteriological control.

The first step in limiting infection in such wounds is accomplished by débridement, that is, excision of the tract with removal of all the devitalized and contaminated tissues, together with foreign bodies, bits of projectile, clothing, etc. Bacteria are greatly diminished though not eradicated by this procedure.

Bacteriological control of the original infection of the wound and its progress under treatment is carried out by a method of systematic smear examination of the wound, supplemented by cultures, first practically developed by Carrel.

The smear method, introduced by Carrel and employed since that time by many surgeons on a large material, is simple, can be carried out by any well trained assistant or technician without the aid of a highly trained bacteriologist, and has apparently yielded results of value. Our description is taken almost entirely from Carrel's own writings. Wounds should be examined every two or three days, and when the time for secondary elosure appears, perhaps every day. The principle consists in the examination of the secretions of the wounds 
by means of smears in such a way that an approximate estimate of the number of bacteria contained in the wounds can be made. Although the method is very inaccurate, its value does not depend upon its revealing slight differences, the significant variations being so widely apart that the necessary error in the comparative enumerations does not render the method useless.

The examination need not begin earlier than twelve hours after the infliction of the wound, since up to that time few bacteria will be found. At the end of this time, when hemorrhage has completely stopped, smears are taken with a platinum loop from different parts of the wound. The points from which cultures are taken should always be those in which bacteria are most likely to be present in large numbers. Thus, Carrel chooses points in contact with foreign bodies, necrotic bits of bone, and from deep in the sinuses and erevices of the wound. Specimens should never be taken from bleeding points. Specimens should always be taken from a considerable number of different places in the same wound. Care should be exercised to avoid taking smears from the skin adjacent to the wound. With the end of a small platinum loop small amounts of secretion are picked up, and smeared upon slides in such a way that approximately the same area is covered by the different loopfuls of secretion. With loops of uniform size and a little practice, a surprising uniformity of technique can he developed.

These smears are allowed to dry, and may be stained in a variety of ways. Carbol thionin has been extensively used, but we believe that a Gram stain which is almost as simple, will give a little more useful information.

The stained slides can now be examined under the microscope and the number of bacteria per field, counted. If the number exceeds fifty or more to the field, more accurate counting will yield no valuable information because the wound still contains too many bacteria to warrant closure or relaxation of the local therapy that is being applied.

Gradually, as the wound improves, less and less bacteria will appear in the daily series of slides, and when it has dropped below fifty per field, careful counting may give an index of daily variations. Eventually, they will drop to only one microorganism per five, ten or twenty fields, in which ease the daily report, can be expressed in fractions, as $1 / 5,1 / 10$, or $1 / 20$, etc. The daily counts can, in this way, be numcrically charted, and constructed into a curve which 
will show the surgeon by a glance the numerical progress of the bacterial infection.

Carrel states that it is useless to take any smears as long as hemorrhage exists. If the wound is being irrigated with Dakin's solution or other antiseptic fluids, the treatment must be omitted for at least two hours before the smears are taken. Smears taken from the surface of smooth muscles are practically useless, since smooth muscle becomes sterile early in the healing process. Therefore, the choosing of the point of smear is of the utmost importance. The depth of the wound may begin to beeome sterile at times when individual little foci around necrotic bone, small pockets, etc., may still contain numerous bacteria. This must be borne in mind and an intelligent survey of the wound made by the bacteriologist who takes the smear. To overlook such dangerous points would seriously imperil the life of the patient, were the wound closed. When absolutely no bacteria are found in such smears, it does not mean that the wound is completely sterile. It is still possible that cultures might reveal organisms, and when the period of secondary closure approaches, especially when streptococei have been present at a previous time, we would regard it of the greatest importance to take a culture aimed particularly at the demonstration of hemolytic streptococei, before the actual suture is carried out.

Cultural examinations should be made at the beginning by taking specimens from parts of the wound selected as indicated above, and smearing them upon fresh blood-agar plates (without glucose). This is primarily aimed at determining whether coeci, and especially hemolytie streptococci or staphylococi, are present. If the smears show a great many bacilli resembling the ordinary anaërobes, it may be well, too, to make anaërobic cultures, but anaërobic analysis is not of great immediate value to the surgeon as far as further procedure is concerned because of the long time consumed by such examinations. Suture is not carried out if hemolytic coeci of any kind are present, and for this season, with a smear as a preliminary indication, frequent culture upon blood plates should be undertaken during the progress of the treatment.

In discussing the subject, it is not possible to give an intelligent survey of the hacteriological methods, without, to some extent, entering into the surgical considerations involved. For this reason we quote from Pool, ${ }^{31}$ whose experience with this type of wound has been extensive.

${ }^{31}$ Pool, E. H., Jour, A. M. A., 73, 1919. 
Débridement should be carried out as soon as possible after infliction of the wound. Primary suture may be employed only during quiet periods in case of war, and in hospitals where the patient may be retained for eareful observation. Otherwise suture of the wound may lead to enclosure, within an imperfectly débrided wound, of various microorganisms, including those which produce gas gangrene. In regard to delayed primary and secondary suture, the following observations of Dr. E. H. Pool are not without interest.

"The determination as to when a wound may be sutured depends on bacteriologic findings and clinical observation. It must be emphasized that the co-operation of a bacteriologist is indispensable in making a decision as to the indications for delayed primary and secondary sutures. The practical function and indisputable importance of the bacteriologist in war surgery lies in this. In the consideration as to whether a wound is suturable or not, reliance must be placed chiefly on cultures, the important feature being the determination of the presence or absence of hemolytic cocei. For this, a routine bloodagar examination is essential.

Bacterial counts are far from exact, yet they give an indication as to the degree of bacterial contamination of a wound, especially the progress from day to day, and are of value especially for one untrained in estimating clinically the indications and contraindications for suture.

From eighteen to forty-eight hours after the original operation of débridement or excision of tissues, the wound is dressed and a culture and a smear are made. A report is returned as soon as possible. This contains the approximate number of organisms per field and the varieties of organisms. If no organisms are found, suture is indicated. If hemolytic cocci are present, suture is not considered. In the absence of hemolytic cocci, if the wound is clinically suturable, the presence of a few anaërobes or other organisms (approximately one in two fields) does not contraindicate suture. A considerable number of organisms of any kind indicates delay of suture, until the bacterial growth declines. A culture and a smear should be repeated at the following dressing; the results of this examination will determine suturing or further delay. If the wound is left open for a considerable period, e.g., over a week, or is definitely infected, a smear is made every two days. It is also advisable to make a culture occasionally. Care must be taken not to touch the skin surface in making the smear, since skin contamination vitiates the value of the report. From the smear a bacterial curve is plotted according to Carrel's plan. When the organisms in two successive counts are few, that is, approximately one per two fields, and a culture shows an absence of hemolytic cocci, the wound is considered susceptible of secondary suture except when the wound has contained hemolytic cocei at any time. In that ease careful cultures are made from granulation tissue and from the discharge 
from all parts of the wound, and absence of hemolytic cocci should be established by two successive negative cultures before suture is made. It has been observed that streptococei are prone to lie dormant in small numbers, but to flare up and cause virulent infection after closure of the wound."

In compound fractures the same principles apply, except that, as stated by Pool, expedition, thoroughness and early closure is particularly important because it means the conversion of open into closed fracture. In such fractures of the long bones, delayed primary suture, that is, suture not later than six days after the infliction of the wound, should be aimed at. He states that it has been demonstrated repeatedly that severe fractures of long bones, except the femur, may be elosed in from three to six days after débridement. If this cannot be done, secondary suture may often be made successfully under proper bacteriological control.

In the case of joints, the principle of treatment consists in complete débridement of the tract of the wound into the soft parts and bone, with the removal of foreign bodies and irrigation of the joint, followed by absolute closure of the joint by suture, with or without closure of the superficial parts.

If a joint becomes distended after the operation and infection is suspected, the effusion should be aspirated and examined by smear and culture. If such examination indicates infection, the joint should be reopened and treatment for suppurative arthritis begun.

In civil surgery the principles worked out with war wounds can be applied with still greater hope of success, since here the nature of the trauma and infection is apt to be less extensive.

As to serological treatment in civilian surgery, this will be applicable chiefly in cases in which there has been a considerable delay in the proper surgical treatment of the wound after its infliction. It seems most probable at the present time that the most hopeful prospect for future therapy will lie in the combination of antitoxie sera against B. Welehii, B. oedematiens and Vibrion Septique, with Tetanus antitoxin, prophylactically injected in the same way in which Tetanus antitoxin alone has been used, hitherto. Whether it will be possible to produce this in polyvalent sera produced in the same horse or whether they will have to be separately injected, will depend upon future investigation in large scale serum production.

Bacillus of Symptomatic Anthrax (Bacillus anthracis symptomatici, Rauschbrand, Charbon symptomatique, Sarcophysematos 
bovis).-Symptomatic anthrax is an infectious disease occurring chiefly among sheep, cattle, and goats. It is spoken of as "quarter-evil" or "blackleg." The disease has never been observed in man. 'It was formerly confused with true anthrax, because of a superficial similarity between the elinical symptoms of the two maladies. Bacteriologically, the two microorganisms are in entirely different classes.

Symptomatic anthrax is of wide distribution and infection is usually through the agency of the soil in which the bacillus is present, in the form of spores which may retain viability for several years.

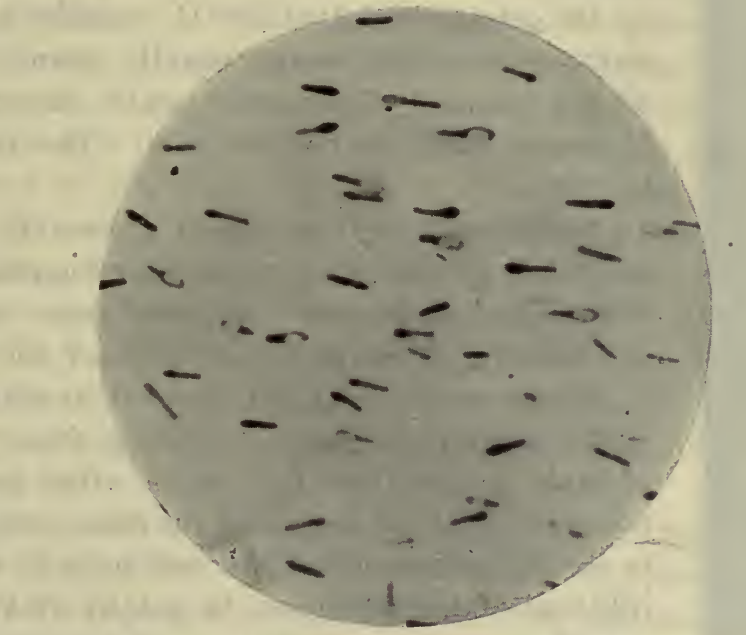

Fig. 76.-Bacillus of Symptomatic Anthrax. After Zettnow.

Morphology and Staining.- - The bacillus of symptomatic anthrax is a bacillus with rounded ends, being about four to six micra long, and five-tenths to six-tenths micra wide. It is usually seen singly and never forms long chains. The bacillus in its vegetative form is actively motile and possesses numerous flagella placed about its periphery. In artificial media it forms spores which are oval, broader than the rod itself, and placed near, though never actually at, the end of the bacillary body. This gives the bacillus a racketshaped appearance.

It is readily stained with the usual anilin dyes, but is easily decolorized by Gram's method of staining. However, von Hibler claims that when, very carefully stained the bacillus can be shown 
to be Gram-positive-at least when taken from the animal body. ${ }^{32}$

Cultivation.-The bacillus is a strict anaërobe. It was obtained in pure culture first by Kitasato. ${ }^{33}$ Under anaërobic conditions it is easily cultivated upon the usual laboratory media, all of which are more favorable after the addition of glucose, glycerin, or nutrose.

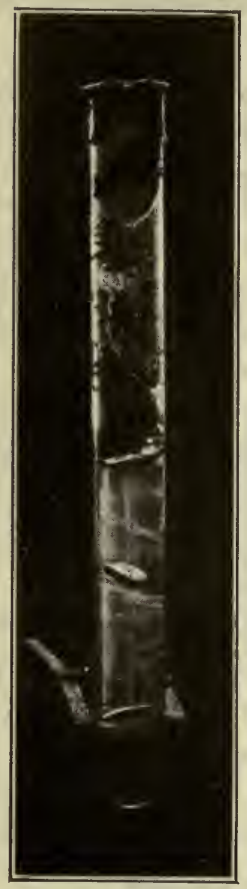

Fig. 77. - $\mathrm{BA}_{-}$ CILlUS OF SyMPtomatic Anthrax. Culture in glucose agar. In all media there is active gas formation, which, owing to an admixture of butyric acid, is of a foul, sour odor. The bacillus is not very delicate in its requirements of a special reaction of media, growing equally well on those slightly acid or slightly alkaline.

On gelatin plates, at $20^{\circ} \mathrm{C}$., colonies appear in about twenty-four hours, usually round or oval, with a compact center about which fine radiating filaments form an opaque halo. The gelatin is liquefied.

Surface colonies upon agar plates are circular and made up of a slightly granular compact center, from which a thinner peripheral zone emanates, containing microscopically a tangle of fine threads.

In agar stabs, at $37.5^{\circ} \mathrm{C}$., growth appears within eighteen hours, rapidly spreading from the line of stab as a diffuse, fine cloud. Gas formation, especially near the bottom of the tube, rapidly leads to the formation of bubbles and later to extensive splitting of the medium. In gelatin stab cultures growth is similar to that in agar stabs, though less rapid.

Pathogenicity.-Symptomatic anthrax bacilli are pathogenic for cattle, sheep, and goats. By far the largest number of cases, possibly the only spontaneous ones, appear among cattle. Guinea-pigs are very susceptible to experimental inoculation. Horses are very little susceptible. Dogs, cats, rabbits, and birds are immune. Man also appears to be absolutely immune. Spontaneous infection occurs by the entrance of infected soil into abrasions or wounds, usually of the lower extremities. Infection depends to some extent upon the relative degree of virulence of the bacillus - a variable factor in this

32 on Hihler, Kolle, Wassermann, etc., p. 792, vol. iv.

${ }^{33}$ Kitasato, Woch. f. Hyg. 1889. 
species. Twelve to twenty-four hours after inoculation there appears at the point of entrance a soft, puffy swelling, which on palpation is found to emit an emphysematous crackling. The emphysema spread rapidly, often reaching the abdomen and chest within a day. The course of the disease is extremely acute, the fever high, the general prostration extreme. Death may result within three or four days after inoculation.

At autopsy the swollen area is found to be infiltrated with a thick exudate, blood-tinged and foamy. Subcutaneous tissue and muscles are edematous and crackle with gas. The internal organs show parenchymatous degeneration and hemorrhagic areas. The bacilli, immediately after death, are found but sparsely distributed in the blood and internal organs, but are demonstrable in enormous numbers in the edema surrounding the central focus.

If carcasses are allowed to lie unburied for some time, the bacilli will attain a general distribution, and the entire body will be found bloated with gas, the organs filled with bubbles. Practically identical conditions are found after experimental inoculation.

Toxins.-According to the investigations of Leclainche and Vallée, ${ }^{34}$ the bacillus of symptomatic anthrax produces a soluble toxin. It is not formed to any extent in ordinary broth, but is formed in considerable quantities in broth containing blood or albuminous animal fluids.

The best medium for obtaining toxin, according to the same authors, is the bouillon of Martin, ${ }^{35}$ made up of equal parts of veal infusion and a pepton solution obtained from the macerated tissues of the stomachs of pigs. The toxin contained in filtrates of such cultures is quite resistant to heat, but rapidly deteriorates if free access of air is allowed.

Immunity.-Active immunization against the bacillus of symptomatic anthrax was first accomplished by Arloing ${ }^{36}$ and his collaborators by the subcutaneous inoculation of cattle with tissueextracts of infected animals. The work of these authors resulted in a practical method of immunization which is carried out as follows :

${ }^{34}$ Leclainche et Vallée, Ann. de l'inst. Pasteur, 1909.

'35 Martin, Ann. de l'inst. Pasteur, 1898.

${ }^{36}$ Arloing, Cornevin, et Thomas, "Le Charbon Sympt.," etc., Paris, 1887. Ref. from Grassberger und Schattenfroh, Kraus und Levaditi, "Handbuch," etc., vol, i, pt. 2 . 
Two vaccines are prepared. Vaccine I consists of the juice of infected meat, dried and heated to $100^{\circ} \mathrm{C}$. for six hours. Vaccine II is a similar meat-juice heated to $90^{\circ} \mathrm{C}$., for the same length of time. By the heating, the spores eontained in the vaceines are attenuated to relatively different degrees. Vaccine $I$ in quantities of 0.01 to 0.02 e.c. is emulsified in sterile salt solutions and injected near the end of the tail of the animal to be protected. A similar quantity of Vaccine II is injected in the same way fourteen days later.

This method has been retained in prineiple, but largely modified in detail by various workers. Kitt ${ }^{37}$ introduced the use of the dried and powdered whole meat instead of the meat juice, and made only one vaceine, heated to $94^{\circ} \mathrm{C}$., for six hours. This method has been largely used in this country. ${ }^{38}$ Passive immunization with the serum $^{39}$ of actively immunized sheep and goats has been used in combination with the methods of active immunization.

${ }^{37} \mathrm{Kitt}$, Ref. from Grassberger und Schattenfroh, loc. cit.

${ }^{38}$ Report of Bureau of Animal Ind., Wash., 1902.

${ }^{39}$ Arloing, Leclainche et Vallée, loc, cit. 


\section{CHAPTER XXXVIII}

\section{BACILLUS ANTHRACIS AND ANTHRAX}

\section{(Milzbrand, Charbon)}

Anthrax is primarily a disease of the herbivora, attacking especially cattle and sheep. Infection not infrequently oceurs in horses, hogs, and goats. In other domestic animals it is exceptional. Man is susceptible to the disease and contracts it either directly from the living animals or from the hides, wool, or other parts of the cadaver used in the industries.

The history of the disease dates back to the most ancient periods and anthrax has, at all times, been a severe seourge upon eattleand sheep-raising communities. Of all infections attacking the domestic animals no other has elaimed so many victims as anthrax. In Russia, where the disease is most 'common, 72,000 horses are said to have suceumbed in one year $(1864) .^{1}$

In Austria-Hungary, Germany, France, and the Eastern countries, each year thousands of animals and numerous human beings perish of anthrax. In England and America the disease is relatively infrequent. No quarter of the globe, however, is entirely free from it.

Especial historical interest attaches to the anthrax bacillus in that it was the first microorganism proved definitely to bear a specific etiological relationship to an infectious disease. The discovery of the anthrax bacillus, therefore, laid, as it were, the cornerstone of modern bacteriology. The bacillus was first observed in the blood of infected animals by Pollender in 1849, and, independently, by Brauell in 1857. Davaine, ${ }^{2}$ however, in 1863, was the first one to produce experimental infection in animals with blood containing the baeilli and to suggest a direet etiologieal relationship between the two. Final and absolute proof of the justice of Davaine's contentions, however, was not brought until the further development of bacteriological technique, by Koch, ${ }^{3}$ had made it possible for

${ }^{1}$ Quoted from Sobernheim, Kolle und Wassermann, vol. ii.

${ }^{2}$ Davaine, Comptes rend. de l'acad. des. sci., lvii, 1863.

${ }^{3}$ Koch, Cohn's “Beitr. z. Biol. d. Pflanzen," ii, 1876. 
this last observer to isolate the bacillus upon artificial media and to reproduce the disease experimentally by inoculation with pure oultures.

Morphology and Staining.-The anthrax bacillus is a straight rod, 5 to 10 micra in length, 1 to 3 miera in width. It is non-motile. In preparations made from the blood of an infected animal, the bacilli are usually single or in pairs. Grown on artificial media they form tangles of long threads. Their ends are cut off squarely, in sharp contrast to the rounded ends of many other bacilli. The

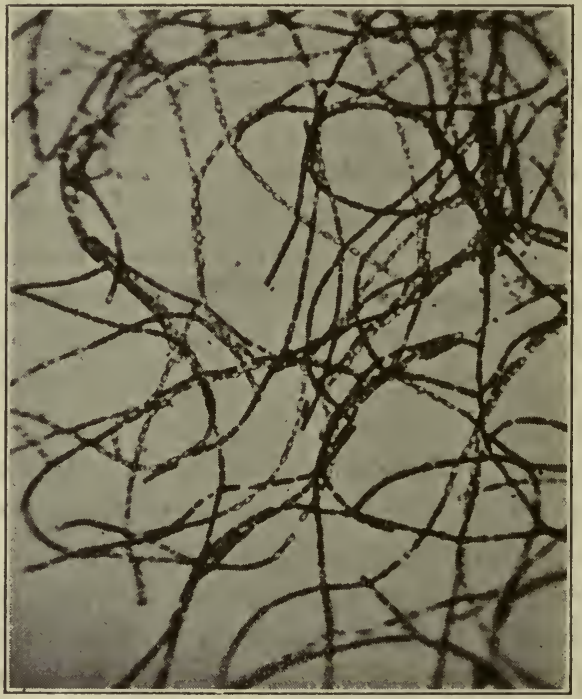

Fig. 78.-Bacillus Anthracis. From pure culture on agar.

corners are often sharp and the ends of bacilli in contact in a chain often touch each other only at these points, leaving in consequence an oval chink between the ends of the organisms. The appearance of a chain of anthrax bacilli therefore, has been not inaptly compared to a rod of bamboo. On artificial media the anthrax bacillus forms spores. Oxygen is necessary for the formation of these spores and they are consequently not found in the blood of infected subjects. The spores are located in the middle of the bacilli and are distinctly oval. They are difficult to stain, but may be demonstrated by any of the usual spore-staining procedures, such as Möller's or Novy's methods. The bacilli themselves are easily stained by the usual anilin dyes, and gentian-violet or fuchsin in aqueous solution 
may be conveniently employed. They are not decolorized by Gram's method.

In preparations from animal tissues or blood, stained by special procedures, the anthrax bacillus may occasionally be seen to possess a capsule. The capsule is never seen in preparations from the ordinary artificial media. Some observers have demonstrated them in cultures grown in fluid blood serum. In chains of anthrax bacilli, the capsule when present seems to envelop the entire chain and not the individual bacteria separately.

Isolation.-Isolation of the anthrax bacillus from infected material is comparatively simple, both because of the ease of its cultivation and because of the sharply characteristic features of its morphological and cultural appearance.

Cultivation.-The anthrax bacillus is an aërobic, facultatively anaërobic bacillus. While it may develop slowly and sparsely under anaërobic conditions, free oxygen is required to permit its luxuriant and characteristic growth.

The optimum temperature for its cultivation ranges about $37.5^{\circ}$ C. It is not, however, delicately susceptible to moderate variations of temperature and growth does not cease until temperatures as low as $12^{\circ} \mathrm{C}$. or as high as $45^{\circ} \mathrm{C}$. are reached. By continuous cultivation at some of the temperatures near either the higher or the lower of these limits, the bacillus may become well adapted to the new environment and attain luxuriant growth. ${ }^{4}$

The anthrax bacillus may be cultivated on all of the usual artificial media, growing upon the meat-extract as well as upon the meat-infusion media.

It may be cultivated also upon hay infusion, various other vegetable media, sugar solutions, and urine. While moderate acidity of the medium does not prevent the growth of this bacillus, the most favorable reaction for media is neutrality or slight alkalinity.

On gelatin plates, colonies develop within twenty-four to fortyeight hours as opaque, white disks, pin-head in size, irregularly round and rather flat. As the colonies increase in size their outlines become less regular and under the microscope they are seen to be made up of a hair-like tangle of threads spreading in thin wavy layers from a more compact central knot. The microscopic appearance of these colonies has been aptly described as resembling a 
Medusa head. Fragments of a colony examined on a slide with a higher power show the individual threads to be made up of parallel chains of bacilli.

After a day or two of further growth, the gelatin about the colonies becomes liquefied.

In gelatin stab cultures, growth appears at first as a thin white line along the course of the puncture. From this, growth proceeds in

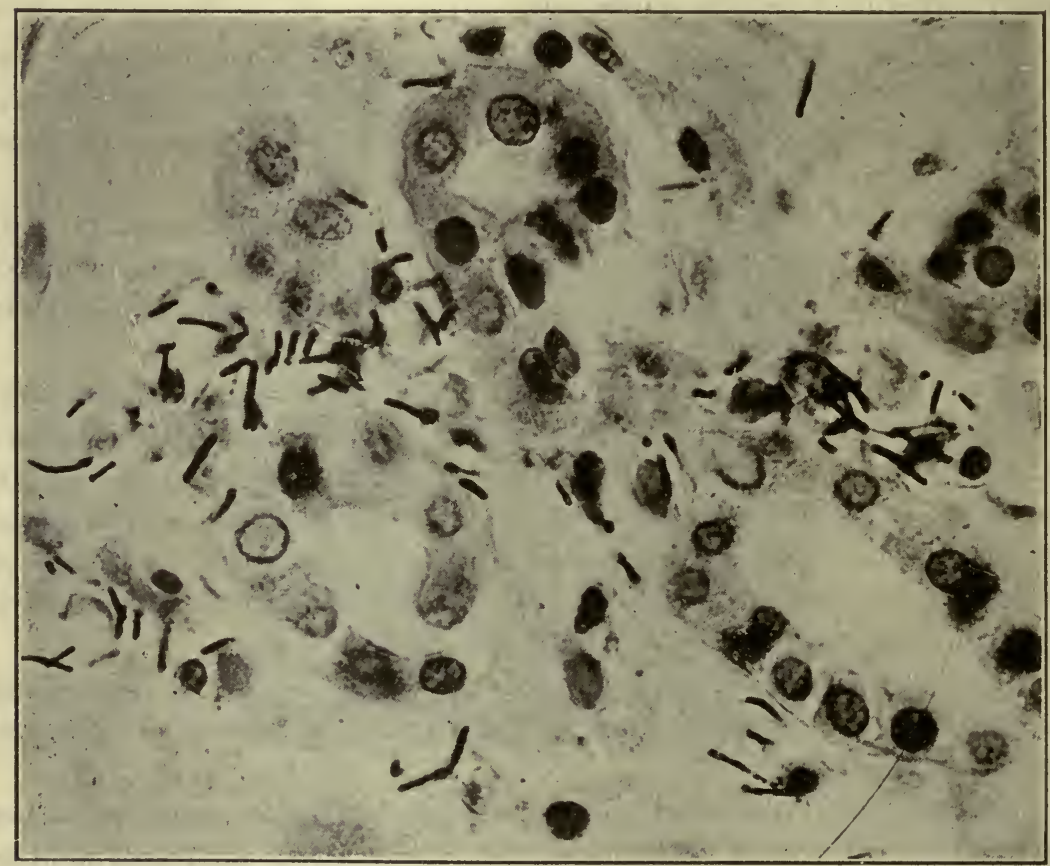

Fig. 79.-Bacillus anthracis. In section of kidney of animal dead of anthrax.

thin spicules or filaments diverging from the stab, more abundantly near the top than near the bottom of the stab, owing to more active growth in well oxygenated environment. The resulting picture is that of a small inverted "Christmas tree." Fluidification bégins at the top, at first a shallow depression filled with an opaque mixture of bacilli and fluid. Later the bacilli sink to the bottom of the flat depression, leaving a clear supernatant fluid of peptonized gelatin.

In broth, growth takes place rapidly, but does not lead to an even, general clouding. There is usually an initial pellicle formation at 
the top where the oxygen supply is greatest. Simultaneously with this a slimy mass appear's at the bottom of the tube, owing to the sinking of bacilli to the bottom. Apart from isolated flakes and threads the intervening broth is clear. Shaken up, the tube shows a tough, stringy mass, not unlike a small cotton fluff, and general clouding is produced only by vigorous mixing.

Upon agar plates, growth at $37.5^{\circ} \mathrm{C}$. is vigorous and colonies appear within twelve to twenty-four hours. They are irregular in

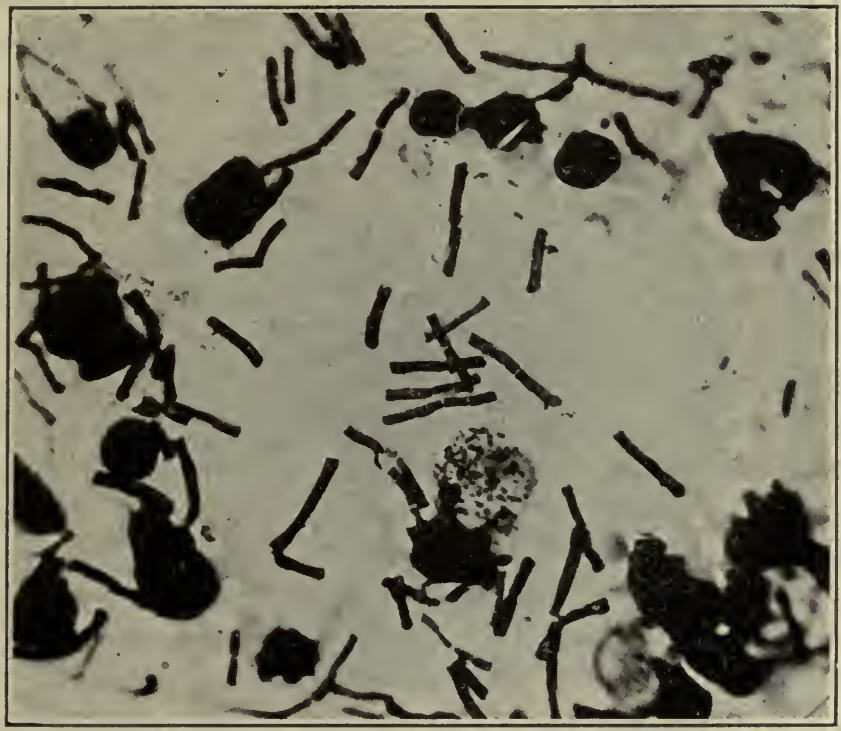

Fig. 80.-Bacillus anthracis. In smear of spleen of animal dead of anthrax.

outline, slightly wrinkled, and show under the microscope the characteristic tangled-thread appearance seen on gelatin, except that they are more compact than upon the former medium. The colonies are slightly glistening and tough in eonsistency.

On agar slants, the colonies usually become confluent, the entire surface soon being covered by a grayish, tough pellicle which, if fished, has a tendency to come away in thin strips or strands.

On potato, growth is rapid, white, and rather dry. Sporulation upon potato is rapid and marked, and the medium is favorable for the study of this phase of development.

Milk is slowly acidified and slowly coagulated. This action is ehiefly upon the easein; very few, if any, changes being produced 
either in the sugars or in the fats of the milk. The acids formed are, according to Iwanow, ${ }^{5}$ chiefly formic, acetic, and caproic acids.

Biological Considerations.-The anthrax bacillus is aërobic and facultatively anaërobic. It is non-motile and possesses no flagella. In the animal body it occasionally forms capsules. In artificial cultures in the presence of oxygen, it sooner or later invariably forms spores. The spores appear after the culture has reached its maximum of development. Sporulation never oceurs in the animal body, probably because of the absence of suffieient free oxygen.

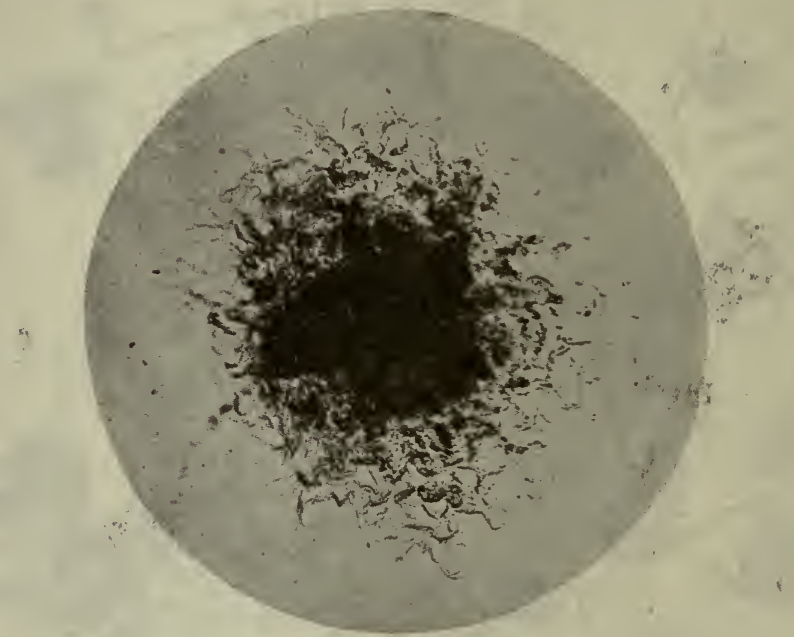

Fig. 81.-Anthrax Colony on Gelatin. (After Günther.)

Spores are formed most extensively ${ }^{6}$ at temperatures ranging from $20^{\circ}$ C. to $30^{\circ} \mathrm{C}$. Spore formation ceases below $18^{\circ} \mathrm{C}$. and above $42^{\circ} \mathrm{C}$. For different strains these figures may vary slightly, as has been shown from the results of various observers. Spores appear most rapidly and regularly upon agar and potato media.

The spore-one in each bacillus-appears as a small, highly refractile spot in the eenter of the individual bacterium. As this enlarges, the body of the bacillus around it gradually undergoes granular degeneration and loses its staining capacity. ${ }^{7}$

${ }^{5}$ Iwanow, Ann. de l'inst. Pasteur, 1892.

${ }^{6}$ Koch, loc. cit.

${ }^{7}$ Behring, Zeit. f. Hyg., vi and vii, 1889; Deut. med. Woch., 1889. 
If anthrax bacilli are cultivated for prolonged periods upon media containing hydrochloric or rosolic acid or weak solutions of carbolic acid, ${ }^{8}$ cultures may be obtained which do not sporulate and which seem permanently to have lost this power, without losing their virulence to the same degree. Similar results may be obtained by continuous cultivation at temperatures above $42^{\circ} \mathrm{C}$. By this procedure, however, virulence, too, is considerably diminished.

Resistance.-Because of its property of spore formation, the anthrax bacillus is extremely resistant toward chemical and physical environment. The vegetative forms themselves are not more resistant than most other non-sporulating bacteria, being destroyed by a temperature of $54^{\circ} \mathrm{C}$. in ten minutes. Anthrax spores may be kept in a dry state for many years without losing their viability. ${ }^{9}$ While different strains of anthrax spores show some variation in their powers of resistance, all races show an extremely high resistance to heat. Dry heat at $140^{\circ} \mathrm{C}$. kills them only after three hours. ${ }^{10}$ Live steam at $100^{\circ}$ kills them in five to ten minutes. Boiling in water destroys them in about ten minutes. Low temperatures have but little effect upon them. Ravenel ${ }^{11}$ found that, frozen by liquid air, they were still viable after three hours.

The variability shown by different strains of spores in their resistance to heat is even more marked in their behavior toward chemicals. ${ }^{12}$ Some strains will retain their viability after exposure to 5 per cent carbolic acid for forty days, ${ }^{13}$ while others are destroyed by the same solution in two days. Corrosive sublimate, $1: 2,000$, kills most strains of anthrax in forty minutes.

Direct sunlight destroys anthrax spores within six to twelve hours. ${ }^{14}$

Pathogenicity.-The anthrax bacillus is pathogenic for cattle, sheep, guinea-pigs, rabbits, rats, and mice. The degrees of susceptibility of these animals differ greatly, variations in this respect existing even among different members of the same species. Thus,

${ }^{8}$ Chamberland et Roux, Comptes rend. de l'acad. des sci., xevi, 1882.

${ }^{9}$ Surmont et Arnould, Ann, de l'inst. Pasteur, 1894.

${ }^{10}$ Koch und Wolffhïgel, Mitt. a. d. kais. Gesundheitsamt, 1881.

11 Ranenel, Medical News, vii, 1899.

12 Fränkel, Zeit. f. Hyg., vi, 1889.

${ }^{13}$ Koch, loc. cit.

14 Momont, Ann. de l'inst. Pasteur, 1892. 
the long-haired Algerian sheep show a high resistance, while the European variety are highly susceptible; and, similarly, the gray rat is much more resistant than the white rat. Dogs, hogs, eats, birds, and the cold-blooded animals are relatively insusceptible. For man the bacillus is definitely pathogenic, though less so than for some of the animals mentioned above.

While separate races of anthrax bacilli may vary much in their degree of virulence, a single individual strain remains fairly constant in this respect if preserved, dried upon threads or kept in sealed tubes, in a cold, dark place. Virulence may be reduced ${ }^{15}$ by various attenuating laboratory procedures which are of importance in that they have made possible prophylactic immunization. Heating the bacilli to $55^{\circ} \mathrm{C}$. for ten minutes considerably reduces their virulence. Similar results are obtained by prolonged cultivation at temperatures of $42^{\circ}$ to $43^{\circ} \mathrm{C}$, or by the addition of weak disinfectants to the culture fluids. ${ }^{16}$ Once reduced, the new grade of virulence remains fairly constant. Increase of virulence may be artificially produced by passage through animals.

Experimental infections in susceptible animals are most easily accomplished by subcutaneous inoculations. The inoculation is followed, at first, by no morbid symptoms, and some animals may appear perfectly well and comfortable until within a few hours or even moments before death, when they suddenly become visibly very ill, rapidly go into collapse, and die. The length of the disease depends to some extent, of course, upon the resistance of the infected subject, being in guinea-pigs and mice from twenty-four to fortyeight hours. The quantity of infectious material introduced, on the other hand, has little bearing upon the final outcome, a few bacilli, or even a single bacillus, often sufficing to bring about a fatal infection. Although the bacilli are not demonstrable in the blood until just before death, they nevertheless invade the blood and lymph streams immediately after inoculation, and are conveyed by these to all the organs. This has been demonstrated clearly by experiments where inoculations into the tail or ear were immediately followed by amputation of the inoculated parts without prevention of the fatal general infection. The bacilli are probably not at first

${ }^{15}$ Toussaint, Comptes rend. de l'acad. des sci., xci, 1880; Pasteur, Chamberlan et Roux, Comptes rend. de l'acad. des sci, xcii, 1881.

${ }^{16}$ Chamberland et Rowx, bid., XCVI, 1882. 
able to multiply in the blood. At the place of inoculation and probably in the organs they proliferate, until the resistance of the infected subject is entirely overeome. At this stage of the disease, no longer held at bay by any antagonistic qualities of the blood, they enter the circulation and multiply within it. Autopsy upon such animals reveals an edematous hemorrhagic infiltration at the point of inoculation. The spleen is enlarged and congested. The kidneys are congested, and there may be hemorrhagic spots upon the serous membranes. The bacilli are found in large numbers in the blood and in the capillaries of all the organs.

The mode of action of Bacillus anthracis is as yet an unsettled point. It is probable that death is brought about to a large extent by purely mechanical means, such as eapillary obstruction. Neither a true secretory toxin nor an endotoxin has been demonstrated for the anthrax bacillus. The decidedly toxemic clinical picture of the disease, however, in some animals and in man, precludes our definitely concluding that such poisons do not exist. It is a matter of fact, however, that neither culture filtrates nor dead bacilli have any noticeable toxic effect upon test animals, and exert no appreeiable immunizing action.

Spontaneous infection of animals takes place largely by way of the alimentary canal, the bacilli being taken in with the food. The bacteria are swallowed as spores, and therefore resist the acid gastric juice. In the intestines they develop into the vegetative forms, increase, and gradually invade the system. The large majority of cattle infections are of this type. Direct subcutaneous infection may also occur spontancously when small punctures̀ and abrasions about the mouth are made by the sharp spicules of the hay, straw, or other varieties of fodder.

When infection upon a visible part occurs, there is formed a diffuse, tense local swelling, not unlike a large carbuncle. The center of this may be marked by a black, necrotic slough, or may contain a pustular depression.

Infection by inhalation is probably rare among animals. Transmission among animals is usually by the agency of the excreta or unburned carcasses of infected animals. The bacilli escaping from the body are deposited upon the earth together with animal and vegetable matter, which forms a suitable medium for sporulation. The spores may then remain in the immediate vicinity, or may be seattered by rain and wind over considerable areas. The danger 
from buried carcasses, at first suspected by Pasteur, is probably very slight, owing to the fact that the bacilli can not sporulate in the anaërobic environment to which the burying-process subjects them. The disease, in infected cattle and sheep, is usually acute, killing within one or two days. The mortality is extremely high, fluctuating about eighty per cent.

In man the disease is usually acquired by cutaneous inoculation. It may also occur by inhalation and through the alimentary tract.

Cutaneous" inoculation occurs usually through small abrasions or seratehes upon the skin in men who habitually handle live-stock, and in butchers, or tanners of hides. Infection occurs most frequently upon the hands and forearms. The primary lesion, often spoken of as "malignant pustule," appears within twelve to twentyfour hours after inoculation, and resembles, at first, an ordinary small furuncle. Soon, however, its center will show a vesicle filled with sero-sanguineous, later sero-purulent fluid. This may change into a black central necrosis surrounded by an angry red edematous areola. Occasionally local gangrene and general systemic infection may lead to death within five or six days. More frequently, however, especially if prompt excision is practiced, the patient recovers. The early diagnosis of the condition is best made bacteriologically by finding the bacilli in the local diseharge.

The pulmonary infection, known as "wool-sorter's disease," occurs in persons who handle raw wool, hides, or horse hair, by the inhalation or by the swallowing of spores. The disease is fortunately rare in this country. The spores, once inhaled, develop into the vegetative forms ${ }^{17}$ and these travel along the lymphatics into the lungs and pleura. The disease manifests itself as a violent, irregular pneumonia, which, in the majority of cases, leads to death. The bacilli in these cases ean often be found in the sputum before death.

Infection through the alimentary canal may occasionally, though rarely, oceur in man, the source of infection being usually ingestion of the uncooked meat of infected animals. This form of infection is rare, because in many cases the bacilli have not sporulated in the animal and the ingested vegetative forms are injured or destroyed by the acid gastric juice. When viable spores enter the gut, however, infection may take place, the initial lesion being localized usually in the small intestine. The clinical picture that follows is one of violent enteritis with bloody stools and great

${ }^{17}$ Eppinger, Wien. med. Woch., 1888. 
prostration. Death is the rule. The diagnosis is made by the discovery of the bacilli in the feces.

General hygienic prophylaxis against anthrax consists chiefly in the destruction of infected animals, in the burying of cadavers, and in the disinfection of stables, etc. The practical impossibility of destroying the anthrax spores in infected pastures, etc., makes it necessary to resort to prophylactic immunization of cattle and sheep.

Immunity Against Anthrax.-Minute quantities of virulent anthrax cultures usually suffice to produce death in susceptible animals. Dead cultures are inefficient in calling forth any immunity in treated subjects. It is necessary, therefore, for the production of active immunity to resort to attenuated cultures. The safest way to accomplish such attenuation is the one originated by Pasteur, ${ }^{18}$ consisting in prolonged cultivation of the bacillus at $42^{\circ}$ to $43^{\circ} \mathrm{C}$. in broth. Non-spore-forming races are thus evolved.

The longer the bacilli are grown at the above temperature the greater is the reduction in their virulence. Koch, Gaffky, and Loeffler, ${ }^{19}$ utilizing the variations in susceptibilities of different species of animals, devised a method by means of which the relative attenuation of a given culture may be estimated and standardized. Rabbits are less susceptible than guinea-pigs, and virulent anthrax cultures, grown for two or three days after the stated conditions, lose their power to kill rabbits, but are less virulent for guinea-pigs. After ten to twenty days of further cultivation at $42^{\circ} \mathrm{C}$. the virulence for the guinea-pig disappears, but the culture is potent against the still more susceptible mouse. Even the virulence for mice may be entircly eliminated by further cultivation at this temperature.

The method of active immunization first practiced by Pasteur, and still used extensively, is carried out as follows: Two anthrax cultures of varying degrees of attenuation are used as vaccins. The premier vaccin is a culture which has lost its virulence for guinea-pigs and rabbits, and is potent only against mice. The deuxième vaccin is a culture which is still definitely virulent for mice and guinea-pigs, but not potent for rabbits. Forty-eight-hour broth cultures of these strains, grown at $37.5^{\circ} \mathrm{C}$, form the vaccin actually employed. Vacein $\mathrm{I}$ is subcutaneously injected into eattle in doses of 0.25 ,c.c., sheep receiving about half this quantity. After twelve days have elapsed similar quantities of Vaccin II are injected.

18 Pasteur, loc. cit.

${ }^{19}$ Koch, Goffky, und Loeffler, Mitt. a. d. kais. Gesundheitsamt, 1884. 
Pasteur's method has given excellent results and confers an immunity which lasts about a year.

Chauveau ${ }^{20}$ has modified Pasteur's method by growing the bacilli in bouillon at $38^{\circ}$ to $39^{\circ} \mathrm{C}$, at a pressure of eight atmospheres. Cultures are then made of races attenuated in this way, upon chicken bouillon and allowed to develop for thirty days. Single injections of 0.1 c.c. each of such eultures are said to protect eattle.

Active immunization of small laboratory animals is very difficult, but can be accomplished by careful treatment with extremely attenuated eultures.

Passive immunization by means of the serum of actively immune animals was first suceessfully accomplished by Sclavo. ${ }^{21}$

The subject of passive immunization has been especially investigated and practically applied by Sobernheim. ${ }^{22}$ The serum used is produced by actively immunizing sheep. It is necessary to earry immunization to an extremely high degree in order to obtain any appreciable protective power in the serum. This is accomplished by preliminary treatment with Pasteur's or other attenuated vaccines, followed by gradually inereasing doses of fully virulent eultures. Treatment continued at intervals of two weeks, for two or three months, usually produces an effective serum. Horses and cattle may also be used for the proeess, but they are believed by Sobernheim to give less active sera than sheep. Bleeding is done about three weeks after the last injection. The sera are stable and easily preserved.

Injections of 20 to 25 c.c. of such a serum have been found to protect animals effectually from anthrax and to confer an immunity lasting often as long as two months. Animals already infeeted are said to be saved by treatment with 25 to 100 e.c. of the serum.

Neither specific bactericidal nor bacteriolytic properties have, so far, been demonstrated in these immune sera. In fact, these properties are distinctly more pronounced against Bacillus anthracis in the normal sera of rats and dogs. Agglutinins have not been satisfactorily demonstrated in sera, partly beeause of the great technical difficulties encountered in the active ehain-formation of the bacillus in fluid media. An increase of opsonic power of such

${ }^{20}$ Chauveau, Comptes rend. de l'acad. des sei., 1884.

21 Sclavo, Cent. f. Bakt., xviii, 1895.

22 Sobernheim, Zeit. f. Hyg., xxv, 1897; xxxi, 1899. 
serum over normal serum has not been satisfactorily demonstrated.

Bacteria Closely Resembling Bacillus Anthracis.-In most laboratory collections there are strains of true anthrax bacilli so attenuated that they are practically non-pathogenic. These do not differ from the virulent strains in any morphological or cultural characteristies. Besides such strains there are numerous non-virulent bacteria cul-

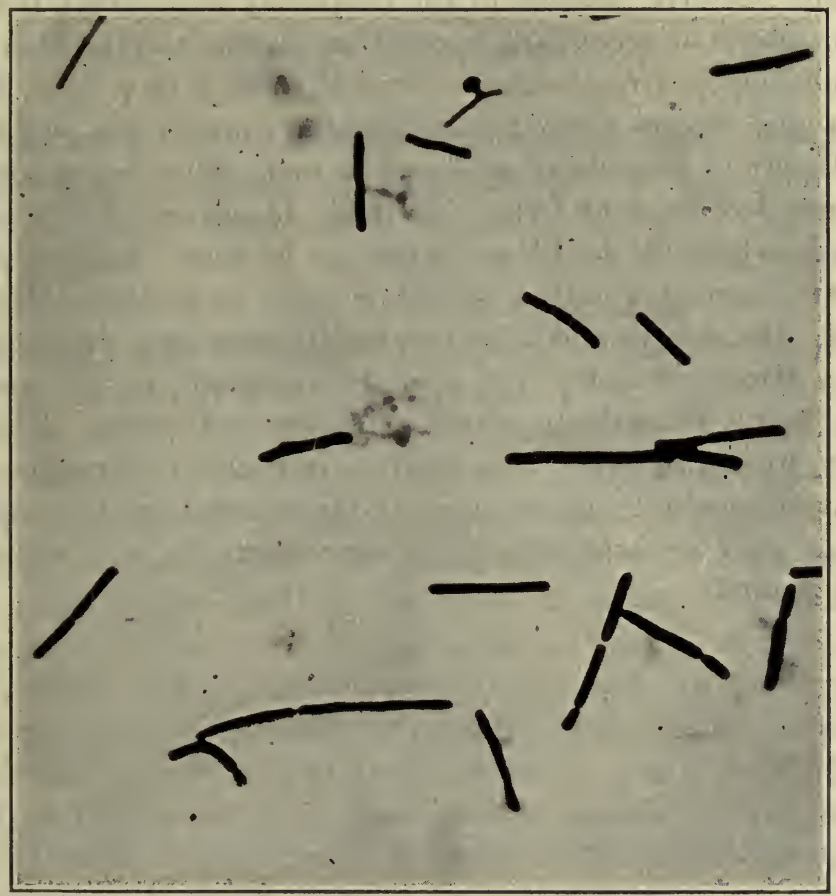

Fig. 82.-Bacillus Subtilis. (Hay Bacillus.)

turally not identical with Bacillus anthracis, but resembling it very (closely.

B. Anthracoides (Hueppe and Wood ${ }^{23}$ ).-A Gram-positive bacillus, morphologically different from B. anthracis in that the ends are more rounded. Culturally, somewhat more rapid in growth and more rapid in gelatin fluidification. Non-pathogenic. Otherwise indistinguishable from B. anthracis.

B. Radicosus (Wurzel Bacillus).-Cultivated from water-city water supplies. Morphologically somewhat larger than Bacillus an-

${ }^{23}$ Hueppe und Wood, Berl. klin. Woch., xvi, 1889. 
thracis, and the individual bacilli more irregular in size. Very rapid fluidification of gelatin and growth most active at room temperature. Non-pathogenic.

B. subTiLis (Hay Bacillus).-Although not very closely related to the anthrax group, this bacillus is somewhat similar and conveniently described in this connection. It is of importance to workers with pathogenic bacteria, because of the frequency with which it is found as a saprophyte or secondary invader in chronic suppurative lesions.

Morphology and Cultivation.-Straight rod, 2 to 8 micra long, 0.7 micron wide. Spores formed usually slightly nearer one pole than the other. Grows in long chains and only in such chains are spores found. It does not decolorize by Gram's method. Is actively motile in young cultures in which the bacilli are single or in pairs. In older cultures chains are formed and the bacilli become motionless. Gelatin is liquefied. On gelatin and agar the bacilli grow as a dry corrugated pellicle. Microscopically, the colonies are made up of interlacing threads, being irregularly round with fringed edges. There is a tendency to confluence. The bacillus is found in brackish water, infusions of vegetable matter, etc., and is practically non-pathogenic, occurring only occasionally as a saprophyte in old sinuses and infected wounds. 


\section{CHAPTER XXXIX}

\section{BACILLUS MALLEI AND GLANDERS}

\section{(Glanders Bacillus)}

GLANDERS is an infectious disease prevalent chiefly among horses, but transmitted occasionally to other domestic animals and to man. The microorganism eausing the disease, though seen and described by several earlicr authors, was first obtained in pure culture and accurately studied by Loeffler and Schütz ${ }^{1}$ in 1882 .

Morphology and Staining.-The glanders bacillus or B. mallei is a rather small rod with rounded ends. ${ }^{2}$ Its length varies from 3 to 4 micra, its breadth from 0.5 to 0.75 micron. Variation in size between separate individuals in the same culture is characteristic. The rods are usually straight, but may show a slight curvature. The bacillus is non-motile. There are no flagella and no spores are formed. The grouping of the bacilli in smears shows nothing very characteristic. Usually they appear as single bacilli lying irregularly parallel, often in chains of two or more. In old cultures, involution forms appear which are short, vacuolated, and almost coccoid.

While the glanders bacillus stains rather easily with the usual anilin dyes, it is so easily decolorized that especial care in preparing specimens must be observed. Stained in the usual manner with methylene-blue, it shows marked irregularity in its staining qualities; granular, deeply staining areas alternating with very faintly stained or entirely unstained portions. This diagnostically helpful characteristic has been variously interpreted as a mark of degeneration or a preparatory stage for sporulation. It is probably neither of the two, but an inherent irregularity in the normal protoplasmic composition of the bacillus, not unlike that of B. diphtheriæ. The bacillus is decolorized by Gram's method of staining.

Cultivation.-The glanders bacillus is easily grown on all of the usual meat-infusion media. It is practically indifferent to moderate

${ }^{1}$ Loeffler und Schütz, Deut. med. Woch., 1882.

${ }^{2}$ Loeffler, Arb. a. d. kais. Gesundheitsamt, 1886. 
variations in reaction, growing equally well upon neutral, slightly acid, or slightly alkaline culture media. Glycerin or small quantities of glucose added to media seem to render them more favorable for the eultivation of this bacillus.

Upon agar the colonies show little that is characteristic. They appear after twenty-four hours at $37.5^{\circ} \mathrm{C}$. as yellowish-white spots, at first transparent, later more opaque. They are round, with an even border, and microscopically appear finely granular. The older the cultures are, the more yellow do they appear.

On gelatin at room temperature, growth is slow, grayish-white, and

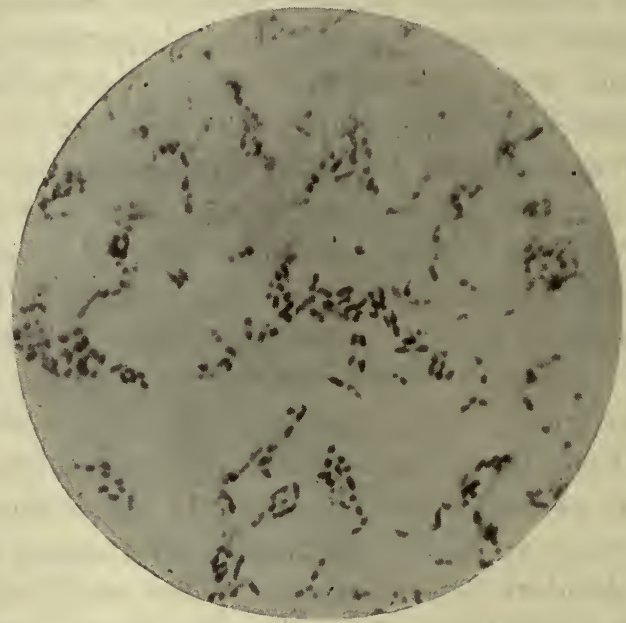

Fig. 83.-Glanders Bacillus. From potato culture. (After Zettnow.)

no liquefaction of the gelatin occurs. Growth upon this medium is never abundant.

In broth, there is, at first, diffuse clouding, later a heavy, tough, slimy sediment is formed. At the same time the surface is covered with a similarly slimy pellicle. The broth gradually assumes a dark brown color.

In milk, coagulation takes place slowly. In litmus milk, acidification appears.

The growth upon potato presents certain features which are diagnostically valuable. On potatoes which are not too acid growth is abundant and within forty-eight hours covers the surface as a yellowish, transparent, slimy layer. This gradually grows darker 
until it has assumed a deep reddish-brown hue. In using this feature of the growth diagnostically, it must not be forgotten that a very similar appearance upon potato occurs in the case of $B$. pyocyaneus.

Biological Considerations.-Bacillus mallei is aërobic. ${ }^{3}$ Growth under anaërobic conditions may take place, but it is slow and impoverished. The most favorable temperature for its cultivation is $37.5^{\circ} \mathrm{C}$. It fails to develop at temperatures below $22^{\circ} \mathrm{C}$. or above $43^{\circ} \mathrm{C}$. On artificial media, if kept cool and in the dark, and in sealed tubes, the glanders bacillus will retain its viability for months and years. On gelatin and in bouillon, it lives for a longer time than on the other media. Exposed to strong sunlight it is killed within twenty-four hours. Heating to $60^{\circ} \mathrm{C}$. kills it in two hours, to $75^{\circ} \mathrm{C}$. within one hour. Thorough drying kills the glanders bacillus in a short time. In water, under the protected conditions that are apt to prevail in watering-troughs, the bacillus may remain alive for over seventy days. The resistance to chemical disinfectants is not very high. ${ }^{4}$ Carbolic acid, one per. cent, kills it in thirty minutes, bichlorid of mercury, 0.1 per cent, in fifteen minutes.

Pathogenicity.- Spontaneous infection with the glanders bacillus occurs most frequently in horses. It occurs also in asses, in cats, and, more rarely, in dogs. In man the disease is not infrequent and is usually contracted by those in habitual contact with horses. Experimental inoculation is successful in guinea-pigs and rabbits. Cattle, hogs, rats, and birds are immune to experimental and spontaneous infections alike.

Spontaneous infection takes place by entrance through the broken skin, through the mucosa of the mouth or nasal passages. Infection in horses not infrequently takes place through the digestive tract. $^{5}$ In all cases, so far as we know, previous injury to either the skin or to the mucosa is necessary for penetration of the bacilli and the development of the disease.

Glanders in horses may occur in an acute or chronic form, depending upon the relative virulence of the infecting culture and the susceptibility of the subject. The more acute form of the disease is usually limited to the nasal mucosa and upper respiratory tract. The more chronic type of the disease is often accompanied by mul-

${ }^{3}$ Loeffler, loc. cit.

${ }^{4}$ Finger, Ziegler's Beitr., vi, 1889.

${ }^{5}$ Nocard, Bull. de la soc. centr. de méd. vét., 1894. 
tiple swellings of the skin and general lymphatic enlargement. This form is often spoken of as "farcy."

Acute glanders in the horse begins violently with fever and prostration. After two or three days there is a nasal discharge, at first serous, later seropurulent. At the same time there is ulceration of the nasal mucosa and acute swelling of the neighboring lymph nodes. These may break down and form deep pus-discharging

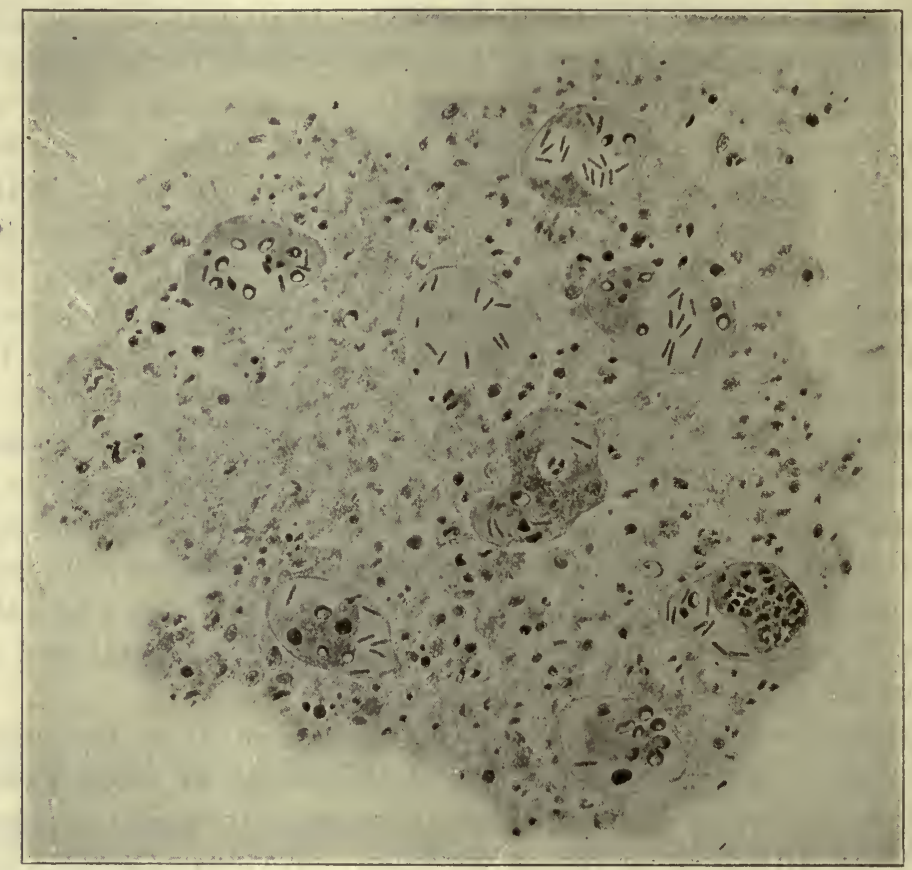

Fig. 84.-Glanders Bacilli in Tissue. (From a drawing furnished by Dr. James Ewing.)

sinuses and uleers. Finally, there is involvement of the lungs and death within four to six weeks.

When the disease takes the chronic form the onset is more gradual. Coneomitant with the nasal inflammation there is a formation of subcutaneous swellings all over the body, some of whieh show a tendency to break down and ulcerate. Together with this the lymphaties all over the body become enlarged. The disease may last for several years, and occasionally may end in complete cure. 
In horses the chronic form of the disease is by far the more frequent. In man the disease is similar to that of the horse exeept that the point of origin is more frequently in some part of the skin rather than in the nasal mucosa, and the elinieal symptoms differ aceordingly. The onset is usually violent, with ferer and systemic symptoms. At the point of infection a nodule appears, surrounded by lymphangitis and swelling. A general papular eruption may oceur. The papules may become pustular, and the clinical features may thus simulate variola. This type of the disease usually ends fatally in eight to ten days. The chronic form of the disease in man is much like that in the horse, but is more frequently fatal.

The histological appearance of the glanders nodules is usually one of diffuse leucocytic infiltration and the formation of young connective tissue which prepond rates more and more as the disease becomes chronic. Virchow has classed these lesions with the granulomata. From the center of such nodules B. mallei may often be obtained in pure culture. The nodules may be generally distributed throughout the internal organs. The bacilli themselves are found, apart from the nodules, in the nasal secretions, and oceasionally in the eirculating blood. ${ }^{6}$

The bacteriological diagnosis of glanders may be made by isolating and identifying the bacilli from any of the above-mentioned sources. When superficial nodules ean be opened for the purpose of diagnosis this may prove an easy task. The most diagnostically helpful medium in such cases is potato. In a majority of eases, however, isolation is extremely difficult and resort must be had to animal inoculation. The most suitable animal for this purpose is the male guinea-pig. Intraperitoneal inoculation of such animals with máterial containing glanders bacilli leads within two or three days to tumefaction and purulent inflammation of the testicles. Such an experiment, spoken of as the "Strauss test," 7 should always be reinforeed by cultural examination of the testicular pus, the spleen, and the peritoneal exudate of the animals employed.

Toxin of Bacillus mallei.-The toxin of B. mallei, or mallein, belongs to the class of endotoxins. The toxic products have been invariably obtained by extraction of dead bacilli. ${ }^{8}$ Mallein differs

- Wassilieff, Deut. med. Woch., 1883.

${ }^{7}$ Strauss, Arch. de méd. exp., 1889.

${ }^{3}$ Kresling, Arch. d. sci. biol., 1892; Preuser, Berl. thierärzt. Woch., 1894. 
from many other bacterial poisons in being extremely resistant. It withstands temperatures of $120^{\circ} \mathrm{C}$. and prolonged storage without noticeable loss of strength. ${ }^{9}$

In its physiological action upon healthy animals, mallein is not a powerful poison. It can be given in considerable doses without causing death. Mallein may be obtained by a variety of methods. Helman and Kalning, the discoverers of this toxin, used filtered aqueous and glycerin extracts of potato cultures. Roux ${ }^{10}$ cultivates virulent glanders bacilli in flasks containing 250 c.c. each of 5 per cent glycerin bouillon. Growth is allowed to continue at $35^{\circ} \mathrm{C}$. for one month. At the end of this time, the cultures are sterilized at $100^{\circ}$ for thirty minutes, and evaporated on a water bath to onetenth their original volume. They are then filtered through paper. This concentrated poison is diluted ten times with 0.5 per cent carbolic acid before use. Concentration is done merely for purposes of conservation. The diagnostic dose of such mallein for a horse is 0.25 c.c. of the undiluted fluid.

At the Washington Bureau of Animal Industry, mallein is prepared by growing the bacilli for five months at $37.5^{\circ} \mathrm{C}$. in glycerinbouillon. This is then boiled for one hour and allowed to stand in a cool place for one week. The supernatant fluid is then decanted and filtered through clay filters by means of a vacuum pump. The filtrate is evaporated to one-third its original volume on a water bath, and the evaporated volume resupplied by a 1 per cent carbolic acid solution containing about 10 per cent of glycerin.

Diagnostic Use of Mallein.-The injection of a proper dose of mallein into a horse suffering from glanders is followed within six to eight hours by a sharp rise of temperature, often reaching $104^{\circ}$ to $106^{\circ} \mathrm{F} .\left(40^{\circ} \mathrm{C}\right.$. + ). The high temperature continues for several hours and then begins gradually to fall. The normal is not usually regained for several days. Locally, at the point of injection, there appears within a few hours a firm, hot, diffuse swelling, which gradually extends until it may cover areas of 20 to 30 centimeters in diameter. The swelling is intensely tender during the first twentyfour hours, and lasts for three to nine days. Together with this there are marked symptoms of general intoxication. In normal animals the rise of temperature following an injection is trifling,

9 Wladimiroff, in Kraus und Levaditi, "Handbuch," etc., 1908.

${ }^{10}$ Roux et Nocard, Bull. d. 1. soc. centr. vét., 1892. 
and the local reaction is much smaller and more transient. Injections are best made into the breast or the side of the neck.

The directions given by the United States Government for using mallein for the diagnosis of glanders in horses are as follows:

"Make the test, if possible, with a healthy horse, as well as with one or more affected or supposed to be affected with glanders. Take the temperature of all these animals at least three times a day for one or more days before making the injections.

"The injection is most conveniently made at 6 or 7 o'clock in the morning, and the maximum temperature will then usually be reached by or before 10 P.M. of the same day.

"Use for each horse one cubic centimeter of the mallein solutior. as sent out, and make the injection beneath the skin of the middle of one side of the neck, where the local swelling can be readily detected.

"Carefully sterilize the syringe after injecting each horse by flaming the needle over an alcohol lamp or, better, use separate syringes for healthy and suspected animals. If the same syringe is used, inject the healthy animals first, and flame the needle of the syringe after each injection.

"Take the temperature every two hours for at least eighteen hours after the injection. Sterilize the thermometer in a 5 per cent solution of carbolic acid, or a 0.2 per cent solution of corrosive sublimate, after taking the temperature of each animal.

"The temperature, as a rule, will begin to rise from four to eight, hours after the injection, and reach its maximum from ten to sixteen hours after injection. On the day succeeding the injection takn the temperature at least three times.

"In addition to the febrile reaction, note the size, appearance, and duration of any local swelling at the point of injection. Note the general condition and symptoms of the animal, both before, during, and after the test.

"Keep the solution in the sealed bottle and in a cool place, and do not use it when it is clouded or if it is more than six weeks old; when it leaves the laboratory of the Bureau it is sterile."

If the result of first injection is doubtful, the horse should be isolated and retested in from one to three months, when the slight immunity conferred by the first injection will have disappeared. The second injection into healthy horses usually shows no reaction whatever. 
Mallein may cause reactions in the presence of other diseases than glanders, such as bronchitis, periostitis, and other inflammatory lesions and is not so specifically valuable as tuberculin for diagnosis.

Complement Fixation in Glanders.-Diagnostic complement fixation for the diagnosis of glanders has been developed by McNeil and Olmstead at the New York Department of Health. The antigen is made by growing the glanders bacilli on a 1.6 per cent glyecrin potato agar. From this stock cultures transplanted are made upor. a neutral meat-free-veal-peptone agar. Twenty-four-hour growths are washed off with distilled water sterilized at $80^{\circ} \mathrm{C}$. for four hours and filtered through a Berkefeld. After filtration the antigen must again be sterilized at $80^{\circ}$ for one hour.

Immunity.-Recovery from a glanders infection does not confer immunity against a second inoculation. ${ }^{11}$ Artificial active immunization has been variously attempted by treatment with attenuated cultures, with dead bacilli, and with mallein, but without convincing results.

The serum of subjects suffering from glanders contains specific agglutinins. ${ }^{12}$ These are of great importance diagnostically if the tests are made with dilutions of, at least, 1 in 500, since normal horse serum may agglutinate B. mallei in dilutions lower than this.

11 Finger, Ziegler's Beiträge, vi, 1899.

12 Galtier, Jour. de méd. vét., 1901. 


\section{CHAPTER XL}

THE BACILLUS MELITENSIS (MICROCOCCUS MELITENSIS), BACILLUS BRONCHISEPTICUS, BACILLUS OF CATTLE ABORTION, BACILLUS OF GUINEA-PIG PNEUMONIA, AND THE BACILLUS PYOCYANEUS.

\section{MALTA FEVER AND THE BACILLUS MELITENSIS}

MaLta fever probably has its endemic focus in the Mediterranean islands and along the Mediterranean coast. From here it appears to have spread into continental Europe, France, Italy and Spain and into the Balkans. Naturally enough it has been found to occur in Northern Africa and cases have been reported along the East African coast. Castellani and Chalmers ${ }^{1}$ state that it has also been found in parts of Russia, in South Africa, in Uganda, China and the Philippines and in North and South America and in the West Indies. Castellani also has reported cases from Ceylon.

It appears, thus, that the disease is very widely distributed, but centralizes chiefly about the Mediterranean, being most common in the warmer temperate and subtropical climates. Studies on its distribution have been made particularly by Bassett-Smith, ${ }^{2}$ who has mapped its distribution throughout the world. A reproduction of his map may be found in Castellani and Chalmer's book.

Morphology.-The micrococcus melitensis is an extremely small bacterium which has been described both as a bacillus and a coccus. It has been the custom of bacteriologists who have studied it more reecntly to regard it as a bacillus and speak of it as the Bacillus Melitensis. Eyre ${ }^{3}$ describes it as an extremely small coccus which has bacillary forms on various media which he regards as involution: forms. It is Gram-negative and non-motile and does not form spores. In eultures it appears both singly and in short chains of two or more. Chains of any considerable length are uncommon.

${ }^{1}$ Castellani and Chalmers, Textbook of Tropical Medicine, William Wood \& Co., N. Y., 1919 .

${ }^{2}$ Bassett-Smith, British Med. Jour., 2, 1904.

${ }^{3}$ Eyre, Kolle and Wassermann Handb., Second Edition, Vol. 4, 421. 
Cultivation.-The organism can usually be cultivated from the spleens of those who have died of the disease, or by spleen puncture, a method by which Bruce ${ }^{4}$ obtained it in his early studies. It can also be obtained from the blood stream in active cases, from the urine and from the milk of infected goats. Eyre states that the optimum is at $37^{\circ} \mathrm{C}$. and that it will grow but slightly and slowly on media at room temperature at $20^{\circ}$ or thereabout. It will grow both aërobically and under conditions of limited anaërobiosis. Growth is relatively slow and does not become luxuriant for three days or more. It does not seem to be very delicate in its nutritive requirements and has been cultivated on most of the ordinary media. It will grow on gelatin without liquefying the gelatin. Its growth on potato is hardly visible.

Animal Pathogenicity.-According to Eyre, the B. melitensis is pathogenic for almost all laboratory animals, although it may take a very long time to kill. Eyre states that guinea pigs will live for as long as 100 or more days after an injection of $\mathrm{B}$. melitensis, but that the virulence of the organism can be greatly enhanced by animal passage. It spontaneously infects goats which, as we shall see, is an important point in its epidemiology, and apparently it may similarly infect horses, cattle and sheep.

The Disease.-In a number of ways, Malta fever is similar to typhoid fever. It probably gets into man in most cases by way of mouth, passing from the mucous membrane of the intestinal canal into the blood without causing any considerable lesions. A bacteriemia follows during which there is a typhoid fever-like temperature and an enlarged spleen. The incubation time seems to be about two weeks, and the onset of the disease in its generalized symptoms again has the indefinite characters, malaise, headache, etc., that are associated with typhoid. There is no leucocytosis and a relative lymphocytosis. Usually cases are protracted, the disease passing through a prolonged febrile period, lasting two, three, or four weeks. There may be acute cases in which the onset is sudden and the course of the disease violent.

Secondary symptoms may consist in neuritis, parenchymatous nephritis and pulmonary congestion, arthritis and orchitis.

Bruce, Practitioner, 1887. 
Diagnosis ean be made by agglutination and isolation of the organisms. According to Wright and Semple, ${ }^{5}$ Bassett-Smith, Eyre ${ }^{6}$ and others, the agglutinins in the blood are very high and may be detected in dilutions of 1:1,000 or over, even as early as the end of the first week.

Blood eultures may be positive very early in the disease. Later, the organisms appear in the urine.

Epidemiology.-It seems to be unquestionable at the present time that the disease is transmitted chiefly by the ingestion of the milk of infected goats, and according to Eyre the goat is the natural host of the infection, keeping the disease going in endemic regions. Apparently a very large percentage of the goats in infected regions are infected. According to Castellani and Chalmers in Malta 50 per cent of the goats are infeeted, and in parts of Northern Africa the percentage of infection in goats as indicated by various researehes ranges from. less than 4 to 34 per cent.

Since goats' milk is.a common human food in these regions, the spread of the disease by this means is natural.

There are probably carriers among goats and human beings that recover. Among other forms of transmission, direct infection from individual to individual probably takes place, and indireet infection through food and flies is not out of question.

It has been suggested that mosquitoes, too, may transmit the disease and Eyre states that it has been possible to demonstrate the organisms in the stomachs of a number of different mosquito species.

However, it seems quite clear that the most important method of transmission is by means of infected milk.

Immunity.-It is the general belief that one attack of Malta fever protects against a second attack. However, there are a great many cases in which the fever recurs several times in the same individual in the form of relapses and recrudescences. The intermissions between such attacks often last for months. Prophylactic vaccination has not yet been carried out on a sufficiently large scale to permit the formulation of conelusions.

${ }^{6}$ Eyre, etc., Report of the Mediterranean Fever Commission, 1907. 
B. Bronchisepticus.-Prior to 1910 a great deal of inconclusive work was done on canine distemper, but in this year Ferry ${ }^{7}$ published a preliminary paper in which he reported the isolation of a bacillus which was often present in pure culture in the smaller bronchii and the tracheæ of dogs killed early in the disease. At almost the same time, and independently of Ferry, McGowan ${ }^{8}$ reported similar observations. Soon after this the subject was very thoroughly studied by Torrey and Rahe. ${ }^{9}$ The B. bronchisepticus was described as a short, Gram-negative organism, occasionally coccoid in appearance, slowly motile, which grows very slowly at first isolation, the colonies being hardly visible on agar in twenty-four hours but definitely visible in forty-eight hours. It grows well on glycerin agar, will grow at $20^{\circ}$ with an optimum at about $37.5^{\circ}$. It is not an obligate aërobe, but grows poorly without oxygen. It does not liquefy gelatin. It renders broth uniformly turbid with a slight deposit and no pellicle. No acid or gas is formed on carbohydrate media, and the media are rendered slightly alkalin in the course of four or five days. Torrey could determine no indol formation. The typical media, according to McGowan, Ferry and Torrey are litmus milk and potato. Torrey states that the use of these two media alone is sufficient for identification in the hands of a practiced observer.

On litmus milk it grows like the B. fecalis alkaligenes. There is a progressive alkalinization, and after about twenty-four hours at $37^{\circ}$ a ring of deep blue appears, extending about $3 / 8$ inch from the surface. In from five to ten days the whole medium has assumed a blue-black color. On potato in twenty-four hours a marked yellowish-brown growth appears with sometimes a greenish darkening of the potato. In this respect again it is very similar to $\mathrm{B}$. fecalis alkaligenes. Torrey also reports that the organism produces a hemolysin for rabbit and guinea pig erythrocytes. He states that typical distemper can be induced in susceptible dogs by injection with pure cultures, and dogs which have recovered from attacks induced by the bacillus are protected by exposure to the disease in other dogs.

7 Ferry, Amer. Veterinary Review, 499, 1910 (quoted from Torrey) Jour. Infec. Dis., 8, 1911, 399.

${ }^{8}$ McGowan, Jour. Path. and Bacter., 15, 1911, 372 and 1916, 257.

9 Torrey and Rahe, Jour. Med. Res., 27, 1912, 291. 
Bacillus of Cattle Abortion (B. Abortus of Bang).-We have already mentioned, in speaking of the organism which causes abortion in mares, that there was another bacillus described by Bang ${ }^{10}$ in 1897 which was found by him to be the etiological factor in abortion of eattle, but which was distinetly different from that subsequently found by Smith and others in the analogous disease of horses. This organism has been observed by a great many writers since Bang, and it was Smith who first pointed out its similarity to the B. bronchisepticus of canine distemper and to a bacillus which causes epidemic pneumonias in guinea pigs. Alice Evans ${ }^{11}$ subsequently pointed out the similarity of B. Abortus and of B. bronchisepticus, to the $\mathrm{B}$. melitensis which causes Malta fever. Evans' description of Bacterium Abortus as studied from strains obtained by the Dairy Division of the Bureau of Animal Industry, United States Department of Agriculture, is as follows:

It is a short, slender pleomorphic rod with rounded ends, sometimes so short as to appear coccoid. Obtained from the condensation water of a twenty-four hour culture on agar, it is non-motile and does not form spores. It is Gram-negative.

It is difficult to grow on artificial media on first isolation, and in these early cultures its growth is favored by partial anaërobiosis, which Evans obtained by incubation in a closed jar in the presence of cultures of B. subtilis. Glycerin or serum agar are favorable media for isolation, but after prolonged cultivation it grows well on ordinary media. Colonies on agar plates develop after about two days in very small dewdrop form. It never heavily clouds broth. Milk is rendered slightly alkaline. On potato there is a slight grayish brown growth after several days, and subsequently, the brownish tinge discolors the potato itself. It forms no acid or gas on any of the sugars, but slightly reduces the hydrogen ion concentration of broth cultures. It produces ammonia from amino acids such as asparagin. It does not liquefy gelatin.

It is easily distinguished from B. bronchisepticus by its lack of motility, and less rapid and abundant growth on artificial media, as well as by agglutination reactions. Evans states from her studies that $B$. melitensis is closely related to $B$. abortus and can be distinguished from it only by means of agglutination tests. Fleischner,

${ }^{10}$ Bang. Zeit. f. Tier Med., 1, 1897, 241.

11 Evans, Jour. Infec. Dis., 22, 1918, 580. 
Meyer and Shaw ${ }^{12}$ have reecntly examined skin reactions in connection with B. abortus bovis and B. melitensis, and claim that this method establishes a very close association between the two.

Of great importance to the sanitarian is the fact that the $B$. abortus may appear in the milk of cattle that have aborted and may also be present in the milk of cattle that are carriers and have suffered no abortion themselves. Cotton ${ }^{13}$ has shown that the Bacillus may persist in the genital organs for forty-six days after abortion has taken place. From the studies of Schroeder and Cotton, ${ }^{14}$ Stafseth $^{15}$ and others, it appears that the B. abortus does not establish itself permanently in the uterine cavity, and Stafseth's recent investigations seem to show that it does not penetrate into the deeper layers of the mucous membrane and remain there as a latent infection.

Theobald Smith ${ }^{16}$ in 1912 called attention to the tuberculosislike lesions in guinea pigs caused by B. abortus, a subject which later was studied in more detail by Smith and Fabyan. ${ }^{17}$ Fabyan, as well as Schroeder demonstrated the presence of the B. abortus in milk by guinea pig injection. Characteristic lesions not unlike tubercles developed in such animals in about eight to ten weeks, and Evans found the Bacillus in the milk of six out of twenty-four cows that had not aborted. Huddleson ${ }^{18}$ has recently studied methods of isolating B. abortus from milk, other than by guineapig injection. He uses a liver or spleen infusion agar, developed by Stafseth, a medium in the preparation of which overheating and filtration through paper is avoided. The optimum hydrogen ion concentration is $\mathrm{P}_{\mathbf{H}}$ 6.6. He adds to this medium gentian violet to a final concentration of $1: 10,000$. Ten c.c. of milk is centrifuged at 2,000 revolutions for about two hours. 0.1 c.c. of the sediment is taken out with a capillary pipette and distributed over the surface of a gentian violet agar plate. These plates are incubated in jars in which about 10 per cent of the air has been displaced by $\mathrm{CO}_{2}$.

12 Fleischner, Meyer and Shaw, Rep. Hooper Foundation, Univ. of Calif., 4, 1918. ${ }^{13}$ Cotton, Amer. Veter. Rev., 44, 1913, 307.

14 Schroeder and Cotton, Jour. Amer. Vet. Assoc., 3, 1916, Quoted from Stafseth (3).

${ }^{15}$ Stafseth, Stud. of Infectious Abortion, Mich. Agric. College, 49, 1920.

${ }^{16}$ Smith, Theobald, Footnote in article by Cotton, quot. from Fabyn, Jour. Med. Res., 28, 85, 1913.

${ }_{17}$ Smith and Fabyan, Cent. f. Bakt. Orig., 61, 1912, 549.

${ }^{18}$ Huddleson, Mich. Agric. College Exper. Station, 49, Nov. 1920, 25. 
By this method he was able to obtain results comparable in regularity to guinea-pig inoculation, and obtained cultures in about four days as against eight weeks by the guinea-pig inoculation.

It is important in making routine guinea-pig injections of milk for the determination of tuberculosis to remember the warning of Theobald Smith ${ }^{19}$ and not to jump at conclusions from mere gross appearance at autopsy of the animals.

Whether or not the presence of $\mathrm{B}$. abortus in milk is a danger to man is not certain, but it seems possible that the organism may cause diarrheal and perhaps other diseases, and the milk of aborted cattle should not be used for some time after abortion, and perhaps subjected to bacteriological test.

The striking similarity described by Evans and others of the B. abortus of cattle and the B. melitensis, taken together with the fact recently observed that other animals, especially the goat, may be infected by the B. melitensis, suggests the possibility of human pathogenicity for B. abortus, though at the present time this can be mentioned merely as a conjecture and a subject for inquiry.

Bacillus of Guinea-Pig Pneumonia.-In 1914 Theobald Smith ${ }^{20}$ called attention to a minute motile bacillus originally described by Tartakowsky which he found to be the cause of epidemic pneumonia in guinea-pigs. He found it to be similar to the organism described by Strade and Traina as the B. pneumoniae caviarum and Selter's B. caviae septicus. This organism he identified with the B. bronchisepticus of McGowan. ${ }^{21}$ He deseribes the bacillus of guinea-pig pneumonia as follows:

It is a minute rod with rounded ends. From agar slants it measures about 0.7 micron in length and about 0.5 micron broad. Longer rods are occasionally seen. The organisms stain solidly and are Gram-negative. They are rapidly motile. They grow moderately well on gelatin at room temperature, but do not liquefy the gelatin. On potato the bacillus makes a rich yellowish-brown color appearing within a week. It clouds broth within twenty-four hours, making a delicate iridescent membrane on the surface after prolonged growth. Later, a ropy deposit appears. It makes no visible change on milk. It does not produce indol. It is strictly aërobic and on sugar media

${ }^{19}$ Smith, Jour. Exper. Med., 30, 4, 325, 1919.

${ }^{20}$ Smith, Theobald, Jour. Med. Res., 29, 1914, 291.

${ }^{21}$ McGowan, Jour. Path. and Bact., 15, 1910, 372. 
does not produce gas or acid, but renders broth slowly alkalin. He states that in its bio-chemical aspects it approaches the pyocyaneous group, especially in regard to its strict aërobiosis and its lack of activity on sugars. In this respect, Smith calls attention to the fact that it is similar to the bacillus of cattle abortion. Smith compared the organism with a culture of the B. bronchisepticus sent him by Torrey which was supposed to be the cause of distemper in dogs, and found that his bacillus agreed with Torrey's strain in every particular, in regard to morphology and cultural characteristics.

He also identified his guinea-pig bacillus with an organism observed by Mallory in the ciliated epithelium of the air tubes in fatal cases of whooping cough.

In his description of the growth of his guinea-pig organism on potato, Smith calls attention to the similarity of this bacillus to the growth on potato of the bacillus of cattle abortion of Bang.

Bacillus Pyocyaneus.-It is a matter of common surgical experience that many suppurating wounds, especially sinuses of long standing, discharge pus which is of a bright green color. The fact that this peculiar type of purulent inflammation is due to a specific chromogenic microorganism was first demonstrated by Gessard ${ }^{22}$ in 1882. The bacillus which was described by Gessard has since become the subject of much careful research and has been shown to hold a not unimportant place among pathogenic bacteria. ${ }^{23}$

Morphology and Staining.-Bacillus pyocyaneus is a short rod, usually straight, occasionally slightly curved, measuring, according to Flügge, about 1 to 2 micra in length by about 0.3 of a micron in thickness. The bacilli are thus small and slender, but are subject to considerable variation from the measurements given, even in one and the same culture. While ordinarily single, the bacilli may be arranged end to end in short chains of two and three. Longer chains may exceptionally be formed upon media which are especially unfavorable for its growth, such as very acid media or those containing antiseptics.

Spores are not found. The bacilli are actively motile and possess each a single flagellum placed at one end.

Bacillus pyocyaneus is stained easily with all the usual dyes, but is decolorized by Gram's method. Irregular staining of the bacillary

22.Gessard, Thèse de Paris, 1882.

26 Charrin, "La maladie pyocyanique," Paris, 1889. 
body is common, but is always an indication of degeneration, and not a normal characteristic, as, for instance, in the diphtheria group.

Cultivation.-The pyocyaneus bacillus is aërobic and facultatively anaërobic. It can be adapted to absolutely anaërobic environments, but does not produce its characteristic pigment without the free access of oxygen. The bacillus grows readily upon the usual laboratory media and is not very sensitive to reaction, growing equally well upon moderately alkaline or acid media. Development takes place at temperatures as low as $18^{\circ}$ to $20^{\circ} \mathrm{C}$., more rapidly and luxuriantly at $37.5^{\circ} \mathrm{C}$.

On agar slants, growth is abundant and confluent, the surface of the agar being covered by a moist, grayish or yellowish, glistening, even layer. The pigment which begins to become visible after about eighteen hours soon penetrates the agar itself and becomes diffused throughout it, giving the medium a bright green fluorescent appearance, which grows darker as the age of the culture increases.

In gelatin stabs, growth takes place much more rapidly upon the surface than in the depths. A rapid liquefaction of the gelatin takes place, causing a saucer-shaped depression. Aś this deepens, pigment begins to form in the upper layers, often visible as a greenish pellicle.

In gelatin plates, the colonies have a characteristic appearance. They are round and are composed of a central dense zone, and a peripheral, loosely granular zone, which extends outward into the peripheral fluidified area in a fringe of fine filaments. When first appearing, they are grayish yellow, later assuming the characteristic greenish hue.

In broth, growth is rapid and chiefly at the surface, forming a thick pellicle. Below this, there is moderate clouding. The pigment is formed chiefly at the top. In old cultures there is a heavy flocculent precipitate. In fluid media containing albuminous material, strong alkalinity is produced.

On potato, growth develops readily and a deep brownish pigment appears, which is not unlike that produced by $B$. mallei upon the same medium.

Milk is coagulated by precipitation of easein and assumes a yellowish-green hue. In older cultures the casein may again be digested and liquefied.

The pigment of Bacillus pyocyaneus has been the subject of much investigation. It was shown by Charrin ${ }^{24}$ and others that 
this pigment had no relation to the pathogenic properties of the bacillus. It is found in cultures as a colorless leukobase which assumes a green color on the addition of oxygen. Conversely, the typical green "pyocyanin," as the pigment is called, may be decolorized by reducing substances. This explains the fact that it is not found in cultures sealed from the air. Pyocyanin may be extracted from cultures with chloroform and crystallized out of such solution in the form of blue stellate crystals. These, on chemical analysis, have been found to belong to the group of aromatic compounds, with a formula, according to Ledderhose, ${ }^{25}$ of $\mathrm{C}_{14} \mathrm{H}_{14} \mathrm{~N}_{2} \mathrm{O}$.

Besides pyocyanin, Bacillus pyocyaneus produces another pigment which is fluorescent and insoluble in chloroform, but soluble in water. ${ }^{26}$ This pigment is common to other fluorescent bacteria, and not peculiar to Bacillus pyocyaneus. The reddish-brown color seen in old cultures ${ }^{27}$ and supposed by some writers to be a third pigment, is probably a derivative from pyocyanin by chemical change.

Chloroform extraction of pyocyanin from cultures may serve occasionally to distinguish the pyocyaneus bacilli from other similar fluorescent bacteria. Ernst has claimed that there are two types of B. pyocyaneus, an $a$-type which produces only the fluorescent, watersoluble pigment, and a $\beta$-type which produces both this and pyocyanin. ${ }^{28}$

Pathogenicity.-Bacillus pyocyaneus is one of the less virulent pathogenic bacteria. It is widely distributed in nature and may be found frequently as a harmless parasite upon the skin or in the upper respiratory tracts of animals and men. It has, however, occasionally been found in connection with suppurative lesions of various parts of the body, often as a mere secondary invader in the wake of another incitant, or even as the primary cause of the inflammation. In most cases where true pyocyaneus infection has taken place, the subject is usually one whose general condition and resistance are abnormally low. ${ }^{29}$ Thus pyocyaneus may be the cause of chronic otitis media in ill-nourished children. It has been cultivated

${ }^{25}$ Ledderhose, quoted from Boland, Cent. f. Bakt., xxv, 1889.

${ }^{20}$ Boland, loc. cit.

${ }^{27}$ Gessard, Ann. de l'inst. Pasteur, 1890, 1891, and 1892.

${ }^{28}$ Ernst, Zeit. f. Hyg., ii, 1887.

${ }^{20}$ Rohner, Cent. f. Bakt., xi, 1892. 
out of the stools of children suffering from diarrhea, and has been found at autopsy generally distributed throughout the organs of children dead of gastro-enteritis. ${ }^{30}$ It has been cultivated from the spleen at autopsy from a case of general sepsis following mastoid operation. The bacillus has been found, furthermore, during life in pericardial exudate and in pus from liver abscesses. ${ }^{31}$

Brill and Libman, ${ }^{32}$ as well as Finkelstein, ${ }^{33}$ have cultivated B. pyocyaneus from the blood of patients suffering from general sepsis. Wassermann ${ }^{34}$ showed the bacillus to have been the etiological factor in an epidemic of umbilical infections in new-born children. Similar examples of B. pyocyaneus infection in human beings might be enumerated in large numbers, and there is no good reason to doubt that, under given conditions, fatal infections may occur. Such cases, however, are still to be regarded as depending more upon the low resistance of the individual attacked than upon the great pathogenicity of B. pyocyaneus.

Many domestic animals are susceptible to experimental pyocyaneus infection, chief among these being rabbits, goats, mice, and guinea-pigs. Guinea-pigs are killed by this bacillus with especial ease. Intraperitoneal inoculation with a loopful of a culture of average virulence usually leads to the death of a young guinea-pig within three or four days.

Toxins and Immunization.-Emmerich and Löw have shown that filtrates of old broth cultures of $B$. pyocyaneus contain a ferment-like substance which possesses the power to destroy some other bacteria, apparently by lysis. They have called this substance "pyocyanase" and claim that, with it, they have succeeded in protecting animals from anthrax infection. During recent years pyocyanase has been employed locally for the removal of diphtheria bacilli from the throats of convalescent eases. Broth-culture filtrates evaporated to one-tenth their volume in vacuo are used for this purpose.

Pyocyanase is exceedingly thermostable, resisting boiling for several hours, and is probably not identical with any of the other toxins or peptonizing ferments produced by B. pyocyaneus.

\footnotetext{
${ }^{20}$ Neumann, Jahrb. f. Kinderheilk., 1890.

${ }^{31}$ Kraunhals, Zeit. f. Chir., xxxvii, 1893.

${ }^{32}$ Brill and Libman, Amer. Jour. Med. Sci., 1899.

${ }^{33}$ Finkelstein, Cent. f. Bakt., 1899.

${ }^{34}$ Wassermann, Virchow's Arch., elxv, 1901.
} 
The toxins proper of B. pyocyaneus have been the subject of much investigation, chiefly by Wassermann. ${ }^{35}$ Wassermamn found that filtrates of old cultures were far more poisonous for guinea-pigs than extracts made of dead bacteria. He concludes from this and other observations that B. pyocyaneus produces both an endotoxin and a soluble secreted toxin. The toxin is comparatively thermostable, resisting $100^{\circ} \mathrm{C}$. for a short time. Animals actively immunized with living cultures of $B$. pyocyaneus give rise in their blood serum to bacteriolytic antibodies only. Immunized with filtrates from old cultures, on the other hand, their serum will contain both bacteriolytic and antitoxic substances. The true toxin of B. pyocyaneus never approaches in strength that of diphtheria or of tetanus. Active immunization of animals must be done carefully if it is desired to produce an immune serum, since repeated injections cause great emaciation and general loss of strength. Specific agglutinins have been found in immune sera by Wassermann $^{36}$ and others. Eisenberg ${ }^{37}$ claims that such agglutinins are active also against some of the fluorescent intestinal bacteria.

Bulloch and Hunter ${ }^{38}$ have recently been able to show that old broth cultures of B. pyocyaneus contain a substance capable of hemolyzing the red blood corpuscles of dogs, rabbits, and sheep. This "pyocyanolysin" seems intimately attached to the bacterial body. Prolonged heating of cultures does not destroy it. Heating of hemolytic filtrates, however, destroys it in a short time. The filtration of young cultures yields very little pyocyanolysin in the filtrate. In old cultures, however, a considerable amount passes into the filtrate. Whether or not the hemolytic power is due to a specific bacterial product or is dependent upon changes in the culture fluid, such as alkalinization, etc., can not yet be regarded as certain.

Gheorghiewski ${ }^{39}$ claims to have found a leucocyte-destroying ferment in pyocyaneus cultures.

\footnotetext{
${ }^{35} \dot{W}$ assermann, Zeit. f. Hyg., xxii, 1896.

se Wassermann, Zeit. f. Hyg., 1902.

${ }^{37}$ Eisenberg, Cent. f: Bakt., 1903.

${ }^{3 s}$ Bulloch und Hunter, Cent. f. Bakt., xxviii, 1900.

${ }^{39}$ Gheorghiewski, Ann. de l'inst. Pasteur, xiii, 1899.
} 


\section{CILAPTER XLI}

\section{PLAGUE AND BACILLUS PESTIS}

\section{(The So-called Haemorrhagic Septicaemia Group)}

Plague.-The history of epidemic diseases has no more terrifying chapter than that of plague. ${ }^{1}$ Sweeping, time and again, over large areas of the civilized world, its scope and mortality were often so great that all forms of human activity were temporarily paralyzed. In the reign of Justinian almost fifty per cent of the entire population of the Roman Empire perished from the disease. The "Black Death" which swept over Europe during the fourteenth century killed about twenty-five million people. Smaller epidemics, appearing in numerous parts of the world during the sixteenth, seventeenth, and eighteenth centuries, have claimed innumerable victims. In 1893 plague appeared in Hong Kong. During the epidemic which followed, Bacillus pestis, now recognized as the etiological factor of the disease, was discovered by Kitasato ${ }^{2}$ and by Yersin, ${ }^{3}$ independently of each other. By both observers the bacillus could invariably be found in the pus from the bubos of afflicted persons. It could be demonstrated in enormous numbers in the cadavers of victims. The constancy of the oceurrence of the bacillus in patients, shown in the innumerable researches of many bacteriologists, would alone be sufficient evidence of its etiological relationship to the disease. This evidence was strengthened, moreover, by accidental infections which oecurred in Vienna in 1898, with laboratory cultures.

Since that time the investigations of plague cases and plague outbreaks by individual bacteriologists and by commissions of many governments have established the relationship between the disease and the bacillus to such a degree that there is not the shadow of a doubt as to its etiological significance.

1 IIirsch, "Handb. d. histor.-geogr. Path.," 1881.

${ }^{2}$ Kitusato, Lancet, 1894.

Yersin, Ann, de l'inst. Pasteur, 1894. 
Morphology and Staining:-Bacillus pestis is a short, thick bacillus with well-rounded ends. Its length is barely two or two and a half times its breadth ( 1.5 to 1.75 micra by 0.5 to 0.7 micron). The bacilli appear singly, in pairs, or, more rarely, in short chains of three or more. They show distinct polar staining. In size and shape these bacilli are subject to a greater degree of variation than are most other microorganisms. In old lesions or in old cultures the bacilli show involution forms which may appear either as swollen coccoid forms or as longer, club-shaped, diphtheroid bacilli. Degenerating individuals appear often as swollen, oval vacuoles. All these involution forms, by their very irregularity, are of diagnostic importance. They appear more numerous in artificial cultures than

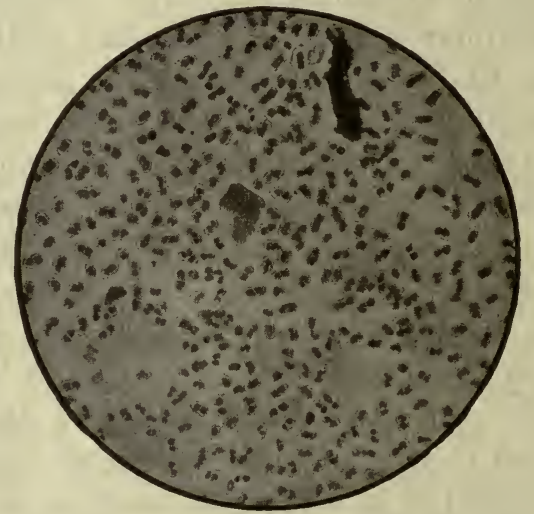

Fig. 85.-Bacillus pestis. (After Mallory and Wright.)

in human lesions. A very important property of the plague bacillus in this connection is the formation within twenty-four to forty-eight hours of vacuolated and swollen involution forms upon salt agar, that is, agar to which 3 to 5 per cent of salt is added. Such a medium is of great value in diagnostic work.

According to Albrecht and Ghon, ${ }^{4}$ the plague bacillus may, by special methods, be shown to possess a gelatinous capsule. It does not possess flagella and does not form spores.

The plague bacillus is easily stained with all the usual anilin dyes. Diluted aqucous fuchsin and methylene-blue are most frequently employed. With these stains the characteristically deeper

Albrecht und Ghon, Wien, 1898. 
staining of the polar portions of the bacillus is usually easy to demonstrate. Special polar stains have been devised by various observers. Most of these depend upon avoidance of the usual heat fixation of the preparations, which, in some way, seems to interfere with good polar staining. Fixation of the dried smears with absolute alcohol is, therefore, preferable. The bacillus is decolorized by Gram's method.

Isolation and Cultivation.-The bacillus is easily isolated in pure culture from the specific lesions of plague patients, during life or at autopsy. It is worth noting that smears from bubos and other plague lesions will often show the typical bacilli in very small

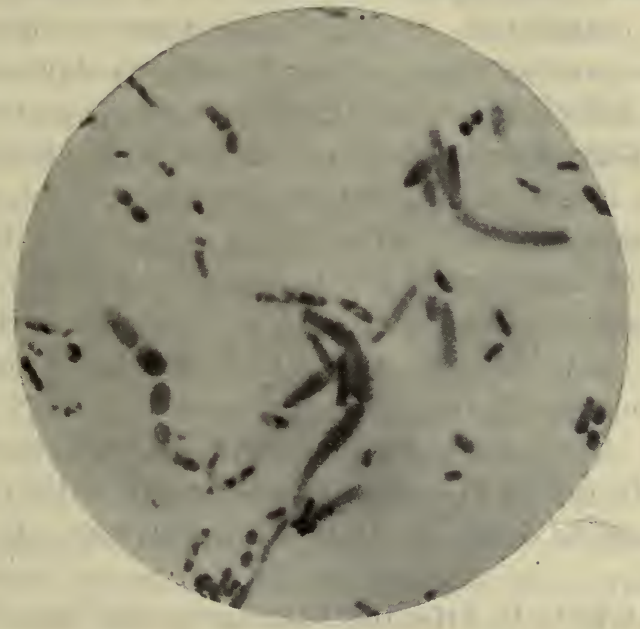

Fig. 86.-Bacillus Pestis, 'Involution Forms. (After Zettnow.)

numbers only, possibly because of the ease with which they undergo degeneration. The bacillus grows readily and luxuriantly upon the meat-infusion media. The optimum temperature for its cultivation is about $30^{\circ} \mathrm{C}$. Below $20^{\circ} \mathrm{C}$. and above $38^{\circ} \mathrm{C}$, growth is sparse and delayed, though it is not entirely inhibited until exposed to temperatures below $12^{\circ} \mathrm{C}$., or above $40^{\circ} \mathrm{C}$. The most favorable reaction of culture media is neutrality or moderate alkalinity, though slight acidity does not prevent development.

On agar, growth appears within twenty-four hours as minute colonies with a compact small center surrounded by a broad, irregularly indented, granular margin. 
On gelatin, similar colonies appear after two or three days at $20^{\circ}$ to $22^{\circ} \mathrm{C}$. The gelatin is not liquefied.

In bouillon, the plague bacilli grow slowly. They usually sink to the bottom or adhere to the walls of the tube as a granular deposit and may occasionally form a delicate pellicle. Chain-formation is not uncommon. In broth cultures, moreover, a peculiar stalactitelike growth is often seen, when the culture fluid is covered with a layer of oil and the flasks are incubated in a place where shaking or vibration can be prevented. Delicate threads of growth hang down from the surface of the medium into its depths like stalactites.

Characteristic involution forms are brought out best when the bacilli are grown upon agar containing 3 to 5 per cent $\mathrm{NaCl}$.

Milk is not coagulated. In litmus-milk there is slight acid formation. On potato and on blood serum the growth shows nothing characteristic or of differential value. On pepton media no indol is formed.

Biological Considerations.-Bacillus pestis is aërobic. Absence of free oxygen is said to prevent its growth, at least under certain conditions of artificial cultivation. It is non-motile. Outside of the animal body the bacilli may retain viability for months and even years if preserved in the dark and in a moist environment. In cadavers they may live for weeks and months if protected from dryness. In pus or sputum frorn patients they may live eight to fourteen days. These facts are of great hygienic importance.

Complete drying in the air kills the baeilli within two or three days. $^{5}$ Thoroughly dried by artificial means they die within four or five hours.

Dry heat at $100^{\circ} \mathrm{C}$. kills the bacillus in one hour. ${ }^{6}$ Live steam or boiling water is effectual in a few minutes. The bacilli possess great resistance against cold, surviving a temperature of $0^{\circ} \mathrm{C}$. for as many as forty days.

Direct sunlight destroys them within four or five hours. The common disinfectants are effectual in the following strengths: carbolic acid, one per eent kills them in two hours, five per cent in ten minutes; bichloride of mercury $1: 1,000$ is effectual in ten minutes.

In a recent eommunication to the New York Pathologieal Society, Dr. Wilson reported that plague cultures which he had kept sealed

Kitasato, Lancet, 1894.

- Abel, Cent. f. Bakt.. xxi. 1897. 
for as long as ten years in the ice chest were found living and virulent at the end of this time.

In regard to the viability of plague bacilli in air at different atmospheric temperatures and conditions of humidity, there are many important sanitary problems involved which are of particular significance in connection with the spread of pneumonic plague. Teague and Barber ${ }^{7}$ worked on this subject in connection with the Manchurian epidemic of pneumonic plague, and found that plague bacilli contained in fine droplets of pneumonic plague sputum would suffer death from drying in a few minutes unless they were suspended in an atmosphere with a very small water deficit; in other words, the humidity or the degree of saturation of the atmosphere with water is a very important factor in determining the length of time for which plague bacilli will remain alive in such dropletspray. Such atmospheres under ordinary circumstances are common in cold climates and droplets of sputum will, therefore, remain infectious longer in cold, wet elimates than in warm ones.

Animal Pathogenicity.-Bácillus pestis is extremely pathogenic for rats, mice, guinea-pigs, rabbits, and monkeys. The most susceptible of these animals are rats and guinea-pigs, in whom mere rubbing of plague bacilli into the unbroken skin will often produce the disease. This method of experimental infection of guinea-pigs is of great service in isolating the plague bacillus from material contaminated with other microorganisms. For the same purpose, infection of rats subcutaneously at the root of the tail may be employed. Such inoculation in rats is invariably fatal.

The studies of $\mathrm{MeCoy}^{8}$ of the United States and Public Health Service upon guinea-pigs and white rats show that individual plague cultures may vary considerably in virulence. The size of the dose, always excepting enormous quantities such as a whole agar culture, seems to make little difference in the speed with which the animals die. There may be considerable variation in the susceptibility of individual animals. Prolonged cultivation on artificial media may gradually reduce the virulence of plague bacilli, though, as stated above, this has not been the experience of all observers.

- Teague and Barber, Philippine Jour. of Science, B, 7, 1912.

${ }^{8} \mathrm{McCoy}$ noted the surprising fact that, in San Francisco a considerable percentage of wild rats-especially old ones, showed a high natural immunity to plague. 
In rats, spontaneous infection with plague is common and plays an important rôle in the spread of the disease. The pneumonic type of the disease is common in these animals and has been produced in them by inhalation experiments. During every wellobserved plague epidemic, marked mortality among the domestic rats has been noticed.

Although it was formerly supposed that rat infection took place because of the gnawing of dead cadavers by other rats, the work of the British Indian Plague Commission has shown that rats, like man, are spontaneously infected by means of fleas which pass from the infected to the uninfected animal.

In his work in California McCoy showed that the weasel and chipmunk are susceptible to plague infection, and therefore, potential means of spread if once infected.

Toxin Formation.-The systemic symptoms of plague are largely due to the absorption of poisonous products of the bacteria. Albrecht and Ghon, ${ }^{9}$ Wernicke, ${ }^{10}$ and others were unable to obtain any toxic action with broth-culture filtrates and concluded that the poisons of $B$. pestis were chiefly endotoxins, firmly attached to the bacterial body. Kossel and Overbeck, ${ }^{11}$ however, on the basis of a careful investigation, came to the conclusion that, in addition to the endotoxin, there is formed in older broth eultures a definite and important true, soluble toxin.

This, however, is unlikely in the light of a general survey of experimental work and conditions as they exist in the disease itself. It is most likely that the toxic symptoms here are those generally spoken of as endotoxin and also, we believe, perhaps some of the proteose substances suggested by us in connection with other bacteria.

Immunization.-A single attack of plague usually protects human beings from reinfection. A second attack in the same individual is extremely rare. Immunization in animals produces specific agglutinating and bacteriolytic substances which are of great importance in the bacteriological diagnosis of the bacillus. The agglutinating action of the serum of patients is clinically important in the diagnosis of the disease, even in dilutions of one in ten, since undiluted normal human serum has no agglutinating effect upon plague bacilli.

\footnotetext{
- Albrecht und Ghon, loc. cit.

10 Wernicke, Cent. f. Bakt., Ref., xxiv, 1898.

${ }^{11}$ Kossel und Overbeck, Arb. a. d. Gesundh., xviii, 1901.
} 
The curative plague serum prepared by Yersin and others by the immunization of horses with plague eultures has been extensively used in practice and though often disappointing, a definitely beneficial influence on the milder eases has been noted. The sera are standardized by their protective power as measured in white rats.

The question of prophylactie vaccination and active immunization will be taken up in connection with plague prevention below.

Plague in Man.-There are two chief methods by which the disease is acquired by man. The first is by entrance of the bacilli through the skin as a consequence of the bite of an infeeted flea. During the act of biting, the flea may either regurgitate blood, or, as is usually the case, deposit feces on the skin. The possibilities of entrance of the plague bacillus through minor injuries in the skin are so great that perhaps the infection may take place through the lesion caused by the fleabite, but more likely is rubbed in by the clothing or by scratehing as the fleabite becomes inflamed and irritated.

The other method by which plague is transmitted to man is by direct inhalation of sputum spray, a mode of infection which causes pneumonic plague. Aceording to Castellani and Chalmers ${ }^{12}$ and others, about 2.5 per cent of the cases occurring during epidemics of bubonic plague are of the pneumonic variety, and there may be special epidemies of pneumonic plague like the one deseribed in another section and studied by Strong, Teague and others ${ }^{13}$ in Manchuria and a more recent one which occurred in Northern China.

The incubation time of the disease is usually less than ten days, and may be no longer than two or three. The organisms entering through the skin may cause a localized lesion at the point of entranee. This may be of negligible size or may show a considerable inflammatory reaction. The organisms enter the lymphaties and cause the so-called bubo. The primary bubos are situated in the glands into which the infected area drains and, for this reason, the most eommon seat for these lesions is in the glands of the groin, but they also may be first seen in the axillary, cervieal or other glands. Secondary bubos may arise in other parts of the body, along the distribution of lymphatics, and the organisms rapidly enter the blood stream, causing septicemia.

${ }^{12}$ Castellani and Chalmers, Manual of Tropical Medicine, W. Wood \& Co., N. Y., 1919.

. ${ }^{13}$ Teague and Strong, Philippine Jour. of Science, Sec. B, No. 7, 1912. 
The onset is usually sudden, with high fever and the general symptoms of a severe toxemia. Castellani states the bacilli ean be found in blood cultures in about 30 per cent of the cases.

The disease may take a considerable number of forms which depend very largely upon the virulence with which the organisms overwhelm the body. It may be relatively mild, or may take an acute septicemic form which is rapidly fatal.

The pneumonic type is very severe and apt to kill rapidly. The onset of the pneumonic type according to Strong and Teague is abrupt, without prodromal symptoms.' There is often a chill, headache, and fever which reaches $103^{\circ}$ or $104^{\circ}$ within a day of the onset, accompanied by a very rapid pulse. Cough appears within twenty-four hours. The expectoration soon becomes abundant and consists of blood-tinged mucus. When later it becomes thick and bright red, it contains enormous numbers of plague bacilli. There are marked signs of cardiac involvement, and delirium and coma frequently appear. The same observers state that plague bacilli may frequently be found in the blood in such numbers that simple microscopical examination suffices for their detection. They state that in the Manchurian epidemic not a single case in which baeteriological diagnosis was complete, was known to have recovered.

The pathology of the lungs in this condition consists of general engorgement and edema. There are hemorrhages under the pleura, often fresh fibrinous pleurisy, and if a ease lasts long enough there may be pneumonic infiltration. The distribution of the pneumonic areas may be cither lobar or lobular. Bacteria are found in enormous numbers in the peribronchial lymph spaces and in the adjoining alveoli. They may also be present in large numbers in the interlobular septa and under the pleura.

Epidemiology.-Owing to the frequency and wide-spread nature of plague epidemies in the history of the world from most aneient times, it is quite impossible to more than very briefly outline the epidemiology of the disease. For fuller treatment of the epidemiological aspects the reader is referred to such books as Rosenau' $\mathbf{s}^{\mathbf{1 4}}$ Preventive Medicine and Castellani and Chalmer' ${ }^{15}$ work on tropical medicine. The prevalence of the disease in ancient tirnes

${ }^{14}$ Rosenau, Preventive Medicine and Hygiene, D. Appleton \& Co., N. Y. \& London, 1921.

${ }^{15}$ Castellani and Chalmers, Manual of Tropical Medicine, William Wood \& Co., N. Y., 1919. 
has been mentioned in the introduction. Through the Middle Ages a number of plague epidemics swept through Europe and frequently reached the commereial ports of Italy, Asia Minor and other parts of Eastern Europe from the Orient. In India it has long been known as a fatal form of epidemie disease and since the early part of the nineteenth century, has probably been endemie there. Castellani and Chalmers ${ }^{15}$ state that it was introdueed into China probably in the first half of the eighteenth eentury by Mohammedans returning from Mecea via Burma to the Provinee of Yunnan. Here it has been epidemie ever sinee. In 1894 the study of the Hongkong epidemic revealed the eausative agent of the disease. There was a very serious epidemic in 1894 which started in China, spread through Bombay to other parts of India, thence to Madagascar, into the Malay States, the Philippine Islands, other islands of the Pacifie, reaching North and South America and Europe; finally in 1900, it appeared in Cape Town and on the British Isles. Clemow, whom we quote from Castellani and Chalmers, stated that in 1900 plague was 'endemic in Mongolia, Southern China, the Himalayas, Mesopotamia, Persia, Uganda, parts of Russia and Northern Africa. In Africa the same author states that there are two endemic areas, one in Tripoli and the other in Uganda from which occasional African epidemics take origin.

The disease is, thus, a constant menace in many different parts of the world and must remain an important source of coneern to national public health organizations. In the United States the problem is perhaps more important than is appreciated by the people at large. In 1903 the disease appeared in California and for several years after that human eases oceurred, though the disease never took on the menace of an epidemic. This was prevented probably by the energetic work of the United States Public Health Service under Rupert Blue, MeCoy, ${ }^{16}$ Curry, and Wherry ${ }^{17}$ who instituted energetic methods of rat extermination, rat proofing and other necessary sanitary measures. More reeently, foei have appeared in Texas and New Orleans and it must never be forgotten that the conditions of elimate and in other respects are not by any means unfavorable to the development and spread of plague in some parts of America.

To eonvey an idea of the prevalence of plague in the world to-day

${ }^{16}$ McCoy, Pub. Health Report., July, 1913, No. 37.

${ }^{17}$ Wherry, Jour. Infec. Dis., 5, 1908. 
we insert a record of plague cases reported from various places in 1911, which we take from Jackson's Book on Plague. ${ }^{18}$ This record appeared in the British Medical Journal for September 16th, 1911:

India.-Deaths from plague in India during the first six months (of 1911), 604,634. Most prevalent (1) United Provinces, 281,317 (2) Punjab, 171,084; (3) Bengal, 58,515; (4) Bombay Presideney, 28,109. Deaths in July, not included above, 8,990 .

Hong Kong.-April 24 to August 21 (1911), 255 cases, 194 deaths.

China.-Since January 1, 1911, plague was reported in varying intensity in (provinees and towns) Manchuria, Peking, Tientsin, Chefoo, Shantung, Shanghai, Amoy, Foochow, Swatow, Canton, Pakhoi and Laichow.

Indo-China.-At Saigon, in March and April (1911), many cases reported. April 17 to May 7, 56 cases; 17 deaths. May 22 to May 28, 37 eases; 12 deaths.

Siam.-In Bangkok plague was more severe during 1911 than in any previous year. March 15 to April 15, 33 cases and 29 deaths.

Java and Sumatra.-In Java, May 25 to June 3 (1911), 105 eases and 62 deaths (one province). In Sumatra plague was present, no statisties.

Straits Settlements.-A few cases mostly imported, reported in 1911.

Japan.-A few cases at Kobe in 1911. In Formosa, from April 2 to April 15, 31 cases; 24 deaths.

Egypt.-Plague reported from Port Said, Suakin (on board ship), Cairo and Alexandria; also from 11 provinces. The province of Kena had a severe outbreak, May 5 to May 31 (1911), 51 cases and 49 deaths.

Persia.-Several cases reported from ports on the Persian Gulf.

Turkey in Asia.-A few cases at Muscat, Basra and at Port of Jeddah.

British East Africa.-Kismayu and Port Florence reported a few cases in April (1911).

Mauritius.-January 1 to April 11 (1911), 110 eases and 70 deaths.

Portuguese East Africa.-Plague was reported present at Nahoria in May (1911).

Russia.-In the Kirgis Steppe in the Astrakan Government in January (1911), 50 cases; 30 deaths.

South America.-Plague prevailed during 1911 in Peru, Ecuador, Brazil, Chile and Venezuela. No severe outbreak except in Peru, where from February to May many cases occurred and died. At Libertad, in March, 60 eases and 23 deaths were reported.

Plague is primarily a disease of rodents. The bacillus is pathogenic for rats, mice, guinea-pigs, rabbits, for the California 
ground squirrel, ${ }^{19}$ and for various species of ground moles such as the Manchurian tarbagan (Aretomys bobac). ${ }^{20}$

The spread of plague by rats has long been recognized and even in ancient times mortality in rats has been associated with large epidemic outbreaks. The most important recent experimental work on this matter was done by the British Indian Plague Commission at Bombay. This commission demonstrated the relationship between rats and plague infection in carefully conducted experiments in which considerable numbers of rats were used. According to the Commission, the most important species of rats are the Epimys norvegicus and Epimys rattus. Over thirteen hundred of some seventeen hundred rats found infected, belonged to these two species. Other rats can also be infected and the danger of plague exists wherever rats are found.

The rat problem is a very important one, not only in connection with plague, but in connection with economic loss as well. Creel of the United States Public Health Service ${ }^{21}$ has ealled attention to the necessity of rat extermination for economic reasons alone. The distribution and number of rats in the world is much greater than anyone ordinarily supposes. Creel states that in the cane producing tropical and semi-tropical countries, Porto Rico, the West Indies, the Hawaiian Islands and the Philippines, there is an enormous rat population. He states that on one cane plantation in Porto Rico where there were less than 500 people, 25,000 rats were killed in six months. He estimates that in the United States the rat population is probably as great as the human population, and the annual economic cost per rodent is higher than $\$ 1.00$ a piece. Computing the upkeep of rats as one-half cent per day and estimating their number as above, Creel says that a sum of $\$ 167,000,000$ is lost annually to the country by rat depredations.

According to the British Plague Commission, the usual way by which rats are infected from others is by means of fleas, and this, as first suggested in 1898 by Simond, is the method by which the disease is carried to man. In the British Indian Plague Commission experiments, when healthy and infected rats, entirely free from fleas were placed together, no plague developed, even when these rats were in contact with the urine and feces of the infected ones and with polluted food. But when fleas were introduced, infection occurred. The most

${ }^{19}$ McCoy, Jour. Infec. Dis., 5, 1909.

${ }^{20} \mathrm{~W} u$ Lien Teh., Jour. Hygiene, 13, 1913.

${ }^{21}$ Creel, Rep. No. 135, U. S. Pub. Health Serv., Vol. 28, No. 27, 1913. 
common flea found on rats is the Xenopsylla cheopis. The disease can also be transmitted by Ceratophyllus fasciatus and by Pulex irritans. Fleas habitually infesting dogs and cats may also infest rats which means that flea extermination must be general. It also indicates that the climatic and geographical distribution of fleas, as well as that of rats, must be taken into account in dealing with the disease.

In rats the first development is a generalized blood infection during which enormous numbers of bacilli may be present in the blood. These are then taken into the intestine of the flea where they can live for a long time, and may be deposted upon the skin of the victim during feeding, since the flea is apt to regurgitate blood and to deposit feces at this time. It may also be that some of the bacilli are directly introduced with the bite, but it is probably more common that the organisms thus distributed will be rubbed in either by the clothing or in scratching the fleabite.

It is thus established with considerable certainty that while contact infection and other means of direct and indirect transmission may, of course, occur, the usual manner of spread of plague is from rat to rat, rat to man, or man to man, by the agency of fleas. It is the Epimys rattus which lives in closest relationship to man, and is perhaps the most dangerous for this reason. The ordinary rat flea leaves the body of the rat within about three days of its death and is capable of remaining alive about three or four weeks. The plague bacilli may multiply tremendously in the intestine of the flea during the period between feedings. In the California outbreak infection from ground squirrels to man was definitely shown in a number of cases, and in Manchuria the tarbagan mentioned above has also been suspected of being the direct source.

$\mathrm{McCoy}^{22}$ in 1921, summarizing the results of recent plague studies, states that in the United States natural infection has taken place among ground squirrels of California, the black rats of Hawaii, and a species of wood rat and field rodent in Louisiana. Human cases have been unquestionably traced to ground squirrels, and almost always, he says, have the peculiarity of showing the primary bubos in the axillae, because the fleas in the course of the infection, attack the upper extremities, whereas when the disease is contracted from rats, the fleas are more apt to bite on the legs. Squirrel

${ }^{22}$ McCoy, Amer. Jour. Hyg., March, 1921. 
infection, however, according to McCoy, form very few cases, not more than about seventeen in all having been found since the squirrel origin was first studied. The squirrel flea can carry plague from squirrel to squirrel and from squirrel to other rodents.

Such transmission does not hold good, however, for the pneumonic form of the disease. Careful studies have been made on the pneumonic form by Strong, Teague, Crowell and Barber ${ }^{23}$ who observed the Manchurian epidemic which occurred during the winter of 1910 to 1911, and during which, within three months, 50,000 people died of the disease. According to these writers the infection here is not as formerly supposed primarily a septicemic condition, during which the lungs become secondarily involved, but occurs by direct inhalation into the bronchi. The organisms either pass along the bronchioles into the alveoli, or through the walls of the bronchioles into the lungs, giving rise first to peribronchial inflammations and later to more diffuse processes, followed by pneumonic changes of the lobar or lobular type. After this, the blood becomes quickly infected and bacteriemia is, therefore, secondary to pneumonia. As mentioned above, the organisms are coughed out with the droplets of sputum, and thus sprayed into the atmosphere. If the atmosphere is dry, they will rapidly die out. If, however, the weather is cold and the atmosphere charged with moisture the organisms may remain alive for considerable periods and inhalation of virulent organisms may take place easily. Acording to the same writers, the organisms are not usually exhaled by the expired air during ordinary respiration or even during the labored respirations of the pneumonic case, but only during coughs when they may be sprayed out in enormous numbers even when the naked eye can detect no visible spray. In this form of plague, then, the transmission is very largely direct.

McCoy states that pneumonic plague rarely occurs from rat infection, and states that it is an interesting and perhaps "significant fact" that in plague squirrels there seems to be a definite tendency to localize in the lungs, a thing which rarely happens in rats. From a study of the plague cases in the United States, he states that except for one single focus of thirteen cases, this form of the disease has not occurred. This pneumonic outbreak originated from a bubonic case of squirrel origin which developed secondary pneu-

${ }^{23}$ Strong, Teague, Crowell and Barber, Philippine Jour. Science, Sec. B, 7, 1912. 
monia and spread through four transmission generations in man in the autumn of 1919 .

Plague Prevention.-From what has been said in regard to the transmission of the disease it is apparent that the prevention of plague becomes very largely a question of rat extermination and protection against fleas. Vigilance in observation of the mortality among rats in endemic centers, for the discovery of early rodent foci is important. International precautions depend upon quarantine against rats which may easily be carried, and have been carried from country to country, by ships and by rail. The disinfestation of ships by $\mathrm{SO}_{2}$ by means of the Clayton apparatus, and by hydrocyanic acid gas as described by Creel and Faget of the United States Public Health Service, are among the important methods in use for the disinfestation of ships, sleeping cars, etc. Quarantine regulations and the supervision of incoming ships is important. In the United States a quarantine of seven days is imposed on ships arriving from plague ports, a period which is probably not long enough. Precautions must be taken to prevent the travel of rats along hawsers when ships are docked at a wharf, and this is usually accomplished by the application of large circular shields along the course of the hawsers in such a way that rats cannot cross.

When foci of plague are discovered in any community, wholesale rat destruction and isolation of the focus, by destruction of buildings, ratproofing of cellars, etc., must be resorted to. Blake has introduced a system of which Castellani and Chalmers speak very highly, the principle of which is that the rat extermination and other precautionary measures are started in a wide circle about the focus, working in toward the center, since work beginning at the focus itself in an outward circle may easily serve to scatter rats, rather than circumscribe them. In the Philippines and in villages in which natives live in primitive huts, actual burning of the houses has been resorted to, but this, too, may easily result in merely scattering the rat population into the neighboring districts. On a large scale, rat extermination is usually carried out by poisons in which phosphorous paste is perhaps the most important method. Of especial importance is the protection of food stores, and particular attention to all depositories of food, grain, etc., about which rats are apt to accumulate.

Bacteriological Diagnosis of Suspected Plague Cases.-Since the bacteriological diagnosis of the earliest cases that occur is one of 
the most important problems of prevention, various governments have laid down methods of collection and shipment of material that should be followed in the case of suspected cases in man and rats. Public Health Reports, Volume 35, Number 37, lays down the method in which material is to be collected for the United States. This we quote in toto from this Bulletin as follows:

To the Officers of the Public Health Service and State and Local Health Officers:

Owing to the appearance of plague in several American ports it is important that all cases of suspected plague, both in man and animals, be subjected to a bacteriological examination.

1. The following material from persons or rodents suffering from plague may be sent to laboratories:

\section{Human Cases (Living)}

(a) Pus or gland fluid from buboes aspirated by syringe or collected after incision, on agar slants.

(b) Portions of tissues affected, removed at operation, in sterilized bottles, securely stoppered.

(c) Blood specimens, in sterilized sealed glass ampules or test tubes.

(d) Cultures of suspected organisms, on agar slants.

\section{Human Cases (Necropsy)}

(a) Portions of the affected tissues-preferably bubo, lung and spleenin sterilized glass bottles, securely stoppered.

\section{Rodents}

(a) The whole rodent carcass, in fruit preserving jar.

2. Do not place tissues or rodents in a preservative. The bacteriological diagnosis of plague rests upon the production of the disease in laboratory animals; and the isolation and growth of the causative organism, Bacillus pestis. Any preservative that kills this organism will defeat the purpose of the examination. If decomposition of the specimen is feared, it may be placed in a tight container and this in turn surrounded by ice in a larger container, preferably of wood. Every specimen should be plainly marked preferably by ordinary peneil, showing the date and the exact location from which it was taken.

3. The shipper must make certain that the specimen is packed in such manner as to prevent possible danger to those handling the same, provided the package is properly handled.

In this connection it is necessary that specimens be wrapped in sufficient cotton or other absorbent material, to prevent leakage of fluid from the container should the glass be broken.

The Following Instructions should be explicitly observed.

1. Ship by express-Federal laws prohibit the shipping of plague-infected material, or cultures, by mail. 
2. Do not make packages too small, as small packages are more likely to be lost in transit, or overlooked.

3. Each package should be marked as follows:

Notice

This package contains perishable specimens

for bacteriological examination

Please Expedite

Careful autopsy must of course be made on all cases, animal or man, and the lesions studied. The lesions in rats have been fully described in another section. Cultures are taken on agar and smears taken from buboes or sputum, stained by Loeffler's methylene-blue, the bipolar appearance and degeneration forms of the organisms looked for. Cultural diagnosis is then made by the appearance of the growing organisms, and their colonies, the staining properties, appearance on salt agar, agglutination in immune sera, and, above all, inoculation of rats and guinea-pigs with observation of the characteristic lesions in these animals.

Since the examination of rats for plague is an important phase of the study of epidemics, it may be well to review the typical lesions in these animals as described by an experienced American student of plague, George W. McCoy. ${ }^{24}$ MeCoy, agreeing with the Indian Plague Commission, states that the naked eye is superior to the microscopical examination. There is engorgement of the subcutaneous vessels and a pink coloration of the muscles. The bubo when present is sufficient for diagnosis. Marked injection surrounds it and sometimes there is hemorrhagic infiltration. The gland itself is firm but usually caseous or occasionally hemorrhagic. In the liver there is apparent fatty change, but this is due to necrosis. Pin-point spots give it a stippled appearance as though it had been dusted with pepper. Pleural effusion is an important sign. The spleen is large, friable, and often presents pin-point granules on the surface. One or two per cent of rats may present no gross lesions. Cultures should of course be made. The method of examination consists in immersing the rat in any convenient antiseptic to kill fleas and other ectoparasites. The rats are nailed by their feet to a shingle and the skin is reflected from the whole front of the body and neek so as to expose the cervical, axillary, and inguinal regions. The thoracic and abdominal eavities are then opened and examined.

${ }^{24}$ McCoy, Jour. of Inf. Dis., vi, 1909; George W. McCoy, Public Health Reports, July, 1912. 
An excellent example of the circumscription of a plague focus at its first discovery is one which we take from a note by Rueker in the United States Public Health Service Report, No. 28, 1915, based largely on the work of Passed Assist. Surg. R. A. Kearny.

In September, 1914, a dead Mus norvegicus was found on a street corner in New Orleans. Laboratory examination proved this plague infected. The district was searched for other rats and on the 16th of September a similar plague rat was found in the neighborhood in a Chinese restaurant located in a ramshackle frame building, situated between a rat-proof brick building and an open lot. Behind the restaurant was a frame shed which was not rat proof. A survey of the district followed, in which thirty-eight infected rats were discovered, all of them of the same species as the preceding. One hundred and thirteen dead rats were found, and two infected rats were found on a neighboring street corner. Twenty-one were found in the Chinese restaurant, and one in the open lot and the other in the neighborhood. Rucker believes that the focus was eliminated largely because there was plenty of food in this particular neighborhood, and rats could not easily leave there without entering the street, a thing which they would have done only under the pressure of shortage of food supply. He calls attention to the fact that if this had been a focus of Mus ratus or Mus alexandrinus which are climbing rats, the original focus would rapidly have been spread. But since the Mus norvegicus is a ground rat, it was closed in by the neighboring brick walls. In the operations following these discoveries, the building ehiefly infested was torn down and the frame sheds behind it were rendered not inhabitable for rats. Many rats were found dead and a considerable number were killed. Fumigation was carried out on the premises, and these and other premises washed down with tank oil for the purpose of killing fleas. Very few rats escaped. An interesting control was carried out which has been introduced into plague campaigns, namely, that guinea-pigs were placed into the fumigated premises after fumigation. One of these contracted plague and died, and the place was, therefore, refumigated. Guinea-pigs which had been used as controls in other places remained alive. Only one human case was attributable to this focus.

A Bulletin published by the United States Public Health Service in November, 1920, (35, No. 45) has laid down ordinances for ratproofing. These we quote in toto directly from this Bulletin.

"The rat-proofing of buildings is generally secured either by elevation of the structure, with the underpinning open and free, or by marginal rat-proof walls of concrete or of stone or brick laid in cement mortar, sunk two feet into the ground, and fitting flush to the floor above. The wall must fit tightly to the flooring and not merely extend to the joists or 
supporting timbers, as this would result in open spaces, permitting the entrance of rodents. Groceries, stables, warehouses, markets, and food depots in general are best rat-proofed by having a concrete floor in addition to concrete walls. In these structures, untenanted as they are at night, rats might well enter by a doorway or window carelessly left open, or be introduced concealed in merchandise, and, gnawing through plank flooring, obtain a well-protected hiding place.

"In addition to concrete floor and walls, these food depots must have tight-fitting doors, and all windows and other openings should be properly screened. A 12-guage wire is preferable on account of its strength and durability, and the mesh should not be larger than one-half inch.

"Rat-proofing by elevation of the building is chiefly applicable to small and medium sized frame dwellings. The purpose is to have a sufficient elevation, about two feet, so that the ground area beneath will be as exposed and free from covert as land unbuilt upon. Marginal rat-proofing will suffice in more pretentious dwellings where sufficient care can be exercised to prevent rats from gnawing through the plank floors.

"Chicken pens can be protected by marginal concrete walls, sunk into the ground two feet or more, and by covering the sides and top with $1 / 2$-inch mesh wire netting. Garbage eans should be made of serviceable metal and should have properly fitting tops.

"Plank sidewalks and plank coverings for yards should be avoided. Cinders and concrete should be used instead. The latter should have marginal protection to prevent rats from burrowing beneath it.

"Double walls, with a dead space between, should be avoided, or, if used, they should be rat-proofed at the top and bottom with heavy wooden timbers, 4 by 4 -inch fillers, or by a concrete fill. Atties should be well opened and kept free of rubbish or other refuge for rats.

"These precautions against rat harborage and for the protection of food supplies, in connection with careful trapping and poisoning, will be attended with considerable success in the destruction of rats.

"The appended model ordinance is applicable, with perhaps slight modifieations, to any urban community. It should be examined by competent local counsel for changes in form, or in substance if necessary, as dictated by special constitutional, legislative, or charter considerations."

Plague Vaccination.-The immunization of animals with suspensions of plague bacilli, killed by moderate heating, $50^{\circ}$ for one hour, was first attempted by Yersin, Calmette and Borrel in 1897. Kolle, ${ }^{25}$ Haffkine ${ }^{26}$ and others studied plague vaccination particularly in the subsequent years. A great many different vaccines have been

${ }^{2}$ Kolle, loc. cit.

*Haffkine, loc. cit. 
introduced since then. The one most extensively used is that of Haffkine, which consists of cultures grown in broth in shallow bottles for six weeks at room temperature and shaken once a day. At the end of this time they are sterilized at $65^{\circ}$ for several hours. The material then consists of degenerated organisms and extracts of the organisms. Glycerinated broth cultures have been introduced, but so far have had little practical application. The German Plague Commission $^{27}$ in 1899 introdueed the use of heated cultures to which 0.5 per cent earbolic acid had been added. Strong ${ }^{28}$ believing that attenuated living bacilli might be more efficient than dead cultures, produced vaceines from a three year's' old laboratory culture, subsequently eultivated at temperatures above $41^{\circ}$. These living cultures after such treatment had lost their virulence for guinea-pigs and monkeys almost completely. He vaccinated forty-two individuals with these cultures without harm, and with resulting development of specific antibodies. The method is probably quite efficient but because of the possible danger involved in it, has not been extensively employed. The so-called nueleo-protein vaccins of Lustig and Galeotti, ${ }^{29}$ that is, plague bacilli extracted with weak alkalin solutions and precipitated with acid in the cold, have been studied extensively by Rowland ${ }^{30}$ and others, and Rowland made several vaceines of his own in one of which he extracted the bacterial mass with sodium sulphate. He also used cultures killed with chloroform.

None of these vaccines have had any extensive application except that of Haffikine which has been used by the British Government Sanitary organization in India on a very large scale. Bannermann, Bitter and more recently, Major Glen Liston ${ }^{31}$ have analyzed the results obtained with Hafflkine's virus of which over eight million doses were distributed in India between 1886 and 1899. Aceording to these studies the Haffkine virus seems to be of definite prophylactic value, though not completely protective as one would cxpect from the nature and virulence of the disease. Major Liston states in his report of the Bombay Bacteriological Laboratory for the years 1913 to 1916, that it is quite impossible to give any positive statement for India, but that in isolated epidemics in which careful figures

${ }^{27}$ German Plague Commission Report, 1899.

${ }^{28}$ Strong, Philip. Jour. Science., Sec. B, 1907 and 1912.

${ }^{20}$ Lustig and Galeotti, Deut. med. Woch., 1897-1912.

${ }^{80}$ Rowland, Journal of Hygiene, 1910-1914.

${ }^{21}$ Liston, Maj. Glen, Bombay Bacter. Lab. Rep., 1913-1916. 
could be secured, the indications are that the vaccine has been of great value. In one town in India, 794 individuals were inoculated, and 286 uninoculated, and among the inoculated there were only twelve cases and three deaths, while among the smaller number of the untreated, thirty cases developed and twenty-five died. In one house there were four vaccinated and three unvaccinated. All of the unvaceinated died, and only one of the vaccinated contracted the disease and he recovered. A number of similar studies are eited by Major Liston. There seems, therefore, to be very little doubt as to the protective value of some form of plague vaccination. Whether or not the Haffkine virus is the most useful and final method, cannot of course be stated at the present time.

\section{THE PLAGUE-LIKE DISEASE OF RODENTS (McCOY) ${ }^{32}$}

\section{Bacterium Tularense (McCoy and Chapin) ${ }^{33}$}

McCoy has described a disease occurring in Californian ground squirrels (Citellus beechyi) which presents lesions very similar to those of plague in these animals. In fact the disease was noticed in the course of the systematic examination of rodents by McCoy at the Federal Laboratory in San Francisco. Although MeCoy was able to transmit the disease to guinea-pigs, mice, rabbits, monkeys, and gophers, and plague-like lesions could be produced in most of the animals, he was at first entirely unable to cultivate any organism from these lesions. In 1912 McCoy and Chapin finally succeeded in growing the specific bacterium on an egg medium made entirely of the yolk. Morphologically it is a very small rod, 0.3 to 0.7 micron in length and often capsulated. The rods stain poorly with methylene blue, better with carbol fuchsin or gentian violet. They are found in large numbers in the spleen of animals dead of the disease.

\section{THE BACILLI OF THE HEMORRHAGIC SEPTICEMIA GROUP}

In many of the lower animals there occur violently acute bacterial infections characterized by general septicemia, usually with petechial hemorrhages throughout the organs and serous membranes and severe intestinal inflammations. These diseases, spoken of as the "'hemorrhagic septicemias," are caused by a group of closely allied

${ }^{22}$ McCoy, U. S. Public Health, Bull. 43, 1911.

${ }^{83}$ McCoy and Chapin, Jour. of Inf. Dis., x, 1912. 
bacilli, first classified together by Hueppe ${ }^{34}$ in 1886 . Some confusion has existed as to the forms which should be considered within Hueppe's group of "hemorrhagic septicemia," a number of bacteriologists including, in this class bacilli such as Loeffler's Bacillus typhi murium, and Salmon and Smith's hog-cholera bacillus, microorganisms which, because of their motility and eultural characteristies, belong more properly to the "Gärtner," "enteritidis," or "paratyphoid" group, intermediate between colon and typhoid.

The organisms properly belonging to this group are short bacilli, more plump than are those of the colon type, showing a marked tendeney to stain more deeply at the poles than at the center. They are non-motile, possess no flagella, and do not form spores. They grow readily upon simple media, but show a very marked preference for oxygen, growing but slightly below the surface of media. By some observers they are characterized as "obligatory aërobes," but this is undoubtedly a mistake.

While showing considerable variations in form and differences in minor cultural characteristies, the species characteristies of polar staining, decolorization by Gram, immobility, lack of gelatin liquefaction, and great pathogenicity for animals, stamp alike all members of the group. Its chief recognized representatives are the bacillus of chicken cholera, the bacillus of swine-plague (Deutsche Schweineseuche), and the Bacillus pleurosepticus which causes an acute disease in eattle and often in wild game.

Because of certain cultural and pathogenic characteristics, it seems best to consider the bacillus of bubonic plague with this group.

\section{BACILLUS OF CHICKEN CHOLERA}

\section{(Bacillus avisepticus)}

The bacillus of chicken cholera was first carefully studied by Pasteur ${ }^{35}$ in 1880. It is a short, non-motile bacillus, measuring from 0.5 to 1 micron in length. Stained with the ordinary anilin dyes, it displays marked polar staining qualities, which often give it the appearance of being a diplococeus. It is decolorized by Gram's method. It does not form spores, but may occasionally form vacuolated degeneration forms, not unlike those described for Bacillus pestis.

${ }^{34}$ Hueppe, Berl. klin. Woch., 1886.

as Pasteur, Comptes rend. de l'acad. des sci., 1880. 
The bacillus is easily cultivated from the blood and organs of infected animals, it grows well upon the simplest media at temperatures varying from $25^{\circ}$ to $40^{\circ} \mathrm{C}$. In broth, it produces uniform clouding with later a formation of a pellicle. Upon agar it forms, within twenty-four to forty-eight hours, minute colonies, white or yellowish in color, which are at first transparent, later opaque. Upon gelatin, it grows without liquefaetion. Upon milk, the growth is slow and does not produce coagulation. According to Kruse, ${ }^{36}$ indol is formed from pepton bouillon. Aeid, but no gas, is formed in sugar broth.

Among barnyard fowl, this disease is widely prevalent, attacking chickens, ducks, geese, and a large variety of smaller birds. The infection is extremely acute, ending fatally within a few days. It is accompanied by diarrhea, often with bloody stools, great exhaustion, and, toward the end, a drowsiness bordering on coma. Autopsy upon the animals reveals hemorrhagic inflammation of the intestinal mucosa, enlargement of the liver and spieen, and often bronchopneumonia.

The specific bacilli may be found in the blood, in the organs, in exudates, if these are present, and in large numbers in the dejecta. Infection takes place probably through the food and water contaminated by the discharges of diseased birds. ${ }^{37}$

Subeutaneous inoculation or feeding of such animals with pure cultures, even in minute doses, gives rise to a quickly developing septicemia which is uniformly fatal. The bacillus is extremely pathogenie for rabbits, less so for hogs, shecp, and horses, if infection is practiced by subcutaneous inoculation. Infection by ingestion does not seem to cause disease in these animals.

Historically, the bacillus of chicken cholera is extremely interesting, since it was with this microorganism that Pasteur ${ }^{35}$ earried out some of his fundamental researches upon immunity, and sueceeded in immunizing chickens with attenuated cultures. The first attenuation experiment made by Pasteur consisted in allowing the bacilli to remain in a broth culture for a prolonged period without transplantation. With minute doses of such a culture (vacein I) he inoculated chickens, following this, after ten days, with a small dose of a fully virulent culture. Although enormously imporfant in principle, the practical results from this method, as applied to

${ }^{86}$ Kruse, in Flügge's "Die Mikroorganismen.",

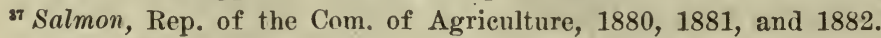


chicken cholera, have not been satisfactory. It was with this bacillus, furthermore, that Pasteur was first able to demonstrate the existence of a free toxin which could be separated from the bacteria by filtration.

\section{BACILLUS OF SWINE PLAGUE}

\section{(Bacillus suisepticus, Schweineseuche)}

This microorganism is almost identical in form and cultural characteristics with the bacillus of chicken cholera. It is non-motile, forms no spores, is Gram-negative, and does not liquefy gelatin. The bacillus causes an epidemic disease among hogs, which is characterized almost regularly by a bronchopneumonia followed by general septicemia. There is often a sero-sanguineous pleural exudate, a swelling of bronchial lymph glands and of liver and spleen. The gastrointestinal tract is rarely affected. The bacilli at autopsy may be found in the lungs, in the exudates, in the liver and spleen, and in the blood. The disease is rarely acute, but, in young pigs, almost uniformly fatal.

It is probable that spontaneous infection usually occurs by inhalation. Experimental inoculation is successful in pigs, both when given subcutaneously and when administered by the inhalation method. Mice, guinea-pigs, and rabbits are also susceptible, dying within three or four days after subcutaneous inoculation of small doses.

Active and passive immunization of animals against Bacillus suisepticus has been attempted by various observers. Active immunization, if carried out with care, may be successfully done in the laboratory. Passive immunization of animals with the serum of actively immunized horses has been practiced by Kitt and Mayr, ${ }^{38}$ Schrieber, ${ }^{39}$ and Wassermann and Ostertag. The last-named observers, working with a polyvalent serum produced with a number of different strains of the bacillus, have obtained results of considerable practical value. The researches of Kitt and Mayr have revealed a fact pointing to the interrelationship of the bacilli of the "hemorrhagic septicemia" group. They were able to show that the serum of horses immunized with chicken cholera bacilli was able to protect, somewhat, against Bacillus suisepticus.

\footnotetext{
${ }^{38}$ Kitt and Mayr, Monatsschr. f. Thier Leilk., vol. 8, 1897.

${ }^{39}$ Schrieber, Berl. Thierärztl. Wochenschr., vol. 10, 1899.
} 
Infection with the bacillus of swine plague, in hogs, is often accompanied by an infection with the hog-cholera bacillus (Schweinepest). The latter, as we have seen, is a microorganism belonging to the enteritidis group, intermediate between Bacillus coli and Bacillus typhosus, and differing from suisepticus in being actively motile, possessing flagella, not showing the polar staining, having a more slender morphology, and producing gas upon dextrose broth. A confusion between the two bacilli frequently occurs because of their nomenclature. Bacteriologically and pathogenically, they are quite distinct. Bacillus suisepticus produces an acute septicemia, accompanied by bronchopneumonia and usually not affecting the gastro-intestinal canal. The bacillus of hog cholera produces an infection localized in the intestinal canal. 


\title{
CHAPTER XLII
}

\author{
ASIATIC CHOLERA AND THE CHOLERA ORGANISM
}

(Spirillum cholerœ asiaticœ, Comma Bacillus)

THE organism of Asiatic cholera was unknown until 1883. In this year, Koch, ${ }^{1}$ at the head of a commission established by the German government to study the disease in Egypt and India, discovered the "comma bacillus" in the defecations of patients, and satisfactorily determined its etiological significance.

Koch's investigations were carried out on a large number of cases and many investigations have since then corroborated his results.

Apart from the evidence of the constant association of the cholera spirillum with the disease, the etiological relationship has been clearly demonstrated by several accurately recorded accidental infections occurring in bacteriological workers, and by the famous experiment of Pettenkofer and Emmerich, who purposely drank water containing cholera spirilla. Both observers became seriously ill with typical clinical symptoms of cholera, and one of them narrowly escaped death.

Morphology and Staining.-The vibrio or spirillum of cholera is a small curved rod, varying from one to two micra in length. The degree of curvature may vary from the slightly bent, comma-like form to a more or less distinct spiral with one or two turns. The spirals do not lie in the same plane, being arranged in corkscrew fashion in three dimensions. The spirillum is actively motile and owes its motility to a single polar flagellum, best demonstrated by Van Ermengem's flagella stain. Spores are not found. In young cultures the comma shapes predominate, in older growths the longer forms are more numerous. Strains which have been cultivated artificially for prolonged periods without passage through the animal body have a tendency to lose the curve, assuming a more bacilluslike appearance. The spirilla are stained with all the usual aqueous anilin dyes. They are decolorized by Gram's method. In histologica]

\footnotetext{
${ }^{1}$ Koch, Deut. med. Woch., 1883 and 1884.
} 
section they are less easily stained, but may be demonstrated by staining with alkaline methylene blue.

Cultivation.-The cholera spirillum grows easily upon all the usual culture media, thriving upon meat-extract as well as upon meat-infusion media. Moderate alkalinity of the media is preferable, though slight acidity does not prevent growth.

In gelatin plates growth appears at room temperature within twenty-four hours as small, strongly refracting yellowish-gray, pinhead colonies. As growth increases the gelatin is fluidified. Under

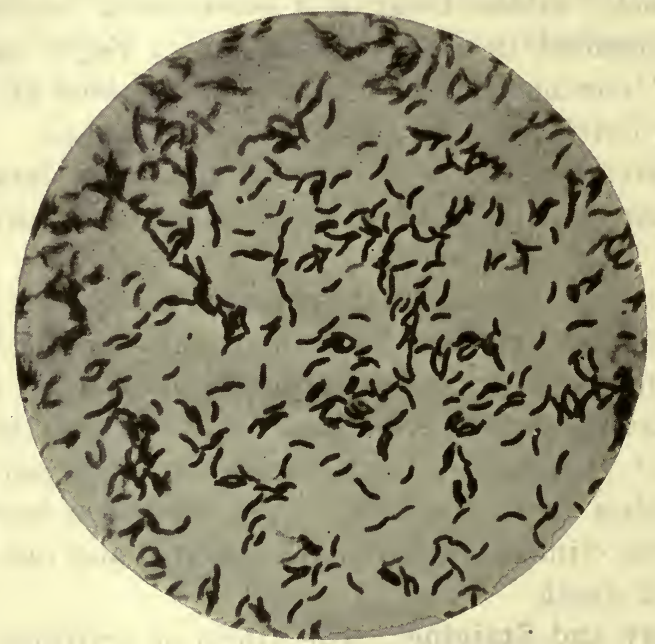

Fig. 87.-Cholera Spirillum. (After Fränkel and Pfeiffer.)

magnification these colonies appear coarsely granular with margins irregular because of the liquefaction. Liquefaction, too, causes a rapid development in such colonies of separate concentric zones of varying refractive power. Old strains, artificially cultivated for long periods, lose much of their liquefying power.

In gelatin stab cultures fluidification begins at the surface, rapidly giving rise to the familiar funnel-shaped excavation.

Upon agar plates, within eighteen to twenty-four hours, grayish, opalescent colonies appear, which are as a rule easily differentiated by their transparency from the other bacteria apt to appear in feces. Agar plates, therefore, are important in the isolation of these organisms. 
Coagulated blood serum is liquefied by the cholera vibrio. On potato, growth is profuse and appears as a brownish coarse layer. In milk, growth is rapid and without coagulation. In broth, general clouding and the formation of a pellicle result. The rapidity and luxuriance of growth of the cholera spirillum upon alkaline pepton solutions render such solutions peculiarly useful as enriching media in isolating this microorganism from the stools of patients. In pepton solution, too, the cholera spirillum gives rise to abundant indol, demonstrated in the so-called "cholera-red" reaction. This reaction has a distinct diagnostic value, but is by no means specific. In the case of the cholera spirillum the mere addition of strong sulphuric acid suffices to bring out the color reaction. This is due

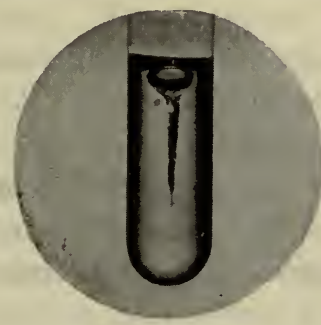

FIG. 88.

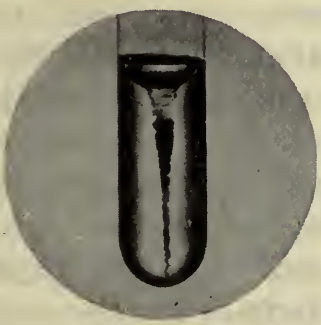

FIG. 89.

Fig. 88.-Cholera Spirillum. Stab Culture in Gelatin, three days old.

Fig. 89.-Cholera Sprrillum. Stab Culture in Gelatin, six days old. (After Fränkel and Pfeiffer.)

to the fact that, unlike some other indol-producing bacteria, the cholera organism is able to reduce the nitrates present in the medium to nitrites, thus itself furnishing the nitrite necessary for the color reaction. The medium which is most suitable for this test is that proposed by Dunham, ${ }^{2}$ consisting of a solution of 1 per cent of pure pepton and .5 per cent $\mathrm{NaCl}$ in water.

Dieudonné has recommended a selective medium upon which cholera spirilla will grow well, but upon which the colon bacillus will grow either very sparsely or not at all. Cocei will produce minute pin-point colonies only and other common bacilli like those of the proteus group will grow hardly more easily than bacillus coli. Its preparation is very simple.

${ }^{2}$ Dunham, Zeit. f. Hyg., ii, 1887.

'Dieudonné A., Cent. Bakt., 1., orig., 1909. 
To seventy parts of ordinary 3 per cent agar, neutralized to litmus, there are added thirty parts of a sterile mixture of equal parts of defibrinated beef blood and normal sodium hydrate.

The latter is sterilized by steam before being added to the agar. This pure alkali agar is poured out in plates and allowed to dry several days at $37^{\circ}$ or five minutes at $60^{\circ}$.

The material to be examined is smeared upon the surface of these plates with a glass rod. If the blood-alkali mixture is prepared beforehand and allowed to stand for four or five weeks, the plates may be used immediately after pouring (Teague).

The principle of this medium is that cholera. will grow in the presence of an amount of alkali which inhibits other fecal bacteria.

For other cholera media see the section on media in the first part of this book.

The rational basis for the isolation of cholera spirilla from fecal or other material is found in two chief properties of the spirilla. These have been described to us by Teague as follows: (1) It grows on media of an alkalinity that retards or completely inhibits the growth of most of the fecal bacteria. (2) It eomes to the surface of fluid media, rich in oxygen, to a greater extent than do the fecal bacteria.

The best results in the practical isolation of the cholera spirilla from stools are obtained by making use of both of these properties from the beginning. A portion of the stool is seeded directly into alkalin-pepton water. The broth used should be distinetly alkalin, titrated to -0.5 , or -1.0 , with phenolphthalein. After six to twelve hours, a loopful from the surface of these pepton water tubes is plated upon plates of Dieudonné's medium, and is also transferred to a second series of alkalin-pepton water tubes. Onee isolated, the spirilla are identified by their morphology and motility, by the appearance of their colonies, by their manner of growth upon gelatin stabs, by the eholera-red reaction, and, finally, by agglutinative tests in immune sera. Owing to the existence of other spirilla morphologically and culturally similar, the serum reactions are the only absolutely positive differential criteria.

For isolation of the bacteria from water, it is, of course, necessary to use comparatively large quantities. Flügge and Bitter advise the distribution of about a liter of water in ten or twelve Erlenmeyer flasks. To each of these they add 10 e.e. of sterile pepton-salt solution (pepton ten per cent, $\mathrm{NaCl}$ five per cent). After eighteen 
hours at $37.5^{\circ} \mathrm{C}$. the surface growths in these flasks are examined both microscopically and culturally as before.

Biological Considerations.-The cholera spirillum is aërobic and facultatively anaërobic. It does not form spores. The optimum temperature for its growth is about $37.5^{\circ} \mathrm{C}$. It grows easily, however, at a temperature of $22^{\circ} \mathrm{C}$. and does not cease to grow at temperatures as high as $40^{\circ}$. Frozen in ice, these bacteria may live for about three or four days. Boiling destroys them immediately. A temperature of $60^{\circ} \mathrm{C}$. kills them in an hour. In impure water, in moist linen, and in food stuffs, they may live for many days. Associated with saprophytes in feces and other putrefying material, and wherever active acid formation is taking place, they are destroyed within several days. Complete drying kills them in a short time. The common disinfectants destroy them in weak solutions and after short exposures (carbolic acid, five-tenths per cent in one-half hour ; bichlorid of mercury, $1: 100,000$ in ten minutes ; mineral acids, $1: 5,000$ or 10,000 in a few minutes).

Cholera in Man.-In man the discase is contracted by ingestion of cholera organisms with water, food, or any contaminated material. The disease is essentially an intestinal one. The bacteria, very sensitive to an acid reaction, may often, if in small numbers, be checked by the normal gastric secretions. Having once passed into the intestine, however, they proliferate rapidly, often completely outgrowing the normal intestinal flora. Fatal cases, at autopsy, show extreme congestion of the intestinal walls. Occasionally ecchymosis and localized necrosis of the mucosa may be present and swelling of the solitary lymph-follicles and Peyer's patches. Microscopically the cholera spirilla may be seen to have penetrated the mucosa and to lie within its deepest layers close to the submucosa. The most marked changes usually take place in the lower half of the small intestine. The intestines are filled with the characteristically fluid, slightly bloody, or "rice-water" stools, from which often pure cultures of the cholera vibrio can be grown. The microorganisms can be cultivated only from the intestines and their contents, and the parenchymatous degenerations taking place in other organs must be interpreted as being purely of toxic origin.

There is at the same time a profound toxemia due, in part, at least to the absorbed cholera substanees.

The incubation time of the disease is usually short, lasting from a few hours to several days. The disease usually begins, with diar- 
rhea which gradually becomes more violent until the colorless typical, rice water stools appear. Castellani and Chalmers describe the further course as follows:

"Vomiting generally appears early, food being first expelled, followed later by watery fluid with which bile and oceasionally blood may be mixed. As the purging and vomiting persist the urine diminishes and may stop, and fluid departs from the subcutaneous tissues, which therefore contract so that the face alters, the nose becoming sharp, the cheekbones prominent, and eyes sunken and the skin of the fingers becomes wrinkled like that of a washerwoman."

A considerable rôle is played in the subsequent course of the disease by the depletion of water, with consequent aneuria, low blood pressure, eyanosis, acidosis, etc. The therapeutic effect of saline infusions is said to be astonishing.

Animal Pathogenicity.-In animals, cholera never appears as a spontaneous disease. Nikati and Rietsch ${ }^{4}$ have succeeded in producing a fatal disease in guinea-pigs by opening the peritoneum and injecting cholera spirilla directly into the duodenum. Koch ${ }^{5}$ succeeded in producing a fatal cholera-like disease in animals by introducing infected water into the stomach through a eatheter after neutralization of the gastric juice with sodium carbonate. At the same time, he administered opium to prevent active peristalsis. A method of infection more closely analogous to the infection in man was followed by Metchnikoff, who successfully produced fatal disease in young suckling rabbits by contaminating the maternal teat.

Subcutaneous inoculation of moderate quantities of cholera spirilla into rabbits and guinea-pigs rarely produces more than a temporary illness. Intraperitoneal inoculation, if in proper quantities, generally leads to death. It will be remembered that when working with intraperitoneal cholera inoculations the phenomenon of bacteriolysis was discovered by Pfeiffer.

Different strains of cholera spirilla vary greatly in their virulence. The virulence of most of them, however, can be enhanced by repeated passages through animals. Most of our domestic animals enjoy considerable resistance against cholera infection, though under experimental conditions successful inoculations upon dogs, cats, and mice have been reported. Doves are entirely insusceptible.

-Nikati und Rietsch, Deut. med. Woch., 1884.

${ }^{5}$ Koch, Deut. med. Woch., 1885. 
Cholera Toxin.-The absence of the cholera spirilla from the internal organs of fatal eases, in spite of the severe general symptoms of the disease, points distinctly to the existence of a strong poison produced in the intestine by the microorganisms and absorbed by the patient. It was in this sense, indeed, that Koch first interpreted the clinical picture of cholera. Numerous investigations into the nature of these toxins have been made, the earlier ones defective in that definite identification of the cultures used for experimentation were not carried out.

Pfeiffer, ${ }^{6}$ in 1892 , was able to show that filtrates of young bouillon cultures of cholera spirilla were but slightly toxie, whereas the dead bodies of carefully killed agar cultures were fatal to guinea-pigs cven in small quantities. In consequence, he regarded the cholera poison as consisting chiefly of an endotoxin. ${ }^{7}$ The opinion as to the endotoxic nature of the cholera poison is not, however, shared by all workers. Metehnikoff, Roux, and Salimbeni, ${ }^{8}$ in 1896, succeeded in producing death in guinea-pigs by introduction into their peritoneal cavities of cholera cultures enclosed in celloidin sacs. Brau and Denier, ${ }^{9}$ and, more recently, Kraus, ${ }^{10}$ claim that they have succeeded not only in demonstrating a soluble toxin in alkaline broth cultures of cholera spirilla, but in producing true antitoxins by immunization with such cultures. It appears, therefore, that the poisonous action of the cholera organisms may depend both upon the formation of true secretory toxins and upon endotoxins. Which of these is paramount in the production of the disease can not be at present definitely stated. In favor of the great importance of the endotoxic elements is the failure, thus far, to obtain successful therapeutic results with supposedly antitoxic sera.

Epidemiology.-Cholera is essentially a disease of man. Endemic in India and other Eastern countries, it has from time to time epidemically invaded large territories of Europe and Asia, not infrequently assuming pandemic proportions and sweeping over almost the entire earth. Five separate cholera epidemies of appalling magnitude occurred during the nineteenth century alone; several of

- Pfeiffer, Zeit. f. Hyg., xi, 1892.

'Pfeiffer und Wassermann, Zeit. f. Hyg., xiv, 1893.

${ }^{8}$ Metchnikoff, Roux, et Salimbeni, Ann. de l'inst. Pasteur, 1896.

' Brau et Denier, Comptes rend. de l'acad. des sci., 1906.

${ }^{10}$ R. Kraus, Cent. f. Bakt., 1906. 
these, spreading from India to Asia Minor, Egypt, Russia, and the countries of Central Europe, reached even to North and South America. The last great epidemic began about 1883, traveled gradually westward, and in 1892 reached Germany where it appeared with especial virulence in Hamburg, and thence, following the highways of ocean commerce, entered America and Africa. During this epidemic in Russia alone 800,000 people fell victims to the disease.

An important epidemiological fact is the existence of certain endemic foci where cholera is always going on and from which epidemics and pandemics originate. The chief endemic focus seems to be located in lower Burmah, and it is, as yet, an unsolved puzzle as to why the disease should remain smouldering in such regions and spread widely only at certain periods, five, ten or more years apart. During recent years important epidemics have occurred between the years 1879 and 1910. In 1879 an epidemic spread to Europe through Egypt and this outbreak is notable because in 1883 in Egypt, Koch, ${ }^{11}$ as head of the German Cholera Commission, isolated the cholera spirillum. In 1891 another great epidemic, originating in India, is stated by Castellani as having started on the occasion of a bathing festival held on the Ganges. It spread among pilgrims and reached Europe in 1892, appearing with particular virulence in Hamburg. From there it spread to America and Africa by ocean commerce. During this epidemic it is said that 800,000 people fell victims in Russia alone. Violle records that in 1908, 1909 and 1910 there were a series of epidemies in Russia. In 1908 there were about 30,000 cases with 14,000 deaths; in 1909 , there were 21,000 cases with 9,700 deaths, and in 1909 to 1910 there were 130,000 deaths. During the Balkan War in 1912 cholera appeared among the armies. During the late war there were cases of cholera in Galicia in the Austrian Army, and there were outbreaks in Bulgaria, Greece and Turkey, and in Mesopotamia.

The prevalence of cholera as an important epidemic disease may be estimated by the following chart of cholera epidemics of the last hundred years which is taken from Violle's recent work on cholera (1918) to which numerous references have been made.

The disease always originates from the dejecta of cholera patients and carriers. At times of epidemic, infection of the water and

${ }^{11}$ Koch, Deut. med. Woch., 1883 and 1884. 
food supplies naturally plays an important rôle, and in such epidemies, as the one in Hamburg, the water supply was primarily responsible. The distribution of the disease here followed definitely

\section{EPIDEMICS OF CHOLERA IN VARIOUS PLACES ${ }^{1}$}

\begin{tabular}{|c|c|c|}
\hline Place & Date & Deaths \\
\hline Havana. . . & 1833 & 8,000 \\
\hline Malta $\ldots \ldots \ldots \ldots \ldots \ldots$ & 1837 & 4,000 \\
\hline London . . . . . . . . . . . & 1832 & 4,000 \\
\hline Paris.................... & 1832 & 7,000 \\
\hline Basra.................... & 1821 & 5,000 \\
\hline Lahore............... & 1845 & 22,000 \\
\hline Tabriz........... & 1852 & 12,000 \\
\hline Teheran $\ldots \ldots \ldots \ldots \ldots \ldots$ & 1852 & 15,000 \\
\hline Bagdad $\ldots \ldots \ldots \ldots \ldots \ldots$ & 1852 & 2,000 \\
\hline Bellary and Mysore, India.... & 1865 & 40,000 \\
\hline Province of Bombay.......... & 1865 & 84,000 \\
\hline Cachemire................ & 1892 & 5,000 \\
\hline England................ & 1854 & 20,000 \\
\hline France $\ldots \ldots \ldots \ldots \ldots \ldots \ldots$ & 1854 & 140,000 \\
\hline Italy $\ldots \ldots \ldots \ldots \ldots \ldots \ldots$ & 1854 & 24,000 \\
\hline Egypt................... & 1865 & 60,000 \\
\hline Egypt.................. & 1883 & 50,000 \\
\hline Egypt.................. & 1831 & 150,000 \\
\hline Cairo.................... & 1831 & 36,000 \\
\hline Cairo..................... & 1902 & 33,000 \\
\hline 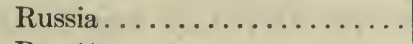 & 1909-10 & 130,000 \\
\hline Rosetta................ & 1865 & 2,168 \\
\hline \multicolumn{3}{|l|}{ Epidemic of Cholera in Russia } \\
\hline In $1908,30,000$ cases....... & & 14,000 \\
\hline In $1909,21,000$ cases. .... & & 9,700 \\
\hline
\end{tabular}

the distribution of the infected water supply and the organisms were isolated from the water. This epidemic is one of the classical water epidemics and has served more than any other water epidemic in impressing medical and health authorities with the importance of water supply supervision. In countries like India and Egypt, etc., where water supplies are often taken from collecting tanks not properly supervised, and from individual wells, and where the supervision of feces disposal is not strict, it is quite natural that distribution by water supplies should be extremely important. Violle adds a number of interesting instances of water transmission which occurred in France in 1885, in which the source was soiled linen 
washed in a stream with distribution of the disease further downstream in other villages, but not in-any of the villages higher up the river. It is also probable that in countries such as India, the custom of throwing dead bodies into rivers may contribute. materially to the constant presence of the disease.

In endemic centers, it is more than likely that the cholera carricr is a very important factor of distribution. The existence of the carrier is proven beyond doubt, and, as in typhoid, individuals may remain earriers for very long periods. Greig has shown that the organisms may live in the gall-bladders of human beings as in the typhoid carrier state. MeLaughlin ${ }^{12}$ has found as many as 7 per cent of the population of an infected district to be cholera carriers.

Again, as in typhoid, distribution of fecal material to food by flies probably plays a very important rôle, and according to Barber the organisms may live for some time in the intestines in such insects as cockroaches. Whether or not domestic animals can act as distributers of the organisms is uncertain. Violle quotes Haffkine as stating that he had found the spirilla in the intestines of cattle, and that they were found by Hahn in the intestines of cows during cholera epidemics. The importance of this, however, is still quite uncertain.

In nature the cholera spirilla may, under favorable conditions, remain alive for considerable periods. In drinking water they have been found alive after several days and they may remain alive for weeks in water supplies. From the investigations of Wernicke, ${ }^{13}$ Shirnoff ${ }^{14}$ and others it would appear that under favorable conditions the spirilla may remain alive in river water and other natural waters for weeks or even months. In milk and other foods, the longevity of the cholera spirilla seems to depend particularly upon the nature and numbers of other bacteria present and on the production of an acid reaction. In cholera stools they will remain alive until considerable putrefaction has taken place and, therefore, may be assumed under favorable conditions to live at least one day, or perhaps three days or longer. In cold weather when bacterial growth is more or less inhibited, they may remain alive much longer than this.

${ }^{12}$ McLaughlin, quoted from Rosenau's Preventive Medicine and Hygiene, D. Appleton and Co., New York and London, 1921.

${ }^{13}$ Wernicke, Hyg. Rundschau, 1895.

${ }^{14}$ Shirnoff, Cent. f. Bakt., 41, 1908. 
Cholera Immunization.-One attack of cholera confers protection against subsequent infection. Active immunization of animals may be accomplished by inoculation of dead eultures, or of small doses of living bacteria. In the serum of immunized animals specific bacteriolytic and agglutinating substances are found. The discovery of bacteriolytic immune bodies, in fact, was made by means of cholera spirilla. Both the bacteriolysins and the agglutinins, because of their specificity, are of great importance in making a bacteriological diagnosis of true cholera organisms.

Prophylactic Vaccination.-Aetive immunization of cholera was one of the first methods of prophylactic vaccination attempted in the bacteriological era of infectious disease study. The work was done by a Spanish bacteriologist, Férran, who had been a pupil of Pasteur, and as early as 1884 earried out immunization experiments with cholera on guinea-pigs. Férran, ${ }^{15}$ in accordance with the methods prevalent at that time, worked with attenuated cholera cultures and developed a method of attenuation which depended upon room-temperature cultivation on gelatin. He tried this method on human beings in Spain in 1885 with results which seemed to him eneouraging. Subsequent to this many different vaccines have been developed. Haffkine ${ }^{16}$ worked intensely on the subject and observed the results of vacination on an enormous number of people in India, over a period of more than ten years. Haffkine's virus has undergone a number of modifications since he first used it. He, too, made use of living cultures, beginning his experiments with attenuation of cholera spirilla by cultivation at temperatures of $40^{\circ}$ and over, using, at first, a less virulent and next a more virulent strain. Later, it was found that the cultures attenuated by cultivation at increased temperatures were not necessary, and it appears at the present time that in most places only cultures of a virulence enhanced by passage through guinea-pigs are used. The extensive experimental work in India mentioned above seems to have shown that there is a distinet prophylactic value in the use of Haffkine's virus.

Other observers have made use chiefly of killed eultures. The French vaccine made at the Pasteur Institute consists of broth cultures killed at $50^{\circ}$. Kolle ${ }^{17}$ grows his cholera spirilla on agar,

15 F'érran, Comptes rend. de l'acad. des sciences, 1885.

${ }^{16}$ Haffkine, Bull. med., 1892.

${ }^{17}$ Kolle and Schurmann, Kolle and Wassermann Handb., Vol. 4, Second Edition. 
suspending them in salt solution, killing at $56^{\circ} \mathrm{C}$. for one hour, then adding one-half per cent carbolic acid. Other observers, like Nicoll and Vincent ${ }^{18}$ killed without heat, by the addition of carbolic acid. Extracts of the cholera spirilla have also been used in various ways. Strong ${ }^{19}$ grows cholera organisms on agar, takes them up in salt solution, kills at $60^{\circ}$ and then allows the suspensions to stand in the incubator for about five days, subsequently filtering through a Berkefeld candle. This filtrate is used for inoculation, after its sterility has been determined by culture. Wassermann ${ }^{20}$ has used materials prepared by precipitation of cultures with alcohol. Castellani $^{21}$ during the last ten years has prepared what he calls a T. A. B. C., or tetravaccine, which is made by mixing agar cultures of typhoid, paratyphoid "A," paratyphoid "B," and cholera in saline emulsion. The emulsion is killed with one-half per cent carbolic acid, preserved for twenty-four hours in this form at room temperature and then standardized by the usual counting chamber method so that 1 c.c. should contain five hundred thousand typhoid, 250 thousand paratyphoid "A," 250 thousand paratyphoid "B," and two thousand million cholera spirilla. 0.5 c.c. of this is injected, three doses being given within two weeks. 'This is the vaccine which we used on the Serbian Army during the war.

The principle underlying all these procedures seems to us to be the same, in that they consist of introducing, subcutaneously, substances derived from the bodies of cholera spirilla. And since the cholera organisms probably do not live very long after subcutaneous introduction, it is not likely that it makes very much difference whether attenuated living cultures, or dead cultures are used.

As far as the available statistics show at the present time, cholera vaccination is of distinct value. This has been the judgment of those who have scrutinized Haffkine's immunization experiments, as well as those who have observed more recent army experiences. The following table which we used in our Nelson article, again taken from Violle, will give some idea of the comparisons made

${ }^{18} \mathrm{Nicoll}$ and $\mathrm{Vincent}$. Cited from Violle loc. cit.

${ }^{10}$ Strong. J. of Exp. Med., Vol. 8, p. 229, 1905.

${ }^{20}$ Wassermann. Festschr. R. Koch, Jena, 1903.

${ }^{21}$ Castellani and Chalmers, Manual of Tropical Medicine, W. Wood \& MG, New York, 1919. 
upon vaccinated and unvaccinated individuals among troops, and in some of the more recent experiments.

EPIDEMIC OF CHOLERA IN THE GREEK ARMY DURING THE SECOND BALKAN WAR (from ARNAUD)

Vaccinated, 2 inoculations

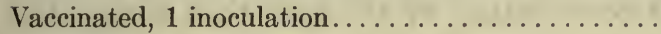

Not vaccinated.

\begin{tabular}{|c|c|c|c}
$\begin{array}{c}\text { Number of } \\
\text { Soldiers }\end{array}$ & $\begin{array}{c}\text { Cases of Cholera, } \\
\text { Per Cent }\end{array}$ \\
\hline 76,652 & 0.43 \\
21,216 & 3.12 \\
14,332 & 5.7 \\
\hline
\end{tabular}

Morbidity, 1,801 (12.5 per cent)

Mortality, 348 (2.5 per cent)

II

EPIDEMIC OF CHOLERA IN RUSSIA, 1912 (from ABRAMow)

\begin{tabular}{|c|c|c|}
\hline 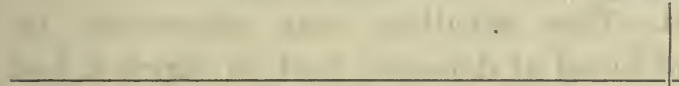 & $\begin{array}{l}\text { Numbers of } \\
\text { Soldiers }\end{array}$ & $\begin{array}{c}\text { Cases of Cholera, } \\
\text { Per Cent }\end{array}$ \\
\hline $\begin{array}{l}\text { Vaccinated } \ldots \ldots \ldots \ldots \ldots \ldots \ldots \ldots \ldots \ldots \ldots \ldots \\
\text { Not vaccinated } \ldots \ldots \ldots \ldots \ldots \ldots \ldots \ldots\end{array}$ & $\begin{array}{c}1,500 \\
\ldots \ldots\end{array}$ & $\begin{array}{l}5=0.3 \\
\ldots \ldots\end{array}$ \\
\hline
\end{tabular}

III

RECENT EPIDEMIC OF CHOLERA IN INDIA (from (HAFFKINE)

\begin{tabular}{|c|c|c|}
\hline & Cases of Cholera & Deaths, Per Cent \\
\hline $\begin{array}{l}\text { Not inoculated............ } \\
\text { Inoculated............. }\end{array}$ & 8,000 & $\begin{array}{r}11 \\
3\end{array}$ \\
\hline
\end{tabular}

IV

EPIDEMIC OF CHOLERA IN THE GREEK ARMY DURING THE THE BALKAN WAR (from SAVAS)

\begin{tabular}{|c|c|c|}
\hline 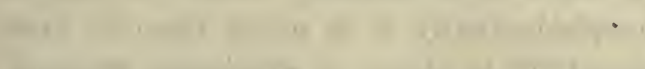 & $\begin{array}{l}\text { Number of } \\
\text { Individuals }\end{array}$ & $\begin{array}{r}\text { Deaths, } \\
\text { Per Cent }\end{array}$ \\
\hline $\begin{array}{l}\text { Not vaccinated } \ldots \ldots \ldots \ldots \ldots \ldots \ldots \ldots \ldots \ldots \ldots \\
\text { Vaccinated once } \ldots \ldots \ldots \ldots \ldots \ldots \ldots \ldots \ldots \ldots \\
\text { Vaccinated twice } \ldots \ldots \ldots \ldots \ldots \ldots \ldots \ldots\end{array}$ & 10,000 & $\begin{array}{r}20 \\
3 \\
1\end{array}$ \\
\hline
\end{tabular}

Cholera vaccination naturally is of relative value only, just as this is the case in typhoid vaccination. Vaccination must be repeated certainly every two years and probably more often in the case of armies in the field. 


\section{CHOLERA-LIKE SPIRILLA}

The biological group of the spirilla, to which the cholera spirillum belongs, is a large one, numbering probably over a hundred separate species. Most of these are of bacteriological importance chiefly because of the difficulties which they add to the task of differentiation, for while some of them simply bear a morphological resemblance to the true cholera vibrio, others can be distinguished only by their serum reactions and pathogenicity for various animals. Additional difficulty, too, is contributed by the fact that within the group of true cholera organisms occasional variations in agglutinability and bacteriolytic reactions may exist. Certain strains, too, the six El Tor cultures isolated by Gottschlich, while in every respect similar to true cholera spirilla, are considered as a separate sub-species by Kraus, ${ }^{22}$ because of their ability to produce hemolytic substances, a function lacking in other cholera strains.

Spirillum Metchnikovi-_This spirillum was discovered by Gamaleia $^{23}$ in the feces and blood of domestic fowl, in which it had caused an intestinal disease. Morphologically and in staining reactions it is identical with Spirillum choleræasiaticæ. It possesses a single polar flagellum, and is actively motile. Culturally it is identical with Vibrio choleræ except for slightly more luxuriant growth and more rapid fluidification of gelatin. It gives the cholera-red reaction in pepton media.

It is differentiated from the cholera vibrio by its power to produce a rapidly fatal septicemia in pigeons after subcutaneous inoculation of minute quantities. ${ }^{24}$ It is much more pathogenic for guinea-pigs than the cholera vibrio. It is not subject to lysis or agglutinated by cholera immune sera.

Spirillum Massaua.-This organism was isolated at Massaua by Pasquale $^{25}$ in 1891 from the feces of a clinically doubtful case of cholera. Culturally and morphologically it is much like the true cholera vibrio, but in pathogenicity is closer to Spirillum Metchnikovi, in that small quantities produce septicemia in birds. It possesses four flagella. It does not give a specific serum reaction with cholera immune serum.

${ }^{22}$ Kraus, Kraus and Levaditi, "Handbuch," vol. i. p. 186.

${ }^{23}$ Gamaleia, Ann. de l'inst. Pasteur, 1883.

${ }^{24}$ Pfeiffer und Nocht, Zeit. f. Hyg., vii, 1889.

${ }^{25}$ Pasquale, Giorn. med. de r. escre. ed. R. Marina, Roma, 1891. 
Spirillum of Finkler-Prior ${ }^{26}$-Isolated by Finkler and Prior from the feces of a ease of cholera nostras. Morphologically it is like the true cholera spirillum, though slightly larger and less uniformly curved. Culturally it is much like the cholera vibrio, but grows more rapidly and thickly upon the usual media. It does not give the cholera-red reaction, nor does it give specific serum reactions with cholera immune serum.

Spirillum Deneke. ${ }^{27}$-A vibrio isolated by Deneke from butter. Much like that of Finkler-Prior. It does not give the eholera-red reaction.

${ }^{26}$ Finkler und Prior, Ergänz. Hefte, Cent. f. allg. ges. Phys., 1884.

${ }^{27}$ Deneke, Deut. med. Woch., iii, 1885. 


\section{CHAPTER XLIII}

DISEASES CAUSED BY SPIROCH ATES (TREPONEMATA), CLASSIFICATION, SYPHILIS AND TREPONEMA PALLIDUM, RELAPSING FEVERS, VINCENT'S ANGINA, YAWS, AND THE SPIROCHATE PER'TENUE, SPIROCH ETE GALLINARUM, RAT BITE FEVER, NONPATHOGENIC SPIROCHATES OF THE HUMAN BODY.

THE microorganisms known as spirochætes are slender, undulating, corkscrew-like threads which show definite variations both structurally and culturally from the bacteria as a class. Most important among them are the spirochæte of relasping fever, Spirochæte pallida of syphilis, the spirillum of Vincent, Spirochæte refringens, Spirillum gallinarum, a microorganism which causes disease in chickens, Spirochæte anserina, which causes a similar condition in geese, and several species which have been found as parasites, both in animals and in man, without having definite etiological connection with disease.

\section{CLASSIFICATION OF SPIRAL ORGANISMS}

Classification of the spiral organisms in general is still unsatisfactory because the difficulties of staining and cultivation have made it impossible to apply to these organisms the same exact criteria which ean be applied to most species of bacteria. We may say, in general, that the word spirillum should be retained for true bacteria of spiral form in which the cell body is rigid and motility is brought about entirely by flagella. Such, for instance, are the spirillum of Asiatic cholera, the spirillum Metchnokovi, the spirillum Deneke and others.

The true spirochæte are probably not true bacteria, and we have no exact eriteria upon which we can base their classification with the protozoa. However, the striking parasitism of most of them, and certain features of their immunological relations would suggest that they either belong to, or are very close to protozoa. Schaudinn, the discoverer of the syphilis organism, classified the treponema pallidum with the protozoa on the basis of morphological study. He believed that stained preparations often showed an undulating 
membrane extending along the long axis of the microorganisms similar to that observed in trypanosomes. He also asserted that most of the spiral forms reproduce by cleavage along the longitudinal axis. On the other hand, Laveran, ${ }^{1}$ Novy and Knapp ${ }^{2}$ and others maintained a close relationship of these microorganisms to the true bacteria. ${ }^{3}$

A review of observed facts seems to show that most of these spiral organisms have the power of multiplication by transverse fission. Many of them possess flagella and in some of them definite immune bodies can be demonstrated in the serum of infected subjects, similar to those produced by bacteria during infection. In others again, like the treponema pallidum, no true circulating antibodies against the virulent parasitic forms can be found. Indeed, in syphilis it seems that immunity exists only so long as the living organisms still persist in the body, an observation which is entirely analogous to that made with certain trypanosomes, and with malaria. Also, with some of them, transmission by an intermediate insect host in which the spirilla undergo multiplication has been definitely shown, a state of affairs which corresponds with conditions in many protozoan infections. Kolle and Hetsch favor a classification midway between the protozoa and the bacteria, a view which is probably as correct as any that we have any justification for holding at the present time.

Noguchi, ${ }^{4}$ who has had extensive experimental experience with the spirochaete has suggested the tentative classification which follows :

He calls attention to the fact that the term "spirochæta" was applied first by Ehrenberg in 1838 to a free living, fresh water or marine form of spiral organism which creeps along the surface of an object but does not swim, divides by transverse fission, and probably has nothing to do with the organism to which we now apply this word.

Noguchi divides the spiral organisms of the group which we are now considering into:

I. Cristispira or Saprospira.-This is a limited group of motile spiral organisms which infest the great crystalline styles of certain

${ }^{1}$ Laveran, Comptes rend. de l'acad. des sci., 1902 and 1903.

Novy and Knapp, Jour. Infec. Dis., 3, 1906.

' Kolle and Hetsch, “Die experimentelle Bakt.,' Berlin, 1906.

- Noguchi, Jour. Exper. Med., 27, 1918, 575. 
mollusea. The term was first proposed by Gross ${ }^{5}$ in 1910. A type of these organisms is found in oysters (Spirochæta balbianii (Certes, ${ }^{6}$ ) 1882). Another genus of the same order, Saprospira, was found by Gross to exist in mussels.

None of these are pathogenic for higher animals. They are characterized by the presence of a membraneous structure which resembles a crista or ridge which runs spirally along the entire length of the body. The body is chambered, that is, transverse bands seem to show along its entire length. There are no terminal filaments and there seems to be a strong flexible membrane. Reproduction, according to Gross, takes place by multiple transverse fission or sporulation, but Noguchi has failed to confirm the occurrence of sporulation.

II. Spironema and Treponema.-This is a large group of parasitic spiral organisms which are commonly spoken of as the "spirochete" in medical nomenclature. The characteristic feature of these is a spiral flexible body with terminal filaments, but no undulating membrane. They may apparently multiply by transverse as well as by longitudinal fission. They move by an undulating movement, a few of them, however, retaining their regular curves during motion. Dobell in an address before the Royal Society in 1912 expressed the belief that the word treponema should be used for all of the small parasitic varieties. Noguchi believes with Gonder that the term treponema should be restricted, as was done by Schaudinn, ${ }^{7}$ to those varieties having great constancy of curves, while spironema should be applied to those with less constant curves, but he, nevertheless, classifies them together under the same main heading since he believes they are closely related.

In this class belong the Treponema pallidum of syphilis and the Treponema or Spirochcete pertenue of Yaus. The class also includes the organisms of relapsing fever, a number of parasites found in rodents, such as the well known organism which invades apparently normal mice (and was once falsely looked upon as the cause of cancer in mice) and various saprophytic types found in the mouth, intestine and genital mucous membranes, such as the Treponema calligyrum

- Gross, Mitt. zool. Station Neapel., 1910-13, 20, 41 and 188, Cent. f. Bakt., Orig., 65, 1912, 83.

- Certes, Bull. Soc. zool. franc., 7, 1882, 347.

'Schaudinn, Deut. med. Woch., 43, 1909, 1728, Arb. a. d. k. Gesundhst., 1904. 
found in smegma, the Treponema microdentium and macrodentium, found in the mouth, especially under the gums, and in the throat.

Among the Spironema in this main group Noguchi places the Spironenta refringens ${ }^{8}$ of smegma, the Spironema vincenti of Vincent's angina, the Spironema recurrentis of Obermeier, ${ }^{9}$ the Spironema Duttoni, ${ }^{10}$ the Spironema Kochi, the Spironema gallinarum and the Spironema Novyi.

III. Leptospira.-The types of this class are the Leptospira ictero hxemorrhagice of Inada and Ido and the Leptospira icteroidis recently isolated by Noguchi from eases of yellow fever, and probably representing the etiological factor of that disease. These organisms are much more easily cultivated than the preceding. They are characterized by closely set regular spirals which remain unchanged during a peculiar rotary spinning motion. As described by Noguchi these organisms, while in motion, draw the entire body together into a straight line, except for a hook formation of one or both ends. When one end is extended and straight and the other semicircularly hooked, the organism progresses in the direction of the straight portion, appearing to be propelled from the rear by the rotary hook. A specimen with both ends hooked remains stationary in spite of its rapid rotary motions. This description is taken verbatim from Noguchi. In this sort of movement the body assumes wide wavy undulations. So far no terminal or peritrichal flagella have been seen.

\section{SYPHILIS AND SPIROCH屟TA PALLIDA}

\section{(Treponema pallidum)}

The peculiar manifestations of syphilis, its mode of transmission, and the fact that its primary lesion was always located at the point of contact with a preceding case, have always stamped it as unquestionably infectious in nature. Until very recently the microorganism which gives rise to syphilis was unknown. Many bacteriologists had studied the problem and many microorganisms for which definite etiological importance was claimed had been described. Most of these announcements, however, aroused little more than a sensational interest and received no satisfactory confirmation. A

8 Schaudinn and Hoffmann, Arb. a. d. w. Gesundhst., 22, 1905.

' Obermeier, Cent. f. d. med. Wiss., 11, 1873.

10 Dutton and Todd, Brit. Med. Jour., 1905. 
bacillus deseribed by Lustgarten ${ }^{11}$ in 1884 seemed, for a time, to have solved the mystery. The Lustgarten bacillus was an acid-fast organism very similar to Bacillus tuberculosis, and found by its discoverer in a large number of syphilitic lesions. The observation, at first, aroused much interest and received some confirmation. Later extensive investigations, however, failed to uphold the etiologieal relationship of this bacillus to the disease but identified it with the smegma bacillus, so often a saprophyte upon the mucous membranes of the normal genitals.

In 1905, Schaudinn, ${ }^{12}$ a German zoologist, working in collaboration with Hoffmann, investigated a number of primary syphilitic

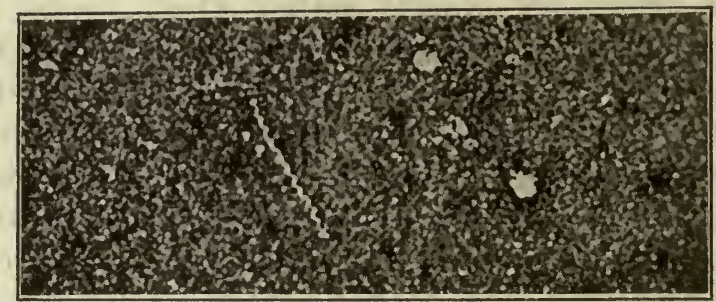

Fig. 90.-Sirocheta Pallida. Smear preparation from chancre stained by the india-ink method.

indurations and secondarily enlarged lymph nodes; and in both lesions diseovered a spirochæte similar to, but easily distinguished from, the spirochætes already known. He failed to find similar microorganisms in uninfected human beings.

The microorganism described by him as "Spirochæta pallida" is an extremely delicate undulating filament measuring from four to ten micra in length, with an average of seven micra, and varying in thickness from an immeasurable delicacy to about 0.5 of a micron. It is thus distinetly smaller and more delieate than the spirochxte of relapsing fever. Examined in fresh preparations it is actively motile, its movements consisting in a rotation about the long axis, gliding movements backward and forward, and, occasionally, a bending of the whole body. Its convolutions, as counted by Schaudinn, vary from three to twelve and differ from those observed in many other spirochætes by being extremely steep, or, in other words, by

${ }^{11}$ Lustgarten, Wien. med. Woch., xxxiv, 1884.

${ }^{12}$ Schaudinn und Hoffmann, Arb. a. d. kais. Gesundheitsamt, 22, 1905. 
forming acute, rather than obtuse, angles. The ends of the microorganism are delicately tapering and come to a point. In his first investigations, Schaudinn was unable to discover flagella and believed that he saw a marginal undulating membrane similar to that noticed in the trypanosomes. Later observations by this observer, as well as by others, revealed a delicate flagellum at each end, but left the existence of an undulating membrane in doubt. Uncertain, in his later investigations, whether the microorganisms described by him could scientifically be classified with the spirochæte proper, Schaudinn suggested the name of "Treponema pallidum."

In the same preparations in which Spirochæta pallida was first seen, other spirochætes were present, which were easily distinguished from the former by their coarser contours, their flatter and fewer undulations, their more highly refractile cell bodies, and, in stained preparations, their deeper color. These microorganisms were not found regularly, and were interpreted merely as fortuitous and unimportant companions. To them Schaudinn gave the name of "Spirochæta refringens."

The epoch-making dis c o very of Schaudinn and Hoffmann was soon

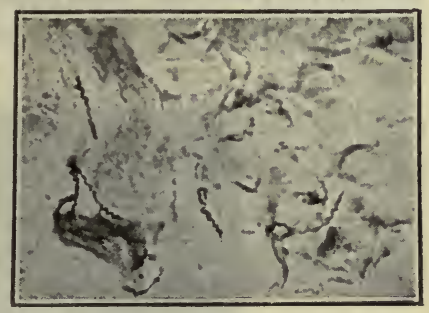

Fig. 91.-SPIrocheta PaLLIDA. Spleen, congenital syphilis. (Levaditi method.) confirmed by many observers, and the etiological relationship of Spirochæta pallida to syphilis may now be regarded as an accepted fact. Although our inability to cultivate the microorganism has made it impossible to carry out Koch's postulates, nevertheless indirect evidence of such a convincing nature has accumulated that no reasonable doubt as to its causative importance can be retained. The spirochætes have been found constantly present in the primary and secondary lesions of all carefully investigated cases, and, so far, have invariably been absent in subjects not afflicted with syphilis.

Schaudinn himself, not long after his original communication, was able to report seventy cases of primary and secondary syphilis in which these microorganisms were found. Spitzer ${ }^{13}$ found them constantly present in a large number of similar cases. Sobernheim and Tomasczewski ${ }^{14}$ found the spirochretes in fifty cases of primary

\footnotetext{
${ }^{13}$ Spitzer, Wien. klin. Woch., 1905.

${ }^{14}$ Sobernhcim und Tomasczewsti, Münch. med. Woch., 1905.
} 
and secondary syphilis, but failed to find them in eight tertiary eases. Mulzer, ${ }^{15}$ who found the microorganisms invariably in twenty cases of clinical syphilis, failed to find them in fifty-six carefully investigated non-syphilitic subjects. The voluminous confirmatory literature which has accumulated upon the subject can not here he reviewed. The presence of these spirochætes in the blood at certain stages of the disease has been demonstrated by Bandi and Simonelli ${ }^{16}$ who found them in the blood taken from the roseola spots, and by Levaditi and Petresco ${ }^{17}$ who found them in the fluid of blisters produced upon the skin.

In tertiary lesions the spirochætes have been found less regularly than in the primary and secondary lesions, but positive evidence of their presence has been brought by Tomasczewski, ${ }^{18}$ Ewing, ${ }^{19}$ and others who succeeded in demonstrating them in gummata.

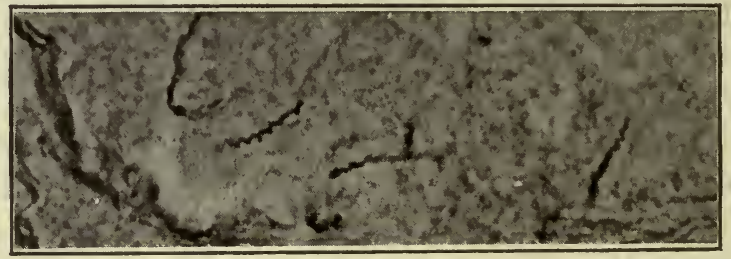

Fig. 92.-Spirocheta Pallida, Liver, congenital syphilis. (Levaditi method.)

Noguchi and Moore ${ }^{20}$ have recently found the Spirochæta pallida in the brain of patients dead of general paresis.

In congenital syphilis, many observers have found Spirochæta pallida in the lungs, liver, spleen, pancreas, and kidneys, and, in isolated cases, in the heart muscle. The organisms were always present in large numbers and practically in pure culture. These results more than any others seem to furnish positive proof of the etiological relationship between the spirochæte and the disease.

Demonstration of Treponema pallidum.-In the living state the spirochætes have been observed in the hanging drop or under a coverslip rimmed with vaseline. It is extremely important, in pre-

${ }^{15}$ Mulzer, Berl. klin. Woch., 1905, and Archiv f. Dermat. u. Syph., 79, 1906.

${ }^{16}$ Bandi und Simonelli, Cent. f. Bakt., 40, 1905.

${ }^{17}$ Levaditi et Petresco, Presse méd., 1905.

18 Tomasczewski, Münch. med. Woch., 1906.

${ }^{10}$ Ewing, Proc. N. Y. Path. Soc., N. S., 5, 1905.

${ }^{20}$ Noguchi and Moore, Jour. Exp. Med., xvii, 1913. 
paring such specimens from primary lesions or from lymph glands, to obtain the material from the deeper tissues, and thus as uncontaminated as possible by the secondary infecting agents present upon the surface of an ulcer, and also as free from blood as possible. It is best to employ a special device known as a "condenser for darkfield illumination" (Dunkel-Kammer-Beleuchtung). This apparatus is screwed into the place of the Abbé condenser. The preparation is made upon a slide and covered with a cover-slip as usual. A drop of oil is then placed upon the upper surface of the condenser and the slide laid upon it so that an even layer of oil, without airbubbles, intervenes between the top of the dark chamber and the bottom of the slide. An are light furnishes the most favorable illumination. In such preparations the highly refractive cell-bodies stand out against the black background, and the motility of the organisms may be observed. ${ }^{21}$

The dark-field condenser is without question the easiest method of finding the Spirochæta pallida. Its use is easily learned and the apparatus is sufficiently cheap so that it lends itself to the use of the clinic and the office. With very little practice it is possible to detect the spirochæte in suspension if care is taken that not too much blood or other solid particles are mixed with the preparation. Should it be impossible to obtain the material seraped from syphilitic lesions in a sufficiently dilute condition it is best to emulsify it in a drop or two of human ascitic fluid.

Examination in Smears.-The Spirochæta pallida can not be stained with the weaker anilin dyes, and even more powerful dyes. such as carbol-fuchsin and gentian-violet, give but a pale and unsatisfactory preparation. The staining method most commonly used is the one originally recommended by Schaudinn and Hoffmann. This depends upon the use of Giemsa's azur-eosin stain employed in various modifications. The most satisfactory method of applying this solution is as follows:

Make smears upon slides or cover-slips, if possible from the depth of the lesions, as free as possible from blood.

Fix in methyl alcohol for ten to twenty minutes and dry.

Cover the preparation with a solution freshly prepared as follows:

Distilled water .............................. 10 e.c.

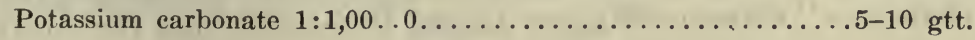

${ }^{21}$ For a critical summary of the various methods of dark-field illumination, the reader is referred to an article by Siedentopf, Zeit. f. wiss. Mikrosc., xxv, 1908. 
Add to this:

Giemsa's solution (für Romanowski Färbung)...........10-12 gtt.

This staining fluid is left on for one to four hours, preferably in a moist chamber. Wash in running water. Blot.

By this method Spirochæta pallida is stained characteristically with a violet or reddish tinge.

A rapid and convenient method for staining such smears consists in the use of azur I and eosin in aqueous solutions as recommended by Wood. The smears are fixed in methyl alcohol as before and are then flooded with the azur I solution. The eosin solution is then dropped on the preparation until an iridescent pellicle begins to form. Satisfactory preparations may be obtained by this method after ten or fifteen minutes of staining.

A fairly satisfactory method of staining the treponema pallidum in smear-preparations is that of Fontana. ${ }^{22}$ For this method, the following solutions are necessary:

1. Acetic acid $\ldots \ldots \ldots \ldots \ldots \ldots \ldots \ldots \ldots \ldots \ldots \ldots \ldots \ldots \ldots, 1$ c.c.

Formalin ................................ 2 c.c.

Distilled water .............................. 100 c.e.

Leave in one minute; wash in water.

2. Phenol 86 per cent (liquefied erystals) ............. 1 c.e. Tannic acid ............................... 5 grams

Distilled water $. . \ldots \ldots \ldots \ldots \ldots \ldots \ldots \ldots \ldots \ldots \ldots, 83$ c.c.

Cover preparation with this and steam gently one-half minute; wash.

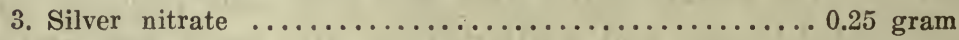

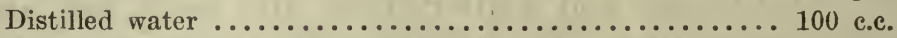

Ammonia q. s.

Add ammonia drop by drop until the precipitate which first appears goes into solution. Steam one-half minute; wash.

Recently a rapid and extremely simple but not very reliable method for the demonstration of Spirochæta pallida in smears, by the use of India ink, has been described.

Smears are prepared in the following way: A drop of the fluid squeezed out of the syphilitic lesion, as free as possible from blood cells, is mixed, on a slide, with a drop of India ink (best variety is "Chin chin" Günther-Wagner Iiquid Pearl ink), and the mixture

${ }^{22}$ See Levaditi and Bankowski: Ann de l'Inst. Past., 1913, XXVII, p. 583. 
smeared with the edge of another slide as in making blood smears. When the smear dries, which takes about a minute, it may be immediately examined with an oil-immersion lens. The organisms are scen unstained on a black background.

Demonstration of SPIROCHetes in Tissues.-Ordinary histological staining methods do not reveal the spirochætes in tissue sections. It is customary, therefore, to employ some modification of Cajal's silver impregnation. The technique most commonly employed is that known as Levaditi's method, ${ }^{23}$ which is carried out as follows :

The fresh tissue is cut into small pieces which should not be thicker than 2 to 4 millimeters.

Fix in $10 \%$ formalin ( $4 \%$ formaldehyde) for twenty-four hours. Wash in water.

Dehydrate in $96 \%$ alcohol twenty-four hours. Wash in water.

Place in a $3 \%$ silver-nitrate solution at incubator temperature $\left(37.5^{\circ} \mathrm{C.}\right)$ and in the dark for 3 to 5 days. Wash in water for a short time.

Place in the following solution (freshly prepared):

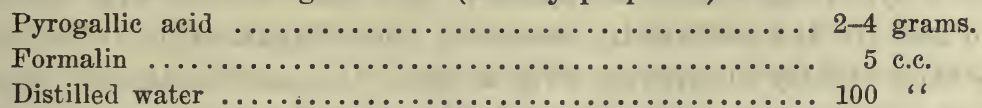

Leave in this for twenty-four to forty-eight hours at room temperature.

Wash in water.

Dehydrate in graded alcohols.

Embed in paraffin and cut thin sections.

The sections may be examined without further staining, or, if desired, may be weakly counterstained with Giemsa's solution or hematoxylin.

A modification of this method which has been much recommended is that of Levaditi and Manouelian. ${ }^{24}$ The directions given by these authors are as follows:

Fix in formalin as in previous method.

Dehydrate in $96 \%$ alcohol twelve to twenty-four hours. Wash in distilled water.

Place in a $1 \%$ silver-nitrate solution to which $10 \%$ of pyridin has been added just before use.

Leave in this solution for two to three hours at room temperature and from four to six hours at $50^{\circ} \mathrm{C}$. approximately.

Wash rapidly in $10 \%$ pyridin.

${ }^{28}$ Levaditi, Comptes rend. de la soc. de biol., 59, 1905.

${ }^{24}$ Levaditi et Manouelian, Comptes rend. de la soc. de biol., 60, 1906. 
Place in a solution containing $4 \%$ of pyrogallic acid to which $10 \%$ of C. P. acetone, and $15 \%$ (per volume) of pyridin have been added just before use. Leave in this solution two to three hours.

Wash in water, dehydrate in graded alcohols, and embed in paraffin by the usual technique.

Examined after treatment by either of these methods, the spirochætes appear as black, untransparent bodies lying chiefly extracellularly. They are characteristically massed about the bloodvessels of the organs and only exceptionally seem to penetrate into the interior of the parenchyma cells.

Attempts at cultivating Spirochæta pallida were at first unsuccessful. In 1909 Schereschewsky ${ }^{25}$ reported that he had succeeded in obtaining multiplication of the organisms on artificial media as follows: Sterile horse serum in centrifuge tubes was coagulated at $60^{\circ} \mathrm{C}$. until it assumed a jelly-like consistency. It was then placed in the incubator at $37.5^{\circ} \mathrm{C}$. for three days before being used. The cultures were planted by snipping off a small piece of tissue from a syphilitic lesion, dropping it into such a tube, and causing it to sink to the bottom by means of centrifugalization. The tube was then tightly stoppered with a cork. In such anaërobic serum cultures Schereschewsky claims to have grown the organisms for several generations, though not in pure culture.

Mühlens also obtained growth of Spirochæta pallida in horse serum agar by a method which is very similar to that of Schereschewsky. The most extensive and convineing work on treponema pallidum has been more recently by Noguchi. Noguchi ${ }^{26}$ began his work in 1910 and 1911. His first successful cultivations were made from the syphilis-infected testicles of rabbits, and after many unsuccessful attempts, with slightly varying media and technique, he finally succeeded in the following way: He prepared tubes $(20 \mathrm{~cm}$. high and $1.5 \mathrm{~cm}$. wide), containing 10 c.c. of a serum-water made of distilled water, three parts; and horse, sheep, or rabbit serum, one part. These were sterilized by the fractional method in the usual way (15 minutes each day). Into them was then placed a small piece of sterile rabbit kidney or testicle and a bit of the testicle of a syphilitic rabbit, in which many spirochætes were present. The fluid was then covered with sterile paraffin oil and placed in an

${ }^{26}$ Schereschewsky, Deut. med. Woch., N. S., xix and xxix, 1909.

${ }^{26}$ Noguchi Jour. Exp. Med., xiv, 1911; xvii, 1913. 
anaërobic jar. After ten days at $33.5^{\circ}$ C. the spirochætes had multiplied considerably, in all but one case, together with bacteria. He obtained pure cultures from these initial cultivations after much difficulty, by a number of methods. At first he suceeeded only by allowing the spirochætes to grow through Berkefeld filters, which they did on the fifth day. A better method more recently adopted by him consists in preparing high tubes of three parts of very slightly alkaline or neutral agar to which a picee of sterile tissue has been added. These tubes are then inoculated from the impure cultures with a long pipette. Close to the tissue and along the stab the spirochætes and bacteria will grow and, after about ten days to two weeks, the spirochætes will have wandered away from the stab and will be visible as hazy colonies. They can then be fished, after cutting the tubes, and directly transplanted to other serum-agar-tissue tubes prepared as before, and eventually will grow in pure culture. By this method Noguchi has also cultivated pure cultures from lesions in monkeys.

The writer, with Hopkins, has suceessfully applied Noguchi's method and has found that, after once cultivated artificially, the treponema pallidum can be obtained in quantity best by cultivation in flasks containing heated or unheated rabbit kidney with ascitic broth and sealed with paraffin. Recently we have been using modifications of a method worked out in our laboratory by Miss Gilbert, in which slanted egg, with or without glycerin, made as for tubercle cultivation, is used instead of kidney tissue. This is put up in high tubes and ascitic broth and paraffin oil added. By this method, large quantities of culture pallida are obtained within two weeks and can be concentrated in large quantities.

Animal Pathogenicity.-Until very recently, all experimental inoculation of animals was unsuccessful. During the year 1903 Metchnikoff and Roux ${ }^{27}$ finally succeeded in transmitting the disease to monkeys. The monkey first used by these observers was a female chimpanzee. At the point of inoculation, the clitoris, there appeared, twenty-six days after inoculation, a typical indurated chancre, which was soon followed by swelling of the inguinal glands. Fifty-six days after the inoculation there appeared a typical secondary eruption, together with swelling of the spleen and of the lymph nodes. Similar successful experiments were made soon after this by Lassar. ${ }^{28}$

${ }^{27}$ Metchnikoff et Roux, Ann. de l'inst. Pasteur, 1903, 1904, and 1905.

${ }^{28}$ Lassar, Berl. klin. Woch., xl, 1903. 
Soon after the experiments of Metchnikoff and Roux, successful inoculations upon lower monkeys (macacus) were carried out by Nicolle. ${ }^{29}$ Since that time, it has been found by various observer's that almost all species of monkeys are susceptible. Simple subcutaneous injection is not sufficient to produce a lesion. The technique which has given the most satisfactory results consists in the cutancous implantation of small quantities of syphilitic tissue obtained by excision or curetting of primary and secondary lesions. A small pocket is made under the mucous membrane of the genitals or of the eyebrows and the tissue placed in this under aseptic precautions. The inoculation may be made directly from the human being, but can also be successfully carried out from monkey to monkey for many generations. Attempts at transmission from tertiary lesions have so far been unsuccessful. The spirochætes can be demonstrated both in the primary lesions of the inoculated animal and in the secondarily enlarged glands. The successful inoculation of rabbits with syphilis has been recently performed by Bertarelli. ${ }^{30}$ He obtained ulcerative lesions by inoculation upon the cornea and into the anterior chamber of the eye and was able to prove the syphilitic nature of these lesions by finding the spirochæte within the tissue. In these animals, as well as in the lower monkeys, the disease usually remains localized.

In 1907 Parodi showed that syphilitic lesions could be produced by direct inoculation into the testicles of rabbits. This method of inoculation has been subsequently studied by many investigators, especially by Uhlenhuth and Mulzer. ${ }^{31}$ It is the easiest method of obtaining spirochæte in any quantity from lesions in man. The spirochæte-containing lesions may be either excised or scraped as conditions permit and rubbed up in a mortar with sterile sand, in a few centimeters of sterile human ascitic fluid. This emulsion is then injected directly into the substance of rabbit testicles. A swelling supervenes which is often noticeable after two weeks, and is usually at its height in five to seven weeks. At this time the testicle is much larger than normal, sometimes evenly swollen and sometimes nodular, and of a firm elastic consistency. When taken out at castration it oozes a sticky fluid, both from testicle and tunica, which is rich in actively motile spirochætes. By continuous

${ }^{29}$ Nicolle, Ann. de l'inst. Pasteur, 1903.

so Bertarelli, Cent. f. Bakt., xli, 1906.

r Uhlenhuth und Mulzer, Arb. a. d. k. Gesundh't's Amt., xxxiii, 1909. 
transinoculation from one rabbit to another such a strain ean be indefinitely carried along. It can be inoculated from rabbits to monkeys and vice versa. This method as well as Noguchi's cultivations have opened a new era of spirochæte investigation. 'It is stated by some observers that intravenous inoculation of rabbits may be followed by localization in the testis and occasionally gummatous infections in other parts of the body have been induced after such inoculation by Uhlenhuth, Mulzer, and others. Brown and Pearce in an elaborate series of recent investigations published in Journal of Experimental Medicine in 1920, have succeeded in reproducing almost all types of syphilitic lesions in rabbits by appropriate methods of inoculation.

Immunization in Syphilis.-It is a well-known fact observed by clinicians that during active syphilis the patient cannot be superinfected. That this resistance develops quite rapidly was shown by Metchnikoff and Roux, who found that reinfection of a monkey was possible if attempted within two weeks of the first inoculation, but was unsuccessful if delayed beyond this period.

On the basis of this knowledge, Metchnikoff, ${ }^{32}$ Finger and Landsteiner, ${ }^{33}$ and others have made attempts to devise some method of immunization. They attempted to attenuate the syphilitic virus by repeated passage through monkeys. These experiments were unsuccessful, the last-mentioned observers finding absolutely no attenuation after twelve generations of monkey inoculation.

Bertarelli and others have shown that the production of a syphilitic lesion on the cornea of one eye does not protect against an inoculation done on the other. Rabbits that have been inoculated with spirochæte material and that have not developed syphilitic disease can be successfully inoculated on subsequent attempts. The offspring of female rabbits with syphilis of the cornea are, according to Muhlens, not immune.

There is no evidence so far that specific therapy or treatment with spirochæte material has had favorable influence upon the disease. Chemotherapy has had results analogous to those obtained in man. ${ }^{34}$

Attempts at passive immunization have been entirely without success.

s: Metchnikoff, Arch. gén. de méd., 1905.

${ }^{23}$ Finger und Landsteiner, Sitzungsber. d. Wien. Akad. d. Wiss., 1905.

34 Von Prowazek, "Handbuch der pathogenen Protozoen," i, 1912, Leipzig, Bartsch. 
Investigations carried on in our own laboratory* in the last three years have shown definitely, we think, that immunization of animals with culture pallida produces antibodies, agglutinins, treponemacidal substances, entirely analogous to similar substances produced against bacteria. However, there is a biological change which takes place when treponema pallidum is cultivated. The antibodies produced with the culture pallida have no action whatsoever upon the virulent organisms. The latter, indeed, seem to be entirely insulated against such antibodies and do not induce antibody formation to any great extent, in either the infected animal or man. Both active and passive immunization with culture pallida and the sera produced with them have no effect. We have obtained some evidence, however, that in rabbits a purely local resistance develops in the tissue previously the site of a lesion.

The occurrence of a Wassermann reaction was formerly supposed to indicate the existence of specific syphilitic antibodies in the serum of patients. More recent information regarding this reaction secms to show that it depends upon the presence in the serum of syphilitic patients of substances produced indirectly because of the presence of syphilitic infection. It may be a relative increase of globulins or, as Schmidt has suggested, a change in the physical state of the globulins or other substances present in the serum. At any rate it has been found that the fixation of complement in the Wassermann reaction does not depend upon the occurrence of a specific antigen-antibody reaction. In the first place the antigens most commonly used, and successfully so, in the Wassermann reactions, are non-specific lipoidal extracts of normal organs.

Again it has been demonstrated that extracts of cultures of the Spirochæta pallida as well as extractions from the testes of syphilitic rabbits do not furnish an antigen suitable for the Wassermann reaction. This has followed especially from the work of Noguchi, ${ }^{35}$ of Craig and Nichols, ${ }^{36}$ and ourselves. This forms a corollary to the other experiments previously mentioned and shows that, whatever the Wassermann reaction may be, it is not a specific complement fixation in the sense of Bordet and Gengou. It must be admitted, therefore, that our knowledge of syphilis immunity is in

* Zinsser and Hopkins, Series of papers, Journ. Exp. Med., 1915 and 1916.

* Noguchi, Jour. Am. Med. Assoc., 1912.

${ }^{36}$ Craig and Nichols, Jour. Exp. Med., xvi, 1912. 
its infancy and that we know very little about the systemic reactions which follow infection with the Spirochæta pallida.

The fact that the syphilitic virus does not pass through a filter has been demonstrated by Klingmüller and Baermann, ${ }^{37}$ who inoculated themselves with filtrates from syphilitic material.

Hopkins and the writer have carried out some seventy filtration experiments with culture treponemata at various stages of growth without ever obtaining filter passage. It is our opinion that the assumption of a filtrable stage of the syphilitic virus is entirely unjustified and devoid of valid experimental support.

\section{THE SPIROCH开TES OF RELAPSING FEVER}

The microorganisms causing relapsing fever were first observed in 1873 , by Obermeier, ${ }^{38}$ who demonstrated them in the blood of patients suffering from this distinct type of fever. Since his time extensive studies by many other observers have proven beyond question the etiological connection between the disease and the organisms.

Morphology and Staining.-The spirochæte of Obermeier is a delicate spiral thread measuring from 7 to 9 micra in length (Novy), and about 1 micron in thickness. While this is its average size, it may, according to some observers, be considerably longer than this, its undulations varying from four to ten or more in number. Compared with the red blood, cells among which they are seen, the microorganisms may vary from one-half to nine or ten times the diameter of a corpuscle. In fresh preparations of the blood, very active corkscrew-like motility and definite lateral oscillation are observed. In stained preparations no definite cellular structure can be made out, the cell body appearing homogeneous, except in degenerated individuals, in which irregular granulation or beading has been observed. Flagella have been described by various observers. Novy and Knapp ${ }^{39}$ believe that the organisms possess only one terminal flagellum. Zettnow, ${ }^{40}$ on the other hand, claims to have demonstrated lateral flagella by special methods of staining.

${ }^{37}$ Elingmüller und Baermann, Deut. med. Woch., 1904.

${ }^{38}$ Obermeier, Cent. f. d. med. Wiss., 11, 1873.

${ }^{39}$ Novy and Knapp, Jour. of Infec. Dis., 3, 1906.

${ }^{4}$ Zettnow, Deut. med. Woch., 32, 1906. 
Norris, Pappenheimer, and Flournoy, ${ }^{41}$ in smears stained by polychrome methods, have described long, filamentous tapering ends which they interpreted as bipolar, terminal flagella, never observing more than one at each end. Spores are not found.

Cultivation.-Innumerable attempts to induce these microorganisms to multiply upon artificial media have been made. Novy and

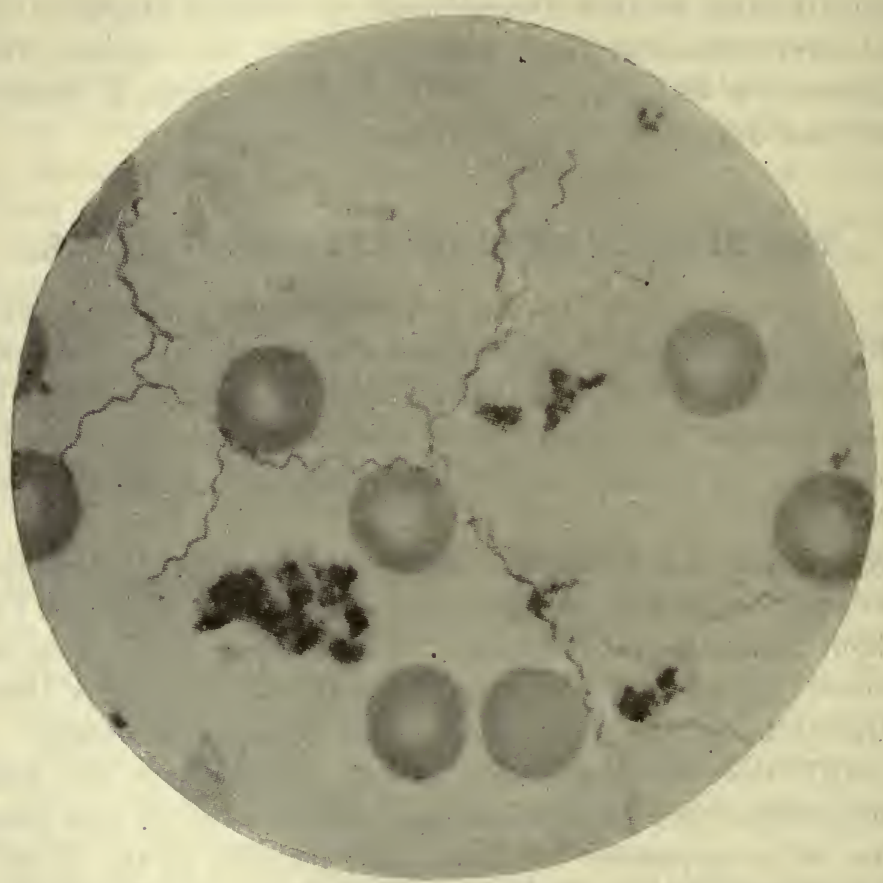

Pig. 93.-Spirochete of Relapsing Fever. (After Norris, Pappenheimer, and Flournoy.)

Knapp succeeded in keeping the microorganisms alive and virulent in the original blood for as long as forty days, and call attention to the fact that the length of time for which they may be kept alive depends to a great extent upon the stage of fever at which the blood is removed from the patient. They do not, however, believe that extensive multiplication, or, in other words, actual cultivation, had taken place in their experiments. Norris, Pappenheimer, and Flournoy, on the other hand, have obtained positive evidence of

${ }^{41}$ Norris, Pappenheimer, and Flournoy, Jour. of Inf. Dis., 3, 1906. 
multiplication of the spirochætes in fluid media. They ontained their cultures by inoculating a few drops of spirochætal rat blood into 3 to 5 e.e. of eitrated human or rat blood. Smears made from these tubes, after preservation for twenty-four hours at room temperature, showed the microorganisms in greater number than in the original infected blood. A similar multiplication could be observed in transfers made from these "first-generation" tubes to other tubes of citrated blood. Attempts at cultivation for a third generation, however, failed.

Noguchi ${ }^{42}$ has lately successfully cultivated the spirochæte of

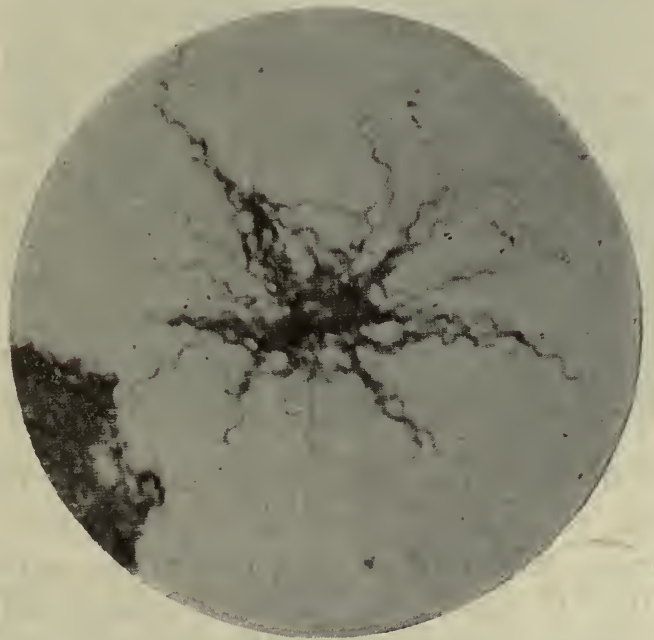

Fig. 94.-Spirochete of Relapsing Fever. Citrated normal rat blood. (After Norris, Pappenheimer, and Flournoy.)

Obermeier in ascitic fluid containing a piece of sterile rabbit's kidney and a few drops of citrated blood under anaërobic conditions.

Four different, probably distinct varieties of spirochæte have been described in connection with relapsing fever, all of which have been cultivated by Noguchi by means of this method. The first is known as the spirochæte of Obermeier mentioned above. Probably distinct are the Spirochæta Duttoni of West African Tick fever described by Dutton and Todd ${ }^{43}$ in 1905 , the Spirochæta Kochi, and

${ }^{43}$ Dutton and Todd, Brit. Med. Jour., 1905. 
the Spirochæta Novyi, ${ }^{44}$ the organism studied by Norris and Flournoy and Pappenheimer, and regarded-as a different species by them.

Pathogenicity.-Inoculation with blood containing these spirochætes produces disease in monkeys, rats, and mice. Attempts to transmit the disease experimentally to dogs, rabbits, and guinea-pigs have so far been unsuccessful. The subcutancous inoculation of monkeys is followed after from two to four days by a rise of temperature which occurs abruptly as is the case in the disease in man and which may last several days. During this time the spirochætes can be found in the blood of the animals just as it is found in that of infected human beings. The temperature subsides after a day or more, when it again rapidly returns to normal. As a rule, the paroxysms are not repeated. Oceasionally, however, two or three attacks may supervene before immunity is established. In rats, an ineubation time of from two to five days occurs. At the end of this time the spirochætes may be found in large numbers in the blood, and the animals show symptoms of a severe systemic infection. The attack lasts from four to five days, at the end of which time the microorganisms again disappear. Oceasionally even in these animals relapses have been observed. Gross pathological changes are not found, with the exception of an enlargement of the spleen.

In man the disease caused by the spirochrte of Oberme1er and allied organisms commonly known as relapsing fever, is common in Eastern Europe, India, Africa, and most of the warmer countries. It has, from time to time, been observed epidemically in Europe, especially in Russia, and a few epidemics have occurred in the Southern United States. The disease comes on abruptly, beginning usually with a chill accompanied by a sharp rise of temperature and generalized pains. Together with the rise of temperature, which often exceeds $104^{\circ} \mathrm{F}$., there are great prostration and occasionally delirium. Early in the disease the spleen becomes palpable and jaundice may appear. The spirochætes are easily detected in the blood during the persistence of the fever, which lasts usually from three to ten days. At the end of this time the temperature usually drops as suddenly as it rose, and the general symptoms rapidly disappear. After a free interval of from one to three weeks a relapse may occur, which is usually less severe and of shorter duration than

* Novy and Fraenkel, eited from Noguchi. 
the original attack. Two, three, or even four attacks may occur, but the disease is not very often fatal. When patients do succumb, however, the autopsy findings are not particularly characteristic. Apart from the marked enlargement of the spleen, which histologically shows the changes indicating simple hyperplasia, and a slight enlargement of the liver, no lesions are found. The diagnosis is easily made during the febrile stage by examination of a small quantity of blood under a cover-slip or in the hanging-drop preparation.

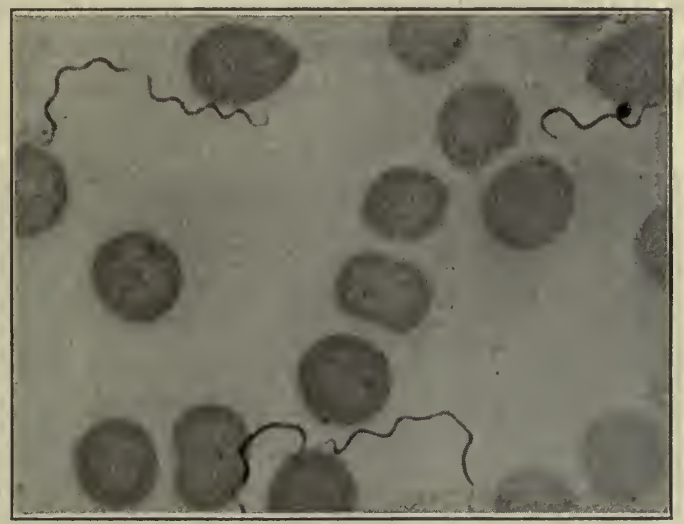

Fig. 95. - Spirochete of Relapsing Fever. (From preparation furnished by Dr. G. N. Calkins.)

Several types of relapsing fever have been deseribed. In Africa the disease has long been prevalent in many regions and the investigations of Ross and Milne, ${ }^{45}$ Koch, ${ }^{46}$ Dutton and Todd, ${ }^{47}$ and others have brought to light that many conditions occurring among the natives, formerly regarded as malarial, are eaused by a species of spirochæte. Whether or not the microorganisms observed in the African disease are exactly identical with the spirochæte observed by Obermeier is yet a question about which several opinions are held. Dutton and Todd believe that the same microorganism is responsible for both discases. Koch, on the other hand, believes that the slightly smaller size of the African spirochrte and the milder course of the clinical symptoms indicate a definite difference

${ }^{45}$ Ross and Milne, Brit. Med. Jour., 1904.

${ }^{46}$ Koch, Deut. med. Woch., xxxi, 1905.

${ }^{\circledR 7}$ Dutton and Todd, Lancet, 1905, and Jour. of Trop. Med., 1905. 
between the two. Animal experiments made with the African organism, furthermore, usually show a much more severe infection than do similar inoculations with the European variety. Tr.e spirochæte found in the African disease is usually spoken of at present as "Spirochæta Duttoni." Novy and Knapp,48 after extensive studies with the microorganisms from various sources, have come to the conclusion that, although closely related, definite species differences exist between the two types mentioned above, and that these again are definitely distinguished from similar organisms described by Turnbull ${ }^{49}$ as occurring in a similar disease observed in India.

The mode of transmission of this disease is not clear for all types.

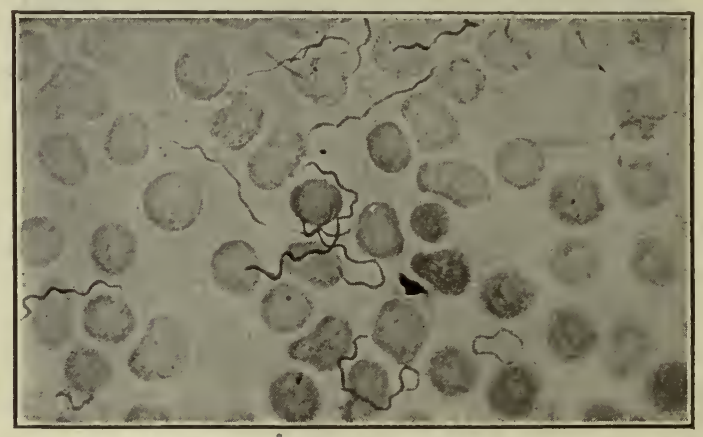

Fig. 96.-Spirochete of Dutton, African Tick Fever. (From preparation furnished by Dr. G. N. Calkins.)

Dutton and Todd, however, were able to show satisfactorily that, in the case of the African disease at least, transmission occurs through the intermediation of a species of tick. The conditions under which such intermediation occurs have been carefully studied by Koch. ${ }^{50}$ The tick (Ornithodorus moubata) infects itself when sucking blood from an infected human being. The spirochæte may remain alive and demonstrable within the body of the tick for as long as three days. Koch has shown, furthermore, that they may be found also within the eggs laid by an infected female tick. He sueceded in producing experimental infection in monkeys by

\footnotetext{
${ }^{4}$ Novy and Knapp, loc. cit.

${ }^{49}$ Turnbull, Indian Med. Gaz., 1905.

so Koch, Berl. med. Woch., 1906.
} 
subjecting the animals to the bites of the infected insects. For the European variety of the disease no such intermediate host has as yet been demonstrated with absolute certainty. It is known, however, that the organism can live in the bodies of bed bugs and it has also been suggested that lice may be the carriers. Lice also are regarded as the transmitting agent of a similar relapsing fever prevalent in North Africa caused by the Spiroschaudinnia berberi.

Immunity.- It has long been a well-known fact that recovery from an attack of relapsing fever usually results in a more or less definite immunity. The blood of human beings, monkeys, and rats which have recovered from an attack of this disease show áefinite and specific bactericidal and agglutinating substances, and Novy and Knapp have demonstrated that the blood serum of such animals may be used to confer passive immunity upon others.

\section{VINCENT'S ANGINA}

The condition known as Vincent's angina consists of an inflammatory lesion in the mouth, pharynx, or throat, situated most frequently upon the tonsils. The disease usually begins as an acute stomatitis, pharyngitis, or tonsillitis, which soon leads to the formation of a pseudo-membrane, which, at this stage, has a great deal of resemblance to that caused by the diphtheria bacillus. At later stages of the disease there may be distinct ulceration, the ulcers having a well-defined margin and "punched-out" appearance, so that clinically they have often been erroneously diagnosed as syphilis. Apart from the localized pain, the disease is usually mild, but occasionally moderate fever and systemic disturbances have been observed. Unlike diphtheria and syphilis, this peculiar form of angina usually yields, without difficulty, to local treatment.

The nature of lesions of this peculiar kind was not clear until Plaut, ${ }^{51}$ Vincent, ${ }^{52}$ and others reported uniform bacteriological findings in cases of this description. These observers have been able to demonstrate in smears from the lesions a spindle-shaped or fusiform bacillus, together with which there is usually found a spirillum not unlike the spirillum of relapsing fever. The two microorganisms are almost always found together in this form of disease and were

"Plaut, Dent. mel. Woch., xlix, 1894.

52 I'incent, Ann. de l'inst. Pasteur, 1896, and Bull. et mém. de la soc. méd. des hôp. de P., 1898. 
regarded by the first observers as representing two distinct forms dwelling in symbiosis.

The fusiform bacilli described by Vincent, Plaut, Babes, and others, are from 3 to 10 micra in length, and have a thickness at the center varying from 0.5 to 0.8 micron. From the center they taper gradually toward the ends, ending in blunt or sharp points. The length of these bacilli may vary greatly within one and the same smear preparation. They are usually straight, sometimes slightly curved. They do not stain very easily with the weaker anilin dyes, but are readily stained by Loeffler's methylene-blue, carbol-fuchsin, or bet-

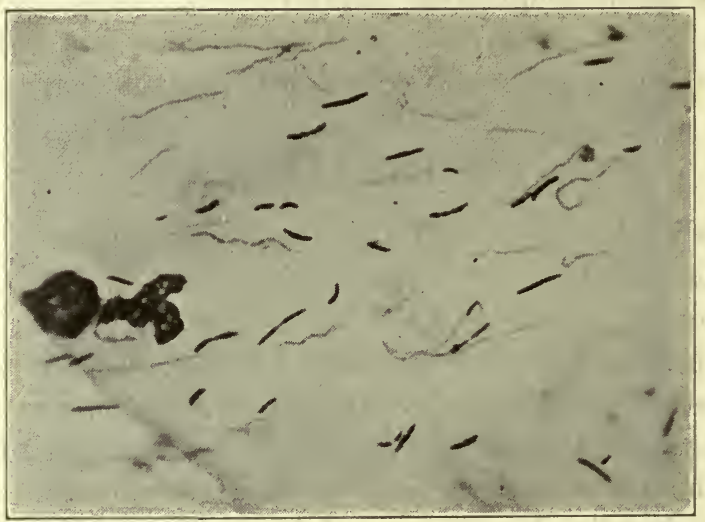

Fig. 97.-Throat Smear, Vincent's Angina. Fusiform bacilli and spirilla.

ter, by Giemsa's stain. Stained by Gram, they are usually decolorized, though in this respect the writers have found them to vary. Stained preparations show a characteristic inequality in the intensity of the stain, the bacilli being more deeply stained near the end, and showing a banded or striped alternation of stained and unstained areas in the central body. Their staining qualities in this respect are not unlike those of the diphtheria bacillus, and according to $\mathrm{Babes}^{53}$ the dark areas are to be interpreted as metachromatic granules. The bacilli are not motile.

The spirilla found in Vincent's angina are usually somewhat longer than the fusiform bacilli, and are made up of a variable number of undulations, shallow and irregular in their curvatures,

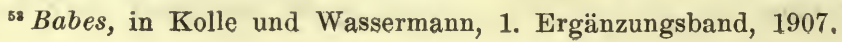


unlike the more regularly steep waves of Spirochæta pallida. They are stained with even more difficulty than are the bacilli and usually appear less distinct in the preparations. The stain, however, is taken without irregularity, showing none of the metachromatism observed in the bacilli.

By the earlier observers cultivation of these microorganisms was attempted without success. Recently, however, it has been shown that cultivation could be carried out under anaërobic conditions. Tunnicliff ${ }^{54}$ has cultivated the organisms anaërobically upon slants of ascitic agar at $37.5^{\circ} \mathrm{C}$. This observer found that in such cultures, before the fifth day, bacilli only could be found, that after this time, however, spirilla gradually appeared and finally constituted the majority of the organisms in the culture. It appeared to Tunnicliff from this study that the spirilla might be developed out of the fusiform microorganisms representing the adult form. This, howevr, is an error.

The microorganisms of Vincent's angina, when occurring in the throat, are rarely present alone, being usually accompanied by other microorganisms, such as staphylococci, streptococci, and not infrequently diphtheria bacilli. When occurring together with diphtheria, they are said, by some German observers, to aggravate the latter condition considerably. This frequent association with other microorganisms renders it impossible to decide conclusively that the fusiform bacilli and spirilla are the primary etiological factors in these inflammations. It has been frequently suggested that they may be present as secondary invaders upon the soil prepared for them by other microorganisms.

Animal inoculation with these microorganisms has led to little result.

Fusiform Bacilli other than those in Vincent's Angina.-Fusiform bacilli morphologically indistinguishable from those found in the angina of Vincent may frequently be found in smears taken from the gums, from carious teeth, and occasionally among the microorganisms in the pus from old sinuses. Several varieties of these bacilli have been described in connection with definite pathological conditions.

Babes, ${ }^{55}$ in 1893 , observed spindle-shaped bacilli not unlike those described above, but somewhat shorter, in histological sections prepared from tissues from the gums of individuals suffering from scurvy. He found similar bacilli

${ }^{64}$ Tunnicliff, Jour. of Infec. Dis., 3, 1906.

${ }_{55}$ Rabes, Deut. med. Woch., xliii, 1893. 
in rabbits intravenously inoculated with material from the patients and was able to eultivate the bacilli for several generations. His deseriptions, however, of the nicroorganisms as found in the secondary cultures vary considerably from those of the original findings in the gums of the patients. His results are not convineing.

In noma, a gangrenous disease of the gums and cheeks, occurring oceasionally in individuals who have been severely run down by acute infectious diseases or great hardship, Weaver and Tunnicliff have found spirilla and fusiform bacilli in large numbers. The organisms were present not only in smears from the surface, but were also found by histological methods, in large numbers, lying in the tissues beyond the area of necrosis. Here again it is not entirely certain whether these microorganisms were the primary etiological factors or whether they are to be regarded merely as secondary invaders of a necrotic focus.

Fusiform bacilli are cultivated with greater ease than formerly supposed; we have found it relatively simple to grow them together with Gram positive cocci in symbiosis in simple broth tubes covered with paraffin oil without the addition of any enriching substance and in similar symbiotic conditions on infusion agar plates under ineomplete anaërobic conditions. In such plates they form curious colonies in which the fusiform bacilli and micrococei are intimately commingled. Krumwiede ${ }^{56}$ has had no difficulty in cultivating them in pure culture in anaërobic plates.

\section{SPIROCH现TA PERTENUE}

In a disease known as "Frambœsia tropica," or popularly "Yaws," occurring in tropical and subtropical countries and much resembling syphilis, Castellani, ${ }^{57}$ in 1905 , was able to demonstrate a species of spirochæte which has a close morphologieal resemblanee to Spirochæta pallida. The mieroorganism was found in a large percentage of the cases examined both in the cutaneous papules and in ulcerations. Confirmatory investigations on a larger series of cases were later carried out by von dem Borne. ${ }^{58}$

The microorganism is from 7 to 20 miera in length with numerous undulations and pointed ends. Examined in fresh preparations, it has an active motility similar to that of Spirochæta pallida. In smears it is easily stained by means of the Giemsa method.

Both the clinical similarity between yaws and syphilis, as well as the similarity between the mieroorganisms causing the diseases,

${ }^{56}$ Krumwiede, Jour. Inf. Dis., 1913.

${ }^{57}$ Castellani, Brit. Med. Jour., 1905, and̉ Deut. med. Woch., 1906.

ss von dem Borne, Jour. Trop. Med., 10, 1907. 
has opened the question as to the identity of the two microorganisms. According to most clinical observers, however, yaws, which is a disease characterized chiefly by a generalized papular eruption, is unquestionably distinct, elinically, from lues, and experiments of Neisser, Baermann, and Halberstadter, ${ }^{59}$ as well as of Castellani ${ }^{60}$ himself, have tended to show that there is a distinct difference between the immunity produced by attacks of the two diseases. The disease is transmissible to monkeys, as is syphilis.

\section{SPIROCHATA GALLINARUM}

An acute infectious disease occurring among chickens, chiefly in South America, has been shown by Marchoux and Salimbeni ${ }^{61}$ to be caused by a spirochæte which has much morphological similarity to the spirochæte of Obermeier.

The disease comes on rather suddenly with fever, diarrhea, and great exhaustion, and often ends fatally. The spirochæte is easily demonstrated in the circulating blood of the animals by staining blood-smears with Giemsa's stain or with dilute carbol-fuchsin.

Artificial cultivation of the microorganism has not yet been accomplished. Experimental transmission from animal to animal is easily carried out by the subcutaneous injection of blood. Other birds, such as geese, ducks, and pigeons, are susceptible; mammals have, so far, not been successfully inoculated. According to the investigation of Leviditi and Manouelian, ${ }^{62}$ the spirochætes are found not only in the blood but thickly distributed throughout the various organs.

Under natural conditions, infection of chickens seems to depend upon a species of tick which acts as an intermediate host and causes infection by its bite. The spirochæte, according to Marchoux and Salimbeni, may be found in the intestinal canal of the ticks for as long as five months after their infection from a diseased fowl.

In the blood of animals which have survived an infection, agglutinating substances appear and active immunization of animals may be carried out by the injection of infected blood in which the spirochates have been killed, either by moderate heat or by preserva-

\footnotetext{
${ }^{59}$ Neisser, Baermann, und Halberstadter, Münch. med. Woch., xxviii, 1906.

${ }^{60}$ Castellani, Jour. of Hyg., 7, 1907.

-1 Marchoux et Salimbeni, Ann. de l'inst. Pasteur, 1903.

${ }^{62}$ Levaditi et Manouelian, Ann. de l'inst. Pasteur, 1906.
} 
tion at room temperature. The serum of immune animals, furthermore, has a protective action upon other birds.

It is not impossible that the Spirochrta gallinarum may be identical with the Spirochrta anserina previously discovered by Sacharoff. ${ }^{63}$ This last-named microorganism causes a disease in geese, observed especially in Russia and Northern Africa, which both clinically and in its pathological lesions corresponds closely to the disease above described as occurring in chickens. The spirochæte is found during the febrile period of the disease in the circulating blood, is morphologically indistinguishable from the spirochæte of

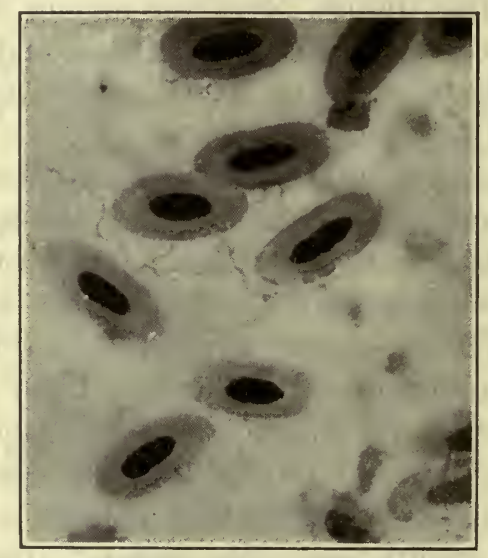

Fig. 98.- SPirochete Gallinarum. (From preparation furnished by Dr. G. N. Calkins.)

chickens, and can not be cultivated artificially. The similarity is further strengthened by the fact that Spirochæta anserina is pathogenic for other birds, but not for animals of other genera. Noguchi has succeeded in cultivating Spirochæta gallinarum by the same method by which he has cultivated the organisms of relapsing fever. Ascitic fluid tubes with a piece of sterile rabbit kidney were inoculated with a few drops of blood containing the spirochætes and cultivated at $37.5^{\circ} \mathrm{C}$. under anaërobic conditions.

Spirochæta phagedenis.-This is an organism cultivated by Noguchi by his ascitic-fluid-tissue method from phagedenic lesions on human external genitals. It is probably a new species.

${ }^{63}$ Sacharoff, Ann. de l'inst. Pasteur, 1891. 
Spirochæta macrodentium.-Cultivated by Noguchi ${ }^{64}$ is believed by him to be identical with the spirochæte found in Vincent's angina.

Spirochæta microdentium.-A similar organism with wide convolutions, eultivated by Noguchi from the tooth deposits chiefly in children. It was grown on mixtures of sheep serum water and sterile tissue in a way similar to that employed by him for other organisms of this group.

Spirochæta calligyrum.-Cultivated by Noguchi ${ }^{65}$ from condylomata-is probably a new species.

Rat-Bite Fever.-Rat-bite fever is a peculiar disease, which, after an incubation period of ten or more days, is characterized by fever, headache and inflammation at the site of the bite, swollen lymph glands, skin eruption and pains. After three to six days the fever ceases and an afebrile period of two or three days ensues. ${ }^{66}$ After this the fever again occurs. Recently Futaki, Takaki, Taniguchi, and $\mathrm{Osumi}^{67}$ have described a treponema which they have ealled Treponema morsus muris. It is a spiral organism, somewhat larger than the Treponema pallidum, and is found in the skin, the lymph nodes, and in the blood. They have succeeded in inoculating rats and have cultivated it in Schimamine medium, which consists of 100 e.e. of horse serum in which 0.5 to 0.75 gram of sodium nucleate is dissolved and carbon dioxide passed through the solution until the serum becomes transparent. It is then heated for three days at $60^{\circ}$, and on the fourth day at $65^{\circ}$ until it coagulates. This medium is deeply inoculated, but no other anaërobic precautions are taken.

Noguchi, Jour. Exp. Med., xv, 1912.

6s Noguchi, Jour. Exp. Med., xvii, 1913.

${ }^{68}$ In connection with Rat-Bite Fever see also Kaneko and Okuda, Journal Exp. Med., vol. xxvi, 1917, p. 363 .

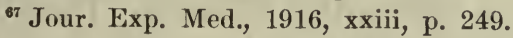




\section{CHAPTER XLIV}

YELLOW FEVER AND THE LEPTOSPIRA ICTEROIDES, WEIL'S DISEASE (INFECTIOUS JAUNDICE) AND LEPTROSPIRA ICTEROHEMORRHAEG $A$

YELLOW fever is an aeute infeetious disease which prevails endemically in the tropical countries of the Western Hemisphere, but occurs also along the western coast of Africa and has exceptionally appeared, in epidemic invasons, in the north temperate United States and Europe. Guiteras, as quoted by Osler, elassifies the distribution of the disease into three areas of infection.

1. The area in which the disease is never absent, including tropical South American ports and Havana.

2. The area of periodic epidemies, including sea-ports of the tropical Atlantic in Ameriea and Africa.

3. The area of accidental epidemics, extending from parallel $45^{\circ}$ north latitude to $35^{\circ}$ south latitude. In the United States severe epidemies have frequently occurred in Louisiana, Mississippi, and Alabama, and oceasional but severe epidemies have oceurred in Philadelphia and Baltimore.

The disease oecurs spontaneously only in man, and experimental inoeulation of lower animals has been suceessful only in the chimpanzee in a single case reported by Thomas. ${ }^{1}$

In man afflicted with the malady the clinical picture is one of a rapidly developing fever with severe gastrointestinal symptoms, vomiting of blood, albuminuria, and often aetive delirium. The mortality is usually high, often reaching eighty per eent or more in the severe epidemics.

Mode of Transmission.-Until comparatively reeent years the mode of transmission of yellow fever was not understood and many erroneous theories were prevalent. It was supposed that yellow fever was contagious, and transmitted from person to person by direct or indirect contact with those afflicted or by fomites. The first to make the definite assertion that yellow fever was transmitted

${ }^{1}$ Thomas, Brit. Med. Jour., 1, 1907. 
by the agency of mosquitoes was Carlos Finlay. Finlay, ${ }^{2}$ as early as 1881 , advanced the theory that mosquitoes were responsible for the transmission of this disease and, furthermore, recognized "Stegomyia fasciata" or "Stegomyia calopus" as the guilty speeies. Finlay's opinion, although later proved to be correct, was at first based only upon such circumstantial evidence as the correspondence of the yellow-fever zones with the distribution of this species of mosquito and the great prevalence of mosquitoes at times during which epidemies occurred. His theory was, therefore, received with much skepticism and was neglected by scientists until its revival in 1900 , when the problem was extensively investigated by a commission of American army surgeons.

Reed, Carroll, Agramonte, and Lazear were the members of this commission. The courage, self-sacrifice, and scientific accuracy which characterized the work of these men have made the chapter of yellow fever one of the most brilliant in the annals of American scientific achievement.

Their work was much facilitated by the experience of Gorgas ${ }^{3}$ and others, who had demonstrated the absolute failure of ordinary sanitary regulations to limit the spread of yellow fever.

They began their researches by investigating carefully the validity of Sanarelli's elaims as to the eiiological significance of his "Bacillus icteroides." The results of this work yielded absolutely no basis for confirmation.

They then proceeded to investigate the possibility of an intermediate host.

In August, 1900, the commission began its work on this subject by allowing mosquitoes, ${ }^{4}$ chiefly those of the stegomyia species, to suck blood from patients, later causing the same insects to feed upon normal susceptible individuals. The first nine experiments were negative. The tenth, of which Carroll was the subject, was successful. Four days after being bitten by the infected insect Carroll became severely ill with an attack of yellow fever, by which

${ }^{2}$ Finlay, Ann. Roy. Acad. d. Havana, 1881.

${ }^{3}$ Gorgas, Jour. of Trop. Med., 1903.

"Reed, Carroll, Agramonte, and Lazear, Phila. Med. Jour., Oct., 1900; also Am. Pub. Health Assn. Rep., 1903; Agramonte, N. Y. Med. News, 1900; Reed, Jour. of Hyg., 1902; Reed, Carroll, and Agramonte, Am. Medicine, July, 1901. Boston Med. and Surg. Jour., 14, 1901; Carroll, Jour. Am. Med. Assn., 40, 1903; Carrol, "Yellow Fever"' in Mense, "Handbuch der Tropen-Krankheiten," ii. 
his life was endangered, and from the effects of which he died several years later.

On the 13th of September, Lazear, while working in the yellowfever wards, noticed that a stegomyia had settled upon his hand, and deliberately allowed the insect to drink its fill. Five days later he beeame ill with yellow fever and died after a violent and short illness.

With these experienees as a working basis, the commission now decided upon a more systematic and thoroughly controlled plan of experimentation.

In November of the same year, 1900, an experiment station, "Camp Lazear," was established in the neighborhood of Havana, about a mile from the town of Quemados. The camp was surrounded by the strictest quarantine. Volunteers from the army of occupation were called for, and twelve individuals were selected for the eamp, three immunes and nine non-immunes. Two of the latter were physicians. The immunes and the members of the commission only were allowed to go in and out. All non-immunes who left the camp were prohibited from.re-entering and their places taken by other non-immune volunteers. During December, five of the nonimmune inmates were suecessfully inoculated with yellow fever by means of infected mosquitoes. During January and February five further successful experiments were made. Clinical observations were made by experienced native physicians, Carlos Finlay among them, and the patients, as soon as they were unquestionably ill with yellow fever, were removed to a yellow-fever hospital. This was done to prevent the possibility of the disease spreading within the eamp itself. The mosquitoes used for the experiments were all cultivated from the larva and kept at a temperature of about $26.5^{\circ} \mathrm{C}$.

A further important experiment was now made. A small house was erected and fitted with absolutely mosquito-proof windows and doors. The interior was divided by wire mosquito netting into two spaces. Within one of these spaces fifteen infected mosquitoes were liberated. Seven of these had fed upon yellow-fever patients four days previously; four, eight days previously; three, twelve days previously; and one, twenty-four hours previously. A non-immune person then entered this room and remained there about thirty minntes, allowing lumself to be bitten by seven mosquitoes. Twice after this the same person entered the room, remaining in it alto- 
gether sixty-four minutes and being bitten fifteen times. After four days this individual came down with yellow fever.

In the other room two non-immunes slept for thirteen nights without any evil results whatever.

It now remained to show that mosquitoes were the sole means of transmission and to exclude the possibility of infection by contact with excreta, vomitus, or fomites. For this purpose another mosquito-proof house was constructed. By artificial heating its temperature was kept above $32.2^{\circ} \mathrm{C}$. and the air was kept moist by the evaporation of water. Clothing and bedding, vessels, and eating utensils, soiled with vomitus, blood, and feces of yellow-fever patients were placed in this house and three non-immune persons inhabited it for twenty days. During this time they were strictly quarantined and protected from mosquitoes. Each evening, before going to bed, they unpacked and thoroughly shook clothing and bedding of yellow-fever patients, and hung and scattered these materials about their beds. They slept, moreover, in contact with linen and blankets soiled by patients. None of these persons contracted yellow fever. The same experiment was twice repeated by other non-immunes, in both eases with like negative results.

All of the non-immunes taking part in these experiments were American soldiers. Four of them were later shown to be susceptible to yellow fever by the agencies of mosquito infection or bloodinjection.

The results obtained by the investigations of this commission may be summarized, therefore, as follows:

Yellow fever is aequired spontaneously only by the bite of the Stegomyia fasciata. It is necessary that the infecting insect shall have sucked the blood of a yellow-fever patient during the first four or five days of the disease, and that an interval of at least twelve days shall have elapsed between the sucking of blood and the reinfection of another human being. Sucking of the blood of patients advanced beyond the fifth day of the disease does not seem to render the mosquito infectious, and at least twelve days are apparently required to allow the parasite to develop within the infected mosquito to a stage at which reinfection of the human being is possible.

The results of the American Commission were soon confirmed by Guiteras ${ }^{5}$ and by Marchoux, Salimbeni, and Simond. ${ }^{6}$ These latter

- Marchoux, Salimbeni, and Simond, Ann. de l'inst. Pasteur, 1903. 
observers, moreover, confirmed the fact that infection could be experimentally produced by injections of blood or blood serum taken from patients during the first three days of the disease. They showed that blood taken after the fourth day was no longer infectious: that 0.1 c.c. of serum sufficed for infection and finally that no infection could take place through excoriations upon the skin. They furthermore confirmed the observation of Carroll that the virus of the disease could pass through the eoarser Berkefeld and Chamberland filters,--passing through a Chamberland candle " $\mathrm{F}$ ", but held back by the finer variety known as " $\mathrm{B}$."

The fundamental factors of yellow-fever transmission thus discovered, we are in possession of logical means of defense. The most important feature of such preventive measures must naturally center upon the extermination of the transmitting species of mosquito.

Stegomyia fasciata or calopus is a member of the group of "Culicidæ." It is more delicately built than most of the other members of the group culicidæ, is of a dark gray color, and has peculiar thorax-markings which serve to distinguish it from other species. The more detailed points of differentiation upon which an exact zoological recognition depends are too technical to be entered into at this place. Briefly described, they consist of lyre-like markings of the back, unspotted wings, white stripes and spots on the abdomen, and bandlike white markings about the metatarsi and tarsi of the third pair of legs. The peculiar power of transmitting yellow fever possessed by this species is explained by Marchoux and $\mathrm{Simond}^{7}$ by the fact that Stegomyia faseiata is unique among culicidæ in that the female lives for prolonged periods after sucking blood. Among other speeies-Culex fatigens, Culex confirmatus, and most others-the female lays its eggs within from two to eight days after feeding on blood and rarely lives longer than the twelfth day - the time necessary for the development of the yellow-fever parasite.

The limitation of yellow fever to tropical countries ${ }^{8}$ is explained by the fact that stegomyia develops only in places where high temperatures prevail. The optimum temperature for this species lies between $26^{\circ}$ and $32^{\circ}$ C. At $17^{\circ}$ C. it no longer feeds, and beomes practically paralyzed at $15^{\circ} \mathrm{C}$. In order to thrive, the

'Marchoux and Simond, Ann. de 1'inst. Pasteur, 1906.

'Otto, in Kolle und Wassermann, "Handbuch,', ete., 11, Ergänzungsband. 
species requires a temperature never going below $22^{\circ} \mathrm{C}$. at night and rising regularly above $25^{\circ} \mathrm{C}$. during the day. The females only are dangerous as sources of infeetion. The inseet, like Anopheles, has the peculiarity of feeding chiefly at night.

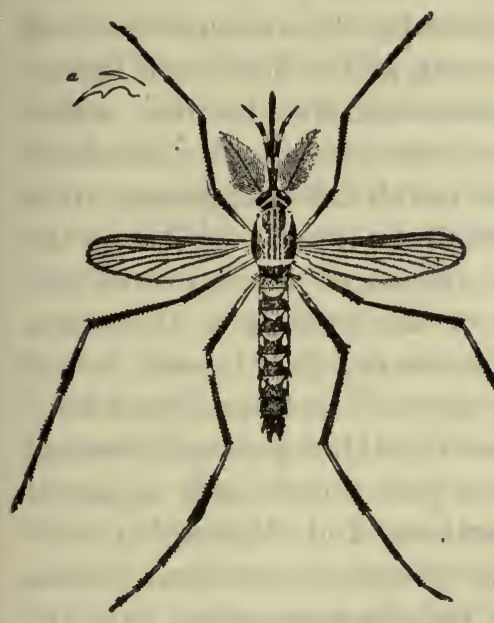

Fig. 99.-Stegomyia Fasciata.

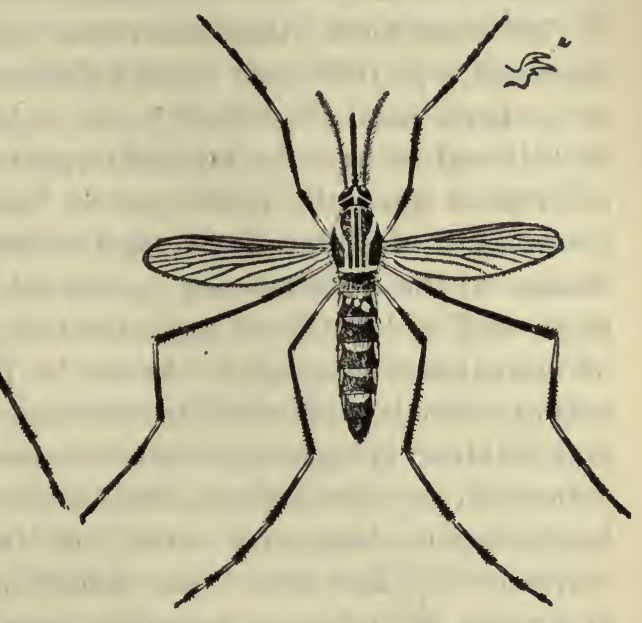

Experiments done by Reed, Carroll, Agramonte, and Lazear, to aseertain whether the power of infecting was hereditarily transmissible from the mosquito to following generations, were negative. A positive result, however, has been reported by Marchoux and Simond. ${ }^{9}$ This question must still await more extensive research.

\section{ETIOLOGY OF YELLOW FEVER}

Numerous researches have been aimed at the elucidation of the problem of etiology and a large number of different microorganisms for which etiologieal significance was elaimed, have been isolated from dejecta, vomitus and secretions of patients. A few of these elaims have only historical importance, but may be mentioned because of the wide interest they aroused among bacteriologists in the past.

- Marchoux and Simond, Comptes rend. de la soc. de biol., 59, 1905. 
Cornil and Babes, ${ }^{10}$ in 1883 , described chained cocci to which they attributed etiological significance, but their contentions have remained entirely unconfirmed. Sternberg, ${ }^{11}$ in 1897 , described a colon-like organism, "bacillus $X$," for which he made very conservative claims, which he himself, later, withdrew.

The most active discussion was roused by the announcement of Sanarelli, ${ }^{12}$ in 1897, that he had discovered, in the blood and tissues of patients dead of yellow fever, a Gram-negative bacillus, which he believed to be the etiological agent of the disease. He based his contention upon the facts that he had isolated the organism from seven cases of yellow fever, had produced symptoms similar to the disease of the human being by the inoculation of pure cultures into dogs, and had obtained agglutination of the bacillus in the serum of convalescent patients. Later he inoculated five human beings subcutaneously with sterilized cultures of this "Bacillus icteroides," and obtained symptoms which he believed simulated closely those of yellow fever. The claims of Sanarelli at first found much apparent confirmation, but later work by Durham and Myers, ${ }^{13}$ Otto, ${ }^{14}$ Agramonte, ${ }^{15}$ and others has definitely refuted his original claims, and there is to-ray no scientific basis for the assumption that the Bacillus icteroides has any etiological relationship to the disease Protozoan incitants, also, have been described by Klebs, ${ }^{16}$ Schüller, ${ }^{17}$ Thayer, ${ }^{18}$ and others, without bringing conviction or even justifying extensive investigation of their claims.

While the earlier etiological investigations, therefore, were inconclusive, much evidence was adduced which seemed to indicate that the virus was filtrable. Reed, Carroll, Agramonte and Lazear, carried out experiments which they thought demonstrated that the infecting agent was present in the blood of patients during the first three days of the disease, and could pass through the pores of

${ }^{10}$ Cornil and Babes, Comptes rend. de l'acad. des sci., 1883.

${ }^{11}$ Sternberg, Cent. f. Bakt., I, xii, 1897.

${ }^{12}$ Sanarelli, Ann. de l'inst. Pasteur, 1897, and Cent. f. Bakt., I, xxii, xxvii, and $\mathrm{xxix}$.

${ }^{13}$ Durham and Myers, Thompson Yates Laboratory Reports, 3, 1902.

${ }^{14}$ Otto, Vierteljahrsch. f. gericht. Medizin, ete., 27, 1904.

${ }^{15}$ Agramonte, N. Y. Med. TJews, 1900.

${ }^{16}$ Klehs, Jour. Am. Med. Assn., April, 1898.

${ }^{17}$ Schüller, Berl. klin. Woch., 7, 1906.

${ }^{18}$ Thayer, Med. Record, 1907. 
a Berkefeld filter. The filtrability of the virus has recently become doubtful in view of the researches of Noguchi.

Recently, Noguchi ${ }^{19}$ has carried out investigations which promise to settle the etiological problem in yellow fever conclusively. Noguchi in 1918 carricd out extensive studies in the Yellow Fever Hospital at Guayaquil, where he began by observing 172 typical cases of the disease, studying them clinically and pathologically. We mention this because the only possible source of error in his investigations seems to us to be that of mistaking of cases of infectious jaundice for yellow fever, which, of course, is possible in view of the clinical similarity between the diseases, as emphasized by Nishi. It is important, therefore, to mention that Noguchi worked on what he considered classical cases of yellow fever in a Yellow Fever Hospital where he was aided by physicians familiar with the disease. He began by injecting blood from these cases in the first week of the disease, into a large number of different animals. The only animals with which he had success, however, were guinea-pigs. With the blood of carly cases he inoculated seventy-four guinea-pigs from twenty-seven eases of yellow fever. Of these, eight, representing six cases, came down with symptoms resembling human yellow fever. After an ineubation period of three to six days, the guinea-pigs showed a marked rise of temperature, conjunctival congestion, leucocytosis followed by progressive leucopenia, and a drop of temperature after a few days. Jaundice was noticed during this period, and hemorrhages from the nose and anus were oecasionally observed. At autopsy the tissues were deeply jaundiced and the organs hyperemic. Hemorrhagic spots were found in the lungs and in the intestinal mucous membrane.

In the blood, liver and kidneys of the guinea-pigs experimentally infected, Noguchi found the organism which he calls the Leptospira Ictero-Homorrhagice. This organism resembles quite closely the causative agent of infectious jaundice. In general, his monkey experiments were negative in all species except the Marmosets (Midas Oedipus and Midas Geoffroyi).

The examination of the blood of patients by the dark field in Noguchi's ${ }^{20}$ hands never yielded large numbers of organisms. In careful examinations made on twenty-seven cases he found them in three only. He never saw them in urine, but one guinea-pig

\footnotetext{
${ }^{19}$ Noguchi, Jour. Exper. Med., 29, 1919, 547-596.

${ }^{20}$ Noguchi, Jour. Exper. Med., 30, 1919, 87.
} 
inoculated with 10 c.c. of urine eame down. Examinations of the organs revealed them in only one case in the kidneys.

Working with mosquitoes, Noguehi allowed Stegomyia ealopus to bite yellow fever patients during the early stages of the discase. He placed the arm of a patient into a cage containing 200 to 300 mosquitoes hatehed from the larvæ, and allowed the mosquitoes to feed until the females were full of blood. Twenty-three days after feeding on the patient, the mosquitoes were allowed to feed on guinea-pigs. By this method he claims that he obtained one positive experiment out of six. In this positive experiment the guinea-pigs developed typical symptoms in about fifteen days.

Noguchi also eultivated the Leptospira three times directly from yellow fever patients. The medium consisted of a mixture of one part of serum from non-immune persons and three parts of Ringer's solution, used both in the liquid form and also in the semi-solid condition, by adding small amounts of melted neutral agar. To this about 1 c.e. of eitrated blood from the median basilic vein of the patient was added, first being mixed with the semi-solid agar, while this was in the fluid eondition at $42^{\circ} \mathrm{C}$. This was allowed to solidify by cooling, and the warm Ringer's solution was then poured on the semi-solid portion and about 0.5 to 1 c.c. of the same blood introduced. The eulture was then covered with paraffin oil.

He also eultivated the organisms from infeeted guinea-pigs.

He described them as follows: The Leptospira is an extremely delicate filament about 4 to 9 miera in length and 0.2 of a micron in width. It tapers gradually toward the extremities and ends in thick sharp points. It is minutely wound at short and regular intervals, each section measuring about 0.25 of a mieron. The windings are so placed as to form a zigzag line at angles of $90^{\circ}$.

It is not visible by ordinary light, but is easily seen with a dark field. It is actively motile, showing a vibratory motion and sometimes twisting parts of the filament. It bores into the semi-solid material and is remarkably flexible.

It is difficult to stain with ordinary dyes, but can be fixed by osmic acid and stained with Giemsa or other polyehrome stains.

In regard to transmission, it is an important fact (Noguchi) that 67 per cent of the wild rats of Guayaquil showed organisms in their kidneys similar in appearance to the leptospira just deseribed; and these inoculated into guinea-pigs produced lesions similar to those produced by the yellow fever blood. 
In regard to the question of the identity of the yellow fever spirochæte with that of Weil's disease, Noguchi states that serological differentiation could be made. Polyvalent immune sera, one specific for icteroides and the other speeifie for icterohemorrhagice, showed a high neutralizing power for eultures of the homologous group. He found, however, that the action of the sera is not absolutely specific, since the injection of a sufficient amount of anti-icteroides serum prevents a fatal outcome in guinea-pigs inoculated with multiple doses of the other organism, and vice versa. Other forms of serum reaction showed the same lack of abṣolute specificity.

Subsequently, Noguchi attempted to protect guinea-pigs against multiple doses by injection of immune sera. He produced polyvalent immune serum by inoculation of a horse, and found that such serum could protect guinea-pigs when administered during the period of incubation, and modified the course of the disease when used in the early stages, but had no perceptible result in the later periods.

Subsequent to the experiments of Noguchi recorded above, Noguchi and Kligler ${ }^{21}$ obtained results of similar experimental significance in Yucatan.

\section{PREVENTION OF YELLOW FEVER}

Success in the prevention of yellow fever has been one of the important factors in the development of South and Central American countries. The conversion of Rio de Janeiro into a healthy port by Oswaldo Cruz, the cleaning up of New Orleans and the work done by Gorgas in Panama indicate the splendid rôle which an understanding of the relations of transmission in this disease have played in the progress of eivilization. Knowing what we do about the Stegomyia and the part played by it in transmission, preventive measures must depend primarily upon the suppression of the mosquito and the isolation of eases from which mosquitoes may aequire the infection. The disease is never, as far as we know, conveyed by direct infection from man to man, or by soiled clothing, etc., and in consequence there is no necessity for care in these matters. The stegmoyia breeds particularly in rather pure water, such as found in rain water eisterns, and fortunately does not, like some other mosquitoes, breed in swamps, ponds and other natural

${ }^{21}$ Noguchi and Kligler, Jour. Exper. Med., 32, 1920, 601. 
surface waters. Its flying radius is apparently not very large. Unlike the Anopheles, its habits are diurnal instead of nocturnal. Rosenau states that experience of the epidemic in New Orleans in 1905 showed that this mosquito does not fly far from its place of birth. We take from the same writer the statement that, in order to hold back the small mosquito, a mesh must be used containing at least twenty strands to the inch.

Prevention may, therefore, be summed up as consisting in screening of patients, screening of houses, destruction of mosquitoes within houses by insecticides and fumigation and the painstaking removal of all stagnant waters in the neighborhood of human habitations with especial care to the screening of drinking water cisterns in places where, as in Bermuda, drinking water is collected in rain water receptacles. In places like Bermuda, the Stegomyia fasciata is common, and yet there has been no case of yellow fever, we understand, since 1869 .

Immunity.-Natural immunity against yellow fever was formerly assumed to exist in the negro race. More recent investigations have not borne out this assumption. The negro soldiers of the American army in Cuba were afflicted equally with the white troops. The relative immunity of dark-skinned races, however, is explained possibly by the fact that the stegomyia prefers to attack light-colored surfaces.

A single attack seems to protect against subsequent infection throughout life.

Relative immunity was produced by Marchoux, Salimbeni, and Simond.$^{22}$ by injections of the serum of convalescents, serum heated to $55^{\circ}$ C., and of defibrinated blood preserved for eight days in vessels sealed with vaseline.

Experimental work is being carried out by Noguchi on both active and passive immunization by the use of his pure cultures of the Leptospira icteroides. These experiments are being actively carried out at the present time, but final results have not yet been achieved. On the whole, however, the promise of this work is great and extremely encouraging. It is especially encouraging in consideration of the great similarity between this Leptospira and the one of Weil's disease with which immunization experiments are also full of promise.

${ }_{22}^{2}$ Marchoux, Salimbeni, and Simond, Ann. de 1'inst. Pastenr, 1903. 


\section{WEIL'S DISEASE; ICTERO-HÆMORRHAGIC FEVER; (EPIDEMIC JAUNDICE)}

This is a disease which has been known for a very long time. It was described by Larrey as early as 1800 and the probability of its being a clinical entity was recognized by a number of other observers between that time and 1886 when Weil gave an accurate description of a number of eases he observed in Germany. The infectious nature of the disease was suspected from the course and symptoms and many etiological "guesses" were made. For a time it was suspected that the disease was particularly associated with butcher shops and occurred especially in individuals following the butcher's trade. During the recent war the disease oceurred in many of the armies at the front, in the trenches. They were first reported from the Italian front and later in the British, German and French armies, oceurring at times in almost epidemic-like form. The disease has been relatively mild in European countries, whereas in Japan where it seems to be particularly a disease of mine workers, it is quite virulent and often has a high death rate. It seems to have been particularly a disease of war, since the cases of Larrey came from the Eygptian army of Napoleon. The recent outbreaks were largely among soldiers and it is stated that a considerable number of eases occurred in the South African and the American Civil Wars. It is important to note in connection with what will be said later about yellow fever that Nishi identified Weil's disease with fellow fever some time ago, but was opposed by Ohno. Inada and his co-workers mention this, and emphasize the difference between the two diseases from the epidemiological point of view, though they admit the similarity of the symptoms. Also, transmission seems to be quite different in the two diseases.

The Disease.-Fiedler, ${ }^{23}$ whom we quote from Inada ${ }^{24}$ divides the disease into three stages, an initial period of two or three days, with fever, followed by a second stage of three days, during which icterus, edema of the liver, enlargement of the spleen and skin hemorrhages oecur. The deferveseent period starts on the seventh day and initiates convaleseence. Inada deseribes it as follows: The

28 Fiedler, Deut. arch. klin. Med., 1892, 1, 232.

${ }^{24}$ Inada, Jour. exper. Med., 26, 1917, 355. 
onset is initiated by chills and fever, intestinal disturbances, headache, muscular pains, hyperemia of the conjunctivx and al. buminuria. In this stage death is rare, and during this time the spirochætes are freely circulating in the blood. Blood injected during this period into guinea-pigs produces a typical reaction. The infectivity of the blood decreases from this time on, according to Inada. From the seventh to the thirteenth day of the icteric period, which, according to Inada, covers a little less than a week. The symptoms decrease, the jaundice and hemorrhages into the skin appear, together with great weakness, nervous and cardiac symptoms. The two stages shade into each other and the second period is the one during which death is most common. In eases dead at this time the spirochaetes have disappeared from the blood, and antibodies can be shown by the Pfeiffer test. During the second stage, the spirochaetes are present in the urine and can be found by the dark field. Inada showed them in 17.4 per cent of his cases on the tenth day, with a gradually growing percentage up to 52.2 per cent. As antibodies develop the spirochaetes disappear from the blood and from the liver.

On the thirteenth and fourteenth day begins the convalescent stage. The jaundice becomes less and anemia and emaciation appear. Antibodies reach their highest point in the blood, the spirochaetes completely disappear from the blood, and become more abundant in the urine. Inada states that the only organ in which the spirochaetes can be found at this time are the kidneys. By the nineteenth and twentieth day, practically all cases show the organisms in the urine.

The incubation period of the disease is five to seven days.

Etiology of Weil's Disease.-A great many different microorganisms have been described in the course of time as the cause of Weil's disease. The question was definitely settled in 1916 by Inada, Yutaka, Hoki, Kaneko and $\mathrm{Ito}^{25}$ who deseribed the Spirochata IcteroHaemorrhagiae. 'They found the organisms in the blood, liver, adrenal glands and kidneys and transmitted it by intraperitoneal injection into guinea-pigs. They also sueceeded in enltivating the organisms by the Noguchi method. There can be no question whatever at the present time eoncerning the etiological importance of this organism in Weil's disease. Inada and his co-workers inoculated

${ }^{25}$ Inada, and coworkers, Jour: Exper. Med., 23, 1916, 377. 
monkeys, rabbits, rats and guinea-pigs with the blood of patients in July, 1913, and found that the guinea-pigs developed albuninuria, Jaundice and hemorrhages when the blood was injected during the first seven days of the illness. Subsequently, they found the spirochaetes in large numbers in the livers of inoculated guinea-pigs, and following this, found them microseopically in six specimens of patients' blood and at autopsies. The jaundice in the guinea-pigs appears about seven or eight days after inoculation, the shortest time being six days and the longest thirteen. The disease can be kept going in guinea-pigs through many generations. Rabbits are insusceptible. Mice and rats seem to be slightly susceptible.

The organism seems always to remain outside the blood cells and is present in the interstitial tissues of organs. It has irregular wavy curves and is from 6 to 9 micra long, the largest being 12 to 13 . Thickness seems to vary with staining methods. Undulations are more irregular than those of treponema pallidum and are usually composed of two or three large irregular and four or five smaller waves.

Cultivation was successful in the hands of Inada and his coworkers by the method used by Noguchi in the case of the spirochæte of recurrent fever. They used guinea-pig instead of rabbit kidney, and covered their deep tubes with liquid paraffin. They found, however, that $37^{\circ} \mathrm{C}$. is not the best temperature for development, but that the cultures grow best at between $22^{\circ}$ and $25^{\circ} \mathrm{C}$. The cultures are odorless and there is no coagulation of the fluid which remains clear, even slight cloudiness indicating contamination. The cultures may live from three to six weeks in the first generation, and in later generations as long as fifty-five days.

Transmission.-Subsequent to the announcement of Inada's discovery, Miyjajima called attention to the fact that he had seen spirochaetes in the kidneys of field mice. This clue was followed up by Ido, Hoki, Ito, Wani ${ }^{26}$ and many others who found spirochaetes similar to the ones described by Inada in house and roof rats, Epymis or Mus alexandrinus and Mus norvegicus. Since that time Stokes ${ }^{27}$ demonstrated the organisms in the kidneys of field rats in the neighborhood of infected districts in Flanders, and Noguchi ${ }^{28}$ has found similar

\footnotetext{
${ }^{26}$ Ido, Hoki, Ito, and Wani, Jour. Exper. Med., 26, 1917, 341.

${ }^{27}$ Stokes, Ryle and Tytler, Lancet, 1, 1917, 142.

${ }^{28}$ Noguchi, Jour. Exper. Med., 25, 1917, 755.
} 
organisms in wild rats in the United States. From these and other experiments, it appears probable that the disease is primarily one. of rats, and is transmitted secondarily to man. It may be transmitted by contact with the urine of the rats and, according to the Japanese investigators, the infected soil may play an important part. Damp, cold mines in which rats abound seem to be particularly favorable. Infection from man to man may occur, since, as we have seen, the spirochrtes pass into the urine, but this is relatively rare. According to Inada and his coworkers, ${ }^{29}$ the spirochretes enter the. human body chiefly through the alimentary canal, but they may also come in through abrasions of the skin. Among fifty-five cases admitted to Inada's clinic, only a few indicated cutaneous origin, but there were certain of these in which cutaneous origin could not be denied.

Serum Treatment.-Inada and $\mathrm{Ido}^{29}$ first attempted to treat the disease with the blood of convalescent cases and observed astonishingly favorable results. Later, they actively immunized horses with pure cultures and finally obtained horses into whom they could inject 800 c.c. of a pure culture containing thirty spirochætes per field. 0.01 c.c. of this serum injected into the peritoneal cavity of guinea-pigs protected them against 1 c.c. of pure culture. In human cases they injected as much as 60 c.c. of this horse serum in twentyfour hours. Of thirty-five patients who received serum, seven died, showing a mortality of 11.4 per cent as against 17.3 per cent of cases dead at the same time, without serum. The serum completely destroyed spirochætes contained in the circulating blood. Further work on specific treatment is in progress.

Prevention.-The prevention of the fever seems from the above facts to rest primarily upon the destruction of rats and the prevention of contact of rats with food, the prevention of the pollution of soil, and isolation and care in regard to transmission in the case of patients suffering from the disease.

${ }^{20}$ Inada and Ido, Jour. Exper. Med., 24, 1916, 465. 


\section{SEGTION IV}

\section{DISEASES CAUSED BY FILTRABLE VIRUS, THE EXANTHE- MATA, AND DISEASES OF UNCERTAIN ETIOLOGY}

\section{CHAPTER XLV}

GENERAL CONSIDERATION OF FILTRABLE VIRUS, SMALLPOX AND RABIES

\section{FILTRABLE VIRUS}

RECENT investigations into the causation of disease have revealed that a considerable number of infections may be eaused by organisms too small to be held back by filters through which even the smallest bacteria cannot pass. The earliest observations of such "filtrable virus" are probably those of Froseh (1898) in foot-and-mouth disease and of Beijerinck in the mosaic disease of tobacco. Since then similar investigations have shown that a large number of diseases are probably caused by such minute organisms; their investigation, long delayed by the belief in their invisibility by even the most powerful microseopic aid, and by our inability to cultivate them, has taken new impetus from the discovery and probable cultivation of minute globoid bodies from the virus of poliomyelitis by Flexner and Noguchi (see below).

Similar bodies have since then been seen in connection with other diseases by many observers, and in lethargic encephalitis recently Strauss and Loewe claim to have cultivated organisms quite similar to those of poliomyelitis eultivated by Noguchi. In thinking of filtrability, however, one must remember that filtrability, like diffusibility, is not an absolute concept, and that much depends upon the nature of the filter used and the amount of suction applied. Thus, there has been much diseussion as to the filtrability of diseases like syphilis, yellow fever, ete., and in conditions eaused by flexible 
organisms like spirochætes it might be quite possible with sufficient suction and filters of sufficient size to mechanically pull through organisms which, by their actual normal dimensions, would be regarded as too large to be classified with filtrable organisms merely in regard to size. The Berkefeld filters of various grades will differ materially in the sizes of the particles they will let through, and filters that are new will act differently from filters that have been used; and in this regard will vary to some extent, according to the nature of the substances that they have been used for at previous filtrations. Also, in a comparative experiment made by us in connection with syphilis some years ago, it was found that new filters of presumably the same manufacturer's grade, differed somewhat in regard to the substances they allowed to pass through, and all experienced investigators will always control their filtration experiments by adding to the substance, the filtrability of which they wish to test, bacteria of a small size (like influenza bacillus or B. pyocyaneus), so that they may know in that particular filtration the filter used was impermeable to the small bacteria. Filtrability, then, is a matter of gradation and not a sharp wall which necessarily separates one kingdom of microorganisms from another.

The following tabulation of the diseases presumably caused by filtrable virus was made by Wolbach ${ }^{1}$ some time ago and is taken from his article by us in its entirety with one or two modifications made necessary by recent developments:

\section{SMALLPOX}

Smallpox or variola is one of the most virulent of infectious diseases. Throughout history it has been a severe scourge of mankind; prevailing in China and other Eastern countries many centuries before Christ and sweeping through medieval Europe, especially at the time of the Crusades, in a series of severe epidemics. All races of men are susceptible and no age fiom childhood to senility is exempt. In modern times the disease is endemic in most uncivilized countries, especially those of the East, and occurs sporadically in all parts of the globe. Owing to rigid enforcement of vaccination and of quarantine laws, however, the disease has been practically eradicated from civilized countries.

${ }^{2}$ Wolbach, Jour. of Med. Res., xxvii, 1912. 


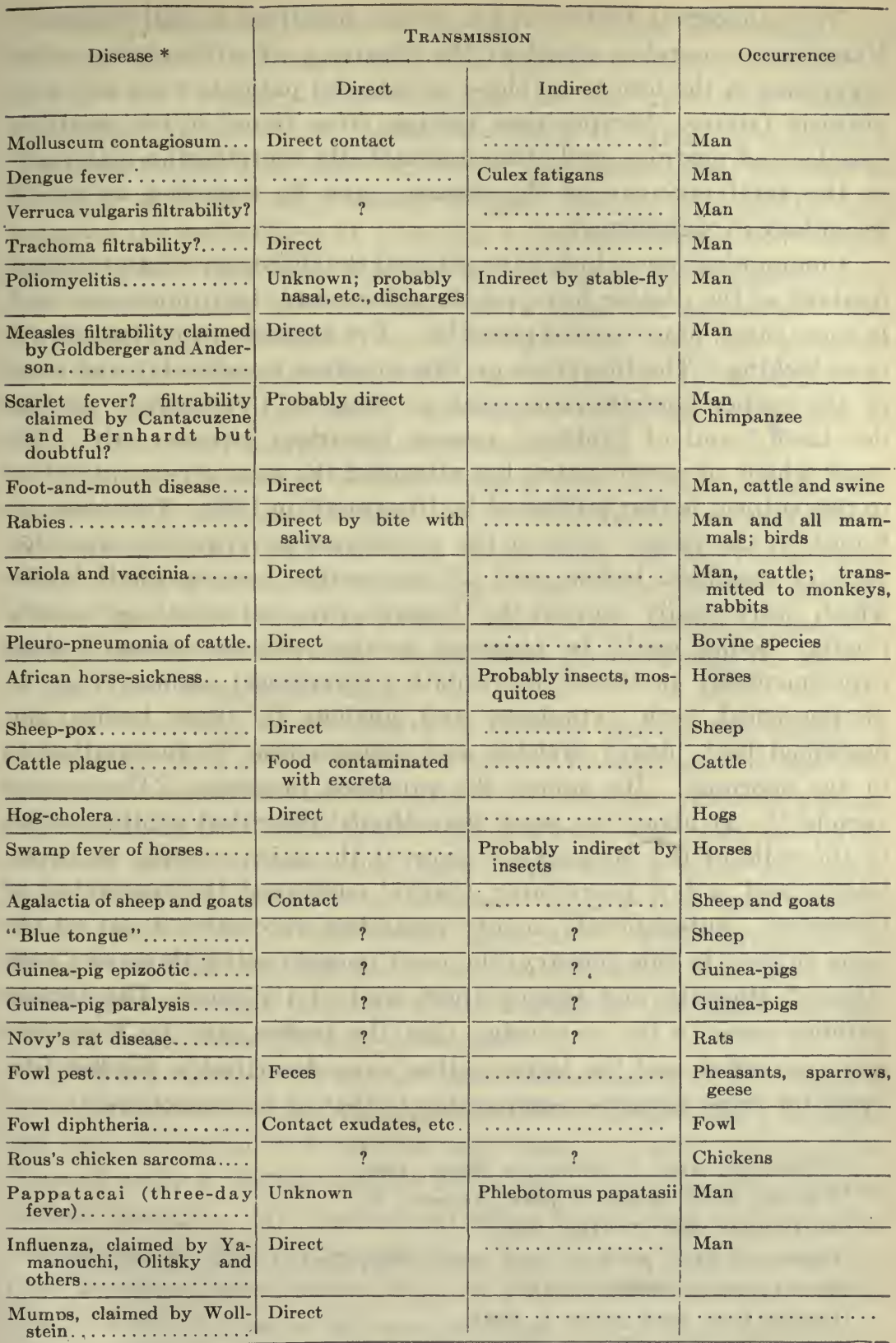

* Table slightly modified from Wolbach, loc. cit. 
The etiological factor which causes smallpox is still unknown. Numerous researches aimed at the discovery of cultivatable microorganisms in the lesions or blood of infected patients have met with uniform failure. Streptococei, though often found in the smallpox vesicles and pustules, and often undoubtedly contributing materially to the fatal outcome of the disease, may be regarded as purely secondary in significance.

Communications which have claimed the discovery of a protozoan incitant of the disease have, on the other hand, been numerous and, in some cases, have seemed plausible. Yet absolute proof has always been lacking. The literature on this question is extensive and some of the earlier contributions, such as those of Grünhagen, ${ }^{2}$ of Van der Loeff, ${ }^{3}$ and of Pfeiffer, ${ }^{4}$ possess historical interest only. The work which, of recent years, has attracted the most serious attention to this subject is that published by Guarnieri ${ }^{5}$ in 1892 . This observer found, in the deeper cells of the epithelium covering the pustules, both of smallpox lesions and of vaccination lesions, small bodies which were easily stained by hematoxylin, safranin, or carmin. Similar bodies could be observed in the cells of corneal lesions experimentally produced in rabbits. Guaranieri claimed that he distinguished both cytoplasm and nucleus in these bodies and described both binary division and reproduction by sporulation as in the sporozoa. He named the supposed protozoan "Cytorryctes variolæ." At about the same time Monti ${ }^{6}$ described similar bodies in the cells of the Malpighian layer of the skin covering smallpox lesions and, a few years later, Clarke ${ }^{7}$ confirmed the researches of Guarnieri. Subsequently, many researches were carried out on the same subject in this country, the most notable being those of Councilman, ${ }^{8}$ Magrath and Brinckerhoff, and of Calkins. ${ }^{9}$ The former authors came to the conclusion that the bodies seen by Guarnieri were parasites, and the latter author even described a distinct lifecycle for these parasites comparable to that of some protozoa.

${ }^{2}$ Grünhagen, Arch. f. Dermat. u. Syph., 1892.

s Van der Loeff, Monat. f. prakt. Dermat., iv.

4L. Pfeiffer, Zeit. f. Hyg., xxiii.

${ }^{5}$ Guarnieri, Arch. per le se. med., xxvi, 1892; Cent. f. Bakt., I, xvi, 1894.

'Monti, Cent. f. Bakt., I, xvi.

${ }^{7}$ Clarke, Brit. Med. Jour., 2, 1894.

${ }^{8}$ Councilman, Magrath, and Brinckerhoff, Jour. Med. Res., xi, 1904.

${ }^{9}$ Calkins, Jour. Med. Res., xi, 1904. 
These researches, however, are by no means convincing, and Ewing, ${ }^{10}$ while admitting that the vaccine bodies are probably specific for variola, calls attention to the fact that specific cell-degenerations or inclusions are found in diphtheria, measles, glanders, rabies, and other infectious processes, which can not be regarded as in any way related to these diseases etiologically, and suggests the probability of a similar interpretation for the vaccine bodies. Much has been said on both sides of the question since that time, and the problem can not be regarded as settled. The burden of proof, of course, rests upon those who claim the discovery of a specific microorganism, and absolute proof will probably be lacking until our experimental methods are such as will permit of other than purely morphological demonstration.

Whatever the actual causative agent may be, it is certain that the disease is transmitted with extreme ease-actual contact, direct or indirect, with a patient being unnecessary for its transmission. While we have no certain knowledge of the portal of entry through which the virus invades the human body; many considerations have made it seem plausible that this may take place through the mucosa of the upper respiratory tract.

Our knowledge of the means of defense against the malady is fortunately more advanced than is that of its etiology. It has been known for centuries that one attack of smallpox protects against subsequent attacks. This knowledge was made use of by the physicians of ancient China and India, who, during mild epidemics, exposed healthy children to infection, hoping that mild attacks would result which would confer immunity. While dangerous in the extreme, such "variolation," nevertheless, was not without some benefit and was even introduced into Europe in the eighteenth century by Lady Mary Wortley Montagu.

Such practices, however, were made unnecessary by the classical investigations of Jenner published in 1798. Jenner, as a student, had been impressed with the fact that country-people who had been infected with a disease known as cowpox, were usually immune against smallpox. His studies and observations came to a practical issue when, in 1796, he inoculated a hoy, James Phipps, with pus from a cowpox lesion on the hand of an infected dairy-maid. Two months later the same boy was inoculated with material from a

${ }^{10}$ Ewing, Jour. Med. Res., xiii, 1905. 
smallpox pustule without subsequent disease. With this experiment the principles of vaccination as in use at the present time were founded.

The question as to the identity of cowpox and smallpox has been the basis oi a long controversy. Many observers claimed from the beginning that the two diseases, though closely related to each other, were essentially different. Others, on the contrary, and this seems to be the prevailing opinion among seientists at the present days maintain that cowpox or vaceinia, as it is called when inoculated into a human being, represents merely an altered and attenuated variety of variola. This latter view is based on the following considerations, which we take from Haccius as quoted by Paul. ${ }^{11}$

1. Variola is invariably transmissible to cattle, when proper methods of inoculation are employed.

2. Variola earried through several animals, in the above way, becomes altered in character, approaching in nature typical vaccinia or cowpox.

3. Such virus, reinoculated into man, gives rise to purely local lesions which are mild and unlike smallpox.

4. Inoculation with such virus protects both man and animals against subsequent inoculation with cowpox, and, in the case of man, against smallpox as well.

Kolmer ${ }^{12}$ has carried out complement-fixations, using as antigens salt solution suspensions of cowpox and smallpox virus, and has demonstrated close biological relationship between the two.

It has been claimed, moreover, that cowpox originally was transmitted to eattle by human beings affected with smallpox. This seems likely both because of the comparative rarity of the former disease and because of its spontaneous occurrence almost invariably upon the teats of cows, although both males and females are equally susceptible to experimental inoculation.

The relationship of variola to chicken-pox or varicella has been more easily determined. Chicken-pox does not proteet against smallpox nor is this the case vice versa. The two diseases are unquestionably quite distinct.

The Production of Vaccine--During the early days of vaccination, it was customary to inoculate human beings with the matter

"Paul, "Vaccination"'; Kraus and Levaditi, "Handbuch," ete., I.

12 Kolmer, Jour, of Immunology, No. 1. Feb., 1916. 
obtained from the pustules of those previously vaccinated. While this method was perfectly satisfactory for the immediate purposes in view, practical difficulties and the occasional accidental transmission of syphilis have rendered this practice undesirable. In consequence, at all institutes at which vaccine is produced for use upon man, the virus is obtained from animals. Horses and mules, both extremely susceptible to vaccine, have been employed, and goats have, at times, been chosen because of their insusceptibility to tuberculosis. Rabbits have also been used more recently by Calmette and Guerin. ${ }^{13}$

The animals almost exclusively employed at the present day, however, are calves, preferably at ages of from six months to two years. Very young suckling calves are unsuitable because of the great speed of development and small size of the lesions produced. The animals should be healthy and at some institutes (Dresden) are subjected, before use, to the tuberculin test; although, according to Paul, ${ }^{11}$ this produces a hypersusceptibility to the vaccine, and can be omitted without danger when careful supervision is observed. Some observers prefer to use light-colored animals rather than darkskinned or black ones, both for reasons of greater ease of cleanliness and because the former are supposed to be more susceptible than the latter. This contention is denied by others. The sex of the animals is immaterial.

During the period of use, the calves are fed, according to age, with either an exclusive milk diet, or they are given, in addition, fresh hay. The greatest cleanliness in regard to the bedding and stalls must be observed and separate stables should be available for the animals under treatment and those under observation before treatment. These stables, if possible, should be so built that they can be easily scoured and flushed with water, and stalls should be disinfected after occupation. If possible, stables should be artificially heated and a comfortable temperature maintained. Halters and fastenings should be so arranged that the animals can not lick the scarified surfaces. Careful veterinary control before vaccination and during the period of treatment must be observed in order to eliminate animals with systemic disease or other complications.

The calves may be vaccinated with material taken from previously vaccinated animals. Again, they may be inoculated with

${ }^{13}$ Calmette and Guerin, Ann. de l'inst. Pasteur, 1902. 
"seed virus" obtained from the vesicles of human vaceinia. This method of using humanized virus for the inoculation of calves for vaceine production is preferred by many workers and is spoken of as "retrovaceination."

Park $^{14}$ believes that the most efficient and reliable seed virus consists of what he calls human-calf-rabbit seed virus. Crusts from healthy children, nineteen days after vaccination, are collected. These are cut up and emulsified with boiled water. With this an area of about six inches square is inoculated on a ealf, the remainder of which is vaceinated the ordinary way. The virus from this space is separately collected and, after being glycerinated, is used in dilution of 1 to $12 \frac{1}{2}$ parts of salt solution to vaceinate rabbits on the shaven skin of the back. The pulp from this rabbit vaccination is then used for ealf vaccination.

Actual vaccination of the animals is done as follows: Calves which have been kept under observation for at least a week are thoroughly washed and cleaned and the abdomen is clipped and shaved over an area extending from the ensiform cartilage to the pubic region, including the entire width of the belly and the inner folds of the thighs. It is best to shave the animal a day or two before vaccination so as to avoid fresh seratches and excoriations. Just before actual operation the animal is strapped to a specially constructed operating table in such a way as to allow free access to the shaved area. This area is now thoroughly washed with soap and water followed by alcohol, or, in some institutes, by a weak solution of lysol. If the latter is used, the field of operation must again be thoroughly rinsed with sterile.water. About a hundred small scarifications are made in this area, preferably by crossed seratches, covering for each searification an area of about $3-4$ square centimeters. Into these areas the virus is rubbed, using for each small area a quantity about sufficient to vaceinate three children. Two to three centimeter spaces are left between the lesions. The lesions are then allowed to dry and may be covered with sterile gauze or, as in Vienna, with a paste made up of beeswax, gum arabie, zine oxid, water, and glycerin. In some iistitutes the lesions are left entirely uneovered.

Ordinarily within about twenty-four hours after vaccination a narrow pink areola appears about the scratches. Within forty-eight

${ }^{14}$ Park and Williams, Path. Microorg., N. Y., 1914, p. 569. 
hours the seratehes themselves become slightly raised and papular, and within four or six days typical vaceina vesicles have usually developed.

To obtain the vaceme from such lesions, the entire operative field is earefully washed with warm water and soap, followed by sterile water. In some cases two per cent lysol is employed, but must again be thoroughly removed by subsequent washing with sterile water. Crusts, if present, are then earefully picked off and the entire contents of the vesicle, sticky serum, and pulpy exudate removed by the single sweep of a spoon-curette. The curetted masses are caught in sterile beakers or tubes and to them is added four times their weight of a mixture of glycerin fifty parts, water forty-nine parts, and carbolic acid one part. ${ }^{15}$ German workers prefer a mixture of glycerin eighty parts, and water twenty parts, omitting the use of carbolic acid. The glycerinated pulp is allowed to stand for three or four weeks in order to allow bacteria, which are invariably present, to die out. After preservation for such a length of time, moreover, thorough emulsification is obtained more easily than when this is attempted immediately after curettage. At the end of three or four weeks, the glycerinated pulp is thoroughly triturated, either with mortar and pestle or by means of specially constructed triturating devices. Pulp so prepared should remain active for at least three months if properly preserved in sealed tubes in a dark and cool place.

From the serum oozing from the bases of the lesions, after curettage, bone or ivory slips may be charged for vaceination with dry virus. The glycerinated pulp is put up in small capillary tubes, sealed at both ends, and distributed in this form. Park states that a calf should yield about 10 grams of pulp (which when made up should suffice to vaceinate 1,500 persons), and. in addition about 200 charged bone slips.

The virus may be tested for its efficiency by a variety of methods. Calmette and Guerin inoculate rabbits upon the inner surfaces of the ears and estimate the potency of the virus from the speed of development and extensiveness of the resulting lesions. Guerin ${ }^{16}$ has estimated the potency of virus quantitatively by a method depending upon the inoculation of rabbits with a series of dilutions.

\footnotetext{
${ }^{15}$ Huddleston, quoted in Park, "Pathogenic Bacteria," N. Y. 1908.

${ }^{16}$ Guerin, Ann. de l'inst. Pasteur, 1905.
} 
Beginning with a mixture containing equal weights of glycerin and vaccine pulp, dilutions are made with sterile water ranging from 1 in 10 to 1 in 100. Rabbits are shaved over the skin of the back and 1 e.c. of each of these dilutions is rubbed into the shaved areas. Fully potent virus should eause closely approximated vesicles in a dilution of 1 in 500, and numerous isolated vesicles in a dilution as high as 1 in 1,000 .

Quantitative estimations of the bacteria in the glycerinated virus should be made by the plating method and the vaccine used only when after several weeks of preservation the numbers of the bacteria have been greatly diminished. In glycerinated pulp the bacteria will often disappear entirely in the course of a month. The vaceine should also be tested for the possible presence of tetanus bacilli, by the inoculation of white mice.

Vaccination of human beings is performed by slightly scarifying the skin of the arm or leg with a sharp sterile needle or lancet and rubbing into the lesion potent vaceine virus. The virus was formerly dried upon wood, bone, or ivory slips and moistened with sterile water before the operation: At the present day the glycerinated pulp is almost universally employed.

Since the ordinary scarification method has not been universally satisfactory, other methods of vaceination have been suggested. Occasional failure of the scarification method may in part be due to the fact that the glycerinated, ripened virus as used in most countries, has lost considerably in potency. We have seen men in the American Army successfully vaccinated with French virus after two vaccinations with American virus had failed. The French virus used had not been allowed to ripen in glycerine, was reasonably fresh (so-called green virus) and was, therefore, probably more potent. The fact that it still contained staphylococei and other organisms did not in these cases lead to infections of any importance. It must, however, always be dangerous, and the use of green virus, while perhaps more efficient from the point of view of vaceination, does not seem to us to be commendable. It is probably better to attempt modification of the present method by a more efficient introduction of the virus. The alternative methods which have been suggested are puncture in which drops of virus are placed on the skin and punctures made through the drops. Scarification or small incisions through the drops have also been recommended. Recent 
studies by Wright ${ }^{17}$ have shown that the intracutaneous injection of 0.1 c.c. of the ordinary glycerinated virus diluted with one part of sterile distilled water, resulted in "takes" when the ordinary scarification methods were unsuccessful. He vaccinated 227 negro soldiers in this way, and obtained over 70 per cent successful vaccinations when only slightly over 8 per cent were obtained on the same series of cases previously, by the incision method.

Sternberg, Kinyoun and others have demonstrated that within two weeks after vaccination the blood serum of the vaccinated patients will neutralize vaccine virus if allowed to stand with it in a test tube overnight.

That vaccination is of incalculable benefit to the human race is no longer a question of opinion, and opposition to the practice is explicable only on the basis of ignorance. Statistical compilations upon this point are very numerous. It may suffice to select from the voluminous literature a single example, taken from Jürgensen, which embodies the statistics of death from smallpox in Sweden, during the periods immediately preceding and following the introduction of vaccination. In that country the first vaccination was done in 1801. By 1810 the practice was generally in use but not enforced. In 1816 it was legally enforced. The years from 1774 to 1855 can thus be divided into three periods.

1. Prevaccinal period, 1774-1801 (25 years). Deaths smallpox per million inhabitants .............................. 2,050

2. Transitional period, $1801-1810$ (9 years) $\ldots \ldots \ldots \ldots \ldots \ldots \ldots 660$

3. Vaccination enforced, $1810-1855$ (35 years) ............. 169 Prevaccinal period death rate 20.00 per mille. Vaccinal period death rate 0.17 per mille.

In considering the benefit of vaccination it must not be forgotten that revaccination is quite as important as the first vaccination, which confers immunity only for from seven to ten years. A child should therefore be vaccinated soon after birth or at least before the eighth month, and the process should be repeated about every seven years thereafter.

${ }^{17}$ Wright, Jour. A. M. A. 71, 1918, 654. 


\section{RABIES}

(Hydrophobia, Rage, Lyssa, Hundswuth)

Rabies is primarily a disease of animals, infectious for practically all the mammalia, but most prevalent among carnivora, dogs, cats, and wolves. It is said also to occur spontaneously among skunks of the Southwestern United States, and is readily inoculable upon guinea-pigs, rabbits, mice, rats, and certain birds, chicken and gecse being especially susceptible. Man is subject to the disease. Infection usually occurs as a consequence of the saliva of rabid animals gaining entrance to wounds from bites or seratches. The disease is more or less widely prevalent in all eivilized countries except England, where the careful supervision of dogs, enforcement of muzzling laws, and rigid legislation regarding the importation of dogs, have caused a practical eradication of the disease. A fair estimate of the prevalence of the disease may be obtained from the statisties of animals dying or killed because of rabies in different countries. In Germany, according to Kolle and Hetsch, during the fifteen years ending in 1901, there were 9,069 dogs, 1,664 eattle, 191 sheep, 110 horses, 175 hogs, 79 cats, 16 goats, 1 mule, and 1 fox affected with rabies. In eastern United States the disease is not uncommon. The statistics of the New York Department of Health, for a period of six months ending December 31, 1907, show seventy-four cases of rabies among dogs in New York City and vicinity. Among human beings the disease is no longer common in civilized countries, since early preventive treatment is successfully applied in almost all infected subjects.

Experimental infection in susceptible animals is best carried out by injections of a salt-solution emulsion of the brain or spinal cord of an afflicted animal, subdurally, through a trephined opening in the skull, but may also be accomplished by injection into the peripheral nerves, the spinal canal, or the anterior chamber of the eye, Intravenous and intramuscular injections are also successful, though less regularly so.

The time of incubation after inoculation varies with the nature of the virus used, the location of the injection, and the quantity injected. In accidental infections of man and animals the incubation is shortest and the disease most severe when the wounds are 
about the head, neek, and upper extremities and are deeply lacerated. This is explained by the fact that the poison is conveyed to the central nervous system chiefly by the path of the nerve trunks. This has been experimentally shown by di Vestea and Zagari ${ }^{18}$ who inoculated animals by injection into peripheral nerves, and showed that the nerve tissue near the point of inoculation becomes infectious more quickly than the parts higher up; thus the lumbar cord of an animal inoculated in the sciatic nerve is infectious several days before virus ean be demonstrated in the medulla.

In man, infected with "street virus," that is, with the virus of a dog or other animal not experimentally inoculated, the incubation period varies from about forty to sixty days. Isolated eases have been reported in which this period was prolonged for several months beyond this.

The virulence of rabic virus may be markedly increased or diminished by a number of methods. By repeated passage of the virus through rabbits, Pasteur ${ }^{19}$ was able to increase its virulence to a more or less constant maximum. Such virus which had been brought to the highest obtainable virulence, he designated as "virus fixe." Inoeulation of rabbits, dogs, guinea-pigs, rats, and mice with such virus usually results in symptoms within six to eight days. The same animals inoculated with street virus may remain apparently healthy for two to three weeks.

In dogs and guinea-pigs inoculation usually results first in a stage of increased excitability, restlessness, and sometimes viciousness. This is followed by depression, torpor, loss of appetite, inability to swallow, and finally paralysis. In rabbits the disease usually takes the form of what is known as "dumb rabies," the animals gradually growing more somnolent and weak, with tremors and gradual paralysis beginning in the hind legs.

In experimentally infected birds the disease is slow in appearing and may show a course of gradually increasing weakness and progressive paralysis extending over a period of two weeks after the appearance of the first symptoms.

In man, the disease begins usually with headaches and nervous depression. This is followed by difficulty in swallowing and spasms of the respiratory muscles. These symptoms occur intermittently,

${ }^{18}$ di Vestea and Zagari, Ann. de l'inst. Pasteur, iii.

${ }^{10}$ Pasteur's work on rabies. Compt. rend. de I'acad. des sciences, 1881, 1882, 1884, 1885, 1886, and Ann. de l'inst. Pasteur, 1887 and 1888. 
the free intervals being marked by attacks of terror and nervous depression. Occasionally there are maniacal attacks in which the patient raves and completely loses self-control. Finally, paralysis sets in, ending eventually in death.

Pathological examination of the tissues of rabid animals and human beings reveals macroscopically nothing but ecchymoses in some of the mucous and serous membranes. Microscopically, however, many abnormal changes have been observed and were formerly utilized in histological diagnosis of the condition. Babes ${ }^{20}$ has deseribed a disappearance of the chromatic element in the nerve cells of the spinal cord. This observation has been confirmed by others, ${ }^{21}$ but is no longer regarded as pathognomonic of rabies. The same observer has deseribed a marked leucocytic infiltration which occurs about the blood-vessels of the brain and about the ganglia of the sympathetic system. These changes are not found in animals infected with virus fixe and are present only in animals and human beings inoeulated with street virus.

In $1903 \mathrm{Negri}^{22}$ of Pavia described peculiar structures which he observed in the cells of the central nervous system of rabid dogs. While present in all parts of the brain, these "Negri bodies" are most regularly present and numerous in the larger cells of the hippocampus major and in the Purkinje cells of the cercbellum. The presence of these structures in rabid animals and man has been confirmed by a large number of workers in various parts of the world, and the specific association of these bodies with the disease is now beyond doubt. In consequence, the determination of "Negri bodies" in the brains of suspected animals has become an extremely important method of diagnosis-more rapid and accurate than the methods previously known.

The demonstration of Negri bodies in tissues is carried out as follows: A small pieee of tissue is taken from the cerebellum or from the center of the hippocampus major (cornu ammonis), and is fixed for twelve hours in Zenker's fluid. It is then washed thoroughly in water and dehydrated as usual in graded alcohols, embedded in paraffin, and sectioned. The sections are best stained by the method of Mann, as follows:

\footnotetext{
${ }^{20}$ Babes, Virch. Arch., 110, and Ann. de 1'inst. Pasteur, 6, 1892.

${ }^{21} V$ an Gehuchten, Bull. de l'acad. de méd. et biol., 1900.

${ }^{22}$ Negri, Zeit. f. Hyg., xliii and xliv.
} 
The sections, attached to slides in the usual way, are immersed in the following solution for from twelve to twenty-four hours:

Methylene-blue (Gruebler 00), 1 per cent............. 35 c.e.

Eosin (Gruebler BA), 1 per cent.................. 35 e.c.

Distilled water ............................. 100 c.e.

They are then differentiated in:

Absolute alcohol ............................ 30 c.e.

Sodium hydrate, 1 per cent in absolute alcohol............ 5 c.c.

In this solution blue is given off and the sections become red. After about five minutes the sections are removed from this solution, are washed in absolute alcohol, and are placed in water where they again become faintly bluish. It is of advantage to immerse them, now, in water slightly acidified with acetic acid. Following this they are dehydrated with absolute alcohol and cleared in xylol, as usual.

In preparations made in this way, the nerve cells are stained a pale blue, and in their cytoplasm, lying either close to the nucleus or near the root of the axis-cylinder process, are seen small oval bodies stained a deep pink. The bodies are variable in size, measuring from 1 to 27 micra in diameter. They are round or oval, show a more deeply stained peripheral zone which has been interpreted as a cell membrane, and, in their interior, often show small vacuolelike bodies. There may be more than one, often as many as three or four, in a single cell.

The rapid demonstration of Negri bodies in smears of brain tissue has recently been advocated by many observers and has been extensively used for diagnosis. It is carried out, according to Van Gieson, ${ }^{23}$ in the following way: A small pin-head-sized piece of brain tissue from the regions indicated above, is placed on one end of a slide under a cover-glass and the cover is gently squeezed with the finger until the tissue is flattened out into a thin layer. The glass cover is then gently shifted across the slide until the brain tissue is smeared along the entire surface. These smears may be fixed in methyl alcohol and stained by the Giemsa method, as described in the chapter on Spirochæta pallida.

Stained in this way, the Negri bodies are stained light blue, in contrast to the darker and more violet cell-bodies.

${ }^{23}$ Van Gieson, Proc. of N. Y. Pathol. Soc., N. S., iv, 1906. 
The smears may also be stained by a method originated by Van Gieson, which gives an excellent contrast stain and reveals more clearly the inner structure of the Negri bodies. Van Gieson's stain is prepared as follows:

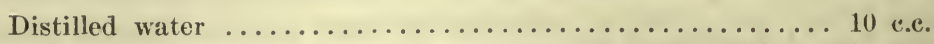

Saturated alcoholic solution of rosanilin violet.......... 2 drops.

Saturated aqueous solution of methylene-blue diluted one-half

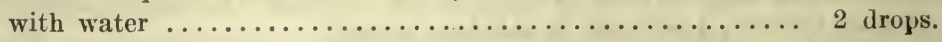

This method has been modified by Williams and Lowden, ${ }^{24}$ who add to 10 c.c. of distilled water three drops of saturated alcoholic basic fuchsin and 2 c.e. of Loeffler's methylene-blue. The slides are fixed in methyl alcohol, washed in water, and covered with the freshly prepared stain. The slide is held over the flame until the solution steams and is then rinsed in water and dried. The Negri bodies assume a brilliant red and contain in their interior darkly stained, irregular particles which have been interpreted as ehromatin bodies. As to the nature of the Negri bodies opinions are still divided. Their constant presence in rabic brain tissue is unquestioned and their diagnostic significanee well established. Cultivation experiments, however, have been uniformly unsuccessful. A number of observers, Negri himself, Calkins, ${ }^{25}$ Williams and Lowden, ${ }^{26}$ and others, believe these bodies to be protozoa. The last-named authors base this opinion upon the definite morphology of the bodies, and their staining properties, which in many respects are similar to those of protozoa. These observers also claim that the morphology of the bodies shows a number of regular cyclic ehanges which are found accompanying different stages of the disease; these changes correspond, according to these workers, to similar eyeles oceurring among known protozoa of the suborders of the class Sporozoa. Many pathologists still look upon them as speeifie degenerations of the nerve cells similar to the ehanges observed by Babes.

It is not possible to decide absolutely from the facts at present at our disposal whether or not the Negri bodies should be regarded as parasites or as specific degeneration products. Their constant presence in rabic animals, and their apparent absence from normal

${ }^{24}$ Williams and Lowden, Jour. Inf. Dis., 3, 1906.

${ }^{25}$ Calkins, Discussion, Proc. N. Y. Pathol. Soc., N. S., vol. vi, 1906.

${ }^{26}$ Williams and Lowden, loc. cit. 
brains and the brains of animals dead of other diseases, would tend to favor the parasitic view. To us it. would seem that added to this the clear outlines, apparent regularity of structure, and curiously consistent grouping of the darkly staining inclusions would add weight to such an assumption. We have triturated rabie tissue and shaken it up in anti-formin and seen many free Negri bodies apparently enueleated from the eells in consequence. Such complete extrusion from the cell also is seen in the ordinary smear preparations. It is at least unlikely that a cell-degeneration area would be expelled from the eytoplasm in so clearly outlined and morphologically unaltered a form. The fact that the virus is filtrable, as shown by Remlinger, ${ }^{27}$ Poor and Steinhardt, ${ }^{28}$ and others, would on the other hand seem to contradict the etiological importance of the Negri bodies unless, with some of the observers named, we assumed them to represent a large stage in the life-cycle of a protozoan parasite, which also occurred in smaller forms. It is a curious fact, also, that Negri bodies are scarce or absent in the spinal cord and cerebrum, though these areas are as virulent or more so than the hippoeampus and eerebellum. They are small and hard to find in virus fixe, largest and most plentiful in cases in which the incubation period has been prolonged-as with street-virus infection. Much can be said on both sides, but in analyzing the present experimental facts, it seems fair to say that neither point of view is certain, though the parasitic nature of the Negri bodies seems very likely.

The eultivation of parasites from rabie tissues has of eourse been attempted by most bacteriologists who have studied the disease since Pasteur. In all attempts, until very recently, either no results were obtained or else the parasites deseribed could be shown to be present because of extraneous contamination. Recently Noguchi announced that he has been able to eultivate the virus by employing a teehnique similar to his methods of eultivating Treponema pallidum and poliomyelitis virus. Into high tubes filled with ascitic fluid a bit of fresh sterile rabbit kidney and a small piece of rabic virus were placed. The ascitic fluid was covered with sterile oil and the tubes incubated at $37.5^{\circ} \mathrm{C}$. After five days' incubation eloudiness appeared and with it, minute globoid bodies not unlike those seen in

${ }^{27}$ Remlinger, Ann. de l'inst. Past., xvii, 1903.

${ }^{28}$ Poor and Steinhardt, Jour. of Inf. Dis., xii, 1913. 
poliomyelitis. After several generations large highly refractile bodies with dark centrål spots appeared in the cultures, and these Noguchi ${ }^{29}$ regards as possibly the parasites and similar to Negri bodies. Opinions are still divided as to the significance of Noguchi's results. However, whatever may be one's opinion regarding the nature of the peculiar bodies visible in his cultures, he has accomplished the feat of preserving the virulence of the virus through twenty-one generations on artificial media, a fact which alone would seem to prove that he had successfully cultivated it, even though his " "nucleated bodies" do not eventually turn out to be anything more than cell degenerations. The possibility that he may have carried original virus through twenty-one generations and that it has remained virulent for about 1.00 days at $37.5^{\circ} \mathrm{C}$. can not be excluded as yet, but seems very remote.

The Specific Therapy of Rabies.-The treatment which is now prophylactically applied to patients infected with or suspected of infection with rabies has been but little altered either in principle or in technical detail since it was first worked out by Pasteur. In principle it consists of an active immunization with virus, attenuated by drying, administered during the long incubation period in doses of progressively increasing virulence.

By the repeated passage of street virus through rabbits, Pasteur obtained a virus of maximum and approximately constant virulence which he designated as virus fixe. By a series of painstaking experiments he then ascertained that such virus fixe could be gradually attenuated by drying over caustic potash at a temperature of about $25^{\circ} \mathrm{C}$, the degree of attenuation varying directly with the time of drying. Thus, while fresh virus fixe regularly caused death in rabbits after six to seven days, the incubation time following the inoculation of dried virus grew longer and longer as the time of drying was increased, until finaliy virus dried for eight days was no longer regularly infectious and that dried for twelve to fourteen days had completely lost its virulence.

The method of active immunization which Pasteur used consisted in injecting, subcutaneously, virus of progressively increasing virulence, beginning with that derived from cords dried for thirteen days and gradually advancing to a strong virus. Thus the patient was immunized to a potent virus several weeks before the incubation time

${ }^{20}$ Noguchi, Jour. Exp. Med., xviii, 1913. 
of his own infection had elapsed. Pasteur suceessfully proved the efficacy of his method upon dogs and finally upon human beings, the first human ease being that of a nine-year-old child-Joseph Meister.

TeChnique of Rabies TheraPY.-The technique developed by Pasteur is still, in the main, followed by those who treat rabies to-day.

I. As a preliminary, it is neeessary to prepare or obtain virus fixe. This may generally be proeured from an established laboratory or may be prepared independently by passing street virus through a series of young rabbits (weighing from 700 to 1,000 gms.). According to Högyes, ${ }^{30}$ the passage of the virus through twenty-one to thirty rabbits, in this way, will reduce its incubation time to seven or eight days. Babes claims to obtain a virus fixe more rapidly by passing the vimus alternately through rabbits and guinea-pigs.

For purposes of inoculation, virus is prejared by emulsifying in sterile salt solution pieces of the medulla or cerebellum of animals dead of a previous inoculation. The brain tissue which is not emulsified may be preserved under sterile glycerin in a dark and cool place for further use.

II. Rabbits are inoculated with virus fixe by intracranial injection. A small incision is made in the shaved scalp in the median line, and the skin is retracted. With a small trephine or a round chisel, Fig. 100.-Метнan opening is made in the skull and in the angle between the coronary and sagittal sutures. Through this opening about 0.2 to 0.3 e.e. of the virus fixe is injected, either directly into the brain substance or simply under the dura.

As soon as a rabbit so inoculated has died it is autopsied. The animal before dissection should be washed in a disinfectant solution -lysol or carbolic acid. The skin is then removed and the animal, lying on its ventral surface, is fastened to a dissecting board. The spinal canal is then laid open with a pair of eurved scissors and the spinal cord carefully removed. This is accomplished by eutting across the cord in the lumbar region, and lifting this with a forceps while the nerve roots are divided from below upward.

The cord is suspended by a sterile thread within a large bottle

2. Högyes, quoted from Kraus and Levaditi, "Handb.," etc., I. 
into the bottom of which pieces of potassium hydrate have been placed. The bottle is then set away in a dark room or closet, the temperature of which is regulated so as to vary little above $25^{\circ} \mathrm{C}$. - Bacteriological controls as to the sterility of the cord should also be made.

After drying, pieces of the cord are prepared for injection. This is done in various ways. At the New York Department of Health $1 \mathrm{~cm}$. of the cord is emulsified in 3 e.c. of sterile salt solution, the dose for injection being usually 2.5 c.c. Marx ${ }^{31}$ emulsifies $1 \mathrm{~cm}$. of the cord in 5 e.c. of sterile bouillon or salt solution, using 1 to 3 c.c. of this for injection according to the age of the cord. For shipment 20 per cent of glycerin and 0.5 per cent of carbolic acid are added.

The scheme of treatment is also subject to variations according to the individual customs of various laboratories. The following scheme is the routine of the Pasteur Institute in Paris, as quoted in Kraus and Levaditi, "Handbuch für Immunitätsforschung," Vol. I, p. 713 .

\begin{tabular}{|c|c|c|c|c|c|c|}
\hline \multirow{2}{*}{$\begin{array}{l}\text { Day of } \\
\text { Treatment }\end{array}$} & Mild Cases & \multirow[b]{2}{*}{ Dose } & Medium Cases & \multirow[b]{2}{*}{ Dose } & Severe Cases & \multirow[b]{2}{*}{ Dose } \\
\hline & $\begin{array}{l}\text { Days of } \\
\text { Drying }\end{array}$ & & $\begin{array}{l}\text { Days of } \\
\text { Drying }\end{array}$ & & Days of Drying & \\
\hline 1 & $14+13$ & 3 c.c. & $14+13$ & 3 c.c. & А.M. $14+13$ P.м. $12+11$ & 3 c.c. \\
\hline 2 & $12+11$ & 3 c.c. & $12+11$ & 3 c.c. & А.м. $10+9$ Р.м. $8+7$ & 3 c.c. \\
\hline 3 & $10+9$ & 3 c.c. & $10+9$ & 3 c.c. & $\begin{array}{lll}\text { А.M. } & 7 & \text { P.M. } 6\end{array}$ & 2 c.c. \\
\hline 4 & $8+7$ & 3 c.c. & $8+7$ & 3 c.c. & ar & 2 c.c. \\
\hline 5 & $6+6$ & 3 c.c. & $6+6$ & 3 c.c. & 5 & 2 c.c. \\
\hline 6 & 5 & 1 c.c. & 5 & 2 c.c. & - & 2 c.c. \\
\hline 7 & 5 & 1 c.c. & 5 & 2 c.c. & 3 & 1 c.c. \\
\hline 8 & 4 & 1 c.c. & 4 & 2 c.c. & $t_{1}$ & 2 c.c. \\
\hline 9 & 3 & 1 c.c. & 3 & 1 c.c. & 3 & 1 c.c. \\
\hline 10 & 5 & 2 c.c. & 5 & 2 c.c. & 5 & 2 c.c. \\
\hline 11 & 5 & 2 c.c. & 5 & 2 c.c. & 5 & 2 c.c. \\
\hline 12 & 4 & 2 c.c. & 4 & 2 c.c. & 4 & 2 c.c. \\
\hline 13 & 4 & 2 c.c. & 4 & 2 c.c. & $=$ & 2 c.c. \\
\hline 14 & 3 & 2 c.c. & 3 & 2 c.c. & $\log 1$ & 2 c.c. \\
\hline 15 & 3 & 2 c.c. & 3 & 2 c.c. & | & 2 c.c. \\
\hline 16 & & & 5 & 2 c.c. & 5 & 2 c.c. \\
\hline 17 & & & 4 & 2 c.c. & 4 & 2 c.c. \\
\hline 18 & & & 3 & 2 c.c. & rets & 2 c.c. \\
\hline 19 & fret & & 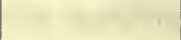 & it & 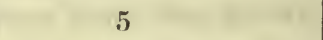 & 2 c.c. \\
\hline 20 & 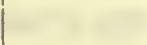 & & 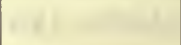 & & 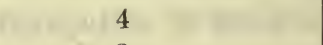 & 2 c.c. \\
\hline 21 & & & & & 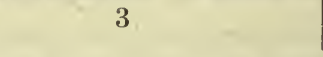 & 2 c.c. \\
\hline
\end{tabular}

${ }^{31}$ Marx, Deut. med. Woch., 1899, 1900. 
The treatment at the New York Department of Health is as follows :

\begin{tabular}{|c|c|c|c|c|c|c|}
\hline \multirow{2}{*}{$\begin{array}{c}\text { Day of } \\
\text { Treatment }\end{array}$} & Mild Cases & \multirow{2}{*}{ Dose } & Medium Cases & \multirow{2}{*}{ Dose } & Severe Cases & \multirow{2}{*}{ Dose } \\
\hline & $\begin{array}{l}\text { Days of } \\
\text { Drying }\end{array}$ & & $\begin{array}{l}\text { Days of } \\
\text { Drying }\end{array}$ & & Days of Drying & \\
\hline 1 & $14+13$ & 4 c.c. & 10 & 4 c.c. & А.M. $10+9$ Р.м. $10+9$ & 4 c.c. \\
\hline 2 & $12+11$ & 4 c.c. & 9 & 4 c.c. & А.м. $8+7$ Р.м. $8+7$ & 4 c.c. \\
\hline 3 & $10+9$ & 4 c.c. & 9 & 4 c.c. & 6 & 4 c.c. \\
\hline 4 & $8+7$ & 4 c.c. & $8+7$ & 4 c.c. & 4 & 4 c.c. \\
\hline 5 & 6 & 2 c.c. & 6 & 2 c.c. & 3 & 2 c.c. \\
\hline 6 & 5 & 2 c.c. & 5 & 2 c.c. & $\sqrt{1}+2=1-1$ & 2 c.c. \\
\hline 7 & 4 & 2 c.c. & 4 & 2 c.c. & 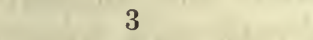 & 2 c.c. \\
\hline 8 & 3 & 2 c.c. & 3 & 2 c.c. & 2 & 2 c.c. \\
\hline 9 & 5 & 2 c.c. & 2 & 2 c.c. & 1 & 2 c.c. \\
\hline 10 & 4 & 2 c.c. & 5 & 2 c.c. & 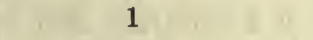 & 2 c.c. \\
\hline 11 & 3 & 2 c.c. & 4 & 2 c.c. & 4 & 2 c.c. \\
\hline 12 & 5 & 2 c.c. & 1 & 2 c.c. & 3 & 2 c.c. \\
\hline 13 & 4 & 2 c.c. & . & 2 c.c. & 10 & 2 c.c. \\
\hline 14 & 3 & 2 c.c. & $\cdot$ & 2 c.c. & $3+1=27$ & 2 c.c. \\
\hline 15 & 5 & 2 c.c. & ta & 2 c.c. & 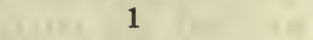 & 2 c.c. \\
\hline 16 & 4 & 2 c.c. & 2 & 2 c.c. & 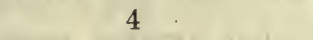 & 2 c.c. \\
\hline 17 & & 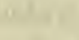 & 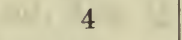 & 2 c.c. & 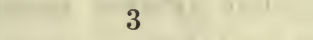 & 2 c.c. \\
\hline 18 & 1 & 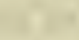 & ( & 2 c.c. & 1 & 2 c.c. \\
\hline 19 & . 1 & trat & , & 2 c.c. & +4 & 2 c.c. \\
\hline 20 & & & if & 4 & 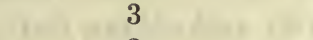 & 2 c.c. \\
\hline 21 & & & ting & & $y^{2}$ & 2 c.c. \\
\hline 22 & & 11 & 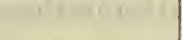 & & 4 & 2 c.c. \\
\hline 23 & , nt & a & in & $\cdot 1$ & $\begin{array}{r}3 \\
\square\end{array}$ & 2 c.c. \\
\hline 24 & -2 & 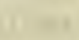 & 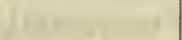 & 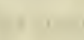 & 2 & 2 c.c. \\
\hline 25 & $x^{2}+2$ & & $x_{1}+2=0$ & (1) & · 4 & 2 c.c. \\
\hline 26 & & & & & 3 & 2 c.c. \\
\hline
\end{tabular}

The severity or mildness of eases is estimated from the depth and degree of laceration of the wounds, also from their locationbites about the face and upper extremities being the most dangerous.

During the eourse of such treatment patients may show troublesome erythema about the point of injection and occasionally backache and museular pains. Treatment need not be omitted unless these symptoms become excessive.

The efficiency of the Pasteur treatment in rabies is no longer problematical. According to Högyes, 50,000 people have been treated within ten years, with an average mortality of 1 per cent.

Although the method described above is the one which is extensively used in all established institutes for the treatment of rabies, 
other methods have been elaborated and used to a slight extent. One of the most important of these is the "dilution method" of Högyes. This method is carried out as follows: A definite quantity of the spinal cord of a rabbit dead of virus fixe is emulsified in 100 c.c. of normal salt solution. Dilutions of this emulsion are made and the patient is injected at first with a dilution of $1: 1,000$, subsequent injections being made of gradually increasing concentration until a concentration of $1: 100$ is reached. This method, so far as it has been used, has been satisfactory, but it has not yet found extensive application.

Harris and Shackell ${ }^{32}$ describe an improved method of desiccating rabic virus which consists in placing the material to be dried in the bottom of a vacuum desiccating jar in the upper part of which is a separate dish containing sulphuric acid. The temperature is reduced by placing the jar in a salt and ice mixture, and after thorough solidification of the material has resulted, a rapid vacuum is produced by a Geryk pump to less than $2 \mathrm{~mm}$. of mercury. The virus so dried will retain its virulence for as long as four months, if guarded against moisture. It will be noted that this method cannot be taken as justifying any particular conclusions as to the nature of the rabic virus since the same method has been applied by Swift and others to the maintenance of the virulence of bacteria.

Harris $^{33}$. believes that the attenuation of a rabic cord in the Pasteur method does not depend primarily upon the loss of water, but rather upon the method of extracting the water. Slow desiccation attenuates the virus, Harris concludes, by reason of the concentration of salt and other substances in solution of the brain and cord, the action being thus essentially a chemical one. Harris has studied the minimal lethal dose of his rapidly dried material on animals, and has developed with this material a modified method of immunizing animals and human beings against rabies.

He prepares suspensions (from the material rapidly dried at zero degrees as above) by emulsifying 10 milligrams in 10 c.c. salt solution. This gives a dilution of 1 milligram to each cubic centimeter of emulsion, and from this basic suspension dilutions are made.

${ }^{32}$ Harris and Shackell, Jour. of Infec. Dis., 8, 1911, 47.

${ }^{83}$ Harris, Jour. of Infec. Dis., 13, 1913, 155. 
He tests virulence by injecting 0.1 c.c. of the dilution into the brain of a rabbit, by trephining and passing a very fine hypodermic needle through the brain to the base or into the lateral ventricle, so that nore of the material may escape when the needle is withdrawn. He finds that, by this method, his material after preservation of three weeks, is equivalent to that of fresh cord. After fifty days it is 25 per cent more infeetive than the same quantity of the "one day" cord of the old method. After 200 days its infectivity is exactly equal to that of the "two day" cord of the old method. After 500 days it is two and one-half times as infective as the "three day" cord. The only precaution that is absolutely essential is that in preparing and preserving the diluted material, the presence of moisture must be absolutely prevented.

Having prepared this material, Harris thought it worth while to follow the suggestion of Högyes who has developed a method of immunization dependent upon the dilutions of virulent virus, instead of using the Pasteur method of quantitative destruction by drying. Högyes had treated 10,000 patients by his method without aceident, giving from 70 to 220 M. I. D., or minimal infective doses for rabbits, the first day and from 200 to 400 M. I. D. on the second, the M. I. D. being, as in Harris's experiments, determined by injeetions into the brains of rabbits. Harris has eonstructed eharts from careful experiments in which he tabulates the proportion of infectious to non-infectious material in a milligram of desiceated brain after preservation for various periods. Unlike Harvey and MeKenderick (quoted from Harris) who believe that when rabic virus has lost its infectiousness, it no longer has the power of conferring immunity, Harris believes that when all the infectivity of rabic material has been destroyed rapidly by light and various temperatures, it still has considerable powers of conferring immunity. It is, of course, impossible to determine the degree of immunity conferred by the non-infective portions of desiceated material.

Harris begins his treatment with material in which the proportion of living to non-infective material is estimated as being about 1 to 25 , that is, material at least six months old. As the treatment continues, he gradually increases until he uses material which contains 100 M. I. D. per milligram. In two years of such treatment, he had no accidents either in patients, dogs or rabbits.

Attempts to treat active rabies with the sera of immunized animals have so far been unsuceessful. 


\section{CHAPTER XLVI}

\section{ACUTE ANTERIOR POLIOMYELITIS}

\section{LETHARGIC ENCEPHALITIS}

THE disease known as acute anterior poliomyelitis has long been recognized as an acute infectious condition, both because of the characteristics of its elinical manifestations and of its epidemic occurrence. For these reasons it was classified with acute infectious diseases by Marie and by Strümpell long before any experimental evidence of infection was obtained.

Its contagiousness, while not a proven fact, seemed very likely from the evidence of its mode of spreading and has been removed from the sphere of mere conjecture by the careful study of a Swedish epidemic, comprising one thousand cases, made by Wickman. ${ }^{1}$

While acute anterior peliomyelitis is almost exclusively a disease of childhood, it is assumed by clinicians that it is etiologically closely related to, possibly identical with, certain diseases of the adult, characterized by bulbar paralysis and acute encephalitis. Into this eategory, also, some observers place the condition known as "Landry's paralysis." The basis for the identification of these conditions with poliomyelitis lies chiefly in the similarity of the pathological lesions and upon the fact that the last-named diseases occur most often during the course of poliomyelitis epidemics. The writer some years ago obtained a typical poliomyelitis infection in a monkey with material from a definite case of Landry's paralysis in a young woman.

The most thorough recent clinical study of acute poliomyelitis of which we know is the one by Peabody, Draper and Dochez. ${ }^{2}$ The incubation period of the disease varies, but appears to be about 10 days. Prodromal symptoms, if they appear at all, come on just before the onset of the disease. They may be very mild, consisting

${ }^{1}$ Wickman, quoted from Landsteiner and Popper, Zeit. f. Immunitätsforch., ii, 1909.

2 Peabody, Draper and Dochez, Monog. Rock Inst., 4, Jan., 1912. 
ot slight fever, sweating, drowsiness, pain in the neek and head, and weakness. Intestinal symptoms are not uncommon. Again eases may begin without prodromal symptoms with sudden illness, chill and fever. This may be all that occurs in the so-called abortive cases wnich in Wickman's studies represent from 25 to 56 per cent of all cases.

During the early periods of the disease the cases may show various types of development. They may simply show signs of general infection, they may resemble influenza, the gastro-intestinal symptoms may be predominant, and others may show signs of meningea irritation. The neck may be stiff, but not with the involuntary stiffness of meningitis, showing rather a reflex resistance when the attempt is made to move the head forward. Peabody, Draper and Dochez state that the best appreciation of the clinical condition in acute poliomyelitis may be had by considering the cases as (1) the abortive cases which never become paralysed, ${ }^{3}$ the cerebral group which is rare and in which involvement of the upper motor neurons with spastic paralysis is the chief characteristic, and (2) the bulbospinal group, which is the largest group, and in which motor neuron involvement and flaceid paralysis occur.

In the pre-paralytic stages the leucocytes are apt to be slightly increased in number and there is a definite increase of the polynuclears by 10 or 15 per cent. A leucocytosis of 15,000 to 30,000 is stated by the writers mentioned above as distinctly suggestive of the disease, especially if the polynuclears are increased at the expense of the lymphocytes. The study of the spinal fluid is of great value. During the first days of the disease, before the paralysis appears, there is an increase of the cellular contents with a total which may run as high as 500 cells per cubic millimeter, but usually they run about 50 per cubic millimeter. The writers above quoted saw two cases in which there were 999 and 650 cells, respectively. During the second week, of 45 cases seen by them, 8 had over 50 , and 23 were above normal. In the later stages of the disease the cell counts come back to normal. During this early stage, most of the cells consist of lymphocytes, rarely showing predominant polynuclears. 'I'he globulin contents during the early stages are practically normal, or slightly increased. This, however, increases later, as the cell counts drop. The fluid reduces Fehling's solution.

${ }^{8}$ For literature, see Landsteiner and Popper, loc. cit. and Wickman, Die HeineMedinsche Krankheit, Berlin, 1911. 


\section{ETIOLOGY}

An important advanee in the study of this discase was made in 1908 when Landsteiner and Popper succeeded in transmitting it to two monkeys (Cynocephalus hamadryas and Macacus rhesus). The transmission was accomplished by intraperitoneal injections of a saline emulsion of the spinal cord of a child that had died during the fourth day of an attack of infantile paralysis-during the stage of acute fever. The first monkey injected became severely ill six days after the injection and died on the eighth day. The second animal became paralyzed seventeen days after the injection and was killed two days later. Cultural experiments with the substance injected were negative, as were also inoculation experiments earried out upon guincapigs, rabbits, and mice. The histological lesions produced in the inoculated monkeys were similar to those occurring in children afflicted with the disease.

An attempt to transmit the disease to another monkey with spinalcord substance of the animal that was killed resulted negatively.

Soon after the successful experiments of Landsteiner and Popper, a similar result was recorded by Knoepfelmacher. ${ }^{4}$ An attempt to transmit the disease from monkey to monkey was again negative.

Similar positive inoculation results were published, a little later than this, by Flexner and Lewis ${ }^{5}$ in November, 1909, and by Strauss and Huntoon ${ }^{6}$ in January, 1910.

Flexner and Lewis, in their work, moreover, succeeded in transmitting the disease through several inoculation-generations of monkeys. The same workers ${ }^{7}$ have ascertained that inoculation may be successfully applied not only by the intraperitoneal route but intracerebrally, subeutaneously, intravenously, and by the path of the larger nerves. They also proved that not only the brain and cord of afflicted animals contains the virus, but that this may be found, during the early days of the disease at least, in the spinal fluid, the blood, the nasopharyngeal mucosa, and lymph nodes near the site of inoculation.

Landsteiner and Levaditi, ${ }^{8}$ meanwhile, experimenting with the

'Knoepfelmacher, Mediz. Klinik, v, 1909.

- Flexner and Lewis, Jour. Am. Med. Assn., 53, 1909.

- Strauss and Huntoon, N. Y. Med. Jour., Jan., 1910.

'Flexner and Lewis, Jour. Exp. Med., 12, 1909.

- Landsteiner and Levaditi, Comptes rend. de la soc. de biol., Nov., 1909, and Dec., 1909. 
virus independently, suceeded in transferring the disease from one animal to others, demonstrated that the virus could pass through the pores of a Berkefeld filter, and showed that the virus was present in the salivary glands-a fact which may prove of great importance in possibly establishing a clew to the mode of contagion among human beings. The same authors, as well as Flexner and Lewis, were able to show that the virus was preservable under glycerin for as long as ten days and retained its virulence for from seven to eleven days when dried. What has been said eoncerning infantile paralysis may also be taken to apply to Landry's paralysis. From a typical case of Landry's paralysis in an adult the writer succeeded in obtaining typical poliomyelitis in monkeys at Stanford University in 1912. The clinical diagnosis in this case was made by Wilbur. The disease in the monkeys was typical of poliomyelitis and the histological sections showed the typical lesions.

According to Flexner and Lewis the virus remains active, when frozen, for as long as forty days, but is extremely sensitive to heat, being destroyed by a temperature of from $45^{\circ}$ to $50^{\circ} \mathrm{C}$. maintained for thirty minutes.

Experiments aimed at the isolation or even morphological detection of a parasite in the virulent material have been entirely without success until recently. Bacteria which in the past have been isolated from nerve substance and spinal fluid in cases of poliomyelitis can of course be excluded from etiological significance by the recent determination of the filtrability of the virus as determined by Flexner and Lewis, and Landsteiner and Levaditi. Small coccoid forms in smears from the nerve tissue recently described by Proescher ${ }^{9}$ are of very uncertain significance. The streptococci recently described by Rosenow are, most probably, secondary invaders. The most important contribution which has been made in the solution of this problem is that of Flexner and Noguchi. ${ }^{10}$ These investigators placed small bits and emulsions of the brain of monkeys, dead of poliomyelitis, in high tubes containing human ascitic fluid together with a piece of fresh sterile rabbit kidney. In all essentials the method was that followed by Noguchi in his cultivation of Treponema pallidum. It was necessary to use fresh unheated ascitic fluid. Heat sterilization rendered it unsuitable.

By this method, after five days opalescence appeared about the

- Proescher, N. Y. Med. Jour., 1913.

${ }^{10}$ Flexner and Noguchi, Jour. of Exp. Med., xviii, 1913. 
pieces of tissue. This increased until the tenth day, when sedimentation began. Microscopical examination by Giemsa's method of staining revealed small globoid bodięs measuring from 0.15 to 0.3 micron in diameter, arranged in pairs, short chains, and masses. Similar bodies could later be found in poliomyelitis tissues. Cultures were obtained from glycerinated as well as from fresh virus and from the filtered as well as the unfiltered material. Typical lesions and death have been produced in monkeys with such cultures in a few eases.

We have few data which throw light upon possible immunity to the disease. Repeated attacks of the disease in the same human being have not been noted; but this, as Flexner and Lewis point out, may be due to the fact that the epidemics are rare, and individuals once afflicted have passed beyond the susceptible age by the time of the second epidemic. As a matter of fact, however, these workers have not succeeded in reinfecting monkeys that had recovered.

In chickens a disease has been observed similar in many ways to poliomyelitis, but further study has shown this to be a polyneuritis.

Of other animals besides monkeys, rabbits only have been successfully inoculated with this disease. Transmission to these animals was first reported by Kraus and Meinicke ${ }^{11}$ and later by Lentz and Huntemüller. ${ }^{12}$ Marks ${ }^{13}$ has studied the disease in rabbits thoroughly, and concludes that there is no doubt that the virus can be cultivated through a limited number of generations in rabbits. He was able to transmit to monkeys from rabbit material. The disease, however, does not resemble that of man or monkeys clinically and no definite lesions of the central nervous system are present. The rabbits seem perfectly well for six or seven days, when rapid weakness and death in convulsions occur.

Animals which have been unsuccessfully injected, even with living virus, do not develop immunity.. However, animals that have been successfully inoculated and recovered are, like human beings, thereafter immune. Levaditi and Landsteiner, Roemer and Joseph, and Flexner and Lewis have shown that the serum of recovered monkeys will protect normal animals from fatal doses of the virus. That the same protective power for monkeys has been shown in the serum of human recovered cases, is shown by the same authors and by Anderson and Frost and consequently the intraspinous injection of the serum of

${ }^{11}$ Kraus and Meinicke, Deut. med. Woch., xxxv, 1909.

${ }^{12}$ Lentz und Huntemüller, Zeitschr. f. Hyg., lxvi, 1910.

${ }^{13}$ Marks, Jour. of Exp. Med., xiv, 1911. 
recently recovered children into patients in early stages of the disease has recently been advocated and is though well of by a number of observers. This work, however, has not reached completion and fiual judgment must be withheld.

Flexner and Amoss have paid particular attention to the problem of passive immunization and found that in protecting monkeys if the quantity of virus injected into the brain is not too great paralysis can be prevented in some eases and delayed in others, by injeeting the serum of recovered monkeys into the subarachnoid space by lumbar puncture. Immunizing sera cannot be produced by treatment of insusceptible animals with virus, but Flexner and his associates have occasionally succeeded in immunizing actively by injecting, subcutaneously, graded doses of erude virus. This method, however, is not very useful, nor is it very safe since some of the animals so treated do not develop a strong immunity and others may become paralyzed. It appears, therefore, that the neutralizing principle, whatever it may be, is present only in animals and man that have recovered from actual infection, and the only method of passive immunization or serum treatment, therefore, available at the present time is that in. which the blood serum of individuals who have recovered from an attack is used.

Epidemiological Facts.-To summarize the important epidemiological facts in poliomyelitis we may say that the work of Flexner and his group, as well as that of European workers, has shown that the poliomyelitis virus is present in the mucous membranes of the nose and throat, in the exeretions from these membranes and in the intestinal contents. It may also be present in the tonsils. It leaves the infected body with the discharges from the nose and throat and the intestines, and when swallowed from the throat can pass into the intestines, resisting the action of the gastric and intestinal secretions. Flexner, Clarke and Dochez ${ }^{14}$ have injected monkeys with filtrates from washings of the intestines after feeding monkeys with spinal cord material from infected monkeys and taking the intestinal fluid two hours after feeding. Outside the human body the virus probably ean survive for some time, though the exact period is not known. Neustaedter and Thro ${ }^{15}$ claim to have been successful in infecting monkeys with dust taken from a sick room.

\footnotetext{
${ }^{14}$ Flexner, Clarlic and Dochez, Journ. A. M. A., vol. 59, 1912.

${ }^{15}$ Neustaedter and Thro, N. Y. Med. Journal, 94, 1911, p. 613, 813
} 
There is a great deal of evidence to show that poliomyelitis carriers exist. Experimental evidence of this carrier state has been advanced by Osgood and Lucas ${ }^{16}$ who found the virus in monkeys five months after convalescence. We ourselves have seen cases in which it could be definitely proven that they had not been in contact with a preceding case, two of them in country districts where the scantily populated surrounding area could be searched without danger of overlooking a case. It is also likely that at times of epidemic a great many very mild attacks of poliomyelitis may occur, which are mistaken for mild influenza or severe colds, and in connection with the occurrence of a recent case seen by us there were a number of indefinitely diagnosed cases of intestinal disturbance, with fever, in the neighborhood. It is not impossible that such eases may be true poliomyelitis of a mild type without paralysis but capable of passing on the virus. Peabody, Dochez and Draper ${ }^{17}$ have cited similar cases.

The virus probably gets into the new patient by direct and indirect contact, and can be carried from place to place, perhaps on the feet of flies, a fact which would be indicated by some experiments done by Flexner.

In 1911 epidemiologists of the State Public Health Service of Massachusetts established a parallelism between the distribution of poliomyelitis cases and the occurrence of the biting stable fly, Stomoxys. Subsequently, M. J. Rosenau published experiments in which he obtained poliomyelitis infection by allowing infected Stomxys flies to bite monkeys. These observations were confirmed by Anderson and Frost, ${ }^{18}$ but all subsequent attempts to repeat the experiments have failed. The ordinary manner of infecting of the human being is probably, then, through the nasopharynx. A great many cases begin with intestinal disturbances which may last a few days before the patient is ill enough to go to bed. It is more than likely, therefore, that the virus may also enter the body by ingestion and that infection of food may play a rôle.

The disease is usually present to some extent in crowded centers of the world, in the spring and summer months. The season of greatest prevalence is May to November. Most cases are in children below five, but adult cases do occur.

${ }^{16}$ Osgood and Lucas, Journal A. M. A., 57, 1911, p. 495.

${ }^{17}$ Peabody, Draper and Dochez, Monog. Rock. Inst., No. 4, Jan., 1912.

${ }^{18}$ Anderson and Frost, Tourn. A. M. A., 56, 1911, p. 663. 
Although a great many studies have been made to trace the infection of one case to exposure to another, such attempts have failed in most instances, and it seems fairly well established that there is great variation in the susceptibility of individuals to the disease. Whether this depends upon previous mild attacks of the varicty spoken of above or whether it is a congenital difference, cannot be stated.

\section{ENCEPHALITIS LETHARGICA}

It is difficult to say whether the disease which we now speak of as Lethargic Encephalitis is identical with the conditions formerly described as "sleeping sickness," "Schlaf Krankheit," etc. Camerarius, whom we quote from Smith, ${ }^{19}$ is said to have described an epidemic disease which occurred in Germany in 1712 which probably represents the same condition. In 1768 and in 1835 similar epidemics seem to have occurred in the trail of influenza outbreaks, a fact which is of considerable importance in view of the fact that recent interest in the disease dates from the oceurrence of many eases of lethargic encephalitis which followed in the train of the last influenza epidemic. After the epidemic of 1889 , relatively few typical cases of what we now speak of as lethargic encephalitis were reported, though nervous complications were apparently very common. During the later stages of the great war epidemic of influenza, eases began to appear in many different places which, at first, were either mistaken for poliomyelitis or undiagnosed before death. We remember ourselves seeing two cases in soldiers during this period in which diagnosis was doubtful and which we now believe to have been lethargic encephalitis.

One of the first systematic reports is that of Economo ${ }^{20}$ who described an outbreak of the disease in Vienna in 1917. In 1918 an outbreak occurred in Great Britain, which was studied and reported by Wilson, ${ }^{21} \mathrm{Hall},{ }^{22}$ Herringham, and others. (The onset of the disease in America was dealt with in an editorial in the Journal of the American Medical Association, 72, 1919, 414.) In speaking of the distribution of the disease during this last epidemic, Smith states that the first cases occurred in Central Europe in 1917, appeared in France,

${ }^{19}$ Smith, U. S. Pub. Health Report, No. 6, Vol. 37, February, 1921.

${ }^{20}$ Economo, Wien. klin. Woch., 30, 1917, 581.

${ }^{21}$ Wilson, Lancet, 2, 1918.

${ }^{22}$ Hall, Brit. Med. Jour., 2, 1918, 467. 
Great Britain and Algeria in late 1917, and early 1918, and reached North America during the latter half of 1918 and in early 1919. The disease spread rapidly throughout the United States, and Smith says that by May, 1919, cases had been reported from twenty of the states. The largest number were reported from Illinois, New York, Louisiana, and Tennessee, a fact which shows the apparent independence of the disease of climatic conditions. The disease spread through the United States from east to west. Smith summarizes the epidemiological features as follows: In almost all outbreaks the appearance of epidemic encephalitis has been preceded by influenza. Evidence of direct communicability is lacking, since in analyzing approximately 900 people exposed in the immediate families of cases, no secondary cases occurred. The age distribution is entirely different from poliomyelitis, so much so that Smith believes that this alone would distinguish the two diseases. In poliomyelitis over 59 per cent. of the cases occur before the fourth year, and 68 per cent of the cases occur below the age of 5 , whereas in epidemic encephalitis 58 per cent. of the cases occurred in individuals 20 years of age and over. As to sex, 60 per cent of the cases of encephalitis occurred in males. Seasonally the apex of the epidemic curve in the United States was reached in March.

The relationship of the disease to influenza is vague, but something to be very seriously considered in view of the recent researches of Olitsky with influenza and of Strauss and Loewe on filtrable virus in encephalitis.

The onset is usually gradual, with headache, lassitude and gradually increasing fever. Occasionally there are vomiting, vertigo and muscular pains. Disturbances of vision may appear early. Following this there may be an acute stage during which the fever rises, vomiting may intensify, there may be disturbances of the eranial nerves and great general weakness which gradually lapses into coma. Occasionally a case may get well in two or three weeks following this, again the coma may persist for a long time. Paralysis of muscular groups and facial paralysis, ptosis, disturbances of pupil reflexes and other muscular reflexes occur.

The spinal fluid shows an increased number of cells in a certain percentages of cases. The leucocytes may be slightly increased, but are usually not very high. The mortality in Smith's study was 29 per cent.

The etiology of the disease has been very carefully worked upon since the last appearance of the disease. A number of bacteria have 
been described and isolated from cases, but very probably have no significanee. We may dismiss the claims of bacterial causative agents as not in any case sufficiently based on reliable evidence. Of importance is a publication by Strauss, Hirschfeld and Loewe in $1919 .^{23}$

These workers obtained naso-pharyngeal mucus of fatal cases of the disease, filtered it through Berkefeld eandles and injected it subdurally and intracranially into monkeys and rabbits. In these animals they produced the disease. A monkey (Macacus Cynomolgus) injected on April 25th developed by May 2d, lethargy, general malaise, temperature and ptosis of the left lid, but recovered. Similar results were obtained with a Rhesus. Rabbits intracranially injected died in 4 or 5 days with punctate hemorrhages in the brain, intense congestion, marked meningitis and mononuclear infiltrations about the vessels. They claim to have repeated these experiments many times since their first publication. In 1920 Levaditi and Harvier ${ }^{24}$ claimed that they confirmed the experiments of Strauss, Hirschfeld and Loewe, both in monkeys, and in addition assert the susceptibility of guinea pigs. In a later publication of Loewe and Strauss ${ }^{25}$ they state that the lesions produced in the brains of such experimental animals are similar to those seen in human eases, that is, showing mononuclear perivascular infiltration, small homorrhages and general congestion, and they add experiments in which they succeeded in transferring the disease with spinal fluid and blood, as well as with material preserved in 50 per cent glycerine for many months.

By means of the Noguchi anaërobic tissue-acetic-fluid method, they report the cultivation of minute filtrable coccoid bodies, virtually identical with the globoid bodies described by Noguchi for poliomyelitis.

It is not possible at the present time to make definite statements concerning the reliability of these claims. Other observers of great experience with a large amount of material have failed to obtain similar results. The confirmation by Levaditi and more recently by Inmann, of Strauss and Loewe's experiments, however, would encourage the hope that they are right. Moreover, the similarity of the disease to poliomyelitis and the general similarity of pathological lesions would incline one to assume the disease to be probably due to a filtrable virus.

${ }^{28}$ Strauss, Hirschfeld and Loewe, N. Y. Med. Jour., 1919, 772; Jour. Infec. Dis., 25, 1919, 378.

${ }^{24}$ Levaditi and Harvicr, Compt. Rend. de la Soc. Biol., 83, 1920, 354.

${ }^{25}$ Loewe and Strauss, Jour. Infec. Dis., 27, 1920, 250. 


\section{CHAPTER XLVII}

MEASLES, SCARLE'T FEVER, MUMPS, DENGUE FEVER, FOO'T AND MOUTH DISEASE

\section{MEASLES}

THE causative agent of measles is unknown to the present day, and it would be a thankless task to review the literature of the many attempts to isolate microorganisms from this disease, none of which has resulted in throwing any light on the etiology.

Attempts to produce the disease experimentally have frequently been made, the earliest recorded being those of Home of Edinburgh, published in 1759. ${ }^{1}$ Home took blood from the arms of patients afflicted with measles, caught it upon cotton, and inoculated normal individuals by placing this blood-stained cotton on wounds made in the arm. Home claimed that in this way he produces measles of a modified and milder type in fifteen individuals. Home's results, however, while at first accepted, were assailed by many writers and it is by no means certain that the disease produced by him was really measles.

A number of other observers after Home attempted experimenta] inoculation of this disease, and positive results were reported by Stewart of Rhode Island (1799), Speranza of Mantua (1822), Katowa of Hungary (1842), and McGirr of Chicago (1850).

The experiments of all these early writers, however, are unsatisfactory, owing to the neecssarily unreliable technique of their methods.

In 1905 Hektoen $^{2}$ succeeded in experimentally producing the disease in two medical students by subcutaneous injection of blood taken from measles patients at the height of the disease (fourth day). The experiments were carefully carried out and the symptoms in the subjects were unquestionable. They demonstrated that the virus of the disease is present in the blood. Attempts at cultivation earried out with the same blood were entirely negative. It was also

${ }^{1}$ Home, “Medical Facts and Experiments,' Edinburgh, 1759.

${ }^{2}$ Hektoen, Jour. Inf. Dis., ii, 1905. 
MEASLES, SCARLET FEVER, MUMPS, DENGUE FEVER, ETC. 923

shown by Hektoen's experiments that the virus of measles may be kept alive for at least twenty-four hours when mixed with ascitic broth.

Similar experiments were recently carried out by Sellards, both on monkeys and on eight volunteers, but entirely without success.

More important than the blood transfer experiments from the point of view of transmission are experiments in which inoculation has been attempted with secretions from the nose and throat. In 1852 Mayer reported the successful inoculation of human beings with mucus from the noses and throats of early measles cases, but complete failure in similar attempts at transfer with skin desquamations following the rash. Anderson and Goldberger ${ }^{3}$ claimed in 1911 that they were able to produce temperature reactions and mild skin changes in monkeys by the injection of nasal and pharyngeal secretions from early cases. This work has been recently elaborated and brought to more convincing conclusions by Blake and Trask. ${ }^{4}$ These writers inoculated monkeys (Macacus Rhesus) intratracheally with filtered and unfiltered washings from patients in the early eruptive stages of measles and showed that the lesion which developed in the skin and buccal mucous membrane during the course of the monkey infection was histologically almost identical with that found in human measles. They successfully transmitted the infection from monkey to monkey and demonstrated that one attack of experimental measles conferred immunity upon the monkeys.

Epidemiology and Prevention.-There are few infectious diseases as common as measles. Crum ${ }^{5}$ has collected statistics which show that measles is responsible for about 1 per cent. of all deaths occurring in the temporate zones. In statistical summaries of 22 countries extending over a period of four years preceding 1910, there were over 366,000 deaths attributable to measles of an aggregate population of $32,625,651$. All races and ages seem to be susceptible, though children are more often infected, and the discrepancy between adults and children is probably due merely to the fact that most adults have had the discase before they attain adult life. Whenever young adults from rural districts come together in camps, epidemics will occur quite comparable and more severe than those occurring among school children and asylum children at an earlier period of life. The disease is com-

${ }^{3}$ Anderson and Goldberger, Jour. A. M. A., 57, 1911, 1612.

${ }^{4}$ Blake and Trask, Jour. Exper. Med., 33, 1921, 385, 413 and 621.

' Crum, Amer. Jour. Pub. Health, 4, 1914, 289. 
mon all over the world and not apparently influenced by climatic conditions.

When it appears first among aboriginal populations, it sweeps through them with a violence unknown among more civilized nations with whom the disease has been endemic for centuries. Such was the great epidemic in the Fiji Islands in 1874, and similar epidemies have occurred in the South Sea Islands and among American Indians and the negro races. The disease occurs more commonly in cities than in rural districts.

Susceptibility of previously uninfected individuals seems to be practically universal. Interesting in this connection are the statisties of concentration camps in the United States during the recent war such as those of Vaughan and Palmer ${ }^{6}$ made at Camp Wheeler. The population of this camp, like that of many others, was made up of young men from rural communities, many of whom had not had measles before. The sick rate week by week which followed the onset of the epidemic is tabulated by Vaughan and Palmer as follows :

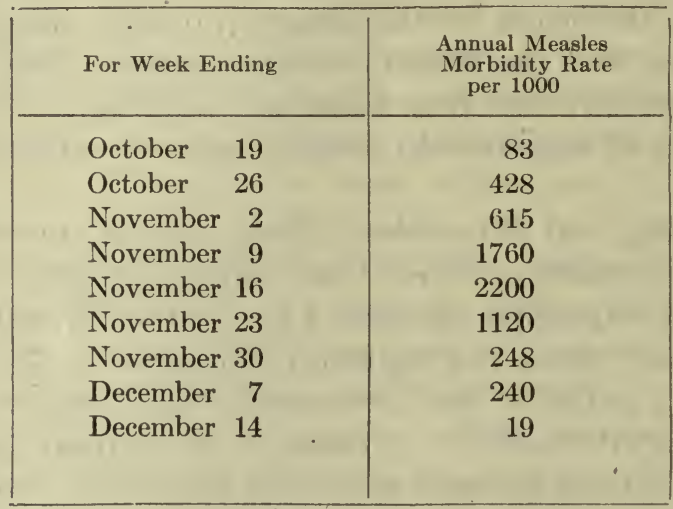

We may assume that the definite exposure to measles of an uninfected human being will almost invariably result in an attack.

Since the disease is probably communicated by the secretions of the nose and throat, reasonable exposure may be taken to imply crowding in sleeping quarters, contact in public vehicles, places of amusement, at meals, at play, in schools and in the ordinary indoor association of work and recreation. Whether or not the disease can be conveyed indirectly to any degree is not certain, but it is very

-Vaughan and Palmer, Jour. Lab. and Clin. Med., 4, 1919, 647. 
likely that infection from secretions on toys, food or other objects that are put into the mouth may take place, so long as the secretion is not dried. Judging from what we know or other filtrable virus, moreover, the virus of measles may offer considerable resistance even to drying.

One of the most important epidemiological facts is the infectiousness of the secretions in the early pre-eruptive stages. According to Levy of Richmond, the disease may be infectious as long as 4 days before the rash appears and since at this time the patients are rarely very sick, this is the dangerous period for transmission.

Uncomplicated measles in itself is not a very fatal disease, but, like influenza, measles seems to bring about a certain susceptibility to various respiratory infections, and measles epidemics are usually accompanied by many fatal post-measles pneumonias. These pneumonias may take the form of pneumococcus or streptococeus infections, according to the nature of the most prevalent mouth and throat flora prevailing in the community. The conditions for a fatal measles epidemic, therefore, are fulfilled when measles breaks out in an industrial community, a camp, a school, etc., during the cold weather when coughs and colds prevail and when virulent pneumococei and streptococci are plentifully scattered about in mucus.

The prevention of measles, in crowded communities or groups, is fraught with many difficulties. However, with vigilance and adequate discipline, much can be accomplished. In schools, industrial communities and in military units, the most important procedure in our opinion is constant inspection and early segregation of all individuals with catarrhal colds. In the army it has been the practice of sani. tarians, a practice which we believe we have seen succeed to an unexpected degree, to inspect entire units once a day upon the appearance of a case of measles. The entire unit is made to pass an inspecting officer in single file in the morning, a few questions as to general health are asked, the conjunctivæ and throats and the skin of the chest and arms are rapidly inspected, and individuals complaining of headache, a restless night, a cold or a cough, or those in whom the conjunctivæ are inflamed, or the nose secreting, are made to step out and, on these, temperatures are taken. All those with a temperature of $100^{\circ}$ or above are isolated and great care is taken to segregate catarrhal cases from the rest of the population. This method makes it possible to inspect a large group in a very short time and will accomplish far greater results than the mere isolation 
of individual suspicious cases which come to the sanitarian of their own accord. Munson ${ }^{7}$ has given this method particular attention in the army with astonishingly favorable results.

Since the incubation time of the disease is about two weeks, the exclusion of children from school need not exceed this period.

\section{SCARLET FEVER}

\section{(Scarlatina)}

The etiology of scarlet fever, like that of measles, is still obscure. Streptococei have been found with striking regularity in the throats of scarlet-fever patients, and a large number of investigations have seemed to furnish evidence for the etiological relationship of these microorganisms with the disease. Aceording to von Lingelsheim, Crooke as early as 1885 demonstrated the presence of streptocoeci in the cadavers of scarlet-fever victims. Baginsky and Sommerfeld ${ }^{8}$ in 1900 examined a number of searlatina cases with reference especially to streptococens infection, and reported the presence of streptococci in the heart's blood of eight patients who had died after a very acute and short illness. 'They expressed the belief that the acuteness of the illness and the rapidity of death in these cases precluded the possibility of the streptococei being merely secondary invaders. A large number of other observers have expressed similar opinions, but we can not, as yet, justly conclude that streptococci are actually the etiological agents of this disease.

Class 9 in 1899 described a diplococcus which he cultivated from a large number of searlatina patients and with which he was able to produce exanthemata and acute fever in pigs. Subsequent investigations seem to show that Class was really working with a streptococcus.

Moser, ${ }^{10}$ working in Escherich's clinic, has recently reported the very favorable influence upon the course of scarlet fever of polyvalent streptococeus antisera. This is not really very strong evidence in favor of the streptococeus etiology of the disease, since there is, of course, no doubt that streptoeocens infection eomplicates the discase,

'Munson, Military Surgeon, 40, 1917, 666.

"Baginsky and Sommerfeld, Berl. klin. Worb., 1900.

'Class, Phila. Merl. Jour., iii, 1899.

in Moser, quoted by Escherich, Wien. klin. Woch., xxiii, 1903. 
and it is to be expected that antistreptococeus scrum should, therefore, benefit the patient's condition by combating this complication.

Mallory ${ }^{11}$ in 1904 published observations on four searlatina eases which suggested that possibily scarlatina may be caused by protozoa. In the skin, between the epithelial cells, he found small bodies which wero easily stained with methylene-blue and which because of their arrangement and form he interpreted as parasites not very unlike the plasmodium of malaria. Subsequent investigations of Field ${ }^{12}$ and others have failed to substantiate Mallory's conclusions. Mallory and Medlar ${ }^{13}$ subsequently described a diphtheroid bacillus which they found in the tonsils, throat and trachea of scarlet fever cases. Another diphtheroid bacillus has been isolated by Mallory and Parker from the middle ear in a number of scarlet fever eases complicated with otitis media. The frequency with which these bacilli were found, and the fact that the organism found by Mallory and Parker produces highly toxic substances in broth, makes it necessary to keep them in mind, though Mallory and Parker, themselves, make no etiological elaims.

In 1911 Landsteiner, Levaditi and Prasek ${ }^{14}$ elaimed that they successfully inoculated chimpanzees with searlet fever by injecting blood from patients and also by rubbing the throats of the animals with swabs taken from scarlet fever throats. Hektoen and Weller in the same year failed in similar experiments upon Macacus Rhesus. Cantacuzene claims successful results on a number of lower monkeys with the blood and lymph-node suspensions of scarlet fever cases. Draper and Hanford ${ }^{15}$ have carefully gone over all these investigations and attempted to confirm them but completely failed.

The question concerning the etiology of scarlet fever is at the present time entirely unsettled. Unquestionably the most interesting line of thought at the present time is that connected with the streptococci. We have mentioned, in the streptococeus chapter, the work of Baginsky and Sommerfeld, ${ }^{16}$ and there is no question about the fact that a severe hemolytic infection of the throat is an invariable accompaniment of scarlet fever. In the series studied by Baginsky and Sommerfeld there were 8 in which the streptococeus infection accom-

${ }^{11}$ Mallory, Jour. Med. Research, x, 1904.

12 Field, Jour. Exper. Med., 7, 1905.

${ }^{13}$ Mallory and Medlar, Jour. Med. Res., 34, 1916.

${ }^{14}$ Landsteiner, Levaditi and Prasek, Ann. de I'Inst. Past., 25, 1911, 754.

${ }^{15}$ Draper and Hanford, Journ. Exp. Med. Vol. 17, 1913, p. 517.

${ }^{16}$ Baginsky and Sommerfeld. 
panying scarlatina was so violent that it could hardly be regarded as a mere secondary infection, and the writer, like many others, has seen a number of cases in which severe streptococcus infection of the throat, rapidly followed by general septicemia, was accompanied by a scarlatiniform rash, and in such cases it is impossible to say whether one is dealing with a fulminating scarlet fever case or with a violent streptococcus infection with a toxic rash. The experiments of Tunieliff ${ }^{17}$ seemed to show definitely that scarlet fever blood contains specific increase of opsonins for hemolytic streptococei and that streptocoeci from scarlet fever cases fall into a homologous group by agglutination reactions. Moser and Von Pirquet ${ }^{18}$ in 1903 had claimed that the blood of scarlet fever patients quite frequently agglutinated streptococci and also stated that the streptococei isolated from scarlet fever blood could be agglutinated specifically in most cases with immune sera produced with such streptococci, while streptococei isolated from other sources were rarely so agglutinated. This apparent specificity of the scarlet fever streptococci has recently been more carefully investigated by Bliss* in Dochez's laboratory, and in spite of the technical difficulties of agglutination experiments with this group, these workers too seemed to have found a certain amount of specificity in the scarlet fever streptococcus group. In view of the toxic substances which Zinsser, Parker and Kuttner ${ }^{19}$ have recently obtained from streptococcus cultures, toxic substances which are not regarded as specific exotoxins and are, therefore, not to be confused with previous claims of exotoxin production by streptococci, and in consideration of the nature of the scarlet fever rash, we do not believe it impossible that the skin manifestations of scarlet fever may be toxic in nature, and that, while many features in the disease would suggest a filtrable virus similar to that of measles, there is still much logic in continuing bacteriological investigations.

Epidemiology and Prevention.-There seems to be no doubt about the fact that the disease is transmitted by the nasal and pharyngeal mucus at all stages of the disease. The disease is probably communicable from the very beginning of the onset of symptoms in

${ }_{17}$ Tunicliff, Jour. Infec. Dis., Journ. A. M. A., 1920, 74, p. 1386 and 75, p. 1339 ; also Journ. Inf. Dis., 29, p. 91, 1921.

${ }^{18}$ Moser and Von Pirquet, Cent. f. Bakt., Orig., 34, 1903, 560 and 714.

* Bliss, Bull. Johns Hopk. Hosp., 31, 1920, p. 173.

${ }^{19}$ Zinsser, Parker and Kuttner, Proc. Soc. for Exper. Biol. and Med., 18, 1920, 49. 
the throat. It remains contagious throughout the disease and far into the convalescent period. In ordinary uncomplicated cases the infectiousness probably ends three or four weeks after disappearance of the rash, but when suppurating ears or other open secondary lesions persist, contagiousness may last throughout the period of the existence of the secondary lesion. Place ${ }^{20}$ has particularly studied such cases, and reports isolated observations in which con. tagiousness has lasted for twenty weeks after convalescence.

The so-called "return" cases are due to this long period of contagiousness, and Rosenau states that in the Boston City Hospital it has been observed that about 1.5 per cent of discharged scarlatina convalescents give rise to "return" cases, although the patients are kept in the Hospital for fifty days. From our own observations in army sanitation, there is very little doubt in our minds about the existence of scarlet fever carriers and this is in keeping with the observations of others.

We have, thus, as dangers for scarlet fever transmission, the typical cases themselves from the beginning of the throat infection until long after convalescence, the cases in which persistent secondary suppurative lesions continue after convalescence, the mild unrecognized cases and, possibly, carriers.

In addition to this, transmission by milk has not been uncommon. Trask $^{21}$ has collected thirty-five scarlet fever epidemics indirectly traceable to milk, and Rosenau speaks of a milk epidemic in Boston which gave rise to 500 cases. This epidemic was suppressed by pasteurization of the milk.

In prevention of the disease attention must, therefore, be chiefly centered upon early recognition and proper quarantine. In schools, asylums and other closely associated units, great care must be exercised to detect the first symptoms of sore throat when scarlet fever has occurred in any member of the community, and daily inspection is as necessary here as in measles. Children from households in which scarlet fever has occurred should be excluded from school and isolated until the incubation period of seven days is over. The incubation in this disease is usually shorter than this, rarely longer. The isolation of cases should be continued for from fifty to sixty

${ }^{20}$ Place, cited from Rosenau's Preventive Medicine and Hygiene, D. Appleton \& Co., New York and London, 1921.

${ }^{21}$ Trask, U. S. Pub. Health Ėulletin, No. 41. 
days, and no case should be returned to ordinary life as long as middle ear disease or any other open suppurative lesion persists.

\section{MUMPS}

Mumps is one of the most difficult infections to circumseribe when onee it has started in a erowded eommunity. Mumps epidemics in the army spread with speed and without yielding to ordinary preventive measures. Ordinarily it is chiefly a disease of children among whom it spreads in schools and institutions.

Prevention is particularly difficult because the susceptibility among children is practically universal and since exposure need not be very close to give rise to infection. Also, difficulties are added to by the fact that it may be passed on to others during the ineubation time before actual symptoms have appearea. Our impression from army experience is that there. may be carriers.

Infection is direct, by the secretions of the mouth, nose and throat, and it enters the new victim probably by the same route. The incubation time after:infection may be anywhere from five days to three weeks. Martha Wollstein ${ }^{22}$ has brought forward evidence which indicates that the saliva and secretions from mumps cases contain a filtrable virus. With such filtered secretions she obtained pathological changes in the testicles and parotid glands of cats which simulated human mumps. The most serious complications are those occurring in the testes which in male adults may have serious consequences.

One attack usually protects, though not always.

Prevention depends upon early recognition and isolation for two weeks after all symptoms have disappeared.

When the disease spreads in a group, it should be remembered that it may be infectious for some time before symptoms oceur and that the incubation time may last as long as three wecks. Protective measures, exclusion of contacts from school, and closure of schools if found necessary, must cover this period.

${ }^{22}$ Wollstein, M., Jour. Exper. Med., 23, 1916, 265. 


\section{DENGUE FEVER}

Dengue fever is of sanitary importance because it spreads rapidly, occurs epidemically and may lead to a very high sick rate, though it rarely kills. It is tropical in its distribution, but has occurred epidemically in the sub-tropical countries. Southern and Eastern Europe have had small epidemies and an oceasional sporadic distribution of eases.

The onset is sudden and begins with severe pains throughout the body, weakness and chilliness. There is conjunctival injection and there may be gastro-intestinal symptoms. The temperature rises to $103^{\circ}$ or $104^{\circ}$ and with this there is headache and the pains in the back and legs increase. The pain seems to be largely localized in the muscles. The fever persists usually for about three days when it drops considerably, and remains down for two or three days when it rises again. Usually at this time there is a rash on the hands which spreads to the arms, trunk and legs. Castellani and Chalmers ${ }^{23}$ describe this as a "measly" eruption in most eases, though in others it may resemble that of scarlet fever. After the second febrile attack, convalescence is usually rapid. The mortality of the disease is negligible.

In 1903 Graham, ${ }^{24}$ working in Syria, made blood examinations on a large number of eases of Dengue and described protozoa-like organisms within the red blood cells. He believed that the disease was transmitted by the ordinary mosquito, Culex fatigans. The geographical distribution of this mosquito corresponds fairly well with that of the disease. In one ease he produced the disease in man by the subcutaneous injection of a suspension of salivary g]ands of infected mosquitoes. He produced the diseases by bites of the mosquitoes that had fed on Dengue patients. In 1906 Bancroft produced typical attacks in two volunteers by the bites of Stegomya fasciata which had been allowed to feed on Dengue patients on the second day of the disease. Of considerable importance are the experiments of Ashburn and Craig ${ }^{25}$ of the United States Army which were carried out in the Philippine Islands in 1907. These inves-

${ }^{23}$ Castellani and Chalmers, Textbook of Tropical Medicine, W. Wood \& Co., New York.

${ }^{24}$ Graham, Jour. Trop. Med., 6, 1903, 209.

${ }^{25}$ Ashburn and Craig, quoted from Craig, Jour. A. M. A., 75, 1920, 1171. 
tigators failed to confirm Graham's work regarding the presence of protozoa-like organisms in the blood. They produced the disease in volunteers with unfiltered blood of Dengue eases. They then injected two volunteers intravenously with diluted defibrinated blood of Dengue patients, filtered through filter candles which held back Micrococcus melitensis and the cholera spirillum. Both of these volunteers developed typical attacks in about three days. They subsequently confirmed Graham's assertion concerning the transmission of the disease by Culex fatigans. They also showed that the disease is not contagious in the ordinary sense. Craig suggests the possibility of the disease being eaused by a spirochrte, basing his opinion on certain analogies with yellow fever as brought out by Noguchi's researches. However, there is still considerable uncertainty concerning this.

\section{FOOT-AND-MOUTH DISEASE}

This malady occurs chiefly in cattle, sheep, and goats, more rarely in other domestic animals. : It is characterized by the appearance of a vesicular eruption localized upon the mucosa of the mouth and upon the delicate skin between the hoofs. In the females similar eruptions may appear upon the udders. With the onset of the eruption there may be increased temperature; refusal of food, and general depression. Usually the disease is mild; the vesicles become small ulcers and pustules. Occasionally the disease is complicated by catarrhal gastroenteritis or an inflammation of the respiratory tract, and death may ensue.

The disease is transmitted from animal to animal by means of virus contained in the vesicular contents. Infection may also take place through the agency of milk.

Rarely the disease may be transmitted to man. Such infection, when it does take place, occurs usually among the milkers and attendants in dairies, and is transmitted by direct contact. The disease in man is usually very mild. Mohler states that the disease may be transmitted to man by the milk of infected animals. $\mathrm{He}^{26}$ adds that in the United States the disease has been practically eradicated.

${ }^{26}$ Mohler, Bull. No. 41, U. S. Pub. Health and Mar. Hosp. Serv., Wash., 1908. 
MEASLES, SCARLET FEVER, MUMPS, DENGUE FEVER, ETC. 933

The causative agent of foot-and-mouth disease is unknown. A number of organisms have been cultivated from the vesicles and mucous membranes of aftlicted animals, but none of these could be shown to have etiological significance. Loeffler and Frosch, ${ }^{27}$ have demonstrated that the virus contained in the vesicles may pass through the pores of a filter. The virus is easily destroyed by heating to $60^{\circ} \mathrm{C}$. and by complete desiccation.

One attack of foot-and-mouth disease protects against subsequent attacks. This immunity in most cases lasts for years, though rare eases of recurrence within a single year have been reported. Loeffler has actively immunized horses and cattle with graded doses of virus obtained from vesicles and with the sera of such animals has produced passive immunity in normal subjects.

${ }^{27}$ Loeffler und Frosch, Cent. f. Bakt., 1, 1908. 


\section{CHAPTER XLVIII}

TYPHUS FEVER, TRENCH FEVER, ROCKY MOUNTAIN SPOTTED FEVER. NOTES ON DELOUSING AND A CONSIDERATION OF THE SO-CALLED RICKETTSIA BODIES.

IN this chapter we have brought together a group of diseases which are insect borne and in which the etiological factor at the present time is uncertain. We have attached to the end of the chapter a short résumé of Rickettsia bodies, appearances to which allusion is made in consideration of all the discases here dealt with. The chapter has not been introduced with these Rickettsia bodies beeause we do not feel at the present time that their relationship to the diseases or even their positive interpretation as living organisms has been fully established. The evicience which connects them with the diseases in question, however, is growing so important that general information as to their nature and appearance cannot be ignored. In reading the chapters, if the reader finds reference to Rickettsia bodies in the sections on the diseases, he is referred for further information to the section on Rickettsia at the end of the chapter.

\section{TYPHUS FEVER}

Typhus fever is an infectious disease which is characterized by an incubation time of five days or more, high temperature, and a petechial rash. It has been characterized as peculiarly a disease of filth and has epidemically disappeared in most of the civilized countries, although it is still endemic in certain parts of Europe, North and South America, and occurs epidemically in Mexico under the name of Tabardillo. In New York it has recently been found to exist not infrequently. It was described as a new clinical entity by Brill, and has been spoken of as Brill's disease, but the work of Anderson and Goldberger has shown that Brill's disease is identical with typhus fever.

During the present war great epidemies occurred in the countries of Eastern Europe, an epidemic of great destructiveness sweeping 
over Serbia during the winter of 1914 to 1915 ; and the disease occurred in the Russian, Hungarian, Austrian, Balkan and Turkish Armies throughout the subsequent years. At the present writing there is much typhus in Russia, Serbia, Poland and in parts of Turkey.

The Disease.-Among clinical descriptions of typhus the first important scientific ones were those of Gerhard, Jenner and Murchison. The differentiation between typhoid and typhus exanthematicus was first made by Gerhard in 1837. Since then, the disease has been clinically well characterized and, indeed, is not easy to mistake for any other malady if once observed in a typical case.

The incubation time may range from five to twenty-one days. A case of autopsy infection published during the war took exactly twelve days to develop.

The onset may vary from extreme abruptness to a more gradual one. We take the main points of our description from a recent study by George C. Shattuck ${ }^{1}$ on cases observed during the Serbian epidemic.

The temperature rises rapidly, often to from $103^{\circ}$ to $104^{\circ}$, with chills, great depression, weakness, pains in the head and limbs. The eruption appears on the fourth or fifth day after the onset and, exeept in times of epidemic, the diagnosis is extremely difficult in the pre-eruptive stage. As the eruption appears the fever is apt to rise. The rash begins to appear usually on the shoulders and trunk, extending secondarily to the extremities, the backs of the hands and feet, and sometimes to the palms and soles. It becomes more abundant during the subsequent second and third days, but it is seen very rarely on the face and forehead. The rash is at first composed of pink spots which disappear on pressure, but soon it becomes purplish, more deeply brownish-red and finally fades into a brown color. Hemorrhagic centers may later develop which persist for eonsiderable lengths of time. Shattuck saw no eruptions on the mucous membranes of the mouth and pharynx. It is important to remember (a thing which Shattuck points out and which we have confirmed) in connection with the differential diagnosis between typhus and purpura hæmorrhagiea that in the purpura eruption the spots are hemorrhagie from the beginning and are more sharply

${ }^{1}$ Shattucl, Typhus Fever, ete., Rep. Amer. Reil. Cross, Serb. Epidemie, Harvard Univ. Press, 1920. 
defined than those of typhus. Fresh flea bites are sometimes hard to distinguish from the typhus eruption.

The heart is usually rapid and may become irregular. The blood pressure is apt to be low and Shattuck believes that myocardial weakness often oceurs. Epistaxis may occur at the height of the disease. Bronchitis often occurs during the later stages, and eough is almost regularly present. Nervous symptoms of various kinds are important accompaniments of the disease. In many cases a state of lethargy resembling that of typhoid fever is present. There may be twitching of the muscles during this stage of stupor. Delirium occurs in severe cases.

The leucocytes, as worked out by Sellards, ${ }^{2}$ are rarely increased in number, ranging in number from 3,000 to 15,000 , the average being between 5,000 and 7,000 . Differential counts show approximately normal percentages.

The most common complications are parotitis, suppurative otitis and mastoiditis, and a peculiar gangrene of the extremities, especially of the feet, which is particularly associated with eases occurring during the cold weather. This gangrene is characteristic of the disease and is probably associated with the vascular changes incident to the localization of the virus. Bronchitis is almost a regular complication. Albuminuria is present, and the urine gives a Diazo reaction.

For a thorough discussion of the pathology of the disease we refer the reader to Wolbach's Harvey Lecture. (Series 1920-1921, New York Harvey Society.)

Epidemiology.-Hirsch ${ }^{3}$ in his Handbook of Geographical and Historical Pathology, associated typhus fever with the dark days of the history of the world, war and famine. Typhus fever epidemies have probably decimated populations and armies far back into the history of the Middle Ages, and probably before. An epidemic of what is probably typhus fever was spoken of in the Chronicles of Joinville as almost destroying the Christian Armies near Salonika. Great epidemies ravaged Central and Eastern Europe in the Eighteenth Century, and the disease was prevalent in England and Ireland at this time. Epidemies occurred in Northern England and

2 Sellaris, Typhus Fever, etc., In the Serbian Epidemic, Red Cross Report, Harvard Univ. Press, 1920.

Hirsch, Indenham Society Publication, London, 1888. 
in Dublin in the Nineteenth Century, and Strong ${ }^{4}$ states that in Dublin alone, in the epidemic of $1846,60,000$ people died of the discase. In Mexico the disease has been endemic since the early part of the Sixteenth Century, and here, as in South America, it is known as Tabardillo.

Hirsch states (we quote from Strong), that of 147 epidemics which occurred in temperate and cold latitudes, 30 reached their heights in the spring, 28 in the winter and spring, 21 in the spring and summer, and 19 in the summer and autumn. As a matter of fact, the disease is one of relatively cold climates and elevated plateau countries. Nevertheless, it is also endemic in such places as the North of Africa, where Nicolle ${ }^{5}$ made his important discoveries. In some large cities of countries that are not ordinarily visited by typhus epidemics, the disease has remained endemically prevalent among those parts of the population living under unclean conditions. This is the case in New York where the disease has been prevalent in a mild form for a great many years.

Transmission is, as far as we know at the present time, entirely by the agency of lice. The idea that lice were concerned in the disease is not a new one. We find in Strong's study of the literature that Murchison suggested it in 1876. Cortezo made a similar statement in 1903, basing the opinion purely on clinical observation. The matter was noc settled until 1909 when Nicolle proved louse transmission by infecting a chimpanzee with typhus blood and subsequently showing that the disease could be transmitted to monkeys by the bites of infected body lice, as well. This important result was confirmed in 1911 by Ricketts and Wilder ${ }^{6}$ in Mexico, and in the same year by Anderson and Goldberger ${ }^{7}$ of the United States Public Health Service. Since that time many confirmatory observations have been made.

Whether or not other methods of transmission are possible is still somewhat in doubt. It is of course certain that direct transmission of blood from an infected case can cause the disease, and

- Strong, Typhus Fever, ete., Rep. Red Cross, Serbian Epidemic, Harv. Univ. Press, 1920.

${ }^{5}$ Nicolle, Compt. rend. Acad. d. Se. 1909, 157, Ann. de 1'Inst. Past., 1910, 1911, 1912.

' Ricketts and Wilder, Jour. Infee. Dis., July, 1911, p. 9.

"Anderson and Goldberger, Pub. Health Rep., Washington, March, 1912 and May 31, 1912. 
autopsy infections have been observed, but this mode of transmission can, of course, play no rôle of importance in epidemic transmission. It was suspected for a time that the sputum of typhus cases during the stage of bronchitis might prove infectious and doctors and nurses in Serbia for a time wore masks. But no elear evidence of any such accident has been brought to our knowledge, and as far as we know at the present time the louse is the only important means by which the disease is conveyed. Indeed, our own experiences with lice would persuade us that it is very difficult to absolutely exclude the bite of a louse in an infected case, for lice may lodge on the body in spite of the most rigid precautions and the bite of a louse may be entirely painless and without noticeable reaction in many individuals.

There has been a considerable amount of discussion as to whether the headlouse ean transmit the disease as well as the body louse. Goldberger and Anderson at one time believed this to be the case, but there is still much uncertainty about it, and the weight of evidence seems to be against it. As a matter of fact, in individuals who are sufficiently lousy, specimens of the body louse variety may be found in the hair of the head, neck and beard, on occasion.

These facts explain clearly why typhus epidemics oceur under conditions of crowding, poverty and war; why they spread so easily from patient to doctor, and why they occur chiefly in cold countries at times of the year when human beings are less apt to bathe and keep clean and more likely to live close together in erowded quarters for the sake of warmth. The louse, also, is not a tropical inseet, but thrives particularly in the cooler countries. In Mexico, for instance, as Ricketts and Wilder found, there was little or no typhus fever in the lowlands near the coast where lice were less common than fleas, but typhus was most prevalent on the cooler plateau country about the City of Mexico, where the population was much more commonly infested with lice.

The Serbian epidemic of 1915 was so severe that it interfered materially with military activities, and it was probably because of the epidemic that the Austrian Armies delayed their second attack upon Serbia. A detailed description of this epidemic is found in the article by Strong in the Red Cross Report referred to above. Typhus appeared in the Serbian Army in October and November of 1914, and it is said by Strong to have been introduced from Albania. It is also believed that typhus was present in the Austrian 
Army during its first invasion of Serbia, and there may well have been endemic cases in Serbia before the invasion. After the Austrian repulse of the late summer of 1914, typhus broke out among Austrian prisoners and Serbian soldiers. Owing to the fighting in the north the Serbian civilian population was forced southward and conditions of personal hygiene, housing, etc., were made particularly difficult. There was insufficient shelter, and the cold autumn weather, the lack of clothing, etc., brought about conditions of crowding and a tendency to remain close in quarters and wear whatever clothes the people had, without changing for long periods at a time. Filth and lousiness naturally resulted, and ideal conditions for typhus dissemination were created. In addition to this, shortage of food and the hardships attendant upon the general conditions reduced resistance. It is natural that under the circumstances very little was done at the beginning of the outbreak to circumscribe the disease, and it is doubtful whether this would have been possible. By January of 1915 the epidemic had begun to spread throughout the country from that period on, and reached its height in March and April. According to Strong, at the height of the epidemic cases were appearing at the rate of 9,000 a day. It is estimated that the mortality at the height of the epidemic ranged between 30 and 60 per cent, and that 150,000 deaths occurred within six months.

Animal Transmission.-One of the most important steps of course in etiological study of an infectious disease is the production of the disease in animals. In the case of typhus this was first accomplished by Nicolle.

In 1909 Nicolle $^{8}$ successfully inoculated an anthropoid ape, and Anderson and Goldberger ${ }^{9}$ in the same year succeeded in inoculating lower monkeys, rhesus and capuchin. Similar successful monkey inoculations were made by Ricketts and Wilder, ${ }^{10}$ by Gavino and Girard. ${ }^{11}$ In these animals inoculation with blood from active cases

Nicolle, Compt. rend. Acad. d. Sc., 1909, p. 157; Ann. de l'inst. Past., 1910, 1911, 1912.

'Anderson and Goldberger, Jour. A. M. A., 1912, p. 49; Jour. Med. Res., 1910, p. 469 ; N. Y. Med. Jour., 1912, p. 976.

10 Ricletts and Wilder, Jour. A. M. A., Feb., 1910, p. 463; ibid., Aprị 16, 1910, p. 1304; ibid., April 23, 1910, p. 1373; ibir., July 23, 1910, p. 309 ; Wilder, Jour. of Inf. Dis., vol. 9, 1911.

${ }^{11}$ Gavino and Girard, cited from Anderson and Goldberger. 
is followed by a rapid rise of temperature after an incubation time of five days or more, and the fever remains high for three to five days, after which it comes down by lysis. Oceasional recrudescences have been noticed in monkeys. Goldberger and Anderson have liad a mortality of 2 per eent in their monkeys. The disease may be transmitted from monkey to monkey with the blood, which is infectious during the febrile period and may be so for as long as thirty-two hours after the temperature returns to normal.

The first successful transmission of the disease to guinea-pigs was accomplished by Ricketts and Wilder. In these animals the only symptoms are fever, a matter which has made experimentation with these animals relatively diffieult. According to Nicolle, guineapig inoculation may occasionally result in no symptoms at all, and yet blood of such animals may produce the fever reaction in others inoculated with it. There seems to be a considerable difference in the degree of susceptibility in guinea-pigs. Anderson found about 44 per cent of his guinea-pigs resistant in the first generation of transmission from the typhus patient. Da Rocha-Lima believes that about 80 to 90 per cent of young guinea-pigs weighing not more than 300 grams, will usually be found susceptible. The typical reaction to guinea-pigs is a rise of two or three degrees of temperature on the sixth, seventh or eighth days.

Although ordinary observation shows only the fever reaction in guinea-pigs, it has been lately claimed by Löwy ${ }^{12}$ that carefu] inspection of the inner surface of the skin of guinea-pigs may reveal small hemorrhagic spots, not unlike the typhus eruptions in human beings.

Etiology.-The disease was at first suspected to be eaused by a filtrable virus, an opinion which is still held by some observers. Most workers agree to-day, especially because of the work of Anderson and Goldberger, that filtered blood will not convey the disease, and, although Nicolle, Conor and Conseil, Ricketts and Wilder, and others have reported that occasionally inoculation with filtered blood renders monkeys refractory to later inoculation, it is generally believed at present that the disease is eaused by some agent too large to pass through the Berkefeld or Chamberland filters.

Work on the etiology of typhus has been very extensive and many microorganisms have been described.

${ }^{12}$ Löwy, Wien. klin. Woch., 18, 1916. 
Ricketts and Wilder saw short bacilli in smear preparations, but were not able to cultivate them. Rabinovitch ${ }^{13}$ described a Gram-positive diplo-bacillus, cultivated from cases of an epidemic in Kieff, and with antigens prepared from this organism, he obtained eomplement-fixation and agglutination. Fürth studied an epidemic in China and obtained short, plump rods which grew aërobically in short chains. P. Th. Muller saw a diplo-bacillus upon which he did not lay much stress etiologically, and Prowezek described inclusions in leucocytes which he regarded as protozoa. It is hardly worth while at the present time to describe in detail the many different findings that have been reported, since in few of them is there sufficient evidence to enable us to come to conclusions.

In 1914 Plotz $^{14}$ described a short Gram-positive bacillus which he obtained by anaërobic cultivation, with considerable regularity, from eases of Brill's disease at the Mt. Sinai Hospital, New York, and which since then has been made the subject of considerable study by Plotz, Olitsky, and Baehr. ${ }^{15}$ They have obtained the bacillus again and again, have succeeded in obtaining positive agglutinations and eomplement-fixation in the blood of endemic typhus cases after the crisis and have obtained a similar bacillus from a number of European typhus eases which have come into quarantine.

The method of eultivation by which this bacillus is grown is relatively simple, eonsisting of taking blood directly from a vein into high tubes containing glucose agar and unheated and unfiltered ascitic fluid of a specific gravity not less than 10.15 .

The American-Red Cross Commission which went to Siberia during the last typhus epidemic-and of which the writer was a member-attempted to work along the lines laid down by Plotz but found it extremely diffieult to do systematic work and obtain reliable materials under the conditions then existing. The undersigned obtained an organism very similar to the Plotz bacillus by Plotz's method in two eases. In the first of these the organism could not be carried further than the second generation, and in the second it did not reach America alive. Hopkins obtained a similar organism later toward the end of the epidemic. However, these organisms were found so rarely that we were forced to the conclusion that these isolated findings, though pointing somewhat in favor of Plotz's organism, did not establish proof.

Petruschky ${ }^{16}$ has recently cultivated a similar but aërobic bacillus from sputum in typhus eases and Arnheim ${ }^{17}$ has aërobically eultivated an organism which in appearance and staining properties is not unlike the Plotz bacillus.

${ }^{13}$ Rabinovitch, Centralbk. Bakt. Orig., 1909, lii, Arch, f. Hyg., 1909.

${ }^{14}$ Plotz, Jour. of A. M. A., Ixii, 20, p. 14.

${ }^{15}$ Plotz, Olitsky and Baehr, Jour. of Inf. Dis., xvii, 1915, p. 1.

${ }^{10}$ Petruschky, Centralbk. f. Bakt., lxxv, 1915, p. 497.

${ }^{17}$ Arnheim, D. Med. Woch., 36, 1916, p. 1060. 
Arnheim obtained his organism from six cases, on ascitic agar plates, on which on the first cultures there appeared a growth hardly visible to the naked eye and which in transplants continued to grow very delicately. He states his organism is not unlike that of Petruschky and he obtained it out of the blood, the sputum and the urine of typhus cases.

A serious objection to the acceptance of the Plotz bacillus is that, despite the fact that a great many workers have been studying this disease during the last few years, the microorganisms which have been described have not been similar one with the other, and the fact that Plotz organism seems to lose its virulence immediately upon artificial cultivation. Also according to Anderson, active immunization with the Plotz bacillus does not render guinea-pigs refractory to virus inoculation.

Careful experiments at the Washington Hygienic Laboratory also have shown that the inoculation of large amounts of living Plotz organisms will neither injure nor immunize guinea-pigs or monkeys, whereas a single injection of typhus blood, after eausing the typical curve, leaves these animals refractory to further inoculation.

In the course of the war a great deal of further etiological research was done on typhus incidental to which, naturally, renewed search for bacterial causative agents was made. Gotschlich ${ }^{18}$ in reviewing this work finds that only two other investigators, Paneth and Popoff, found organisms similar to the Plotz bacillus. Gotschlich believes, as we do, that the etiological relationship of the Plotz bacillus has by no means been proven and is unlikely. The indirect evidence adduced by Plotz and his associates with agglutination reactions, etc., has lost a great deal of its value in view of the more recent work done on the Weil-Felix reaction in which a species of proteus is agglutinated with considerable regularity by typhus blood.

It is quite impossible to review completely the enormous bacteriological literature that has grown up about claims of causative relationship for many isolated organisms. In none of them could absolute proof be adduced and such claims seem to become less and less important as we follow the more recent developments concerning the so-called Rickettsia bodies.

In 1909 Ricketts $^{19}$ saw small bacillus-like bodies in the blood of guinea-pigs he had infected with Rocky Mountain Spotted Fever,

${ }^{18}$ Gotschlich, Erg. d. Hyg. Bakt., Berlin, 245, 1917.

${ }^{10}$ Ricketts, Medical Record, 76, 1909, 842. 
and when in 1910 he studied typhus in Mexico, he found similar short bacillus-like forms in the blood of typhus fever cases. They were extremely small and stained well only with Giemsa. In 1910, also, Gavin and Girard ${ }^{20}$ saw similar bodies in the blood of typhus cases in Mexico. Prowazek ${ }^{21}$ working in Serbia in 1910, also studied the blood of typhus fever cases and saw, within leucocytes, many smali rod shaped bodies, not unlike those described by Ricketts and Wilder and by Gavin and Girard. In 1914 Sergent, Foley and Vialatte ${ }^{22}$ observed similar bodies in lice taken from typhus infected people, and this was confirmed by Nicolle, Blanc and Conseil. ${ }^{23}$

A considerable number of similar observations were made by other workers and a very thorough study was published in 1916 by da Rocha-Lima. ${ }^{24}$ Da Rocha-Lima found these small bodies in the contents of the alimentary canals of lice which had fed on typhus fever patients. At first he did not find similar bodies in lice fed on normal people, and he definitely concluded that these "organisms" were etiologically related to the disease and thought that they were probably protozoa. It was he who suggested that they be known as "Rickettsia-prowazeki" in honor of the two men who had died in the study of the disease. Since that time numerous investigations have been published by da Rocha-Lima, Toepfer ${ }^{25}$ and others. Among the most important investigations that have followed are those of Brumpt. ${ }^{26}$ Brumpt obtained evidence that Rickettsia-like bodies could be found in lice taken from healthy individuals and that these organisms could remain in lice throughout the entire life of the louse, while the typhus virus did not seem to be active in the lice for longer than about eight or nine days. Arkwright, Bacot and Duncan ${ }^{27}$ using lice bred from a clean stock and working with trench fever, showed that Rickettsia-like bodies could be found in the lice after feeding on trench fever patients. Strong has tabulated on pages 77 to 80 of the Red Cross Report referred to above, all the various observations that have been made upon Rickettsia-like bodies in lice in connection with various dis-

${ }^{20}$ Gavin and Girard, Bull. de 1'Inst. Past., 8, 1910, 841.

${ }^{21}$ Prowazeli.

${ }_{22}^{22}$ Sergent, Foley and Vialatte, Compt. rend. de la Soc. Biol., 77, 1914, 101.

${ }^{23}$ Nicolle, Blane and Conseil, Compt. rend. de la Acad. de Sciences, 159, 1914.

${ }^{24}$ da Rocha-Lima, Berl. klin. Woch., 21, 1916, and Munch. med. Woch., 39, 1916.

${ }^{20}$ Toepfer, Berl. klin. Woch., 53, 1916, 323, and Mrd. Klinik, 13, 1917, 678.

${ }^{26}$ Brumpt, quoted from Strong. loc. cit.

${ }^{27}$ Arkwright, Bacot and Duncan, Proc. Roy. Soc. Med., 13, 1919, 23. 
eases. It appears from this literature that Rickettsia-like microorganisms may be present in lice fed upon healthy people, as well as in lice fed upon typhus eases, upon trench fever and upon healthy individuals. Recent and important investigations by Wolbach, Todd and collaborators seem to show that all these observations are correct, but that it is likely that the $R$. prowazeki associated by da Rocha-Lima with typhus differs materially from some of the other Rickettsia bodies.

All this elaborate work leaves us still considerably in the dark. It is not at all conclusively definite that the Rickettsia bodies are microorganisms, but those who have' studied them most carefully seem to feel reasonably sure that they are. Since the organisms cannot be cultivated, positive differentiation between the various observed forms is not possible. The finding, however, of similar appearances in the blood of typhus patients and in tissue sections gives a certain amount of basis for tentative etiological suggestions. In a separate section taken largely from Wolbach's description, we have given the general characterization of Rickettsia bodies as a class. The $R$. prowazeki of da Rocha-Lima, like the others, is very small, being rarely more than two micra in length, and often much less, is non-motile, is hard to stain, gives a reddish-blue color with Giemsa and is found intracellularly particularly in the gastric and intestinal epithelium of the mouse. In typhus patients we have already mentioned the similar bodies seen by Ricketts and Wilder in the blood, and Prowazek found similar bodies within the leucocytes of typhus blood. This has been confirmed by Lipschütz and others.

Attempts to identify the Plotz bacillus and other organisms with the Rickettsia do not seem logical at the present time since the Plotz bacillus can be cultivated with relative ease, while Rickettsia bodies cannot be cultivated. It is very difficult to stain them, and they decolorize by Gram. The painstaking work of Wolbach, Todd and their associates is not yet published. It will be very shortly published, however, in Wolbach's Harvey Lecture and the reader is referred to this article.

Prevention of Typhus Fever.-From what has been said above, it is plain that the prevention of typhus fever must be centered chiefly upon delousing, both of patients and of the population as a whole. Delousing methods will be dealt with in speaking of the general prevention of louse-borne diseases at the end of this section. 
Immunity. - A single attack of typhius fever seems to protect permanently. Monkeys and guinea-pigs that have once passed through the febrile reaction are thereafter refractory.

Prophylactic vaccines made from various bacteria isolated from typhus cases have not in our opinion given convincing proof of success. Attempts to immunize prophylactically with inactivated typhus blood have not so far had sufficient test. Specific therapy with the serum of convalescents attempted by Nicolle and others have not yet borne sufficient fruit to warrant very much hope.

The Weil-Felix Reaction.-This reaction is of peculiar interest in that it represents a diagnostic serum reaction in typhus with an organism which quite surely has no etiological relationship to the disease. It was first described by Weil and $\mathrm{Felix}^{28}$ in 1916, after isolation, from the urine of a case of typhus fever, of an organism which agglutinated in the serum of the patient in a dilution of $1: 200$. The organism apparently belonged to the proteus type and was designated by them as "Proteus $\mathbf{X}_{2}$." Further study with it showed that similar cases also agglutinated this organism. Later another bacillus $\mathrm{X}_{19}$, very similar to the first one, was isolated from another ease.

The organism is a Gram-negative, motile bacillus which ferments glucose and lactose, coagulates milk with acid formation, liquefies gelatin and in colony appearance resembles the proteus group. Bengston ${ }^{29}$ of the United States Public Health Service has studied the two organisms (" $\mathrm{X}_{2}$ " and " $\mathrm{X}_{19}$ " of Weil and Felix) bacteriologically, and has compared them to laboratory cultures of proteus. She found that they were very slow gelatin liquefiers, that they were extremely slow in digesting coagulated blood serum, but that they did not ferment lactose. In this, the organisms of Weil and Felix which she studied resembled one reported by Fairley. ${ }^{30}$ Agglutination reactions, against proteus sera produced with other strains, were in some cases active against these strains, and conversely sera produced with the Weil-Felix strains were more active against some other proteus organisms.

Apparently the agglutination of proteus " $\mathrm{X}$ " strains is of distinct value in typhus diagnosis. Fairley observed positive agglutination of these organisms in 97 per cent of the typhus sera which

${ }^{28}$ Weil and Felix, Wien. klin. Woch., No. 2, 1916.

${ }^{29}$ Bengston, Jour. Infec. Dis., 24, 1919, 428.

so Fairley, Jour. of Hyg., 18, 1919, 203. 
he examined. He never obtained agglutination with the blood of non-typhus cases, using dilutions of $1: 20$ in all the tests. Weil and Felix found that almost all their clinically typical typhus cases agglutinated this organism, whereas very few sera of non-typhus patients and no norral sera showed agglutination in dilutions of $1: 25$. Fairley repored that of thirty-five cases examined during the febrile stage, all agglutinated the organisms in dilutions of 1:20 and $1: 1200$ after the fifth day and throughout the second week.

The test is carried out by growing the organisms on agar, suspending them in salt solution and testing with dilutions of $1: 25$ and 1:50 of the serum of suspected cases. The agglutination titer in true typhus cases may rise as high as 1:200 or higher by the end of the second week. Bengston states that in one test made on a typhus case there was complete agglutination of the Weil-Felix organisms in diluton of $1: 400$, while eultures of the Rawlings typhoid and of Proteus vulgaris were not agglutinated in dilutions of $1: 50$.

The explanation of this reaction is doubtful. It may be assumed quite definitely that this organism has no etiological relationship to typhus. It is possible that in typhus fever secondary, non-specific agglutinating antibodies for a variety of organisms may be present. We need only call attention to the antibody reactions carried out with the Plotz bacillus and with some of the other organisms for which etiological relationship has been claimed in this connection. Incidentally, the Weil-Felix reaction adds to our scepticism about Plotz's claims.

\section{TRENCH FEVER (WOLHYNIAN FEVER)}

In 1919 there appeared among the Armies at the front a disease which did not clinieally resemble the ordinary well-known febrile diseases. Cases of this condition were seen among British troops by Graham and Herringham ${ }^{31}$ and on the German front in Poland and Wolhynia similar ones were deseribed by His ${ }^{32}$ and by Werner. ${ }^{33}$ Apparently the disease had been-noticed by Grätzer ${ }^{34}$ as early as 1914. Cases appeared in enormous numbers and because the disease

\footnotetext{
${ }^{31}$ Graham, Lancet, 2, 1915, 703 ; Herringham, Lancet, 9, 1916, 429.

32 His, Berl. klin. Woch., 53, 1916, 738.

${ }^{33}$ Werner, Münch. Med. Woch., 63, 402, 1916.

" Grätzer, Wien. klin. Woch., 29, 295, 1916.
} 
seemed to arise almost entirely from the front areas was spoken of as Trench fever.

The Disease.-Work on the clinical differentiation of the disease was done by a great many army surgeons. An accurate report was made by MeNee, Brunt and Renshaw, ${ }^{35}$ by a number of German workers, and finally by a British and by an American commission, the American group organized under Strong, and including as clinician Homer Swift. In a Harvey Lecture by Swift ${ }^{36}$ printed in the Archives of Internal Medicine, July, 1920, an accurate summary of available facts concerning this disease to date may be found.

The disease is sudden in onset with fever, headache and pains in the muscles. The onset resembles that of influenza. In a few days, pain and tenderness of the joints appears, and the temperature shows peculiar remissions which Swift characterizes as being of the "spiky" type.

Characteristic of the disease are the bone pains which are not accompanied by any signs of inflammation. There may be continued hyperesthesia. There may be sensory disturbances with increase of the tendon reflexes. The fever curves are very irregular, some showing the intermittent "spiky" type referred to above, others developing the typhoid-like ladder type. Another characteristie is the frequency of relapses, in which, after remissions of varying intervals, a second rise of temperature comes on. The relapses may come on after weeks or months. A case of which we have personal knowledge has developed two relapses in the course of two years after the original attack. In other cases Swift states that the manifestations may assume a subacute or chronic form with low grade fever which may continue for months.

Transmission and Etiology.-MeNee, Brunt and Renshaw, in 1916, succeeded in transferring the disease from man to man by intravenous and intramuseular injections of whole blood. In these early experiments they found that the plasma if entirely free from hemoglobin, was not infectious, but that the red cells contained the virus even after repeated washings. They did not succeed in passing the virus through a Berkefeld filter. In 1917, Werner, whom we quote from Swift, allowed himself to be bitten by lice that had previously fed on trench fever patients, and is said to have con-

${ }^{25}$ McNee, Brunt and Renshaw, Brit. Med. Jour., 1, 1916, 295.

${ }^{86}$ Swift, Harvey Lecture, Harvey Soc., New York, Jan. 10, 1920. 
tracted a mild form of the disease. A similar observation was made by Kuezynski ${ }^{37}$ on himself. Davies and $\mathrm{Weldon}^{38}$ in the same year earried out a similar experiment, allowing themselves to be bitten by lice immediately after the lice had fed on trench fever patients. One of them developed trench fever twelve days later. A similar experiment on a volunteer was successfully made by Pappenheimer and Mueller $^{39}$ in 1917, but in criticising all these experiments Swift believes that the proof brought was not sufficiently conclusive because of inadequate control. In 1918 two commissions were formed for the purpose of studying the disease. The American commission was aided by a British entomologist, Captain Peacock. The first result of these investigations was that McNee's observation about transmission with whole blood was confirmed, but it was found, in contrast to McNee's results, that the plasma as well as the red blood cells was infectious. It seemed that the blood taker as early as the fourth day of the disease was more infectious than that obtained later. Transmission after filtration through Berkefeld filters did not succeed at first, but Swift records that later, when infectious urine was used, filtration was successful. It was found at this time that, as weil as the blood, the urine of patients is also infectious.

Carefui experiments were made with lice, all of which were reared from eggs and fed on normal subjects, and the non-infectiousness of these lice was proven by allowing them to feed on eleven different uninfected people. Such lice were allowed to feed several times on trench fever patients and subsequently allowed to feed on twenty-three volunteers, 78 per cent of whom developed trench fever. It did not seem necessary for the lice to be in contact with the skin while feeding, nor was it necessary to produce scarification. In two instances the mere bite of the louse through the meshes of gauze covering the box produced the disease. The incubation time in these louse transmitted cases, varied from fourteen to thirtyeight days, the average being twenty-one. Meanwhile, the British eommission found that the excreta of infected lice applied to scarified skin could also produce the disease, thus showing that the

${ }^{27}$ Kuczynski, Reported from Jungmann, Deut. med. Woch., 64, 1917, 359.

${ }^{3}$ Davies and Weldon, Lancet, 1, 1917, 183.

${ }^{39}$ Pappenheimer and Mueller, Amer. Red Cross Committee Report, London, 1918, Oxford Press. 
excrement of lice that have bitten trench fever patients may bo infectious when it comes in contact with lesions on the skin.

Byam $^{40}$ showed that as late as 300 to 400 days after the onset of the disease, trench fever patients can still infect lice. This is of great importance in appraising the epidemiological possibilities of carriers. Lice could also be infected by patients during the periods of remission.

Both the British and the American commission showed that the virus is probably not transmitted through the eggs of the louse. The Pritish commission reported that the headlouse can transmit the disease through its excreta in the same way as the body louse. Other insects, however, did not seem to carry the disease.

As to the causative agent, little is definitely known. Toepfer, ${ }^{41}$ da Rocha-Lima ${ }^{42}$ and other German observers who have studied Rickettsia bodies in typhus fever were encouraged to undertake similar studica in connection with trench fever because of the similarity of the means of conveyance of the two diseases. These observers, as well as Jungmann ${ }^{43}$ succeeded in finding Rickettsia bodies in the intestines of lice fed on trench fever patients. Da Rocha-Lima comparing lice that had bitten individuals who did not have trench fever with those fed on trench fever patients found that 72 per cent of the insects found on the trench fever patients showed Rickettsia bodies, but 20 per cent of those fed on normal people showed similar ones. Arkwright, Bacot and Duncan ${ }^{44}$ found. similar Rickettsia bodies in a large number of lice that had fed several times on sixty-four trench fever patients. They found the bodies in only one out of many lots of insects fed on normal people. Their experiments seem to indicate that when Rickettsia bodies appear in the excrement of lice after feeding, these excrements were infectious.

It is quite clear that no positive conclusions can be drawik, especially in view of the frequent finding of Rickettsia-like bodies in lice that have had no connection with this disease. The clue furnished by the finding of Rickettsia, however, must be followed,

${ }^{40}$ Byam, et al., Trench Fever, Brit. War Office Commit. Rep., Oxford Press, 1919 ; Byam, Proc. Roy. Soc. Merl., 13, 1919, 19.

${ }^{41}$ Toepfer, Munch. med. Woch., 63, 1916, 1495.

42 da Rocha-Lima, Münch. med. Woch., 64, 1917.

${ }^{43}$ Jungmann, Deut. med. Woch., 64, 1917, 359.

"Arkwright, Bacot and Duncan, Proc. Roy. Soc. Med., 13, 1919, 23. 
since the possibilities here are the only ones that seem to show great etiological promise at the present time.

Prevention of trench fever, like the prevention of typhus, depends upon delousing.

\section{ROCKY MOUNTAIN SPOTTED FEVER}

Rocky Mountain Spotted Fever is a disease which has long existed in the United States. A thorough review of the entire subject has been made by Wolbach ${ }^{45}$ who states that authentic cases were reported as early as 1873 . The disease has been pretty well limited to the mountainous regions, most of the cases being reported from Idaho and Montana.

The Disease.-The onset of the disease is usually abrupt, though occasionally it may be preceded by a few days of general malaise. It not infrequently begins with a chill, followed by a rapid rise of temperature which reaches $102^{\circ}$ or $104^{\circ}$. The temperature may show morning remissions with rises of one or two degrees in the evening, and gradually increasing, reaching its height during the second week. ()n the third or fourth day after the onset of the disease, a rash appears first on the wrists, ankles and back, later upon the arms, legs and chest, extending to the forehead and abdomen. It is always least marked on the abdomen, according t,o Wolbach. It comes out in the course of about thirty-six hours and may also involve the mucous membranes of the mouth and pharynx. The temperature remains up after the appearance of the rash: The rash consists of little red patches about 4 or $5 \mathrm{~mm}$. in diameter which at first disappear on pressure. Like the typhus rash, they become darker red, then purplish and later hemorrhagic in charaeter. Small petechial spots may appear in the eenter. They fade into pigmented spots later. During recovery desquamation oceurs.

There may be violent nervous symptoms. The blood picture is not altered materially, the leueoeytes slightly inerease in number, but the differential count remains approximately normal.

The mortality of the disease as estimated from various sources by Wolbach for 1915 and 1916, ranged between 7 and 13 per cent.

The ineubation time seems to vary between three and nine days.

${ }^{45}$ Wolbach, Jour. Med, Res., 41, 1919, 1. 
Epidemiology.-The distribution of the disease follows that of the wood tick, Dermacentor venustus. The disease oceury in Idaho, Montana, Nevada, Wyoming, California, Colorado and Washington. Wolbach notes that the distribution of cases in various states seems to be restrieted to definite localities. In Idaho, he finds that the cases are particularly frequent in the Snake River Valley and in Montana in the Bitter Root Valley where there seem to be infectious foei. Seasonally the disease occurs almost entirely in the spring.

The wood tick named above was associated with the disease first by Wilson and Chowning ${ }^{46}$ in 1902 . Ricketts brought proof of this in $1906,{ }^{47}$ showing that the disease could be produced in guinea-pigs by allowing wild ticks of this species to feed upon them. McClintic ${ }^{48}$ confirmed this and his investigations with those of Ricketts, centralized attention upon this particular species, the Dermacentor venustus. Larvæ, fed upon infected ticks, remain infective into the nymph stage and the nymph once infeeted remains infected into the adult stage. He also showed that eggs from infected females would produce the disease when injected into guinea-pigs and that both male and female ticks would transmit it. Wolbach 's ${ }^{40}$ investigations have confirmed most of these points.

Guinea-pigs infected by ticks develop a temperature in about three to seven days. Injeeted with blood from other guinea-pigs, the disease may begin at the end of forty-eight hours. Death, which often follows, occurs on the sixth or seventh day.- As the disease progresses there is swelling and reddening of the skin of the scrotum, loss of appetite and general signs of illness. There may be redness and swelling of the eye-lids, cars and paws, and ulcers of the paws may form. On autopsy, there may be edema and hemorrhages of the skin and subcutancous tissues of the scrotum. Male guinea-pigs show the disease most characteristically because of the scrotal lesions. Rabbits are suseeptible, although not regularly so. Foot ${ }^{50}$ has studied this under Wolbach's direction. When it does occur in rabbits, the disease is virtually the same as that occurring in guinea-pigs, except that in addition to the other signs of illness,

${ }^{46}$ Wilson and Chowning, Jour. A. M. A., 39, 1902.

${ }^{47}$ Rucketts, Jour. A. M. A., 46, 1906.

${ }^{48}$ McClintic, U. S. Pub. Health and Marine Hosp. Serv., Weekly Bulletin, No. 20, 27, 1912.

46 Wolbach, Jour. Med. Research, 41, 1919-1920, 3.

sc Hoot, Jour Med., Res., 39, 1919. 
the ears are often inflamed, and in white rabbits thrombosed vessels in the ears can be seen.

Monkeys are susceptible and usually die at the end of seven days.

Etiology.-The lesions in the blood vessels in animals and man indicate the presence of the causative agent in these locations. Wolbach $^{49}$ who studied histological material from this point of view, found within the endothelial cells of the vascular lesions, in smooth muscle cells of the media, as well as occasionally in detached endothelial cells present in thrombi extremely minute small "diplococcus like" organisms. These, he states, stain with eosin-methyleneblue. The organisms were best stained with Giemsa solution with which they appear as slender pale blue rods. There was a distinct contrast between the appearance of these organisms and that of accidentally introduced bacteria, in that the shape of the former was vaguely outlined and less sharp than that of the bacteria. Ricketts ${ }^{51}$ had described similar organisms in the blood of man and guinea-pigs which he described as "lanceolate chromatin-staining bodies" in sets of two, a small amount of eosin-staining substance appearing between the two individuals. The same organism was seen in smears of the intestinal contents of ticks. Wolbach has also seem them in smear preparations made from the eggs of infected ticks.

In the case of these bodies, as well as in those described in connection with typhus fever, definite conclusions cannot be reached as yet. A careful analysis of the entire subject will be found in Wolbach's paper of 1919. The organisms, if they are organisms, probably belong to the class of Rickettsia. They have not been cultivated.

The prevention of the disease depends largely upon the prevention of tick bites and the suppression of the wood tick, a matter which is very difficult in the countries in which they abound.

${ }^{51}$ Ricketts, Jour. A. M. A., 47, 1906, 33; Jour. A. M. A., 47, 1906, 358 ; Jour. A. M. A., 47, 1906, 1067; Jour. A. M: A., 49, 1907, 1278; Jour. Infec. Dis., 4, 1907, 141; Jour. A. M. A., 49, 1907, 24; Trans. Chicago Path. Soe., 1907 ; Jour. Infer. Dis., 5, 1908, 221; Jour. A. M. A., 52, 1909, 379; Medical Record, 76, $1909,842$. 


\section{LICE AND DELOUSING}

The sanitation of typhus fever, of trench fever and of some forms of relapsing fever is so definitely dependent upon processes of louse extermination that a few paragraphs on the habits of lice and the means for their destruction will contain the most important principles upon which sanitary efforts in the prevention of these diseases must be based. The lice which infest the human body are of two types, the Pediculus humanus which includes the body louse and the head louse, and the Phthyrius pubis, or the pubic or crab louse. It is the first two of these, the body louse and the head louse with which sanitarians are most concerned.

For an anatomical description of lice we must refer the reader to Nuttall's comprehensive Monograph in the British Journal of Hygiene of 1917, and to textbooks on medical entomology. The following facts, important for the sanitarian, are compiled from various sources.

The ordinary life of a louse is about four to six weeks. The female louse begins to lay eggis about eight or nine days after hatching. It is stated that such lice, well fed, and in normal environment, will lay altogether about 300 eggs at the rate of ten or so a day. It takes about one week to eight days for these eggs to hatch. On this basis, a single generation of lice takes about sixteen to eighteen days.

The louse prefers to lay its eggs upon little threads or hairs, more readily upon rougher cloth than upon silk. It was suspected in the early part of the war that silk underclothing gave some protection, but apparently this is not of very much use. The body louse prefers to lay its eggs on the inner surfaces of underclothing and other clothing, preferably along the seams, and on blankets, though most of the louse eggs are probably laid on underclothing. It should not be forgotten that in arranging for disinfestation, the outer clothing, overcoats, blankets, etc., may also be infested. In addition to this, both the head louse and the body louse may lay their eggs on the hairs of the body. The louse, being an habitual parasite on animals and man, prefers to lay its eggs at a temperature little below that of the body, a temperature which is stated as ranging about $30^{\circ} \mathrm{C}$. If the temperature is lower than that, it takes them two weeks or more to hatch. According to studies made by British Army sanitarians, they will not hatch below $22^{\circ} \mathrm{C}$. and 
the most favorable temperature for hatching is at $35^{\circ} \mathrm{C}$, when the hatching time is very much speeded up and may be less than eight days. It is important to know that louse eggs are destroyed by temperatures slightly above $60^{\circ} \mathrm{C}$. But it is not safe to rely upon such low temperatures for disinfestation. While the nits are quite susceptible to temperature, they are much harder to destroy by insecticides than are the adult lice. It is important to remember that many of the insecticide substances which are applied to the body and clothing for the prevention of lousiness may keep lice away but will not kill them when once present.

It is also important to remember that, although the adult louse must feed with some regularity in order to thrive and lay its eggs, the eggs may remain alive on clothing, underwear, etc., for a month at least, away from the human body, and may be hatched out when this clothing is put on. Thus, clothing, underwear, blankets, etc., of louse infested durouts, huts, ships, etc., must be taken care of even if it has not been worn for some time.

Although the louse, like the bed bug in the song, has no wings "at all," and is not a wanderer, it is astonishing how easily it can pass from one individual to another. Lice may be easily acquired during the examination of a case, in passing through a crowd, or in handling underwear and clothing in laundry work or disinfesting operations.

The adult louse feeds about twice a day, and the louse bites, while they may be quite annoying to some individuals, may cause practically no reaction or annoyance in habitually lousy persons. They are apt to leave the body of the sick and usually do leave the body of the dead as soon as possible. When removed from human sources of food, they may die in anywhere from one or two days to a week. Nine or ten days is stated as the probable limit to which the adult may live in clothing that has been hung up or put away.

Delousing depends upon early discovery of lousiness in a community, regiment or other unit, personal cleanliness, disinfestation of those who are lousy and disinfestation of clothing, blankets, etc., and quarters.

In armies and in eommunities during the existence of louse-borne diseases, inspection for lousiness of bodies, underelothing, ete., should be carried out at frequent intervals. The individual who attempts to protect himself should inspect his own body and underclothing on going to bed at night. 
The most efficient individual protection against louse infestation consists in frequent baths, preferably hot showers, in which a free use of soap is made, and all the hairy parts of the body very thoroughly soaped. The best type of soap is a soft soap. A British Army preparation which was very useful during the war was made by slowly warming three pounds of soft soap with one-half pint of water and, after removal from the fire, this was mixed with five and one-half pounds of crude paraffin oil. Two and one-half per cent cresol was added to this mixture. This formula is taken from D. G. M. S. Cireular Memorandum, No. 16, of the British Army. After bathing, a complete change of underelothing should be made. In the American Army bathing establishments were arranged from ordinary Adrian huts, which were applicable to delousing on a large scale. Bath houses were so arranged that men undressed in an anteroom, tying up their outer elothing into bundles with tags attached and throwing their soiled underelothing into large wire baskets which were immediately taken to the steam sterilizers. They then passed into the shower rooms and eame out into a dressing room, into a window of which the outer clothing after sterilization was returned to them, and into which from another window clean underclothing was passed. We have described the arrangement as used in the American Army in an article on army sanitation. ${ }^{52}$

For the purpose of keeping lice away from the body, naphthalin sprinkled through the underelothing probably has some effeet.

Kerosene or gasoline when applied to clothing in small quantities may keep insects from lodging in elothing.

Various soaps and oir'tments have been made in which petroleum, kerosene or naphthalin have been used as ingredients, and these have been applied by smearing along the seams of the elothing, under the arm pits, etc.

An excellent method for personal prophylaxis has been the spraying of crude creosote oil on the inner and outer clothing. This has been used successfully by Pappenheimer and Mueller. A good way of killing insects that may have wandered into the elothing during hospital or other duties in the course of the day is to drop the elothing into a dress suit bag or other fairly tight container and pouring in an ounce or two of chloroform, closing it for the night. This will not always kill nits.

82. Zinsser, Military Surgenn. November, 1918. 
Much might be written about the various substances that can be applied to the skin and clothing to keep insects away, but none of these means are infallable or sufficiently safe to be relied upon. The best possible method, after all, is the use of shower baths of hot water, the plentiful use of soap, combined with steam disinfestation of the clothing, and clipping of hair and beard, etc.

Gaseous Disinfectants for Rooms, Clothing, etc.-It is an important practical fact that formaldehyd, in spite of its powerful action upon bacteria, is a weak insecticide and cannot be relied upon to kill lice, mosquitoes, or fleas.

Better than formaldehyd is $\mathrm{SO}_{2}$ gas used in quantities of two to three pounds of sulphur per 1,000 eubic feet, with the simultaneous evaporation of water. (Clayton apparatus.)

Hydrocyanic acid gas is also very efficient, but of course extremely poisonous and dangerous unless used in a proper way.

The most important facts concerning these gases, their generation and application, have been dealt with in a preceding section.

For the wholesale disinfestation of clothing, fomites, etc., in connection with louse infested populations, clothing, blankets, etc., the experience of the late war has shown that the most practical systems are those depending upon the application of heat.

Heat may be applied as dry heat or moist heat.

Our own experience has taught us that the surest and most foolproof method of disinfesting large quantities of material during epidemics is by the use of large autoclave drums placed on trucks such as the Foden-Thresh autoclave lorries, which consist of large autoclaves with steam jackets so arranged that clothing, etc., can be exposed to steam under slight pressure (about five pounds), then the connection between the inner and outer jackets closed and the material dried in the same chamber before removal. The steam is supplied from the motor since such lorries are usually steam driven.

In the field, dry heat chambers can be constructed consisting of well sealed huts within which small brick furnaces or tin stoves, with stovepipe arrangements surrounding the walls are used for heating purposes. These dry heat disinfestors are not as uniformly practical or foolproof as are the methods in which steam under pressure is applied, but it may be necessary to use them when other means are not available. Detailed descriptions cannot be given here, but a little ingenuity with attention to proper size in relation to heating apparatus, proper distribution of heat with tin pipes, and 
provision for the circulation of air by proper vent holes will yield good results. In the so-called "Canadian" type of hut the heat is applied from below by digging a hole in the floor of the hut which connects with the outside through a small tunnel, in which a furnace, constructed in a variety of ways, can be placed, and a glowing coal fire maintained. According to $\mathrm{Bacot}^{53}$ and others it has been found that nits protected by a single layer of khaki cloth are killed in fifteen minutes at $52^{\circ} \mathrm{C}$. The heat of such huts must be carefully controlled, a matter which can be done either by thermometers or, as advised by Bacot, by hanging in various places small tubes containing paraffin or stearin, with a melting point of $60^{\circ}$ or over.

Excellent methods of applying the various forms of disinfestation by heat are those which were originated by Dr. Richard Strong in Serbia, in which disinfestation trains with a shower bath ear, a steam sterilizing car made of a converted refrigerator truck, were drawn by an engine, which supplied the steam for the disinfestors, the hot water for the baths and the motor power.

\section{THE RICKETTSIA BODIES}

In 1910 during their work in Mexico, Ricketts and Wilder observed small ovoid bacterium-like bodies in the intestinal canals of lice which had fed on typhus cases. They described them as showing polar staining, with slightly stained or entirely unstained centers and as having the general appearance of very small ${ }^{\circ}$ bacilli. Similar observations were made by Prowazek and by Sergent, Foley and Vialatte in 1913, though the identity of the bodies seen by these observers with those of Ricketts and Wilder was not, at first clear. Most of the original observations were made on typhus material, but subsequently Wolbach saw similar appearances in the endothelial cells and vessel walls of animals infected with Rocky Mountain Spotted Fever which he believed to be probably identical with diplococcus-like structures described in the blood in the same disease by Ricketts a few years earlier. Still later bodies of the same general appearance were noticed in lice taken from Trench Fever cases and in lice eollected from the bodies of normal human beings. The peculiar staining properties, frequently intracellular position, minute size and pleomorphic structure of these peculiar bodies suggested to many of these workers the possibility that they

${ }^{53}$ Bacot, Brit. Med. Jour., 2, 1917, 151. 
might represent a group of parasitic, and perhaps pathogenic, organisms not hitherto observed. Von Prowazek did not believe these small bodies to be bacteria, and from the beginning took the position that they were more likely to belong to, or be closely related to the protozoa. Da Rocha-Lima, who has studied them particularly in their relationship to typhus fever, gave the appearances which he saw in typhus lice the name of Rickettsia prowazeki. In the course of numerous investigations upon the etiological significance of these peculiar appearances, especially in connection with Typhus fever, Rocky Mountain Spotted Fever, and Trench Fever, many workers have confirmed the observations of the earlier observers, and while it is quite impossible at the present time to classify them with any degree of certainty either with the bacteria or the protozoa, the various forms described possess sufficient similarity to each other to warrant the establishment of a tentative group. In describing them in a separate section we do not mean to imply that, at the present time, it is absolutely certain that they are parasites. But this seems so likely, and their etiological relationship to the diseases mentioned has been suggested by so many careful investigations, that clearness of treatment at the present time fully justifies such segregation into a separate group.

The appearances which are classified together as Rickettsia are minute, ovoid or bacterium-like bodies. They are, as a rule, extremely small, the smallest forms being more minute than the smallest known bacteria, measuring about 0.3 to 0.5 of a micron. Larger forms more bacillary in appearance, may be observed, and it is suggested that the Rickettsia bodies go through a developmental cycle. The small forms often appear in the "diplo" form and some German observers have described capsule-like halos around groups of two.

They are all very difficult to stain. The ordinary aniline dyes stain them either very faintly or not at all. Prolonged staining with Giemsa gives them a faint reddish blue tinge. They do not retain the Gram stain.

They are non-motile.

Up to the present time none of the Rickettsia have been cultivated, with the exception of one form observed in the sheep louse which grows aërobically on glucose-blood-agar.

All of them have an insect host which acts, in the case of the pathogenic Rickettsia, as the transmitting agent. 
According to experiments of Ricketts and Wilder, da RochaLima, Sergent and his co-workers, some of the Rickettsia can pass into the egg of the louse and thus be inherited from one generation to the other.

Most of them, unlike bacteria and more resembling protozoa, appear to enter the eells of the host as intracellular parasites.

The sub-classification of the Rickettsia has been tentatively attempted by Wolbach by whose courtesy we are enabled to insert the following table:

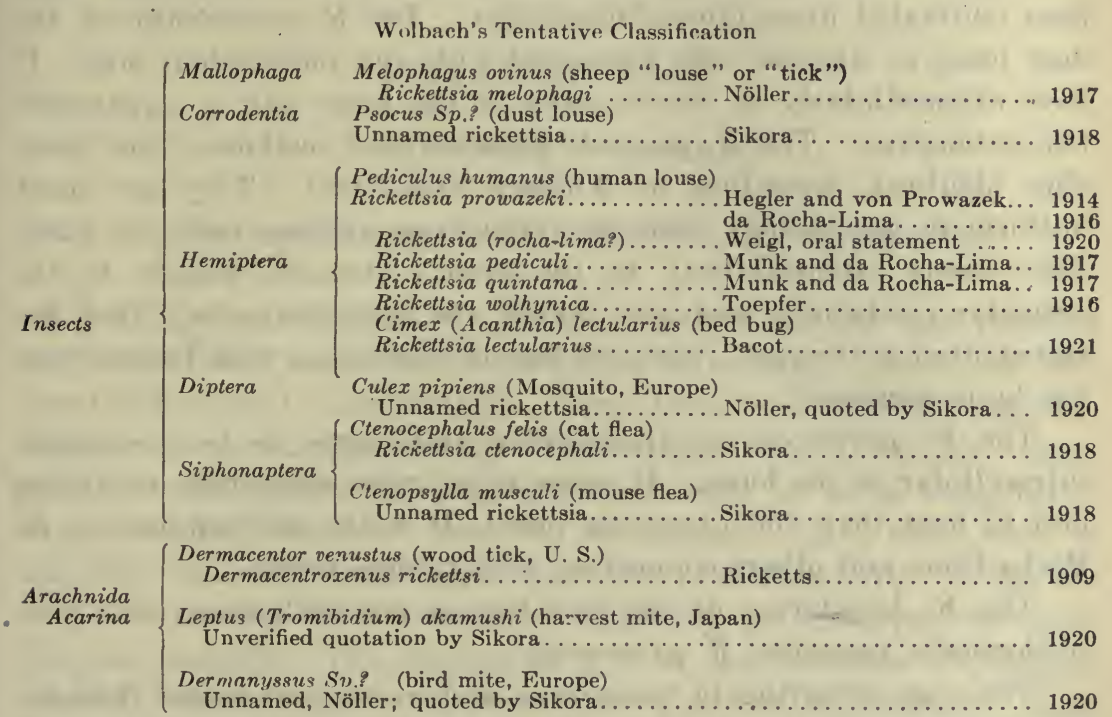

In proposing this elassification, however, it should be said in justice to Wolbach that he introduces it by stating definitely that a reliable classification of the Rickettsia is impossible at the present time, and that he believes that there have already been included under this heading a number of unrelated forms. He states that the Rickettsia of the sheep louse has little to distinguish it from bacteria and that the Rickettsia seen in eonnection with typhus fever has peeuliarities which separate it from others. The Rickettsia studied by him in connection with Rocky Mountain Spotted Fever resembles somewhat the Rickettsia-prowazeki seen in typhus, and both of them are quite unlike the "morphologieally simple" one observed in connection with trench fever. Wolbach summarizes his reasons for constructing a table of elassification by saying that he believes it warranted since "they are forms of microorganisms 
primarily adapted to insect tissues, with occasional representatives pathogenic for animals."

For more detailed analysis of the Rickettsia, we refer the reader to the Harvey Lecture and to the articles on typhus investigations in Poland now being prepared for publication by Wolbach, Todd and their associates.

Abstracting from the further analysis of the Rickettsia sent us with this table by Wolbach, we may mention the following points. The $R$. melophagia is not pathogenic and is the only one that has been cultivated upon glucose-blood-agar. The $R$. corrodentia of the dust louse is, likewise, not associated with any mammalian host. It lives extracellularly in the stomach of the louse and is apparently non-pathogenic. The $R$. pediculi quintana and wolhynica are probably identical, according to Wolbach and Todd. They are more uniform in morphology than the typhus one and are easier to stain. They occur extracellularly in the louse's stomach, adhere to the cuticular epithelium, and may invade the epithelial cells. They are transmitted to the egg. Their etiological association with trench fever has been suggested.

The $R$. prowazeki is pleomorphic and seems to be exclusively intracellular in the louse. It seems to be more susceptible to drying and to heat than the preceding ones. It is the one studied by da Rocha-Lima and others connection with typhus fever.

The $R$. lectularius of the bed bug is non-pathogenic, but mor-. phologically resembles $R$. prowazeki.

The ones occurring in mosquitoes and in eat and mouse fleas are non-pathogenic.

The Dermacentroxenus rickettsi is the Rickettsia body which has been associated by a number of writers with Rocky Mountain Spotted Fever. Wolbach includes it in the general classification, though he has found many differences between it and the other Rickettsia. Wolbach states that it is less bacterium-like than any of the other Rickettsia and many forms show red and blue staining materials. Unlike the Prowazeki, it does not show the thread-like forms. In the louse he states that the Prowazeki continues to multiply in the gastric epithelium and eventually causes the death of the louse by interfering with digestion. The Dermacentroxenus, however, after multiplying within the nucleus chiefly, floods all the tissues of the tick and then diminishes in numbers, leaving behind in the salivary gland and some other tissues, forms which Wolbach regards as a resistant stage. 


\title{
SECTION V*
}

\section{THE HIGHER BACTERIA, MOLDS AND FUNGI}

\section{CHAPTER XLIX}

\author{
THE HIGHER BACTERIA
}

(Chlamydobacteriaceœ, Trichomycetes, Microsiphonales)

STANDING midway between the true bacteria and the more complex molds, there are a number of pathogenic microorganisms which offer great difficulties to classification. These forms resemble the hyphomycetes in the gross appearance of the cultures, which are dry, tough, wrinkled and sometimes covered with a down of aerial outgrowths. Morphologically they are made up of filaments which often show at the ends chains of round bodies analogous to arthrospores. In the size and structure of their component cells they are, however, far more like the bacteria. The component cells of the filaments are usually about 0.3 micron and rarely more than 1 micron in diameter. They frequently stain unevenly but show no definite nuclei and the round spore-like cells are about the size of micrococci. In the classification of Migula most of these forms have been placed in a rather heterogeneous group, the Chlamydobacteriaceæ. By other authors, notably Lachner-Sandoval, ${ }^{1}$ Berestnew, ${ }^{2}$ and by Petruschky, ${ }^{3}$ the close relationship of these forms to the. higher hyphomycetes has been emphasized and they have been grouped as a subdivision of the true fungi under the family name of Trichomycetes.

* For a careful revision of this Section we are indebted to Dr. J. Gardner Hopkins.

'Lachner-Sandoval, “Ueber Strahlenpilze.' Diss. Strassburg, 1898.

${ }^{2}$ Berestnew, Ref. Cent. f. Bakt., xxiv, 1898.

'Petruschky, in Kolle und Wassermann, "Handbuch," ete. 
Petruschky proposes the following clear schematization, which, even though possibly defective from a purely botanical point of view, is at least serviceable for the purposes of the bacteriologist.

\section{Hyphomycetes}

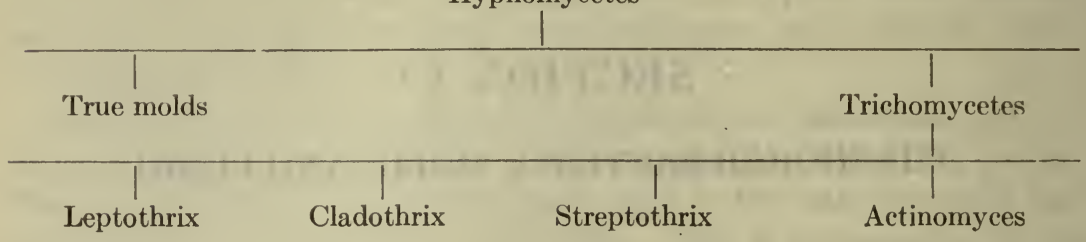

Leptothrix is used to designate those forms which appear as simple threads without branching.

Cladothrix is a thread-like form in which false branching may be recognized. By false branching is meant an appearance resulting from the fragmentation of threads. The terminal cell breaks away from the main stem, is set at an angle by the elongation of the thread

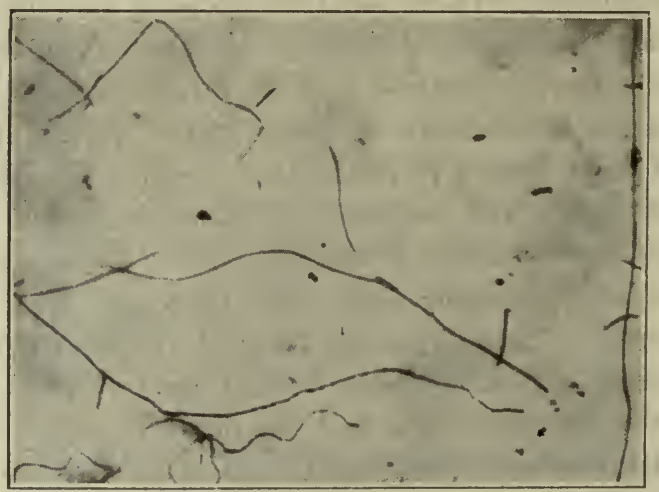

Fig. 101.-Cladothrix, Showing False Branching.

itself, and, as both continue dividing, the simulation of true branching is produced.

Streptothrix denotes forms with numerous true branches and spores which usually appear in chains.

Actinomyces is of more eomplicated structure, characterized by the formation of elub-shaped ends and the stellate arrangement of its threads.

Concerning the use of the last three generie names there has been much controversy, which has recently been discussed with a full 
bibliography by Brced and Conn (J. Baeteriol., 1919, iv, 585). The outcome of the matter seems to be that the genus leptothrix may stand as representing filamentous forms without branehing, of which our knowledge is very ineomplete, and that there are two distinet groups of pathogenic speeies-Nocardice, which are aërobie, and the actimomyces, which are anaërobic. The former, at least, includes a large number of related species.

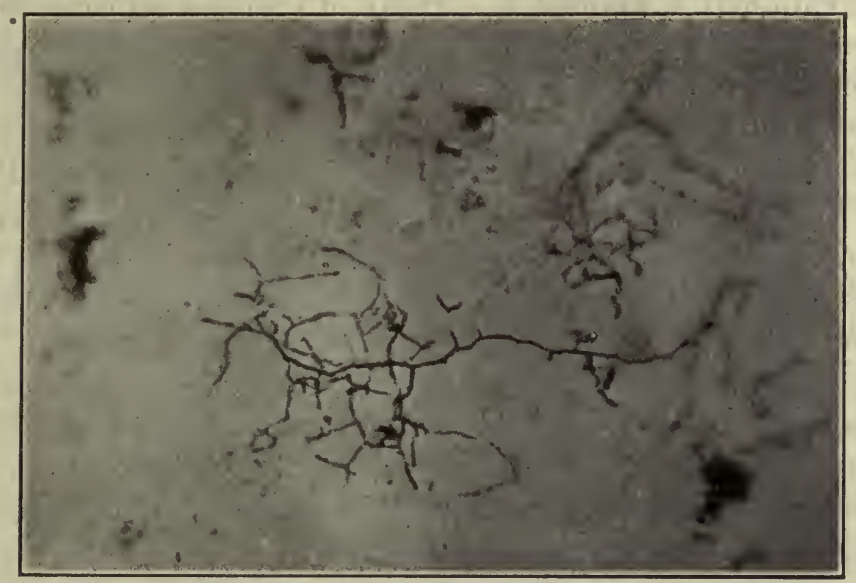

Fig. 102. - Streptothrix, Showing True Branching.

\section{LEPTOTHRIX}

Members of the leptothrix group have been observed in connection with inflammations of the mouth and pharynx by Fränkel, ${ }^{4}$ Michelson, Epstein, ${ }^{6}$ and others. In many of these cases the organism was identified by morphology chiefly, pure cultures not having been obtained. The disease in none of these cases was aeeompanied by severe systemic symptoms and it is likely that when found in human beings the organisms may be regarded simply as eomparatively harmless saprophytes appearing in connection with some other specifie inflammation.

Cultivation of the Leptothrices is not easy and has been suceessful only in the hands of Vignal ${ }^{7}$ and Arustamoff. ${ }^{8}$

"Fränkel, Eulenburg's “Realencyel. d. gesam. Heilkunde," 1882.

'Michelson, Berl. klin. Woch., ix, 1889.

- Epstein, Prag. med. Woch., 1900.

'Vignal, Ann. de phys., viii, 1886.

${ }^{8}$ Arustamoff, Quoted from Petruschky, loc. cit. 


\section{NOCARDIA}

Streplothrix, Cladothrix, Oospora, Discomyces.-This genus includes a large group of aërobic organisms which grow in branching filaments made up of bacteria-like units. English medical writers more frequently refer to them as streptothrices, but, as the name streptothrix is applied to a group of common saprophytjc fungi with coarse filaments, it cannot be properly used for these organisms. Saprophytic varieties of nocardia are numerous and pathogenic strains have also been reported as the cause of varied infections in man and animals. Nocard ${ }^{9}$ described a member of this group as the etiological factor in a glanders-like disease, "farcin du boeuf," occurring in Guadeloupe, which he called actinomyces farcinica. The first human case was that of Eppinger, ${ }^{10}$ who cultivated from a brain abscess an organism which he called Cladothrix asteroides on account of the star-like appearance of the young colonies on agar. He also found the organisms in sections of the bronchial lymph-nodes and believed the invasion had occurred through the respiratory tract. Since then a number of fatal systemic infections due to similar organisms have been described by Petruschky, ${ }^{11}$ Berestnew, ${ }^{12}$ Flexner, ${ }^{13}$ MacCallum, ${ }^{14}$ Norris and Larkin ${ }^{15}$ and others, and an apparently identical organism was isolated by Musgrave and Clegg in a case of Madura foot. A summary of the various cases up to 1921 has been made by Henrici and Gardner (J. Infect. Dis., 1921, xxviii, 232). In most of these cases the portal of entry was the respiratory tract, but a few began as wound infections. Strains from various cases have been considered by some to be identical, by others to represent a number of closely related species.

Morphology. - Morphologically the nocardia show considerable variation. In material from infectious lesions they have most oficu appeared as rods and filaments with well-marked branching. Occasionally the filaments are long and intertwined, and branches have

- Nocard, Ann. de l'inst. Pasteur, ii, 1888.

${ }^{10}$ Eppinger, Wien. klin. Woch., 1890.

${ }^{11}$ Petruschky, Verhanil. đ. Kongr. f. innere Mediz., 1898.

${ }^{12}$ Berestneff, Zeit. f. Hyg., xxix, 1898.

13 Flexner, Jour. Exp. Med., iii, 1896.

"MacCallum, W. E., Centralbl. f. Bakt., I, O, 1902, xxxi, 529.

${ }^{15}$ Norris and Larkin, Proc. of N. Y. Path. Soc., March, 1899. 
shown bulbous or club-shaped ends. In Norris and Larkin's case, the young cultures in the first generations seem to have consisted chiefly of rod-shaped forms not unlike bacilli of the diphtheria group, showing marked metachromatism when stained with Loeffler's methylene-blue. They are easily stained with this dye or with aqueous fuchsin. Many strains are acid-fast, but decolorize somewhat more readily than do tubercle bacilli. In tissue sections they may be demonstrated by the Gram-Weigert method.

Cultivation.-The organism develops slowly on ordinary agar or gelatin plates, forming visible colonies in from two to five days. Later it forms a membrane somewhat adherent to the surface which soon becomes wrinkled. It is at first white but later turns yellow or even a brilliant orange. On broth they grow as a thick pellicle or, occasionally as a floceulent precipitate. Most strains have not liquefied gelatin or altered litmus milk, but liquefying strains have been described. All strains have proved highly virulent for guineapigs and somewhat less so for rabbits, producing in the animals lesions indistinguishable from tuberculosis.

Nocardie in Rat-Bite Fever.-In the cases of fatal septicemia following rat bites, Schottmueller ${ }^{16}$ and Blake ${ }^{17}$ have recovered nocardiæ from the blood. It has since been shown, however, that this disease is due to infection by treponemata.

Streptothrix of Rosenbach.-A species of nocardia undoubtedly different from the asteroides group has been deseribed by Rosenbach $^{18}$ as the cause of an indolent dermatitis of the fingers and toes known as erysipeloid.

\section{ACTINOMYCES}

(Streptothrix Israeli, Kruse; Discomyces bovis, Brumpt; Cohnistreptothrix Israeli, Pinoy)

Among the diseases caused by the Trichomycetes or higher bacteria, the most important is actinomycosis. Occurring chiefly in some of the domestic animals, notably in cattle, the disease is observed in man with sufficient frequency to make it of great clinical importance. In eattle the specific microorganism which gives rise

${ }^{16}$ Schottmueller, Dermat. Wehnschrft., 1914, LVIII, Sup. 77.

${ }^{17}$ Blake, F. G., Jour. Exp. Med., 1916, XXIII, 39.

${ }^{18}$ Rosenbach, Areh. f. klin. Chirurg. 1887, xxiv, 346. 
to the disease was first observed by Bollinger ${ }^{19}$ in 1877 . In the following year Israel ${ }^{20}$ diseovered a similar microorganism in human cases.

The parasites appear in the pus from discharging lesions as small granular bodies, plainly visible to the naked eye and somewhat resembling sulphur granules, of a grayish or of a pale yellow color. In size they measure usually a fraetion of a millimeter. Ordinarily they are soft and easily erushed under a cover-slip, but occasionally, especially in old lesions, they may be quite hard, owing to ealcification.

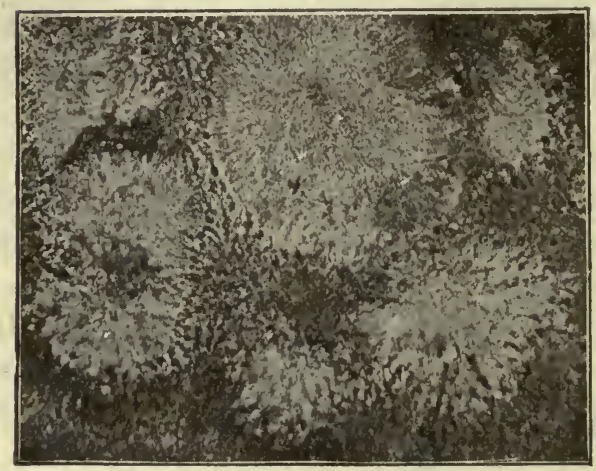

Fig. 103.-Actinomyces Grandle Crushed Beneath a Cover-glass. Unstained. Low power. Shows radial striations. (After Wright and Brown.)

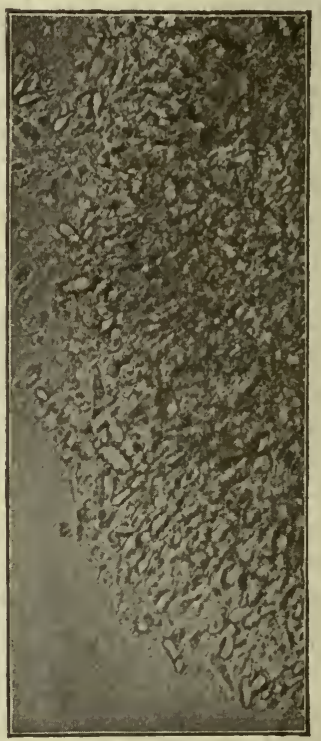

Fig. 104.-А C TINOM Y CES Granule Crushed BeNEATH A COVER-GLASS. Instained. The preparation shows themargin of the granule and the "clubs." (After Wright and Brown).

Microscopically they are most easily recognized in fresh preparations prepared by crushing the granules upon the slide under a cover-slip and examining them without staining. They may be rendered more clearly visible by the addition of a drop or two of 20 per cent potassium hydrate. When the granules are calcareous, the addition of a drop of concentrated acetic acid will facilitate

19 Bollinger, Deutsch. Zeit. f. Thiermed., iii, 1877.

${ }^{20}$ Israel, Virch. Arch., 74, 1878 and 1879, LXXVIII, 421. 
examination. Fresh preparations may be examined after staining with Gram's stain. Observed under the microscope, the granules appear as rosette-like masses, the centers of which are quite opaque and dense, appearing to be made up of a closely meshed network of filaments. Around the margins there are found radially arranged striations which in many cases end in characteristically club-shaped bodies. Inside of the central network there are often seen coccoid or spore-like bodies which have been variously interpreted as spores, as degeneration products, and as separate coeci fortuitously found in symbiosis with the-actinomyces. Individually considered, the central filaments have approximately the thickness of an anthrax bacillus and are, aceording to Babes, ${ }^{21}$ composed of a sheath within

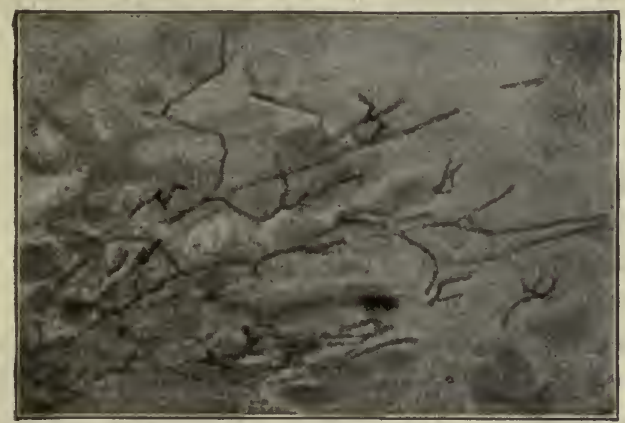

Fig, 105.-Branching Filaments of Aetinomyces. (After Wright and Brown.)

which the protoplasm contains numerous and different sized granules.

About the periphery of the granules the free ends of the filaments become gradually thickened to form the so-ealled actinomycosis "clubs." These clubs, according to most observers, must be regarded as hyaline thiekenings of the sheaths of the threads and are believed to represent a form of degeneration and not, as some of the earlier observers believed, organs of reproduction. They are homogeneous, and in the smaller and presumably younger granules are extremely fragile and soluble in water. In older lesions, especially in those of eattle, the clubs are more resistant and less easily destroyed.

They appear only in the parasites taken from active lesions in animals or man, or, as Wright ${ }^{22}$ has found, from eultures to which

${ }^{21}$ Babes, Virch. Arch., 105, 1886.

${ }^{22}$ J. H. Wright, Jour. Med. Res., 1905, vii, 349. 
animal serum or whole blood has been added. In cultures from media to which no animal fluids have been added, such as glucose agar or gelatin, no clubs are found. In preparations stained by Gram's method the clubs give up the gentian-violet and take counterstains, such as eosin.

The coccus-like bodies found occasionally lying between the filaments of the central mass, most observers now agree, do not represent anything comparable to the spores of the true hyphomycetes. In many cases they are unquestionably contaminating cocci; in others again they may represent the results of degeneration of the threads.

In tissue sections, the mieroorganisms may be demonstrated by Gram's method of staining or by a special method devised by Mallory. ${ }^{23}$ This is as follows for paraffin sections:

1. Stain in saturated aqueous eosin ten minutes.

2. Wash in water.

3. Anilin gentian-violet, five minutes.

4. Wash with normal salt solution.

5. Gram's iodin solution one minute.

6. Wash in water and blot.

7. Cover with anilin oil until section is clear.

8. Xylol, several changes.

9. Mount in balsam.

Cultivation.-The isolation of actinomyees from lesions may be easy or difficult according to whether the pus is free from contamination or whether it contains large numbers of other bacteria. In the latter case it may be almost impossible to obtain cultures. The descriptions of methods of isolation and of cultural characteristics given by various writers have shown considerable differences. The most extensive cultural work has been done by Bostroem, ${ }^{24}$ Wolff and Israel, and by J. H. Wright. Bostroem has described his eultures as aërobie, but Wolff and Israel $^{25}$ and Wright ${ }^{26}$ agree in finding that the microorganisms isolated by them from actinomycotic lesions grow but sparsely under aërobic conditions and favor an 1908 .

${ }^{23}$ Mallory, Methor No. 1, Mallory and Wright, "Path. Technique," Phila.,

\footnotetext{
${ }^{24}$ Bostroem, Beitr. z. path. Anat. u. z. allg. Path., ix, 1890.

${ }^{25}$ Wolff und Israel, Virch. Areh., 1891, exxvi, 4.

${ }^{26}$ J. H. Wright, Jour. Med. Res., viii, 1905.
} 
environment which is entirely free from oxygen, or at least contains it only in small quantities. The method for isolation recommended by Wright is, briefly, as follows: Pus is obtained, if possible, from a closed lesion and washed in sterile water or broth. The granules are then crushed between two sterile slides and examined for the presence of filaments. If these are present in reasonable abundance, the material is distributed in tubes of glucose agar, which are then allowed to solidify. If these first cultivations show a large number of contaminations, Wright recommends the preservation of other. washed granules in test tubes for several weeks, in the hope that contaminating microorganisms may thus be killed by drying before the actinomyces lose their viability.

If cultivation is successful colonies will appear, after two to four days at $37.5^{\circ} \mathrm{C}$., as minute white specks, which, in Wright's cultures, appeared most abundantly within a zone situated 5 to 10 millimeters below the surface of the medium. Above and below this zone they are less numerous, indicating that a small amount of oxygen furnishes the best cultural environment. Upon the surface of agar slants, growth, if it takes place at all, is not luxuriant.

In alkaline meat-infusion broth growth takes place in the form of heavy, flocculent masses which appear at the bottom of the tubes. Surface growth and clouding do not take place.

Milk and potato have been used as culture media but are not particularly favorable.

Pathogenicity.-As stated above, actinomycosis occurs spontaneously most frequently among eattle and human beings. It may also occur in sheep, dogs, eats, and horses. Its locations of predilection are the various parts adjacent to the mouth and pharynx. It occurs also, however, in the lungs, in the intestinal canal, and upon the skin. When occurring in its most frequent location, the lower jaw, the disease presents, at first, a hard nodular swelling which later becomes soft because of eentral necrosis. It often involves the bone, causing a rarefying osteitis. As the swellings break down, sinuses are formed from which the granular pus is discharged. The neighboring lymph nodes show painless, hard swellings. Histologically, about the filamentous knobs or granules, there is a formation of epithelioid cells and a small round-cell infiltration. In older cases there may be an encapsulation in connective tissue and a calcification of the necrotic masses, leading to spontaneous cure. As a rule, this process is extremely chronic. Infection in the lungs 
or in the intra-abdominal organs is, of course, far more serious. When death occurs acutely, it is often due to secondary infection. The disease is acquired probably by the agency of hay, straw, and grain.

Berestnew $^{27}$ has succeeded in isolating actinomyces from straw and hay which he covered with sterile water in a potato jar and placed in the incubator. After a few days small white specks looking like chalk powder appeared upon the stalks which upon further cultivation developed a growth which he considered identical with the pathogenic species of the Bostroem type. Typical anaërobic actinomyces have never been isolated except from cases of the disease.

Animal inoculation has given conflicting results. Bostroem with his aërobic cultures was unable to produce lesions. Israel and Wolff did, on the other hand, produce nodules resembling those seen in spontaneous infections with their anaërobic cultures, but the lesions were not progressive and healed spontaneously. Wright could produce lesions with anaërobic but never with aërobic strains. He concluded that the anaërobic organism was the true cause of actinomyces, and that Bostroem was probably dealing with a contamination. On the other hand Pinoy, Castellani, Brumpt, and others reviewing the subject state that the disease actinomycosis, though usually produced by the anaërobic organism is in some cases caused by aërobic organisms belonging to the genus nocardia. Others have attempted to distinguish between strains by the presence or absence of clubs in the infected tissue, reserving the name actinomyces for those parasites which produce clubs and calling all others streptothrices or nocardice. This is an unsatisfactory criterion, however, as MacCallum was able to produce clubs by intraperitoneal injection of his strain which, in every other respect, was a typical nocardia asteroides.

B. actinomycetum comitans.-As in other mycoses the isolation of the parasite in this disease is made difficult by the presence in the lesions: of numerous bacteria which overgrow primary cultures. The contaminants are frequently pyogenic cocei and saprophytic intestinal bacilli, One unusual type of organism has, however, been found in these lesions with sufficient frequency to deserve mention. Wolf and Israel $^{28}$ mention the presence in the granules of numerous

${ }^{27}$ Berestnew, Ref. Cent. Bakt., 24, 1898.

${ }^{28}$ Wolf, M., and Israel, J., loc. cit. 
pleomorphic bodies resembling micrococci. Similar organisms have been noted by Klingler ${ }^{29}$ and reeently in twenty-four eases by Colebrook $^{30}$ on whose paper the following description is based. In the granules they appear as minute Gram-negative cocco-bacilli fused together into sheets. In eulture they grow aërobieally or anaërobieally as minute coherent colonies which on agar are smooth and glistening, in broth, starlike and frequently adherent to the sides of the tubes. They grow on simple media but quickly die out in culture. They form acid but no gas on sugars. Guinea-pigs and rabbits may be killed by large injections but no lesions resembling actinomyeosis are produeed. It is doubtful if these bacteria play an important rôle in spontaneous cases of the disease.

Actinobacillosis.-A disease of cattle simulating actinomycosis, in which granules with rays oeeur in the exudates has been deseribed in the Argentine by Lignières and Spitz and in England by Griffith. ${ }^{31}$ Definite filaments were not found in the lesions and eultures yielded a Gram-negative strepto-bacillus. One human case has been reported by Ravaut and Pinoy. ${ }^{32}$

\section{MYCETOMA (MADURA FOOT)}

The disease known by this name is not unlike actinomycosis. It is more or less strietly limited to warmer elimates and was first reeognized as a clinieal entity, in India, by Carter. ${ }^{33}$ Clinically it eonsists of a chronie produetive inflammation most frequently attacking the foot, less often the hand, very infrequently other parts of the body. Nodular swellings oceur, which break down in their centers, leading to the formation of abscesses, later of sinuses. Often the bones are involved and a progressive rarefying osteitis results. From the sinuses a purulent fluid exudes, in which are found charaeteristie granular bodies. These may be hard, brittle, and black, resembling grains of gunpowder, or may be grayish-white or yellow and soft and grumous. Aceording to the appearanee of these granules different varieties of the disease have been described as mycetoma

${ }^{29}$ Klingler, $R$., Centr. f. Bact., I, O; 1912, LXII, 191.

3n Colebrook, L., Brit. Jour. Exp. Path., 1920, I, 197.

${ }^{31}$ Grifill, $F$., Jour. of Hyg., 1916, XV, 195.

${ }^{32}$ Ravaut and Pinoy, Presse Mérl., 1911, XIX, 49.

${ }^{33}$ Carter on Myeetoma, etc., London, 1874. 
with black, white, yellow, or red granules. These different varieties of the disease though elinically similar may apparently be produced by a large number of diffeernt parasites all belonging to the fungi or higher bacteria. We will mention below the few cases from which aspergilli and allied molds have been isolated. In the majority of eases, organisms similar to the nocardia have been found. Brumpt divides them into two genera: the madurella having septate mycelia and the discomyces which is without septa, but this distinction is not generally recognized. For a discussion of the characteristics of the various strains one should refer to the works of Brumpt and of Castellani.

The parasite of the commoner black variety which certainly seems to be a distinct disease has been carefully studied by Wright from whose description the following points are taken.

The small, brittle granules observed under the microseope show a dark, almost opaque center along the edges of which, filaments, or hyphæ, may be seen in a thickly matted mass. By crushing the granules under a cover-slip in a drop of sodium hypochlorite or of strong sodium hydrate, the black amorphous pigment is dissolved and the structural elements of the fungus may be observed. They seem to be composed of a dense meshwork of mycelial threads which are thick and often swollen, and show many branches. Transverse partitions are plaeed at short distances and the individual filaments may be very long. Spores were not observed by Wright. In a series of over fifty eultivations on artificial media from the original lesion, Wright obtained growth in a large percentage.

In broth, he obtained at first a rapid growth of long hyphæ which eventually formed a structure which he compares in appearance to a powder-puff.

On agar, growth appeared within less than a week and spread over the surface of the medium as a thick meshwork of spreading hyphw of a grayish color. In old cultures black granules appeared among the mycelial meshes.

On potato, he observed a dense velvety membrane, eentrally of a pale brown, white at the periphery. Small brown droplets appeared on the growth in old cultures.

Animal inoeulation with this mieroorganism has so far been unsuceessful. 


\section{CHAPTER I}

\section{THE PATHOGENIC FUNGI ${ }^{1}$}

THE earliest demonstrations of microorganisms as the causes of disease were the discovery of a fungus in the scutula of favus by Schoenlein in 1839 and Langenbeck's discovery of the thrush parasite in the same year. Later the work of Pasteur and his followers showed the far greater importance of bacteria as disease-producers and the study of these simpler forms has since been given greater attention. We must, however, briefly consider a group of diseases known as the mycoses which are due to infection by the fungi.

In the broader sense the term fungi is used to include all thallophyta (plants without stems, roots, or leaves) which are devoid of chlorophyl or its analogues, and which consequently are limited to a saprophytic or parasitic existence. In this sense the fungi form a class of which the bacteria, or fungi which reproduce by simple fission, are the simplest types. In the narrower sense the term is applied only to forms which reproduce by means of spores. The cells of these latter organisms are somewhat larger than those of the bacteria and are usually enclosed in a well differentiated membrane. They contain, as a rule, a demonstrable nucleus, granules of various types and often vacuoles. Some fungi are unicellular, as are the bacteria, but most are made up of many cells which are interdependent and show some differentiation in form and function.

Typical fungi are made up of cylindrical cells, joined into filaments, from which smaller rounded cells called spores are developed. From these two elements, filaments and spores, the fungi build up a structure that differs immensely in complexity in the different species. The unicellular types such as the common yeasts grow in easily dissociated masses like bacteria and each cell combines the functions of absorbing food-stuffs, of building them up into its own substanee and of reproducing new individuals. In the molds, which represent simpler multicellular fungi, the filaments lie distinct in

1 This chapter has been rewritten for us by Dr. J. G. Hopkins. 
a loose meshwork, without definite arrangement except that certain of them are thrust up vertically and develop spores. In the higher types, of which the mushrooms are familiar examples, certain filaments form a cobweb-like net-work which is spread through the soil on which they grow. These serve to absorb and pass on nourishment. They connect with other filaments which are welded together to form the firm umbrella-like structure which projects above the ground. This is covered with a tough protective membrane also made up of closely cohering filaments. Along the gills on the under side of the cap are rows of characteristic cells (basidia) on which the spores are born. These different portions are analogous in function to the roots, stems, bark, fruit and seeds of higher plants.

The gross appearance, the microseopic structure and especially the type of spores produced are relied on for the identification and classification of the various species of fungi. Consequently, it will be necessary to define some of the morphological terms used before proceeding to a description of the different pathogenic types.

\section{MORPHOI.OGICAL DEFINITIONS}

The Thallus.-The entire vegetative portion of a fungus is called the thallus; the individual filaments of which it is composed, hyphae. When the hyphae lie in a loose meshwork without definite arrangement, the mass is termed a mycelium and sometimes this term is applied to the entire thallus, even when it develops a characteristic morphology. In some species the hyphae are continuous tubes with multiple nuclei; in others they are divided by septa into chains of eylindrical cells.

Hyphae which have special functions are often differentiated from the rest of the mycelium. In most of the pathogenic fungi the thallus is rudimentary and it is not necessary to discuss here the elaborate and somewhat confused terminology used in describing the more complex forms. In the species to be considered only the fertile hyphae, i.e., those that give rise to spores are differentiated. They are called sporophores or conidiophores, these terms being applied sometimes to one specialized cell, sometimes to a multicellular or branched filament or to a bundle of filaments. A small cell, or even a conical process from a cell, which serves as a point of attachment for spores is called a sterigma; flask-shaped cells of this type are called phialides.

Spores.-The term spore in the stricter sense means a rounded reproductive cell analogous in function to the multicellular seed of a higher plant. As a rule, a spore differs in form from the parent cell, and does not divide until it becomes separated from the thallus, then after a latent period it 
germinates and produces a new thallus. This meaning is quite different from that in which the word is used when describing bacteria. The same term is, however, often loosely used for any rounded cell of a fungus, whether it be a part of the body of the organism, or an encysted resting form, or a true reproductive type. In fact the function of a cell in these rudimentary plants is often hard to define, as one. which seems at first to be merely a component unit of the thallus may if it becomes detached reproduce an entire organism. We may, however, divide all these rounded cells into two classes: (a) True spores, the sole function of which is reproduction; and (b) Vegetative spores, remembering that the latter may also serve to reproduce the species.

Spores differ in their mode of origin and in their arrangement on or within the thallus. Almost every species of fungus produces several types. There are two groups of reproductive spores, one sexually produced, the other asexually. Of the many names given to different forms we will attempt to define only those which it will be necessary to use in this chapter. It will be found that many of these are used in a somewhat varying sense by different writers.

ReProductive SPORES.-An Oospore is a sexual type produced by the fertilization of a female cell by a differentiated male cell.

A Zygospore is a sexual type produced by the fusion of two undifferentiated cells.

Conidium is a general term applied to all asexual reproductive spores. The term is by some writers restricted to exospores or those formed by a bud which protrudes from the membrane of the parent cell.

Endospore is a general term applied to any spore formed within the membrane of the parent cell.

Ascospores are a special class of endospores which are formed in a membrane known as the ascus, the number of spores in the sack being limited to two, four, or eight, and constant for the particular species producing them. The parent eell from which ascospores are produced has originally two nuclei which fuse into one before again dividing to form the ascospores. This fusion is regarded by Dangeard as a rudimentary sexual process. It has also been shown by Harper and others that in certain species the parent cell is the result of the fertilization of a female cell by a differentiated male cell. This is not true of some of the simpler types such as yeasts.

Basidiospores are exospores produced on a special type of sporophore, known as a basidium. The number of spores on a basidium is limited and constant for a given species. Like the ascus, the basidium has in certain species been found to have two nuclei which fuse before the spores are produced.

Vegetative Spores.-Thallospore is a general term applied to cells morphologically resembling the above types which are essentially a part of the vegetative portion of the fungus. 
Blastospores are thallospores which develop by budding from the end or side of the parent cell and which may in turn throw out another bud or a mycelial filament without becoming detached, and without any period of latency. The buds of yeast eells are familiar examples.

Arthrospores are thallospores formed by the segmentation of a hypha into a chain of cells at first eubical and later rounded.

Chlamydospores are single thalospores formed by the concentration of the protoplasm of a hypha into a swollen portion of the filament, the membrane of which becomes thickened. They are purely resting spores and are closely analogous in function to the spores of bacteria. They appear as roughly spherical thick-walled cells much greater in diameter than the hyphæ and are ealled intercalary if they develop in the course of a continuous filament; terminal, if at the end of a long hypha or a short lateral branch.

\section{CLASSIFICATION}

The whole subject of the classification of the fungi is in confusion and the phylogenetic relationship of the various groups is obscure. Even the identification of a species is often difficult, partly because some simpler types show little that is distinctive in their structure but chiefly because their morphology varies greatly with changed environment. For example, some of the blastomyees which grow in the animal body as round cells reproducing by means of blastospores, when placed on artificial media develop a mycelium and conidia. Many of the higher forms, too, which are parasitic on plants assume on different hosts forms which bear no resemblance to each other. This pleomorphism has made the study of the fungi a difficult field. A species may be observed for years before it exhibits characteristies which show elearly its relationship to certain other species.

The modes of spore production are the chief characteristics according to which the fungi are grouped-especially those modes which seem to represent a sexual process. The various species are classed as those which form oospores, or ascospores, etc. Many, however, produce only conidia and it is generally considered that they are degenerate types which have lost the power of even rudimentary sexual reproduction. Such varieties are often grouped together as the fungi imperfecti. As to relationships among these organisms, there is little agreement and almost every writer has brought forward a new grouping and a new classification. There are, however, certain large groups which are generally recognized, the main characteristics of which are shown in the following table: 

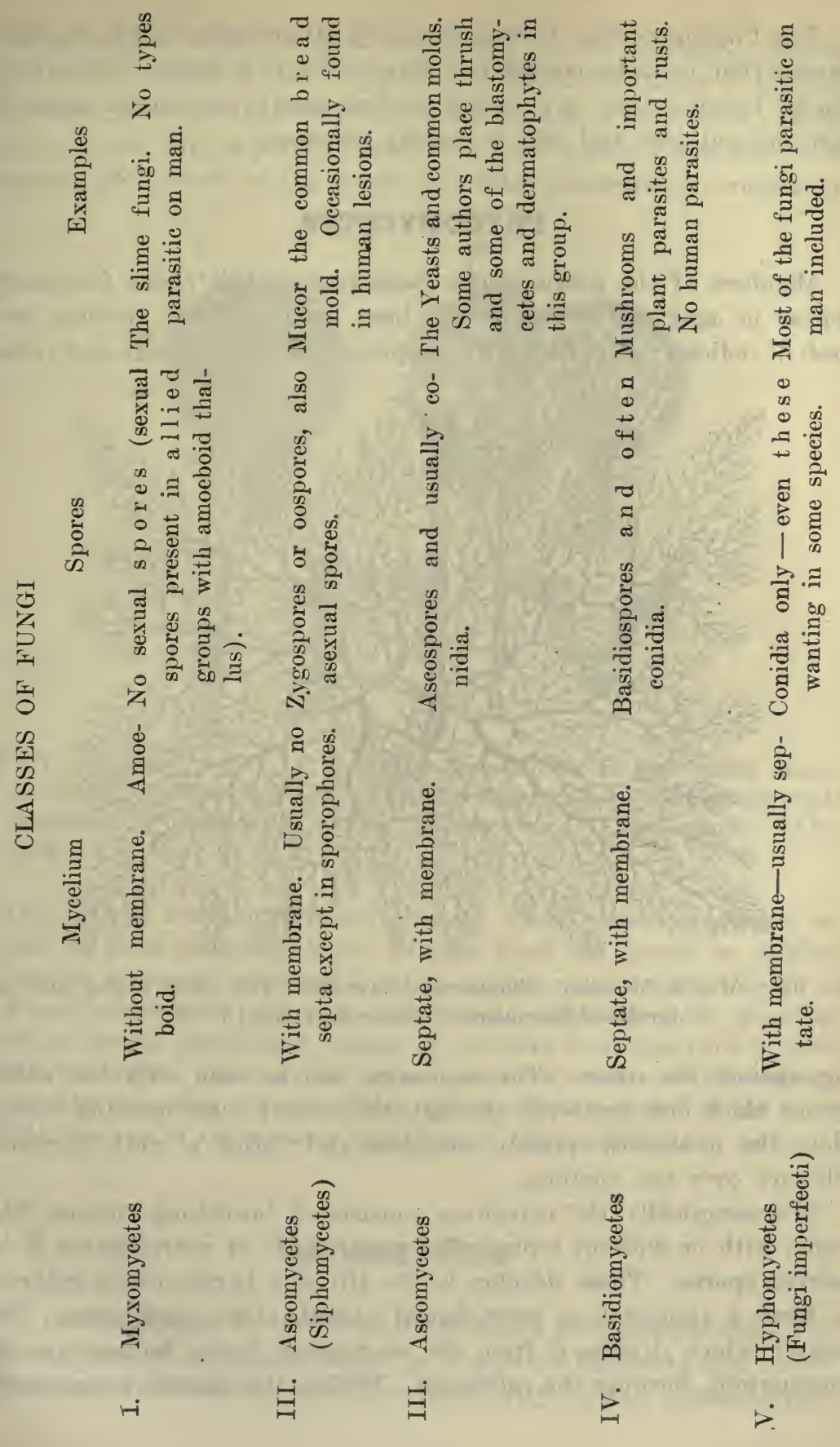
The Phycomycetes, Ascomyectes and Hyphomyeetes are the only groups that need be considered here. The two former concern us chiefly because they are found so frequently as contaminants in bacterial eultures and will be briefly discussed.

\section{PHYCOMYCETES}

Members of the genus Mucor belonging to this order frequently appear in agar plates which have been opened. They develop as a mesh of delicate white filaments completely filling the plate and press-

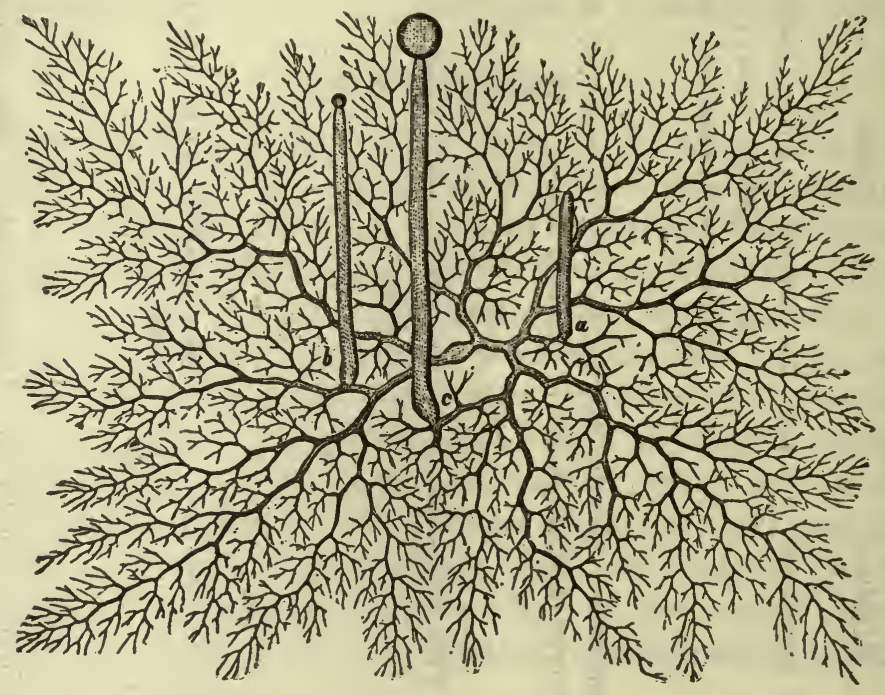

Fig. 106.-Mucor Mucedo. Single-celled mycelium with three hyphæ and one developed sporangium. (After Kny, from Tuvel.)

ing against the cover. The sporangia can be seen with the naked eye as black dots scattered through the growth. On opening such a plate the meshwork quickly collapses and forms a white speckled feltwork over the medium.

Microscopically the mycelium consists of branching tubular filaments with or without septa. The usual mode of reproduction is by asexual spores. These develop in the tip of a hypha which enlarges to form a spherical or pear-shaped capsule, the sporangium. The septum which divides it from the supporting hypha bulges into the sporangium, forming the columella. Within the capsule innumerable 
sporangiospores develop which are freed by its rupture. Sexual reproduction, which is less frequent, consists in the fusion of the tips or lateral processes of two neighboring hyphæ, which form a large spore covered with a warty membrane, known as a Zygospore. No exospores are formed by the mucors but chlanydospores are numerous.

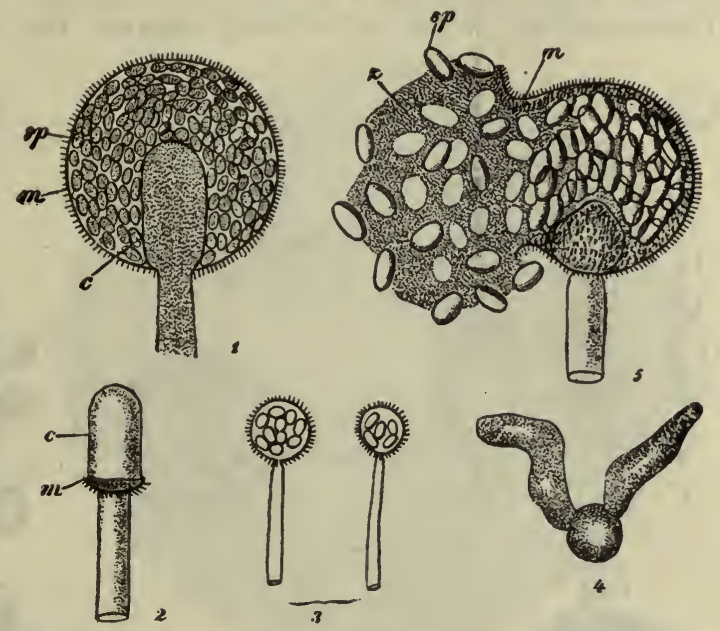

Fig. 107.-Mucor Mucedo. 1. Sporagium, c. columella, m. sporangium capsule sp. spores. 2. Columella, after bursting of sporangium. 3. Poorly developed sporangia. 4. Germinating spore. 5. Emptying of sporangium. (After Brefeld.)

The common laboratory contaminants are Mucor mucedo a constant inhabitant of horse dung, and Mucor pusillus, which ean usually be obtained by 'allowing a piece of moistened bread to stand in a covered Petri dish.

Mucor corymbifer (Lichteimia corymbifera) differs from the preceding species in having pear-shaped instead of spherical sporangia born in loose clusters on hyphae which are not raised above the surface of the medium. This species is pathogenic for rabbits, and has been reported as the cause of inflammations of the auditory canal and of other infections in man.

\section{ASCOMY CETES}

In this group are included all fungi which form ascospores. The majority have a mycelium made up of septate filaments and reproduce by means of conidia which are frequently born on characteristic 
structures. In the lower types met with as laboratory contaminants ascospore formation is rarely observed.

The Yeasts.-The simplest forms of ascomycetes are the exoasci of which the yeasts or saccharomycetes are familiar examples. These develop on laboratory media as moist masses of separate round or oval cells usually from 10 to 20 microns in diameter. These send out buds (blastospores) which gradually assume the size of the
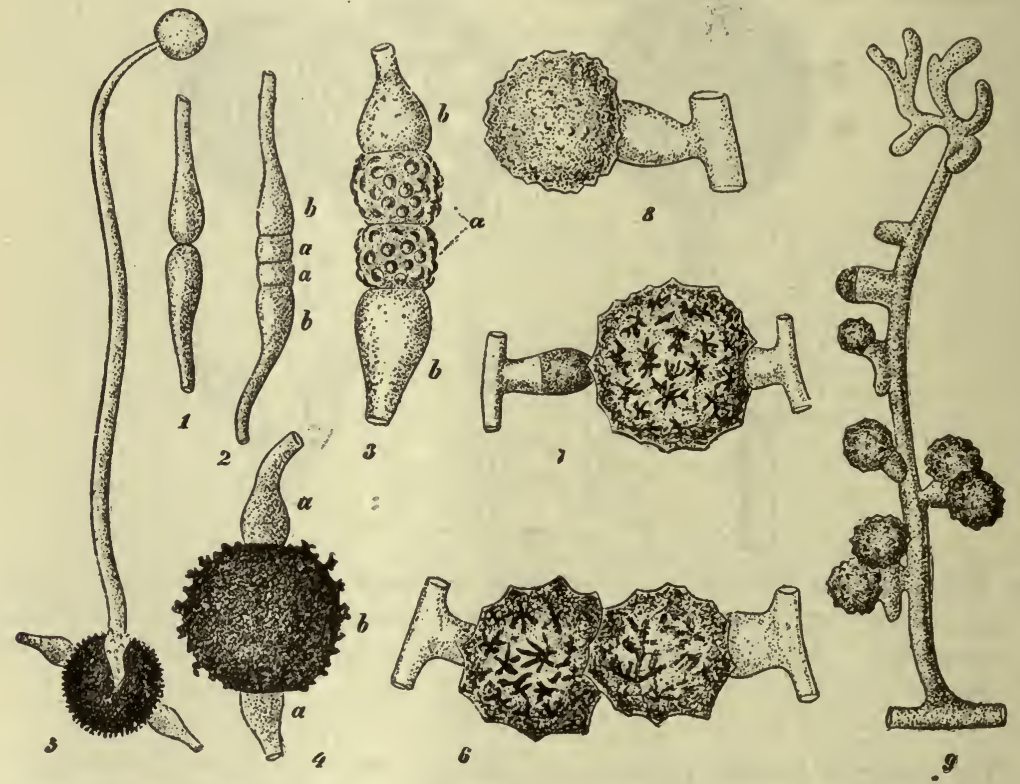

Fig. 108.-Mucor Mucedo. Formation of zygospore. 1. Two branches coalescing. 2 and 3. Process of conjugation. 4. Ripe zygospore. 5. Germination of zygospore. 6 and 7. Mucor erectus. Azygo sporulation. No two branches meet, but form spores withoùt conjugation. 8 and 9. Mucor tenuis. Azygo sporulation. The spores grow out from side branches without sexual union. (1-5 after Brefeld; 6-9 after Banier, from Tavel.)

parent cells from which they quickly separate. When grown on a dry surface some of the cells divide into ascospores which remain for a while enclosed in the membrane of the parent cell or ascus. Typical yeasts are unicellular but in some species the cells successively produced by budding adhere to the parent cells and form mycelial filaments, which consist of chains of round or oval units. In other cases the individual cells of such a chain elongate and form a hypha with eylindrical elements. 
The yeasts have been studied most extensively in connection with fermentation and are industrially of great importance in the production of wine and beer. Much of our knowledge of the life-processes of bacteria is based on these early investigations of the yeasts. Although Schwann, as early as 1837 , recognized the fact that many fermentations could not oceur without the presence of yeast, it was not until considerably later that the study of such fermentations was put upon a scientific basis. The typical fermen-

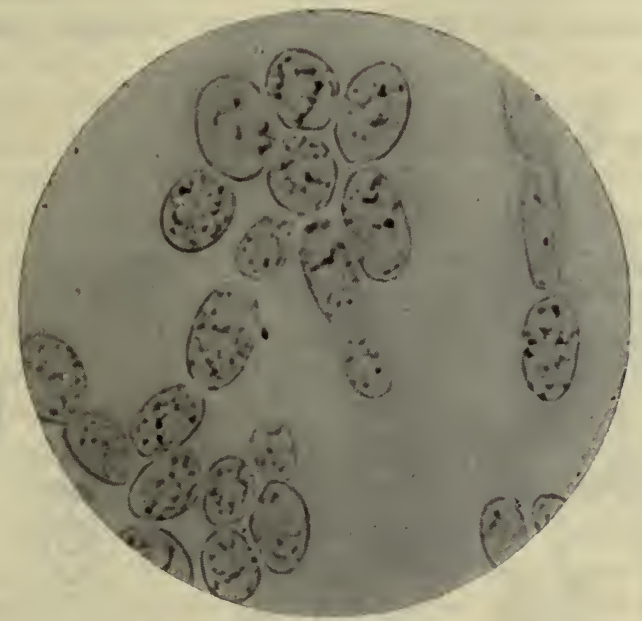

Fig. 109.-Yeast Cells. Young culture unstained. (After Zettnow.)

tative action consists in the transformation of sugar into ethyl alcohol according to the following formula:

$$
\mathrm{C}_{6} \mathrm{H}_{12} \mathrm{O}_{6}=2 \mathrm{C}_{2} \mathrm{H}_{5} \mathrm{OH}+2 \mathrm{CO}_{2} \text {. }
$$

The enzyme by which this fermentation is produced is known as "zymase," and is, according to Buchner, in most cases, an endo-enzyme which may be procured from the cell by expression in a hydraulic press. In addition to this, however, the various yeasts also produce other ferments by means of which they may split higher carbohydrates, such as saccharose, maltose, and even stareh, and prepare them for action of the zymase. The manner in which this is accomplished, and the by-products which are formed during the process, vary among different species, and it is for this reason that the employment of pure cultures is of such great importance in the wine and beer industries where differences in flavor and other qualities may be directly dependent upon the particular species of yeast employed for the

${ }^{2}$ Pasteur, Études sur ia bière, Paris, 1876. 
fermentation. It is due to the work ehiefly of Pasteur ${ }^{2}$ and of Hansen ${ }^{3}$ that the beer and wine industries have been carried on along exact and scientific lines.

Many observers include in the yeast family certain pathogenic fungi causing thrush and blastomyeosis. These will be described in the section on the hyphomycetes.

The MoLds.-The powdery molds which so frequently appear in Petri dish cultures and which even grow through cotton plugs and

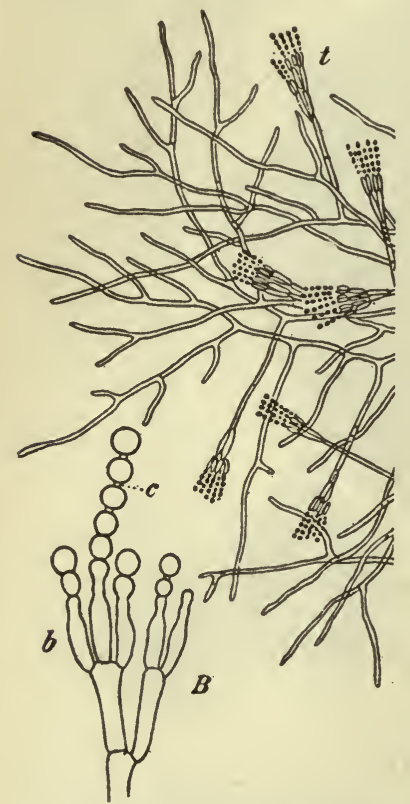

Fig. 110.-Penicillium Glaucum. A. Showing septate mycelia. B. End of a hypha -branching into two conidiophores, from which are given off the sterigmata. From the ends of these are developed the round conidia. (After Zopf.)

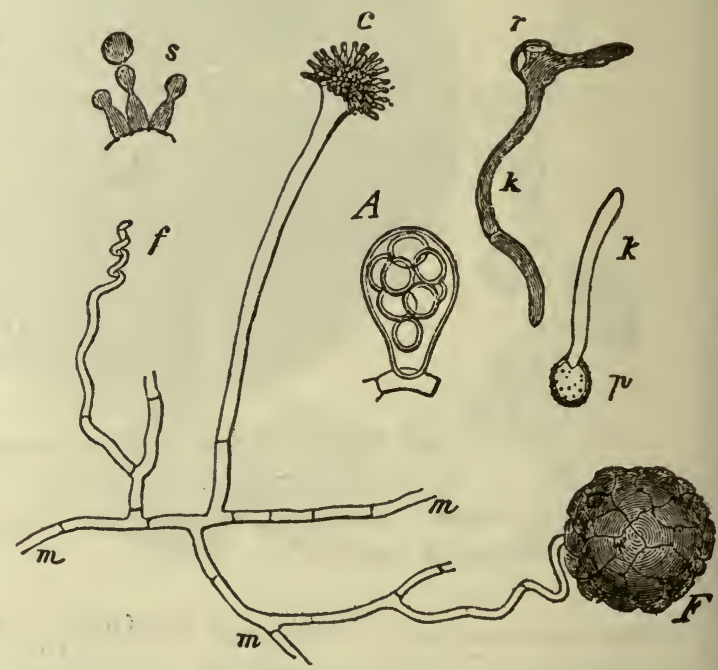

Fig. 111.-Aspergillus Glaucus. m. Mycelial threads. s. Sterigmata. r. Ascospore. p. Germinating condium. A. Ascus. (After de Bary.)

invade tube cultures also belong to the ascomycetes. The two commonest genera have characteristic conidiophores which make their identification easy.

\footnotetext{
Hansen, Prac. Studies in Fermentation, London, 1896.
} 
In the Penicillium glaucum (or crustaceum) the fertile hyphae show numerous branches toward their upper extremities and terminate in a radiating group of flask-shaped cells (phialides) from the tips of which chains of conidia develop-the structure somewhat resembling a broom. Typical cultures have a dusty green color.

The Aspergilli are equally common and troublesome contaminants. They' appear on culture media as a white feltwork often thickly dotted with black points becoming in older cultures diffusely black or yellow or green. The conidia are born on hyphae which terminate in a large rounded head from which phialides project in all directions. From the tips of these extend chains of conidia, often so densely packed together that the supporting structure is hidden and the whole appears as a spherical mass of pigmented spores. These mold are active producers of oxalic and other organic acids. The Sterigmocystis differs from the aspergillus in that secondary phialides each bearing a chain of spores project from each primary phialide.

Pathogenicity.-Many species of these molds are pathogenic for laboratory animals if the spores are injected intravenously. A number of human infections have also been aseribed to them. So-called pulmonary asperigillosis is a condition clinically resembling tuberculosis, in which an aspergillus, usually the species fumigatus, is found abundantly in the sputum. In most cases the fungus merely invades tissue previously infected with tuberculosis, in other cases (notably in the infection which has been described in the pigeon feeders of Paris by Dieulafoy and by Chantemesse and Widal) the disease is apparently primary and spontaneous eure may result. Madura foot, a disease which will be referred to in discussing the actinomyces group, has been in certain cases attributed to infection with these molds.

Pinta or Caraté, a disease of tropical America characterized by superficial colored patches on the skin is thought to be caused in some cases by aspergilli, in others by a species of trichophyton. Aspergilli have also been reported as infecting the eye, nose, auditory canal and wounds in various regions.

\section{HYPHOMYCETES}

\section{(Fungi imperfecti)}

Most of the fungi which have definitely been shown to be the cause of human disease are rudimentary types in which one can detect no distinetive reproductive processes. This makes impossible their classification in the well defined groups of fungi. The thallus is, as a rule, without characteristic structure and reproduction is by means of simple conidia. Certain types do show close analogy to saprophytie fungi with specialized spores. For example, some 
strains of blastomycetes grow as a mass of round cells developing by means of blastospores, which indicates a close relationship to the true yeasts, and although ascospore formation has never been observed Brumpt and others group them among the ascomycetes. Other strains producing the same type of disease are considered by all to be hyphomycetes.

As the botanical classification of these organisms is still the subject of controversy it has seemed clearer for the purposes of this chapter to arrange them according to the diseases which they produce. For discussion of their botanical relationships as well as for an account the numerous species which have been described, one is referred to the works of Brumpt, ${ }^{4}$ Castellanni, ${ }^{5}$ and Plant. ${ }^{6}$ Only the more important types can be mentioned here.

\section{BLASTOMYCOSIS}

Busse $^{7}$ in 1894 reported a case of fatal, generalized yeast infection beginning in an abscess of the tibia. Pus from the lesions contained numerous giant cells in which he observed round or oval, double-contoured bodies surrounded by a wide capsule. They varied in size from that of a red corpuscle to that of a liver cell. Many showed small cells projecting as buds from the larger parasites. The parasite grew readily on ordinary media in budding forms, often surrounded by a capsule as in the tissues. It was pathogenic for laboratory animals especially for white mice. In glucose solutions it produced carbon dioxide and alcohol. In 1896 Gilchrist $^{8}$ described a similar organism isolated from a patient with a severe chronic cutaneous disease which he described as pseudo lupus vulgaris. This organism also was found in giant cells, was capsulated, and showed budding forms. In culture, however, it produced mycelia and conidia and did not ferment glucose. Oxalic acid crystals were seen in the cultures. In the same year Curtis ${ }^{9}$ in

'Brumpt, Precis de Parisitologie, Masson et Cie, Paris.

${ }^{5}$ Chalmers and Castellani, Handbook of Tropical Medicine.

- Plant, H. C., Handb. d. Path. Microorg., Kolle u. Wassermann, vol. V.

'Busse, Centralbl. f. Bakteriol., I, 1894, xvi, 175.

${ }^{8}$ Gilchrist, Bull. Johns Hopkins Hosp., 1896, vii.

- Curtis, Ann. de l'Inst. Pasteur, 1896, x. 
France isolated a similar parasite from a myxomatous tumor of the leg.

Numerous cases of infection with yeast-like organisms have sinee been described. In a case reported by Zinsser ${ }^{10}$ the lesion was an abscess of the back involving the spine. The parasite corresponded morphologically to Busse's. Animal inoculation in rabbits and guinea-pigs proved positive and the organism seemed to show a selective preference for the lungs and spleen. In the lungs of the animals, especially, lesions were found with surprising regularity even when the inoculation was made intraperitoneally.

Clinically there are two distinct types of this disease, the blastomyeetic dermatitis of Gilchrist and the systemic blastomycosis. The former is by far the more common. Of the latter a collection of forty-seven cases was made in $1916 .^{11}$ The portal of entry in many of these has seemed to be the respiratory tract, the earlier lesions being in the lungs. In other cases the general infection has followed a cutaneous lesion. The skin and subcutaneous tissues show the most numerous secondary lesions but the bones, liver, spleen, kidney and brain have each been involved in several instances.

\section{BLASTOMYCES HOMINIS}

(Saccharomyces hominis, Busse; Cryptococcus Gilchristi, Vuillemin; Zymonema Gilchristi, de Beurmann and Gougerot; Mycoderma dermatitis, Brumpt; Oidium Hektoenii, Rieketts)

The parasites causing this disease probably represent a group of allied organisms rather than an individual species. As seen in the tissues or exudates they have shown a marked similarity in all eases. In the abscesses of the generalized form they are very numerous, in the cutaneous form somewhat more difficult to find and are best sought in the small epidermal abscesses which border the lesion. In an unstained moist film of pus spread out between a cover-slip and slide they appear as round or occasionally oval highly refractive bodies containing granules of various sizes and often vacuoles and surrounded by a hyalin eapsule. They vary greatly in diameter usually from 10 to 20 microns. Budding forms

${ }^{10} Z$ insser, Proc. New York Path. Soc., 1907, vii.

${ }^{11}$ Wade and $\mathrm{Bel}$, Arch. Int. Med., 1916, xviii, 103. 
and pairs united in a figure eight can usually be found. They stand out more definitely if the pus is cleared by mixing with a 10 per cent potassium hydrate solution. They stain irregularly. As a rule with the stronger aniline dyes they are overstained so that the details are obseured. With hemotoxylin eosine the capsule usually remains unstained and the body takes a pale stain with deep blue granules but in some cells the capsule may stain deeply. Thionine, polychrome methylene blue, and Wright's blood stain have been recommended. In tissue sections stained with hemotoxylin and eosin, or better with thionine, or methylene blue the clean cut, circular parasites are easily recognized, lying, as a rule, within multinuclear giant cells.

Isolation.-The isolation of the organism is rendered difficult by the frequent presence in the lesions of bacteria which develop more rapidly than the fungus. Gilchrist and Zinsser encountered Gram-positive cocci, others diphtheroid bacilli. No special methods for facilitating isolation have been devised but success will often attend painstaking and repeated plating of the cultures in high dilution. The most favorable medium is glucose agar and the organisms develop well at room or at incubator temperature.

Cultural Characteristics. - On agar or glucose agar the colonies appear after two to four days as minute glistening white hemispherical spots which are not unlike colonies of staphylococeus albus. In older cultures the appearance of different strains shows marked variations, some remaining smooth and pasty, others changing to a tough wrinkled membrane firmly adherent to the agar, and still others becoming covered with white aerial hyphae. All become brown with age. In agar stab cultures the organisms show their preference for a well oxygenated environment by growing but slightly along the course of the stab and by heaping up a thick creamy layer on the surface of the medium. Most strains fail to liquefy gelatine. In broth cultures the medium remains clear, the organisms growing as a stringy sediment, as a pellicle, or as tufted masses in the depth of the medium. On blood serum, potato and bread growth is easily obtained. Some strains ferment carbohydrates but most do not.

Morphology.-In freshly isolated eultures the growth consists of large round cells with blastospores like those seen in the tissues. Capsules are often formed. Most strains sooner or later develop coarse, irregular, branching mycelial filaments. These are divided hy septa and produce ehains of arthrospores and terminal or lateral conidia. Hamburger ${ }^{12}$ in a rareful culture study of four strains

${ }^{12}$ Hamburger, Jour. Inf. Dis., 1907, IV, 201. 
from systemic infections was impressed with the effect of the temperature of incubation on the morphology of the organism. All of his strains grown in the incubator tended to multiply by budding, while at room temperature all were filamentous, and two produced aerial hyphæ.

Pathogenicity.-All strains which have been tested have proved pathogenie for laboratory animals. White mice seem the most suseeptible.

Classification.-Cultures from the various eases have shown little uniformity. It is impossible to be certain that they represent distinct species as they are somewhat pleomorphic and change their morphology with varying cultural conditions and after long preservation on artificial media.

The most serious attempt to systematize their varying characteristics is that of Rieketts. ${ }^{13}$ He considered the seventeen strains on which he based his report all to be closely related speeies of one genus. He described three main types under which in spite of minor differences he grouped the various strains.

His elassification, is not however, satisfactory. Stober ${ }^{14}$ has observed the three types of agar growth to occur successively in the same strain.

A definite classification of these organisms is as yet impossible but there seem to be two types which the various strains more or less closely resemble.

I. The fermenting type described by Busse and called Cryptococeus hominis by Vuillemin, Brumpt and Castellani. These organisms resemble the true yeasts in their morphology and zymogenie properties.

II. The non-fermenting type deseribed by Gilehrist and called Cryptococeus Gilchristi by Vuillemin, Cryptococeus dermatitidis by Castellani and Myeoderma dermatitis by Brumpt. These types usually produce mycelium and reproduce by means of thallospores and conidia. They are related to the sporothrices and trichophyta deseribed below. There is no eorrelation between the type of organism and the type of lesion it produces.

\section{COCCIDIOIDAL GRANULOMA}

There is a group of cases resembling elosely systemic blastomycosis, the first observation of which was that of Wernicke and Posadas. ${ }^{15}$

\footnotetext{
${ }^{13}$ Ricketts, J. Med. Res., 1901, vi, 373.

${ }^{14}$ Stober, Arch. Int. Med., 1914, xiii, 509.

${ }^{15}$ Wernicke, $R$., Centralbl. f. Bakteriol., I, 1892, xii, 859.
} 
Their case presented numerous pea sized cutaneous tumors and clinically resembled mycosis fungoides. In the lesions they discovered intracellular spherical hyalin bodies which they thought to be protozoan cysts. These eysts were surrounded by hyalin eapsules and some of the larger forms contained a great number of the daughter cysts. Later Rixford and Gilchrist ${ }^{16}$ reported a similar case and since then a number of other cases have been reported, most of them from the San Joaquin Valley, California.

Ophuls described three clinical types: (1) with primary cutaneous lesions, and later generalization; (2) with primary pulmonary lesions and later generalization but no skin lesions; (3) with primary pulmonary lesions and secondary subcutaneous lesions.

The disease runs a more acute and severe course than blastomycosis and of twenty-four cases collected by MacNeal and Taylor, but two recovered. The cutaneous lesions consist of large rather painless granulomatous abscesses; there is usually marked lymphadenitis and the lungs, bones, liver, kidney, and meninges have been found to be involved. Histologically, the lesions both in man and in experimentally infected animals very closely resemble those of tuberculosis.

\section{Coccidioides Immitis}

(Oidium coccidioides, Ophuls; Mycoderma Immite, Brumpt)

The distinction between this parasite and that of blastomyces is recognized by Ophuls, ${ }^{17}$ Wolbach $^{18}$ and MacNeal, ${ }^{19}$ but no typical cases have been described by Europeans. The parasite seen in the tissues resembles the blastomyces, but does not show buds and reproduces by the formation of endopores. These appear as a mass of minute round bodies_each of which may be capsulated-within the membrane of the parent cell. The parasites vary greatly in size and some are much larger than those usually found in blastomycosis, reaching 50 microns in diameter.

Cultures.-The colonies appear in surface plants in from two to seven days as small slightly raised disks distinctly penetrating the media. Older cultures become covered with a dusty white layer

\footnotetext{
${ }^{16}$ Rixford and Gilchrist, Johns Hopkins Hosp. Reports, 1896, I.

${ }^{17}$ Ophuls, W., J. Exper. M., 1905, vi, 443.

${ }^{18}$ Wolbach, J. M. Res., 1904, N. S. viii, 53.

${ }^{19}$ MacNeal, W. J., and Taylor, R. M., J. M. Res., 1914, xxv, 261.
} 
of aerial hypha. The cultures become brown with age. In broth they grow as a fluffy mass which sinks to the bottom. Sugars are not fermented. Gelatin is slowly liquefied and milk slowly peptonized.

Morphology.-In culture the spherical bodies immediately throw out filaments 2 to 8 microns in diameter which are branched and septate. In older cultures they develop large chlamydospores which resemble the forms seen in culture and also conidia which are usually arranged in chains at the end of the hyphæ. In anaërobic cultures in Noguchi's ascitic agar, rabbit kidney medium, MacNeal and Taylor observed the formation of endospores like those found in the lesions. The organism is pathogenic for dogs, rabbits and guinea-pigs.

Cooke $^{20}$ has studied the immune reactions in a human case of this disease and found precipitins which reacted up to a 1:160 dilution of the serum against extracts of the cultures. The serum did not react with extracts of cultures of blastomyces. He was unable to obtain complement fixation or positive skin reactions with his extracts.

\section{THRUSH}

Thrush is a localized disease of the mouth, occurring most frequently in children suffering from malnutrition, but also in cachectic adults. It is characterized by the development of creamy patches on an area of catarrhal inflammation, usually on the tongue.

\section{Monilia Albicans}

(Oidium albicans, Robin; Saccharomyces albicans, Rees; Endomyces albicans, Vuillemin)

The microorganism which causes thrush was first described by Langenbeck in 1839. It is found abundantly in the false membrane eovering the lesion where it appears as a mass of simple or branched mycelial filaments made up of irregular units of about four by twenty microns. Oval cells of somewhat larger diameter are also found, attached to the ends of the filaments, or lying free and throwing out buds. 
The parasite grows readily on all ordinary media. On agar it forms a creamy layer, in broth a floceulent deposit. It forms gas on certain sugars. Most strains do not liquefy gelatin or clot milk. The morphology of the organism has been most thoroughly studied by Roux and Linoissier. Commonly it develops as a mass of oval cells reproducing by budding but often, especially in fluid media forms filaments like those seen in the lesions, with terminal or lateral spores. Large globoid chlamy-

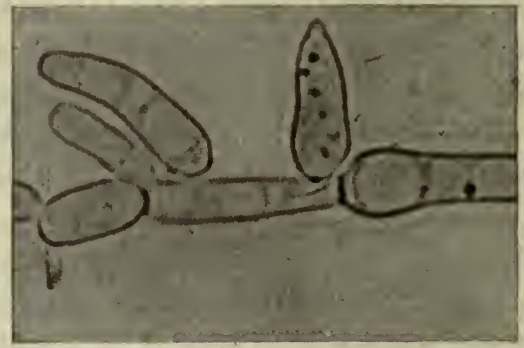

Fig. 112.-Thrush. Oidium albicans, unstained. (After Zettnow.)

dospores at the end of short lateral branches are also found. These characteristies have led most authors to call the parasite a monilia.

Monilia is a rather ill-defined genus of hyphomycetes in which are included yeast-like organisms which reproduce by blastospores but which also form filaments of short irregular units which are easily detached. The filaments give rise to large oval cells frequently appearing as short terminal chains which are called by some conidia, by others, blastospores. Castellani includes in this genus only organisms which ferment sugars with the formation of gas.

Several authors have reported the presence of ascospores in cultures of the thrush fungus and have classed it with the saccharomyces. Vuillemin found also endospores within the mycelial filaments. It seems probable that the disease is not always caused by the same organism. Fischer and Brebeck described two varieties, a largespored type with endospores which liquefied gelatine, and a smallspored type, without endospores which did not liquefy. Castellani concludes that several species of moniliae and also members of other genera may cause thrush. On the other hand Fineman ${ }^{21}$ studied five strains from thrush and found them identical. They were however indistinguishable from eight strains found incidentally

${ }^{21}$ Fineman, B. C., Jour. Inf. Dis., 1921, XXVIII, 185. 
in throat cultures from diphtheria suspects. All produeed acid and gas on dextrose, levulose and maltose; acid only on galactose and saccharose; and failed to ferment lactose and mannite. ${ }^{22}$

\section{SPRUE}

Sprue is an important disease of subtropical countries which is characterized by progressive wasting with profuse anemia and a white frothy diarrhea. There is an inflammation of the entire intestinal tract and the lesions on the tongue are frequently characteristic.

\section{Monilia Psilosis}

Ashford ${ }^{23}$ has made an elaborate study of cases occurring in Porto Rico. He isolated from the tongue and stools of two hundred patients an organism which he calls monilia psilosis. This is apparently identical with the monilia enterica of Castellani.

Ashford's monilia is a large round organism 4 to 7 microns in diameter with a granular and usually vacuolated protoplasm. On Sabouraud's agar it grows as a faintly greenish ereamy elevated mass with mycelium which usually penetrates the medium.

In gelatin it invariably produces hyphæ which spread laterally from the stab and give the growth an appearance which Ashford describes as that of an inverted Christmas tree. Monilia Psilosis produces acid and gas on dextrose, levulose, maltose and usually on saccharose but does not ferment lactose nor mannit. The fungus turns milk faintly alkaline without further change and does not liquefy gelatin.

On passage through laboratory animals (rabbits and guineapigs) it produces a systemic mycosis and gradually increases in virulence. With these passage strains Ashford could produce stomatitis and diarrhea by feeding.

The etiological relationship of moniliae to sprue is not universally accepted. Castellani has isolated six different species from the stools typical cases. He believes that they are not the primary cause of the disease, though they may be responsible for the frothy diarrhea.

${ }^{22}$ Roux and Linoissier, Arch. de méd. exp. et d'anat. path. 1890, II, 62; Compt. rend. de l'acad. d. se., 1889, 109, 752.

${ }^{23}$ Ashford, B. K., Am. J. Med. Sci., 1917, cliv, 159. 


\section{OTHER YEAST-LIKE PARASITES}

Other diseases caused by members of the yeast family have been reported by a number of observers. Tokishige ${ }^{24}$ found a very minute type in a skin disease, pseudoglanders, occurring among horses in Japan. Brumpt states that similar cases have been found in many countries and that the infection may attack man.

According to Mesnil, ${ }^{25}$ the histoplasma capsulatus of Darling, ${ }^{26}$ which he found in three cases of splenomegaly in Panama, is closely related to the above parasite and should be classed as a cryptococcus.

The fact that blastomycetes have frequently been found in tumor tissue has led several Italian observers ${ }^{27}$ to assume an etiological relationship between these microorganisms and malignant growths. Absolutely no satisfactory evidence in favor of such a belief has been advanced, however, and the yeasts in these conditions must be regarded as purely fortuitous findings.

In considering the possible origin of blastomycetic infections in animals and man, it is, of course, important that we should have some knowledge as to the pathogenic properties of yeasts met with and handled in daily life.

Rabinowich ${ }^{28}$ has investigated the pathogenic properties of fifty different speeies of yeast and among them found only seven varieties that had any pathogenicity for rabbits, mice and guinea-pigs. In most of those successfully inoculated the disease produced in laboratory animals had but very little resemblance to blastomycetic infectious conditions observed in man.

\section{SPOROTRICHOSIS}

Sporotrichosis is a chronic infection usually limited to the skin, the subcutaneous tissues, and lymphaties, oeeasionally involving the muscles, bones and joints-and exeeptionally the viscera. The lesions resemble closely syphilitic gummata and in typical cases occur in

${ }^{24}$ Tokishige, Centralbl. f. Bakteriol., 1896, i, 19.

${ }^{25}$ Mesnil, Quoted by Brumpt, loc. eit.

${ }^{20}$ Darling, S. T., J. Exper. Med., 1901, 11, 515.

${ }^{27}$ San Felice, Centralbl. f. Bakteriol., I, 1902, xxxi, Ztsehr. f. Hyg., 1895, xxi, 1895, xviii.

${ }^{28}$ Rabinowich, Ztsehr. Hyg., 1895, xxi. 
a chain extending up the arm, connected by thickened lymphatic vessels. They slowly soften and ulcerate.

The first cases described were those of Schenck ${ }^{29}$ and of Hektoen and Perkins ${ }^{30}$ in this country. It is a rare infection here, but twenty-eight cases being reported up to 1912. ${ }^{31}$ In France and Switzerland it is relatively common and our present knowledge of the disease is based ehiefly on the work of de Beurmann and Crougerot. $^{32}$ It may be caused by several species of hyphomycetes belonging to the genus sporotrichum.

\section{SPOROTRICHUM}

It is difficult and often impossible to demonstrate the parasites by direct examination. When found in smears of the pus, or in sections of the lesions, they appear as oval or cigar-shaped cells varying in length from 10 to 2 and in breadth from 3 to 1 microns. These are frequently within large mononuclear phagocytes. The parasites may be demonstrated by clearing the pus with 40 per cent $\mathrm{NaOH}$ or by staining with thionine or other basic stains. They are Gram-positive.

Cultural Charasteristics.-As a rule the organism can be found only by making cultures from the pus or from the bloody fluid aspirated from firmer lesions. Tubes of Sabouraud's test medium or of 4 per cent glucose agar should be heavily inoculated on the surface and incubated at room temperature. Taylor ${ }^{33}$ recommends glycerine-glucose-agar to which acetic or eitric acid (1-1500) is added. The colonies appear in four days or more as minute gray flecks, soon surrounded by a delicate fringe. The centers as they enlarge become raised and wrinkled and darken to a buff, or chocolate, or in some species to a black color. In flask cultures they may attain a diameter of $10 \mathrm{~cm}$. and more. At the periphery is a smooth flat zone with delicate radiating outgrowths, which, if they reach the side of the tube, grow upward along the dry glass. The surface is usually hard and glistening but in old cultures may show hairy or powdery outgrowths from the surface.

The sporothrix grows on media of very simple composition but more

${ }^{29}$ Schenck, B. R., J. Hopkins Hosp. Bull. 1898, ix, 286.

3o Hektoen, L., and Perkins, C. F., Jour. Exp. Med., 1900, V, 77.

${ }^{31}$ Hamburger, $W$. W., Jour. Am. Med. Assn., 1912, LIX, 1590. (Bibliography.)

${ }^{22}$ de Beurmann and Gougerot, Les Sporotrichoses, Felix Alcan., Paris, 1912.

ss Taylor, Kenneth, Jour. Am. Med. Assn., 1913, LX, 1142. 
luxuriantly on those containing sugar. On glueose broth it forms a thick membrane. It liquefies glucose gelatine but does not elot or digest milk. On hexoses it forms lactic acid but does not ferment mannite or dulcite. Species vary in their action on disaccharides.

Morphology.-This is best studied in hang drop cultures. The growth is made up of interlacing, branching septate hyphæ. These are delicate (about 2 microns) and of uniform diameter. The spores

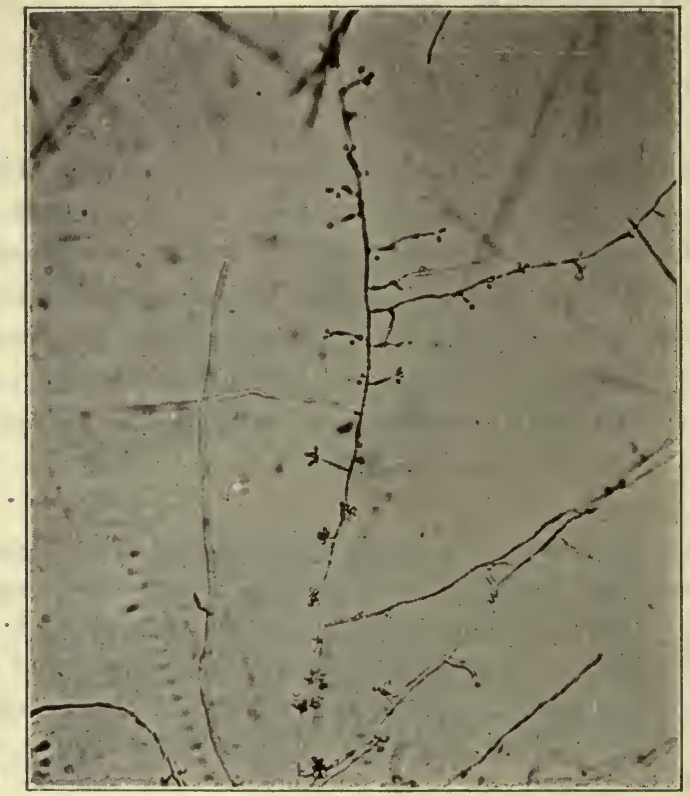

Fig. 113.--Sporotrichum Beurmanni in Hang Drop (Hopkins).

are oval or pear-shaped (about 3 by 4 microns) and are found on any part of the hyphæ to which they are usually attached by short sterigmata. In de Beurmann's species they are very numerous and form thick clusters at the tips of the hyphæ or sheaths along their course. Chlamydospores are also found.

Species.-Sporotrichum Schencki is the species found in most American cases. The eultures are white or slightly brownish. They ferment lactose but not saccharose. The spores are not numerous and have no sterigmata.

Sporotrichum Beurmanni is the eommonest species in France. The cultures soon darken to a chocolate brown. They ferment saccharose but not 
lactose. Spores are very numerous and often provided with short sterigmata.

Five other pathogenic varieties are listed by de Beurmann and there are many saprophytic species. Wolbach ${ }^{34}$ has described a species isolated from an arthritis of the knee which he named Sp. Councilmanni.

Pathogenicity.-Spontaneous infections due to the sporotrichum Beurmanni have been observed in rats, dogs and horses. Experimentally cultures have been shown to be virulent for rats and other laboratory animals.

The pathogenic species seem capable also of saprophytic existence. De Beurmann recovered his species from the normal throats of patients with sporotrichosis and of others who had recovered from the disease. Gougerot found in oat grains, and in other plants sporothrices which he regarded as identical with the human species and which were virulent for rats.

Immunity.-Widal and Abrami ${ }^{35}$ found that suspensions of sporothrix spores were agglutinated by the sera of patients in dilutions 1-200 or above and have used this reaction in diagnosis. They also obtained fixation of complement. Others report that patients give skin reactions with eulture extracts. In human eases there is no evidence of the development of an immunity but in animals successful immunization, both active and passive, has been reported.

\section{DISEASES OF THE RINGWORM GROUP}

There is a group of common and relatively trivial fungus infections called dermatomycoses. In it are included a number of diseases clinically distinct, all of which are, however, characterized by the fact that the invasion of the parasite rarely penetrates deeper than the epidermis and its appendages - the hair and nails. The fungi causing these infections are known as dermatophytes. They are filamentous organisms resembling in structure the hyphomycetes, in which group they are usually placed.

Brumpt, Castellani and others prefer to group them with the ascomyeetes. This decision is based partly on their general struetural resemblanee to these higher fungi but ehiefly on the observation of Matruchot

3t Wolbach, Sisson and Meier, Jour. Med. Res., 1917, CXXXVI, 337.

${ }^{35}$ Quoted by de Beurmann and Gougerot, loc. eit. 
and Dassonville of ascospore formation in Eidamella spinosa, a parasite isolated from a dermatomycosis of the dog. In no other member of the group, however, have ascospores been found.

The fact is that ringworm-like diseases may be caused by various fungi which show little resemblance to each other and that the dermatophytes as a group are defined not by any common botanical characteristies but by the type of lesions they produce.

Our knowledge of the dermatophytes dates from Schoenlein's discovery of the favus fungus in 1839. Two years later Gruby discovered the fungus of ringworm and distinguished between the large and small spored types. Since then many workers have contributed to our knowledge of these parasites but by far the most thorough and extended investigation has been made by Sabouraud. ${ }^{36}$

Cultural Characteristics.-The more important varieties of dermatophytes have been cultivated and show certain common characteristics. All of them grow as leathery masses of closely interwoven hyphæ. From a point of inoculation on solid media they spread out symmetrically over the surface at the same time sending down numerous short branches which penetrate the substrate and bind the growth firmly to it. As the membranous disc extends peripherally the central mycelium continues to grow increasing in thickness and forcing the less adherent portions upward. This produces on the surface a series of humps and ridges, with corresponding hollows and grooves on the under side, which often form striking geometrical patterns. The surface may be smooth and hard but most species sooner or later develop a duvet-a covering of aerial hyphæ which according to their length give the surface a powdery or velvety or hairy appearance. The majority develop a yellow or brown pigment and some are characterized by brilliant red and violet colors which appear late and are most marked in those portions of the membrane which are raised above the surface of the medium.

The rapidity of growth varies greatly in the different species but their evolution is always a matter of weeks. Some varieties such as the microspora attain a diameter of ten centimeters or more. Others do not extend beyond one or two centimeters from the center, but may pile up a mass a centimeter in thickness.

${ }^{86}$ Sabouraud, R., Les Teignes, Masson et Cie., Paris, 1910. 
On broth they usually form a thick membrane which spreads over the surface, but if the fragment planted sinks it develops 'only a delicate mesh of filaments in the bottom. On potato the growtl is less vigorous and often moist. Gelatin is liquefied by most strains. Glucose and mannite are usually completely consumed but no gas is formed and the medium is not acidified. Lactose, saccharose and maltose are less favorable to growth. Like many other fungi the dermatophytes grow well on the simpler synthetic media without peptone or protein, if glucose be added.

The optimum reaction is somewhat more acid than that for bacteria, about $\mathrm{P}_{\mathrm{H}} 7.0$; but they develop readily throughout a range of reaction from 4.0 to 8 and above. Although obligate aërobes, they will grow feebly with scanty oxygen supply as in the depth of a broth culture. They develop well at room temperature and most species also in the incubator.

Morphology.-Morphologically as has been said there is little that characterizes the ringworm fungi as a group. In the infected hairs or skin, they appear as masses of spores or as filaments, the latter often consisting of chains of spore-like cells. In culture the growth is made up of branching hyphæ which are always septate but may be coarse or delicate, straight or crooked, cylindrical or irregularly bulging. Under favorable conditions most species produce conidia. Chlamydospores are far more common and arthrospores are also found. From the common molds which they somewhat resemble these parasites are distinguished by the absence of ascospores or of the specialized conidiophores such as are found in the aspergilli and penicillii. These characteristics do not, however, serve to distinguish them from other hyphomycetes.

Individual species do develop in culture peculiar spores and mycelial structures which help to distinguish them. The following are some to which Sabouraud has given descriptive names:

Morphological Definitions.-Clubs.-These are swollen mycelial tips which vary greatly in size. They are not markedly differentiated from the hyphae which bear them but when occurring in great numbers as in the achorion of favus they present a striking and characteristic appearance.

Fuseaux.-These are large elongated chambered bodies considered as chlamydospores by some, by others as specialized conidia. Sabouraud applies this name to two different types, one a lenticular spore with a tapering pointed tip and thick doubly contoured wall, covered with warty or hairy outgrowths, the other a blunt club-shaped spore with thin smooth walls. Both are divided into segments by parallel transverse septa.

Conidia.-These are irregular in shape, size, and arrangement. In the 
microspora they are attached to the fertile hyphae by a flattened facet so that they resemble abortive branches (Acladium type). In the tricophyta they are more frequently attached by a pointed tip (Botrytis type). Often they are scattered irregularly along the hyphae, but oceasionally show a characteristic arrangement. Groups of conidia formed along the sides of an unbranched terminal hypha Sabouraud calls thyrses, larger groups born on branched conidiophore clusters (grappes).

Pectinate bodies are swollen and usually curved ends of hyphae which give off a row of abortive branches from one side-the structure vaguely resembling a comb.

Spirals.-These are simply convoluted hyphae which may take all the forms of a tendril from a spirillum-like form to a close set coil.

Nodular organs.-These are chains of large rounded cells knotted together into small dense masses suggesting the sclerotia of higher fungi.

Pleomorphism.-The gross appearance and microscopic structure of the dermatophytes varies greatly with the cultural conditions. After a period of growth on sugar containing media they seem to undergo a permanent change, ceasing to produce conidia and developing a thick covering of very long aerial hyphæ. This gives to these altered cultures a smooth white downy appearance. Sabouraud refers to cultures of this type as Pleomorphic using the term here in a special sense. The altered forms of different species resemble each other closely so that the identification of a culture by its gross or microscopic appearance is often almost impossible once this change has taken place.

Pathogenicity.-The ringworm fungi are found with such regularity and in such profusion in many of the human lesions as to leave little doubt as to their causative relationship to the disease. Many of the same species are found, too, in similar lesions of domestic animals. These latter will also induce lesions in guineapigs with more or less regularity if culture fragments are inserted in the skin. Such experimental lesions are often transitory but lesemble somewhat the spontaneous disease. Other species such as the Microsporon Audouim and Epidermophyton inguinale, mentioned below, although found abundantly in characteristic human lesions, have not been found in animals and are innocuous when experimentally injected.

Concerning the power of the dermatophytes to produce systemic disease we have little information. Their entire lack of invasive power in the spontaneous infections is quite striking. The isolated reports of Sabrazes 
and of Stavino on the production of tuberculoid lesions by intravenous injections of cultures of these fungi have little significance in view of the fact that many types of foreign bodies produce similar results when so introduced.

Immunity.-Jadassohn ${ }^{37}$ has noted that patients who have recovered from deep ringworm infections seem immune from subsequent attacks. A number of experimental observations especially those of Bloch and Massini ${ }^{38}$ and of Kusunoki ${ }^{39}$ bear this out. It appears that animals which have been infected with the more virulent types which produce suppurative lesions resist reinoculation and that the protection is valid against other species than those first injected. On the other hand both clinical and experimenta] observations show that infections with less virulent species induce no immunity.

All attempts to immunize animals by the injections of killed cultures and extracts have failed. The reports ${ }^{40}$ on the favorable therapeutic action of vaccines are inconclusive on account of the variable course of the disease. Plato $^{41}$ was able to produce in patients with suppurative ringworm both cutaneous and generalized reactions by the injection of extracts of trichophyton cultures. Skin reactions have also been obtained in animals and seem to be nonspecific in regard to the species of fungus concerned but are, as a rule, seen only in animals with those more severe infections which confer immunity. Kolmer and Strickler ${ }^{42}$ report that serum from ringworm cases gives complement fixation with extracts of cultures but that the reactions were again not highly specific as to species.

Methods of Examination.-The demonstration of the spores and mycelium in lesions is most readily made by placing the infected hairs or scrapings from the skin in caustic soda or potash and covering with a cover slip. The alkali renders the hair and epidermis transparent but does not attack the fungus and renders it easily visible. (A satisfactory solution for this purpose is a mixture of equal parts 30 per cent aqueous sodium hydroxide and glycerin.)

It is possible to stain the parasites with borax methylene blue and by modifications of Gram's method, but none of the staining methods suggested

${ }^{37}$ Quoted by Sabouraud.

${ }^{3 s}$ Bloch, B., and Massini, R., Ztschr. f. Hyg., 1909, lxxiii, 68.

${ }^{39}$ Kusunoki, F., Arch. f. Dermatol. u. Syph., 1912, exiv, 1.

40 Strickler, Jour. Am. Med. Assn., 1xv, 225.

${ }^{41}$ Reported by Neisser, Arch. f. Dermatol. u. Syph., 1902, Ix, 65.

"Kolmer and Strickler, Jour. Am. Med. Assn., 1915. 
have proved as satisfactory for diagnosis as the simple treatment with caustic soda. The process of clearing may be hastened by gently heating the slide.

Cultivation.-Cultures may be obtained from the lesions by distributing fragments of the infected epidermis or hairs over the surface of agar slants. Four per cent glueose agar or Sabouraud's test medium mentioned below are suitable for this purpose but cultures can also be obtained on plain agar. The chief difficulty is to avoid overgrowth of the parasites by bacteria and molds. The ordinary plating procedures are inapplicable. Skin fragments may be soaked for a few minutes, and hairs for hours, in 95 per cent alcohol before planting without killing the fungi. Increasing the acidity of the media to $P_{H} 5.0$; adding to it 1 part in 80,000 of methyl violet, or $1-200,000$ brilliant green are of some assistance. Any of these procedures inhibit the bacteria considerably but they are of little avail against the molds. Main reliance must be placed on making a large number of plants from each case.

As the gross appearance of the cultures varies greatly with the cultural conditions, Sabouraud has suggested a standard test meäum on which cultures should be planted for the purpose of identification. This has the following composition:

\section{(Sabouraud's Test Medium)}

Maltose brute de Chanut................... $40 \mathrm{gm}$.

Peptone granulée de Chassaing................. 10 gm.

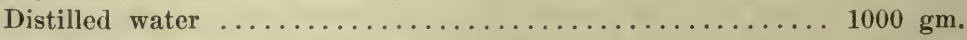

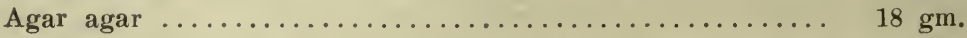

The mixture is dissolved in the autoclave, filtered through paper, poured into Erlenmeyer flasks to a depth of about $1 \mathrm{~cm}$. and sterilized once in the autoclave, allowing the temperature to rise slowly to 120 degrees. Sabouraud does not define the reaction of the medium and it cannot be exactly reproduced except by employing the brands of reagents which he suggests. It has, however, a reaction of about $\mathrm{P}_{\mathbf{H}} 5.5$ and similar though not identical results can be obtained by employing domestic peptone and malt extract and adjusting the reaction to this point.

In order to prevent the pleomorphic changes in the cultures, Sabouraud recommends that stock strains should be preserved on a medium of the following composition:

(Sabouraud's Conservation Medium)

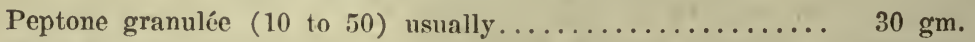

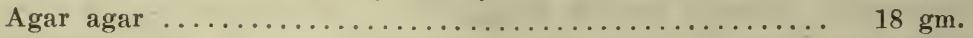

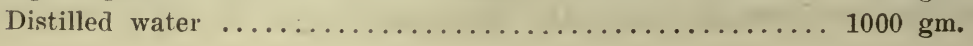


The study of the morphology of cultures is made difficult by the fact that in detaching pieces for observation from the tough mass of growth the spores are usually detached and characteristic mycelial structures deformed or broken up. Certain points ean be made out by examining such fragments mounted in water in an unstained condition but more information can be gained from the study of hang-drop eultures. These are made by placing a large drop of 4 per cent maltose broth on a sterile cover slip inoculating it with a fragment of the culture and inverting over a hollow slide with a deep concavity. This may be sealed with sterile oil or petrolatum.

\section{FAVUS}

Favus is a disease usually limited to the scalp but which occasionally attacks the glabrous skin and the nails. It is characterized by the formation at the mouths of the hair follicles of small yellow cupshaped crusts known as scutula. It is found in adults as well as in children and in this country occurs chiefly among immigrants from eastern and southern Europe where it is endemic.

\section{Achorion Schoenleini}

Favus of the scalp is caused by one species of fungus the Achorion Schoenleini. A scrutulum when crushed in alkali is found

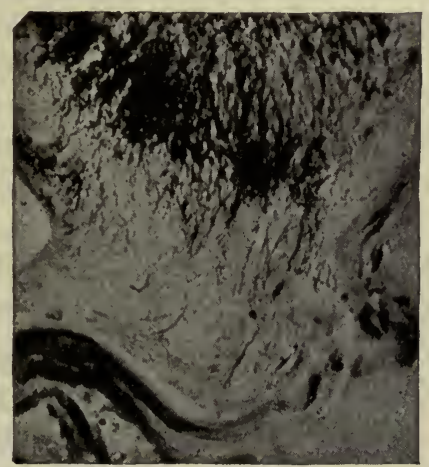

Fig. 114.-Achorion Schoenteinir. Section of favus crust. Stained by Gram (After Fraenkel and Pfeiffer.)

to be composed almost entirely of the fungus. The central portion is made up of rounded sporelike bodies of varying size without definite arrangement. Toward the periphery similar elements are 
seen strung out in filaments, and mixed with them hyphæ of thicker elongated elements with irregular contours. Within the diseased hairs are filaments, sometimes of cubical, sometimes of elongated elements. They differ from those found in the hairs of ringworm, chicfly in that cells of different sizes and forms are found in the same case.

The isolation of the achorion is rendered difficult by its frequent assiociation in lesions with pyogenic cocei and molds. It develops slowly on agar and the growth attains a maximum diameter of 2 to $3 \mathrm{~cm}$. in three to four weeks. It forms a remarkably tough brownish membrane with deep irregular folds the general outline being rounded upward toward the center. The

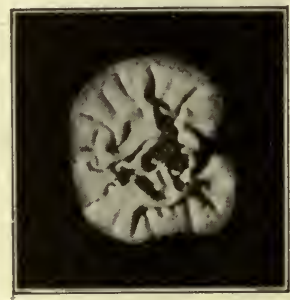

Fig. 115.-Achorion Schoenleini (Eight Weeks Culture on Sabouraud's Test Medium $\frac{1}{2}$ Natural Size. Hopkins.)

surface is waxy at first and later shows a whitish powdery duvet. In most strains however after long cultivation on artificial media subcultures grow more rapidly, attain a larger size and become covered with a white velvety layer of aerial hyphae (pleomorphism). On gelatin a small surface growth quickly fluidifies the entire tube but the achorion utilizes sugars slightly if at all.

Microscopically the growth is made up of crooked hyphæ of irregular contour often made up of chains of oval cells. Pearshaped conidia may be found scattered on the sides of the more delicate filaments but never in clusters. Chlamydospores are always numerous sometimes attached singly to the clubbed tips of hyphæ but more often occurring in chains in their course. These chains may be knotted into a small nodular mass.

About the periphery of a culture are numerous clubs either pearshaped or notched at the tip. These may occur singly (têtes de clous) or in clusters (chandeliers faviques).

Inoculation of fragments of scutula into the skin of guinea-pigs 
produces transitory ringworm like lesions. With some culture strains similar results have been obtained with others inoculation has been unsuccessful.

Other Species of Achoria.-Fungi have also been cultivated from favus-like lesions in animals. The Achorion Quinckeanum which frequently infects house mice has occasionally been found in human cases of favus of the body. In culture these so-called achoria show little in common with Schoenlein's parasite but resemble somewhat trichophyta of the gypseum group (V. infra). Plaut concludes that there is no reason for placing them in this genus except that they form scutula in lesions.

\section{RINGWORM OR TINEA}

The common form of this disease is tinea of the scalp which affects only children. It is highly contagious and in children's schools and institutions may assume epidemic proportions. The infection begins in the epidermis where it is often transitory but the parasite soon invades the hairs and there remains.

There is usually slight evidence of inflammation but some species of the ringworm fungi cause suppuration in and about the hair follicle. This may result in the formation of large indolent subcutaneous abscesses known as kerions.

Tinea of the body may occur secondarily to scalp lesions in children or as a primary infection at any period of life. The lesions often assume a circular form-the disease progressing at the periphery and clearing at the center. There may be only slight thickening and desquamation of the epidermis but usually there are superficial vesicles which quickly dry into crusts, and occasionally follicular pustules and kerions. Some species invade the nails.

Ringworm is also a common disease in domestic animals and human cases can often be traced to infection from these sources. The fungi of animal origin when they infect man either directly from a diseased animal or indirectly from another human case, usually produce more inflammatory lesions than do those species which affect man only.

\section{MIICROSPORON}

The small spored ringworm fungi are in this locality the commonest cause of ringworm of the scalp. The species of animal 
origin also attack the glabrous skin especially in children. In the epidermis of the diseased scalp they appear as curved branching hyphæ made up of elongated elements. The stumps of the diseased hairs are covered with a mosaic of small round spores of uniform diameter (about 2 microns) which completely envelops the hair. If the hair is crushed, mycelial threads are also seen which grow along the medulla. From these central filaments branches project out through the cortex and give rise to the sheath of spores.

Cultures.-These parasites are easily cultivated. They grow rapidly on agar producing large flat colonies which from the first are covered with a duvet of aerial hyphæ. At the center is a raised papilla and from this folds in the membrane radiate out. The color of the duvet varies from snow-white to deep buff and of the mem-

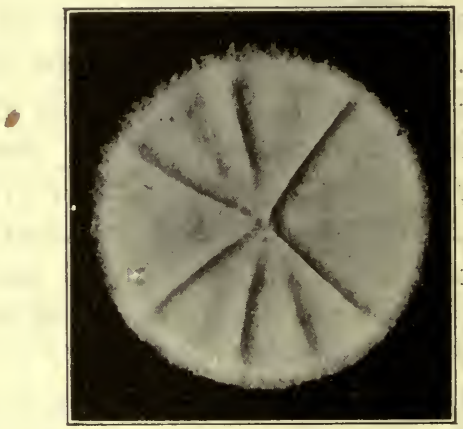

Fig. 116.-Microsponon Lanosum (Six Weeks Culture on Sabouraud's Test Medium $\frac{1}{2}$ Natural Size. Hopkins).

brane from buff to brilliant orange or even a russet brown. The pigment, which is well developed only on glucose-containing media, is diffusable. Gelatin is very slowly liquefied.

Morphology.-In young cultures, long straight coarse trunks radiate out from the center giving off frequent branches which later form an inextricable tangle of threads running in all direetions. Some terminal branches bear conidia attached to their sides by a flattened faects (Ácladium type). They also form chlamydospores, and fuseaux.

Sabouraud deseribes eleven species of microspora which he divides into two groups: one affecting man only and one affecting both animals and man.

Microsporon Audouini.-This is the type species of the former group. In gross appearance the eultures are distinguished by their slower growth, 
their velvety white or faintly buff duvet and less active pigment production. Microsporon Audouini produces only oceasional and atypical fuseaux but numerous chalmydospores which are frequently sub-terminal the hypha projecting beyond them like a spine. It also produces typical pectinate bodies. Animal inoculation has been unsuccessful. Closely allied to this species are: Microsporon umbonatum, M. tardum, and M. velveticum.

Microsporon lanosum (M. Audouini, var. canis).-This is the type species of the microspora of animal origin. It produces ringworm in the dog. The cultures grow very rapidly and on glucose are deeply pigmented. The duvet is long, shaggy, and in older growths assumes a dark tan color. The upper

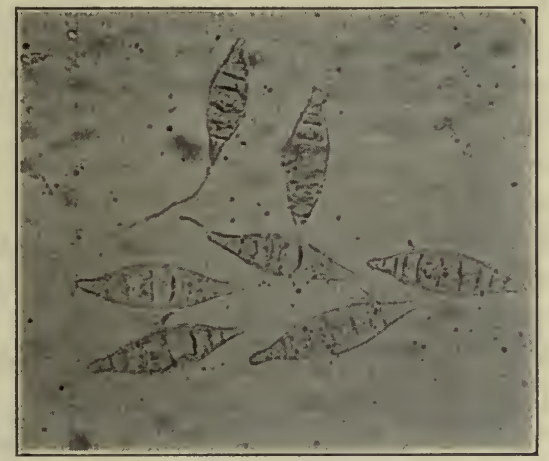

Fig. 117.-Fuseaux of Microsporon Lanosum $\times 200$.

surface is covered with a thick crop of typical lenticular fuseaux which alone serve to identify cultures as belonging to this group. Experimental lesions in the guinea-pig can be produced by the inoculation of cultures. Other members of this group are: Microsporon felineum, M. equinum, M. fulvum, M. villosum, M. pubescens, and M. tomentosum.

\section{TRICHOPHYTON}

The trichophyta, like the microspora, may produce ringworm of the sealp but they also produce lesions on the glabrous skin and nails. The genus is defined chiefly by the fact that its members produce lesions without seutula and appear in infected hairs as chains of spore-like elements. This alignment of the so-called spores (which are in reality short mycelial elements) distinguishes them from those of the preceding genus. In most trichophyta, too, the spores are large but in a few they are of about the same size as those of the microspora (3 microns). In eulture most species produce conidia attached to the hyphæ by pointed tips and frequently 
arranged in clusters. A few species (Tr. violaceum) which bear no conidia are, however, included in the genus. On artificial media the growths are much buckled and raised as compared to the flat cultures of the microspora. The cultures, however, vary so greatly among themselves in appearance that no description ean be given which would apply to the genus as a whole.

Classification.-Sabourand lists thirty species. His classification is based primarily on the appearance of the parasite in the infected hairs. Such a scheme has the disadvantage that it groups together forms which have diverse cultural characteristics and separates others which eulturally are similar. He divides them first into three divisions:

I. The Trichophyton endothrix in which the fungus is found only within the medulla of the hair.

II. The Trichophyton neo-endothrix in which some infected hairs show also a few filaments on their surface.

III. The Trichophyton ectothrix in which besides invading the substance of the hair the fungus proliferates actively on its surface.

Of this third division there are two groups:

A. The microid endothrices, of which the spore-like elements about the infected hairs are from 3 to 4 microns in diameter. They are again divided into two sub-groups, differing culturally: (a) The Gypseum Group and (b) The Niveum Group.

$B$. The megalospora, of which the rounded elements are from 6 to 8 microns in diameter. These Sabouraud again divides into: (a) A group forming downy cultures, and (b) A group in which the cultures are faviform.

The typical members of the Endothrix Group ( $\mathrm{Tr}$. erateriforme and Tr.' acuminatum) form small raised colonies less than $4 \mathrm{~cm}$. in diameter. They are white or slightly yellowish and are covered from the first with a short powdery or velvety duvet but show little tendency to pleomorphic change. They produce numerous conidia in thyrses or small clusters but show no other characteristic structures. Sabouraud describes many variants of these species which differ in the contour of their colonies or in pigment production. They are frequently found in mild ringworm of the scalp in France but so far we have not found them in New York.

Trichophyton violaceum, which is also an endothrix, is a very different organism. It develops slowly, forming small wrinkled colonies with a hard glistening surface, which slowly develop a black-violet color. The mycelium is made up of short crooked elements and no conidia are formed. It is found with relative frequency in tinea of the scalp, body and nails. A variant (Tr. glabrum) differs only in the absence of pigment.

Trichophyton cerebriforme, the type of the Neo-Endothrix, Group, resembles in culture the Tr. crateriforme. 
The Gypseum Group (Tr. astèroides, radiolatum, etc.) grow more vigorously forming large colonies up to $10 \mathrm{~cm}$. in diameter. The surface is powdery or plaster-like, but the parasites soon become pleomorphic and produce a long velvety suvet. They produce conidia in large clusters, rudimentary fuseaux, and numerous spirals. The lesions are inflammatory, with folliculitis and kerion formation. They cause ring-worm in horses.

The Niveum Group resemble the pleomorphic forms of the gypseum group. They form conidia only.

Trichophyton rosaceum (the type of the Downy Megalospora) forms a colony of medium size resembling a folded dise of white velvet. The deep portion develops a crimson or violet pigment which gives a rose tint as seen through the white duvet. It forms long thyrses and rudimentary fuseaux.

The Faviform Strains (Tr. ochraceum, Tr. album, etc.) resemble culturally the Achorion Schoenleini. They are grouped with the trichophyta chiefly because they form no scutula in lesions.

A description of all the individual species would exceed the scope of this chapter.

\section{ECZEMA MARGINATUM AND POMPHOLYX}

Eczema marginatum or ringworm of the groin is a common dermatosis. In the lesions Castellani and Sabouraud found a fungus to which the latter gave the name Epidermophyton inguinale. More recently Ormsby and Mitchell ${ }^{43}$ and others in this country have found the same fungus in eczematous lesions of the hands and feet and also in the vesicular eruptions in these regions formerly called pompholyx. Sabouraud has also found various species of trichophyta in palmar eczemas.

\section{EPIDERMOPHYTON INGUINALE}

\section{(Trichophyton cruris)}

In the epidermis the parasite is seen as long interlacing filaments made up of oblong or oval cells with double contours. It develops slowly in culture as a greenish buff colony with folds radiating from a central or slightly eccentric peak, attaining a diameter of perhaps two centimeters in a month. The surface at first is dry and powdery but on sugar media it quickly becomes pleomorphic, developing a 
thick white duvet. Related varieties are also found which form huge erateriform eolonies.

The cultures form no conidia but are readily identified by the innumerable blunt fuseaux which are born on aerial hyphæ. These are often in elusters which have been eompared to a hand of bananas. Older cultures show also many intercalary chlamydospores. The fuseaux are not found in cultures which have become downy.

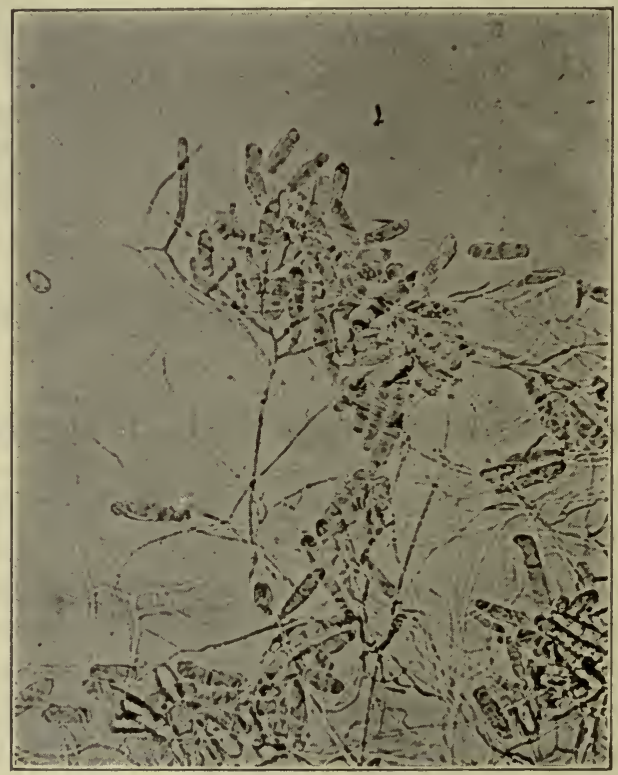

Fig. 118.-Fuseaux of Epidermophyton Inguinale $\times 200$.

Inoculations of epidermophyton cultures into men, dogs and guinea-pigs have been uniformly unsueeessful.

\section{TINEA VERSICOLOR}

\section{(Pityriasis versicolor, Chromophytosis)}

Tinea versicolor appears as large fawn-colored patehes on the covered parts of the body. These show slight superficial scaling but no evidenee of inflammation. The causative fungus is found abundantly in the horny epidermis where it seems to grow as a saprophyte. 
Microsporon furfur (Malassezia furfur). This fungus bears little resemblance to the microspora which cause ring-worm. It appears as septate filaments of very irregular contour 3 to 4 microns wide. They are usually unbranched but interlace, forming a meshwork in which are found masses of spore-like bodies. Most attempts to isolate the organism have failed. A few investigators ${ }^{44}$ have reported successful cultures but their work lacks confirmation.

\section{ERYTHRASMA}

Erythrasma is a superficial infection of the epidermis which produces round scaling patches usually located in the axillae or groins. There is no evidence of inflammatory reaction except a hyperemia which gives the lesions a characteristic red color. A parasite is found in the epidermis which appears as minute twisted threads which are easily broken up into elements about the size of bacilli. Cultures have been described by Michele and by Ducrey and Reale but according to others the organism cannot be cultivated. It has been given various names: Microsporon minutissimum, sporothrix minutissimum, and nocardia minutissimum.

${ }^{44}$ Kotjar, Ref. Baugartens Jahresbericht, 1892. 


\title{
SECTION VI
}

\section{BACTERIA IN AIR, SOIL, WATER, AND MILK}

\section{CHAPTER LI}

\author{
BACTERIA IN THE AIR AND SOIL
}

\section{BACTERIA IN THE AIR}

BACTERIA reach the air largely from the earth's surface, borne aloft by currents of air sweeping over dry places. Their presence in air, therefore, is largely dependent upon atmospheric conditions; humidity and a lack of wind decreasing their numbers, dryness and high winds increasing them. Multiplication of bacteria during transit through the air probably does not take place.

Apart from these considerations the presence of bacteria in air also depends upon purely local conditions prevailing in different places. They arè most plentiful in densely populated areas and within buildings, such as theaters, meeting halls, and other places where large numbers of people congregate. On mountain tops, in deserts, over oceans, and in other uninhabited regions, the air is comparatively free from bacteria. A classical illustration of this fact is found in the experiments which Pasteur carried out in his refutation of the doctrine of spontaneous generation. Tyndall also, in working upon the same subject, demonstrated this fact. From the surface of the ground and other places where bacteria have been deposited, they reach the air only after complete drying. It is a fact of much importance, both in bacteriological work and in surgery, that bacteria do not rise from a moist surface. From dry surfaces they may rise, but only when the air is agitated either by wind or by air-currents produced in other ways. In closed rooms, therefore, even when bacteria are plentiful and the walls and floors are perfectly dry, there is little danger of the inhalation of bacteria unless the air is agitated in some way. The most favorable condi- 
tions for the occurrence of many bacteria in air are the existence of a prolonged drought followed by a dry wind. Under such conditions, even the dark places and unlighted corners of streets and habitations are thoroughly dried out, and bacteria are taken up and carried about together with particles of dust. At such times the dangers from inhalation are much multiplied. By experiments made in balloons, it has been found that bacteria are plentiful below altitudes of about fifteen hundred feet and may be present, though much reduced in numbers, as high up as a mile above the earth's surface. The species of bacteria found in the air are, of course, subject to great variation, depending upon locality. Molds and spore-forming bacteria, being more regularly resistant to the effects of sunlight and drying than bacteria possessing only vegetative forms, are naturally more generally distributed.

Out of air thus laden with bacteria, they may again settle when the wind subsides and the air becomes quiescent. The process of settling, however, is extremely slow, since the weight of a bacterium is probably less than a billionth of a gram, and it may be held in suspension in air for considerable periods. Rains, snow, or even the condensation of moisture from a humid atmosphere, hastens this process considerably and large quantities of bacteria may settle out from air, in a comparatively short time, in ice chests, in operating rooms, or in other places in which much condensation of water vapor takes place.

The importance of the air as a means of conveying disease is still a problem upon which much elucidation is needed. The importance of this manner of conveyance in smallpox, in measles, in scarlet fever, and in other exanthemata, can not be denied. As regards the diseases of known bacterial origin, conveyance by air is of importance in the case of tuberculosis, where infection by inhalation may take place, and in the case of anthrax, where inhaled anthrax spores may give rise to the pulmonary form of the disease. The importance of air conveyance for any great distance in pneumonia, in influenza, in diphtheria, and in meningitis is by no means clear and requires much further study. The expulsion of bacteria from the lungs and naso-pharynx does not take place during simple expiration, since an air-current passing over a moist surface is not sufficient to dislodge microorganisms. Expulsion of bacteria in these conditions must take place together with small particles of moisture carried out in sneezing, coughing, or any forced expiration. 
The bacteria thus diseharged are then subject to the process of drying and often are exposed to direct sunlight for a considerable period before they are again taken up in the air.

The methods of estimating the bacterial contents of the air are not entirely satisfactory. The simple exposure of uncovered gelatin or agar plates for a definite length of time, and subsequent estimation of the colonies upon the plates, yield a result which is variable according to the air-currents and the degree of moisture in the atmosphere, and furnish no volume standard for comparative results. The methods which are in use at the present time depend upon the suction of a definite quantity of air by means of a vacuum-pump through some substance which will catch the bacteria. One of the first devices used for this purpose was that of Hesse, who sucked air through a piece of glass tubing, about $78 \mathrm{~cm}$. long and about $3.5 \mathrm{~cm}$. in diameter, the inner surface of which had been coated with gelatin in the manner of an Esmarch roll tube. This method is not efficient, since a large number of the bacteria may pass entirely through the tube without settling upon the gelatin. One of the most satisfactory methods at present in use is that in which definite volumes of air are sucked through a sand-filter. Within a small glass tube, a layer of sterilized quartz sand, about $4 \mathrm{~cm}$. in depth, is placed. The sand is kept from being dislodged by a small wire screen. After the air has been, sucked through the filter the sand is washed in a definite volume of sterile water or salt solution, and measured fractions of this are planted in agar or gelatin in Petri plates. The colonies which develop are counted. Thus, if two liters of air have been sucked through the filter, and the sand has been washed in 10 c.c. of salt solution, and 1 c.c. of this is planted, with the result of fifteen colonies, then the two liters of air have contained one hundred and fifty bacteria.

\section{BACTERIA IN SOIL}

Besides the normal bacterial inhabitants of the soil, bacteria reach the soil from the air, in contaminated waters, in the dejecta, excreta, and dead bodies of animals and human beings, and in the substance of decaying plants. It is self-evident, therefore, that the distribution of bacteria in soil depends largely upon the density of population and the use of the soil for agricultural or other 
purposes. Thus, bacteria are most plentiful in the neighborhood of cess-pools or in manured fields and gardens. Such conditions, however, may be regarded as abnormal. Even in uncultivated fields there is a constant bacterial flora in the soil which is of great importance in its participation in the nitrogen eycle, a phase of the bacteriology of soil which has been discussed in detail in another section.

There are, thus, regular and normal inhabitants of the soil which fulfill a definite function and may be found wherever plant life flourishes. In addition to these, innumerable varieties of saprophytes and pathogenic germs may be present, which vary in species and in number with lecal conditions. Numerous investigations into the actual numerical contents of the soil have been made. Houston ${ }^{1}$ found an average of 1,500,000 bacteria per gram in garden soil, and about 100,000 bacteria per gram in the arid soil of uncultivated regions. Fraenkel, ${ }^{2}$ in studying the horizontal distribution of bacteria in the earth, has found that they are most numerous near the surface, a gradual diminution occurring down to a depth of about two yards. Beyond this, the soil may be often practically sterile.

Pathogenic bacteria may at times be found in the surface layers, and these are often of the spore-bearing varieties. Most important among them from the medical standpoint are the bacillus of tetanus, of malignant edema, and the Welch bacillus. If a guinea-pig is inoculated subcutaneously with an emulsion of garden soil, death will result almost invariably with enormous bloating and swelling of the body due to gas production. This is due to the fact that the spore-bearing, gas-producing anaërobic bacilli are commonly present and are actively pathogenic for these animals. The frequent occurrence of tetanus in persons sustaining wounds of the bare feet and hands in fields and excavations, is a matter of common knowledge. Anthrax, also, may be easily conveyed by soil in localities where animals are suffering from this infection. It is not probable that pathogenic germs which are not spore-bearers survive in the soil for any great length of time. Unless the soil is specially prepared by the presence of defecations or other organic material, the nutrition at their disposal is not at all suitable for their needs, since rapid decomposition of organic materials by saprophytes is always

${ }^{1}$ Houston, Report Med. Officer, I.ocal Govern. Bd., London, 1897

${ }^{2}$ Fraenkel, Zeit. f. Hyg., ii, 1887. 
going on in the upper layers. Furthermore, in the deeper layers the conditions of temperature and possibly oxygen supply are not at all favorable for the growth of most pathogenic bacteria. Within a short distance from the surface the temperature of the soil usually sinks below $14^{\circ}$ or $15^{\circ} \mathrm{C}$. An interesting series of experiments by Fraenkel $^{3}$ have demonstrated this point. This investigator buried freshly inoculated agar and gelatin cultures of cholera spirilla and of typhoid and anthrax bacilli at different levels, and examined them for growth after two weeks had elapsed. The anthrax bacilli hardly ever showed growth at a depth below about two yards, and cholera and typhoid developed colonies at these depths only during the summer months. Under natural conditions it must be remembered that, at these levels, suitable nutritive material is not found.

A consideration of practical importance in this connection is the possibility of infection by means of buried cadavers. An elaborate series of experiments has been carried out upon this subject in Germany, with results which demonstrate that the danger from the burial of persons dead of infectious diseases was formerly much exaggerated. Experiments ${ }^{4}$ usually failed to reveal the presence of cholera and typhoid bacilli within two to three weeks after burial, and tubercle bacilli were never found after three months had elapsed. It was only in the case of sporulating microorganisms, such as the anthrax bacillus, that the living incitants could be found for as long as two years after burial. The dangers of infection of human being through the agency of soil, therefore, are chiefly those arising from the spore-bearing bacteria which are able to remain alive in spite of the unfavorable cultural conditions. It has been found by some observers, ${ }^{5}$ however, that, under special conditions, nonsporulating bacteria, more especially the typhoid bacillus, may remain alive in soil for several months. Although these bacteria, as well as those of cholera, diphtheria, etc., can not proliferate under the conditions found in the soil, the fact that they can remain viable for such prolonged periods in the upper layers suggests the possibility of danger from the use of unwashed vegetables, such as lettuce or radishes or other soil and sewage contaminated food products. The examination of soil for colon bacilli, while demon-

Fraenkel, Zeit. f. Hyg., xi, 1887.

"Petri, Arb. a. d. kais. Gesundheitsamt, vii.

'Firth and Horrocks, Brit. Med. Jour., Sept., 1902. 
strating the presence or absence of manure or sewage contamination, has no practical value, since colon bacilli are found in the dejecta of animals.

Examination of specimens of soil for their numerical bacterial contents is extremely unsatisfactory because the bacteria there found can hardly ever all be cultivated together under one and the same cultural environment. A large number are anaërobic, others again thrive at low temperatures, while again another class may require unusually high temperatures. When such examinations are made, however, specimens of the soil from the surface layer may be taken in a sterile platinum spoon. When taken from the lower levels, a drill, such as that devised by Fraenkel, may be used. This consists of an iron rod the lower end of which is pointed. Just above the point a movable collar is fitted. This collar has a slit-like opening. The rod beneath the collar has a deep longitudinal groove corresponding to the slit in the collar. A flange on the collar permits opening and closing of the groove while the instrument is below the ground. The drill is forced into the earth to the desired depth, the groove is opened and earth is forced into the chamber by twisting the rod. In the same manner the groove may be closed. The soil obtained in this way is taken out of the chamber and a definite quantity, say one gram, is dissolved and washed thoroughly in a measured volume of sterile water or sterile salt solution. Fractions of this are then mixed with the culture medium, plated, and cultivated aërobically or anaërobically as desired. 


\section{CHAPTER LII}

\section{BACTERIA IN WATER}

ALL natural waters contain a more or less abundant bacterial flora. This fact, combined with our knowledge that the incitants of several epidemic diseases and a number of minor ailments of a diarrheal character are water borne, gives the bacteriological investigation of water a place of great importance in hygiene. In nature, there are very few sources of water supply which do not contain bacteria of one or another description. While pathogenic bacteria are usually not present except in those waters which are directly contaminated from human sources, a thorough understanding of the quantitative and qualitative bacterial contents of all natural waters is necessary in order that we may intelligently gather comparative data as to the fitness of any given water for human consumption.

The gross appearances of water is rarely, if ever, an indication of its danger. The turbid waters of running streams in sparsely populated agricultural districts may be safe, while perfectly clear well waters subjected to the dangers of contamination from neighboring sinks or cess-pools may contain large numbers of pathogenic germs.

The diseases which are known to be more directly connected with water supply are typhoid fever and cholera.

Typhoid germs discharged from the bowel or from the urine of typhoid patients or convalescents may be carried by the sewage or from the neighboring soil into a river or lake and lead to infection of the population derivirg its drinking water from this source. There are a great many investigations on record in which severe typhoid epidemics have been traced to such sources.

In the case of cholera, where the germs are discharged from the bowels in enormous numbers, conveyance of the disease by water is even more apparent, and the discoverers of the cholera germ themselves, in their early work in Egypt and India, were able to isolate the bacteria from contaminated water supplies. 
In regard to the less clearly understood diarrheal diseases, dysentery, cholera infantum, etc., the direct relation to water supply has not been so definitely proven, and can be deduced only from the diminution of such infections after the substitution of pure water for the previously used impure supply. It is thus seen that water bacteriology is one of the most important branches of the science of hygiene, and has led, and is constantly leading, to enormous diminution of the death rate in all communities where an intelligent study of the conditions has been made.

The bacterial purity of natural waters, although dependent upon special and local conditions in relation to possible contamination, differs widely, according to the source from which such waters are derived.

Rain water and snow water are usually contaminated with bacteria by the dust which they gather on their way to the ground, and are especially rich in bacteria when taken during the first few hours of a rain or snow storm when the air is still dusty and filled with floating particles. During the later hours of prolonged storms, rain water and snow water may be comparatively sterile. Miquel, ${ }^{1}$ who made extensive experiments in France on the bacterial contents of rain water, found that in country districts, where the air is less dusty, rain water contained an average of about 4.3 bacteria to the cubic centimeter.

The bacterial counts of snow water are usually somewhat higher than those of rain.

The waters of streams, ponds, and lakes are usually spoken of as surface waters, and these of all natural supplies contain the largest number of bacteria. In each case, of course, the quantitative and qualitative bacterial flora of such waters is intimately dependent upon the conditions of the surrounding country, the density of the population, and the relation of these waters to sewage. It is also, and to no less important degree, dependent upon weather conditions, the influence of light and temperature, and the food supply contained within the waters in the form of decayed vegetation. In all such surfaee waters there is eonstantly going on a process of self-purifieation. The chief factor in this proeess is sedimentation. In stagnant ponds and lakes with but sluggish currents there is a constant sedimentation of the heavier particles, which gradually but steadily

${ }^{1}$ Miquel, Revue d'hyg., viii, 1886. 
leads to a diminution of the number of bacteria in the upper layers of the water. In rivers where sedimentation is to a certain extent prevented by rapidity of current, the effectiveness of such sedimentation is, of course, entirely dependent upon the speed of the current.

The influence of light in purifying surface waters is important chiefly in ponds, lakes, and sheets of water which expose a large surface to the sunlight, and where the surroundings are such that the sun has free access throughout the day. According to the researches of Buchner, ${ }^{2}$ the bactericidal effects of light penetrate through water to a depth of three feet.

The effects of temperature in purifying surface waters under natural conditions are probably not great. There is, however, a general tendency toward diminution of the bacterial flora as the temperature of such waters becomes lower.

The presence of protozoa in natural waters as purifying agents has recently been emphasized by Huntemüller, ${ }^{3}$ who claims that these organisms by phagocytosis greatly diminish the number of bacteria in any given body of water. It is self-evident that the number of bacteria in any of these waters is never constant, since all factors which tend to a diminution or increase in volume, such as drying up of tributary streams or the occurrence of heavy rains. would lead to differences of dilution which would materially change numerical bacterial estimations. The influence of rains, furthermore, may be a twofold one. On the one hand, heavy rain-falls, by washing a large amount of dirt into the rivers and lakes from the surrounding land, have a tendency to increase the bacterial flora. This influence would be especially marked in all bodies of water which are surrounded by cultivated land where manured fields and grazing-meadows supply a plentiful source of bacteria. On the other hand, in regions where arid, uninhabited lands surround any given river or lake, the rain would carry with it very little living contamination and would act chiefly as a diluent and diminish the actual proportion of bacteria in the water.

Another and extremely important source of water supply is that spoken of technically as "ground water." The "ground waters" include the shallow wells employed in the country districts, springs, and deep or artesian wells. The shallow wells that form the water

${ }^{2}$ Buchner, Arch. f. Hyg., xvii, 1895.

Huntemüller, Arch. f. Hyg., liv, 1905. 
supply for a large proportion of farms in the eastern United States are usually very rich in bacteria and are by no means to be regarded as safe sources, except in cases where great care is observed as to cleanliness of the surroundings. In such wells the filtration of the water entering the well may be subject to great variation according to the geological conditions of the surrounding ground. The proximity of barns and sinks may lead to dangerous contamination of such waters.

Examinations by various bacteriologists have shown that such wells frequently contain as many as five hundred bacteria to the cubic centimeter.

Perennial spring waters are usually pure. Examinations by the Massachusetts State Board of Health ${ }^{4}$ in 1901 showed an average of about forty bacteria per cubic centimeter. As sources of water supply for general consumption, however, springs can hardly be very important because of the insignificant quantities usually derived from them.

Of much greater practical importance are deep artesian wells, which, under ordinary conditions, are largely free from bacterial contamination.

Quantitative Estimations of Bacteria.-The quantitative estimation of bacteria in water is of necessity inexact, because of the difficulty of always securing fair average samples from any large body of water, and because of the large variations in cultural requirements of the flora present in them. All these methods depend upon colony enumeration in plates of agar or gelatin, preferably of both. For the sake of gaining some basis of comparison for results which, at best, can never be entirely accurate, an attempt has been made by the American Public Health Association ${ }^{5}$ to standardize the methods of analysis.

Water for analysis should always be collected in clean, sterile bottles, preferably holding more than 100 c.c. If water is to be taken from a running faucet or a well supplied with piping, it is important that it should be allowed to run for some time before the sample is taken, in order that any change in bacterial content occurring inside of the pipes may be excluded. It is obvious that in water pipes through which the flow is not constant, bacteria may

4 Mass. State Bd. of Health, 33d Annual Report for 1901.

' Fuller, Trans. Amer. Public Health Assn., xxvii, 1902. Report of Com. on Standard Methods of Water Analysis, Jour. Inf. Dis., Suppl. 1, 1905. 
find favorable conditions for growth and such a sample would not represent fairly the supply to be tested. When the water is taken from a pond, lake, or cess-pool, the bottle may be lowered into the water by means of a weight, or may be plunged in with the hand, great care being exercised not to permit contamination from the fingers to occur.

After the water has been collected it is important to plate it before the bacteria in it have a chance to increase. The changes taking place during transportation, even when packing in ice has been resorted to, have been found by Jordan and Irons ${ }^{6}$ to be considerable. It is imperative, therefore, that plating of the water, if possible, shall not be delayed for longer than one or two hours after collection.

Bacteriological Examination of Water--In describing the methods of bacteriological examinations of water, we adhere strictly to the recommendations of the Committee on Standard Methods of the American Public Health Association, ${ }^{7}$ taking the following paragraphs with slight changes from their report of 1915 :

It should be remembered that quantitative estimations of bacteria in water are of most value when repeatedly done and a "normal" for the particular water supply has been established, so that deviation from this "normal" can be easily recognized. Single isolated determinations may easily lead to error.

The following paragraphs are taken without change from the Public Health Association's report:

"Since gelatine does not give the total number of bacteria in the water, the committee has thought it wise to use agar incubated at $37^{\circ}$ C. as a standard medium. 'This adnits of counts in one day instead of two, and gives results on the kind of bacteria growing at blood temperature and therefore more nearly related to pathogenic types.

"Media.-The standard medium for determining the number of bacteria in water shall be nutrient agar. All variations from this medium shall be considered special media. If any medium other than standard agar is used, this fact shall be stated in the report.

"For general work the standard reaction shall be +1 per cent, but for long continued work upon water from the same source the optimum reaction shall be ascertained by experiment and thereafter adhered to. If the reaction used, however, is different from the standard, it shall be so stated in the report.

- Jordan and Irons, Reports of the Amer. Fub. Health Assn., xxv, 1889.

${ }^{7}$ Amer. P. H. A., Stand. Meth. Exam. Water and Sewage, 1915. 
"Procedure.-Shake at least twenty-five times the bottle which contains the sample. Withdraw 1 c.c. of the sample with a sterilized pipette and deliver it into a sterilized Petri dish, $10 \mathrm{~cm}$. in diameter. If there be reason to suspect that the number of bacteria is more than 200 per c.c., mix 1 c.c. of the sample with 9 c.c. of sterilized tap or distilled water. Shake twenty-five times and measure 1 c.c. of the diluted sample into a Petri dish. If a higher dilution be required proceed in the same manner, e.g., 1 c.c. of the sample to 99 c.e. of sterilized water, or 1 c.c. of the once diluted sample to 9 c.c. of sterilized water, and so on. In the case of an unknown water or a sewage it is advisable to use several dilutions for the same sample. To the liquid in the Petri dish add 10 c.e. of standard agar at a temperature of about $40^{\circ} \mathrm{C}$. Mix the medium and water thoroughly by tipping the dish back and forth, and spread the contents uniformly over the bottom of the plate. Allow the agar to cool rapidly on a horizontal surface and transfer to the $37^{\circ} \mathrm{C}$. ineubator as soon as it is hard. Incubate the culture for twenty-four hours at a temperature of $37^{\circ} \mathrm{C}$. in a dark, well-ventilated incubator where the atmosphere is practically saturated with moisture. ${ }^{8}$ After the period of incubation place the Petri dish on a glass plate suitably ruled and count the colonies with the aid of a lens which magnifies at least five diameters. So far as practicable the number of colonies upon the plate shall not be allowed to exceed 200. The whole number of colonies upon the plate shall be counted, the practice of counting a fractional part being resorted to only in case of necessity.

"It will be found advantageous to use Petri dishes with porous earthenware covers in order to avoid the spreading of colonies by the water of condensation. ${ }^{9}$

"Expression of Results.-In order to avoid fictitious accuracy and yet to express the numerical results by a method consistent with the precision of the work the rules given below shall be followed:

"Numbers of Bacteria per c.c.

\begin{tabular}{|c|c|c|c|c|c|c|c|c|}
\hline From & 1 & to. & 50 & Recorded & as & four & & \\
\hline 66 & 51 & & 100 & 6 & to & the & nearest & 1 \\
\hline 66 & 101 & & 250 & 66 & "6 & 6 & 6 & 10 \\
\hline “6 & 251 & & 500 & “6 & 66 & 66 & ، & 25 \\
\hline 66 & 501 & 66 & 1,000 & 66 & 66 & 66 & 66 & 50 \\
\hline 66 & 1,001 & “6 & 10,000 & “6 & 66 & 66 & 6 & 100 \\
\hline 66 & 10,001 & “6 & 50,000 & 6 & 66 & 66 & 6 & 500 \\
\hline 66 & 50,001 & & 100,000 & 6. & 6 & 66 & 6 & 1,000 \\
\hline "6 & 100,001 & “6 & 500,000 & 6 & 6 & 66 & 6 & 10,000 \\
\hline " & 500,001 & & $1,000,000$ & 6" & “6 & 66 & “ & 50,000 \\
\hline 66 & $1,000,001$ & 6 & $10,000,000$ & 66 & 6 & 66 & 6 & 100,000 ", \\
\hline
\end{tabular}

Qualitative Bacterial Analyses of Water.-Of far greater importance than quantitative analysis is the isolation of bacteria either

${ }^{8}$ Whipple, Tech. Quar., 1899, 12, p. 276.

- Hill, Jour. Med. Res., 1904, N. S., 8, p. 93. 
distinctly pathogenic, such as the cholera spirillum and the typhoid bacillus, or of other species probably emanating from contaminating sources, such as a B. coli. Unfortunately there are no reliable methods by which typhoid and cholera germs can be isolated from water with any degree of regularity or certainty. Although frequently the isolation of such organisms is possible, a negative result in these cases is by no means eliminative of their presence.

The isolation of typhoid bacilli from water is very difficult, chiefly because of the great dilution which contaminations undergo upon entering any large body of water. The difficulty of isolating typhoid bacilli, even from the stools of infected patients, makes it clear that such difficulties are enhanced when a considerable dilution of the excreta takes place. Furthermore, water is by no means a favorable medium for the typhoid bacillus. Russell and Fuller ${ }^{10}$ have shown that typhoid bacilli may die in water within five days, and it is unquestionable that the rate of increase of these bacteria is by no means equal to that of many other microorganisms for which polluted water at the temperature encountered in streams and lakes forms a much more favorable medium.

It is thus clear that even in infected waters the number of typhoid bacilli encountered can never be very great. ${ }^{11}$

A large number of methods for the isolation of the typhoid bacillus from water have been devised. Most of the media used are identical with those employed for the isolation of these bacteria from the stools. These media have been discussed in the chapter dealing with the typhoid bacillus. Success is rendered more likely if 10 c.c. of the water is first planted into lactose-bile in fermentation tubes holding 40 c.c. After forty-eight hours at $37.5^{\circ}$ there will be an enrichment of typhoid bacilli which can be then isolated by plating in the usual manner on Endo's medium, Conradi Drigulski or any of the other usual differential media.

A method which has proved useful in the hands of Adami and Chapin $^{12}$ is one which depends upon the phenomenon of agglutination. They attempt to agglutinate the bacilli out of liter samples of water by adding powerful agglutinating serum.

Vallet and others have attempted to precipitate typhoid bacilli out of water by chemical means. To two liters of water add 20 c.c.

${ }^{10}$ Russell and Fuller, Jour. Inf. Dis., Suppl. 2, 1908.

${ }^{11}$ Laws and Anderson, Rep. of Med. Officer, London County Council, 1894.

${ }^{12}$ Adami and Chapin, Jour. Med. Res., xl, 1904. 
of a 7.75 per cent solution of sodium hyposulphite and 20 c.c. of a 10 per cent solution of lead nitrate. When the precipitate has settled. the clear supernatant fluid is decanted and the precipitate dissolved in a saturated sodium hyposulphite solution. This clear solution is then plated. Willson ${ }^{13}$ has modified this method by adding to the water $0.5 \mathrm{gm}$. of alum to each liter. The supernatant fluid is removed and the precipitate plated.

The isolation of the vibrio of cholera is less difficult than that of B. typhosus, primarily because of the much greater numbers of these microorganisms discharged into sewage. The number of cholera spirilla in the excreta of cholera patients is enormously higher than is that of B. typhosus in the stools of typhoid-fever patients. It is not infrequent, therefore, that the source of a cholera infection may be directly traced to the water supply. Koch, ${ }^{14}$ the discoverer of the cholera vibrio, has indicated a method which has frequently found successful application.

To 100 c.c. of the infected water are added one per cent of pepton and one per cent of salt. This mixture is then incubated at $37.5^{\circ}$ C., and after ten, fifteen, and twenty hours, specimens from the upper layers are examined microscopically and are plated. The scum from the surface of such a medium may be plated on the starch agar of Stokes and Haechtel,${ }^{15}$ on which colonies of intestinal spirilla will appear pink and spreading.

Because of the great difficulties outlined above in isolating specific pathogenic germs from polluted waters, bacteriologists have attempted to form an approximate estimation of pollution by the detection of other microorganisms which form the predominating flora of sewage. Chief among these is B. coli. The isolation and numerical estimation of $\mathrm{B}$. coli in polluted water has been for a long time one of the criteria of pollution. This so-called colon test, however, should always be approached with conservatism and should never be carried out qualitatively only. Careful quantitative estimation should be made in every case.

B. coli in water is by no means always the result of human contamination, since this bacillus is found in great abundance in the

${ }^{13}$ Willson, Jour. of Hyg., v, 1905.

14 Koch, Zeit. f. Hyg., xiv, 1893.

${ }^{15}$ Stokes and Haechtel, see Report 1915 A. P. H. A., on Water Analysis. The medium is an agar with 5.5 grams agar, 5.0 meat extract, 10 Pepton and $8.5 \mathrm{NaCl}$ to liter to which is added 10 grams of soluble stareh. 
intestines of domestic animals. According to Poujol, B. coli does not even always point to fecal contamination, since this author was able to find the bacillus in the water of a number of wells where no possible contamination of any sort could be traced. Prescott ${ }^{16}$ explains this, as well as similar cases, by the fact that organisms of the colon group may occasionally be parasitic upon plants.

The opinions of hygienists are widely at variance as to the value of the colon test. While the discovery of isolated bacilli of the colon group may therefore be of little value, it is nevertheless safe to follow the opinion of Houston, ${ }^{17}$ who states that the discovery of B. coli in considerable numbers invariably points to sewage pollution, and that the absolute absence of $\mathrm{B}$. coli is, as a rule, reliable evidence of purity.

Rosenau states that a ground water should be condemned even if only a few colon bacilli are found, for, as he put it, "these bacteria have no business in a soil-filtered and properly protected well or spring-water." Surface waters, however, may easily contain a few colon bacilli without necessarily having been exposed to contamination by human forces. The limit of safety, Rosenau states, is one colon bacillus per c.c. If more are present the water should be regarded as suspicious. If more than 10 per c.c. are found the water must be regarded as dangerous and unqualifiedly condemned.

Presumptive Colon Tests.-For this purpose, a large number of methods have been devised. In examining sewage or other polluted waters in which the number of colon bacilli is comparatively large, the direct use of lactose litmus agar plates yields excellent results. The method advised by the American Public Health Association is as follows:

"Add the quantities of water or sewage to be tested to fermentation tubes holding at least forty cubic centimeters of lactose bile, ${ }^{18}$ incubate at $37^{\circ} \mathrm{C}$. and note the production of gas. The standard time for observing gas production is forty-eight hours. Small numbers of somewhat attenuated B. coli may require three days to form gas. Attenuated B. coli does not represent recent contamination and all $B$. coli not attenuated grows readily in lactose bile. No other organism except $\mathrm{B}$. Welchii gives such a test in lactose bile. B. Welchii is of rather rare occurrence in water, is of fecal origin, is almost invariably accompanied by B. coli, and the sanitary significance is the same.

16 Prescott, Science, xv, 1903.

${ }^{17}$ Houston, Rep. Medical Officer, Local Gov. Board, London, 1900.

18 Prescott, Science, xvi, 1902. 
"A comparison of the positive results obtained in the various dilutions of the water or sewage planted into the lactose bile gives a good idea of the relative amount of contamination in the various samples examined.

"Quantities of Water Tested.-For ordinary waters, $0.1,1.0$ and 10.0 c.c. shall be used for the colon test. For sewage and highly polluted surface waters, smaller quantities shall be used; and for ground waters, filtered waters, ete., the quantities shall be larger, if necessary to obtain positive results. The quantities shall vary preferably in the tenfold manner indicated. Single tests with quantities which give ordinarily a positive result or ordinarily a negative result are in themselves of but little account for quantitative determinations. The range in quantities studied shall be sufficient to allow the quantities needed for both a positive and a negative result to be recorded for each sample. When this is done, the results of several tests allow an approximate estimate of the number of B. coli per c.e."

The identification of colon bacilli so obtained should then be undertaken. The following table, again taken from the report of the A. P. H. A., will be of help: 


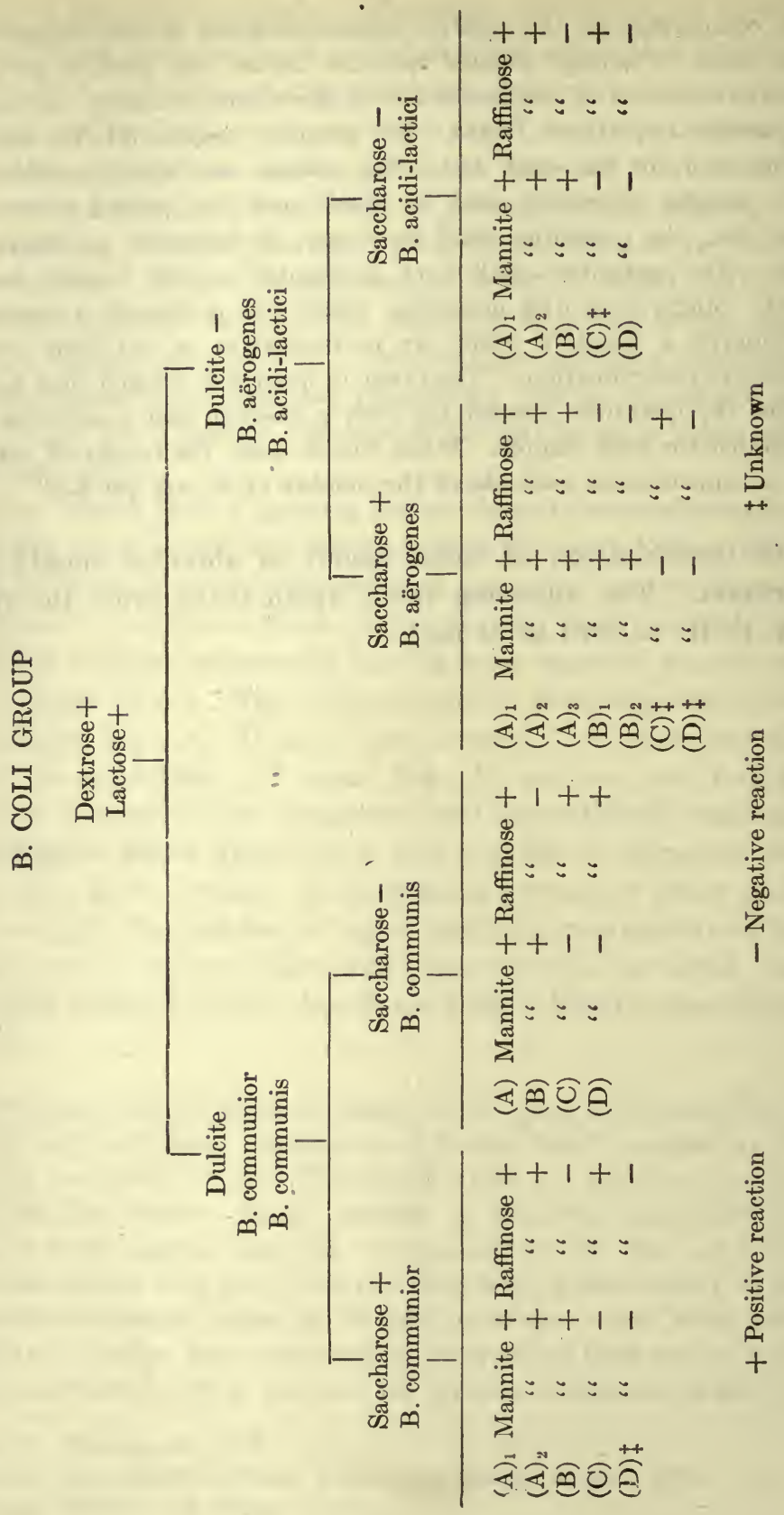




\section{CHAPTER LIII}

\section{BAC'TERIA IN MILK AND MILK PRODUCTS, BACTERIA IN THE INDUSTRIES}

\section{BACTERIA IN MILK}

THE universal use of cows' milk as a food, especially for the nourishment of infants, has necessitated its close study by bacteriologists and hygienists. It furnishes an excellent culture medium for bacteria and is, therefore, pre-eminently fitted to convey the germs of infectious disease. The many changes which take place in milk, furthermore, and which add or detract from its nutritive value, are due largely to bacterial growth and have been elucidated by bacteriological methods.

Within the udder of the healthy cow, milk is sterile. If pyogenic or systemic diseases of bacterial origin exist in the cow, the milk may, under certain circumstances, be infected even within the mammary glands. In the milk ducts and in the teats, however, even in perfectly healthy animals, a certain number of bacteria may be found. For this reason, even when all precautionary measures are followed, the milk as received in the pail is usually contaminated. As a matter of fact, the anatomical location of the udder and the mechanical difficulties of milking make it practically impossible to collect milk under absolutely aseptic conditions, and, under the best circumstances, from 100 to 500 microorganisms per c.c. may usually be found in freshly taken milk. Withdrawn under conditions of ordinary cleanliness, the bacterial contents of milk are considerably higher than this. After the process of milking, in spite of all practicable precautions, the chances for the contamination of milk are considerable; but even could these be eliminated, the bacterial contents of a given sample would ultimately rapidly increase because of the rich culture medium which the milk provides for bacteria. Whether large increases shall take place or not depends, in the first place, upon the temperature at which milk is kept, and, in the second place, upon the length of time which intervenes before 
its consumption. Though fresh milk possesses slight bactericidal powers, ${ }^{1}$ these are by no means sufficient to be of practical importance in the inhibition of bacterial growth. Kept at or about freezing-point, the bacterial contents of milk do not appreciably increase. At higher temperatures, however, a rapid propagation of bacteria takes place which, especially during the summer months, speedily leads to enormous numbers. In a ease reported by Park, ${ }^{2}$ where milk, containing at the first examination 30,000 microorganisms per eubic centimeter, was kept at $30^{\circ} \mathrm{C}$. $\left(86^{\circ} \mathrm{F}\right.$.) for twentyfour hours, the count at the end of this time yielded fourteen billions of bacteria for the same quantity.

It is of much importance, therefore, that the cleanliness of dairies, of eattle, and in the handling of milk should be reinforced by the utmost care in chilling and icing during shipment and before sale.

Because of its great importance, especially for the health of the children in large cities during the summer months, the milk question has, of recent years, received much attention from health officers. In the city of New York, the question has been made the subject of many careful studies by Park $^{3}$ and his associates. Commissions, working in Chicago, ${ }^{4}$ Boston, ${ }^{5}$ and other large towns, have placed the sale of milk under more or less exact bacteriological supervision. Park has determined that the milk, as sold in New York stores during the cold weather, not infrequently averages seven hundred and fifty thousand bacteria per cubic centimeter; during the hot summer months, the bacterial contents of similar milk not infrequently average one million and more, for the same quantity. ${ }^{6}$ In consequence of these and other researches, large dairies have introduced bacteriological precautions into their method of milk production. They have attempted the reduction of the bacterial contents of milk by serupulous cleanliness of the barns and of the udders and teats of the cow, by the elimination of diseased cattle, by sterilization of the vessels in which the milk is received, and of the hands of the milker; also by the immediate filtering and cooling of the milk and the packing of the milk eans in ice, where they

${ }^{1}$ Rosenau and McCoy, Jour. Med. Res., 18, 1908.

${ }^{2}$ Park, W. H., 'Pathogenic Bacteria,', New York, 1905, p. 463.

${ }^{3}$ Park, Jour. of Hygiene, 1, 1901.

"Jordan and Heinemann, Rep. of the Civic Federation of Chicago, 1904.

${ }^{5}$ Sedgwick and Batchelder, Bost. Med. and Surg. Jour., 126, 1892.

- Escherich, Fort. d. Medizin, 16 and 17, 1885. 
remain until delivered to the consumer. In consequence of such measures, it is possible for cities to be supplied with milk containing no more, and often less, than fifty thousand bacteria to the cubic centimeter. A standard of cleanliness has been established in various towns by the introduction of the so-called "certified milk," which, by the New York Milk Commission, is required to contain no more than thirty thousand bacteria per cubic centimeter. Great stress is laid upon such numerical counts simply in that they are approximate estimates of cleanliness. Most of the bacteria, however, contained in milk are non-pathogenic, and numbers much larger than the maximum required for certified milk may be present without actual disease or harm following its consumption.

The various species of bacteria which may be found in milk include almost all known varieties. Whether there are special, so-called milk bacteria or not is a question about which investigators have expressed widely differing opinions. It is probable that many of the species, formerly regarded as specifically belonging to milk, are there simply by virtue of their inhabitual presence in fodder, straw, or bedding, or upon cattle. It is likely, furthermore, that some of these species are found with great regularity because of their power to outgrow other species under the cultural conditions offered them in milk.

Under normal conditions, milk always undergoes a process which is popularly known as souring and curdling. This is due to the formation of lactic acid from the milk sugar and is the result of the enzymatic activities of several varieties of bacteria commonly found in milk. Most common among these bacteria is the so-called Bacillus lactis aërogenes, an encapsulated bacillus closely related to the colon-bacillus group. (See page 637.) The transformation of the lactose into lactic acid may oceur either directly by hydrolytic cleavage:

$$
\mathrm{C}_{12} \mathrm{H}_{22} \mathrm{O}_{11}+\mathrm{H}_{2} \mathrm{O}=4 \mathrm{C}_{3} \mathrm{H}_{6} \mathrm{O}_{3} ;
$$

or indirectly through a monosaccharid,

$$
\mathrm{C}_{12} \mathrm{H}_{22} \mathrm{O}_{11}+\mathrm{H}_{2} \mathrm{O}=2 \mathrm{C}_{6} \mathrm{H}_{12} \mathrm{O}_{6}=4 \mathrm{C}_{3} \mathrm{H}_{6} \mathrm{O}_{8} \text {. }
$$

Other microorganisms which may cause lactic-acid fermentation in milk are the so-called Streptococcus lacticus, the common pyogenic streptococcus, the Staphylococeus aureus, Bacillus coli communis and communior, and many other species. Most commonly concerned in 
lactic-acid production, however, according to Heinemann, ${ }^{7}$ are the two first-mentioned, Bacillus lactis aërogenes and Streptococcus lacticus. The secret of the regularity with which some of these bacteria are present in sour milk is probably found in the ability of these varieties to withstand a much higher degree of acidity of the culture medium than other species. In consequence, they are able to persist and develop when cultural conditions are absolutely unsuited to other bacteria.

Consequent upon acidification of the milk by lactic-acid formation, there is coagulation of casein. Casein precipitation, however, may also be due to a non-acid coagulation caused by a bacterial ferment. Casein precipitated in this way may be redissolved by a bacterial trypsin or casease, produced by the same or other bacteria, the milk again becoming entirely liquid, transparent, and of a yellowish color. The casein precipitated by lactic-acid formation, however, is never thus redissolved, because the high acidity does not permit the proteolytic ferments to act. ${ }^{8}$

Butyric-acid fermentation in milk, a common phenomenon, is also an evidence of bacterial growth. As a rule, it is produced by the anaërobic bacteria, and is a process developing much more slowly than other fermentations. A large number of bacteria have been described which are capable of producing such changes, the chemical process by which they are produced being, as yet, not entirely understood. It is probable that the process takes place after hycrolysis of the dissaccharid somewhat according to the following formula :

$$
\mathrm{C}_{6} \mathrm{H}_{12} \mathrm{O}_{6}=\mathrm{C}_{4} \mathrm{H}_{8} \mathrm{O}_{2}+2 \mathrm{CO}_{2}+2 \mathrm{H}_{2} \text {. }
$$

Special bacteria have been described in connection with this form of milk fermentation, ${ }^{9}$ most of them non-pathogenic. It is unquestionable, however, that many of the well-known pathogenic bacteria, such as Bacillus aërogenes capsulatus, Bacillus œdematis maligni, possess the power of similar butyric-acid formation. While less commonly observed in milk, because milk is rarely kept long enough to permit of the action or development of these enzymes, the butyricacid fermentation is of importance in connection with butter, where it is one of the causes producing rancidity.

"Heinemann, Jour. of Inf. Dis., 3, 1906.

${ }^{8}$ Conn, Exper. Stat. Rep., 1892.

' Schattenfroh und Grasberger, Arch. f. Hyg., 37, 1900. 
Alcoholic fermentation may take place in milk as a result of the activities of certain yeasts. Upon the occurrence of such fermentations depends the production of kefyr, koumys, and other beverages which have been in common use for many years, especially in the region of the Caucasus. The characteristic quality of these beverages is contributed by the feeble alcoholic fermentation produced by members of the saccharomyces group, but side by side with this process lactic-acid formation also takes place. Beijerinck, ${ }^{10}$ who has carefully studied the so-called kefyr seeds, used for the production of kefyr in the East, has isolated from them a form of yeast similar in many respects to the ordinary beer yeast, and a large bacillus to which he attributes the lactic-acid formation.

Occasional but uncommon changes which occur in milk lead to the formation of the so-called "slimy milk," yellow and green milk, and bitter milk. These may be due to a number of bacteria. A microorganism which is commonly found in connection with the slimy changes in milk is the so-called Bacillus lactis viscosus. According to the researches of Ward, ${ }^{11}$ this microorganism is frequently derived from water and it is the water supply which should attract attention whenever such trouble occurs in dairies.

The so-called blue, green, and yellow changes are usually due to chromogenic bacteria, such as Bacillus cyanogenes, Bacillus prodigiosus, and others.

"Bitter milk," a condition which has occasionally been observed epidemically, is also the consequence of the growth of microorganisms. Conn, ${ }^{12}$ in 1891, isolated from a specimen of bitter cream a diplococcus which occasionally forms chains and which in sterilized milk develops rapidly, producing an extremely bitter taste. The organism of Conn differs from a similar diplococcus described by Wagmann ${ }^{13}$ in that it possesses the ability of producing butyric acid.

Milk in Relation to Infectious Disease.-As a source of direct infection, milk is second only to water, and deserves close hygienic attention. A large number of infectious diseases have been traced to milk, although the actual proof of the etiological part played by it in such cases has often been difficult to adduce and has necessarily been indirect. Nevertheless, even when indirect proof only

\footnotetext{
${ }^{10}$ Beijerinck, ('ent. f. Bakt., vi, 1889.

${ }^{11}$ Thard, Bull. 165, Cornell Univ. Agri, Exp. Stat., 1899.

12 Conn, Cent. f. Bakt., ix, 1891.

1s Wagmann, Milchztg., 1890.
} 
has been brought, it has been sufficiently convineing to necessitate the most careful investigation into milk supplies whenever epidemics of certain infectious maladies occur.

Typhoid-fever epidemics have been frequently traced to milk infection, and, in this disease, milk is, next to water, the most frequent etiological factor. Schüder, ${ }^{14}$ in an analysis of six hundred and fifty typhoid epidemics, found four hundred and sixty-two attributed to water, one hundred and ten to milk, and seventy-eight to all other eauses.

Trask $^{\mathbf{1 5}}$ compiled statistics of one hundred and seventy-nine typhoid epidemics supposed to have been caused by milk, in various parts of the world. In all such epidemics the origin of infection was generally traceable to diseased or convalescent persons employed in dairies, to contaminated well water used in washing milk utensils, or to the use of cans and bottles returned from dwellings where typhoid fever had existed. Actual bacteriological proof of the infectiousness of milk by the isolation of Bacillus typhosus is rare, but has been accomplished in isolated instances. In the case of one epidemic, Conradi ${ }^{16}$ isolated the bacillus from the milk on sale at a bakery at which a large number of the infected individuals had purchased their milk. The examination of market milk at Chicago, through a period of eight years, revealed the presence of typhoid bacilli but three times.

In spite of the few cases in which actual bacteriological proof has been brought, it is not unlikely that careful and systematic researches would reveal a far greater number, since many writers have shown that typhoid bacilli may remain alive in raw milk for as long as thirty days, ${ }^{17}$ and may actively proliferate in the milk during this time. One peculiarity of epidemics which may aid in arousing the suspicion that they have originated in milk is that, in such cases, women and children are far more frequently attacked than men. ${ }^{18}$

A feature which adds considerably to the dangers of milk infection is the unfortunate absence of any gross changes, such as coagulation, by the growth of typhoid bacilli.

${ }^{14}$ Schïder, Zeit. f. Hyg., xxxviii, 1901.

${ }^{15}$ Trask, Bull. No. 41, U. S. Pub. Health and Mar. Hosp. Serv., Wash.

${ }^{16}$ Conradi, Cent. f. Bakt., I, xl, 1905

${ }^{17}$ IIeim, Arb. a. d. kais. Gesundheitsamt, v.

${ }^{18}$ Wilckens, Zeit. f. Hyg., xxvii, 1898. 
Scarlet fever, ${ }^{19}$ though as yet of unknown etiology, has in many eases been traced indirectly to milk infection. Trask has collected fifty-one epidemics of scarlet fever presumably due to milk. In one epidemic occurring in Norwalk, Conn., ${ }^{20}$ twenty-nine cases were distributed among twenty-five families living in twenty-four different houses. The individuals affected did not attend the same school, and were of entirely different social standing, the only factor common to all of them being the milk supply.

Diphtheria has been frequently traced to the use of infected milk. In most of the epidemics reported as originating in this way, the proof has been necessarily indirect. In two out of twenty-three epidemics reported by Trask, however, Bacillus diphtheriæ was isolated from the milk directly. The ability of the Klebs-Loeffler bacillus to proliferate and remain alive for a long while in raw milk has been demonstrated by Eyre ${ }^{21}$ and others.

Whether or not cholera asiatica may be transmitted by means of milk has been a disputed question. Hesse ${ }^{22}$ claims that cholera spirilla die out in raw milk within twelve hours. This statement, however, has not been borne out by other observers. ${ }^{23}$ Unquestionable cases of direct transmission of cholera by means of milk have been reported by a number of writers, notably by Simpson. ${ }^{24}$.

The relation of milk to the diarrheal diseases of infants has, of late years, received a great deal of attention. In large cities, during the summer months, numerous eases of infantile diarrhea anong bottle-fed babies occur, which, in many instances, are attributed to feeding with contaminated milk. Park and Holt, ${ }^{25}$ who have made extensive researches upon this question in New York City, have come to the conclusion that the harmful effects of contaminated milk upon babies can not be ascribed to any given single microorganism in the milk. Specifically toxic properties were found by these writers for none of the one hundred and thirty-nine different species of bacteria isolated from unsterilized milk. It is unlikely, therefore, that the diarrheal diseases among babies have a uniform bacteriolog-

19 Trask, loc. eit.

${ }^{20}$ Herbert E. Smith, Rep. Conn. State Bd. of Health, 1897.

${ }^{21}$ Eyre, Brit. Med. Jour., 1899.

${ }^{22}$ Hesse, Zeit. f. Hyg., xvii, 1894.

${ }^{23}$ Basenau, Arch. f. Hyg., xxiii, 1895.

${ }^{24}$ Simpson, Indian Med. Gaz., 1887.

${ }^{25}$ Park and Holt, Arch. of Ped., Dec., 1903. 
ical cause. Whether or not these diarrheal conditions depend entirely upon the bacterial contents of milk or, in a large number of eases at least, upon the inability of the child to digest cow's milk because of chemical conditions, must be left undecided. Park and Holt, in analyzing their extensive data, conclude that milk containing "over one million bacteria to the cubic centimeter is certainly harmful to the average infant."

The significance of the presence of streptococei in milk, as an element of danger, has recently received much attention in the literature. Heinemann, ${ }^{26}$ who has made a careful comparison of Streptococcus lacticus (formerly spoken of as Bacillus acidi lactici [Krusel]), with other streptococei, has shown that, essentially, this streptococcus does not differ from streptococci from other sources, and is practically indistinguishable by cultural methods from Streptococcus pyogenes. Similar comparisons made by Schottmüller, ${ }^{27}$ Müller, ${ }^{28}$ and others have led to like results. Since streptococci may be found in milk from perfectly normal cows and are almost regularly associated with lactic-acid fermentation, it is unlikely that these microorganisms hold ordinarily any specific relationship to disease.

Recently, however, a number of epidemics of sore throat caused by streptococci have bcen traced to milk upon reasonably reliable evidence. Accounts of such epidemics in Chicago and in Baltimore have been published by Capps and Miller ${ }^{29}$ and by Hamburger. ${ }^{30}$

The presence of pus cells and leucocytes in milk, together with streptococci, was also formerly regarded as of great importance. Enumerations of leucocytes in milk were first made by Stokes and Weggefarth. ${ }^{31}$ Their method of enumeration consisted in centrifugalizing a definite volume of milk, spreading the entire sediment over a definite area on a slide, and counting the leucocytes found in a number of fields. Calculations from this may then be made as to the number of leucocytes per cubic centimeter. This method, and modifications of it, have been used by a large number of observers, but the value of the conclusions drawn from them has been much exaggerated. Normal milk may contain leucocytes in

\footnotetext{
${ }^{26}$ Heinemann, Jour. Inf. Dis., 3, 1906.

${ }^{27}$ Schottmüller, Münch. med. Woch., 1903.

${ }^{28}$ Müller, Arch. f. Hyg., lvi, 1906.

${ }^{20}$ Capps and Miller, Jour. A. M. A., June, 1912, p. 1848.

${ }^{30}$ Hamburger, Bull. of the Johns Hopk. Hosp., xxiv, Jan., 1913.

${ }^{*}$ Stokes and Weggefarth, Med. News, 91, 1897.
} 
moderate numbers, and importance may be attached to such leucocyte counts only when their number largely exceeds that present in other specimens of perfectly normal milk. Whenever such high leucocyte counts are found, of course, a careful veterinary inspection and examination for pyogenic disease should be made.

Foot-and-mouth disease, an infectious condition prevailing among cattle, characterized by a vesicular rash on the mouth and about the hoofs, has, in a number of cases, been definitely shown to be transmitted to man through the agency of milk. Notter and Firth ${ }^{32}$ reported an epidemic occurring among persons supplied with milk from a single dairy in which foot-and-mouth disease prevailed among the cows. In this epidemic, two hundred and five individuals were affected with vesicular eruptions of the throat, with tonsillitis and swellings of the cervical lymph nodes. Similar cases have been reported by Pott. $^{33}$

Although anthrax has never been definitely shown to have been conveyed by milk, Boschetti ${ }^{34}$ succeeded in isolating living anthrax bacilli from a sample of milk two weeks after its withdrawal from the cow.

Mitk and Tuberculosis.-The question of the conveyance of tuberculosis by means of milk is a subject which, because of its great importance, has been extensively investigated by bacteriologists. A large number of observers have succeeded in proving the presence of tubercle bacilli in the milk of tuberculous cows by intraperitoneal inoculation of rabbits and guinea-pigs with samples of milk. Such positive results have been obtained by Bang, ${ }^{35}$ Hirschberger, ${ }^{36}$ Ernst, ${ }^{37}$ and many others. A number of these observers, notably Ernst, have shown that tubercle bacilli may be present in the milk without tuberculous disease of the udders. In an examination of the milk supply of Washington, D. C., ${ }^{38} 6.72$ per cent of the samples contained tubercle bacilli.

The path of entrance of the bacilli from the cow into the milk

${ }^{32}$ Notter and Firth, quoted from Harrington, "Theory and Practice of Hygiene.',

${ }^{33}$ Pott, Münch. med. Woch., 1899.

${ }^{34}$ Boschetti, Giorn. med. vet., 1891.

3s Bang, Deut. Zeit. f. Tierchem., xi, 1884.

${ }^{36}$ Hirschberger, Deut. Areh. f. klin. Med., xliv, 1889.

${ }^{37}$ Errist, H. C., Amer. Jour. Med. Sei., xeviii, 1890.

${ }^{38}$ Anderson, Bull. No. 41, U. S. Pub. Health and Mar. Hosp. Serv., Wash., 1908. 
has long been a subject of controversy. That the bacilli may easily enter the milk, when tuberculous disease of the udder is present, stands to reason and is universally conceded. It is now believed also, on the basis of much experimentation, that in systemically infected cows tubercle bacilli may pass through the mammary glands into the milk, without evidence of local disease in the secreting gland. An experiment performed by the Royal British Tuberculosis Commission $^{39}$ illustrates this point. A cow, injected subcutaneously with tubercle bacilli behind the shoulder, began to discharge tubercle bacilli in the milk within seven days after inoculation and continued to do so until death from generalized tuberculosis.

Milk may become indirectly contaminated, furthermore, with tubercle bacilli emanating from the feces of cows. It has been shown that tubercle bacilli are present in the feces of cattle so early in the disease that diagnosis can be made only by a tuberculin test. ${ }^{40}$

Whether or not contaminated milk is common as an etiological factor in human tuberculosis, must be considered at present as an unsettled question. Behring, at the Congress of Veterinary Medicine, at Cassel, in 1903, advanced the view that pulmonary tuberculosis in adults may be a late manifestation of a milk infection contracted during infancy. He stated as his own opinion, moreover, that most cases of tuberculosis in man are traceable to this origin. The problem is as difficult of solution as it is important. In bottle-fed infants, infection by means of milk unquestionably occurs with considerable frequency. Smith, ${ }^{41}$ Kossel, Weber, and Huess, ${ }^{42}$ and others, have isolated tubercle bacilli of the bovine type from the mesenteric lymph nodes of many infected children. Animal experimentation has, furthermore, revealed that lesions in the mesenteric nodes, as well as later in the bronchial lymph nodes, may occur as a consequence of feeding tubercle bacilli, without any demonstrable lesions in the intestinal mucosa. It is thus certain that infection by the ingestion of tuberculous milk may occur, especially among young children who, as is well-known, are comparatively susceptible to bacilli of the bovine type. Whether or not such infection will

${ }^{39}$ Quoted from Mohler, P. H., and Mar. Hosp. Serv. Bull. 41, 1908.

${ }^{40}$ Schrocder and Cotton, Bull. Bureau Animal Industry, Wash., 1907.

${ }^{41}$ Smilh, Trans. Assu. Amer. Physic., 18, 1903.

42 Kossel, Weber, and Huess, Tuberkul. Arb. a. d. kais. Gesundheitsamt, 1904, 1905, Hft. 1 and 3 . 
account for many cases of tuberculosis in adults is a question which, for final solution, will require much more investigation. The sole reliable method of approaching it lies in determining the type, human or bovine, of the bacilli present in a large number of cases. Experience thus far seems to indicate that the bovine type is comparatively rare in the pulmonary disease of adults.

The value of the tubereulin reaction for diagnosis, and the elimination of all eattle showing a positive reaction, for the prevention of tuberculosis, can not be overestimated. The failure of the test in diseased animals is rare, and an aceurate diagnosis ean be established in over 90 per cent of diseased animals. ${ }^{43}$ The assertion that the cattle are permanently injured by tuberculin injections is without scientific basis. If this test were conscientiously carried out, and infected cattle condemned, the dangers from bovine bacillus infection would be practically eliminated, for there are but few instances in which science has been able to furnish such definite information for absolute protection. It is needless to say, however, that the carrying out of such precautions is subject to great expense and great difficulties of organization.

Dairy inspection is practiced in the vicinity of many of our larger cities, and the movement is daily gaining ground. Until fully established, however, upon a financial basis which brings the best products within the means of the poorer classes, other inexpensive measures to render milk safe must often be resorted to.

Sterilization by high temperatures is objected to by pediatricians because of the physical and chemical changes produced in the milk which are said to detract from its nutritive value.

The development of scurvy and rickets in infants has often been attributed to the use of such milk. These objections, however, do not apply to the use of milk which has been subjected to the process of "pasteurization." By this term is meant the heating of any substance to $60^{\circ} \mathrm{C}$. for twenty to thirty minutes. The process, first devised by Pasteur for the purpose of destroying germs in wine and beer in which excessive heating was supposed to injure flavor, brings about the death of all microorganisms which do not form spores-in other words, of all the bacteria likely to be found in milk which ean give rise to infection per os. At the same time the chemical and physical constitution of the milk is not appreciably

48 Mohler, loc. cit. 
changed, at least not to an extent which renders it less valuable as a food. Statistics by Park and Holt have shown strikingly the advantages of pasteurized over raw milk in infant feeding. Of fifty-one children fed with raw milk during the summer months, thirty-three had diarrhea, two died, and only seventeen remained entirely well. Of forty-one receiving pasteurized milk, but ten had diarrhea, one died, and thirty-one remained entirely well throughout the summer. The actual diminution of the living bacterial contents of milk by pasteurization is enormous, the milk so treated often containing not more than one thousand, usually less than fifteen thousand, living bacteria to each cubic centimeter.

Methods of Estimating the Number of Bacteria in Milk.-In estimating the number of bacteria in milk, colony counting in agar or gelatin plates is resorted to. Great care must be exercised in obtaining the specimens. If taken from a can, the contents of the can should be thoroughly mixed, since the cream usually contains many more bacteria than the rest of the milk. The specimen is then taken into a sterile test tube or flask. If the milk is supplied in an ordinary milk bottle, this should be thoroughly shaken before being opened, and the specimen for examination taken out with a sterile pipette. Dilutions of the specimen ean then be made in sterile broth or salt solution. If an initial dilution of $1: 100$ is made, quantities ranging from 1 c.c. to 0.1 c.c. of this will furnish 0.01 c.c. to 0.001 c.c. of the milk, respectively. Inoculation of properly cooled tubes of melted neutral agar and gelatin, with varying quantities of these dilutions, are then made and plates poured. After twenty-four to forty-eight hours at room temperature or in the incubator, colony counting is done, and the proper calculation is made. In samples in which few bacteria are expected, direct transference of $1 / 20$ or $1 / 40$ of a c.c. of the whole milk into the agar may be made. This method saves time but is less accurate.

Direct Methods of Counting Bacteria.-Direct methods of counting bacteria in milk have recently been advised, the one most extensively tried being that of Prescott and Breed. By this method a capillary tube is marked to measure accurately 0.01 c.c. This amount of the milk is spread over a square $\mathrm{cm}$. on a microscope slide. It is dried in the air and fixed with methyl alcohol, after which the fatty constituents can be dissolved with xylol. It can then be stained lightly with the Jenner stain. The bacteria are counted under an oil immersion lens, the tube Iength and magnification being so 
arranged that the microscopic field covers $1 / 50$ sq. mm. A standardized eyepiece micrometer may be usea. The average number of bacteria found in such fields may be multiplied by 5,000 to give the number of bacteria contained in 0.01 c.c. of milk. This method has not yet displaced the one of plating and does not promise to do so for some time.

For the isolation of special pathogenic bacteria from milk, no rules can be laid down, since, in every case, the method adapted to the particular organism sought for must be chosen.

Tubercle bacilli can be isolated from milk with success only by guinea-pig injection. The milk is centrifugalized and 5 c.c. of the sediment, together with some of the cream that has risen to the top, is intraperitoneally or subcutaneously injected.

The control of milk in the market depends upon careful regulations, which must include care of cattle, dairy inspection and bacteriological control of the delivered milk. This is a subject which is too extensive to touch upon in a book of this kind. However, a general idea of the methods employed may be obtained by studying the accompanying table, which is taken from the New York City Department of Health Regulation for the Sale of Milk and Crcam.

Bacteria and Butter.-Butter is made from cream separated from milk either by standing or by centrifugalization. After this, the cream is agitated by churning, which brings the small fat-globules into mutual contact, allows them to adhere to each other and form clumps of butter. It has been a matter of common experience, however, that unless the cream is allowed to "ripen" for a considerable period before churning, the resulting butter lacks the particular quality of flavor which gives it its market value. The interval of ripening, at first a necessity upon small farms where cream must be collected and allowed to accumulate, has now been recognized as an essential for the production of the best grades of butter, and it has been shown that the changes taking place in the cream during this period are referable to the action of bacteria. Cream, which before the ripening process contains but 50,000 bacteria to each cubic centimeter, at the end of a period of "ripening"' will often contain many millions of microorganisms. At the same time, the cream becomes thick and often sour.

The species of bacteria which take part in this process and which, therefore, must determine to a large extent the quality of the end product, are various and, as yet, incompletely known. Usually some 
REXULATIONS GOVERNING THE GRADES AND DESIGNATION OF MILK

The following classifications apply to milk and cream. The regulations regarding

\begin{tabular}{|c|c|c|c|}
\hline $\begin{array}{c}\text { GRADES OF } \\
\text { MILK OR } \\
\text { CREAM } \\
\text { WHICH MAY } \\
\text { BE SOLD IN } \\
\text { THE CITY OF } \\
\text { NEW YORK }\end{array}$ & DEFINITION & $\begin{array}{l}\text { TUBERCULIN } \\
\text { TEST AND } \\
\text { PHYSICAL } \\
\text { CONDITION }\end{array}$ & BACTERIAL CONTENTS \\
\hline $\begin{array}{l}\text { GRADE A } \\
\text { Milk or Cream } \\
\text { (Raw) }\end{array}$ & $\begin{array}{l}\text { Grade A milk or cream (raw) is milk or } \\
\text { cream produced and handled in accord- } \\
\text { ance with the minimum requirements, } \\
\text { rules and regulations as herein set forth. }\end{array}$ & $\begin{array}{l}\text { 1. Only such } \\
\text { cows shall be ad- } \\
\text { mitted to the } \\
\text { herd as have not } \\
\text { react ed to a } \\
\text { diagnostic injec- } \\
\text { tion of tubercu- } \\
\text { lin and are in } \\
\text { good phy sical } \\
\text { condition. } \\
2 \text {. All cows } \\
\text { shall be tested } \\
\text { annually w it h } \\
\text { tuberculin and } \\
\text { all reacting ani- } \\
\text { mals shall be ex- } \\
\text { cluded from the } \\
\text { herd. }\end{array}$ & $\begin{array}{l}\text { Grade A milk (Raw) shall not eon- } \\
\text { tain more than } 60,000 \text { bacteria per } \\
\text { c.c. and cream more than } 300,000 \\
\text { bacteria per c.c. when delivered to } \\
\text { the consumer or at any time prior to } \\
\text { such delivery. }\end{array}$ \\
\hline $\begin{array}{l}\text { Milk or Cream } \\
\text { (Pasteurized) }\end{array}$ & $\begin{array}{l}\text { Grade A milk or cream (pasteurized) is } \\
\text { milk or cream handied and sold by dealers } \\
\text { holding permits therefor from the Board } \\
\text { of Health, and produced and handled in } \\
\text { accordance with the requirements, rules } \\
\text { and regulations as herein sct forth. }\end{array}$ & $\begin{array}{l}\text { No tuberculin } \\
\text { test required but } \\
\text { cows must be } \\
\text { healthy as dis- } \\
\text { closed by phys- } \\
\text { ical examination } \\
\text { made annually. }\end{array}$ & $\begin{array}{l}\text { Grade A milk (pasteurized) shall } \\
\text { not contain more than } 30,000 \text { bacteria } \\
\text { per c.c. and Cream (pasteurized) more } \\
\text { than } 150,000 \text { bacteria per c.c. when } \\
\text { delivered to the consumer or at any } \\
\text { time after pasteurization and prior } \\
\text { to such delivery. } \\
\text { No milk supply averaging more } \\
\text { than } 200,000 \text { bacteria per c.c. shall be } \\
\text { pasteurized for sale under this desig- } \\
\text { nation. }\end{array}$ \\
\hline $\begin{array}{l}\text { GRADE B } \\
\text { Milk or Cream } \\
\text { (Pasteurized) }\end{array}$ & $\begin{array}{l}\text { Grade B milk or cream (pasteurized) is } \\
\text { milk or cream produced and handled in } \\
\text { accordance with the minimum require- } \\
\text { monts, rules and regulations herein set } \\
\text { forth and which has been pasteurized in } \\
\text { accordance with the requirements and } \\
\text { rules and regulations of the Department } \\
\text { of Health for pasteurization. }\end{array}$ & $\begin{array}{l}\text { No tuberculin } \\
\text { tests required but } \\
\text { cows must be } \\
\text { healthy as dis- } \\
\text { closed by phys- } \\
\text { ical examination } \\
\text { made annually. }\end{array}$ & $\begin{array}{l}\text { No milk under this grade shall con- } \\
\text { tain more than } 100,000 \text { bacteria per } \\
\text { c.c. and no cream shall contain more } \\
\text { than } 500,000 \text { bacteria per c.c. when } \\
\text { delivered to the consumer or at any } \\
\text { time after pasteurization and prior to } \\
\text { such delivery. } \\
\text { No milk supply averaging more } \\
\text { than } 1,500,000 \text { bacteria per c.c. shall } \\
\text { be pasteurized in this city for sale } \\
\text { under this designation. } \\
\text { No milk supply averaging more } \\
\text { than } 300,000 \text { bacteria per c.c. shall be } \\
\text { pasteurized outside of this city for } \\
\text { sale under this designation. }\end{array}$ \\
\hline $\begin{array}{l}\text { GRADE C } \\
\text { Milk or Cream } \\
\text { (Pastcurized) } \\
\text { (For cooking } \\
\text { a nd m a n u - } \\
\text { facturing pur- } \\
\text { poses only.) }\end{array}$ & $\begin{array}{l}\text { Grade C milk or cream is milk or cream } \\
\text { not conforming to the requirements of } \\
\text { any of the subdivisions of Grade A or } \\
\text { Grade B and which has been pasteurized } \\
\text { according to the requirements and rules } \\
\text { and regulations of the Board of Health or } \\
\text { boiled for at least two (2) minutes. }\end{array}$ & $\begin{array}{l}\text { No tuberculin } \\
\text { test required but } \\
\text { cows must be } \\
\text { healthy as dis- } \\
\text { closed by phys- } \\
\text { ical examination } \\
\text { made annually. }\end{array}$ & $\begin{array}{l}\text { No milk of this grade shall contain } \\
\text { more than } 300,000 \text { bacteria per c.c. } \\
\text { and no cream of this grade shall con- } \\
\text { tain more than } 1,500,000 \text { bacteria } \\
\text { per c.c. after pasteurization. }\end{array}$ \\
\hline
\end{tabular}

NOTE-Sour milk, buttermilk, sour cream, kumyss, matzoon, zoolac and similar products shall not be made the process of souring. Sour cream shall not contain a less percentage of fats than that designated for cream.

No other words than those designated herein shall appear on the label of any container containing milk or cream

The term "certified" milk is usually defined for each region by a special commission of the County Med. Soc., County, N. Y.:

CerTified Mruk must have every charactoristic of pure, clean, fresh, wholesome cow's milk. The milk must Nothing must be added to the milk and nothing taken awav.

CERTIFIE MuK shall not contain less than 4 por cent of butter fat.

* Table taken from Rules and Regulations of N. Y. City Department of Health, 1914, applying to sale of milk 


\section{AND CREAM WHICH MAY BE SOLD IN THE CITY OF NEW YORK *}

bactcrial content and time of delivery shall not apply to sour eream

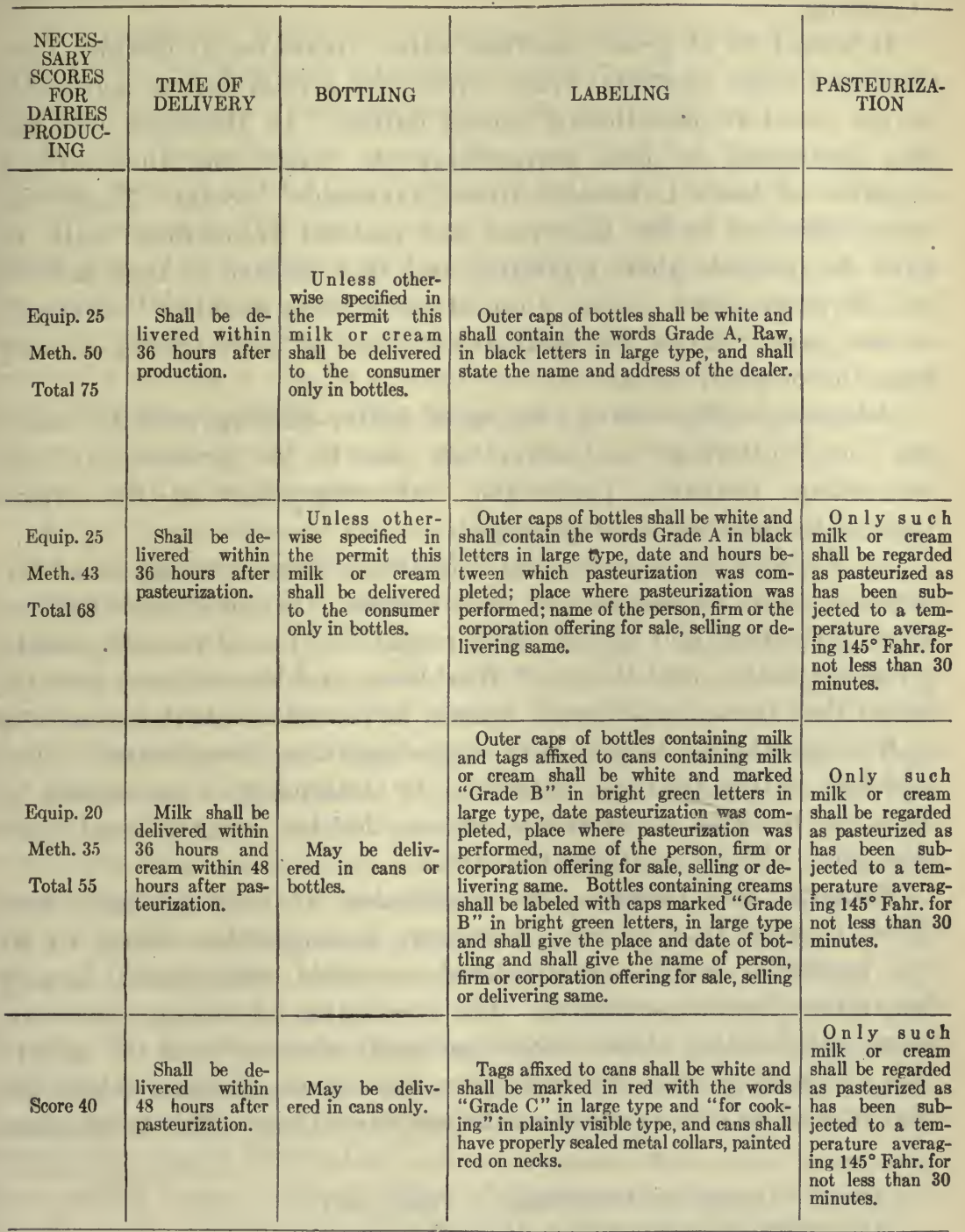

from any milk of a less grade than that designated for "Grade B" and shall be pasteurized before being put through or milk or cream products except the word "certified" when authorized under the State laws.

sanction by State Law. The following is the definition of certified milk given by the Milk Commission of Kings be in its natural state, not having been heated and without the addition of coloring matter or preservatives.

and cream. 
variety of lactic-acid bacilli is present and these, as in milk, outgrow other species and, according to Conn, ${ }^{44}$ are probably essential for "ripening."

It would be of great practical value, therefore, if definite pure cultures of the bacteria which favor the production of agreeable flavors could be distributed among dairies. In Denmark this has been attempted by first pasteurizing the cream and then adding a culture of bacteria isolated from "favorable" cream. These cultures, delivered to the dairyman, are planted in sterilized milk, in order to increase their quantity, and this culture is then poured into the pasteurized cream. In most cases, these so-called "starters" are not pure cultures, but mixtures of three or more species derived from the original cream.

Adverse accidents in the course of butter-making, such as "souring" or "bittering" of butter, are due to the presence of contaminating, probably proteolytic, microorganisms in the cream during the process of "ripening."

As a means of transmitting infectious disease, butter is of importance only in relation to tuberculosis. Obermüller, ${ }^{45}$ Rabinowitch, ${ }^{46}$ Boyce, ${ }^{47}$ and others, have repeatedly found tubercle bacilli in market butter, and Mohler, ${ }^{48}$ Washburn, and Rogers have recently shown that these bacilli could remain alive and virulent for as long as five months in butter kept at refrigerator temperature. The acid-fast butter bacillus, described by Rabinowitch as similar to the true Bacillus tuberculosis, shows decided cultural and morphological differences from the latter.

Bacteria and Cheese.-The conversion of milk products into cheese consists in a process of protein decomposition which, by its end products, leucin, tyrosin, and ammonia compounds, largely determines the cheese-flavors. The production of cheese, therefore, is due to the action of proteolytic bacterial enzymes ${ }^{49}$ and the variety of a cheese is largely determined by the microorganisms which are present and by the cultural conditions prevailing. The sterilization

${ }^{44}$ Conn, "Agricultural Bacteriology," Phila., 1901.

${ }^{45}$ Obermüller, Hyg. Rundschau, 14, 1897.

4 Rabinowitch, Zeit. f. Hyg., xxvi, 1897.

${ }^{47}$ Boyce and Woodhead, Brit. Med. Jour., 2, 1897.

${ }^{48}$ Mohler, U. S. P. H. and Mar. Hosp. Serv. Bull. 41, 1908.

49 Freudenreich, Koch's Jahresbericht, ete., 135, 1891. 
of cream, or the addition of antiseptics, absolutely prevents cheese production.

The organisms which are concerned in such processes have been extensively studied and attempts have been made, with moderate success, to produce a definite flavor with pure cultures.

In the production of cheese the two varieties, hard and soft cheeses, depend not so much upon the bacterial varieties as upon the differences in the treatment of the curds before bacterial action has begun. In the former case, a complete freeing of the curds from the whey furnishes a culture medium which is comparatively dry and of almost exclusively protein composition; in the latter, retention of whey gives rise to eultural conditions in which more rapid and complete bacterial action may take place. The holes, which are so often observed in some of the hard cheeses, are due to gas production during the process of "ripening."

As to the varieties of microorganisms present in various cheeses, much careful work has been done. Duclaux ${ }^{50}$ attributed the "ripening" of some of the soft cheeses to a microorganism closely related to Bacillus subtilis. V. Freudenreich ${ }^{51}$ in part substantiated this, but laid particular stress upon the action of Oïdium lactis, a mold, and upon several varieties of yeast. Conn, ${ }^{52}$ more recently, in a bacteriological study of Camembert cheese, has demonstrated that the production of this cheese depends upon the united action of two microorganisms, one an oïdium, like the Oïdium lactis of Freudenreich, which is found chiefly in the interior softened areas, the other a mold belonging to the penicillium variety, found in a matted feltwork over the surface and penetrating but a short distance. In spite of the scientific basis upon which the work of these men and of others has seemed to place cheese production, attempts at uniformity in cheese production have met with almost insuperable obstacles because of the presence of a variety of adventitious microorganisms which, depending in species and proportion upon the local conditions under which the various cheeses have been produced, have added minor characteristics of flavor which have determined market value. Occasional failure of good results in cheese produc-

"Duclaux, “Le Lait,", Paris, 1887.

${ }^{*}$ V. Freudenreich, Cent. f. Bakt., II, i, 1895.

62 Conn, Bull. Statis. Agri. Exp. Stat. 35, 1905. 
tion $^{53}$ is due to contamination with other chromogenic or putrefactive bacteria.

In its relationship to the spread of infectious disease, cheese is relatively unimportant except in regard to tuberculosis. Typhoid and other non-spore forming pathogenic germs can not survive the conditions existing during cheese-ripening for any length of time. Tubercle bacilli, both of the human and bovine types, have been found in cheese by Harrison ${ }^{54}$ and others, and Galtier has shown experimentally that tubercle bacilli may remain alive and virulent in both salted and unsalted cheese for as long as ten days.

\section{THE LACTIC-ACID BACILLI AND METCHNIKOFF'S* BACTERIOTHERAPY}

A problem which has occupied clinical investigation for many years is that of gastrointestinal autointoxication. There are a number of conditions occurring in man, in which symptoms profoundly affecting the nervous system, the circulation, and, in a variety of ways, the entire body, can be elinically traced to the intestines, and can, in many cases, be relieved by thorough purgation and careful diet. In some of these conditions, specific microorganisms can be held accountable for the diseases (B. enteritidis, B. botulinus, etc.). In other cases, however, etiological investigations have met with but partial success because of the large variety of microorganisms present in the intestinal tract and because of the complicated symbiotic conditions thereby produced. Intestinal putrefaction, recognized as the cardinal feature of such maladies, has been attributed to Bacillus proteus vulgaris, ${ }^{55}$ to Bacillus aërogenes capsulatus, to Bacillus putrificus, ${ }^{56}$ and to a number of other bacteria, but definite and satisfactory proof as to the etiological importance of any of these germs has not yet been advanced. The fact remains, however, that, whatever may be the

* See also B. Acidophilus, etc., in another section of this book.

ss Beijerinck, Koch's Jahresber, ete., 82, 189.

4 Harrison and Galtier, quoted from Mohler, U. S. Pub. H. and Mar. Hosp. Serv., Hygiene Lab. Bull. 41, 1908.

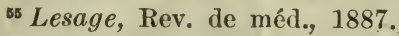

on Tissier, Ann. de l'inst. Pasteur, 1905. 
specific cause, the disease itself, a grave and often fatal affliction, may be clinically traced, in a number of cases, to the absorption of poisons from the intestinal canal, and it is more than likely that these poisons are the products of bacterial activity. Reason dictates, furthermore, that the bacteria primarily responsible for the production of these toxic substances do not belong to the varieties which attack carbohydrates only, but must belong to that class of aërobic and anaërobic germs which possess the power of breaking up proteins -in other words, the bacteria of putrefaction.

On the basis of the mutual antagonism existing in culture between many acid-producing bacteria and those of putrefaction-a phenomenon recognized by some of the earliest workers in this field, many investigators have suggested the possibility of combating. intestinal putrefaction by adding acid-forming bacteria together with carbohydrates to the diet of patients suffering from this condition. The first to suggest this therapy was Escherich ${ }^{57}$ who proposed the use, in this way, of Bacillus lactis aërogenes; with the same end in view, Quincke, ${ }^{58}$ a little later, suggested the use of yeastsOidium lactis. The reasoning underlying these attempts was meanwhile upheld by experiments carried out both in vitro and upon the living patient. Thus Brudzinski ${ }^{59}$ was able to demonstrate that Bacillus lactis aërogenes, in culture, inhibited the development of certain races of the proteus species and succeeded in obtaining markedly favorable results by feeding pure cultures of Bacillus lactis aërogenes to infants suffering from fetid diarrhea. Similar experiments $^{60}$ carried out with the Welch bacillus (aërogenes capsulatus) and Bacillus coli, however, had no such corroboratory results, since this anaërobe possesses a considerable resistance against an acid reaction. In considering the difficulties of the problems involved in this question, it occurred to Metchnikoff ${ }^{61}$ that much of the practical failure of therapy, based upon the principles stated above, might be referred to insufficent powers of acid production on the part of Bacillus coli, Bacillus lactis aërogenes, and other germs

${ }^{57}$ Escherich, Therapeut. Monatshefte, Oct., 1887.

${ }^{58}$ Quincke, Verhandl. des Congress f. Inn. Med., Wiesbaden, 1898.

${ }^{59}$ Brudzinski, Jahrbuch f. Kinderheilkunde, 52, 1900 (Ergänzungsheft).

* Tissier and Martelly, Ann. de 1'inst. Pasteur, 1906.

"Metchnikoff, "Prolongation of Life," G. P. Putnam's Sons, N. Y.; also in "'Bactériothérapie," ete. "'Bibliothèque de thérapeutique," Gilbert and Carnot, Paris, 1909. 
previously used. In searching for more powerful acid producers, his attention was attracted to Bacillus bulgaricus, isolated from milk by Massol ${ }^{62}$ and Cohendy ${ }^{63}$ in 1905 . This bacillus, according to the researches of Bertrand and Weisweiller, ${ }^{64}$ produces as much as 25 grams of lactic acid per liter of milk. In addition to this, it manufactures, from the same

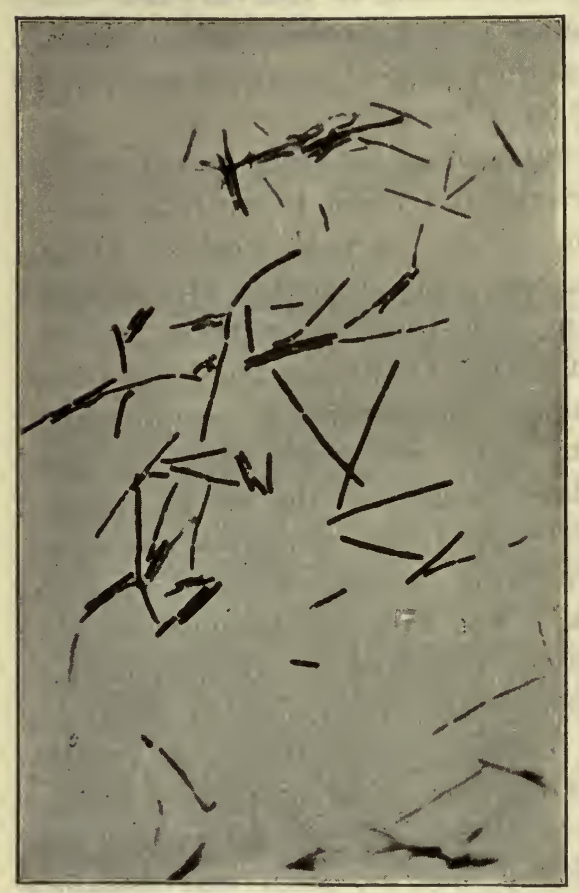

Fig. 119.-Bacillus bUlgaricus. quantity of milk, about 50 centigrams of acetic and succinic acids and exerts no putrefactive action upon proteins. Added to these characters, it is especially adapted to therapeutic application by its complete lack of pathogenicity.

The administration of the bacillus to patients suffering from intestinal putrefaction, first suggested by Metchnikoff in 1906, has, since that time, been extensively practiced and often with remarkable success. In spite of sharp criticism, especially by Luersen and Kühn, ${ }^{65}$ who deny much of the antiputrefactive activity of the bacillus, the treatment of Metchnikoff has found many adherents, upon the basis of purely clinical experiment. It is not possible to review completely the already extensive literature. Among the more valuable contributions may be mentioned the articles by Grekoff, ${ }^{66}$ by Wegele, ${ }^{67}$ and by Klotz. ${ }^{68}$ In Metchnikoff's experiments and in the work of his immediate successors, the

${ }^{62}$ Massol, Revue médicale de la Suisse romande, 1905.

${ }^{63}$ Cohendy, Comptes rend. de la soc. de biol., 60, 1906.

- Bertrand and Weisweiller, Ann. de l'inst. Pasteur, 1906.

${ }^{65}$ Luersen and Kühn, Cent. f. Bakt., II, xx, 1908.

"Grekoff, "Observations cliniques sur l'effet du lact. agri.," etc., St. Petersburg, 1907.

${ }^{67}$ Wegele, Deut. med. Woch., xxxiv, 1908.

${ }^{6}$ Klotz, Zentralbl. f. innere Med., 1908. 
bacillus was used either in milk eulture or in broth in which it was induced to grow in symbiosis with other microorganisms.

\section{BACTERIOLOGICAL EXAMINATION OF OYSTERS}

On account of the danger of the transmission of typhoid fever by oysters which have been bred or stored in eontaminated water, standard methods ${ }^{69}$ have been devised for the estimation of the bacterial content of oysters. These are similar in principle and method to those used for the examination of water, and a most important index of sewage contamination and consequent danger of typhoid infection is the number of colon bacilli present in the shell fish. The shell liquor is used for examination, and in examining oysters in the shell the following procedure is followed: Five oysters having deep bowls and elosed shells are selected. Lips of the shell are sterilized in the flame or by burning with alcohol. The liquor is obtained by opening the shell with a sterilized knife, or better, by drilling a hole through the flame surface with a sterilized gimlet. For determining the total number of bacteria the shell liquor is withdrawn with a sterilized pipette, diluted with 1 per cent salt solution, and placed in agar. More important, however, is the presumptive colon test, which is earried out by inoculating three lactose bile tubes with 1.0 c.e., 0.1 c.c., and 0.1 c.c., respectively, from each of the five oysters. The tubes are incubated for three days, and the development of over 10 per cent of gas in the closed arm is considered a positive reaction. The seore is recorded as the approximate number of colon bacilli contained in the 5.55 c.e. of shell liquor from the five oysters, and is estimated in the following way: A positive reaction in a tube inoculated in 1 c.c. is recorded as 1.0 , a positive reaction in 0.1 c.c. is 10 , and in 0.01 is recorded as 100 . The sum of these figures is the score for the batch of oysters from which the five have been taken. In examining shucked oysters a well-mixed sample of oysters and the surrounding fluid are put in a sterilized vessel and lactose bile tubes inoculated in triplicate with 1.0 c.c., 0.1 c.c., 0.01 c.c., 0.001 c.e. of the liquor. No definite standard seore has been adopted, but the United States Pure Food Board has condemned unshucked stock having a score of 32 or higher.

${ }^{69}$ Amer. Jour. Pub. Health, 1913, ii, 34. 


\title{
SEGTION VII
}

\section{PATHOGENIC PROTOZOA}

\author{
Frederick F. Russela, M.D.
}

\section{INTRODUCTION}

IN the practice of his profession the physician requires a knowledge of the pathogenic protozoa found in man and the domestic animals and of their closely related non-pathogenic forms. Quite commonly in the diagnosis of fevers it is necessary to examine the blood of the same patient for both malaria and bacteria, therefore a working knowledge of the principal pathogenic protozoa is essential. In this work it will be possible to describe the forms only of medical interest, and the reader is referred to other works for further information.

The protozoa are unicellular animal organisms that occur singly or in temporary colonies. The functions of the animals are carried out by the protoplasm of the single cell, parts of which may be differentiated for special purposes and are then called organellæ.

\section{CLASSIFICATION OF THE PROTOZOA}

Class I. Sarcodina (Rhizopoda).-The body is naked or encased and the animal moves by means of protruding temporary prolongations of the body ealled pseudopods. They possess one or many nuclei and reproduce by fission or multiplication in a cyst.

Order I. Amoebce (Lobosa).-Naked or with a simple shell, the pseudopia are lobose or finger-shaped, the nucleus is usually single and there is sometimes a contractile vacuole. Example, the amobre.

Class II. Mastigophora (F'lagellata).-They possess flagella for locomotion and for obtaining food; they may be naked or fur. 
nished with a membrane; many forms possess nucleus, contractile vacuole and a small groove spoken of as the eytostome. Examples, the trypanosomes and intestinal flagellates.

Class III. Sporozoa.-They live parasitically in the tissues of other animals, ingesting food by osmosis; they have no cilia in the adult stage but may form pseudopoda, one or more nuclei, no contractile vacuole, reproduction by spores. They are divided into two subclasses, telesporidia and neosporidia. Examples, gregarinida, coccidiidea, hemosporidia, sarcosporidia, etc.

Class IV. Infusoria (Ciliata).-The body is generally uniform in shape, with eilia and contractile vacuole, and usually with macroand micronucleus. Examples, paramecium, balantidium. 


\section{CHAPTER LIV}

\section{CLASS I-SARCODINA (RHIZOPODA)}

\section{THE AMOEB}

THESE organisms belong to the order Amœbina (Ehrenberg). They are characterized during the vegetative stage by a semifluid consistence, permitting rapid changes of form, amœboid movements, and progression by means of pseudopods. There is no internal skeleton and the protoplasm is naked and may be differentiated into endo- and ectoplasm, and in some cases a contractile vacuole is present. All forms possess one or more nuclei. Multiplication takes place by division into two or more daughter cells. Fertilization possibly takes place by the conjugation of two gametes.

Since some flagellates possess an amœboid stage, it is necessary to know most of the life cycle of an organism before classifying it as an ameba. The protoplasm varies greatly in its consistency, depending on the species as well as the stage of the life cycle, and the environment and food supply. Most amœbæ, including all the parasitic forms (entamœbæ), possess a single nucleus, yet $A$ mœba diploidea and $A m œ b a$ binucleata always have two, and the other species may show more. The nucleus of all types possesses a karyosome. The nucleus is well developed and in it may be followed either a simple or typical mitosis. The cytoplasm is usually at some stage divided into a granular endoplasm and a clear or hyalin ectoplasm, the latter forming the pseudopods by which the animal moves from place to place.

Until recent years all amœboid organisms were placed in the genus $A m œ b a$, but Schaudinn established a genus, the Entamœba, for the parasitic species which have many points of difference from the free living varieties. Of the free living forms, the easiest to study is the Amœba proteus (Pallas), a very large organism, 200 microns in diameter, found frequently in stagnant water; it, however, has no direct importance in medicine. Another group of free living amobx is of some interest, because of the confusion they have eaused in the study of narasitic amoba; they are the so-ealled "limax amohæ," 
which have been cultivated on agar, and for this genus Chatton (1912) has proposed the name Vahliampfia. They are small organisms, 5 to 30 microns in diameter, provided with fingerlike or spinous pseudopodia, and characterized by a nucleus with a large karyosome and a single nucleated resistance eyst in which no multiplication oceurs. They have repeatedly been cultivated from human dysenteric stools, from the air, and apparently from liver abscess pus. It has been shown, beyond doubt, that they are harmless to man, and that they pass through the intestinal tract with food and water in the cyst

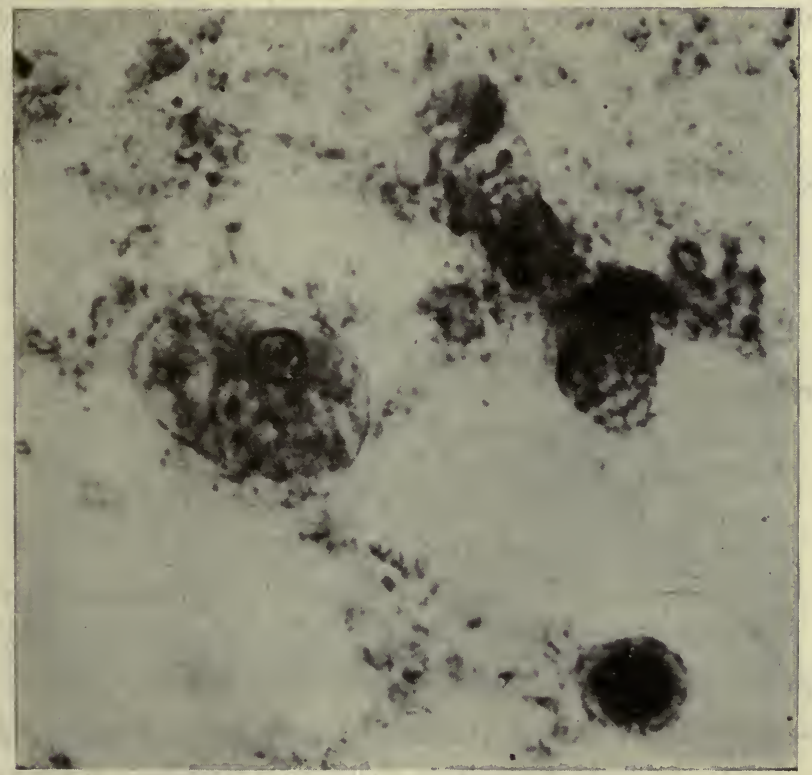

Fig. 120--Entambea histolytica. Vegetative form showing histolytica type of nucleus. Stained with iron hematoxylin. (Army Medical School Collection, Washington, D. C.)

form. While they will develop in cultures at body temperature, a better growth is obtained at the temperature of the room. Since the true parasitic amœbæ have never been cultivated on artificial media, the Vahlkampfia may be dismissed with the statement that they are not pathogenic.

The genus Entamœbæ includes all human parasitic forms, and is characterized, among other things, by the absenee of a contractile vacuole, which is always present in Amœba and Vahlkampfia. The 
species of importance to physicians are Entamcba histolytica, Entamoba coli and Entamoba gingivalis.

Leidy of Philadelphia established the genus endamoba which may possibly be closely related to or identical with the genus entamoba, for the large amœba which is parasitic in the cockroach, and called it Endamœba blattc; this genus presents; some points of resemblance to those present in man, but its life history has not been sufficiently studied by our present methods and the merging of the two at this period seems scarcely justifiable.

The nomenclature of the human parasitic entamœbæ is shown in the following table which has been taken from Dobell (1919):

SYNOPSIS OF GENERA AND SPECIES OF AMEBE LIVING IN MAN

Genus I. ENTAMEBA (Casagrandi and Barbagallo, 1895.

Synonyms :

$$
\text { s. (nee Endamoba Leidy, 1879.) }
$$

Poneramœba Lühe; 1908.

Liereckia $\{$ Chatton and Lalung-Bonnaire, 1912.

Proctambrba Alexeieff, 1912

(Amœba (pro parte), Endamœba, Entameba, Endameba, En, tamöba, Auctt.)

Type: E. coli (Grassi) Casagrandi and Barbagallo.

Species in Man: E. coli (Grassi) Casagrandi and Barbagallo.

E. histolytica Schaudinn (emend. Walker).

E. gingivalis (Gros) Brumpt.

Genus II. ENDOLIMAX Kuenen and Swellengrebel, 1917.

Only species, hence type: E. nana (Wenyon and O'Connor) Brug.

Genus III. IODAMEBA nov, gen.

Only species, hence type: I. bütschlii (Prowazek) Dobell.

Genus IV. DIENTAMEBA Jepps and Dobell, 1918.

Only species, hence type: D. fragilis Jepps and Dobell. 


\section{ENTAMOEBA HISTOLYTICA}

(Entamœba tetragena [Viereck], Entamœba africana [Hartmann] Entamœba nipponica [Koidzumi, pro parte], Entamoba tropicalis [Lesage, pro parte])

It has long been customary to say that amœbæ as a cause of disease were first described by Lambl of Prague, in 1860, who found them present in the stools from a case of severe diarrhea in a child, but some zoölogists, Leuckart (1863), Grasśi (1888) and Dobell (1919) believe that the organisms he described were degenerated trichomonads.

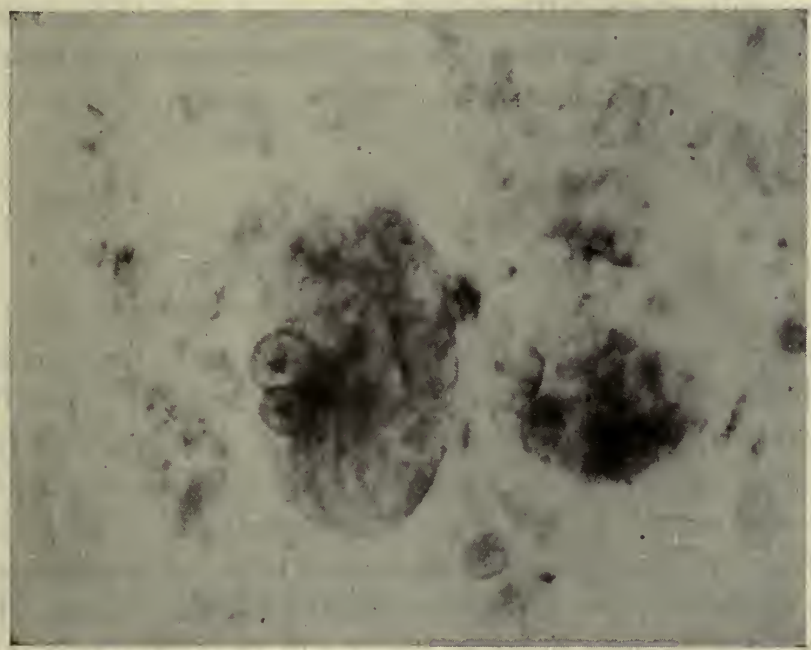

Fig. 121.-Entamoeba histolytica. Vegetative form, simple division. $(\times 1300$. (Army Medical School Collection, Washington, D. C.)

In 1870 Lewis and Cunningham found amœbæ in 20 per eent of the stools of cholera patients, but attached no pathogenic importance to them. The first accurate description we owe to Loesch of Petrograd, who in 1875 studied an undoubted case of amœbic dysentery with relapses, and he named the organism Amœba coli. He was further successful in reproducing the disease in a dog, and thus began its experimental investigation. Not much progress was made until Kartulis in Egypt began, in 1886, the publication of a long series of studies which has continued up to the present time, and because of the rich clinical and pathological material at his disposal his work has been of the greatest value. In 1890 Osler published the first paper 
- in America. He was followed by Musser and Stengel and, in 1891, by Dock, and Councilman and Lafleur. The work of the last two authors was especially complete and firmly established the entity of this disease in America. In 1902 Jürgens differentiated the pathogenic amœeba from the harmless, and in 1903 the work of Schaudinn appeared. This author, who was a zoölogist by training, showed clearly that there were two forms of parasitic amœbæ and he followed out most of the details in their life history, renaming them Entamoba histolytica and Entamœba,coli. Schaudinn accepted the name for the genus proposed by Casagrandi and Barbagallo (1895) but named his pathogenic species histolytica and the non-pathogenic coli. These names are still in use although the work of later investigators has shown that many of his observations were erroneous. Our present knowledge of this organism we owe to the work of Craig, Whitmore, Walker, Sellards, Darling, Dobell and others.

\section{CLINICAL DYSENTERY}

Dysentery as a disease has been known from the earliest times and referenees are found to it in Sanserit and Egyptian literature and in early Greek and Roman writings. Until recent years its etiology was obseure, but we now recognize two separate forms, bacillary and amœbic; the former has already been deseribed under the dysentery bacilli. Amœbic dysentery is a distinct clinical entity, and runs a course quite different from the bacillary form. It begins gradually, and in some cases is chronic in character from the start. Usually there is no rise in temperature nor any great change in weight or health until the disease has existed some time. The bowel movements become gradually more frequent and the fecal matter is aceompanied by larger and larger amounts of mueus and blood. As the disease progresses and more and more of the colon is involved the amount of blood and mucus increases until the stool contains little else. The colieky pains increase in frequency and severity and there is added tenesmus and finally nausea and vomiting. The patient loses flesh and strength and when the stools increase to twenty and thirty daily, becomes bed-ridden. The abdomen is concave and tender on pressure, especially over the colon. The eourse of the disease, if untreated, tends to progress with periods of remission, and spontaneous cure probably does not occur. Bacillary 
dysentery, it will be remembered, is a disease with a short incubation period and an acute onset; after two or three days' illness the bacillary case is confined to bed, is pale, weak, emaciated and presents every evidence of profound toxemia; an amobic ease, sick the same length of time, will be up and about and perhaps will not have applied for treatment.

Complications.-A common and most dangerous complication is abscess of the liver. The amœbæ travel from the ulcers in the colon by way of the lymphaties to the liver and there set up a liquefying necrosis of the parenchyma. The liquefied portion contains a reddish or chocolate-colored fluid, which is not pus in the ordinary sense, although it may become a pus-containing abseess if secondary bacterial infection oceurs. Liver abseesses may be single, but are much more often multiple, and at times the whole liver may be riddled with large and small abscess eavities; both right and left lobes may be involved. If surgical interference be withheld, the abscess increases in size, approaches the surface, and finally ruptures into the lung through the diaphragm or into the peritoneal cavity. A few cases of amcbic abscess of the brain have been reported. (Kartulis, 1904.)

At autopsy the lesions are found in the colon, principally at the sigmoid flexure and in the cecum, though in chronic cases the whole colon is involved, showing ulcers with undermined edges, swollen solitary follicles and a hemorrhagic-catarrhal inflammation of the mucous membrane. The ulcers, readily differentiated from those caused by the tubercle bacillus, are of all sizes, shallow or deep, and are characterized by irregular margins and undermined edges. Fresh smears made at autopsy will show vegetative amœbæ. In chronic cases, the colon is a mass of scars and ulcers and acutely inflamed, swollen and thickened mucous membrane resting on a hypertrophied submucosa. The severe and chronic forms of the disease are now as rare as they were formerly common as a result of the present specific treatment with emetin.

Geographical Distribution.-Although amœbic dysentery is classed among the tropical diseases, it is by no means confined to the tropies. In the United States, for example, it is endemic as far north as Baltimore and Washington, and eases are not very infrequent in the northern tier of states; hence one must examine the stools for amœeæ in dysenteric cases regardless of the location of the patient's home. 
Diagnosis.-While the history of the case may suggest amœbic infection, the diagnosis can only be made with certainty by microscopic examination of the stool. For this purpose the examination should be made as soon after the stool is passed as possible; and in this disease it is usually practicable to have the patient come to the hospital, clinic or office and pass a stool there. It may then be examined immediately. If this is impracticable, the stool may be kept warm and sent to the laboratory in a small glass jar inside a tin pail partly filled with water at body heat; a little cloth or absorbent cotton will hold the hot water and prevent splashing during transit. The stool will show bloody mucous masses, and small drops of this are placed on slides, protected with a cover glass and ringed with warm vaseline to prevent evaporation. The preparation, to be of value, must be thin, and the bloody mucus may be diluted with salt solution if necessary. Except in hot weather, the slide should be examined on a warm stage, or the slide may be warmed by placing heated coins on it, near the cover glass. At least half a dozen slides should be examined before reporting a negative result. Since the entamœbæ degenerate and die soon after the stool is passed, it is particularly important, when studying the life history, to use only the very freshest material. : Dobell ${ }^{1}$ believes that most of the mistakes which have been made in studying the life history of these organisms have been caused by the examination of degenerated or dead parasites, in which both nucleus and cytoplasm may have been abnormal.

For the study of living amœbæ and cysts it is helpful to mix a particle of stool in a drop of salt solution on one slide and in a drop of iodine solution on another. (Iodine should be used as a strong aqueous solution, in potassium iodide-the stronger the better. Dobell.) The iodine penetrates the cyst wall, and if glycogen be present in the vacuoles, gives it the characteristic color; it also acts as a fixative and renders the nuclei easily visible, so that they may be counted and the details of structure made out fairly well.

Stained preparations are not difficult to prepare, although the process requires some time and care. As in most zoölogical work, wet, rather than dry, fixation is used. Thin smears are made on cover glasses or slides and before they can dry'are covered with or immersed in Schaudinn's fluid. This is a mixture of two parts

${ }^{1}$ Dobell, Clifford, The Amœbæ Living in Man, London, 1919. 
of a saturated solution of bichlorid of mercury in normal salt solution and one part of absolute aleohol. The mercuric solution is prepared by adding to boiling normal salt solution a little more mercury than will dissolve; on cooling, some of the bichlorid crystallizes out. At no stage of the process must the preparation become dry or the smear is worthless.

1. Fix in hot $\left(60^{\circ}\right.$ C. ) Schaudinn's fluid, five to ten minutes.

2. Harden in 70 per cent alcohol ten to thirty minutes, then wash in 70 per cent alcohol to which a few drops of tincture of iodin have been added until it is distinctly colored-ten minutes; store in 70 or 80 per cent alcohol until ready to stain.

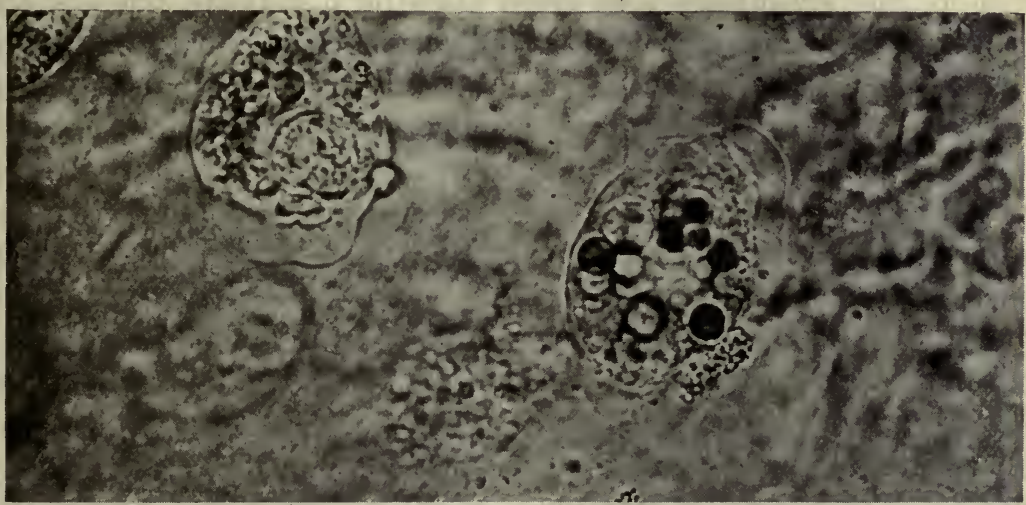

Fig. 122.-Entamoba histolytica. Motile forms showing ingested blood cells and clear rectopiasm. (Army Medical School Collection, Washington, D. C.)

3. The Rosenbusch hematoxylin is quite satisfactory. Transfer the slides to distilled water and change several times until they are free from alcohol, then immerse in 3.5 per cent iron-alum solution for from half an hour to overnight.

4. Stain in the following solution, after rapid washing in distilled water :

(a) 1 per cent hematoxylin in 95 per cent alcohol.

(b) Saturated aqueous solution of lithium carbonate.

Solution $(b)$ is added to solution $(a)$ until the mixture is a cherry red, four or five drops of lithium to 10 c.c. of hematoxylin is sufficient.

The solution is either pipetted onto the slides or they are immersed in it. Stain from twenty minutes to overnight.

5. Wash thoroughly in distilled water.

6. Differentiate with a weak iron-alum solution (three parts of 
distilled water to one of the iron-alum solution is satisfactory), until the slide under the microscope shows the structure of the nucleus; the examination is made in water under a cover glass.

7. When the differentiation is complete the slide is washed in distilled water and passed through graded alcohols, 80, 95 and absolute into xylol and xylol-balsam. This stain is permanent.

Romanowski stains on dried smears may be used, but are not so good.

In fresh specimens Entamœba histolytica presents the following appearance: the vegetative forms are pale, unstained with bile, and are seen to be large bodies, 20 to 30 microns in diameter, consisting of endo- and ectoplasm, and often showing a delicate nucleus and also many inclusions in the digestive vacuoles, principally red blood cells. The organisms for several hours after the stool is passed remain actively motile, pushing out clear, glass-like pseudopods, into which the granular endoplasm pours as the amœba progresses across the field. Even when there is no progression the pseudopods are protruded or retracted first in one then in another direction. There is usually, during motion, a distinct separation of the clear ectoplasm from the granular endoplasm, and the latter, in acute cases especially, contains many red blood cells, oceasional examples showing as many as twenty or thirty. The presence of red blood cells either entire or partly digested is characteristic of Entamœba histolytica. The amœba is sometimes greenish, and it is supposed that this color is due to hemaglobin liberated from the ingested red cells. The pseudopods of this species are clear, glassy and evidently viscid and dense and have given it its name "histolytica," since Schaudinn states that he saw the amoba penetrate the mucous membrane, the pseudopods dissecting apart the epithelial cells. It is much more probable however, that the parasite secretes a strong ferment, which first softens and then dissolves the tissue cells. The nucleus, when the endoplasm is packed with inclusions, may not be visible, but further search will reveal amœbæ showing a nucleus. It is vesicular, with a delicate limiting membrane, and as it is highly refractile, may appear as a clear bright spot. $\Lambda$ s the specimen grows older the amœbæ lose much of their motility and the nucleus may become clearly visible, revealing small chromatic dots or masses adherent to its inner surface and a small central karyosome.

The motile amœbæ cannot be confused with anything else, but when in the resting stage they have been mistaken for swollen and 
edematous epithelial cells. A little attention to the nucleus will prevent this error, since the tissue-cell nucleus is large, distinct, and entirely different from the nucleus of an amœba.

In specimens stained with hematoxylin the finer details, especially in the nucleus, may be studied, but stained preparations are never necessary for clinical diagnosis. In smears from fresh cases vegetative forms only are found, later many degenerative forms appear and during convalescence only eysts may be seen. In stained specimens there is rarely any separation of ectoplasm and endoplasm, but the nucleus is always visible. The cytoplasm is granular and has a coarse honey-combed appearance. The nucleus shows a dis-

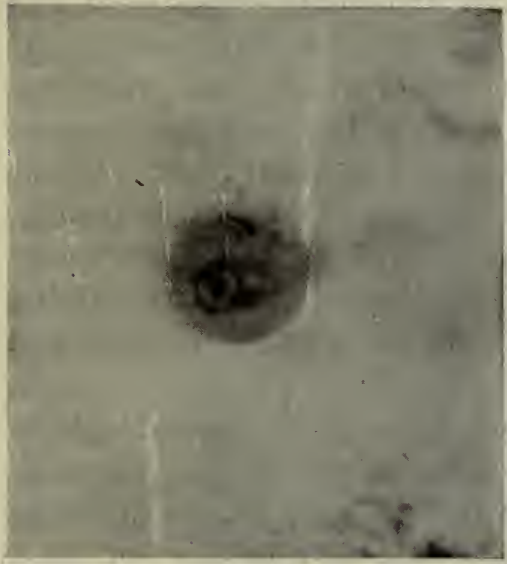

Fig. 123. - Entamceba histoltitica. (Army Med. School Collection, Washington, D. C.)

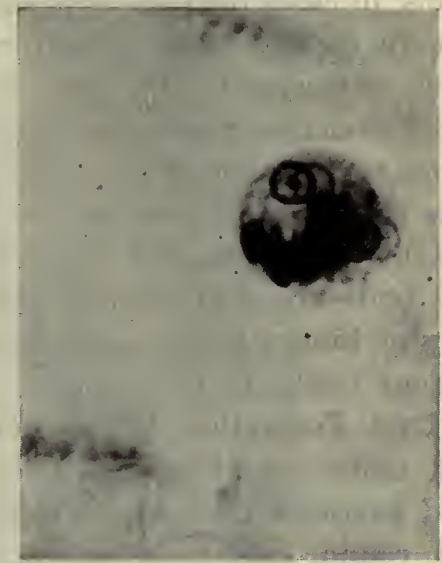

Fig. 124.-Entamosa histolytic.$(\times 1150)$ Cyst, showing four nuclei, two of which are very distinct, and large chromatoid body. (Army Med. School Collection, Washington, D. C.)

tinct, though delicate, limiting membrane, on the inner surface of which are few or many chromatin dots. In the center is a small karyosome, which may show a central body or centriole. The outer zone of the nucleus has a honey-comb structure, in which are imbedded granules of chromatin.

Multiplication in the vegetative stage is by division into two daughter cells; in the intestinal contents, it is difficult to find forms which are undergoing division, but a few have been described and pictured. In experimental dysentery, in the eat, however, all stages of the process may be followed by removing the intestine 
from an infected animal which has been killed and making serial sections of the ulcers. In such sections it can be seen that the chromatin of the nucleus migrates from the nuclear membrane toward the center, and the nucleus elongates, being first oval and later spindle shaped; although the chromatin dots and threads and the achromatic fibers ean be readily seen in the well stained specimens they do not show the usual typical figures of a typical mitosis, but are arranged in an atypical and irregular manner. The elongated nucleus becomes constricted in the middle and a little later divides and the cytoplasm soon follows, leaving two daughter cells.

Degenerative Forms.-These are extremely common in stale stools, in cases during convalescence, or under active treatment, and also in experimental dysentery in the cat, and they have leu to much confusion in the past. The nucleus breaks up into fraguents and chromatin masses are extruded into the cytoplasm in irregular forms, and parts of the cells are apparently budded off. At one time the budding process was looked upon as normal by Schaudinn and his followers, but there is now little doubt that both spores and buds are degenerative changes and that the animal multiplies only by binary fission in the vegetative forms or by the development of four nuclei in the cysis.:

Cyst Formation.-The encystment follows the general rule in that under suitable conditions, an amœba comes to rest, ejects all food particles from the cytoplasm which becomes finally granular, and round, and then secretes a cyst wall, and in this condition passes out on the body with the feces. The cyst of Entamœeba histolytica was first described by Quincke and Roos (1903), and again by Huber (1903), but without making any real impression on the medical or zoölogical opinion of the day. They were rediscovered by Viereck (1907) and called by him Entamœba tetragena, and for a time was believed to be a new species. In fact, Hartmann described a vegetative stage of Entamœba tetragena as Entamœba africana, afterwards accepting the name "tetragena," but it is now apparent that tetragena is merely the end, or cyst stage, of Entamœba histolytica, which had formerly been overlooked by Schaudinn and his followers. Cysts are not easily found in all cases, and it is possible that when treatment is vigorous they never develop. They are, without doubt, the form in which the parasite leaves the body to infect new victims; because of their heavy cyst wall they are quite resistant. The protoplasm of the cyst and the precystic 
stage is granular, but shows no vacuoles nor cell inclusions. The nucleus undergoes division by mitosis first into two, and then four small ring-like nuclei, and the presence of these four nucleated cysts is pathognomonic of the disease. They may be found most abundantly, not in the small amount of mucus which may adhere to the formed feces, but in surface scrapings from the fecal mass. In addition to the four small ring-like nuclei, the cysts contain few or many clumps of chromatin; these in total mass may be many times greater than the nucleus, and it is impossible, therefore, that

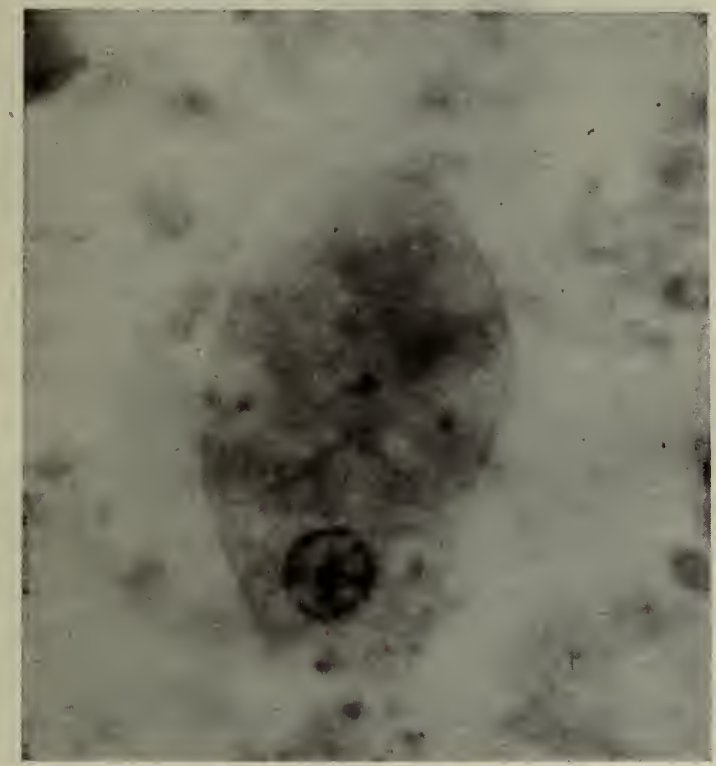

Fig. 125.-Entamoba coli. (Army Med. School Collection, Washington, D. C.)

they are simply extruded from the nucleus; evidently, the chromatin grains, while in the eytoplasm, increase in size and number. In hematoxylin stains no structure in these masses is discernible and their function is unknown; it is possible that they are merely reserve food material; after a time they disappear and one finds cysts quite free of them. The presence, however, of many large chromatin masses in the cysts is quite characteristic of Entamœba histolytica. These masses, which stain deeply with iron-hæmatoxylin, have been given various names: chromatin, chromatoid masses, ehromedia, crystalloids, inclusions, ete. 
Fertilization inside the eyst has not been demonstrated, nor has any other sexual process or conjugation been shown to occur.

In size the cysts from different patients vary considerably, so much so that well recognized races or pure lines occur: Dobell and Jepps (1918) describe five such races, the eysts of which have average diameters of 6.6 microns, 8.3 microns, 11.6 microns, 13.3 microns and 15.0 mierons.

The Dysentery Carrier.-Both convalescent and healthy contact

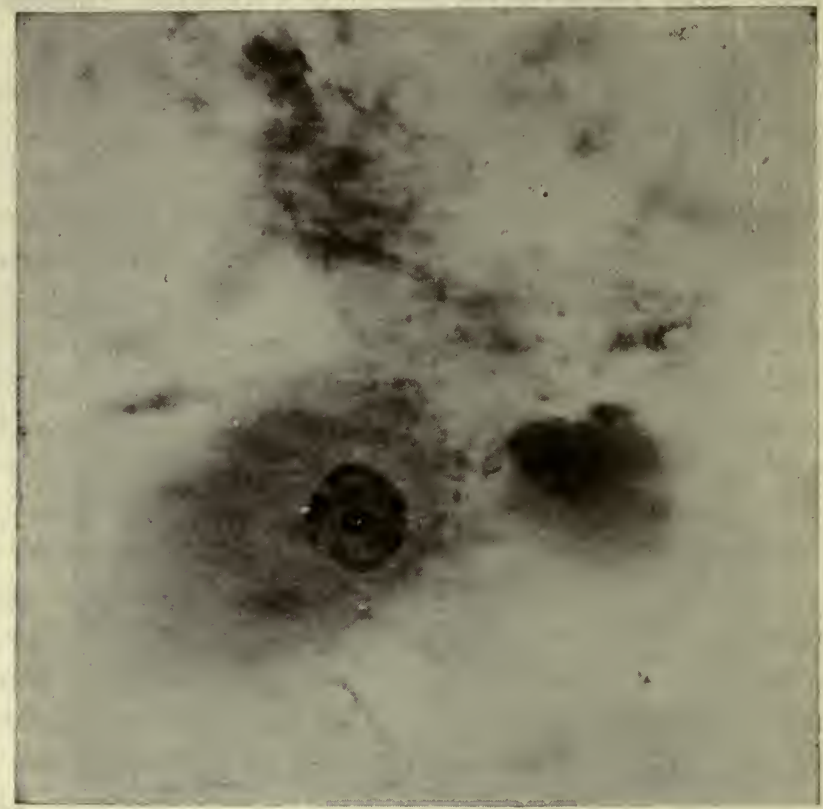

Fig. 126.-Entamceba coli. Typical nucleus. (Army Med. School Collection, Washington, D. C.)

carriers are known, and recent experiments have shown that they are not infrequent, even in the absence of eases of chemical dysentery. To explain the carrier state, it is of course necessary to predicate some insignificant and silent lesions in the colon of the apparently healthy man. There the first part of the life history is lived through and the parasites which eome to lie in the lumen of the intestine eneysts and are found in the feces.

The treatment of amœbic dysentery, to be effective, must be radical and persistent, and may be compared to the treatment of malaria with quinine. For many years the English in India, with a few 
followers in other parts of the world, had treated dysentery with ipecac in massive doses, with wonderful results in some cases and failure in others. The treatment was quite disagreeable and not entirely satisfactory. Vedder, in 1911, examined the various alkaloids of ipecac and found that emetin was strongly amœbacidal, and he recommended its use for this disease. Rogers, in India, following out this suggestion, soon reported excellent resuits, and the drug is now accepted as a specific. It is administered hypodermically in 1/3-grain doses three times a day at first, then twice and later once daily until a total of ten grains has been administered (Vedder). In addition, the patient is put to bed and placed on a milk diet. During convalescence large doses of bismuth subnitrate, a heaping teaspoonful suspended in water or milk, may be given (Deeks). Relapses are of course treated in the same manner as primary infections. As a resuit of the emetin treatment and exact diagnosis the clinical picture of amœbic dysentery has completely changed, and we no longer see the weak and emaciated dysenterics who formerly crowded the wards of tropical hospitals.

Epidemiology.-One significant fact appears in the epidemiology of the disease-it always occurs sporadically and never in explosive. epidemics such as we see in water-borne diseases, like typhoid and cholera; house epidemics are, on the contrary, not uncommon. This fact points to the importance of contact; and flies, as the chief agents in its spread. Buxton ${ }^{2}$ and others have cxamined the droppings and intestinal contents of flies caught in latrines. Buxton found 0.3 per cent of a thousand flies harboring $E$. histolytica cysts, so that there remains little doubt but that the house fly is one of the principal carriers of dysentery. Extreme cleanliness among the servants and in the kitchen will prevent the transfer of histolytica cysts from the ill to the well. The disease has disappeared from the Panama Canal Zone, where it formerly was common, since the introduction of good water and sewer systems and better hygienic conditions.

\section{ENTAMCEBA COLI (grassi 1879) casa grandi et Barbagallo 1895}

This is a haimless parasite of man, and its presence in stools, at one time, gave rise to much confusion, and in the minds of many,

${ }^{2}$ Buxton, P. A., The Importance of the House Fly as a Carrier of $\boldsymbol{E}$. histoly. tica. Brit. Med. Jour., London, 1920, 142. 
threw doubt upon the existence of a form of dysentery due to amœba, since it was found not infrequently in healthy individuals. Schaudinn found it present in the stools of 50 per cent of the persons examined in East Prussia, in Berlin in 20 per cent, and in Istria in 60 per cent. Craig, and Craig and Ashburn found it present in 176 , or 58 per cent, of 307 examinations of healthy American soldiers. Craig was able to follow some individuals for four to six years, during which time they constantly. showed Entamœba coli in the feces, yet never developed dysentery. The organism seems to be

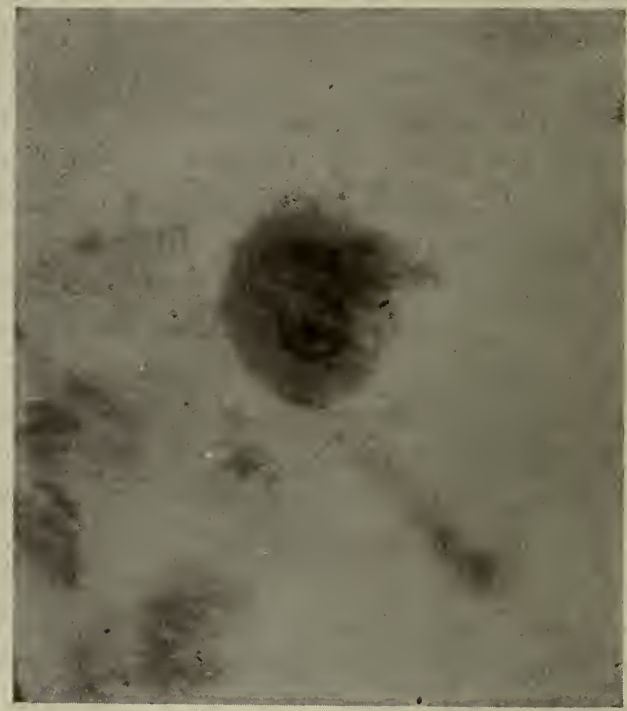

Fig. 127.-Entamciba coli. Small precystic form. (Army Med. School Collection, Washington, D. C.)

found in all countries, regardless of climate. Its recognition and separation from histolytica we owe to Schaudinn, Jürgens, Craig and others.

In size it varies from ten to forty microns, the average being between twenty and thirty. The ectoplasm is never seen except. during movement, and it is then hyaline, and only slightly refractile, and much more fluid than in histolytica. The digestive vacuoles rarely, if ever, contain red blood cells, but are filled with cocci and bacilli, a form of food not scen in healthy histolytica. In general, the vacuoles are larger and more numerous in coli than in histolytica, and the motility is feebler. In fresh specimens the nueleus is rather 
easier to find than in histolytica, and is distinctly outlined by a heavy, double-contoured membrane. The nucleus, as in all amœba, is vesicular, and shows a small eceentric karyosome and dots of chromatin on the nuclear membrane and imbedded in the nuclear network.

Multiplication in the vegetative stage is by binary fission of the nucleus and the cytoplasm, resulting in two daughter cells.

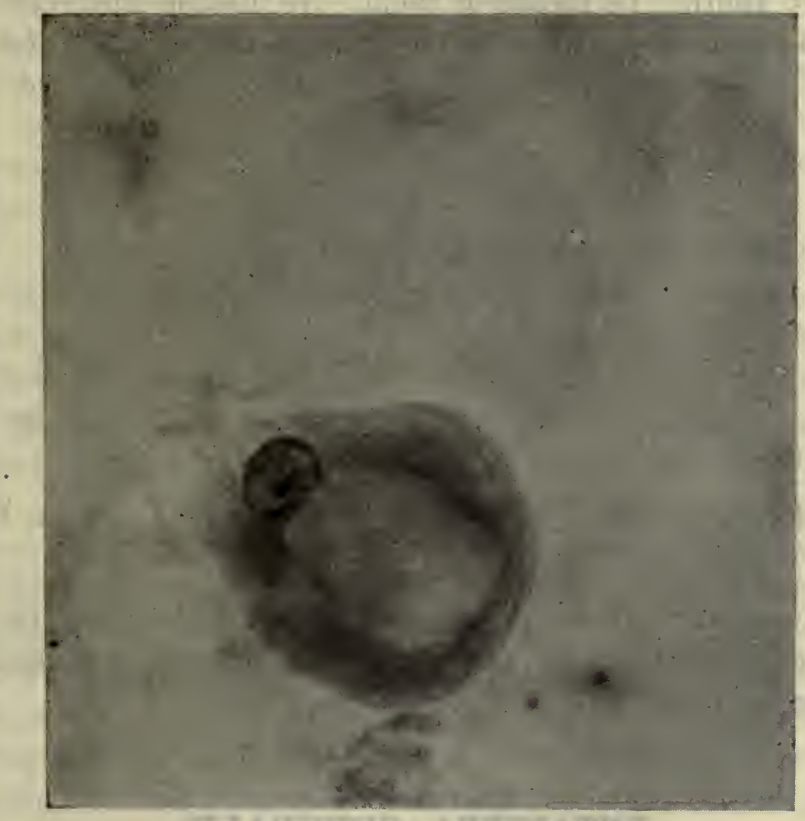

Fig. 128.-Entamoba coli. Cyst showing a large vacuole. (Army Med. School Collection, Washington, D. C.)

Cyst Formation.-This is characteristic of the species, and it furnished one of the prineipal reasons for the separation of coli and histolytica. Before encysting the animal frees itself of all inclusions and becomes clear, transparent, and assumes a spherical form, and secretes a cyst wall. The nucleus divides first into two, then four, and finally eight daughter nuclei; there is a large vacuole containing glycogen which reaches its maximum size in the double nucleus stage; it later disappears and is not seen in the mature cyst. Schaudinn described a complicated autogamy in the cyst, yet later researches by Hartmann and Whitmore show nothing more than 
repeated binary division of the nucleus. The normal number of nuclei in a coli cyst is eight, yet occasionally cysts.are seen in which division has gone on until there are as many as sixtecn. In size the cyst measures 10 to $30 \mu$ or more.

Cats or human beings may be parasitized by feeding material containing coli cysts, and in nature, as the cysts are the resistant forms of the parasite, the infection is probably transmitted from one host to another by means of them. No disease, however, results, though the amobæ continue to be present in the stools for years.

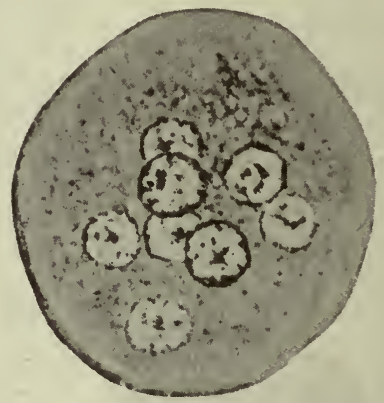

Fig. 129.-Entamceba coli. Cyst showing eight nuclei. (Arch. für Protistenkunde, 1912, xxiv.)

- It is possible that fertilization takes place between the young amœbæ (gametes?), which are liberated when the cyst dissolves in a new host, as is the ease with Entamœba blattæ.

\section{ENTAMEBA GINGIVALIS}

(Gros 1849, emend. von Prowazek 1904)

This amœba is found in the human mouth both in health and disease. It has been described at different times under various names (bucalis, dentalis) by Gros, Steinberg, von Prowazek, Lewald, Smith and Barrett, Chiavaro and Craig, and quite recently has been suggested as the cause of pyorrhea alveolaris by Smith and Barrett and Bass and Johns. It is widely distributed, and has been reported from all quarters of the world.

The organism is easily found in the tartar at the base of the teeth, in cavities in the teeth, and even at the gum margin in healthy mouths. It varies in size from seven to thirty-five microns, averaging 
between twelve and twenty (Craig). Motility is well marked, though it is not so active an organism as histolytica, the pseudopods, mostly short and blunt, being formed of the clear, slightly refractile ectoplasm. The endoplasm is granular, contains the nucleus and many food vacuoles containing nuclei of leucocytes and granular matter, and possibly a few red blood cells. Although Dobell and others maintain that red cells are never found. The nucleus is small, and in fresh specimens is usually invisible. In structure, it is not unlike the nuclei of coli and histolytica; the chromatin granules form a compact and distinct ring obscuring the nuclear membrane. The karyosome is small but distinct and is either central or slightly excentric and is surrounded by a clear achromatic halo. There is no chromatin visible between the karyosome and the nuclear margin.

Before encysting the parasites are much reduced in size, and the cytoplasm frees itself from all inclusions and becomes clear, spherical and immobile. The cysts are small, eight to ten microns, circular and definitely outlined, sometimes with a double contour, and in stained specimens the nucleus is always visible. It is small, averaging only three microns (Craig), making it smaller than in histolytica and coli. The limiting membrane, while not heavy, is distinct, and encloses a nuclear body having very little chromatin other than the small centrally located karyosome. Multiplication occurs only in the vegetative state and by binary fission.

Cyst Formation.-Cysts are rarely observed, and then in small numbers ; the cyst wall is not heavy, but may show a double contour; the protoplasm is clear, free from all inclusions and vacuoles and shows a single small nucleus, but without any signs of multiplication. It is apparent, therefore, that the cyst is a protective stage and has nothing to do with reproduction, which occurs in the vegetative state only. In this respect it resembles the Vahlkampfia. The finding of eysts is so rare that many investigators doubt their existence altogether, and suggest that cysts when found may pertain to some other organism accidentally present in the mouth. Further studies are necessary.

Transmission of the infection could occur directly by contact from person to person by kissing, and a eyst stage is not, therefore, essential to the survival of the parasite.

Although the organism is almost constantly present in pyorrhea alveolaris it is also found in healthy months, and in the absence of all experimental proof, it is doubtful if the organism is of patholog- 
ical importance. Emetin has a decided effect upon many cases of pyorrhea alveolaris, and under that treatment alone the disease may disappear; the nature of its therapeutic action is not yet clear, and does not necessarily indicate any etiological relationship.

\section{ENDOLIMAX NANA (Wenyon and O'Connor, 1917) Burg, 1918}

(Amcba limax, Wenyon, 1916. Entamœba nana, Wenyon and O'Connor, 1917. Endolimax intestinalis, Kuenen and Swellengrebel, 1917.)

This organism was first described in 1917 by Wenyon and O'Connor, by Swellengrebel and Mongkoe. Winoto, by Kuenen and Swellengrebel and by Dobell and Jepps. The last mentioned authors believe it to be the commonest inhabitant of the human bowel. It is agreed by all investigators that it is not pathogenic and that its principal importance is due to the possibility of confusing it with $\mathrm{E}$. histolytica. In the vegetative stage it is quite small, usually measuring 6 to 12 microns in diameter. It usually contains food vacuoles filled with bacteria but no blood cells and never contractile vacuoles. Its movements are sluggish, resembling those of E. coli, the differentiation between ecto- and endoplasm is not clear cut and the pseudopods are few and blunt. Outside the body all movement soon ceases.

The nucleus is seen with difficulty in the living specimen but when the organisms are properly fixed and stained with iron haematoxylin the nucleus becomes the most characteristic feature of the parasite. It is vesicular and 1 to 3 microns in diameter averaging between 2 and 2.5 microns. The karyosome is large and pleomorphic. It consists of a principal mass, which is excentrically located and provided with few or many lobes, which are often almost detached from the main body, being connected only by a narrow isthmus. It is necessary to have well differentiated stains or the details of structure will be overlooked.

If the stool is not fresh the amœbæ may degenerate and the nucleus present quite a different appearance; the segments of the karyosome can no longer be distinguished and the total mass may come to lie on the outer ring of chromatin granules, giving rise to a signet ring appearance. It is best therefore to obtain the freshest possible material for study. 
Cysts.-Before encystment the parasite extrudes all food particles and the protoplasm becomes clear, all movement ceases and the parasite assumes a round or oval form and the secretion of a cyst wall bégins. The mature eyst has four nuclei, whose internal structure resembles that of the vegetative form; that is, there is a karyosome made up of a central mass and more or less detached lobules of chromatin. There are no chromatoid rods in the eytoplasm as in histolytica but there are granules which give the staining reactions of volutin. In iodin solution the eysts are stained yellow; in some may be noted masses of glycogen, but this substance is

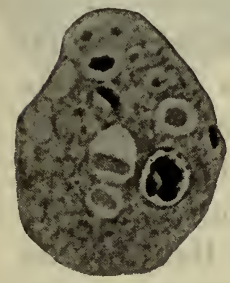

$a$

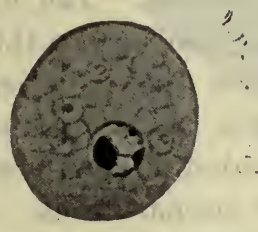

$b$

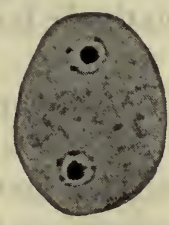

$c$

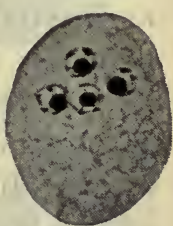

$d$

Fig. 130.-Endolimax Nana. This figure contains figures Nos. 23, 25, 26 and 27 of plate No. 2, Dobell's "Amœbæ Living in Man." The first figure on the left, represents an active amœboid form. The next three show uninucleate, binucleate and quadrinucleate (mature) cysts, respectively. Plate 11 of "Amobæ of Living Man” by Clifford Dobell, published by John Bale, Sons \& Danielsson, London.

commoner in the vegetative, precystic and young cysts than in those which are mature.

Pathogenicity.-It is not believed that the parasite causes any disease; it may however be found in connection with histolytica and thus cause confusion. No treatment so far given has had any influence on the organism.

IODAMOEBA BÜTSCHLII (Prowazek, 1912, Emend. Dobell, 1919)

(Entamœba bütschlii, Prowazek, 1912. Iodin Cysts or I. Cysts, Wenyon, 1916. Pseudolimax, Kuenen and Swellengrebel, 1917.)

Wenyon in 1915 described quite briefly some spherical bodies containing an inclusion which stained with iodin solutions. Later he called these I or iodin cysts and under that name they have been recognized by many as not very rare. They are always found 
in eonnection with some other amœba and never alone, but it is not believed that they have any pathological importance.

Until recently the vegetative stage had not been recognized, but in 1919 Dobell published a complete description, and identified the parasite with the one incompletely described by Prowazek as Entamœba bütsehlii in 1912 .

In size the organism is small, averaging 9 to 13 microns, and it resembles E. coli in general appearance exeept that the nucleus is almost if not quite invisible, particularly in organisms with food vaeuoles filled with bacteria and granular matter.

In stained speeimens the structure of the nueleus serves to distinguish it from other intestinal amœbæ. The nucleus is vesieular and contains a relatively large karyosome, one-third to one-half the diameter of the nucleus. Between the karyosome and the well developed nuclear membrane lies a row of granules of peripheral chromatin which Dobell has succeeded in counterstaining with eosin in well differentiated hematoxlyn preparations. It is the presence of this large karyosome and the layer of peripheral ehromatin dots that permit the differentiation of the organism from E. coli; the two organisms are alike in their food habits.

The cysts are peculiar: and quite unlike those of any other intestinal amœbæ. In shape they are irregular although some are round or oval. In measuring them Dobell averaged the two principal dimensions and found that the average size is 9 or 10 microns, with extreme of 6 to 16 microns. The nucleus of the cysts is single, and is distinguished by having the karyosome, which has already been described, pass to the periphery and come to rest against the nuclear membrane, giving rise to a well marked signet ring appearanee. Peripheral granules of chromatin may still be made out in well stained specimens. Oecasional eysts may have two nuclei but they may be interpreted in the same way that we interpret more than the usual number of nuclei in E. histolytica and coli, as an abnormality.

The iodin masses in the cysts which give this organism its name are well brought out by staining the fresh specimens in iodin solution. As a rule the glycogen mass is single, large and has well defined borders, but its appearance varies with its age; the preeystic amoba shows merely a diffuse brown stain, older specimens appear as above, although occasional specimens may show more than one iodin staining mass. Throughout the cytoplasm may be seen granules 
of volutin and the granules increase in visability as encystment proceeds.

The cysts, like those of other amœbæ, do not withstand drying but they remain alive in feces or water for two or three weeks without undergoing any further development.

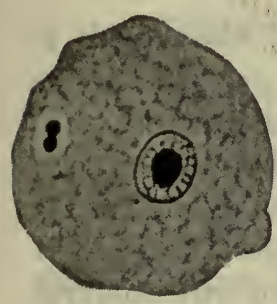

35

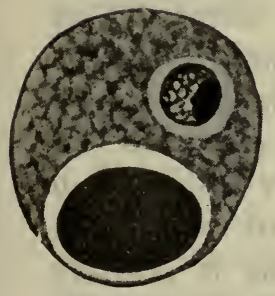

39

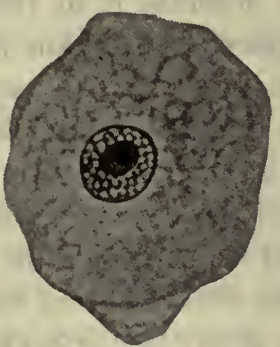

36

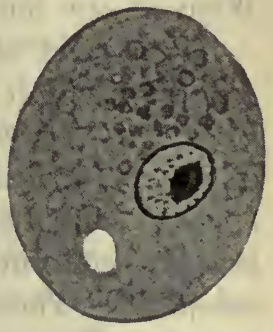

37

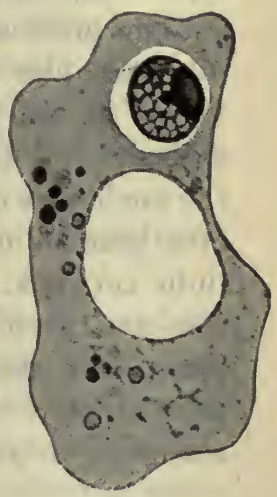

42

Fig. 131.-Iodamcéa Bütschlir. Taken from Dobell as above. The numerals on the plate are Dobell's figure numbers. Nos. 35 and 36 represent precystic amobæ. Observe changes taking place in nuclear structure, freedom from cytoplasmic inclusions, etc. No. 37 is an organism just encystic, volutin granules, pink in cytoplasm, small clear space representing glycogen. No. 39 is a mature cyst, large red glycogen mass. Nos. 40, 41 and 42 are mature cysts showing typical structure of nucleus, volutin granules and glycogen vacuole. Nos. 41 and 42 are cysts of irregular shape often formed by this species and not artifacts. Plate 11 of "Amøbæ of Living Man" by Clifford Dobell, published by John Bale, Sons \& Danielsson, London.

This parasite has been found in the healthy as well as in dysentery cases and there is no evidence that it has any pathogenic power, it disappeared under the administration of emetin and in this point alone resembles E. histolytica. 


\section{DIENTAMCEA FRAGILIS (Jepps and Dobell, 1918)}

This is the only species of the genus and was discovered and described by Dobell and Jepps in 1918, although it is probable that Wenyon saw but did not describe it in 1909 .

Only eight cases of proved infection are known, although it is probable that it is commoner than this would lead one to suppose since the organism dies quickly and the degerierated forms aro soon unrecognizable. It is quite small the average size being 8 or 9 microns with extremes of 3.5 to 12 . The er:0- and endoplasm is sharply differentiated and the pseudopods, consist almost entirely of ectoplasm. The cytoplasm contains food vacuoles filled with bacteria but no red cells are ever seen; the organism is probably, therefore, a pure saprophyte and without pathological importance.

The nucleus is characteristic and is typically double, although a few examples with only one nucleus have been seen. The nucleus varies in size with the size of the cell; its nuclear membrane is delicate and free from chromatin particles. All the chromatin of the nucleus is accumulated in the central karyosome where it consists of a number of granules of varying distinctness all imbedded in a linin ketwork. The discoverers believe that the cell grown to full size and then divides into two uninucleate daughter celis, which increase in size and the single nucleus divides into two. Up to the present time no cysts have been found, and in this it possibly resembles $E$. gingivalis, in which the presence of cysts is still doubtful. 
CLASS II-MASTIGOPHORA (DIESING)

\section{SUB-CLASS-FLAGELLATA (COHN EMEND. BUTSCHLI)}

\section{ORDER I-POLYMASTIGINA (Blochmann)}

THEse are flagellates, possessing three to eight flagella.

Technic of the examination for intestinal flagellates: It is not necessary to administer a cathartic unless motile vegetative forms are desired; cysts are found in formed stools. Small particles of feces are mixed with a drop of water, or dilute stain and the slide is examined with a high, dry lens, after a cover glass has been applied. The stains most used are Gram's iodin and dilute eosin; the iodin besides staining the parasite, colors the iodophilic inclusions. Thin smears may be stained with iron hematoxylin and with eosin methylene blue mixtures.

The flagellates may be cultivated on media used for the cultural amœbæ.

Genus 1.-Trichomonas (Donné, 1837).-These have pyroform (pear-shaped) bodies, rounded in front and tapering to a point behind, provided with three long flagella, often matted together at the anterior end. An internal supporting structure, known as the axial filament or axostyle, is present. There is an undulating membrane bordered by a trailing flagellum that begins anteriorly and runs obliquely backwards.

Trichomonas vaginalis (Donné).-The organism is fifteen to twenty-five microns long and seven to twelve wide; it is provided with three flagella and an undulating membrane. It is found in the vaginal secretion only when it is acid, and in three instances it has been transmitted to the male.

Trichomonas intestinalis ( $R$. Leuckard, 1879).-This parasite is practically indistinguishable from Trichomonas vaginalis. It occurs in the small intestine and appears in the stools during diarrheal attacks, but is probably non-pathogenic. It is readily found in the intestine and colon of mice and guinea-pigs. In fresh specimens 
(protected with a cover glass and vaseline) it is actively motile, but the undulating membrane is difficult to detect until the movement has slowed down.

The presence of cystic forms has been questioned, and two quite different forms have been called resistance or dauer eysts. The earlier one, described by Ucke (1908),

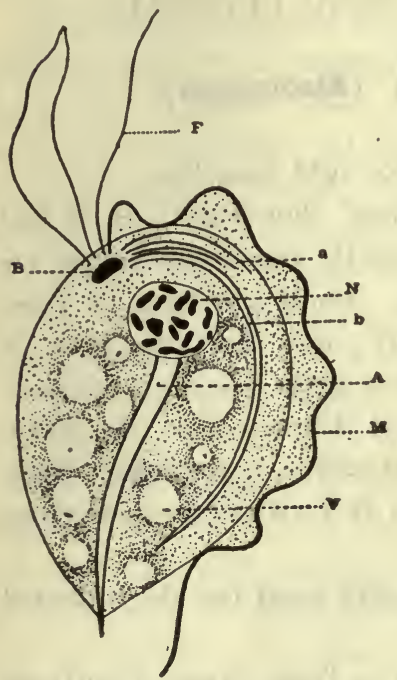

Fig. 132.-Trichomonas INTESTINALis. (After Brumpt, "Précis de Parasitologie," 1914 ed.) Bohne and von Prowazek (1908), and Benson (1910), is a fairly large body, showing a double contour and a central homogeneous mass, perhaps food material, and an outer ring-like body containing two or more nuclei. Brumpt and Alexieff believe this form to be a fungus, having no relation to the trichomonad, and have called it "Blastocystis hominis." Lynch" agrees with these authors, and describes an altogether different body as the resistant form. It is six by eight microns in size and perfectly symmetrical in shape. The wall is distinct, and there is a clear space between it and the body of the parasite. The nucleus, undulating membrane and flagella remain visible in the cyst, but Lynch was unable to detect any change in the parasite indicating intracystic multiplication.

Infection takes place probably by contact, and, as in typhoid fever, food, fingers and flies carry the resistant forms from one individual to another. Among the natives of tropical countries infection is almost universal, but the parasites are rarely seen in the large eities of the North.

Genus 2.-Tetramitis mesnili (Wenyon, 1910).-Macrostoma mesnili, Chilomastix mesniti, Fanapapea intestinalis. This organism, first described by Wenyon, from a native of the Bahamas, differs from trichomonas by the possession of a deep groove or cystostome, in which is found the undulating membrane. It is present in diarrheal discharges, but its pathogenicity is doubtful.

Genus 3.-Giardia intestinalis (synonym, Lamblia intestinalis). -The giardia are bilaterally symmetrical, pear-shaped organisms,

'Lynch, Kenneth M., Jour. Parasitol., Urbana, 1916, iii, 28. 
provided with a sucking disk anteriorly. There are eight pairs of flagella, the two posterior ones being continuations of longitudinal axostyles. The nueleus is first dumb-bell shaped and later divided into two separate nuclei. Cysts are found, and according to Schaudinn conjugation occurs in them with the development of four nuclei. The young parasites attach themselves to the surface of epithelial cells of the small intestine by the sucking disk, but even when present in large numbers do not produce any characteristic symptoms. Giardia infestation is quite common in children in the United States, and not uncommon in adults. Maxcy* found 20 per
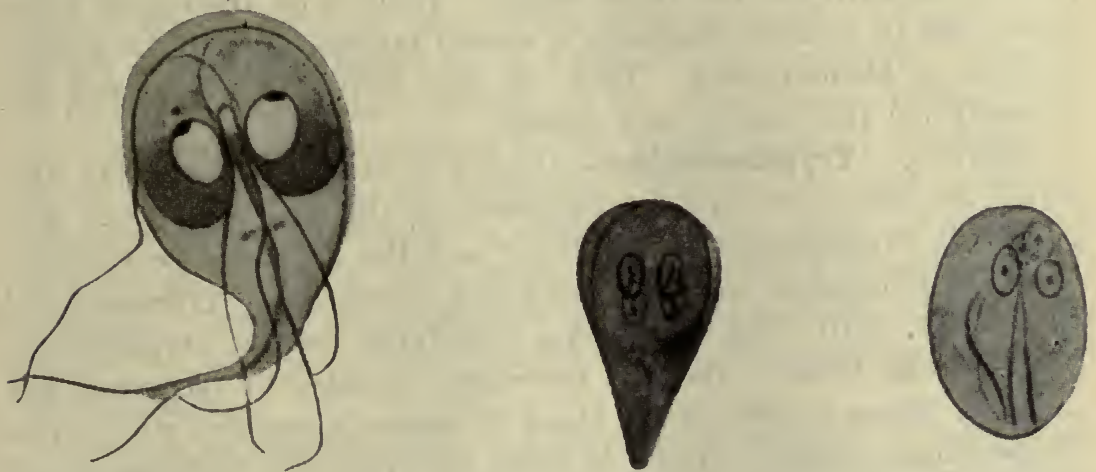

Fig. 133.-Lamblia intestinalis. Cyst formation. (After Doflein, "Lehrbuch der Protozoenkunde.")

cent of children infested and Kofoid found 6 per cent of young healthy soldiers harboring the parasite. The same or like parasites are present in mice, rats, dogs, eats, and sheep. Transmission is by contact, as in trichomonad infections.

\section{ORDER II-PROTOMONADINA}

The Protomonadina, another order of the Flagellata, have less than three flagella, and are divided into the Cercomonadida, Bodonida and the Trypanosomidae.

GENus 1.-Cercomonadidæ-Cercomonas hominis (Davaine, 1854).-As originally described, this organism has a pear-shaped body,

* Maxcy, Kenneth F., Johns Hopkins Hospital Bull., 1921, 32, 166. 
drawn out to a point posteriorly, is armed with a single flagellum in front, and has no undulating membrane. It is a doubtful species and of no present importance.

Genus 2.-Bodonidæ-Prowazekia (Hartmann and Chagas).These organisms, the only examples of the Bodonida of medical interest, are of some importance, since they have been cultivated from human feces on agar plates. The genus was founded for Prowazekia cruzi, a species discovered in human feces in Brazil. Other species are urinaria, asiatica, parva, weinbergi and javanensis. There are two flagella, arranged in the heteromastigote manner, that is, one flagellum projects forward and one trails behind. There is no undulating membrane, but in stained specimens a second nucleus is seen, the kinetonucleus or blepharoplast. They are also found in water, and are probably not the cause of any disease.

Genus 3.-Trypanosomidæ.-History of the genus.-In 1841 Valentine discovered the first hemoflagellate in the blood of a trout now known as Trypanoplasma valentini,* and the following year Gruby described a flagellate in frog's blood and named it a "trypanosome." It was not. until 1878 that Lewis discovered the rat trypanosome, Trypanosoma lewisi. The first pathogenic member of the genus was noted by Evans in 1889 in the blood of Indian horses sick with surra, Trypanosoma evansi. Bruce in 1894 described the trypanosome of Nagana, Trypanosoma brucei a horse disease of Zululand, and also demonstrated its transmission by the tsetse fly, glossina palpalis. In 1894 to 1899, Rouget, Schneider and Buffard found the trypanosome of Dourine, or "mal de coit," among Algerian horses. Elmassian described, in 1901, the South American horse disease, "mal de caderas," and discovered the parasite, Trypanosoma equinum. Since this time a large number of new species have been discovered, the more important of which will be described.

Morphology.-The morphology of the trypanosomes, while subject to many variations in detail, is still uniform as to the characteristics of the genus, so that there is little difficulty in immediately recognizing the parasite. The body is long and sinuous, tapering anteriorly to a fine point called the flagellum; the posterior end is never so delicate and is often quite blunt. All contain two nuclei, the larger being called the trophonucleus and the smaller the kinetonucleus. The trophonucleus is usually located midway in the length of the body,

* Gauthier, M. C. R. Acad. Sci., 1920, 170, 69. 
and the kinetonucleus behind it, often at the posterior extremity. 'The flagellum arises from a centriole (blepharoplast), which is located close to or in the kinetonucleus, and quickly reaches the surface of the body, when it turns forward and forms the border of the undulating membrane, a thin fold of periblast running the entire length of the body, and is often continued further forward as delicate filament. During life the undulating membrane has a constant wave-like motion.

Transmission from one animal to another is usually by means of some blood-sucking invertebrate. Two possible forms of transmission have been recognized, the direct and indirect or cyclical; the direet form is used in the laboratory when transferring blood with a hypodermic syringe from an infected animal to a healthy one, and it also occurs in nature, although not so frequently as the second. Dourine, or mal de coit, is the best example of the natural direet method. The cyclical method is exemplified in the transmission of Trypanosoma lewisi by the rat flea, Ceratophyllus fasciatus, in which inseet the trypanosome passes through a complicated life cycle. Whether the parasite in the inseet ever passes from parent to offspring is still doubtful. Among fishes, reptiles and amphibians the parasites are carried by leeches, in. whose intestinal tract they undergo a cycle of development.

Just as in malaria, there is usually an alternation of hosts, from invertebrate to vertebrate, a part of the life cycle being passed in eaeh. In the blood of the vertebrate is found the fully developed trypanosome, and in the intestinal tract of the invertebrate, crithidial. and trypanomonad types, which are characterized by having the kinetonucleus placed in front of or close beside the trophonucleus and by having a rudimentary undulating membrane.

Cultivation.-In 1903 Novy and $\mathrm{MacNeal}^{2}$ first obtained pure cultures of trypanosomes on artificial media. The medium devised by them is prepared by equal parts of nutrient agar and defibrinated rabbit blood. After the agar has been melted and cooled to about $50^{\circ}$ C. an equal quantity of rabbit blood is added, mixed and allowed to cool. "The tubes thus prepared are allowed to set in an inclined position, after which they are at once inoculated. It is essential that the surface of the medium be moist and soft, and if this is not the case, the tubes should be placed in an upright position until some water of condensation accumulates at the bottom. The initial

${ }^{2}$ Novy and MacNeal, Contrib. Med. Research (Vaughan), Ann Arbor, 1903, p. 549 . 
culture usually requires a week or more, although not infrequently fairly rich growths may be obtained in three or four days" (Novy).

Trypanosoma rotatorium.-Gruby deseribed and named this hemoflagellate in 1843, and it is, therefore, the type species of the genus. The organism is widely distributed throughout the world, and is found in Rana esculenta, Rana temporaria and Hyla arborea: the organisms are, however, not very numerous in any single frog. It is most often found during the spring and summer months, rarely in winter.

Morphology.-Both body and undulating membrane are broad, the eytoplasm is granular, and toward the straight side shows striæ, probably indicating the presence of myonemes. The trophonucleus
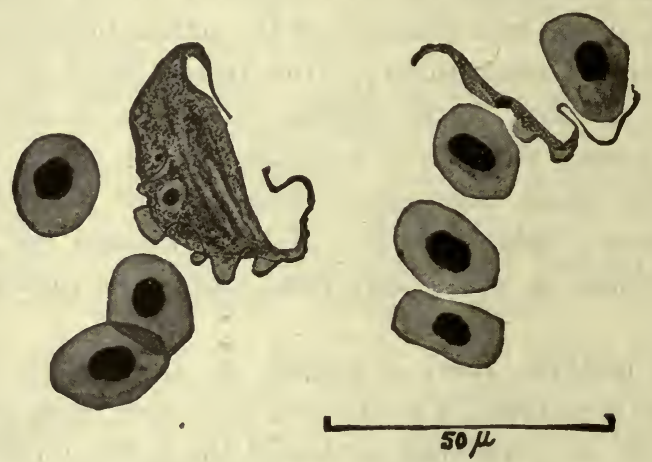

Fig. 134.-Trppanosoma Rotatorium in Blood of Frog. (After MacNeal "Pathogenic Microorganisms," published by P. Blakiston's Son \& Co.)

is large, lies near the middle. of the body and near the undulating membrane; the kinetonueleus is smaller, lies posteriorly and stains deeply; the flagellum which originates near the kinetonucleus turns forward, forming the border of the undulating membrane, and is continued forward as a short flagellum. The posterior end is usually drawn out to a stubby point. The fully developed organism is large, being 40 to 80 mierons long by 5 to 40 wide. One striking thing about this parasite is its tendeney to pleomorphism.

Multiplieation in the blood stream of the frog is by binary fission; in addition, a form of multiple division occurs in the viscera, preceded, aceording to Machado, by conjugation of sexually differentiated forms. The merozoites liberated from the, mother cell are small trypanosomes, which in turn grow to large size, thus explaining the pleomorphism of the parasite. 
Cultures have been obtained by Lewis and Williams on the blood agar of Novy and MacNeal in which a great variety of forms may be seen; the method of transmission is unkown, but the infection is probably conveyed by leeches. Many other trypanosomes have been found in fishes, frogs, and reptiles all over the world.

Trypanosoma lewisi (Kent).-This, one of the longest known and commonest forms, has been studied more completely than any other organism of its class. It oecurs in a large proportion of rats throughout the world, twenty-five to one hundred per eent being infected, and since it is non-pathogenie, it is a convenient organism íor research. It may be passed from wild to white rats without difficulty, by inoculating the latter with a small quantity of citrated blood containing the organisms. At first the parasites are few, but after the lapse of three or four days, lakge numbers may be found; the condition of rapid multiplication lasts from eight to fourteen days, and is sueceeded by a period of a month or more, during which time the parasites gradually diminish in number, finally disappearing completely, rendering
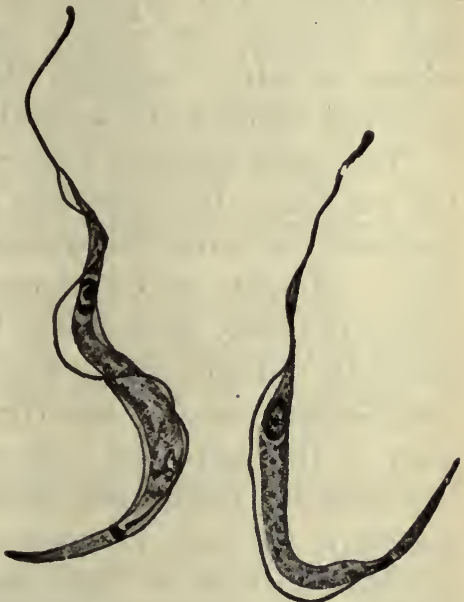

Fig. 135.-Trypanosoma Le w is I. (After Doflein and Minchin. MacNeal, "Pathogenic Microorganisms," published by P. Blakiston's Son \& Co.) the animal immune from further infection, the immunity being eomplete. The serum of an immune rat has a certain protective power, and when inoculated simultaneously with blood eontaining trypanosomes, may prevent the infeetion. No other animals are susceptible.

The blood should be examined in both fresh and stained specimens. In fresh specimens, because of the rapid, lashing movements of the parasite, the organisms are particularly easy to find. The details of structure, however, do not appear except in spreads stained with some of the modifications of the Romanowski stain, such as Wright's or MacNeal's.

In the adult stage the organisms are quite uniform in size and shape, being 27 or 28 microns long and 1.5 to 2.0 microns broad; the posterior end is long, tapering and pointed; the kinetonucleus 
oval and flattened; the trophonucleus is located near the anterior end, and the undulating membrane, while distinct, is relatively narrow. The endoplasm is fincly granular, and by careful focusing the bodywall or periblast may be seen.

Multiplication in the rat is rapid, and many young forms are seen; these are smaller, stain more deeply, and vary much more in size than the adults. Dividing forms are common, the division being longitudinal and unequal, the parent retaining the flagellum. Multiple division also occurs, resulting in the production of rosettes, whose structure suggests that repeated longitudinal division has occurred without the separation of the daughter cells.

The inscet hosts are two: the rat fiea, Ceratophyllus fasciatus, and the rat louse, Homatopinus spinulosus; the former being the right host and the latter the wrong one, since in it development is incomplete. Minchin and Thompson ${ }^{3}$ have studied the cycle in the flea, which is briefly as follows: When the injected blood and parasites reach the midgut of the flea, the trypanosomes lose their flexibility and become more or less rigid, and are able to penetrate the outer wall of the epithelial cells of the stomach. Once inside the cell, the parasite folds upon itself and grows to large size; the nuclei multiply, the body becomes spherical and divides up within its own periblast into six or eight daughter cells, all actively moving within their common envelope. This becomes tense and finally bursts, liberating the young trypanosomes within the epithelial cell, through whose wall they soon escape into the lumen of the stomach. This form of multiplication may be several times repeated, after which the young trypanosomes pass down the intestine to the lower end to begin the rectal phase. There the parasites in large numbers are found attached to the epithelial cells by their flagella. Rapid multiplication takes place by repeated fission and the parasite becomes crithidial in form, that is, it loses its undulating membrane, becomes short and stubby, and the kinetonucleus moves forward close to or in front of the trophonucleus. Ultimately some change back to minute trypanosomes, and these, when regurgitated or passed in the feces, serve to infect the next victim. The rectal phase, when once established, lasts for several months or perhaps indefinitely, making every infected flea a chronic carrier.

Trypanosoma evansi.-Surra is a disease of horses and mules, camels, elephants, buffaloes, and dogs, which prevails in India and

${ }^{3}$ Minchin and Thompson, Quarter. Jour. Mier. Se., Lond., 1915, 1x, 463. 
other parts of Asia, and also in the Philippines and Northern Australia. The Philippine outbreak was traced to animals returned from China after the Boxer outbreak; for at that time American troops came into contact with native Indian troops and their animals.

The trypanosome causing the disease was discovered by Evans in 1880 . The clinical course of the disease is marked by an irregular recurring fever, with many remissions, during which the parasite cannot be demonstrated in the blood, although it is not difficult to find during the febrile period. The animal is anemic, weak, emaciated, and may show an ecchymotic eruption on the abdomen. The course of the disease may be either short or long, but leads almost invariably to death. In camels it lasts from two to four years, often without symptoms until near the end, and these animals probably act as chronic carriers.

Morphology.-Morphologically, the parasite is very like the Trypanosoma brucei of Nagana, yet, as a rule, the trophonucleus lies nearer the anterior end than in brucei, although it may be impossible to distinguish in smears between the two.

The disease is carried by biting flies, tabanido and stomoxys, and also by fleas.

Trypanosoma brucei.-Nagana is a well-known horse and animal disease of Africa, which causes an enormous economic loss and has greatly interfered with the development of the country. The parasite was discovered by Bruce in 1895. Among the natives it is known as tsetse fly disease, and investigation has incriminated Glossina morsitans as the carrier. Clinically, the disease in horses is much like the Surra of India; the native name for the disease, nagana, means weakness. Nearly all the larger animals are susceptible to either natural or artificial infection, yet man is apparently immune,

Iorphology.-Morphologically, it resembles closely most of the other pathogenic trypanosomes, and Minchin makes it the type of a group of pathogenic trypanosomes, all closely resembling one another and possibly descended from one common ancestor: the group consists of brucei, gambiense, evansi, equiperdum, rhodesiense, and hippicum. The organism is less slender than lewisi and has a wider undulating membrane. The posterior end is relatively short, the trophonucleus lies in the middle of the body and the kinctonucleus at the extreme posterior end; a vacuole is placed just in front of the latter. In length the parasite measures twenty-five to thirty-five microns and is one and 
a half to two and a half microns in width; inultiplication in the blood stream is by binary fission.

Transmission is by means of the tsetse fly, Glossina morsitans, and perhaps Glossina pallidipes and Glossina fusca. The fly may transmii: the disease directly after infection, acting as a mere mechanical carrier, but it is more probable that a cyclical development of the parasite takes place in the fly, after which it remains infectious for
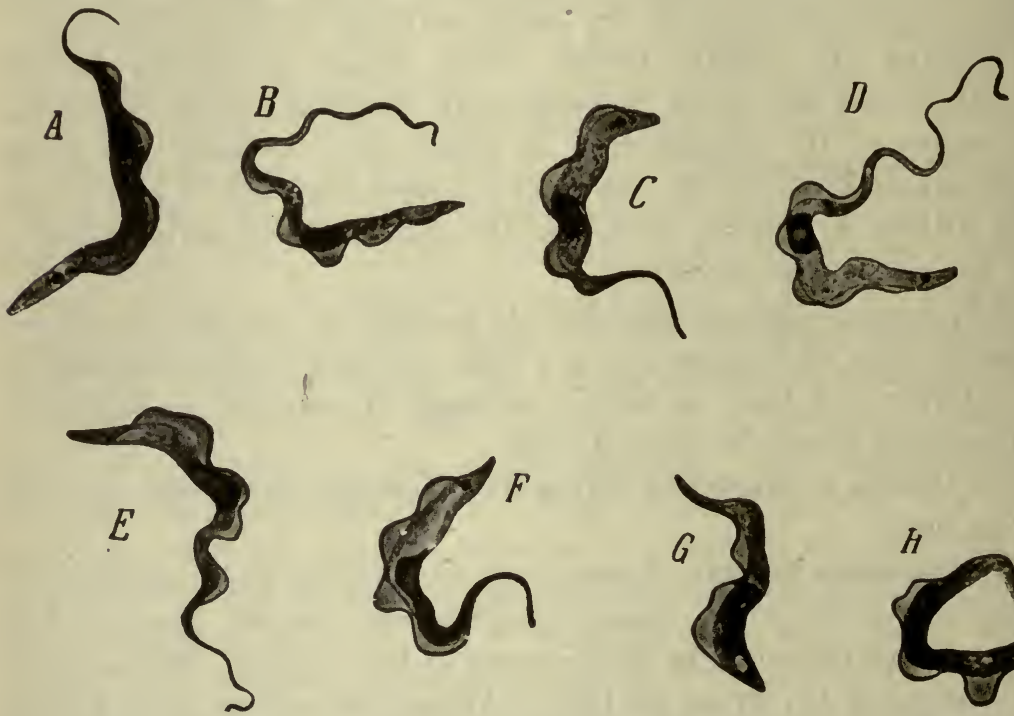

Fig. 136.-The Most Important Trypanosomes Parasitic in Vertebrates. $A$, Tr. lewisi; $B$, Tr. evansi (India); $C$, Tr. evansi (Mauritius); $D$, Tr. brucci; $E$, Tr. equiperdum; $F$, Tr. equinum; $G$, Tr. dimorphon; $H$, Tr. gambiense. ( $\times 1500$.) (From Doflein after Novy. MacNeal, "Pathogenic Microorganisms," published by P. Blakiston's Son \& Co.)

a long period. It has been shown that after the first few hours the fly is not infectious again until the lapse of eighteen days, when its bite onee more conveys the disease, and trypanosomes may be found in the intestinal eanal, the body cavity, the salivary glands and in the proboseis. Studies of the cycle in the fly show that only about five per cent of the flies permitted to feed on sick animals beeome ehronie earriers. The work of Bruee and others has shown that the trypanosomes are more or less harmless parasites of the big game animals of Africa, which therefore are believed to act as a reservoir, from which the disease is transferred to the domestic 
animals by the tsetse fly. The distribution of Glossina is not uniform, as they are only present in ecrtain definite areas called fly belts. Since the disease does not spread in the absence of the larger wild animals, it has been proposed that all big game be exterminated as a prophylactic measurc. Mice and rats are susceptible and die in six to fourteen days after inoculation; guincapigs are more resistant, and may show one or more relapses within two to ten weeks. It has not been possible to immunize larger animals, although a certain degree of success has been obtained with the smaller animals used in the laboratory.

Cultures have been grown on artificial media, yet not so readily as with lewisi and avian trypanosomes. The medium recommended by MacNeal contains the extractives of one hundred and twenty-five grams of meat, ten of pepton, five of salt, and twenty-five of agar to the liter; to this is added twice its volume of warm, defibrinated rabbit's blood. The blood agar slants should be soft and moist when inoculated. Filtrates from cultures are not toxic, the toxin apparently being liberated, according to MacNeal, from the body of the disintegrating trypanosome.

Trypanosoma hippicum (Darling).-The disease eaused by this trypanosome in horses and mules has been known in Panama for many years under the name of "Murrina de caderas" or "Derrengadera de caderas," the latter term being used when paralysis of the posterior extremities is the dominant symptom; both names indicate a weakness of the hind quarters. The symptoms are weakness, emaciation, and, sooner or later, conjunctivitis and subconjunctival ecchymosis, and anemia. The horses and mules affected are obviously weak, and while in the stall, pull back on the halter, or stand with straddling hind legs.

The incubation period in animals used for experiment is less than a week, a few animals lose weight rapidly and die within a few days, others live for several weeks.

Treatment, including the use of arsenical preparations, is without effect, and all infected animals should be destroyed.

The disease is apparently transmitted directly by flies, which carry blood and serum from ulcers and abrasions on infected to healthy animals.

Morphology of the Parasite.-The trypanosome is sixteen to eighteen microns long and two microns wide. The kinetonucleus is about two microns from the posterior end, the trophonucleus 
about eight to ten microns from the same point. The posterior end is blunt and the cytoplasm usually contains numerous basophile granules; the undulating membrane is well developed and a chromatin filament runs from the kinetonucleus to the tip of the flagellum. 'The large kinetonucleus distinguishes this organism from Trypanosoma equinum.

Pathological Anatomy.-Aside from the emaciation, edema of the belly wall, conjunctivitis and subconjunctival ecchymosis, there is usually excessive fluid in the body cavities, an enlarged spleen, and, what is more characteristic, small petechial spots on the capsule of the spleen and in the cortex of the kidney and in the endo- and pericardium and occasionally on the pleural surfaces.

Prophylaxis consists in the destruction of all infected animals; the protection of wounds and ulcers in otherwise healthy animals by dressings and, wherever possible, the use of fly screens about the stables.

Trypanosoma equiperdum (Dourine).-This organism is the cause of dourine, a disease of horses and donkeys, which is usually transmitted by coitus, but may be carried by biting flies, stomoxys. The organism was first described by Rouget in 1894; it resembles bruce i in many ways and produces a progressive, fatal disease of great economic importance. Formerly it was present throughout the greater part of Europe, but is now almost limited to the shores of the Mediterranean. From time to time it has been introduced into the United States and Canada by blooded French stallions and has spread into parts of the Northwest.

The clinical course may be divided into a stage of edema, lasting about a month, during which there is a painless, soft swelling, limited to the genitalia and the belly wall. This is followed by the stage of eruption, during which plaques, or round edematous areas, are found under the hide on the flanks and hind quarters, and sometimes on thighs, shoulders and neck; this stage is short, lasting about a week. It is followed by the third stage of paralysis and anemia; the animal loses flesh and strength, develops superficial ulcers, conjunctivitis, keratitis, and ultimately paralysis, leading to death in two to eighteen months.

The trypanosome is found most readily in the serous exudate from the ulcers, as it is infrequent in the peripheral circulation; in this respect it resembles the treponema of lues. The organism is about twenty-five microns in length and possesses a clear cyto- 
plasm, free from granules, except when propagated in white mice, when they are plentiful.

Diagnosis by Complement Fixation.-E. A. Watson, ${ }^{4}$ of Canada, has shown that it is possible not only to diagnose the disease when the clinical signs are clear, but also to determine the existence of its non-clinical, obscure and latent forms. Horses may tolerate an infection for one to three years, during which time they are capable of conveying the disease and yet remain normal in health and

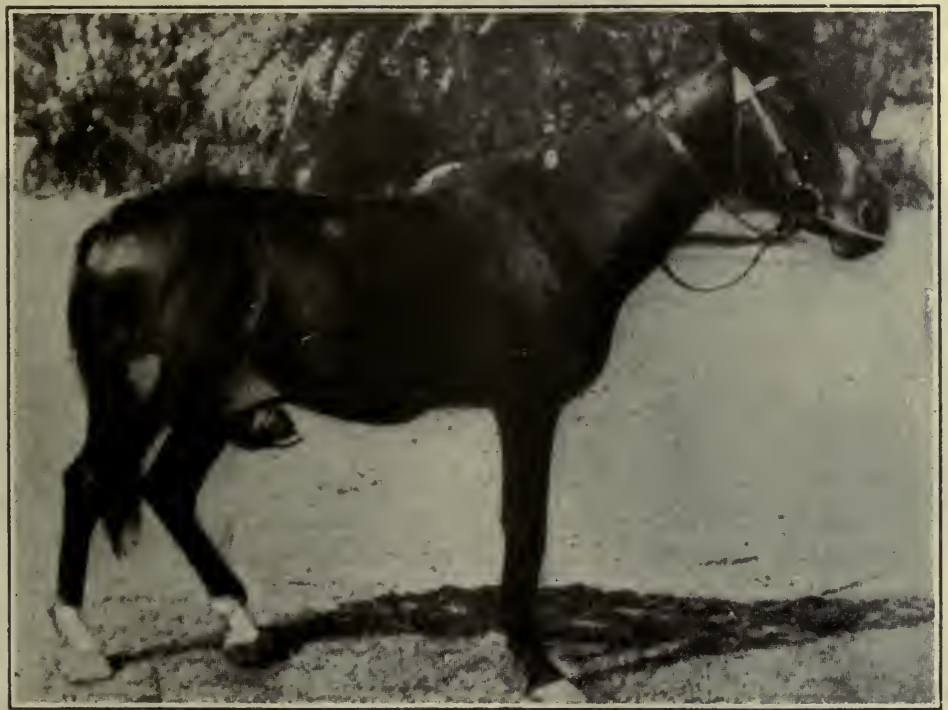

Fig. 137.-Dourine. Showing swelling of genitalia and plaques on the skin. (After Kolle and Wassermann, "Handbuch der Pathogenen Mikro-organismen," 2te Aufl., 1913.)

general appearance, and this method of diagnosis is, therefore, invaluable.

Watson obtains the antigen by inoculating a large number of white rats with Trypanosoma equiperdum, collecting their blood when teeming with trypanosomes, and separating them from the erythrocytes and plasma by washing and centrifuging. Each of ten to twenty rats receive 0.3 c.c. of blood rich in trypanosomes intraperitoneally, and at about the end of the third day, when the organisms are very numerous, the rats are bled into eitrate solution. By

E. A. Watson, Parasitology, Cambridge, Eng., 1915, VIII, 156. 
repeated washing the organisms may be separated, as a pure white layer overlying the erythrocytes. This mass of organisms is killed and preserved by a formalin-glycerin mixture, after which its antigenic strength is standardized by titration in the usual way. The test, a pure culture of trypanosomes being used as antigen, is specific and is not positive in any other disease of horses.

Trypanosoma avium.-This parasite was first described by Danilewski in 1885. In 1905 Novy and MacNeals found trypanosomes in 8.8 per cent of 431 American birds. Although there are doubtless several species, the most common is Trypanosoma avium, a para-
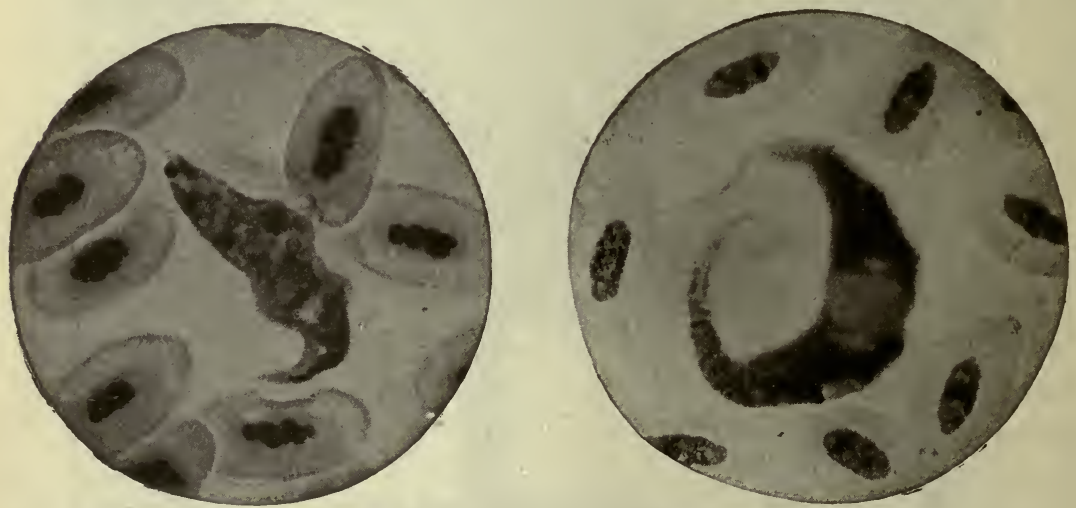

Fig. 138.-Trypanosoma Avium in Blood of Common Wild Birds. (After Novy and MacNeal. MacNeal, "Pathogenic Microorganisms," published by P. Blakiston's Son \& Co.)

site twenty to seventy microns long and four to seven microns wide. They are found in the blood over long periods of time and do not appear to be pathogenic. Cultures are easily made and kept alive for long periods by weekly transfers. The mode of transmission is unknown.

This was the parasite which was confounded in 1904 by Schaudinn with developmental stages in the life eycle of Hemorproteus noctua and Hemorproteus ziemani with resulting confusion in the study of trypanosomes and hemoeytozoa, and it is only reeently that the error has been generally acknowledged.

Trypanosoma gambiense (Sleeping Sickness).-Two names have been given to the disease eaused by this parasite, both of which are

'Novy and MacNeal, Jour. Infect. Dis., Chicago, 1905, ii, 256. 
now recognized as stages in one and the same infection, human trypanosomiasis : they were trypanosome fever, and slceping sickness. It is a chronic infection characterized by fever, lassitude, weakness, wasting, and, in its terminal stages, by a protracted lethargy. Sleeping sickness and trypanosome fever had long been known in tropical Africa, and the disease at present is widespread and the

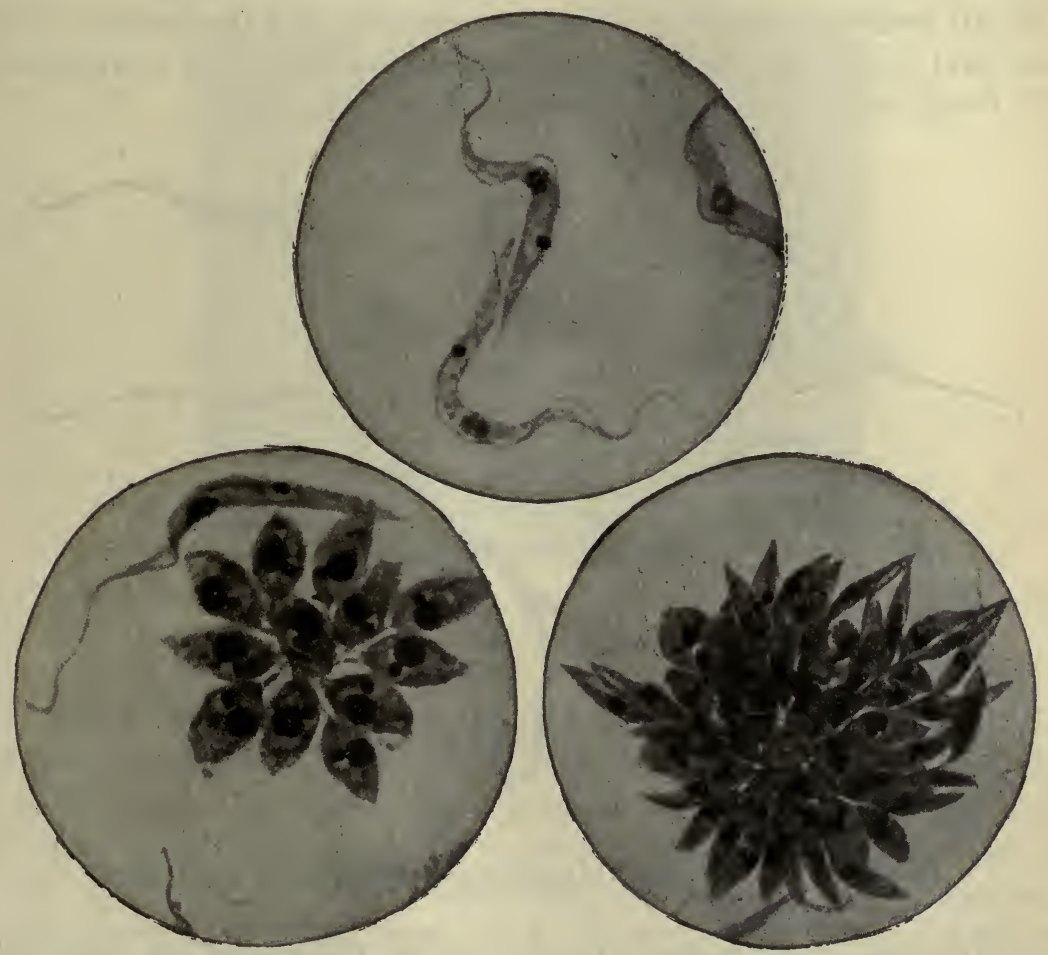

Fig. 139.-Trypanosoma Avium in Culture on Blood Agar. ( $\times 1500)$. (After Novy and MacNeal. MacNeal, "Pathogenic Microorganisms," published by P. Blakiston's Son \& Co.)

cause of tremendous mortality. It is estimated that one hundred thousand deaths occurred during the ten years ending in 1910. It is endemic in the lake region of Central Africa, and in the Congo basin. It was early introduced into Martinique in the West Indies, but did not spread and has now died out.

Dutton and Todd found the parasite in 1901 in the blood of an Englishman in Gambia, who died after a febrile illness of two years' 
duration; Castellani in 1903 found the parasite in the cerebro-spinal fluid of well-marked cases of sleeping sickness occurring among natives of Uganda.

Clinical Signs.-The disease begins with slight febrile attacks, headache and increasing weakness, emaciation, swelling of the eyelids and enlargement of the lymph nodes. The temperature increases, edema of the extremities appears and the spleen enlarges. During the last stages nervous symptoms predominate and the patient sleeps day and night, but may have periods of excitement or convulsions, yet finally sinks into deep coma and dies of exhaustion.

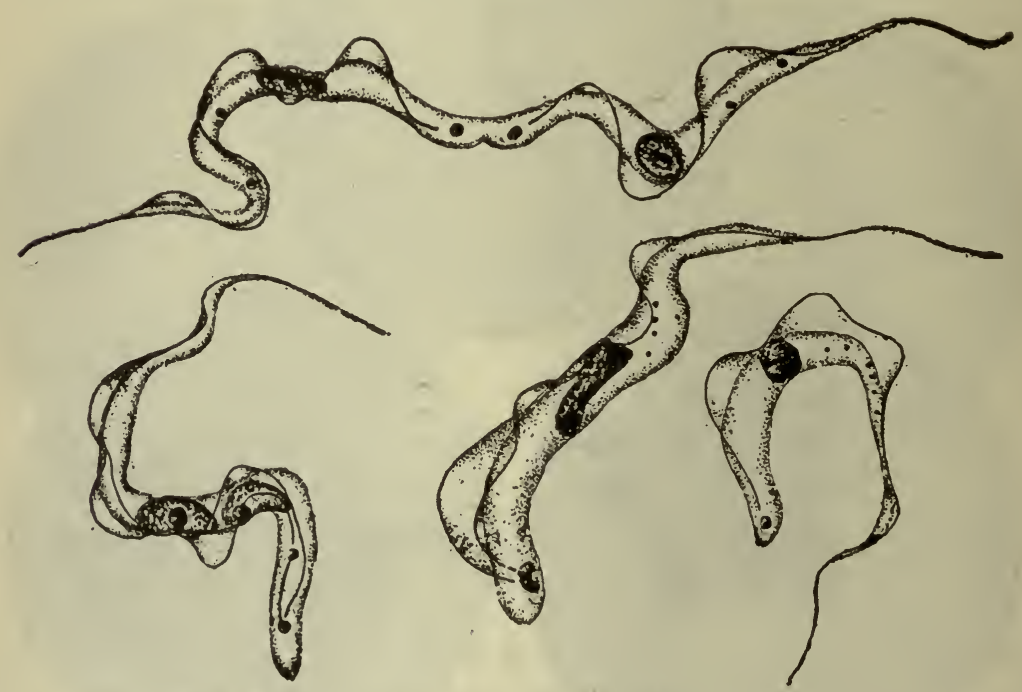

Fig. 140.-Trypanosoma Gambiense. Calkin, "Protozoölogy."

Etiology.-The disease is transmitted by the bite of the tsetse fly, Glossina palpalis, which is apparently able to transmit the infection mechanically immediately after biting an infected host, yet in most flies the trypanosomes disintegrate and disappear from the intestinal tract within four or five days. In from five to ten per cent of the flies, however, the trypanosomes multiply in the intestinal tract, and after eighteen to fifty-three days they again become infectious and remain so for a long period, the parasites being found regularly in the salivary glands and in the proboscis.

It is possible that the disease is transmitted in other ways than by Glossina palpalis; blood-sucking insects, such as stomoxys, 
anopheles, mansonia and perhaps fleas, may act as mechanical earriers. It is also possible that the disease is transmitted by coitus. Without some such explanation it is difficult to understand certain house epidemics which have occurred outside the fly belts.

The animal host of the Trypanosoma gambiense is believed to be the big game animals, particularly the antelope.

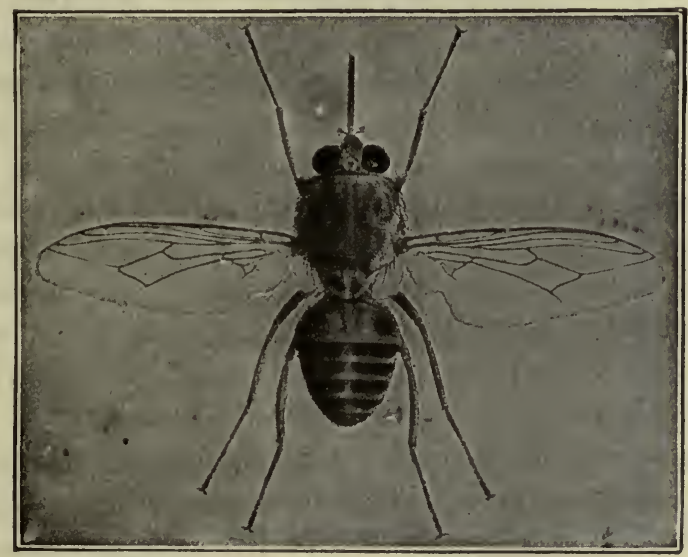

Fig. 141.-T'setse Fly (Glossina Palpalis). (From Rosenau, "Preventive Medicine and Hygiene.")

Morphology.-The organism belongs to the brucei group, and its differentiation on morphology is difficult, yet, on the average, the posterior end is somewhat more pointed than the brucei. In length it varies from fifteen to thirty microns, and in thickness from one to three microns. In fresh preparations the motility is not marked; both plump and slender forms are found in the blood, but in the cerebro-spinal fluid slender forms only are seen.

Cultures on blood agar have been made by Thompson and Sinton, yet they died out after a few weeks, and were never virulent. The pathogenicity varies somewhat with the strain used, but apes are easily infected. In white rats there may be two or three relapses before death occurs, while when inoculated with brucei death follows within two weeks.

Pathogenicity.-Although eultures vary greatly in virulence, it is possible to infect rats, dogs and monkeys with a fatal trypanosomiasis; cattle, sheep and goats continue to show a few parasites for months 
after inoculation but without sickening. In no animal, however, is it possible to reproduce the sleeping sickness stage as it occurs in man.

Trypanosoma rhodesiense.-This species was established by Stephens and Fantham. ${ }^{6}$ It is transmitted by the Glossina morsitans, a fly which is widespread over large tracts of country, independently of the presence of water. It is becoming generally recognized that there are two forms of sleeping sickness, one of which is caused by this trypanosome. This form of the disease is more acute and is less amenable to treatment; the trypanosome is also more virulent for animals and may be differentiated from gambiense on its morphology. As both parasites are found in the antelope, the prophylaxis is the same. Bruce ${ }^{7}$ is of the opinion that rhodesiense and brucei are identical, but Taute and Huber,* by inoculating themselves and 129 natives with the blood of naturally infected animals with out reproducing the disease, seem to have shown that the parasites are not identical.

Diagnosis.-When the disease is well developed in an endemic area, the diagnosis is easily made. During the early stages the examination of the cerebro-spinal fluid, puncture fluid from the lymph nodes and the peripheral blood may all show the trypanosome; since the parasites are scarce the use of the thick film method of Ross may be necessary. When direct examination is unsuccessful, enrichment in the blood of susceptible animals, rats and mice will establish the diagnosis.

Treatment.-Treatment is based upon the observation of Bruce and Lingard, that arsenious acid is trypanocidal. The best results have been obtained with atoxyl, in half gram doses, repeated at intervals of ten days or more for not less than four months. Light cases become trypanosome free and are apparently cured, yet many relapse on cessation of treatment. Well marked cases may show improvement yet ultimately grow worse and die. Better results are obtained when atoxyl and tartar emetic are both used and also when the treatments are repeated every six months. The successful treatment of an intractable case of gambiense infection with stibenyl (the sodium salt of p-acetylaminophenyl-stibinic acid) has been reported by Manson-Bahr

- Stephens and Fantham, Proc. Roy. Soc., 1910; Ser. B., lxxxiii, 28.

'Bruce, Bull. Trop. Dis., 1916, vii, 68.

* Taute, M., aud Hubcr, 'r., Abstracted in Tropical Diseases. Bull. London, 1920. 
(Brit. Med. Jl., 1920, 235). Other arsenical preparations have been used but none are entirely successful. Salvarsan drives the parasite from the peripheral blood but not from the cerebro-spinal fluid. The prognosis as to ultimate recovery is unfavorable. The most hopeful cases are those which are brought under treatment in the earliest stage of the disease.

Prophylaxis.-Prophylaxis is quite complicated and is carried out along several different lines. Infected fly belts are depopulated, the inhabitants being removed to a fly-free district where they may be treated at hospital stations. The fly breeding may be greatly diminished by clearing off the forest and brush, especially along the river courses, since the glossina needs abundant moisture for its propagation. Since the fly bites only during the day, all traveling in infected districts is best done at night. A prophylactic measure of prime importance is the search for and the treatment of the cases, which are also carriers. While the treatment may not cure, it does bring about improvement and lessens the number of heavily infected carriers.

Trypanosome cruzi, Chagas (Schizotrypanum cruzi).-This parasite, which differs from all other trypanosomes, is the cause of a
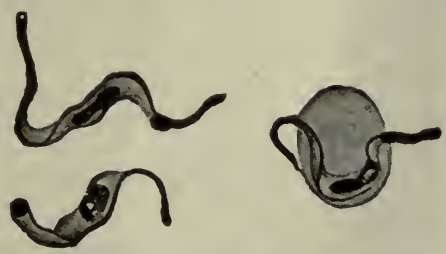

Fig. 142. - Schizotrypanum Cruzi in Human Blood. (From Doflein after Chagas. MacNeal, "Pathogenic Microorganisms," published by P. Blakiston's Son \& Co.)

form of human trypanosomiasis occurring in Brazil. It is transmitted by a bug, Triatoma magista (Conorhinus megistus), in which the parasite passes part of its cycle of development. Brumpt† believes the infection is transmitted by the dejecta of the bug as well as by its bite, and thinks the first method is probably the more usual one. In the human being, multiplication takes place in endothelial cells, lymphocytes and parenchymatous cells of the viscera; and also in the skeletal and heart muscles. While in this stage the parasite 


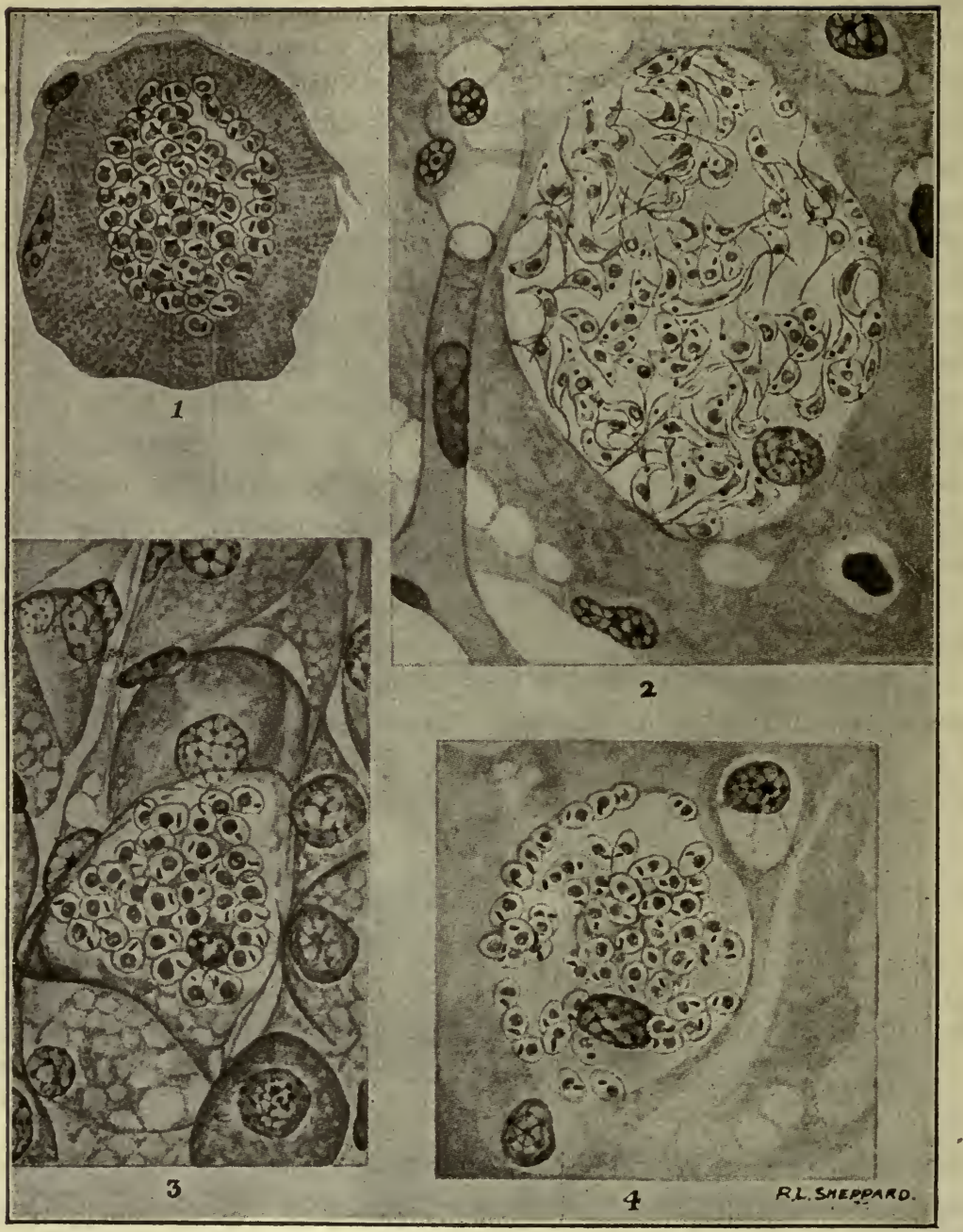

Fig. 143.-Schizotrypanum Cruzi Developing in Tissues of Guinea-pig. 1. Cross-section of fibers of striated muscle containing Schizotrypanum cruzi; 2, Section of brain showing cyst in a neuroglia cell containing chiefly flagellated forms; 3, Section through suprarenal, fascicular zone; 4, Section of brain showing neuroglia cell filled with round forms. (After Low and Vianna. MacNeal, "Pathogenic Microorganisms," published by Blakiston's Son \& Co.) 
has no flagellum and resembles the leishmania; only after escape into the blood does it take on the trypanosome form.

Guinea-pigs, rats, mice and monkeys are susceptible; the bedbug, cimex, is also capable of transmitting the disease.

Cultures were obtained by Chagas and proved virulent for animals. The human disease is found both in children and adults and is regularly fatal. It is characterized by an irregular fever, severe anemia, swelling of the lymph nodes, edema and disturbance of the nervous system. Two quite distinct forms are recognized, the acute, which is usually found in young children, and the chronic, found in adults. The acute form is characterized by fever, a myxedematous swelling of the face and neck or even the whole body, and the presence of trypanosomes in the circulating blood. The nervous system may be involved in the acute cases, in which event they end fatally. The chronic form has no trypanosomes in the blood, but has foci, or cysts in the heart, the voluntary muscles or in the viscera or the nervous system. Disturbances of almost any part of the body may result. While the foci may be generalized they do not follow the blood vessels.

Leishmania.-This genus was founded by Ross in 1903 for the Leishman-Donovan and Wright bodies found in kala-azar and Delhi boil, to which Nicolle added another in 1909, the parasite of infantile splenomegaly. Leishman, Donovan and Wright, working independently, described the first two parasites in 1903, and, although they have received various names, leishmania is now the accepted term. Rogers, Calkins and others, however, class them as herpetomonads, because of the elongated, flagellated form all take in cultures on the Novy-MacNeal-Nicolle blood agar medium. It is, however, best to consider them as a separate genus, because of their natural parasitic habits in human beings. Leveran, Fantham and others have shown that it is possible in the laboratory to induce the herpetomonads parasitic in the intestine of various insects to become parasitic in various vertebrates.

Leishmania donovani (Kala-azar).-This parasite is the cause of kala-azar, a disease characterized by irregular fever, weakness, anemia, cachexia and a remarkable enlargement of the spleen, and occasionally of the liver. It is chronic, progressive and frequently fatal, the mortality being about 80 to 90 per cent. This disease is common in tropical Asia and in northeastern Africa.

Morphology.-The parasite is intracellular, and is found prin- 
cipally in the endothelial cells of the spleen and liver, and in the bone marrow. It is oval, two to four microns in diameter, finely granular and occasionally vacuolated. It contains a large, round nucleus and a smaller blepharoplast which is oval or rod shaped; a third body, a slender short thread, may sometimes be recognized, which is presumably the undeveloped flagellum. Stained specimens

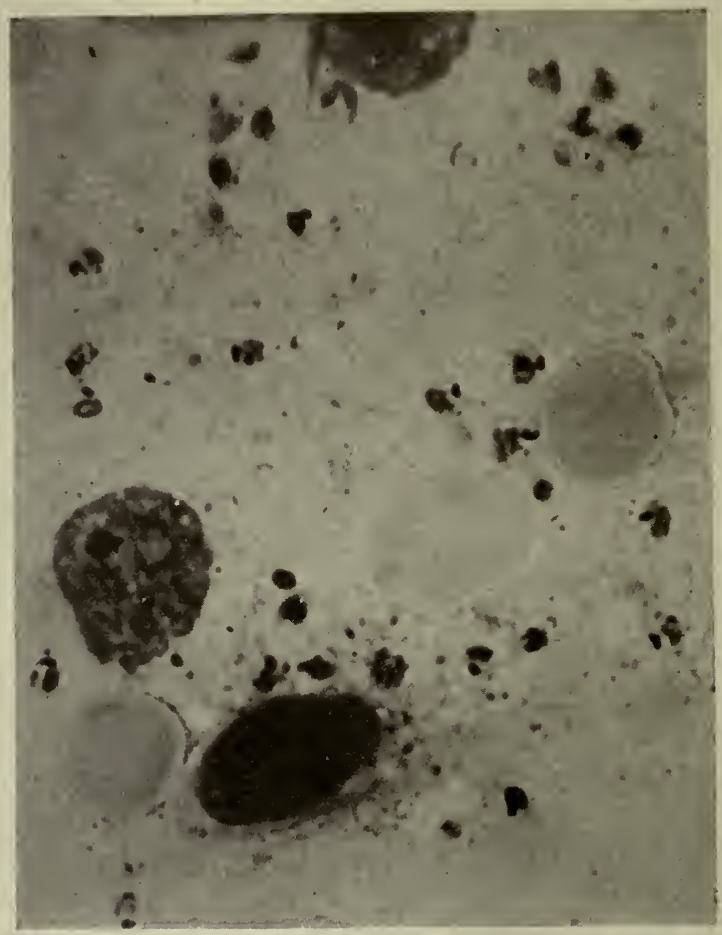

Fig. 144.-Leishmania donovani. (Army Med. School Collection, Washington, D. C.)

of blood, spleen and liver pulp, and bone marrow, usually show large endothelial cells or leucocytes closely packed with parasites, one to two hundred to a single cell. Multiplication in the body is by simple division, and incompletely divided pairs of organisms are frequently seen. Cultures have been obtained in citrated blood and on the usual N. N. N. medium. When fully grown the cultural organisms are typical herpetomonads (leptomonads); the cell body elongates and the rudimentary whip develops into a true flagellum. Both dogs and monkeys are susceptible to artificial inoculations. 
The parasite is probably transmitted by some insect, either cimex (Rogers), or by the dog flea, Ctenocephalus canis (Wenyon), or a plant-feeding bug, Conorhinus, which occasionally sucks blood.

Animal Pathogenicity.-Wenyon in $1913^{8}$ inoculated a dog with splenic emulsion from a man who died in London of kala-azar contracted in Calcutta. The parasite has been successfully carried through five animals, and in 1915 an examination of the bone marrow showed not only typical leishmania, but also a few large, well-marked leptomonad forms. Similar forms were described by Escomel in 1911, from South American dermal lesions. Monkeys may also be infected.

Leishmania tropica (Delhi or Aleppo boil) is the organism found in a local skin affection variously termed Delhi boil, Aleppo boil or tropical ulcer. While it is probably transmitted by some insect, there is as yet no definite proof. The incubation period is about two months, while the disease, once manifèst, lasts twelve to eighteen months and is followed by immunity for life.

The parasite, which was first described by J. H. Wright, ${ }^{9}$ shows minor differences from leishmania donovani, particularly a variable morphology, all gradations, from the usual oval to elongated narrow forms with pointed ends, being found.

Cultures may be obtained on the N.N.N. blood agar, which develop into leptomonads, as with Leishmania donovani. Dogs and monkeys are susceptible to artificial inoculation, and it is possible that in nature the disease is carried from dogs to human beings by some insect.

Leishmania infantum (Infantile Splenomegaly) was described by Nicolle in 1909 from cases of infantile splenomegaly occurring in Northern Africa. It is, however, not limited to this region, but occurs throughout the whole Mediterranean District. Javarone ${ }^{10}$ described 110 cases observed in Naples from 1913 to 1920. The disease resembles kala-azar in all respects, except that the patients are young children, and it is possible it is the same disease. Without treatment the disease, like kala-azar runs a progressive course almost always leading to death. The parasites are found in abundance in the liver, spleen and bone marrow at autopsy and may be cultivated in the usual way on the N. N. N. blood agar.

${ }^{8}$ Wenyon, Jour. Trop. Med. and Hyg., London, 1915, xviii, 218.

' Wright, J. H., Jour. Med. Res. Bost. 1903, x, 472.

${ }^{10}$ Javarone, $N$., Infantile Leishmaniasis in Naples and Neighborhood, Tropical Diseases Bulletin, London, 1920, 16, 454. 
Animal Pathogenicity.-The disease occurs naturally in African dogs, and they are probably the source of infection, the parasite being carried by a flea or some other insect. Dogs, monkeys and guinea-pigs are susceptible to artificial inoculation.

Treatment.-The use of tartar emetic in the treatment of kala-azar is due to the success of the treatment of infantile leishmaniasis introduced by Di Christina and Caronia. In kala-azar it has now been

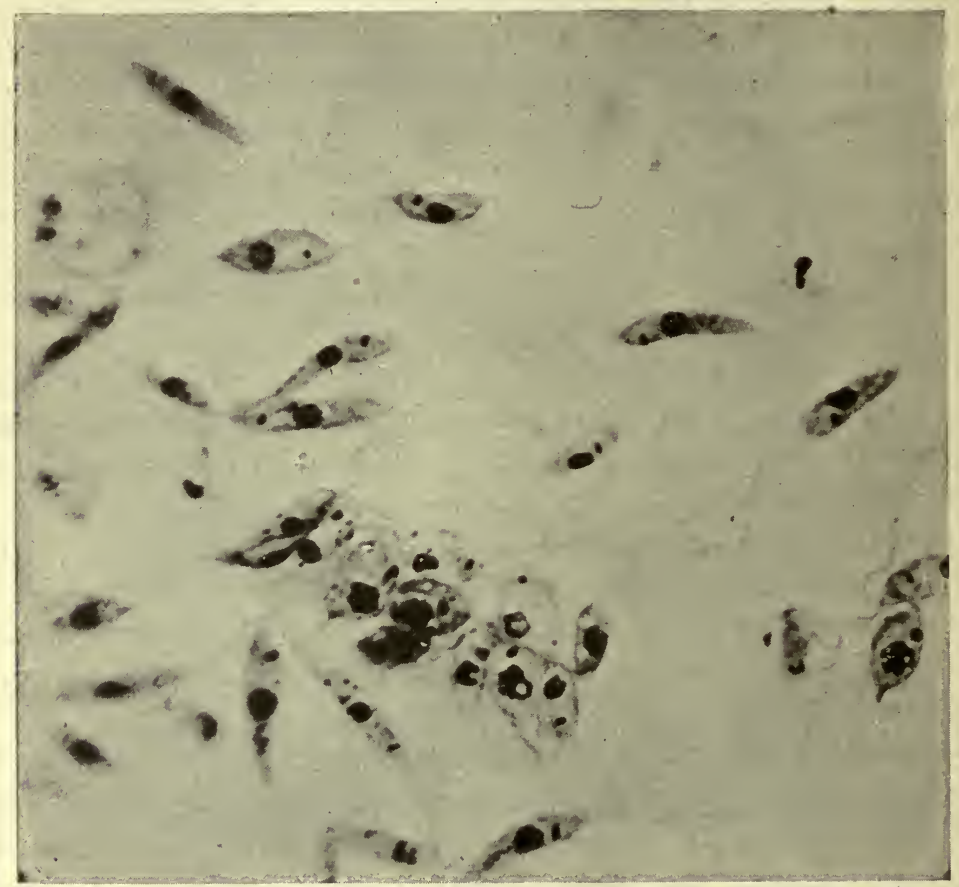

Fig. 145.-Leishmania infantum. (Army Med. School Collection, Washington, D. C.)

used successfully by many and when properly given is without danger and gives a large number of recoveries.

The untreated disease has a mortality of about $90 \%$. DobbsPrice $^{11}$ has reported 2000 injections with $67 \%$ of recoveries. He used a $11 \frac{2}{2} \%$ solution of sodium antimony tartrate, in a weekly dosage increasing from 1 to 8 c.c.

${ }^{11}$ Dodds-Price, J., Kala-Azar in Europeans in the Nowgong District of Assam -Indian Medical Gazette, 1920, Vol. 55, No. 3, pp. 87-89. 
Since intravenous treatment of children with any drug is difficult, a search has been made for preparations which can be given into the muscles, without producing pain or necrosis. Some promising results have been obtained by Spagnolio and Manson-Bahr, ${ }^{12}$ with acetyl-pamino-phenyl-stibiate of sodium, and "stibenyl" a related drug. The drug is dissolved in distilled water and "stibenyl" according to Manson-Bahr, may be given in doses up to 0.6 gram for an adult. The treatments are given weekly over a period of 4 or 5 months with resulting cure. The time of treatment may be shortened by using the intravenous route when possible.

The prognosis, as a result of the new treatment with antimony, may now be considered good, particularly in acute cases.

Two other forms of dermal leishmaniasis have been described; the first, due to Leishmania braziliensis, accurs in many parts of South America. The parasite is morphologically identical with Leishmania tropica. Since the disease is always contracted in the virgin forest, one name for the affection is forest yaws; uta and espundia are probably different clinical forms of the same disease. The transmitting insect cannot well belong to the household vermin or domestic insects; sylvan insects such as the ixodides, tabanides, simulids, mosquitoes and Conorhinus are all suspected of being carriers.

The second form is called Leishmania nilotica (Brumpt, 1913). and is found in non-ulcerating keloid nodules in Egyptian negroes. Morphologically, the parasite is indistinguishable from Leishmania tropica.

${ }^{12}$ Spagnolio, Giuseppe, Tropical Diseases Bulletin, London, 1920, 16, 455, and Manson-Bahr, Philip, Brit. Med. Jour. 1920, Aug. 14, 235. 


\title{
CHAPTER LVI
}

\author{
CLASS III-SPOROZOA
}

\section{SUB-CLASS-TELOSPORIDIA}

\section{HEMOSPORIDIA}

THE Hemosporidia and Sarcosporidia are the only members of this order of medical interest. The hemosporidia belong to the sub-class Telosporidia of the Sporozoa, because spore formation begins at the end of the life cycle.

The systematists have not yet agreed upon the proper classification of this group of parasites; consequently the older arrangement will be followed. They are, like the coccidia, parasites of cells, at least during the schizogenous cycle; all change hosts to some insect for the sporogenous cycle. As the name implies, they live in blood cells and are rapidly growing ameboid bodies, which, beginning as sporozoites, penetrate the host cells arid develop into trophozoites. These grow rapidly to adult segmenting parasites, in which case they are called schizonts, or to sexual forms, or gametes, when they are termed sporonts. In the course of their development, most species produce melanin from the destruction of the hemaglobin.

The nucleus, which is readily stained, is single and posesses a karyosome; the mature schizont divides into many small forms called merozoites, and these, when freed by the rupture of the degenerated erythrocyte, escape into the blood plasma, and if not phagocyted, penetrate other erythrocytes and repeat the asexual or schizogenous cycle. The pigment and undivided portion (restkörper) of the cytoplasm of the mother cell accumulate in the bone marrow, spleen and other viscera.

After a number of cycles of asexual multiplication have been lived through, a new development takes place and sexual forms begin to appear in the circulation. These grow to large size, yet show no indication of division into merozoites and were at one time considered

\footnotetext{
${ }^{1}$ For classification, see page 1049.
} 
degeneration forms. Two varieties may be distinguished, one with a dark staining cytoplasm and fine granular melanin, and the other witb. light staining, hyalin cytoplasm and coarse pigment; the former, loaded with reserve food material, is the female or macrogametocyte; the latter, the male or microgametocyte. The gametes do not develop further until taken into the digestive tract of the insect host. For purposes of study, however, the microgametocytes may be made to exflagellate on the slide, dampened a little by breathing upon it, to stimulate the condition in the insect host. In such a preparation, the flagella, or microgametes, may be seen actively moving inside the cell body, whose wall they ultimately rupture, and all, four to eight, escape and whip about until they come in contact with a macrogametocyte, when one microgramete enters through the micropyle and finally fuses with the female nucleus.

Hemoproteus columbæ (Halteridium).-This parasite of the red blood cells of doves was described in 1891 by Celli and Sanfelice. It is widely distributed in nature and has been reported from Europe, Asia and North and South America. The organism is found within the cytoplasm of the erythrocyte; the nucleus, which is not regularly displaced, is surrounded by the growing parasite like a halter, and for this reason it was named halteridium by Labbe. It is sluggishly ameboid and produces an abundance of melanin, and when the blood is drawn the ripe male sporonts, the microgametocytes, rupture easily, liberating the active flagella, or microgametes. Under favorable circumstances the fertilization of the macrogametocyte by the microgametes may be observed on the slide, and it was while working with this parasite that Macallum first followed out the whole process of fertilization in the hemosporidia and gave the proper explanation of the flagellate stage seen in the malarial parasite.

In the blood of the dove this parasite is usually seen as a large or small crescent, partly encircling the nucleus; the gametes are readily recognized by the usual marks, that is, the female, or macrogametocyte, is rich in reserve material and the stained specimen takes a deep color; the male, or microgametocyte, being poor in reserve material stored in the cytoplasm, appears relatively pale in stained specimens.

The invertebrate host of the parasite is Lynchia maura (Bigot), or Lynchia lividocolor, a biting hippoboseid fly of louse-like habits which lives in the nest and in the plumage. The cycle in the fly has

²Adie, Helen, Indian Jour. Med. Research, Calcutta, 1915. 
been successfully worked out by Adie, ${ }^{2}$ who has demonstrated the oökinetes, zygotes and oöcysts in the lower portion of the midgut. As

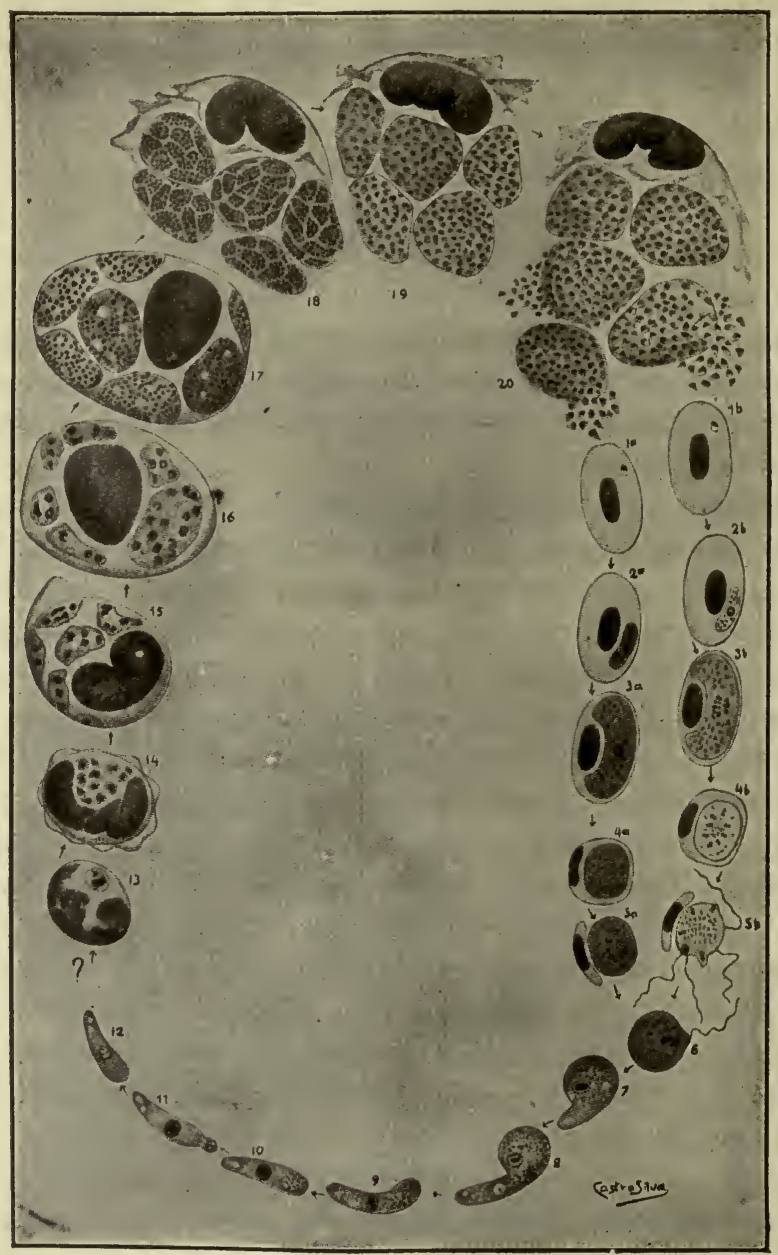

Fig. 146.-Hжморroteus Columbes. $1 a$ to $3 a$, Development of female parasite in blood of dove; $1 b$ to $3 b$, Development of male parasite in blood of dove; $4 a$, $4 b, 5 b, 6$ to 12 , Development in the digestive tube of the fly (Lynchia); 13 to 20, Development of the parasite inside leucocytes in the lung of the dove. (After Avagao. MacNeal, "Pathogenic Microorganisms," published by P. Blakiston's Son \& Co.)

the oöcyst grows, it stands out from the gut wall and finally shows the striations indicative of the presence of sporozoites; after rupture of 
the mature eyst, these collect in large numbers in the salivary glands and ducts.

The life history of the parasite is seen to be like that of proteosoma and malaria, except that the asexual or schizogenous cycle appears to be lacking.

Proteosoma (plasmodium) præcox.-This parasite is a typical representative of the sporozoa, and is interesting historically, since it was the one with which Ross worked in 1898, when he first demonstrated the part played by the mosquito in "bird malaria."

Grassi and Feletti described the parasite in 1890 under the name of hemameba precox. It is widely distributed geographically, and is common in the blood of small birds, sparrows, robins and larks. It can be propagated in the laboratory in the blood of canaries with-
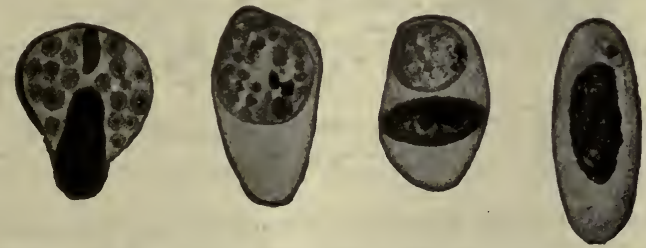

Fig. 147.-Proteosoma Precox in Blood of Field Lark. $A$. Young parasite in blood cell; $B$, Half-grown parasite which has pushed aside nucleus of blood cell; $C$, Parasite with clump of pigment and many nuclei; $D$, Division into many merozoites. (After Doflein and Wasielewski. MacNeal, "Pathogenic Microorganisms," published by P. Blakiston's Son \& Co.)

out great difficulty; sparrows, however, do not long survive in captivity unless kept in round glass jars, where they cannot injure themselves by dashing against the walls. The blood for examination is obtained from the cephalic wing vein, close to the body, which is nicked with a razor, and the blood taken up in a capillary glass tube containing a little citrate solution. To inoculate a new bird, it is sufficient to inject a small quantity of citrated blood from an infected canary into the breast muscles of the new bird, transferring to a new host at intervals of a month or less. Because it is not difficult to keep on hand, this organism may be used for class study in localities where malarial cases are infrequent. There is no apparent reason for placing it in a different genus from the malarial parasites.

The entire asexual cycle, schizogony, may be studied in the peripheral circulation, as in quartan malarial fever.

In nature it is transmitted by both culex and stegomyia (Aedes 
calopus), and its development is briefly as follows: The bird is inoculated by the mosquito with spindle-shaped young forms known as sporozoites. These possess the power of ameboid motion, and rapidly penetrate into an erythrocyte, in which they grow quickly; they constantly move about inside the cell until nearly full grown, and are during this stage ealled trophozoites. The substanee of the erythrocyte is rapidly consumed by the parasite and a dark pigment, melanin or hemozoin, is formed from the destroyed hemaglobin. The mature parasite divides into many small forms called merozoites, and these, when freed by the rupture of the degenerated erythrocyte, eseape into the blood plasma, and if not phagocyted, penetrate other erythroeytes and repeat the asexual or schizogenous eycle. The pig-

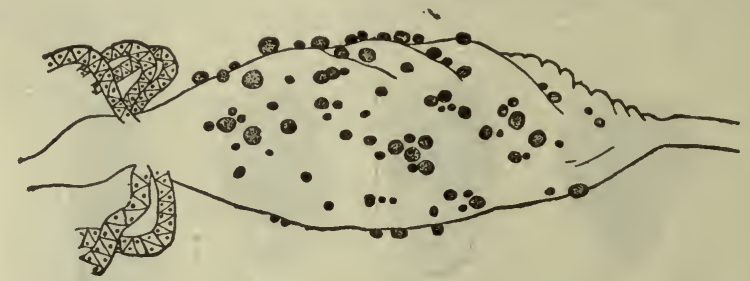

Fig. 148.-Midgut of Culex Mosquito, Covered with Oöcysts of Proteosoma Precox. V, Vasa MalPighir. (After Doflein and Ross. MacNeal, "Pathogenic Microorganisms," published by P. Blakiston's Son \& Co.)

ment and undivided portion (restkörper) of the cytoplasm of the mother cell accumulate in the bone marrow, spleen and other viscera.

\section{MALARIA}

This is one of the most common and widespread of preventable human diseases, and in some localities is the cause of a greater mortality and morbidity than tuberculosis. It is caused by one or more of the three forms of the malarial plasmodium. As a rule the infeetions are simple, yet in the tropies it is not uncommon to find two or even three species of plasmodia in the same patient, and this condition is ealled a mixed infection.

History.-The disease under various names, as chills and fever, Roman fever, Chagres fever, has been known since the greatest antiquity. The cause was not discovered until 1880, when Laveran, a French military surgeon stationed in Algeria first saw the organism and described it as the cause of malaria. He saw and described not 
only the pigmented trophozoite, but also the crescentic gametocytes and flagellating microgametes, and, because of the activity of the flagella, ealled the parasite Oscillaria malarice, a name afterwards given up. Later, in 1885, Celli and Marchifava described the parasite with greater accuracy and named it Plasmodium malarice, a poor name, since it describes merely a condition assumed by some fungi and mycetozoa, yet, according to the rules of zoölogical nomenclature, it must stand. In the same year, Golgi described the quartan parasite and in the following year demonstrated the relation of the various stages of the life cycle of the tertian parasite to the temperature curve.

Even in antiquity many had noted the curious distribution of malaria, and its intimate relation to swamps and marshy places. Manson, who had already shown the rôle played by an infected mosquito in transmitting filarial disease, in 1894, suggested that the epidemiology of the disease could best be explained by the hypothesis that it was conveyed by the bite of some blood-sucking insect, probably the mosquito.

For years the interpretation of the flagella was a subject of controversy. They were regarded as degeneration products by some and as living elements by others. In 1897, MacCallum, working with halteridium, was able to show that they were, in fact, spermatozoa, as he saw them penetrate and fertilize the macrogametes, or large spherical forms without flagella.

In 1897, Ross, of the British. Indian Medical Service, described the beginning of the sporogenous cycle in what he called a dapple-winged mosquito, which we now recognize as an anopheline. Following out further Manson's hypothesis, he was able the same year after long and laborious research to clear up the method of transmission of bird malaria, proteosoma, an analogous disease. Grassi and Bignami and Bastianelli, in 1898, succeeded in demonstrating the complete lifo cycle of the human form of malaria in the anopheles mosquito.

Geographical Distribution.-The disease is found in a belt round the world extending from 40 degrees S. latitude to 60 degrees N.; it is, however, not equally distributed throughout this zone, and even in the tropies there are many malaria-free areas, principally in the regions of higher altitudes, since the special home of malaria is in the lowlying, swampy and torrid coastal districts and river basins. Islands at a distance from the main land may he entirely free. Malaria reaches its maximum intensity in the tropies, where the anopheline mosquitoes breed continuously throughout the year. and new infec- 
tions may oceur at any time; while in the sub-tropies and temperate regions it is a seasonal disease, appearing soon after the onset of hot weather with its new erop of anophelines and continuing until the first cold weather which destroys most of the infected mosquitoes. It is possible that the disease may be earried over from season to season by the hibernating mosquito although definite proof of the importance of this is lacking. It is earried over to the next season by the human earrier, in whom the disease may be latent or who may have suffered from clinical relapses throughout the year. Modern times have seen it disappear from many regions where it was formerly

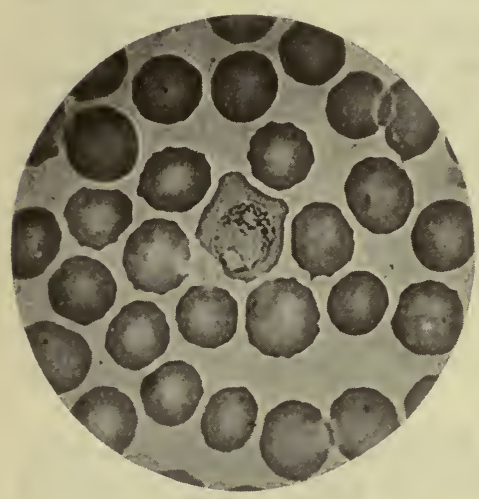

Fig. 149.-Plasmodium viva (Army Med. School Collection, Washington, D. C.)

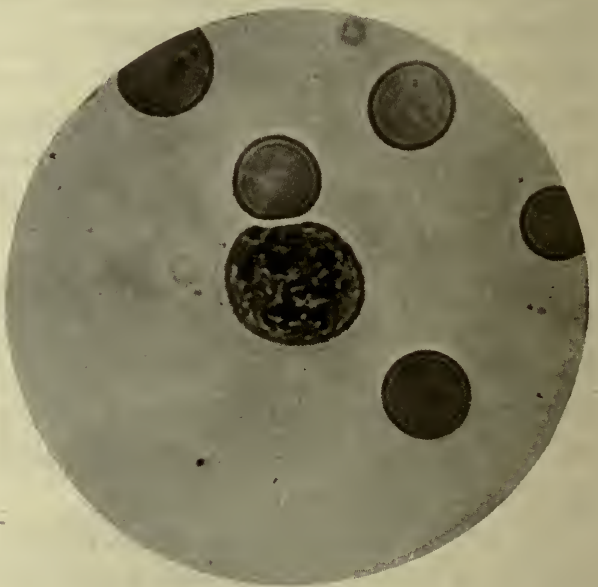

Fig. 150.-Plasmodium vivax. (Gamete.) (Army Med. School Collection, Washington, D. C.)

endemic, because of increased cultivation of the soil and better surface drainage, as, for example, in England and the Ohio river valley.

In the registration area of the United States there were 1565 deaths from malaria in 1913 ; in Italy, up to 1900 , the average number of deaths from this cause annually was 16,000 . One cannot obtain a true picture of the importance of the disease, however, from mortality statistics, since it is not often fatal, and the morbidity is out of proportion to the mortality. In many villages, where it is endemic, onethird to one-half the population may have parasites in the blood, most of them without elinical symptoms, yet they are not able to work and the children remain undeveloped and backward. Much of the illness attributed to hookworm infection is, in reality, due to latent malaria. 
During the first part of the acute attack the predominant forms are schizonts, but later in the course of the disease, as a result of increasing immunity or of treatment, the sporogenous cycle becomes evident. The trophozoites, in increasing numbers, develop into sporonts rather than schizonts. These forms do not develop a vacuole but increase in size and remain round or oval bodies. When mature they occupy most of the red cell but show no sign of nuclear change or of segmentation and they are then called gametocytes. The sex can be distinguished in well stained specimens by remembering that the microgametocyte, or male form, is rich in nuclear chromatin, of which the fiagella or spermatozoa will be formed after the gametocyte has been ingested by the proper mosquito. The macrogametocyte, or female form, is distinguished, on the other hand, by the store of nutrient material in the cytoplasm, causing it to stain deeply.

After ingestion by a susceptible mosquito the red cells undergo dissolution and the contained gametocytes are liberated. The microgametocyte sends out several microgametes (flagella) one of which fertilizes a macrogamete by penetrating the cytoplasm and uniting with its nuclear chromatin. The fertilized cells change its shape from round to ovoid and becomes motile and is called a traveling vermicule, or oökinette. It travels to the stomach wall which it penetrates and comes to rest on its outer surface and is then called an oöcyst or zygate. The cyst or zygate increases in size with each successive segmentation of its nucleus until it is ripe. It then ruptures and discharges a mass of sporozoites into the body cavity. These wander to all parts of the body of the mosquito but many reach the salivary glands and later the saliva, and when next the mosquito bites they are injected into the new host.

In the state of Mississippi Bass has shown that the age distribution is such that 23 per cent of the population under 20 years of age showed parasites in the blood, whereas only 19 per cent of persons over 20 years were infected, and that the five-year period from 5 to 9 years of age showed the greatest number of infections.

Race and color are important, and negroes showed 36 per cent more infections than whites, and the high point is reached much earlier in black than in white children. (South Med. Jour., 1919, 12, 456.)

The parasites belong to the class of hemosporidia, and are closely related to the coccidia, which are parasites of epithelial cells, while 
the plasmodia are parasitic on red-blood cells. There are two divisions of the life cycle; that which occurs in man, the endogenous, asexual or schizogenous, and that which occurs in the mosquito, the exogenous, sexual or sporogenous; for this reason the mosquito is the definitive and the man the intermediate host.

Although the life cycle of the parasite varies in details in the different forms of malaria, certain stages are common to all, and in general the life cycle is usually described as follows: an infected mosquito bites a warm blooded animal, often a human being, in order to obtain its meal of blood. As it bites, it infects the wound with its saliva which contains sporozoites coming from the salivary glands. The sporozoites, in the new host, soon gain access to the blood and attach themselves to the erythrocytes upon which they become parasitic. In shape they are long and slender spindles, and when stained with the usual eosin-methylene blue dyes, show a blue cytoplasm and a compact dot of red nuclear chromatin in the center. When the sporozoite escapes the phagocytes, and succeeds in establishing itself on a red cell it soon changes its shape to the ring form and grows rapidly and during this growing stage is known as a trophozoite. This last named form may develop in either of two ways: in the asexual or schizogenous cycle when it is called a schizont, or in the sexual or sporogenous cycle, when it is called a sporont.

The schizont goes on to full development in the human host; the sporont cannot complete its cycle until taken into the stomach of a suitable anopheline mosquito, capable of conveying malaria.

The trophozoit which ends its life as a schizont grows rapidly at the expense of the red cell and develops a characteristic vacuole which increases the area of the parasite in contact with the host cell. When mature its nucleus undergoes mitotic changes and the parasite divides into a more or less definite number of segments called merozoites; these when liberated by the disintegration of the host cell, attack new erythrocytes and develop during the second and subsequent generations in the same manner as the sporozoite.

There are three well-recognized forms of the plasmodia, (1) Plasmodium vivax (Grassi and Filetti), causing tertian fever (also called "benign tertian"); (2) Plasmodium malarioe (Laveran), causing quartan fever; (3) Plasmodium falciparum (immaculatum) (Welch), causing the tropical form of malaria, the so-called rstivo-autumnal or subtertian. As the details of development cannot be made out 
easily in fresh specimens, the following description applies to those stained with some form of the Romanowski stain.

Plasmodium vivax.-The parasite of tertian fever has a life cycle lasting forty-eight hours and is easily recognized only when full grown, that is, twenty-four to forty-eight hours after the chill. While a diagnosis may be made on younger forms, it is not so readily made. As its name implies, the Plasmodium vivax is actively ameboid, and pseudopods and irregular outlines characterize the wellgrown parasite; the infected erythrocyte is swollen, often to twice

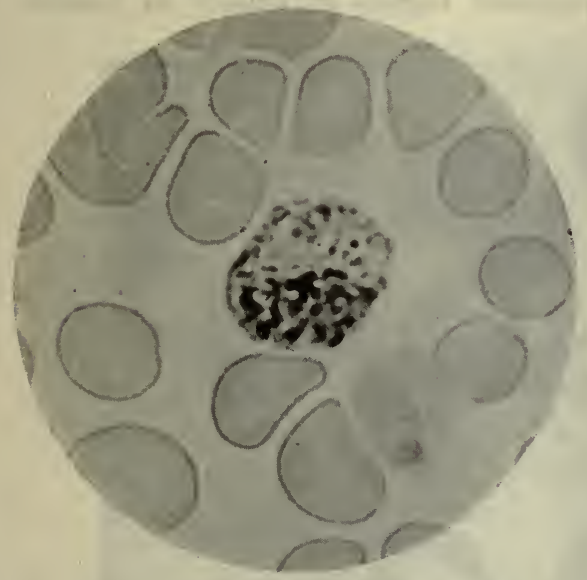

Fig. 151.-Plasmodium vivax, an Atypical Macrogametocyte. Form interpreted by Schaudinn as undergoing parthenogenesis. (Army Med. School Collection, Washington, D. C.)

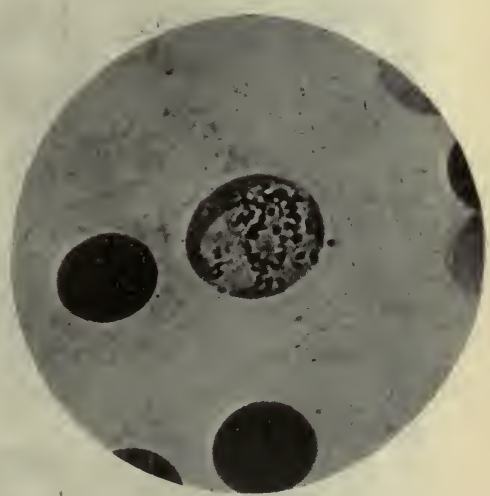

FIG. 152.-PLASMODIUM VIVAX (Army. Med. School Collection, Washington, D. C.)

its normal size, the hemoglobin is pale and, especially in spreads in which Manson's stain has been used, it is so much paler than in the surrounding cells that the infected cell stands out clearly. The part of the cell unoccupied by the parasite is stippled, that is, dotted with reddish granules called Schuffner's dots, and, as the swollen red cell and Schuffner's dots are found in no other form of malaria, their presence is pathognomonic of tertian.

The youngest form, the free merozoite, is rarely seen, but young comet-like forms composed of a particle of red chromatin and a little blue cytoplasm may readily be detected at the height of the fever; that is, a few hours after the chill and sporulation. The round, young schizont as it grows develops early a central vacuole 
and assumes the shape of a signet ring, the red chromatin dot being the stone. This small tertian ring grows rapidly as the fever subsides, and at the same time the infected cell increases in size. Twenty-four hours after the chill the ring has grown so much that it is referred to as the large tertian ring, and its tendency to irregularities of shape and ameboid form becomes apparent, and fine granules of pigment, called melanin or hemozoin, begin to be visible. After thirty-six hours the rings will all have grown into large ameboid forms. After about forty hours the parasite occupies almost the entire cell and the pigment begins to collect in masses

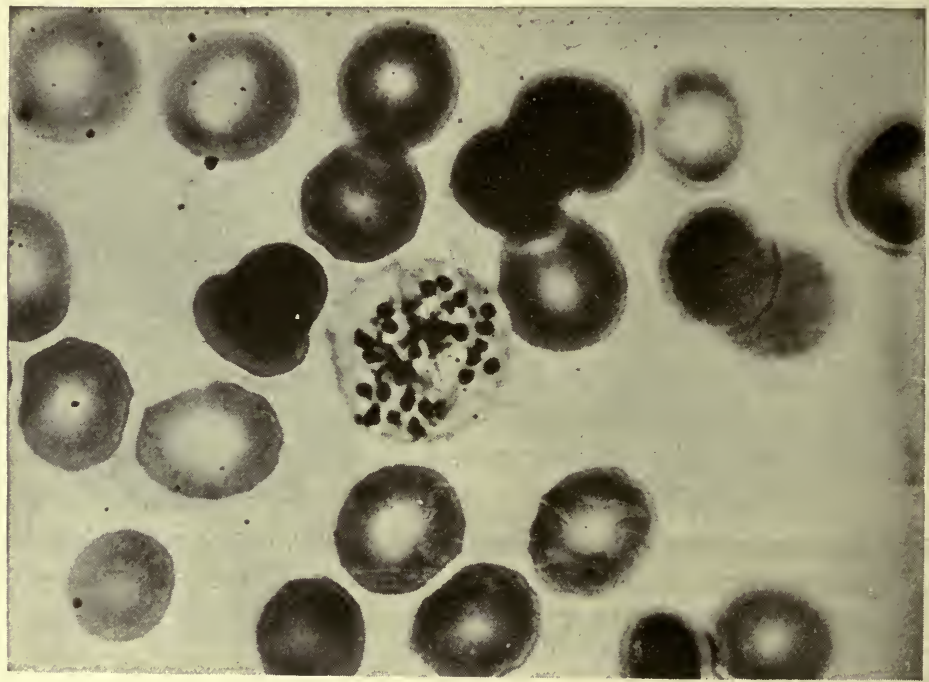

Fig. 153.-Piasmodium vivax. (Army Med. School Collection, Washington, D. C.)

toward the center. Soon after the first signs of segmentation appear, which becomes more and more distinct until fifteen to twenty separate segments or merozoites are seen, each composed of nucleus and cytoplasm. The pigment of the adult parasite and the unused portion of the cytoplasm are cast off after segmentation as a restkörper, which is promptly phagocyted and such masses accumulate in the spleen, bone marrow and viscera. With rupture of the erythrocyte, at the time of the chill, the merozoites are set free, and if not phagocyted, immediately attack new erythrocytes and the asexual or schizogenous cycle is repeated, until treatment or increasing immunity halts or alters the cycle. 
In practice it is not unusual to find parasites of diflerent ages in the same film, as some individuals seem to develop in advance of other's; in this ease, however, there will not be much differenee in their appearance. When extreme difference of age is noted in films it is probable that there have been several different inoculations, producing double or triple infections with quotidian or irregular fever curves, and such eases are not uncommon.

As all the forms so far deseribed belong to the schizogenous cycle, they may be called schizonts, or trophozoites of the schizo-

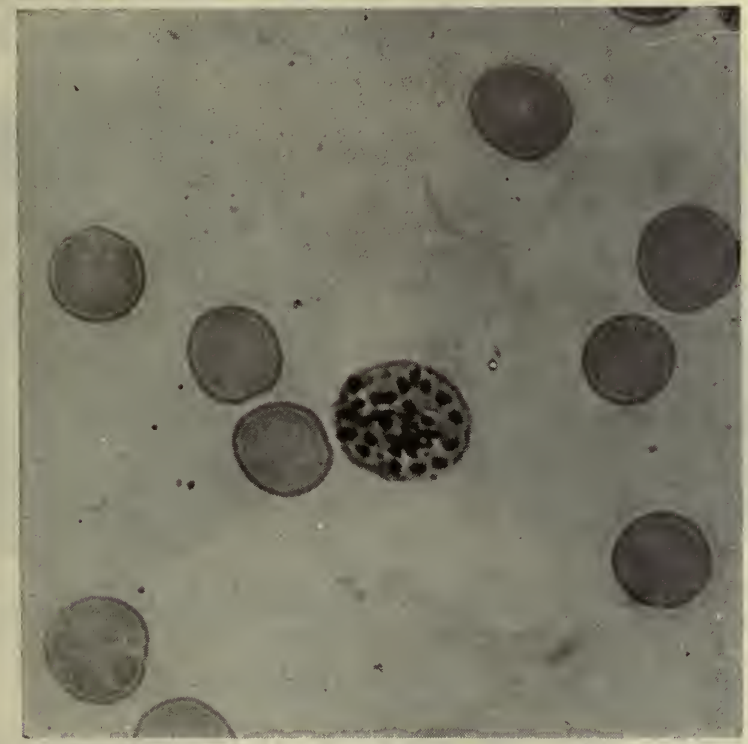

Fig. 154.-Plasmogium vivax. (Army Med. School Collection, Washington, D.C.)

genous eycle. The sporogenous eycle begins in man and is completed in the mosquito. The earliest sexual forms noted were the so-called "spheres," large adult parasites, first seen in wet preparations, which did not segment with the schizonts. They are now called gametocytes and after the disease has lasted some time are found in films made at all stages of the fever; that is, they are incapable of further development until taken into the stomach of the mosquito. The possibility of parthenogenesis will be referred to later. In appearance they are round or oval, and in this fever may be twice the size of the red eell. As a rule a narrow margin of red cell is visible after Romanowski stains, although the gamete 
may lie free in the plasma. Unlike the schizonts, the gametocytes have the pigment uniformly distributed throughout the body and there is no indication of segmentation. The young sporonts are distinguished from schizonts by the absence of the vacuole, and, when a little older, by a larger amount of hemozoin.

Plasmodium malariæ.-The quartan parasite has a life cycle of seventy-two hours, or twenty-four hours longer than the tertian, and the paroxysms eome on every third day, or, according to the Italian method of reckoning time, on the fourth day. The young rings of the plasmodium malarice are indistinguishable from young tertian rings, but the diagnosis may be made on older forms. The bleach-

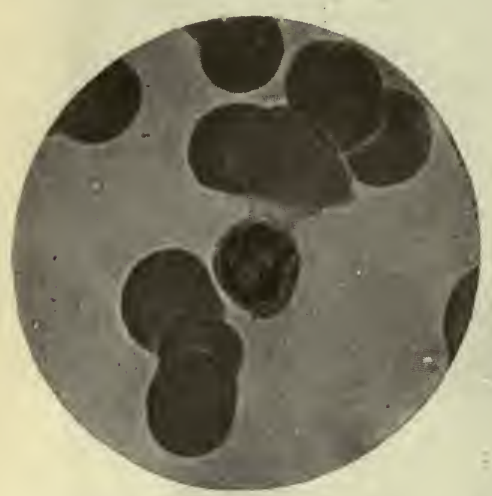

Fig. 155. - Plasmodidm MaLÁria . (Army Med. School Collection, Washington, D. C.)

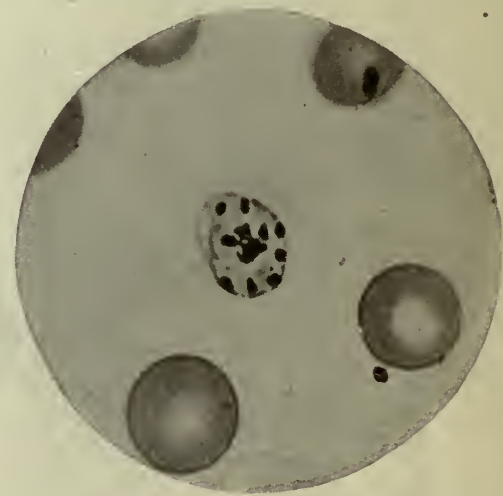

Fig. 156.-Plasmodium malarie. (Army Med. School Collection, Washington, D. C.)

ing, enlargement and stippling of the erythrocyte characteristic of tertian is never found in quartan fever, the infected erythrocyte being almost normal in appearance. The well-grown quartan parasite does not show amœbic changes but assumes a band form, more or less wide, stretehing across the red cell from border to border; with inereasing age the band widens until the parasite is nearly square and the hemozoin accumulates toward the center. Segmentation gives rise to almost symmetrical "daisy" forms, showing six to eight or, rarely, fourteen merozoites. Parasites of different ages may be found, as in tertian, and it is characteristic of quartan fever that examples of all stages of the life cycle may be found at the proper time in the peripheral circulation. Gametocytes differ from tertian mainly in size, since they are never larger than 
the normal erythrocyte until after the latter has ruptured, but when free in the plasma it is practically impossible to distinguish them from tertians.

Plasmodium falciparum.-The parasite of restivo-autumnal fever, Plasmodium falciparum, differs considerably from the two forms already described; the life cycle varies between twenty-four and fortyeight hours, and, at least in new infections, only ring forms are found in the peripheral blood, although at a later stage crescentic gametocytes may be present. The youngest æstivo-autumnal rings, found at the height of the fever, are more delicate than the young tertians. As the temperature falls the rings increase in size, but

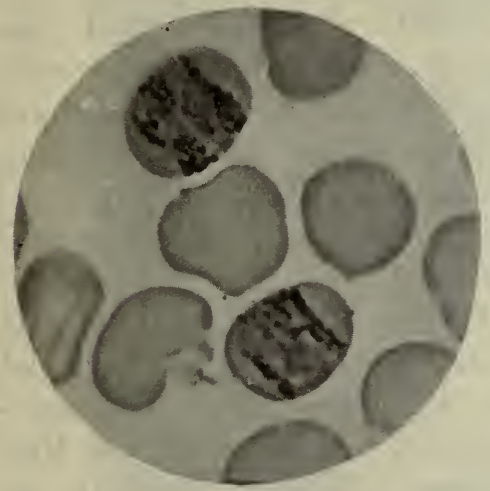

Fig. 157.-P L A S M O D I U M Malarie. (Army Med. School Collection, Washington, D. C.)

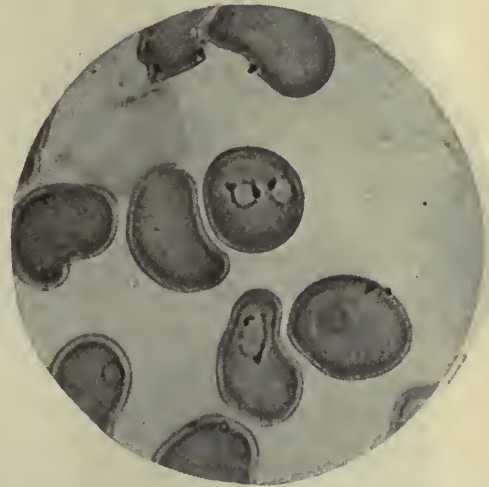

Fig. 158. - Plasmodium Falciparum. $(\times 150.0) \quad$ (Army Med. School Collection, Washington, D. C.)

without change of form; the growth is not uniform, but occurs as a thick crescentic swelling on the convex surface of the ring, and occasionally more than one such swelling is present. The large æstivo-autumnal ring, found after the febrile paroxysm has passed, occupies one-third to one-half the red cell, which is never swollen nor stippled, as in tertian, and the parasite is never band-like, as in quartan. Segmenting parasites are almost never seen in the peripheral blood in æstivo-autumnal fever, though in tertian they are common and in quartan numerous. If, however, films are prepared at autopsy from the spleen, liver, bone marrow and brain, enormous numbers of segmenting forms, together with all other stages of the parasite, may be found. The full grown segmenter occupies onethird to one-half the cell and shows a collection of hemozoin in large 
blocks in the center. The merozoites vary in number from eight to twenty-five. In addition to the small and large rings the peripheral blood shows, after the fever has lasted sufficiently long, the sexual forms or gametocytes. The infected erythrocyte is never stippled nor swollen, but, on the contrary, may appear shrunken. Both the micro- and macrogametocytes in æstivo-autumnal fever are crescentie in shape, their length being about one and one-half and the width about one-half that of an erythrocyte; the pigment is collected toward the center, which is rather paler in stained specimens than the poles. At first sight the gametocytes appear to lie free in the plasma, yet in stained specimens a rim or rib of the pale

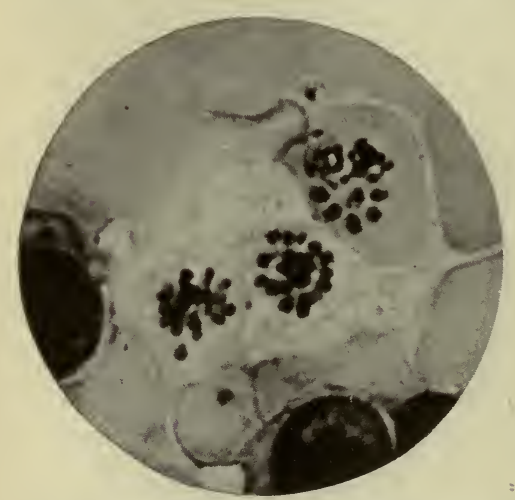

Fig. 159.-Plasmodidm Falcipardm. $(\times 1500$.) (Army Med. School Collection, Washington, D. C.) red cell may be seen on the concave side. When liberated from the erythrocyte the gametocyte becomes first spindle-shaped and finally oval or round. The male crescent is short and broad, and the female relatively long and slender.

The Finer Structure of the Plasmodia.-The finer details, which are only hinted at in fresh specimens and in those stained with Manson's stain, ean be studied to advantage in those stained with some one of the many modifications of the Romanowski stain, such as that

of Wright, Hastings, MacNeal or Giemsa.

The tertian parasite, which lies in a red cell, may be seen to be divided into a blue cytoplasm and a brilliant red nucleus, and it would be well for the novice to remember that these three conditions must be satisfied before the diagnosis of malaria can be made; the principal stumbling-block is the blood platelet, often found overlying a red cell, but it, although possessing a ragged blue eytoplasm, has always a relatively large purple nucleus. The chromatin of the young rings is usually present as a single dot, but two such dots are frequently seen. In older forms of the tertian and quartan parasites the various changes found in mitosis may be followed in the nueleus. The whole schizogenous eycle may be followed by taking blood smears from a single ease of malaria at intervals of 
three or four hours for forty-eight hours for tertian and æstivoautumnal, and for seventy-two hours for quartan.

Two forms of sporonts or gametocytes may be seen; in one the quantity of chromatin is large and the cytoplasm pale blue; while in the other the reverse is found, the nuclear ehromatin is comparatively s'nall in quartity and the cytoplasm, being rich in nutrient material, stains deeply. The first form, with abundant chromatin, is the male, or microgametocyte, and the latter the

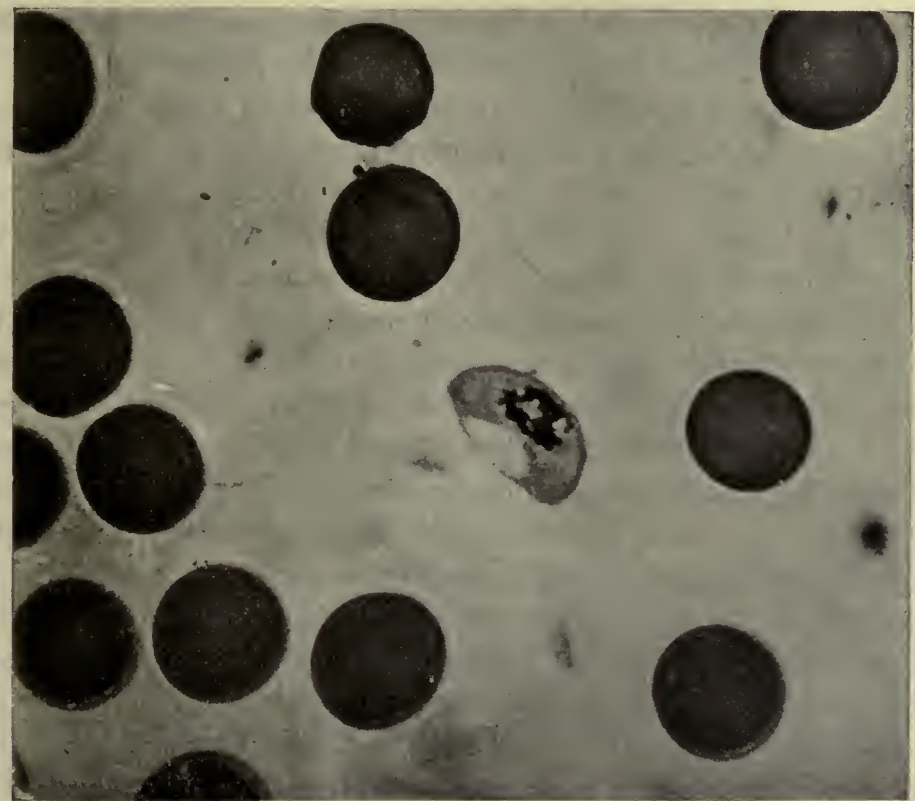

Fig. 160.-Plasmodium falciparum, Male Crescent. (Army Med. School Collection, Washington, D. C.)

female, or macrogametocyte. The differentiation between schizont and sporont may be made while the parasites are still quite young, since the schizont is eharacterized by the presence of a nutrient vacuole, and the sporont, of equal age, while lacking the vacuole, shows a greater amount of hemozoin, which is never concentrated in the center of the parasite but is seattered equally throughout the body. The eytoplasm of the sporont is less fluid than that of the schizont and shows no tendency to ameboid motion. The chromatin is relatively large in amount and, although broken up more or less 
into granules and threads, shows no real tendency to segment or disperse, but remains a compact mass.

The quartan parasite, when stained with Wright's or a similar preparation, shows quite regular and symmetrical segmentation, usually into eight merozoites. The distinction between schizont and sporont and between male and female gametocytes may be made on the same grounds as in tertian.

In æastivo-autumnal fever the chromatin dot in the young ring is often doubled, or even trebled, and in general is large and stains brilliantly. The adult and half-grown gametes may be differentiated into male and female by the criteria already given.

The Examination of Fresh Blood.-Directions have already been given (Chap. LIX) for making wet preparations and if, by ringing the cover-glass with vaseline, drying be prevented, the preparations will keep and may be studied for hours. In tertian fever the young ring forms are at first difficult to detect, unless the amount of light going through the microscope be cut down to the minimum. As the parasite grows older, an increasing number of hemozoin granules appear, and since they are in constant motion the parasite is readily detected. Its cytoplasm is delicate, and with very young parasites is difficult to distinguish from the red cell itself; older parasites, however, develop pseudopods, which are constantly projected and retracted, and the entire organism shows active movements, rendering it easy to see. The pigment continues to increase, and in the gametes is abundant and in constant motion; the gametes, however, fail to show any ameboid changes, and the protoplasm is stiff and rigid with a regular, unbroken margin. At times a clear refractile spot is seen, which is the nucleus. The infected erythrocyte is pale and swollen. Even in unstained preparations the sexes may be distinguished; the microgametocyte is about the size of a red cell, the cytoplasm is hyalin, and after the preparation has been made ten to twenty minutes the flagella, or microgametes, may be seen thrashing about in the parasite. After repeated attempts four to eight microgametes rupture the cell and emerge. The macrogamete is larger than a red cell and is finely granular and no exflagellation of microgametes occurs.

In quartan malaria the differenees already deseribed in stained blood may be easily followed.

In astivo-autumnal fever the diagnosis with fresh blood is much more difficult in new infections because of the relative scarcity of 
the parasites in the peripheral blood and the exceedingly small size of the young rings, the absence of hemozoin in them, and the very slight ameboid motion. The older rings are larger, contain some pigment and are more easily secrl. The infected erythrocyte is never pale nor swollen, but, on the contrary, may be shrunken and brassy in color. The crescentic gametocytes are readily detected, and the sexes may be differentiated by their shape and the hyaline or granular character of the cytoplasm.

Incubation Period of the Malarial Fevers.-Two methods have been used to determine this point-the injection of infected malarial blood, and biting experiments with infected anophelines. By the first method the incubation period was eighteen days (the longest) for quartan, three days (the shortest) for æstivo-autumnal, and ten days for tertian. By the second method æstivo-autumnal was nine to twelve days, tertian fourteen to nineteen days, and quartan eighteen to twenty-one days. Since æstivo-autumnal is the only parasite which can complete its cycle in twenty-four hours, the short incubation period is easily understood; on the other hand, the long life eycle of quartan, seventy-two hours, explains its slower development.

Clinical descriptions of the malarial fevers may be found in the standard text-books on medicine, and it is only necessary here to refer briefly to the various forms found in practice. The classical malarial fever consists of a series of paroxysms, following one another with a definite periodicity, daily, every other day, or every third day. Each paroxysm is ushered in by a pronounced chill, which is sometimes preceded by malaise, headache and lassitude. The chill lasts from ten minutes to an hour or more, and the patient wraps himself up in heavy blankets. During the ehill the temperature begins to rise and within a few hours reaches its high point, $103^{\circ}$ to $106^{\circ}$, and then falls slowly to normal during the next few hours. The decline of the fever is accompanied by a profuse perspiration. Successive paroxysms may oceur at exactly the same hour of the day, or may anticipate, febris anteponens, or be delayed an hour or more, febris postponens. The sequence of events, therefore, in a typical malarial paroxysm is malaise, chill, fever and sweat, followed by a period of apparent well-being.

There, is, of course, a typical symptomatology and clinical course in the various forms of malarial fever, but it must not be forgotten that there are many atypical cases, and that malaria is a protein 
disease, mimicking many other infections, and that without a proper examination of the blood that a correct diagnosis is frequently impossible. Pernicious malaria is often mistaken for typhoid, for gastric, renal or cerebral disease, and malaria in children may end fatally without presenting any of the classical symptoms. Blood examinations in all febrile eases in malarious districts are therefore necessary to a correct diagnosis.

The most important sequel is marked secondary anaemia, which comes on rapidly, even in eases so mild that their nature is unsuspected; in severe cases the red cell count may drop to three, two or even one million red cells per'cubic millimeter. The occurrence of anemia, in malarious regions, should always lead one to suspect this disease. It disappears rapidly under proper quinine treatment but recovery can be hastened by the administration of iron and arsenic.

1. Tertian malaria is distinguished by a chill and fever oecurring every other day, the patient feeling quite well on fever-free days. A double tertian infection occurs not infrequently, giving a daily, or quotidian, chill and fever with no free day.

2. Quartan fever, which is relatively rare, gives a chill and fever every third day, with two fever-free days. In this disease also there may be double or even triple infections, giving a quotidian or irregular type of fever.

3. Astivo-autumnal fever (subtertian, or malignant tertian) shows an irregular temperature curve, the cycle varying from twenty-four to forty-eight hours. By some authors this type of malaria is divided into two forms, quotidian and tertian, the former giving a minute ring, the latter a larger one. As multiple infections are common the resulting fever curve may be irregular or continuous and the chill entirely absent. In contrast to the regular intermittency of tertian and quartan this form is often remittent, the temperature curve never dropping to normal.

4. Mixed infections with any two of the above fevers are often found in bad malarial regions in the tropies.

5. Latent malaria is also not infrequent, in which the patient, having no symptoms of the disease, consults a physician for some other reason.

In a region where the disease is very prevalent, Bass* has shown

* C. C. Bass, South. Med. Journ., 1919, 12, p. 460. 
that almost half (45 per cent) the infections are latent, give rise to no symptoms and have negative histories.

6. The carrier state is found among natives or persons long resident in malarial regions, and, aside from the presence of a large spleen and some secondary anemia, may present no symptoms. It is particularly common among native children, tramps and vagabonds. It is not uncommon to find fifty to one hundred per cent of the children in a native village harboring the parasite.

\section{THE DEVELOPMENT OF THE HUMAN MALARIAL PARASITE IN THE MOSQUITO}

\section{(Sexual half of the life cycle, Sporogonie)}

For this stage to be successful the mosquito must bite a malarial patient with gametes in his blood, for if the patient be one in the first stage of the disease, with only schizonts in his blood, no infection of the mosquito will take place, since the schizonts all perish in its stomach. On the contrary, if the mosquito takes blood from a person who has been ill with malaria for some time, or from an apparently healthy carrier, the schizonts die as usual, but the gametes find in the mosquito stomach for the first time conditions suitable for their further development.

The various stages may be studied by causing suitable species of anophelines to bite persons with many gametes in their blood, and then dissecting the stomach and observing the changes which take place there. The development is visible in unstained specimens with high, dry lenses. Since there is no essential difference in the development of the three forms of malaria-in the mosquito, they will be considered together. The first stage has already been described in discussing the appearance and behavior of gametes in fresh blood. In the mosquito the process may be followed further; the macrogamete, freed from its enveloping red cell, projects a little mound on its surface, and this apparently attracts the microgametes to its neighborhood. Into this microphyle one, but never more, of the flagella penetrates, following which the mound is instantly retracted. The fertilized macrogamete, now called a "zygote," soon develops the power of vermicular motion, then being called an oökinet, and travels to the wall of the stomach, and, like the coceodia, penetrates an epithelial cell and there encysts, making the oöcyst. This grows 
rapidly and soon escapes from its host eell and comes to lie in the outer layers of the stomach wall, and as it grows projects into the body cavity of the mosquito. The nucleus divides repeatedly, always accompanied by some of the eytoplasm, forming numerous sporoblasts, and these, in turn, subdivide into innumerable sporozoites; these last escape, with the rupture of the oöcyst, into the body cavity. From there they pass to all parts of the mosquito, but especially, perhaps because of chemotaxis, to the salivary glands and ducts, and when next the mosquito bites a warm-blooded host the sporozoites enter the blood stream and start life anew.

Cultivation of the Malarial Parasites in Vitro.-Bass and Johns in 1911 announced the cultivation of a few generations of plasmodium vivax in vitro under strict anaërobic conditions. Ten c.c. or more of blood from a malarial patient is defibrinated and distributed in small test tubes in one c.c. quantities and to it is added one per cent of a fifty per cent solution of glucose. The red cells settle so that they are covered with one-half $\mathrm{cm}$. of serum; the parasites grow in a thin layer near the top of the cell mass; beneath this they die, or are phagocyted. The optimum temperature is $19^{\circ}$ to $40^{\circ} \mathrm{C}$.

Bass states that he has eultivated all three species of plasmodia by destroying the complement by heating one-quarter to one-half hour at $40^{\circ} \mathrm{C}$. Under strict anaërobiasis it was possible to transfer the cultures and to keep them alive for twenty days.

Transmission.-Transmission is solely by various species of anopheles mosquitoes. Although there are fifty or more recognized species, only sixteen have been proved malarial carriers. The more important are Anopheles quadrimaculatus in the United States, Anopheles albimanus in the American tropies, Anopheles maculipennis in Europe, Anopheles senensis in India, and Anopheles costalis in Afriea.

Description of the Mosquito.-It is impraetical to give more than a hasty deseription of mosquitoes, and the reader is referred to larger works on the subject (Howard, Dyer and Knab; Theobald or "Medical Entomology," Patton and Cragg, London, Madras and ('alcutta, 1913). Malaria at Home and Abroad, S. P. James, 1920. It may be noted, however, that the Culicide, or mosquitoes, belong to the Diptera, or two-winged insects. They pass through four distinct. stages in their development, the agg, larval, pupal and adult or imago stage. The eggs are invariably laid in water where, if the temperature is warm, they hatch in one to four days. Anopheles eggs are single. 
and oval, supported on the surface of the water by ornamental air cells; those of culex are cemented together when laid in raft-like masses.

The larva are aquatic and die quickly out of water. They are both bottom and top feeders, eat voraciously, consuming alga and other vegetable natter, and some varieties are camnibalistic. The larvæ are provided with a breathing tube or respiratory siphon projecting upward from the dorsal surface at the caudal end, and in breathing this is thrust upward to the surface of the water and the larva hangs suspended from the surface film. In the anophelines the breathing tube is short and its angle with the body is such that the larva lies parallel with the surface; with eulex and other genera the body lies at an angle with the surface. The larval stage lasts about six to fourteen days, depending upon the temperature and food supply, and is followed by the pupal stage, during which no feeding occurs; the pupa, however, needs air and is provided with a short respiratory tube at each side of the head. The habit of coming to the surface of the water to obtain air, which obtains in all mosquitoes except the Mansonia, gives a point of attack in combating them, since a layer of mineral oil on the surface of the water occludes the respiratory siphon and so kills them.

The adult, or imago, emerges from the pupa when the latter is one to three days old; the pupal case ruptures along its dorsum and the emerging imago rests on the floating pupal ease until its wings are dry. Since this is a critical stage in its life history and demands quiet water, it is evident that the least wave action is fatal to the mosquito.

The nature of the breeding place is characteristic, to a certain extent, of each genus of culicida; stegomyia (cdes calopus), the earrier of yellow fever, for example, is strietly domestic and breeds in water jars, tin cans, old beer bottles and other artificial eollections of water, wyeomyia breeds exelusively in the fluid at the base of the leaves of air plants (bromeliads); the anophelines, while domestic to the extent that they live near human habitations, require natural collections of water for breeding places, such as sheltered spots along the overgrown banks of streams, temporary puddles and even in water in the footprints of man and animals.

A proper classification of mosquitoes requires considerable training, but the following points will suffice to separate the anophelines from other mosquitoes. On either side of the proboscis, as seen 

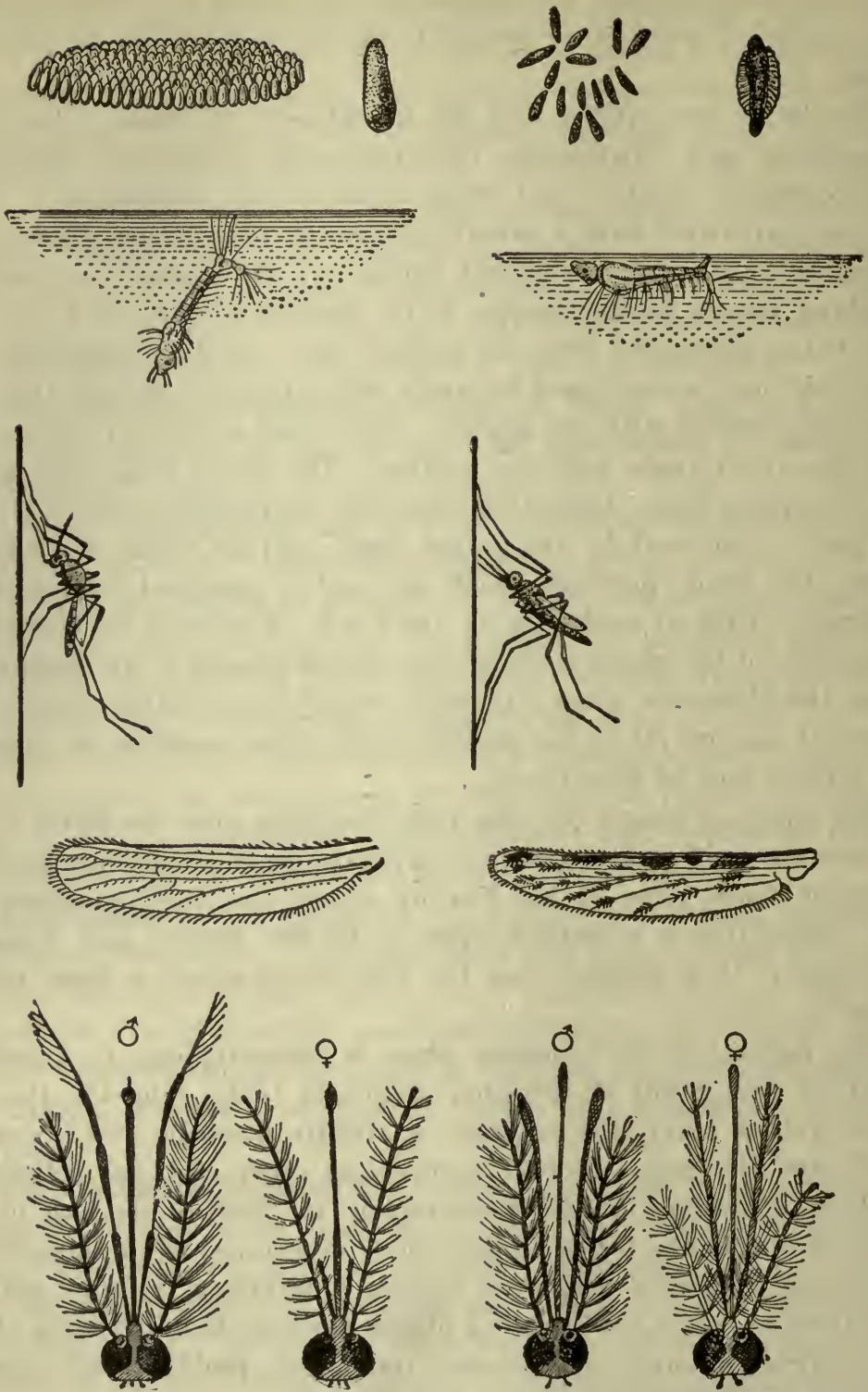

Fig. 161.-Comparison of Culex (left) and Anopheles (Right). Eggs; larvæ (note position); position of insects at rest; wings; heads showing antennæ and palpi. (After Kolle and Hetsch. Jordan, "General Bacteriology," Saunders.) 
under a hand lens, are two pairs of organs; next to the proboscis are the palpi, and outside of these the antennæ; the latter serve to distinguish the sexes, the antennæ of the male being heavily ornamented with a bushy, hairy investment (plumose); the female antennæ, on the contrary, are provided with relatively few, short hairs, arranged in rings at the joints (pilose). In the anophelines the palpi in both sexes are long, at least as long as the probocis, while in all other mosquitoes they are short in the female or in both sexes. This is the principal differential point. The wing markings are of some help, since anopheline wings are almost always spotted. Quite characteristic also is the position assumed by both genera while at rest; among the anophelines the head, thorax and abdomen are all in a straight line and the insect makes an angle with the surface upon which it rests; while the culicidæ are humpbacked, the thorax and head are bent on the abdomen so that the latter lies parallel to the surface. By these characteristics it is easy to identify a mosquito as an anopheline, but the further classification intu species is less simple, and works on entomology must be consulted.

The life history of the anophelines is not yet completely known; they fly and bite at dusk and dawn and during the night, and thus differ from stegomyia and most other culicidæ, which are day-time biters. Their breeding places have already been described; of special importance are the temporary collections of water in which the larvæ, unhampered by their natural enemies, quickly reach maturity in large numbers. With abundance of food and warm weather, the larvæ may require no more than ten days to complete their three molts. The female alone sucks blood, the male living on fruit and vegetable juices. Egg-laying does not take place until after a meal of blood, and it is possible that the female journeys fairly long distances to obtain this food, and that the first flight, from breeding place to human habitations, may be longer than subsequent flights. In general the flight is short, not over three hundred yards, and Gorgas found, in Panama, that a clearing of that width about houses gave ample protection.

The incubation period of the parasite in the mosquito is about twelve days, after which the insect remains a carrier during the rest of its life, and as the health of the mosquito is unaffected, this may be for two months or more. 
Epidemiology.-Since malaria is conveyed solely by the bite of an infected anopheline, the epidemiology is comparatively simple. In practice, nevertheless, the prevention of the disease is extremely difficult; but it is the same for all forms of malaria. It must be attacked from all possible angles and the following are the main points to be observed:

1. Screened houses afford, perhaps, the simplest form of protection, and in beginning work in a new, badly infected place, should be the first thing provided, since they afford a place of security in an otherwise dangerous area, where the workers may take refuge until the situation is under control. This method alone has given magnificent results in Italy (Celli) since it was first used experimentally by Sambon and Low in the Roman Campagna. The screens, to be durable, must be of bronze and not iron, and of a fine mesh (20 strands to the inch), and should be placed, not on windows and doors, but on the outside of porches and balconies; doorways should have screened vestibules.

In default of metallic house screens, bed nets may be used, although they are not very satisfactory, since one must retire at dusk to be protected. In default of both screens and bed nets, something may be accomplished, temporarily, by daily mosquito catching, and in Panama the method has given remarkable results. A native, armed with a small acetylene lantern and a few catching bottles, soon becomes expert, and can capture each day all the mosquitoes in a number of dwellings. In this way very few anophelines escape capture long enough to become infective for man. Chloroform eatching bottles are easily prepared by packing a half ounce of small rubber bands, eut up finely, into the bottom, and pouring in as much chloroform as the rubber will absorb and covering it over with dry blotting paper. The wide-mouthed catching bottle is uncorked and inverted over the resting mosquito, which is killed by the chloroform. A convenient trap bottle has been described by La Prince. Below the cork is placed a funnel trap, making it possible to pass from one spot to another without waiting for the chloroform to act upon the mosquito.

2. The second measure is the prevention of mosquito breeding; this is a large but not a hopeless undertaking, if carried out intelligently. In the first place, it is to be remembered that comparatively few mosquitoes are disease carriers, and that measures need be directed against them only. It was first shown, for example, by 
Gorgas, in Havana, that stegomyia could be practically exterminated by doing away with water in artificial containers in the neighborhood of dwellings; this so reduced their numbers that when a case of yellow fever was introduced into a community the disease would not spread. In the same way malaria-carrying mosquitoes may be fought, without regard to the presence of non-disease carriers. An aecurate mosquito survey is first made, both by examination of the catch of adults and by a hunt for larvæ in eollections of water throughout the district. A puddle or stream is examined by dipping with a white saucer or a long-handled dipper, along the margins; after a little practice it is possible to obtain any larvæ which may be present, and to decide by their appearance and behavoir whether they are anophelines. It is, of course, unneecssary to waste time and money destroying collections of water free from anophelines. When the brecding places have been loeated, they may be destroyed by the use of oil or larvacide, by draining or by filling.

The use of oil and larvacides, while usually a temporary measure, is, nevertheless, of great importance; any light fuel oil may be used by spraying, by mopping the sides of ditches or margins of ponds, or by a drip barrel at the head of a water course. Since in the malarial season the mosquito develops rapidly the oil must be applied regularly once a week. It is better in the long run to begin in the center of the settlement, with permanent improvements, working outwards as money becomes available, and not to depend on oiling except as a temporary measure.

Wherever possible, the swampy areas and pools must be drained, and for this purpose both agricultural tile and open ditches may be used; in the tropies it is necessary to line the latter with concrete to prevent overgrowth by rank vegetation and to protect the banks against eaving. Where, because of the lay of the land, drainage is impracticable the area may be filled, or sometimes flooded, or irrigated with sea water, in which most anophelines do not survive.

3. The infection is kept alive in a community by human carriers, and these are especially common among natives and the poor and ignorant, and especially among the native children. Neweomers should not live within five hundred yards of the dwellings of these classes, as infected anophelines can easily travel shorter distances. Only when it is possible to protect the natives also by these measures is it safe to live among them; and that this is possible has been shown many times, partieularly in Panama. 
4. The proper treatment and cure of all eases will not only prevent relapses and the carrier state (malarial eachexia), but is one of the most important means of exterminating the disease, since every neglected ease becomes a focus for new mosquito and in consequence new human infections. It is just as important a part of the prevention of the disease as any other single measure, and in general has been given too little consideration. Between 50 and 90 per cent of all malarial attacks are relapses and not new infections, and these cases can be cured and the sick rates be reduced accordingly by giving proper and adequate treatment during the acute attack and during convalescence. This means, in effect, that the education of the physicians and of the community in general must be undertaken by the health authorities. It is not sufficient merely to obtain the support of the physicians in any given locality, for perhaps not more than 25 per cent of all cases of malaria ever consult a physician. Many now buy various advertised remedies most of which are of little value, but so far as they are curative, the good effect is without doubt due to the quinine which they contain. The general public can be influenced to buy and take quinine in adequate doses over a considerable period by the educational division of the Health Department. Bass in Mississippi has obtained excellent results by using and advocating the use of a standard treatment of ten grains a day, after the acute attack is past, for a period of eight weeks. While this will not cure all eases, it is a great improvement upon the system of treatment commonly used. To provide as far as possible for the continuation of the treatment for the regular period of eight weeks, the standard package sold and recommended by the local health authorities contains enough quinine for the complete treatment, and it is impossible to buy a part of it at the official price. The immediate effect upon the morbidity and mortality rates of adequate treatment of malarial attacks is immediate and very satisfactory. It is not so much a question of educating the public to take medicine for their illness, they already do that in some form or other, as it is to edueate them to take quinine in proper doses. The influence upon the amount of malaria in a community of proper treatment of all cases is difficult to estimate, because of the shifting of population in malarious regions, but it promises to diminish the number of carriers and correspondingly the number of foci of infection. 
5. Quinine prophylaxis is an unsatisfactory measure which must be used by travelers, explorers and troops. There is no method of using quinine which will entirely prevent malaria when the chances for infection are many; the following methods have all been used: (1) The so-called gram prophylaxis, in which one gram of quinine is taken intermittently every tenth day as the minimum to every fourth day as the maximum. The gram may be taken in a single dose at bed-time, or in four divided doses during the day-time. The double gram prophylaxis, in which the dose is taken on two successive days, as, for example, on the tenth and eleventh, or on the fifth and sixth. (3) The half gram prophylaxis, as proposed by A. Plehn, was 0.5 gram every fifth day; experience has shown that it is of little value. (4) A daily dose of 0.4 to 0.8 gram gives better results than any other method, since the patient suffers less from cinchonism than when larger doses are taken intermittently, and takes his quinine more faithfully. The size of the dose depends on the form of fever present and the number of chances for infection; 0.4 gram will often protect against tertian and quartan, while even 0.8 may fail to prevent rstivo-autumnal. Latent infections and relapses among "prophylacticers" are common and black water fever is not an infrequent sequel.

It is well to remember that quinine is rapidly absorbed from the intestinal tract and also as rapidly excreted, and that so far as possible the doses should be properly timed.

It is probable that quinine is already in the blood within half an hour of the time it is taken, and that the whole dose is absorbed within six hours and that most of it has already been excreted in the same period. To provide quinine in the blood in suffieient concentration to be effective in killing the sporozoites injected by the mosquito, it is most reasonable to take one dose of five grains an hour before sunset, and a second dose, if one is compelled to be out, just before midnight. Ten grains taken in this way will provide against infection at dusk and at dawn, the two principal periods during which the anophelines bite, better than one dose at bed time, of the same size.

6. Personal prophylaxis by means of head nets, gloves, suitable clothing, and the use of essential oils, such as eitronella, on exposed parts of the body, is helpful in emergeneies.

7. The protection of human beings by means of animal barriers has been recommended by several writers. The subject has been 
reviewed by Roubaud,* who has been much impressed by the practical disappearance of malaria from France without a corresponding diminution of anophelines capable of acting as carriers. In La Vendee, a Department of France, are regions where there are many swamps and few eattle or domestic animals and it is in such regions that human malaria remains; in other regions, abundantly supplied with domestic animals the disease has practically disappeared although anophelines still may be found in the animal shelters. He believes that the insects prefer the blood of cattle and horses to that of human beings, and that a sufficient number of animals will protect the human inhabitants against infection in an otherwise malarious locality. The method is of sufficient importance to merit further study and trial.

In conclusion, it may be affirmed that any district, no matter how notorious, may be freed from malaria, if necessity demand it and money be fortheoming, by means of the above measures.

Pathology.-The pathological features of the disease are quite definite; at autopsy there is evidence of some secondary anemia, due to the destruction of enormous numbers of erythrocytes; the hemozoin, in well-marked cases, accumulates in the viscera until they are chocolate or slate colored; the spleen is enlarged and friable, and the liver and kidneys may show cloudy swelling. In smears prepared from the spleen, liver, kidneys, brain and bone marrow, parasites and hemozoin will be found, although the former may not be numerous. The distribution of the parasites is usually unequal, but they are often present in the spleen and brain in greatest numbers.

The origin of the pigment has already been described; it is phagocyted and accumulates in the viseera, but after a time disappears in some unknown way; its presence, therefore, is an indication of malaria in recent years. It must be distinguished from hemosiderin, a yellowish pigment found in the viscera after extensive destruction of red blood cells. Hemozoin is soluble in alkalis and insoluble in acids, water, ehloroform, aleohol and ether, while hemosiderin is insoluble in acids and alkalis but soluble in alcohol. Both contain iron, yet the former (hemozoin) does not give a Berlin blue reaction, while it is present with the latter.

* Roubaud, E., Les Conditions de nutrition des anopheles en France et le rôle du Betail dans la prophylaxie du palurlisme. Ann. Inst. Pasteur., Paris, 1920, $34,181$. 
The leucocytes, although increased during paroxysm, are soon diminished so that leucopenia is characteristic of the disease; in addition, there is a relative increase in the mononuclear leucocytes. The loss of hemoglobin is very great, yet is quickly recovered from during convalescence.

In fatal cases of æstivo-autumnal fever a striking feature is the presence of innumerable infected red cells in the capillaries of the brain or abdominal viscera. A smear from a pigmented brain may show a capillary thrombus made up of infected erythrocytes, most of them showing the sporulating stage. In deaths after repeated malarial attacks the kidneys will show, in addition to the presence of many parasites, a marked chronic diffuse nephritis, one of the most important sequelæ of the disease.

Immunity.-The disease is strictly confined to human beings, as none of the lower animals are susceptible; there appears to be some racial and acquired immunity, although it is incomplete; in native settlements the number of children showing parasites in the blood is much greater than the number of adults, yet the latter are not absolutely immune; one attack certainly gives no protection against a new infection. The evidence from different regions is quite conflicting in regard to immunity and has been most plausibly explained in this way; where the disease prevails throughout the year there is a constant reinfection and under this stimulus the body is able to keep up an immunity strong enough to kill off the parasites before any symptoms arise; in other regions, however, where malaria is a seasonal disease, the constant stimulus is lacking and there is less evidence of immunity. It has also been noted that the three varieties of the disease are distinct, and that no immunity against the whole group is obtained by an infection with one species.

Since many mild cases recover without treatment, it is apparent that some little immunity is produced by an infection, yet it is temporary and does not protect against repeated relapses. Before the days of radical treatment with quinine, relapses were the rule and were considered an essential feature of the disease (Manneberg).

Clinical experience teaches that relapses occur when treatment has been insufficient, and especially after fatigue, getting wet and catching cold, or after over-heating in the tropical sun, and particularly after a sea voyage or a long journey. They may continue to occur in a region free from malaria, for about three years; in a malarial region it is difficult to differentiate between relapses and 
reinfections. They are most frequent after quartan, then tertian, and least following restivo-autumnal.

The cause of the relapse is still a matter of diseussion; Schaudiun explained it as due to parthenogenesis, the macrogamete changing to a schizont after expelling a part of its nucleus and cytoplasm and so starting a new eycle of asexual parasites. Craig has suggested an intra-corpuscular conjugation as the beginning of a new crop of parasites. It is possible that the old theory, that parasites survive for long periods in the viscera, is correct and that the relapse is brought about by any condition which temporarily reduces the convalescent's immunity, such as fatigue, ete. H. C. Clark, in Panama, has examined a large series of placentas from apparently healthy women and found that not infrequently they contained plasmodia in large numbers, even in the absence of recent malarial attacks. There was, evidently, sufficient immunity to hold the parasites in check under ordinary circumstances.

Under ordinary conditions the disease prevails more extensively and in a more severe form among recent arrivals in a district than among the older residents; while this is no doubt partly due to an acquired immunity, it is not improbable that education in the proper methods of treatment of the acute disease, to prevent relapses; in the avoidance of notoriously dangerous areas, and in the selection for favorable sites for dwelling, and the protection of the individual by screens, are of equal importance in protecting the older residents.

A specially severe form of malaria has been called "campaign malaria," which develops among troops in endemic areas. In such cases the housing, and food conditions are usually poor, the men are engaged in hard and dangerous work at all hours of the day and night, with the result that they are repeatedly kitten by anophelines, and their blood shows a multiple infection.

\section{BLACK WATER FEVER}

This condition follows malaria and occurs only in malarial districts, and by most authorities is believed to be a sequel of the disease, due to some unknown factor. It is characterized by the passage of urine containing hemoglobin, albumin and casts. In color the urine varies from a pale red to a black. Castellani and Chalmers, however, separate it into three forms, symptomatic, toxic 
and specific hemoglobinuria, examples of which are, respectively, malarial and quinine, hemoglobinuria and black water fever. In none of these, however, is the etiology clear; the disease does not occur independently of malaria, and an attack may be precipitated or aggravated by quinine. Leishman has described certain cell inclusions, possibly chlamydozoa, as the eause. The etiology is, at the present time, far from elear. Malarial parasites may be present in the blood up to time of the appearance of the hemaglobinuria, but then they rapidly disappear since it is the infected parasites which first undergo destruction. To determine the presence of hemaglobin with certainty the urine should be examined with a spectroscope; small models are made which are suitable. The hemaglobin in the blood falls rapidly, to twenty-five per cent or even less; the number of red cells is also diminished, often to three million per cubic millimeter or and in severe cases to a much lower figure.

In these cases quinine must be stopped until the hemaglobinuric attack is over, when the parasites reappear and the malaria must again be treated. In the mean time the patient should be kept in bed and be given the best possible care and nursing.

\section{TREATMENT OF MALARIA}

In quinine we have a true chemical specific for malaria, and when given early enough and in sufficient doses, will cure the disease with certainty. It is usually given in the form of the sulphate or dihydrochlorate, preferabiy in solution, but may be administered in freshly prepared capsules; pills and tablets, while convenient, are unsatisfactory because of their relative insolubility. It acts vigorously on the merozoites and young trophozoites, but has almost no direct effect upon the gametes. The size of the dose depends on the form of the fever and its severity. In ordinary cases five grains three times a day is sufficient, while in severe infections not less than thirty grains a day must be given; the best time is immediately after meals, without regard to the time of the ehill. To prevent relapses, the treatment of the original infeetion must be thorough, and the patient should be kept in bed, upon a light diet and attention paid to the condition of the bowels. During and after convaleseence the treatment must be continued for three months, though the daily 
dose may be decreased gradually, beginning a few days after the subsidence of the fever. In exeeptional cases a relapse will oceur while the patient is still taking massive doses of quinine, and by some this has been looked upon as an evidence of immunity of the parasite to the drug, but it is possible that it is merely due to nonabsorption of the quinine, and carminatives should be added to the dose to assist in its absorption.

The addition of pepsin to the quinine will reduce the unpleasant effects of the drug. To ninety parts of quinine add 6 parts of laetose and 4 parts of pepsin.

The proportionate dose required for children is greater than indicated by Cowling's rule. Bass finds that for children of fifteen or over, the full adult dose is necessary. From eleven to fourteen 0.8 of the adult dose, from eight to ten 0.6 , from five to seven 0.4 , for three and four year old children 0.3 , two year old 0.2 , one year old 0.1 of the adult dose is necessary.*

In selected cases quinine is occasionally given subcutaneously or intravenously although the method is not free from the risk of abscesses in the subcutaneous tissue, or death after intravenous use.

For intravenous use a freshly sterilized solution consisting of 12 to 15 grains of bihydrochlorid of quinine dissolved in 10 to 20 c.c. of normal salt solution can be injected into a vein at the rate of half a c.e. per minute. For subeutaneous use the same quantity of quinine may be used but it must be dissolved in a few cubic centimeters of fluid. The injection must be given into the loose arcolar tissue but even then it is often painful and apt to be followed by necrosis and abseess.

Treatment with arsphenamine and related preparations.

It has been shown that the administration of half doses of the 606 group of remedies can be given to selected eases of severe malaria in addition to the usual treatment with quinine, with benefit; for ehronic resistant infections and for malarial cachexia three or four injections at weekly intervals are recommended. The tonic effect of these drugs is quite marked and the duration of illness is shortened by the combined treatment.

*Pratt-Johnson, J., Gilchrist, Kenneth, and Hay-Michel. On the Action of Certain Special Preparations on Malarial Parasites and their Fmployment in the Treatment of Malaria. 
The gametocytes, when once formed resist all forms of treatment, but under energetic quinine medication the crescents usually cease to be produced and disappear from the circulating blood in about fifteen days.

\section{OTHER MALARIAL PARASITES}

From time to time additional malarial parasites have been described, only two of which are of any importance at present: Plasmodium vivax, variety minuta, and Plasmodium tenue. The former was described by Ahmed Emin in 1914. It resembles the usual tertian in general, but differs in the following points; it is smaller, the infected erythrocyte is not enlarged, and the number of merozoites is small (four to ten). The pigment is fine and motility is not marked. Multiple infection of the erythrocytes is not uncommon. Craig ${ }^{3}$ described a similar parasite in 1900 , and he suggests that the parasite has been confounded with Plasmodium malariæ.

In 1914 Stephens $^{4}$ deseribed an organism from one slide, which he calls Plasmodium tenue. It is said to be defieient in pigment, markedly motile, and rich in chromatin. Since the parasite was described from one slide, it is very doubtful if it ean at present be accepted as a valid species.

Piroplasmidæ (Franca).-This is a provisional family belong to the hemosporidia, the type of which is Babesia bigeminum, Smith and Kilborne (pirosoma, piroplasma), the cause of Southern cattle fever.

The parasite was first described by Smith and Kilborne in 1889 , and correctly placed by them among the protozoa; they also demonstrated its transmission by the cattle tick, and this achievement marks the beginning of medical protozoölogy. To the original parasite, the cause of Texas fever, has, in course of time, been added other forms until now we have a family consisting of Babesia bigeminum, bovis, canis, equi, ovis, mutans, quadrigeminum, and a closely related parasite, Theileria parva.

Morphology. - The parasites are pear-shaped, round, oval, or ameboid, inhabiting mammalian red hlood cells, which they destroy but without produeing pigment. In cattle, sheep, horses and dogs, the

${ }^{3}$ Craig, Jour. Parasitol., Urbana, IIl., 1914, 1, 88.

'Stephens, Proc. Roy. Soc., Lond., Series B, 87, p. 375. 
freed hemoglobin is excreted by the kidneys, producing the disease variously known as red water fever, Texas or Southern cattle fever, tick fever, bovine malaria, hemoglobinuria, and others. The parasite is small, two to four microns long and one to two wide, and characteristically occurs in pairs, the narrow ends being united. When the parasite is mature the two daughter cells separate and when liberated by the degenerated erythrocyte attack now red cells. The pear-shaped babesia enters a new cell by its broad end, becomes rounded or ringlike, then ameboid in form, and finally the nucleus sends out a bud; this divides into two by forking, and as the nuclear matter continues

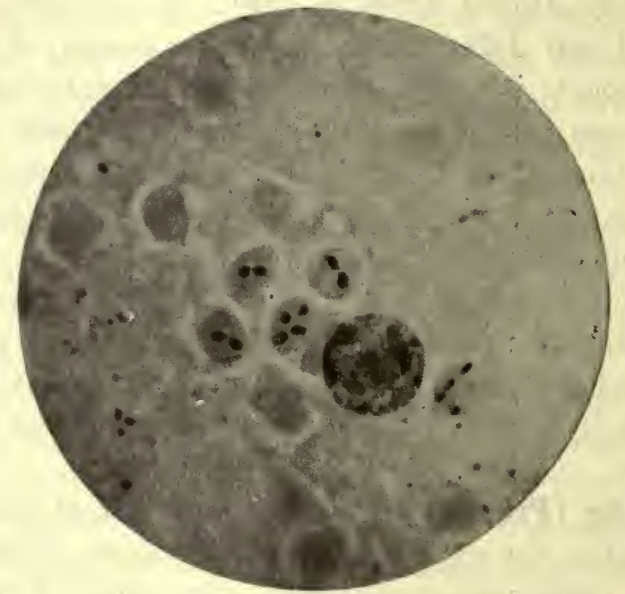

Fig. 162.-Babesia Bigeminum. (Army Med. School Collection, Washington, D. C.)

to grow each portion becomes surrounded by cytoplasm and ultimately the two new daughter cells separate from one another. Multiple infections of single red cells are common, as many as sixtcen pairs having been seen in a single cell.

Good preparations for clinical work are obtained by some one of the Romanowski stains, but the finer details of the nucleus and cell division can only be studied after iron hemotoxylin staining.

The parasites, with the exception of Theileria parva, do not disappear completely from the blood after the animal recovers from its illness, but remain indefinitely in the circulation, and their continued presence and virulence may be demonstrated by the inoculation and non-immune animals with blood from an animal which has recovered. 
Transmission.-Transmission from host to host is by means of ticks. Margaropus annulatus carries Southern cattle fever in the United States, but other ticks are carriers in South America and the West Indies. In general, each species of babesia is specific for a particular animal, and each is carried by a separate group of ticks.

Clinical Observations.-Clinical observations have shown an incubation period of about fourteen days; the onset is with fever and the cattle look weak and ill, neither eat nor chew the cud, but stand with sunken head and relaxed ears. A bloody diarrhea sets in early and the urine, which is small in amount, is deep red in color and contains much albumin. The blood shows few parasites at first, but they soon increase and the number of red cells falls rapidly. The mortality varies in different epidemies from five to sixty per cent, but has been as high as ninety in some herds. Young cattle, under a year, have a mild form of the disease, and remain thereafter immune.

The treatment, once the disease has appeared, is unsatisfactory, sinee we have no specific, but much may be done by prophylaxis. Quarantine of cattle from infected regions is absolutely necessary to prevent the spread of the disease, since all immune animals are also carriers. Young animals, between nine and twelve months of age, may be inoculated with the blood of those which have recovered, five to ten c.c. being given in a single dose; the disease lasts two to three days, and the mortality is not great, as eighty to ninety per cent of the inoculated animals recover and remain immune. Inoculation, however, is a measure of doubtful value and some means of tick eradication should be used if the disease is to be permanently stamped out.

\section{Life History of the Tick (Margaropus annulatus) ${ }^{5}$}

The tick's life is divided into two stages, one part passed on eattle and another part passed on the ground. The mature female, as found on cattle, is about half an inch in length, plump and olive green. When fully engorged with blood from its host, it drops to the ground, seeks a sequestered hiding place and if it escapes birds, ants and other enemies, begins after a few days of warm weather to lay eggs. These are small, elliptical, at first light, later dark brown, and are cemented together in irregular masses by a sticky secretion; in numbers they vary from a few hundred to more than five thousand for

'Farmers' Bull. No. 498, U. S. Dept. of Agri. 
each female. The female tick dies in a few days after the egg-laying has been eompleted. The eggs soon hatch (after nineteen days in summer to one hundred and eighty-eight in winter), and a small, oval, six-legged larva or seed tick appears, and promptly climbs up on the nearest vegetation, grass, weeds or bushes to lie in wait for a warmblooded host. Although while on vegetation seed ticks do not take food nor grow, their endurance is great, and during the colder parts of the year they may live for eight months. The next stage begins after the seed tick has found a host, when it sucks blood, increasing in size and soon (five to twelve days) molts and a new form, the eight-

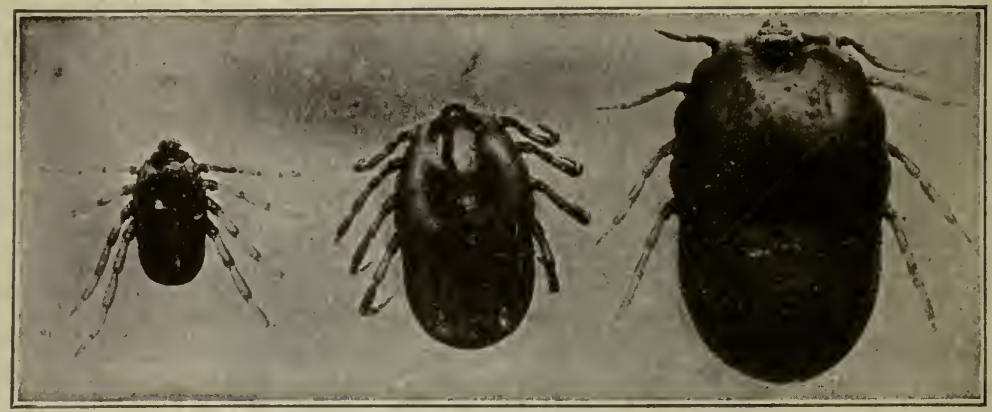

Fig. 163.-The Texas Fever Tick (Margaropus annulatus). (Rosenau, "Preventive Medicine and Hygiene.")

legged nymph, appears. In five to eleven days more a second molt occurs and the tick is then sexually mature and males can be distinguished from females. The female does not move about on the animal, but the male seeks her out, and after fertilization growth goes on rapidly until the engorged female drops to the ground. To summarize: On the ground is the engorged female, the eggs and seed ticks; on the animal is the seed tick; the nymph, the sexually mature adult and finally the engorged female. The infected female tick transmits the babesia to the larvæ through the eggs, but does not herself bite nor convey the disease directly to another animal.

It is evident that the tick may be attacked in the pasture or on the cattle. Pasture rotation is one of the methods recommended by the Agricultural Department, and its rests upon the fact that all the ticks will die from starvation in an unused pasture in from six to twelve months, varying with the elimate, the shorter period holding true for warmer localities. By changing pastures a farm may be freed 
of ticks in four and a half or eight months, depending on the plan followed. For cconomic reasons, lack of sufficient pasture land, etc., this plan has not been widely adopted. A second plan, that of dipping, has been more successful. The cattle are driven through a large dipping vat at intervals of two weeks (never more than three must elapse) until they and the pasture are free from ticks. The fluid in the dipping vats is an alkaline solution of arsenic; oil dips are little used at present. Arsenical dips are cheap, easily prepared and efficacious, two or at most three dippings, at ten-day intervals, being sufficient to free heavily infected cattle, and if they can then be put on tick-free pastures the problem is solved. If no tick-free pastures are available, dipping must be continued, as above, until the animals remain permanently free.

The prevention of tick-borne disease is to be solved, therefore, by tick eradication, and experience in the Southern states has shown this to be a practical measure.

\section{SUB-CLASS-NEOSPORIDA}

\section{SARCOSPORIDIA}

These organisms belong to the sub-class Neosporidia of the Sporo$z o a$, because the spore formation commences before the completion of growth. They have been known since 1843, when Miescher discovered "tubes" in muscle fibers, visible to the naked eye as fine, white, opaque filaments. They have been found in deer, cattle, sheep, swine, rabbits and man, and occasionally in birds and reptiles. A]though long known, our knowledge of them is still defective. In sheep they are the cause of severe epizoöties, and in mice they are fatal: otherwise they seem to be harmless parasites and in rare instances have been accidentally found at autopsy in man.

The method of transmission is not definitely known. Theobald Smith was able to infeet mice with Sarcocystis muris by feeding muscles from infected mice, and Darling was able to infect guinea-pigs in the same mammer. In nature it is probable that infection oceurs through the intestinal tract. They are exceedingly common in some localities and may be seen in most abattoirs; they have been found in ninety-eight per cent of swine, ninety-eight per cent of sheep, and commonly in deer and mice. 
To the naked eye they appear as whitish, opaque, cylindrical bodies in the muscles lying parallel to the fibres. In the sheep, Sarcocystis tenella reaches a length of sixteen mm., and in the deer cysts of fifty $\mathrm{mm}$. are found. In structure the "tube" is seen under the microseope to be composed of many sickle-shaped spores, ealled Rainy's eapsules. The tube has not a single eavity but a honey-comb or alveolar structure, and the spores are found in small aggregations in the chambers, completely walled off from one another. The tube itself has a heavy striated wall, either secreted by the organism or composed of altered muscle fiber of the host. The spores are sickle- or kidneyshaped and vary both among themselves and with the host. They contain a nucleated trophozoit, and at one pole either a elear or an obliquely striated body.

Laveran and Mesnil $^{6}$ (1899) obtained a toxin from Sarcocystis tenella and named it sarcocystin. Darling, working in Panama, found this organism in the biceps muscle of a negro from Barbados. Not more than five human eases are known.

'Trop. Diseases Bull. London, 1920, 16, 96. 


\section{CHAPTER LVII}

\section{CLASS IV-INEUSORIA}

SUB-CLASS-(CILIATA)

THIS class is sharply distinguished from all other protozoa by the presence of numerous cilia, distributed in various ways over the ectoplasm, which serve as organs of locomotion, and by the presence of two or more nuclei. They fall into two classes, the ciliata, which are provided with cilia during the entire life cycle, and the suctoria, which lose their cilia on entering the adult stage; the latter are of no interest, since none are holozoic.

Only one of the ciliates is of importance in medicine, Balantidium coli. Balantidium minutum and Nyctotherus faba, having been reported only once in man by Schaudinn, are too rare to be considered here.

\section{BALANTIDIUM COLI (Malstan)}

This parasite, first described in 1857 , is commonly found in the lower intestinal tract of swine; while usually harmless, it may cause a moderate mortality in them from subacute and chronic dysentery. It has frequently been reported as present in man, and is readily diagnosed on examination of the stools.

The organism is much like paramecium and is actively motile when obtained from fresh stools, or from scrapings from the uleerated cecum at autopsy. The body is ovoid and rather stumpy toward the anterior end, distinguished by the triangular peristome; average measurements are about eighty by sixty microns. The ectoplasm is covered with thick, parallel bands of active cilia, giving the animal a striated appearance; the macronucleus is kidney shaped and lying close to it the micronucleus may usually be distinguished. At one side of the organism are found two contractile vacuoles, and, in the endoplasm, food vacuoles and fat droplets. 
Multiplication in its simplest form consists of binary division, though onnjugation also occurs. In the feces of the host encysted forms are common, and by means of these the infection is transferred to new hosts.

Although long believed to be harmless, pathological lesions have been found by Strong and others in man and monkeys, consisting of

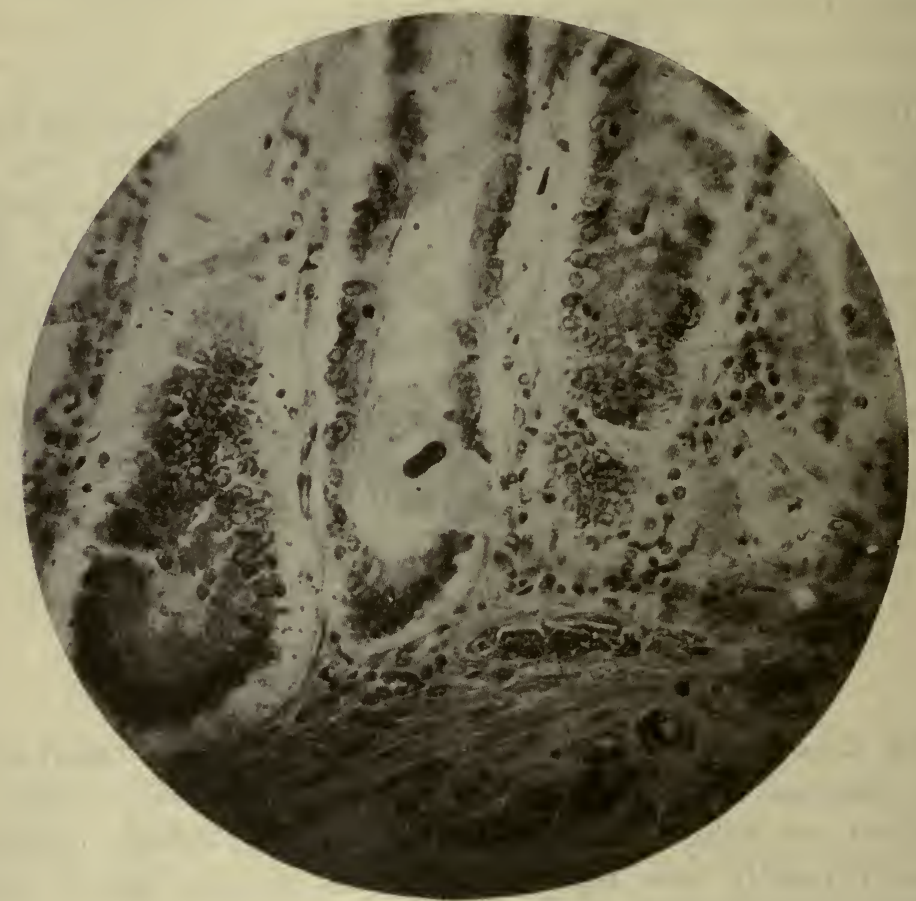

Fig. 164.-Balantidium coli in a Follicle of the Colon, Breaking throvgh THE Mucosa. (Army Med. School Collection, Washington, D. C.)

thickening and ulceration of the infected intestine, with penetration of the balantidium through the gut wall to the subserous layer. Extensive lesions may be found at autopsy, even in the absence of a history of dysentery or diarrhea.

The disease is transmitted, in all probability, by ingesting the cysts from swine which undergo development in the human being to the cyst stage: this stage, however, is never found in human infections and man, therefore, is probably incapable of spreading the disease. 
Treatment has not been satisfactory : Brug, ${ }^{1}$ however, has reported a case which responded to arsphenamine after simaruba, iodoform enemas and oil of chenopodium had failed.

\section{CHLAMIDOZOA}

There is a small group of very minute intracellular organisms which are believed by many to be protozoa, and for them von Prowazek has created the class named "chlamydozoa." He believes that such organisms" produce smallpox, vaccinia, hydrophobia, trachoma, scarlatina, Molluscum contagiosum, avian plague, the contagious epithelioma of birds, hoof and mouth disease, jaundice of silk worms and others. The delicate organisms are first noticed as fine dots of chromatin lying in the cytoplasm of the infected cell (the so-called elementary bodies). When larger they are called initial bodies and are believed to set up a reaction in the cell, which causes an extrusion of the plastin from the nucleus; this envelops the initial bodies like a mantle, hence the name "chlamydozoa," from the Greek stem "chlamys," meaning mantle. Inside of this covering they multiply, in some instances invading the nucleus.

Their very existence is a matter of dispute, yet at the present time the consensus of belief is that they constitute a group of parasites and are not merely forms of cell inclusions or degenerations.

Noguchi ${ }^{2}$ has shown that in trachoma these organisms may be found alone without any other pathogenic organisms being present, and that they may be transferred to the conjunctiva of the baboon and higher apes, producing a mild form of the disease. Smears of the exudate, taken from the infected animals and stained with Giemsa, show cell inclusions without the presence of bacteria.

${ }^{1}$ Brug, S. L., Tropical Diseases Bulletin, London, 1921, 17, 181.

${ }^{2}$ Noguchi, Jour. Exp. Med., N. Y., 1915, xxii, 304. 


\section{CHAPTER LVIII}

\section{TECHNIQUE OF BLOOD EXAMINATIONS FOR PROTOZOA}

St_IDES and cover glasses must be serupulously clean, and are best kept in covered glass jars which are, dust-proof. For most purposes stained preparations on slides are satisfactory and much easier to handle than cover glasses.

In all investigations, both wet and dry preparations should be made; some practice is, of course, necessary to obtain satisfactory ones, thin enough to show details. As Manson says, in looking for dates on coins, one would not pile one coin on top of another; so, in searching blood eells for parasites, it is necessary to have the cells lying flat, and in a single layer. Wet preparations are made with a drop of blood not much larger than the head of a pin, on which a cover glass is dropped, and gently pressed down; it is advantageous to lute the margin of the cover glass with warm vaseline (warmed by holding the eamel's hair pencil over a flame for a few seconds) to prevent evaporation and consequent "eurrents under the cover glass. A properly sealed wet preparation may be examinea at intervals during several days, and this is often necessary in studying movements and the life eycle. Malarial parasites, trypanosomes and others are quickly deteeted by their movements.

Stained preparations are necessary for the study of details of structure, and for elinical work are invaluable, since smears may be collected at the bedside, and examined later at home, either by daylight or artifieial light. In general, a one hundred watt concentratedfilament nitrogen bulb will be found the best source of illumination for the study of both living and stained protozoa. The Welsbach mantle, acetylene gas or even a kerosene lamp are satisfactory substitutes. At times a color sereen, made by interposing a round, glass flask, filled with diluts copper sulphate solution, between the light and the mirror, will prove advantageous. It is particularly in the South and in the tropies that artifieial illumination has been most valuable.

To prepare stained preparations, a small drop of. blood from the ear is placed on a slide near one end, while with another clean slide 
the spread is made by holding its narrow end in the drop until the blood has flowed between the two, and then pushing the spreader, or second slide, held at an angle of about $45^{\circ}$, toward the opposite end of the first slide. The drop of blood follows closely behind the spreader, and the thickness of the film may be varied by changing the angle of the spreader. The thin film of blood dries quickly in the air. It is never fixed in the flame, but always in methyl alcohol or in the stain itself.

Many varieties of blood stains have been proposed, but the simplest and best are those of Wright and MacNeal. Wright's stain is prepared as follows:

Dissolve $0.5 \mathrm{gm}$. of sodium bicarbonate in 100 c.c. distilled water, and add $1 \mathrm{gm}$. of methylene blue (Gruebler). Any of the methyleme blues of Gruebler known as “BX," Koch's or Ehrlich's rectified may be used. It seems to be important that the bicarbonate of soda be all dissolved before adding the methylene blue.

The mixture is next to be steamed in an ordinary steam sterilizer for one hour, counting the time after "the steam is up." The heating should not be done in a pressure sterilizer, or in a water-bath, or in any other way than as stated. This steaming of the alkaline solution: of methylene blue effects certain changes in the methylene blue whereby a polychromatic quality is given to it, so that the compound with eosin, which is later to be formed with it, has the property not only of differentially staining the chromatin of the malarial parasite, but also of differentiating and bringing out more sharply the nuclei and granules of the white blood corpuscles.

When the steaming is completed, the mixture is removed from the sterilizer and allowed to cool, the flask being placed in cold water if desired. When it is cold, without filtering, pour it into a large dish or flask, and.add to it, stirring or shaking meanwhile, a sufficient quantity of a 1:1000 solution of eosin (Gruebler, yellowish, soluble in water) until the mixture, losing its blue color, becomes purplish, and a scum with yellowish metallic luster forms on the surface, while on close inspection a finely granular black precipitate appears in suspension. This will require about 500 c.c. of the eosin solution for 100 c.c. of alkaline methylene blue solution. (The proper amount of eosin to add may be determined hy placing a drop of mixture on white filter paper, and adding eosin until the blue spot shows a distinct halo of pink.)

The precipitate is collected on a filter, and, without washing, is 
allowed to dry thereon; when thoroughly dry, dissolve this precipitate in pure methyl alcohol in the proportion of three-tenths of a gram to one hundred cubie centimeters of alcohol. This alcoholic solution is the staining fluid.

MacNeal ${ }^{1}$ has shown that both methylene azure and methylene violet participate in the nuclear staining, and that an excellent stain, equal to any, may be made directly by mixing the pure dyes according to the following formula:

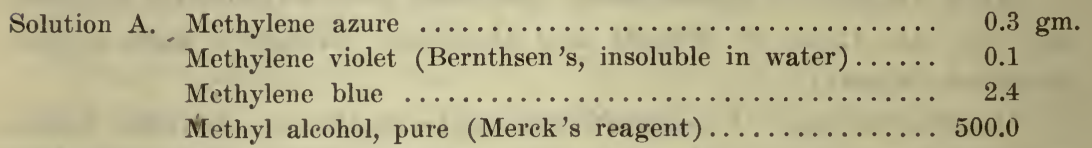

Solution B. Eosin, yellowish, water soluble............... 2.5

Methyl alcohol, pure................. 500.0

These stock solutions will keep for at least a year. They are mixed as needed in equal parts, and diluted by the addition of 25 c.e. of methyl alcohol to each 100 c.c. of the mixture. The mixture will keep for several months.

The method of staining is the same, whatever methyl alcohol stain be used. The cover glass or slide is flooded with the stain, which is allowed to act for one minute; next, as much distilled water is added, drop by drop, as the slide will hold, or until a yellowish metallic scum is formed on the surface, and the mixture is allowed to act from three to five minutes. A convenient staining dish is made by laying a pair of glass rods on top of a flat oblong dish; the rods may be held in place with adhesive plaster or perforated strips of wood. Fixation is accomplished by the undiluted solution, but the actual staining does not occur until water is added; by repeated washing in distilled water, any desired differentiation may be made.

It is sometimes convenient, when searching for malarial parasites in clinical cases, to stain as follows : ${ }^{2}$

Use four bottles or Coplin jars; in the first, put the undiluted stain, and in it immerse the slide for one minute; in the second, put distilled water and transfer the slide to it for four or five minutes; in the third jar put diluted Manson's stain, about 0.5 c.c. to 50 c.c. water, and in this the smear remains one-half minute; in the fourth

${ }^{1}$ MacNeal, Jour. Inf. Dis., Chicago, 1906, iii, 412.

${ }^{2}$ Russell, F. F., Jour. Amer. Med. Ass., Chicago, 1915, 1xiv, 2131. 
bottle or tumbler put distilled water, in which the slide is washed quickly and then dried. This method, for the average man, is more economical and simpler than the open method. Stained smears are examined directly in immersion oil without the use of cover glass.

Manson's stain is prepared as follows:

T'wo grams of methylene blue, medicinally pure (Höchst), is added to 100 e.c. of a boiling 5 per cent solution of borax. This stain, though not permanent, will last a long time. It is used chiefly for the diagnosis of malaria. It is diluted before use. Stain for ten to fifteen seconds.

When parasites are few, as in latent malaria, thick films may be used, as first proposed by Ross. A large drop of blood is spread thickly on the side, with the needle or pen used in puncturing the ear; after drying, it is put into (1) 95 per cent methyl aleohol to which 1 per eent of hydrochlorie acid has been added, until the smear has been hemolized; (2) it is then thoroughly washed in running water, (3) and then stained in the usual way with a methyl alcohol stain. Although the method takes considerable practice, it is a valuable procedure. 


\section{+}

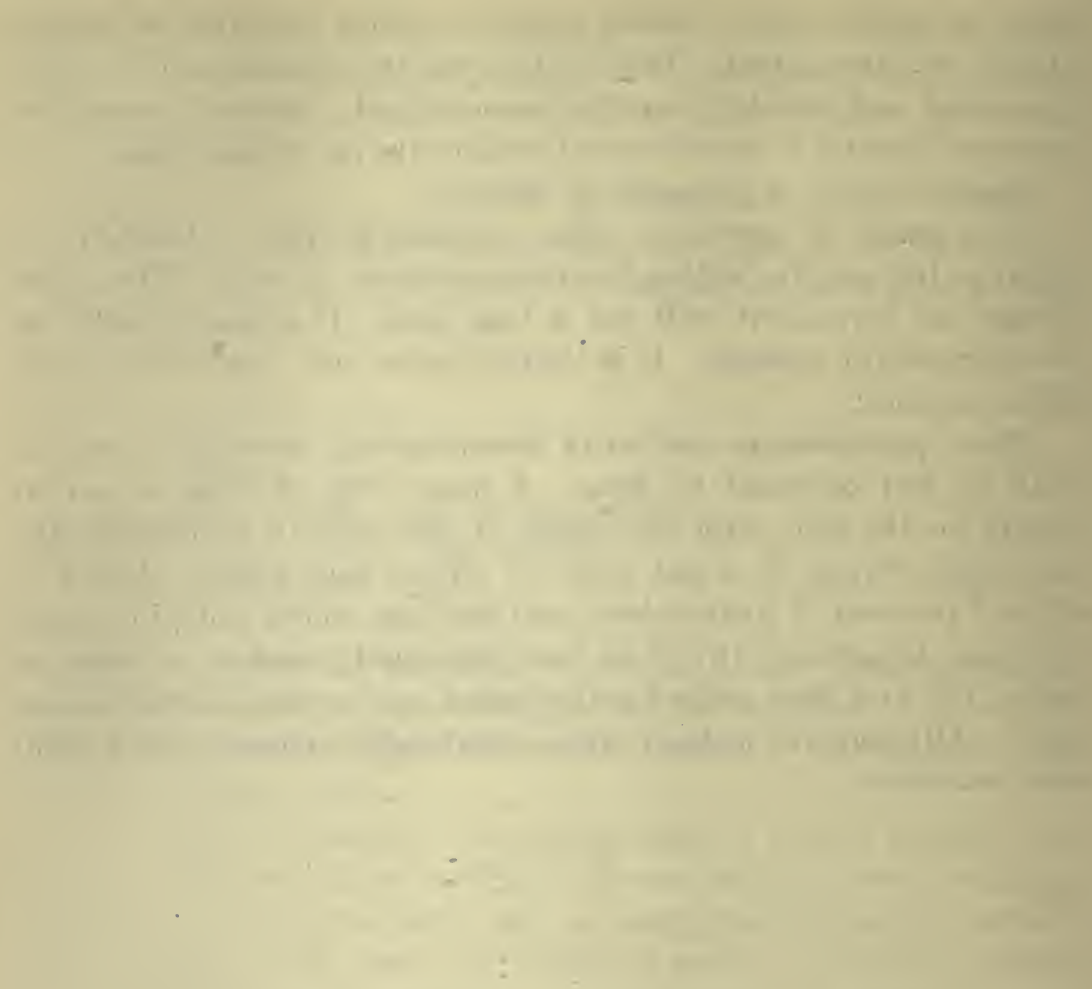




\section{INDEX OF ACTHORS}

Аввотт, 116, 389

АвEL, 240, 724, 726, 810

ACHARd AND Bensaude, 688

ADAMI, 222, 631

AdAMI AND CHAPIN, 1022

Adie, Helen, 1099

Albrecht ANd Giron, 518, 523, 566, 808

Alvarez and Tavel, 618

АМАKО, 712

Anderson, 596, 1035

Anderson And Frost, 916, 918

Anderson AND Goldberger, 923, 937, 939

Anderson ANd McClintic, 99

ANDREWES AND HARDER, 409

ANTHONY, 419

Aristothe, 2

ArKWright, Bacot and DunCan, 943, 949

ArLoing, 77

Arloing, Carnevin and Thomas, 771

Armstrong, 746

Arnheim, 941

Arning, 624

Aronson, 108, 166, 411, 425, 430, 451

D'Arsonville and Charrin, 79

Arthus, 355

Arustamoff, 963

Ascoli ANd Figari, 253

Asiburn and Craig, 931

AsHFord, 991

AsoKAWA, 268

Avery, 156, 447, 497

Avery, Chickering, Cole and Dochez, $439,458,462$

AVERY AND DOCIIEZ, 45.5, 463

AXENFFi, $50 \mathrm{~S}$

BABES, 11, 563, 625, 868, 869, 902, 967

BABES AND LEPP, 248

\section{ВАсот, 957}

BAGINSKY, 568, 734

BAGINSKY AND SOMMERFELD, 412, 419, 425,926

BAIL, 349, 394

BANDELIER AND ROEPKE, 611

BANDI AND SIMONELLI, 852

BANG, 693, 799, 1035

BALDWIN, 372

BARBER, 178, 750

BARKER AND COLE, 667

BARSIEKOW, 164

BASCHETTI, 1035

BASENAU, 688, 1033

Bass, C. C., 1116

BASSETT-SMITH, 540, 437, 795

BAUMGARTEN, 125, 593

BechmanN, 95

BECK, 563, 566

Behring and Kitasato, 248, 250

Behring, 93, 95, 98, 246, 257, 274, 353, 580,778

Behring ANd Wernicke, 248, 250

BeiJERINCK, 28, 30, 1031, 1044

BELFANTI, 729

Belfanti and Carbone, 252

Belpaeff, 249

BENDA, 96

Bengstan, 945, 755

Benger, 521

Benians, 120

BERANECK, 606

Berestafef, 964

Berestnew, 961, 970

Bertareli, 858

Berthelot, 61

Bertrand AND Weisweillere, 1046

Bessat, 709

BesRedKa, 369, 657, 684

deBeurmann and Gougerot, 975, 993 
BezanCON, 399

Bezancon, Griffon and LeSoukd, 511 BIENSTOCK, 228, 587, 632

BiggS AND PARK, 284

BILlRoth, 7

BirCh-HirsChFELd, 632

BITTER, 35, 201, 618

BLAKE, 965

Blake and Cecil, 459

Blaise and Sambac, 78

Blake and Trask, 923

Blanc and Pozerski, 761

BuISS, 928

Block, B., and Massini, 999

BLUMENTHAL, 672

BLUMER, 655

Bogart and Bernard, 252

BogGS, 499

BolLINGER, 966

BoLton, 26

BonNey AND BRowning, 97

BORDET, 252, 264，278，279，290，293, 294, 507

BORDET AND CUICA, 72, 74

BORDET AND GENGOU, 298, 315, 595

BORDONI-UFFREDUZZI, 449

BoRIsSOW, 393

VON DEM BOME, 870

Bostroem, 968

BOYCE AND WoODHEad, 1042

BOYLE, ROBERT, 3

BRADFord, BashFord aNd WILSON, 487

Branell, 6

Brau and Denier, 837

Braun, 425, 427

BREM, 596

Brieger, 234, 656

Brieger aNd Boer, 573,732

BRIEger AND CoHN, 731, 732

Brieger and Frankel, 573

Brieger and Kempner, 743

Brill and Libman, 805

Brosdifurst, 409, 435

Browning, Gulbsauen, Kennaway and THornton, 96

BROWNLEE, 492

Bruce, 796, 1090

Brudzinski, 1045
BRUG, 1139

BRUMPT, 943, 984, 1091

v. BRUNN, 107

BUCHNER, 22, 77, 250, 254, 277, 278, 628, 1018

BUCHNER AND HAHN, 606

Buchner AND Meisenheimer, 57

BUDD, 643

BUDINGER, 391

BUERGER, 117

BuFFoN, 4

BULL, 286

Bull and Pritchett, 648, 754

Bullock ANd AtKin, 337, 339

BULloch AND Hunter, 806

Bullock and Western, 337

BULly, 676

BUмм, 547, 548

BURger AND WyNTENBERG, 434

Burgers, 120

BURKE, 743, 745

BURKHOLTZ, 77

Burnet and Weissenbach, 163

Busse, 984

Butschil, 11, 12

Buxton, 686, 1063

Buxton and Coleman, 214, 689

BYAM, 949

Cala mida and Bertarelli, 222

CALKINS, 892, 904

CALMette, 250, 255, 607

Calmette and Guerin, 895

CANFORA, 223, 735

Cantani, 498

Cantacuzene, 335

CAPPS AND MILLER, 421, 1034

Carlo and Rattone, 727

Carrel, Dakin, Daufresne, Dehelly, and Dumas, 93

CARriere, 54

CARTER, 971

CASTEllani, 288, 651, 870, 871

('asteldani and Chalmers, 712, 795, $813,814,842,931,984$

CAULfifeld, 754

Cecil and Austin, 481

Celli, 78 
CERTES, 39, 848

Chamberland and Roux, 246, 779, 780 Charrin, 79, 802

Charrin and Roger, 28:

Chantemesse, 668, 684

Chantemesse and Widal, 655, 659

Chauveau, 246, 784

Chesney, 456

CHIARI, 671

Снiск, 89

Chickering, 466

Christen, 82,83

Chudiokow, 29

Churchman, 170

Christmas, 553

Citron, 327, 351

Clairmont, 724

Clark, Bayard, 559 .

Clark, W. M., 146

Clark and Fenton, 425

Clark and Lubs, 138, 140, 141

Clarke, 892

Class, 926

Chegg, 621

Clovgh, 467

CoBReTt, 583

CocA, 353, 363, 371

Cogniard-Latour, 3,6

Cohendy, 1046

CoHn, 5, 41, 43

CoLE, 370, 445, 455

Cole ANd Lloyd, 149

Colebrook, 971

Coleman and BuXton, 651

Coleman and Shaffer, 227

Coles, 619

Conn, 679, 1030, 1031, 1042, 1043

Conradi, 163, 651, 675, 708, 714, 1032

Conradi and Drigalski, 158

COOKe and VANderVeer, 370

Corril and Babes, 880

Councilman, Magrath and BrinckerHOFF, 892

Councilman, Mallory and Wright, 515,516

Courmont ANd Goyen, 753

Craig, 1131

Craig and Nichols, 860
Cranes, 22

Creel, 817

Creel, Fagot and Wrightson, 109, 110

Creite, 735

Crum, 923

Cruz, Oswaldo, 883

Cuica, 72, 74

Cushing, 689

CUSHING AND Livingood, 631

CURTis, 984

DAKIN, 90, 92, 93

DALE, 361

DANSYZ, 265

Darling, 992

Davaine, 6, 773

Davies and Weldon, 948

DAVIS, 422, 435

Davis and Rosenow, 421

DEAN, 338, 625

Debrand, 731

DelezenNe, 252

DeNeKe, 845

Denys, 430, 604, 606, 611

Denys and Laclef, 336

Denys and Marchand, 430

Denys and Van de Velde, 395

DESLONGCham PS, 389

Deutsch and Feistmantel, 349

DiCKson, 742, 745, 746, 747

Dickson AND HowitT, 747

DieudonNe, 77, 78, 166, 775, 269, 833

DOBELL, Clifford, 1056

Dochez, Avery and Lancefield, 412, 419, 432

Dodds-Price, 1096

DOERr, 709, 352, 649

DokrR AND Russ, 360

DOCHEZ, 467

DOChez AND Avery, 474

Dochez AND Gillespie, 446

DONATH AND LaNDSTEINER; 300

Donitz, 272, 239

DOPTER, 522

DORSET, 24

DORYLAND, 27

Draper and Hanford, 927

Dreyer and Madsen, 261 
Drigalski, 670

Drigalski aNd Conradi, 652, 670

DoRR, 674

Drury, 601

Dubarke and Terke, 615

Duclaux, 1043

DUJARRIC DE LA Riviere, 486

DUNBAR, 370

VON DUNGERN, 252

Dunham, 523, 560, 686, 694, 833

DUtTon AND TodD, $849,863,865$

Durham and Myers, 880

Dusch, 4

Duval, 621, 622

Duval AND SHaRer, 715

EBERTH, 643

Eсопомо, 919

EHRENBERG, 2

EHRLICH, 124, 238, 250, 255, 256, 257, $258,262,263,266,587,734$

Ehrlich, Kossel aNd Wassermani , 258

Ehrlici ANd Morgenroth, 279, 299

EISENBERG, 44, 806

EISENBERG AND VoLK, 662

v. Eisler ANd PRIBRAM, 729

Elser, 527

Elser and Huntoon, 519, 523, 533

ENDO, 159

ENGELMAN, 28, 68

EPPINGER, 782, 964

Epstein, 94, 213, 651

VAN Ermengen, 14, 119, 740, 688

ERNST, 804, 11, 1035

v. ESMARCH, 77, 82, 177, 180

Escherich, 224, 628, 228, 564, 1045

Evans, 799

Evans ANd Russell, 109

EWING, 852, 893

ExNer aNd Heywarski, 674

EykmanN, 54, 55

Eyre, 198, 419, 795, 797, 1033

FABER, 424

Fairley, 945

FARNeT, 656

Faure-Beaulieu, 552
Fehleisen, 401, 414

FEJES, 487

Fermi and Pernassi, 732

F'erran, 31, 246, 729, 841

Ferri and Celli, 78

FerRy, 798

Ficker, 76, 283, 666

FiedLeR, 885

FIELD, 927

FiNGER, 789, 794

Finger and Iandsteiner, 859

Finlay, 875

FINK, 357

Finkelstein, 805

Finkler and Prior, 845

Firth and Horrock, 1014

Fiscier, A., 9, 10, 19, 24, 26, 64, 66, 388

Fischer and Proskauer, 106

Flandrin, 693

Fleischner; Meyer and Shaw, 800

Fleming, 33

FleXNER, 522, 526, 531, 533, 656, 701, 709,964

Flexner and Amoss, 917

Flexner, Clarke and Dochez, 917

Flexner and Jobling, 529, 530

Flexner and Lewis, 914

Flexner and Noguchi, 423, 732, 915

FlugGe, 103, 104, 388

FOA, 456

Foa and Carbone, 454

v. FodAR, 249, 277

FooT, 951

Foote, 679

FORD, 689

Foges, 734

ForNaEcA, 399

Fornet ANd Muller, 305

Forssman and Lunstrom, 747

Forster, F., 36

Foster, 437

Fostes and Cookson, 491

FraEnKeL, 619, 752, 1013, 1014

FrANKeL, A., 440, 456, 464, 655, 664, $720,779,649$

Franzott, 389

Fraser, 596 
Freer, 78

Freundenreich, 1042, 1043

Fineman, 990

Friedberger and Hartoch, 361, 365

Friedlander, 439, 720

Friedmann, 616, 226, 362

FRIEDRICH, 490

Frisch, 724

Frocastor, 2

Frosch AND Kolle, 405

Frost, 489

Fumrmann, 49

Fuller, 1019

GABBET, 125,588

Gaethigens, 674

GAFFKY, 80, 643

GAFFSKY, 398

GALTIER, 794

GAMALEIA, 844

GARBAT, 673

Garbat and Meyer, 685

GARRE, 391

GARTNER, 676, 687

Gates, 518

GAUTHIER, 1076

Gavina and Gerard, 939, 943

GAY, 296, 379, 419, 668

Gay and Chickering, 468

Gay and Claypole, 650, 685

GAY AND SoUthard, 359

GepPaRT, 105

GengoU, 294, 296, 327

Van Gehuchten, 902

Geronne, 676

Gess $A R D, 802,804$

GHearghiewSKI, 806

Ghon and H. Pfeiffer, 559

GHON AND SACHS, 756

Gibson, 272

Gibson and Collins, 272

Giebert, 688

Giems, 127

VAN Gieson, 903

Gilcinist, 984

Globig, 37

Glover, 541

GMINDER, 693
Goldberger, Williams and Hacktel, 575

GordoN, 153, 522, 540, 409

Gordon, Flack and Hines, 520

GoRGAS, 875, 883

GotTsCHLICII, 25, 942

Got'tstein, 79

GOULD, 756

GRAнам, 931, 946

GraM, 56, 120

GraMENITSKI, 295

GrassbergER, 498

Gratzer, 946

GraUCher ANd Ledoux-Lebard, 593

GrekofF, 1046

GRIFFITH, 971

Gross, 848

GRUBER, 81, 95, 664

GRUber AND DURHAM, 251, 282, 288

GRUNHAGEN, 892

GUARNIERI, 445, 449, 892

Guerin, 897

Guiteras, 877

GUMPRECHT, 733

GUNTHER, 439

GWYN, 688

HAAS, 80

HAFFKINE, $841=$

HАHN, 242, 243, 574, 657

HALL, 919

HALLier, 7

HAMburger, 986, 993, 1034

Hamburger aNd Sluka, 595

HAMMERSCHLAG, 603

HAMmerstein, 53

HANEL, 389

Hankin and Leumann, 21

Hankin AND WestbrooK, 202

HANSEN, 982, 619

Harding and OstenberG, 159

HARRIS, 909

Harris and Shackell, 908

Harrison ANd Galtier, 1044

HAUSER, 640

HEIM, 1032

Heiman, 548, 552

Heinemann, 1030, 1034 
HeKToen, 922, 338, 428

Hektoen and Perkins, 993

HEKTOEN AND RUEDIGER, 337

HELLER, 758

HELLRIEgel AND WILFARTh, 62, 63

VAN Helmont, 4

HeNle, 3

HENRY, 753

HeNRY ANd LaCy, 763

HeNRIJEAN, 730

D 'Herelle, 70, 71

Hermanies, 551

HERRICK, 538

HERRINGHAM, 946

Herter, 224, 211, 634

Hesse, 592, 1033

HEYFELDER, 490

VON HIBLER, 750

von Hibler, Kolle and Wassermann, 770

Hilbert, 35, 572

HILDERBRANDT, 221

Hill, 105, 112, 1021

HiNe, 524

HIRSCH, 807, 936

HIRSCHBERGER, 1035

Hiss, 13, 116, 346, 403, 405, 406, 433, $444,445,449,453,467,652,654,704$, 705,946

Hiss ANd AtKinson, 249, 272

Hiss AND Russell, 703

HOFER, 726

HOFFMANN, 287

HoFrmanN-WellenhoFf, 582

HoG YES, 907, 909

HoLLER, 347

Holman, 409

Holmes, 5

HoLST, 688

HOOKER, 397

HoPKINS AND LANG, 409

HORTON-SMITH, 652, 654

Houston, 409, 673, 1013, 1024

Houston aNd THOMAS, 675

HowARD, 724

Howard and Perkins, 448

Howe, 922

HUbER ANd KOESSLER, 363
HUDDLESTON, 897

HUDDLESTON, 800

HUEPPE, 827

HUEPPE AND WOOD, 785

HUNTEMULLER, 1018

Huntoon, 33, 149

ICHIKAWA, 685

Ido, Hoki, Iтo AND Woni, 887

INADA, 885, 886

INADO AND IDO, 888

IROUS AND MARINE, 420

IRWIN AND Houston, 675

ISRAEL, 966

IWANOFF, 24

IWANO, 778

JACKSON, 163, 816

JACKSON AND MELIA, 164

J AEGER, 515

J AEHLE, 499

JAGER, 724

JANOWSKI, 77

$J_{\text {AVORONE, } 1095}$

JENNER, 127

JENSEN, 122

ЈоАСНIM, 249

JoAs, 287

Jobling And Petersen, 247, 366

JOHNSTON, 649

JoNES, 23,88

DE JoNG, 293

JORDAN, 51, 690

JoRdan AND Heinemann, 1028

JORDAN AND IRONS, 1020

JoRDAN AND SHARP, 493

JoRGENSEN AND MADSEN, 287

JUNGMANN, 949

Kabeshima, 72

KALBERLAH, 676

KAMEN, 633

KANEKO AND OKUDA, 873

KAPPES, 22

KARLIN SKY, 654

Kastle and Elvove, 159

KEM PNER, 250, 747

KeMPNer AND Pollack, 744, 745 
KEMPNER AND SCHEPILEWSKY, 268

KEXDALL, 160, 224, 229

KENDALL AND DAY, 227

KENDALL, DAY AND W ALKER, 642

Kinyous, 899

KINSELLA AND SWIFT, 411, 412

KIRCHER, 1

KISTER AND Wolfy, 292

KrTASATO, $727,729,732,770,807,810$

Kitasato ANd WeYL, 29

KITT, 772

KITT AND MAYR, 829

KLEBS, $7,439,880,561,607$

KLEIN, 211, 67i, 587, 649, 667

KLEMPERER, 618,454

KLIGLER, 165

KLINGLER, 971

KLINGMULLER AND BAERMANN, 861

KLOTZ, 1046

KLOSE, 754

KNAPP, 583

KNOEPFELMACHER, 914

KNORR, 25i, 3อ̄3

KOBERT AND STILLMARCK, 256

Косн, 3, $7,8,77,86,94,104,105,106$, $401,439,502,590,604,605,606,60 \pi$, $610,611,612,649,670,756,773,779$, $836 ; 838,865,866,1023$

Kock, GAFTKY AND LOEFFLER, 80 , 81, 783

Koch ANd Petrescher, 429

KOCH AND RaBINovitSCH, 615

Koch AND WOLFFHCgR, 80, 779

KOESSLleg ANd HANKe, 52

KOHER, 599, 600

Kolle AND Hetsch, 847

Kolle AND OTTO, 396

Kolle AND SCHCBMANA, 841

KOLle AND WASSERMANN, 523, 616

KOLMER, 894

KOLMER AND STRICKLER, 999

KORBRIDE, 455

Kors, 617

KorsCHUN, 346

KosSeL, 257

KossEL AND OtERBack, 812

Kosset, WEBER AND HUESS, 1036

Kratemals, 805
KRAUS, 251，299，292，394，667，672， $685,708,83 \bar{\top}, 844$

KraUS, R., 304

Krats ANd DOERr, 710, 708

KRAUS AND Low, 286, 636

Kraus and Meinicke, 916

KraUs ANd v. Pirquet, 291

Kraus ANd Stenitzer, 657

KraUse, 372

KresLiNg, 791

KRETZ, 270

Kronig and Paut, 87, 89, 96, 93

DE KRUIP, 751

de Kruif and Ireland, 427

KRTMWIEDE, 870

KRUMWIEDE AND KoHN, 689, 695

KRUMWIEDE, KoHN AND VAlENTiNe, 648

KrUMWIEde ANd PRATT, 170

KrUmwirde, Pratt and Kohn, 690

KRUSE, 349, 437, 701, 702, 710, 828

KUCZYNSRI, 948

Кㄴтн, 689

Kusoxoki, 999

KUSTER, 222, 225, 672

KUTSCHER, 415, 670

KUTSCHER, KOLLE AND WASSERMANN, 535.

KUTSCHERT AND NEISSER, 584

LaMar ANd Meltzer, 457

LANDMANN, 743

LANDSTEINER, 252

LANDSTEINER AND JAGIC, 294

LANDSTEINER AND LEVADITI, 914

LANDSTEINER AND POPPER, 913, 914

Landsteiner, Levaditi and Prasek, 927

LASSAR, 857

LARREY, 885

LAUBENHEIMER, 672

LAVERAN, $84 \pi$

LAWS AND ANDERSON, 1022

LEACH, 23

VAN LEEUWENHOEK, 2,4

LECHNER-SANdotaL, 961

Leclain aNd Vallee, 771

LEDDERHASE, 804 
LEICHTENSTERN, 515, 489

LEISHMAN, 336

LENTZ, 107, 697, 703

Lentz and Huntemuleki, 916

LEPIEIRE, 526

LESAGE, 1044

LESCHKE, 487

LEUCHS, 743, 747

LEVADITI, 332, 338, 855

LEVADITI AND BANKOWSKI; 854

LeVAditi ANd HaRrier, 901

LEVADITI AND INMANN, 338

Levaditi ANd Manouelian, 871

Levaditi and Petresco, 852

LEVENE, 23

LEVY, 925

Levy and Alexander, 420

LEWITH, 80

LiBMAN, 405, 689, 698

LiBMAN AND ROSENTHAL, 453

LiBORIUS, 180

LIEBERMEISTER, 588

v. Lingelsheim, 393, 403, 430, 535, 407

LISTER, 6, 480, 448

LISTER AND TAYLOR, 487

LISTON, 825

LLOYD, 33

LOEB, 386

VAN DER LOEFF, 892

LOEFFLER, 561，571，14，80，81，119, 129

LOEFFLER AND FrosCh, 933

LOEFFLER AND SCHUTZ, 787

Loewe and Strauss, 921

LOEW, 27

LOHLEIN, 337

LONGCOPE, 697

Lowy, 940

LOWENSTEIN, 615

LUBARSCH, 241

LUBBERT, 389

LUDKE, 685

LUERSEN AND KUHN, 1046

LUSTGARTEN, 618, 850

Lustig and Galeotti, 825

LYNCH, KENNETH, 1074

MaAsen, 59
MacCallum, 416, 964

MacCallum, Cole and Dochez, 420

Mackensie and LongCope, 368

Matileis, 423

MACFA YDEN, 454

MaCfayden AND Rowland, 657

MACNEAL, 491, 988, 1142

Madison, 500

MADSEN, 271

MAFUCCI, 587, 604, 614

MaLloRY, 927, 968

MALLORY AND HORNER, 507

MaLlory aNd MEdLAR, 927

MALLORY AND WRIGHT, 132, 639

MANSON-BAHR, 1097

ManWaring, 363

Maragliano, 612

Marbaix, 414, 407, 416

Marchiafava and Celli, 515

Marchoux and Salimbent, 871

Marchoux, Salimbeni and Simond, 877,884

Marchoux and Simond, 878, 879

MaRINESCO, 744

MARKS, 916

MARMIER, 425

MARMOREK, 411, 425, 612

Marschal, 514

MarTin, 5, 72, 771

Martin ANd Cherry, 256

Martini AND Lentz, 702

MarX, 431, 909

MASSOL, 1046

Mathers aNd Herrold, 540

MAYER, 745

Mayer and Ahreiner, 673

MCClintic, 99, 951

McCoy, 625, 815, 816, 818

McCoy and Chapin, 826

MCGowaN, 798, 801

McIntosh and Fildes, 187, 749

MCLEOD, 427

McNee, Brunt and Renshaw, 947

Meleney and Ray, 541

MEISENHEIMER, 57

Mennes, 454, 467

MESNIL, 286, 992

Messea, 16 
MESSERSCHMidT, 715

METCHNIKOFF'S BACTERIOTHERAPY, 1044

МетснNiкоFF, 239, 250, 252, 278, 282, $330,762,958,1045$

MEtChNiKOFF . AND BesRedKa, 650, 683

METCHNikofF AND Roux, 857

METChNiKoff, Roux and SALIMbeni, 837

MEYER, 238, 692, 759

Meyer and Ransom, 733

Meyer, F. K., ANd Stickel, 163

Michaelis, 114, 305

MICHEL, 565

Mignesco, 77

Migula, 13, 41, 388

MikULICZ, 725

MilleR, H. R., 608

Minchin and Thompson, 1080

Miquel, 37, 1017

MITCHELL, 200

MiYjayima, 887

MOELLER, 116

MOHLER, 609, 932, 1036, 1042

Moldovan and Doerr, 366

Moltschanoff, 553

MoMonT, 779

MonTI, 892

MOORE, 415, 694

Morax, 508

Morax AND Marie, 732, 733

Morgan, 649, 717

MorgenRoth, 253

Morgenroth AND SACHS, 296, 320

Morishima, 648, 662

MoRo, 228, 608

Moser, 926, 928

Moser and von Pirquet, 412, 419

Moss, Guthrie and Gelien, 437

MonTon, 330

MUCH, 588

MUELleR, J. H., 32

MUIR AND RITCHIE, 756

MULLER, 619, 398, 1034

Muller, Fr., 388

MUller, OTto FriedriciI, 2

MUlZER, 852

Munson, 926
Muntz, 27

Muntz AND Schlossing, 64

MYERS, 268, 304

NANKANISHI, 10,11, 18, 19, 112

NAKAYAMA, 428

NASTJUKOFF, 498

NeEdha M, 4

Negri, 902

NeISSER, 11, 126, 394, 620, 547, 563, 999

Neisser, Baermann, Halberstadter, 871

Neisser, Kolle and WassermanN, 388

Neisser and Friedmann, 294

NeIsser AND SACHS, 298, 327

Neisser and Shiga, 708

Neisser ANd Wechsberg, 252, 297, 393, 394

NENki AND SCheffer, 22

NEUFELD, 412，429，453，466，467，655

NeUfELd AND HAENDEL, 474, 446

Neufeld and Hune, 338

Neufeld and Rimpau, 337, 467

NeUfeld AND TOPFER, 338

Neumann, 221，654，805

Neustaedter and Thro, 917

NeWSholm, 574

NiCHOLS, 672

Nicolaier, 727

Nicoll and VinCent, 842

NicolLE, 287, 356, 359, 360, 622, 524, $858,937,939$

Nicolle, Blanc and Conseil, 943

Nicolle and Lebailly, 486

Nikati ANd Rietsch, 836

NIKOLAYSEN, 553

NisHI, 881, 885

Nishimura, 24

NoCARD, 688, 694, 789, 964

Nocard aNd Roux, 587, 614, 615

NoGuchI, $319,847,856,860,863,873$, $881,887,905,906,1139$

Nogúchi and Kligler, 883

NOGUCIII AND MOORE, 852

NoRrIS, 291，292，304，667

NORRIS AND LARKIN, 964 
Norris, Pappenheimer and F'lournoy, 862

NotTER AND Firth, 1035

Novy, 759

Novy AND FraenKel, 864

Novy AND FreEr, 78

Novy AND KNAPP, 847

NOVY AND KNAPP, 861, 866

Novy and DE. Kruiff, 366

Novy and MacNeal, 1077, 1086

NuttalL, 249, 250, 277, 292

NUtTall AND Thierfelder, 632

Obermeier, 7, 849, 861

OBERMUELLER, 1042

OGSTON, 384, 401

Онко, 704, 710, 885

OLitsky and Gates, 488

OLitsky aND Kligler, 709

OLMSTEAD, 447

OMELIANSKI, 56, 65

OMeltshenko, 96

OPHULS, 988, 744

OPIE, 595, 500

OPPENHEIMER, 48,53

OSGOOD AND LUCAS, 918

OSTWALD, 47, 146

Ostertag, 693

Отто, $355,359,360,880,878$

OTTOLENGHI, 450

OVERTON, 238

PAKE, 198

PANE, 454,467

PAPASOTIRIN, 631

PAPPENHEIM, 125, 589

PAPPENheimer AND MUeller, 948

PARK, 106, 269, 270

PARK ANd CAREY, 704

Park and Dunham, 702

Park and Holt, 1033

Park and Krumwiede, 600

Park aNd Nicolle, 740

Park and Thorne, 273

Park and Williams, 472, 896

PARK AND Zingher, 578, 580

PARKER, 498

PARSONS, 490
PASQUALe, 403, 844

PASSET, 389, 402

Pasteur, 3, 5, 6, 8, 29, 47, 245, 246, $440,827,901,981$

Pasteur and Chamberland, 144

Pasteur, Chamberland and Roux, 245,780

Pasteur ANd Jourbet, 756

PAUL, 87, 894

Peabody, Draper and Dochez, 209, 912, 918.

Peabody and Pratt, 163

Pearce, 253, 550

Pearce and Eisenberg, 361

Pearl, 492

Perez, 726

PeNFold, 44

Perkins, 723

Perrone, 404

Petersen, 348

Petri, 617, 1014

Petruschky, 416, 639, 649, 654, 961, 964, 981

Petroff, 590

Petruski, 675

Petterson, 346

PFAUndler, 283, 286

PFEFFER, 61, 63

PFeiffer, 235, 250, 285, 494, 499, 502, $657,837,892$

Pfeiffer and Beck, 501

PFEIFFer ANd IsAeff, 277

Pfeiffer and Kolle, 285, 659, 681

Pfeiffer and Nocitt, 844

Pfeiffer and Ungermann, 709

Pfeiffer and WassermanN, 837

Pfluger, 66

Pfunl, 188, 641

Pierrallini, 331

v. Pirquet, 608

v. Pirquet and Schick, 354, 356

PITT, 14

Place, 929

Plant, 867, 984

Plenciz, 2, 3

Plotz, 942

Plotz, Olitsky and Baehr, 941

POLLENDER, 6 
Poels AND DHont, 688

POOLE, 766

Poor ANd SteinhardT, 905

PoRges, 14, 287

PoTt, 1035

Poynton And Paine, 422

PratT, 656, 671

Prescott, 631, 1024

Pratt-Johnson, Gilchrist, Hay-MICHEL, 1130

Prigge, 673

Pritchett and Stillman, 500

Proescher, 915, 936

Proskauer and Beck, 32

Von ProwazeK, 859, 943

Prudden, 649

Prudden ANd Hodenpyl, 604

QUigley, 423

QUINCKE, 1045

Rabinovitsch, 617, 625, 941, 992, 1042

RANSOM, 733

Ransome and Fullerton, 106

Ravant and Pinoy, 971

RAVENEL, 613, 779

voN Recklingshausen, 7

REDTENBACHER, 652

ReEd and Carroll, 688

Reed, Carroll, Agramonte and LAZEAR, 875

REMLINGER, 905

RETTGER, 226, 695, 753

RetTger AND Koser, 695

RetTger aNd Newell, 50

Richet ANd Hericourt, 248, 396

Richardson, 78, 654, 656

RicketTS, 942, 951, 952, 987

RicketTS AND WiLdER, 937, 939

RIDEAL-WALKER, 98

RIEDER, 79

RINDFLEISCH, 7

Rixford AND Gilchrist, 988

ROBERTSON, 168, 757

Robinson AND RetTGer, 160

da Rocha-Lima, 943, 949

RoEmer AND JosePH, 916

ROHNER, 804
ROGER, 425

ROMANOWSKY, 127

ROMER, 741

RosenaU, 590, 504, 814

Rosenau ANd ANderson, 275, 355, 356, 570

Rosenau, Lumsden and Kastle, 679

Rosenau and McCoy, 1028

RosenbacH, 384, 401, 965

ROSENBERger, 596

Rosenow, 44, 434, 454, 423

ROSENOW AND TOWNE, 423

ROSENOW AND WHEELER, 423

ROSENTHAL, 708

Ross ANd Milne, 865

Rost, 621, 624

ROTHBERGER, 646

Roux, 11, 78, 180

ROUX AND LiNOISSIER, 991

Roux AND Nocard, 792

Roux AND YeRsin, 561, 571, 573

ROWLAND, 825, 1126

RUBNER, 26

RUEDIGER, 428

RUPPEL, 23, 605

RUSSELL, 164, 682, 1142

Russell aNd Fuller, 1022

SAATHOF, 115

SABouraud, 996, 999

SACHAROFF, 872

SACHS AND GEORGI, 329

SACHS, 238, 250, 253

SACHSE, 61

SACQUEPEE, 670,759

SAHLI, 607

SALMON, 828

SALMON AND SMITH, 247, 691

SANARELLI, 880

SANFELICE, 992

SAUERBECK, 350

SAUL, 94

SAVAGE, 284

SchaEFFER AND STEINSCHNEIDER, 552

SCHAFER, 35

SchatTenfroH, 346, 393

SChatTenfroh aNd Grassberger, 1030

SchaudinN, 848 
SchaUdinn and HoffmanN, 850

SCHEFFER, 22

SChell and FishCer, 593

SCHELLER, 673

SCHENK, 993

SCHERESCHEWSKX, 856

SCHERING, 109

SCheurlen and Spiro, 87, 88, 89, 95

Schick, 574

SCIILD, 631

SCHim Meliusch, 391

Schistovitch, 290

Schlossing, 64

SchlossmanN, 107

SCHNEIDER, 388

SCHотTELIUS, 632

Scholtz, 547

Schone, 674

SCHоттмÜLLER, 403, 407, 448, 453, 651, $689,965,1034$

SCHREIBER, 580, 829

SChroeder AND Cotton, 800, 1036

Schroeder AND DUSCH, 4

SCHROETER, 67

SCHUDER, 677, 1032

SCHUITZLER, 641

SCHule, 379

SCHULLER, 880

SchULTz, 361

SchUlze, 4

SCHUTZ, 414

Schutze, 253, 290

SchwanN, 3, 4, 6

DE Schweintz ANd DoRset, 24, 603

Sclavo, 445, 784

SEDGWick AND BATChILDER, 1028

Sembac, 78

Sellards, 936

SELTER, 487

Sergent, Foley and Vialotte, 943

ShaRNOSKY, 121

SнатTоск, 935

SHERMAN, 121

SHIBAJAMA, 692

SHIRNOFF, 840

SIIG $\Lambda, 700,702,710,716$

SigNORELLI, 170

SILBERSCHMIDT, 641
Simon, Lamar and Bispham, 341

SimondS, 226, 359, 753

Simons, 425

SIMPSON, 1033

Simpson and Hewlett, 99

Sмітн， 119，355，572，591，593，612, 613, 919, 1036

Sмітн, G., 583

Smith, Herbert E., 1033

Sмітн, Тн., 29, 30, 39

SMith AND BRown, 408

Smith, Brown and WaLKer, 729

Smith, L., Drennan, Rettie and CAM PBell, 93

Smith, Th., AND Kilborne, 246, 693

Smith, Th., AND MOORE, 688

Smith and Ten Broeck, 690, 695

SoBERNHEIM, 773, 784

Sobernheim and Tomasczewski, 851

SOMMERViLle, 99

SOPER, 679

Sophian, 531

Sorensen, 139

Spallanazani, 4

SPAgNolio, 1097

SPEAKMAN, 56

SPENGLER, 607

SPILKER ANd Got'TSTEIN, 29

SPITZER, 851

SPROUCK, 702

SPRONK, 572

STAFSETH, 800

STANLEY, 491

Steensma, 199

STEFANSKY, 625

Stephen and Fontham, 1090

STEPHENS, 1131

Stephens ANd Myers; 257

STERN, 291, 664

Stern AND KoRte, 311, 661

STERNBERG, 38, 388, 406, 448, 440, 564, 880,899 .

STTLLMAN, 474

STICKER, 623

STOBER, 987

STOKEs AND HaEChtel, 1023

Stokes, Ryle AND Tytler, 887

Stokes aNd Weggefarth, 1034 
Strauss, 791

Strauss and Gamaleia, 604, 615

Strauss, Hirschfeld and Loewe, 921

Strauss and Huntoon, 914

Strelitz, 568

STRICHT, 744

Strong, 723, 825, 842, 937

Strong and Musgrave, 701

Strong, Teague, Crowell and Barber, 819

SURMONT, 252

Surmont AND ARNould, 779

SUZUKI AND TAKAKI, 597

SwiFT, 371, 947

SWIFT ANd KINSELla, 423

TACKE, 61

TALAMON, 439

TAMURA, 23, 24

TAROZZI, 735

Tarozzi aNd SMith, 167

TAute and Huber, 1090

Taylor, KenNeth, 993

Teague and Barber, 811

Teague and DeIBert, 512

Teague and Strong, 813

Teague and Torrey, 550

Teague and Travis, 166

TEN BRoeCK, 357

TERIN, 390

THAYER, 880

Thom, Edmonson and Giltner, 743

THomas, 874

Thomson ANd Hewlett, 220

TIDSWELL, 675

Tissier, 225, 1044

Tissier and Martelly, 632, 1045

Tizzoni, 735

ToDD, 394, 708, 709

TOEPFER, 943, 949

TOKISHIGE, 992

DE TOMa, 593

TORINI, 53

ToRrey, 223, 228, 550, 553

TORREY AND RAHE, 228, 798

Totsuka, 662

TOUSSAINT, 245,780

Trask, 929, 1032
Trillat, 106

TrOoMSDORF, 692

TSIKLIMSKI, 37

TUlLOCH, 522, 525, 737, 378

TUNNICLIFF, 413, 869

TURNBULL, 866

TURRO, 74

TworT, 44, 69, 70, 74

UHLENHUTH, 291, 590

UHLENHUTH AND HUBENER, 698

Uhlenhuth AND MUlzer, 858

UlLMANN, 552

USCHINSKI, 31, 151, 573

VAGEDES, 613

VAILLARD AND DOPTER, 708

VAILLARD AND ROUGET, 734

VAILLARD AND ViNCENT, 731, 732

VALNTINE AND COOPER, 501

VALLARI-RADOT, 4

VAUGHAN, 44, 356

Vaughan and Palmer, 537, 924

VAUGHAN AND WhEELER, 365

VEDDER, 549

VedDER ANd DUVAL, 702

VEEDER, 679

VEILLON, 398

VEILLON AND ZUBER, 752

VAN DE VELDE, 394, 430

di Vestea aNd Zagari, 901

VigNAL, 963

VILLEMIN, 586

VINCENT, 867

Vincent ANd MURATET, 707, 712

Voges, 32

VOTTALER, 728

WADSWORTH, $118,445,450,457,467$

WADSWORTH AND KIRKBRIDE, 469

Wagmann, 1031

DE Waele and Sugg, 412

WALDEYER, 7

WALKER, 662

WARD, 78, 1031

WASHBURN, 467

WASSERMANN, $250,256,290,529,549$, $553,805,842$ 
WASSERMANN AND BRUCK, 298, 316

WASSERMANN AND CITRON, 350

WassermanN, Neisser and BRUCK, 317

Wassermann and Proskaur, 573

WASSERMANN AND SCHUTZE, 291

WASSERMANN AND TAKAKI, 238, 268, 334, 733

WASSILIEF, 791

WATAHIKI, 550

WATSON, E. A., 1085

WEEKS, 502

WEENY, 662 .

WEGELE, 1046

WeICHSELBAUM, 515, 560, 398, 440, 720

WEIGERT, $7,130,267$

WEIL, 359, 360, 367, 885

Weil and Felix, 945

WeISS, 589

WeLCH, 116, 154, 634, 445

WELCH AND BLACHSTEIN, 649

WELCH AND NUTTALL, 211, 752

WELLS, 120, 356, 368

WENGON, 1095

WERNER, 946

WENICKE, 602, 840, 812, 987

WERTHEIM, 548

WESENBERG, 641

WestPhal AND UHLENHUT, 623

WEYL, 29

WHEELER, 23

WHERRY, 625, 815

WHERRY AND ERWIN, 518

WHERRY AND OLIVER, 189

WHIPPLE, 1021

WiCkMAN, 912

WIDAL, 663

Widal AND NobeCOURT, 688

WILBUR, 745

WILCKENS, 1032

WILDE, 724

WILLAMON, 33

Williams, 33, 501
Williams AND LOWDEN, 904

WILlSON, 1023

WILTSCHOUR, 652

Wilson, 919

Wilson and Chowning, 951

WINOGRADSKY, 14, 27, 61, 64, 65

WinsLOW, 421

Winslow, Kligler and Rothenberg, 647

Winslow and Palmer, 409

WINTERNITZ AND HIRSCHFELDER, 457

WLADIMIROFF, 792

WolbaCH, 890, 936, 950, 951, 988

WoLBACH AND ERNST, 613

Wolbach, Sisson and Meier, 995

WoLFF, 350, 635, 760

WOLF-EISNER, 356, 365, 607

WOLFF AND ISRAEL, 969

WOLFHUGEL, 80

WoLLSTEIN, 500, 506, 508, 522, 715, 930

WooD, 113, 128, 450

WORONIN, 62

WriGHT, 127, 221, 181, 183, 681, 899

WRIGHT, J. H., 967, 968, 1095

Wright and Douglas, 336, 337

Wright aNd MoRgan, 480

Wright ANd SEMPLe, 681, 979

Wu LiEN TEH, 817

WUTZDORFF, 492

WYNEKOOP, 499

YERSIN, 807

ZetTNOW, 861

ZiNSSER, 16, 185, 346, 570, 583, 656, 705, 955

Zinsser and CARY, 626

ZINSSER AND DWYER, 351

ZiNSSER AND HOPKINS, 860

Zinsser, Parker and Kutther, 237

Zinsser ANd Tsen, 346 


\section{INDEX OF SUBJECTS}

Аввотт's spore stain, 116

Absorption method in agglutination, 288

Acetone produced by the fermentation of starch, 55 :

Achorion Schoenleini, 1001, 1002 other species, 1003

Acid añd alkäli production by bacteria, 198

acids commonly found, 199

Acid-fast bacteria, staining of, 124 definition of, 587

Actinobacillosus, 971

Actinomyces, 965-971. See also Nocardia, 964

animal inoculation, 970

appearance of in lesion, 906

cultivation of, 968

definition of, 962

diseases caused by, 965

microscopical examination and morphology, 966, 967

pathogenicity, 969

Wright's method of cultivation, 969

Addison's disease, 596

Aerobic organisms, obligatory and facultative, 8

Aestivo autumnal fever, 1107, 1110

- Agglutination, 282

absorption method is, 288

acid, 284

agglutinins, nature of, 285

action on dead bacteria, 283

discovery of, 251

major and minor, 288

partial studied by absorption method, 288

production of, 286

specificity of, 288

theoretical considerations, 292

"group," 288
Agglutination, macroscopic, 283

method of bacterial differentiation, 282

microscopic, 282

practical application in typhoid, 282 physical phenomenon of, 285

presence of electrolyte necessary for, 285

proagglutinoid zone, 289

test, method of performing, 302

macroscopic, 303

microscopic, 303

with capsulated organisms, 14, 287

with tubercle bacillus, 302

Agglutinogen, 287

Aggressin theory, 349

arguments against, 350

relation to anaphylatoxin, 351

Air, bacteria in, 1010

in the conveyance of disease, 1011

method of estimating the number of

bacteria in, 1012

Alcoholic fermentation, 58

Alcohols as disinfectants, 94, 103

Alexin and sensitizing antibodies, 277 281

discovery of, 250 .

facts concerning, 295, 296

Aleuronat used for injecting rabbits to obtain leucocytes, 345

Alkali production by bacteria, 198

Allantiasis, 740-748

Allepo boil, 1095

Allergy defined, 353

Amboceptor, quantitative relation to complement, 296

Amœba, 1050

binucleata, 1050

diploidea, 1050

proteus, 1050 
Amœba, synopsis of genera, 1052 valk amphia, 1051

Amylase, 55

Anabolic activities of bacteria, 60 liberation of energy, 66 light production by, 66 nitrogen fixation, 60 pigment formation, 66 sulphur production, 67

Anaphylatoxin, 365

Anaphylaxis, 352-372

anaphylatoxin theories, 365,366 antianaphylaxis, 364

antigen in, 367

Arthus phenomenon, 355

eriteria for, 356,357

Well's summary of, 356

desensitization, 364

methods for, 369,370

Doerr's classification, 352, 353

drug idiosyncrasies, 371

hay fever, 370

historical background, 354-356

observations by Arthus, 355

Hericourt and Richet, 354

Nicolle, 356

Otto, 355

Portier and Richet, 354

Richet, 355

Rosenau and Anderson, 355

Theobald Smith, 355

Vaughan and Wheeler, 356

in infectious diseases, 372

sensitization, active methods for, 358 , 359

incubation period in, 358

sensitization, passive, 359

incubation period in, 360

transmission by inheritance, 360

serum sickness, 366, 367

skin reactions, 368

delayed, 368

for determining hypersensitiveness for injecting horse serum therapeutically, 369

immediate, 368

site of reaction, 361,362

symptoms in various animals, 362,363
Anaerobic bacilli, 727-772

associated with traumatic injuries,

749-763

method of identifying, 763

proteolytic group, 750, 761

B. histolyticus, 762

B. putrificus, 762

B. sporogenes, 761

saccharolytic group, 750

B. Fallax, 760

B. oedematiens, 759

B. Welchii, 751

Vibrion Septique, 756

B. anthraci, symptomatici, 768-772

B. botulinus, 740-748

B. Tetanus, 727-740

Anaerobic blood cultures, 214

Anaerobic indicator, methylene blue, 187

Anaerobic methods of cultivation, 179189

displacement of air by hydrogen, 182 indicator for, 187

McIntosh and Fildes palladium asbestos method, 187

mechanical exclusion of air, 180

4 Esmarch's method, 180

fluid media covered with oil, 180

Liborius method, 180

pyrogallol in, 183

Roux's method, 180

Wright's method, 181

Method for combining exhaustion, hydrogen replacement and oxygen absorption, 185

Pyrogallic acid for oxygen absorption, 183

Buchner tube, 183

Wright's modification, 183

Plate cultures of anaerobes by simple method, 187

tissues in culture media, 189

Anaerobic organisms, obligatory and facultative, 28

methods of cultivating, 179

Andrade indicator, 147

Anilin dyes, as staining agents, 113

introduction of, 7 
Anilin dyes, selective action of in eulture media, 170, 161

Animal experimentation, autopsies in, 204

bleeding of animals in, 204

from the carotid artery, 205

from the heart, 205

methods of inoculation, 202, 203

intraperitoneal, 203

intravenous, 203

Kolle vaceination method, 204 subcutaneous, 203

Animal holders, 204

Animal inoculation, 203

Antagonism of bacteria, 34

used in bacteriotherapy of autointoxication, 1045

Anthrax, 773-784

attenuation of, 780,783

bacteria resembling, 785

B. anthrocoides, 785

B. radicosus, 785

B. subtilis, 786

biological considerations, 778

cultivation of, 775-777

historical interest of, 773

immunity against, 783

active (Pasteur's work), 783, 784

passive, 784

morphology, 774

pathogenicity, 779

experimental inoculation, 780

in animals, 780,781

in man, 782

susceptibility of animals, 779

spore formation, 778

resistance of, 779

vaccination, 783,784

virulence, 780

Anthrax symptomatic, bacillus of, 768 772

cultivation, 770

immunity, 771

morphology, 769

staining, 769

toxins, 771

Antibodies and the substances giving rise to them, 249
Antibodies, experimentation and diseovery of, 249

agglutinins, 251

antiferments, 253

antitoxins, 251

bacteriolysins, 251

cytotoxins, 252

precipitins, 252

antibodies sensitizing, 227-281

Bordet's views concerning, 279

Ehrlich's views concerning, 279

Antiferments, 253

antilab, 253

antilactase, 253

antipepsin, 253

antisteapsin, 253

Antiformin for concentrating tubercle bacilli in sputum, 589

Antigen, anaphylactic, 357

definition of, 254

in the Wassermann test, 317, 318

Antileucocidin in staphylococcus immune sera, 396

Antiricin, 250

Antisepties, inhibition strengths, 104

Antitoxin, diphtheria, 269-273

concentration of, 272

immunization of horses, 270, 271

prophylactic doses, 273

prophylactic immunization, 580-582

regulation of by law, 272

specific therapy in, 579

dosage, 579

standardization of, 271

therapeutic doses, 273

toxin-antitoxin reaction, theoretical

considerations, $255-268$

discovery of, 250

tetanus, 274

immunization of horses, 274

prophylactic dose, 736

$\mathrm{L}+$ dose, definition of, 276

standardization of, 275-276

therapeutic use of, 738

administration of, 740

unit of, 275

Antivenin, 256

"Arnold" sterilizer, 83 
Arrhenius and Madsen, theories concerning toxin-antitoxin, 264, 265

Arthus' phenomenon, 355

Arthrospores, not related to true spores, 16

Ash, bacterial, 24

Aspergillus, 61, 983

Attenuated cultures in active immunization, methods for, 245

with anthrax, 780

Autoclave, 85

table of relation between temperature and pressure, 86

Autointoxication, gastro-intestinal, 1044

Metchnikoff's bacteriotherapy in, 1045

Autolysins, 300

Autopsies of experimental animals, 204

BABES,-ERNST, granules, discussion of, 11

special stains for, 126

Babesia bigeminum, 1131

Bacilli, 9 genus of, 41

Bacillus abortus (Bang), 799-801 isolation from mlik, 800

lesions produced by, in guinea pigs, 800

See also B. abortus equi, 693

Bacillus abortus equi, 693

See also cattle abortion, 799

Bacillus acidi lactici, 639

Bacillus acidophilus in the normal intestinal tract, 225

description of, 228

Bacillus acidophilus aerogenes, description of, 228

Bacillus actinomycetum comitans, occurrence of in mycosis, 970

Bacillus aerogenes capsulatus, 752 Sec B. Welchii

Bacillus anthracis, 773. See also Anthrax

See also Symptomatic anthrax, 768

Bacillus anthracis symptomatici, 768

Bacillus anthrocoides, 785

Bacillus avisepticus, 827
Bacillus avisepticus, Pasteur's early work with, 828

Bacillus Bellonenis, 759

Bacillus bifidus in the normal intestinal tract, 225,227

description of, 228

Bacillus botulinus, $740-748$

antitoxin, 747

clinical manifestations, 746

cultivation of, 741

isolation of, 742

morphology, 741

occurrence of, 744-746

pathology of, 744

prevention of, 748

specific therapy, 747

staining of, 741

toxin, 742-744

not produced in the tissues of mammals, 744

thermolability of, 743

transmission of, 744

relation to limberneck of chickens, 746

types of, 744, 748

Bacillus bovis morbificans (Basenau), 688

B. bronchisepticus in canine distemper, 798

Bacillus bulgaricus, 1046

Bacillus butryicus, 617

Bacillus chauvaci, 759. See also Symptomatic anthrax

Bacillus cloacae, 641

Bacillus coli communior, 637

Avery type, 637

Malia type, 637

Bacillus eoli communis, 628-637

cultivation of, 629

distribution of, 631

immunization with, 635

normal agglutinins for, 636

morphology of, 628

occurrence of in the intestinal tract, 225

occurrence of in the normal nose, 221 occurrence of in water, 1023

presumptive test, 1024 
Bacillus coli communis, occurrence of in water, table for identification, 1026 pathogenicity of, 632-634

septicemia due to, 633 poisonous products of, 635 varieties of, 636

Winkel's disease due to, 633

Bacillus dentifricans, 60

Bacillus diphtheriæ, 561-582. See also Diphtheria bacillus

Bacillus enteritidis (Gaertner), 687 meat poisoning due to, 698

clinical picture in, 699

Bacillus fallax, 760

Bacillus fecalis alkaligenes, 639

Bacillus granulobacter pectinovarum, 55

Bacillus histolyticus, 762

Bacillus Hoffmanni, 582 occurrence of in the normal mouth, 219

Bacillus icteroides, 688

Bacillus influenzæ, 482. See also Influenza

Bacillus lactis ærogenes, 637-639 in the normal intestine, 225

in Metchnikoff's Bacteriotherapy, 1044

in the mouth, 219

in the nose, 221

Bacillus lepræ, 620. See also Leprosy

Bacillus Mallei, 787-794. See also Glanders

Bacillus maximus baccalis in the normal mouth, 220

Bacillus melitensis, 795-797

animal pathogenicity, 796

cultivation of, 796

disease caused by, 796

epidemiology, 797

immunity, 797

morphology, 795

Bacillus Morseele (van Ermengen), 688

Bacillus of meat poisoning, 686, 698

Bacillus mueosus eapsulatus, 720

cultivation, 721

general characteristies of group, 722 morphology, 720
Bacillus mucosus capsulatus, pathogenicity, 723

staining, 722

Bacillus œdematiens, 759

antitoxin, 760

method of identifying, 763

pathogenicity, 760

toxin, 760

Bacillus œdematiens maligni II, 759

Bacillus of eattle abortion, 799-801

isolation from milk, 800

lesions produced by, in guinea pigs, 800

Bacillus of chicken cholera, 827

Pasteur's early work with, 828

Bacillus of fowl typhoid, 694

Bacillus of Ghon and Sachs, 756

Bacillus of guinea pig pneumonia, 801 . 802

Bacillus of swine plague, 829

Bacillus of whooping eough, 504-508

Bacillus ozænæ, 726

Bacillus perfringens, 752. See also B. Welchii

Bacillus pestis, 807. See also Plague

Bacillus pestis caviæ, 692

Bacillus phlegmonis emphysematosæ, 752. See also B. Welehii

Bacillus prodigiosus, quantitative ehemical analysis of, 22

Bacillus proteus, 640, 641

agglutination of in typhus serum, 642, 945

pathogenicity of, 641

Bacillus psittacosis, 694

Bacillus putrificus in the normal intes-

tinal tract, 225

deseription of, 229,762

Bacillus pullorum, 695

Bacillus pyocyaneus, $802-806$

antitoxic substances produced by, 806 eultivation, 803

pigment production by, 803,804

immunization, 805

morphology, 802

pathogenicity, 804

staining, 802

toxins, 805 
Bacillus radicicola, 62

Bacillus radicosus, 785

Bacillus Rhinoseleroma, 724

pathogenicity of, 725

Bacillus sanguinarium, 694

Bacillus smegmatis, 618-619

Bacillus sporogenes, 761

Bacillus subtilis, 786

Bacillus suisepticus, 829

Bacillus tetanus, 727-740. See also 'Te. tanus

Bacillus III of von Hibler, 756

Bacillus tubereulosis, 586. See also Tuberculosis

Bacillus typhi abdominalis, 643. Sec also Typhoid bacillus

Bacillus typhi murium, 691

Bacillus typhosus, 643-685. See also Typhoid bacillus

Bacillus Welchii, 751-756

agglutinin production, 753

antitoxin, 755

cultivation, 752-753

fermentations of, 753

hemolysin production, 753

importance of in intestinal autointoxication, 1044

importance of in the normal intestinal flora, 226

isolation of, 755,756

method of identifying, 763

morphology of, 752

pathogenicity of, 753

spore formation, 752

staining of, 752

toxin production, 754

Bacillus Xerosis, 584

Bacteria (see also Bacterial cell)

acid and alkali formation by, 198

anabolic or synthetic activities of, 60 antagonism of, 34

chemical agents injurious to, 86

classification, 39

based on organs of motility, 16

by Migula, 41

counting of, 194-195

in milk, 1038

cultivation of, 172
Bacteria, cultivation of, by anaërobic methods, 179

degenerative forms, 20

denitrifying, 59

destruction of, 76

differentiation by fermentation, 54

enzymes produced by, 54, 201

gas formation, 196

Gram negative, 123

Crram positive, 123

in air, 1010

in milk, 1027

in the intestinal tract, 2

at different ages, 224, 225

in relation to diet, 226,227

in the normal mouth, 217-220

in the normal nose, $220-222$

in the tissues, 223

in water, 1020

katobolic activities of, 46

microscopic study of, 111

nitrifying, 64

nutrition of, 27

parasitic, 33

pathogenic, 231

physical agents injurious to, 76

relation of to moisture, 39

relation of to pressure, 39

relation of to temperature, 36

relationship to other plants, 40

reproduction of, 19

saprophytic, 33

size of, 9

staining of, 113

sulphur, 67

symbiosis, 34

thermal death points of, 38

variations in form of, 20

Bacteriæmia, definition of, 233

Bacteriaceæ, definition of, 41

Bacterial cell, ash in, 24

Babes-Ernst granules in, 11

capsule in, 12

chemical constituents of, 21

fats in, 24

proteins in, 22,25

membrane of, 12

metachromatic granules, 11 
Bacterial cell, morphology of, 10 motility of, 14

uucleus in, 10

osmotic properties of, 25

plasmolysis, 25

plasmoptysis, 25

specific gravity of, 26

spores in, 16

water, 22

Bacteriocidal and bacteriolytic tests. 307-311

bacteriolysins, discovery of, 251

determination of the bacteriolytic power of serum against a known organism in vivo, 308

identification of a microörganism by observing its susceptibility to lysis in a known immune serum, 309

reaction in test tubes, 310

"Bacteriophage"' phenomenon, 69

Bordet and Cuica's work, 72

early work of Twort, 70

discovery of lytic principle by d'Herelle in filtrates from the stools of dysentery and typhoid convalescents, 71

Bacterium aceti, 57

Bacterium clostridium pasteurianum, 61

Bacterium, genus, 41

Bacterium pasteurianum, 57

Bacterium pneumoniæ, 720. See also

Bacillus mucosus eapsulatus

Bacterium tularesne, 826

Bail's aggressin theory, 349

arguments against, 350

relation to anaphylatoxin, 351

Balantidium Coli, 1137

Bang Bacillus abortus bovis, 799

Barber pipette method of isolating a single organism, 178

Baumgarten's stain for differentiating between the tubercle bacillus and the bacillus of leprosy, 125

Beggiatoa, genus, 42

Beggiatoaceæ, definition of, 42

Berkefeld filter, 144

Biological activities of bacteria, 45

Black death, 807
Blackleg, 769. See also Symptomatic anthrax

Black water fever, 1128

Blastomycetes, relationship to other plants, 40

Blastomycosis, 984-987

blastomyces hominus, 985

classification of, 987

cultivation, 986

isolation, 986

microseopic examination, 985, 986

morphology, 986

pathogenicity, 987

staining, 986

Bleaching powder as a disinfectant, 89

Bleeding of animals, 204

from the heart, 204

from the carotid artery, 205

from the external jugular vein, 205

Blood cultures, technique of taking, 212-

215

method of obtaining blood, 212

media for, 213, 214

from typhoid patients, 214

anaërobic, 214

Blood examination for protozoa, 11401143

Blood grouping, 313-315

Jansky's classification, 313

Moss' classification, 313

“'universal donor,'” 314

Blood, method of obtaining, 204, 205

defibrinated, method of obtaining, 205

enriching. substance to be added to media, 169

ether adiled as a preservative, 205, 169

Bodonidæ, 1076

Boiling as a method of sterilization, 83

Bordet-Gengou bacillus, 504-508

antibody production by, 508

cultivation of, 506

epidemiology, 504-505

morphology of, 505

pathogenicity of, 507

staining of, 506

Bordet-Gengou phenomenon, 298 
Bordet's interpretation of toxin antitoxin reaction, 265

Botulismus, 740-748. See also Bacillus botulinus

Brilliant green as a disinfectant, 97 in culture media, 163

Brill's disease, 934. See also 'Typhus fever

Brownian movement, 14

Buboes, 809, 813

Buchner tube for anaërobic cultivation, 183

"Buffers', in culture media, definition of, 138

Burning, as a method of sterilizing, 82 Butter bacillus, 617

Butter, bacteria in, 1039-1042

Tubercle bacilli in, 1042

Butyl alcohol produced by fermentation of starch, 55

\section{Cadaverine, 51}

Capsules, description of, 12, 13 relation to virulence, 14 special stains for, 116

Carbolic acid, 95 coefficient of, 98 determination, of, 101

Carbon dioxide produced by bacteria, 196

Carbon in the nutrition of bacteria, 27

Carrel smear method of examining wounds, 764

Carriers as a source of disease, 377

in diphtheria, 575-577

in dysentery amœbic, 1062

in dysentery bacillary, 714

in malaria, 1117, 1123

in meningitis, 542

in typhoid, 669-676

Cellulose, 12, 24, 56

Carcomonadidæ, 1075

Bodonidæ, 1076

Trypanosomidæ, 1076

Cercomonas homini, 1055

Chagas (Schizotrypanum eurzi), 1091

Chaneroid, Ducrey bacillus in, 512

Charbon, 773. See also Anthrax
Charbon symtomatique, 768. See also Symptomatic anthrax

Chaulmoogra oil, in treating lepers, 625

Cheese, hard and soft, bacteria in, 1043

Chemical constituents of the bacterial cell, 21

bacterial ash, 24

lipoidal constituents, 24

nature of the antigenic proteins, 25 quantitative chemical analysis of mass cultures, 22

types of bacterial protein, 22, 23

Chemotaxis in phogocytosis, 332

Chicken cholera, bacillus of, 827-829

Chicken' pox, distinct from small pox, 894

China blue indicator, 148

Chitin in bacteria, 24

Chlamidozoa, 1139

Chlamydor bacteriaceæ, definition of, 42 description of, 961

Chloramine T, 93

Chloride of lime as a disinfectant, 89

Cholera, Asiatic, spirillum of, 831

animal pathogenicity, 836

biological considerations, 835

cholera-red reaction, 833

cultivation of, $832-834$

Dieudonne's selective medium for, 833,166

disease caused by, in man, 835

epidemiology, 837

endemic foci, 838,840

immunization, 841

isolation of from feces, 834

isolation of from water, 834

morphology, 831

prophylactic vaccination of, $841-843$

staining, 831

toxin, 837

Cholera-like spirilla, 845

Spirillum Deneke, 845

Spirillum Finkler Prior, 845

Spirillum Massaua, 844

Spirillum Metchnikovi, 844

Chorea, streptococeus viridans associated with, 423

Chromobacteria, 66 
Chromophytosis, 1008

Cladothrix asteroides, 964 defined, 692 genus, 42

Classification of bacteria, 41 according to flagella (Massea), 16 according to Migula, 41 of the more important pathogenic bacteria according to Gram's stain, 123

Clinic relation to bacteriology, 373

Coceaeæ, definition of, 41

Cocci, description of, 9

Coccidiodal granuloma, 987 coccidiodes immunitis, 988

Cohni streptothrix israeli, 965

Cold, the common, 436-438 filtrable virus in, discussion of, 437 epidemiological considerations, 488

Colon bacillus, group of, 627-640

cultivation of, 629

definition of, 637

differentiation of, by sugar fermentations, 637

distribution of, 631

immunization with, 635

in the intestinal tract, appearance of, 225

in the normal nose, 221

in water, 1023

presumptive test, 1024

table for identification, 1026

pathogenicity, 632-634

poisonous products of, 635

varieties of, 636

Colony fishing, technique of, 177

Colony study, 193

colony counting, 194

Color standards for colorimetric method of titrating media, preparation of, 140

Comma bacillus, 831. See also Cholera Complement deviation (Neisser-Wechsberg phenomenon), 297

Complement, facts concerning, 295 effect of concentration, 295

filtration of, 295

inactivation of, 295
Complement, inhibition of, 295

separation into fractions, 296

Complement fixation by precipitates, 296

determinatoin of antibodies by, 315 , 316

determination of antigen by, 325

for protein differentiation, 327

in glanders, 794

in the Wassermann test, 317

in tuberculosis, 608

Cowpox relation to small pox, 894

Crenothrix, genus, 42

Cristispira, 847

Crytococcus Gilehristi, 985

Culex fatigans in the transmission of Dengue fever, 931

Culture media, 133-171. See also Media Cytorryctes variolæ, 892

Cytotoxins, discovery of, 252

nephrotoxins, 253

DAKIN's solution, production of, 90

Dansyz, effect, 265

Dansyz type, 692

Dark-field in the demonstration of spirochæta pallida, 853

Delousing, 953 crude creosote oil in personal prophylaxis, 955 bath house, arrangement of, 955 gaseous disinfection, 956

formaldehyde, 956

sulphur dioxide, 956

hydrocyanic gas, 956

heat, aplication of, 956, 957

Degeneration forms of bacteria, 20

variations not degeneration forms, 20

Delhi boil, 1095

Dengue fever, 931-932

transmission by culex fatigans, 931 , 932

clinical symptoms, 931

Denitrifying bacteria, 59

Denitrification, 59

Dermatophytes, type of fungi causing ringworm, 995-1009 
Dermentor Venustus in. the transmission of Rocky Mountain Spotted fever, 951

Desensitization, 364

methods for, 369,370

Destruction of bacteria, 76

by chemical agents, 86

gaseous, 105

in solution, 86

inorganie, 87

organic, 94

by physical agents, 76

drying, 76

light, 77

electricity, 79

heat, 79

Diastase. See Amylase

Dichloramine T, 93

Dientamœba fragilis, 1072

Diet, effect on intestinal flora, 224, 226

Diphtheria bacillus, 561-582

antitoxin, 269-273

concentration of, 272

immunization of horses, $270,271$.

prophylactic doses, 273

prophylactic immunization, 580-582

regulation of by law, 272

specific therapy, $578-580$

dosage in, 579

standardization of, 271

therapeutic doses, 273

toxin-antitoxin reaction, theoretical considerations, 255-268

unit, definition of, 271

biological characteristies, 564

carrier problem in, 575-577

cultivation of, 565

Loeffler's medium, 565

diagnosis, 567

diphtheria-like bacilli, 582-584

bacillus Hoffmanni, 582

bacillus Xerosis, 584

diphtheria bacilli, 584-585

differentiation by sugar fermentations, 585

discovery of, 561

epidemioiogy of, 574-577

earriers in, 575-577
Diphtheria bacillus, isolation, 566

morphology, 562

pathogenicity, 567-570

causes of death, 569

for animals, 570

"'pseudomembranes," 567

resistance of, 564

Schick reaction, 577

staining, 562

- Neisser stain, 563

Toluidin blue, 564

thermal death point, 564

toxin, 571-574

in antitoxin reaction, theoretical considerations, 225-268

chemical and physical properties of, 573

Ehrlich's analysis of, 257-263

deterioration of, 258

expitoxoid or toxon, definition of, 261

Limes death or $\mathrm{L}+$ dose, definition of, 260

Limes zero in $\mathrm{L}_{0}$ dose, definition of, 259

method of partial absorption, 262

standardization, 258

toxoid, 259

unit of, 257

method of production, 571

thermolability, 572

virulence determination

Diphtheroids, description of, 584, 585

in the normal nose, 221

Diplococeus crassus, 535

Diplococcus gonorrhœæ, 547. See also Gonococeus

Diplococcus lanceolatus, 438 . S'ee also Pneumococeus

Diplococcus mucosus, 536

Diplococeus pneumoniæ, 438. See also Pneumococcus

Discomyces, 964. See also Nocardiæ

bovis, 965

in madura foot, 972

Disinfectants, 86-109 
Disinfectants, bactericidal strengths of, 105

gaseous for fumigation, 105

chlorin, 106

formaldehyde, 106

hydrocyanic aeid gas for rodents, 109

oxygen, 106

sulphur dioxide, 106

inhibition strengths of, 104

inorganic, 87

efficiency of acids, salts and bases proportional to degree of dissociation, 87

halogens, 89

ehloride of lime, or bleaching powder, 89

eusol, 93

terchloride of iodin, 93

tincture of iodin, 93

iodoform, 93

oxydizing agents, 93

peróxide of hydrogen, 93

potassium permanganate, 94

organic, 94

aleohols, 94

earbolic acid, 95

essential oils, 96

flavine dyes, 96

formaldehyde, 95

formalin, 95

lysol, 95

tricresol, 95

triphenylmethane dyes, 97

brilliant green, 97

malacite green, 97

testing efficiency of, methods for, 97 98

Rideal Walker, 98

U. S. Public Health Service, 99

Distemper, eanine, 798

Doderlein's bacillus, occurrence of in vagina, 230

Dourine, 1084

Drying, resistance of bacteria to, 76

Duerey bacillus, 510-513

cultivation in rabbit's blood, 167

etiologieal faetor in chancroid, 512
Ducrey bacillus, isolation of, 511

pathogenicity, 513

Drug idiosynerasies, 371, 352

DTN $M^{250}$, meaning of, 257

Dyes, anilin, as staining arents, 113

selective action of in media, 170, 161

Dysentery, amœbic (Entamœba histoly-

tica), 1054-1062

autopsy findings, 1055

earriers in, 1062

complications following, 1055

cyst formation, 1060

degenerative forms, 1060

diagnosis, 1056

distribution, 1055

epidemiology of, 1063

multiplication, 1057

observation of fresh specimen, 1058

staining methods, 1057

treatment in, 1062

Dysentery, bacillary, 700-717

earriers in, 714

elinical description of, 711

epidemiology of, 711.715

transmission of, 713,714

fermentations of the bacilli causing, 707

compared with typhoid-paratyphoid group, 718

general characteristies of bacilli eausing, 706

historical survey, 700-704

immunization with dysentery bacilli, 710

poisonous products of dysentery baeilli, 708

action on animals, 709

prevention of, 711

prophylactic vaccination, 716

resistanee of dysentery bacilli, 709

serum treatment of, 717

thermal death point, 707

types of bacilli eausing, 704-707

Flexner, 704, 707

Shiga, 700, 704, 707

Strong, 704, 707

"'Y," 704, 707 
EHRLICH's analysis of diphtheria antitoxin, 257-263

Ehrlich's side chain theory, 216-268

Electricity in the destruction of bacteria, 79

Empyema, following pneumonia, 465

Endocarditis, gonococcus in, 553 streptococcus viridans in, 422, 423

Encephalitis lethargica, 919

clinical picture in, 920

etiology, 920, 921

discovery of globoid bodies in, 921 relation to influenza, 920

spinal fluid in, 920

Endoenzymes, 49

Endolimax, nana, 1068

Endotoxins, 235

probably protein, 24

Energy of bacteria, source of, 29, 30 derived from oxidation of ammonia, 65

derived from oxidation of hydrogen sulphide, 68

Entamœba, 1051

Entamœba Butschlii, 1068 (iodamreba Butschlii)

Entamœba Coli, 1063

cyst formation, 1065

infection, experimental with, 1066

morphology, 1064

multiplication, 1065

Entamœba gingavalis, 1066

morphology of, 1067

cyst formation of, 1067

histolytica, 1054-1062

(etiological factor in amœbic dysen.

tery, see Dysentery amœbic)

nana, see Endolimax nana, 1068

tetragena africana, 1060

Enteritidis, bacillus of (Gaertner), 687 meat poisoning due to, 698

clinical picture in, 699

Enterococcus, 399

in the normal intestinal tract, 225, 227

Enzymes, bacterial, as katalysers, 47 amylase or diastase, 55

method of testing for, 212
Enzymes, cellulase, 56

gelase, 56

invertase, 56

method of testing for, 202

lab, 53

lactase, 56

lactic acid fermentation, 56

lipase, 53

maltase, 56

of fermentation, 54

oxydases, 57

proteolytic, 48

method of testing for, 49, 201, 202

xymase, in alcoholic fermentation, 58

Epidemiology of infectious diseases, 377-383

van Ermengen's stain for flagella, 119

Erysipelas, streptococcus in, 416

treatment with leucocyte extracts, 433

Erythrasma, 1009

Esmarch roll tubes for obtaining single colonies, 177

Essential oils as disinfectants, 96

Eusol, 93

Exudates, examination of, 207, 208

FARCY, 790

Fats in bacterial cell, 24

Fat-splitting enzymes produced by bacteria, 53

Favus, 1001

Ferments. See Enzymes, 47

Fermentation, 54

as a source of energy, 30

alcoholic, 58

ammoniacal, 52

caused by both yeasts and bacteria, 46

due to bacterial enzymes, 47

lactic acid, 56

Feces, examination of, 211

isolation of tubercle bacilli from, 212

isolation of typhoid bacilli from, 653

kinds of organisms present, 211

isolation of anaërobes from, by rab-

bit inoculation, 211

number of bacteria in, 211 
Filters, types of :

Berkefeld, 144

Chamberland, 144

cleaning and sterilization of filters, 145

Filtrable virus, general considerations, 889-890

diseases caused by, Wolbach's chart of, 891

in connection with the common cold, 437

in influenza, $486-488$

Flagella, 15

arrangement and structure, 15

staining of, 118

Flavine dyes as disinfectants, 96

Flexner bacillus in bacillary dysentery, 704,707

Foot-and-mouth disease, 932-933

due to a filtrable virus, 933

Formaldehyde, disinfectant action of, 95 methods for producing for fumigation, 107, 108

Breslau apparatus, 107

v. Brunn apparatus, 118

Lentz apparatus using glycerin to prevent polynecrization, 107

"lime" method, 108

potassium permanganate method, 109

Trillat autoclave, 109

\section{Formalin, 95}

bactericidal strength of, 104

inhibition strength of, 103

methods of producing formaldehyde

from, 107. See also Formaldehyde.

Fowl typhoid, 694

Frambœsia tropica, 870

Friedlander's bacillus, 720

occurrence of in the normal mouth, 219

occurence of in the normal nose, 221 pathogenicity of, 723

Fumigation, 105-110

chlorine in, 106

formaldehyd, 107-109

hydrocyanic gas for rodents, 109

oxygen, 106
Fumigation, ozone, 106

sulphur dioxide, 106

Fungi, pathogenic, 973-984

ascomycetes, 979

yeastes, 980

classification of, 975

liyphomycetes (fungi imperfecti), 983-1009

morphological definitions, 974

phycomycetes, 978

mucor, 978,979

Furunculosis, 391

Fusiform bacilli in Vincent's angina, 867

Fusiform bacilli other than those found in Vincent's angina, 869

GABBET's stain for the tubercle bacillus, 124

Gaertner bacillus, 687

meat poisoning due to, 698

clinical picture in, 699

Gas bacillus, 751-756. See also B. Welchii

Gas formation by bacteria, 196

analysis of, 197

determined by Smith fermentation tube, 196

hydrogen sulphide, 197

Gelase, 56

Genitalia, examination of lesions on, 216 syphilitic, 216

chancroid, 216

Gentian violet, selective action in media, 170

Geryk pump for producing a vacuum, 908

Giardia intestinalis, 1074

Giemsa's stain, 127

Glanders bacillus, 787-794

biological considerations, 789

complement fixation, 794

cultivation, 787-788

diagnosis of, 791

Strauss test in, 791

immunity, 794

morphology, 787

pathogenicity, 789 
Glanders bacillus, pathogenicity, in horses, 789-791

in $\operatorname{man}, 789,790$

nodule formation in the chronic

form of the disease, 791

staining, 587

toxins of, 791

mallein, diagnositic use of, 792, 793

mallein, preparation of, 792

Glassware, method of cleaning and sterilizing, 133

Globoid bodies in poliomyelitis, 915

Globulin in spinal fluids, method of testing for, 209

Glycerin in culture media, 169

for growth of tubercle bacillus, 592

Gonococeus, 547-559

antibodies to, 553

complement fixation with, 553

cultivation of, $548-550$

endocarditis due to, 553

fermentation reactions, 535

identification of, 551, 552

by sugar fermentations, 551

morphology of, 547

ophthalmia due to, 555

pathogenicity, 552

prophylaxis, 559

rheumatism due to, 553

resistance, 552

thermal death point, 552

types of, 550

vaccine therapy, 554

vulvovaginitis due to, 556

Gram-negative bacteria, important pathogenic list of, 123

Gram-negative cocci, table of fermentations, 535

Gram-positive bacteria, important pathogenic list of, 123

Gram's stain, 121

Gram-Weigert method of staining Gram positive organisms in tissues, 130

Group agglutination, 282. See also Agglutination.

Grubor-Widal reaction, 302

HæMOPFILIC group of organisms, 482
Haffkine's virus, 825, 826

Halogens as disinfectants, 83, 104, 103

"Hanging block method" for studying living bacteria, 112

"Hanging drop" method for studying living bacteria, 111

Halteridium, 1099-1101

Hay fever, 370

Heat, in the destruction of bacteria, 79 dry and moist, comparison of, 80

low efficiency of "superheatell" steam, 82

live steam, 83

steam under pressure, 85

Helber counting chamber for bacteria, 195

Hemorrhagic-Septicæmia group, 807-829

bacillus of chicken cholera, 827

bacillus of plague-like diseases in rodents, 826

bacillus Pestis, 807

bacillus of swine plague, 829

Hemolysin, 280

discovery of, 252

immune, discovery of, 279

specificity of, 299

production by staphylococcus, 393

production by streptococeus hemoly. ticus, 426

Hemolytic tests, 311

Hemoproteus columbæ, 1099-1101

Hemosporida, 1098

d'Herelle's "'Bacteriophage" phenomenon, 70,71

Hermann's stain for the tubercle bacillus, 126

Higher bacteria for trychomycetes, 761

Hiss' capsule stain, 116

Hiss' leucocytic extract, 344-346

Histamine, 52

liberated by abnormal proteolytic activity in the intestinal tract, 227

produced in bacterial cultures, 237

Hoffmann bacillus, 582

Hog-cholera, bacillus of, 691

Hot air sterilization, 82

Huntoon's capsule stain, 117 
Hydrocyanic acid gas, destruction of rodents by fumigation with, 109

Hydrogen in the nutrition of bacteria, 31

Hydrogen ion determination, 138-142

by colorimetric method, 138-142

$\mathrm{Ph}$, meaning of, 138, 138

preparation of color standards for, 141

indicators for various ranges of $\mathrm{Ph}, 140,141$

actual steps in, 142

"'buffer," definition of, 138

comparator, for reading, 142

Hydrogen sulphide produced by bacteria, determination of, 197

Hydrophobia, 900. See also Rabies

Hypersusceptibility, definition of, 352

Doerr's classification, 352, 353

Hypomycetes, 983

in relationship to other plants, 40

Imмunity, defined, 240, 241

natural, 241

racial, 242

species, 241

acquired, 243

active, 244

produced with attenuated cultures, 245

produced with sublethal doses, 246

produced with dead bacteria, 246 produced with bacterial products, 247

passive, 247, 248

antitoxic sera, 247

antibacterial sera, 247

Impetigo contagiosum, 392

Ietero-hæmorrhagic fever, 885. See also Weil's disease.

Incubators, 190

thermo regulators, 191

Indicators, theory of, 146

for hydrogen ion determination, 140, 141

phenol red or phenolsulphonephtha-

lein, 141
Indicators for hydrogen ion determination, brom-thymol blue, 141 cresol red, 141

for addition to culture media, 147

Andrade, 147

china blue, 148

litmus, 147

neutral red, 148

Indol production by bacteria, 199

method of testing for, 199

Vanillin test, 199

Infantile paralysis, 912. See also Poliomyelitis

Infantile splenomegaly, 1095

Infection, definition of, 230

factors determining, 232, 233

path of, 232

Infectious diseases, transmission of by direct contact, 380

by insects, 380

in intestinal group, 379

through respiratory channels, 378

Influenza bacillus, $482-501$

biology of, 498

clinical picture, 482, 483

cultivation of, $495-498$

preservation of, 497

epidemiology, 488

characteristics of the epidemics, 489

history of the last War epidemies, 493,494

secondary and tertiary waves, char. acteristics of, 492

transmission, 490

etiology of, 484

arguments for and against Influenza bacillus, 485,486

filtrable virus theory, 486-488

in trachoma, 500, 501

isolation of, 495

Avery sodium oleate agar, 496

morphology, 494

occurrence of in inter-epidemic per-

iods, 499

pathogenicity for animals, 501

stains, 494

toxin formation, 499 
Influenza bacillus, varieties of, 500

Infusoria, 1137

Inhibition strength, of various antisepties, 103

Inoeulation of media, 172

type of wire for, 173

technique of transferring cultures, 175

Intestinal tract, bacterial flora of, 223228

influence of age, 224, 225

influence of diet on, 224, 226

influence of B. Welehii in, 226, 227

Intravital method of studying living bacteria, 112

Invertase, 56

Iodamœba Butschlii, 1069

Iodine, tincture of, as a disinfectant, 93

Iodoform, 93

Isoagglutinins in human blood, 313-315 Jansky's classification, 313

Moss classification, 313

" universal donor," 314

Isolation of bacteria in pure culture by, 174

pour plates, 175

technique of, 175

colony fishing, technique of, 177

streak plates, technique of, 178

Isolysins, 299, 300

JAUNDICE, epidemic, 885-888

elinical description, $885-886$

etiology, spirochaeta icterohæmorrhagiæ, 886

cultivation of, 886,887

prevention, 888

serum treatment, 888

transmission, 887

Jenner's stain, 127

Jensen's modification of Gran's stain, 122

KALA-AZAR, 1093

Katabolic activities of bacteria, 46

Kefyr, 1031

Kernig sign in meningitis, 527
Klebs-Loeffler bacillus, 561. See also

Diphtheria bacillus

Koch-Weeks bacillus, 502

Kolle vaccination method for $B$. pestis, 204

Koumys, 1031

Kreatoxismus, 51

L+ DoSE, diphtheria toxin, 260

$\mathrm{L}_{0}$ dose, diphtheria toxin, 259

Labferment, 53

Lactase, 56

Lactic acid bacilli in bacteriotherapy, 1044

Lactic acid fermentation, 56

Lambia intestinalis, 1074. See also Gicrdia intestinalis, 1074

Landry's paralysis, 912, 915

Lateney of bacteria in tissues and circulation, 223

Leischmania, 1093

Leischmania braziliansis, 1097

Leischmania donovani, 1093

animal pathogenicity, 1095

morphology, 1093

Leischmania infantum, 1095

Leischmania nilotica, 1097

Leisclımania tropica, 1095

Leprolin, 624

Leprosy, bacillus of, $620-625$

cultivation of, 621

discovery of, 620

distribution of, 622,623

morphology of, 620

pathogenicity, 622

clinieal varieties of, 623

contagiousness of, 624

reaction of lepers to tuberculin, 625

therapeutic use of Chaulmogra oil, 625

Leprosy, rat, 625,626

acid-fast bacilli in, 626

cultivation of, 626

Leptothrix, 962, 963

in the normal mouth, 220

Leptospira, elass of spiral organisms, 849 
Leptospira icterohaemorrhágiæ, causative agent of yellow fever, 881-883 description of, 882

immunization with, 884

medium for cultivation of, 882

relation to Weil's disease, 883

Leucocidin, Nakayama's method of testing for, 429

production by staphylococeus, 394

production by streptococcus, 428

specificity of, 238

Leucocytic substances (Hiss), effect of injections of, $344-346$

method of obtaining, 345

Lice, 953

baths for individual proctection against, 955

crude creosote oil for prophylaxis, 955 habits of, 953,954

heat, in delousing, 956, 957

in the transmission of typhus fever, 937

Rickettsia bodies in, 943

varieties of, 953

Light in the destruction of bacteria, 77

Light production by bacteria, 66

Limber neck in chickens, relation to botulismus, 746

Lipase, enzyme produced by bacteria, 53

Lipoidal constituents of the bacterial cell, 24

Lipovaccine, typhoid, 481, 683

Litmus indicator, 147

Lockjaw, 727. See also Tetanus

Loeffler's medium for diphtheria, 565

Loeffler's method for staining bacteria in tissues, 129

Loeffler's stain for flagella, 119

Ludwig's angina, 417

Lysins, 278, 279

Lysol, 95, 104

Lytic agents, transmissible in series, 69

Lyssa, 900-909. See also Rabies

MACROPHAGE, 331

Madura foot, 971, 972

Madurella in madura foot, 972
Malachite green as a disinfectant, 97 in culture media, 163

Malaria, 1102-1131

carrier state in, 1117, 1123

clinical description of, 1115-1117

cultivation of in vitro, 1118

description of the mosquito, 1118

development of the larva, 1119

incubation period in, 1121

life history, 1121

development of human malarial para-

sites in the mosquito, 1117

epidemiology, 1122

earriers in, 1123

prevention of mosquito breeding, 1122

quinine prophylaxis, 1125

screening, 1122

treatment, 1124

examination of fresh blood, 1114

geographical distribution, 1103

history of, 1102

immunity in, 1127

life cycle of parasite, 1106

other malarial parasites, 1131

pathology, 1126

plasmodia finer structure of, 1112

plasmodium falliparum causing aes-

tivo autumnal fever, 1111

staining of, 1114

plasmodium malariæ causing quar$\tan$ fever, 1110

staining of, 1114

plasmodium vivax, eausing tertian fever, 1107-1110

susceptibility of negroes to, 1105

transmission by various species, anopheles mosquitoes, 1118

treatment of, 1129

Malignant edema, bacillus of. See Vibrion septique; B. sporogenes

Mallein, 791

diagnostic use of, 792

preparation of, 791

Mallory's eosin methylene-blue stain for bacteria in tissues, 131

Malta fever, 795. See also B. melitensis

Maltase, 56 
Marmosets, species of monkey, susceptible to Leptospira icterohæmorrhagiæ, 881

Mastigophora (class of protozoa), 1073

Measles, 922-926

virus in blood, 922

virus in nasal and pharyngeal wash-

ings, 923

epidemiology, 923-926

infectious period, 925

incubation period, 926

pneumonias following, 925

prevention of, 925

Meat poisoning due to B. botulinus, 740

Meat poisoning due to Gaertner baccillus, 698

Media, 133-171

anaërobic tissue tubes for spirochæte cultivation, 167

cooked meat medium for eultivating anaërobes, 168

dyes, selective action in media, 170 , also see 161,162

enriching substances added to, 168 ascitic, hydrocele or pleural fluids, 169

blood, 169

method of obtaining, 169

preservation of blood by adding ether, 169

Formulæ of media in general use, 148-156

agar, hormone or vitamine, 149

lactose litmus (Wurtz), 153

meat extract, 148

meat infusion, 148

sodium oleate for the influenza

bacillus, 156

starch (Vedder), 154

trypager, 153

broth, ealcium carbonate, 156

for streptococeus agglutination (Avery), 150

glycerine, 150

hormone or vitamine, 149

meat extract, 148

meat infusion, 148

potato, 155
Media, formulæ in general use, sugarfree broth, 150

"chocolate"' media, 158

Dorset egg medium, 154

Duuham's solution, 151

Gelatin, meat extract, 151

gelatine, meat infusion, 152

glycerine egg (lubenau), 154

hormone media, 149

Loeffler's medium, 156

medium for cultivating nitrifying

bacteria, 65

milk media, 156

nitrate broth, 151

pepton salt solution (Dunham), 151

serum water for fermentation

(Hiss), 157

synthetic media for tubercle bacilli, 155

Uschinsky's protein free, 151

Welch's modification of Guanieri's

medium, 154

General methods of preparing, 133

clearing with egg, 143

filtration, 143

ingredients, 135

meat extract and meat infusion

defined, 135

slanting of, 146

sterilization of, by 144

autoclaving, 144

filtration through Berkefeld or Chamberland filters, 144

fractional method, 144

titration of, 136

colorimetric method, 138

actual steps in, 142

"'buffer," definition of, 138

color standards, preparation of, 140

indicators for various ranges of $\mathrm{Ph}, 141$

$\mathrm{Ph}$, meaning of, 138,139

phenolphthalein, old method, 136

Indicators added to, 147-148

Rabbits' blood for Ducrey bacillus, 
Media, formulæ in general use, special for colon-typhoid differentiation, 158

Barsickow's medium, 164

bile medium, 163

brilliant green agar (Krumwiede), 161

brilliant green eosin agar (Teague and Clurman), 162

Conradi-Drigalski, 158

Endo's, 159

Kendall's modification of, 160

Robinson's and Rettger's modification, 160

Jackson's lactose bile medium, 163 lead acetate agar for Partyphoid A and $\mathrm{B}$ differentiation, 163

lead acetate added to Russell double sugar, 165

Mackonkey's bile salt, 164

malachite green broth (Peabody and Pratt), 163

Neutral red medium, 164

Russell double sugar, 164 with lead acetate, 165

special, for isolation of eholera spiralla, 166

Aronson's, 166

Dieudonne, 166

Teague and Travis, 167

Meningitis, acute primary, 514

due to meningococeus or pneumococeus, 514

epidemic, 514

secondary, 514

Meningococeus, 514, 546

agglutination, 523

agglutinin absorption, 522

diagnostic, 543, 544

method of produeing agglutinating serum for laboratory use, 523

technique of, 524

animal pathogenicity, 526

carrier determination, 542

diagnostie agglutination, 543, 544

media for, 542

West tube for taking nasopharngeal swabs, 542

cultivation of, 516
Meningococcus, eultivation of, influence of $\mathrm{CO}_{2}$ on, 518

media for fermentation reactions, 520

optimum $\mathrm{Ph}, 518$

optimum temperature, 518

special media for, 519,520

diagnosis of, 527

differentiation from other Gram neg-

ative cocci, 533

epidemiology, 536-546

earriers, 539

cure of, 545

determination of, $542-544$

rate of, 540,541

seasonal prevalence, 537

susceptibility of negroes, 537

susceptibility, variability of, 539

fermentation reactions of, 535

medium for, 520

morphology of, 515

resistance of, 520

septicæmia due to, 527,529

serum therapy in, 529,533

serum, administration of, 530

dosage in, 531

intravenous injections, 532

effect of, 532, 533

standardization by agglutination, 530

spinal fluid, method of obtaining, 527

spinal fluid, examination of, 528, 529

staining of, 515

toxic products, 521

types of, 522

virulence, 546

Metchnikoff's bacteriotherapy, 1044

Metchnikoff's work on phagocytosis, 330,334

Methylene-blue, anaërobic indicator, 187

Micrococei. See Staphlyococei, 384

Microcoecus, genus, 41

Micrococens candicans in the normal mouth, 218

Microcoecus eatarrhalis, 553, 559-560

differentiation from gonococeus and meningococeus, 559

fermentation reactions of, 535 
Micrococcus catarrhalis, oceurrence of in the normal mouth, 218

Micrococeus crassus, 535

Micrococeus flavus, 534

in the normal mouth, 218

Micrococeus intracellularis meningitidis, 504. See also Meningococeus

Micrococeus melitensis, 795. See also B. melitensis

Micrococeus pharyngis siccus, 535 occurrence of in the normal mouth, 218

Micrococeus tetragenous, 398

present in the normal mouth, 218

Microphage, 331

Microscopic study of bacteria, 111

in fixed preparations, 112

method of making a smear, 113

see staining, 113

in living state,

by "'hanging drop" method, 111

by "'hanging block" method, 111

by intravital method of Nakanishi, 112

Microsiphonales, 961

Microspira, genus, 42

Mierosporon, 1003. See also Microsporon furfur and Microsporon minutissum, 1009

Milk, alcoholic fermentation in, 1031

bacteria in, 1027

“'bitter," 1031

butyric acid formation in, 1030

" certified," 1029

counting bacteria in, 1038

dairy inspection, 1037

pasteurization, 1037

regulation concerning, 1040

relation to infectious disease, 1031

anthrax, occurrence of, in, 1035

cholera traced to, 1033

diphtheria traced to, 1033

foot-and-mouth disease transmitted

by, 1035

infantile diarrhea attributed to, 1034

scarlet fever traced to, 1033

streptococei in, 1034
Milk, streptococei in, epidemic of sore throat caused by, 1034

tuberculosis transmitted by, 10351037

tubereulin test for eattle, 1037

species of bacteria in, 1029

source of bacteria in, 1027

souring of, 1029

Kefyr, 1031

Koumys, 1031

sterilization of, 1037

Milzbrand, 773. See also Anthrax

Moeller's spore stain, 116

Moisture, importance of in bacterial growth, 39

Molds, in the normal nose, 221

occurrence of, 982

pencillium glaucum, 983

aspergilli, 983

sterignocystis, 983

Monilia albicans, 989

Monilia psilosis, 991

relation to sprue, 991

Morax-Axenfeld bacillus, 508

Morgan bacilli in infantile diarrhea, 717

Motility of bacteria, 14

conditions favoring, 15

organs of, 14

true motility distinguished from

Brownian movement, 14

Mount Desert bacillus in bacillary dysentery (Park), 704, 707

Mouth, bacteria in, 217, 218, 219

Much's granules in tubercle bacilli, 588

Mucor, 979

Mumps, 930

incubation period, 930

Muriseptus, bacillus of, 503

Mutation, bacterial, 43

problem of, in connection with streptococcus, 434

Mycetoma or Madura foot, 971, 972

Mycoderma dermatitis, 985

NAgANA, 1081

Nakayoma's method for testing for leucocidin, 429 
Negri bodies in rabies, 902 cultivation of, by Noguchi, 905, 906 diagnosis of rabies by, 903

van Gieson's method for rapid demonstration, 903, 904

significance of, 904, 905

staining in tissues, 902

Neisser's stain for polar bodies, 126

Neisser-Wechsberg phenomenon, 297

Nephrotoxin, 253

Neutral red indicator, 148

Nitrifying bacteria, 64

Nitrite formation by bacteria, 200 method of testing for, 200

Nitrogen fixation by bacteria, 60

Nitrogen in the nutrition of bacteria, 31

Nitromonas or nitrosomonas, 64

Nocardia, 963-965

in rat-bite fever, 965

in Madura foot, 972

minutissimum, 1009

Noguchi's butyric acid test for globulin, 209

Noguchi's globoid bodies in poliomyelitis, 915,916

Noguchi's method of eultivating spirochæta pallida, 856

Non-specific protein therapy, 347

elinical pieture following, 348

Nose, normal bacterial flora, 220

filtering action of nasal mucous membrane, 221

pereentage findings of various bacteria, 221

Novy jar in anaërobic cultivation, 185

Nutrition of bacteria, elements necessary, 27

carbon, 27

oxygen, 28

nitrogen, 31

hydrogen, 31

salts, 31

substances of unknown composition, 32

vitamines, 33

Ordrum albicans, 989

Oidium Hektoenii, 985
Oidium lactis, in cheese, 1042

therapeutic use of, 1045

Oospira, 964

Ophthalmia due to gonococcus, 555

Opsonic index, 341

Opsonic test, 339

in judging effect of vaccine therapy, 344

Simon, Lamar and Bispham's method, 341

Wright's technique of, 339

nethod of obtaining bacterial emulsion, 339

method of obtaining blood serum, 339

method of obtaining leucocytes, 340

Opsonins, 336

multiplicity of, 337

Neufeld's and Rimpau's work on, 337 relation to antibodies and complement, 338, 339

specificity, 337

thermolability of, 338

Wright's work on, 336, 337

Osteomyelitis, caused by staphylococcus, 392

caused by streptococeus, 416

Osmotic properties of bacterial cell, 25 Oxydases, 57

Oxydising agents as disinfectants, 93, 103, 104

Oxygen, in the nutrition of bacteria, 28 bactericidal action of, 106 partial tension for the growth of bac-

teria, 189

Oysters, examination of, 1047

Ozænæ, bacillus of, 726

Perez bacillus of ozænæ, 726

Pasteurization of milk, 1037

Paltauf's modification of Gram's stain, 121

Pappenheim-Saathof stain, 115

for gonococcus smears, 548

Pappenheim's stain for differentiating between the tubercle bacillus and the smegma bacillus, 125 
Pappenheimer's method for staining Gram positive bacteria in tissues, 131 Parasites, defined, 33, 231

facultative parasites, 34

not necessarily pathogenic, 231,232

Paratyphoid-enteritidis group, 686-699 differential considerations, within group, 669

fermentation reactions, chart of, 690. See also 718

differentiation of from B. coli and typhoid, 687

general survey of, 687-689

Paratyphoid A, defined, 689

pathogenicity for man, 696

Paratyphoid B, heterologous group, 698

pathogenicity for animals, 691-695

B. abortus equi, 693

distinguished from B. abortus bovis, 693,799

B. enteritidis (Gaertner bacillus), 687, 698

B. of fowl typhoid (B. sanguinarium), 694

B. Pestis caviæ, 692

B. psittacosis, 694

B. pullorum, 695

B. typhi murium, 691

Danysz type, 692

hog cholera, 691

pathogenicity in man, 697

meat poisoning due to Gaertner ba-

cillus (B. enteritidis), 698

elinical picture in, 699

typhoid-like fever due to, 697

Paratyphoid C, 688

preventive measures, 699

Paronychia, 391

Penicillium, 983

in cheese, 1043

Perez bacillus of Ozæna, 726

Peripneumonia of eattle, 10

Perlsucht, tuberculosis of eattle, 612

Peroxide of hydrogen, as a disinfectant, 93, 104

Pertussis bacillus, 504-508

antibody production, 508
Pertussis bacillus, cultivation of, 506

epidemiology, 504, 505

morphology of, 505

pathogenicity, 507

staining of, 506

Petroff's method of isolating tubercle bacilli from sputum, 591

Petruschky's scheme of the higher bacteria, 962

Pfeiffer bacillus, 482. See Influenza.

Pfeiffer phenomenon, 251

$\mathrm{Ph}$, meaning of, 138

Phagocytic index, in opsonic tests, 341.

Phagocytosis, 330-335

chemotaxis, 332

"fixed" cells, 331

of dead and living matter, 332

theoretical considerations, 334, 335

Metchnikoff's work, 330, 334

variations in phagocytic response, 333 variations of susceptibility of bacteria to, 333

"wandering", cells, 331

macrophages, 331

microphages, 331

Phenol production by bacteria, 200

method of testing for, 200

Phenol, red indicator in hydrogen ion determination, 141

Phenol-sulphon-phthalein, 141

Phenolphthalein method of titrating media, 136

Pharynx, normal bacterial flora, 217

Phragmidiothrix, genus, 42

Phycomycetes, group of pathogenic fungi, 978

Physical properties of bacteria, 26

Pigment formation, by bacteria, 66 condition necessary for, 67 red pigment of thiobacteria, $6 \mathrm{~s}$

Piroplasmidæ (Faunea), 1131

clinical observations, 1133

morphology, 1132

transmission by ticks, 1133

life history of the tick, 1133-1135

Pityriasis versicolor, 1008

Plague bacillus, $807-825$

animal pathogenicity, 815 
Plague bacillus, autopsy findings in rats, 822

bacteriological diagnosis, 820

biological considerations, 810

cultivation, 809

on salt agar to obtain involution

forms, 810

disease, caused by, in man, 813

incubation period, 813

pneumonic form, 814

transmission by flea bite or inhala-

tion, 813

epidemiology, 814

pneumonic form, transmission of, 819

prevalence of, 815

rats in the transmission of, 816

due to flea, 817

immunization, 812

isolation, 809

morphology, 808

prevention of, 820

eircumvention of a focus, 823

rat destruction in, 820

rat proofing in, 823

staining, 808

toxin formation, 812

vaccination, $824-826$

Haffkine's virus, 825

Plague-like disease of rodents, 826

Planococeus, genus, 41

Planocarcina, genus, 41

Plasmodia, finer structure of, 1112

Plasmodium falciparum, 1111, 1106

Plasmodium malariæ, 1110, 1106

Plasmodium vivax, 1106, 1107-1110

minuta, 1131

Plasmolysis, 12, 25

Plasmoptysis, 26

Pleuropneumonia of rabbits, bacillus of, 503

Plotz bacillus, association with typhus fever, 941, 942, 944

P'neumonobacillus, 720. See also B. mucosus capsulatus

Pneumococeus, 438-481

antibody formation, 466, 467
Pneumococcus, as the cause of primary meningitis, 514

bile test for identification, 453

blood cultures for, 464

capsule formation, 440, 441

complications following pneumonia, 465

cultivation of, $442-446$

description of on blood agar, 445

on inulin serum water, 445

epidemiology of, 472-480

primary pneumonias, $473-478$

autoinfection, discussion of, 474, 475

prevention of, 477

susceptibility factor in, 475,476

secondary pneumonias, 478

prevention of, 479

etiological factor in lobar pneumonia, 434

hemolysin production by, 445

inulin fermentation by, 445,453

immunity, 466

immunization, active, 480

immunization, passive, 467

serum production, 468

serum treatment, $470-472$

standardization of serum, 467,470

in the normal mouth, 218

in the normal nose, 221

morphology of, 440-442

mucosus Type II (streptococeus mucosus), 448

pathogenicity of, 455

intratracheal method of injecting rabbits, 457,459

protection tests, 469,470

resistance to disinfectants, 450

staining of, 442

in tissues, 442

capsules, 442

serum treatment, $470-472$

thermal death point, 450

types of, $446-448$

subgroups of type II, 447,448

incidence in lobar pneumonia, 463

incidence in normal persons, 459

typing from sputum, 460 
Pneumococcus, typing from sputum, mouse injection, 461

agglutination of peritoneal exudate, 462

precipitins in peritoneal exudate, 463

by protection experiments, 463

Avery method, 462

urine, precipitins in, method of determining, 463

viability of, 449

virulence of, 455

method of increasing, 456

Poisons, bacterial, 234-239

endotoxins, 235

mode of action of, 238

toxins, definition of, 234

" $\mathrm{x}$ ", substances, 237

Polar bodies, 11

Poliomyelitis, acute anterior, 912-918

clinical picture, 913

epidemiology, 917

earrier state, 918

fly transmission discussed, 918

etiology, 914, 915

globoid bodies in, 915,916

filtrability of virus, 915

immunity, 916, 917

spinal fluid in, 913

transmission to monkeys, 914

transmission to rabbits, 916

Polymastigna (order of protozoa), 1073

Pompholyx, 1007

Phosphorescence, produced by bacteria, 66

Potassium permanganate, disinfectant action, 94

Pour plates, technique of, 175

Precipitation, 289

precipitins, 290

absence of in normal sera, 292

discovery of, 252

specificity of, 290

tests, 304

preparation of bacterial filtrates

and protein solutions for, 306

theoretical considerations, 292
Precipitation, precipitins, production of precipitating sera, 304, 305

Pressure, effect of, on bacteria, 39

Protein cleavage by bacteria, 46

Proteins, as antigens, 254. See also

Antigen of the bacterial cells, 22, 23,25

racemized, not antigenic, 357

Proteolytic enzymes, 48

method of testing for, 49

Proteosoma praecox, 1101

Proteus group of bacilli, 640

agglutination in typhus serum, 642, 945

occurrence of in the normal mouth, 219

pathogenicity, 641

Protomonadina, 1075

Cercomonadidæ, 1075

Protozoa, pathogenic, 1048

classification, 1048

technique of blood examination for, 1140,1143

Pseudomones, genus, 42

Pseudodiphtheria bacillus, 582

Pseudoinfluenza bacillus, 501

Pseudo-membranes in diphtheria, 567

Pseudo-tuberculosis in guinea pigs due to B. pestis carviæ, 692

Ptomaines, 50 chemical constitution of, 51

discovery of, 234

Public health management of venereal diseases, 556

Purpura hemorrhagica, clinical differentiation from typhus fever, 935

Pus, examination of, 207

Putrefaction, as a source of energy, 30 definition of, 50

Putrescine, 51

Pyemia, defined, 233

Pyocyanin, pigment produced by $B$. pyomaneus, 804

Pyocyanolysin, 806

Pyrogallic acid in anaërobic cultivation, 183

Pyorrhea, 1066

amœba in, 1066 
QUARTAN fever, 1106

clinical description, 1115-1117

Quarter-evil, 769. See also Symptomatic anthrax

RABBIT inoculation for isolation of anaërobes from feces, 211

method of obtaining leucocytes from, 345

Rabies, 900-909

filtrability of virus, 905

incubation period in, 900, 901

Negri bodies, cultivation of by No-

guchi, 905, 906

diagnosis by, 902

significance of, 904, 905

staining of, 902-904

specific therapy, 906-909

dosage of, 908, 909

preparation of the cord, 907, 908

"street" virus, 901

symptoms in animals, 901

symptoms in man, 901

"virus fixe," 901

attenuation of, 906

Rage, 900. See also Rabies

Rat-bite fever, 873

Ratin, 692

Rat leprosy, 625, 526

Rauschbrand, 768. See also Symptommatic anthrax

Reducing powers of bacteria, 200 method of testing for, 200

Refractive index of bacteria, 26

Relapsing fever, 861

cultivation of Obermeier's spirochæte, 862

immunity, 867

morphology, 861

pathogenicity, 864

staining, 861

transmission of, 866

types of, 865

Reproduction of bacteria, 19 rate of growth, 19

"Resistance" defined, 240

variations in, 243

Respiration of bacteria, 29
Rheumatism, gonorrheal, 553

streptococcus viridans in, 422,423

Rhinoscleroma, bacillus of, 724

pathogenicity of, 725

Rhizopoda, 1050

Rhusiopathiæ, bacillus of, 503

Rickettsia bodies, 957-960

description of the group, 958

classification of, 959

R. Prowazeki, description of, 960

Dermacentroxenus richettsi, 960

staining of, 958

inheritance of, 959

in typhus fever, 942, 943, 944

in Rocky Mountain spotted fever, 953

in trench fever, 949

Rideal-Walker method of testing disinfectants, 98

Ringworm or tinea,

eczema marginatum and pompholyx, 1007

epidermophyton inguinale, trichophyton cruris, 1007

microsporon, 1003

cultures, 1004

morphology, 1004, 1005

other members of this group, 1005

trichophyton, 1005

classification, 1006

Ringworm group, 995-1000

cultivation of, 1000

conservation medium (Sabouraud), 1000

test medium (Sabouraud), 1000

cultural characteristics, 996, 997

immunity, 999

methods of examination, 999

morphology, 997

pathogenicity, 998

pleomorphism, 998

Rocky Mountain spotted fever, 950

clinical description, 950

incubation period in, 950

epidemiology, 951

transmission by Dermacentor venistus, 950

transmission to guinea pigs, 951

transmission to rabbit, 951, 952 
Rocky Mountain spotted fever, epidemiology,transmission to monkeys, 925 Rickettsia in, 952

Root tubercles in the leguminosæ, 61

SABouraUd's medium for members of the ringworm group, 1000

Saccharomyces albieans, 989

Saccharomyees hominis, 985

Saccharomyeetes or yeasts, 980

Sachs-Georgi reaction for syphilis, 328 Salts in the nutrition of bacteria, 31

Saprophytes defined, 33, 231

Saprospira, 847

Sarcina, genus, 41

in the normal nose, 221

Sareodina (Rhizopoda), 1050

Sareophysematos bovis, 768. See also Symptomatic anthrax

Sarcosporidia, 1135

Searlatina, 926-930. See also Scarlet fever

Scarlet fever, 926-930

diphtheroids as the causative agent, 927

streptococci as the causative agent, 925, 927, 298

epidemiology of, 928

transmission by nasal and pharyn-

geal mucous, 928

period of infectiousness, 929

"return" cases, 929

incubation period, 929

Schick reaction, 577

Schizomycetes, definition of, 41

Schweineseuche, 829

Sepsine, 52

Septicæmia, definition of, 233

Serum, methods of obtaining from man, 301,205

from rabbits, 301

from large animals, 302

Serum sickness, 366,367

Shiga's bacillus in bacillary dysentery, $700,704,707$

Side chain theory, Ehrlich, 216-268

Sitotoxismus, 51

Size of bacteria, 9
Skin reactions, 368

delayed, 368

for determining hypersensitiveness, 369

immediate, 368

Sleeping sickness, African, 1086

Sleeping sickness. See Eneephalitis lethargica

Smallpox, 890-899

etiology, 892-893

relation to cowpox, 894

vaccination, $898-899$

vaccine production, 894

calf inoculation, 896

collection and sterilization of the pulp, 897

human-calf-rabbit seed virus, 896

method of testing, 897-898

" retro-vaceination," 896

"'seed virus," 896

Smegma bacillus, 618

identification of, 619

morphology of, 618

occurrence of, 618

Smith fermentation tube, 196

Soil, bacteria in, 1012

numerical estimation of, 1015

pathogenic bacteria in, 1013

persistence of after the burial of

infected cadavers, 1014

Specific gravity of bacteria, 26

Specimens from patients, methods of obtaining, 206, 207

Spinal fluid, examination of, 208-210

in acute meningitis, 209, 528, 529

in poliomyelitis, 209

in syphilis, 209

in tuberculosis, 209

Spiral organisms, classification of, 847

Spirillaceæ, definition of, 42

Spirillum, description of, 9

genus, 42

Spirillum choleræ asiaticæ, 831. See also Cholera
S. Deneke, 845
S. Finkler-Prior, 845 ,
S. Massaua, 844
S. Metchnikovi, 844 
Spirillum Milleri in the normal mouth, 219

Spirochæta anserina, 872

Spirochæta calligyrum, 873

Spirochæta Duttoni, 866

Spirochæta gallinarum, 871

Spirochrte, genus, 42

anaërobic tissues tubes for cultivation of, 167

Spirochæte Ieterohaemorrhagiæ, 885-888 causative agent of Weil's disease, 886 eultivation of, 886-887

Spirochæte macrodentium, 873

microdentium, 873

Spirochæte of Obermeier, 861

Spirochæta pallida, 849-861

animal pathogenicity, 857

cultivation of, $856-857$

demonstration of, 852-855

Wassermann reaction with, 866

Spirochæta pertenue, 870

Spirochæta phagedenis, 872

Spirochæta refringens, 846,849

Spirochætes, diseases 'caused by, 846

Rat-bite fever, 873

relapsing fever, 861

syphilis, 849

Vincent's angina, 867

Yaws, 867

Spironema, 848

Vincenti in the normal mouth, 219

Spontaneous generation, 4, 5, 6

Spores, 16

conditions necessary for formation, 16

destruction of by boiling, 83

destruction of by fractional sterilization, 84

destruction of by steam under pressure, 85

discovery of, 6

germination of, 18

loss of ability to form spores, 37

position of, 17

resistance to temperature changes, 36

not related to arthrospores, 12,16

special stain for, 116

Sporothroichosis, 992
Spirosoma, genus, 42

Sporotrichum (sporothrix), 993

minutissimum, 1009

Spine, 991

Sputum, examination of, 215

collection of, 215

for pneumococcus typing, 215

for tuberculosis examination, 215

Sputum, Petroff's method of isolating

tubercle bacilli from, 591

methods of concentrating tubercle bacilli in, 589

antiformin, 589

Staining of bacteria, 113

acid-fast, 124

Baumgarten's, 125

Ehrlich's, 124

Gabbet's, 124

Hermann's, 126

Pappenheim 's, 125

Zichl-Neilson, 124

capsule stains, 116

Buerger, 117

Hiss, 116

Huntoon, 116

Wadsworth's for smear and section, 118

Welchi, 116

differential stains, Gram's method, 121

classification by, 123

discussion of chemical basis for, 120

Jensen's modification, 122

Paltauf's modification, 121

Sterling's modification, 122

discussion of chemical principles underlying, 114

flagella stains, 118

van Ermengen's, 119

Loeffler's, 119

Smith's modification of Pitfield'g method, 119

in tissues, 129

Gram-Weigert, 130

Loeffler's, 129

Mallory's eosin methylene blue, 131 method for staining acitomyces, 132 
Staining of bacteria, in tissues, Pappenheimer's method for staining Gram positive bacteria, 131

polychrome stains, 127

Giemsa, 127

Jenner, 127

Wood's, 128

Wright's modification of Leishman 's, 127

special stains for polar hodies, 126

earbol thionin, 126

Neisser, 126

toluidin blue, 126

spore stains, 116

Abbott's, 116

Moeller's, 116

stains in common use, 115

earbol-fuchsin, 115

Loeffler's alkaline methylene blue, 115

Pappenheim-Saathof methyl green, 115

toluidin blue, 115

Staphylococcus epidermidis albus, 397.

Staphylococeus pyogenes albus, 397

Staphylococeus pyogenes aureus, 384397

agglutinins in immune serum, 396

cultural characteristies, 385

immunization against, 396

active, 396

passive, 397

in the mouth, 217

in the nose, 221

morphology, 384

pathogenicity, 390

in animals, 390

in man, 391, 392

impetigo contagiosum, 392

osteomyelitis, 392

paronychia, 391

pigment formation, 388

resistance of, 388

staining, 384

thermal death point, 388

toxic products, 393

hemolysins, 393

thermolability of, 394
Staphylococcus pyogenes aureus, toxic products, leucocidin, 392

thermolability of, 395

differentiation from leucotoxin, 395

virulence of, 389

Staphlycococeus pyogenous citreus, 397

Sterigmocystis, 983

Stomxys, flies in poliomyelitis transmission, 918

Strangles, 415

Strauss test for diagnosis of glanders, 791

Streak plate, technique of, 178

Streptothrix, 962. See also Nocardia, 964, and Actinomyces, 965

genus, 42

in the normal mouth, 220

of Israeli, 965

of Rosenbach, 965

Steam, in the destruction of bacteria, 80 live, 83

saturated, 82

"superheated," 82

under pressure, 85

Stegomyia fasciata or calopas, 877,884

habits of, $883-884$

Sterling's modification of Gram 's stain, 122

Sterilization, fractional, 84

practical methods, 82

" Arnold"' sterilizer, 83

autoclave, 85

boiling, 83

burning, 82

hot air, 82

Streptococcus erysipelatis, 416

genus, 41

Streptococeus hemolyticus (Beta type), 408. See also Streptococcus pyogenes below

as secondary invaders in small pox and diphtheria

grouping by agglutination, 412, 413

hemolysin production by, 426

thermolability of, 427

hemogeneity of group as determined by complement fixation, 411,412 
Streptococcus hemolyticus, in bronchopneumonia following influenza, 419 in bronchopneumonia following measles, 419

in epidemic sore throat, 421

in puerperal sepsis, 417

in scarlet fever, 419

interference of with suture of wounds, 766

septicæmia caused by, 418

tonsilitis caused by, 418

toxic substances produced by, 425

Streptococcüs mucosus (pneumococcus mucosus, Type III), 403, see also 448

Streptococeus pyogenes (streptococcus

hemolyticus and viridans), 401-445

agglutinin production by, 433

technique of agglutination with spontaneously agglutinating strains, 433, 434

antibody production, 429, 430

antistreptococcic sera, 430,434 standardization of by protection experiments, 431

capsule formation by, 403

elassification, 407

by fermentation reactions, 409, 410

Type Alpha, 408

Type Beta, 408

Type Gamma, 408

eultivation, 404, 405

differentiation from pneumococcus, 450

in the mouth, 217

in the nose, 221

leucocidin production by, 429

morphology, 402

pathogenicity, 413

nature of lesions in animals, 414

nature of lesions in man, 415-423

preservation of, 407

resistance of, 406

to chemicals, 407

staining, 402, 403

thermal death point, 406

variations in size, 403

virulence of, 413,414
Streptococcus viridans (Alpha type), 408, 409. See also Streptococeus pyogenes

heterogenity of group, 411, 412

in endocarditis, 422, 423

relation to chorea, 423

relation to rheumatic fever, 422,423 relation to poliomyelitis, 423

tissue specificity of, 424

Strong bacillus in bacillary dysentary, 704,707

Substances of unnkown eomposition in bacterial nutrition, 32

Sulphur dioxide as a fumigating agent, 106

Sulphur bacteria, 67

Surra, 1080

Symbiosis of bacteria, 35

Symptomatic anthrax, bacillus of, 758 772

cultivation, 770

differentiation from vibrion septique, 759

immunity, 771

morphology, 769

pathogenicity, 770

staining, 769

toxins, 771

vaccines, 772

Syphilis, 849-861

spirochæta pallida in, 850

animal pathogenicity, 857

cultivation of, 856,857

demonstration of, 852,855

dark field, 853

in smear, 853-855

immunization with, 859

Wassermann reaction with, 866

TARBardillo, 934, 937

Temperature, relation of, to bacteria, 36-39

arlaptation to, 37

effect of changes in, 37

effect of low temperatures on bacteria, 39

resistance of spores, 38 
Temperature, relation of, to bacteria, thermal death point of non-spore bearers, 38

Terchloride of iodin, 93

Tertian fever, 1106 clinical description, 1115-1117

Tetanus, bacillus of, 727-740 antitoxin, 736-740

immunization of horses, 274, 275

prophylactic use of, 736

standardization of, 276

$\mathrm{L}+$ dose of toxin, 276

therapeutic use of, 738

administration of, 740

biological considerations, 729

distribution, 728

morphology of, 727

pathogenicity of, 734

acute form of, 735

"chronic" form of, 735

incubation period after infection,

735

symptoms in man, 735,736

atypical cases, 726

spores, latency of, 735

resistance of, 736

staining of, 727

tetanolysin, 734

toxin, 731

action of, 723

incubation period with, 733

L+ dose, 276

medium for the production of, 731

M. L. D. for guinea pigs, 275

M. L. D. for mice, 274,732

precipitation of, 731,732

thermolability of, 732

destruction of by cosin, 732

types of, 737

relation to toxin production, 738

Tetramitis mesnili, 1074

Theileria parva, 1131

Thermoregulators, 193

Thiothrix, genus, 42

Thread reaction of $P$ faundler, 283

Throat, smears and examinations, 216

for diphtheria, 216

for Vincent's angina, 216
Thrush, 989

Timothy bacillus, 617

Tinea or ringworm, 1003-1008

Tinea vervicolor, 1009

Tissue in culture media, reducing action of, 189

Tissues, latency of bacteria in, 222, 223

Tissue specificity, discussion of, 424

Titration, 136

colorimetric method, 138

actual steps in, 142

"'buffer," definition of, 138

indicators for various ranges of $\mathrm{Ph}$, 141

preparation of color standards

$\mathrm{Ph}$, meaning of, 138, 139

comparator, for reading, 142

phenolphthalein, old method, 136

Toluidin blue stain for polar bodies, 115

Tonsilitis, streptococcus in, 418

Toxin antitoxin reaction, nature of, 255 268

analogy to colloidal reactions, 265

Arrheinus and Madsen, theories concerning, 264, 265

Ehrlich's analysis of, 257-263

side chain theory, 266-268

Toxin, definition of, 234

Toxin, diphtheria, 571-574

antitoxin reaction, theoretical considerations, 255-268

chemical and physical properties of, 573

Ehrlich's analysis of, 257-263

deterioration of, 258

epetoxoid or toxin, definition of, 261

$\mathrm{L}+$ dose, 260

$\mathrm{L}_{0}$ dose, 259

standardization, 258

toxoid, 259

unit of, 257

method of production, 571

thermolability, 572

Toxin, tetanus, 731-733

action of, 733

incubation period with, 733

L+ doses, 276

medium for the pioduction of, 731 
Toxin, tetanus, M. L. D. for guinea pigs, 274,732

M. L. D. for mice, 274

precipitation of, 731,732

thermolability of, 732

destruction of by eosin, 732

Trachoma, influenza bacillus in, 500, 501

Transferring cultures, technique of, 173

Trench fever, 946-950

elinical description, 947

transmission and etiology, 947-950

incubation period of louse transmitted cases, 948

louse transmission, 948, 949

Rickettsia bodies in, 949

virus contained in the blood and urine, 948

Treponema, 848

Treponema macrodentium, 849

occurrence of in dirty mouths, 219

Treponema pallidum, 851-861. See also Spirochæta pallida

as an antigen for the Wassermann test, 317,860

Trichomonas, 1073

vaginalis, 1073

intestinalis, 1073

Trichomycetes, definition of, 961

classification of, 962

Trichophyton, 1005

Trichophyton eruris, 1007

Tricresol, 95

Trillat autoclave for the generation of formaldehyde, 107

Triphenylmethane dyes as disinfectants, 97

Trypanosoma brucei, 1081

cultivation of, 1083

morphology of, 1081

Nagana eaused by, 1081

transmission of, 1082

Trypanosoma eruzi, 1091

Trypanosoma equiperdum, 1084 complement fixation for diagnosis, 1085
Trypanosoma evansi, 1080

infection in horses, mules, etc., 1080

transmission of, 1080

Trypanosoma gambiense (sleeping sick-

ness), 1086

clinical signs, 1088

etiology, 1088

transmission by insects, 1088

morphology, 1089

pathogenicity, 1089

Trypanosoma hippicum, 1083

morphology, 1083

pathology, 1083

Trypanosoma lewisi, 1079

insect hosts of, 1080

morphology of, 1079

multiplication in rats, 1080

Trypanosoma rhodosiense, 1090

diagnosis, 1090

prophylaxis, 1091

treatment, 1091

Trypanosoma rotatorium, 1078

Trypanosoma valenti, 1076

Trypanosomidæ, 1076

Tubercle bacillus (avian), 614

Tubercle bacillus (bovine type), 612614

differentiation of from human type, 514

Tubercle bacillus (human type), 586612

bacilli elosely related to, 612

bacillus of bovine tuberculosis, 612 614

cultural differentiation from human type, 613 .

differentiation by virulence, 613 summary of differentiation between bovine and human type, 614

bacillus of avian tubereulosis, 614 bacillus of tuberculosis in coldblooded animals, 615

immunization with, 616

bacillus, timothy, 617

bacillus butyricus (Butter bacillus), 617

biological eonsiderations, 593 
Tubercle bacillus (human type), chemical analysis of tubercle bacillus, 603 complement fixation with, 608 cultivation of, 590-593

Hesse's medium for, 593

discovery of, 586

frequency of, 597

immunization, passive, 611

Maragliano's serum, 612

Marmorek's serum, 612

isolation of, 590

from feces, 212

Petroff's method for, from sputum, 591

milk in the transmission of, 598 morphology of, 586

pathogenicity, 593-597

for animals (human type), 597

for animals, human and bovine

type compared, 613, 614

lesions produced by, 595, 596

manner of invasion, 594, 595

occurrence of in blood, 596

tubercles, description of, 593

prevention of, 602,603

sputum, method of concentrating tu-

bercle bacilli in, 589

staining, 587-589

antiformin for concentrating tu-

bercle bacilli in sputum, 589

Gabbett's method, 588, 124

Herman's method, 588, 126

in sections, 132

Much's method for Much's granules, 588

Weiss' modification of, 589

Pappenheim's stain for differentia-

tion from smegma bacillus, 589, 125

susceptibility to bovine and human types, 600

thermal death point, 593

toxins produced by, 604. See also

Tubereulins

transmission, 597

by butter, 1042, 1044

by dust, 601

by milk, 598
Tubercle bacillus (human type), transmission, inheritance of, 602 predisposing factors, 601

tuberculins, 604

bouillon filtre (Denys), 606

diagnostic use of, 607

eutaneous tuberculin reaction, 608

on cattle, 609,610

- ophthalmo-tuberculin reactions, 607

subcutaneous, 607

new, 605

new tuberculin-bacillary emulsion, 606

old, 604

reaction to, by lepers, 625

therapeutic uses of, 610

Tubercle bacillus in cold-blooded animals, 615

immunization with, 616

Tuberculins, diagnostic use of, 607

for testing cattle, 1037

preparation of, 604-606

therapeutic use of, 610

Typhoid bacillus of, 643-685

antibodies produced by, 659

agglutinins, 662

Widal reaction, 663-667, 282, 302

Dryer's method, 666

method of obtaining patient's serum, 665

serum dilutions used, 663

bactericidal and bacteriolytic, 661 opsonins, 667

precipitins, 667

biological considerations, 648

blood cultures for, 214, 651

carriers, 669-675

chronic, 670-675

gall-bladder carriers, 671

" "healthy" carriers, 673

intestinal carriers, 672

liver duct earriers, 671

urinary carriers, 672

pathological lesions caused by carrier state, 674 
Typhoid, bacillus of, carriers, stool examinations for the detection of, 653

temporary carriers, 670

treatment of, 675

characteristics of, 648

cultivation of, 643-648

differential media for, 647, 158-165

brilliant green agar (Krumwiede), 161

brilliant green eosin agar ('Teague), 162

Conradi-Drigalski medium, 158

Endo's medium, 159

discovery of, 643,8

fermentations of, 646,648 , chart of, 718

cultural differences within typhoid group, 648

immunity, active, 659

after one attack, 660

method of immunizing animals, 659

vaccination, $680-684$

duration, 684

lipovaceine, 683

sensitized typhoid vaccine, 683

isolation of from feces, 653

morphology of, 643

pathogenicity of, 649-656

for animals, 649-650.

method of inducing carrier state

in animals, 650

suppurative lesions due to typhoid bacillus, 655

typhoid fever in man, 650-656

septicæmia in, 650

media for isolating typhoid

from blood, 651

typhoid bacilli in the rose spots, 655

typhoid bacilli in the sputum, 655 typhoid bacilli in the stool, 652

typhoid fever without intestinal lesions, 656

poisons of, 656,659

endotoxins, method of obtaining, 657

prevention of, 680
Typhoid, bacillus of, sanitary cousiderations, 668-680

transmission of, 676

by contact infection, 679

by flies, 679

by milk, 678

by oysters, 1047

by water, 677

isolation of typhoid from, 1023

specific treatment of typhoid fever, 684

staining, 643

thermal death point, 648

Typhi Murium, bacillus of, 691,688

Typhus fever, 934-945

clinical description, 935, 936

differential diagnosis from purpura

hemorrhagica, 935

epidemiology, 936

Serbian epidemic, 938, 939

transmission by lice, 937,938

etiology, 940

Plotz bacillus, discussion of, 941 , 942, 944

probably not due to a filtrable virus, 940

Rickettsia bodies in, 942, 943, 944

Rickettsia prowazeki, 944

immunity in, 945

incubation period, 935

prevention of, 944

transmission to guinea pigs, 940

transmission to monkeys, 939

Weil-Felix reaction in diagnosis of, 945

Tryamin, produced in bacterial cultures, 237

Tyrotoxismus, 51

Urobacillus liquefaciens septicus, 641

Urine, examination of, 210

Urticaria, 370

U. S. Public Health Service, method of testing disinfectants, 99

VACCINATION, small-pox, 898-899

Vaccination, typhoid bacillus, 680-684 
Vaccine production (small-pox), 894 calf inoculation, 896 collection and sterilization of pulp, 897

human-calf-rabbit seed virus, 896

method of testing, 897-898

"retrovacination," 896

"'seed virus," 896

Vaceine therapy (Wright), 342

autogenous, 342

dosage in staphylococeus infection, 344

method of counting, 343

negative phase in, 344

positive phase in, 344

production of, 342

Vaceinia, or cowpox, relation to smallpox, 894

Vanillin test for indol, 199

Variola or small pox, 890-899. See Smallpox

Varicella or chicken pox distinct from smallpox, 894

Venereal diseases, Public Health management of, 556

Vibrion septique, 756

antitoxin, 758

diseovery of, 756

differentiation from symptomatic anthrax, 759

method of identifying, 753

occurrence of in war wounds, 756

pathogenicity of, 738

toxin production, 757

types of, 757

Vincent's Angina, 867-869

fusiform bacilli, in, 868

in throat smears, 216

spirilla in, 868

Vinegar, making of, 57

Virulence,

relation to eapsulation, 14

loss of due to cultivation at high temperatures, 39

definition of, 232

problem of, 348

Bail's aggressin theory, 349

arguments against, 350
Virulence, problem of, Bail's agressin

theory, relation to anaphylatoxin, 351

"Virus fixe" in rabies therapy, 901

Vitamines in bacterial nutrition, 33

Vulvovaginitis due to gonococcus, 556

WADSWORTH eapsule stain for smear and sections, 118

Wassermann test for syphilis, 317

actual test, 322

antigen, 317

alcoholic extract of heart muscle, 318

cholestrinized, 318

Noguchi's acetone insoluble lipoid, 318

spirocheta pallida, 860

complement in, 321

hemolytic serum, $319-320$

production of, 319

titration of, 320

unit of, 320

modifications of, 323

serum to be tested for syphilitic anti-

body, 321

sheep corpuscles, 321

Water, bacteria in, 1016

pathogenic bacteria in, 1016, 1017

in surface water, 1017

in rain and snow, 1017

in "ground" water, 1018, 1019

quantitative estimation of, 1019, 1020

standard medium for, 1020

procedure, 1021

expression of results, 1021

qualitative estimation, 1021

typhoid isolation from, 1022

Weil's disease, 885-888

clinical description, 885-886

etiology, spirochæta icterohæmor-

rhagiæ, 886

eultivation of, 886,887

prevention, 888

serum treatment, 888

transmission, 887

Weil-Felix reaction, non specificity of, 942 
Weil-Felix reaction, in the diagnosis of typhus fever, 945,946

Welch bacillus, 751-756. See also B. Welchii

Welch's eapsule stain, 116

Welch and Nuttall, method of rabbit inoculation for the isolation of anaërobes, 211

West tube in taking nasopharyngeal cultures, 542

Whooping eough, bacillus of, 504-508 antibody production by, 508 cultivation of, 506

epidemiology, 504, 505

pathogenicity, 507

morphology of, 505

staining of, 506

Widal reaction, 282 method of performing, 302

Winckel's disease, 633

Wolbach's table of disease caused by filtrable viruses, 891

Woolsorter's disease, 782

Wolfhugel counting plate, 195

Wohlhynin fever, 946. See also Trench fever

Wood's polychrone stain, 128

Wood tieks in the transmission of Rocky Mountain Spotted fever, 952

Wounds, bacteriological eontrol of, 763 . 768 anaërobic bacilli found in, 749-763 cultural examination, 766 serological treatment, 768 smear method, Carrel, 754 surgical considerations, 767

Wright's method of counting bacteria, 195
Wright's stain, 127

Wright's technique of the opsonic test, 339

vaceine therapy, 341

dose in staphylococeus infections, 344

method of eounting bacteria, 343

negativ phase, 344

positive phase, 344

" $\mathrm{X}$ " substances, bacterial poisons, 237

Xerosis bacillus, 584

YAWS, 870

" $\mathrm{Y}$ ', bacillus in bacillary dysentery, 704,707

Yeasts, 980

diseases caused by, 984, 985

in aleoholic fermentation, 981

in the normal nose, 221

other yeast-like parasites, 992

Yellow fever, 874-884

etiology, 879-883

early elaims, 880

Noguchi's work, $881-883$

distribution of, 874

immunity in, 884

prevention of, 883

transmission of, 874-878

ZIEHL-NEILSON stain for the tubercle bacillus, 124

Zur Nedden's bacillus, 509

Zymase, enzyme of aleoholic fermentation, 58, 981

Zymonema Gilehristi, 985 


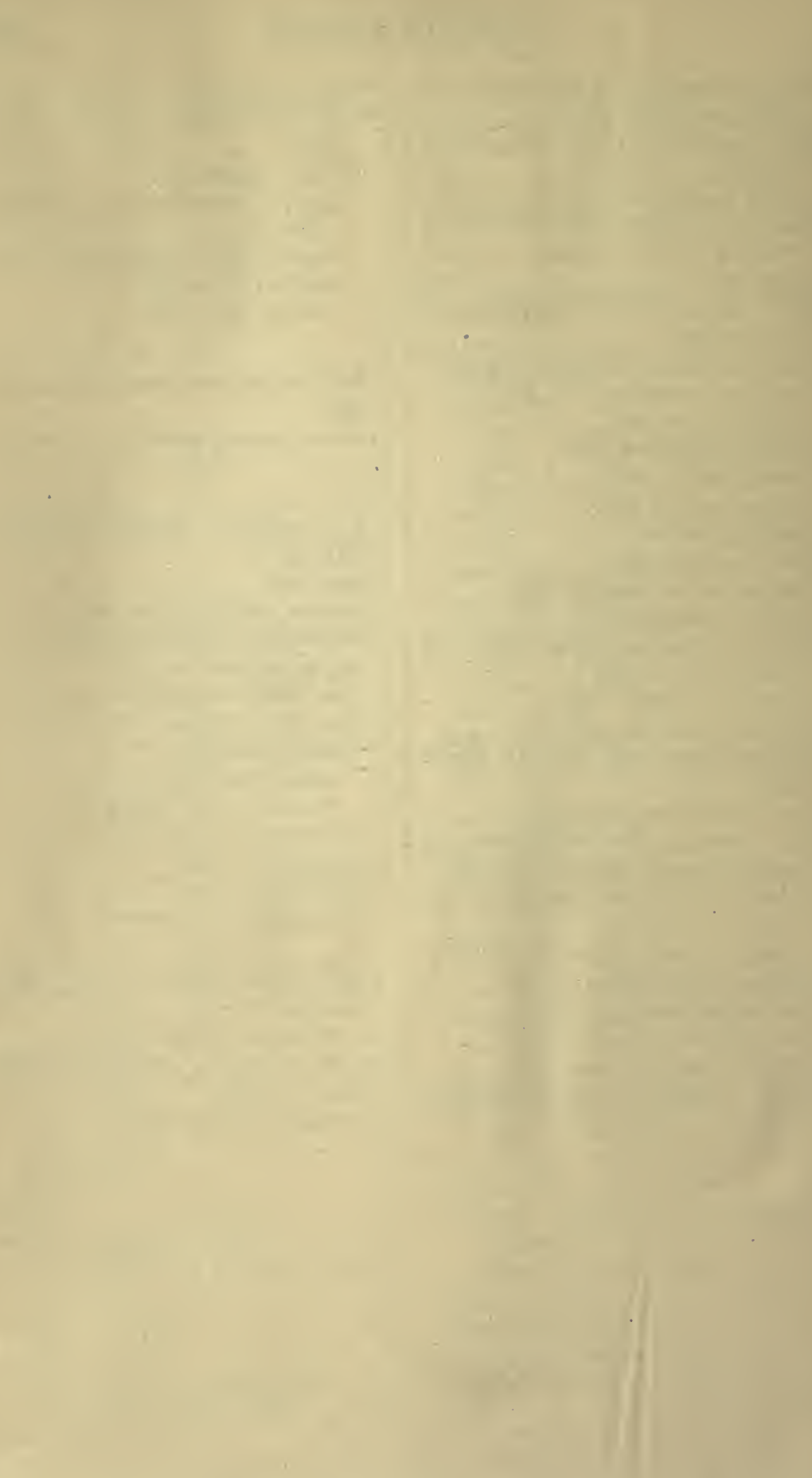





\section{THIS BOOK IS DUE ON THE LAST DATE STAMPED BELOW}

\section{AN INITIAL FINE OF 25 CENTS}

WILL BE ASSESSED FOR FAILURE TO RETURN THIS BOOK ON THE DATE DUE. THE PENALTY WILL INCREASE TO 50 CENTS ON THE FOURTH DAY AND TO \$1.00 ON THE SEVENTH DAY OVERDUE.

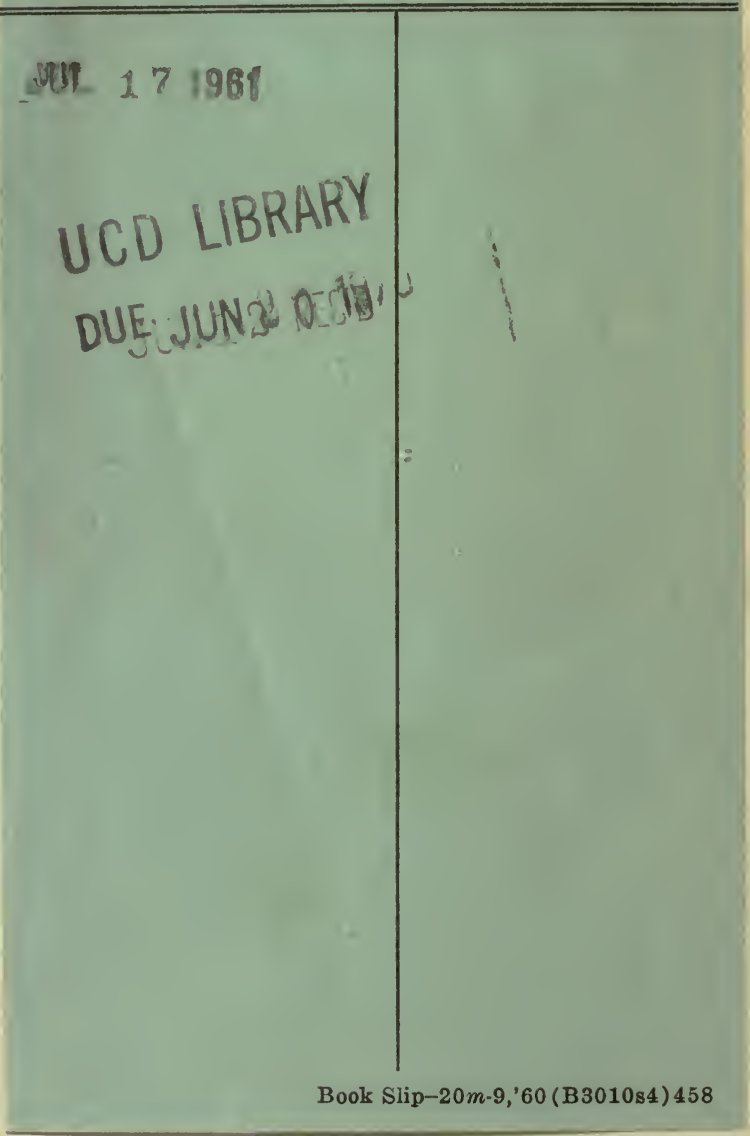




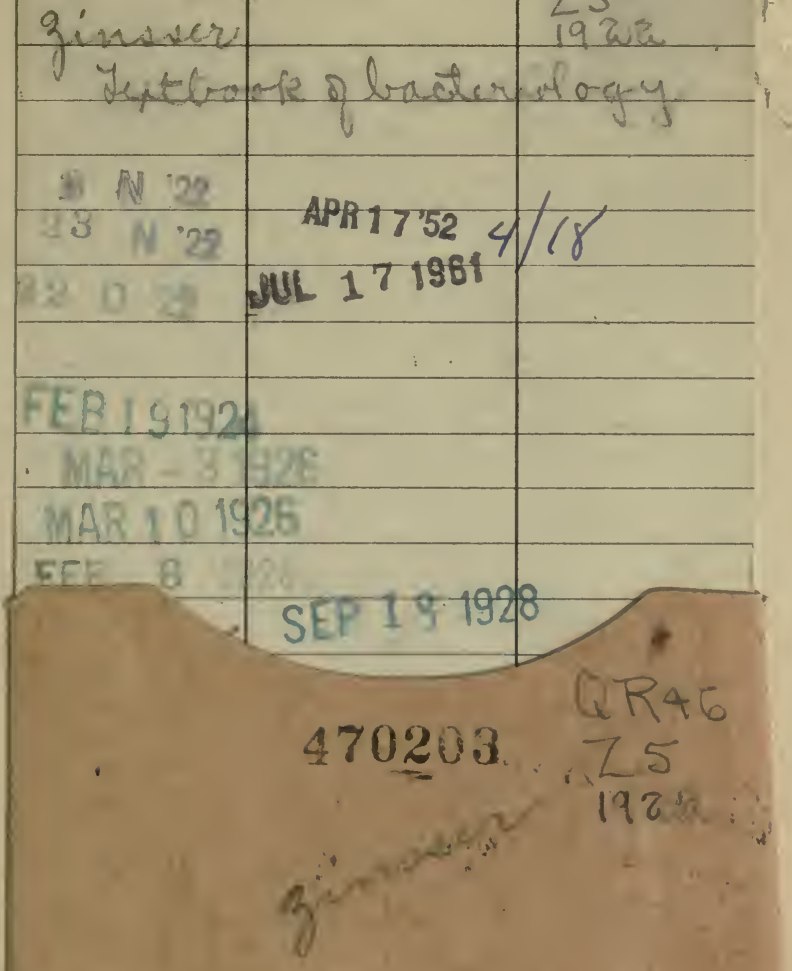

UNIVERSITY OF CALIFORNIA LIBRARY 
